\title{
$\mathbf{J} \mathbf{A} \mathbf{C} \mid \mathbf{S}$
}

Supporting information for

\section{Hydrogen Bonding Phase-Transfer Catalysis with Ionic Reactants: Enantioselective Synthesis of $\gamma$-Fluoroamines}

Giulia Roagna, ${ }^{\S}, \dagger$ David M. H. Ascough,${ }^{\S} \dagger$ Francesco Ibba,${ }^{\dagger}$ Anna Chiara Vicini,,${ }^{\dagger}$ Alberto Fontana,,${ }^{\dagger}$ Kirsten

E. Christensen, ${ }^{\dagger}$ Aldo Peschiulli, $\|$ Daniel Oehlrich, $\|$ Antonio Misale, ${ }^{\ddagger}$ Andrés A.Trabanco, ${ }^{\ddagger}$ Robert S. Paton, ${ }^{\dagger}, f$ Gabriele Pupo, ${ }^{\dagger^{*}}$ and Véronique Gouverneur ${ }^{{ }^{* *}}$

${ }^{\dagger}$ University of Oxford, Chemistry Research Laboratory, 12 Mansfield Road, Oxford, OX1 3TA, UK

* Janssen Cilag Research and Development, Calle Jarama 75A, Toledo 45007, Spain

$\|_{\text {Discovery Sciences Medicinal Chemistry, Janssen Research \& Development, Janssen Pharmaceutica N.V., }}$

Turnhoutseweg 30, Beerse B-2340, Belgium

${ }^{f}$ Department of Chemistry, Colorado State University, Fort Collins, CO 80523, USA

$\S$ These authors contributed equally to this work.

To whom correspondence should be addressed: gabriele.pupo@chem.ox.ac.uk and veronique.gouverneur@chem.ox.ac.uk. 


\section{$\underline{\text { Table of Contents }}$}

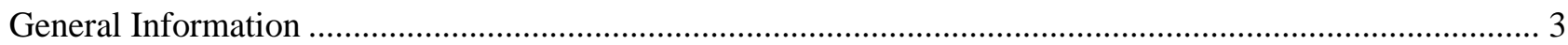

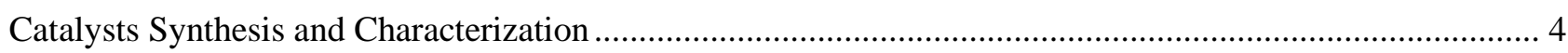

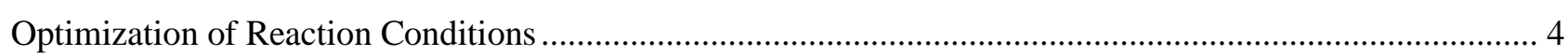

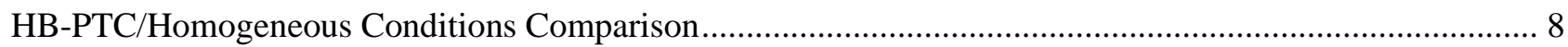

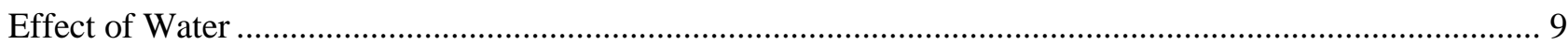

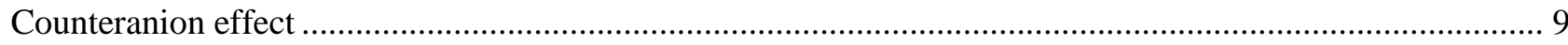

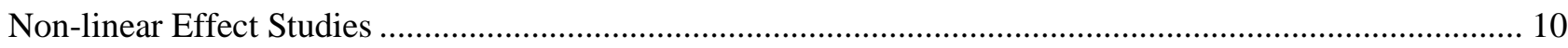

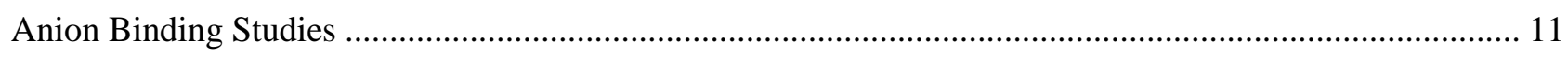

Fluorination under Chiral Anion Phase Transfer (CAPT) …....................................................................... 16

General Procedure for the Asymmetric Nucleophilic Fluorination of Azetidinium Ions................................. 17

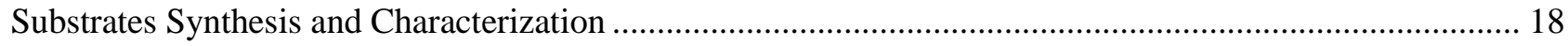

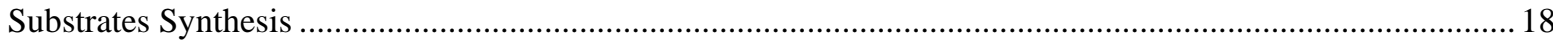

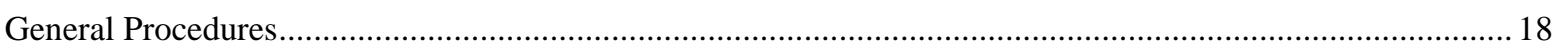

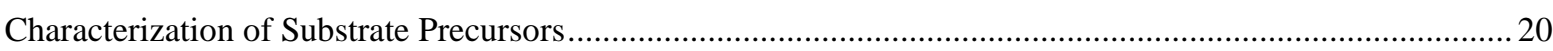

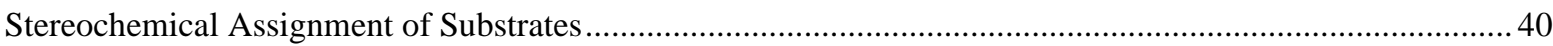

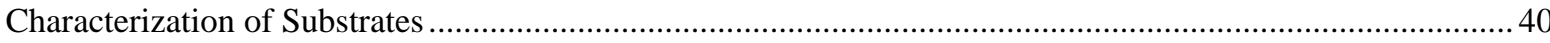

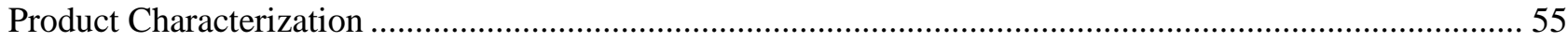

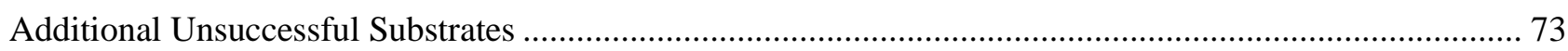

Determination of the Absolute Configuration (Single Crystal X-ray Diffraction Studies) ............................ 74

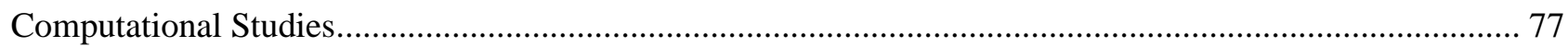

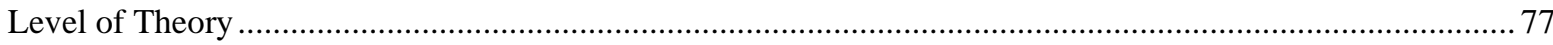

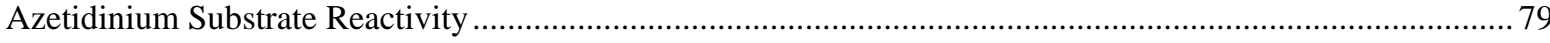

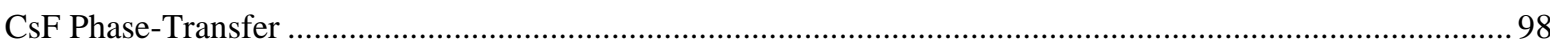

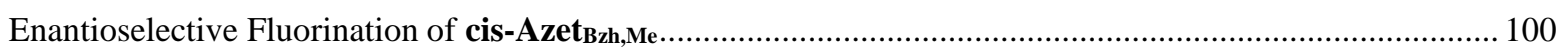

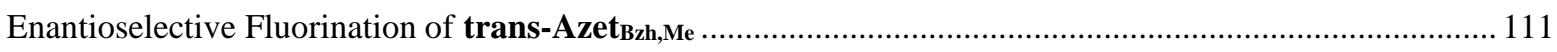

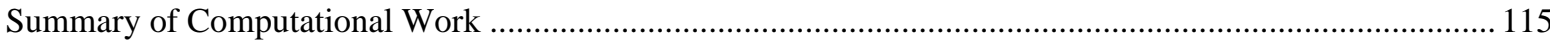

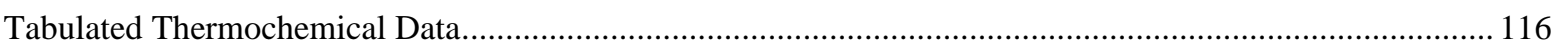

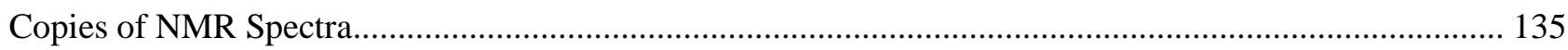

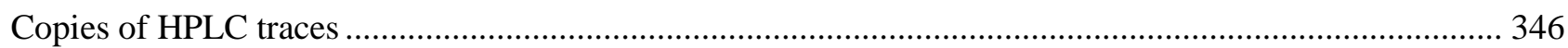

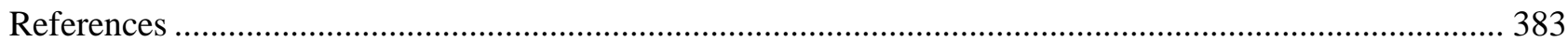




\section{General Information}

Unless otherwise stated, all reagents were purchased from commercial suppliers (Sigma-Aldrich, Alfa Aesar, Fluorochem, Apollo Scientific and CombiBlock) and used without further purification. Unless otherwise stated, solvents were used without prior drying/degassing. Reactions requiring anhydrous conditions are clearly stated and were conducted after flame-drying of the appropriate reaction vessel (round-bottom twoneck flasks, Schlenks or microwave vials) and under an inert atmosphere of $\mathrm{N}_{2}$. Dry solvents were purchased from commercial suppliers. The dry 1,2-dichloroethane (DCE) employed in the fluorination was purchased from Sigma-Aldrich (99.8\%, anhydrous). CsF (99.9\% trace metal basis from Sigma-Aldrich) was ground prior to the reaction and used without pre-drying. KF (99.9\% trace metal basis from Alfa Aesar) was used as provided by the supplier (fine powder) and used without pre-drying. Reactions were monitored by thin layer chromatography (TLC) on silica gel pre-coated aluminium sheets (Merck Kieselgel $60 \mathrm{~F}_{254}$ plates). Visualization was accomplished by irradiation with UV light at $254 \mathrm{~nm}$, and/or phosphomolybdic acid (PMA) stain, and/or cerium ammonium molybdate (CAM) stain, and/or permanganate stain. Flash column chromatography (FCC) was performed on Merck silica gel (60, particle size 0.040-0.063 mm). Optical rotations were measured on an Autopol L 2000 (Schmidt-Haensh) or a Rudolf Autopol V plus at $589 \mathrm{~nm}$, $25{ }^{\circ} \mathrm{C}$. Data are reported as: $[\alpha]_{\mathrm{D}}{ }^{\mathrm{T}}$, concentration $(\mathrm{c}$ in $\mathrm{g} / 100 \mathrm{~mL}$ ), and solvent. The absolute configuration was determined by $\mathrm{X}$-ray analysis of compounds $(S)$-2 ma $\cdot \mathrm{HCl}$ and $(R)-\mathbf{3 a b}$. The configuration of the other products was assigned by analogy. All NMR spectra were recorded on Bruker AVIIIHD 400, AVIIIHD 500 or VII 500. ${ }^{1} \mathrm{H}$ and ${ }^{13} \mathrm{C}$ NMR spectral data are reported as chemical shifts $(\delta)$ in parts per million (ppm) relative to the solvent peak using the Bruker internal referencing procedure (edlock). ${ }^{19} \mathrm{~F}$ NMR spectra are referenced relative to $\mathrm{CFCl}_{3}$. Data are reported as follows: chemical shift, multiplicity $(\mathrm{s}=$ singlet, $\mathrm{d}=$ doublet, $\mathrm{t}=$ triplet, $\mathrm{q}=$ quartet, pent $=$ pentet, sept $=$ septet, $\mathrm{br}=$ broad, $\mathrm{m}=$ multiplet $)$, coupling constants $(\mathrm{Hz})$ and integration. $\mathrm{NMR}$ spectra were processed with MestReNova 11.0 or Topsin 3.5. High resolution mass spectra (HRMS, $m / z$ ) were recorded on a Thermo Exactive mass spectrometer equipped with Waters Acquity liquid chromatography system using the heated electrospray (HESI-II) probe for positive electrospray ionization $\left(\mathrm{ESI}^{+}\right)$. Infrared spectra were recorded as the neat compound or in solution using a Bruker tensor 27 FT-IR spectrometer. Absorptions are reported in wavenumber $\left(\mathrm{cm}^{-1}\right)$. Melting points of solids were measured on a Griffin apparatus and are uncorrected. The enantiomeric ratios were determined by HPLC analysis on a Shimadzu $i$-Prominence LC-2030 (PDA detector) or SFC analysis on an Agilent Aurora SFC-1260 Infinity system (DAD detector) or a Waters SFC UPC2 (PDA and QDa MS detector), employing a chiral stationary phase column specified in the individual experiment by comparing the samples with the appropriate racemic mixtures. 


\section{Catalysts Synthesis and Characterization}

Catalyst A-D were prepared according to literature procedures and their analytical data were in agreement with the literature values. ${ }^{1}$

\section{Optimization of Reaction Conditions}

General reaction conditions: $0.05 \mathrm{mmol}$ of $\mathbf{1}, \mathrm{CsF}$ and the organocatalyst were stirred in the appropriate solvent at $900 \mathrm{rpm}$ at r.t. Yields determined by ${ }^{19} \mathrm{~F}$ NMR using 4-fluoroanisole as internal standard; e.r. = enantiomeric ratio, determined by HPLC using a chiral stationary phase.

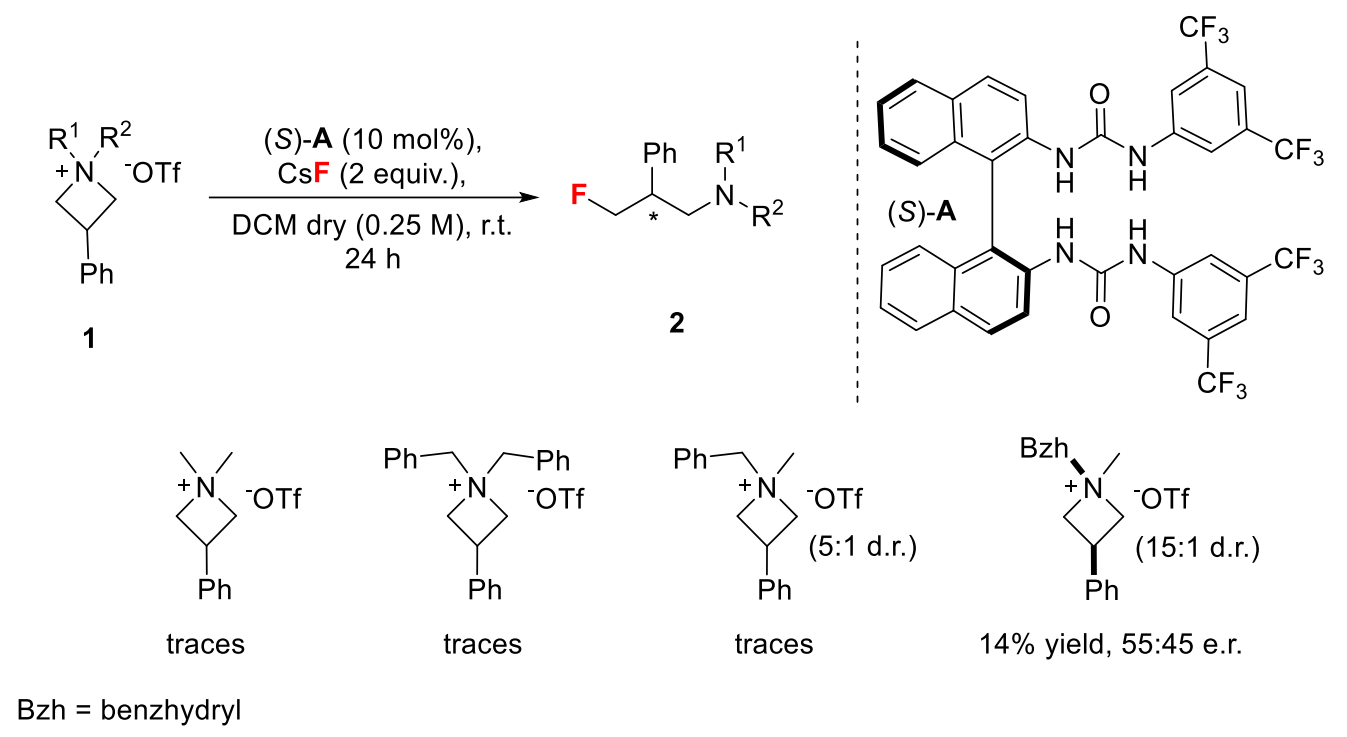

Scheme S1: Preliminary N-protecting groups screening

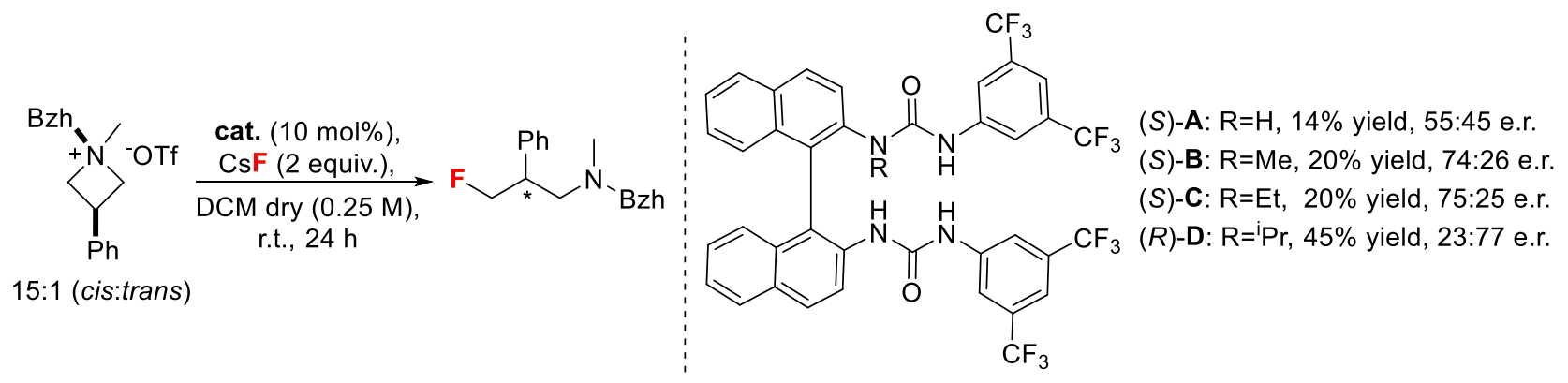

Scheme S2: Catalyst Screening 
Table S1: Solvent Screening (using catalyst $(R)-D)$

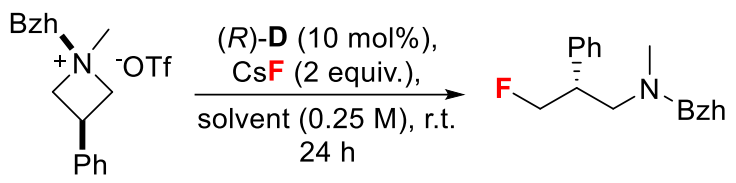

$15: 1$ (cis:trans)<smiles>O=C(Nc1cc(C(F)(F)F)cc(C(F)(F)F)c1)Nc1ccc2ccccc2c1-c1c(NC(=O)Nc2cc(C(F)(F)F)cc(C(F)(F)F)c2)ccc2ccccc12</smiles>

\begin{tabular}{ccccc}
\hline Entry & Solvent & NMR Yield & e.r. & Background reactivity $^{[\mathbf{1}]}$ \\
\hline $\mathbf{1}$ & $\mathrm{CH}_{2} \mathrm{Cl}_{2}(\mathrm{dry})$ & $45 \%$ & $77: 23$ & $6 \%$ \\
$\mathbf{2}$ & $\mathrm{CH}_{2} \mathrm{Cl}_{2}$ & $34 \%$ & $76: 24$ & $6 \%$ \\
$\mathbf{3}$ & $\mathrm{CHCl}_{3}$ & $56 \%$ & $67: 33$ & $33 \%$ \\
$\mathbf{4}^{[2]}$ & $1,2-\mathrm{DCE}(\mathrm{dry})$ & $55 \%$ & $81: 19$ & $8 \%$ \\
$\mathbf{5}^{[3]}$ & $1,2-\mathrm{DCE}(\mathrm{dry})$ & $51 \%$ & $81: 19$ & -- \\
$\mathbf{6}$ & $1,2-\mathrm{DCE}$ & $37 \%$ & $81: 19$ & $8 \%$ \\
\hline $\mathbf{7}$ & Toluene & $51 \%$ & $79: 21$ & $4 \%$ \\
$\mathbf{8}$ & $1,2-\mathrm{DFB}$ & $47 \%$ & $79: 21$ & $12 \%$ \\
$\mathbf{9}$ & $\alpha, \alpha, \alpha-$ trifluorotoluene (dry) & $69 \%$ & $78: 22$ & $0 \%$ \\
$\mathbf{1 0}$ & Fluorobenzene & $63 \%$ & $76.5: 23.5$ & $0 \%$ \\
\hline \hline $\mathbf{1 1}$ & $\mathrm{CH}{ }_{3} \mathrm{CN}$ & $4 \%$ & $61.5: 38.5$ & traces \\
$\mathbf{1 2}$ & EtOAc & $9 \%$ & $77.5: 22.5$ & $0 \%$
\end{tabular}

${ }^{[1]}$ Background reactivity $={ }^{19} \mathrm{~F}-\mathrm{NMR}$ yield of the corresponding reaction in the absence of catalyst. ${ }^{[2]}$ Under otherwise identical reaction conditions, when $\mathrm{KF}$ (5 equiv.) was employed instead of $\mathrm{CsF}$ ( 2 equiv.), only traces of product were obtained; ${ }^{[3]} \mathrm{CsF}$ was dried for $3 \mathrm{~d}$ at $190{ }^{\circ} \mathrm{C}$ prior to usage. 1,2-DFB $=1,2$-difluorobenzene; 1,2 -DCE $=1,2$-dichloroethane.

Table S2: Nucleophile Equivalents Screening (using catalyst $(R)$-D)

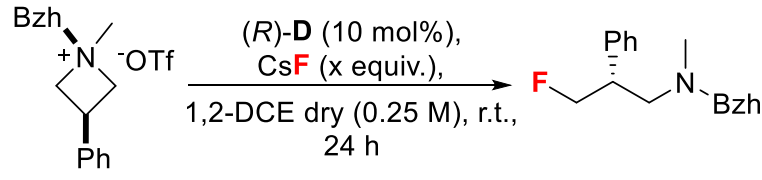

$15: 1$ (cis:trans)<smiles>O=C(Nc1cc(C(F)(F)F)cc(C(F)(F)F)c1)Nc1ccc2ccccc2c1-c1c(NC(=O)Nc2cc(C(F)(F)F)cc(C(F)(F)F)c2)ccc2ccccc12</smiles>

\begin{tabular}{ccc}
\hline CsF equiv. & NMR Yield & e.r. \\
\hline 1.2 & $28 \%$ & $82: 18$ \\
2 & $55 \%$ & $80.5: 19.5$ \\
3 & $69 \%$ & $70: 30$ \\
5 & $68 \%$ & $70: 30$
\end{tabular}


Table S3: Concentration and Catalyst Loading Screening (using catalyst $(R)$-D)

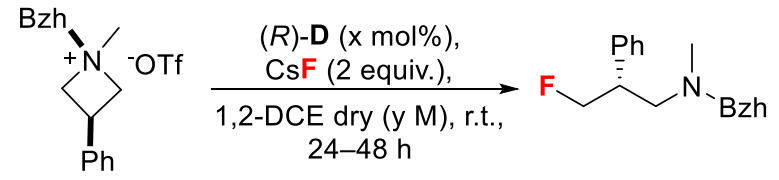

$15: 1$ (cis:trans)

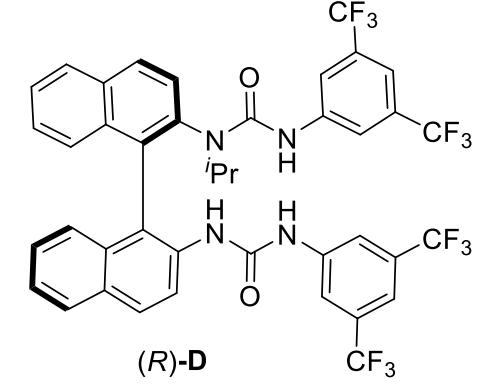

\begin{tabular}{ccccc}
\hline Molarity [M] & Cat. Loading & Time & NMR Yield & e.r. \\
\hline 0.1 & $10 \mathrm{~mol} \%$ & $24 \mathrm{~h}$ & $50 \%$ & $80: 20$ \\
0.25 & $10 \mathrm{~mol} \%$ & $24 \mathrm{~h}$ & $55 \%$ & $80.5: 19.5$ \\
0.5 & $10 \mathrm{~mol} \%$ & $24 \mathrm{~h}$ & $34 \%$ & $81.5: 18.5$ \\
\hline \hline 0.25 & $10 \mathrm{~mol} \%$ & $48 \mathrm{~h}$ & $93 \%$ & $80: 20$ \\
0.25 & $5 \mathrm{~mol} \%$ & $48 \mathrm{~h}$ & $46 \%$ & $79.5: 20.5$
\end{tabular}

Table S4: Reaction Time and Diastereomeric Ratio of Substrate (using catalyst $(R)-D$ )

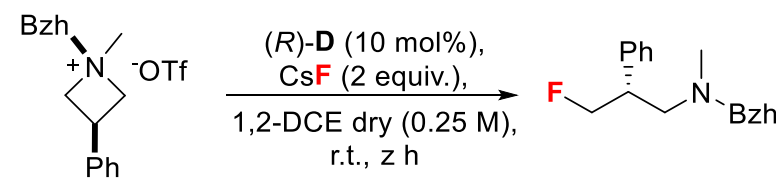

$\mathrm{x}: \mathrm{y}$ (cis:trans)<smiles>N=C(c1ccc2ccccc2c1NC(=O)Nc1cc(C(F)(F)F)cc(C(F)(F)F)c1)c1c(NC(=O)Nc2cc(C(F)(F)F)cc(C(F)(F)F)c2)ccc2ccccc12</smiles>

NMR

e.r.

$55 \% \quad 80.5: 19.5$

$93 \% \quad 80: 20$

$15: 1$

$48 \mathrm{~h}$

$>95 \% \quad 80.5: 19.5$

$72 \mathrm{~h}$

$57 \% \quad 80.5: 19.5$

>99:1 (cis)

$72 \mathrm{~h}$

$>95 \% \quad 80.5: 19.5$

$>1: 99($ trans $)$

$24 \mathrm{~h}$

$35 \%$

$80: 20$

$>1: 99$ (trans)

$72 \mathrm{~h}$

$53 \%$

$81.5: 19.5$ 

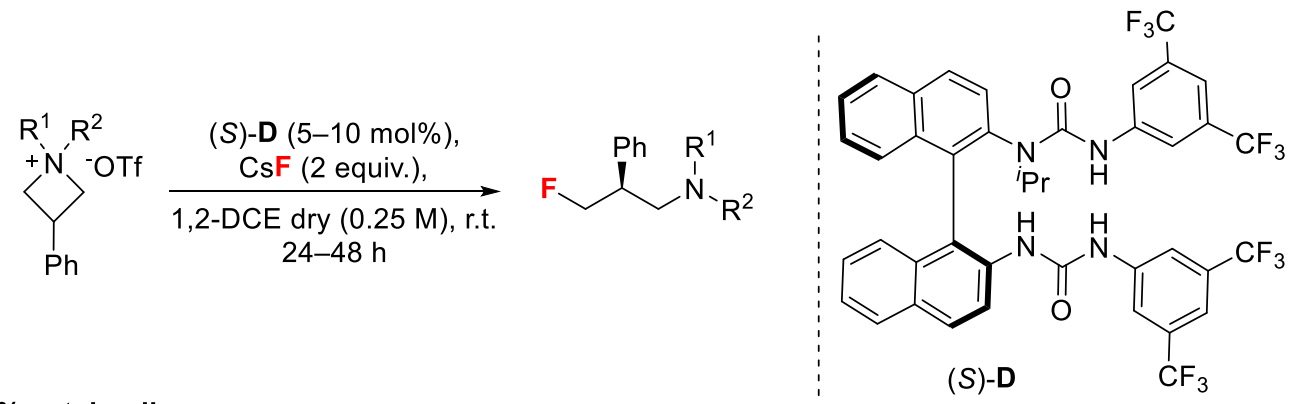

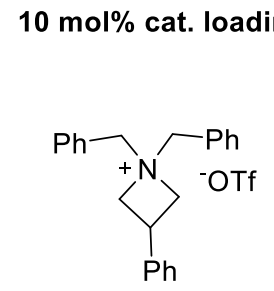

$5 \%$ yield, $77.5: 22.5$ e.r. (24 h)
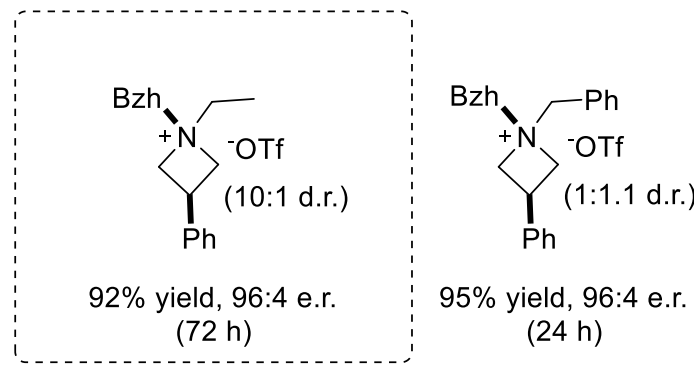

95\% yield, $96: 4$ e.r. $(24 \mathrm{~h})$

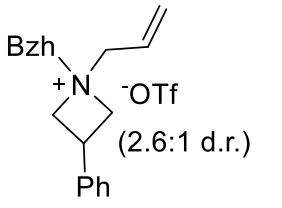

$96 \%$ yield, 93.5:6.5 e.r. (36 h)

\section{5 mol\% cat. loading}
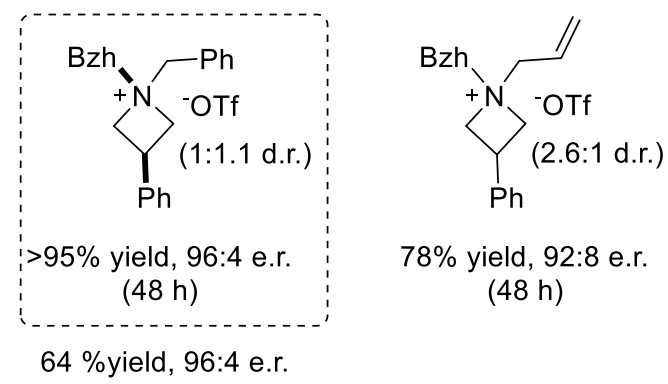

$78 \%$ yield, $92: 8$ e.r.

$(48 \mathrm{~h})$

$(24 \mathrm{~h})$

Scheme S3: Final $N$-protecting groups screening and determination of the optimized conditions for the substrate scope using catalyst $(S)$-D. Reaction times are optimized to achieve full conversion. 


\section{HB-PTC/Homogeneous Conditions Comparison}

A.
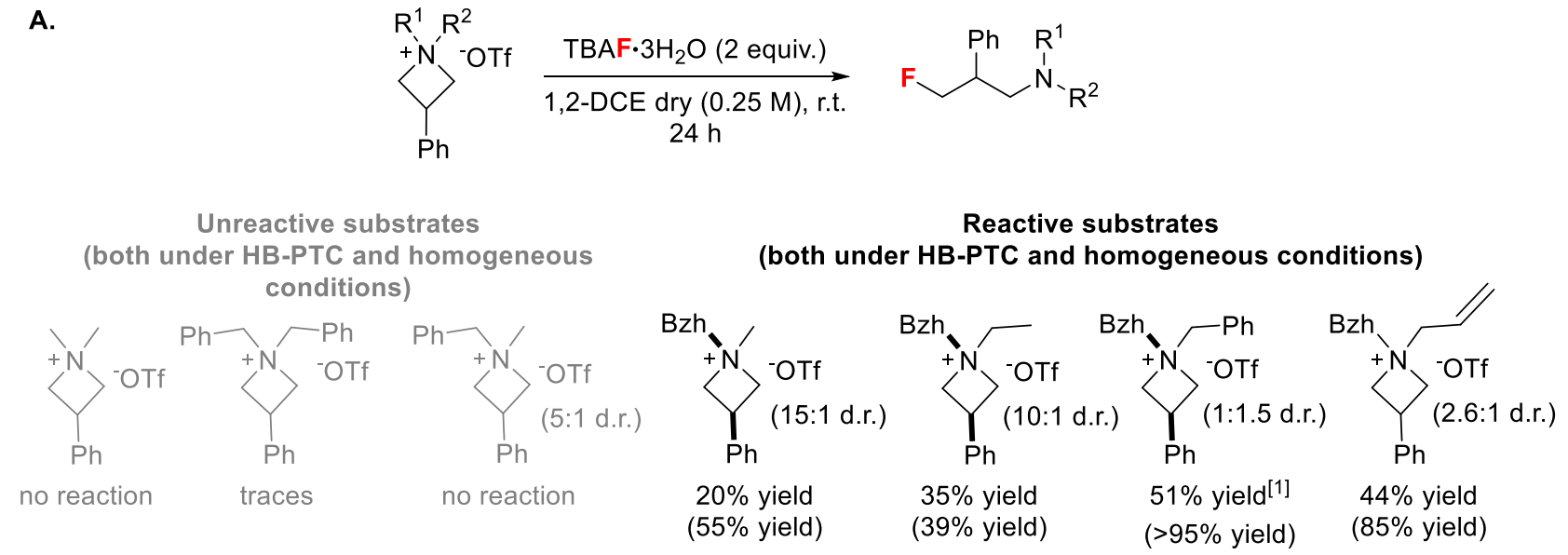

B.
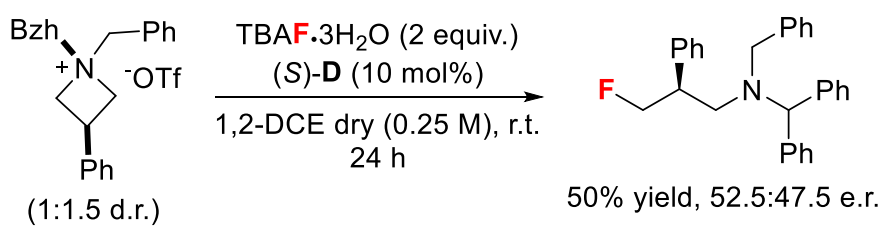<smiles>CCCNc1ccc2ccccc2c1-c1c(NC(=O)Nc2cc(C(F)(F)F)cc(C(F)(F)F)c2)ccc2ccccc12</smiles>

Scheme S4: A) Comparison between HB-PTC and homogeneous reaction conditions. In parenthesis, the yield obtained under the optimized HB-PTC conditions using $10 \mathrm{~mol} \%$ of (S)-D and 2 equiv. of CsF (24 h); B) Fluorination under homogeneous conditions with the addition of $10 \mathrm{~mol} \%$ of $(S)$-D.

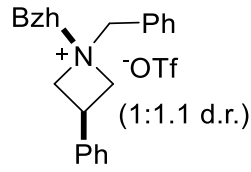

$$
\begin{aligned}
& \text { (S)-D TBAF complex } \\
& \text { (1 equiv.) } \\
& \text { 1,2-DCE dry (0.25 M), } \\
& \text { r.t., } 24 \mathrm{~h}
\end{aligned}
$$<smiles>O=C(CN(Cc1ccccc1)C[C@@H](CF)C(=O)O)OCc1ccccc1</smiles>

Comparable results were obtained when 1 equiv. (S)-D was pre-mixed with 1 equiv. of TBAF $3 \mathrm{H}_{2} \mathrm{O}$ (12h stirring) and then the substrate was added (additional $12 \mathrm{~h}$ ).

Scheme S5: Asymmetric reaction using stoichiometric amounts of $(S)$-D·TBAF complex. 


\section{Effect of Water}

Table S5: Effect of water on reactivity and enantioselectivity.

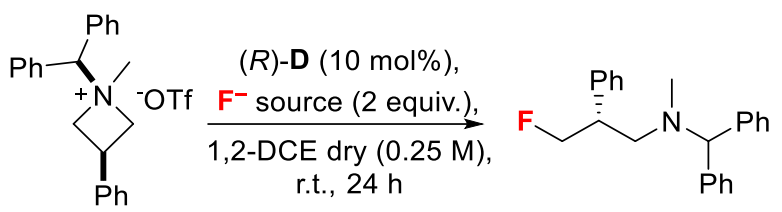
15:1 (cis:trans)<smiles>O=C(Nc1cc(C(F)(F)F)cc(C(F)(F)F)c1)Nc1ccc2ccccc2c1C(=O)Nc1ccc2ccccc2c1NC(=O)Nc1cc(C(F)(F)F)cc(C(F)(F)F)c1</smiles>

\begin{tabular}{ccccccc} 
Entry & $\mathbf{F}^{-}$source & $\mathbf{H}_{2} \mathbf{O}$ added (equiv.) & Catalyst & Yield & e.r. & Background reactivity \\
\hline $\mathbf{1}$ & $\mathrm{CsF}$ & $/ /$ & $(R)-\mathbf{D}$ & $55 \%$ & $80.5: 19.5$ & $8 \%$ \\
$\mathbf{2}$ & $\mathrm{CsF}$ & 1 equiv. & $(R)-\mathbf{D}$ & $40 \%$ & $81: 19$ & $0 \%$ \\
$\mathbf{3}$ & $\mathrm{CsF}$ & 5 equiv. & $(R)-\mathbf{D}$ & $2 \%$ & $82: 18$ & $0 \%$ \\
\hline $\mathbf{4}$ & $\mathrm{TBAF} \cdot 3 \mathrm{H}_{2} \mathrm{O}$ & $/ /$ & $/ /$ & $51 \%$ & $/ /$ & $/ /$ \\
$\mathbf{5}$ & $\mathrm{TBAF} \cdot 3 \mathrm{H}_{2} \mathrm{O}$ & 1 equiv. & $/ /$ & $32 \%$ & $/ /$ & $/ /$ \\
$\mathbf{6}$ & $\mathrm{TBAF} \cdot 3 \mathrm{H}_{2} \mathrm{O}$ & 5 equiv. & $/ /$ & $16 \%$ & $/ /$ & $/ /$
\end{tabular}

\section{Counteranion effect}

Table S6: Effect of the counteranion on reactivity and enantioselectivity.

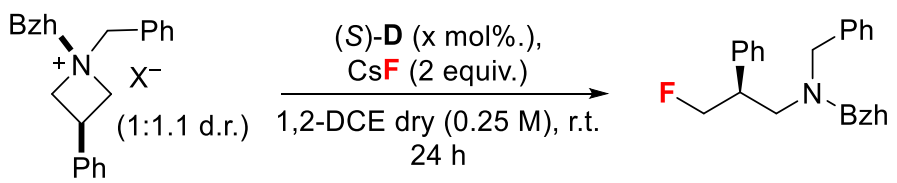

\begin{tabular}{cccccc} 
Entry & $\mathbf{X}^{-}$ & Cat. loading & Yield & e.r. & Background reactivity \\
\hline $\mathbf{1}$ & OTf & $5 \mathrm{~mol} \%$ & $64 \%$ & $96: 4$ & $17 \%$ \\
$\mathbf{2}^{\mathrm{a}}$ & OTf & $10 \mathrm{~mol} \%$ & $>95 \%$ & $96: 4$ & $17 \%$ \\
$\mathbf{3}$ & $\mathrm{BF}_{4}$ & $5 \mathrm{~mol} \%$ & $>95 \%$ & $85: 15$ & $95 \%^{\mathrm{b}}$ \\
$\mathbf{4}$ & $\mathrm{BF}_{4}$ & $10 \mathrm{~mol} \%$ & $>95 \%$ & $90: 10$ & $95 \%^{\mathrm{b}}$ \\
$\mathbf{5}$ & $\mathrm{BF}_{4}$ & $20 \mathrm{~mol} \%$ & $>95 \%$ & $95: 5$ & $95 \%^{\mathrm{b}}$ \\
$\mathbf{6}$ & $\mathrm{PF}_{6}$ & $5 \mathrm{~mol} \%$ & $31 \%$ & $96: 4$ & $9 \%$
\end{tabular}

a $48 \mathrm{~h}$ reaction; $^{\text {b }}$ In the absence of $\mathrm{CsF}$ no product was obtained. Background reactivity due to the tetrafluoroborate anion itself as a fluoride source is therefore absent. 


\section{$\underline{\text { Non-linear Effect Studies }}$}

The non-linear effect study was conducted with $(R)$ and $(S)$ mixtures of catalyst $\mathbf{D}$ by employing the previously described protocol for reaction optimization (see detailed conditions in scheme below). Under these conditions (1,2-DCE as solvent, r.t.), (S)-3d affords the product in $92 \%$ ee (96:4 e.r.).

Table S7. Non-linear effect study

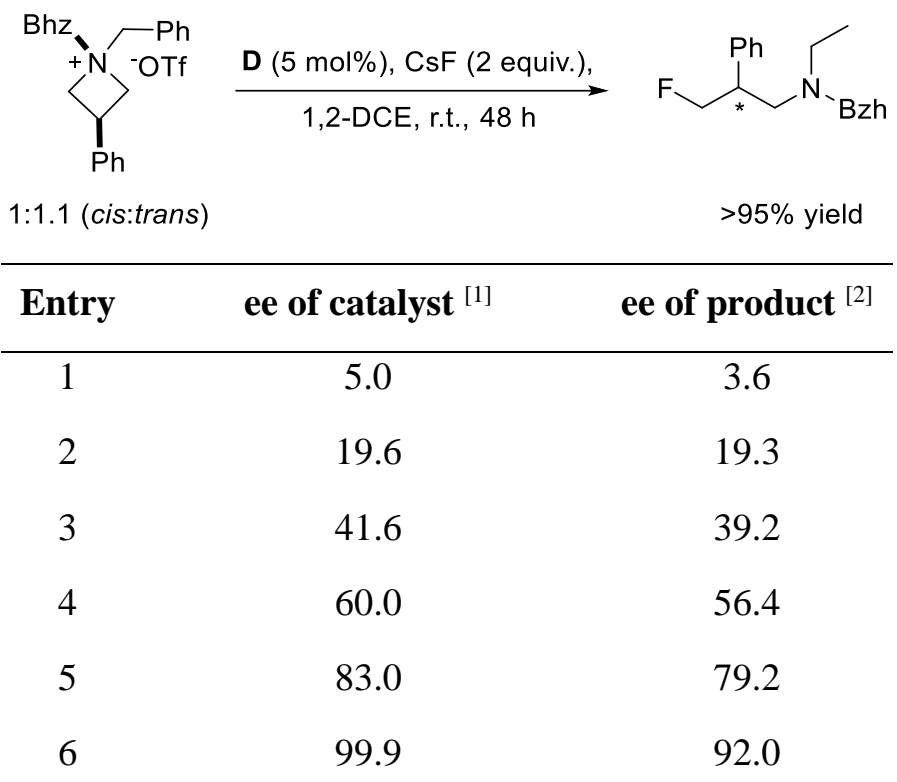

${ }^{[1]}(R)$-D and $(S)$-D were premixed in a ratio between 50:50 and 0:100, respectively. The precise ee of the catalyst was determined by HLPC analysis using a chiral stationary phase at $254 \mathrm{~nm}$ (DAICEL CHIRALPAK ${ }^{\circledR}$ IG, 96:4 Heptane:EtOH, 1mL/min).

${ }^{[2]}$ The ee of the product was determined by HLPC analysis using a chiral stationary phase at $254 \mathrm{~nm}$ (DAICEL CHIRALCEL ${ }^{\circledR}$ OJ-H, 95:5 Heptane:EtOH, 1mL/min).

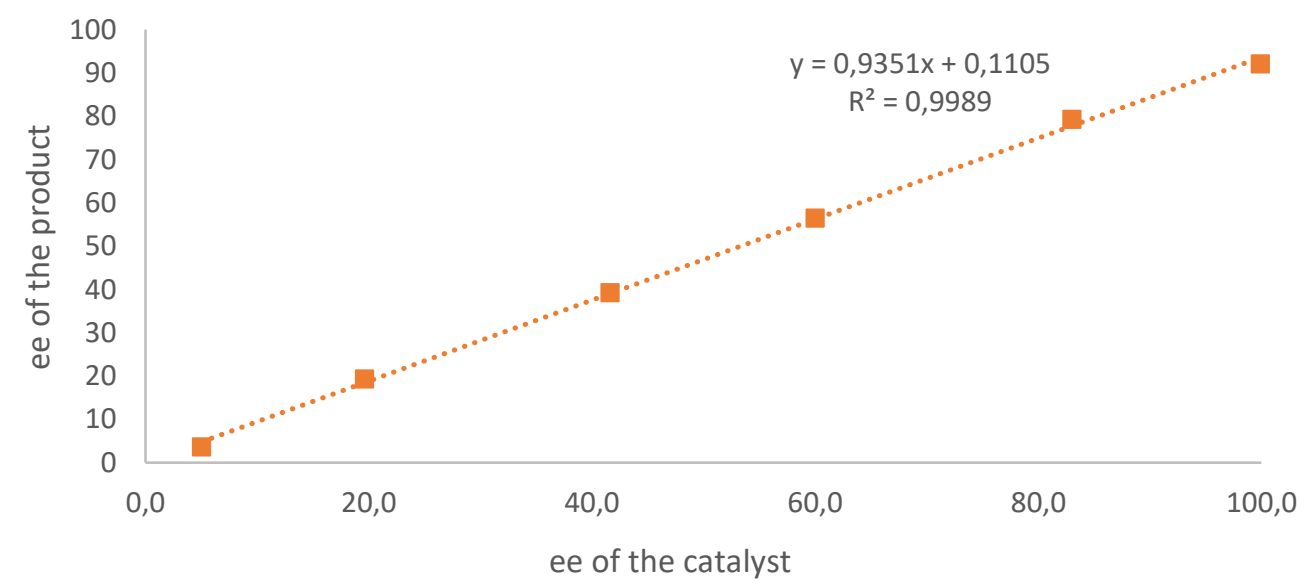

Conclusion: No non-linear effect was observed. The computed transition state model (see computational analysis section) involving only one molecule of the urea catalyst is thus consistent with the experimental data. 


\section{$\underline{\text { Anion Binding Studies }}$}

A stock solution of urea $(S)$-D $(2 \mathrm{mM})$ in $\mathrm{CH}_{2} \mathrm{Cl}_{2}: \mathrm{CD}_{2} \mathrm{Cl}_{2}$ (3:2) was prepared in a volumetric flask. A ca. $80 \mathrm{mM}$ solution of the appropriate tetrabutylammonium salt $\left(\mathrm{OTf}^{-}, \mathrm{BF}_{4}^{-}{\mathrm{or} \mathrm{PF}_{6}}^{-}\right)$was then prepared by accurately weighting the solid and dissolving it in the previously prepared urea solution using a volumetric flask. An aliquot of $0.5 \mathrm{~mL}$ of stock solution was transfered into an NMR tube to which increasing amounts of TBA salt $(1-200 \mu \mathrm{L})$ were added using a microsyringe. After every addition a ${ }^{1} \mathrm{H}$ NMR spectrum was acquired, using a dedicated pulse sequence to suppress the $\mathrm{CH}_{2} \mathrm{Cl}_{2}$ resonance at $5.37 \mathrm{ppm}$ (perfect echo excitation sculpting sequence). ${ }^{2}$ The chemical shift variation of three diagnostic aromatic protons was monitored and plotted against the concentration of added anion. The association constants were determined from the experimental data by non-linear curve fitting using Bindfit (http://supramolecular.org) ${ }^{3}$ and expressed as an averaged value of three replicated experiments. Different binding model were evaluated, 1:1 resulting in the one giving consistently better fit. For every experiment a representative example of data analysis (in each case, only replica $\mathbf{I}$ is shown) is reported below. The full data set are made available open access on http://supramolecular.org.

Tetrabutylammonium trifluoromethanesulfonate (TBAOTf)

Table S8: Input data for the titration of (S)-D with TBAOTf (variation of three distinct protons in replica I).

\begin{tabular}{cccccc}
$\begin{array}{c}(\boldsymbol{S})-\mathbf{D} \\
\text { concentration }[\mathbf{M}]\end{array}$ & $\begin{array}{c}\text { TBAOTf } \\
\text { concentration } \\
{[\mathbf{M}]}\end{array}$ & Equivalents & $\begin{array}{c}\boldsymbol{\delta}^{\mathbf{1}} \mathbf{H}-(\mathbf{A}) \\
{[\mathbf{p p m}]}\end{array}$ & $\begin{array}{c}\boldsymbol{\delta}^{\mathbf{1}} \mathbf{H}-(\mathbf{B}) \\
{[\mathbf{p p m}]}\end{array}$ & $\begin{array}{c}\boldsymbol{\delta}^{\mathbf{1}} \mathbf{H}-(\mathbf{C}) \\
{[\mathbf{p p m}]}\end{array}$ \\
\hline 0.0019 & 0.0000 & 0.00 & 8.178 & 7.879 & 6.943 \\
0.0019 & 0.0002 & 0.09 & 8.181 & 7.876 & 6.933 \\
0.0019 & 0.0003 & 0.17 & 8.183 & 7.874 & 6.926 \\
0.0019 & 0.0005 & 0.26 & 8.185 & 7.871 & 6.918 \\
0.0019 & 0.0008 & 0.43 & 8.189 & 7.867 & 6.906 \\
0.0019 & 0.0012 & 0.60 & 8.192 & 7.863 & 6.896 \\
0.0019 & 0.0015 & 0.76 & 8.195 & 7.859 & 6.887 \\
0.0019 & 0.0018 & 0.93 & 8.198 & 7.857 & 6.879 \\
0.0019 & 0.0023 & 1.18 & 8.201 & 7.853 & 6.869 \\
0.0019 & 0.0032 & 1.66 & 8.206 & 7.848 & 6.854 \\
0.0019 & 0.0048 & 2.45 & 8.212 & 7.842 & 6.836 \\
0.0019 & 0.0077 & 3.93 & 8.218 & 7.835 & 6.818 \\
0.0019 & 0.0140 & 7.21 & 8.224 & 7.830 & 6.800 \\
0.0019 & 0.0195 & 9.98 & 8.225 & 7.827 & 6.794
\end{tabular}



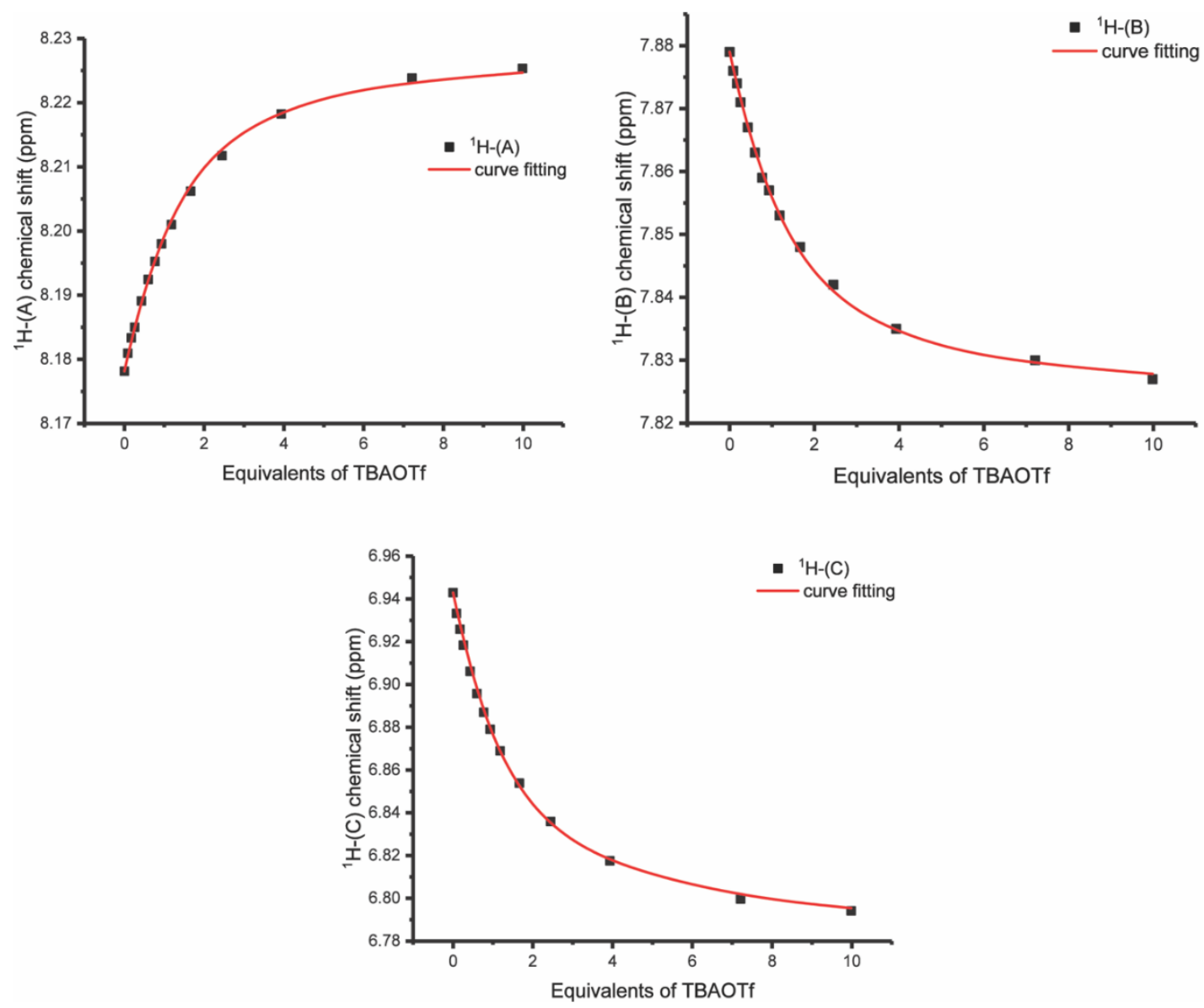

Figure S1: Titration profiles of diagnostic protons in replica I, experimental data point and curve fitting.

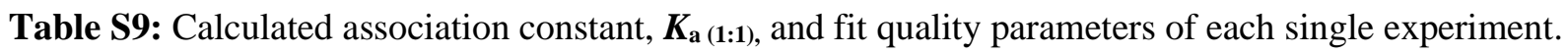

\begin{tabular}{cccc} 
& $\boldsymbol{K}_{\mathbf{a}(\mathbf{1 : 1})}\left[\mathbf{M}^{-1}\right]$ & $\begin{array}{c}\text { Residual sum of } \\
\text { squares }\end{array}$ & Cov $_{\text {fit }}$ \\
\hline Replica I & 617 & $6.9469 \times 10^{-5}$ & $7.0739 \times 10^{-4}$ \\
Replica II & 545 & $6.0937 \times 10^{-5}$ & $7.0157 \times 10^{-4}$ \\
Replica III & 528 & $6.9531 \times 10^{-5}$ & $7.5339 \times 10^{-4}$
\end{tabular}

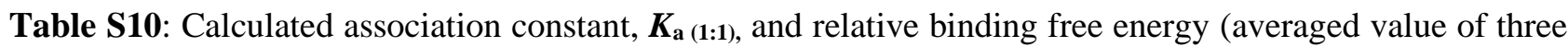
replicated experiments).

$K_{\mathbf{a}(1: 1)}\left[\mathbf{M}^{-1}\right]$

$\Delta \mathbf{G}[\mathrm{kJ} / \mathbf{m o l}]$

$560 \pm 47$

$-15.7 \pm 0.20$ 
Tetrabutylammonium tetrafluoroborate $\left(\mathrm{TBABF}_{4}\right)$

Table S11: Input data for the titration of $(S)$-D with $\mathrm{TBABF}_{4}$ (variation of three distinct protons in replica I).

\begin{tabular}{cccccc}
$\begin{array}{c}(\boldsymbol{S})-\mathbf{D} \\
\text { concentration } \\
{[\mathbf{M}]}\end{array}$ & $\begin{array}{c}\mathbf{T B A B F}_{\mathbf{4}} \\
\text { concentration } \\
{[\mathbf{M}]}\end{array}$ & Equivalents & $\begin{array}{c}\boldsymbol{\delta}^{\mathbf{1}} \mathbf{H}-(\mathbf{A}) \\
{[\mathbf{p p m}]}\end{array}$ & $\begin{array}{c}\boldsymbol{\delta}^{\mathbf{1}} \mathbf{H}-(\mathbf{B}) \\
{[\mathbf{p p m}]}\end{array}$ & $\begin{array}{c}\boldsymbol{\delta}^{\mathbf{1}} \mathbf{H}-(\mathbf{C}) \\
{[\mathbf{p p m}]}\end{array}$ \\
\hline 0.0020 & 0.0000 & 0.00 & 8.178 & 7.879 & 6.942 \\
0.0020 & 0.0002 & 0.09 & 8.180 & 7.877 & 6.937 \\
0.0020 & 0.0003 & 0.17 & 8.181 & 7.876 & 6.934 \\
0.0020 & 0.0005 & 0.26 & 8.183 & 7.874 & 6.931 \\
0.0020 & 0.0009 & 0.43 & 8.186 & 7.873 & 6.925 \\
0.0020 & 0.0012 & 0.60 & 8.188 & 7.871 & 6.920 \\
0.0020 & 0.0015 & 0.77 & 8.190 & 7.870 & 6.916 \\
0.0020 & 0.0019 & 0.94 & 8.193 & 7.869 & 6.912 \\
0.0020 & 0.0024 & 1.19 & 8.195 & 7.867 & 6.907 \\
0.0020 & 0.0033 & 1.67 & 8.200 & 7.865 & 6.899 \\
0.0020 & 0.0049 & 2.46 & 8.206 & 7.862 & 6.888 \\
0.0020 & 0.0079 & 3.96 & 8.215 & 7.859 & 6.874 \\
0.0020 & 0.0145 & 7.25 & 8.224 & 7.855 & 6.856 \\
0.0020 & 0.0200 & 10.04 & 8.228 & 7.854 & 6.848
\end{tabular}
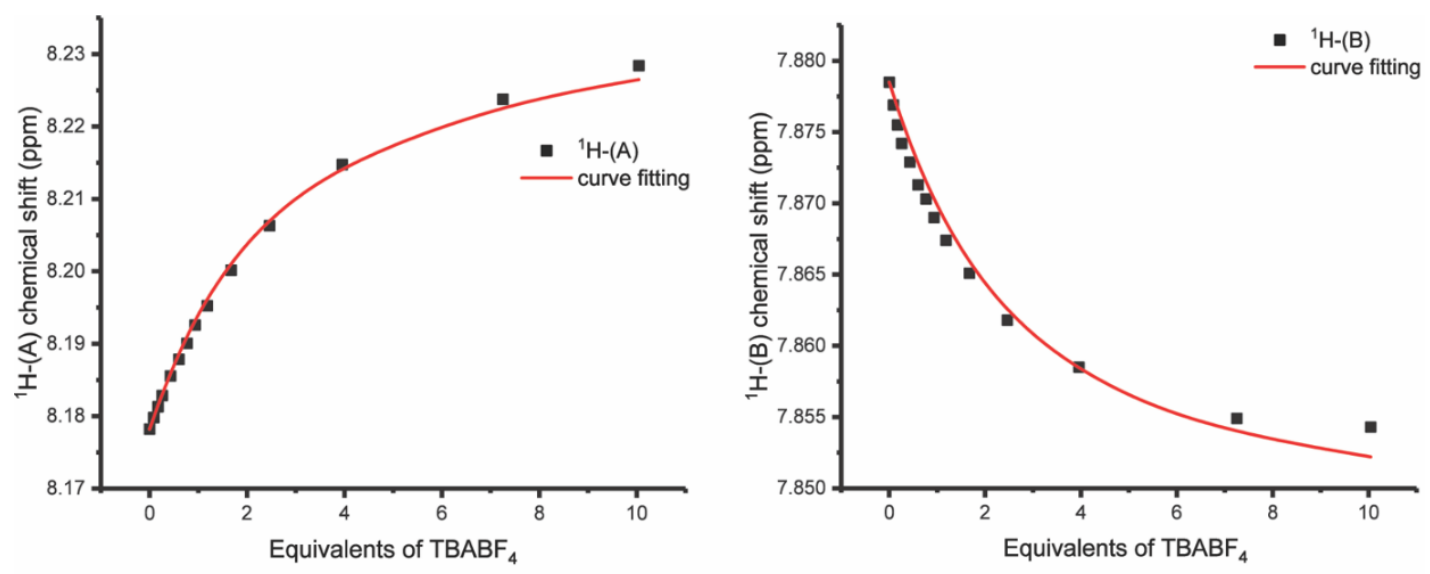


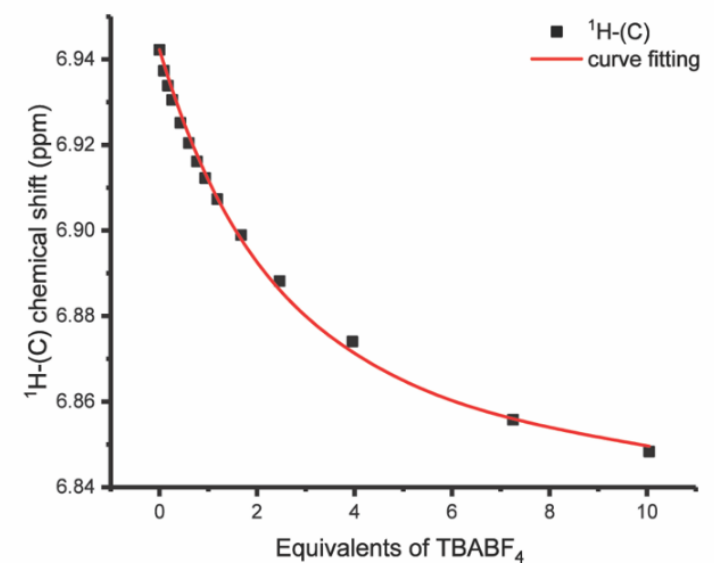

Figure S2: Titration profiles of diagnostic protons in replica I, experimental data point and curve fitting.

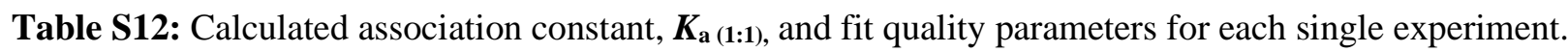

\begin{tabular}{cccc} 
& $\boldsymbol{K}_{\mathbf{a}(\mathbf{1}: \mathbf{1})}\left[\mathbf{M}^{-\mathbf{1}}\right]$ & $\begin{array}{c}\text { Residual sum of } \\
\text { squares }\end{array}$ & $\mathbf{C o v}_{\text {fit }}$ \\
\hline Replica I & 254 & $6.8255 \times 10^{-5}$ & $1.55384 \times 10^{-3}$ \\
Replica II & 248 & $3.9894 \times 10^{-5}$ & $1.12533 \times 10^{-3}$ \\
Replica III & 287 & $1.4294 \times 10^{-4}$ & $3.32541 \times 10^{-3}$
\end{tabular}

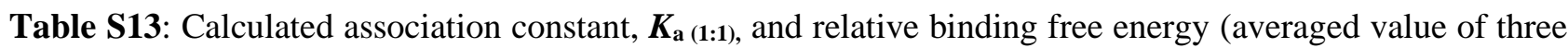
replicated experiments).

$\boldsymbol{K}_{\mathrm{a}(1: 1)}\left[\mathbf{M}^{-1}\right]$

$260 \pm 20$
$\Delta \mathbf{G}[\mathrm{kJ} / \mathrm{mol}]$

$-13.8 \pm 0.19$ 
Tetrabutylammonium hexafluorophosphate $\left(T B A P F_{6}\right)$

Table S14: Input data for the titration of $(S)$-D with $\mathrm{TBAPF}_{6}$ (variation of three distinct protons in replica I).

(S)-D concentration

[M]

TBAPF $_{6}$

concentration

$[\mathrm{M}]$

0.0019

0.0019

0.0019

0.0019

0.0019

0.0019

0.0019

0.0019

0.0019

0.0019

0.0019
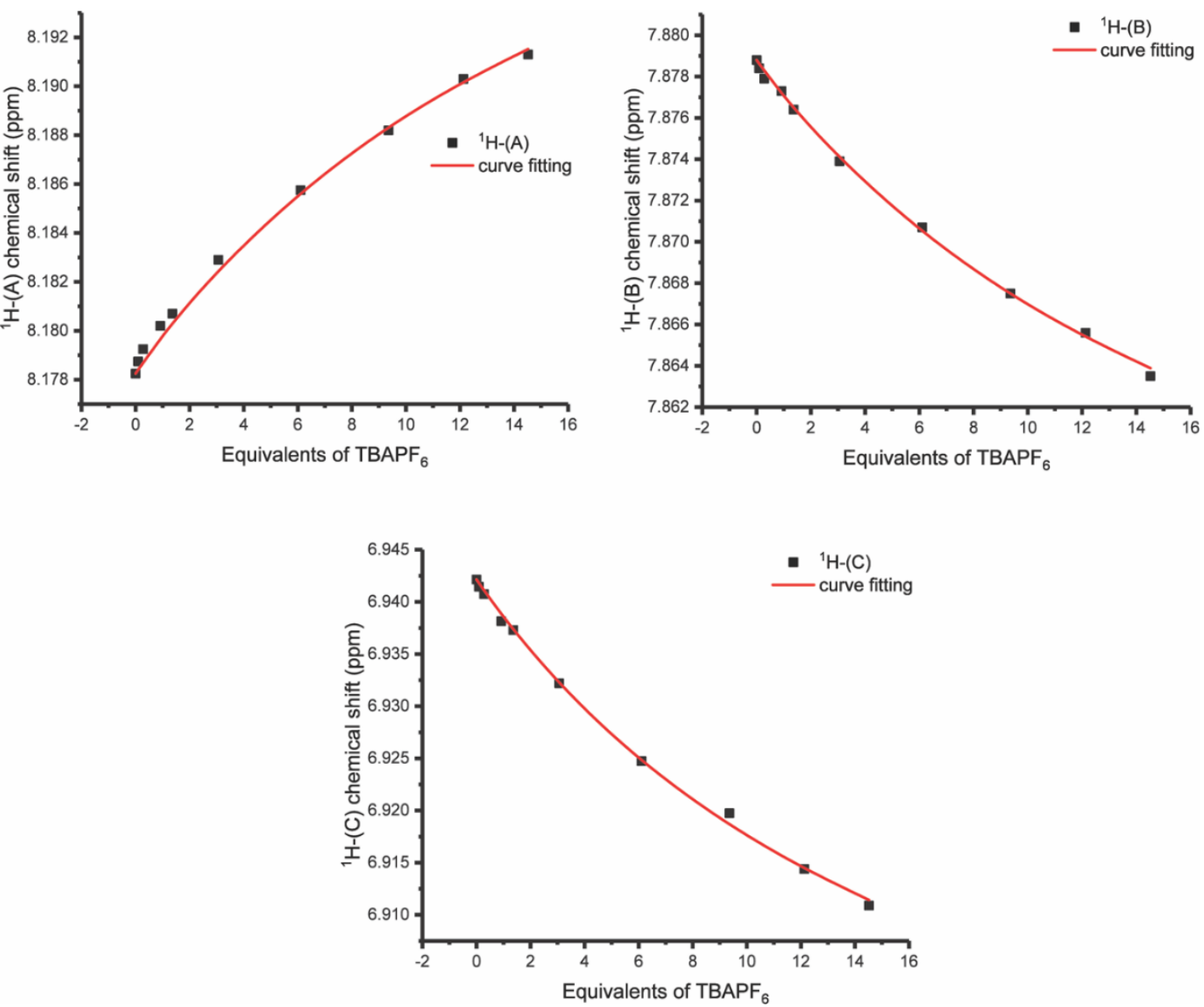

Figure S3: Titration profiles of diagnostic protons in replica I, experimental data point and curve fitting. 


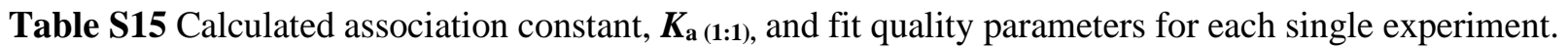

\begin{tabular}{cccc} 
& $\boldsymbol{K}_{\mathbf{a}(\mathbf{1 : 1})}\left[\mathbf{M}^{-\mathbf{1}}\right]$ & $\begin{array}{c}\text { Residual sum of } \\
\text { squares }\end{array}$ & Cov $_{\text {fit }}$ \\
\hline Replica I & 30 & $3.8713 \times 10^{-6}$ & $1.16848 \times 10^{-3}$ \\
Replica II & 32 & $5.8417 \times 10^{-6}$ & $1.73996 \times 10^{-3}$ \\
Replica III & 30 & $6.0144 \times 10^{-6}$ & $2.13053 \times 10^{-3}$
\end{tabular}

Table S16: Calculated association constant, $\boldsymbol{K}_{\mathbf{a}(1: 1)}$, and relative binding free energy (averaged value of three replicated experiments).

$$
K_{\mathbf{a}(1: 1)}\left[\mathbf{M}^{-1}\right] \quad \Delta \mathbf{G}[\mathrm{kJ} / \mathbf{m o l}]
$$

$\mathbf{3 1} \pm \mathbf{1 . 2}-8.51 \pm 0.096$

\section{Conclusion}

The binding affinity of $(S)$-D towards three different anions, encountered as counter ions in the desymmetrization of aziridininium salts $\left(\mathrm{OTf}^{-}, \mathrm{BF}_{4}^{-}\right.$and $\left.\mathrm{PF}_{6}^{-}\right)$, were evaluated to assess whether these counterions could be involved in competitive binding interactions to $\mathrm{F}^{-}\left(\mathrm{K}_{\mathrm{a}\left(\mathrm{U}: \mathrm{F}^{-}\right)}\right.$was previously measured by UV-Vis titrations). ${ }^{1}$ The experimental data shows the following trend:

$$
\mathrm{K}_{\mathrm{a}\left(\mathrm{U}: \mathrm{X}^{-}\right)}: \mathrm{F}^{-}\left(10^{6} \mathrm{M}^{-1}\right)>>\operatorname{OTf}^{-}\left(10^{2} \mathrm{M}^{-1}\right)>\sim \mathrm{BF}_{4}^{-}\left(10^{2} \mathrm{M}^{-1}\right)>\mathrm{PF}_{6}^{-}\left(10^{1} \mathrm{M}^{-1}\right) \text {. }
$$

This shows that hydrogen bonding to fluoride is at least four order of magnitude higher than the hydrogen bonding interactions with the other studied anions. These findings combined with the reactivity/enantioselectivity data of the three salts (Table S6) suggest that an anion binding/ion pairing catalysis mechanism ${ }^{4}$ is unlikely.

\section{Fluorination under Chiral Anion Phase Transfer (CAPT)}

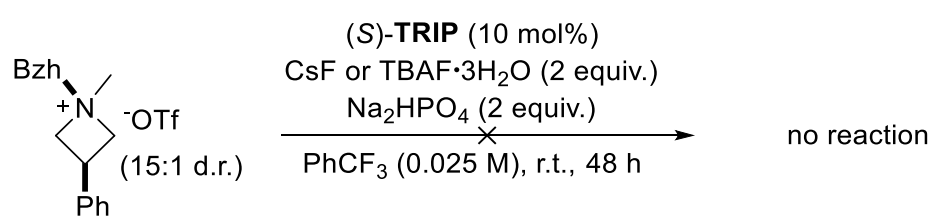

Scheme S6: Fluorination under CAPT conditions (Sun et al.), ${ }^{5}$ no product formation was observed. 


\section{General Procedure for the Asymmetric Nucleophilic Fluorination of Azetidinium Ions}

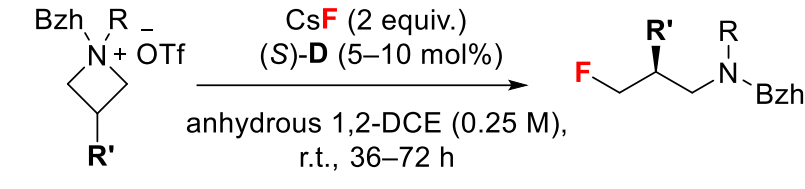

$\mathrm{R}=\mathrm{Bn}, \mathrm{Et}, \mathrm{All}$

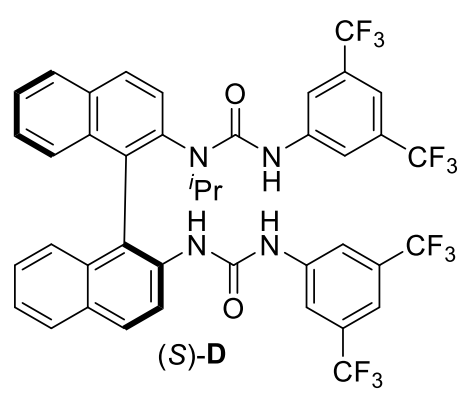

(S)-D

In a screw-capped vial equipped with a magnetic stirring bar were added CsF ( 2 equiv., $0.2 \mathrm{mmol}$ ), organocatalyst $(S)$-D (5-10 mol\%) and the substrate (1 equiv., $0.1 \mathrm{mmol})$. Anhydrous 1,2-DCE (0.25M, $0.4 \mathrm{~mL}$ ) was added, the vial was sealed and the reaction mixture was stirred at $900 \mathrm{rpm}$ at r.t. for the indicated time. The crude mixture was then directly purified by flash column chromatography (FCC).

For reaction optimization was filtered through a small plug of silica, evaporated to dryness and the crude mixture was analyzed by ${ }^{1} \mathrm{H}$ and ${ }^{19} \mathrm{~F}$ NMR (4-fluoroanisole as internal standard) to determine the yield; an aliquot of the reaction mixture was purified by preparative TLC and analyzed by HPLC (chiral stationary phase) to determine the e.r.

Note: CsF (99.9\% trace metal basis from Sigma-Aldrich) was ground prior to the reaction and used without pre-drying.

Racemate synthesis: The racemic reference products for HPLC analysis were obtained using stoichiometric amounts of TBAF $3 \mathrm{H}_{2} \mathrm{O}$ in THF $(0.25 \mathrm{M}$, r.t., $12 \mathrm{~h})$. Alternatively, the substrate was reacted with $\mathrm{CsF}$ ( 2 equiv.) in 1,2-DCE $(0.25 \mathrm{M})$ at $60{ }^{\circ} \mathrm{C}$ for $6 \mathrm{~h}$. 


\section{Substrates Synthesis and Characterization}

\section{Substrates Synthesis}

Substrate precursors 5 and $\mathbf{6}$ (1-Boc and 1-benzhydryl azetidines) were commercially available and used without further purification or synthesized according to the following route:

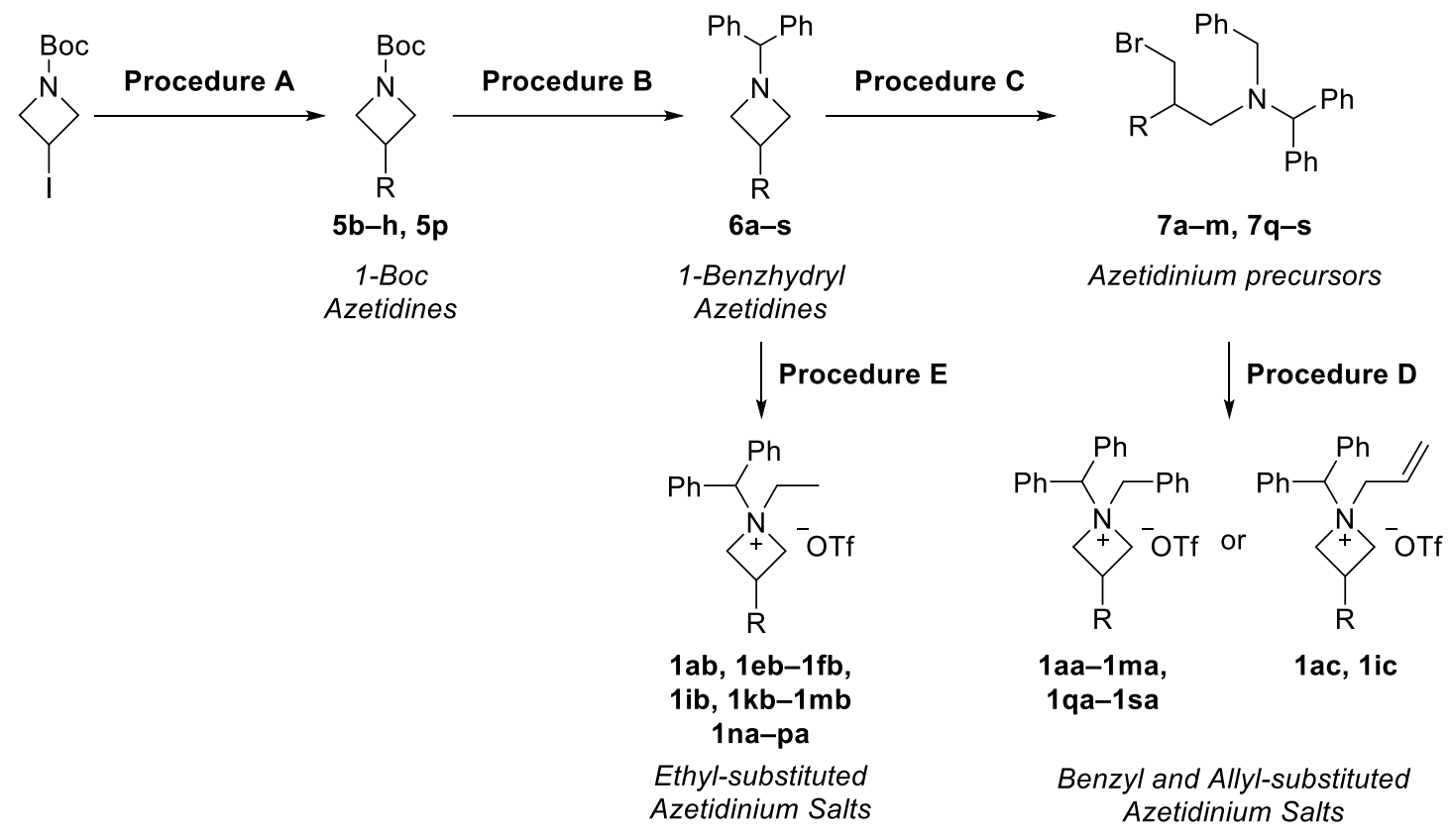

\section{General Procedures}

\section{Azetidines $(5,6)$ and Brominated Precursors (7)}

\section{General Procedure A: Synthesis of 1-Boc Azetidines (5)}

The appropriate boronic acid (2 equiv.), $\mathrm{NiI}_{2}(6 \mathrm{~mol} \%)$ and trans-2-aminocyclohexanol hydrochloride (6 mol\%) were weighed into a microwave vial and placed under an inert atmosphere. Commercially available 1-Boc-3-iodoazetidine (1 equiv.) and dry isopropanol $(2 \mathrm{~mL} / \mathrm{mmol})$ were then added, followed by NaHMDS (1M in THF, 2 equiv.) at r.t. The mixture was heated at $80{ }^{\circ} \mathrm{C}$ for $30 \mathrm{~min}$ under microwave irradiation. After cooling to r.t., the mixture was diluted with $\mathrm{EtOH}(0.25 \mathrm{mmol} / \mathrm{mL})$ and filtered through a celite plug. The filter cake was rinsed with EtOH and the filtrate was concentrated in vacuo. The crude mixture was purified by flash column chromatography (Heptane:EtOAc as eluent) to afford the desired 1-Boc-azetidine.

\section{General procedure B: Synthesis of 1-benzhydryl Azetidines (6)}

The appropriate 1-Boc-azetidine (1 equiv.) was dissolved in $\mathrm{CH}_{2} \mathrm{Cl}_{2}(10 \mathrm{~mL} / \mathrm{mmol})$ and TFA $(5.8 \mathrm{~mL} / \mathrm{mmol})$ was slowly added at $0{ }^{\circ} \mathrm{C}$. The reaction was stirred at r.t. for $12 \mathrm{~h}$, the solvent was removed in vacuo and the obtained oil was washed twice with pentane to obtain the desired azetidinium·TFA salt. The salt was then dissolved in dry $\mathrm{CH}_{3} \mathrm{CN}(1 \mathrm{M})$ before DIPEA (5 equiv.) was added dropwise. Bromodiphenylmethane (1 equiv.) was then added and the reaction mixture was stirred at $100{ }^{\circ} \mathrm{C}$ for $2 \mathrm{~h}$. The reaction mixture was then concentrated in vacuo and the residual slurry was purified by flash column chromatography (Heptane:EtOAc as eluent) to afford the desired 1-benzhydryl azetidines. 


\section{General procedure C: Synthesis of brominated azetidinium precursors (7)}

Under a flow of $\mathrm{N}_{2}$, an oven-dried flask was charged with 1-benzhydryl-azetidine and dry $\mathrm{CH}_{3} \mathrm{CN}$ $(1.0 \mathrm{~mL} / \mathrm{mmol})$. Benzyl or allyl bromide (1.6 equiv.) were added and the mixture was stirred at $60{ }^{\circ} \mathrm{C}$ for $12 \mathrm{~h}$. The solvent was removed under vacuum and the crude mixture was purified by flash column chromatography (Hexane:EtOAc) to afford the desired azetidinium precursor.

\section{Azetidinium substrates (1)}

\section{General procedure D: Synthesis of 1-Benzyl and 1-Allyl Azetidinium·TFA Salts*}

Under a $\mathrm{N}_{2}$ atmosphere, to an oven-dried flask charged with AgOTf (1 equiv.), a solution of 1-benzhydryl-1benzyl-3-bromo-propan-1-amine (1 equiv.) in dry $\mathrm{CH}_{2} \mathrm{Cl}_{2}(10 \mathrm{~mL} / \mathrm{mmol})$ was added. The reaction mixture was stirred at r.t. for $12 \mathrm{~h}$ in the dark. The mixture was then filtered through celite and the filtrate was evaporated under reduced pressure. The resulting powder was washed with cold $\mathrm{Et}_{2} \mathrm{O}$ to afford the desired azetidinium salt.

* The tetrafluoroborate and hexafluorophosphate salts (Table S6) were prepared according to the same procedure by employing $\mathrm{AgBF}_{4}$ and $\mathrm{AgPF}_{6}$ respectively instead of $\mathrm{AgOTf}$.

\section{General procedure E: Synthesis of 1-Methyl and 1-Ethyl Azetidinium Salts}

To a solution of 1-benzhydryl-azetidine in dry $\mathrm{CH}_{2} \mathrm{Cl}_{2}(2 \mathrm{M})$ at $0{ }^{\circ} \mathrm{C}$, methyl or ethyl triflate (1.5 equiv.) were added dropwise and the reaction was stirred overnight at r.t. The solvent was then evaporated, the white precipitate was washed with $\mathrm{Et}_{2} \mathrm{O}$ and filtered. The solid was further washed with cold $\mathrm{Et}_{2} \mathrm{O}$ to afford the desired compound. 


\section{Characterization of Substrate Precursors}

\section{Azetidines, Brominated Precursors and Intermediates}

\section{1-benzhydryl-1-benzyl-3-bromo-2-phenylpropan-1-amine (7aa)}

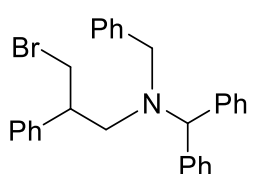

7 aa

Prepared according to a slightly modified version of general procedure C. Under a flow of $\mathrm{N}_{2}$, an oven-dried flask was charged with commercially available 1-benzhydryl-3phenylazetidine ( $8.48 \mathrm{mmol}, 2.52 \mathrm{~g}$ ) in dry $\mathrm{CH}_{3} \mathrm{CN}(1 \mathrm{M}, 8.4 \mathrm{~mL})$. Benzyl bromide $(0.95$ equiv., $8.06 \mathrm{mmol}, 0.95 \mathrm{~mL}$ ) was added and the mixture was stirred at $60{ }^{\circ} \mathrm{C}$ for $12 \mathrm{~h}$. The

crude mixture was purified by flash column chromatography (Pentane:EtOAc $=100: 0$ to 98:2, gradient) to afford the desired product in $85 \%$ yield $(7.21 \mathrm{mmol}, 3.36 \mathrm{~g})$ as a colorless oil. ${ }^{1} \mathbf{H} \mathbf{~ N M R}\left(400 \mathrm{MHz}, \mathrm{CDCl}_{3}\right) \delta$ ppm: $7.42-7.20(\mathrm{~m}, 18 \mathrm{H}), 7.01-6.91(\mathrm{~m}, 2 \mathrm{H}), 4.96(\mathrm{~s}, 1 \mathrm{H}), 3.76(\mathrm{dd}, J=10.0,5.6 \mathrm{~Hz}, 1 \mathrm{H}), 3.65(\mathrm{~s}, 2 \mathrm{H})$, $3.34(\mathrm{dd}, J=10.0,8.6 \mathrm{~Hz}, 1 \mathrm{H}), 3.19-3.07$ (m, 1H), $2.89(\mathrm{dd}, J=13.3,7.6 \mathrm{~Hz}, 1 \mathrm{H}), 2.75(\mathrm{dd}, J=13.3,7.2$ $\mathrm{Hz}, 1 \mathrm{H}) ;{ }^{13} \mathbf{C}$ NMR (101 MHz, $\left.\mathrm{CDCl}_{3}\right) \delta$ ppm: 141.58, 140.72, 140.51, 139.40, 129.51, 129.22, 129.11, 128.52, $128.44,128.40,128.31,128.04,127.34,127.17,127.15,69.67,55.77,55.72,46.96,36.58$. IR (thin layer film) $v\left(\mathrm{~cm}^{-1}\right)=3027,2834,1601,1493,1452,1265,1122,1076,1029,920,741,697$. HRMS $\left(\mathrm{ESI}^{+}\right) \mathrm{m} / z$ $\mathrm{C}_{29} \mathrm{H}_{29} \mathrm{BrN}^{+}[\mathrm{M}+\mathrm{H}]^{+}$: calculated 470.1483 , found 470.1487. Spectroscopic data were in agreement with the ones previously reported in literature. ${ }^{5}$

\section{1-benzhydryl-1-(3-bromo-2-phenylpropyl)prop-2-en-1-amine (7ab)}

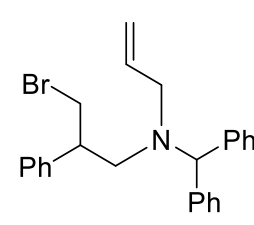

$7 a b$

Prepared according to general procedure $\mathrm{C}$ using commercially available 1-benzhydryl-3phenylazetidine $(6.68 \mathrm{mmol}, 2.0 \mathrm{~g})$ and allyl bromide $(11.13 \mathrm{mmol}, 0.96 \mathrm{~mL})$ The crude mixture was purified by flash column chromatography (Heptane 100\%) to afford the desired product in $75 \%$ yield $(5.00 \mathrm{mmol}, 2.10 \mathrm{~g})$ as a colorless oil. ${ }^{1} \mathbf{H}$ NMR $(500 \mathrm{MHz}$, $\left.\mathrm{CDCl}_{3}\right) \delta$ ppm: $7.33-7.27$ (m, 6H), $7.26(\mathrm{dt}, J=2.8,1.4 \mathrm{~Hz}, 1 \mathrm{H}), 7.25-7.17(\mathrm{~m}, 6 \mathrm{H})$, $7.08-7.02(\mathrm{~m}, 2 \mathrm{H}), 5.83(\mathrm{ddt}, J=17.0,10.2,6.6 \mathrm{~Hz}, 1 \mathrm{H}), 5.12(\mathrm{ddt}, J=10.2,2.2,1.2 \mathrm{~Hz}, 1 \mathrm{H}), 5.05(\mathrm{dq}, J=$ 17.1, $1.5 \mathrm{~Hz}, 1 \mathrm{H}), 4.86(\mathrm{~s}, 1 \mathrm{H}), 3.86$ (dd, $J=10.0,5.4 \mathrm{~Hz}, 1 \mathrm{H}), 3.50$ (dd, $J=10.0,8.6 \mathrm{~Hz}, 1 \mathrm{H}), 3.25-3.15$ (m, 2H), $3.13-3.05(\mathrm{~m}, 1 \mathrm{H}), 2.85(\mathrm{dd}, J=13.4,8.3 \mathrm{~Hz}, 1 \mathrm{H}), 2.69(\mathrm{dd}, J=13.3,6.7 \mathrm{~Hz}, 1 \mathrm{H}) ;{ }^{13} \mathbf{C} \mathbf{N M R}(126$ $\left.\mathrm{MHz}, \mathrm{CDCl}_{3}\right) \delta \mathrm{ppm}: 141.74,141.63,141.61,135.36,128.92,128.84,128.51,128.44,128.41,128.02,127.21$, $127.17,127.15,118.01,70.69,55.48,53.96,46.92,36.69$. IR (thin layer film) $v\left(\mathrm{~cm}^{-1}\right)=3062,3027,2980$ 1600, 1493, 1452, 1249, 1155, 1078, 1030, 996, 922, 759, 699, 611. HRMS (ESI $\left.{ }^{+}\right) m / z \mathrm{C}_{25} \mathrm{H}_{27} \mathrm{BrN}^{+}[\mathrm{M}+\mathrm{H}]^{+}$: calculated 420.1321 , found 420.1325 .

\section{tert-butyl 3-(m-tolyl)azetidine-1-carboxylate (5b)}

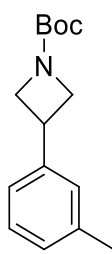

$5 \mathbf{b}$

Prepared according to general procedure A using commercially available 1-Boc-3-iodoazetidine (7.56 mmol, $2.14 \mathrm{~g})$ and 3-methylphenylboronic acid (15.1 mmol, $2.06 \mathrm{~g})$. The crude mixture was purified by flash column chromatography (Heptane:EtOAc $=100: 0$ to 90:10, gradient) to give the desired product in $57 \%$ yield $(4.34 \mathrm{mmol}, 1.07 \mathrm{~g})$ as a colorless oil. ${ }^{\mathbf{1}} \mathbf{H} \mathbf{~ N M R}\left(500 \mathrm{MHz}, \mathrm{CDCl}_{3}\right) \delta$ ppm: $7.24(\mathrm{t}, J=7.6 \mathrm{~Hz}, 1 \mathrm{H}), 7.15-7.04(\mathrm{~m}, 3 \mathrm{H}), 4.31(\mathrm{t}, J=8.6 \mathrm{~Hz}, 2 \mathrm{H}), 4.03-3.94(\mathrm{~m}, 2 \mathrm{H})$, 
$3.70(\mathrm{tt}, J=8.7,6.0 \mathrm{~Hz}, 1 \mathrm{H}), 2.36(\mathrm{~s}, 3 \mathrm{H}), 1.47(\mathrm{~s}, 9 \mathrm{H}) ;{ }^{13} \mathbf{C ~ N M R}\left(126 \mathrm{MHz}, \mathrm{CDCl}_{3}\right) \delta \mathrm{ppm}: 156.45,142.23$, 138.40, 128.61, 127.70, 127.48, 123.81, 79.49, 56.74, 33.44, 28.45, 21.43. IR (thin layer film) $v\left(\mathrm{~cm}^{-1}\right)=2973$, 2883, 1702, 1609, 1479, 1392, 1366, 1296, 1255, 1163, 1133, 863, 781, 702. HRMS (ESI $\left.{ }^{+}\right) \mathrm{m} / z \mathrm{C}_{15} \mathrm{H}_{22} \mathrm{NO}_{2}{ }^{+}$ $[\mathrm{M}+\mathrm{H}]^{+}$: calculated 248.1645, found 248.1646.

\section{1-benzhydryl-3-(m-tolylphenyl)azetidine (6b)}

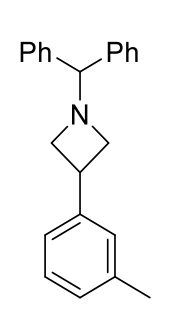

$6 b$

Prepared according to general procedure B using 1-Boc-3-(m-tolylphenyl)azetidine $\mathbf{5 b}$ $(4.04 \mathrm{mmol}, 1.00 \mathrm{~g})$. The crude mixture was purified by flash column chromatography (Heptane:EtOAc $=100: 0$ to $85: 15$, gradient $)$ to give the desired product in $65 \%$ yield $(2.63 \mathrm{mmol}$, $0.82 \mathrm{~g})$ as a colorless oil. ${ }^{1} \mathbf{H}$ NMR $\left(500 \mathrm{MHz}, \mathrm{CDCl}_{3}\right) \delta \mathrm{ppm}: 7.56-7.50(\mathrm{~m}, 4 \mathrm{H}), 7.35(\mathrm{dd}, J=$ 8.4, $6.9 \mathrm{~Hz}, 4 \mathrm{H}), 7.29-7.24(\mathrm{~m}, 3 \mathrm{H}), 7.20-7.13(\mathrm{~m}, 2 \mathrm{H}), 7.10(\mathrm{~d}, J=7.5 \mathrm{~Hz}, 1 \mathrm{H}), 4.48(\mathrm{~s}, 1 \mathrm{H})$, $3.81-3.69(\mathrm{~m}, 3 \mathrm{H}), 3.27-3.19(\mathrm{~m}, 2 \mathrm{H}), 2.41(\mathrm{~s}, 3 \mathrm{H}) ;{ }^{13} \mathbf{C ~ N M R}\left(126 \mathrm{MHz}, \mathrm{CDCl}_{3}\right) \delta \mathrm{ppm}:$ 143.06, 142.35, 138.05, 128.56, 128.44, 127.84, 127.67, 127.20, 127.14, 124.00, 78.42, 60.73, 34.86, 21.57. IR (thin layer film) $v\left(\mathrm{~cm}^{-1}\right)=3025,2945,2826,1607,1490,1452,1346,1275,1204,1147,1075,1029,927$, 778, 742, 698, 639, 621, 611. HRMS $\left(\mathrm{ESI}^{+}\right) \mathrm{m} / z \mathrm{C}_{23} \mathrm{H}_{24} \mathrm{~N}^{+}[\mathrm{M}+\mathrm{H}]^{+}$: calculated 314.1903, found 314.1902. Spectroscopic data were in agreement with the ones previously reported in literature. ${ }^{6}$

\section{1-benzhydryl-1-benzyl-3-bromo-2-(m-tolyl)propan-1-amine (7ba)}

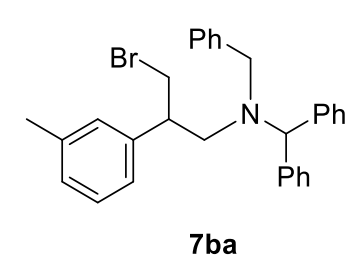

7 ba Prepared according to general procedure $\mathrm{C}$ using 1-benzhydryl-3-(m-tolyl)azetidine 6b $(1.28 \mathrm{mmol}, 0.40 \mathrm{~g})$ and benzyl bromide $(2.04 \mathrm{mmol}, 0.24 \mathrm{~mL})$. The crude mixture was purified by flash column chromatography (Pentane:EtOAc $=100: 0$ to $98: 2$, gradient) to afford the desired product in $46 \%$ yield $(0.58 \mathrm{mmol}, 0.28 \mathrm{~g})$ as a colorless oil. ${ }^{1} \mathbf{H}$ NMR $\left(500 \mathrm{MHz}, \mathrm{CDCl}_{3}\right) \delta$ ppm: $7.38(\mathrm{~d}, J=4.4 \mathrm{~Hz}, 4 \mathrm{H}), 7.34-7.22$ (m, $11 \mathrm{H}), 7.22-7.16(\mathrm{~m}, 1 \mathrm{H}), 7.12-7.08(\mathrm{~m}, 1 \mathrm{H}), 6.81-6.77(\mathrm{~m}, 1 \mathrm{H}), 6.75(\mathrm{~d}, J=1.9 \mathrm{~Hz}, 1 \mathrm{H}), 4.97(\mathrm{~s}, 1 \mathrm{H})$, $3.73(\mathrm{dd}, J=10.0,5.9 \mathrm{~Hz}, 1 \mathrm{H}), 3.70-3.61(\mathrm{~m}, 2 \mathrm{H}), 3.35(\mathrm{dd}, J=10.0,8.5 \mathrm{~Hz}, 1 \mathrm{H}), 3.13(\mathrm{qd}, J=7.5,5.8 \mathrm{~Hz}$, $1 \mathrm{H}), 2.89(\mathrm{dd}, J=13.3,7.3 \mathrm{~Hz}, 1 \mathrm{H}), 2.74(\mathrm{dd}, J=13.3,7.4 \mathrm{~Hz}, 1 \mathrm{H}), 2.33(\mathrm{~s}, 3 \mathrm{H}) ;{ }^{13} \mathbf{C ~ N M R}\left(126 \mathrm{MHz}, \mathrm{CDCl}_{3}\right)$ $\delta$ ppm: 141.51, 140.81, 140.41, 139.49, 138.02, 129.58, 129.22, 129.07, 128.77, 128.41, 128.40, 128.38, $128.27,127.97,127.33,127.12,127.11,125.14,69.51,55.67,55.65,46.90,36.56,21.63$. IR (thin layer film) $v\left(\mathrm{~cm}^{-1}\right)=3026,2921,2838,1604,1493,1451,1247,1076,1029,970,910,784,763,743,700$. HRMS $_{\left(\text {ESI }^{+}\right)}$ $m / z \mathrm{C}_{30} \mathrm{H}_{31} \mathrm{BrN}^{+}[\mathrm{M}+\mathrm{H}]^{+}$: calculated 484.1634 , found 484.1634 .

\section{tert-butyl 3-(3-methoxyphenyl)azetidine-1-carboxylate (5c)}

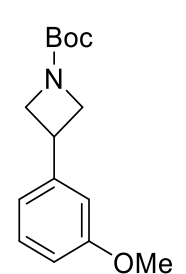

$5 c$

Prepared according to general procedure procedure A using commercially available 1-Boc-3iodoazetidine (7.50 mmol, $2.12 \mathrm{~g}$ ) and 3-methoxyphenylboronic acid (15.0 mmol, $2.28 \mathrm{~g})$. The crude mixture was purified by flash column chromatography (Heptane:EtOAc $=100: 0$ to 90:10, gradient) to give the desired product in $69 \%$ yield $(5.20 \mathrm{mmol}, 1.97 \mathrm{~g})$ as a colorless oil. ${ }^{1}$ H NMR (400 MHz, $\left.\mathrm{CDCl}_{3}\right) \delta$ ppm: $7.26(\mathrm{t}, J=7.9 \mathrm{~Hz}, 1 \mathrm{H}), 6.92-6.87(\mathrm{~m}, 1 \mathrm{H}), 6.86-6.83$ 
(m, 1H), $6.82-6.77(\mathrm{~m}, 1 \mathrm{H}), 4.35-4.25(\mathrm{~m}, 2 \mathrm{H}), 4.01-3.94(\mathrm{~m}, 2 \mathrm{H}), 3.81(\mathrm{~s}, 3 \mathrm{H}), 3.70(\mathrm{tt}, J=8.7,6.0 \mathrm{~Hz}$, 1H), 1.47 (s, 9H); $\left.{ }^{13} \mathbf{C ~ N M R ~ ( 1 2 6 ~ M H z , ~} \mathrm{CDCl}_{3}\right) \delta \mathrm{ppm:} \mathrm{160.03,} \mathrm{156.51,} \mathrm{143.99,} \mathrm{129.85,} \mathrm{119.18,} \mathrm{112.70,}$ 112.27, 79.62, 56.53, 55.35, 33.63, 28.53. IR (thin layer film) $v\left(\mathrm{~cm}^{-1}\right)=2971,2884,1698,1603,1586,1481$, 1456, 1390, 1366, 1293, 1254, 1158, 1130, 1041, 980, 918, 863, 774, 697. HRMS $\left(\mathrm{ESI}^{+}\right) \mathrm{m} / 2 \mathrm{C}_{15} \mathrm{H}_{22} \mathrm{NO}_{3}{ }^{+}$ $[\mathrm{M}+\mathrm{H}]^{+}$: calculated 264.1594, found 264.1595. Spectroscopic data were in agreement with the ones previously reported in literature. ${ }^{6,7}$

\section{1-benzhydryl-3-(3-methoxyphenyl)azetidine (6c)}

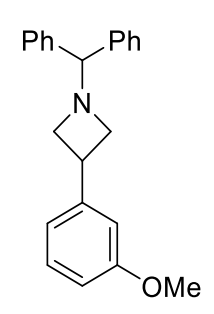

$6 \mathrm{c}$

Prepared according to general procedure procedure B using 1-Boc-3-(3methoxyphenyl)azetidine 5c (4.44 mmol, $1.17 \mathrm{~g})$. The crude mixture was purified by flash column chromatography (Heptane:EtOAc $=100: 0$ to $85: 15$, gradient) to give the desired product in $45 \%$ yield $(2.03 \mathrm{mmol}, 0.67 \mathrm{~g})$ as a colorless oil. ${ }^{1} \mathbf{H} \mathbf{~ N M R}\left(500 \mathrm{MHz}, \mathrm{CDCl}_{3}\right) \delta$ ppm: $7.45(\mathrm{dd}, J=8.2,1.4 \mathrm{~Hz}, 4 \mathrm{H}), 7.29(\mathrm{dd}, J=8.3,6.9 \mathrm{~Hz}, 4 \mathrm{H}), 7.24(\mathrm{t}, J=7.9 \mathrm{~Hz}, 1 \mathrm{H})$, $7.22-7.17(\mathrm{~m}, 2 \mathrm{H}), 6.92-6.85(\mathrm{~m}, 2 \mathrm{H}), 6.80-6.74(\mathrm{~m}, 1 \mathrm{H}), 4.40(\mathrm{~s}, 1 \mathrm{H}), 3.81(\mathrm{~s}, 3 \mathrm{H}), 3.73$ - 3.61 (m, 3H), 3.19 - $3.12(\mathrm{~m}, 2 \mathrm{H}) ;{ }^{13} \mathbf{C}$ NMR (126 MHz, $\left.\mathrm{CDCl}_{3}\right) \delta$ ppm: 159.83, 144.97, 142.35, 129.52, $128.59,127.66,127.23,119.46,112.99,111.54,78.39,60.66,55.33,34.96$; IR $\left(\right.$ thin layer film) $v\left(\mathrm{~cm}^{-1}\right)=$ 3026, 2942, 2832.34, 1601, 1583, 1489, 1451, 1434, 1290, 1267, 1207, 1158, 1076, 1046, 927, 868, 778, 744, 697, 638. HRMS $\left(\mathrm{ESI}^{+}\right) \mathrm{m} / z \mathrm{C}_{23} \mathrm{H}_{24} \mathrm{NO}^{+}[\mathrm{M}+\mathrm{H}]^{+}$: calculated 330.1852, found 330.1851. Spectroscopic data were in agreement with the ones previously reported in literature. ${ }^{6}$

\section{1-benzhydryl-1-benzyl-3-bromo-2-(3-methoxyphenyl)propan-1-amine (7ca)}

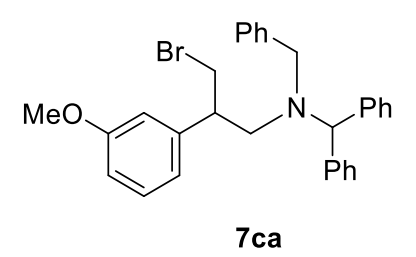

Prepared according to general procedure C using 1-benzhydryl-3-(3methoxyphenyl)azetidine $\mathbf{6 c}(1.21 \mathrm{mmol}, 0.40 \mathrm{~g}$ ) and benzyl bromide (1.94 mmol, $0.23 \mathrm{~mL})$. The crude mixture was purified by flash column chromatography (Pentane:EtOAc $=100: 0$ to $98: 2$, gradient) to afford the desired product in $83 \%$ yield $(1.00 \mathrm{mmol}, 0.50 \mathrm{~g})$ as a colorless oil. ${ }^{\mathbf{1}} \mathbf{H} \mathbf{~ N M R}\left(500 \mathrm{MHz}, \mathrm{CDCl}_{3}\right) \delta$ ppm: $7.40-7.35(\mathrm{~m}, 4 \mathrm{H})$, $7.34-7.24(\mathrm{~m}, 11 \mathrm{H}), 7.24-7.19(\mathrm{~m}, 1 \mathrm{H}), 6.82(\mathrm{dd}, J=8.0,2.6 \mathrm{~Hz}, 1 \mathrm{H}), 6.60-6.55(\mathrm{~m}, 1 \mathrm{H}), 6.48(\mathrm{t}, J=2.1$ Hz, 1H), 4.96 (s, 1H), 3.76 (s, 4H), $3.65(\mathrm{~d}, J=1.7 \mathrm{~Hz}, 2 \mathrm{H}), 3.32$ (dd, $J=10.0,8.6 \mathrm{~Hz}, 1 \mathrm{H}), 3.09$ (qd, $J=7.4$, $5.4 \mathrm{~Hz}, 1 \mathrm{H}), 2.89(\mathrm{dd}, J=13.4,7.5 \mathrm{~Hz}, 1 \mathrm{H}), 2.75(\mathrm{dd}, J=13.3,7.1 \mathrm{~Hz}, 1 \mathrm{H}) ;{ }^{13} \mathbf{C} \mathbf{~ N M R}\left(126 \mathrm{MHz}, \mathrm{CDCl}_{3}\right)$ $\delta$ ppm: 159.68, 143.28, 140.77, 140.57, 139.43, 129.51, 129.49, 129.22, 129.10, 128.44, 128.41, 128.32, $127.35,127.18,127.17,120.41,113.80,112.44,69.77,55.84,55.80,55.25,47.10,36.39$. IR (thin layer film) $v\left(\mathrm{~cm}^{-1}\right)=3027,2934,2834,1600,1492,1453,1262,1156,1047,970,873,764,744,699,616$. HRMS $_{\left(\text {ESI }^{+}\right)}$ $\mathrm{m} / \mathrm{z} \mathrm{C}_{30} \mathrm{H}_{31} \mathrm{BrNO}^{+}[\mathrm{M}+\mathrm{H}]^{+}$: calculated 500.1583, found 500.1581 . 


\section{tert-butyl 3-(3-chlorophenyl)azetidine-1-carboxylate (5d)}

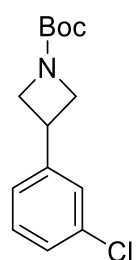

Prepared according to general procedure procedure A using commercially available 1-Boc-3iodoazetidine (15.1 mmol, $4.28 \mathrm{~g}$ ) and 3-chlorophenylboronic acid (30.3 mmol, $4.73 \mathrm{~g}$ ). The crude mixture was purified by flash column chromatography (Heptane:EtOAc $=100: 0$ to 90:10, gradient) to give the desired product in $71 \%$ yield $(10.7 \mathrm{mmol}, 2.86 \mathrm{~g})$ as a colorless oil. ${ }^{1} \mathbf{H}$ NMR $(400 \mathrm{MHz}$, $\left.\mathrm{CDCl}_{3}\right) \delta$ ppm: $7.32-7.27$ (m, 2H), $7.25-7.15(\mathrm{~m}, 2 \mathrm{H}), 4.37-4.28(\mathrm{~m}, 2 \mathrm{H}), 3.99-3.89(\mathrm{~m}, 2 \mathrm{H})$, $3.69(\mathrm{tt}, J=8.8,5.9 \mathrm{~Hz}, 1 \mathrm{H}), 1.47(\mathrm{~s}, 9 \mathrm{H}) ;{ }^{13} \mathrm{C}$ NMR $\left(101 \mathrm{MHz}, \mathrm{CDCl}_{3}\right) \delta \mathrm{ppm:} \mathrm{156.48,} \mathrm{144.46,}$ $134.75,130.14,127.31,127.17,125.09,79.85,56.50,33.36,28.55$. IR (thin layer film) $v\left(\mathrm{~cm}^{-1}\right)=2974,2885$, 1698, 1599, 1573, 1479, 1390, 1366, 1296, 1255, 1129, 1082, 999, 977, 913, 860, 775, 693. HRMS (ESI ${ }^{+}$ $m / z \mathrm{C}_{14} \mathrm{H}_{19} \mathrm{ClNO}_{2}{ }^{+}[\mathrm{M}+\mathrm{H}]^{+}$: calculated 268.1099, found 268.1099. Spectroscopic data were in agreement with the ones previously reported in literature. ${ }^{8}$

\section{1-benzhydryl-3-( $m$-chlorophenyl)azetidine (6d)}

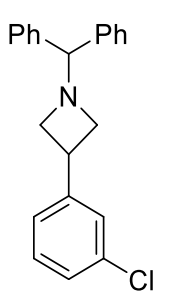

Prepared according to general procedure procedure B using 1-Boc-3-( $m$-chlorophenyl)azetidine 5d $(2.05 \mathrm{mmol}, 0.55 \mathrm{~g})$. The crude mixture was purified by flash column chromatography (Heptane:EtOAc $=100: 0$ to $85: 15$, gradient) to give the desired product in $40 \%$ yield $(0.75 \mathrm{mmol}, 0.25 \mathrm{~g})$ as a colorless oil. ${ }^{1} \mathbf{H} \mathbf{N M R}\left(500 \mathrm{MHz}, \mathrm{CDCl}_{3}\right) \delta \mathrm{ppm:} 7.53-7.39(\mathrm{~m}, 4 \mathrm{H})$, $7.32-7.27(\mathrm{~m}, 5 \mathrm{H}), 7.25-7.17(\mathrm{~m}, 5 \mathrm{H}), 4.40(\mathrm{~s}, 1 \mathrm{H}), 3.72-3.57(\mathrm{~m}, 3 \mathrm{H}), 3.18-3.08(\mathrm{~m}, 2 \mathrm{H})$; 6d ${ }^{13}$ C NMR (126 MHz, $\left.\mathrm{CDCl}_{3}\right) \delta$ ppm: 145.40, 142.19, 134.38, 129.81, 128.63, 127.64, 127.33, 127.30, 126.62, 125.33, 78.36, 60.53, 34.70. IR (thin layer film) $v\left(\mathrm{~cm}^{-1}\right)=3026,2953,2828,2360,1598$, 1571, 1489, 1452, 1432, 1347, 1275, 1198, 1153, 1078, 1029, 1000, 927, 876, 782, 745, 702, 694, 637, 621, 611. HRMS $\left(\mathrm{ESI}^{+}\right) \mathrm{m} / z \mathrm{C}_{22} \mathrm{H}_{21} \mathrm{ClN}^{+}[\mathrm{M}+\mathrm{H}]^{+}$: calculated 334.1357, found 334.1356.

\section{1-benzhydryl-1-benzyl-3-bromo-2-(3-chlorophenyl)propan-1-amine (7da)}

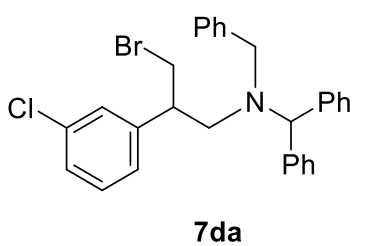

Prepared according to a slightly modified version of general procedure C. Under a flow of $\mathrm{N}_{2}$, an oven-dried flask was charged with 1-benzhydryl-3-(3chlorophenyl)azetidine $\mathbf{6 d}(5.72 \mathrm{mmol}, 1.91 \mathrm{~g})$ in dry $\mathrm{CH}_{3} \mathrm{CN}(0.8 \mathrm{M}, 7.0 \mathrm{~mL})$. Benzyl bromide (1.2 equiv., $6.87 \mathrm{mmol}, 0.82 \mathrm{~mL}$ ) was added and the mixture was stirred at $60{ }^{\circ} \mathrm{C}$ for $12 \mathrm{~h}$. The crude mixture was purified by flash column chromatography (Pentane:EtOAc $=100: 0$ to $98: 2$, gradient) to afford the desired product in $89 \%$ yield $(5.09 \mathrm{mmol}, 2.62 \mathrm{~g})$ as a colorless oil. ${ }^{1} \mathbf{H}$ NMR (400 MHz, $\left.\mathrm{CDCl}_{3}\right) \delta \mathrm{ppm}: 7.45-7.18(\mathrm{~m}, 17 \mathrm{H}), 6.91(\mathrm{t}, J=$ $1.9 \mathrm{~Hz}, 1 \mathrm{H}), 6.87-6.80(\mathrm{~m}, 1 \mathrm{H}), 4.94(\mathrm{~s}, 1 \mathrm{H}), 3.72-3.58(\mathrm{~m}, 3 \mathrm{H}), 3.28(\mathrm{dd}, J=10.1,8.4 \mathrm{~Hz}, 1 \mathrm{H}), 3.14-$ $3.03(\mathrm{~m}, 1 \mathrm{H}), 2.88(\mathrm{dd}, J=13.4,7.0 \mathrm{~Hz}, 1 \mathrm{H}), 2.75(\mathrm{dd}, J=13.3,7.7 \mathrm{~Hz}, 1 \mathrm{H}) ;{ }^{13} \mathbf{C} \mathbf{N M R}\left(101 \mathrm{MHz}, \mathrm{CDCl}_{3}\right)$ $\delta$ ppm: 143.66, 140.85, 140.23, 139.21, 134.32, 129.72, 129.55, 129.08, 129.06, 128.51, 128.47, 128.39, $128.15,127.46,127.38,127.30,127.25,126.44,69.98,55.95,55.61,46.80,35.84$. IR (thin layer film) $v\left(\mathrm{~cm}^{-1}\right)$ $=3060,3027,2924,2842,1598,1573,1493,1452,1246,1079,1029,809,784,763$, 744, 699, 623. HRMS $\left(\mathrm{ESI}^{+}\right) \mathrm{m} / z \mathrm{C}_{29} \mathrm{H}_{28} \mathrm{BrClN}^{+}[\mathrm{M}+\mathrm{H}]^{+}$: calculated 504.1088, found 504.1087. 


\section{tert-butyl 3-(4-fluorophenyl)azetidine-1-carboxylate (5e)}

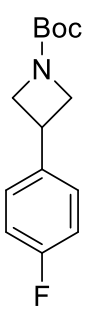

Prepared according to general procedure procedure A using 4-fluorophenylboronic acid (30.3 mmol, $4.24 \mathrm{~g}$ ). The crude mixture was purified by flash column chromatography (Heptane:EtOAc $=100: 0$ to $90: 10$, gradient) to give the desired product in $84 \%$ yield $(12.6 \mathrm{mmol}, 3.18 \mathrm{~g})$ as a colorless oil. ${ }^{1} \mathbf{H}$ NMR $\left(500 \mathrm{MHz}, \mathrm{CDCl}_{3}\right) \delta$ ppm: 7.31 - $7.24(\mathrm{~m}, 2 \mathrm{H}), 7.07-6.99(\mathrm{~m}, 2 \mathrm{H}), 4.38-4.29(\mathrm{~m}, 2 \mathrm{H})$, $3.97-3.89(\mathrm{~m}, 2 \mathrm{H}), 3.71(\mathrm{tt}, J=8.7,6.0 \mathrm{~Hz}, 1 \mathrm{H}), 1.47(\mathrm{~s}, 9 \mathrm{H}) ;{ }^{13} \mathbf{C} \mathbf{N M R}\left(126 \mathrm{MHz}, \mathrm{CDCl}_{3}\right) \delta \mathrm{ppm}$ :

5e $\quad 161.93(\mathrm{~d}, J=245.4 \mathrm{~Hz}), 156.51,138.13(\mathrm{~d}, J=3.1 \mathrm{~Hz}) 128.42(\mathrm{~d}, J=8.0 \mathrm{~Hz}), 115.66(\mathrm{~d}, J=$ $21.5 \mathrm{~Hz}), 79.74,56.65,33.01,28.54 ;{ }^{19} \mathbf{F}$ NMR $\left(377 \mathrm{MHz}, \mathrm{CDCl}_{3}\right) \delta \mathrm{ppm}:-115.78$ (tt, $\left.J=8.8,5.4 \mathrm{~Hz}, 1 \mathrm{~F}\right)$. IR (thin layer film) $v\left(\mathrm{~cm}^{-1}\right)=3658,3492,2980,2927,2344,2116,1983,1703,1607,1513,1457,1392,1228$, 1158, 1138, 953, 832, 774, 705, 640, 621. HRMS $\left(\mathrm{ESI}^{+}\right) \mathrm{m} / \mathrm{C}_{14} \mathrm{H}_{18} \mathrm{FNNaO}_{2}{ }^{+}[\mathrm{M}+\mathrm{Na}]^{+}$: calculated 274.1214, found 274.1215 . Spectroscopic data were in agreement with the ones previously reported in literature. ${ }^{7}$

\section{1-benzhydryl-3-(4-fluorophenyl)azetidine (6e)}

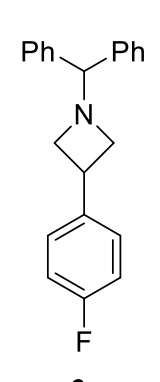

Prepared according to general procedure procedure B using 1-Boc-3-(4-fluorophenyl)azetidine 5e (12.3 mmol, $3.1 \mathrm{~g}$ ). The crude mixture was purified by flash column chromatography (Heptane:EtOAc $=100: 0$ to $85: 15$, gradient) to give the desired product in $39 \%$ yield $(4.8 \mathrm{mmol}$, $1.50 \mathrm{~g})$ as a colorless oil. ${ }^{1} \mathbf{H}$ NMR $\left(500 \mathrm{MHz}, \mathrm{CDCl}_{3}\right) \delta$ ppm: $7.49-7.44(\mathrm{~m}, 4 \mathrm{H}), 7.34-7.27(\mathrm{~m}$, $6 \mathrm{H}), 7.23-7.18(\mathrm{~m}, 2 \mathrm{H}), 7.05-6.98(\mathrm{~m}, 2 \mathrm{H}), 4.42(\mathrm{~s}, 1 \mathrm{H}), 3.71-3.61(\mathrm{~m}, 3 \mathrm{H}), 3.18-3.09(\mathrm{~m}$, 2H); ${ }^{13} \mathbf{C ~ N M R ~}\left(126 \mathrm{MHz}, \mathrm{CDCl}_{3}\right) \delta \mathrm{ppm:} 161.62(\mathrm{~d}, J=244.4 \mathrm{~Hz}), 142.23,138.97$ (d, $\left.J=3.2 \mathrm{~Hz}\right)$, 128.61, $128.54(\mathrm{~d}, J=8.0 \mathrm{~Hz}), 127.63,127.27,115.27$ (d, $J=21.3 \mathrm{~Hz}), 78.37,60.91,34.35$; ${ }^{19} \mathbf{F ~ N M R ~}\left\{{ }^{1} \mathrm{H}\right\}\left(470 \mathrm{MHz}, \mathrm{CDCl}_{3}\right) \delta \mathrm{ppm}:-116.85(\mathrm{~s}, 1 \mathrm{~F})$. IR (thin layer film) $v\left(\mathrm{~cm}^{-1}\right)=3027,2947,2827$, 1600, 1510, 1491, 1452, 1223, 1157, 1098, 1076, 1029, 924, 830, 743, 702. HRMS $\left(\mathrm{ESI}^{+}\right) \mathrm{m} / 2 \mathrm{C}_{22} \mathrm{H}_{21} \mathrm{FN}^{+}$ $[\mathrm{M}+\mathrm{H}]^{+}$: calculated 318.1653 , found 318.1652 . Spectroscopic data were in agreement with the ones previously reported in literature. ${ }^{6}$

\section{1-benzhydryl-1-benzyl-3-bromo-2-(3-fluorophenyl)propan-1-amine (7ea)}

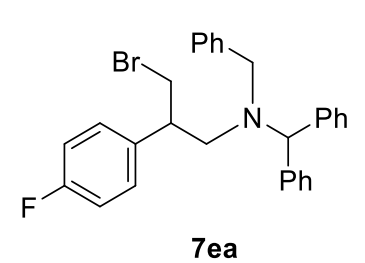

Prepared according to a slightly modified version of general procedure C. Under a flow of $\mathrm{N}_{2}$, an oven-dried flask was charged with 1-benzhydryl-3-(3fluorophenyl)azetidine 6 e (2.52 mmol, $0.80 \mathrm{~g})$ in dry $\mathrm{CH}_{3} \mathrm{CN}(1 \mathrm{M}, 2.5 \mathrm{~mL})$. Benzyl bromide ( 0.95 equiv., $42.39 \mathrm{mmol}, 0.28 \mathrm{~mL}$ ) was added and the mixture was stirred at $60{ }^{\circ} \mathrm{C}$ for $12 \mathrm{~h}$. The crude mixture was purified by flash column chromatography (Pentane:EtOAc = 100:0 to 98:2, gradient) to afford the desired product in 89\% yield $(2.24 \mathrm{mmol}, 1.09 \mathrm{~g})$ as a colorless oil. ${ }^{1} \mathbf{H}$ NMR $\left(400 \mathrm{MHz}, \mathrm{CDCl}_{3}\right) \delta$ ppm: $7.53-7.33(\mathrm{~m}, 15 \mathrm{H}), 7.15-7.06(\mathrm{~m}, 2 \mathrm{H}), 7.01$ (ddd, $J=$ 8.7, 5.3, 2.6 Hz, 2H), $5.08(\mathrm{~s}, 1 \mathrm{H}), 3.88-3.79(\mathrm{~m}, 1 \mathrm{H}), 3.77$ (d, J = 5.6 Hz, 2H), 3.40 (dd, J = 10.1, 8.5 Hz, 1H), $3.27-3.15(\mathrm{~m}, 1 \mathrm{H}), 3.00(\mathrm{dd}, J=13.3,7.3 \mathrm{~Hz}, 1 \mathrm{H}), 2.85(\mathrm{dd}, J=13.3,7.4 \mathrm{~Hz}, 1 \mathrm{H}) ;{ }^{13} \mathbf{C}$ NMR $(101$ $\left.\mathrm{MHz}, \mathrm{CDCl}_{3}\right) \delta \mathrm{ppm}: 161.99(\mathrm{~d}, J=245.1 \mathrm{~Hz}), 140.80,140.35,139.31,137.26$ (d, $\left.J=3.2 \mathrm{~Hz}\right), 129.52,129.46$ (d, $J=7.9 \mathrm{~Hz}), 129.14,129.11,128.47,128.44,128.36,127.42,127.25,127.23,115.31$ (d, $J=21.2 \mathrm{~Hz}), 69.88$, 
55.93, 55.75, 46.22, 36.55; ${ }^{19} \mathbf{F}$ NMR (377 MHz, $\left.\mathrm{CDCl}_{3}\right) \delta$ ppm: -115.63 (tt, $\left.J=8.7,5.3 \mathrm{~Hz}, 1 \mathrm{~F}\right)$. IR (thin layer film) $v\left(\mathrm{~cm}^{-1}\right)=3027,2833,1603,1509,1493,1451,1225,1159,1123,1076,1028,968,919,830,764$, 738, 698, 627. HRMS $\left(\mathrm{ESI}^{+}\right) \mathrm{m} / z \mathrm{C}_{29} \mathrm{H}_{28} \mathrm{BrFN}^{+}[\mathrm{M}+\mathrm{H}]^{+}$: calculated 488.1384, found. 488.1385.

\section{tert-butyl 3-(4-(trifluoromethyl)azetidine-1-carboxylate (5f)}

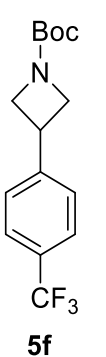

Prepared according to general procedure A using commercially available 1-Boc-3-iodoazetidine (15.1 mmol, $4.28 \mathrm{~g}$ ) and 4-(trifluoromethylphenylboronic acid (30.3 mmol, $5.75 \mathrm{~g}$ ). The crude mixture was purified by flash column chromatography (Heptane:EtOAc $=100: 0$ to $90: 10$, gradient) to give the desired product in $69 \%$ yield $(10.5 \mathrm{mmol}, 3.16 \mathrm{~g})$ as a colorless oil. ${ }^{1} \mathbf{H} \mathbf{~ N M R}\left(500 \mathrm{MHz}, \mathrm{CDCl}_{3}\right) \delta$ ppm: $7.61(\mathrm{~d}, J=8.0 \mathrm{~Hz}, 2 \mathrm{H}), 7.43(\mathrm{~d}, J=8.1 \mathrm{~Hz}, 2 \mathrm{H}), 4.36(\mathrm{~m}, J=8.7 \mathrm{~Hz}, 2 \mathrm{H}), 3.97(\mathrm{~m}, 2 \mathrm{H}), 3.78$ $5 f \quad(t \mathrm{t}, J=8.8,5.9 \mathrm{~Hz}, 1 \mathrm{H}), 1.47(\mathrm{~s}, 9 \mathrm{H}) ;{ }^{13} \mathbf{C ~ N M R}\left(126 \mathrm{MHz}, \mathrm{CDCl}_{3}\right) \delta \mathrm{ppm:} 156.47,146.45,129.48(\mathrm{q}$, $J=32.5 \mathrm{~Hz}), 127.30,125.84(\mathrm{q}, J=3.8 \mathrm{~Hz}), 124.24(\mathrm{q}, J=271.9 \mathrm{~Hz}), 79.93,56.55,33.45,28.53 ;{ }^{19} \mathbf{F}$ NMR $\left(470 \mathrm{MHz}, \mathrm{CDCl}_{3}\right) \delta-62.51$ (s, 3F). IR (thin layer film) $v\left(\mathrm{~cm}^{-1}\right)=2976,1702,1620,1393,1367,1326,1256$, 1164, 1125, 1069, 1017, 909, 836, 774. HRMS $\left(\mathrm{ESI}^{+}\right) \mathrm{m} / z \mathrm{C}_{15} \mathrm{H}_{19} \mathrm{~F}_{3} \mathrm{NO}_{2}{ }^{+}[\mathrm{M}+\mathrm{H}]^{+}$: calculated 302.1362, found 302.1363. Spectroscopic data were in agreement with the ones previously reported in literature. ${ }^{7}$

\section{1-benzhydryl-3-(4-(trifluoromethyl)phenyl)azetidine (6f)}

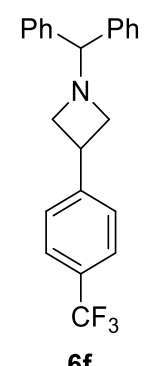

Prepared according to general procedure B using 1-Boc-3-(4-(trifluoromethyl)phenyl) azetidine $\mathbf{5 f}$ (10.5 mmol, $3.16 \mathrm{~g})$. The crude mixture was purified by flash column chromatography (Heptane:EtOAc $=100: 0$ to 90:10, gradient) to give the desired product in $45 \%$ yield $(6.68 \mathrm{mmol}$, $1.36 \mathrm{~g})$ as a colorless oil. ${ }^{1} \mathbf{H}$ NMR $\left(500 \mathrm{MHz}, \mathrm{CDCl}_{3}\right) \delta \mathrm{ppm}: 7.58(\mathrm{~d}, J=8.1 \mathrm{~Hz}, 2 \mathrm{H}), 7.48-7.42$ $(\mathrm{m}, 6 \mathrm{H}), 7.33-7.26(\mathrm{~m}, 4 \mathrm{H}), 7.24-7.17(\mathrm{~m}, 2 \mathrm{H}), 4.41(\mathrm{~s}, 1 \mathrm{H}), 3.75-3.68(\mathrm{~m}, 1 \mathrm{H}), 3.68-3.61$ 6f $\quad(\mathrm{m}, 2 \mathrm{H}), 3.20-3.14(\mathrm{~m}, 2 \mathrm{H}) ;{ }^{13} \mathbf{C} \mathbf{~ N M R}\left\{{ }^{1} \mathrm{H},{ }^{19} \mathrm{~F}\right\}\left(126 \mathrm{MHz}, \mathrm{CDCl}_{3}\right) \delta \mathrm{ppm}: 147.49,142.12$, $128.75,128.65,127.60,127.43,127.33,125.50,124.42,78.30,60.43,34.78 ;{ }^{19} \mathbf{F}$ NMR $\left(470 \mathrm{MHz}, \mathrm{CDCl}_{3}\right)$ $\delta$ ppm: -62.48 (s, 3F). IR (thin layer film) $v\left(\mathrm{~cm}^{-1}\right)=2954,2830,1619,1491,1453,1326,1164,1121,1069$, 1018, 835, 744, 704, 641. HRMS $\left(\mathrm{ESI}^{+}\right) \mathrm{m} / z \mathrm{C}_{23} \mathrm{H}_{21} \mathrm{~F}_{3} \mathrm{~N}^{+}[\mathrm{M}+\mathrm{H}]^{+}$: calculated 368.1621, found 368.1620.

\section{1-benzhydryl-1-benzyl-3-bromo-2-(4-(trifluoromethyl)phenyl)propan-1-amine (7fa)}<smiles>FC(F)(F)c1ccc(C(CBr)CN(Cc2ccccc2)C(c2ccccc2)c2ccccc2)cc1</smiles>

$7 f a$

Prepared according to a slightly modified version of general procedure C. Under a flow of $\mathrm{N}_{2}$, an oven-dried flask was charged with 1-benzhydryl-3-(4(trifluoromethyl) phenylazetidine $\mathbf{6 f}(2.18 \mathrm{mmol}, 0.80 \mathrm{~g})$ in dry $\mathrm{CH}_{3} \mathrm{CN}$ (1 M, $2.1 \mathrm{~mL})$. Benzyl bromide ( 0.95 equiv., $2.07 \mathrm{mmol}, 0.25 \mathrm{~mL}$ ) was added and the mixture was stirred at $60{ }^{\circ} \mathrm{C}$ for $12 \mathrm{~h}$. The crude mixture was purified by flash column chromatography (Pentane: $\mathrm{Et}_{2} \mathrm{O}=100: 0$ to $98: 2$, gradient) to afford the desired product in $87 \%$ yield (1.90 mmol, $1.02 \mathrm{~g}$ ) as a colorless oil. ${ }^{1} \mathbf{H}$ NMR (400 MHz, $\left.\mathrm{CDCl}_{3}\right) \delta \mathrm{ppm:} 7.41(\mathrm{~d}, J=8.0 \mathrm{~Hz}, 2 \mathrm{H}), 7.33-$ $7.07(\mathrm{~m}, 15 \mathrm{H}), 6.90(\mathrm{~d}, J=8.0 \mathrm{~Hz}, 2 \mathrm{H}), 4.83(\mathrm{~s}, 1 \mathrm{H}), 3.64-3.53(\mathrm{~m}, 2 \mathrm{H}), 3.50(\mathrm{~d}, J=13.7 \mathrm{~Hz}, 1 \mathrm{H}), 3.18$ $(\mathrm{dd}, J=10.1,8.6 \mathrm{~Hz}, 1 \mathrm{H}), 3.08-2.96(\mathrm{~m}, 1 \mathrm{H}), 2.79(\mathrm{dd}, J=13.4,7.1 \mathrm{~Hz}, 1 \mathrm{H}), 2.66(\mathrm{dd}, J=13.4,7.4 \mathrm{~Hz}$, 
1H); ${ }^{13} \mathbf{C}$ NMR $\left\{{ }^{1} \mathrm{H},{ }^{19} \mathrm{~F}\right\}\left(126 \mathrm{MHz}, \mathrm{CDCl}_{3}\right)$ [overlapping signals] $\delta \mathrm{ppm:} \mathrm{145.70,} \mathrm{140.79,} \mathrm{140.19,} \mathrm{139.14,}$ 130.22, 129.52, 129.38, 129.11, 129.05, 128.51, 128.40, 127.51, 127.34, 127.30, 125.42, 124.35, 70.09, 56.12, 55.77, 46.92, 35.65; ${ }^{19} \mathbf{F}$ NMR (377 MHz, $\left.\mathrm{CDCl}_{3}\right) \delta \mathrm{ppm}$ : -62.38. IR (thin layer film) $v\left(\mathrm{~cm}^{-1}\right)=3028,1619$, 1494, 1452, 1325, 1165, 1123, 1069, 1018, 836, 764, 745, 700. HRMS $\left(\mathrm{ESI}^{+}\right) \mathrm{m} / z \mathrm{C}_{30} \mathrm{H}_{28} \mathrm{BrF}_{3} \mathrm{~N}^{+}[\mathrm{M}+\mathrm{H}]^{+}$: calculated 538.1352, found. 538.1350.

\section{tert-butyl 3-([1,1'-biphenyl]-4-yl)azetidine-1-carboxylate (5g)}

Boc Prepared according to general procedure A using commercially available 1-Boc-3-iodoazetidine<smiles>CN1CC(c2ccc(-c3ccccc3)cc2)C1</smiles>
(7.57 mmol, $2.14 \mathrm{~g}$ ) and [1,1'-biphenyl]-4-ylboronic acid (15.1 mmol, $3.00 \mathrm{~g})$. The crude mixture was purified by flash column chromatography (Heptane:EtOAc $=100: 0$ to $90: 10$, gradient) to give the desired product in $77 \%$ yield $(5.82 \mathrm{mmol}, 1.80 \mathrm{~g})$ as a colorless oil. ${ }^{1} \mathbf{H}$ NMR $\left(500 \mathrm{MHz}, \mathrm{CDCl}_{3}\right) \delta$ ppm: $7.62-7.57(\mathrm{~m}, 4 \mathrm{H}), 7.45(\mathrm{dd}, J=8.4,7.0 \mathrm{~Hz}, 2 \mathrm{H}), 7.42-7.38(\mathrm{~m}, 2 \mathrm{H}), 7.38-7.33(\mathrm{~m}, 1 \mathrm{H})$,

5g $\quad 4.43-4.31(\mathrm{~m}, 2 \mathrm{H}), 4.10-3.99(\mathrm{~m}, 2 \mathrm{H}), 3.78(\mathrm{tt}, J=8.8,6.0 \mathrm{~Hz}, 1 \mathrm{H}), 1.49(\mathrm{~s}, 9 \mathrm{H}) ;{ }^{13} \mathbf{C} \mathbf{N M R}(126$ $\left.\mathrm{MHz}, \mathrm{CDCl}_{3}\right) \delta$ ppm: 156.54, 141.43, 140.82, 140.08, 128.91, 127.55, 127.43, 127.34, 127.15, 79.66, 56.95, 33.35, 28.56. IR (thin layer film) $v\left(\mathrm{~cm}^{-1}\right)=2972,2884,1697,1485,1391,1366,1296,1254,1130,1008,966$, 909, 834, 764, 730, 698. HRMS $\left(\mathrm{ESI}^{+}\right) \mathrm{m} / z \mathrm{C}_{20} \mathrm{H}_{24} \mathrm{NO}_{2}{ }^{+}[\mathrm{M}+\mathrm{H}]^{+}$: calculated 310.1802, found 310.1802.

\section{1-benzhydryl-3-([1,1'-biphenyl]-4-yl)azetidine (6g)}

$\mathrm{Ph}_{Y} \mathrm{Ph}$ Prepared according to general procedure B using 1-Boc-3-([1,1'-biphenyl]-4-yl)phenyl)azetidine 5g (4.95 mmol, $1.53 \mathrm{~g})$. The crude mixture was purified by flash column chromatography (Heptane:EtOAc $=100: 0$ to $85: 15$, gradient) to give the desired product in $46 \%$ yield $(2.28 \mathrm{mmol}$, $0.85 \mathrm{~g})$ as a colorless oil. ${ }^{1} \mathbf{H}$ NMR $\left(500 \mathrm{MHz}, \mathrm{CDCl}_{3}\right) \delta \mathrm{ppm}: 7.63-7.54(\mathrm{~m}, 4 \mathrm{H}), 7.51-7.47(\mathrm{~m}$, 4H), $7.47-7.42(\mathrm{~m}, 2 \mathrm{H}), 7.40(\mathrm{~d}, J=2.1 \mathrm{~Hz}, 2 \mathrm{H}), 7.38-7.33(\mathrm{~m}, 1 \mathrm{H}), 7.33-7.28(\mathrm{~m}, 4 \mathrm{H}), 7.24$ $-7.18(\mathrm{~m}, 2 \mathrm{H}), 4.44(\mathrm{~s}, 1 \mathrm{H}), 3.80-3.71(\mathrm{~m}, 1 \mathrm{H}), 3.71-3.66(\mathrm{~m}, 2 \mathrm{H}), 3.24-3.18(\mathrm{~m}, 2 \mathrm{H}) ;{ }^{13} \mathrm{C}$ NMR (126 MHz, $\mathrm{CDCl}_{3}$ ) [overlapping signals] $\delta$ ppm: 142.35 (br), 141.10, 139.44, 128.89, 128.60, 127.68, 127.54, 127.29, 127.25, 127.16, 78.42, 60.81, 34.72. IR (thin layer film) $v\left(\mathrm{~cm}^{-1}\right)=3658,2980,2889,1462$, 1383, 1252, 1152, 1073, 955, 816. HRMS $\left(\mathrm{ESI}^{+}\right) \mathrm{m} / z \mathrm{C}_{28} \mathrm{H}_{26} \mathrm{~N}^{+}[\mathrm{M}+\mathrm{H}]^{+}$: calculated 376.2060, found 376.2059. Spectroscopic data were in agreement with the ones previously reported in literature. ${ }^{6}$

\section{2-([1,1'-biphenyl]-4-yl)-1-benzhydryl-1-benzyl-3-bromopropan-1-amine (7ga)}

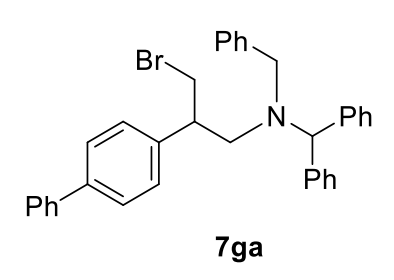

Prepared according to a slightly modified version of general procedure C. Under a flow of $\mathrm{N}_{2}$, an oven-dried flask was charged with 1-benzhydryl-3-([1,1'-biphenyl]4-yl)azetidine $6 \mathrm{~g}$ (2.13 mmol, $0.80 \mathrm{~g}$ ) in dry $\mathrm{CH}_{3} \mathrm{CN}$ (1 M, $\left.2.5 \mathrm{~mL}\right)$. Benzyl bromide (1.2 equiv., $2.56 \mathrm{mmol}, 0.30 \mathrm{~mL}$ ) was added and the mixture was stirred at $60^{\circ} \mathrm{C}$ for $12 \mathrm{~h}$. The crude mixture was purified by flash column chromatography (Pentane $100 \%)$ to afford the desired product in $43 \%$ yield $(0.92 \mathrm{mmol}, 0.50 \mathrm{~g})$ as a colorless oil. ${ }^{1} \mathbf{H}$ NMR $(400 \mathrm{MHz}$, $\left.\mathrm{CDCl}_{3}\right) \delta$ ppm: $7.56-7.49(\mathrm{~m}, 2 \mathrm{H}), 7.49-7.10(\mathrm{~m}, 20 \mathrm{H}), 6.96-6.89(\mathrm{~m}, 2 \mathrm{H}), 4.89(\mathrm{~s}, 1 \mathrm{H}), 3.68(\mathrm{dd}, J=$ 
$10.0,5.7 \mathrm{~Hz}, 1 \mathrm{H}), 3.57(\mathrm{~d}, J=3.1 \mathrm{~Hz}, 2 \mathrm{H}), 3.28(\mathrm{dd}, J=10.1,8.5 \mathrm{~Hz}, 1 \mathrm{H}), 3.13-3.02(\mathrm{~m}, 1 \mathrm{H}), 2.83(\mathrm{dd}, J=$ 13.3, 7.3 Hz, 1H), $2.69(\mathrm{dd}, J=13.3,7.3 \mathrm{~Hz}, 1 \mathrm{H}) ;{ }^{13} \mathbf{C ~ N M R}\left(101 \mathrm{MHz}, \mathrm{CDCl}_{3}\right)$ [overlapping signals] $\delta$ ppm: $141.05,140.83$, 140.72, 140.45, 140.04, 139.43, 129.57, 129.21, 129.13, 128.92, 128.46, 128.43, 128.33, $127.39,127.35,127.23,127.20,127.18,69.75,55.86,55.82,46.69,36.47$. IR (thin layer film) $v\left(\mathrm{~cm}^{-1}\right)=3059$, 3027, 2922, 2837, 1600, 1487, 1451, 1247, 1122, 1075, 1029, 1008, 918, 834, 764, 743, 699, 653. HRMS $\left(\mathrm{ESI}^{+}\right) \mathrm{m} / z \mathrm{C}_{35} \mathrm{H}_{33} \mathrm{BrN}^{+}[\mathrm{M}+\mathrm{H}]^{+}$: calculated 546.1791, found 546.1790.

\section{tert-butyl 3-(p-tolyl)azetidine-1-carboxylate (5h)}

Boc Prepared according to general procedure A using commercially available 1-Boc-3-iodoazetidine (3.75 $\left\langle\hat{N}^{N}\right.$ mmol, $\left.1.06 \mathrm{~g}\right)$ and 4-methyphenylboronic acid $(7.50 \mathrm{mmol}, 1.02 \mathrm{~g})$. The crude mixture was purified by flash column chromatography (Heptane:EtOAc $=100: 0$ to $90: 10$, gradient) to give the desired product in $75 \%$ yield $(2.81 \mathrm{mmol}, 0.66 \mathrm{~g})$ as a colorless oil. ${ }^{1} \mathbf{H}$ NMR $\left(500 \mathrm{MHz}, \mathrm{CDCl}_{3}\right) \delta$ ppm: 7.23 $-7.18(\mathrm{~m}, 2 \mathrm{H}), 7.18-7.13(\mathrm{~m}, 2 \mathrm{H}), 4.31(\mathrm{t}, J=8.7 \mathrm{~Hz}, 2 \mathrm{H}), 3.95(\mathrm{dd}, J=8.6,6.1 \mathrm{~Hz}, 2 \mathrm{H}), 3.74-$

5h $3.65(\mathrm{~m}, 1 \mathrm{H}), 2.34(\mathrm{~s}, 3 \mathrm{H}), 1.47(\mathrm{~s}, 9 \mathrm{H}) ;{ }^{13} \mathbf{C ~ N M R}\left(126 \mathrm{MHz}, \mathrm{CDCl}_{3}\right) \delta$ ppm: $156.58,139.39,136.73$, 129.51, 126.80, 79.59, 56.73, 33.32, 28.58, 21.18. IR (thin layer film) $v\left(\mathrm{~cm}^{-1}\right)=2980,2885,1701,1517$, 1479, 1456, 1392, 1366, 1296, 1252, 1161, 1132, 966, 909, 860, 813, 773. HRMS (ESI $\left.{ }^{+}\right) m / z \mathrm{C}_{15} \mathrm{H}_{23} \mathrm{NNaO}_{2}{ }^{+}$ $[\mathrm{M}+\mathrm{Na}]^{+}$: calculated 270.1465 , found 270.1466 . Spectroscopic data were in agreement with the ones previously reported in literature. $^{7}$

\section{1-benzhydryl-3-(4-methyphenyl)azetidine (6h)}

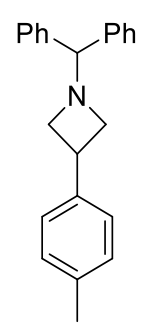

Prepared according to general procedure B using 1-Boc-3-(4-methyphenyl)azetidine 5h (5.43 mmol, $1.34 \mathrm{~g}$ ). The crude mixture was purified by flash column chromatography (Heptane:EtOAc $=100: 0$ to $85: 15$, gradient) to give the desired product in $58 \%$ yield $(3.2 \mathrm{mmol}, 0.99 \mathrm{~g})$ as a colorless oil. ${ }^{1} \mathbf{H}$ NMR (500 MHz, $\left.\mathrm{CDCl}_{3}\right) \delta$ ppm: 7.49 - 7.44 (m, 4H), 7.33 - 7.27 (m, 4H), 7.25 - 7.18 (m, $4 \mathrm{H}), 7.14(\mathrm{~d}, J=8.0 \mathrm{~Hz}, 2 \mathrm{H}), 4.42(\mathrm{~s}, 1 \mathrm{H}), 3.74-3.62(\mathrm{~m}, 3 \mathrm{H}), 3.19-3.11(\mathrm{~m}, 2 \mathrm{H}), 2.35(\mathrm{~s}, 3 \mathrm{H})$;

$6 \mathrm{~h}$ ${ }^{13}$ C NMR (126 MHz, $\mathrm{CDCl}_{3}$ ) $\delta$ ppm: 142.41, 140.18, 135.97, 129.20, 128.57, 127.67, 127.20, 126.98, 78.42, 60.92, 34.66, 21.16. IR (thin layer film) $v\left(\mathrm{~cm}^{-1}\right)=3025,2922,2826,1599,1515,1490,1452$, 1344, 1309, 1273, 1197, 1151, 1113, 1076, 1029, 924, 811, 742, 702, 6218, 611. HRMS (ESI $\left.{ }^{+}\right) \mathrm{m} / \mathrm{z} \mathrm{C}_{23} \mathrm{H}_{24} \mathrm{~N}^{+}$ $[\mathrm{M}+\mathrm{H}]^{+}$: calculated 314.1903 , found 314.1903 . Spectroscopic data were in agreement with the ones previously reported in literature. ${ }^{6}$

\section{1-benzhydryl-1-benzyl-3-bromo-2-(4-methyphenyl)propan-1-amine (7ha)}

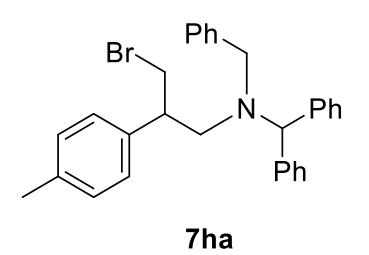

Prepared according to a slightly modified version of general procedure C. Under a flow of $\mathrm{N}_{2}$, an oven-dried flask was charged with 1-benzhydryl-3-(4methylphenyl)azetidine $\mathbf{6 h}(1.82 \mathrm{mmol}, 0.57 \mathrm{~g})$ in dry $\mathrm{CH}_{3} \mathrm{CN}(1 \mathrm{M}, 1.8 \mathrm{~mL})$. Benzyl bromide (1.2 equiv., $2.18 \mathrm{mmol}, 0.26 \mathrm{~mL}$ ) was added and the mixture was stirred at $60{ }^{\circ} \mathrm{C}$ for $12 \mathrm{~h}$. The crude mixture was purified by flash column chromatography (Pentane 100\%) to afford 
the desired product in $67 \%$ yield $(1.21 \mathrm{mmol}, 0.59 \mathrm{~g})$ as a colorless oil. ${ }^{1} \mathbf{H}$ NMR $\left(400 \mathrm{MHz}, \mathrm{CDCl}_{3}\right) \delta$ ppm: $7.44-7.22(\mathrm{~m}, 15 \mathrm{H}), 7.11(\mathrm{~d}, J=7.8 \mathrm{~Hz}, 2 \mathrm{H}), 6.90-6.83(\mathrm{~m}, 2 \mathrm{H}), 4.97(\mathrm{~s}, 1 \mathrm{H}), 3.79(\mathrm{dd}, J=10.0,5.5 \mathrm{~Hz}$, 1H), $3.73-3.59(\mathrm{~m}, 2 \mathrm{H}), 3.33(\mathrm{dd}, J=10.0,8.8 \mathrm{~Hz}, 1 \mathrm{H}), 3.16-3.04(\mathrm{~m}, 1 \mathrm{H}), 2.88(\mathrm{dd}, J=13.3,7.9 \mathrm{~Hz}, 1 \mathrm{H})$, $2.73(\mathrm{dd}, J=13.3,6.9 \mathrm{~Hz}, 1 \mathrm{H}), 2.37$ (s, 3H); ${ }^{13} \mathbf{C ~ N M R}\left(101 \mathrm{MHz}, \mathrm{CDCl}_{3}\right.$ ) [overlapping signals] $\delta$ ppm: $140.69,140.64,139.45,138.51,136.72,129.46,129.29$, 129.24, 129.11, 128.44, 128.39, 128.30, 127.86, 127.31, 127.16, 69.67, 55.85, 55.79, 46.57, 36.83, 21.27. IR (thin layer film) $v\left(\mathrm{~cm}^{-1}\right)=3026,2919,2850$, $1738,1635,1600,1514,1493,1452,1371,1244,1121,1076,1028,918,812,763,743,700$. HRMS (ESI $\left.{ }^{+}\right)$ $m / z \mathrm{C}_{30} \mathrm{H}_{31} \mathrm{BrN}^{+}[\mathrm{M}+\mathrm{H}]^{+}$: calculated 484.1634, found 484.1635.

\section{1-benzhydryl-1-benzyl-3-bromo-2-phenoxypropan-1-amine (7ia)}

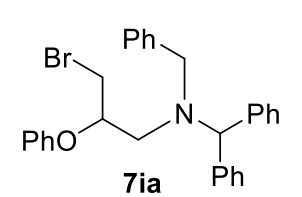

Prepared according to a slightly modified version of general procedure C. Under a flow of $\mathrm{N}_{2}$, an oven-dried flask was charged with commercially available 1-benzhydryl-3phenoxyazetidine $(4.75 \mathrm{mmol}, 1.5 \mathrm{~g})$ in dry $\mathrm{CH}_{3} \mathrm{CN}(5.8 \mathrm{~mL})$. Benzyl bromide $(1.2$ equiv., $5.71 \mathrm{mmol}, 0.68 \mathrm{~mL}$ ) was added and the mixture was stirred at $60{ }^{\circ} \mathrm{C}$ for $12 \mathrm{~h}$.

The residue was purified by flash column chromatography (Pentane:EtOAc $=98: 2$ ) to afford the desired product in quantitative yield $(4.75 \mathrm{mmol}, 1.94 \mathrm{~g})$ as a colorless oil. ${ }^{1} \mathbf{H} \mathbf{~ N M R}\left(400 \mathrm{MHz}, \mathrm{CDCl}_{3}\right) \delta \mathrm{ppm:} 7.50$ - 7.21 (m, 17H), $7.02-6.93(\mathrm{~m}, 1 \mathrm{H}), 6.77-6.69(\mathrm{~m}, 2 \mathrm{H}), 5.03(\mathrm{~s}, 1 \mathrm{H}), 4.37-4.27(\mathrm{~m}, 1 \mathrm{H}), 3.84-3.70(\mathrm{~m}$, 2H), $3.52(\mathrm{dd}, J=10.8,5.0 \mathrm{~Hz}, 1 \mathrm{H}), 3.36(\mathrm{dd}, J=10.7,5.2 \mathrm{~Hz}, 1 \mathrm{H}), 2.98(\mathrm{dd}, J=6.0,1.1 \mathrm{~Hz}, 2 \mathrm{H}) ;{ }^{13} \mathbf{C} \mathbf{~ N M R}$ $\left(101 \mathrm{MHz}, \mathrm{CDCl}_{3}\right) \delta$ ppm: 157.63, 141.34, 140.91, 139.50, 129.69, 129.38, 129.15, 129.11, 128.57, 128.53, $128.51,127.43,127.35,127.31,121.58,116.19,76.80,71.15,56.92,53.55,33.29$. IR (thin layer film) $v\left(\mathrm{~cm}^{-}\right.$ $\left.{ }^{1}\right)=3027,2835,1598,1492,1452,1237,1172,1077,1029,921,745,698$. HRMS $\left(\mathrm{ESI}^{+}\right) \mathrm{m} / \mathrm{z} \mathrm{C}_{29} \mathrm{H}_{29} \mathrm{BrNO}^{+}$ $[\mathrm{M}+\mathrm{H}]^{+}:$calculated 486.1427 , found 486.1426 .

\section{1-benzhydryl-1-(3-bromo-2-phenoxypropyl)prop-2-en-1-amine (7ic)}

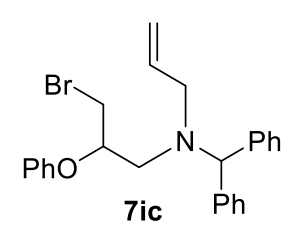

Prepared according to a slightly modified version of general procedure C. Under a flow of $\mathrm{N}_{2}$, an oven-dried flask was charged with commercially available 1-benzhydryl-3phenoxyazetidine ( $2.53 \mathrm{mmol}, 0.8 \mathrm{~g}$ ) in $\mathrm{CH}_{3} \mathrm{CN}$ (2.3 mL). Benzyl bromide (1.2 equiv., $2.28 \mathrm{mmol}, 0.20 \mathrm{~mL}$ ) was added and the mixture was stirred at $60^{\circ} \mathrm{C}$ for $12 \mathrm{~h}$. The residue was purified by flash column chromatography (Pentane: $\mathrm{Et}_{2} \mathrm{O}=98.5: 1.5$ ) to afford the desired product in $62 \%$ yield $(0.79 \mathrm{~g}, 1.57 \mathrm{mmol})$ as colorless oil. ${ }^{1} \mathbf{H}$ NMR (400 MHz, $\left.\mathrm{CDCl}_{3}\right) \delta \mathrm{ppm}: 7.32-7.10(\mathrm{~m}, 12 \mathrm{H}), 6.88$ (tt, $J=7.3,1.1 \mathrm{~Hz}, 1 \mathrm{H}), 6.75-6.67(\mathrm{~m}, 2 \mathrm{H}), 5.84(\mathrm{ddt}, J=16.8,10.2,6.5 \mathrm{~Hz}, 1 \mathrm{H}), 5.11-4.97(\mathrm{~m}, 2 \mathrm{H}), 4.87$ (s, $1 \mathrm{H}), 4.40-4.30(\mathrm{~m}, 1 \mathrm{H}), 3.59(\mathrm{dd}, J=10.7,4.8 \mathrm{~Hz}, 1 \mathrm{H}), 3.44(\mathrm{dd}, J=10.7,5.4 \mathrm{~Hz}, 1 \mathrm{H}), 3.20-3.10(\mathrm{~m}, 2 \mathrm{H})$, $2.84(\mathrm{~d}, J=6.2 \mathrm{~Hz}, 2 \mathrm{H}) ;{ }^{13} \mathbf{C} \mathbf{N M R}\left(101 \mathrm{MHz}, \mathrm{CDCl}_{3}\right) \delta \mathrm{ppm}: 157.82,141.87,141.67,135.38,129.69,129.03$, $128.75,128.54,128.53,127.37,127.24,121.63,118.26,116.36,77.48,71.50,55.37,52.95,33.53$. IR (thin layer film) $v\left(\mathrm{~cm}^{-1}\right)=3061,3027,2834,1598,1587,1492,1452,1418,1336,1289,1237.1171,1077,1029$, 995, 922, 823, 750, 704, 610. HRMS $\left(\mathrm{ESI}^{+}\right): \mathrm{m} / z \mathrm{C}_{25} \mathrm{H}_{27} \mathrm{BrNO}^{+}[\mathrm{M}+\mathrm{H}]^{+}$: calculated 436.1271, found 436.1270 . 


\section{1-benzhydryl-1-benzyl-3-bromo-2-(4-fluorophenoxy)propan-1-amine (7ja)}

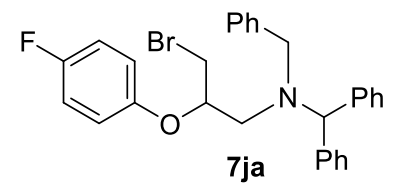

Prepared according to general procedure $\mathrm{C}$ using commercially available 1-benzhydryl-3-(4-fluorophenoxy)azetidine $(0.60 \mathrm{mmol}, 0.20 \mathrm{~g})$ and benzyl bromide $(0.96 \mathrm{mmol}, 0.11 \mathrm{~mL})$. The residue was purified by flash column chromatography (Heptane:EtOAc $=95: 5)$ to afford the desired product in $62 \%$ yield $(0.19 \mathrm{~g}, 0.38 \mathrm{mmol})$ as colorless oil. ${ }^{1} \mathbf{H}$ NMR $\left(400 \mathrm{MHz}, \mathrm{CDCl}_{3}\right) \delta$ ppm: $7.44-7.26(\mathrm{~m}, 15 \mathrm{H}), 6.95-6.84(\mathrm{~m}, 2 \mathrm{H}), 6.66-6.56(\mathrm{~m}$, 2H), $4.98(\mathrm{~s}, 1 \mathrm{H}), 4.16-4.06(\mathrm{~m}, 1 \mathrm{H}), 3.79-3.66(\mathrm{~m}, 2 \mathrm{H}), 3.50(\mathrm{dd}, J=10.8,4.6 \mathrm{~Hz}, 1 \mathrm{H}), 3.30$ (dd, $J=10.8$, $5.5 \mathrm{~Hz}, 1 \mathrm{H}), 2.99-2.85(\mathrm{~m}, 2 \mathrm{H}) 1 ;{ }^{13} \mathbf{C}$ NMR $\left(101 \mathrm{MHz}, \mathrm{CDCl}_{3}\right) \delta \mathrm{ppm:} 157.80$ (d, $\left.J=239.4 \mathrm{~Hz}\right), 153.76$, $141.30,140.89,139.42,129.36,129.16,129.07,128.60,128.57,128.56,127.49,127.42,127.37,117.68$ (d, $J$ $=7.9 \mathrm{~Hz}), 116.02(\mathrm{~d}, J=23.0 \mathrm{~Hz}), 78.06,71.41,57.14,53.72,33.40 ;{ }^{19} \mathbf{F} \mathbf{N M R}\left(470 \mathrm{MHz}, \mathrm{CDCl}_{3}\right) \delta \mathrm{ppm}:-$ 78.22. IR (thin layer film) $v\left(\mathrm{~cm}^{-1}\right)=3065,1498,1459,1257,1224,1155,1030,902,851,754,700,638$. HRMS (ESI ${ }^{+}$: $m / z \mathrm{C}_{29} \mathrm{H}_{28} \mathrm{BrFNO}^{+}[\mathrm{M}+\mathrm{H}]^{+}$: calculated 504.1333, found 504.1332.

\section{1-benzhydryl-3-isopropoxyazetidine (6k)}

$\mathrm{Ph} Y^{\mathrm{Ph}}$ Prepared according to a slightly modified version of general procedure B. To a solution of ${ }^{N} \quad$ commercially available 3-isopropyloxyazetidine hydrochloride (10.02 mmol, $\left.1.52 \mathrm{~g}\right)$ in dry $\mathrm{CH}_{3} \mathrm{CN}(9.2 \mathrm{~mL})$, DIPEA (2.7 equiv., $27.32 \mathrm{mmol}, 4.7 \mathrm{~mL}$ ) and bromodiphenylmethane (0.9 equiv., $9.10 \mathrm{mmol}, 2.25 \mathrm{~g}$ ) were subsequently added. The reaction mixture was stirred at $100{ }^{\circ} \mathrm{C}$

6k for $2 \mathrm{~h}$ after which it was concentrated in vacuo. The crude mixture was purified by flash column chromatography (Heptane:EtOAc $=100: 0$ to $85: 15$, gradient) to give the desired product in $98 \%$ yield $(8.88$ mmol, $2.6 \mathrm{~g})$ as a colorless oil. ${ }^{1} \mathbf{H}$ NMR $\left(400 \mathrm{MHz}, \mathrm{CDCl}_{3}\right) \delta \mathrm{ppm}: 7.45-7.37(\mathrm{~m}, 4 \mathrm{H}), 7.32-7.22(\mathrm{~m}, 4 \mathrm{H})$, $7.22-7.13(\mathrm{~m}, 2 \mathrm{H}), 4.37(\mathrm{~s}, 1 \mathrm{H}), 4.25-4.11(\mathrm{~m}, 1 \mathrm{H}), 3.63-3.49(\mathrm{~m}, 3 \mathrm{H}), 2.97-2.80(\mathrm{~m}, 2 \mathrm{H}), 1.11(\mathrm{~d}, J=$ $6.1 \mathrm{~Hz}, 6 \mathrm{H}) ;{ }^{13} \mathrm{C}$ NMR $\left(101 \mathrm{MHz}, \mathrm{CDCl}_{3}\right) \delta$ ppm: 142.38, 128.54, 127.63, 127.21, 78.68, 71.32, 66.35, 62.25, 22.67. IR (thin layer film) $v\left(\mathrm{~cm}^{-1}\right)=3061,3027,2972,2833,1599,1491,1452,1379,1334,1273,1195$, 1174, 1143, 1074, 1029, 1000, 970, 938, 834, 745, 703, 637, 612. HRMS $\left(\mathrm{ESI}^{+}\right) \mathrm{m} / z \mathrm{C}_{19} \mathrm{H}_{24} \mathrm{NO}^{+}[\mathrm{M}+\mathrm{H}]^{+}$: calculated 282.1852, found 282.1852 .

\section{1-benzhydryl-1-benzyl- 3-bromo-2-isopropyloxypropan-1-amine (7ka)}

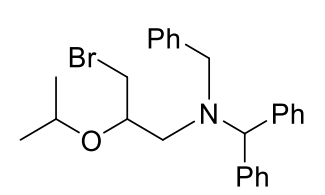

Prepared according to general procedure $\mathrm{C}$ using 1-benzhydryl-3-isopropyloxy-azetidine 6k $(3.01 \mathrm{mmol}, 0.85 \mathrm{~g})$ and benzyl bromide $(4.83 \mathrm{mmol}, 0.57 \mathrm{~mL})$. The crude mixture was purified by flash column chromatography (Pentane:EtOAc $=100: 0$ to 95:5, gradient) to afford the desired product in $89 \%$ yield $(2.67 \mathrm{mmol}, 1.36 \mathrm{~g})$ as a colorless oil. ${ }^{1} \mathbf{H}$ NMR $\left(500 \mathrm{MHz}, \mathrm{CDCl}_{3}\right) \delta$ ppm: $7.58-7.14(\mathrm{~m}, 15 \mathrm{H}), 4.97(\mathrm{~s}, 1 \mathrm{H}), 3.68(\mathrm{~d}, J=2.1 \mathrm{~Hz}, 2 \mathrm{H})$, $3.57-3.43(\mathrm{~m}, 2 \mathrm{H}), 3.42-3.29(\mathrm{~m}, 1 \mathrm{H}), 3.16(\mathrm{dd}, J=10.5,6.2 \mathrm{~Hz}, 1 \mathrm{H}), 2.71(\mathrm{dd}, J=13.7,7.5 \mathrm{~Hz}, 1 \mathrm{H}), 2.62$ $(\mathrm{dd}, J=13.8,5.4 \mathrm{~Hz}, 1 \mathrm{H}), 1.04(\mathrm{dd}, J=13.9,6.1 \mathrm{~Hz}, 6 \mathrm{H}) ;{ }^{13} \mathbf{C ~ N M R}\left(126 \mathrm{MHz}, \mathrm{CDCl}_{3}\right)$ [overlapping signals] $\delta$ ppm: 141.04, 140.95, 139.58, 129.37, 129.28, 129.11, 128.52, 128.43, 128.40, 127.31, 127.27, 75.95, 71.27, 70.81, 56.98, 54.77, 35.63, 22.91, 22.67. IR (thin layer film) $v\left(\mathrm{~cm}^{-1}\right)=3027,2970,2927,1600,1493,1452$, 
1379, 1332, 1247, 1120, 1047, 1029, 970, 922, 763, 743, 699, 667, 621. HRMS (ESI $\left.{ }^{+}\right) \mathrm{m} / z \mathrm{C}_{26} \mathrm{H}_{31} \mathrm{BrNO}^{+}$ $[\mathrm{M}+\mathrm{H}]^{+}$: calculated 452.1584, found 452.1581.

\section{1-benzhydryl-3-methoxyazetidine (61)}

$\mathrm{Ph} Y^{\mathrm{Ph}}$ Prepared according to a slightly modified version of general procedure B. To a solution of commercially available 3-methoxyazetidine hydrochloride $(10.02 \mathrm{mmol}, 1.24 \mathrm{~g})$ in dry acetonitrile (9.2 mL), DIPEA (2.7 equiv., $27.32 \mathrm{mmol}, 4.7 \mathrm{~mL}$ ) and bromodiphenylmethane (0.9 equiv., 9.10 mmol, $2.25 \mathrm{~g}$ ) were subsequently added. The reaction mixture was stirred at $100{ }^{\circ} \mathrm{C}$ for $2 \mathrm{~h}$ after which it was concentrated in vacuo. The crude mixture was purified by flash column chromatography (Heptane:EtOAc $=100: 0$ to $85: 15$, gradient) to give the desired product in $98 \%$ yield $(8.88$ mmol, $2.25 \mathrm{~g}$ ) as a colorless oil. ${ }^{1} \mathbf{H} \mathbf{~ N M R}\left(400 \mathrm{MHz}, \mathrm{CDCl}_{3}\right) \delta \mathrm{ppm:} 7.53-7.43(\mathrm{~m}, 4 \mathrm{H}), 7.37-7.29(\mathrm{~m}, 4 \mathrm{H})$, $7.28-7.21(\mathrm{~m}, 2 \mathrm{H}), 4.42(\mathrm{~s}, 1 \mathrm{H}), 4.11(\mathrm{~m}, 1 \mathrm{H}), 3.60-3.52(\mathrm{~m}, 2 \mathrm{H}), 3.29(\mathrm{~s}, 3 \mathrm{H}), 3.02-2.94(\mathrm{~m}, 2 \mathrm{H})$; ${ }^{13} \mathrm{C}$ NMR (101 MHz, $\left.\mathrm{CDCl}_{3}\right) \delta$ ppm: 142.27, 128.56, 127.60, 127.26, 78.66, 69.49, 60.63, 56.01. IR (thin layer film) $v\left(\mathrm{~cm}^{-1}\right)=3027,2936,2829,1599,1491,1452,1368,1307,1276,1222,1177,1155,1120,1075$, 1029, 1012, 822, 745, 703, 634. HRMS $\left(\mathrm{ESI}^{+}\right) \mathrm{m} / z \mathrm{C}_{17} \mathrm{H}_{20} \mathrm{NO}^{+}[\mathrm{M}+\mathrm{H}]^{+}$: calculated 254.1539, found 254.1544. Spectroscopic data were in agreement with the ones previously reported in literature. ${ }^{5}$

\section{1-benzhydryl-1-benzyl-3-bromo-2-methoxypropan-1-amine (7la)}<smiles>COC(CBr)CN(Cc1ccccc1)C(c1ccccc1)c1ccccc1</smiles>

7la

Prepared according to general procedure $\mathrm{C}$ using 1-benzhydryl-3-(methoxy)azetidine $\mathbf{6} \mathbf{I}$ (3.01 mmol, $0.76 \mathrm{~g}$ ) and benzyl bromide $(5.01 \mathrm{mmol}, 0.6 \mathrm{~mL})$. The crude mixture was purified by flash column chromatography (Heptane:EtOAc $=100: 0$ to $95: 5$, gradient) to afford the desired product in $99 \%$ yield (3.00 mmol, $1.27 \mathrm{~g}$ ) as a colorless oil. ${ }^{1} \mathbf{H}$ NMR $\left(400 \mathrm{MHz}, \mathrm{CDCl}_{3}\right) \delta \mathrm{ppm}: 7.37-7.26(\mathrm{~m}, 9 \mathrm{H}), 7.26-7.13(\mathrm{~m}, 6 \mathrm{H}), 4.91(\mathrm{~s}, 1 \mathrm{H}), 3.68-$ $3.55(\mathrm{~m}, 2 \mathrm{H}), 3.43-3.34(\mathrm{~m}, 1 \mathrm{H}), 3.22-3.10(\mathrm{~m}, 5 \mathrm{H}), 2.69-2.59(\mathrm{~m}, 2 \mathrm{H}) ;{ }^{13} \mathbf{C} \mathbf{~ N M R}\left(101 \mathrm{MHz}, \mathrm{CDCl}_{3}\right) \delta$ ppm: 141.05, 140.86, 139.60, 129.33, 129.29, 129.08, 128.51, 128.44, 128.42, 127.31, 127.29, 127.25, 79.99, 70.62, 57.90, 56.65, 53.24, 34.35. IR (thin layer film) $v\left(\mathrm{~cm}^{-1}\right)=3060,3027,2929,2826,1600,1493,1452$, 1094, 1029, 977, 763, 744, 699, 670. HRMS $\left(\mathrm{ESI}^{+}\right) \mathrm{m} / \mathrm{z} \mathrm{C}_{24} \mathrm{H}_{27} \mathrm{BrNO}^{+}[\mathrm{M}+\mathrm{H}]^{+}$: calculated 424.1271, found 424.1269.

\section{1-benzhydryl-3-(benzyloxy)azetidine (6m)}

$\mathrm{Ph}$ In a round-bottom flask under a flow of $\mathrm{N}_{2}, \mathrm{NaH} 60 \mathrm{wt} \%$ in mineral oil $(15.0 \mathrm{mmol}, 0.60 \mathrm{~g})$ was
added to a solution of commercially available 1-benzhydrylazetidin-3-ol $(10.0 \mathrm{mmol}, 2.40 \mathrm{~g})$ in
PHF $(20 \mathrm{~mL})$ at $0{ }^{\circ} \mathrm{C}$. The mixture was stirred at r.t. for $30 \mathrm{~min}$ before benzyl bromide $(13.0 \mathrm{mmol}$,
to cool to r.t. A saturated aqueous $\mathrm{NH}_{4} \mathrm{Cl}$ solution $(20 \mathrm{~mL})$ was then added. The layers were separated, and the aqueous layer was extracted with EtOAc $(2 \times 50 \mathrm{~mL})$. The combined organic layers were washed with brine $(20 \mathrm{~mL})$, dried over $\mathrm{Na}_{2} \mathrm{SO}_{4}$, and concentrated under reduced pressure. The crude mixture 
was purified by flash column chromatography (Heptane:EtOAc $=100: 0$ to 90:10, gradient) to give the desired compound in $90 \%$ yield $(9.1 \mathrm{mmol}, 3.0 \mathrm{~g})$ as a colorless oil. ${ }^{1} \mathbf{H}$ NMR $\left(500 \mathrm{MHz}, \mathrm{CDCl}_{3}\right) \delta$ ppm: $7.41-7.36$ (m, 4H), $7.34-7.22(\mathrm{~m}, 9 \mathrm{H}), 7.21-7.14(\mathrm{~m}, 2 \mathrm{H}), 4.40(\mathrm{~s}, 2 \mathrm{H}), 4.36(\mathrm{~s}, 1 \mathrm{H}), 4.23(\mathrm{~m}, 1 \mathrm{H}), 3.49$ (dd, J = 6.2, $2.0 \mathrm{~Hz}, 2 \mathrm{H}), 2.94(\mathrm{dd}, J=6.2,2.0 \mathrm{~Hz}, 2 \mathrm{H}) ;{ }^{13} \mathbf{C} \mathbf{~ N M R}\left(126 \mathrm{MHz}, \mathrm{CDCl}_{3}\right) \delta \mathrm{ppm}: 142.20,137.74,128.46$, $128.44,127.95,127.84,127.48,127.12,78.51,70.96,67.65,60.94$. IR (thin layer film) $v\left(\mathrm{~cm}^{-1}\right)=3027,2942$, 2833, 1599, 1492, 1452, 1354, 1308, 1274, 1207, 1172, 1112, 1074, 1027, 912, 821, 742, 696, 636, 621, 611. HRMS $\left(\mathrm{ESI}^{+}\right) \mathrm{m} / z \mathrm{C}_{23} \mathrm{H}_{24} \mathrm{NO}^{+}[\mathrm{M}+\mathrm{H}]^{+}$: calculated 330.1858, found 330.1862. Spectroscopic data were in agreement with the ones previously reported in literature. ${ }^{9}$

\section{1-benzhydryl-1-benzyl-2-(benzyloxy)-3-bromopropan-1-amine (7ma)}

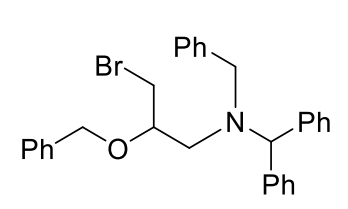

Prepared according to general procedure $\mathrm{C}$ using 1-benzhydryl-3-( benzyloxy)azetidine $7 \mathrm{ma}$ 6m $(3.01 \mathrm{mmol}, 0.99 \mathrm{~g})$ and benzyl bromide $(4.82 \mathrm{mmol}, 0.6 \mathrm{~mL})$. The crude mixture was purified by flash column chromatography (Heptane:EtOAc $=100: 0$ to 95:5, gradient) to afford the desired product in $99 \%(3.00 \mathrm{mmol}, 1.50 \mathrm{~g})$ as a colorless oil. ${ }^{1}$ H NMR (400 MHz, $\left.\mathrm{CDCl}_{3}\right) \delta$ ppm: $7.33-7.12(\mathrm{~m}, 20 \mathrm{H}), 4.88(\mathrm{~s}, 1 \mathrm{H}), 4.43$ (d, $J=$ $11.4 \mathrm{~Hz}, 1 \mathrm{H}), 4.34(\mathrm{~d}, J=11.4 \mathrm{~Hz}, 1 \mathrm{H}), 3.59(\mathrm{~s}, 2 \mathrm{H}), 3.48-3.38(\mathrm{~m}, 2 \mathrm{H}), 3.26-3.16(\mathrm{~m}, 1 \mathrm{H}), 2.68(\mathrm{~d}, J=$ $6.0 \mathrm{~Hz}, 2 \mathrm{H}) ;{ }^{13} \mathbf{C ~ N M R}\left(101 \mathrm{MHz}, \mathrm{CDCl}_{3}\right.$ ) [overlapping signals] $\delta$ ppm: 140.98, 140.89, 139.48, 138.16, 129.33, 129.27, 129.09, 128.51, 128.42, 128.10, 127.90, 127.31, 127.27, 127.24, 77.75, 72.42, 70.58, 56.60, 53.84, 34.70. IR (thin layer film) $v\left(\mathrm{~cm}^{-1}\right)=3061,3028,2842,1600,1493,1453,1339,1062,1028,921,764$, 742, 698. HRMS $\left(\mathrm{ESI}^{+}\right) \mathrm{m} / z \mathrm{C}_{30} \mathrm{H}_{31} \mathrm{BrNO}^{+}[\mathrm{M}+\mathrm{H}]^{+}$: calculated 500.1581, found 500.1583.

\section{1-benzhydrylazetidin-3-yl benzoate (6n)}

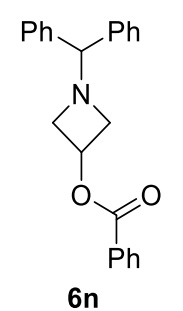

Benzoic anhydride (13.8 mmol, $3.12 \mathrm{~g})$ was added to a pre-stirred mixture of 1-benzhydrylazetidin-3-ol (9.19 mmol, $2.20 \mathrm{~g}$ ) and N,N-dimethylaminopyridine (18.4 mmol, 2.25 g) in $\mathrm{CH}_{2} \mathrm{Cl}_{2}(48 \mathrm{~mL})$, and the resulting mixture was stirred for $12 \mathrm{~h}$. An aqueous $\mathrm{NaHCO}_{3}$ solution $(8 \mathrm{wt} \%, 50 \mathrm{~mL})$ was added and the reaction was stirred for a further $30 \mathrm{~min}$. The phases were separated, and the aqueous layer extracted with $\mathrm{CH}_{2} \mathrm{Cl}_{2}(50 \mathrm{~mL})$. The combined organic layers were concentrated and purified by flash column chromatography (Heptane:EtOAc $=100: 0$ to 90:10, gradient) to afford the desired compound in $99 \%$ yield $(9.19 \mathrm{mmol}, 3.16 \mathrm{~g})$ as yellow oil. ${ }^{1} \mathbf{H} \mathbf{~ N M R}\left(500 \mathrm{MHz}, \mathrm{CDCl}_{3}\right)$ $\delta$ ppm: 8.09 - 8.04 (m, 2H), 7.61 - $7.54(\mathrm{~m}, 1 \mathrm{H}), 7.49-7.42$ (m, 6H), $7.34-7.25$ (m, 4H), 7.25 - 7.18 (m, $2 \mathrm{H}), 5.33(\mathrm{~m}, 1 \mathrm{H}), 4.45(\mathrm{~s}, 1 \mathrm{H}), 3.78-3.72(\mathrm{~m}, 2 \mathrm{H}), 3.22-3.16(\mathrm{~m}, 2 \mathrm{H}) ;{ }^{13} \mathbf{C}$ NMR $\left(126 \mathrm{MHz}, \mathrm{CDCl}_{3}\right) \delta$ ppm: $166.07,142.01,133.25,129.91,129.77,128.62,128.50,127.55,127.36,78.46,64.40,60.37$. IR (thin layer film) $v\left(\mathrm{~cm}^{-1}\right)=3061,3027,2963,2837,1718,1601,1585,1490,1451,1344,1314,1268,1208,1171$, $1115,1070,1025,925,847,827,745,703,666,634,621,611 . \mathbf{H R M S}\left(\mathrm{ESI}^{+}\right) \mathrm{m} / z \mathrm{C}_{23} \mathrm{H}_{22} \mathrm{NO}_{2}{ }^{+}[\mathrm{M}+\mathrm{H}]^{+}$: calculated 344.1650 , found 344.1649 . 


\section{1-benzhydryl-5'-bromo-3'H-spiro[azetidine-3,1'-isobenzofuran] (6o)}

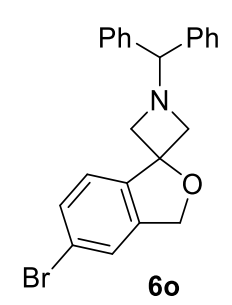

Prepared according to general procedure procedure B using commercially available 1-Boc-5'bromo-3'H-spiro[azetidine-3,1'-isobenzofuran] (1.393 mmol, $0.47 \mathrm{~g}$ ) The crude mixture was purified by flash column chromatography (Heptane:EtOAc $=100: 0$ to $85: 15$, gradient) to give the desired product in $62 \%$ yield $(0.86 \mathrm{mmol}, 0.35 \mathrm{~g})$ as a white solid. ${ }^{1} \mathbf{H}$ NMR (400 MHz, $\left.\mathrm{CDCl}_{3}\right) \delta$ ppm: $7.75(\mathrm{~d}, J=8.0 \mathrm{~Hz}, 1 \mathrm{H}), 7.53(\mathrm{~m}, 1 \mathrm{H}), 7.50-7.44(\mathrm{~m}, 4 \mathrm{H}), 7.33-7.26(\mathrm{~m}$, $5 \mathrm{H}), 7.21-7.14(\mathrm{~m}, 2 \mathrm{H}), 5.04(\mathrm{~d}, J=1.0 \mathrm{~Hz}, 2 \mathrm{H}), 4.43(\mathrm{~s}, 1 \mathrm{H}), 3.51-3.43(\mathrm{~m}, 2 \mathrm{H}), 3.43-3.35(\mathrm{~m}, 2 \mathrm{H}) ;{ }^{13} \mathrm{C}$ NMR $\left(101 \mathrm{MHz}, \mathrm{CDCl}_{3}\right) \delta$ ppm: 142.51, 142.40, 140.91, 131.37, 128.61, 127.55, 127.31, 123.93, 122.63, 121.84, 82.64, 78.20, 72.25, 67.53. IR (thin layer film) $v\left(\mathrm{~cm}^{-1}\right)=3049,3026,2947,2830,1600,1492,1472$, 1452, 1414, 1350, 1313, 1271, 1249, 1208, 1194, 1175, 1154, 1074, 1027, 1006, 857, 821, 743, 703, 672, 641, 614. HRMS $\left(\mathrm{ESI}^{+}\right) \mathrm{m} / z \mathrm{C}_{23} \mathrm{H}_{21} \mathrm{BrNO}^{+}[\mathrm{M}+\mathrm{H}]^{+}$: calculated 406.0801, found 406.0800. MP $148-150{ }^{\circ} \mathrm{C}$.

\section{tert-butyl 3-(thiophen-3-yl)azetidine-1-carboxylate (5p)}

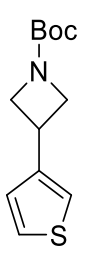

To a pre-formed solution of (1-(tert-butoxycarbonyl)azetidin-3-yl)zinc(II) iodide ${ }^{10}$ in THF $(0.3 \mathrm{M}$, $28.6 \mathrm{~mL}$ ), 3-bromothiophene (4.29 mmol, $0.70 \mathrm{~g}$ ), bis(dibenzylideneacetone) palladium (5 mol\%, $0.22 \mathrm{mmol}, 123 \mathrm{mg}$ ) and XPhos (10 mol\%, $0.43 \mathrm{mmol}, 205 \mathrm{mg}$ ) were added. The mixture was stirred at $50{ }^{\circ} \mathrm{C}$ for $2 \mathrm{~h}$. After cooling the mixture to r.t., the solvent was evaporated in vacuo and the crude

5p product was purified by flash column chromatography (Heptane:EtOAc $=100: 0$ to 90:10, gradient) to afford the desired compound in $64 \%$ yield $(2.75 \mathrm{mmol}, 0.66 \mathrm{~g})$ as a colorless oil. ${ }^{1} \mathbf{H} \mathbf{~ N M R}\left(400 \mathrm{MHz}, \mathrm{CDCl}_{3}\right)$ $\delta$ ppm: 7.33 (dd, $J=4.7,3.2 \mathrm{~Hz}, 1 \mathrm{H}), 7.11-7.05(\mathrm{~m}, 2 \mathrm{H}), 4.34-4.25(\mathrm{~m}, 2 \mathrm{H}), 3.99-3.89(\mathrm{~m}, 2 \mathrm{H}), 3.86-$ $3.74(\mathrm{~m}, 1 \mathrm{H}), 1.46$ (s, 9H); ${ }^{13} \mathbf{C}$ NMR (101 MHz, $\left.\mathrm{CDCl}_{3}\right) \delta$ ppm: 156.56, 143.25, 126.68, 126.42, 120.73, 79.68, 56.56, 29.36, 28.56. IR (thin layer film) $v\left(\mathrm{~cm}^{-1}\right)=2973,2884,1698,1479,1456,1390,1366,1294$, 1253, 1161, 1131, 929, 849, 774, 647. HRMS $\left(\mathrm{ESI}^{+}\right) \mathrm{m} / \mathrm{C}_{12} \mathrm{H}_{18} \mathrm{NO}_{2} \mathrm{~S}^{+}[\mathrm{M}+\mathrm{H}]^{+}$: calculated 240.1053, found 240.1054 .

\section{1-benzhydryl-3-(thiophen-3-yl)azetidine (6p)}

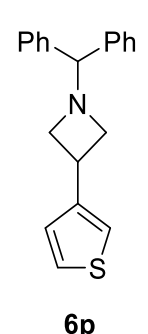

Prepared according to a slightly modified version of general procedure B. To a solution of tertbutyl 3-(thiophen-3-yl)azetidine-1-carboxylate 5p (4.18 mmol, $0.74 \mathrm{~g}$ ) in dry $\mathrm{CH}_{3} \mathrm{CN}(3.8 \mathrm{~mL}$ ), DIPEA (3.6 equiv., $15.21 \mathrm{mmol}, 2.6 \mathrm{~mL}$ ) and bromodiphenylmethane (0.9 equiv., $3.80 \mathrm{mmol}$, $0.94 \mathrm{~g}$ ) were subsequently added. The crude mixture was purified by flash column chromatography (Heptane:EtOAc $=100: 0$ to 85:15, gradient) to give the desired product in $80 \%$ yield (3.35 mmol, $0.93 \mathrm{~g})$ as a colorless oil. ${ }^{1} \mathbf{H}$ NMR $\left(400 \mathrm{MHz}, \mathrm{CDCl}_{3}\right) \delta \mathrm{ppm:} 7.48-7.43(\mathrm{~m}, 4 \mathrm{H}), 7.33-7.24(\mathrm{~m}$, $5 \mathrm{H}), 7.22-7.16(\mathrm{~m}, 2 \mathrm{H}), 7.10(\mathrm{dd}, J=5.0,1.3 \mathrm{~Hz}, 1 \mathrm{H}), 7.05-7.01(\mathrm{~m}, 1 \mathrm{H}), 4.40(\mathrm{~s}, 1 \mathrm{H}), 3.72(\mathrm{p}, J=7.2 \mathrm{~Hz}$, $1 \mathrm{H}), 3.66-3.57$ (m, 2H), $3.16-3.08(\mathrm{~m}, 2 \mathrm{H}) ;{ }^{13} \mathbf{C ~ N M R}\left(101 \mathrm{MHz}, \mathrm{CDCl}_{3}\right) \delta \mathrm{ppm}: 144.33,142.32,128.58$, $127.65,127.23,127.18,125.85,120.12,78.43,61.03,30.79$. IR (thin layer film) $v\left(\mathrm{~cm}^{-1}\right)=3025,2948,2825$, 1598, 1490, 1451, 1076, 1029, 822, 778, 742, 702, 643, 610. HRMS (ESI $\left.{ }^{+}\right) \mathrm{m} / z \mathrm{C}_{20} \mathrm{H}_{20} \mathrm{NS}^{+}[\mathrm{M}+\mathrm{H}]^{+}$: calculated 306.1311 , found 306.1310 . 


\section{1-(1-benzhydrylazetidin-3-yl)-4-bromo-1H-pyrazole (6q)}

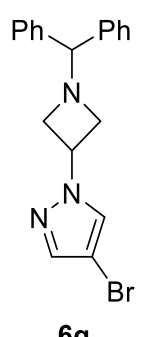

Prepared according to general procedure B using commercially available 1-(1-Boc-azetidin-3-yl)4-bromo-1 $\mathrm{H}$-pyrazole $(3.32 \mathrm{mmol}, 1.00 \mathrm{~g})$. The crude mixture was purified by flash column chromatography (Heptane:EtOAc $=100: 0$ to 80:20, gradient) to afford the desired product in $29 \%$ yield (0.87 mmol, $0.32 \mathrm{~g}$ ) as a colorless oil. ${ }^{1} \mathbf{H}$ NMR $\left(500 \mathrm{MHz}, \mathrm{CDCl}_{3}\right) \delta \mathrm{ppm}: 7.65(\mathrm{~s}, 1 \mathrm{H}), 7.49$

$\mathrm{Br} \quad(\mathrm{s}, 1 \mathrm{H}), 7.46-7.40(\mathrm{~m}, 4 \mathrm{H}), 7.28(\mathrm{dd}, J=8.4,6.9 \mathrm{~Hz}, 5 \mathrm{H}), 7.24-7.16(\mathrm{~m}, 2 \mathrm{H}), 4.95-4.86(\mathrm{~m}$, $1 \mathrm{H}), 4.50(\mathrm{~s}, 1 \mathrm{H}), 3.69-3.62(\mathrm{~m}, 2 \mathrm{H}), 3.47-3.41(\mathrm{~m}, 2 \mathrm{H}) ;{ }^{13} \mathbf{C}$ NMR $\left(126 \mathrm{MHz}, \mathrm{CDCl}_{3}\right) \delta \mathrm{ppm}$ : $141.71,140.14,128.72,128.55,127.54,127.49,93.48,77.95,60.21,51.89$. IR (thin layer film) $v\left(\mathrm{~cm}^{-1}\right)=$ 3026, 2960, 2848, 1598, 1491, 1451, 1383, 1310, 1263, 1206 1180, 1149, 1076, 1029, 1004, 952, 843, 804, 744, 704, 644, 613. HRMS $\left(\mathrm{ESI}^{+}\right) \mathrm{m} / 2 \mathrm{C}_{19} \mathrm{H}_{19} \mathrm{BrN}_{3}{ }^{+}[\mathrm{M}+\mathrm{H}]^{+}$: calculated 368.0757, found 368.0761.

\section{1-benzhydryl-1-benzyl-3-bromo-2-(4-bromo-1H-pyrazol-1-yl)propan-1-amine (7qa)}

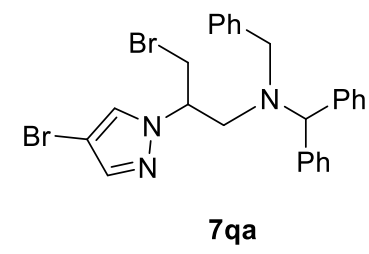

$7 q a$

Prepared according to a slightly modified version of general procedure C. Under a flow of $\mathrm{N}_{2}$, an oven-dried flask was charged with 1-benzhydryl-3-(4-bromo-1Hpyrazol-1-yl)azetidine $6 \mathbf{q}(0.92 \mathrm{mmol}, 0.34 \mathrm{~g})$ in dry $\mathrm{CH}_{3} \mathrm{CN}(1 \mathrm{M}, 0.9 \mathrm{~mL})$. Benzyl bromide ( 0.98 equiv., $0.90 \mathrm{mmol}, 0.11 \mathrm{~mL}$ ) was added and the mixture was stirred at $60{ }^{\circ} \mathrm{C}$ for $12 \mathrm{~h}$. The crude mixture was purified by flash column chromatography (Heptane:EtOAc $=100: 0$ to $98: 2$, gradient) to afford the desired product in $96 \%$ yield $(0.88 \mathrm{mmol}, 0.48 \mathrm{~g}$ ) as a colorless oil. ${ }^{1} \mathbf{H}$ NMR $\left(500 \mathrm{MHz}, \mathrm{CDCl}_{3}\right) \delta$ ppm: $7.43(\mathrm{~s}, 1 \mathrm{H}), 7.40-7.22(\mathrm{~m}, 15 \mathrm{H}), 7.09(\mathrm{~s}, 1 \mathrm{H}), 4.83(\mathrm{~s}$, 1H), $4.03-3.94(\mathrm{~m}, 1 \mathrm{H}), 3.62(\mathrm{~s}, 2 \mathrm{H}), 3.53(\mathrm{dd}, J=10.7,4.4 \mathrm{~Hz}, 1 \mathrm{H}), 3.41(\mathrm{dd}, J=10.7,8.8 \mathrm{~Hz}, 1 \mathrm{H}), 3.11-$ 2.98 (m, 2H); ${ }^{13} \mathbf{C ~ N M R ~ ( 1 2 6 ~ M H z , ~} \mathrm{CDCl}_{3}$ ) [overlapping signals] $\delta$ ppm: 140.79, 140.51, 140.45, 138.96, $130.24,129.32$, 129.12, 128.92, 128.72, 128.66, 128.62, 127.63, 127.49, 92.54, 71.27, 63.73, 57.02, 55.06, 32.85. IR (thin layer film) $v\left(\mathrm{~cm}^{-1}\right)=3061,3029,2845,1600,1493,1452,1381,1313,1179,1121,1077$, 1028, 976, 952, 913, 846, 765, 745, 700, 611. HRMS (ESI $\left.{ }^{+}\right) m / z \mathrm{C}_{26} \mathrm{H}_{26} \mathrm{Br}_{2} \mathrm{~N}_{3}{ }^{+}[\mathrm{M}+\mathrm{H}]^{+}$: calculated 538.0488, found 538.0486 .

\section{1-benzhydrylazetidin-3-yl 4-methylbenzenesulfonate (8r)}

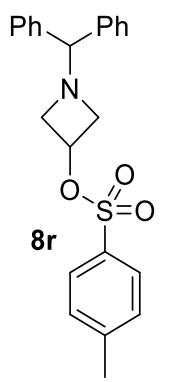

In a flame-dried round bottom flask under a flow of $\mathrm{N}_{2}, \mathrm{NaH} 60 \mathrm{wt} \%$ in mineral oil $(62.7 \mathrm{mmol}$, $2.50 \mathrm{~g})$ and 4-methylbenzenesulfonyl chloride $(50.1 \mathrm{mmol}, 9.55 \mathrm{~g})$ were subsequently added to a solution of commercially available 1-benzhydrylazetidin-3-ol $(41.8 \mathrm{mmol}, 10.0 \mathrm{~g})$ in dry THF $(100 \mathrm{~mL})$ at $0{ }^{\circ} \mathrm{C}$. The reaction mixture was stirred for $12 \mathrm{~h}$ at r.t. After completion of the reaction (LC-MS monitoring), the reaction mixture was quenched with water $(50 \mathrm{~mL})$. The aqueous phase was extracted with EtOAc ( 2 x $250 \mathrm{~mL})$. The organic phases were combined, washed with brine $(200 \mathrm{~mL})$, dried over $\mathrm{Na}_{2} \mathrm{SO}_{4}$, filtered and concentrated in vacuo. The crude mixture was purified by flash column chromatography (Heptane:EtOAc $=100: 0$ to 90:10, gradient) to give the desired product as a white solid in $32 \%$ yield $(13.7 \mathrm{mmol}, 5.40 \mathrm{~g}) .{ }^{1} \mathbf{H}$ NMR (500 MHz, $\left.\mathrm{CDCl}_{3}\right) \delta$ ppm: $7.81-7.75(\mathrm{~m}, 2 \mathrm{H}), 7.39$ $-7.32(\mathrm{~m}, 6 \mathrm{H}), 7.30-7.15(\mathrm{~m}, 6 \mathrm{H}), 4.91(\mathrm{p}, J=6.0 \mathrm{~Hz}, 1 \mathrm{H}), 4.35(\mathrm{~s}, 1 \mathrm{H}), 3.52-3.45(\mathrm{~m}, 2 \mathrm{H}), 3.11-3.04$ 
(m, 2H), 2.45 (s, 3H); $\left.{ }^{13} \mathbf{C ~ N M R ~ ( 1 2 6 ~ M H z , ~} \mathrm{CDCl}_{3}\right) \delta$ ppm: 145.22, 141.62, 133.36, 130.07, 128.65, 128.00, 127.46, 127.43, 78.22, 68.26, 60.20, 21.80. IR (thin layer film) $v\left(\mathrm{~cm}^{-1}\right)=3028,2965,2841,1598,1492,1452$, 1366, 1308, 1191, 1177, 1097, 1079, 1049, 1001, 952, 922, 864, 839, 815, 755, 705, 661. HRMS (ESI ${ }^{+} \mathrm{m} / \mathrm{z}$ $\mathrm{C}_{23} \mathrm{H}_{24} \mathrm{NO}_{3} \mathrm{~S}^{+}[\mathrm{M}+\mathrm{H}]^{+}$: calculated 394.1471 , found. 394.1470. MP $106-108{ }^{\circ} \mathrm{C}$.

\section{1-(1-benzhydrylazetidin-3-yl)-3-methyl-1H-indole (6r)}

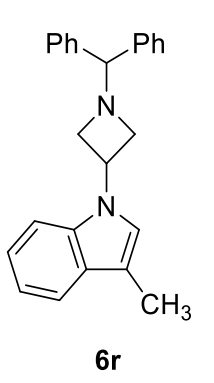

In a flame-dried round bottom flask under a flow of $\mathrm{N}_{2}, \mathrm{NaH} 60 \mathrm{wt} \%$ in mineral oil (15.8 mmol, $0.63 \mathrm{~g})$ was added portion-wise to a solution of 1-benzhydrylazetidin-3-yl 4methylbenzenesulfonate $\mathbf{8 r}$ (7.88 mmol, $3.10 \mathrm{~g})$ in dry DMF (15.5 mL). The mixture was stirred for $30 \mathrm{~min}$, then 3-methylindole $(9.45 \mathrm{mmol}, 1.24 \mathrm{~g})$ was added. The reaction mixture was stirred for $24 \mathrm{~h}$ at r.t. After completion, a saturated aqueous $\mathrm{NH}_{4} \mathrm{Cl}$ solution $(20 \mathrm{~mL})$ was added. The layers were separated, and the aqueous layer was extracted with EtOAc $(2$ x $50 \mathrm{~mL})$. The combined organic layers were washed with brine $(20 \mathrm{~mL})$, dried over $\mathrm{Na}_{2} \mathrm{SO}_{4}$, and concentrated under reduced pressure. The crude mixture was purified by flash column chromatography (Heptane:EtOAc = 100:0 to 95:5, gradient) to afford the desired compound in $70 \%$ yield $(5.51 \mathrm{mmol}, 1.94 \mathrm{~g})$ as a colorless oil. ${ }^{1} \mathbf{H}$ NMR $\left(500 \mathrm{MHz}, \mathrm{CDCl}_{3}\right) \delta \mathrm{ppm}: 7.55(\mathrm{dt}, J=7.8,1.0 \mathrm{~Hz}, 1 \mathrm{H}), 7.48-7.42(\mathrm{~m}, 4 \mathrm{H}), 7.32-7.23(\mathrm{~m}, 5 \mathrm{H}), 7.23-7.12$ (m, 4H), 7.09 (ddd, $J=7.9,6.9,1.0 \mathrm{~Hz}, 1 \mathrm{H}), 5.00$ (p, $J=6.8 \mathrm{~Hz}, 1 \mathrm{H}), 4.45(\mathrm{~s}, 1 \mathrm{H}), 3.80-3.73$ (m, 2H), 3.38 $-3.32(\mathrm{~m}, 2 \mathrm{H}), 2.34(\mathrm{~d}, J=1.1 \mathrm{~Hz}, 3 \mathrm{H}) ;{ }^{13} \mathbf{C} \mathbf{N M R}\left(126 \mathrm{MHz}, \mathrm{CDCl}_{3}\right) \delta \mathrm{ppm:} \mathrm{141.93,} \mathrm{136.47,} \mathrm{129.00,} \mathrm{128.71,}$ $127.55,127.46,122.53,121.72,119.24,119.13,111.32,109.36,78.32,60.55,45.20,9.84$. IR (thin layer film) $v\left(\mathrm{~cm}^{-1}\right)=3026,2917,2838,1599,1491,1463,1452,1388,1371,1317,1278,1234,1210,1076,1029,1014$, 923, 739, 703, 641, 612. HRMS (ESI $\left.{ }^{+}\right) m / z \mathrm{C}_{25} \mathrm{H}_{25} \mathrm{~N}_{2}{ }^{+}[\mathrm{M}+\mathrm{H}]^{+}$: calculated 353.2012, found. 353.2011.

\section{1-benzhydryl-1-benzyl-3-bromo-2-(3-methyl-1H-indol-1-yl)propan-1-amine (7ra)}

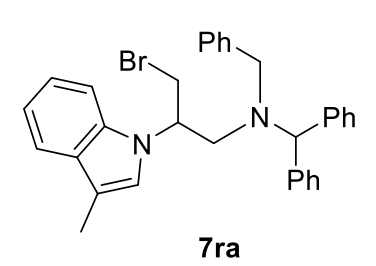

Prepared according to a slightly modified version of general procedure C. Under a flow of $\mathrm{N}_{2}$, an oven-dried flask was charged with 1-benzhydryl-3-(3-methyl-1Hindol-1-yl)azetidine 6r (1.53 mmol, $0.54 \mathrm{~g})$ in dry $\mathrm{CH}_{3} \mathrm{CN}(1 \mathrm{M}, 1.5 \mathrm{~mL})$. Benzyl bromide ( 0.95 equiv., $1.45 \mathrm{mmol}, 0.2 \mathrm{~mL}$ ) was added and the mixture was stirred at $60{ }^{\circ} \mathrm{C}$ for $12 \mathrm{~h}$. The crude mixture was purified by flash column chromatography (Heptane:EtOAc $=100: 0$ to $98: 2$, gradient) to afford the desired product in $68 \%$ yield $(1.04 \mathrm{mmol}, 0.54 \mathrm{~g})$ as a colorless oil. ${ }^{1} \mathbf{H}$ NMR $\left(400 \mathrm{MHz}, \mathrm{CDCl}_{3}\right) \delta$ ppm: $7.56-7.48(\mathrm{~m}, 1 \mathrm{H}), 7.37-7.26(\mathrm{~m}, 14 \mathrm{H}), 7.14-7.03$ (m, 2H), $6.89-6.80(\mathrm{~m}, 1 \mathrm{H}), 6.51-6.46(\mathrm{~m}, 1 \mathrm{H}), 4.84(\mathrm{~s}, 1 \mathrm{H}), 4.40-4.29(\mathrm{~m}, 1 \mathrm{H}), 3.74$ (dd, $J=10.7,4.9$ $\mathrm{Hz}, 1 \mathrm{H}), 3.65$ (d, $J=1.9 \mathrm{~Hz}, 2 \mathrm{H}), 3.35$ (dd, $J=10.7,8.4 \mathrm{~Hz}, 1 \mathrm{H}), 3.18$ (dd, $J=13.9,7.4 \mathrm{~Hz}, 1 \mathrm{H}), 2.98$ (dd, $J=13.9,6.8 \mathrm{~Hz}, 1 \mathrm{H}), 2.26(\mathrm{~d}, J=1.1 \mathrm{~Hz}, 3 \mathrm{H}) ;{ }^{13} \mathbf{C} \mathbf{~ N M R}\left(101 \mathrm{MHz}, \mathrm{CDCl}_{3}\right)$ [overlapping signals] $\delta$ ppm: 141.12 , 140.90, 139.22, 129.30, 129.19, 128.96, 128.87, 128.69, 128.67, 128.58, 127.60, 127.53, 127.37, $122.05,121.56,119.17,119.03,111.41,109.40,71.53,57.16,56.19,54.67,34.10,9.85$. IR (thin layer film) $v$ $\left(\mathrm{cm}^{-1}\right)=3061,1498,1461,1389,1258,1225,1159,1030,742,706,638$. HRMS $\left(\mathrm{ESI}^{+}\right) \mathrm{m} / z \mathrm{C}_{32} \mathrm{H}_{31} \mathrm{BrN}_{2}{ }^{+}$ $[\mathrm{M}+\mathrm{H}]^{+}$: calculated 523.1743, found 523.1741. 


\section{1-benzhydrylazetidin-3-yl methanesulfonate (8s)}

$\mathrm{Ph} \mathrm{Ph}^{\mathrm{Ph}}$ In a flame-dried round bottom flask under a flow of $\mathrm{N}_{2}$, commercially available 1-benzhydrylazetidin-3-ol $(8.36 \mathrm{mmol}, 2.0 \mathrm{~g})$ was dissolved in dry $\mathrm{CH}_{2} \mathrm{Cl}_{2}(20.0 \mathrm{~mL})$ and a premixed solution of methanesulfonyl chloride $(10.0 \mathrm{mmol}, 1.15 \mathrm{~g})$ and triethylamine $(11.7 \mathrm{mmol}$, $1.64 \mathrm{~mL})$ in dry $\mathrm{CH}_{2} \mathrm{Cl}_{2}(5.0 \mathrm{~mL})$ was slowly added to it at $0{ }^{\circ} \mathrm{C}$. The solution was stirred at r.t. $8 \mathrm{~s} \quad \mathrm{~S}=0$ for $12 \mathrm{~h}$, then water $(20 \mathrm{~mL})$ was added and the mixture was extracted with $\mathrm{CH}_{2} \mathrm{Cl}_{2}(3 \times 20 \mathrm{~mL})$. The organic phases were combined, washed with brine $(20 \mathrm{~mL})$, dried over $\mathrm{Na}_{2} \mathrm{SO}_{4}$, filtered and concentrated in vacuo to afford the desired product in quantitative yield $(8.86 \mathrm{mmol}, 2.64 \mathrm{~g})$ as a pale yellow solid. ${ }^{1} \mathbf{H}$ NMR (400 MHz, $\left.\mathrm{CDCl}_{3}\right) \delta$ ppm: $7.43-7.35(\mathrm{~m}, 4 \mathrm{H}), 7.28(\mathrm{tq}, J=8.2,1.1 \mathrm{~Hz}, 4 \mathrm{H}), 7.23-7.16(\mathrm{~m}, 2 \mathrm{H}), 5.10(\mathrm{p}, J=5.8 \mathrm{~Hz}$, 1H), 4.40 (s, 1H), $3.67-3.59$ (m, 2H), $3.24-3.15$ (m, 2H), $2.98(\mathrm{~s}, 3 \mathrm{H}) ;{ }^{13} \mathbf{C} \mathbf{N M R}\left(101 \mathrm{MHz}, \mathrm{CDCl}_{3}\right) \delta \mathrm{ppm}$ : $141.55,128.71,127.54,127.45,78.32,68.11,60.33,38.27$. IR (thin layer film) $v\left(\mathrm{~cm}^{-1}\right)=3027,2937,2842$, 1599, 1491, 1452, 1358, 1207, 1177, 1161, 1079, 1051, 1002, 971, 947, 924, 866, 841, 819, 749, 706, 636, 612. HRMS $\left(\mathrm{ESI}^{+}\right) \mathrm{m} / z \mathrm{C}_{17} \mathrm{H}_{20} \mathrm{NO}_{3} \mathrm{~S}^{+}[\mathrm{M}+\mathrm{H}]^{+}$: calculated 318.1158 , found 318.1158 . MP $110-114{ }^{\circ} \mathrm{C}$.

\section{2-(1-benzhydrylazetidin-3-yl)isoindoline-1,3-dione (6s)}

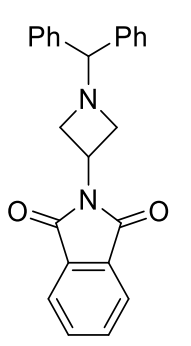

$6 s$

In a flame-dried round bottom flask under a flow of $\mathrm{N}_{2}$, tributylhexadecylphosphonium bromide $(0.84 \mathrm{mmol}, 0.43 \mathrm{mg})$ and phthalimide potassium salt $(10.0 \mathrm{mmol}, 1.82 \mathrm{~g})$ were added to a solution of 1-benzhydrylazetidin-3-yl methane sulfonate $8 \mathrm{~s}$ ( $8.35 \mathrm{mmol}, 2.65 \mathrm{~g})$ in toluene (45 mL). The reaction was refluxed for $24 \mathrm{~h}$, cooled to r.t. and filtered. The residual solid was washed with $\mathrm{CH}_{2} \mathrm{Cl}_{2}$ and the combined filtrate was evaporated under vacuum. The crude mixture was purified by flash column chromatography (Pentane:EtOAc $=100: 0$ to $95: 5$, gradient) to afford the desired product in $65 \%$ yield $(5.43 \mathrm{mmol}, 1.99 \mathrm{~g}) .{ }^{1} \mathbf{H} \mathbf{~ N M R}\left(400 \mathrm{MHz}, \mathrm{CDCl}_{3}\right) \delta \mathrm{ppm}: 7.74(\mathrm{dd}, J$ $=5.5,3.0 \mathrm{~Hz}, 2 \mathrm{H}), 7.67-7.58(\mathrm{~m}, 2 \mathrm{H}), 7.42-7.35(\mathrm{~m}, 4 \mathrm{H}), 7.25-7.16(\mathrm{~m}, 4 \mathrm{H}), 7.17-7.07(\mathrm{~m}, 2 \mathrm{H}), 4.80$ $(\mathrm{p}, J=7.9 \mathrm{~Hz}, 1 \mathrm{H}), 4.59(\mathrm{~s}, 1 \mathrm{H}), 3.66-3.52(\mathrm{~m}, 4 \mathrm{H}) ;{ }^{13} \mathbf{C} \mathbf{N M R}\left(101 \mathrm{MHz}, \mathrm{CDCl}_{3}\right) \delta \mathrm{ppm}: 168.34,142.22$, $134.25,131.98,128.59,127.68,127.28,123.36,77.90,57.38,39.85$. IR (thin layer film) $v\left(\mathrm{~cm}^{-1}\right)=3027$, 2836, 1600, 1487, 1450, 1266, 1076, 1029, 1008, 918, 834, 764, 735, 698, 652. MP 120 - $122{ }^{\circ} \mathrm{C}$. HRMS $\left(\mathrm{ESI}^{+}\right) \mathrm{m} / z \mathrm{C}_{24} \mathrm{H}_{21} \mathrm{~N}_{2} \mathrm{O}_{2}{ }^{+}[\mathrm{M}+\mathrm{H}]^{+}$: calculated 369.1598, found 369.1599. Spectroscopic data were in agreement with the ones previously reported in literature. ${ }^{11}$

\section{2-(1-(benzhydryl(benzyl)amino)-3-bromopropan-2-yl)isoindoline-1,3-dione (7sa)}

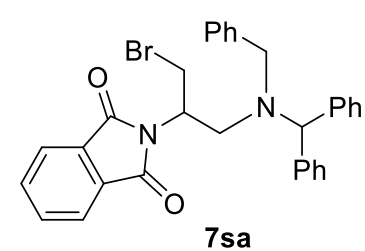

chromatography (Heptane:EtOAc $=100: 0$ to $95: 5$, gradient) to afford the desired product in $88 \%$ yield (3.27 mmol, $1.76 \mathrm{~g})$ as a colorless oil. ${ }^{1} \mathbf{H}$ NMR (400 MHz, $\left.\mathrm{CDCl}_{3}\right) \delta$ ppm: $7.82-7.76(\mathrm{~m}, 2 \mathrm{H}), 7.76-7.71$ 
(m, 2H), $7.40-7.21(\mathrm{~m}, 9 \mathrm{H}), 7.21-7.08(\mathrm{~m}, 6 \mathrm{H}), 4.93(\mathrm{~s}, 1 \mathrm{H}), 4.67-4.55(\mathrm{~m}, 1 \mathrm{H}), 3.82-3.71(\mathrm{~m}, 2 \mathrm{H}), 3.54$ $(\mathrm{d}, J=13.5 \mathrm{~Hz}, 1 \mathrm{H}), 3.48(\mathrm{dd}, J=10.5,5.4 \mathrm{~Hz}, 1 \mathrm{H}), 3.23(\mathrm{dd}, J=14.0,8.2 \mathrm{~Hz}, 1 \mathrm{H}), 2.95(\mathrm{dd}, J=14.0,4.4$ $\mathrm{Hz}, 1 \mathrm{H}) ;{ }^{13} \mathbf{C ~ N M R}\left(101 \mathrm{MHz}, \mathrm{CDCl}_{3}\right) \delta$ ppm: 167.97, 140.90, 140.14, 138.83, 134.02, 132.03, 129.40, 129.24, $129.15,128.59,128.43,128.31,127.49$, 127.24, 127.22, 123.47, 69.74, 55.95, 52.16, 51.09, 30.96. IR (thin layer film) $v\left(\mathrm{~cm}^{-1}\right)=3027,1773,1711,1494,1452,1378,1083,1029,874,764,746,720,700$. HRMS $\left(\mathrm{ESI}^{+}\right)$ $m / z \mathrm{C}_{31} \mathrm{H}_{28} \mathrm{BrN}_{2} \mathrm{O}_{2}^{+}[\mathrm{M}+\mathrm{H}]^{+}$: calculated 539.1334, found 539.1328 .

\section{Tert-butyl 3-(benzhydryl(benzyl)amino)-2-(bromomethyl)propanoate (7ta)}<smiles>CC(C)(C)OC(=O)C(CBr)CN(Cc1ccccc1)C(c1ccccc1)c1ccccc1</smiles>

7 ta

Prepared according to general procedure B using tert-butyl 1-benzhydrylazetidine-3carboxylate* $(2.69 \mathrm{mmol}, 862 \mathrm{mg})$. The crude mixture was purified by flash column chromatography (Pentane: $\mathrm{Et}_{2} \mathrm{O}=100: 0$ to $95: 5$, gradient) to afford the desired product in $85 \%$ yield $(2.28 \mathrm{mmol}, 1.13 \mathrm{~g})$ as a colorless oil. ${ }^{1} \mathbf{H} \mathbf{~ N M R}\left(400 \mathrm{MHz}, \mathrm{CDCl}_{3}\right) \delta$ ppm: $7.45-7.25(\mathrm{~m}, 15 \mathrm{H}), 5.01(\mathrm{~s}, 1 \mathrm{H}), 3.72(\mathrm{~d}, J=13.9 \mathrm{~Hz}, 1 \mathrm{H}), 3.63(\mathrm{~d}, J=13.9$

$\mathrm{Hz}, 1 \mathrm{H}), 3.46$ (dd, $J=9.8,4.3 \mathrm{~Hz}, 1 \mathrm{H}), 3.37$ (dd, $J=9.8,8.8 \mathrm{~Hz}, 1 \mathrm{H}), 2.99-2.88(\mathrm{~m}, 1 \mathrm{H}), 2.83(\mathrm{dd}, J=13.3$, $6.3 \mathrm{~Hz}, 1 \mathrm{H}), 2.66(\mathrm{dd}, J=13.2,7.8 \mathrm{~Hz}, 1 \mathrm{H}), 1.48(\mathrm{~s}, 9 \mathrm{H}) ;{ }^{13} \mathbf{C ~ N M R}\left(101 \mathrm{MHz}, \mathrm{CDCl}_{3}\right) \delta \mathrm{ppm}: 171.71,140.65$, $140.13,138.85,129.41,129.22,129.14,128.49,128.44,128.40,127.38,127.35,127.31,81.56,69.37,55.20$, $51.73,48.28,31.83,28.19$. IR (thin layer film) $v\left(\mathrm{~cm}^{-1}\right)=3028,2977,1728,1600,1494,1452,1392,1368$, 1250, 1216, 1147, 1076, 1029, 972, 910, 843, 763, 739, 700, 649, 601, 517. HRMS (ESI $\left.{ }^{+}\right) \mathrm{m} / \mathrm{z} \mathrm{C}_{28} \mathrm{H}_{33} \mathrm{BrNO}_{2}{ }^{+}$ $[\mathrm{M}+\mathrm{H}]^{+}$: calculated 494.1689, found 494.1688.

* Prepared by transesterification from the corresponding commercially available methyl 1-benzhydrylazetidine-3-carboxylate, according to a known procedure (5 equiv. $t \mathrm{BuONa}, 50{ }^{\circ} \mathrm{C}, 24 \mathrm{~h}$; US Patent 2013 n. 0225552)

\section{1-benzhydryl-3-(benzyloxy)-3-methylazetidine (6u)}

$\mathrm{Ph} Y \mathrm{Ph}$ In a flame-dried round-bottom flask under a flow of $\mathrm{N}_{2}, \mathrm{NaH} 60 \mathrm{wt} \%$ in mineral oil (1.2 equiv., $12.0 \mathrm{mmol}, 0.48 \mathrm{~g}$ ) was added portionwise to a solution of commercially available 1-benzhydryl-3methylazetidin-3-ol (1 equiv., $9.9 \mathrm{mmol}, 2.50 \mathrm{~g})$ in THF $(20 \mathrm{~mL})$ at $0{ }^{\circ} \mathrm{C}$. The mixture was stirred at r.t. for $30 \mathrm{~min}$ then benzyl bromide (1.3 equiv., $12.8 \mathrm{mmol}, 1.49 \mathrm{~mL}$ ) was added dropwise. The reaction mixture was stirred at $60{ }^{\circ} \mathrm{C}$ for $18 \mathrm{~h}$ and then allowed to cool to r.t. A saturated aqueous

$\mathrm{NH}_{4} \mathrm{Cl}$ solution $(20 \mathrm{~mL})$ was then added. The layers were separated, and the aqueous layer was extracted with EtOAc $(2 \times 50 \mathrm{~mL})$. The combined organic layers were washed with brine $(20 \mathrm{~mL})$, dried over $\mathrm{Na}_{2} \mathrm{SO}_{4}$, and concentrated under reduced pressure. The crude mixture was purified by flash column chromatography (Hexane:EtOAc $=90: 10$ to 80:20, gradient) to give the desired compound in 77\% yield (7.6 mmol, $2.6 \mathrm{~g})$ as a pale yellow solid. ${ }^{1} \mathbf{H}$ NMR (400 MHz, $\left.\mathrm{CDCl}_{3}\right) \delta$ ppm: $7.45-7.39(\mathrm{~m}, 4 \mathrm{H}), 7.33$ (d, J=4.4 Hz, 4H), $7.29-$ $7.23(\mathrm{~m}, 5 \mathrm{H}), 7.20-7.14(\mathrm{~m}, 2 \mathrm{H}), 4.40(\mathrm{~s}, 1 \mathrm{H}), 4.38(\mathrm{~s}, 2 \mathrm{H}), 3.22-3.15(\mathrm{~m}, 2 \mathrm{H}), 3.08(\mathrm{~d}, J=8.0 \mathrm{~Hz}, 2 \mathrm{H})$, 1.62 (s, 3H); ${ }^{13} \mathrm{C}$ NMR (101 MHz, $\left.\mathrm{CDCl}_{3}\right)$ [overlapping signals] $\delta$ ppm: 142.66, 138.87, 128.53, 127.67, 
127.64, 127.61, 127.19, 78.11, 72.39, 65.54, 64.51, 22.98. IR (thin layer film) $v\left(\mathrm{~cm}^{-1}\right)=3027,2933,2837$, 1599, 1492, 1452, 1371, 1344, 1314, 1226, 1055, 1029, 964, 883, 741, 702, 637, 622, 545, 482, 422, 409. MP $71-72{ }^{\circ} \mathrm{C}$. HRMS $\left(\mathrm{ESI}^{+}\right) \mathrm{m} / z \mathrm{C}_{24} \mathrm{H}_{26} \mathrm{NO}^{+}[\mathrm{M}+\mathrm{H}]^{+}$: calculated 344.2009, found 344.2003.

\section{1-benzhydryl-1-benzyl-2-(benzyloxy)-3-bromo-2-methylpropan-1-amine (7ua)}

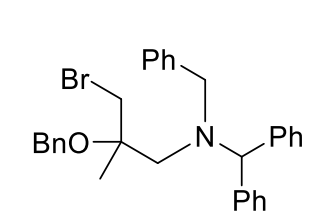

7ua

Prepared according to general procedure C using 1-benzhydryl-3-(benzyloxy)-3methylazetidine $6 \mathbf{u}(2.91 \mathrm{mmol}, 1.0 \mathrm{~g})$ and benzyl bromide $(2.85 \mathrm{mmol}, 0.34 \mathrm{~mL})$. The

crude mixture was purified by flash column chromatography (Heptane:EtOAc $=100: 0$ to $95: 5$, gradient) to afford the desired product in $64 \%$ yield $(1.8 \mathrm{mmol}, 0.95 \mathrm{~g})$ as a colorless oil. ${ }^{1} \mathbf{H}$ NMR (400 MHz, $\left.\mathrm{CDCl}_{3}\right) \delta$ ppm: 7.47 - $7.16(\mathrm{~m}, 20 \mathrm{H}), 5.38(\mathrm{~s}, 1 \mathrm{H})$, $4.48-4.36(\mathrm{~m}, 2 \mathrm{H}), 3.86(\mathrm{~d}, J=13.6 \mathrm{~Hz}, 1 \mathrm{H}), 3.62(\mathrm{~d}, J=13.6 \mathrm{~Hz}, 1 \mathrm{H}), 3.45-3.35(\mathrm{~m}, 2 \mathrm{H}), 2.95-2.82(\mathrm{~m}$, 2H), 1.28 (s, 3H); ${ }^{13} \mathbf{C}$ NMR (101 MHz, $\mathrm{CDCl}_{3}$ ) [overlapping signals] $\delta$ ppm: 141.04, 140.02, 139.87, 138.87, 130.29, 129.70, 129.45, 128.40, 128.38, 128.16, 127.55, 127.44, 127.18, 127.11, 127.01, 78.17, 68.57, 64.35, 56.14, 55.88, 39.59, 20.86. IR (thin layer film) $v\left(\mathrm{~cm}^{-1}\right)=3220,2835,1493,1452,1064,1028,741,699,542$, 465, 418. HRMS $\left(\mathrm{ESI}^{+}\right) \mathrm{m} / z \mathrm{C}_{31} \mathrm{H}_{33} \mathrm{BrNO}^{+}[\mathrm{M}+\mathrm{H}]^{+}$: calculated 514.1740, found 514.1738.

\section{1-benzhydryl-3-methylazetidine-3-carbonitrile (6v)}

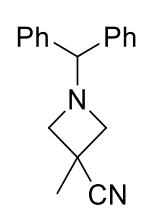

6v

A flame-dried round-bottom flask under a flow of $\mathrm{N}_{2}$ was charged with commercially available 1benzhydrylazetidine-3-carbonitrile (1 equiv., $20.1 \mathrm{mmol}, 5.0 \mathrm{~g})$ in dry THF (0.90 mol, $73.2 \mathrm{~mL})$, and a solution of LiHMDS (1.06 M in THF, 1.1 equiv., $22.1 \mathrm{mmol}, 22.1 \mathrm{~mL})$ was added at $-78^{\circ} \mathrm{C}$. While keeping the same temperature, the reaction was first stirred for $30 \mathrm{~min}$, then $\mathrm{CH}_{3} \mathrm{I}$ ( 1.5 equiv., $30.2 \mathrm{mmol}, 1.88 \mathrm{~mL}$ ) was added dropwise. After stirring for further $45 \mathrm{~min}$ at $-78{ }^{\circ} \mathrm{C}$, the reaction was quenched with $\mathrm{NH}_{4} \mathrm{Cl}(10 \mathrm{~mL})$ and extracted with EtOAc $(3 \times 20 \mathrm{~mL})$. The combined organic layers were dried over $\mathrm{Na}_{2} \mathrm{SO}_{4}$ and concentrated under reduced pressure. The crude mixture was purified by flash column chromatography (Hexane:EtOAc $=100: 0$ to 90:10, gradient) to give the desired compound in $91 \%$ yield (18.3 mmol, $4.8 \mathrm{~g}$ ) as white solid. ${ }^{1} \mathbf{H}$ NMR (400 $\left.\mathrm{MHz}, \mathrm{CDCl}_{3}\right) \delta$ ppm: $7.43-7.36(\mathrm{~m}, 4 \mathrm{H}), 7.32-7.24$ (m, 4H), $7.23-7.17(\mathrm{~m}, 2 \mathrm{H}), 4.38(\mathrm{~s}, 1 \mathrm{H}), 3.49-3.43(\mathrm{~m}, 2 \mathrm{H}), 3.14-3.07(\mathrm{~m}, 2 \mathrm{H}), 1.66(\mathrm{~s}, 3 \mathrm{H}) ;{ }^{13} \mathrm{C} \mathrm{NMR}(101 \mathrm{MHz}$, $\left.\mathrm{CDCl}_{3}\right) \delta$ ppm: $141.26,128.75,127.60,127.45,123.63,77.40,62.94,26.45,23.05$. IR (thin layer film) $v\left(\mathrm{~cm}^{-1}\right)$ $=3061,3027,2958,2840,2238,1599,1493,1452,1380,1348,1313,1256,1227,1076,1029,927,863,745$, 705, 623, 599, 549. MP $86-88{ }^{\circ} \mathrm{C}$. HRMS $\left(\mathrm{ESI}^{+}\right) \mathrm{m} / \mathrm{z} \mathrm{C}_{18} \mathrm{H}_{19} \mathrm{~N}_{2}{ }^{+}[\mathrm{M}+\mathrm{H}]^{+}$: calculated 263.1543, found 263.1545 . 


\section{Tert-butyl 3-fluoro-3-phenylazetidine-1-carboxylate (8w)}

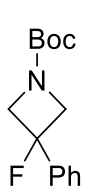

In a flame-dried round bottom flask, tert-butyl 3-oxoazetidine-1-carboxylate (1 equiv., $17.5 \mathrm{mmol}, 3$ g) was dissolved in anhydrous THF $(55 \mathrm{~mL})$ and the solution cooled to $0^{\circ} \mathrm{C}$. Phenylmagnesium bromide ( $3 \mathrm{M}_{\text {in }} \mathrm{Et}_{2} \mathrm{O}, 1.3$ equiv., $22.75 \mathrm{mmol}, 8 \mathrm{~mL}$ ) was added dropwise at the same temperature.

8w The reaction was stirred for $2 \mathrm{~h}$ at r.t. then quenched at $0{ }^{\circ} \mathrm{C}$ by addition of aqueous $\mathrm{NH}_{4} \mathrm{Cl}_{\text {(sat) }}(20 \mathrm{~mL})$ and extracted with $\mathrm{Et}_{2} \mathrm{O}(3 \times 20 \mathrm{~mL})$. The combined organic layers were dried over $\mathrm{MgSO}_{4}$ and concentrated in vacuo to afford the crude alcohol as a pale-yellow solid. This residue was re-dissolved in anhydrous $\mathrm{CH}_{2} \mathrm{Cl}_{2}$ $(120 \mathrm{~mL})$ and the solution cooled to $-78^{\circ} \mathrm{C}$. Deoxo-fluor ${ }^{\circledR}(2.7 \mathrm{M}$ in Toluene, 1.2 equiv., $21 \mathrm{mmol}, 8 \mathrm{~mL})$ was added dropwise at $-78{ }^{\circ} \mathrm{C}$ and the reaction was stirred for $20 \mathrm{~h}$ at r.t. The reaction was quenched at $0{ }^{\circ} \mathrm{C}$ by addition of aqueous $\mathrm{NaHCO}_{3}$ (sat) $(15 \mathrm{~mL})$ followed by aqueous $\mathrm{NaOH}(1 \mathrm{M}, 10 \mathrm{~mL})$ and extracted with $\mathrm{Et}_{2} \mathrm{O}$ ( 3 x $20 \mathrm{~mL}$ ). The combined organic layers were dried over $\mathrm{MgSO}_{4}$ and concentrated under reduced pressure. The crude product was purified by flash column chromatography (Pentane: $\mathrm{Et}_{2} \mathrm{O}=100: 0$ to $94: 6$, gradient) to afford the desired compound as a colorless oil in $44 \%$ yield $(7.7 \mathrm{mmol}, 1.95 \mathrm{~g}) .{ }^{1} \mathbf{H}$ NMR $\left(400 \mathrm{MHz}, \mathrm{CDCl}_{3}\right)$ $\delta$ ppm: $7.50-7.32(\mathrm{~m}, 5 \mathrm{H}), 4.40(\mathrm{ddd}, J=22.4,10.1,1.3 \mathrm{~Hz}, 2 \mathrm{H}), 4.27$ (ddd, $J=19.3,10.1,1.3 \mathrm{~Hz}, 2 \mathrm{H}), 1.48$ (s, 9H); ${ }^{13}$ C NMR (101 MHz, $\left.\mathrm{CDCl}_{3}\right) \delta \mathrm{ppm:} 156.39$ (d, $\left.J=2.8 \mathrm{~Hz}\right), 139.20(\mathrm{~d}, J=23.2 \mathrm{~Hz}), 128.80,128.73$ (d, $J=1.7 \mathrm{~Hz}), 124.22$ (d, $J=8.3 \mathrm{~Hz}), 91.49$ (d, $J=206.7 \mathrm{~Hz}), 80.37,62.69$ (br), 28.48; ${ }^{19} \mathbf{F}$ NMR $(377 \mathrm{MHz}$, $\left.\mathrm{CDCl}_{3}\right) \delta \mathrm{ppm}:-148.75--149.07(\mathrm{~m}, 1 \mathrm{~F})$. IR (thin layer film) $v\left(\mathrm{~cm}^{-1}\right)=2978,2885,1705,1475,1450,1390$, 1365, 1290, 1253, 1163, 1115, 1045, 1020, 935, 863, 760, 698. HRMS (ESI $\left.{ }^{+}\right) m / z \mathrm{C}_{14} \mathrm{H}_{18} \mathrm{FNO}_{2} \mathrm{Na}^{+}[\mathrm{M}+\mathrm{Na}]^{+}$: calculated 274.1214, found 272.1215.

\section{1-benzhydryl-3-fluoro-3-phenylazetidine (6w)}

$\mathrm{Ph} Y^{\mathrm{Ph}}$ In a flame-dried round bottom flask, tert-butyl 3-fluoro-3-phenylazetidine-1-carboxylate 8w N (1 equiv., $6.30 \mathrm{mmol}, 2.0 \mathrm{~g}$ ) was dissolved in $2 \mathrm{~mL}$ of $\mathrm{Et}_{2} \mathrm{O}$. The solution was cooled to $0{ }^{\circ} \mathrm{C}$ and $\mathrm{HCl}\left(6 \mathrm{M}\right.$ in ${ }^{\mathrm{P}} \mathrm{PrOH}, 4.7$ equiv., $30.0 \mathrm{mmol}, 5 \mathrm{~mL}$ ) was added dropwise. The reaction was stirred for $18 \mathrm{~h}$ at r.t. then volatiles were removed in vacuo to afford the azetidinium hydrochloride salt as a white solid. The compound was directly re-dissolved in dry $\mathrm{CH}_{3} \mathrm{CN}(7 \mathrm{~mL})$. The solution was cooled to $0{ }^{\circ} \mathrm{C}$ and DIPEA (5 equiv., $31.5 \mathrm{mmol}, 5.5 \mathrm{~mL}$ ) was added dropwise. The reaction was stirred for $10 \mathrm{~min}$ at $0{ }^{\circ} \mathrm{C}$ then bromodiphenylmethane (1.2 equiv., $\left.7.56 \mathrm{mmol}, 1.9 \mathrm{~g}\right)$ was added portionwise. The solution was refluxed for $3 \mathrm{~h}$ then cooled to r.t. and volatiles were evaporated in vacuo. The crude product was purified by flash column chromatography (Pentane: $\mathrm{Et}_{2} \mathrm{O}=100: 0$ to $99: 1$, gradient) to afford the desired product as a white solid in 99\% yield (6.23 mmol, $2.0 \mathrm{~g}) .{ }^{1} \mathbf{H}$ NMR (400 MHz, $\left.\mathrm{CDCl}_{3}\right) \delta$ ppm: $7.64(\mathrm{~d}, J=7.2 \mathrm{~Hz}, 2 \mathrm{H})$, $7.55-7.14(\mathrm{~m}, 13 \mathrm{H}), 4.53(\mathrm{~s}, 1 \mathrm{H}), 3.73-3.46(\mathrm{~m}, 4 \mathrm{H}) ;{ }^{13} \mathbf{C}$ NMR $\left(101 \mathrm{MHz}, \mathrm{CDCl}_{3}\right) \delta \mathrm{ppm}: 142.06,140.89$ $(\mathrm{d}, J=23.2 \mathrm{~Hz}), 128.69,128.60,128.22(\mathrm{~d}, J=1.0 \mathrm{~Hz}), 127.54,127.44,124.34(\mathrm{~d}, J=8.7 \mathrm{~Hz}), 90.93(\mathrm{~d}, J=$ 206.5 Hz), 78.20, 65.79 (d, $J=22.9 \mathrm{~Hz}) ;{ }^{19} \mathbf{F}$ NMR (377 MHz, $\left.\mathrm{CDCl}_{3}\right) \delta \mathrm{ppm}:-157.07$ - -157.45 (m, 1F). IR $\left(\right.$ thin layer film) $\vee\left(\mathrm{cm}^{-1}\right)=3028,2956,2843,1599,1493,1450,1346,1305,1213,1179,1076,1057,1028$, 914, 858, 754, 670, 637, 622, 613, 550. MP $88-90{ }^{\circ} \mathrm{C}$. HRMS $\left(\mathrm{ESI}^{+}\right) \mathrm{m} / z \mathrm{C}_{22} \mathrm{H}_{21} \mathrm{FN}^{+}[\mathrm{M}+\mathrm{H}]^{+}$: calculated 318.1653 , found 318.1653 . 


\section{1-benzhydryl-1-benzyl-3-bromo-2-fluoro-2-phenylpropan-1-amine (7wa)}<smiles>FC(F)(Br)CN(Cc1ccccc1)C(c1ccccc1)c1ccccc1</smiles>

7wa

Prepared according to a slightly modified version of general procedure C. Under a flow of $\mathrm{N}_{2}$, an oven-dried flask was charged with 1-benzhydryl-3-fluoro-3-phenylazetidine $\mathbf{6 w}$ (4.70 mmol, $1.49 \mathrm{~g})$ in dry $\mathrm{CH}_{3} \mathrm{CN}(0.5 \mathrm{M}, 6.5 \mathrm{~mL})$. Benzyl bromide (1.6 equiv., $7.52 \mathrm{mmol}, 0.90 \mathrm{~mL}$ ) was added and the mixture was stirred at $60{ }^{\circ} \mathrm{C}$ for $24 \mathrm{~h}$. Additional benzyl bromide ( $2 \times 0.3$ equiv.) was added after $24 \mathrm{~h}$ and $48 \mathrm{~h}$, respectively. After $72 \mathrm{~h}$, the volatiles were evaporated in vacuo. The crude product was purified by flash column chromatography (Pentane: $\mathrm{CH}_{2} \mathrm{Cl}_{2}=100: 0$ to $90: 10$, gradient) to afford the desired product in $68 \%$ yield ( $3.19 \mathrm{mmol}, 1.56 \mathrm{~g}$ ) as a colorless oil. ${ }^{1} \mathbf{H}$ NMR (400 MHz, $\left.\mathrm{CDCl}_{3}\right) \delta$ ppm: $7.43-7.14(\mathrm{~m}, 18 \mathrm{H}), 7.07-6.98(\mathrm{~m}, 2 \mathrm{H}), 5.23(\mathrm{~s}, 1 \mathrm{H})$, $4.15-4.05(\mathrm{~m}, 1 \mathrm{H}), 3.85(\mathrm{~d}, J=13.4 \mathrm{~Hz}, 1 \mathrm{H}), 3.57(\mathrm{~d}, J=13.4 \mathrm{~Hz}, 1 \mathrm{H}), 3.30(\mathrm{dd}, J=31.9,11.8 \mathrm{~Hz}, 1 \mathrm{H})$, $3.16(\mathrm{~m}, 1 \mathrm{H}), 3.10(\mathrm{~m}, 1 \mathrm{H}) ;{ }^{13} \mathbf{C}$ NMR (101 MHz, $\left.\mathrm{CDCl}_{3}\right) \delta \mathrm{ppm}: 140.34,140.24$ (d, $\left.J=21.2 \mathrm{~Hz}\right), 139.34$, 138.84, 129.88, 129.72, 129.70, 128.49, 128.40, 128.36 (d, $J=1.9 \mathrm{~Hz}), 128.30,128.00,127.42,127.39$, 127.33, $124.88(\mathrm{~d}, J=10.0 \mathrm{~Hz}), 99.50(\mathrm{~d}, J=183.6 \mathrm{~Hz}), 68.50(\mathrm{~d}, J=3.5 \mathrm{~Hz}), 57.06(\mathrm{~d}, J=21.2 \mathrm{~Hz}), 55.46$ $(\mathrm{d}, J=3.3 \mathrm{~Hz}), 38.46$ (d, $J=25.4 \mathrm{~Hz}) ;{ }^{19} \mathbf{F}$ NMR $\left(377 \mathrm{MHz}, \mathrm{CDCl}_{3}\right) \delta \mathrm{ppm}:-157.26$ (dddd, $J=32.9,26.4$, $21.3,12.5 \mathrm{~Hz})$. IR (thin layer film) $v\left(\mathrm{~cm}^{-1}\right)=3060,3028,2840,1600,1494,1449,1266,1127,1047,1029$, 979, 918, 836, 761, 744, 699, 603, 562. MP $118-120^{\circ} \mathrm{C} . \mathbf{H R M S}\left(\mathrm{ESI}^{+}\right) \mathrm{m} / z \mathrm{C}_{29} \mathrm{H}_{28} \mathrm{BrFN}^{+}[\mathrm{M}+\mathrm{H}]^{+}$: calculated 488.1384 , found 488.1384 . 


\section{$\underline{\text { Stereochemical Assignment of Substrates }}$}

The relative stereochemistry of the cis or trans diasteromer has been assigned by analysis of the ${ }^{1} \mathrm{H}-\mathrm{NOESY}$ spectrum of the mixtures (see NMR copies section) according to the following scheme:

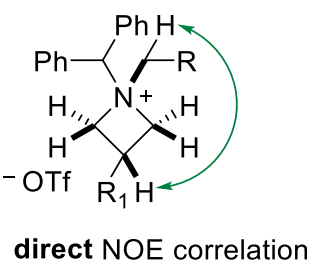

cis-azetinidium salt

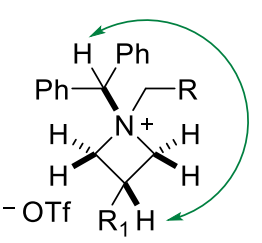

direct NOE correlation trans-azetinidium salt

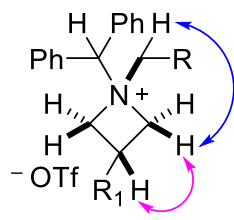

indirect NOE correlation cis-azetinidium salt

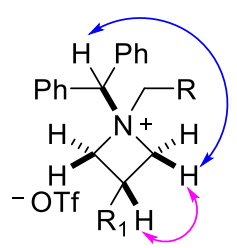

indirect NOE correlation trans-azetinidium salt

Each ${ }^{1} \mathrm{H}-\mathrm{NOESY}$ spectrum indicates if direct or indirect NOE correlation was used for assignment (see NMR copies section). For substrate 1wa, the assignment was based on ${ }^{1} \mathrm{H}-{ }^{19} \mathrm{~F}$ HOESY correlation instead.

For substrates in which the d.r. > 5:1, only the major diastereomer has been reported and characterized. For mixtures, the characterization distinguishes between $\mathrm{H}_{\text {major }}$ (protons of the major diastereomer), $\mathrm{H}_{\text {minor }}$ (protons

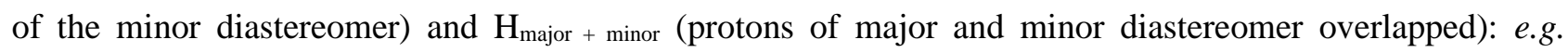
$2 \mathrm{H}_{\text {major }+ \text { minor }}$ corresponds to the signal of $2 \mathrm{H}_{\text {major }}+2 \mathrm{H}_{\text {minor. }}$.

\section{Characterization of Substrates}

\section{1-benzhydryl-1-methyl-3-phenylazetidin-1-ium trifluoromethanesulfonate (1a)}<smiles>[O-]C1CC(c2ccccc2)[N+]2([O-])CC1c1ccccc12</smiles>

1a

Prepared according to general procedure E using commercially available 1-benzhydryl-3phenylazetidine (13.40 mmol, $4.0 \mathrm{~g}$ ) and methyl triflate (1.3 equiv., $17.37 \mathrm{mmol}, 1.9 \mathrm{~mL})$ to afford the desired product in $96 \%$ yield $(12.77 \mathrm{mmol}, 5.92 \mathrm{~g}$, d.r. $15: 1$ cis:trans $)$ as a white powder. ${ }^{1} \mathbf{H}$ NMR $\left(400 \mathrm{MHz}, \mathrm{CDCl}_{3}\right) \delta$ ppm: $7.67-7.60(\mathrm{~m}, 4 \mathrm{H}), 7.56-7.44(\mathrm{~m}, 6 \mathrm{H}), 7.18$ $-7.13(\mathrm{~m}, 1 \mathrm{H}), 7.13-7.07$ (m, 2H), $6.53-6.46(\mathrm{~m}, 2 \mathrm{H}), 6.06$ (s, 1H), 4.87 (dd, $J=9.6,2.2$ $\mathrm{Hz}, 2 \mathrm{H}), 4.57(\mathrm{dd}, J=8.9,2.3 \mathrm{~Hz}, 2 \mathrm{H}), 4.42(\mathrm{~m}, 1 \mathrm{H}), 3.54(\mathrm{~s}, 3 \mathrm{H}) ;{ }^{19} \mathbf{F} \mathbf{N M R}\left(377 \mathrm{MHz}, \mathrm{CDCl}_{3}\right) \delta \mathrm{ppm}:-78.22$ (s, 3F). IR (thin layer film) $v\left(\mathrm{~cm}^{-1}\right)=2361,1498,1454,1256,1224,1153,1029,989,896,853,782,755$, 730, 700, 636. HRMS (ESI $\left.{ }^{+}\right) \mathrm{m} / z \mathrm{C}_{23} \mathrm{H}_{24} \mathrm{~N}^{+}$[M-OTf] $]^{+}$calculated 314.1909, found 314.1908.

cis-1aa and trans-1aa were separated by SFC purification (Stationary phase: Chiralpak IG $5 \mu \mathrm{m} 250 * 20 \mathrm{~mm}$, Mobile phase: $\left.\mathrm{CO}_{2}: \mathrm{MeOH}=70: 30+0.01 \% \mathrm{CF}_{3} \mathrm{SO}_{3} \mathrm{H}\right)$. The fractions were evaporated at $25^{\circ} \mathrm{C}$ and freeze dried. Alternatively, flash column chromatography (EtOAc: $\mathrm{MeOH}=100: 0$ to 95:5) can also be employed. Spectroscopic data were in agreement with the ones previously reported in literature. ${ }^{8}$ 
A single recrystallization of the diasteromeric mixture (15:1 cis:trans) in $\mathrm{CH}_{2} \mathrm{Cl}_{2} / \mathrm{Et}_{2} \mathrm{O}$ at $5{ }^{\circ} \mathrm{C}$ affords cis-1a as a single diastereomer $(>50: 1)$.

\section{cis-1-benzhydryl-1-methyl-3-phenylazetidin-1-ium trifluoromethanesulfonate (cis-1a)}

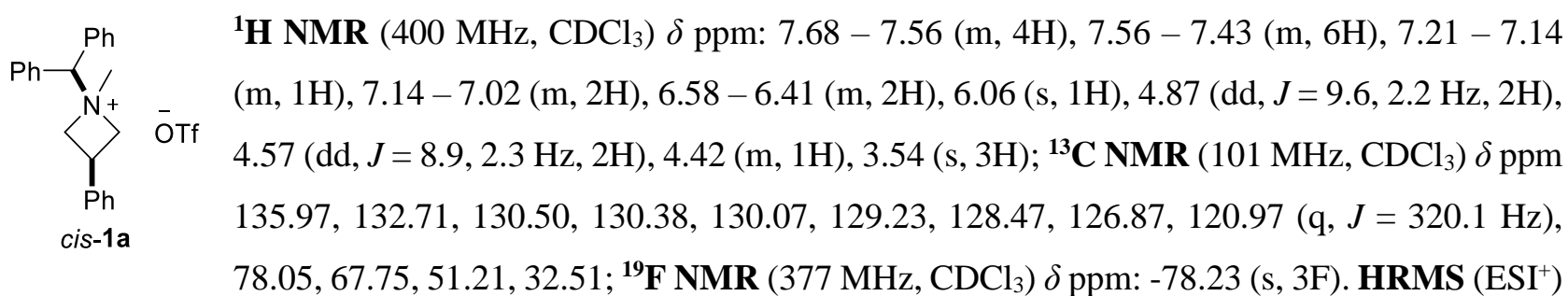
$m / z \mathrm{C}_{23} \mathrm{H}_{24} \mathrm{~N}^{+}[\mathrm{M}-\mathrm{OTf}]^{+}$: calculated 314.1909, found 314.1908.

\section{trans-1-benzhydryl-1-methyl-3-phenylazetidin-1-ium trifluoromethanesulfonate (trans-1a)}

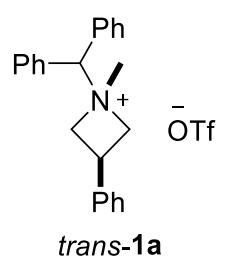

${ }^{1} \mathbf{H}$ NMR (400 MHz, $\left.\mathrm{CDCl}_{3}\right) \delta$ ppm: $7.75-7.66(\mathrm{~m}, 4 \mathrm{H}), 7.56-7.41(\mathrm{~m}, 6 \mathrm{H}), 7.41-7.32(\mathrm{~m}$, $2 \mathrm{H}), 7.33-7.27(\mathrm{~m}, 1 \mathrm{H}), 7.19-7.10(\mathrm{~m}, 2 \mathrm{H}), 6.37(\mathrm{~s}, 1 \mathrm{H}), 5.46(\mathrm{dd}, J=11.7,9.8 \mathrm{~Hz}, 2 \mathrm{H})$, $4.47-4.31(\mathrm{~m}, 2 \mathrm{H}), 3.62-3.48(\mathrm{~m}, 1 \mathrm{H}), 3.30(\mathrm{~s}, 3 \mathrm{H}) ;{ }^{13} \mathbf{C ~ N M R}\left(101 \mathrm{MHz}, \mathrm{CDCl}_{3}\right) \delta \mathrm{ppm}$ : $137.35,132.53,130.47,130.24,130.00,129.58,128.31,126.18,120.95$ (q, $J=320.1 \mathrm{~Hz}$ ), 77.94, 68.98, 52.25, 30.77; ${ }^{19} \mathbf{F}$ NMR $\left(377 \mathrm{MHz}, \mathrm{CDCl}_{3}\right) \delta \mathrm{ppm:}-78.20$ (s, 3F). HRMS (ESI $\left.{ }^{+}\right)$ $m / z \mathrm{C}_{23} \mathrm{H}_{24} \mathrm{~N}^{+}[\mathrm{M}-\mathrm{OTf}]^{+}$: calculated 314.1909, found 314.1908.

\section{1-benzhydryl-1-benzyl-3-phenylazetidin-1-ium trifluoromethanesulfonate (1aa)}

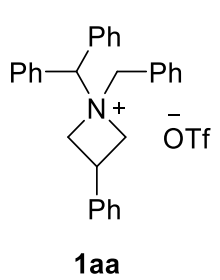

Prepared according to general procedure D using 1-benzhydryl-1-benzyl-3-bromo-2phenylpropan-1-amine 7aa (1.06 mmol, $0.50 \mathrm{~g})$ to afford the desired product in $95 \%$ yield (1.00 mmol, 1.01 g, d.r. 1.5:1 cis:trans) as a white powder. ${ }^{1} \mathbf{H}$ NMR $\left(500 \mathrm{MHz}, \mathrm{CDCl}_{3}\right)$

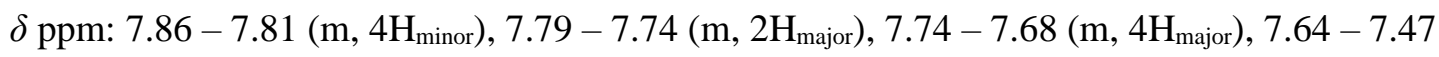
$\left(\mathrm{m}, 11 \mathrm{H}_{\text {minor }}+9 \mathrm{H}_{\text {major }}\right), 7.12-7.04\left(\mathrm{~m}, 1 \mathrm{H}_{\text {major }+ \text { minor }}\right), 7.02-6.95\left(\mathrm{~m}, 2 \mathrm{H}_{\text {major }+ \text { minor }}\right), 6.48(\mathrm{~s}$, $\left.1 \mathrm{H}_{\text {minor }}\right), 6.30\left(\mathrm{~s}, 1 \mathrm{H}_{\text {major }}\right), 6.17-6.12\left(\mathrm{~m}, 2 \mathrm{H}_{\text {minor }}\right), 6.12-6.04\left(\mathrm{~m}, 2 \mathrm{H}_{\text {major }}\right), 5.15-5.07\left(\mathrm{~m}, 2 \mathrm{H}_{\text {minor }}\right), 5.02-$ $4.94\left(\mathrm{~m}, 2 \mathrm{H}_{\text {major }}\right), 4.88-4.79\left(\mathrm{~m}, 2 \mathrm{H}_{\text {major }+ \text { minor }}\right), 4.76\left(\mathrm{~s}, 2 \mathrm{H}_{\text {major }}\right), 4.61\left(\mathrm{~s}, 2 \mathrm{H}_{\text {minor }}\right), 2.72\left(\mathrm{p}, J=9.6 \mathrm{~Hz}, 1 \mathrm{H}_{\text {major }}\right)$, 2.49 (p, $\left.J=9.6 \mathrm{~Hz}, 1 \mathrm{H}_{\text {minor }}\right) ;{ }^{13} \mathbf{C}$ NMR $\left(126 \mathrm{MHz}, \mathrm{CDCl}_{3}\right) \delta \mathrm{ppm:} \mathrm{136.01,} \mathrm{135.98,} \mathrm{134.05,} \mathrm{133.63,} \mathrm{133.21,}$ $132.85,131.28,131.12,130.80,130.38,130.35,130.23,130.19,130.12,129.99,129.86,129.05,128.99$, 128.77, 128.54, 128.32, 128.12, 126.56, 126.38, 120.99 (q, $J=319.9 \mathrm{~Hz}), 77.87,77.83,65.52,65.44,63.65$, 63.26, 32.86, 31.89; ${ }^{19} \mathbf{F}$ NMR (470 MHz, $\left.\mathrm{CDCl}_{3}\right) \delta \mathrm{ppm}$ : -77.11 (s, 3F). IR (thin layer film) $v\left(\mathrm{~cm}^{-1}\right)=3061$, 1498, 1456, 1259, 1224, 1155, 1030, 908, 757, 725, 701, 638. HRMS (ESI $\left.{ }^{+}\right) \mathrm{m} / \mathrm{z}_{2} \mathrm{C}_{29} \mathrm{H}_{28} \mathrm{~N}^{+}$[M-OTf] calculated 390.2221, found 390.2218 .

Multi-gram scale reaction (10 mmol scale, $4.70 \mathrm{~g}$ ) and subsequent purification by flash column chromatography $\left(\mathrm{CH}_{2} \mathrm{Cl}_{2}: \mathrm{MeOH}=100: 0\right.$ to 95:5, gradient), afforded the title compound (7.5 mmol, $4.05 \mathrm{~g}$, d.r. 1:1.1 cis:trans) as a white powder. 


\section{1-benzhydryl-1-ethyl-3-phenylazetidin-1-ium trifluoromethanesulfonate (1ab)}

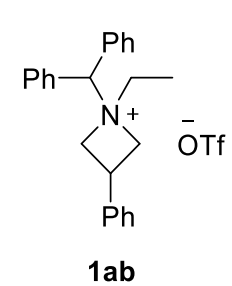

Prepared according to general procedure E using commercially available 1-benzhydryl-3phenylazetidine $(2.67 \mathrm{mmol}, 0.80 \mathrm{~g})$ with ethyl triflate $(4.01 \mathrm{mmol}, 0.52 \mathrm{~mL})$ to afford the desired product in $94 \%$ yield $(2.51 \mathrm{mmol}, 1.20 \mathrm{~g}$, d.r. 8:1 cis:trans) as a white powder. ${ }^{1} \mathbf{H}$ NMR major $\left(500 \mathrm{MHz}, \mathrm{CDCl}_{3}\right) \delta$ ppm: 7.67 - $7.47(\mathrm{~m}, 10 \mathrm{H}), 7.17$ - $7.11(\mathrm{~m}, 1 \mathrm{H}), 7.09-$ $7.02(\mathrm{~m}, 2 \mathrm{H}), 6.45-6.26(\mathrm{~m}, 2 \mathrm{H}), 5.71(\mathrm{~s}, 1 \mathrm{H}), 4.94-4.81(\mathrm{~m}, 2 \mathrm{H}), 4.75-4.63(\mathrm{~m}, 2 \mathrm{H}), 4.36$ (p, $J=9.4 \mathrm{~Hz}, 1 \mathrm{H}), 3.94(\mathrm{q}, J=7.1 \mathrm{~Hz}, 2 \mathrm{H}), 1.52(\mathrm{t}, J=7.1 \mathrm{~Hz}, 3 \mathrm{H}) ;{ }^{1} \mathbf{H} \mathbf{N M R}$ minor $\left(500 \mathrm{MHz}, \mathrm{CDCl}_{3}\right)$ $\delta$ ppm: 7.43 - 7.65 (m, $10 \mathrm{H}), 6.99$ - 7.18 (m, $3 \mathrm{H}), 6.31$ - 6.42 (m, $2 \mathrm{H}), 5.97$ (s, $1 \mathrm{H}), 5.26$ - 5.36 (m, $2 \mathrm{H})$, $4.59-4.66$ (m, $2 \mathrm{H}), 3.66$ (q, $J=7.22 \mathrm{~Hz}, 2 \mathrm{H}), 2.91(\mathrm{q}, J=9.18 \mathrm{~Hz}, 1 \mathrm{H}), 1.37(\mathrm{t}, J=7.1 \mathrm{~Hz}, 3 \mathrm{H}) ;{ }^{13} \mathbf{C} \mathbf{N M R}$ major (126 MHz, $\left.\mathrm{CDCl}_{3}\right) \delta$ ppm: 136.24, 132.38, 130.59, 130.35, 130.09, 129.12, 128.29, 126.82, 121.24 (q, $J=320.0 \mathrm{~Hz}), 74.52,64.80,57.64,32.74,8.96 ;{ }^{19} \mathbf{F}$ NMR $\left(470 \mathrm{MHz}, \mathrm{CDCl}_{3}\right) \delta$ ppm: -78.24 (s, 3F). IR (thin layer film) $v\left(\mathrm{~cm}^{-1}\right)=3061,1498,1456,1260,1224,1154,1030,781,755,728,701,637$. HRMS (ESI $\left.{ }^{+}\right)$ $m / z \mathrm{C}_{24} \mathrm{H}_{26} \mathrm{~N}^{+}$[M-OTf] $]^{+}$calculated 328.2065, found 328.2061.

\section{1-allyl-1-benzhydryl-3-phenylazetidin-1-ium trifluoromethanesulfonate (1ac)}

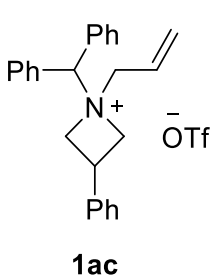

Prepared according to general procedure D using 1-benzhydryl-1-allyl-3-bromo-2phenylpropan-1-amine 7ab $(5.00 \mathrm{mmol}, 2.1 \mathrm{~g})$ to afford the desired product in $94 \%$ yield (4.70 mmol, 2.30 g, d.r. 2:1 cis:trans) as a white powder. ${ }^{1} \mathbf{H}$ NMR $\left(500 \mathrm{MHz}, \mathrm{CDCl}_{3}\right) \delta \mathrm{ppm}$ :

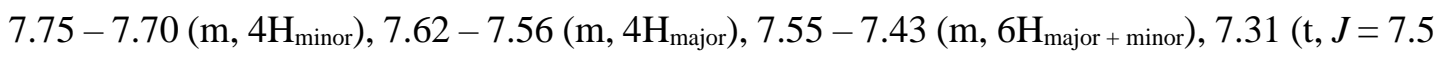
$\left.\mathrm{Hz}, 2 \mathrm{H}_{\text {minor }}\right), 7.24\left(\mathrm{~d}, J=7.4 \mathrm{~Hz}, 1 \mathrm{H}_{\text {minor }}\right), 7.14\left(\mathrm{t}, J=7.4 \mathrm{~Hz}, 1 \mathrm{H}_{\text {major }}\right), 7.09-7.02(\mathrm{~m}$, $\left.2 \mathrm{H}_{\text {major + minor }}\right), 6.39-6.33\left(\mathrm{~m}, 2 \mathrm{H}_{\text {major }}\right), 6.31-6.19\left(\mathrm{~m}, 1 \mathrm{H}_{\text {major }}\right), 6.04-5.93\left(\mathrm{~m}, 2 \mathrm{H}_{\text {minor }}\right), 5.82\left(\mathrm{~s}, 1 \mathrm{H}_{\text {major }}\right), 5.74$ $\left(\mathrm{dd}, J=10.1,1.0 \mathrm{~Hz}, 1 \mathrm{H}_{\text {major }}\right), 5.68\left(\mathrm{dd}, J=16.9,1.3 \mathrm{~Hz}, 1 \mathrm{H}_{\text {major }}\right), 5.61\left(\mathrm{~d}, J=10.2 \mathrm{~Hz}, 1 \mathrm{H}_{\text {minor }}\right), 5.43(\mathrm{dd}, J=$

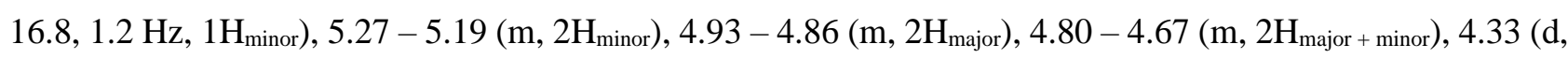
$\left.J=7.3 \mathrm{~Hz}, 2 \mathrm{H}_{\text {major }}\right), 4.20$ (p, $\left.J=9.5 \mathrm{~Hz}, 1 \mathrm{H}_{\text {major }}\right), 4.11$ (d, $\left.J=7.2 \mathrm{~Hz}, 2 \mathrm{H}_{\text {minor }}\right), 2.95$ (p, $J=9.4 \mathrm{~Hz}, 1 \mathrm{H}_{\text {minor }}$ ); ${ }^{13} \mathrm{C}$ NMR (126 MHz, $\mathrm{CDCl}_{3}$ ) [overlapping signals] $\delta \mathrm{ppm:} \mathrm{136.65,} \mathrm{136.17,} \mathrm{132.80,} \mathrm{132.53,} \mathrm{130.65,} \mathrm{130.44,}$ $130.39,130.05,130.02,129.35,129.16,128.35,128.19,126.79,126.34,124.80,121.00$ (q, $J=320.3 \mathrm{~Hz})$, 76.70, 76.53, 65.92, 64.80, 64.38, 64.35, 32.65, 31.08; ${ }^{19}$ F NMR (470 MHz, $\left.\mathrm{CDCl}_{3}\right) \delta$ ppm: -78.21 (s, 3F). IR (thin layer film) $v\left(\mathrm{~cm}^{-1}\right)=3062,1604,1498,1455,1256,1224,1153,1029,954,913,875,842,802,781$, 754, 735, 699, 636. HRMS (ESI $\left.{ }^{+}\right) \mathrm{m} / z \mathrm{C}_{25} \mathrm{H}_{26} \mathrm{~N}^{+}$[M-OTf] $]^{+}$: calculated 340.2065, found 340.2064.

\section{1-benzhydryl-1-benzyl-3-( $m$-tolyl)azetidin-1-ium trifluoromethanesulfonate (1ba)}

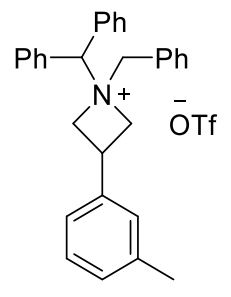

$1 \mathrm{ba}$

Prepared according to general procedure D using 1-benzhydryl-1-benzyl-3-bromo-2-(mtolyl)propan-1-amine $7 \mathbf{b a}(0.58 \mathrm{mmol}, 0.28 \mathrm{~g}$,) to afford the desired product in $80 \%$ yield (0.46 mmol, $257.6 \mathrm{mg}$, d.r. 2:1 cis:trans) as a white powder. ${ }^{1} \mathbf{H} \mathbf{~ N M R}\left(500 \mathrm{MHz}, \mathrm{CDCl}_{3}\right)$ $\delta$ ppm: $7.89-7.42$ (m, 15 $\left.\mathrm{H}_{\text {major + minor }}\right), 6.94-6.83$ (m, 2 $\left.\mathrm{H}_{\text {major + minor }}\right), 6.47$ (s, $\left.1 \mathrm{H}_{\text {minor }}\right), 6.29$ $\left(\mathrm{s}, 1 \mathrm{H}_{\text {major }}\right), 6.03-5.95\left(\mathrm{~m}, 1 \mathrm{H}_{\text {major }+ \text { minor }}\right), 5.86\left(\mathrm{~d}, J=2.2 \mathrm{~Hz}, 1 \mathrm{H}_{\text {minor }}\right), 5.82(\mathrm{~d}, J=2.0 \mathrm{~Hz}$, $\left.1 \mathrm{H}_{\text {major }}\right), 5.18-5.03\left(\mathrm{~m}, 2 \mathrm{H}_{\text {minor }}\right), 5.03-4.92\left(\mathrm{~m}, 2 \mathrm{H}_{\text {major }}\right), 4.89-4.78\left(\mathrm{~m}, 2 \mathrm{H}_{\text {major }+ \text { minor }}\right), 4.74$ 
(s, $2 \mathrm{H}_{\text {major }}$ ), 4.59 (s, $2 \mathrm{H}_{\text {minor }}$ ), 2.64 (p, $J=9.6 \mathrm{~Hz}, 1 \mathrm{H}_{\text {major }}$ ), 2.43 (p, $J=9.7 \mathrm{~Hz}, 1 \mathrm{H}_{\text {minor }}$ ), 2.06 (s, 3 $\mathrm{H}_{\text {major + minor }}$ ); ${ }^{13} \mathrm{C}$ NMR (126 MHz, $\mathrm{CDCl}_{3}$ ) [overlapping signals] $\delta$ ppm: 138.81, 138.74, 135.88, 135.82, 134.19, 133.68, $133.28,132.83,131.25,131.21,130.86,130.42,130.31,130.19,130.10,129.92,129.84,129.11,128.91$, 128.80, 128.77, 128.72, 128.63, 126.77, 126.71, 124.13, 123.89, 121.01 (q, $J=320.0 \mathrm{~Hz}$ ), 77.90, 77.84, 65.44, 63.68, 63.25, 32.97, 32.00, 21.17; ${ }^{19} \mathbf{F}$ NMR (470 MHz, $\left.\mathrm{CDCl}_{3}\right) \delta$ ppm: -78.00 (s, 3F). IR (thin layer film) $v\left(\mathrm{~cm}^{-1}\right)=3057,1609,1497,1456,1259,1224,1156,1030,862,757,702,638$. HRMS $\left._{(E S I}\right)^{+} \mathrm{m} / z \mathrm{C}_{30} \mathrm{H}_{30} \mathrm{~N}^{+}$ [M-OTf] $]^{+}$calculated 404.2373, found 404.2373.

\section{1-benzhydryl-1-benzyl-3-(3-methoxyphenyl)azetidin-1-ium trifluoromethanesulfonate (1ca)}

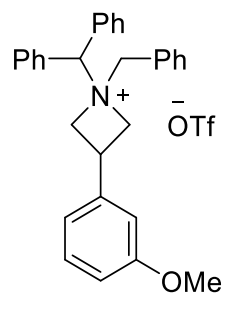

$1 \mathrm{ca}$

Prepared according to general procedure D using 1-benzhydryl-1-benzyl-3-bromo-2-(3methoxyphenyl)propan-1-amine 7ca $(0.81 \mathrm{mmol}, 0.41 \mathrm{~g})$ to afford the desired product in $82 \%$ yield (0.66 mmol, $379.9 \mathrm{mg}$, d.r. 2.2:1 cis:trans) as a white powder. ${ }^{1} \mathbf{H} \mathbf{~ N M R}(500 \mathrm{MHz}$, $\left.\mathrm{CDCl}_{3}\right) \delta$ ppm: $7.91-7.37$ (m, 15 $\left.\mathrm{H}_{\text {major + minor }}\right), 6.87\left(\mathrm{t}, J=8.0 \mathrm{~Hz}, 1 \mathrm{H}_{\text {major + minor }}\right), 6.63-6.55$ $\left(\mathrm{m}, 1 \mathrm{H}_{\text {major }+ \text { minor }}\right), 6.35\left(\mathrm{~s}, 1 \mathrm{H}_{\text {minor }}\right), 6.17\left(\mathrm{~s}, 1 \mathrm{H}_{\text {major }}\right), 5.86\left(\mathrm{t}, J=2.2 \mathrm{~Hz}, 1 \mathrm{H}_{\text {minor }}\right), 5.81(\mathrm{t}, J=$ $\left.2.1 \mathrm{~Hz}, 1 \mathrm{H}_{\text {major }}\right), 5.75\left(\mathrm{dd}, J=7.6,1.7 \mathrm{~Hz}, 1 \mathrm{H}_{\text {minor }}\right), 5.64\left(\mathrm{dd}, J=7.6,1.7 \mathrm{~Hz}, 1 \mathrm{H}_{\text {major }}\right), 5.16-$ 5.08 (m, 2 $\left.2 \mathrm{H}_{\text {minor }}\right), 4.99-4.91$ (m, 2 $\left.\mathrm{H}_{\text {major }}\right), 4.89-4.76\left(\mathrm{~m}, 2 \mathrm{H}_{\text {major }+ \text { minor }}\right), 4.72\left(\mathrm{~s}, 2 \mathrm{H}_{\text {major }}\right), 4.54\left(\mathrm{~s}, 2 \mathrm{H}_{\text {minor }}\right), 3.60$ (s, 3H $\left.\mathrm{H}_{\text {major }+ \text { minor }}\right), 2.75$ (p, $\left.J=9.6 \mathrm{~Hz}, 1 \mathrm{H}_{\text {major }}\right), 2.54\left(\mathrm{p}, J=9.6 \mathrm{~Hz}, 1 \mathrm{H}_{\text {minor }}\right) ;{ }^{13} \mathbf{C ~ N M R}\left(126 \mathrm{MHz}, \mathrm{CDCl}_{3}\right)$ [overlapping signals] $\delta$ ppm: 159.89, 159.85, 137.55, 137.39, 133.79, 133.56, 133.13, 132.79, 131.20, 131.09, $130.69,130.34,130.24,130.20,130.14,130.06,130.03,129.94,129.80,128.70,128.35,121.07$ (q, $J=320.3$ Hz), 118.26, 118.15, 113.44, 113.35, 112.69, 112.26, 78.07, 77.95 , 65.57, 65.41, 63.42, 63.12, 55.44, 55.41, 32.75, 31.60; ${ }^{19} \mathbf{F}$ NMR (470 MHz, $\left.\mathrm{CDCl}_{3}\right) \delta \mathrm{ppm}:-78.12$ (s, 3F). IR (thin layer film) $v\left(\mathrm{~cm}^{-1}\right)=1602,1497$, 1456, 1256, 1224, 1156, 1029, 859, 756, 704, 637. HRMS (ESI $\left.{ }^{+}\right) m / z \mathrm{C}_{30} \mathrm{H}_{30} \mathrm{NO}^{+}[\mathrm{M}-\mathrm{OTf}]^{+}$: calculated 420.2322 , found 420.2323 .

\section{1-benzhydryl-1-benzyl-3-(3-chlorophenyl)azetidin-1-ium trifluoromethanesulfonate (1da)}

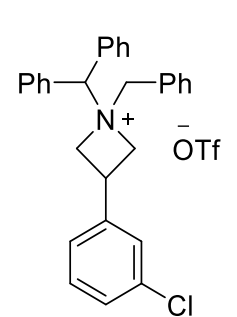

$1 \mathrm{da}$

Prepared according to general procedure E using 1-benzhydryl-1-benzyl-3-bromo-2-(3chlorophenyl)propan-1-amine $7 \mathbf{d a}(0.33 \mathrm{mmol}, 0.16 \mathrm{~g})$ to afford the desired product in $80 \%$ yield ( $0.26 \mathrm{mmol}, 0.15 \mathrm{~g}$, d.r. $1.8: 1$ cis:trans) as a white powder. ${ }^{1} \mathbf{H} \mathbf{~ N M R}(500 \mathrm{MHz}$, $\left.\mathrm{CDCl}_{3}\right) \delta$ ppm: $7.88-7.45\left(\mathrm{~m}, 15 \mathrm{H}_{\text {major }+ \text { minor }}\right), 7.10-7.02\left(\mathrm{~m}, 1 \mathrm{H}_{\text {major }+ \text { minor }}\right), 6.96-6.89(\mathrm{~m}$, $\left.1 \mathrm{H}_{\text {major + minor }}\right), 6.47\left(\mathrm{~s}, 1 \mathrm{H}_{\text {minor }}\right), 6.24\left(\mathrm{~s}, 1 \mathrm{H}_{\text {major }}\right), 6.14-6.07\left(\mathrm{~m}, 1 \mathrm{H}_{\text {major }+ \text { minor }}\right), 6.03(\mathrm{t}, J=1.9$ $\left.\mathrm{Hz}, 1 \mathrm{H}_{\text {minor }}\right), 5.98\left(\mathrm{t}, J=1.9 \mathrm{~Hz}, 1 \mathrm{H}_{\text {major }}\right), 5.19-5.07\left(\mathrm{~m}, 2 \mathrm{H}_{\text {minor }}\right), 5.02-4.92\left(\mathrm{~m}, 2 \mathrm{H}_{\text {major }}\right)$, $4.92-4.83\left(\mathrm{~m}, 2 \mathrm{H}_{\text {major }}\right), 4.83-4.70\left(\mathrm{~m}, 2 \mathrm{H}_{\text {major }+ \text { minor }}\right), 4.57$ (s, 2 $\left.\mathrm{H}_{\text {minor }}\right), 2.73\left(\mathrm{p}, J=9.6 \mathrm{~Hz}, 1 \mathrm{H}_{\text {major }}\right), 2.50$ (p, $\left.J=9.6 \mathrm{~Hz}, 1 \mathrm{H}_{\text {minor }}\right) ;{ }^{13} \mathbf{C ~ N M R}\left(126 \mathrm{MHz}, \mathrm{CDCl}_{3}\right)$ [overlapping signals] $\delta$ ppm: 137.97, 137.94, 134.95, 134.83 , 134.01, 133.47, 132.97, 132.84, 131.65, 131.35, 130.74, 130.71, 130.39, 130.25, 130.20, 130.15, 129.98, 129.90, 128.59, 128.58, 128.33, 128.25, 126.33, 126.25, 125.29, 124.96, 121.00 (q, $J=319.9 \mathrm{~Hz})$, 78.14, 77.94, 65.46, 63.29, 62.72, 32.39, 31.33; ${ }^{19} \mathbf{F}$ NMR (470 MHz, $\left.\mathrm{CDCl}_{3}\right) \delta$ ppm: -78.13 (s, 3F). IR (thin 
layer film) $v\left(\mathrm{~cm}^{-1}\right)=3656,2980,2888,1600,1457,1383,1258,1225,1155,1088,1030,954,751,707,638$. HRMS $\left(\mathrm{ESI}^{+}\right) \mathrm{m} / z \mathrm{C}_{29} \mathrm{H}_{27} \mathrm{ClN}^{+}[\mathrm{M}-\mathrm{OTf}]^{+}$: calculated 424.1827, found 424.1822.

\section{1-benzhydryl-1-benzyl-3-(4-fluorophenyl)azetidin-1-ium trifluoromethanesulfonate (1ea)}

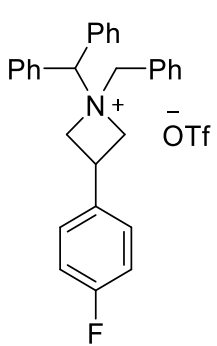

1ea

Prepared according to general procedure D using 1-benzhydryl-1-benzyl-3-bromo-2- (4fluorophenyl)propan-1-amine 7ea $(2.04 \mathrm{mmol}, 0.97 \mathrm{~g})$ to afford the desired product in $85 \%$ yield $\left(1.73 \mathrm{mmol}, 0.97 \mathrm{~g}\right.$, d.r. $1.8: 1$ cis:trans) as a white powder. ${ }^{1} \mathbf{H}$ NMR (400 MHz, $\left.\mathrm{CDCl}_{3}\right) \delta$ ppm: $8.08-7.28$ (m, 15 $\left.\mathrm{H}_{\text {major + minor }}\right), 6.77-6.50$ (m, 2 $\left.\mathrm{H}_{\text {major }+ \text { minor }}\right), 6.36\left(\mathrm{~s}, 1 \mathrm{H}_{\text {minor }}\right)$, $6.26-5.98\left(\mathrm{~m}, 2 \mathrm{H}_{\text {minor }}+3 \mathrm{H}_{\text {major }}\right), 5.22-5.06\left(\mathrm{~m}, 2 \mathrm{H}_{\text {minor }}\right), 5.03-4.66\left(\mathrm{~m}, 2 \mathrm{H}_{\text {minor }}+6 \mathrm{H}_{\text {major }}\right)$, 4.55 (s, 2 $\left.\mathrm{H}_{\text {minor }}\right), 2.78$ (p, $\left.J=9.5 \mathrm{~Hz}, 1 \mathrm{H}_{\text {major }}\right), 2.52$ (p, $\left.J=9.7 \mathrm{~Hz}, 1 \mathrm{H}_{\text {minor }}\right) ;{ }^{13} \mathbf{C} \mathbf{N M R}(101$ $\left.\mathrm{MHz}, \mathrm{CDCl}_{3}\right)$ [overlapping signals] $\delta$ ppm: $162.28(\mathrm{~d}, J=247.9 \mathrm{~Hz}), 133.98,133.47,133.10$, 132.82, 131.95 (d, $J=2.8 \mathrm{~Hz}$, minor), 131.87 (d, $J=3.3 \mathrm{~Hz}$, major), 131.26, 131.07, 130.76, 130.35, 130.31, 130.21, 130.10, 129.92, 129.83, 128.58, 128.47, 128.30 (d, $J=8.2 \mathrm{~Hz}$, major), 128.12 (d, $J=8.1 \mathrm{~Hz}$, minor), 121.01 (q, $J=320.1 \mathrm{~Hz}), 115.90$ (d, $J=21.7 \mathrm{~Hz}$, major), 115.81 (d, $J=21.6 \mathrm{~Hz}$, minor), 78.14, 77.92, 65.50, 65.42, 63.78, 63.34, 32.00, 31.00; ${ }^{19} \mathbf{F}$ NMR $\left(470 \mathrm{MHz}, \mathrm{CDCl}_{3}\right) \delta$ ppm: -78.14 (s, 3F), -113.23 (s, 1F, major), -113.54 (s, 1F, minor). IR (thin layer film) $v\left(\mathrm{~cm}^{-1}\right)=3065,1606,1514,1456,1258,1225,1161,1030$, 834, 757, 706, 638. HRMS $\left(\mathrm{ESI}^{+}\right) \mathrm{m} / z \mathrm{C}_{29} \mathrm{H}_{27} \mathrm{FN}^{+}$[M-OTf] ${ }^{+}$: calculated 408.2122, found 408.2116.

\section{1-benzhydryl-1-ethyl-3-(4-fluorophenyl)azetidin-1-ium trifluoromethanesulfonate (1eb)}

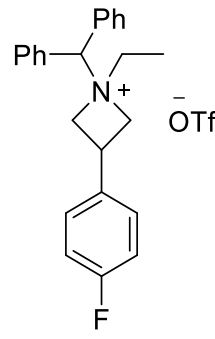

$1 e b$

Prepared according to general procedure E using 1-benzhydryl-3-(4-fluorophenyl)azetidine 7eb $(1.26 \mathrm{mmol}, 0.40 \mathrm{~g})$ to afford the desired product in $96 \%$ yield $(1.20 \mathrm{mmol}, 0.60 \mathrm{~g}$, d.r. $=16: 1$ cis:trans $)$ as a white powder. ${ }^{1} \mathbf{H}$ NMR major $\left(500 \mathrm{MHz}, \mathrm{CDCl}_{3}\right) \delta$ ppm: $7.60(\mathrm{~d}$, $J=6.8 \mathrm{~Hz}, 4 \mathrm{H}), 7.53-7.46(\mathrm{~m}, 6 \mathrm{H}), 6.73-6.67(\mathrm{~m}, 2 \mathrm{H}), 6.32(\mathrm{dd}, J=8.3,5.0 \mathrm{~Hz}, 2 \mathrm{H}), 5.69$ (s, 1H), $4.84-4.76(\mathrm{~m}, 2 \mathrm{H}), 4.72-4.64(\mathrm{~m}, 2 \mathrm{H}), 4.43-4.33(\mathrm{~m}, 1 \mathrm{H}), 3.89$ (q, $J=7.1 \mathrm{~Hz}$, 2H), $1.44(\mathrm{t}, J=7.1 \mathrm{~Hz}, 3 \mathrm{H}) ;{ }^{13} \mathbf{C}$ NMR major $\left(126 \mathrm{MHz}, \mathrm{CDCl}_{3}\right) \delta \mathrm{ppm:} 162.32(\mathrm{~d}$, $J=247.8 \mathrm{~Hz}), 132.36,132.22(\mathrm{~d}, J=3.0 \mathrm{~Hz}), 130.59,130.32,130.05,128.56(\mathrm{~d}, J=8.2 \mathrm{~Hz})$, $121.01(\mathrm{q}, J=320.4 \mathrm{~Hz}), 115.93(\mathrm{~d}, J=21.7 \mathrm{~Hz}), 74.37,64.93,57.63,31.84,8.81 ;{ }^{19} \mathbf{F} \mathbf{N M R}$ major $(470 \mathrm{MHz}$, $\left.\mathrm{CDCl}_{3}\right) \delta \mathrm{ppm}:-78.19$ (s, 3F), -113.58 (m, 1F). IR (thin layer film) $v\left(\mathrm{~cm}^{-1}\right)=3063,1605,1515,1455,1260$, 1225, 1161, 1030, 931, 833, 780, 753, 725, 705, 638. HRMS $\left(\mathrm{ESI}^{+}\right) \mathrm{m} / \mathrm{z}_{24} \mathrm{C}_{25} \mathrm{FN}^{+}$[M-OTf] $]^{+}$calculated 346.1966, found 346.1960. 


\section{1-benzhydryl-1-benzyl-3-(4-(trifluoromethyl)phenyl)azetidin-1-ium trifluoromethanesulfonate (1fa)}

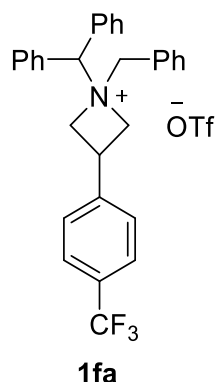

Prepared according to general procedure D using 1-benzhydryl-1-benzyl-3-bromo-2-(4(trifluoromethyl)phenyl)propan-1-amine $7 \mathbf{f a}(1.70 \mathrm{mmol}, 0.92 \mathrm{~g})$ to afford the desired product in $88 \%$ yield (1.50 mmol, 0.91 g, d.r. 5.3:1 cis:trans) as a white powder. ${ }^{1} \mathbf{H} \mathbf{~ N M R}(500 \mathrm{MHz}$, $\left.\mathrm{CDCl}_{3}\right) \delta$ ppm: $7.89-7.84$ (m, 4H $\left.\mathrm{H}_{\text {minor }}\right), 7.77$ (dd, $J=7.0,1.8 \mathrm{~Hz}, 2 \mathrm{H}_{\text {major }}$ ), 7.71 (dd, $J=7.0$, $\left.1.9 \mathrm{~Hz}, 4 \mathrm{H}_{\text {major }}\right), 7.61-7.36\left(\mathrm{~m}, 11 \mathrm{H}_{\text {minor }}+9 \mathrm{H}_{\text {major }}\right), 7.24-7.18\left(\mathrm{~m}, 2 \mathrm{H}_{\text {major }+ \text { minor }}\right), 6.36(\mathrm{~d}, J$ $\left.=4.2 \mathrm{~Hz}, 2 \mathrm{H}_{\text {minor }}\right), 6.34\left(\mathrm{~s}, 1 \mathrm{H}_{\text {minor }}\right), 6.27\left(\mathrm{~d}, J=8.1 \mathrm{~Hz}, 2 \mathrm{H}_{\text {major }}\right), 6.16\left(\mathrm{~s}, 1 \mathrm{H}_{\text {major }}\right), 5.28-5.17$

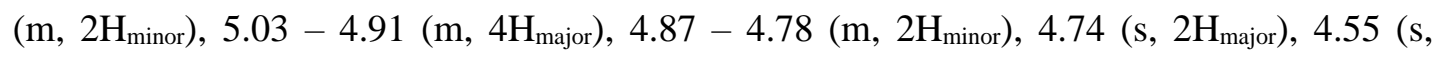
$\left.2 \mathrm{H}_{\text {minor }}\right), 2.91$ (p, $\left.J=9.5 \mathrm{~Hz}, 1 \mathrm{H}_{\text {major }}\right), 2.67$ (p, $\left.J=9.4 \mathrm{~Hz}, 1 \mathrm{H}_{\text {minor }}\right) ;{ }^{13} \mathbf{C} \mathbf{N M R}\left\{{ }^{19} \mathrm{~F}\right.$ at $\left.-62 \mathrm{ppm}\right\}(126 \mathrm{MHz}$, $\left.\mathrm{CDCl}_{3}\right) \delta$ ppm: 140.26, 140.14, 133.86, 133.33, 132.96, 132.84, 131.32, 131.10, 130.71, 130.44, 130.39, $130.34,130.22,130.12,129.87,129.84,128.42,128.26,126.98,126.72,125.86,125.84,125.81,125.78$, 125.71, 125.69, 124.84, 124.80, 121.03 (q, $J=320.1 \mathrm{~Hz}), 78.45,78.05,65.97,65.53,63.23,62.71,32.06$,

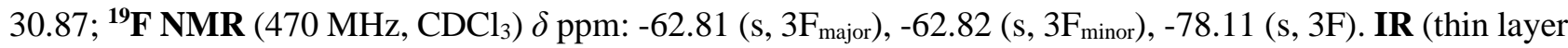
film) $v\left(\mathrm{~cm}^{-1}\right)=3065,1621,1499,1456,1326,1257,1225,1159,1124,1070,1030,1018,930,902,836,757$, 705, 638, 608. HRMS (ESI $\left.{ }^{+}\right) m / z \mathrm{C}_{30} \mathrm{H}_{27} \mathrm{~F}_{3} \mathrm{~N}^{+}[\mathrm{M}-\mathrm{OTf}]^{+}$: calculated 458.2090, found 458.2087.

\section{1-benzhydryl-1-ethyl-3-(4-(methoxl)phenyl)azetidin-1-ium trifluoromethanesulfonate (1fb)}

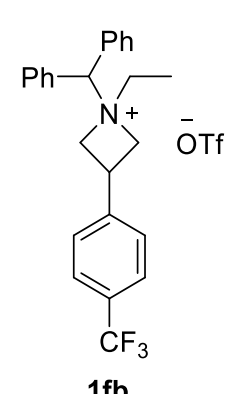

Prepared according to general procedure E using 1-benzhydryl-3-(4(methoxl)phenyl)azetidine $7 \mathbf{f b}(1.09 \mathrm{mmol}, 0.40 \mathrm{~g})$ to afford the desired product in $94 \%$ yield (1.02 mmol, 0.56 g, d.r. $\geq 20: 1$ cis:trans) as a white powder. ${ }^{1} \mathbf{H}$ NMR major (400 MHz, $\left.\mathrm{CDCl}_{3}\right) \delta$ ppm: $7.67-7.58(\mathrm{~m}, 4 \mathrm{H}), 7.58-7.47(\mathrm{~m}, 6 \mathrm{H}), 7.28(\mathrm{~d}, J=7.9 \mathrm{~Hz}, 2 \mathrm{H}), 6.52(\mathrm{~d}$, $J=8.0 \mathrm{~Hz}, 2 \mathrm{H}), 5.68(\mathrm{~s}, 1 \mathrm{H}), 4.93-4.83(\mathrm{~m}, 2 \mathrm{H}), 4.82-4.72(\mathrm{~m}, 2 \mathrm{H}), 4.51(\mathrm{p}, J=9.1 \mathrm{~Hz}$, $1 \mathrm{H}), 3.94(\mathrm{q}, J=7.1 \mathrm{~Hz}, 2 \mathrm{H}), 1.46(\mathrm{t}, J=7.0 \mathrm{~Hz}, 3 \mathrm{H}) ;{ }^{13} \mathbf{C} \mathbf{N M R}$ major $\left(126 \mathrm{MHz}, \mathrm{CDCl}_{3}\right)$ [overlapping signals] $\delta$ ppm: $140.50,132.20,130.53,130.35,130.05,127.24,125.86,123.80$ $(\mathrm{q}, J=280.0 \mathrm{~Hz}), 121.02(\mathrm{q}, J=317.2 \mathrm{~Hz}), 74.34,64.46,57.78,31.90,8.72 ;{ }^{19} \mathbf{F}$ NMR $\left(470 \mathrm{MHz}, \mathrm{CDCl}_{3}\right)$ $\delta$ ppm: -62.82 (s, 3F), -78.25 (s, 3F). IR (thin layer film) $v\left(\mathrm{~cm}^{-1}\right)=3061,1621,1498,1455,1326,1256,1224$, 1158, 1123, 1070, 1029, 964, 931, 834, 782, 737, 705, 637, 607. HRMS (ESI $\left.{ }^{+}\right) m / z \mathrm{C}_{25} \mathrm{H}_{25} \mathrm{~F}_{3} \mathrm{~N}^{+}[\mathrm{M}-\mathrm{OTf}]^{+}$: calculated 396.1933, found 396.1929.

\section{3-([1,1'-biphenyl]-4-yl)-1-benzhydryl-1-benzylazetidin-1-ium trifluoromethanesulfonate (1ga)}

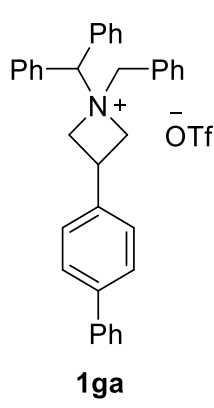

Prepared according to general procedure D using 1-benzhydryl-1-benzyl-3-bromo-2-([1,1'biphenyl]-4-yl)propan-1-amine $7 \mathbf{g a}(0.91 \mathrm{mmol}, 0.50 \mathrm{~g})$ to afford the desired product in $68 \%$ yield ( $0.62 \mathrm{mmol}, 0.38$ g, d.r. $1.8: 1$ cis:trans) as a white powder. ${ }^{1} \mathbf{H}$ NMR $(500 \mathrm{MHz}$, $\left.\mathrm{CDCl}_{3}\right) \delta$ ppm: 7.85 (d, $\left.J=7.6 \mathrm{~Hz}, 4 \mathrm{H}_{\text {minor }}\right), 7.78\left(\mathrm{~d}, J=6.8 \mathrm{~Hz}, 2 \mathrm{H}_{\text {major }}\right), 7.73(\mathrm{~d}, J=7.0$ $\left.\mathrm{Hz}, 4 \mathrm{H}_{\text {major }}\right), 7.63\left(\mathrm{~d}, J=6.8 \mathrm{~Hz}, 2 \mathrm{H}_{\text {minor }}\right), 7.61-7.28\left(\mathrm{~m}, 14 \mathrm{H}_{\text {major }+ \text { minor }}\right), 7.19(\mathrm{~d}, J=8.0$ $\left.\mathrm{Hz}, 2 \mathrm{H}_{\text {major }+ \text { minor }}\right), 6.45\left(\mathrm{~s}, 1 \mathrm{H}_{\text {minor }}\right), 6.26\left(\mathrm{~s}, 1 \mathrm{H}_{\text {major }}\right), 6.22\left(\mathrm{~d}, J=8.1 \mathrm{~Hz}, 2 \mathrm{H}_{\text {minor }}\right), 6.16(\mathrm{~d}$, $\left.J=8.3 \mathrm{~Hz}, 2 \mathrm{H}_{\text {major }}\right), 5.19-5.11\left(\mathrm{~m}, 2 \mathrm{H}_{\text {minor }}\right), 5.04-4.96\left(\mathrm{~m}, 2 \mathrm{H}_{\text {major }}\right), 4.92-4.81(\mathrm{~m}$, 
$2 \mathrm{H}_{\text {major + minor }}$ ), $4.76\left(\mathrm{~s}, 2 \mathrm{H}_{\text {major }}\right), 4.61\left(\mathrm{~s}, 2 \mathrm{H}_{\text {minor }}\right), 2.80\left(\mathrm{p}, J=9.6 \mathrm{~Hz}, 1 \mathrm{H}_{\text {major }}\right), 2.56\left(\mathrm{p}, J=9.6 \mathrm{~Hz}, 1 \mathrm{H}_{\text {minor }}\right)$; ${ }^{13} \mathrm{C} \mathrm{NMR}\left(126 \mathrm{MHz}, \mathrm{CDCl}_{3}\right.$ ) [overlapping signals] $\delta$ ppm: 141.24, 140.99, 140.11, 140.06, 135.00, 134.93, $134.05,133.60,133.20,132.85,131.28,131.10,130.81,130.37,130.33,130.24,130.21,130.12,129.99$, $129.86,128.93,128.72,128.54,127.78,127.75,127.65,127.58,127.05,127.02,126.85,121.02$ (q, $J=320.1$ $\mathrm{Hz}), 78.00,77.88,65.54,65.45,63.70,63.30,32.54,31.54 ;{ }^{19} \mathbf{F}$ NMR (376 MHz, $\left.\mathrm{CDCl}_{3}\right) \delta$ ppm: $-78.11(\mathrm{~s}$, 3F). IR (thin layer film) $v\left(\mathrm{~cm}^{-1}\right)=3063,1488,1456,1259,1224,1155,1030,840,765,702$, 638. HRMS $\left(\mathrm{ESI}^{+}\right) \mathrm{m} / z \mathrm{C}_{35} \mathrm{H}_{32} \mathrm{~N}^{+}$[M-OTf] $]^{+}$: calculated 466.2529, found 466.2526 .

\section{3-([1,1'-biphenyl]-4-yl)-1-benzhydryl-1-ethylazetidin-1-ium trifluoromethanesulfonate (1gb)}

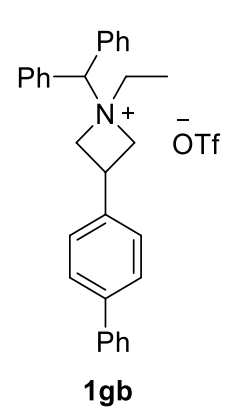

Prepared according to general procedure E using 1-benzhydryl-3-([1,1'-biphenyl]-4yl)phenyl)azetidine $\mathbf{7 g b}(0.25 \mathrm{mmol}, 94.0 \mathrm{mg}$, ) to afford the desired product in $93 \%$ yield $(0.23 \mathrm{mmol}, 138.7 \mathrm{mg}$, d.r. cis:trans $=8: 1)$ as a white powder. ${ }^{1} \mathbf{H}$ NMR major $(400 \mathrm{MHz}$, $\left.\mathrm{CDCl}_{3}\right) \delta$ ppm: $7.65-7.58(\mathrm{~m}, 4 \mathrm{H}), 7.55-7.50(\mathrm{~m}, 5 \mathrm{H}), 7.46-7.43(\mathrm{~m}, 2 \mathrm{H}), 7.42-7.37$ $(\mathrm{m}, 2 \mathrm{H}), 7.36-7.30(\mathrm{~m}, 1 \mathrm{H}), 7.28-7.24(\mathrm{~m}, 3 \mathrm{H}), 6.46-6.38(\mathrm{~m}, 2 \mathrm{H}), 5.72(\mathrm{~s}, 1 \mathrm{H}), 4.97-$ $4.85(\mathrm{~m}, 2 \mathrm{H}), 4.76-4.63(\mathrm{~m}, 2 \mathrm{H}), 4.43(\mathrm{p}, J=9.5 \mathrm{~Hz}, 1 \mathrm{H}), 3.96(\mathrm{q}, J=7.0 \mathrm{~Hz}, 2 \mathrm{H}), 1.52$ (t, $J=7.1 \mathrm{~Hz}, 3 \mathrm{H}) ;{ }^{13} \mathbf{C}$ NMR major (101 MHz, $\left.\mathrm{CDCl}_{3}\right) \delta$ ppm: 141.29, 140.18, 135.17, 132.38, 130.61, 130.44, 130.16, 128.96, 127.78, 127.76, 127.31, 127.10, 120.99 (q, $J=319.1 \mathrm{~Hz}$ ), 74.70, 64.86, 57.72, 32.49, 9.04; ${ }^{19} \mathbf{F}$ NMR major (470 MHz, $\left.\mathrm{CDCl}_{3}\right) \delta$ ppm: -78.19 (s, 3F). IR (thin layer film) $v\left(\mathrm{~cm}^{-1}\right)=3063$, 1488, 1456, 1259, 1224, 1155, 1030, 840, 765, 702, 638. HRMS $\left(\mathrm{ESI}^{+}\right) \mathrm{m} / z \mathrm{C}_{30} \mathrm{H}_{30} \mathrm{~N}^{+}$[M-OTf] ${ }^{+}$: calculated 404.2373, found 404.2378.

\section{1-benzhydryl-1-benzyl-3-(4-methyphenyl)azetidin-1-ium trifluoromethanesulfonate (1ha)}

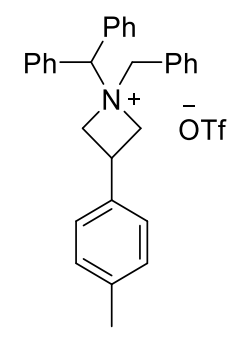

1ha

Prepared according to general procedure D using 1-benzhydryl-1-benzyl-3-bromo-2-(4methylphenyl)propan-1-amine 7 ha $(1.45 \mathrm{mmol}, 0.70 \mathrm{~g})$ to afford the desired product in $78 \%$ yield (1.86 mmol, 0.65 g, d.r. 2.2:1 cis:trans) as a white powder. ${ }^{1} \mathbf{H}$ NMR (400 MHz, $\left.\mathrm{CDCl}_{3}\right) \delta \mathrm{ppm:} 7.83\left(\mathrm{~d}, J=7.0 \mathrm{~Hz}, 4 \mathrm{H}_{\text {minor }}\right), 7.75\left(\mathrm{~d}, J=6.8 \mathrm{~Hz}, 2 \mathrm{H}_{\text {major }}\right), 7.71(\mathrm{~d}, J=6.2 \mathrm{~Hz}$, $\left.4 \mathrm{H}_{\text {major }}\right), 7.60\left(\mathrm{~d}, J=6.8 \mathrm{~Hz}, 2 \mathrm{H}_{\text {minor }}\right), 7.58-7.43\left(\mathrm{~m}, 9 \mathrm{H}_{\text {major }+ \text { minor }}\right), 6.76(\mathrm{~d}, J=7.7 \mathrm{~Hz}$, $\left.2 \mathrm{H}_{\text {major }+ \text { minor }}\right), 6.38\left(\mathrm{~s}, 1 \mathrm{H}_{\text {minor }}\right), 6.20\left(\mathrm{~s}, 1 \mathrm{H}_{\text {major }}\right), 6.02\left(\mathrm{~d}, J=7.9 \mathrm{~Hz}, 2 \mathrm{H}_{\text {minor }}\right), 5.96(\mathrm{~d}, J=8.0$

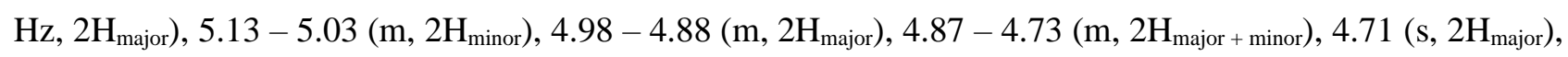
4.56 (s, $2 \mathrm{H}_{\text {minor }}$ ), 2.69 (p, $J=9.6 \mathrm{~Hz}, 1 \mathrm{H}_{\text {major }}$ ), 2.45 (p, $\left.J=9.6 \mathrm{~Hz}, 1 \mathrm{H}_{\text {minor }}\right), 2.16$ (s, $3 \mathrm{H}_{\text {major }+ \text { minor }}$ ); ${ }^{13} \mathbf{C ~ N M R}$ $\left(101 \mathrm{MHz}, \mathrm{CDCl}_{3}\right)$ [overlapping signals] $\delta$ ppm: 138.11, 137.88, 134.01, 133.62, 133.21, 133.03, 132.95, $132.80,131.19,131.01,130.78,130.29,130.25,130.18,130.06,129.93,129.79,129.64,129.58,128.73$, 128.55, 126.44, 126.30, 120.95 (q, $J=319.7$ Hz), 77.95, 77.87, 65.48, 65.38, 63.86, 63.51, 32.59, 31.66, 20.99; ${ }^{19}$ F NMR $\left(376 \mathrm{MHz}, \mathrm{CDCl}_{3}\right) \delta \mathrm{ppm}:-78.09$ (s, 3F). IR (thin layer film) $v\left(\mathrm{~cm}^{-1}\right)=3063,1488,1456,1259$, 1224, 1155, 1030, 840, 765, 702, 638. HRMS $\left(\mathrm{ESI}^{+}\right) \mathrm{m} / z \mathrm{C}_{30} \mathrm{H}_{30} \mathrm{~N}^{+}[\mathrm{M}-\mathrm{OTf}]^{+}$: calculated 404.2373, found 404.2372 . 


\section{1-benzhydryl-1-benzyl-3-phenoxyazetidin-1-ium trifluoromethanesulfonate (1ia)}

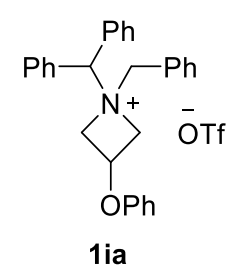

Prepared according to general procedure D using 1-benzhydryl-1-benzyl-3-bromo-2phenoxypropan-1-amine 7ia (2.94 mmol, $1.20 \mathrm{~g}$ ) to afford the desired product in $65 \%$ yield (1.91 mmol, 1.06 g, d.r. 1:1.6 cis:trans) as a white powder. ${ }^{1} \mathbf{H} \mathbf{~ N M R ~}\left(400 \mathrm{MHz}, \mathrm{CDCl}_{3}\right)$ $\delta$ ppm: 7.78 (dd, $J=7.3,1.8 \mathrm{~Hz}, 4 \mathrm{H}_{\text {major }}$ ), $7.75-7.68$ (m, 2 $\mathrm{H}_{\text {minor }}$ ), $7.68-7.60$ (m, $4 \mathrm{H}_{\text {minor }}$ ), $7.58-7.39\left(\mathrm{~m}, 9 \mathrm{H}_{\text {minor }}+11 \mathrm{H}_{\text {major }}\right), 7.18-7.08\left(\mathrm{~m}, 2 \mathrm{H}_{\text {major }+ \text { minor }}\right), 6.90(\mathrm{t}, J=7.4 \mathrm{~Hz}$, $\left.1 \mathrm{H}_{\text {major + minor }}\right), 6.40\left(\mathrm{~s}, 1 \mathrm{H}_{\text {major }}\right), 6.29-6.19\left(\mathrm{~m}, 2 \mathrm{H}_{\text {major }+ \text { minor }}\right), 6.16\left(\mathrm{~s}, 1 \mathrm{H}_{\text {minor }}\right), 5.31-5.21\left(\mathrm{~m}, 2 \mathrm{H}_{\text {major }}\right), 5.08$ $4.98\left(\mathrm{~m}, 2 \mathrm{H}_{\text {minor }}\right), 4.87-4.78\left(\mathrm{~m}, 2 \mathrm{H}_{\text {minor }}\right), 4.76\left(\mathrm{~s}, 2 \mathrm{H}_{\text {minor }}\right), 4.70-4.61\left(\mathrm{~m}, 4 \mathrm{H}_{\text {major }}\right), 3.90-3.81$ (m, $1 \mathrm{H}_{\text {major }+ \text { minor }}$ ); ${ }^{13} \mathbf{C} \mathbf{~ N M R}\left(126 \mathrm{MHz}, \mathrm{CDCl}_{3}\right)$ [overlapping signals] $\delta$ ppm: 155.00, 154.93, 133.35, 133.04, 132.99 , 132.77, 131.43, 131.22, 130.48, 130.42, 130.38, 130.17, 130.10, 129.93, 129.77, 128.36, 127.94, 122.61, 121.03 (q, $J=320.2 \mathrm{~Hz}), 114.65,114.60,78.35,65.97,65.83,64.18,64.16,62.46,62.27$; ${ }^{19} \mathbf{F}$ NMR $\left(376 \mathrm{MHz}, \mathrm{CDCl}_{3}\right) \delta \mathrm{ppm}:-78.12$ (s, 3F). IR (thin layer film) $v\left(\mathrm{~cm}^{-1}\right)=3064,1600,1496,1457,1373,1256$, 1225, 1153, 1079, 1029, 859, 755, 735, 704, 636. HRMS (ESI $\left.{ }^{+}\right) \mathrm{m} / z \mathrm{C}_{29} \mathrm{H}_{28} \mathrm{NO}^{+}$[M-OTf] ${ }^{+}$: calculated 406.2165, found 406.2165.

\section{1-benzhydryl-1-ethyl-3-phenoxyazetidin-1-ium trifluoromethanesulfonate (1ib)}

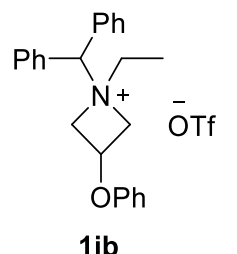

Prepared according to a slightly modified general procedure E. To a solution of commercially available 1-benzhydryl-3-phenoxyazetidine ( $4.76 \mathrm{mmol}, 1.50 \mathrm{~g})$, ethyl triflate (1.1 equiv., $0.68 \mathrm{~mL}, 5.23 \mathrm{mmol}$ ) was added dropwise and the reaction was stirred overnight at r.t. The solvent was then evaporated, the white precipitate was washed with $\mathrm{Et}_{2} \mathrm{O}$ and filtered. The solid was further washed with cold $\mathrm{Et}_{2} \mathrm{O}$ to afford the desired compound in $98 \%$ yield (4.65 mmol, 2.30 g, d.r. 1:50 cis:trans) as a white powder. ${ }^{1} \mathbf{H}$ NMR major (500 $\left.\mathbf{M H z}, \mathrm{CDCl}_{3}\right) \delta$ ppm: $7.66-7.73$ $(\mathrm{m}, 4 \mathrm{H}) 7.40-7.54(\mathrm{~m}, 6 \mathrm{H}) 7.16-7.36(\mathrm{~m}, 2 \mathrm{H}), 6.96-7.04(\mathrm{~m}, 1 \mathrm{H}), 6.59-6.66(\mathrm{~m}, 2 \mathrm{H}), 6.12(\mathrm{~s}, 1 \mathrm{H}), 5.39$ $-5.31(\mathrm{~m}, 2 \mathrm{H}), 4.48-4.41(\mathrm{~m}, 2 \mathrm{H}), 4.36-4.28(\mathrm{~m}, 1 \mathrm{H}), 3.75(\mathrm{q}, J=7.2 \mathrm{~Hz}, 2 \mathrm{H}) 1.47(\mathrm{t}, J=7.17 \mathrm{~Hz}, 3 \mathrm{H})$; ${ }^{13} \mathrm{C}$ NMR major $\left(126 \mathrm{MHz}, \mathrm{CDCl}_{3}\right) \delta$ ppm: 155.19, 132.13, 130.47, 130.37, 130.28, 130.04, 123.02, 120.60 $(\mathrm{q}, J=320.3 \mathrm{~Hz}), 114.85,75.71,66.14,63.45,59.56,8.92 ;{ }^{19} \mathbf{F}$ NMR $\left(470 \mathrm{MHz}, \mathrm{CDCl}_{3}\right) \delta \mathrm{ppm}:-78.16(\mathrm{~s}$, 3F). IR (thin layer film) $v\left(\mathrm{~cm}^{-1}\right)=3062,1600,1491,1456,1258,1224,1152,1029,916,754,704,637$. HRMS $\left(\mathrm{ESI}^{+}\right) m / z \mathrm{C}_{24} \mathrm{H}_{26} \mathrm{NO}^{+}[\mathrm{M}-\mathrm{OTf}]^{+}$: calculated 344.2014, found 344.2017.

\section{1-allyl-1-benzhydryl-3-phenoxyazetidin-1-ium trifluoromethanesulfonate 7a (1ic)}

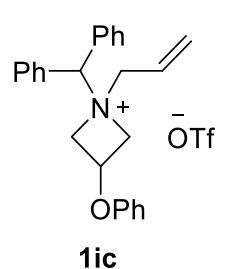

Prepared according to general procedure D, 1-benzhydryl-1-allyl-3-bromo-2-phenoxypropan1-amine 7ic (1.55 mmol, $0.68 \mathrm{~g})$ to afford the desired product in $70 \%$ yield (1.08 mmol, $0.55 \mathrm{~g}$, d.r. 1:1.2 cis:trans) as a white powder. ${ }^{1} \mathbf{H}$ NMR $\left(500 \mathrm{MHz}, \mathrm{CDCl}_{3}\right) \delta$ ppm: $7.76-7.62(\mathrm{~m}$, $\left.4 \mathrm{H}_{\text {minor }}\right), 7.58-7.38\left(\mathrm{~m}, 6 \mathrm{H}_{\text {minor }}+10 \mathrm{H}_{\text {major }}\right), 7.30-7.20\left(\mathrm{~m}, 2 \mathrm{H}_{\text {minor }}\right), 7.20-7.12\left(\mathrm{~m}, 2 \mathrm{H}_{\text {major }}\right)$, $6.97\left(\mathrm{t}, J=7.4 \mathrm{~Hz}, 1 \mathrm{H}_{\text {minor }}\right), 6.92\left(\mathrm{t}, J=7.4 \mathrm{~Hz}, 1 \mathrm{H}_{\text {major }}\right), 6.62\left(\mathrm{~d}, J=8.3 \mathrm{~Hz}, 2 \mathrm{H}_{\text {minor }}\right), 6.47$ (d, $\left.J=8.0 \mathrm{~Hz}, 2 \mathrm{H}_{\text {major }}\right), 6.23-6.10\left(\mathrm{~m}, 1 \mathrm{H}_{\text {major }}\right), 6.10-5.99\left(\mathrm{~m}, 2 \mathrm{H}_{\text {minor }}\right), 5.77-5.58\left(\mathrm{~m}, 1 \mathrm{H}_{\text {minor }}+3 \mathrm{H}_{\text {major }}\right), 5.44$ 
$\left(\mathrm{d}, J=16.7 \mathrm{~Hz}, 1 \mathrm{H}_{\text {minor }}\right), 5.30\left(\mathrm{dd}, J=13.1,6.8 \mathrm{~Hz}, 2 \mathrm{H}_{\text {minor }}\right), 5.12\left(\mathrm{p}, J=5.9 \mathrm{~Hz}, 1 \mathrm{H}_{\text {major }}\right), 5.03-4.92(\mathrm{~m}$, $\left.2 \mathrm{H}_{\text {major }}\right), 4.78\left(\mathrm{dd}, J=12.6,5.5 \mathrm{~Hz}, 2 \mathrm{H}_{\text {major }}\right), 4.54\left(\mathrm{dd}, J=13.2,4.2 \mathrm{~Hz}, 2 \mathrm{H}_{\text {minor }}\right), 4.33-4.26\left(\mathrm{~m}, 1 \mathrm{H}_{\text {minor }}+\right.$ $\left.2 \mathrm{H}_{\text {major }}\right), 4.22\left(\mathrm{~d}, J=7.2 \mathrm{~Hz}, 2 \mathrm{H}_{\text {minor }}\right) ;{ }^{13} \mathbf{C ~ N M R}\left(101 \mathrm{MHz}, \mathrm{CDCl}_{3}\right)$ [overlapping signals] $\delta$ ppm: 155.21 , $155.04,132.21,130.55,130.52,130.48,130.36,130.24,130.08,130.02,129.96,129.82,124.74,124.54$, 122.94, 122.60, 120.96 (q, $J=320.0 \mathrm{~Hz}$ ), 114.97, 114.86, 77.22. 77.09, 65.82, 65.71, 65.17 (br), 63.56, 62.62; ${ }^{19}$ F NMR (376 MHz, $\left.\mathrm{CDCl}_{3}\right) \delta \mathrm{ppm:}-78.19$ (s, 3F). IR (thin layer film) $v\left(\mathrm{~cm}^{-1}\right)=3063,1589,1492,1455$, 1425, 1376, 1257, 1225, 1153, 1079, 1029, 954, 867, 754, 704, 637. HRMS (ESI $\left.{ }^{+}\right) m / z \mathrm{C}_{25} \mathrm{H}_{26} \mathrm{NO}^{+}[\mathrm{M}-\mathrm{OTf}]^{+}$: calculated 356.2009, found 356.2004.

\section{1-benzhydryl-1-benzyl-3-(4-fluorophenoxy)azetidin-1-ium trifluoromethanesulfonate (1ja)}

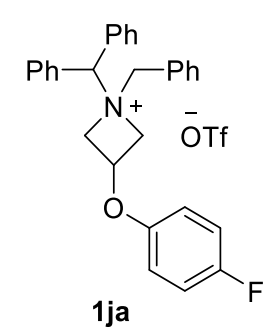

Prepared according to general procedure D using 1-benzhydryl-1-benzyl-3-bromo-2- (4fluorophenoxy)-1-amine $7 \mathbf{j a}(0.19 \mathrm{~g}, 0.38 \mathrm{mmol})$ to afford the desired product in $93 \%$ yield (0.20 g, 0.35 mmol, d.r. 1:1.2 cis:trans) as a white powder. ${ }^{1} \mathbf{H}$ NMR $\left(500 \mathrm{MHz}, \mathrm{CDCl}_{3}\right)$

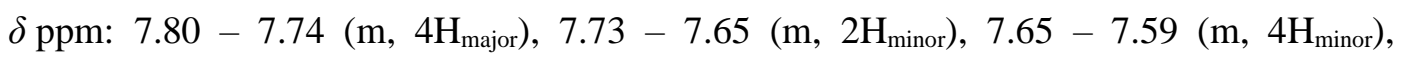

$7.58-7.43\left(\mathrm{~m}, 9 \mathrm{H}_{\text {minor }}+11 \mathrm{H}_{\text {major }}\right), 6.88-6.78\left(\mathrm{~m}, 2 \mathrm{H}_{\text {major }+ \text { minor }}\right), 6.40\left(\mathrm{~s}, 1 \mathrm{H}_{\text {major }}\right), 6.27-$ $6.16\left(\mathrm{~m}, 2 \mathrm{H}_{\text {major }+ \text { minor }}\right), 6.10\left(\mathrm{~s}, 1 \mathrm{H}_{\text {minor }}\right), 5.30-5.22\left(\mathrm{~m}, 2 \mathrm{H}_{\text {major }}\right), 5.09-5.01\left(\mathrm{~m}, 2 \mathrm{H}_{\text {minor }}\right)$,

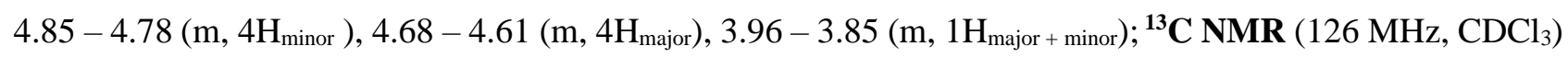
$\delta$ ppm: $158.25(\mathrm{~d}, J=241.3 \mathrm{~Hz}), 151.06(\mathrm{~d}, J=2.3 \mathrm{~Hz}), 151.02(\mathrm{~d}, J=2.4 \mathrm{~Hz}), 133.37,132.99,132.89,132.73$, $131.50,131.31,130.56,130.51,130.41,130.39,130.21,130.14,129.97,129.83,128.25,127.93,120.99$ (q, $J$ $=320.6 \mathrm{~Hz}), 116.47(\mathrm{~d}, J=23.4 \mathrm{~Hz}), 116.02(\mathrm{~d}, J=8.0 \mathrm{~Hz}), 115.96(\mathrm{~d}, J=7.9 \mathrm{~Hz}), 78.36,78.10,65.87,65.84$, 64.25, 64.23, 63.03, 62.90; ${ }^{19} \mathbf{F}$ NMR $\left\{{ }^{1} \mathrm{H}\right\}\left(470 \mathrm{MHz}, \mathrm{CDCl}_{3}\right) \delta$ ppm: -78.15 (s, 3F), -120.98 (s, $\left.1 \mathrm{~F}_{\text {major }}\right),-121.02\left(\mathrm{~s}, 1 \mathrm{~F}_{\text {minor }}\right)$. IR (thin layer film) $v\left(\mathrm{~cm}^{-1}\right)=3657,2981,2888,1505,1458,1382,1258,1224$, 1154, 1090, 1030, 953, 831, 757, 734, 706, 638. HRMS $\left(\mathrm{ESI}^{+}\right) \mathrm{m} / z \mathrm{C}_{29} \mathrm{H}_{27} \mathrm{FNO}^{+}$[M-OTf] $]^{+}$calculated 424.2071, found 424.2069.

\section{1-benzhydryl-1-benzyl-3-(isopropyloxy)azetidin-1-ium trifluoromethanesulfonate (1ka)}

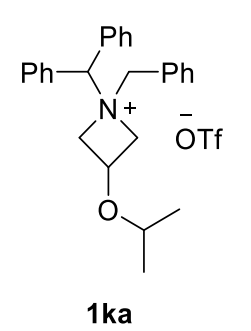

Prepared according to general procedure D, 1-benzhydryl-1-benzyl-3-bromo-2isopropyloxyamine $7 \mathbf{k a}(1.10 \mathrm{mmol}, 0.50 \mathrm{~g})$ to afford the desired product in $90 \%$ yield (0.99 mmol, 0.52 g, d.r. 1:1.2 cis:trans) as a white powder. ${ }^{1} \mathbf{H} \mathbf{~ N M R}\left(400 \mathrm{MHz}, \mathrm{CDCl}_{3}\right)$ $\delta$ ppm: $7.73-7.62\left(\mathrm{~m}, 5 \mathrm{H}_{\text {major }+ \text { minor }}\right), 7.55-7.40\left(\mathrm{~m}, 10 \mathrm{H}_{\text {major }+ \text { minor }}\right), 6.14-6.08(\mathrm{~m}$, $\left.1 \mathrm{H}_{\text {major + minor }}\right), 5.06-4.97\left(\mathrm{~m}, 2 \mathrm{H}_{\text {major }}\right), 4.82-4.72\left(\mathrm{~m}, 2 \mathrm{H}_{\text {minor }}\right), 4.67-4.57(\mathrm{~m}$, $\left.4 \mathrm{H}_{\text {minor }}+2 \mathrm{H}_{\text {major }}\right), 4.54-4.45\left(\mathrm{~m}, 2 \mathrm{H}_{\text {major }}\right), 3.25-3.04\left(\mathrm{~m}, 2 \mathrm{H}_{\text {major }+ \text { minor }}\right), 0.78(\mathrm{~d}, J=6.1 \mathrm{~Hz}$, $\left.6 \mathrm{H}_{\text {major }}\right), 0.71\left(\mathrm{~d}, J=6.1 \mathrm{~Hz}, 6 \mathrm{H}_{\text {minor }}\right) ;{ }^{13} \mathbf{C ~ N M R}\left(101 \mathrm{MHz}, \mathrm{CDCl}_{3}\right)$ [overlapping signals] $\delta \mathrm{ppm:} 133.30$, 133.20, 133.04, 131.16, 131.10, 130.49, 130.31, 130.29, 130.06, 129.98, 129.76, 129.63, 128.61, 128.18, $121.06(\mathrm{q}, J=320.1 \mathrm{~Hz}), 78.28,77.45,71.71,71.67,66.19,65.87,65.52,65.50,62.48,62.45,21.99 ;{ }^{19} \mathbf{F ~ N M R}$ $\left(470 \mathrm{MHz}, \mathrm{CDCl}_{3}\right) \delta \mathrm{ppm}:-78.14(\mathrm{~s}, 3 \mathrm{~F})$. IR (thin layer film) $v\left(\mathrm{~cm}^{-1}\right)=2975,1499,1457,1384,1338,1257$, 
1224, 1154, 1030, 860, 757, 706, 637. HRMS $\left(\mathrm{ESI}^{+}\right) \mathrm{m} / 2 \mathrm{C}_{26} \mathrm{H}_{30} \mathrm{FNO}^{+}$[M-OTf] $]^{+}$calculated 372.2322, found 372.2325 .

\section{1-benzhydryl-1-ethyl-3-isopropoxyazetidin-1-ium trifluoromethanesulfonate (1kb)}

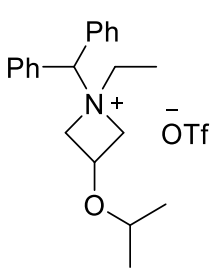

$1 \mathrm{~kb}$

Prepared according to general procedure E, 1-benzhydryl-3-isopropyloxyazetidine $7 \mathbf{k b}$ $(1.78 \mathrm{mmol}, 0.50 \mathrm{~g})$ to afford the desired product in $99 \%$ yield $(1.75 \mathrm{mmol}, 0.81 \mathrm{~g}$, d.r. 1:1.2 cis:trans) as a white powder. ${ }^{1} \mathbf{H}$ NMR $\left(500 \mathrm{MHz}, \mathrm{CDCl}_{3}\right) \delta 7.67-7.61\left(\mathrm{~m}, 2 \mathrm{H}_{\text {major + minor }}\right)$, $7.55-7.40\left(\mathrm{~m}, 8 \mathrm{H}_{\text {major }+ \text { minor }}\right), 5.94\left(\mathrm{~s}, 1 \mathrm{H}_{\text {major }}\right), 5.57\left(\mathrm{~s}, 1 \mathrm{H}_{\text {minor }}\right), 5.15-5.08\left(\mathrm{~m}, 2 \mathrm{H}_{\text {major }}\right), 4.72$ - $4.65\left(\mathrm{~m}, 2 \mathrm{H}_{\text {minor }}\right), 4.65-4.59$ (m, $\left.1 \mathrm{H}_{\text {minor }}\right), 4.59-4.54\left(\mathrm{~m}, 2 \mathrm{H}_{\text {minor }}\right), 4.29-4.22\left(\mathrm{~m}, 2 \mathrm{H}_{\text {major }}\right)$,

$3.78-3.70\left(\mathrm{~m}, 2 \mathrm{H}_{\text {major }+ \text { minor }}\right), 3.62-3.55\left(\mathrm{~m}, 1 \mathrm{H}_{\text {major }}\right), 3.51$ (sept, $\left.J=6.1 \mathrm{~Hz}, 1 \mathrm{H}_{\text {major }}\right), 3.42$ (sept, $\left.J=6.1 \mathrm{~Hz}, 1 \mathrm{H}_{\text {minor }}\right), 1.45\left(\mathrm{t}, J=7.2 \mathrm{~Hz}, 3 \mathrm{H}_{\text {major }}\right), 1.41\left(\mathrm{t}, J=7.1 \mathrm{~Hz}, 3 \mathrm{H}_{\text {minor }}\right), 1.05\left(\mathrm{~d}, J=6.1 \mathrm{~Hz}, 6 \mathrm{H}_{\text {major }}\right.$ ), $0.89\left(\mathrm{~d}, J=6.1 \mathrm{~Hz}, 6 \mathrm{H}_{\text {minor }}\right) ;{ }^{13} \mathbf{C}$ NMR $\left(126 \mathrm{MHz}, \mathrm{CDCl}_{3}\right)$ overlapping signals: $\delta 132.25,130.39,130.30$, 130.24, 129.94, 129.91, 121.01 (q, $J=320.4 \mathrm{~Hz}$ ), 75.60, 74.73, 72.61, 72.01, 67.79, 66.76, 63.39, 63.04, 59.85, 58.43, 22.27, 22.19, 9.06, 8.76; ${ }^{19} \mathbf{F}$ NMR $\left(470 \mathrm{MHz}, \mathrm{CDCl}_{3}\right) \delta-78.25(\mathrm{~s}, 3 \mathrm{~F})$. IR (thin layer film) $v\left(\mathrm{~cm}^{-1}\right)=$ $2977,1499,1456,1384,1336,1258,1224,1154,1030,932,780,754,708,637$. HRMS $\left(\mathrm{ESI}^{+}\right) \mathrm{m} / z \mathrm{C}_{21} \mathrm{H}_{28} \mathrm{NO}^{+}$ [M-OTf] $]^{+}$calculated 310.2165 , found 310.2173 .

\section{1-benzhydryl-1-benzyl-3-(methoxy)azetidin-1-ium trifluoromethanesulfonate (1la)}

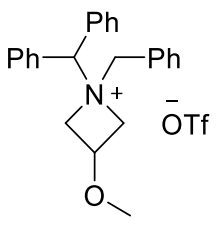

$11 a$

Prepared according to general procedure D using 1-benzhydryl-1-benzyl-3-bromo-2-methoxy1-amine $7 \mathbf{l a}(1.62 \mathrm{mmol}, 0.69 \mathrm{~g})$ to afford the desired product in $88 \%$ yield $(1.43 \mathrm{mmol}, 0.70 \mathrm{~g}$, d.r. 1:1.3 cis:trans) as a white powder. ${ }^{1} \mathbf{H}$ NMR $\left(500 \mathrm{MHz}, \mathrm{CDCl}_{3}\right) \delta \mathrm{ppm:} 7.69-7.62(\mathrm{~m}$, $\left.5 \mathrm{H}_{\text {major }+ \text { minor }}\right), 7.55-7.41\left(\mathrm{~m}, 10 \mathrm{H}_{\text {major }+ \text { minor }}\right), 6.13\left(\mathrm{~s}, 1 \mathrm{H}_{\text {minor }}\right), 6.02\left(\mathrm{~s}, 1 \mathrm{H}_{\text {major }}\right), 5.05-4.96$

(m, $\left.2 \mathrm{H}_{\text {major }}\right), 4.83-4.75$ (m, 2 $\mathrm{H}_{\text {minor }}$ ), 4.69 (s, 2 $\mathrm{H}_{\text {major }}$ ), 4.64 (d, J=5.8 Hz, $4 \mathrm{H}_{\text {minor }}$ ), $4.59-4.52$ (m, $\left.2 \mathrm{H}_{\text {major }}\right), 3.17-3.08\left(\mathrm{~m}, 1 \mathrm{H}_{\text {major }}\right), 3.01\left(\mathrm{p}, J=6.6 \mathrm{~Hz}, 1 \mathrm{H}_{\text {minor }}\right), 2.94\left(\mathrm{~s}, 3 \mathrm{H}_{\text {major }}\right), 2.81\left(\mathrm{~s}, 3 \mathrm{H}_{\text {minor }}\right) ;{ }^{13} \mathbf{C ~ N M R}$ $\left(126 \mathrm{MHz}, \mathrm{CDCl}_{3}\right)$ [overlapping signals] $\delta$ ppm: 133.14, 133.09, 133.00, 132.93, 131.23, 130.46, 130.39, 130.35, 130.30, 130.12, 130.03, 129.82, 129.67, 128.44, 127.99, 121.05 (q, $J=320.3 \mathrm{~Hz}$ ), 78.41, 66.30, 66.16, 65.84, 65.67, 64.97, 64.61, 56.73, 56.68; ${ }^{19} \mathbf{F}$ NMR (470 MHz, $\left.\mathrm{CDCl}_{3}\right) \delta$ ppm: -78.15 (s, 3F). IR (thin layer film) $v\left(\mathrm{~cm}^{-1}\right)=2981,1498,1456,1360,1255,1224,1153,1029,1002,858,736,701,636$. HRMS $\left(\mathrm{ESI}^{+}\right) \mathrm{m} / \mathrm{z}$ $\mathrm{C}_{24} \mathrm{H}_{26} \mathrm{NO}_{+}[\mathrm{M}-\mathrm{OTf}]^{+}$: calculated 344.2009, found 344.2010.

\section{1-benzhydryl-1-ethyl-3-methoxyazetidin-1-ium trifluoromethanesulfonate (1lb)}

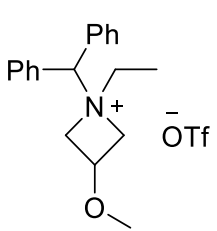

$1 \mathrm{lb}$

Prepared according to general procedure E using 1-benzhydryl-3-methoxyazetidine 7lb $(1.97 \mathrm{mmol}, 0.50 \mathrm{~g})$ to afford the desired product in quantitative yield $(1.96 \mathrm{mmol}, 0.85 \mathrm{~g}$, d.r. 1:2.6 cis:trans) as a white powder. ${ }^{1} \mathbf{H}$ NMR $\left(500 \mathrm{MHz}, \mathrm{CDCl}_{3}\right) \delta$ ppm: $7.65-7.57(\mathrm{~m}$, $\left.3 \mathrm{H}_{\text {major }+ \text { minor }}\right), 7.54-7.41\left(\mathrm{~m}, 7 \mathrm{H}_{\text {major }+ \text { minor }}\right), 5.88\left(\mathrm{~s}, 1 \mathrm{H}_{\text {major }}\right), 5.59\left(\mathrm{~s}, 1 \mathrm{H}_{\text {minor }}\right), 5.13-5.05(\mathrm{~m}$, $\left.2 \mathrm{H}_{\text {major }}\right), 4.72-4.65$ (m, $2 \mathrm{H}_{\text {minor }}$ ), $4.61-4.53$ (m, $\left.2 \mathrm{H}_{\text {minor }}\right), 4.49$ (p, J=6.4 Hz, $\left.1 \mathrm{H}_{\text {minor }}\right), 4.33$ $4.26\left(\mathrm{~m}, 2 \mathrm{H}_{\text {major }}\right), 3.80-3.70\left(\mathrm{~m}, 2 \mathrm{H}_{\text {major }+ \text { minor }}\right), 3.58-3.50\left(\mathrm{~m}, 1 \mathrm{H}_{\text {major }}\right), 3.25\left(\mathrm{~s}, 3 \mathrm{H}_{\text {major }}\right), 3.07\left(\mathrm{~s}, 3 \mathrm{H}_{\text {minor }}\right), 1.50$ 
- 1.39 (m, $\left.3 \mathrm{H}_{\text {major + minor }}\right) ;{ }^{13} \mathbf{C ~ N M R}\left(126 \mathrm{MHz}, \mathrm{CDCl}_{3}\right)$ [overlapping signals] $\delta$ ppm: 132.22, 132.16, 130.31, 130.25, 129.91, 129.85, 124.84, 122.30,121.02 (q, $J=320.3 \mathrm{~Hz}), 119.75,117.20,75.52,74.46,67.00,66.45$, 66.41, 65.77, 59.87, 58.57, 57.06, 56.88, 8.94, 8.50; ${ }^{19} \mathbf{F}$ NMR (470 MHz, $\left.\mathrm{CDCl}_{3}\right) \delta$ ppm: -78.26 (s, 3F). IR (thin layer film) $v\left(\mathrm{~cm}^{-1}\right)=2982,1499,1456,1382,1258,1224,1154,1082,1030,935,781,754,709,637$. HRMS $\left(\mathrm{ESI}^{+}\right) \mathrm{m} / z \mathrm{C}_{19} \mathrm{H}_{24} \mathrm{NO}^{+}[\mathrm{M}-\mathrm{OTf}]^{+}$: calculated 282.1852, found 282.1860.

\section{1-benzhydryl-1-benzyl-3-(benzyloxy)azetidin-1-ium trifluoromethanesulfonate (1ma)}

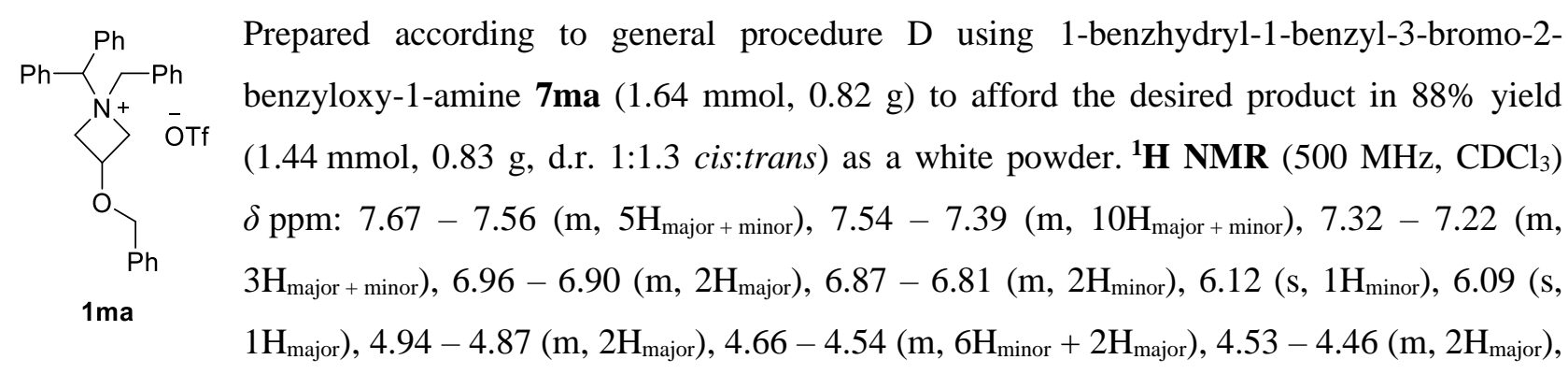

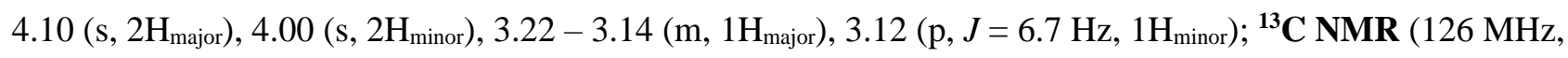
$\left.\mathrm{CDCl}_{3}\right) \delta$ ppm: 136.01, 135.99, 133.27, 133.06, 132.98, 132.94, 131.16, 130.46, 130.35, 130.31, 130.24, $130.06,130.04,129.74,129.72,128.68,128.61,128.54,128.43,128.14,128.09,128.07,121.04$ (q, $J=320.3$ $\mathrm{Hz}), 78.22,71.72,71.68,66.08,65.50,64.97,64.64,64.11,64.07 ;{ }^{19} \mathbf{F ~ N M R}\left(470 \mathrm{MHz}, \mathrm{CDCl}_{3}\right) \delta \mathrm{ppm}:-78.12$ (s, 3F). IR (thin layer film) $v\left(\mathrm{~cm}^{-1}\right)=3064,1499,1456,1377,1256,1224,1153,1029,991,858,757,704$, 637. HRMS $\left(\mathrm{ESI}^{+}\right) \mathrm{m} / z \mathrm{C}_{30} \mathrm{H}_{30} \mathrm{NO}^{+}[\mathrm{M}-\mathrm{OTf}]^{+}$: calculated 420.2322, found 420.2323 .

\section{1-benzhydryl-3-(benzyloxy)-1-ethylazetidin-1-ium trifluoromethanesulfonate (1mb)}

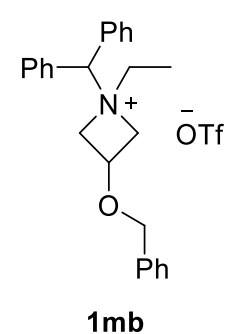

Prepared according to general procedure E using 1-benzhydryl-3-benzyloxyazetidine $\mathbf{7 m b}$ (3.95 mmol, $1.30 \mathrm{~g}$,) was reacted with ethyl triflate $(0.77 \mathrm{~mL}, 5.92 \mathrm{mmol})$ to afford the desired product in $99 \%$ yield (3.94 mmol, $2.00 \mathrm{~g}$, d.r. 1:5 cis:trans) as a white powder. ${ }^{1} \mathbf{H}$ NMR major $\left(400 \mathrm{MHz}, \mathrm{CDCl}_{3}\right) \delta$ ppm: 7.65 - $7.56(\mathrm{~m}, 3 \mathrm{H}), 7.50-7.38(\mathrm{~m}, 7 \mathrm{H}), 7.35-7.27(\mathrm{~m}, 3 \mathrm{H}), 7.25$ $-7.20(\mathrm{~m}, 2 \mathrm{H}), 5.84(\mathrm{~s}, 1 \mathrm{H}), 5.09-4.97(\mathrm{~m}, 2 \mathrm{H}), 4.41(\mathrm{~s}, 2 \mathrm{H}), 4.35-4.25(\mathrm{~m}, 2 \mathrm{H}), 3.73(\mathrm{q}$, $J=7.2 \mathrm{~Hz}, 2 \mathrm{H}), 3.69-3.57(\mathrm{~m}, 1 \mathrm{H}), 1.40(\mathrm{t}, J=7.2 \mathrm{~Hz}, 3 \mathrm{H}) ;{ }^{13} \mathbf{C}$ NMR major (101 MHz, $\left.\mathrm{CDCl}_{3}\right) \delta$ ppm: 136.06, 132.16, 130.32, 130.26, 129.95, 128.87, 128.67, 128.36, 121.06 (q, $\left.J=320.3 \mathrm{~Hz}\right)$, 74.72, 72.07, 66.77, 65.03, 59.80, 8.65; ${ }^{19} \mathbf{F}$ NMR (470 MHz, $\left.\mathrm{CDCl}_{3}\right) \delta$ ppm: -78.17 (s, 3F). IR (thin layer

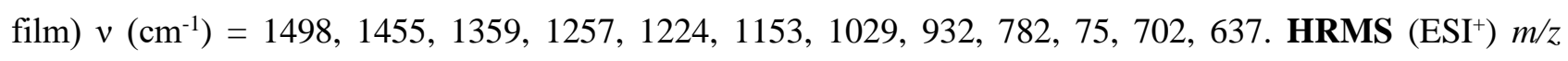
$\mathrm{C}_{25} \mathrm{H}_{28} \mathrm{NO}^{+}[\mathrm{M}-\mathrm{OTf}]^{+}$: calculated 358.2171, found 358.2178. 


\section{1-benzhydryl-3-(benzoyloxy)-1-ethylazetidin-1-ium trifluoromethanesulfonate (1na)}

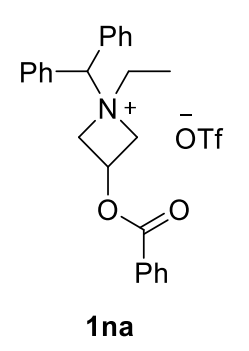

Prepared according to general procedure E using 1-benzhydrylazetidin-3-yl benzoate (2.43 mmol, $0.83 \mathrm{~g})$ to afford the desired product in $95 \%$ yield $(2.30 \mathrm{mmol}, 1.27 \mathrm{~g}$, d.r. 1:1.1 cis:trans) as a white powder. ${ }^{1} \mathbf{H}$ NMR $\left(400 \mathrm{MHz}, \mathrm{CDCl}_{3}\right) \delta$ ppm: $8.02-7.95$ (m, $\left.1 \mathrm{H}_{\text {major + minor }}\right), 7.70-7.31\left(\mathrm{~m}, 14 \mathrm{H}_{\text {major }+ \text { minor }}\right), 6.03\left(\mathrm{~s}, 1 \mathrm{H}_{\text {major }}\right), 5.87\left(\mathrm{~s}, 1 \mathrm{H}_{\text {minor }}\right), 5.44(\mathrm{p}, J=$ $\left.6.5 \mathrm{~Hz}, 1 \mathrm{H}_{\text {minor }}\right), 5.40-5.31$ (m, 2 $\left.\mathrm{H}_{\text {major }}\right), 5.03-4.97$ (m, $\left.4 \mathrm{H}_{\text {minor }}\right), 4.76-4.67$ (m, $2 \mathrm{H}_{\text {major }}$ ), $4.48-4.38\left(\mathrm{~m}, 1 \mathrm{H}_{\text {major }}\right), 3.87-3.76\left(\mathrm{~m}, 2 \mathrm{H}_{\text {major }}+\right.$ minor $), 1.56-1.47$ (m, $\left.3 \mathrm{H}_{\text {major }+ \text { minor }}\right) ;{ }^{13} \mathbf{C ~ N M R}$ $\left(126 \mathrm{MHz}, \mathrm{CDCl}_{3}\right)$ [overlapping signals] $\delta$ ppm: 165.61, 165.18, 134.42, 134.11, 132.29, 132.11, 130.56, 130.46, 130.33, 130.19, 130.13, 130.08, 129.92, 129.87, 128.91, 128.48, 127.81, 120.87 (q, $J=319.8 \mathrm{~Hz}$ ), $75.82,75.17,65.72,64.75,61.01,60.80,60.49,59.04,9.06,8.92 ;{ }^{19} \mathbf{F ~ N M R}\left(470 \mathrm{MHz}, \mathrm{CDCl}_{3}\right) \delta$ ppm: -78.27 (s, 3F). IR (thin layer film) $v\left(\mathrm{~cm}^{-1}\right)=3062,1722,1602,1498,1454,1426,1260,1225,1154,1114,1072$, 1030, 934, 754, 711, 637. HRMS $\left(\mathrm{ESI}^{+}\right) \mathrm{m} / \mathrm{z} \mathrm{C}_{25} \mathrm{H}_{26} \mathrm{NO}_{2}{ }^{+}$[M-OTf] ${ }^{+}$: calculated 372.1964, found 372.1964.

\section{1-benzhydryl-5'-bromo-1-ethyl-3' H-spiro[azetidine-3,1'-isobenzofuran]-1-ium trifluoromethanesulfonate (10a)}

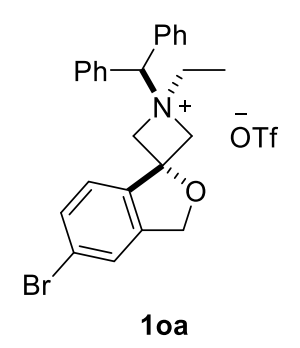

Prepared according to general procedure E using 1-benzhydryl-5'-bromo-3'Hspiro[azetidine-3,1'-isobenzofuran] $(0.74 \mathrm{mmol}, 0.30 \mathrm{~g})$ to afford the desired product in 97\% yield (0.72 mmol, $0.42 \mathrm{~g}$, single diastereomer) as a white powder. ${ }^{1} \mathbf{H}$ NMR (400 MHz, DMSO- $\left.d_{6}\right) \delta$ ppm: $7.75(\mathrm{dd}, J=6.7,3.0 \mathrm{~Hz}, 4 \mathrm{H}), 7.62-7.54(\mathrm{~m}, 7 \mathrm{H}), 7.26(\mathrm{~d}$, $J=8.1 \mathrm{~Hz}, 1 \mathrm{H}), 5.96(\mathrm{~s}, 1 \mathrm{H}), 5.15-5.03(\mathrm{~m}, 5 \mathrm{H}), 4.86(\mathrm{~d}, J=13.4 \mathrm{~Hz}, 2 \mathrm{H}), 3.82(\mathrm{q}$, $J=7.0 \mathrm{~Hz}, 2 \mathrm{H}), 1.32(\mathrm{t}, J=7.0 \mathrm{~Hz}, 3 \mathrm{H}) ;{ }^{13} \mathbf{C ~ N M R}\left(101 \mathrm{MHz}, \mathrm{DMSO}-d_{6}\right) \delta$ ppm: 141.80 , $136.33,132.44,130.76,130.69,130.03,129.51,124.74,122.46,121.92,120.68$ (q, $J=322.4 \mathrm{~Hz}), 81.53$, 72.08, 71.40, 71.01, 58.42, 8.06; ${ }^{19} \mathbf{F}$ NMR (470 MHz, $\left.\mathrm{CDCl}_{3}\right) \delta \mathrm{ppm:}-78.32$ (s, 3F). IR (thin layer film) $v\left(\mathrm{~cm}^{-1}\right)=3657,2981,2888,1462,1383,1254,1152,1071,1030,955,817,705,638$. HRMS $\left(\mathrm{ESI}^{+}\right) \mathrm{m} / \mathrm{z}$ $\mathrm{C}_{25} \mathrm{H}_{25} \mathrm{BrNO}^{+}$[M-OTf] $]^{+}$c calculated 434.1119, found 434.1119.

\section{1-benzhydryl-1-ethyl-3-(thiophen-3-yl)azetidin-1-ium trifluoromethanesulfonate (1pa)}

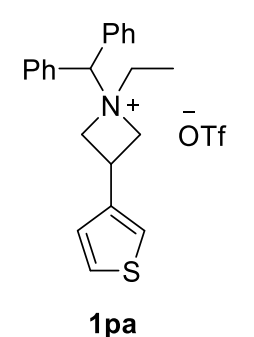

Prepared according to a slightly modified general procedure E. To a solution of , 1-benzhydryl3-(thiophen-3-yl)azetidine 7pa $(0.66 \mathrm{mmol}, 0.20 \mathrm{~g})$, ethyl triflate (1 equiv., $0.65 \mathrm{mmol}$, $0.08 \mathrm{~mL}$ ) was added dropwise and the reaction was stirred overnight at r.t. The solvent was then evaporated, the white precipitate was washed with $\mathrm{Et}_{2} \mathrm{O}$ and filtered. The solid was further washed with cold $\mathrm{Et}_{2} \mathrm{O}$ to afford the desired compound in $95 \%$ yield $(0.30 \mathrm{~g}, 0.62 \mathrm{mmol}$, d.r. 8.2:1 cis:trans) as a white powder. ${ }^{1} \mathbf{H}$ NMR major $\left(500 \mathrm{MHz}, \mathrm{CDCl}_{3}\right) \delta$ ppm: $7.63-7.55$ (m, 4H), $7.55-7.45(\mathrm{~m}, 6 \mathrm{H}), 7.04(\mathrm{dd}, J=5.0,3.0 \mathrm{~Hz}, 1 \mathrm{H}), 6.48(\mathrm{dd}, J=3.0,1.4 \mathrm{~Hz}, 1 \mathrm{H}), 5.92(\mathrm{dd}, J=5.1$, $1.4 \mathrm{~Hz}, 1 \mathrm{H}), 5.68(\mathrm{~s}, 1 \mathrm{H}), 4.88-4.72(\mathrm{~m}, 2 \mathrm{H}), 4.72-4.57(\mathrm{~m}, 2 \mathrm{H}), 4.46$ (p, J= 9.3 Hz, 1H), 3.87 (q, $J=7.1$

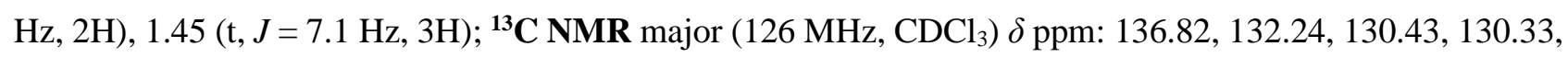
129.99, 127.07, 125.13, 122.78, 120.83 (q, $J=320.4 \mathrm{~Hz}), 74.44,64.86,57.43,28.17,8.82 ;{ }^{19} \mathbf{F}$ NMR $(470$ 
$\left.\mathrm{MHz}, \mathrm{CDCl}_{3}\right) \delta \mathrm{ppm}$ - -78.21 (s, 3F). IR (thin layer film) $v\left(\mathrm{~cm}^{-1}\right)=1498,1455,1259,1224,1155,1030,934$, 784, 753, 721, 705, 637. HRMS (ESI $\left.{ }^{+}\right) \mathrm{m} / z \mathrm{C}_{22} \mathrm{H}_{24} \mathrm{NS}^{+}[\mathrm{M}-\mathrm{OTf}]^{+}$: calculated 334.1624, found 334.1628.

\section{2-((1-benzhydryl-3-(4-bromo-1H-pyrazol-1-yl)azetidin-1-ium-1-yl)methyl)benzen-1-ide} trifluoromethanesulfonate salt (1qa)

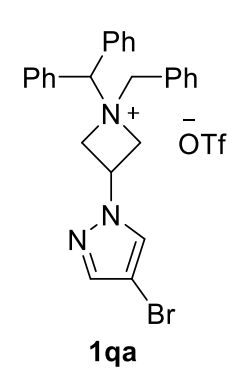

Prepared according to general procedure D using 1-benzhydryl-1-benzyl-3-bromo-2-(4bromo-1H-pyrazol-1-yl)propan-1-amine 7qa $(0.88 \mathrm{mmol}, 0.48 \mathrm{~g}$,$) to afford the desired$ product in $90 \%$ yield $\left(0.79 \mathrm{mmol}, 0.48 \mathrm{~g}\right.$, d.r. 1:2.1 cis:trans) as a white powder. ${ }^{1} \mathbf{H}$ NMR $\left(500 \mathrm{MHz}, \mathrm{CDCl}_{3}\right) \delta$ ppm: $7.71-7.64\left(\mathrm{~m}, 3 \mathrm{H}_{\text {major }+ \text { minor }}\right), 7.64-7.42\left(\mathrm{~m}, 12 \mathrm{H}_{\text {major + minor }}\right), 7.27$ $\left(\mathrm{s}, 1 \mathrm{H}_{\text {minor }}\right), 7.08\left(\mathrm{~s}, 1 \mathrm{H}_{\text {major }}\right), 7.04\left(\mathrm{~s}, 1 \mathrm{H}_{\text {minor }}\right), 6.87\left(\mathrm{~s}, 1 \mathrm{H}_{\text {major }}\right), 6.09\left(\mathrm{~s}, 1 \mathrm{H}_{\text {major }}\right), 6.06(\mathrm{~s}$, $\left.1 \mathrm{H}_{\text {minor }}\right), 5.36-5.23\left(\mathrm{~m}, 2 \mathrm{H}_{\text {major + minor }}\right), 5.13-5.06\left(\mathrm{~m}, 2 \mathrm{H}_{\text {minor }}\right), 5.02-4.95\left(\mathrm{~m}, 2 \mathrm{H}_{\text {major }}\right), 4.90$

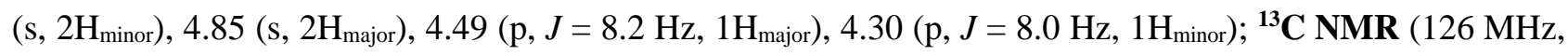
$\left.\mathrm{CDCl}_{3}\right) \delta$ ppm: 141.51, 141.30, 133.10, 132.92, 132.47, 132.39, 131.64, 131.48, 130.71, 130.61, 130.57, 130.40, 130.26, 130.23, 130.21, 130.14, 129.98, 129.57, 127.84, 127.55, 120.92 (q, $J=320.1 \mathrm{~Hz}), 94.69$, 94.60, 78.16, 77.32, 65.67, 65.29, 64.34, 63.51, 48.13, 47.77; ${ }^{19}$ F NMR (470 MHz, $\left.\mathrm{CDCl}_{3}\right) \delta$ ppm: -78.19 (s, 3F). IR (thin layer film) $v\left(\mathrm{~cm}^{-1}\right)=3063,1498,1456,1384,1324,1256,1224,1156,1030,989,950,868,757$, 703, 637, 608. HRMS (ESI $\left.{ }^{+}\right) m / z \mathrm{C}_{26} \mathrm{H}_{25} \mathrm{BrN}_{3}{ }^{+}$[M-OTf] $]^{+}$calculated 458.1226, found 458.1228.

\section{1-benzhydryl-1-benzyl-3-(3-methyl-1H-indol-1-yl)azetidin-1-ium trifluoromethanesulfonate (1ra)}

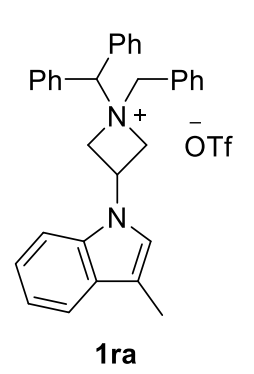

Prepared according to general procedure D using 1-benzhydryl-1-benzyl-3-bromo-2-(3methyl-1H-indol-1-yl)propan-1-amine 7ra $(0.90 \mathrm{mmol}, 0.46 \mathrm{~g})$ to afford the desired product in $90 \%$ yield (0.81 mmol, 0.48 g, d.r. 1:1.9 cis:trans) as a white powder. ${ }^{1} \mathbf{H}$ NMR (500 $\left.\mathrm{MHz}, \mathrm{CDCl}_{3}\right) \delta$ ppm: 7.93 - 7.88 (m, 2 $\mathrm{H}_{\text {major }}$ ), $7.88-7.82\left(\mathrm{~m}, 4 \mathrm{H}_{\text {minor }}\right), 7.81-7.75$ (m, $\left.4 \mathrm{H}_{\text {major }}\right), 7.75-7.70\left(\mathrm{~m}, 2 \mathrm{H}_{\text {minor }}\right), 7.69-7.53\left(\mathrm{~m}, 9 \mathrm{H}_{\text {major }+ \text { minor }}\right), 7.36-7.30\left(\mathrm{~m}, 1 \mathrm{H}_{\text {major }+ \text { minor }}\right)$, $7.04-6.94\left(\mathrm{~m}, 2 \mathrm{H}_{\text {major }+ \text { minor }}\right), 6.60\left(\mathrm{~s}, 1 \mathrm{H}_{\text {minor }}\right), 6.46\left(\mathrm{~s}, 1 \mathrm{H}_{\text {major }}\right), 6.38-6.30\left(\mathrm{~m}, 1 \mathrm{H}_{\text {major }+ \text { minor }}\right)$, $5.27-5.19$ (m, 2 $\left.\mathrm{H}_{\text {minor }}\right), 5.14-5.07$ (m, 2 $\left.\mathrm{H}_{\text {major }}\right), 5.05\left(\mathrm{~s}, 1 \mathrm{H}_{\text {minor }}\right), 5.03-4.96\left(\mathrm{~m}, 3 \mathrm{H}_{\text {major }}\right), 4.96-4.90$ (m, $2 \mathrm{H}_{\text {minor }}$ ), 4.76 (s, 2 $\mathrm{H}_{\text {major }}$ ), 4.66 (s, $\left.2 \mathrm{H}_{\text {minor }}\right), 3.90$ (p, $\left.J=8.7 \mathrm{~Hz}, 1 \mathrm{H}_{\text {major }}\right), 3.71$ (p, $\left.J=8.8 \mathrm{~Hz}, 1 \mathrm{H}_{\text {minor }}\right), 1.98$ (d, $J$ $\left.=1.0 \mathrm{~Hz}, 3 \mathrm{H}_{\text {major }}\right), 1.97\left(\mathrm{~d}, J=1.1 \mathrm{~Hz}, 3 \mathrm{H}_{\text {minor }}\right) ;{ }^{13} \mathbf{C ~ N M R}\left(126 \mathrm{MHz}, \mathrm{CDCl}_{3}\right)$ [overlapping signals] $\delta$ ppm: 136.43, 136.36, 134.39, 133.17, 133.06, 132.82, 131.66, 131.39, 130.66, 130.62, 130.46, 130.37, 130.30, 130.10, 128.66, 128.64, 128.44, 128.40, 122.64, 122.55, 120.98 (q, $J=320.1 \mathrm{~Hz}$ ), 120.16, 120.11, 119.39, 118.99, 118.75, 114.66, 114.47, 107.85, 107.75, 78.45, 78.41, 65.71, 65.49, 64.09, 63.73, 41.60, 40.98, 9.42, 9.40; ${ }^{19}$ F NMR (470 MHz, $\left.\mathrm{CDCl}_{3}\right) \delta \mathrm{ppm}$ : -78.11 (s, 3F). IR (thin layer film) $v\left(\mathrm{~cm}^{-1}\right)=3062,1498,1461$, 1390, 1258, 1225, 1159, 1030, 742, 706, 638. HRMS (ESI $\left.{ }^{+}\right) \mathrm{m} / 2 \mathrm{C}_{32} \mathrm{H}_{31} \mathrm{~N}_{2}{ }^{+}$[M-OTf $]^{+}$: calculated 443.2482, found 443.2482 . 


\section{1-benzhydryl-1-benzyl-3-(1,3-dioxoisoindolin-2-yl)azetidin-1-ium trifluoromethanesulfonate (1sa)}

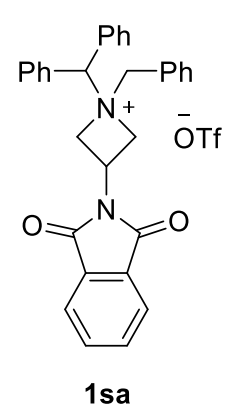

Prepared according to general procedure D using 2-(1-(benzhydryl(benzyl)amino)-3bromopropan-2-yl)isoindoline-1,3-dione 7sa $(1.0 \mathrm{~g}, 1.85 \mathrm{mmol})$ to afford the desired product in $69 \%$ yield (0.77 g, $1.28 \mathrm{mmol}$, d.r. 1: 2.2 cis:trans) as a white powder. ${ }^{1} \mathbf{H} \mathbf{~ N M R}(400 \mathrm{MHz}$,

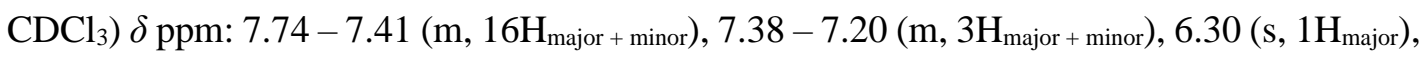
$5.88\left(\mathrm{~s}, 1 \mathrm{H}_{\text {minor }}\right), 5.56-5.42\left(\mathrm{~m}, 2 \mathrm{H}_{\text {major }+ \text { minor }}\right), 5.28-5.18\left(\mathrm{~m}, 2 \mathrm{H}_{\text {minor }}\right), 4.99-4.89(\mathrm{~m}$, $\left.2 \mathrm{H}_{\text {major + minor }}\right), 4.71\left(\mathrm{~s}, 2 \mathrm{H}_{\text {major }}\right), 3.78\left(\mathrm{p}, J=8.3 \mathrm{~Hz}, 1 \mathrm{H}_{\text {major }}\right), 3.67$ (p, $\left.J=8.4 \mathrm{~Hz}, 1 \mathrm{H}_{\text {minor }}\right) ;{ }^{13} \mathbf{C}$ NMR (101 MHz, $\left.\mathrm{CDCl}_{3}\right) \delta$ ppm: 167.15, 166.91, 134.75, 134.73, 132.76, 132.60, 132.54, $132.50,131.50,131.21,131.11,131.02,130.50,130.34,130.23,130.07,130.04,129.63,128.02,127.74$, $123.71,123.62,120.92(\mathrm{q}, J=320.2 \mathrm{~Hz}), 79.51,76.68,67.01,64.88,62.55,61.13,38.20,37.20 ;{ }^{19} \mathbf{F} \mathbf{~ N M R}$ $\left(377 \mathrm{MHz}, \mathrm{CDCl}_{3}\right) \delta \mathrm{ppm}:-78.17$ (s, 3F). IR (thin layer film) $v\left(\mathrm{~cm}^{-1}\right)=3065,1779,1716,1498,1456,1388$, 1258, 1224, 1156, 1089, 1030, 876, 755, 717, 637. HRMS (ESI $\left.{ }^{+}\right) m / z \mathrm{C}_{31} \mathrm{H}_{27} \mathrm{~N}_{2} \mathrm{O}_{2}{ }^{+}$[M-OTf] $]^{+}$calculated 459.2067, found 459.2066 .

\section{1-benzhydryl-1-benzyl-3-(tert-butoxycarbonyl)azetidin-1-ium trifluoromethanesulfonate (1ta)}

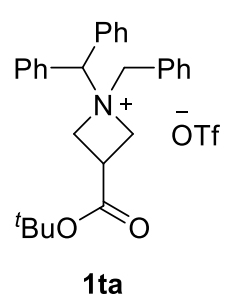

Prepared according to general procedure D using tert-butyl 3-(benzhydryl(benzyl)amino)-2(bromomethyl)propanoate $7 \mathrm{ta}(1.13 \mathrm{~g}, 2.28 \mathrm{mmol})$ to afford the desired product in $61 \%$ yield (785 mg, 1.39 mmol, d.r. 1.3:1 cis:trans) as a white powder. ${ }^{1} \mathbf{H}$ NMR $\left(400 \mathrm{MHz}, \mathrm{CDCl}_{3}\right)$

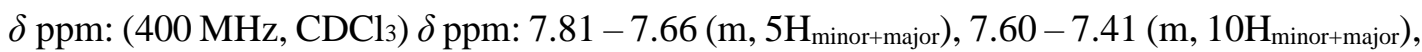
6.31 (s, 1 $\left.1 \mathrm{H}_{\text {minor }}\right), 6.17$ (s, $\left.1 \mathrm{H}_{\text {major }}\right), 5.04-4.91\left(\mathrm{~m}, 2 \mathrm{H}_{\text {minor+major }}\right), 4.86-4.76\left(\mathrm{~m}, 2 \mathrm{H}_{\text {minor }}\right), 4.73$ $-4.62\left(\mathrm{~m}, 4 \mathrm{H}_{\text {major }}\right), 4.54\left(\mathrm{~s}, 2 \mathrm{H}_{\text {minor }}\right), 2.37-2.08\left(\mathrm{~m}, 1 \mathrm{H}_{\text {minor }}\right.$-major $), 1.16\left(\mathrm{~s}, 9 \mathrm{H}_{\text {minor }}\right), 1.13\left(\mathrm{~s}, 9 \mathrm{H}_{\text {major }}\right) ;{ }^{13} \mathbf{C ~ N M R}$ $\left(101 \mathrm{MHz}, \mathrm{CDCl}_{3}\right) \delta$ ppm: 167.24, 167.17, 133.49, 133.08, 132.88, 132.83, 131.28, 131.17, 130.47, 130.36, 130.20, 130.12, 130.08, 129.89, 129.82, 128.24, 127.92, 121.02 (q, $J=320.1 \mathrm{~Hz}$ ), 83.37, 83.29, 77.99, 77.92, $65.57,65.46,58.85,58.73,31.19,30.64,27.70,27.65 ;{ }^{19} \mathbf{F} \mathbf{N M R}\left(377 \mathrm{MHz}, \mathrm{CDCl}_{3}\right) \delta$ ppm: $\left(377 \mathrm{MHz}, \mathrm{CDCl}_{3}\right)$ $\delta$ ppm: -78.14 (s, 3F). IR (thin layer film) $v\left(\mathrm{~cm}^{-1}\right)=2980,1733,1498,1457,1370,1256,1224,1153,1068$, 1029, 843, 800, 760, 734, 705, 637, 573, 413. HRMS (ESI $\left.{ }^{+}\right) \mathrm{m} / z \mathrm{C}_{28} \mathrm{H}_{32} \mathrm{NO}_{2}{ }^{+}[\mathrm{M}-\mathrm{OTf}]^{+}$: calculated 414.2428, found 414.2426.

\section{1-benzhydryl-1-benzyl-3-(benzyloxy)-3-methylazetidin-1-ium trifluoromethanesulfonate (1ua)}

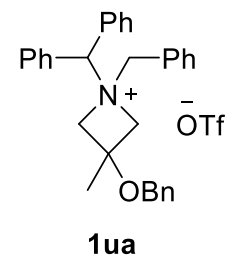

Prepared according to general procedure D using 1-benzhydryl-1-benzyl-2-(benzyloxy)-3bromo-2-methylpropan-1-amine $7 \mathbf{u a}(0.97 \mathrm{mmol}, 0.50 \mathrm{~g})$ to afford the desired product in $76 \%$ yield ( $0.74 \mathrm{mmol}, 0.43$ g, d.r. 1.5:1 cis:trans) as a white powder. ${ }^{1} \mathbf{H}$ NMR (400 MHz, $\left.\mathrm{CDCl}_{3}\right) \delta$ ppm: $7.71-7.16\left(\mathrm{~m}, 18 \mathrm{H}_{\text {minor+ major }}\right), 7.13\left(\mathrm{dd}, J=7.5,2.1 \mathrm{~Hz}, 2 \mathrm{H}_{\text {major }}\right), 6.82$ (dd, $\left.J=6.5,3.0 \mathrm{~Hz}, 2 \mathrm{H}_{\text {minor }}\right), 6.14$ (s, $\left.1 \mathrm{H}_{\text {minor }}\right), 5.69$ (s, $\left.1 \mathrm{H}_{\text {major }}\right), 5.02$ (d, $\left.J=12.6 \mathrm{~Hz}, 2 \mathrm{H}_{\text {minor }}\right), 4.94$

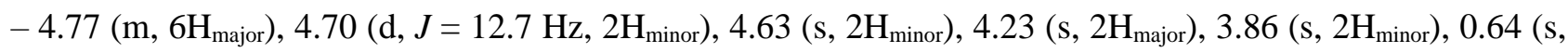
$3 \mathrm{H}_{\text {major }}$ ), 0.52 (s, $\left.3 \mathrm{H}_{\text {minor }}\right) ;{ }^{13} \mathbf{C ~ N M R}\left(101 \mathrm{MHz}, \mathrm{CDCl}_{3}\right.$ ) [overlapping signals] $\delta$ ppm: 137.09, 136.85, 133.84, $133.46,132.81,132.76,131.31,131.04,130.55,130.42,130.37,130.12,130.07,129.82,129.48,128.56$, 
128.49, 128.18, 127.98, 127.91, 127.73, 127.69, 127.39, 121.14 (q, $J=319.5 \mathrm{~Hz}), 79.36,76.62,70.99,69.42$, 68.60, 67.74, 67.02, 65.99, 65.84, 65.34, 19.51, 18.67; ${ }^{19} \mathbf{F}$ NMR (377 MHz, $\left.\mathrm{CDCl}_{3}\right) \delta$ ppm: -78.00 (s, 3F). IR $\left(\right.$ thin layer film) $v\left(\mathrm{~cm}^{-1}\right)=2919,1498,1456,1260,1224,1154,1030,907,758,731,702,638,577,569,558$, 539, 473, 415. HRMS $\left(\mathrm{ESI}^{+}\right) \mathrm{m} / z \mathrm{C}_{31} \mathrm{H}_{32} \mathrm{NO}^{+}$[M-OTf] $]^{+}$: calculated 434.2478, found 434.2477.

\section{1-benzhydryl-3-cyano-1-ethyl-3-methylazetidin-1-ium trifluoromethanesulfonate (1va)}

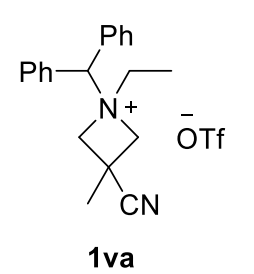

Prepared according to a slightly modified procedure E. Under a $\mathrm{N}_{2}$ atmosphere, to an ovendried flask charged with AgOTf (1 equiv., $0.57 \mathrm{mmol}, 72.3 \mathrm{mg}$ ), a solution of 1-benzhydryl3-methylazetidine-3-carbonitrile $7 \mathbf{v a}$ (1 equiv., $0.57 \mathrm{mmol}, 0.30 \mathrm{~g}$ ) in dry $\mathrm{CH}_{2} \mathrm{Cl}_{2}(5.7 \mathrm{~mL}$ ) was added. The reaction mixture was stirred at $40{ }^{\circ} \mathrm{C}$ for $12 \mathrm{~h}$ in the dark. The mixture was then filtered through celite and the filtrate was evaporated under reduced pressure. The resulting powder was washed with cold $\mathrm{Et}_{2} \mathrm{O}$ to afford the desired product in $50 \%$ yield $(1.02 \mathrm{mmol}, 0.16 \mathrm{~g}$, d.r. $>1$ 1:50) as a white powder. ${ }^{1} \mathbf{H}$ NMR $\left(400 \mathrm{MHz}, \mathrm{CDCl}_{3}\right) \delta$ ppm: $7.65-7.58(\mathrm{~m}, 4 \mathrm{H}), 7.57-7.43(\mathrm{~m}, 6 \mathrm{H})$, $5.80(\mathrm{~s}, 1 \mathrm{H}), 4.91(\mathrm{~s}, 4 \mathrm{H}), 3.84(\mathrm{q}, J=7.1 \mathrm{~Hz}, 2 \mathrm{H}), 1.52(\mathrm{t}, J=7.1 \mathrm{~Hz}, 3 \mathrm{H}), 0.79(\mathrm{~s}, 3 \mathrm{H}), ;{ }^{13} \mathbf{C ~ N M R}(101 \mathrm{MHz}$, $\left.\mathrm{CDCl}_{3}\right) \delta$ ppm: $131.67,130.80,130.53,130.20,120.11,120.95$ (q, $\left.J=319.9 \mathrm{~Hz}\right), 75.65,66.36,60.36,25.83$, 21.23, 8.86; ${ }^{19} \mathbf{F}$ NMR (377 MHz, $\left.\mathrm{CDCl}_{3}\right) \delta$ ppm: -78.00 (s, 3F). IR (thin layer film) $v\left(\mathrm{~cm}^{-1}\right)=3060,1499$, $1455,1423,1259,1225,1157,1030,779,754,729,706,638.574,543$. HRMS $\left(\mathrm{ESI}^{+}\right) \mathrm{m} / z \mathrm{C}_{20} \mathrm{H}_{23} \mathrm{~N}_{2}{ }^{+}[\mathrm{M}-$ $\mathrm{OTf}^{+}$: calculated 291.1856, found 291.1857 .

\section{1-benzhydryl-1-benzyl-3-fluoro-3-phenylazetidin-1-ium trifluoromethanesulfonate (1wa)}

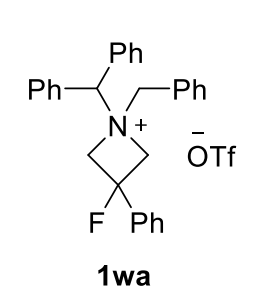

Prepared according to general procedure D using 1-benzhydryl-1-benzyl-3-bromo-2-fluoro2-phenylpropan-1-amine $7 \mathbf{w a}(3.20 \mathrm{mmol}, 1.56 \mathrm{~g})$ to afford, following a silica plug purification $\left(\mathrm{CH}_{2} \mathrm{Cl}_{2}: \mathrm{Et}_{2} \mathrm{O}=80: 20\right)$, the desired product in $80 \%$ yield $(2.56 \mathrm{mmol}, 1.43 \mathrm{~g}$, d.r. 1:2 cis:trans)* as a white powder. ${ }^{1} \mathbf{H}$ NMR $\left(400 \mathrm{MHz}, \mathrm{CDCl}_{3}\right) \delta$ ppm: $7.84-7.73(\mathrm{~m}$,

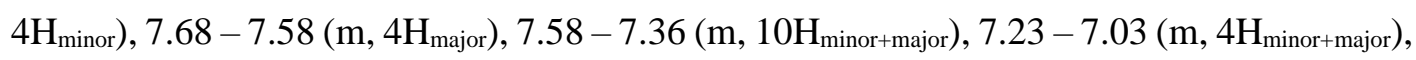

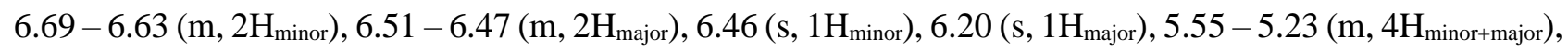
4.99 (s, 2 $\mathrm{H}_{\text {major }}$ ), 4.68 (s, 2 $\mathrm{H}_{\text {minor }}$ ); ${ }^{13} \mathbf{C}$ NMR (101 MHz, $\mathrm{CDCl}_{3}$ ) [overlapping signals] $\delta$ ppm: 133.54, 133.51, 133.30, 133.26, 133.07, 132.46, 131.36, 131.02, 130.57, 130.54, 130.41, 130.39, 130.17, 130.03, 129.80, 129.65, 129.44, 128.82, 128.75, (d, $J=1.9 \mathrm{~Hz}), 127.88,127.29,125.31(\mathrm{~d}, J=4.0 \mathrm{~Hz}), 124.55(\mathrm{~d}, J=6.1 \mathrm{~Hz})$, $121.10(\mathrm{~d}, J=320.3 \mathrm{~Hz}), 89.26(\mathrm{~d}, J=202.3 \mathrm{~Hz}), 79.58,77.83(\mathrm{~d}, J=1.9 \mathrm{~Hz}), 68.19$ (d, $J=30.2 \mathrm{~Hz}), 67.17$, $67.14(\mathrm{~d}, J=32.3 \mathrm{~Hz}), 66.23(\mathrm{~d}, J=1.8 \mathrm{~Hz}) .{ }^{19} \mathbf{F} \mathbf{N M R}\left(377 \mathrm{MHz}, \mathrm{CDCl}_{3}\right) \delta \mathrm{ppm}:-78.16(\mathrm{~s}, 3 \mathrm{~F}),-128.22(\mathrm{p}$, $\left.J=18.6 \mathrm{~Hz}, 1 \mathrm{~F}_{\text {minor }}\right),-140.29\left(\mathrm{p}, J=20.0, \mathrm{~Hz}, 1 \mathrm{~F}_{\text {major }}\right)$. IR (thin layer film) $v\left(\mathrm{~cm}^{-1}\right)=3064,1499,1454,1413$, 1351, 1256, 1224, 1155, 1065, 1030, 1003, 887, 757, 699, 637, 574. HRMS (ESI $\left.{ }^{+}\right) m / z \mathrm{C}_{29} \mathrm{H}_{27} \mathrm{FN}^{+}[\mathrm{M}-\mathrm{OTf}]^{+}$: calculated 408.2122, found 408.2122.

* Assignment of the major diastereoisomer by ${ }^{1} \mathrm{H}-{ }^{19} \mathrm{~F}$ 1D-HOESY. A correlation between the $\mathrm{F}$ atom and the benzylic protons was detected in the major cis diastereomer but not in the minor trans diastereomer.
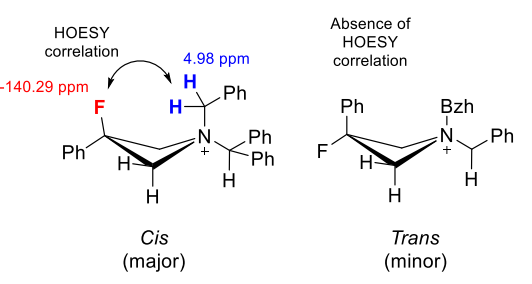


\section{Product Characterization}

(S)- $N$-benzhydryl-3-fluoro- $N$-methyl-2-phenylpropan-1-amine (2a)

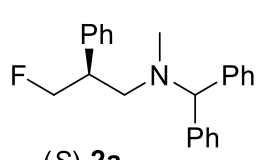

(S)-2a

(S)-2a was prepared from 1a (1 equiv., $0.1 \mathrm{mmol}, 46.4 \mathrm{mg}$ ), according to the general procedure using $10 \mathrm{~mol} \%$ of catalyst $(S)$-D $(0.01 \mathrm{mmol}, 8.4 \mathrm{mg})$ and stirring for $72 \mathrm{~h}$.

Purification FCC eluent $=$ Pentane: $\mathrm{Et}_{2} \mathrm{O}$ (90:0 to 70:30, gradient). Colorless oil, $27.5 \mathrm{mg}$, 82\% yield, e.r. $=80.5: 19.5 .{ }^{1} \mathbf{H}$ NMR $\left(400 \mathrm{MHz}, \mathrm{CDCl}_{3}\right) \delta$ ppm: $7.33-7.10(\mathrm{~m}, 15 \mathrm{H}) 4.80-4.57(\mathrm{~m}, 2 \mathrm{H})$, $4.38(\mathrm{~s}, 1 \mathrm{H}), 3.30-3.15$ (m, 1H), 2.75 (dd, $J=12.6,8.2 \mathrm{~Hz}, 1 \mathrm{H}), 2.53$ (dddd, $J=12.6,7.1,2.3 \mathrm{~Hz}, 1 \mathrm{H}), 2.22$ (s, 3H); ${ }^{13}$ C NMR (101 MHz, $\left.\mathrm{CDCl}_{3}\right) \delta$ ppm: 142.87, 142.79140 .66 (d, $\left.J=3.1 \mathrm{~Hz}\right), 128.51,128.50,128.46$, 128.34, 128.31, 128.22, 127.05, 127.03, 127.01, 85.5 (d, $J=171.1 \mathrm{~Hz}), 76.15,57.48$ (d, $J=6.1 \mathrm{~Hz}), 45.00(\mathrm{~d}$, $J=18.3 \mathrm{~Hz}), 40.62 ;{ }^{19} \mathbf{F}$ NMR $\left(376 \mathrm{MHz}, \mathrm{CDCl}_{3}\right) \delta \mathrm{ppm}:-222.24$ (tdd, $\left.J=47.5,21.3,1.5 \mathrm{~Hz}, 1 \mathrm{~F}\right)$. HRMS $\left(\mathrm{ESI}^{+}\right) \mathrm{m} / \mathrm{z} \mathrm{C}_{23} \mathrm{H}_{25} \mathrm{FN}^{+}[\mathrm{M}+\mathrm{H}]^{+}$: calculated 334.1966, found 334.1956. IR (thin layer film) $v\left(\mathrm{~cm}^{-1}\right)=3061$, $3027,2958,2781,1599,1492,1452,1263,1201,1080,1016,926,760,746,700 .[\alpha]_{\mathbf{D}}{ }^{25^{\circ} \mathrm{C}}=+14.6^{\circ}(\mathrm{c}=0.5$, $\mathrm{CHCl}_{3}$, e.r. $\left.=80.5: 19.5\right)$. HPLC separation DAICEL CHIRALPAK ${ }^{\circledR}$ IB-3, Heptane: ${ }^{i} \operatorname{PrOH}=99.75: 0.25,1$ $\mathrm{mL} / \mathrm{min} ; \mathrm{t}_{1}=4.57 \min$ (minor), $\mathrm{t}_{2}=4.96 \min$ (major).

\section{(S)- $N$-benzhydryl- $N$-benzyl-3-fluoro-2-phenylpropan-1-amine (2aa)}

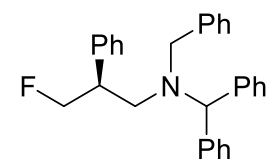

(S)-2aa

(S)-2aa was prepared from 1aa (1 equiv., $0.1 \mathrm{mmol}, 53.9 \mathrm{mg}$ ), according to the general procedure using $5 \mathrm{~mol} \%$ of catalyst $(S)$-D $(0.005 \mathrm{mmol}, 4.2 \mathrm{mg})$ and stirring for $48 \mathrm{~h}$. Purification FCC eluent $=$ Pentane: $\mathrm{Et}_{2} \mathrm{O}$ (100:0 to 90:10, gradient). Colorless oil, 40.1 $\mathrm{mg}, 98 \%$ yield, e.r. $=96: 4$. Gram scale reaction using opposite enantiomer of the catalyst

(R)-D: In a round-bottom flask, 1aa (1 equiv., $1.85 \mathrm{mmol} 1.0 \mathrm{~g}$ ), CsF (2 equiv., $3.70 \mathrm{mmol}, 562 \mathrm{mg}$ ) and 3 mol\% of catalyst $(R)$-D $(0.056 \mathrm{mmol}, 46.4 \mathrm{mg})$ were stirred at r.t. in dry 1,2-DCE $(0.25 \mathrm{M}, 7.4 \mathrm{~mL})$ at 900 rpm. After $72 \mathrm{~h}$, the reaction mixture was directly purified by flash column chromatography. Purification: FCC eluent $=$ Pentane: $\mathrm{Et}_{2} \mathrm{O}$ (100:0 to 90:10, gradient) to elute the pure product as the $(R)$-enantiomer (colorless oil, $696.1 \mathrm{mg}, 92 \%$ yield, e.r. $=96: 4$ e.r.); then Pentane: $\mathrm{Et}_{2} \mathrm{O}$ (90:10 to 50:50, gradient) to recover the catalyst (46.0 mg, 99\% catalyst recovery). ${ }^{1} \mathbf{H}$ NMR (400 MHz, $\left.\mathrm{CDCl}_{3}\right) \delta$ ppm: $7.40-7.36(\mathrm{~m}, 4 \mathrm{H}), 7.22-7.22$ (m, 14H), $7.07-7.02(\mathrm{~m}, 2 \mathrm{H}), 5.00(\mathrm{~s}, 1 \mathrm{H}), 4.62(\mathrm{ddd}, J=47.6,9.0,5.5 \mathrm{~Hz}, 1 \mathrm{H}), 4.52$ (ddd, $J=47.5,9.0,6.4 \mathrm{~Hz}$, 1H), $3.69(\mathrm{~d}, J=1.0 \mathrm{~Hz}, 2 \mathrm{H}), 3.23-3.12(\mathrm{~m}, 1 \mathrm{H}), 2.91(\mathrm{dd}, J=13.3,7.3 \mathrm{~Hz}, 1 \mathrm{H}), 2.75(\mathrm{ddd}, J=13.3,7.5,2.1$ $\mathrm{Hz}, 1 \mathrm{H}) ;{ }^{13} \mathbf{C}$ NMR $\left(101 \mathrm{MHz}, \mathrm{CDCl}_{3}\right.$ ) [overlapping signals] $\delta \mathrm{ppm}: 140.80,140.62$ (d, $\left.J=3.7 \mathrm{~Hz}\right), 140.47$, $139.56,129.51,129.26,129.03,128.54,128.44,128.40,128.35,128.25,127.27,127.10,127.03,85.70(\mathrm{~d}, J=$ $171.4 \mathrm{~Hz}), 69.40,55.57,52.85(\mathrm{~d}, J=6.1 \mathrm{~Hz}), 45.14(\mathrm{~d}, J=18.4 \mathrm{~Hz}) ;{ }^{19} \mathbf{F} \mathbf{N M R}\left(377 \mathrm{MHz}, \mathrm{CDCl}_{3}\right) \delta \mathrm{ppm}:-$ 221.09 (tdd, $J=47.5,21.1,1.7 \mathrm{~Hz}$ ). HRMS $\left(\mathrm{ESI}^{+}\right) \mathrm{m} / z \mathrm{C}_{29} \mathrm{H}_{29} \mathrm{FN}^{+}[\mathrm{M}+\mathrm{H}]^{+}[\mathrm{M}+\mathrm{H}]^{+}$: calculated 410.2279, found 410.2278. IR (thin layer film) $v\left(\mathrm{~cm}^{-1}\right)=3028,2926,2834,1601,1493,1452,1261,1027,762,743$, 698. $[\alpha]_{\mathrm{D}}{ }^{25}{ }^{\circ} \mathrm{C}=+10.1^{\circ}\left(\mathrm{c}=0.3, \mathrm{CHCl}_{3}\right.$, e.r. $\left.=96: 4\right)$. HPLC separation DAICEL CHIRALCEL ${ }^{\circledR}$ OJ-H, Heptane:EtOH $=95: 5,1 \mathrm{~mL} / \mathrm{min} ; \mathrm{t}_{1}=8.83 \mathrm{~min}$ (minor), $\mathrm{t}_{2}=13.52 \mathrm{~min}$ (major). 
(S)- $N$-benzhydryl- $N$-ethyl-3-fluoro-2-phenylpropan-1-amine (2ab)

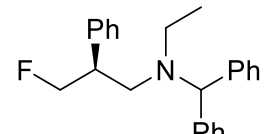

(S)-2ab

(S)-2ab was prepared from 1ab (1 equiv., $0.1 \mathrm{mmol}, 47.8 \mathrm{mg}$ ), according to the general procedure using $10 \mathrm{~mol} \%$ of catalyst $(S)-\mathbf{D}(0.01 \mathrm{mmol}, 8.4 \mathrm{mg})$ and stirring for $72 \mathrm{~h}$.

Purification: FCC eluent $=$ Pentane: $\mathrm{Et}_{2} \mathrm{O}$ (100:0 to 90:10, gradient). Colorless oil, 32.3 $\mathrm{mg}, 93 \%$ yield, e.r. = 96:4. Gram scale reaction: In a round-bottom flask, 1ab (1 equiv., $2.11 \mathrm{mmol} 1.01 \mathrm{~g})$, CsF (2 equiv., $4.22 \mathrm{mmol}, 641 \mathrm{mg})$ and $10 \mathrm{~mol} \%$ of catalyst $(S)-\mathbf{D}(0.21 \mathrm{mmol}, 176 \mathrm{mg})$ were stirred at r.t. in dry 1,2-DCE $(0.25 \mathrm{M}, 8.4 \mathrm{~mL})$ at $900 \mathrm{rpm}$. After $72 \mathrm{~h}$, the reaction mixture was directly purified by flash column chromatography. Purification FCC eluent $=$ Pentane: $_{2} t_{2} \mathrm{O}$ (100:0 to 98.5:1.5, gradient) to elute the pure product (colorless oil, $601 \mathrm{mg}, 82 \%$ yield, e.r. $=96: 4$ e.r.); then Pentane: $\mathrm{Et}_{2} \mathrm{O}$ (90:10 to 50:50, gradient) to recover the catalyst (174 mg, $99 \%$ catalyst recovery). ${ }^{1} \mathbf{H} \mathbf{~ N M R}\left(500 \mathrm{MHz}, \mathrm{CDCl}_{3}\right) \delta \mathrm{ppm}: 7.34-7.15$ (m, 13H), $7.13-7.08(\mathrm{~m}, 2 \mathrm{H}), 4.82(\mathrm{~s}, 1 \mathrm{H}), 4.70(\mathrm{dd}, J=47.5,5.5 \mathrm{~Hz}, 2 \mathrm{H}), 3.14-2.99(\mathrm{~m}, 1 \mathrm{H}), 2.86(\mathrm{dd}, J=$ 13.5, 8.6 Hz, 1H), $2.73-2.52(\mathrm{~m}, 3 \mathrm{H}), 0.96(\mathrm{t}, J=7.0 \mathrm{~Hz}, 3 \mathrm{H}) ;{ }^{13} \mathbf{C ~ N M R}\left(126 \mathrm{MHz}, \mathrm{CDCl}_{3}\right)$ [overlapping signals] $\delta$ ppm: 142.38, 142.15, 140.94 (d, $J=2.8 \mathrm{~Hz}), 128.85,128.79,128.51,128.33,128.31,127.04,127.01$, 126.94, 86.23, 85.57 (d, $J=171.2 \mathrm{~Hz}), 70.90,52.24(\mathrm{~d}, J=6.4 \mathrm{~Hz}), 45.41$ (d, $J=18.2 \mathrm{~Hz}), 44.40,10.87$; ${ }^{19} \mathbf{F}$ NMR $\left(470 \mathrm{MHz}, \mathrm{CDCl}_{3}\right) \delta$ ppm: -222.84 (tdd, $\left.J=47.4,23.0,1.9 \mathrm{~Hz}\right)$. HRMS $\left(\mathrm{ESI}^{+}\right) \mathrm{m} / z \mathrm{C}_{24} \mathrm{H}_{27} \mathrm{FN}^{+}$ $[\mathrm{M}+\mathrm{H}]^{+}$: calculated 348.2122, found 348.2120. IR (thin layer film) $v\left(\mathrm{~cm}^{-1}\right)=3026,2966,1493,1452,1028$, $759,699,623 .[\alpha]_{\mathrm{D}}{ }^{25^{\circ} \mathrm{C}}=+8.8^{\circ}\left(\mathrm{c}=0.5, \mathrm{CHCl}_{3}\right.$, e.r. $\left.=96: 4\right)$. HPLC separation DAICEL CHIRALCEL ${ }^{\circledR}$ OJ-H, Heptane: $\mathrm{EtOH}=96: 4,1 \mathrm{~mL} / \mathrm{min} ; \mathrm{t}_{1}=10.43 \mathrm{~min}$ (minor), $\mathrm{t}_{2}=12.59 \mathrm{~min}$ (major).

\section{(S)- $N$-benzhydryl- $N$-(3-fluoro-2-phenylpropyl)prop-2-en-1-amine (2ac)}

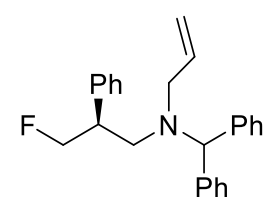

(S)-2ac

(S)-2ac was prepared from 1ac (1 equiv., $0.1 \mathrm{mmol}, 48.9 \mathrm{mg}$ ), according to the general procedure using $10 \mathrm{~mol} \%$ of catalyst $(S)$-D $(0.01 \mathrm{mmol}, 8.4 \mathrm{mg})$ and stirring for $36 \mathrm{~h}$. Purification: FCC eluent $=$ Pentane:Et ${ }_{2} \mathrm{O}$ (100:0 to 90:10, gradient). Colorless oil, 34.5 mg, 96\% yield, e.r. $=94: 6 .{ }^{1} \mathbf{H}$ NMR $\left(400 \mathrm{MHz}, \mathrm{CDCl}_{3}\right) \delta$ ppm: $7.34-7.18(\mathrm{~m}, 13 \mathrm{H})$, $7.14-7.10(\mathrm{~m}, 2 \mathrm{H}), 5.92-5.81(\mathrm{~m}, 1 \mathrm{H}), 5.16-5.04(\mathrm{~m}, 2 \mathrm{H}), 4.90(\mathrm{~s}, 1 \mathrm{H}), 4.78-4.59(\mathrm{~m}, 2 \mathrm{H}), 3.26-3.08$ $(\mathrm{m}, 3 \mathrm{H}), 2.88(\mathrm{dd}, J=13.3,8.4 \mathrm{~Hz}, 1 \mathrm{H}), 2.72(\mathrm{dddd}, J=13.3,7.0,2.3 \mathrm{~Hz}, 1 \mathrm{H}) ;{ }^{13} \mathbf{C} \mathbf{N M R}\left(101 \mathrm{MHz}, \mathrm{CDCl}_{3}\right)$ [overlapping signals] $\delta$ ppm: 141.75, 141.61, $140.75(\mathrm{~d}, J=3.0 \mathrm{~Hz}), 135.45,128.90,128.84,128.49,128.37$, $128.33,127.12,127.07,126.96,117.76,85.57$ (d, $J=171.0 \mathrm{~Hz}), 70.52,53.89,52.55$ (d, $J=6.4 \mathrm{~Hz}), 45.09(\mathrm{~d}$, $J=18.1 \mathrm{~Hz}) ;{ }^{19} \mathbf{F}$ NMR $\left(376 \mathrm{MHz}, \mathrm{CDCl}_{3}\right) \delta \mathrm{ppm}:-222.34$ (tdd, $\left.J=47.1,21.2,1.7 \mathrm{~Hz}, 1 \mathrm{~F}\right) . \mathbf{H R M S}\left(\mathrm{ESI}^{+}\right)$ $\mathrm{m} / z \mathrm{C}_{25} \mathrm{H}_{27} \mathrm{FN}^{+}[\mathrm{M}+\mathrm{H}]^{+}$: calculated 360.2122, found 360.2123. IR (thin layer film) $v\left(\mathrm{~cm}^{-1}\right)=3061,3027$, 2956, 2831, 1600, 1493, 1452, 1079, 1005, 922, 760, 735, 699. $[\alpha]_{\mathbf{D}}{ }^{2{ }^{\circ} \mathrm{C}}=+6.5^{\circ}\left(\mathrm{c}=0.5, \mathrm{CHCl}_{3}\right.$, e.r. $\left.=94: 6\right)$. HPLC separation DAICEL CHIRALCEL ${ }^{\circledR}$ OJ-H, Heptane:EtOH $=99.5: 0.5,1 \mathrm{~mL} / \mathrm{min} ; \mathrm{t}_{1}=16.91 \mathrm{~min}$ (minor), $\mathrm{t}_{2}=22.62 \min$ (major). 
(S)- $N$-benzhydryl- $N$-benzyl-3-fluoro-2-(m-tolyl)propan-1-amine (2ba)

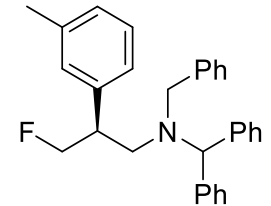

(S)-2ba

$(S)$-2ba was prepared from $1 \mathbf{b a}$ (1 equiv., $0.1 \mathrm{mmol}, 55.4 \mathrm{mg}$ ), according to the general procedure using $5 \mathrm{~mol} \%$ of catalyst $(S)$-D $(0.005 \mathrm{mmol}, 4.2 \mathrm{mg})$ and stirring for $48 \mathrm{~h}$.

Purification: FCC eluent $=$ Pentane: $\mathrm{Et}_{2} \mathrm{O}$ (100:0 to 90:10, gradient). Colorless oil, 37.2 $\mathrm{mg}, 88 \%$ yield, e.r. $=97.5: 2.5 .{ }^{1} \mathbf{H}$ NMR $\left(400 \mathrm{MHz}, \mathrm{CDCl}_{3}\right) \delta \mathrm{ppm}: 7.36-7.13(\mathrm{~m}, 16 \mathrm{H})$, $7.09-7.05(\mathrm{~m}, 1 \mathrm{H}), 6.84-6.77(\mathrm{~m}, 2 \mathrm{H}), 4.96(\mathrm{~s}, 1 \mathrm{H}), 4.64-4.39(\mathrm{~m}, 2 \mathrm{H}), 3.68-3.58(\mathrm{~m}, 2 \mathrm{H}), 3.18-3.05$ (m, 1H), $2.85(\mathrm{dd}, J=13.3,7.0 \mathrm{~Hz}, 1 \mathrm{H}), 2.72(\mathrm{dddd}, J=13.2,7.7,1.9 \mathrm{~Hz}, 1 \mathrm{H}), 2.30(\mathrm{~s}, 3 \mathrm{H}) ;{ }^{13} \mathbf{C ~ N M R}(101$ $\left.\mathrm{MHz}, \mathrm{CDCl}_{3}\right)$ [overlapping signals] $\delta$ ppm: 140.87, $140.46(\mathrm{~d}, J=3.8 \mathrm{~Hz}), 140.38,139.6,138.0,129.56$, $129.25,129.16,129.00,128.41,128.37,128.31,128.21,127.80,127.25,127.05,125.53,85.78$ (d, $J=171.4$ $\mathrm{Hz}), 69.27,55.47,52.82(\mathrm{~d}, J=5.8 \mathrm{~Hz}), 45.03(\mathrm{~d}, J=18.1 \mathrm{~Hz}), 21.59 ;{ }^{19} \mathbf{F} \mathbf{N M R}\left(376 \mathrm{MHz}, \mathrm{CDCl}_{3}\right) \delta \mathrm{ppm:} \mathrm{-}$ 220.64 (tdd, $J=47.3,21.1,1.4 \mathrm{~Hz}, 1 \mathrm{~F})$. HRMS $\left(\mathrm{ESI}^{+}\right) \mathrm{m} / 2 \mathrm{C}_{30} \mathrm{H}_{31} \mathrm{FN}^{+}[\mathrm{M}+\mathrm{H}]^{+}$: calculated 424.2435, found 424.2434. IR (thin layer film) $v\left(\mathrm{~cm}^{-1}\right)=2980,1492,1452,1381,1252,1154,1073,954,764,743,700$; $[\boldsymbol{\alpha}]_{\mathbf{D}}{ }^{25^{\circ} \mathrm{C}}=+7.7^{\circ}\left(\mathrm{c}=0.3, \mathrm{CHCl}_{3}\right.$, e.r. $\left.=97.5: 2.5\right)$. HPLC separation DAICEL CHIRALCEL ${ }^{\circledR}$ OJ-H, Heptane: $\mathrm{PrOH}=96: 4,1 \mathrm{~mL} / \mathrm{min} ; \mathrm{t}_{1}=6.89 \mathrm{~min}$ (minor), $\mathrm{t}_{2}=9.69 \mathrm{~min}$ (major).

\section{(S)- $N$-benzhydryl- $N$-benzyl-3-fluoro-2-(3-methoxyphenyl)propan-1-amine (2ca)}

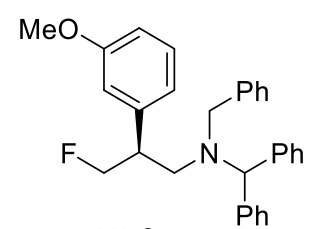

(S)-2ca

(S)-2ca was prepared from 1ca (1 equiv., $0.1 \mathrm{mmol}, 57.0 \mathrm{mg}$ ), according to the general procedure using $5 \mathrm{~mol} \%$ of catalyst $(S)-\mathbf{D}(0.005 \mathrm{mmol}, 4.2 \mathrm{mg})$ and stirring for $48 \mathrm{~h}$.

Purification: FCC eluent $=$ Pentane: $\mathrm{Et}_{2} \mathrm{O}$ (100:0 to 90:10, gradient). Colorless oil, 42.2 mg, 96\% yield, e.r. $=97: 3 .{ }^{1} \mathbf{H}$ NMR $\left(400 \mathrm{MHz}, \mathrm{CDCl}_{3}\right) \delta$ ppm: $7.36-7.15(\mathrm{~m}, 16 \mathrm{H})$, $6.81-6.76(\mathrm{~m}, 1 \mathrm{H}), 6.61(\mathrm{~d}, J=7.6 \mathrm{~Hz}, 1 \mathrm{H}), 6.52(\mathrm{t}, J=2.1 \mathrm{~Hz}, 1 \mathrm{H}), 4.95(\mathrm{~s}, 1 \mathrm{H}), 4.65-4.38(\mathrm{~m}, 2 \mathrm{H}), 3.72$ (s, 3H), $3.64(\mathrm{~s}, 2 \mathrm{H}), 3.15-3.00(\mathrm{~m}, 1 \mathrm{H}), 2.86(\mathrm{dd}, J=13.3,7.2 \mathrm{~Hz}, 1 \mathrm{H}), 2.75(\mathrm{dddd}, J=13.2,7.4,2.1 \mathrm{~Hz}$, $1 \mathrm{H}) ;{ }^{13} \mathbf{C}$ NMR (101 MHz, $\mathrm{CDCl}_{3}$ ) [overlapping signals] $\delta \mathrm{ppm:} \mathrm{159.74,} 142.28$ (d, $\left.J=3.7 \mathrm{~Hz}\right), 140.84,140.51$, $139.57,129.49,129.24,129.01,128.40,128.34,128.25,127.27,127.10,120.80,114.08,112.46,85.64(\mathrm{~d}, J=$ $171.7 \mathrm{~Hz}), 69.49,55.62,55.25,52.94(\mathrm{~d}, J=6.1 \mathrm{~Hz}), 45.23(\mathrm{~d}, J=18.2 \mathrm{~Hz}) ;{ }^{19} \mathbf{F} \mathbf{N M R}\left(376 \mathrm{MHz}, \mathrm{CDCl}_{3}\right) \delta$ ppm: -220.94 (tdd, $J=47.3,21.01 .5 \mathrm{~Hz}, 1 \mathrm{~F}$ ). HRMS $\left(\mathrm{ESI}^{+}\right.$) $\mathrm{m} / \mathrm{z}_{30} \mathrm{C}_{31} \mathrm{FNO}^{+}[\mathrm{M}+\mathrm{H}]^{+}$: calculated 440.2384, found 440.2382. IR (thin layer film) $v\left(\mathrm{~cm}^{-1}\right)=3027,2956,2836,1600,1492,1452,1262,1159,1021,764$, 743, 699. $[\alpha]_{\mathbf{D}}{ }^{25^{\circ} \mathrm{C}}=+3.8^{\circ}\left(\mathrm{c}=0.3, \mathrm{CHCl}_{3}\right.$, e.r. $\left.=97: 3\right)$. HPLC separation DAICEL CHIRALCEL ${ }^{\circledR}$ OJ-H, Heptane:EtOH $=90: 10,1 \mathrm{~mL} / \mathrm{min} ; \mathrm{t}_{1}=9.30 \mathrm{~min}$ (minor), $\mathrm{t}_{2}=15.39$ min (major).

\section{(S)- $N$-benzhydryl- $N$-benzyl-2-(3-chlorophenyl)-3-fluoropropan-1-amine (2da)}

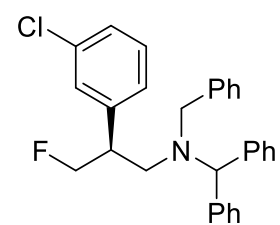

(S)-2da

(S)-2da was prepared from 1da (1 equiv., $0.1 \mathrm{mmol}, 57.4 \mathrm{mg}$ ), according to the general procedure using $5 \mathrm{~mol} \%$ of catalyst $(S)-\mathbf{D}(0.005 \mathrm{mmol}, 4.2 \mathrm{mg})$ and stirring for $48 \mathrm{~h}$. Purification: FCC eluent $=$ Pentane: $\mathrm{Et}_{2} \mathrm{O}$ (100:0 to 90:10, gradient). Colorless oil, 40.0 mg, 90\% yield, e.r. $=96: 4$. For the synthesis of F-Lorcaserin (gram scale): In a roundbottom flask, 1 da (1 equiv., $1.74 \mathrm{mmol} 1.0 \mathrm{~g}$ ), CsF (2 equiv., $3.48 \mathrm{mmol}, 521 \mathrm{mg}$ ) and $5 \mathrm{~mol} \%$ of catalyst $(R)$ D $(0.17 \mathrm{mmol}, 73 \mathrm{mg})$ were stirred at r.t. in dry 1,2-DCE $(0.25 \mathrm{M}, 7.0 \mathrm{~mL})$ at $900 \mathrm{rpm}$. After $48 \mathrm{~h}$, the reaction 
mixture was directly purified by flash column chromatography. Purification: $\mathrm{FCC}$ eluent $=\mathrm{Pentane}_{\mathrm{Et}} \mathrm{O}_{2}$ (100:0 to 90:10, gradient). Colorless oil, $749 \mathrm{mg}$, 97\% yield, e.r. $=96: 4{ }^{1} \mathbf{H}$ NMR (400 MHz, $\left.\mathrm{CDCl}_{3}\right) \delta$ ppm: $7.38-7.16(\mathrm{~m}, 17 \mathrm{H}), 6.97-6.93(\mathrm{~m}, 1 \mathrm{H}), 6.87(\mathrm{~d}, J=6.5 \mathrm{~Hz}, 1 \mathrm{H}), 4.93(\mathrm{~s}, 1 \mathrm{H}), 4.61-4.34(\mathrm{~m}, 2 \mathrm{H}), 3.68(\mathrm{~d}$, $J=14.0 \mathrm{~Hz}, 1 \mathrm{H}), 3.59(\mathrm{~d}, J=14.0 \mathrm{~Hz}, 1 \mathrm{H}), 3.14-2.99(\mathrm{~m}, 1 \mathrm{H}), 2.84(\mathrm{dd}, J=13.3,6.8 \mathrm{~Hz}, 1 \mathrm{H}), 2.75$ (dddd, $J=13.3,8.0,1.6 \mathrm{~Hz}, 1 \mathrm{H}) ;{ }^{13} \mathbf{C}$ NMR $\left(101 \mathrm{MHz}, \mathrm{CDCl}_{3}\right)$ [overlapping signals] $\delta$ ppm: $142.63(\mathrm{~d}, J=3.3 \mathrm{~Hz})$, $140.87,140.13$, 139.31, 134.29, 129.71, 129.52, 129.08, 128.99, 128.54, 128.46, 128.41, 128.33, 127.36, 127.22, 127.18, 126.81, 85.32 (d, $J=172.1 \mathrm{~Hz}), 69.68,55.72,52.67(\mathrm{~d}, J=6.4 \mathrm{~Hz}), 45.95(\mathrm{~d}, J=18.4 \mathrm{~Hz})$; ${ }^{19}$ F NMR $\left(376 \mathrm{MHz}, \mathrm{CDCl}_{3}\right) \delta$ ppm: -221.53 (td, $\left.J=47.2,21.0 \mathrm{~Hz}, 1 \mathrm{~F}\right)$. HRMS $\left(\mathrm{ESI}^{+}\right) \mathrm{m} / z \mathrm{C}_{29} \mathrm{H}_{28} \mathrm{ClFN}^{+}$ $[\mathrm{M}+\mathrm{H}]^{+}$: calculated 444.1889, found 444.1883. IR (thin layer film) $v\left(\mathrm{~cm}^{-1}\right)=3060,3026,2923,2838,1597$, $1573,1493,1451,1080,1028,785,763,744,698 ;[\alpha]_{\mathbf{D}}{ }^{25}{ }^{\circ} \mathbf{C}=+1.6^{\circ}\left(\mathrm{c}=0.3, \mathrm{CHCl}_{3}\right.$, e.r. $\left.=96: 4\right) . \mathbf{H P L C}$ separation DAICEL CHIRALCEL ${ }^{\circledR}$ OJ-H, Heptane:EtOH $=90: 10,1 \mathrm{~mL} / \mathrm{min} ; \mathrm{t}_{1}=6.69 \mathrm{~min}(\operatorname{minor}), \mathrm{t}_{2}=$ 12.75 min (major).

\section{(S)- $N$-benzhydryl- $N$-benzyl-3-fluoro-2-(4-fluorophenyl)propan-1-amine (2ea)}

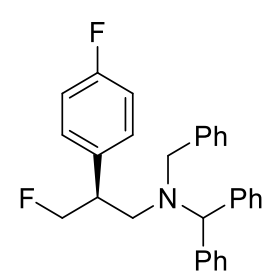

(S)-2ea

(S)-2ea was prepared from 1ea (1 equiv., $0.1 \mathrm{mmol}, 55.8 \mathrm{mg}$ ), according to the general procedure using $5 \mathrm{~mol} \%$ of catalyst $(S)$-D $(0.005 \mathrm{mmol}, 4.2 \mathrm{mg})$ and stirring for $48 \mathrm{~h}$. Purification : FCC eluent $=$ Pentane: $\mathrm{Et}_{2} \mathrm{O}$ (100:0 to 90:10, gradient). Colorless oil, 42.0 $\mathrm{mg}$, 98\% yield, e.r. $=94.5: 5.5 .{ }^{1} \mathbf{H}$ NMR $\left(400 \mathrm{MHz}, \mathrm{CDCl}_{3}\right) \delta \mathrm{ppm:} 7.38-7.19(\mathrm{~m}, 15 \mathrm{H})$, $6.99-6.88(\mathrm{~m}, 4 \mathrm{H}), 4.95(\mathrm{~s}, 1 \mathrm{H}), 4.61-4.35(\mathrm{~m}, 2 \mathrm{H}), 3.67$ (d, J=13.8 Hz, 1H), 3.60 (d, $J=13.8 \mathrm{~Hz}, 1 \mathrm{H}), 3.14-3.00(\mathrm{~m}, 1 \mathrm{H}), 2.84(\mathrm{dd}, J=13.3,7.1 \mathrm{~Hz}, 1 \mathrm{H}), 2.75(\mathrm{dddd}, J=13.2,7.8,1.7 \mathrm{~Hz}, 1 \mathrm{H})$; ${ }^{13} \mathrm{C}$ NMR (101 MHz, $\mathrm{CDCl}_{3}$ ) [overlapping signals] $\delta$ ppm: $161.99(\mathrm{~d}, J=245.0 \mathrm{~Hz}), 140.86,140.27,139.45$, $136.28(\mathrm{~m}), 129.86(\mathrm{~d}, J=7.8 \mathrm{~Hz}), 129.51,129.17,129.02,128.42,128.38,128.29,127.34,127.17,115.28$ $(\mathrm{d}, J=21.0 \mathrm{~Hz}), 85.63(\mathrm{~d}, J=172.0 \mathrm{~Hz}), 69.58,55.70,52.90(\mathrm{~d}, J=6.0 \mathrm{~Hz}), 44.43(\mathrm{~d}, J=18.1 \mathrm{~Hz}) ;{ }^{19} \mathbf{F ~ N M R}$ $\left(376 \mathrm{MHz}, \mathrm{CDCl}_{3}\right) \delta$ ppm: $-116.12--116.26(\mathrm{~m}, 1 \mathrm{~F}),-221.61(\mathrm{td}, J=47.7,21.8 \mathrm{~Hz}, 1 \mathrm{~F})$. HRMS $\left(\mathrm{ESI}^{+}\right) \mathrm{m} / \mathrm{z}$ $\mathrm{C}_{29} \mathrm{H}_{28} \mathrm{~F}_{2} \mathrm{~N}^{+}[\mathrm{M}+\mathrm{H}]^{+}$: calculated 428.2184, found 428.2186. IR (thin layer film) $v\left(\mathrm{~cm}^{-1}\right)=3027,2891,1603$, $1510,1493,1452,1381,1224,1160,1136,1077,1028,967,832,764,744 .[\alpha]_{\mathbf{D}}{ }^{25}{ }^{\circ} \mathbf{C}=+8.2^{\circ}\left(\mathrm{c}=0.5, \mathrm{CHCl}_{3}\right.$, e.r. $=94.5: 5.5)$. HPLC separation DAICEL CHIRALPAK ${ }^{\circledR} \mathrm{IB}-3$, Heptane $:{ }^{i} \mathrm{PrOH}=99.75: 0.25,1 \mathrm{~mL} / \mathrm{min}$; $\mathrm{t}_{1}=5.32 \min$ (minor), $\mathrm{t}_{2}=5.77 \min$ (major).

\section{(S)- $N$-benzhydryl- $N$-ethyl-3-fluoro-2-(4-fluorophenyl)propan-1-amine (2eb)}

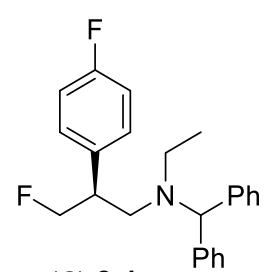

(S)-2eb

$(S)$-2eb was prepared from 1eb (1 equiv., $0.1 \mathrm{mmol}, 55.8 \mathrm{mg}$ ), according to the general procedure using $5 \mathrm{~mol} \%$ of catalyst $(S)$-D $(0.01 \mathrm{mmol}, 8.4 \mathrm{mg})$ and stirring for $48 \mathrm{~h}$. Purification: FCC eluent $=$ Pentane: $\mathrm{Et}_{2} \mathrm{O}$ (100:0 to 90:10, gradient). Colorless oil, 35.3 mg, 97\% yield, e.r. $=95: 5 .{ }^{1} \mathbf{H}$ NMR $\left(400 \mathrm{MHz}, \mathrm{CDCl}_{3}\right) \delta$ ppm: $7.33-7.16(\mathrm{~m}, 10 \mathrm{H})$, $7.09-7.02(\mathrm{~m}, 2 \mathrm{H}), 7.01-6.94(\mathrm{~m}, 2 \mathrm{H}), 4.81(\mathrm{~s}, 1 \mathrm{H}), 4.74-4.56(\mathrm{~m}, 2 \mathrm{H}), 3.09-2.95$ $(\mathrm{m}, 1 \mathrm{H}), 2.83(\mathrm{dd}, J=13.4,8.4 \mathrm{~Hz}, 1 \mathrm{H}), 2.71-2.51(\mathrm{~m}, 3 \mathrm{H}) ; 0.96(\mathrm{t}, J=7.0 \mathrm{~Hz}, 3 \mathrm{H}) ;{ }^{13} \mathrm{C} \mathrm{NMR}(101 \mathrm{MHz}$, $\left.\mathrm{CDCl}_{3}\right)$ [overlapping signals] $\delta$ ppm: $161.94(\mathrm{~d}, J=247.2 \mathrm{~Hz}), 142.26,142.14,136.62(\mathrm{~m}), 129.74(\mathrm{~d}, J=7.9$ 
Hz), 128.87, 128.73, 128.37, 127.12, 127.07, 115.28 (d, $J=21.6 \mathrm{~Hz}), 85.47$ (d, $J=170.6 \mathrm{~Hz}), 70.96,52.27$ (d, $J=6.0 \mathrm{~Hz}), 44.73(\mathrm{~d}, J=18.2 \mathrm{~Hz}), 44.51,10.86 ;{ }^{19} \mathbf{F}$ NMR $\left(376 \mathrm{MHz}, \mathrm{CDCl}_{3}\right) \delta \mathrm{ppm}:-116.25--116.36(\mathrm{~m}$, 1F), -223.39 (tdd, $J=48.1,23.8,1.6 \mathrm{~Hz}, 1 \mathrm{~F})$. HRMS $\left(\mathrm{ESI}^{+}\right) \mathrm{m} / z \mathrm{C}_{24} \mathrm{H}_{26} \mathrm{~F}_{2} \mathrm{~N}^{+}[\mathrm{M}+\mathrm{H}]^{+}$: calculated 366.2028, found 366.2025. IR (thin layer film) $v\left(\mathrm{~cm}^{-1}\right)=3026,2970,1603,1510,1492,1452,1375,1225,1160,1080$, $1015,832,761,745,706 .[\alpha]_{\mathbf{D}}{ }^{25}{ }^{\circ} \mathrm{C}=+3.6^{\circ}\left(\mathrm{c}=0.4, \mathrm{CHCl}_{3}\right.$, e.r. $\left.=95: 5\right)$. HPLC separation DAICEL CHIRALPAK ${ }^{\circledR}$ IB-3, Heptane: $:^{i} \operatorname{PrOH}=99.9: 0.1,1 \mathrm{~mL} / \mathrm{min} ; \mathrm{t}_{1}=5.85 \mathrm{~min}$ (minor), $\mathrm{t}_{2}=6.51$ min (major).

( $S$ )- $N$-benzhydryl- $N$-benzyl-3-fluoro-2-(4-(trifluoromethyl)phenyl)propan-1-amine (2fa)

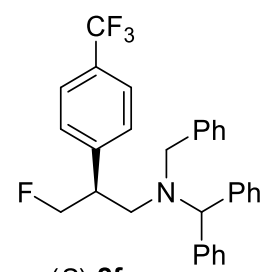

(S)-2fa

$(S)$-2fa was prepared from 1fa (1 equiv., $0.1 \mathrm{mmol}, 60.8 \mathrm{mg}$ ), according to the general procedure using $5 \mathrm{~mol} \%$ of catalyst $(S)$-D $(0.005 \mathrm{mmol}, 4.2 \mathrm{mg})$ and stirring for $48 \mathrm{~h}$. Purification: FCC eluent $=$ Pentane:Et $\mathrm{E}_{2} \mathrm{O}$ (100:0 to 90:10, gradient). Colorless oil, 47.2 mg, 99\% yield, e.r. $=95: 5 .{ }^{1} \mathbf{H}$ NMR $\left(400 \mathrm{MHz}, \mathrm{CDCl}_{3}\right) \delta$ ppm: $6.50(\mathrm{~d}, J=7.9 \mathrm{~Hz}, 2 \mathrm{H})$, $7.38-7.20(\mathrm{~m}, 15 \mathrm{H}), 7.07(\mathrm{~d}, J=8.1 \mathrm{~Hz}, 2 \mathrm{H}), 4.95(\mathrm{~s}, 1 \mathrm{H}), 4.64-4.38(\mathrm{~m}, 2 \mathrm{H}), 3.69(\mathrm{~d}$, $J=13.9 \mathrm{~Hz}, 1 \mathrm{H}), 3.60(\mathrm{~d}, J=13.9 \mathrm{~Hz}, 1 \mathrm{H}), 3.19-3.05(\mathrm{~m}, 1 \mathrm{H}), 2.90(\mathrm{dd}, J=13.4,7.1 \mathrm{~Hz}, 1 \mathrm{H}), 2.79$ (dddd, $J=13.3,7.8,1.7 \mathrm{~Hz}, 1 \mathrm{H}) ;{ }^{13} \mathbf{C} \mathbf{~ N M R}\left(101 \mathrm{MHz}, \mathrm{CDCl}_{3}\right)$ [overlapping signals] $\delta$ ppm: 144.83, 140.88, 140.18 , 139.29, 129.51, 129.29 (q, $J=32.3 \mathrm{~Hz}$ ), 129.14, 129.08, 129.04, 128.81, 128.46, 128.35, 127.44, 127.27, $127.24,125.36(\mathrm{q}, J=3.6 \mathrm{~Hz}), 124.41(\mathrm{q}, J=271.5 \mathrm{~Hz}), 85.17(\mathrm{~d}, J=171.9 \mathrm{~Hz}), 69.90,55.96,52.86(\mathrm{~d}, J=$ $6.1 \mathrm{~Hz}), 45.15(\mathrm{~d}, J=18.4 \mathrm{~Hz}) ;{ }^{19} \mathbf{F}$ NMR $\left(376 \mathrm{MHz}, \mathrm{CDCl}_{3}\right) \delta \mathrm{ppm}:-62.36$ (s, 3F), -221.93 (td, $J=48.0,28.1$ $\mathrm{Hz}, 1 \mathrm{~F})$. HRMS $\left(\mathrm{ESI}^{+}\right) \mathrm{m} / \mathrm{z} \mathrm{C}_{30} \mathrm{H}_{28} \mathrm{~F}_{4} \mathrm{~N}^{+}[\mathrm{M}+\mathrm{H}]^{+}$: calculated 478.2152, found 478.2150. IR (thin layer film) $v$ $\left(\mathrm{cm}^{-1}\right)=3062,3028,2952,2834,1619,1600,1493,1452,1422,1326,1165,1123,1069,1017,837,764,745$, 700. $[\alpha]_{\mathrm{D}}{ }^{25}{ }^{\circ} \mathrm{C}=+4.0^{\circ}\left(\mathrm{c}=0.4, \mathrm{CHCl}_{3}\right.$, e.r. $\left.=95: 5\right)$. HPLC separation DAICEL CHIRALPAK ${ }^{\circledR}$ IB-3, Heptane: $: \mathrm{PrOH}=99: 1,1 \mathrm{~mL} / \mathrm{min} ; \mathrm{t}_{1}=3.54 \mathrm{~min}$ (minor), $\mathrm{t}_{2}=4.35 \mathrm{~min}$ (major).

\section{$(S)$ - $N$-benzhydryl- $N$-ethyl-3-fluoro-2-(4-(trifluoromethyl)phenyl)propan-1-amine (2fb)}

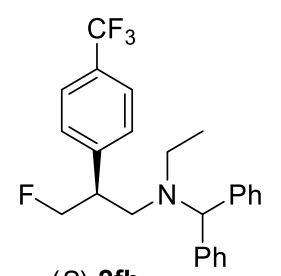

$(S)-2 \mathrm{fb}$

$(S)$-2fb was prepared from $\mathbf{1 f b}$ (1 equiv., $0.1 \mathrm{mmol}, 47.8 \mathrm{mg}$ ), according to the general procedure using $10 \mathrm{~mol} \%$ of catalyst $(S)-\mathbf{D}(0.01 \mathrm{mmol}, 8.4 \mathrm{mg})$ and stirring for $72 \mathrm{~h}$. Purification : FCC eluent $=$ Pentane:Et $\mathrm{O}_{2} \mathrm{O}$ (100:0 to 90:10, gradient). White solid, $39.5 \mathrm{mg}$, 95\% yield, e.r. $=95: 5 .{ }^{1} \mathbf{H}$ NMR $\left(400 \mathrm{MHz}, \mathrm{CDCl}_{3}\right) \delta \mathrm{ppm}: 7.54(\mathrm{~d}, J=8.1 \mathrm{~Hz}, 2 \mathrm{H}), 7.30$ $-7.17(\mathrm{~m}, 12 \mathrm{H}), 4.81(\mathrm{~s}, 1 \mathrm{H}), 4.79-4.59(\mathrm{~m}, 2 \mathrm{H}), 3.16-3.00(\mathrm{~m}, 1 \mathrm{H}), 2.87$ (dd, $J=13.4$, $8.3 \mathrm{~Hz}, 1 \mathrm{H}), 2.74-2.54(\mathrm{~m}, 3 \mathrm{H}), 0.97(\mathrm{t}, J=7.0 \mathrm{~Hz}, 3 \mathrm{H}) ;{ }^{13} \mathbf{C} \mathbf{N M R}\left(101 \mathrm{MHz}, \mathrm{CDCl}_{3}\right) \delta \mathrm{ppm}: 144.95,141.97$, $141.90,128.81(\mathrm{q}, J=32.3 \mathrm{~Hz}), 128.73,128.57,128.53,128.28,128.27,127.06,127.00,125.26(\mathrm{q}, J=3.8$ $\mathrm{Hz}), 124.25(\mathrm{q}, J=271.4 \mathrm{~Hz}), 84.90(\mathrm{~d}, J=172.0 \mathrm{~Hz}), 70.89,51.97(\mathrm{~d}, J=6.5 \mathrm{~Hz}), 45.32(\mathrm{~d}, J=18.1 \mathrm{~Hz})$, 44.53, 10.70; ${ }^{19}$ F NMR (376 MHz, $\left.\mathrm{CDCl}_{3}\right) \delta$ ppm: -62.42 (s, 3F), -223.56 (td, $\left.J=47.3,24.1,1.4 \mathrm{~Hz}, 1 \mathrm{~F}\right)$. HRMS $\left(\mathrm{ESI}^{+}\right) \mathrm{m} / z \mathrm{C}_{25} \mathrm{H}_{26} \mathrm{~F}_{4} \mathrm{~N}^{+}[\mathrm{M}+\mathrm{H}]^{+}$: calculated 416.1996, found 416.1993. IR (thin layer film) $v\left(\mathrm{~cm}^{-1}\right)=$ $3027,2966,2833,1619,1492,1452,1421,1326,1165,1124,1070,1018,839,706 . \mathbf{M P} 85-87^{\circ} \mathrm{C} .[\boldsymbol{\alpha}]_{\mathbf{D}}{ }^{2{ }^{\circ} \mathbf{C}}$ $=-2.6^{\circ}\left(\mathrm{c}=0.4, \mathrm{CHCl}_{3}\right.$, e.r. $\left.=95: 5\right)$. HPLC separation DAICEL CHIRALPAK ${ }^{\circledR}$ IB-3, Heptane: ${ }^{i} \mathrm{PrOH}=$ 99.75:0.25, $1 \mathrm{~mL} / \mathrm{min} ; \mathrm{t}_{1}=4.29 \min$ (minor), $\mathrm{t}_{2}=4.85 \mathrm{~min}$ (major). 
(S)-2-([1,1'-biphenyl]-4-yl)- $N$-benzhydryl- $N$-benzyl-3-fluoropropan-1-amine (2ga)

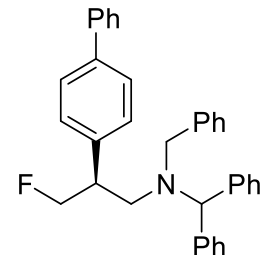

$(S)-2$ a

$(S)$-2ga was prepared from 1ga (1 equiv., $0.1 \mathrm{mmol}, 61.6 \mathrm{mg}$ ), according to the general procedure using $5 \mathrm{~mol} \%$ of catalyst $(S)-\mathbf{D}(0.005 \mathrm{mmol}, 4.2 \mathrm{mg})$ and stirring for $48 \mathrm{~h}$. Purification : $\mathrm{FCC}$ eluent $=$ Pentane: $\mathrm{Et}_{2} \mathrm{O}$ (100:0 to 90:10, gradient). Colorless oil, 39.7 mg, $82 \%$ yield, e.r. $=97: 3 .{ }^{1} \mathbf{H}$ NMR $\left(400 \mathrm{MHz}, \mathrm{CDCl}_{3}\right) \delta$ ppm: $7.65-7.60(\mathrm{~m}, 2 \mathrm{H}), 7.55$ $-7.44(\mathrm{~m}, 4 \mathrm{H}), 7.41-7.35(\mathrm{~m}, 4 \mathrm{H}), 7.35-7.20(\mathrm{~m}, 12 \mathrm{H}), 7.12-7.07(\mathrm{~m}, 2 \mathrm{H}), 5.01(\mathrm{~s}$, 1H), $4.72-4.45(\mathrm{~m}, 2 \mathrm{H}), 3.72(\mathrm{~d}, J=13.9 \mathrm{~Hz}, 1 \mathrm{H}), 3.67(\mathrm{~d}, J=13.9 \mathrm{~Hz}, 1 \mathrm{H}), 3.25-3.12(\mathrm{~m}, 1 \mathrm{H}), 2.93(\mathrm{dd}$, $J=13.2,7.2 \mathrm{~Hz}, 1 \mathrm{H}), 2.82$ (dddd, $J=13.2,7.5,1.8 \mathrm{~Hz}, 1 \mathrm{H}) ;{ }^{13} \mathbf{C} \mathbf{~ N M R}\left(101 \mathrm{MHz}, \mathrm{CDCl}_{3}\right)$ [overlapping signals] $\delta$ ppm: 141.15, 140.87, 140.40, 139.96, 139.72 (d, $J=3.8 \mathrm{~Hz}), 139.56,129.54,129.24,129.04,128.92$, 128.86, 128.42, 128.37, 128.27, 127.33, 127.31, 127.25, 127.19, 127.13, 85.68 (d, $J=171.7 \mathrm{~Hz}), 69.47,55.65$, $52.93(\mathrm{~d}, J=5.9 \mathrm{~Hz}), 44.83(\mathrm{~d}, J=17.9 \mathrm{~Hz}) ;{ }^{19} \mathbf{F} \mathbf{~ N M R}\left(376 \mathrm{MHz}, \mathrm{CDCl}_{3}\right) \delta \mathrm{ppm}:-221.04(\mathrm{td}, J=47.7,20.9$ $\mathrm{Hz}, 1 \mathrm{~F}$ ). HRMS (ESI $\left.{ }^{+}\right) \mathrm{m} / z \mathrm{C}_{35} \mathrm{H}_{33} \mathrm{FN}^{+}[\mathrm{M}+\mathrm{H}]^{+}$: calculated 486.2592, found 486.2587. IR (thin layer film) $v$ $\left(\mathrm{cm}^{-1}\right)=3059,3027,2928.2834,1600,1488,1450,1076,1008,835,764,743,699 .[\alpha]_{\mathbf{D}}{ }^{2{ }^{\circ} \mathrm{C}}=+25.5^{\circ}(\mathrm{c}=$ 0.4, $\mathrm{CHCl}_{3}$, e.r. $\left.=97: 3\right)$. HPLC separation DAICEL CHIRALPAK ${ }^{\circledR}$ IB-3, Heptane: ${ }^{i} \mathrm{PrOH}=99: 1,1 \mathrm{~mL} / \mathrm{min}$; $\mathrm{t}_{1}=5.61 \mathrm{~min}$ (major), $\mathrm{t}_{2}=8.22 \min$ (minor).

\section{(S)-2-([1,1'-biphenyl]-4-yl)- $N$-benzhydryl- $N$-ethyl-3-fluoropropan-1-amine (2gb)}<smiles>CCC(c1ccccc1)N(CC)CC(CF)c1ccccc1</smiles>

$(S)-2 g b$

(S)-2gb was prepared from 1 gb (1 equiv., $0.1 \mathrm{mmol}, 55.4 \mathrm{mg}$ ), according to the general procedure using $10 \mathrm{~mol} \%$ of catalyst $(S)-\mathbf{D}(0.01 \mathrm{mmol}, 8.4 \mathrm{mg})$ and stirring for $72 \mathrm{~h}$. Purification: FCC eluent $=$ Pentane: $\mathrm{Et}_{2} \mathrm{O}$ (100:0 to 90:10, gradient). Colorless oil, $36.4 \mathrm{mg}, 86 \%$ yield, $=97: 3 .{ }^{1} \mathbf{H}$ NMR $\left(400 \mathrm{MHz}, \mathrm{CDCl}_{3}\right) \delta \mathrm{ppm}: 7.61-7.55(\mathrm{~m}, 2 \mathrm{H}), 7.55$ $-7.49(\mathrm{~m}, 2 \mathrm{H}), 7.46-7.40(\mathrm{~m}, 2 \mathrm{H}), 7.36-7.15(\mathrm{~m}, 13 \mathrm{H}), 4.84(\mathrm{~s}, 1 \mathrm{H}), 4.74(\mathrm{dd}, J=47.6$, $5.6 \mathrm{~Hz}, 2 \mathrm{H}), 3.18-3.03(\mathrm{~m}, 1 \mathrm{H}), 2.89(\mathrm{dd}, J=13.3,8.5 \mathrm{~Hz}, 1 \mathrm{H}), 2.76-2.54(\mathrm{~m}, 3 \mathrm{H}), 0.99(\mathrm{t}, J=7.1 \mathrm{~Hz}, 3 \mathrm{H})$; ${ }^{13}$ C NMR (101 MHz, $\left.\mathrm{CDCl}_{3}\right)$ [overlapping signals] $\delta$ ppm: 142.38, 142.16, 141.12, $140.02(\mathrm{~d}, J=2.5 \mathrm{~Hz})$, $139.89,128.89,128.87,128.80,128.72,128.35,127.30,127.24,127.19,127.07,127.04,85.54$ (d, $J=170.1$ Hz), 70.96, $52.28(\mathrm{~d}, J=6.2 \mathrm{~Hz}), 45.10(\mathrm{~d}, J=18.4 \mathrm{~Hz}), 44.49,10.92 ;{ }^{19} \mathbf{F} \mathbf{N M R}\left(376 \mathrm{MHz}, \mathrm{CDCl}_{3}\right) \delta \mathrm{ppm:} \mathrm{-}$ $222.83(\mathrm{td}, J=47.5,23.2,1.6 \mathrm{~Hz}, 1 \mathrm{~F})$. HRMS $\left(\mathrm{ESI}^{+}\right) \mathrm{m} / z \mathrm{C}_{30} \mathrm{H}_{31} \mathrm{FN}^{+}[\mathrm{M}+\mathrm{H}]^{+}$: calculated 424.2435, found 424.2433. IR (thin layer film) $v\left(\mathrm{~cm}^{-1}\right)=2980,1487,1451,1382,1252,1147,2079,935,833,764,732,698$. $[\boldsymbol{\alpha}]_{\mathbf{D}}{ }^{25}{ }^{\circ} \mathrm{C}=+9.7^{\circ}\left(\mathrm{c}=0.3, \mathrm{CHCl}_{3}\right.$, e.r. $\left.=97: 3\right)$. HPLC separation DAICEL CHIRALCEL ${ }^{\circledR}$ OJ-H, Heptane:EtOH $=60: 40,1 \mathrm{~mL} / \mathrm{min} ; \mathrm{t}_{1}=22.06 \mathrm{~min}$ (major), $\mathrm{t}_{2}=34.84 \mathrm{~min}$ (minor).

(S)- $N$-benzhydryl- $N$-benzyl-3-fluoro-2-(p-tolyl)propan-1-amine (2ha)

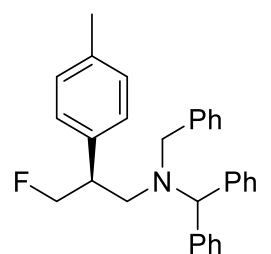

(S)-2ha

(S)-2ha was prepared from 1ha (1 equiv., $0.1 \mathrm{mmol}, 57.0 \mathrm{mg}$ ), according to the general procedure using $5 \mathrm{~mol} \%$ of catalyst $(S)$-D $(0.005 \mathrm{mmol}, 4.2 \mathrm{mg})$ and stirring for $48 \mathrm{~h}$. Purification: FCC eluent $=$ Pentane: $\mathrm{Et}_{2} \mathrm{O}$ (100:0 to 90:10, gradient). Colorless oil, 35.6 mg, 84\% yield, e.r. $=96.5: 3.5 .{ }^{1} \mathbf{H}$ NMR $\left(400 \mathrm{MHz}, \mathrm{CDCl}_{3}\right) \delta$ ppm: $7.37-7.19(\mathrm{~m}, 15 \mathrm{H})$, $7.08(\mathrm{~d}, J=7.9 \mathrm{~Hz}, 2 \mathrm{H}), 7.90$ (d, $J=7.9 \mathrm{~Hz}, 2 \mathrm{H}), 4.97$ (s, 1H), $4.66-4.39$ (m, 2H), 3.64 
(s, 2H), $3.16-3.01(\mathrm{~m}, 1 \mathrm{H}), 2.86(\mathrm{dd}, J=13.3,7.5 \mathrm{~Hz}, 1 \mathrm{H}), 2.73(\mathrm{dddd}, J=13.2,7.3,2.2 \mathrm{~Hz}, 1 \mathrm{H}), 2.34(\mathrm{~s}$, $3 \mathrm{H}) ;{ }^{13} \mathbf{C}$ NMR $\left(101 \mathrm{MHz}, \mathrm{CDCl}_{3}\right)$ [overlapping signals] $\delta \mathrm{ppm:} 140.75,140.62,139.62,137.54(\mathrm{~d}, J=3.8$ $\mathrm{Hz}), 136.55,129.48,129.31,129.23,128.39,128.33,128.27,128.24,127.24,127.08,85.79$ (d, $J=171.1 \mathrm{~Hz})$, 69.38, 55.58, $52.96(\mathrm{~d}, J=6.0 \mathrm{~Hz}), 44.70(\mathrm{~d}, J=18.1 \mathrm{~Hz}), 21.21 ;{ }^{19} \mathbf{F} \mathbf{N M R}\left(376 \mathrm{MHz}, \mathrm{CDCl}_{3}\right) \delta \mathrm{ppm:}-220.99$ (tdd, $J=48.0,21.0,1.5 \mathrm{~Hz}, 1 \mathrm{~F})$. HRMS $\left(\mathrm{ESI}^{+}\right) \mathrm{m} / z \mathrm{C}_{30} \mathrm{H}_{31} \mathrm{FNO}^{+}[\mathrm{M}+\mathrm{H}]^{+}$: calculated 424.2435, found 424.2435. IR (thin layer film) $v\left(\mathrm{~cm}^{-1}\right)=3059,3026,2951,2838,1600,1515,1493,1451,01133,1077,1028$, 813, 763, 743, 700. $[\boldsymbol{\alpha}]_{\mathbf{D}}{ }^{25}{ }^{\circ} \mathbf{C}=+14.8^{\circ}\left(\mathrm{c}=0.5, \mathrm{CHCl}_{3}\right.$, e.r. $\left.=96.5: 3.5\right)$. HPLC separation DAICEL CHIRALCEL ${ }^{\circledR}$ OJ-H, Heptane: ${ }^{i} \mathrm{PrOH}=96: 4,1 \mathrm{~mL} / \mathrm{min} ; \mathrm{t}_{1}=7.77 \mathrm{~min}$ (minor), $\mathrm{t}_{2}=10.38 \mathrm{~min}$ (major).

\section{(S)- $N$-benzhydryl- $N$-benzyl-3-fluoro-2-phenoxypropan-1-amine (2ia)}

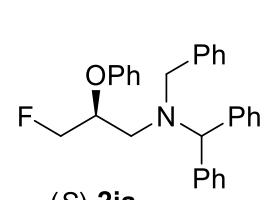

(S)-2ia

$(S)$-2ia was prepared from 1ia (1 equiv., $0.1 \mathrm{mmol}, 55.6 \mathrm{mg}$ ), according to the general procedure using $5 \mathrm{~mol} \%$ of catalyst $(S)$-D $(0.005 \mathrm{mmol}, 4.2 \mathrm{mg})$ and stirring for $48 \mathrm{~h}$. Purification: FCC eluent $=$ Pentane: $\mathrm{Et}_{2} \mathrm{O}$ (100:0 to 90:10, gradient). Colorless oil, 42.0 mg, 99\% yield, e.r. $=88: 12 .{ }^{1} \mathbf{H}$ NMR $\left(400 \mathrm{MHz}, \mathrm{CDCl}_{3}\right) \delta$ ppm: $7.44-7.17(\mathrm{~m}, 17 \mathrm{H})$, $6.96-6.90(\mathrm{~m}, 1 \mathrm{H}), 6.70-6.63(\mathrm{~m}, 2 \mathrm{H}), 4.99(\mathrm{~s}, 1 \mathrm{H}), 4.64-4.22(\mathrm{~m}, 3 \mathrm{H}), 3.76(\mathrm{~d}, J=13.8 \mathrm{~Hz}, 1 \mathrm{H}), 3.70(\mathrm{~d}$, $J=13.8 \mathrm{~Hz}, 1 \mathrm{H}), 2.96-2.75(\mathrm{~m}, 2 \mathrm{H}) ;{ }^{13} \mathbf{C ~ N M R}\left(101 \mathrm{MHz}, \mathrm{CDCl}_{3}\right)$ [overlapping signals] $\delta$ ppm: 157.95, $141.23,140.84,139.41,129.61,129.36,129.14,129.10,128.57,128.52$, 127.44, 127.38, 127.33, 121.37, 116.02, $83.57(\mathrm{~d}, J=173.7 \mathrm{~Hz}), 75.86(\mathrm{~d}, J=18.4 \mathrm{~Hz}), 70.98,56.86,50.87(\mathrm{~d}, J=6.7 \mathrm{~Hz}) ;{ }^{19} \mathbf{F}$ NMR $(376$ MHz, $\left.\mathrm{CDCl}_{3}\right) \delta$ ppm: $-229.37(\mathrm{td}, J=47.6,20.5 \mathrm{~Hz}, 1 \mathrm{~F})$. HRMS $\left(\mathrm{ESI}^{+}\right) \mathrm{m} / z \mathrm{C}_{29} \mathrm{H}_{29} \mathrm{FNO}^{+}[\mathrm{M}+\mathrm{H}]^{+}$: calculated 426.2228, found 426.2225. IR (thin layer film) $v\left(\mathrm{~cm}^{-1}\right)=3061,3028,2920,2849,1598,1493,1452,1299$, 1239, 1174, 1082, 1026, 925, 747, 700. $[\boldsymbol{\alpha}]_{\mathbf{D}}{ }^{25^{\circ} \mathrm{C}}=+21.2^{\circ}\left(\mathrm{c}=0.6, \mathrm{CHCl}_{3}\right.$, e.r. $\left.=88: 12\right)$. HPLC separation DAICEL CHIRALCEL ${ }^{\circledR}$ OJ-3, Heptane: ${ }^{i} \mathrm{PrOH}=95: 5,1 \mathrm{~mL} / \mathrm{min} ; \mathrm{t}_{1}=12.51 \mathrm{~min}$ (minor), $\mathrm{t}_{2}=20.42 \mathrm{~min}$ (major).

\section{(S)- $N$-benzhydryl- $N$-ethyl-3-fluoro-2-phenoxypropan-1-amine (2ib)}

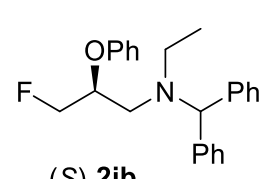

(S)-2ib

$(S)$-2ib was prepared from 1ib (1 equiv., $0.1 \mathrm{mmol}, 49.4 \mathrm{mg}$ ), according to the general procedure using $10 \mathrm{~mol} \%$ of catalyst $(S)-\mathbf{D}(0.01 \mathrm{mmol}, 8.4 \mathrm{mg})$ and stirring for $72 \mathrm{~h}$.

Purification: FCC eluent $=$ Pentane:Et $2 \mathrm{O}$ (100:0 to 90:10, gradient). Colorless oil, 30.5 $\mathrm{mg}, 84 \%$ yield, e.r. $=$ 93.5:6.5. ${ }^{1} \mathbf{H}$ NMR $\left(400 \mathrm{MHz}, \mathrm{CDCl}_{3}\right) \delta \mathrm{ppm:} 7.40-7.33(\mathrm{~m}, 4 \mathrm{H})$, $7.32-7.26(\mathrm{~m}, 4 \mathrm{H}), 7.26-7.17(\mathrm{~m}, 4 \mathrm{H}), 6.94(\mathrm{tt}, J=7.3,0.9 \mathrm{~Hz}, 1 \mathrm{H}), 6.74-6.68(\mathrm{~m}, 2 \mathrm{H}), 4.86(\mathrm{~s}, 1 \mathrm{H}), 4.80$ $-4.53(\mathrm{~m}, 2 \mathrm{H}), 4.41-4.30(\mathrm{~m}, 1 \mathrm{H}), 2.92-2.77(\mathrm{~m}, 2 \mathrm{H}), 2.75-2.59(\mathrm{~m}, 2 \mathrm{H}), 1.01(\mathrm{t}, J=7.0 \mathrm{~Hz}, 3 \mathrm{H}) ;{ }^{13} \mathrm{C}$ NMR (101 MHz, $\left.\mathrm{CDCl}_{3}\right) \delta$ ppm: 158.10, 142.33, 142.30, 129.62, 128.94, 128.62, 128.55, 128.51, 127.32, $127.19,121.40,116.15,82.57(\mathrm{~d}, J=172.1 \mathrm{~Hz}), 76.45(\mathrm{~d}, J=18.2 \mathrm{~Hz}), 71.71,49.99$ (d, $J=6.7 \mathrm{~Hz}), 46.03$ 11.12; ${ }^{19}$ F NMR (376 MHz, $\mathrm{CDCl}_{3}$ ) $\delta$ ppm: -230.23 (td, $\left.J=47.5,21.1,2.0 \mathrm{~Hz}, 1 \mathrm{~F}\right)$. HRMS $\left(\mathrm{ESI}^{+}\right) \mathrm{m} / \mathrm{z}$ $\mathrm{C}_{24} \mathrm{H}_{27} \mathrm{FNO}^{+}[\mathrm{M}+\mathrm{H}]^{+}$: calculated 364.2071, found 364.2071. IR (thin layer film) $v\left(\mathrm{~cm}^{-1}\right)=2969,1598,1493$, $1453,1240,1174,1082,1025,937,793,753,707,620 .[\alpha]_{D^{25}}{ }^{\circ} \mathrm{C}=+21.0^{\circ}\left(\mathrm{c}=0.5, \mathrm{CHCl}_{3}\right.$, e.r. $\left.=93.5: 6.5\right)$. 
HPLC separation DAICEL CHIRALCEL ${ }^{\circledR}$ OJ-H, Heptane:EtOH $=96: 4,1 \mathrm{~mL} / \mathrm{min} ; \mathrm{t}_{1}=13.57 \mathrm{~min}$ (minor), $\mathrm{t}_{2}=16.82 \min$ (major).

\section{(S)- $N$-benzhydryl- $N$-(3-fluoro-2-phenoxypropyl)prop-2-en-1-amine (2ic)}

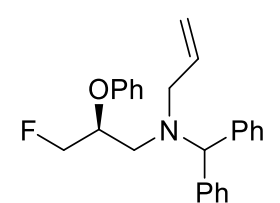

(S)-2ic

$(S)$-2ic was prepared from 1ic (1 equiv., $0.1 \mathrm{mmol}, 50.6 \mathrm{mg}$ ), according to the general procedure using $10 \mathrm{~mol} \%$ of catalyst $(S)$-D $(0.01 \mathrm{mmol}, 8.4 \mathrm{mg})$ and stirring for $36 \mathrm{~h}$.

Purification: FCC eluent $=$ Pentane: $\mathrm{Et}_{2} \mathrm{O}$ (100:0 to 90:10, gradient). Colorless oil, 34.2 mg, $91 \%$ yield, e.r. $=85: 15 .{ }^{1} \mathbf{H}$ NMR $\left(400 \mathrm{MHz}, \mathrm{CDCl}_{3}\right) \delta$ ppm: $7.38-7.19(\mathrm{~m}, 12 \mathrm{H})$, $6.94(\mathrm{tt}, J=8.3,1.0 \mathrm{~Hz}, 1 \mathrm{H}), 6.78-6.73(\mathrm{~m}, 2 \mathrm{H}), 5.97-5.85(\mathrm{~m}, 1 \mathrm{H}), 5.18-5.13(\mathrm{~m}, 1 \mathrm{H}), 5.12-5.05(\mathrm{~m}$, 1H), $4.93(\mathrm{~s}, 1 \mathrm{H}), 4.79-4.50(\mathrm{~m}, 2 \mathrm{H}), 4.51-4.38(\mathrm{~m}, 1 \mathrm{H}), 3.22(\mathrm{~d}, J=6.3 \mathrm{~Hz}, 2 \mathrm{H}), 2.93-2.81(\mathrm{~m}, 2 \mathrm{H})$; ${ }^{13} \mathrm{C}$ NMR (101 MHz, $\mathrm{CDCl}_{3}$ ) [overlapping signals] $\delta \mathrm{ppm:} \mathrm{158.11,} \mathrm{141.86,} \mathrm{141.75,} \mathrm{135.28,} \mathrm{129.62,} \mathrm{129.00,}$ $128.73,128.57,128.54,127.39,127.28,121.43,118.25,116.19,83.66(\mathrm{~d}, J=172.2 \mathrm{~Hz}), 76.32(\mathrm{~d}, J=18.5$ $\mathrm{Hz}), 71.55,55.41,50.17(\mathrm{~d}, J=6.9 \mathrm{~Hz}) ;{ }^{19} \mathbf{F}$ NMR $\left(376 \mathrm{MHz}, \mathrm{CDCl}_{3}\right) \delta \mathrm{ppm:}-229.74(\mathrm{tdd}, J=47.4,27.2,1.5$ $\mathrm{Hz}, 1 \mathrm{~F})$. HRMS $\left(\mathrm{ESI}^{+}\right) \mathrm{m} / \mathrm{z} \mathrm{C}_{25} \mathrm{H}_{27} \mathrm{FNO}^{+}[\mathrm{M}+\mathrm{H}]^{+}$: calculated 376.2071, found 376.2067. IR (thin layer film) $v$ $\left(\mathrm{cm}^{-1}\right)=3061,3027,2922,2848,1597,1493,1453,1289,1240,1173,1083,1026,928,795,753,706 .[\alpha]_{\mathbf{D}}{ }^{25^{\circ} \mathrm{C}}$ $=+18.9^{\circ}\left(\mathrm{c}=0.4, \mathrm{CHCl}_{3}\right.$, e.r. $\left.=85: 15\right)$. HPLC separation DAICEL CHIRALPAK ${ }^{\circledR}$ IB-3, Heptane $: \mathrm{PrOH}=$ 99.75:0.25, $1 \mathrm{~mL} / \mathrm{min} ; \mathrm{t}_{1}=5.26 \mathrm{~min}$ (minor), $\mathrm{t}_{2}=5.70 \mathrm{~min}$ (major).

\section{(S)-N-benzhydryl- $N$-benzyl-3-fluoro-2-(4-fluorophenoxy)propan-1-amine (2ja)}

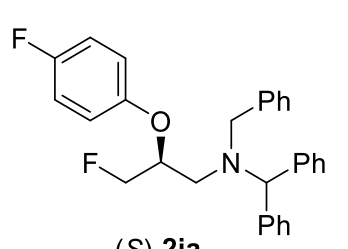

(S)-2ja

$(S)-\mathbf{2 j a}$ was prepared from $\mathbf{1 j a}$ (1 equiv., $0.1 \mathrm{mmol}, 57.4 \mathrm{mg}$ ), according to the general procedure using $10 \mathrm{~mol} \%$ of catalyst $(S)-\mathbf{D}(0.01 \mathrm{mmol}, 8.4 \mathrm{mg})$ and stirring for $48 \mathrm{~h}$. Purification : FCC eluent $=$ Pentane: $\mathrm{Et}_{2} \mathrm{O}$ (100:0 to 90:10, gradient). Colorless oil, 27.5 mg, 62\% yield, e.r. $=88: 12 .{ }^{1} \mathbf{H}$ NMR $\left(400 \mathrm{MHz}, \mathrm{CDCl}_{3}\right) \delta$ ppm: 7.42- $7.24(\mathrm{~m}, 15 \mathrm{H})$, $6.90-6.83(\mathrm{~m}, 2 \mathrm{H}), 6.60-6.54(\mathrm{~m}, 2 \mathrm{H}), 4.96(\mathrm{~s}, 1 \mathrm{H}), 4.75(\mathrm{ddd}, J=47.5,10.1,2.9$ $\mathrm{Hz}, 1 \mathrm{H}), 4.36(\mathrm{ddd}, J=47.6,10.1,5.5 \mathrm{~Hz}, 1 \mathrm{H}), 4.22-4.10(\mathrm{~m}, 2 \mathrm{H}), 3.73(\mathrm{~d}, J=13.7 \mathrm{~Hz}, 1 \mathrm{H}), 3.68(\mathrm{~d}, J=$ $13.7 \mathrm{~Hz}, 1 \mathrm{H}), 2.92-2.81(\mathrm{~m}, 2 \mathrm{H}) ;{ }^{13} \mathbf{C ~ N M R}\left(101 \mathrm{MHz}, \mathrm{CDCl}_{3}\right)$ [overlapping signals] $\delta$ ppm: $157.42(\mathrm{~d}, J=$ $239.2 \mathrm{~Hz}), 154.09$ (d, $J=2.3 \mathrm{~Hz}), 141.20,140.79,139.31,129.34,129.15,129.05,128.60,128.55,127.49$, 127.45, 127.38, $117.40(\mathrm{~d}, J=8.0 \mathrm{~Hz}), 115.90(\mathrm{~d}, J=23.0 \mathrm{~Hz}), 83.64(\mathrm{~d}, J=172.6 \mathrm{~Hz}), 77.16(\mathrm{~d}, J=18.4$ $\mathrm{Hz}), 71.19,57.0250 .87$ (d, $J=7.1 \mathrm{~Hz}) ;{ }^{19} \mathbf{F}$ NMR (376 MHz, $\left.\mathrm{CDCl}_{3}\right) \delta$ ppm: -123.00 - -123.09 (m, 1F), $223.31(\mathrm{td}, J=47.5,19.9 \mathrm{~Hz}, 1 \mathrm{~F})$. HRMS $\left(\mathrm{ESI}^{+}\right) \mathrm{m} / z \mathrm{C}_{29} \mathrm{H}_{28} \mathrm{~F}_{2} \mathrm{NO}^{+}[\mathrm{M}+\mathrm{H}]^{+}$: calculated 444.2133, found 444.2126. IR (thin layer film) $v\left(\mathrm{~cm}^{-1}\right)=2980,1503,1453,1381,1247,1206,1154,1072,1026,953,827$, $744,701 .[\alpha]_{\mathbf{D}}{ }^{25^{\circ} \mathrm{C}}=+15.7^{\circ}\left(\mathrm{c}=0.9, \mathrm{CHCl}_{3}\right.$, e.r. $\left.=88: 12\right)$. HPLC separation DAICEL CHIRALCEL ${ }^{\circledR}$ OJ-3, Heptane: ${ }^{i} \mathrm{PrOH}=80: 20,1 \mathrm{~mL} / \mathrm{min} ; \mathrm{t}_{1}=6.80 \mathrm{~min}$ (minor), $\mathrm{t}_{2}=13.43 \mathrm{~min}$ (major). 
(S)- $N$-benzhydryl- $N$-benzyl-3-fluoro-2-isopropoxypropan-1-amine (2ka)

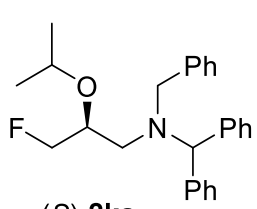

(S)-2ka

(S)-2ka was prepared from 1ka (1 equiv., $0.1 \mathrm{mmol}, 52.1 \mathrm{mg}$ ), according to the general procedure using $5 \mathrm{~mol} \%$ of catalyst $(S)$-D $(0.005 \mathrm{mmol}, 4.2 \mathrm{mg})$ and stirring for $48 \mathrm{~h}$. Purification : FCC eluent $=$ Pentane: $\mathrm{Et}_{2} \mathrm{O}$ (100:0 to 90:10, gradient). Colorless oil, $34.0 \mathrm{mg}$, 87\% yield, e.r. $=93: 7 .{ }^{1} \mathbf{H}$ NMR $\left(400 \mathrm{MHz}, \mathrm{CDCl}_{3}\right) \delta$ ppm: $7.43-7.21(\mathrm{~m}, 15 \mathrm{H}), 4.97(\mathrm{~s}$, 1H), 4.48 (ddd, $J=47.5,9.7,3.1 \mathrm{~Hz}, 1 \mathrm{H}), 4.36(\mathrm{ddd}, J=48.1,9.7,6.3 \mathrm{~Hz}, 1 \mathrm{H}), 3.70(\mathrm{~d}, J=13.7 \mathrm{~Hz}, 1 \mathrm{H}), 3.65$ $(\mathrm{d}, J=13.7 \mathrm{~Hz}, 1 \mathrm{H}), 3.54$ (sept, $J=6.1 \mathrm{~Hz}, 1 \mathrm{H}), 3.52-3.42(\mathrm{~m}, 1 \mathrm{H}), 2.69-2.56(\mathrm{~m}, 2 \mathrm{H}), 2.75(\mathrm{dd}, J=6.1$, $2.7 \mathrm{~Hz}, 6 \mathrm{H}) ;{ }^{13} \mathbf{C} \mathbf{N M R}\left(101 \mathrm{MHz}, \mathrm{CDCl}_{3}\right)$ [overlapping signals] $\delta$ ppm: 140.99, 140.80, 139.54, 129.35, 129.30, 129.06, 128.50, 128.40, 128.35, 127.27, 85.28 (d, $J=170.6 \mathrm{~Hz}), 74.75$ (d, $J=17.4 \mathrm{~Hz}), 71.32,70.42$, 56.73, $51.87(\mathrm{~d}, J=8.0 \mathrm{~Hz}), 22.82,22.69 ;{ }^{19} \mathbf{F}$ NMR (376 MHz, $\left.\mathrm{CDCl}_{3}\right) \delta \mathrm{ppm:}-227.86(\mathrm{tdd}, J=48.3,19.3$, $2.3 \mathrm{~Hz}, 1 \mathrm{~F}) . \mathbf{H R M S}\left(\mathrm{ESI}^{+}\right) \mathrm{m} / z \mathrm{C}_{26} \mathrm{H}_{31} \mathrm{FNO}^{+}[\mathrm{M}+\mathrm{H}]^{+}$: calculated 392.2384, found 392.2385. IR (thin layer film) $v\left(\mathrm{~cm}^{-1}\right)=3061,3028,2967,2926,2848,1600,1494,1452,1368,1330,1180,1123,985,763,744,700$. $[\boldsymbol{\alpha}]_{\mathbf{D}}{ }^{25}{ }^{\circ} \mathrm{C}=+10.5^{\circ}\left(\mathrm{c}=0.5, \mathrm{CHCl}_{3}\right.$, e.r. $\left.=93: 7\right)$. HPLC separation DAICEL CHIRALPAK ${ }^{\circledR}$ IC-3, Heptane: ${ }^{i} \mathrm{PrOH}=99: 1,1 \mathrm{~mL} / \mathrm{min} ; \mathrm{t}_{1}=2.78 \mathrm{~min}$ (minor), $\mathrm{t}_{2}=3.09 \mathrm{~min}$ (major).

\section{(S)- $N$-benzhydryl- $N$-ethyl-3-fluoro-2-isopropoxypropan-1-amine (2kb)}

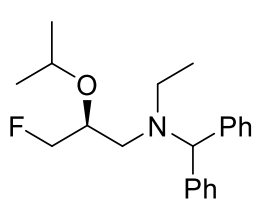

(S)-2kb

(S)-2kb was prepared from 1 $\mathbf{k b}$ (1 equiv., $0.1 \mathrm{mmol}, 46.0 \mathrm{mg}$ ), according to the general procedure $10 \mathrm{~mol} \%$ of catalyst $(S)-\mathbf{D}(0.01 \mathrm{mmol}, 8.4 \mathrm{mg})$ and stirring for $72 \mathrm{~h}$.

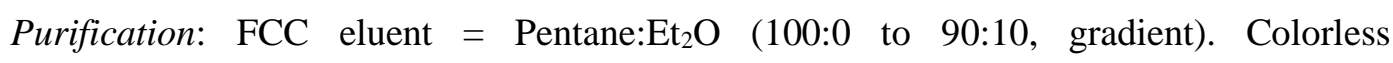
oil, $31.3 \mathrm{mg}, 95 \%$ yield, e.r. $=95: 5 .{ }^{1} \mathbf{H}$ NMR $\left(400 \mathrm{MHz}, \mathrm{CDCl}_{3}\right) \delta$ ppm: $7.39-7.33(\mathrm{~m}$, $4 \mathrm{H}), 7.33-7.24(\mathrm{~m}, 4 \mathrm{H}), 7.25-7.17(\mathrm{~m}, 2 \mathrm{H}), 4.83(\mathrm{~s}, 1 \mathrm{H}), 4.60$ (ddd, $J=47.8,9.7,3.0 \mathrm{~Hz}, 1 \mathrm{H}), 4.38$ (ddd, $J=48.0,9.6,6.1 \mathrm{~Hz}, 1 \mathrm{H}), 3.57(\mathrm{sept}, J=6.1 \mathrm{~Hz}, 1 \mathrm{H}), 3.54-3.42(\mathrm{~m}, 1 \mathrm{H}), 2.69-2.52(\mathrm{~m}, 4 \mathrm{H}), 1.08(\mathrm{dd}$, $J=6.1,3.7 \mathrm{~Hz}, 6 \mathrm{H}), 1.00(\mathrm{t}, J=7.1 \mathrm{~Hz}, 3 \mathrm{H}) ;{ }^{13} \mathbf{C ~ N M R}\left(101 \mathrm{MHz}, \mathrm{CDCl}_{3}\right) \delta$ ppm: 142.34, 142.27, 128.96, 128.71, 128.42, 128.36, 127.15, 127.10, 85.23 (d, $J=170.3 \mathrm{~Hz}), 75.17$ (d, $J=17.2 \mathrm{~Hz}), 71.55,71.40,51.20$ $(\mathrm{d}, J=7.9 \mathrm{~Hz}), 45.75,22.82,22.77,11.24 ;{ }^{19} \mathbf{F}$ NMR $\left(376 \mathrm{MHz}, \mathrm{CDCl}_{3}\right) \delta$ ppm: -229.02 (td, $J=47.8,20.3$ $\mathrm{Hz}, 1 \mathrm{~F})$. HRMS $\left(\mathrm{ESI}^{+}\right) \mathrm{m} / z \mathrm{C}_{21} \mathrm{H}_{29} \mathrm{FNO}^{+}[\mathrm{M}+\mathrm{H}]^{+}$: calculated 330.2228, found 330.2228. IR (thin layer film) $v$ $\left(\mathrm{cm}^{-1}\right)=3027,2970 ; 1492,1453,1379,1333,1122,1021,760,746,731,706 .[\alpha]_{\mathrm{D}}{ }^{25^{\circ} \mathrm{C}}=+12.5^{\circ}(\mathrm{c}=0.5$, $\mathrm{CHCl}_{3}$, e.r. = 95:5). HPLC separation DAICEL CHIRALCEL ${ }^{\circledR}$ OJ-3, Heptane: ${ }^{i} \mathrm{PrOH}=99: 1,1 \mathrm{~mL} / \mathrm{min} ; \mathrm{t}_{1}=$ $4.93 \min$ (major), $\mathrm{t}_{2}=6.63 \mathrm{~min}$ (minor).

\section{(S)- $N$-benzhydryl- $N$-benzyl-3-fluoro-2-methoxypropan-1-amine (2la)}

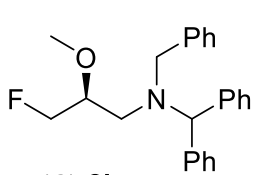

(S)-2la

$(S)$-2la was prepared from 1la (1 equiv., $0.1 \mathrm{mmol}, 49.3 \mathrm{mg}$ ), according to the general procedure using $10 \mathrm{~mol} \%$ of catalyst $(S)$-D $(0.01 \mathrm{mmol}, 8.4 \mathrm{mg})$ and stirring for $48 \mathrm{~h}$. Purification FCC eluent $=$ Pentane: $\mathrm{Et}_{2} \mathrm{O}$ (100:0 to 90:10, gradient). Colorless oil, $30.9 \mathrm{mg}$, $85 \%$ yield, e.r. $=88: 12 .{ }^{1} \mathbf{H}$ NMR $\left(400 \mathrm{MHz}, \mathrm{CDCl}_{3}\right) \delta$ ppm: $7.41-7.22(\mathrm{~m}, 15 \mathrm{H}), 4.98(\mathrm{~s}$, 1H), 4.48 (ddd, $J=47.4,10.0,2.8 \mathrm{~Hz}, 1 \mathrm{H}), 4.23$ (ddd, $J=48.1,10.0,6.2 \mathrm{~Hz}, 1 \mathrm{H}), 3.72$ (d, $J=13.8 \mathrm{~Hz}, 1 \mathrm{H})$, $3.64(\mathrm{~d}, J=13.8 \mathrm{~Hz}, 1 \mathrm{H}), 3.40-3.31(\mathrm{~m}, 1 \mathrm{H}), 3.31(\mathrm{~s}, 3 \mathrm{H}), 2.71-2.59(\mathrm{~m}, 2 \mathrm{H}) ;{ }^{13} \mathbf{C} \mathbf{N M R}\left(101 \mathrm{MHz}, \mathrm{CDCl}_{3}\right)$ 
[overlapping signals] $\delta \mathrm{ppm:} 141.05,140.75,139.55,129.29,129.03,128.50,128.42,128.38,127.30,127.25$, $84.71(\mathrm{~d}, J=170.9 \mathrm{~Hz}), 79.20(\mathrm{~d}, J=17.3 \mathrm{~Hz}), 71.35,58.20,56.46,50.50(\mathrm{~d}, J=8.2 \mathrm{~Hz}) ;{ }^{19} \mathbf{F}$ NMR $(376$ $\mathrm{MHz}, \mathrm{CDCl}_{3}$ ) $\delta$ ppm: -227.91 (tdd, $\left.J=48.2,19.8,2.0 \mathrm{~Hz}, 1 \mathrm{~F}\right)$. HRMS $\left(\mathrm{ESI}^{+}\right) \mathrm{m} / z \mathrm{C}_{24} \mathrm{H}_{27} \mathrm{FNO}^{+}[\mathrm{M}+\mathrm{H}]^{+}$: calculated 364.2071, found 364.2070. IR (thin layer film) $\vee\left(\mathrm{cm}^{-1}\right)=3027,2931,2832,1493,1453,1099$, $1028,764,744,700 .[\mathbf{\alpha}]_{\mathbf{D}}{ }^{25}{ }^{\circ} \mathbf{C}=+11.6^{\circ}\left(\mathrm{c}=0.5, \mathrm{CHCl}_{3}\right.$, e.r. $\left.=88: 12\right)$. HPLC separation DAICEL CHIRALPAK ${ }^{\circledR}$ IB-3, Heptane: $:$ PrOH $=99: 1,1 \mathrm{~mL} / \mathrm{min} ; \mathrm{t}_{1}=3.41 \mathrm{~min}$ (minor), $\mathrm{t}_{2}=4.24$ min (major).

(S)- $N$-benzhydryl- $N$-ethyl-3-fluoro-2-methoxypropan-1-amine (2lb)

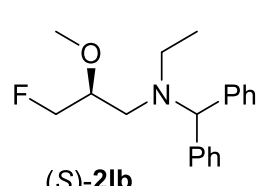

(S)-2lb

$(S)$-2lb was prepared from 1lb (1 equiv., $0.1 \mathrm{mmol}, 46.0 \mathrm{mg}$ ), according to the general procedure $10 \mathrm{~mol} \%$ of catalyst $(S)$-D $(0.01 \mathrm{mmol}, 8.4 \mathrm{mg})$ and stirring for $72 \mathrm{~h}$. Purification: FCC eluent $=$ Pentane: $\mathrm{Et}_{2} \mathrm{O}$ (100:0 to 90:10, gradient). Colorless oil, $29.8 \mathrm{mg}$, 99\% yield, e.r. = 91:9. ${ }^{1} \mathbf{H}$ NMR $\left(400 \mathrm{MHz}, \mathrm{CDCl}_{3}\right) \delta$ ppm: $7.38-7.17(\mathrm{~m}$, $10 \mathrm{H}), 4.85(\mathrm{~s}, 1 \mathrm{H}), 4.63(\mathrm{ddd}, J=47.7,9.9,2.6 \mathrm{~Hz}, 1 \mathrm{H}), 4.41(\mathrm{ddd}, J=48.9,9.9,5.9 \mathrm{~Hz}, 1 \mathrm{H}), 3.42-3.29$ (s + m overlapped, $4 \mathrm{H}), 2.71-2.52(\mathrm{~m}, 4 \mathrm{H}), 1.00(\mathrm{t}, J=7.0 \mathrm{~Hz}, 3 \mathrm{H}) ;{ }^{13} \mathbf{C} \mathbf{~ N M R}\left(101 \mathrm{MHz}, \mathrm{CDCl}_{3}\right) \delta$ ppm: 142.22, 142.11, 128.90, 128.73, 128.43, 128.38, 127.16, 127.14, 84.57 (d, $J=170.8 \mathrm{~Hz}), 79.36$ (d, $J=17.2 \mathrm{~Hz})$, $71.43,58.18,49.81(\mathrm{~d}, J=8.1 \mathrm{~Hz}), 45.54,11.31 ;{ }^{19} \mathbf{F}$ NMR $\left(376 \mathrm{MHz}, \mathrm{CDCl}_{3}\right) \delta \mathrm{ppm:}-229.07$ (tdd, $J=48.0$, 21.0, $2.5 \mathrm{~Hz}, 1 \mathrm{~F})$. HRMS $\left(\mathrm{ESI}^{+}\right) \mathrm{m} / z \mathrm{C}_{19} \mathrm{H}_{25} \mathrm{FNO}^{+}[\mathrm{M}+\mathrm{H}]^{+}$: calculated 302.1915, found 302.1916. IR (thin layer film) $v\left(\mathrm{~cm}^{-1}\right)=2970,1493,1453,1375,1263,1120,1013,760,746,731,706,620 .[\alpha]_{\mathbf{D}}{ }^{2{ }^{\circ} \mathrm{C}}=+15.3^{\circ}$ (c $=0.6, \mathrm{CHCl}_{3}$, e.r. $\left.=91: 9\right)$. HPLC separation DAICEL CHIRALCEL ${ }^{\circledR}$ OJ-3, Heptane: ${ }^{i} \operatorname{PrOH}=98: 2$, $1 \mathrm{~mL} / \mathrm{min} ; \mathrm{t}_{1}=10.04 \mathrm{~min}$ (major), $\mathrm{t}_{2}=11.85 \mathrm{~min}$ (minor).

\section{(S)- $N$-benzhydryl- $N$-benzyl-2-(benzyloxy)-3-fluoropropan-1-amine (2ma)}

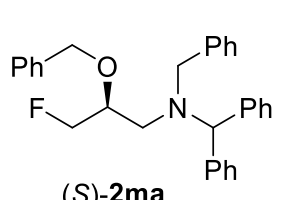

(S)-2ma

(S)-2ma was prepared from 1ma (1 equiv., $0.1 \mathrm{mmol}, 57.0 \mathrm{mg}$ ), according to the general procedure using $5 \mathrm{~mol} \%$ of catalyst $(S)-\mathbf{D}(0.005 \mathrm{mmol}, 4.2 \mathrm{mg})$ and stirring for $48 \mathrm{~h}$. Purification: FCC eluent $=$ Pentane: $\mathrm{Et}_{2} \mathrm{O}$ (100:0 to 90:10, gradient). Colorless oil, 42.1 mg, 96\% yield, e.r. $=95: 5 .{ }^{1} \mathbf{H}$ NMR $\left(400 \mathrm{MHz}, \mathrm{CDCl}_{3}\right) \delta$ ppm: $7.39-7.21(\mathrm{~m}, 20 \mathrm{H})$, $4.97(\mathrm{~s}, 1 \mathrm{H}), 4.60-4.43(\mathrm{~m}, 3 \mathrm{H}), 4.30(\mathrm{ddd}, J=48.2,10.0,6.3 \mathrm{~Hz}, 1 \mathrm{H}), 3.72-3.56(\mathrm{~m}, 3 \mathrm{H}), 2.76-2.61(\mathrm{~m}$, $2 \mathrm{H}) ;{ }^{13} \mathrm{C}$ NMR $\left(101 \mathrm{MHz}, \mathrm{CDCl}_{3}\right.$ ) [overlapping signals] $\delta$ ppm: 140.97, 140.76, 139.43, 138.48, 129.28, $129.03,128.49,128.41,128.38,127.98,127.78,127.29,127.27,127.23,85.22$ (d, $J=171.2 \mathrm{~Hz}), 76.80$ (d, $J$ $=17.3 \mathrm{~Hz}), 72.57,70.24,56.34,51.03(\mathrm{~d}, J=8.3 \mathrm{~Hz}) ;{ }^{19} \mathbf{F}$ NMR $\left(376 \mathrm{MHz}, \mathrm{CDCl}_{3}\right) \delta \mathrm{ppm}:-227.18(\mathrm{tdd}, J=$ 47.2, 19.5, $2.3 \mathrm{~Hz}, 1 \mathrm{~F})$. HRMS $\left(\mathrm{ESI}^{+}\right) \mathrm{m} / z \mathrm{C}_{30} \mathrm{H}_{31} \mathrm{FNO}^{+}[\mathrm{M}+\mathrm{H}]^{+}$: calculated 440.2384, found 440.2383; IR $\left(\right.$ thin layer film) $\vee\left(\mathrm{cm}^{-1}\right)=3030,2921,1494,1453,1262,1094,1027,765,742,699,613 .[\alpha]_{\mathbf{D}}{ }^{25}{ }^{\circ} \mathbf{C}=+4.7^{\circ}$ $\left(\mathrm{c}=0.2, \mathrm{CHCl}_{3}\right.$, e.r. $\left.=95: 5\right)$. HPLC separation DAICEL CHIRALCEL ${ }^{\circledR}$ OJ-H, Heptane:EtOH = 96:4, 1 $\mathrm{mL} / \mathrm{min} ; \mathrm{t}_{1}=17.70 \mathrm{~min}$ (major), $\mathrm{t}_{2}=23.32 \mathrm{~min}$ (minor). 
(S)- $N$-benzhydryl-2-(benzyloxy)- $N$-ethyl-3-fluoropropan-1-amine (2mb)

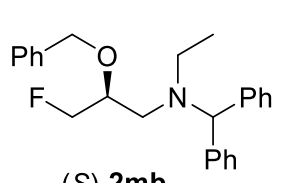

$(S)-\mathbf{2 m b}$ was prepared from $1 \mathrm{mb}$ (1 equiv., $0.1 \mathrm{mmol}, 50.8 \mathrm{mg}$ ), according to the general procedure using $10 \mathrm{~mol} \%$ of catalyst $(S)-\mathbf{D}(0.01 \mathrm{mmol}, 8.4 \mathrm{mg})$ and stirring for $72 \mathrm{~h}$. Purification: FCC eluent $=$ Pentane:Et ${ }_{2} \mathrm{O}$ (100:0 to 90:10, gradient). Colorless oil, 37.4 mg, 99\% yield, e.r. $=97: 3 .{ }^{1} \mathbf{H}$ NMR $\left(400 \mathrm{MHz}, \mathrm{CDCl}_{3}\right) \delta$ ppm: $7.38-7.17(\mathrm{~m}, 15 \mathrm{H})$, $4.83(\mathrm{~s}, 1 \mathrm{H}), 4.75-4.38(\mathrm{~m}, 4 \mathrm{H}), 3.69-3.56(\mathrm{~m}, 1 \mathrm{H}), 2.74-2.51(\mathrm{~m}, 4 \mathrm{H}), 0.98(\mathrm{t}, J=7.0 \mathrm{~Hz}, 3 \mathrm{H}) ;{ }^{13} \mathbf{C} \mathbf{N M R}$ $\left(101 \mathrm{MHz}, \mathrm{CDCl}_{3}\right) \delta$ ppm: 142.20, 142.03, 138.60, 128.86, 128.76, 128.49, 128.41, 128.37, 127.91, 127.76, 127.14, 127.12, 85.07 (d, $J=170.3 \mathrm{~Hz}), 77.03$ (d, $J=17.6 \mathrm{~Hz}), 72.51,71.30,50.36$ (d, $J=8.0 \mathrm{~Hz}), 45.43$, 11.24; ${ }^{19} \mathbf{F}$ NMR $\left(376 \mathrm{MHz}, \mathrm{CDCl}_{3}\right) \delta$ ppm: -228.42 (td, $\left.J=48.0,20.1,2.7 \mathrm{~Hz}, 1 \mathrm{~F}\right) . \mathbf{H R M S}\left(\mathrm{ESI}^{+}\right) \mathrm{m} / z$ $\mathrm{C}_{25} \mathrm{H}_{29} \mathrm{FNO}^{+}[\mathrm{M}+\mathrm{H}]^{+}$: calculated 378.2228, found 378.2227. IR (thin layer film) $v\left(\mathrm{~cm}^{-1}\right)=3028,2967,1493$, $1453,1375,1105,1028,734,699 .[\alpha]_{\mathbf{D}}{ }^{2{ }^{\circ} \mathrm{C}}=+11.2^{\circ}\left(\mathrm{c}=0.5, \mathrm{CHCl}_{3}\right.$, e.r. $\left.=97: 3\right)$. HPLC separation DAICEL CHIRALCEL ${ }^{\circledR}$ OJ-3, Heptane: ${ }^{2} \mathrm{PrOH}=95: 5,1 \mathrm{~mL} / \mathrm{min} ; \mathrm{t}_{1}=9.11 \mathrm{~min}$ (major), $\mathrm{t}_{2}=13.37 \mathrm{~min}$ (minor).

\section{(S)-1-(benzhydryl(ethyl)amino)-3-fluoropropan-2-yl benzoate (2na)}

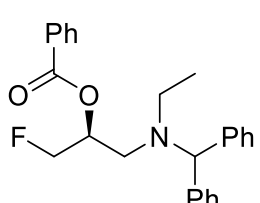

(S)-2na

$(S)$-2na was prepared from $1 \mathrm{na}$ (1 equiv., $0.1 \mathrm{mmol}, 58.4 \mathrm{mg}$ ), according to the general procedure using $5 \mathrm{~mol} \%$ of catalyst $(S)-\mathbf{D}(0.005 \mathrm{mmol}, 4.2 \mathrm{mg})$ and stirring for $48 \mathrm{~h}$. Purification : FCC eluent $=$ Pentane: $\mathrm{Et}_{2} \mathrm{O}$ (100:0 to 90:10, gradient). Colorless oil, 34.0 $\mathrm{mg}, 87 \%$ yield, e.r. $=86.5: 13.5 .{ }^{1} \mathbf{H}$ NMR $\left(400 \mathrm{MHz}, \mathrm{CDCl}_{3}\right) \delta$ ppm: $8.01-7.97(\mathrm{~m}, 2 \mathrm{H})$, $7.50(\mathrm{t}, J=7.4 \mathrm{~Hz}, 1 \mathrm{H}), 7.37(\mathrm{t}, J=7.6 \mathrm{~Hz}, 2 \mathrm{H}), 7.29-7.10(\mathrm{~m}, 10 \mathrm{H}), 5.37-5.24(\mathrm{~m}, 1 \mathrm{H}), 4.85(\mathrm{~s}, 1 \mathrm{H}), 4.59$ $(\mathrm{ddd}, J=47.7,10.2,2.6 \mathrm{~Hz}, 1 \mathrm{H}), 4.79(\mathrm{ddd}, J=47.2,10.2,4.6 \mathrm{~Hz}, 1 \mathrm{H}), 2.85-2.48(\mathrm{~m}, 4 \mathrm{H}), 0.96(\mathrm{t}, J=7.0$ $\mathrm{Hz}, 3 \mathrm{H}) ;{ }^{13} \mathbf{C} \mathbf{N M R}\left(101 \mathrm{MHz}, \mathrm{CDCl}_{3}\right)$ [overlapping signals] $\delta$ ppm: 166.07, 141.91, 141.66, 133.27, 129.96, 128.91, 128.75, 128.51, 128.44, 128.41, 127.19, $82.97(\mathrm{~d}, J=171.7 \mathrm{~Hz}), 71.71$ (d, $J=18.4 \mathrm{~Hz}), 70.84,49.18$ $(\mathrm{d}, J=6.9 \mathrm{~Hz}), 44.85,11.41 ;{ }^{19} \mathbf{F}$ NMR $\left(376 \mathrm{MHz}, \mathrm{CDCl}_{3}\right) \delta \mathrm{ppm}:-232.00(\mathrm{td}, J=47.1,23.3 \mathrm{~Hz}, 1 \mathrm{~F}) . \mathbf{H R M S}$ $\left(\mathrm{ESI}^{+}\right) \mathrm{m} / z \mathrm{C}_{25} \mathrm{H}_{27} \mathrm{FNO}_{2}{ }^{+}[\mathrm{M}+\mathrm{H}]^{+}$: calculated 392.2020, found 392.2019. IR (thin layer film) $v\left(\mathrm{~cm}^{-1}\right)=2980$, $1720,1492,1452,1270,1113,1070,1026,953,762,708 .[\alpha]_{D}{ }^{25}{ }^{\circ} \mathrm{C}=+11.4^{\circ}\left(\mathrm{c}=0.4, \mathrm{CHCl}_{3}\right.$, e.r. $\left.=86.5: 13.5\right)$. HPLC separation DAICEL CHIRALPAK ${ }^{\circledR}$ IC-3, Heptane: ${ }^{i} \mathrm{PrOH}=99.55: 0.45,1 \mathrm{~mL} / \mathrm{min} ; \mathrm{t}_{1}=5.22 \mathrm{~min}$ (minor), $\mathrm{t}_{2}=5.94 \min$ (major).

\section{$N$-benzyl- $N$-((5-bromo-1-(fluoromethyl)-1,3-dihydroisobenzofuran-1-yl)methyl)-1,1-}

\section{diphenylmethanamine (2oa)}

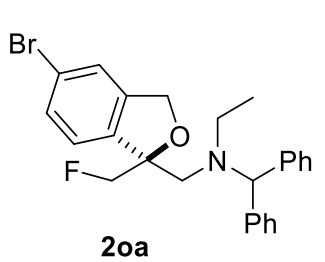

Absolute configuration not assigned

20a was prepared from 10 a ( 1 equiv., $0.1 \mathrm{mmol}, 58.4 \mathrm{mg}$ ), according to the general procedure using $10 \mathrm{~mol} \%$ of catalyst $(S)-\mathbf{D}(0.01 \mathrm{mmol}, 8.4 \mathrm{mg})$ and stirring for $72 \mathrm{~h}$. Purification: FCC eluent $=$ Pentane: $\mathrm{Et}_{2} \mathrm{O}$ (100:0 to 90:10, gradient). White solid, $23.1 \mathrm{mg}, 51 \%$ yield, e.r. $=83: 17 .{ }^{1} \mathbf{H}$ NMR $\left(400 \mathrm{MHz}, \mathrm{CDCl}_{3}\right) \delta \mathrm{ppm}: 7.43-7.13(\mathrm{~m}$, $12 \mathrm{H}), 6.91(\mathrm{~d}, J=8.1 \mathrm{~Hz}, 1 \mathrm{H}), 5.25(\mathrm{~s}, 1 \mathrm{H}), 5.09(\mathrm{~s}, 2 \mathrm{H}), 4.67(\mathrm{dd}, J=47.1,9.5 \mathrm{~Hz}$, $1 \mathrm{H}), 4.60(\mathrm{dd}, J=48.4,9.5 \mathrm{~Hz}, 1 \mathrm{H}), 2.96-2.83(\mathrm{~m}, 2 \mathrm{H}), 2.71-2.59(\mathrm{~m}, 1 \mathrm{H}), 2.56-$

$2.44(\mathrm{~m}, 1 \mathrm{H}), 0.95(\mathrm{t}, J=7.2 \mathrm{~Hz}, 3 \mathrm{H}) ;{ }^{13} \mathbf{C}$ NMR (101 MHz, $\left.\mathrm{CDCl}_{3}\right)$ [overlapping signals] $\delta$ ppm: 142.60, 
$141.73,140.89,139.72(\mathrm{~d}, J=2.4 \mathrm{~Hz}), 130.58,129.47,129.01,128.21,128.19,127.08,126.94,124.43$, 123.91, 122.30, 90.65 (d, $J=17.6 \mathrm{~Hz}), 85.72(\mathrm{~d}, J=175.1 \mathrm{~Hz}), 72.45,69.80,54.19,44.73,12.03$; ${ }^{19}$ F NMR $\left(376 \mathrm{MHz}, \mathrm{CDCl}_{3}\right) \delta$ ppm: $-229.02(\mathrm{t}, J=48.0 \mathrm{~Hz}, 1 \mathrm{~F})$. HRMS $\left(\mathrm{ESI}^{+}\right) \mathrm{m} / z \mathrm{C}_{25} \mathrm{H}_{26} \mathrm{BrFNO}^{+}[\mathrm{M}+\mathrm{H}]^{+}$: calculated 454.1176, found 454.1175. IR (thin layer film) $v\left(\mathrm{~cm}^{-1}\right)=2924,2852,1600,1492,1453,1413,1375,1262$, 1191, 1070, 1030, 818, 761, 731, 703. MP $95-97^{\circ} \mathrm{C} .[\mathbf{\alpha}]_{\mathbf{D}}{ }^{25^{\circ} \mathbf{C}}=+11.7^{\circ}\left(\mathrm{c}=0.3, \mathrm{CHCl}_{3}\right.$, e.r. $\left.=83: 17\right) . \mathbf{H P L C}$ separation DAICEL CHIRALPAK ${ }^{\circledR}$ IC-3, Heptane: $\operatorname{PrOH}=99.5: 0.5,1 \mathrm{~mL} / \mathrm{min} ; \mathrm{t}_{1}=3.35 \mathrm{~min}($ major$), \mathrm{t}_{2}=$ $3.92 \mathrm{~min}$ (minor).

\section{(S)- $N$-benzhydryl- $N$-ethyl-3-fluoro-2-(thiophen-3-yl)propan-1-amine (2pa)}

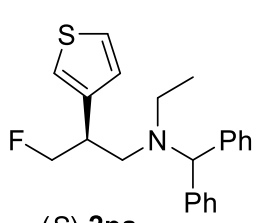

(S)-2pa

$(S)$-2pa was prepared from 1pa (1 equiv., $0.1 \mathrm{mmol}, 48.3 \mathrm{mg}$ ), according to the general procedure using $10 \mathrm{~mol} \%$ of catalyst $(S)$-D $(0.01 \mathrm{mmol}, 8.4 \mathrm{mg})$ and stirring for $72 \mathrm{~h}$. Purification : FCC eluent $=$ Pentane: $\mathrm{Et}_{2} \mathrm{O}$ (100:0 to 90:10, gradient). Colorless oil, $21.6 \mathrm{mg}$, 61\% yield, e.r. $=93.5: 6.5 .{ }^{1} \mathbf{H}$ NMR $\left(400 \mathrm{MHz}, \mathrm{CDCl}_{3}\right) \delta$ ppm: $7.34-7.17(\mathrm{~m}, 11 \mathrm{H}), 6.97$ $(\mathrm{d} \mathrm{br}, J=2.2 \mathrm{~Hz}, 1 \mathrm{H}), 6.87(\mathrm{~d} \mathrm{br}, J=4.9 \mathrm{~Hz}, 1 \mathrm{H}), 4.83(\mathrm{~s}, 1 \mathrm{H}), 4.73(\mathrm{ddd}, J=47.4,8.8,5.4 \mathrm{~Hz}, 1 \mathrm{H}), 4.67$ (ddd, $J=47.8,8.8,4.5 \mathrm{~Hz}, 1 \mathrm{H}), 3.22-3.06(\mathrm{~m}, 1 \mathrm{H}), 2.84(\mathrm{dd}, J=13.5,9.0 \mathrm{~Hz}, 1 \mathrm{H}), 2.73-2.50(\mathrm{~m}, 3 \mathrm{H})$, $0.98(\mathrm{t}, \mathrm{J}=7.0 \mathrm{~Hz}, 3 \mathrm{H}) ;{ }^{13} \mathbf{C} \mathbf{~ N M R}\left(101 \mathrm{MHz}, \mathrm{CDCl}_{3}\right)$ [overlapping signals] $\delta$ ppm: 142.40, 142.16, 140.37 (d, $J=2.6 \mathrm{~Hz}), 128.87,128.79,128.36,127.72,127.05,125.27,121.43,85.15(\mathrm{~d}, J=173.0 \mathrm{~Hz}), 71.01,52.12(\mathrm{~d}$, $J=6.2 \mathrm{~Hz}), 44.66,40.91(\mathrm{~d}, J=18.6 \mathrm{~Hz}), 11.01 ;{ }^{19} \mathbf{F}$ NMR $\left(376 \mathrm{MHz}, \mathrm{CDCl}_{3}\right) \delta \mathrm{ppm}:-223.77$ (tdd, $J=48.5$, 25.9, $1.3 \mathrm{~Hz}, 1 \mathrm{~F})$. HRMS $\left(\mathrm{ESI}^{+}\right) \mathrm{m} / z \mathrm{C}_{22} \mathrm{H}_{25} \mathrm{FNS}^{+}[\mathrm{M}+\mathrm{H}]^{+}$: calculated 354.1686, found 354.1687. IR (thin layer film) $v\left(\mathrm{~cm}^{-1}\right)=3024,2965,1492,1452,1373,1179,1138,1028,914,778,760,745,732,705 .[\alpha]_{\mathbf{D}}{ }^{25}{ }^{\circ} \mathbf{C}=$ $+3.7^{\circ}\left(\mathrm{c}=0.4, \mathrm{CHCl}_{3}\right.$, e.r. $\left.=93.5: 6.5\right)$. SFC separation DAICEL CHIRALCEL ${ }^{\circledR}$ OJ-3, $\mathrm{CO}_{2}: \mathrm{MeOH}(95: 5$ to $40: 60$, gradient $)+0.3 \%$ isopropylamine, $3 \mathrm{~mL} / \mathrm{min} ; \mathrm{t}_{1}=2.13 \mathrm{~min}$ (minor), $\mathrm{t}_{2}=2.41 \mathrm{~min}$ (major).

\section{(S)- $N$-benzhydryl- $N$-benzyl-2-(4-bromo-1H-pyrazol-1-yl)-3-fluoropropan-1-amine (2qa)}

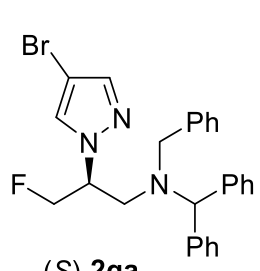

(S)-2qa

(S)-2qa was prepared from 1qa (1 equiv., $0.1 \mathrm{mmol}, 60.8 \mathrm{mg}$ ), according to the general procedure using $5 \mathrm{~mol} \%$ of catalyst $(S)$-D $(0.005 \mathrm{mmol}, 4.2 \mathrm{mg})$ and stirring for $48 \mathrm{~h}$. Purification : FCC eluent $=$ Pentane: $\mathrm{Et}_{2} \mathrm{O}$ (100:0 to 90:10, gradient). Colorless oil, $46.9 \mathrm{mg}$, $98 \%$ yield, e.r. $=87: 13 .{ }^{1} \mathbf{H}$ NMR $\left(400 \mathrm{MHz}, \mathrm{CDCl}_{3}\right) \delta$ ppm: $7.44(\mathrm{~s}, 1 \mathrm{H}), 7.37-7.20(\mathrm{~m}$, $15 \mathrm{H}), 7.11(\mathrm{~s}, 1 \mathrm{H}), 4.89(\mathrm{~s}, 1 \mathrm{H}), 4.47(\mathrm{dd}, J=47.0,5.1 \mathrm{~Hz}, 2 \mathrm{H}), 4.29-4.16(\mathrm{~m}, 1 \mathrm{H}), 3.69$ -3.59 (m, 2H), 3.07 (ddd, $J=14.0,7.6,2.0 \mathrm{~Hz}, 1 \mathrm{H}), 2.95$ (ddd, $J=14.0,5.9 \mathrm{~Hz}, 1 \mathrm{H}) ;{ }^{13} \mathrm{C}$ NMR $(101 \mathrm{MHz}$, $\mathrm{CDCl}_{3}$ ) [overlapping signals] $\delta$ ppm: $140.74,140.34,140.06,138.93,129.85,129.30,129.06,128.94,128.65$, 128.61, 128.55, 127.57, 127.55, 127.43, 93.00, 83.10 (d, $J=173.2 \mathrm{~Hz}), 70.69,61.80$ (d, $J=18.2 \mathrm{~Hz}), 56.49$, $51.69(\mathrm{~d}, J=6.1 \mathrm{~Hz}) ;{ }^{19} \mathbf{F}$ NMR $\left(376 \mathrm{MHz}, \mathrm{CDCl}_{3}\right) \delta \mathrm{ppm}:-226.12(\mathrm{td}, J=47.7,20.1 \mathrm{~Hz}, 1 \mathrm{~F}) . \mathbf{H R M S}\left(\mathrm{ESI}^{+}\right)$ $m / z \mathrm{C}_{26} \mathrm{H}_{26} \mathrm{BrFN}_{3}{ }^{+}[\mathrm{M}+\mathrm{H}]^{+}$: calculated 478.1289, found 478.1286. IR (thin layer film) $v\left(\mathrm{~cm}^{-1}\right)=3063,3028$, 2963, 2191, 2174, 2020, 1980, 1600, 1493, 1452, 1381, 1381, 1310, 1261, 1173, 1078, 1026, 953, 799, 765, $745,701 .[\alpha]_{\mathbf{D}}{ }^{25^{\circ} \mathrm{C}}=-7.7^{\circ}\left(\mathrm{c}=0.4, \mathrm{CHCl}_{3}\right.$, e.r. $\left.=87: 13\right)$. HPLC separation DAICEL CHIRALPAK ${ }^{\circledR}$ IA-3, Heptane: $: \mathrm{PrOH}=99: 1,1 \mathrm{~mL} / \mathrm{min} ; \mathrm{t}_{1}=7.96 \mathrm{~min}$ (minor), $\mathrm{t}_{2}=9.68 \mathrm{~min}$ (major). 
(S)-N-benzhydryl- $N$-benzyl-3-fluoro-2-(3-methyl-1H-indol-1-yl)propan-1-amine (2ra)

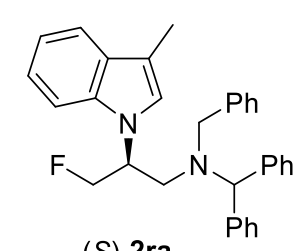

(S)-2ra

(S)-2ra was prepared from 1ra (1 equiv., $0.1 \mathrm{mmol} 59.2 \mathrm{mg}$ ), according to the general procedure using $5 \mathrm{~mol} \%$ of catalyst $(S)$-D $(0.005 \mathrm{mmol}, 4.2 \mathrm{mg})$ and stirring for $48 \mathrm{~h}$. Purification : FCC eluent $=$ Pentane: $\mathrm{Et}_{2} \mathrm{O}$ (100:0 to 90:10, gradient). White solid, $46.0 \mathrm{mg}$, 99\% yield, e.r. = 94:6. ${ }^{1} \mathbf{H}$ NMR $\left(400 \mathrm{MHz}, \mathrm{CDCl}_{3}\right) \delta$ ppm: $7.57-7.51(\mathrm{~m}, 1 \mathrm{H}), 7.37-$ $7.20(\mathrm{~m}, 15 \mathrm{H}), 7.13-7.05(\mathrm{~m}, 2 \mathrm{H}), 6.86-6.80(\mathrm{~m}, 1 \mathrm{H}), 6.67$ (s br, 1H), $4.92(\mathrm{~s}, 1 \mathrm{H})$, $4.64(\mathrm{ddd}, J=47.1,9.6,5.3 \mathrm{~Hz}, 1 \mathrm{H}), 4.58(\mathrm{ddd}, J=47.4,9.6,3.6 \mathrm{~Hz}, 1 \mathrm{H}), 4.44-4.30(\mathrm{~m}, 1 \mathrm{H}), 3.75(\mathrm{~d}, J=$ $13.8 \mathrm{~Hz}, 1 \mathrm{H}), 3.66(\mathrm{~d}, J=13.8 \mathrm{~Hz}, 1 \mathrm{H}), 3.18(\mathrm{dd}, J=13.9,7.5 \mathrm{~Hz}, 1 \mathrm{H}), 3.18$ (ddd, $J=13.9,6.8,1.8 \mathrm{~Hz}, 1 \mathrm{H})$, $2.27(\mathrm{~d}, J=0.9 \mathrm{~Hz}, 3 \mathrm{H}) ;{ }^{13} \mathbf{C} \mathbf{N M R}\left(101 \mathrm{MHz}, \mathrm{CDCl}_{3}\right) \delta \mathrm{ppm}: 141.09,140.62,139.27,136.54,129.33,129.11$, 129.02, 128.69, 128.62, 128.59, 128.49, 127.49, 127.44, 127.30, 123.02 (d, $J=2.5 \mathrm{~Hz}), 121.49,119.08$, 118.94, 111.31, 109.18, $83.90(\mathrm{~d}, J=173.8 \mathrm{~Hz}), 70.85,56.73,54.38(\mathrm{~d}, J=18.8 \mathrm{~Hz}), 51.98(\mathrm{~d}, J=5.3 \mathrm{~Hz})$, 9.80; ${ }^{19} \mathbf{F}$ NMR $\left(376 \mathrm{MHz}, \mathrm{CDCl}_{3}\right) \delta \mathrm{ppm}:-225.77(\mathrm{td}, J=46.7,23.8 \mathrm{~Hz}, 1 \mathrm{~F})$. HRMS $\left(\mathrm{ESI}^{+}\right) \mathrm{m} / z \mathrm{C}_{32} \mathrm{H}_{32} \mathrm{FN}_{2}{ }^{+}$ $[\mathrm{M}+\mathrm{H}]^{+}$: calculated 463.2544, found 463.2547. IR (thin layer film) $v\left(\mathrm{~cm}^{-1}\right)=3061,3027,2919,2844,1599$, $1493,1460,1385,1357,1262,1199,1128,1073,0127,797,739,700 . \mathbf{M P} 47-49^{\circ} \mathrm{C} \cdot[\alpha]_{\mathbf{D}}{ }^{25^{\circ} \mathrm{C}}=+26.4^{\circ}(\mathrm{c}$ $=0.5, \mathrm{CHCl}_{3}$, e.r. $\left.=94: 6\right)$. HPLC separation DAICEL CHIRALPAK ${ }^{\circledR}$ IB-3, Heptane: ${ }^{i} \operatorname{PrOH}=99: 1,1$ $\mathrm{mL} / \mathrm{min} ; \mathrm{t}_{1}=6.63 \min$ (minor), $\mathrm{t}_{2}=8.47 \min$ (major).

\section{(S)-2-(1-(benzhydryl(benzyl)amino)-3-fluoropropan-2-yl)isoindoline-1,3-dione (2sa)}

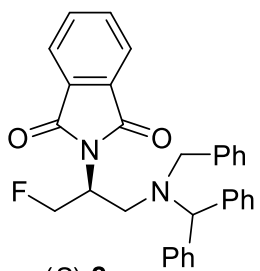

(S)-2sa $\mathrm{Ph}$

(S)-2sa was prepared from 1sa (1 equiv., $1 \mathrm{mmol}, 608.6 \mathrm{mg}$ ), according to the general procedure using $5 \mathrm{~mol} \%$ of catalyst $(S)-\mathbf{D}(0.05 \mathrm{mmol}, 41.8 \mathrm{mg})$ and stirring for $36 \mathrm{~h}$. Purification: FCC eluent $=$ Pentane: $\mathrm{Et}_{2} \mathrm{O}$ (90:0 to 70:30, gradient). White solid, $440 \mathrm{mg}$, $92 \%$ yield, e.r. $=82.5: 17.5$. A single recrystallization in hexane/THF (reflux to r.t.) afforded (S)-2sa as a single enantiomer, e.r. > 99.5:0.5 $(320 \mathrm{mg}, 0.67 \mathrm{mmol}, 73 \%$ recrystallization yield). ${ }^{1} \mathbf{H}$ NMR $\left(400 \mathrm{MHz}, \mathrm{CDCl}_{3}\right) \delta$ ppm: $7.82-6.67(\mathrm{~m}, 4 \mathrm{H}), 7.39-7.07(\mathrm{~m}, 15 \mathrm{H}), 4.94$ (s, 1H), $4.80-4.55(\mathrm{~m}, 2 \mathrm{H}), 4.55-4.37(\mathrm{~m}, 1 \mathrm{H}), 3.79(\mathrm{~d}, J=13.5 \mathrm{~Hz}, 1 \mathrm{H}), 3.55(\mathrm{~d}, J=13.5 \mathrm{~Hz}, 1 \mathrm{H}), 3.22-$

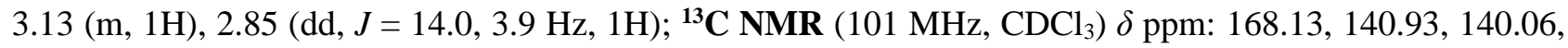
$138.86,134.00,132.13,129.39,129.21,129.08,128.55,128.40,128.28,127.46,127.21,127.16,123.38,81.01$ $(\mathrm{d}, J=174.1 \mathrm{~Hz}), 69.51,55.74,50.02(\mathrm{~d}, J=19.1 \mathrm{~Hz}), 48.27(\mathrm{~d}, J=5.9 \mathrm{~Hz}) ;{ }^{19} \mathbf{F} \mathbf{N M R}\left(376 \mathrm{MHz}, \mathrm{CDCl}_{3}\right) \delta$ ppm: -223.10 (td, $J=46.5,13.6 \mathrm{~Hz}, 1 \mathrm{~F})$. HRMS $\left(\mathrm{ESI}^{+}\right) \mathrm{m} / z \mathrm{C}_{31} \mathrm{H}_{28} \mathrm{FN}_{2} \mathrm{O}_{2}{ }^{+}[\mathrm{M}+\mathrm{H}]^{+}$: calculated 479.2129, found 479.2128. IR (thin layer film) $v\left(\mathrm{~cm}^{-1}\right)=3028,2843,1775,1711,1494,1467,1453,1387,1193,1056$, 1016, 875, 746, 721, 700. MP $48-50{ }^{\circ} \mathrm{C} .[\alpha]_{\mathbf{D}}{ }^{25}{ }^{\circ} \mathbf{C}=+19.6^{\circ}\left(\mathrm{c}=0.5, \mathrm{CHCl}_{3}\right.$, e.r. > 99.5:0.5). HPLC separation DAICEL CHIRALPAK ${ }^{\circledR} \mathrm{IC}-3$, Heptane: ${ }^{i} \mathrm{PrOH}=95: 5,1 \mathrm{~mL} / \mathrm{min} ; \mathrm{t}_{1}=4.67 \mathrm{~min}$ (major), $\mathrm{t}_{2}=5.56$ $\min$ (minor).

* N.B. The recrystallization affords the enantiopure product in the mother liquor. 
tert-butyl (R)-3-(benzhydryl(benzyl)amino)-2-(fluoromethyl)propanoate (2ta)

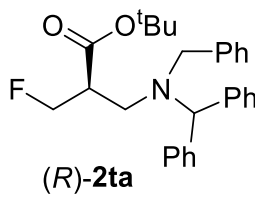

$(R)$-2ta was prepared from 1 ta (1 equiv., $0.5 \mathrm{mmol}, 281.8 \mathrm{mg}$ ), according to the general procedure using $10 \mathrm{~mol} \%$ of catalyst $(S)$-D $(0.05 \mathrm{mmol}, 41.8 \mathrm{mg})$ and stirring for $24 \mathrm{~h}$. Purification: FCC eluent $=$ Pentane:Et $2 \mathrm{O}$ (100:0 to 80:20, gradient). Colorless oil, $212 \mathrm{mg}$, $98 \%$ yield, e.r. $=92: 8$. After treatment with $2 \mathrm{M} \mathrm{HCl}$ in $\mathrm{Et}_{2} \mathrm{O}$ and removal of the volatiles in vacuo, $2 \mathbf{t a} \cdot \mathrm{HCl}$ was recrystallized in hexane/THF/EtOH (reflux to r.t.). After basification $\left(\mathrm{NaHCO}_{3 \text { sat. }}\right),(R)$ 2ta was obtained as a single enantiomer, e.r. $=99.5: 0.5(180 \mathrm{mg}, 0.42 \mathrm{mmol}, 85 \%$ recrystallization yield $) . *$ ${ }^{1}$ H NMR (400 MHz, $\left.\mathrm{CDCl}_{3}\right) \delta$ ppm: $7.42-7.24(\mathrm{~m}, 15 \mathrm{H}), 5.00(\mathrm{~s}, 1 \mathrm{H}), 4.52(\mathrm{~d}, J=6.1 \mathrm{~Hz}, 1 \mathrm{H}), 4.40(\mathrm{~d}, J=$ $6.1 \mathrm{~Hz}, 1 \mathrm{H}), 3.71(\mathrm{~d}, J=14.0 \mathrm{~Hz}, 1 \mathrm{H}), 3.60(\mathrm{~d}, J=14.0 \mathrm{~Hz}, 1 \mathrm{H}), 3.00-2.84(\mathrm{~m}, 1 \mathrm{H}), 2.81$ (ddd, $J=13.2,6.4$, $2.5 \mathrm{~Hz}, 1 \mathrm{H}), 2.61(\mathrm{dd}, J=13.3,7.7 \mathrm{~Hz}, 1 \mathrm{H}), 1.45(\mathrm{~s}, 9 \mathrm{H}) ;{ }^{13} \mathrm{C}$ NMR $\left(101 \mathrm{MHz}, \mathrm{CDCl}_{3}\right) \delta \mathrm{ppm}: 171.72(\mathrm{~d}$, $J=3.6 \mathrm{~Hz}), 140.72$, 140.10, 138.97, 129.42, 129.18, 129.06, 128.47, 128.40, 128.37, 127.36, 127.31, 127.26, $83.11(\mathrm{~d}, J=171.0 \mathrm{~Hz}), 81.31,69.23,55.09,48.35(\mathrm{~d}, J=8.4 \mathrm{~Hz}), 46.39(\mathrm{~d}, J=19.1 \mathrm{~Hz}), 28.18 ;{ }^{19} \mathbf{F} \mathbf{~ N M R}$ $\left(376 \mathrm{MHz}, \mathrm{CDCl}_{3}\right) \delta$ ppm: -222.42 (tdd, $J=46.8,18.1,2.5 \mathrm{~Hz}$ ). HRMS $\left(\mathrm{ESI}^{+}\right) \mathrm{m} / z \mathrm{C}_{28} \mathrm{H}_{33} \mathrm{FNO}_{2}{ }^{+}[\mathrm{M}+\mathrm{H}]^{+}$: calculated 434.2490, found 434.2487. IR (thin layer film) $v\left(\mathrm{~cm}^{-1}\right)=3027,2977,1728,1600,1493,1452$, $1392,1367,1250,1209,1154,1077,1028,1009,916,846,763,743,699,621,602 .[\alpha]_{\mathbf{D}}{ }^{2{ }^{\circ}} \mathbf{C}=-2.9^{\circ}(\mathrm{c}=1.1$, $\mathrm{CHCl}_{3}$, e.r. $\left.=99.5: 0.5\right)$. HPLC separation DAICEL CHIRALPAK ${ }^{\circledR}$ IB-3, Heptane: ${ }^{i} \mathrm{PrOH}=99.6: 0.4,1$ $\mathrm{mL} / \mathrm{min} ; \mathrm{t}_{1}=4.63 \min$ (minor), $\mathrm{t}_{2}=4.97 \min$ (major).

* N.B. The recrystallization affords the enantiopure product in the mother liquor.

\section{$N$-benzhydryl- $N$-benzyl-2-(benzyloxy)-3-fluoro-2-methylpropan-1-amine (2ua)}

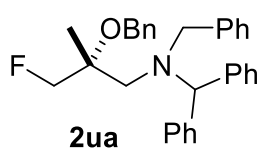

Absolute configuration not assigned

$(R)$-2ua was prepared from 1 ua (1 equiv., $0.1 \mathrm{mmol}, 58.3 \mathrm{mg}$ ), according to the general procedure using $20 \mathrm{~mol} \%$ of catalyst $(S)$-D $(0.02 \mathrm{mmol}, 16.8 \mathrm{mg})$ and stirring for $72 \mathrm{~h}$. Purification: FCC eluent $=$ Heptane: $\mathrm{Et}_{2} \mathrm{O}$ (100:0 to 95:5, gradient). Colorless oil, $40.0 \mathrm{mg}, 88 \%$ yield, e.r. $=87: 13 .{ }^{1} \mathbf{H}$ NMR $\left(400 \mathrm{MHz}, \mathrm{CDCl}_{3}\right) \delta$ ppm: $7.39-7.24(\mathrm{~m}$, 20H), $5.40(\mathrm{~s}, 1 \mathrm{H}), 4.51(\mathrm{~s}, 2 \mathrm{H}), 4.48(\mathrm{dd}, J=48.4,10.0 \mathrm{~Hz}, 1 \mathrm{H}), 4.25(\mathrm{dd}, J=47.9,10.0 \mathrm{~Hz}, 1 \mathrm{H}), 3.81(\mathrm{~d}, J$ $=13.6 \mathrm{~Hz}, 1 \mathrm{H}), 3.65(\mathrm{~d}, J=13.6 \mathrm{~Hz}, 1 \mathrm{H}), 2.86(\mathrm{dd}, J=14.3,2.5 \mathrm{~Hz}, 1 \mathrm{H}), 2.75(\mathrm{~d}, J=14.2 \mathrm{~Hz}, 1 \mathrm{H}), 1.17(\mathrm{~d}$, $J=2.3 \mathrm{~Hz}, 3 \mathrm{H}) ;{ }^{13} \mathbf{C} \mathbf{~ N M R}\left(101 \mathrm{MHz}, \mathrm{CDCl}_{3}\right.$ ) [overlapping signals] $\delta$ ppm: 140.72, 140.03, 140.00, 139.38, $130.13,129.82,129.36,128.43,128.38,128.15,128.14,127.41,127.34,127.12,127.06,87.69(\mathrm{~d}, J=$ $175.1 \mathrm{~Hz}), 78.80(\mathrm{~d}, J=16.1 \mathrm{~Hz}), 68.39,65.26(\mathrm{~d}, J=2.4 \mathrm{~Hz}), 56.06,54.96(\mathrm{~d}, J=5.2 \mathrm{~Hz}), 18.19(\mathrm{~d}, J=5.3$ $\mathrm{Hz}) ;{ }^{19} \mathbf{F}$ NMR $\left(376 \mathrm{MHz}, \mathrm{CDCl}_{3}\right) \delta$ ppm: $-226.99(\mathrm{t}, J=48.1 \mathrm{~Hz}, 1 \mathrm{~F})$. HRMS $\left(\mathrm{ESI}^{+}\right) m / z \mathrm{C}_{31} \mathrm{H}_{33} \mathrm{FNO}^{+}[\mathrm{M}+\mathrm{H}]^{+}$: calculated 454.2541, found 454.2540. IR (thin layer film) $v\left(\mathrm{~cm}^{-1}\right)=3028,1494,1453,1371,1093,1067$, 1028. $763,740,699,535 .[\alpha]_{\mathbf{D}}{ }^{25}{ }^{\circ} \mathrm{C}=+3.3^{\circ}\left(\mathrm{c}=0.6, \mathrm{CHCl}_{3}\right.$, e.r. $\left.=87: 13\right)$. SFC separation DAICEL CHIRALCEL ${ }^{\circledR}$ OJ-3, $\mathrm{CO}_{2}: \mathrm{MeOH}(85: 15$ to $50: 50$, gradient $)+0.2 \%$ isopropylamine, $2.5 \mathrm{~mL} / \mathrm{min} ; \mathrm{t}_{1}=4.51$ $\min$ (minor), $\mathrm{t}_{2}=4.88 \min$ (major). 


\section{3-(benzhydryl(ethyl)amino)-2-(fluoromethyl)-2-methylpropanenitrile (2va)}

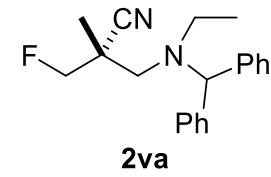

Absolute configuration

not assigned

2va was prepared from 1va (1 equiv., $0.1 \mathrm{mmol}, 44.0 \mathrm{mg}$ ), according to the general procedure using $10 \mathrm{~mol} \%$ of catalyst $(S)-\mathbf{D}(0.01 \mathrm{mmol}, 8.4 \mathrm{mg})$ and stirring for $24 \mathrm{~h}$. Purification: FCC eluent $=$ Heptane: $\mathrm{Et}_{2} \mathrm{O}$ (100:0 to 95:5, gradient). Colorless oil, $28.0 \mathrm{mg}, 90 \%$ yield, e.r. $=86: 14 .{ }^{1} \mathbf{H}$ NMR $\left(400 \mathrm{MHz}, \mathrm{CDCl}_{3}\right) \delta$ ppm: $7.36-7.22(\mathrm{~m}$, $10 \mathrm{H}), 5.15(\mathrm{~s}, 1 \mathrm{H}), 4.56-4.37(\mathrm{~m}, 1 \mathrm{H}), 4.44-4.26(\mathrm{~m}, 1 \mathrm{H}), 2.86(\mathrm{~s}, 2 \mathrm{H}), 2.72(\mathrm{q}, J=7.1 \mathrm{~Hz}, 2 \mathrm{H}), 1.34(\mathrm{~d}, J$ $=1.6 \mathrm{~Hz}, 3 \mathrm{H}), 1.05(\mathrm{t}, J=7.0 \mathrm{~Hz}, 3 \mathrm{H}) ;{ }^{13} \mathbf{C ~ N M R}\left(101 \mathrm{MHz}, \mathrm{CDCl}_{3}\right)$ [overlapping signals] $\delta \mathrm{ppm}: 140.87$, 140.67, 129.31, 129.24, 128.45, 127.41, 127.38, 122.26 (d, $J=6.5 \mathrm{~Hz}), 85.03$ (d, $J=181.3 \mathrm{~Hz}), 70.48,53.98$ $(\mathrm{d}, J=3.6 \mathrm{~Hz}), 45.35,39.09(\mathrm{~d}, J=19.6 \mathrm{~Hz}), 19.94(\mathrm{~d}, J=4.0 \mathrm{~Hz}), 12.34 ;{ }^{19} \mathbf{F} \mathbf{N M R}\left(376 \mathrm{MHz}, \mathrm{CDCl}_{3}\right) \delta$ ppm: -223.20 (t, $J=47.1 \mathrm{~Hz}$ ). ). HRMS (ESI $\left.{ }^{+}\right) m / z \mathrm{C}_{25} \mathrm{H}_{23} \mathrm{FN}_{2} \mathrm{Na}^{+}[\mathrm{M}+\mathrm{Na}]^{+}$: calculated 333.1737, found 333.1738. IR (thin layer film) $v\left(\mathrm{~cm}^{-1}\right)=3328,2973,2883,1485,1452,1420,1380,1088,1046,880,605$. $[\boldsymbol{\alpha}]_{\mathbf{D}}{ }^{25}{ }^{\circ} \mathrm{C}=-242.6^{\circ}\left(\mathrm{c}=0.3, \mathrm{CHCl}_{3}\right.$, e.r. $\left.=99.5: 0.5\right)$. HPLC separation DAICEL CHIRALCEL ${ }^{\circledR}$ OJ-3, Heptane: $\mathrm{EtOH}=95: 5,1 \mathrm{~mL} / \mathrm{min} ; \mathrm{t}_{1}=6.88 \mathrm{~min}$ (major), $\mathrm{t}_{2}=7.79 \mathrm{~min}$ (minor).

\section{$N$-benzhydryl- $N$-benzyl-2,3-difluoro-2-phenylpropan-1-amine (2wa)}

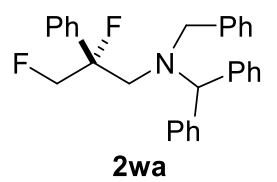

Absolute configuration not assigned

2wa was prepared from 1wa (1 equiv., $0.75 \mathrm{mmol}, 418.2 \mathrm{mg}$ ), according to the general procedure using $5 \mathrm{~mol} \%$ of catalyst $(S)-\mathbf{D}(0.05 \mathrm{mmol}, 31.4 \mathrm{mg})$ and stirring for $24 \mathrm{~h}$. Purification: FCC eluent $=$ Pentane: $\mathrm{Et}_{2} \mathrm{O}$ (100:0 to 90:10, gradient). Dense oil, $280 \mathrm{mg}$,

was treated with $2 \mathrm{M} \mathrm{HCl}$ in $\mathrm{Et}_{2} \mathrm{O}$ and volatiles were removed in vacuo. $2 \mathbf{w a} \cdot \mathrm{HCl}$ was recrystallized in $\mathrm{EtOH} / \mathrm{H}_{2} \mathrm{O}$ (reflux to r.t.). After basification $\left(\mathrm{NaHCO}_{3}\right.$ sat.), 2wa was obtained with an e.r. = 95:5 (92 mg, 0.21 mmol, $50 \%$ recrystallization yield). ${ }^{1} \mathbf{H}$ NMR (400 MHz, $\left.\mathrm{CDCl}_{3}\right) \delta$ ppm: $7.32-6.94(\mathrm{~m}, 20 \mathrm{H}), 5.10(\mathrm{~s}, 1 \mathrm{H})$, 4.70 (ddd, $J=48.9,17.6,10.6 \mathrm{~Hz}, 1 \mathrm{H}), 4.29$ (ddd, $J=46.9,26.8,10.6 \mathrm{~Hz}, 1 \mathrm{H}), 3.67$ (d, $J=13.5 \mathrm{~Hz}, 1 \mathrm{H}), 3.47$ $(\mathrm{d}, J=13.5 \mathrm{~Hz}, 1 \mathrm{H}), 3.08-2.91(\mathrm{~m}, 2 \mathrm{H}) ;{ }^{13} \mathbf{C} \mathbf{N M R}\left(101 \mathrm{MHz}, \mathrm{CDCl}_{3}\right)$ [overlapping signals] $\delta$ ppm: 140.03, $139.48,138.95,138.46$ (dd, $J=25.6,3.8 \mathrm{~Hz}), 129.86,129.77,129.62,128.44,128.39$ (d, $J=1.3 \mathrm{~Hz}), 128.36$, 128.24, 128.04, 127.35, 127.27, $125.11(\mathrm{~d}, J=9.9 \mathrm{~Hz}) ; 100.09$ (dd, $J=178.2,15.9 \mathrm{~Hz}), 85.98(\mathrm{dd}, J=178.3$, $27.8 \mathrm{~Hz}), 68.42(\mathrm{~d}, J=3.6 \mathrm{~Hz}) ; 55.45(\mathrm{~d}, J=3.3 \mathrm{~Hz}) ; 54.91(\mathrm{dd}, J=21.0,4.0 \mathrm{~Hz}) ;{ }^{19} \mathbf{F} \mathbf{N M R}(376 \mathrm{MHz}$, $\left.\mathrm{CDCl}_{3}\right) \delta$ ppm: $-164.20(\mathrm{qt}, J=24.8,15.6 \mathrm{~Hz}, 1 \mathrm{~F}),-230.90(\mathrm{td}, J=47.9,14.0 \mathrm{~Hz}, 1 \mathrm{~F}) . \mathbf{H R M S}\left(\mathrm{ESI}^{+}\right) \mathrm{m} / z$ $\mathrm{C}_{29} \mathrm{H}_{28} \mathrm{~F}_{2} \mathrm{~N}^{+}[\mathrm{M}+\mathrm{H}]^{+}:$calculated 428.2184 , found 428.2185. IR (thin layer film) $v\left(\mathrm{~cm}^{-1}\right)=3028,1794,1453$, $1371,1093,1067,1028,[\alpha]_{\mathrm{D}}{ }^{25}{ }^{\circ} \mathrm{C}=+30.9^{\circ}\left(\mathrm{c}=0.7, \mathrm{CHCl}_{3}\right.$, e.r. $\left.=95: 5\right)$. HPLC separation DAICEL CHIRALPAK ${ }^{\circledR}$ AS-3, Heptane: ${ }^{i} \mathrm{PrOH}=98: 2+0.2 \% \mathrm{BuNH}_{2}, 1 \mathrm{~mL} / \mathrm{min} ; \mathrm{t}_{1}=2.95 \mathrm{~min}$ (minor), $\mathrm{t}_{2}=3.35 \mathrm{~min}$ (major).

\section{(S)-3-fluoro-2-phenylpropan-1-aminium chloride (3aa·HCl)}<smiles>C[C@@H]([Pb])CF</smiles>

$\mathrm{Cl}^{-}$

$(S)-3 \mathbf{a a} \cdot \mathbf{H C l}$ In a Schlenk flask, $(S)$-2aa (1 equiv., $1.70 \mathrm{mmol}, 696 \mathrm{mg}$, e.r = 96:4) was dissolved in a 4:1 mixture of $\mathrm{MeOH}: \mathrm{CH}_{2} \mathrm{Cl}_{2}(0.08 \mathrm{M}, 21 \mathrm{~mL})$ and $\mathrm{Pd}(\mathrm{OH})_{2} 20 \mathrm{wt} \%$ loading (0.1 equiv., 0.17 mmol, $134 \mathrm{mg}$ ) was added. The flask was then purged with $\mathrm{H}_{2}$ three times and the reaction 
was stirred overnight at r.t. under an atmosphere of $\mathrm{H}_{2}$ (balloon). The mixture was filtered on celite, the solvents were evaporated in vacuo and the free amine was stirred in a $1 \mathrm{M} \mathrm{HCl}$ solution in methanol for $5 \mathrm{~min}$. After evaporation of the volatiles, the corresponding chloride salt was obtained as a pale yellow solid in $79 \%$ yield $(1.34 \mathrm{mmol}, 254 \mathrm{mg})$ and e.r. $=96: 4$. A successive recrystallization on $1.05 \mathrm{mmol}$ scale $(200 \mathrm{mg})$ in hexane/EtOH (reflux to $-20^{\circ} \mathrm{C}$ ) afforded $(S)$-3aa as a white solid with an e.r.= 99:1 (140 mg, $0.73 \mathrm{mmol}, 70 \%$ recrystallization yield). ${ }^{1} \mathbf{H}$ NMR $\left(400 \mathrm{MHz}, \mathrm{CD}_{3} \mathrm{OD}\right) \delta \mathrm{ppm:} 7.34-7.21(\mathrm{~m}, 5 \mathrm{H}), 4.76$ (s br, 3H), 4.54 (dd, $J=47.1,5.9 \mathrm{~Hz}, 2 \mathrm{H}), 3.40-3.18(\mathrm{~m}, 3 \mathrm{H}) ;{ }^{13} \mathbf{C} \mathbf{N M R}\left(101 \mathrm{MHz}, \mathrm{CD}_{3} \mathrm{OD}\right) \delta \mathrm{ppm}: 137.27(\mathrm{~d}, J=6.5 \mathrm{~Hz})$, 130.35, 129.42, 129.36, $86.02(\mathrm{~d}, J=172.8 \mathrm{~Hz}), 45.91(\mathrm{~d}, J=19.0 \mathrm{~Hz}), 42.33$ (d, $J=4.6 \mathrm{~Hz}) ;{ }^{19} \mathbf{F}$ NMR (376 MHz, $\left.\mathrm{CD}_{3} \mathrm{OD}\right) \delta \mathrm{ppm:}-221.28(\mathrm{td}, J=47.1,17.9 \mathrm{~Hz}, 1 \mathrm{~F})$. HRMS $\left(\mathrm{ESI}^{+}\right) \mathrm{m} / z$ : calculated for $\mathrm{C}_{9} \mathrm{H}_{13} \mathrm{FN}^{+}$ $[\mathrm{M}-\mathrm{Cl}]^{+}$154.1027, found 154.1029. IR (thin layer film) $v\left(\mathrm{~cm}^{-1}\right)=3032,2917,1626,1518,1496,1455,1397$, 1157, 1082, 934, 761, 702. MP $152-151{ }^{\circ} \mathrm{C} .[\alpha]_{\mathbf{D}}{ }^{25}{ }^{\circ} \mathbf{C}=-41.0^{\circ}(\mathrm{c}=0.6, \mathrm{MeOH}$, e.r. $=99: 1)$. HPLC separation (free amine) DAICEL CHIRALPAK ${ }^{\circledR} \mathrm{IF}-3$, Heptane $:{ }^{i} \mathrm{PrOH}=90: 10+0.2 \%$ diethylamine, $1 \mathrm{~mL} / \mathrm{min} ; \mathrm{t}_{1}=7.58 \min$ (major), $\mathrm{t}_{2}=12.33 \min$ (minor).

\section{(R)-N-ethyl-3-fluoro-2-phenylpropan-1-aminium chloride (3ab·HCl)}

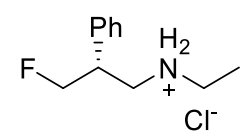

$(R)-3 \mathrm{ab} \cdot \mathrm{HCl}$

In a Schlenk flask, $(R)$-2ab (1 equiv., $1.73 \mathrm{mmol}, 601 \mathrm{mg}$, e.r. $=96: 4)$ was dissolved in a 2:1 mixture of $\mathrm{MeOH}: \mathrm{CH}_{2} \mathrm{Cl}_{2}(0.15 \mathrm{M}, 12 \mathrm{~mL})$ and $\mathrm{Pd} / \mathrm{C} 10 \mathrm{wt} \%$ loading (0.1 equiv., 0.173 mmol, $184 \mathrm{mg}$ ) was added. The flask was then purged with $\mathrm{H}_{2}$ three times and the reaction was stirred overnight at r.t. under an atmosphere of $\mathrm{H}_{2}$ (balloon). After filtration on celite and solvent evaporation in vacuo, the crude mixture was purified by FCC (eluent = Pentane: $\mathrm{Et}_{2} \mathrm{O}: \mathrm{MeOH}$, 93:5:2 to 90:5:5 gradient). The purified amine was then stirred for $5 \mathrm{~min}$ in $1 \mathrm{M} \mathrm{HCl}$ solution in methanol to afford the corresponding chloride salt as a colorless solid in 74\% yield $(1.28 \mathrm{mmol}, 279 \mathrm{mg})$ and e.r. $=96: 4$. A successive recrystallization on $0.23 \mathrm{mmol}$ scale $\left(50 \mathrm{mg}\right.$ ) in $\mathrm{CH}_{2} \mathrm{Cl}_{2} / \mathrm{Et}_{2} \mathrm{O}$ (reflux to r.t.) afforded $(R)-3 \mathbf{a b}$ with an e.r. $=98.5: 1.5\left(43.5 \mathrm{mg}, 0.20 \mathrm{mmol}, 87 \%\right.$ recrystallization yield). ${ }^{1} \mathbf{H} \mathbf{~ N M R}\left(500 \mathrm{MHz}, \mathrm{CDCl}_{3}\right) \delta$ ppm: $9.61(\mathrm{~s} \mathrm{br}, 2 \mathrm{H}), 7.40-7.28(\mathrm{~m}, 5 \mathrm{H}), 4.91-4.65(\mathrm{~m}, 2 \mathrm{H}), 3.86-3.85(\mathrm{~m}, 1 \mathrm{H}), 3.53(\mathrm{dd}, J=13.0,8.0 \mathrm{~Hz}, 1 \mathrm{H})$, $3.53(\mathrm{dd}, J=13.0,6.7 \mathrm{~Hz}, 1 \mathrm{H}), 3.00-2.91(\mathrm{~m}, 2 \mathrm{H}), 1.14(\mathrm{t}, J=7.3 \mathrm{~Hz}, 3 \mathrm{H}) ;{ }^{13} \mathbf{C} \mathbf{~ N M R}\left(126 \mathrm{MHz}, \mathrm{CDCl}_{3}\right) \delta$ ppm: 137.07 (d, $J=4.5 \mathrm{~Hz}), 129.39,128.40,128.18,84.71$ (d, $J=175.0 \mathrm{~Hz}), 48.58$ (d, $J=5.1 \mathrm{~Hz}), 43.79$, $43.22(\mathrm{~d}, J=18.7 \mathrm{~Hz}), 11.21 ;{ }^{19} \mathbf{F}$ NMR $\left(470 \mathrm{MHz}, \mathrm{CDCl}_{3}\right) \delta \mathrm{ppm}:-220.84(\mathrm{td}, J=47.2,22.0 \mathrm{~Hz}, 1 \mathrm{~F})$. HRMS $\left(\mathrm{ESI}^{+}\right) \mathrm{m} / \mathrm{z}$ : calculated for $\mathrm{C}_{11} \mathrm{H}_{17} \mathrm{FN}^{+}[\mathrm{M}-\mathrm{Cl}]^{+} 182.1340$, found 182.1340. IR (thin layer film) $v\left(\mathrm{~cm}^{-1}\right)=2962$, 2774, 2737, 2515, 2433, 2396, 2360, 2342, 1597, 1496, 1457, 1405, 1386, 1133, 1066, 1017, 986, 965, 889, 805, 762, 704, 668. MP $178-181^{\circ} \mathrm{C} .[\alpha]_{\mathrm{D}}{ }^{25}{ }^{\circ} \mathrm{C}=+49.2^{\circ}(\mathrm{c}=0.5$, MeOH, e.r. $=98.5: 1.5)$. HPLC separation (free amine) DAICEL CHIRALPAK ${ }^{\circledR}$ IF-3, Heptane: ${ }^{i} \operatorname{PrOH}=97: 3+0.1 \%$ diethylamine, $1 \mathrm{~mL} / \mathrm{min} ; \mathrm{t}_{1}=3.98$ $\min$ (minor), $\mathrm{t}_{2}=4.69 \min$ (minor). 


\section{(R)-2-(3-chlorophenyl)-3-fluoropropan-1-amine}

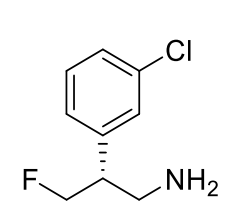

$4 a$

Adapted from a literature procedure. ${ }^{11 \mathrm{~b}}$ In a round bottom flask, a solution of sodium bromate (3 equiv., $4.257 \mathrm{mmol}, 642 \mathrm{mg}$ ) in water $(14.2 \mathrm{~mL})$ was added to a pre-stirred solution of $(R)$ 2da (1 equiv., $1.419 \mathrm{mmol}, 630 \mathrm{mg})$ in EtOAc $(19.0 \mathrm{~mL})$ and stirred at room temperature for $10 \mathrm{~min}$. A solution of sodium dithionite (2.5 equiv., $3.55 \mathrm{mmol}, 618 \mathrm{mg}$ ) in water $(25.8 \mathrm{~mL})$ was then added. The reaction was stirred at r.t. for $2 \mathrm{~h}$. The organic solvent was evaporated in vacuo (bath temperature below $30^{\circ} \mathrm{C}$ ) and the water solution was freeze-dried overnight. The crude mixture was basified using ammonia in $\mathrm{MeOH} 7 \mathrm{M}$, diluted in $\mathrm{CH}_{2} \mathrm{Cl}_{2}$, filtered and purified by flash column chromatography (eluent $\mathrm{CH}_{2} \mathrm{Cl}_{2}:\left[\mathrm{CH}_{2} \mathrm{Cl}_{2} / \mathrm{NH}_{3}\right.$ in MeOH $\left.9 / 1\right]=100: 0$ to $70: 30$, gradient) to afford $4 \mathbf{a}$ as a colorless oil in $72 \%$ yield $(1.02 \mathrm{mmol}, 191.0 \mathrm{mg})$ and with an e.r. $=96: 4$ e.r. as colorless oil. ${ }^{1} \mathbf{H} \mathbf{~ N M R}\left(400 \mathrm{MHz}, \mathrm{CDCl}_{3}\right) \delta \mathrm{ppm:} 7.32$ $-7.21(\mathrm{~m}, 3 \mathrm{H}), 7.13(\mathrm{dt}, J=6.7,1.9 \mathrm{~Hz}, 1 \mathrm{H}), 4.67(\mathrm{qd}, J=9.4,5.3 \mathrm{~Hz}, 1 \mathrm{H}), 4.56(\mathrm{qd}, J=9.2,5.7 \mathrm{~Hz}, 1 \mathrm{H})$, $3.19-3.09(\mathrm{~m}, 1 \mathrm{H}), 3.08-2.91(\mathrm{~m}, 2 \mathrm{H}), 1.25(\mathrm{~s}, 2 \mathrm{H}) ;{ }^{13} \mathbf{C ~ N M R}\left(101 \mathrm{MHz}, \mathrm{CDCl}_{3}\right) \delta \mathrm{ppm}: 141.56(\mathrm{~d}, J=$ $5.4 \mathrm{~Hz}), 134.76,130.16,128.45,127.63,126.55,85.01(\mathrm{~d}, J=172.2 \mathrm{~Hz}), 49.72(\mathrm{~d}, J=18.7 \mathrm{~Hz}), 43.70(\mathrm{~d}, J=$ $5.4 \mathrm{~Hz}) ;{ }^{19} \mathbf{F}$ NMR $\left(376 \mathrm{MHz}, \mathrm{CDCl}_{3}\right) \delta$ ppm: $-222.20(\mathrm{td}, J=47.2,21.1 \mathrm{~Hz}, 1 \mathrm{~F})$. HRMS $\left(\mathrm{ESI}^{+}\right) \mathrm{m} / z$ calculated for $\mathrm{C}_{9} \mathrm{H}_{12} \mathrm{ClFNO}^{+}[\mathrm{M}+\mathrm{H}]^{+}:$188.0637, found 188.0638. $[\alpha]_{\mathbf{D}}{ }^{20}{ }^{\circ} \mathrm{C}=+12.7(\mathrm{c}=0.05$, DMF, e.r. $=96: 4)$. SFC separation: DAICEL CHIRALPAK ${ }^{\circledR}$ ID-3, $40{ }^{\circ} \mathrm{C}, \mathrm{CO}_{2}: \mathrm{EtOH}(85: 15$ to $50: 50$, gradient $)+0.2 \%$ isopropylamine, $\mathrm{t}_{1}=1.48 \min$ (minor), $\mathrm{t}_{2}=1.95 \min$ (major).

\section{(R)-2-(3-chlorophenyl)-N-(2,2-dimethoxyethyl)-3-fluoropropan-1-amine}

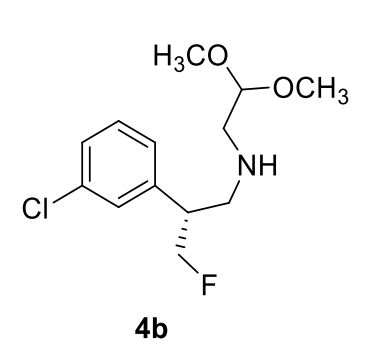

A round bottom flask was charged with $\mathbf{4 a}(0.74 \mathrm{mmol}, 139.0 \mathrm{mg})$ and a solution of dimethoxyacetaldehyde (60 wt\% in water, 1.2 equiv., $0.89 \mathrm{mmol}, 154.2 \mathrm{mg}$ ) in $0.45 \mathrm{~mL}$ of $\mathrm{MeOH}$ was added. The reaction mixture was stirred at r.t. for $12 \mathrm{~h}$. After that time, the mixture was diluted with additional $4.0 \mathrm{~mL}$ of $\mathrm{MeOH}$ and $\mathrm{NaBH}_{3} \mathrm{CN}$ (2 equiv., $1.48 \mathrm{mmol}, 93.1 \mathrm{mg}$ ) was added portionwise. The reaction mixture was stirred at r.t. for additional $12 \mathrm{~h}$. The solvent was then evaporated (bath temperature below $30^{\circ} \mathrm{C}$ ) and the $\mathrm{pH}$ adjusted to $8-9$. The crude mixture was then purified by flash column chromatography (eluent $\mathrm{CH}_{2} \mathrm{Cl}_{2}:\left[\mathrm{CH}_{2} \mathrm{Cl}_{2} / \mathrm{NH}_{3}\right.$ in MeOH $\left.9 / 1\right]=100: 0$ to $70: 30$, gradient) to afford $\mathbf{4 b}$ as a yellow oil in $81 \%$ yield $(0.60 \mathrm{mmol}, 165.9 \mathrm{mg})$ and with an e.r. $=96: 4 .{ }^{1} \mathbf{H}$ NMR $\left(400 \mathrm{MHz}, \mathrm{CDCl}_{3}\right) \delta$ ppm: $7.29-7.22(\mathrm{~m}, 3 \mathrm{H}), 7.13$ $(\mathrm{dt}, J=6.5,2.1 \mathrm{~Hz}, 1 \mathrm{H}), 4.66(\mathrm{qd}, J=9.1,5.7 \mathrm{~Hz}, 1 \mathrm{H}), 4.54(\mathrm{qd}, J=9.1,5.6 \mathrm{~Hz}, 1 \mathrm{H}), 4.41(\mathrm{t}, J=5.4 \mathrm{~Hz}, 1 \mathrm{H})$, $3.34(\mathrm{~s}, 6 \mathrm{H}), 3.21-2.88(\mathrm{~m}, 3 \mathrm{H}), 2.79-2.66(\mathrm{~m}, 2 \mathrm{H}), 1.47(\mathrm{~s}, 1 \mathrm{H}) ;{ }^{13} \mathbf{C} \mathbf{N M R}\left(101 \mathrm{MHz}, \mathrm{CDCl}_{3}\right) \delta$ ppm: $141.75(\mathrm{~d}, J=5.0 \mathrm{~Hz}), 134.73,130.14,128.35,127.62,126.47,103.83,85.30$ (d, $J=172.6 \mathrm{~Hz}), 54.11(\mathrm{~d}, J=$ $3.6 \mathrm{~Hz}), 51.31,51.11(\mathrm{~d}, J=5.4 \mathrm{~Hz}), 46.68(\mathrm{~d}, J=18.7 \mathrm{~Hz}) ;{ }^{19} \mathbf{F} \mathbf{N M R}\left(376 \mathrm{MHz}, \mathrm{CDCl}_{3}\right) \delta \mathrm{ppm}:-222.02(\mathrm{td}$, $J=47.4,20.6 \mathrm{~Hz}, 1 \mathrm{~F})$. HRMS $\left(\mathrm{ESI}^{+}\right) \mathrm{m} / z$ calculated for $\mathrm{C}_{13} \mathrm{H}_{20} \mathrm{ClFNO}_{2}{ }^{+}[\mathrm{M}+\mathrm{H}]^{+}:$276.1161, found 276.1161. $[\alpha]_{\mathrm{D}}{ }^{20} \mathrm{C}=+11.78(\mathrm{c}=0.14$, DMF, e.r. $=96: 4)$. SFC separation DAICEL CHIRALPAK ${ }^{\circledR}$ IG, $40^{\circ} \mathrm{C}$, $\mathrm{CO}_{2}: \mathrm{MeOH}(85: 15$ to $50: 50$, gradient $)+0.2 \%$ isopropylamine, $\mathrm{t}_{1}=1.32 \mathrm{~min}$ (minor), $\mathrm{t}_{2}=1.42$ min (major). 


\section{(R)-8-chloro-1-(fluoromethyl)-2,3,4,5-tetrahydro-1H-benzo[d]azepine (Fluorinated Lorcaserin)}

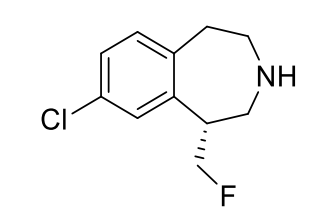

Fluorinated Lorcaserin

In a round bottom flask, $4 \mathbf{b}$ ( 1 equiv., $0.44 \mathrm{mmol}, 120.3 \mathrm{mg}$ ) was dissolved in 1.85 $\mathrm{mL}$ of $\mathrm{CH}_{2} \mathrm{Cl}_{2}$ and trifluoromethanesulfonic acid (5 equiv., $2.18 \mathrm{mmol}, 0.19 \mathrm{~mL}$ ) was added dropwise at $0{ }^{\circ} \mathrm{C}$. The reaction was stirred at r.t. for $30 \mathrm{~min}$. The reaction mixture was then basified with $\mathrm{NaHCO}_{3}$ sat. $(30 \mathrm{~mL})$ and extracted in $\mathrm{CH}_{2} \mathrm{Cl}_{2}(3 \times 15$ $\mathrm{mL}$ ). The organic phase was evaporated, and the crude mixture was dissolved in THF $(21.5 \mathrm{~mL})$. Sodium phosphate monobasic $(0.5 \mathrm{M}$ water solution, $2.15 \mathrm{~mL})$ was added, followed by portionwise addition of sodium cyanoborohydride (9.9 equiv., $4.35 \mathrm{mmol}, 273.2 \mathrm{mg}$ ). The mixture was stirred for 4 hours at r.t. after which the volatiles were evaporated in vacuo (bath temperature below $30^{\circ} \mathrm{C}$ ). After addition of $\mathrm{NaHCO}_{3}$ sat. $(30 \mathrm{~mL})$ the crude product was extracted with $\mathrm{CH}_{2} \mathrm{Cl}_{2}(3 \times 15 \mathrm{~mL})$ and the solvent was evaporated in vacuo. The crude mixture was then purified by flash column chromatography (eluent $\mathrm{CH}_{2} \mathrm{Cl}_{2}:\left[\mathrm{CH}_{2} \mathrm{Cl}_{2} / \mathrm{NH}_{3}\right.$ in MeOH $\left.9 / 1\right]=100: 0$ to $70: 30$, gradient) to afford fluorinated Lorcaserin in $86 \%$ yield $(0.37$ mmol, $80.2 \mathrm{mg})$ and an e.r. $=96: 4 .{ }^{1} \mathbf{H}$ NMR $\left(400 \mathrm{MHz}, \mathrm{CDCl}_{3}\right) \delta$ ppm: $7.15-6.99(\mathrm{~m}, 3 \mathrm{H}), 4.88(\mathrm{ddd}, J=$ $46.9,9.1,7.5 \mathrm{~Hz}, 1 \mathrm{H}), 4.69$ (ddd, $J=46.9,9.1,6.0 \mathrm{~Hz}, 1 \mathrm{H}), 3.27-3.10$ (m, 2H), $3.10-2.90$ (m, 3H), $2.90-$ $2.78(\mathrm{~m}, 2 \mathrm{H}), 1.81(\mathrm{~s}, 1 \mathrm{H}) ;{ }^{13} \mathrm{C}$ NMR $\left(101 \mathrm{MHz}, \mathrm{CDCl}_{3}\right) \delta \mathrm{ppm}: 142.08(\mathrm{~d}, J=6.2 \mathrm{~Hz}), 140.10,132.08$, 131.84, 129.19, 126.90, 83.39 (d, $J=170.8 \mathrm{~Hz}), 49.53$ (d, $J=5.2 \mathrm{~Hz}), 48.99$ (d, $J=18.9 \mathrm{~Hz}), 48.67,39.21$; ${ }^{19} \mathbf{F}$ NMR $\left(376 \mathrm{MHz}, \mathrm{CDCl}_{3}\right) \delta$ ppm: $-220.86(\mathrm{td}, J=46.6,17.0 \mathrm{~Hz})$. HRMS $\left(\mathrm{ESI}^{+}\right) \mathrm{m} / z$ calculated for $\mathrm{C}_{11} \mathrm{H}_{14} \mathrm{ClFN}^{+}[\mathrm{M}+\mathrm{H}]^{+}: 214.0793$, found 214.0795. $[\alpha]_{\mathrm{D}}{ }^{20} \mathrm{C}=+1.4(\mathrm{c}=0.14$, DMF, e.r. = 96:4). SFC separation DAICEL CHIRALPAK ${ }^{\circledR} \mathrm{IG}, 40{ }^{\circ} \mathrm{C}, \mathrm{CO}_{2}: \mathrm{MeOH}(85: 15$ to $50: 50$, gradient $)+0.2 \%$ isopropylamine, $\mathrm{t}_{1}=2.46$ $\min$ (major), $\mathrm{t}_{2}=3.78 \mathrm{~min}$ (minor). 


\section{$\underline{\text { Additional Unsuccessful Substrates }}$}<smiles>CCC(CF)CN(Cc1ccccc1)C(=O)c1ccccc1</smiles>

$85 \%$ yield

$54: 46$ e.r.

$$
\underbrace{\mathrm{MnO}}_{\text {Bzh }}
$$

$20 \%$ yield 90:10 e.r.<smiles>CN(C[C@](C)(CF)c1ccccc1)[13C](=O)c1ccccc1</smiles>

$80 \%$ yield $55: 45$ e.r.<smiles>C=CC(CF)CN(Cc1ccccc1)C(=O)c1ccccc1</smiles>

$90 \%$ yield 63:37 e.r.

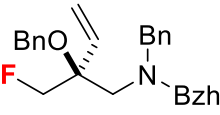

$40 \%$ yield 73:27 e.r.<smiles>CCC(=O)N(CC)CC(CF)C(=O)OC</smiles>

$99 \%$ yield 75:25 e.r.<smiles>FCC(CO[Ga])CN(Cc1ccccc1)Cc1ccccc1</smiles>

$69 \%$ yield $67: 33$ e.r.<smiles>C=CC(CF)(CBr)CN(CC)CCCC</smiles>

$13 \%$ yield 80:20 e.r.

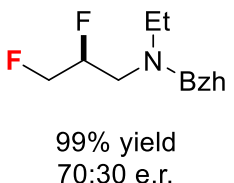<smiles>FCC(Cc1ccccc1)CN(Cc1ccccc1)Cc1ccccc1</smiles>
$30 \%$ yield $60: 40$ e.r.<smiles>C=CC[C@](C)(CF)CN(C)[13CH3]</smiles>
$75 \%$ yield 69:31 e.r.<smiles>CCN(Cc1ccccc1)CC(CF)c1n[nH]c2ccccc12</smiles>

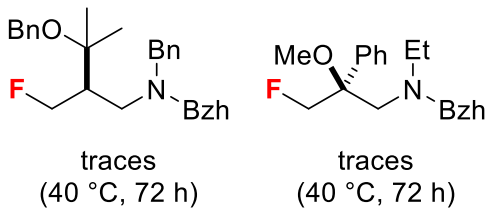

$\underbrace{\mathrm{CN}}_{\text {Bzh }}$

$84 \%$ yield $76: 24$ e.r.

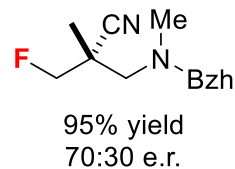

$70: 30$ e.r.

Scheme S7: Additional substrates tested under optimized conditions: 10 mol\% (S)-D, 2 equiv. CsF, 1,2-DCE $(0.25 \mathrm{M})$, r.t., 24-72 h. Absolute configuration not assigned. 


\section{Determination of the Absolute Configuration (Single Crystal X-ray Diffraction Studies)}

Low temperature ${ }^{12}$ single crystal X-ray diffraction data were collected using a Rigaku Oxford Diffraction SuperNova diffractometer. Raw frame data were reduced using CrysAlisPro and the structures were solved using 'Superflip ${ }^{\text {'13 }}$ before refinement with CRYSTALS ${ }^{14}$ as per the CIF. Full refinement details are given in

the Supporting Information (CIF); Crystallographic data have been deposited with the Cambridge Crystallographic Data Centre (CCDC 1973306-07) and can be obtained via www. ccdc.cam.ac.uk/data_request/cif.

Data for $(S)-\mathbf{2 m a} \cdot \mathrm{HCl}$ were collected at $150 \mathrm{~K}$, whereas data for $(R)-\mathbf{3 a b} \cdot \mathrm{HCl}$ were collected at $300 \mathrm{~K}$. A variable temperature experiment using both $\mathrm{Mo}$ - and $\mathrm{Cu}$ radiation was performed for crystals of $(R)-\mathbf{3 a b} \cdot \mathrm{HCl}$, but all crystals split upon cooling to $150 \mathrm{~K}$. Therefore only the $300 \mathrm{~K}$ data collected with $\mathrm{Cu}$-radiation were used for refinement. 


\section{$\underline{(S)-\mathbf{2 m a} \cdot \mathrm{HCl}}$}

Empirical formula

Formula weight

Temperature

Wavelength

Crystal system and Space group

Unit cell dimensions

Volume

$\mathrm{Z}$

Density (calculated)

Absorption coefficient

$\mathrm{F}(000)$

Crystal size

Theta range for data collection

Index ranges

Reflections collected

Independent reflections

Completeness to theta $=74.707^{\circ}$

Absorption correction

Max. and min. transmission

Refinement method

Data / restraints / parameters

Goodness-of-fit on $\mathrm{F}^{2}$

Final $\mathrm{R}$ indices [I>2sigma(I)]

$\mathrm{R}$ indices (all data)

Absolute structure parameter

Largest diff. peak and hole

\section{$\mathrm{C} 25 \mathrm{H} 29 \mathrm{Cl} \mathrm{F} \mathrm{N} \mathrm{O}$}

413.96

$150 \mathrm{~K}$

$1.54184 \AA$

Monoclinic $P 21$

$$
\begin{aligned}
& \mathrm{a}=9.98040(10) \AA \quad \alpha=90^{\circ} \text {. } \\
& \mathrm{b}=8.61600(10) \AA \quad \beta=102.1108(12)^{\circ} . \\
& \mathrm{c}=12.82200(10) \AA \quad \gamma=90^{\circ} \text {. }
\end{aligned}
$$$$
\text { 1078.039(19) } \AA^{3}
$$$$
2
$$

$1.275 \mathrm{Mg} / \mathrm{m}^{3}$

$1.759 \mathrm{~mm}^{-1}$

439.997

$0.25 \times 0.22 \times 0.09 \mathrm{~mm}^{3}$

3.526 to $76.232^{\circ}$.

$-12<=\mathrm{h}<=12,-10<=\mathrm{k}<=10,-16<=\mathrm{l}<=10$

10448

$4454[\mathrm{R}(\mathrm{int})=0.027]$

$99.6 \%$

Semi-empirical from equivalents

0.85 and 0.74

Full-matrix least-squares on $\mathrm{F}^{2}$

4454 / 1 / 263

0.9949

$\mathrm{R} 1=0.0265, \mathrm{wR} 2=0.0700$

$\mathrm{R} 1=0.0268, \mathrm{wR} 2=0.0706$

$-0.010(5)$

0.05 and -0.06 e. $\AA^{-3}$

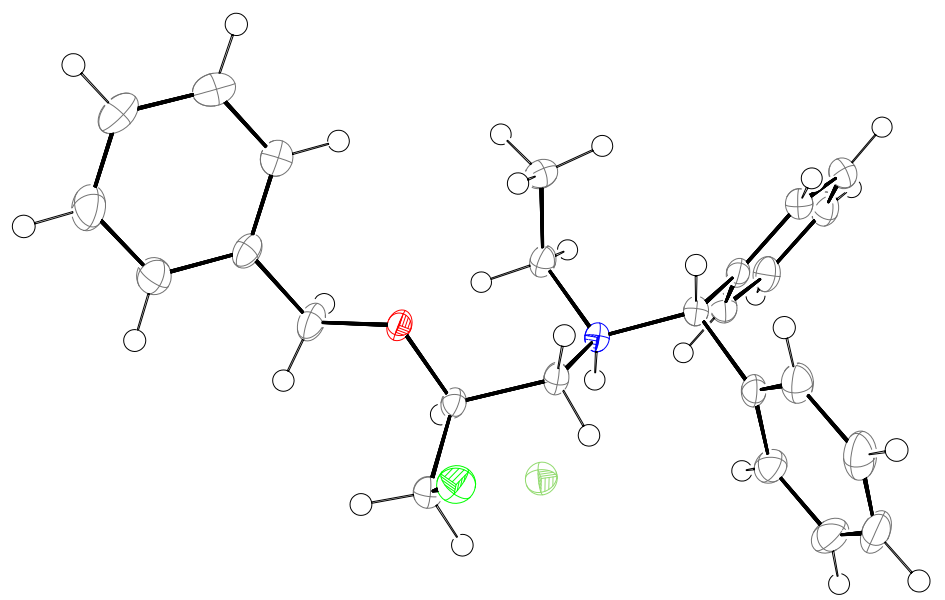

Figure S4. X-ray crystal structure (ellipsoid representation, probability level of 50\%) for $(S)-2 \mathrm{ma} \cdot \mathrm{HCl}$. 


\section{$\underline{(R)-\mathbf{3 a b} \cdot \mathrm{HCl}}$}

Empirical formula

Formula weight

Temperature

Wavelength

Crystal system and Space group

Unit cell dimensions

Volume

$\mathrm{Z}$

Density (calculated)

Absorption coefficient

$\mathrm{F}(000)$

Crystal size

Theta range for data collection

Index ranges

Reflections collected

Independent reflections

Completeness to theta $=77.272^{\circ}$

Absorption correction

Max. and min. transmission

Refinement method

Data / restraints / parameters

Goodness-of-fit on $\mathrm{F}^{2}$

Final $\mathrm{R}$ indices [I $>2 \operatorname{sigma}(\mathrm{I})]$

$\mathrm{R}$ indices (all data)

Absolute structure parameter

Largest diff. peak and hole

\section{C11 H17 Cl F1 N}

217.71

$300 \mathrm{~K}$

\section{$1.54184 \AA$}

Orthorhombic $P 2_{1} 2_{1} 2_{1}$

$\begin{array}{ll}\mathrm{a}=9.95040(10) \AA & \alpha=90^{\circ} . \\ \mathrm{b}=10.52650(10) \AA & \beta=90^{\circ} . \\ \mathrm{c}=23.7547(4) \AA & \gamma=90^{\circ} .\end{array}$

2488.14(5) $\AA^{3}$

8

$1.162 \mathrm{Mg} / \mathrm{m}^{3}$

$2.544 \mathrm{~mm}^{-1}$

928.000

$0.40 \times 0.25 \times 0.10 \mathrm{~mm}^{3}$

3.721 to $77.272^{\circ}$

$-11<=\mathrm{h}<=12,-13<=\mathrm{k}<=13,-30<=1<=29$

49083

$5262[\mathrm{R}(\mathrm{int})=0.060]$

$99.8 \%$

Semi-empirical from equivalents

0.78 and 0.41

Full-matrix least-squares on $\mathrm{F}^{2}$

5262 / 48 / 307

1.0011

$\mathrm{R} 1=0.0343, \mathrm{wR} 2=0.0944$

$\mathrm{R} 1=0.0382, \mathrm{wR} 2=0.0997$

$0.001(6)$

0.12 and -0.16 e. $\AA^{-3}$

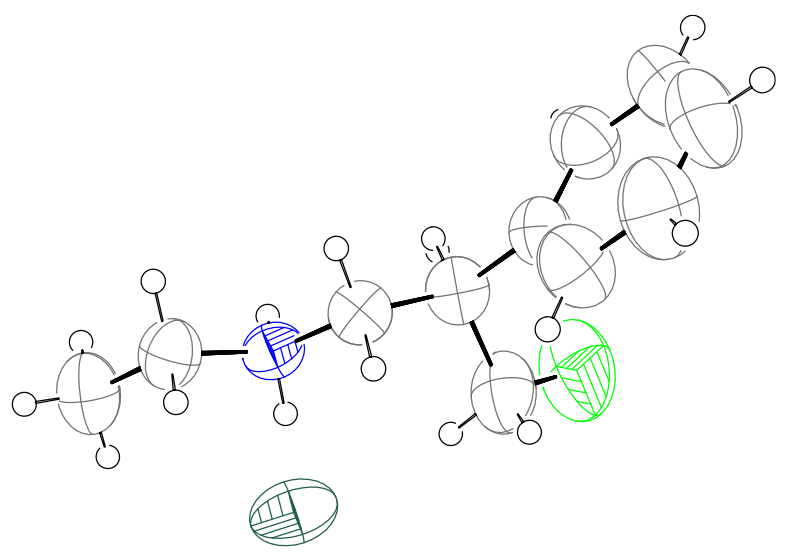

Figure S5. X-ray crystal structure (ellipsoid representation, probability level of 50\%) for $(R)-3 \mathbf{a b} \cdot \mathrm{HCl}$. 


\section{Computational Studies}

\section{Level of Theory}

\section{Density Functional Theory (DFT)}

Geometry optimization and frequency calculations were performed in Gaussian 16 Rev. A.03. ${ }^{15}$ The Minnesota M06-2X functional ${ }^{16}$ was used in combination with the def2-SVP(D) basis set, corresponding to def2-SVPD on electronegative atoms, and def2-SVP otherwise. ${ }^{17,18}$ Cs makes use of an effective core potential (ECP). ${ }^{19}$ The ultrafine integration grid was used. ${ }^{20}$ Solvation in 1,2-dichloroethane (DCE) was modelled using the conductor-like polarizable continuum model $(\mathrm{CPCM}) .{ }^{21-23}$

Single point energy corrections were implemented using the ORCA 4.2.0 software package ${ }^{24,25}$ with the $\omega$ B97X-D3 functional ${ }^{26,27}$, incorporating the D3 dispersion correction by Grimme ${ }^{28}$, in combination with the (ma)-def2-TZVPP basis set, consisting of ma-def2-TZVPP ${ }^{29}$ on heteroatoms and def2-TZVPP otherwise. Integration utilized the Lebedev302 grid for SCF cycles and Levedev590 grid for final energy evaluation. Solvation in DCE was accounted for using the CPCM solvation model, using relative permittivity of 10.5 and refractive index of 1.4448, with gaussian charge scheme and scaled van der Waal cavity. Thermochemistry was evaluated at $298.15 \mathrm{~K}$, and a standard concentration of $1 \mathrm{M}$ using GoodVibes python script. ${ }^{30}$ The entropic contribution of low vibrational modes was corrected using a free-rotor description below a $100 \mathrm{~cm}^{-1}$ cutoff. $^{31}$

Conformational sampling of azetidinium ions and ion pairs was performed using the Conformer-Rotamer Ensemble Sampling Tool (CREST) version 2.7, based on the GFN-XTB method of Grimme and Pracht. ${ }^{32}$ Conformational sampling was run in $\mathrm{CH}_{2} \mathrm{Cl}_{2}$ with default parameters, however a larger energy window of 12.0 $\mathrm{kcal} / \mathrm{mol}$ was used in combination with more stringent duplicate detection criteria (-rthr 0.0625, -ethr 0.05, bthr 0.01) to preserve conformations of the 4-membered ring. Ion pairs were sampled using the built in nci mode, which applies an ellipsoid potential. Only the lowest energy conformers of highly flexible structures were optimized, guided by thorough sampling of the less flexible analogs.

\section{Molecular Dynamics (MD)}

Molecular dynamics simulations (MD) were performed in GROMACS 5.1.4. software package. The optimized potential for liquid simulations (OPLS-AA 2005) forcefield ${ }^{33,34}$ with restrained electrostatic potential charges ${ }^{35,36}$ was used, with compatible parameters generated in Schrödinger Maestro software (ffld_server utility, version 14), ${ }^{37}$ and RESP charges generated by fitting the HF/6-31G(d) potential in Ambertools. ${ }^{38}$ All simulations were performed in the reaction solvent of 1,2-dichloroethane, with topology from virtualchemistry.org. ${ }^{39,40}$

The system of interest was centered in a cubic box, with 3-dimensional periodic boundary conditions (PBC), and minimum molecule-boundary distance of $15 \AA$. System temperature and pressure were maintained with the velocity rescaling method (time constant $=100 \mathrm{fs}$ ) ${ }^{41}$ and the Parrinello-Rahman barostat (time constant $=$ $2 \mathrm{ps}$, references pressure $=1$ bar, compressibility $\left.=4.5 \times 10^{-5} \mathrm{bar}^{-1}\right)^{42,43}$ respectively. The particle mesh Ewald method and van der Waals interactions were implemented with $1 \mathrm{~nm}$ cutoff. The linear constraint solver algorithm (LINCS) was used. ${ }^{44}$

Simulations were first minimized by steepest-descents method of 5000 steps. Equilibration was then performed, firstly by constant volume NVT simulation ( $200 \mathrm{ps}$, timestep $=1 \mathrm{fs}$, heavy atom position restraints, initial velocities derived from a $173 \mathrm{~K}$ Maxwell-Boltzmann distribution). Temperature was raised during the simulation to the required production temperature. Secondly, a constant pressure, NPT, equilibration was run ( $400 \mathrm{ps}$, timestep $=2 \mathrm{fs}$, production temperature) and the stability of the system volume verified. The production run results from a continuation of NPT simulation with data derived from 10 ps snapshots.

Transition states were sampled, using the same parameters as the ion pair, by constraining the length of the forming $\mathrm{C}-\mathrm{F}$ bond and the breaking $\mathrm{C}-\mathrm{N}$ bond, and restraining the $\mathrm{F}---\mathrm{C}---\mathrm{N}$ angle with a harmonic potential. Simulations were performed at elevated temperature of $423.15 \mathrm{~K}$ to enhance sampling. For the cis substrate, bonds were constrained to $2.2 \AA$ and $1.98 \AA$ A respectively, and angle restrained to $180^{\circ}$ with $k_{\theta}=1.5 \mathrm{~kJ} / \mathrm{mol}^{\prime} / \mathrm{deg}^{2}$. 
For the trans substrate, bonds were constrained to $2.0 \AA$ and $1.98 \AA$ respectively, with angle restraint of with $k_{\theta}=3.0 \mathrm{~kJ} / \mathrm{mol} / \mathrm{deg}^{2}$. Constraint distances were based off distances from trial DFT computed TSs, with C-F bond elongated to enhance sampling and system stability. A stronger angle restraint was employed for the trans substrate due to persistence of unproductive conformations with large F---C---N angle.

Trajectories were clustered using the GROMOS algorithm, ${ }^{45}$ with RMSD matrix accounting for molecular symmetry. Extraneous groups (e.g. $\mathrm{CF}_{3}$ ) were excluded from RMSD calculation to ensure chemically relevant clustering.

\section{Kinetics}

The kinetics of the catalytic fluorination of azetidinium ions is interpreted within the framework of the energetic span model. For an overview of the model, its derivation and applicability, the reader is referred to the literature. ${ }^{46-50}$ The primary result is that the turnover frequency of a catalytic cycle can be described by the energetic span of the reaction - defined as the difference in energy between the turnover determining intermediate (TDI) and the turnover determining transition state (TDTS). The TDI and TDTS are the two states of the energetic profile identified such that the energetic span is maximized. 
To investigate the difference in reactivity of the different azetidinium substrates under both HB PTC and homogeneous (TBAF) conditions, the barrier heights to fluorination with seven cis-substrates were computed. The seven substrates are given in Figure S6 and correspond to the ions in Scheme S4. Cis-azetidinium ions were computed for consistency and as this is the major diastereomer in most cases.

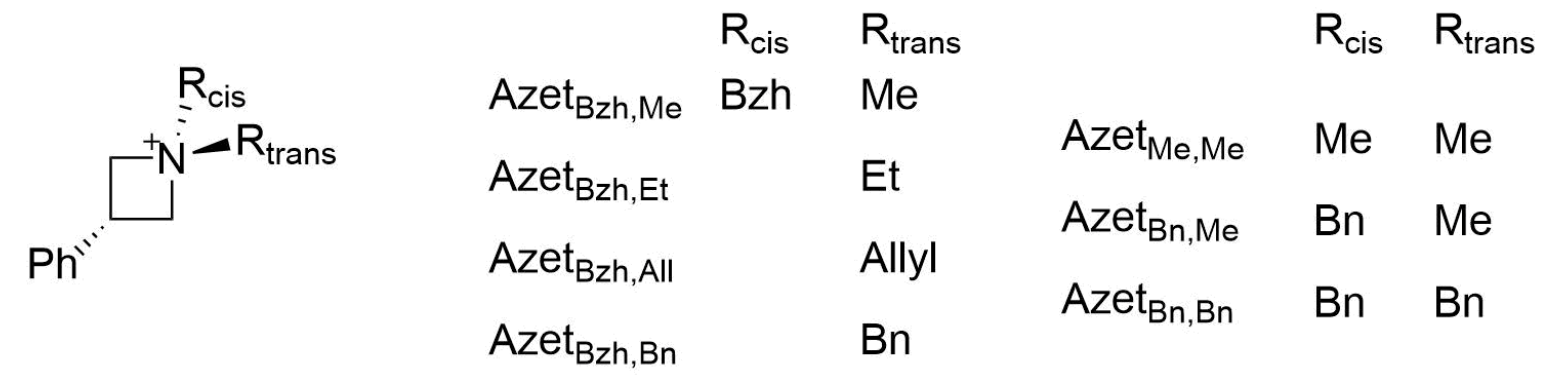

Figure S6: Structures of azetidinium ions used for computational reactivity study.

\section{Structure of azetidinium ions}

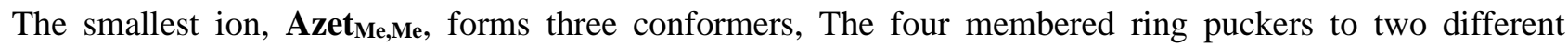
conformations, and the phenyl group can adopt two different conformations relative to the azetidinium ring (Figure S7). In ions with different $\mathrm{N}$ substitution, the azetidinium ring may take a planar conformation, rather than puckered.

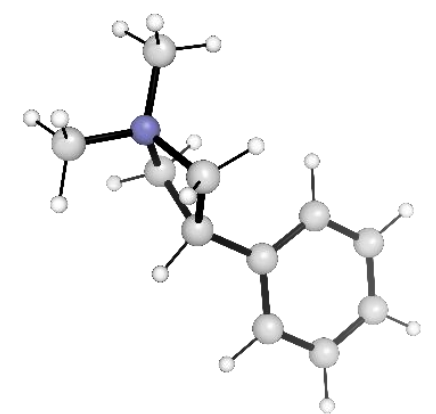

Azet $_{\text {Me,Me }} 1$

$\mathrm{G}_{\mathrm{rel}}=0 \mathrm{~kJ} / \mathrm{mol}$

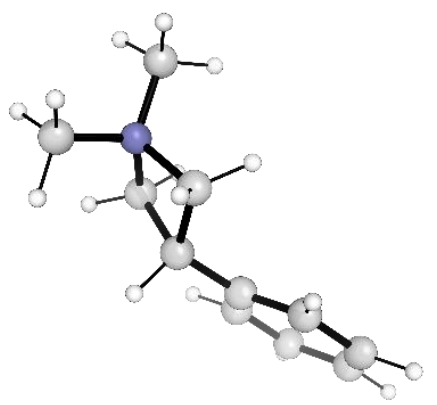

Azet $_{\mathrm{Me}, \mathrm{Me}} 2$

$\mathrm{G}_{\mathrm{rel}}=+1.3 \mathrm{~kJ} / \mathrm{mol}$

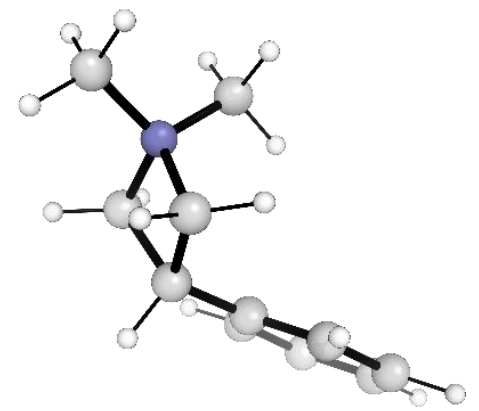

Azet $_{\mathrm{Me}, \mathrm{Me}} 3$

$\mathrm{G}_{\text {rel }}=+4.3 \mathrm{~kJ} / \mathrm{mol}$

Figure S7: Conformations of Azet $_{M e, M e}$

All other ions studied have a cis aromatic group, enabling $\pi$-interactions. This is exemplified by the lowest energy conformers of Azet $_{\text {Bzh,Me }}$ in Figure S8 (mirror image conformers are not shown). The T-shape CH- $\pi$ interaction is favored, facilitated in part by the pre-organization of the phenyl ring eclipsing the azetidinium ring. 

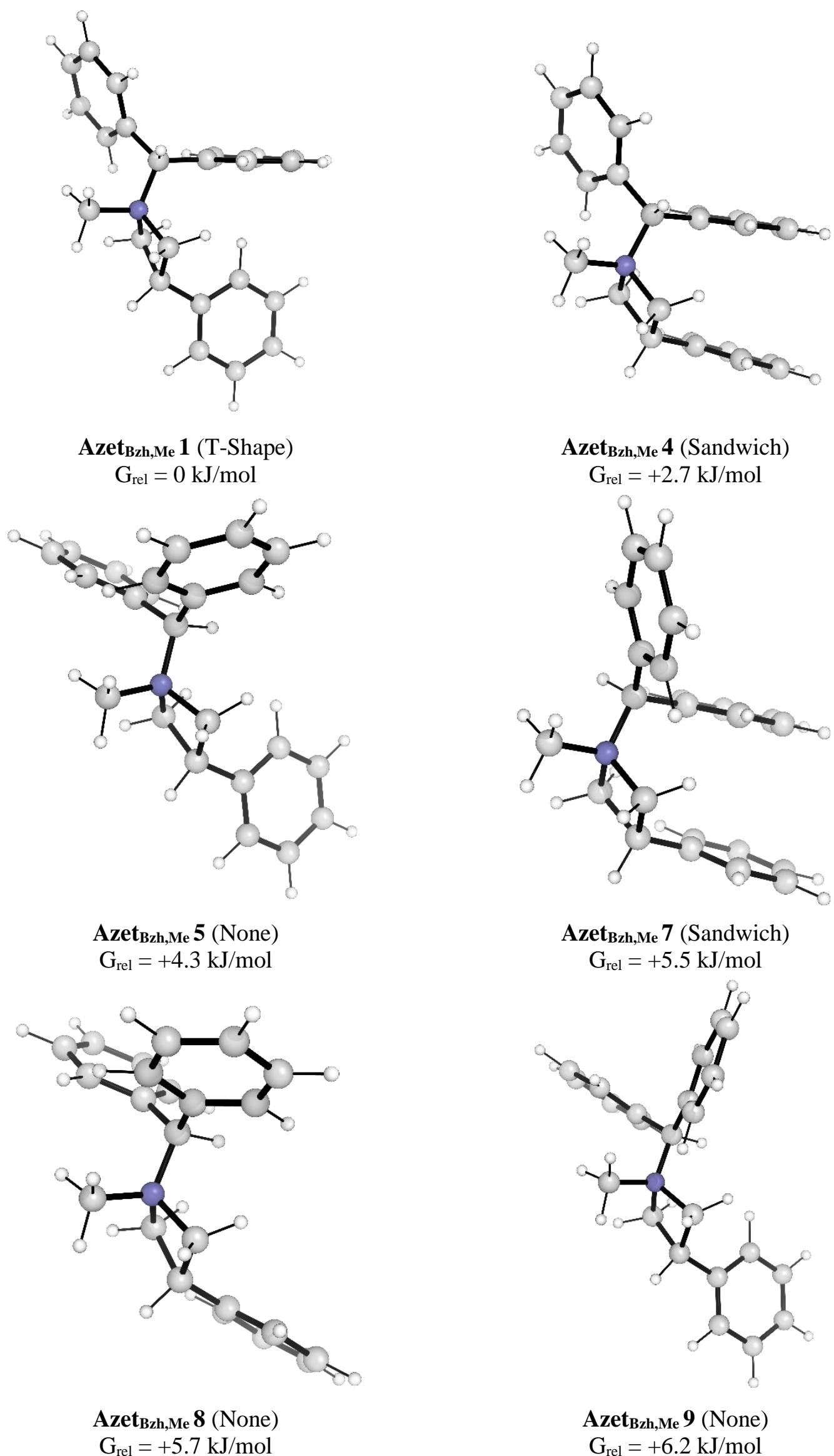

Azet $_{\text {Bzh,Me }} 8$ (None)

$\mathrm{G}_{\mathrm{rel}}=+5.7 \mathrm{~kJ} / \mathrm{mol}$

$\mathrm{G}_{\mathrm{rel}}=+6.2 \mathrm{~kJ} / \mathrm{mol}$

Figure S8: Conformations of Azet $_{\text {Bzh,Me }}$ illustrating $\pi$-interactions 
The lowest energy conformations of all of the ions studied are given in Figure S9. It should be noted that, with

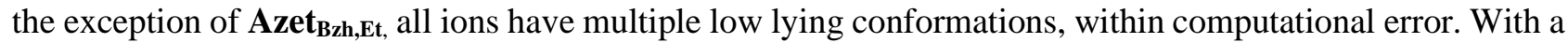
benzhydryl substituent, formation of the $\mathrm{CH}-\pi$ interaction leads to additional conformational rigidity - the benzhydryl group in turn restricts the conformations available to the other nitrogen substituent, which chooses to eclipse the Bzh hydrogen rather than the bulkier Bzh phenyl ring (see Azet $_{\text {Bzh,Et, }}$, Azet Bzh,Allyl, and Azet $_{\text {Bzh,Bn }}$ for orientation of ethyl, allyl and benzyl respectively).
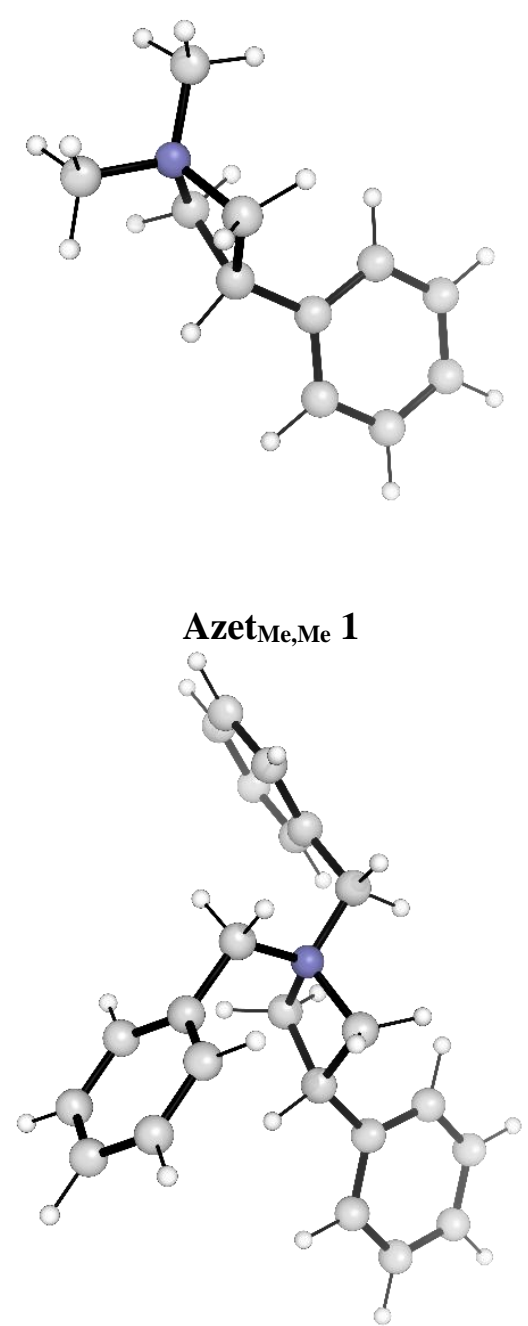

Azet $_{B n, B n} 1$

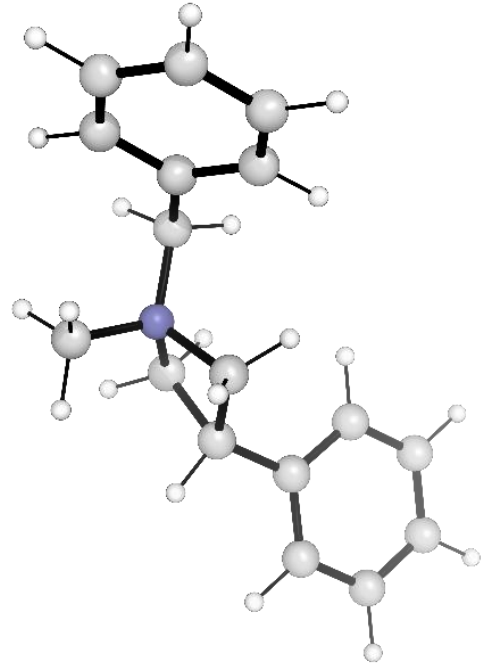

Azet $_{\text {Bn,Me } 1}$

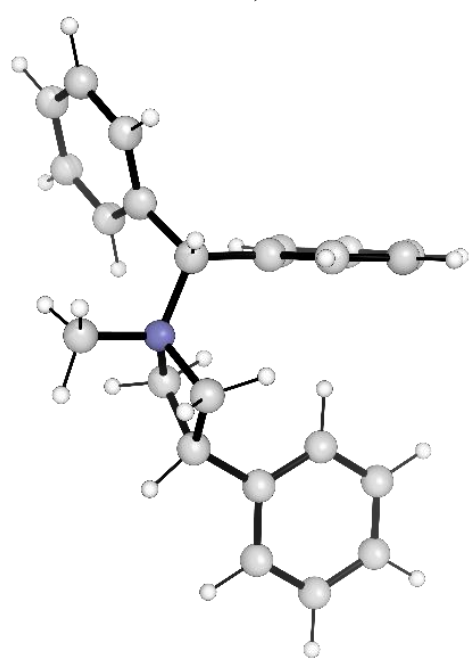

Azet $_{\text {Bzh,Me } 1}$ 


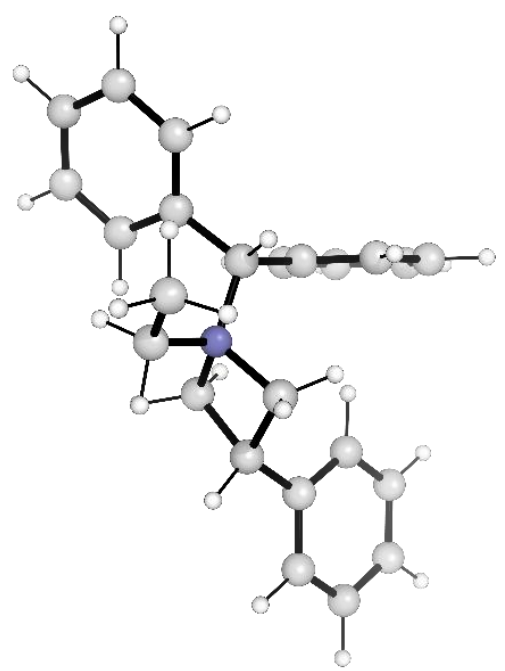

Azet $_{\text {Bzh,Et } 1}$

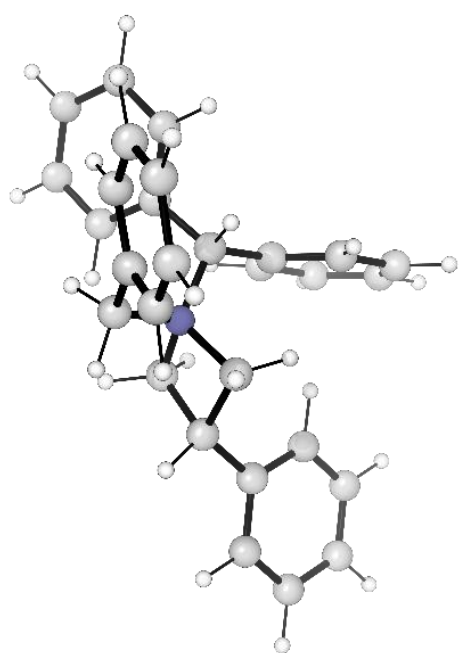

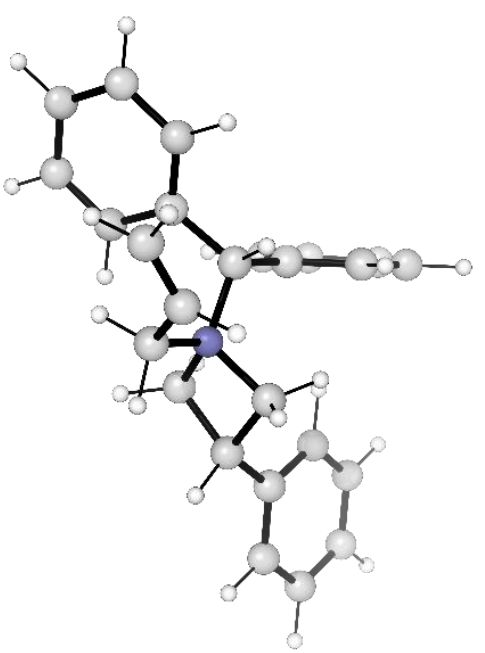

Azet $_{\text {Bzh,Allyl } 1}$

\section{Azet $_{\text {Bzh,Bn }} 1$}

Figure S9: Lowest energy conformations of the studied azetidinium ions

\section{Azetidinium-Fluoride Ion Pairs}

Ion pairs with fluoride favor proximity of the fluoride to the tetracoordinate nitrogen. Sampling with Azet Me,Me $_{\text {en }}$ shows the ion pair favors geometries with the fluoride either to the side, or against the face of, the four membered ring (Figure S10), with the azetidinium ion in its intrinsically favored conformations. Many geometries are located within computational error of the minimum energy geometry (illustrated). Selected higher energy geometries are also shown. 


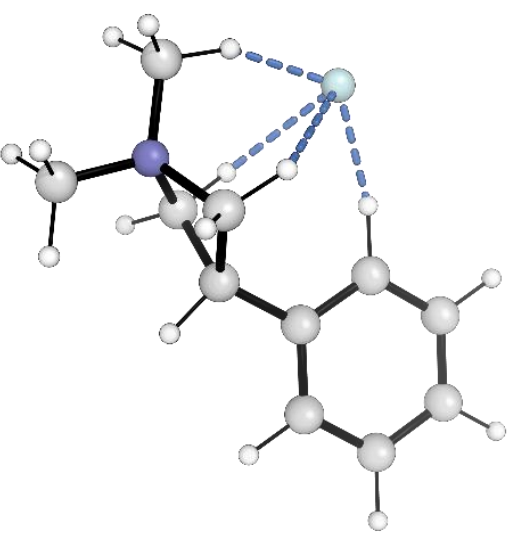

Azet $_{\mathrm{Me}, \mathrm{Me}-\mathrm{F}} 1$

$\mathrm{G}_{\text {rel }}=0.0 \mathrm{~kJ} / \mathrm{mol}$

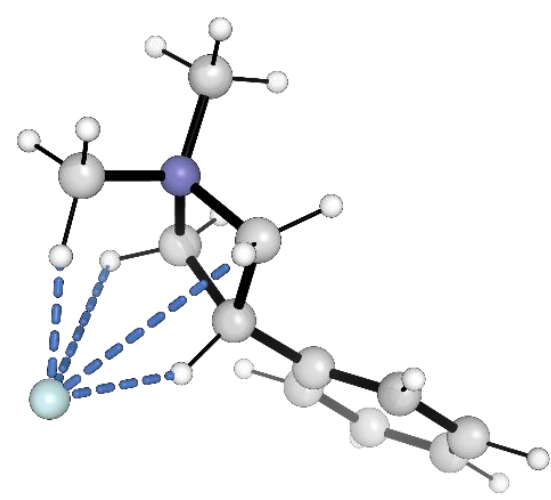

Azet $_{\mathrm{Me}, \mathrm{Me}-\mathrm{F}} 3$

$\mathrm{G}_{\mathrm{rel}}=+3.4 \mathrm{~kJ} / \mathrm{mol}$

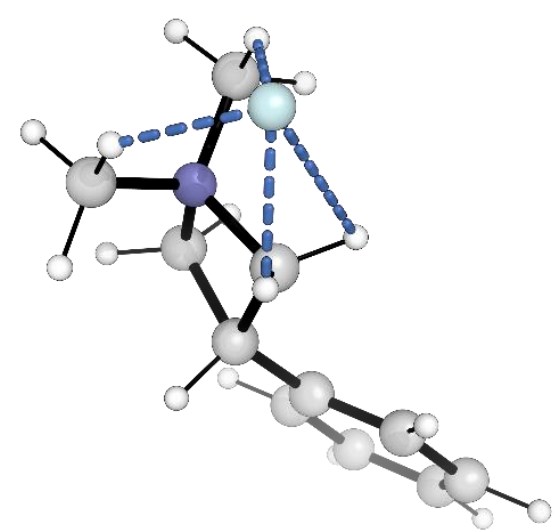

Azet $_{\mathrm{Me}, \mathrm{Me}}-\mathbf{F} 6$

$\mathrm{G}_{\mathrm{rel}}=+5.7 \mathrm{~kJ} / \mathrm{mol}$

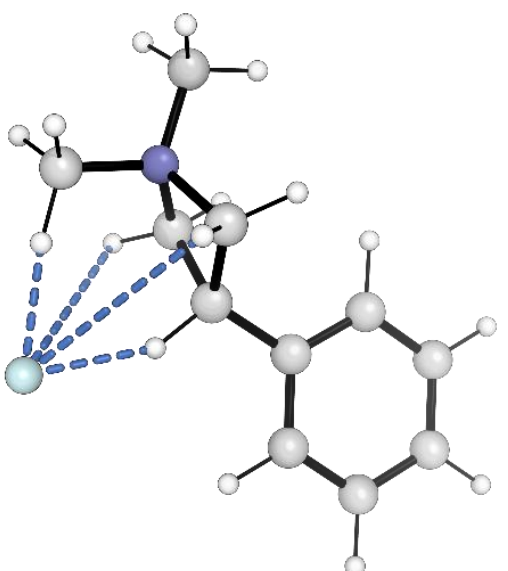

Azet $_{\mathrm{Me}, \mathrm{Me}-\mathrm{F}} 2$

$\mathrm{G}_{\mathrm{rel}}=+1.5 \mathrm{~kJ} / \mathrm{mol}$

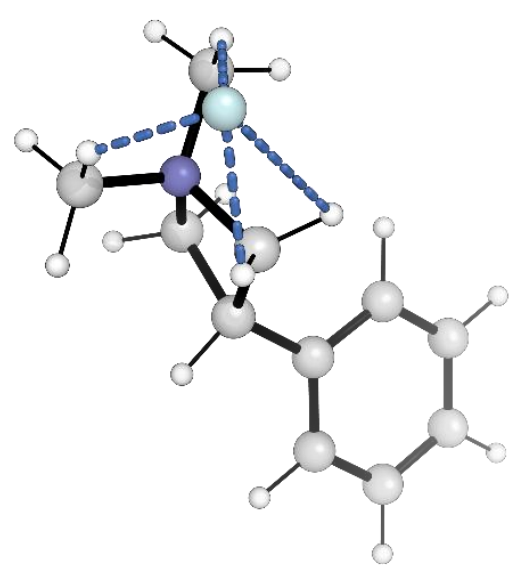

Azet $_{\mathrm{Me}, \mathrm{Me}-\mathrm{F}} 4$

$\mathrm{G}_{\mathrm{rel}}=+4.3 \mathrm{~kJ} / \mathrm{mol}$

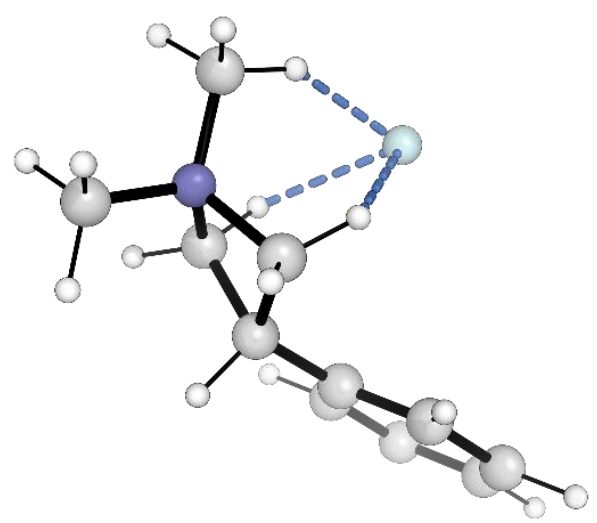

Azet $_{\mathrm{Me}, \mathrm{Me}}-\mathbf{F} 7$

$\mathrm{G}_{\text {rel }}=+6.8 \mathrm{~kJ} / \mathrm{mol}$ 


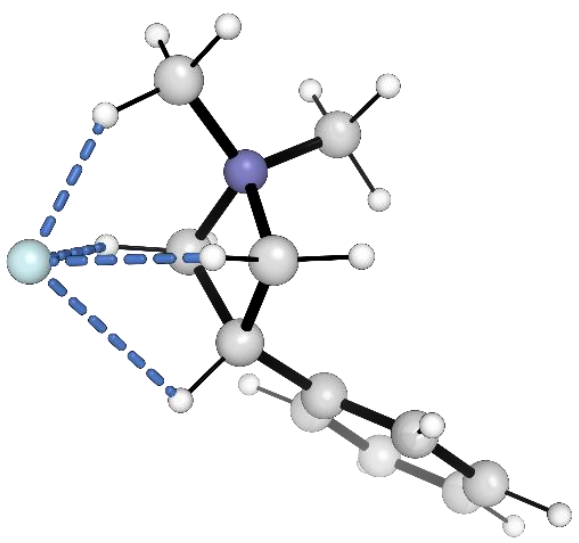

Azet $_{M e, M e-F ~} 8$ $\mathrm{G}_{\text {rel }}=+6.8 \mathrm{~kJ} / \mathrm{mol}$

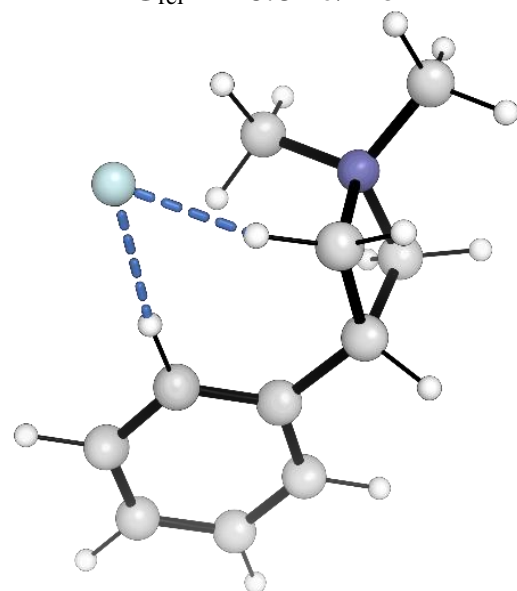

Azet $_{\mathrm{Me}, \mathrm{Me}}-\mathrm{F} 11$ $\mathrm{G}_{\mathrm{rel}}=+9.7 \mathrm{~kJ} / \mathrm{mol}$

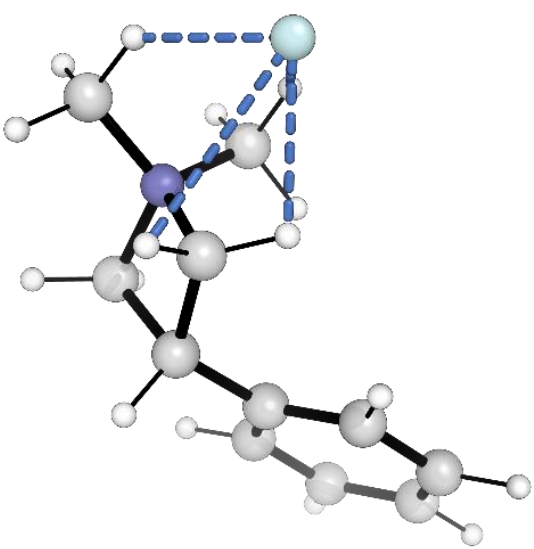

Azet $_{\mathrm{Me}, \mathrm{Me}-\mathrm{F}} 9$ $\mathrm{G}_{\text {rel }}=+8.9 \mathrm{~kJ} / \mathrm{mol}$

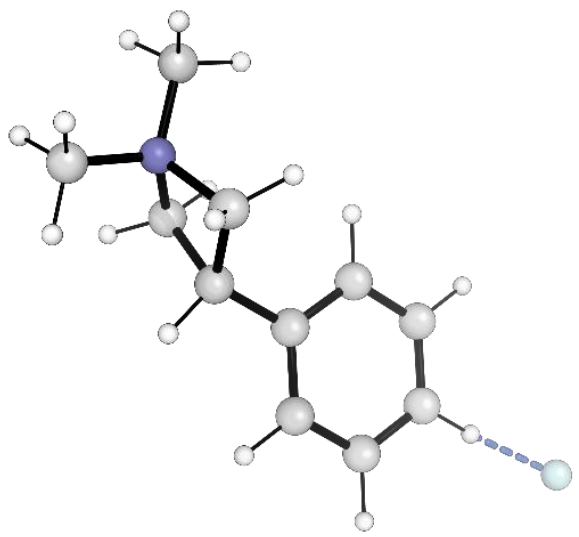

Azet $_{\mathrm{Me}, \mathrm{Me}}-\mathrm{F} \mathbf{1 3}$

$\mathrm{G}_{\mathrm{rel}}=+26.4 \mathrm{~kJ} / \mathrm{mol}$

Figure S10: Representative geometries of Azet $_{\mathrm{Me}, \mathrm{Me}}$ ion pair with fluoride

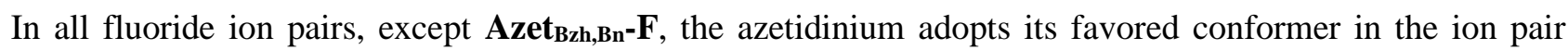
(Figure S11). The azetidinium ion that binds fluoride most weakly is Azet $_{\mathbf{B z h}, \mathbf{E t}}$ as the ethyl group prevents close approach of fluoride. For azetidinium ions without the benzhydryl group, fluoride interacts with azetidinium phenyl group in addition to being proximal to nitrogen. In ions with benzhydryl group, the intramolecular $\mathrm{CH}-\pi$ interaction is retained and fluoride positioned elsewhere.

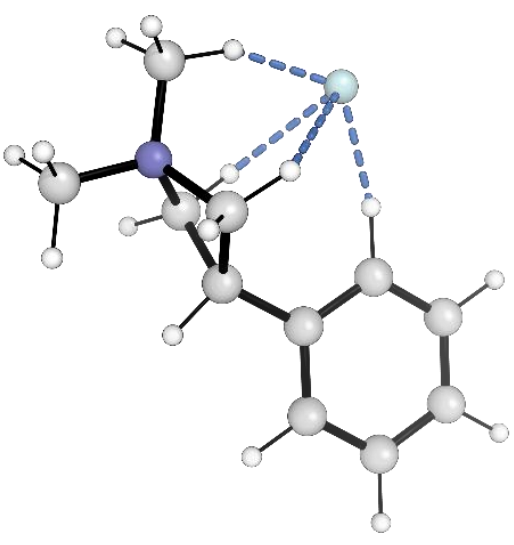

Azet $_{\mathrm{Me}, \mathrm{Me}}$-F 1

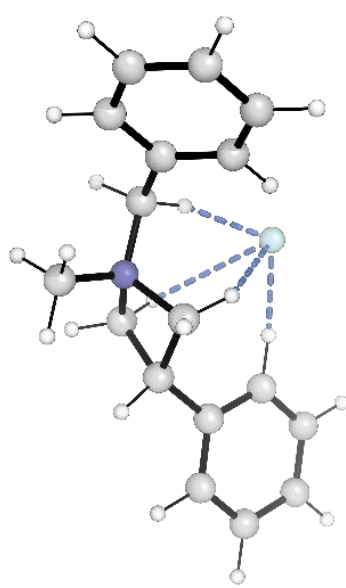

Azet $_{\text {Bn,Me-F } 1}$ 

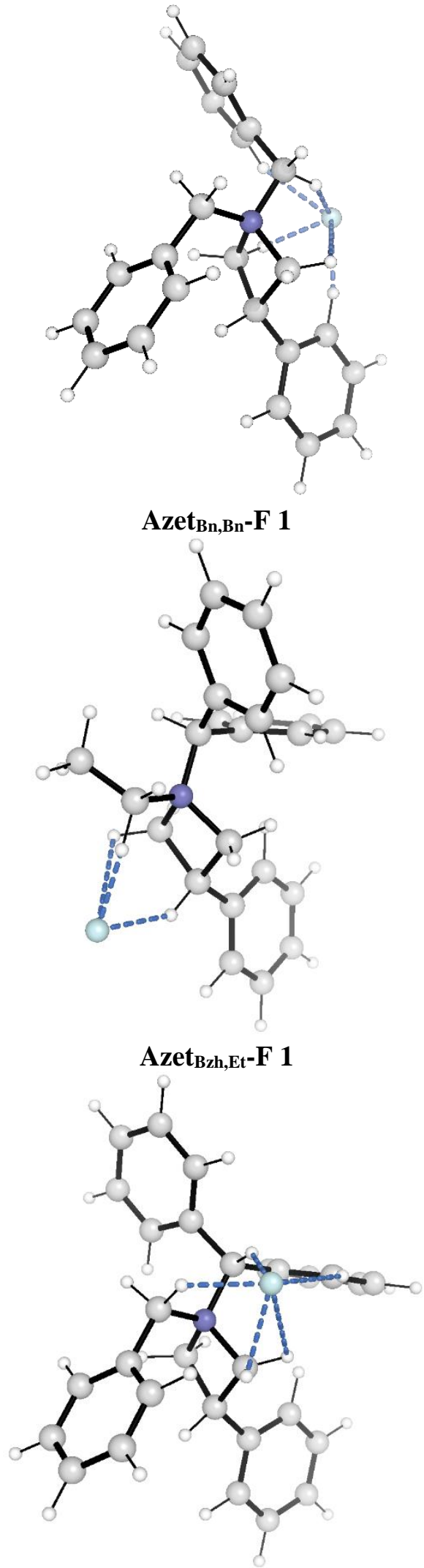

Azet $_{\text {Bzh,Bn-F } 1}$

Figure S11: Geometries of lowest energy ion pairs with fluoride
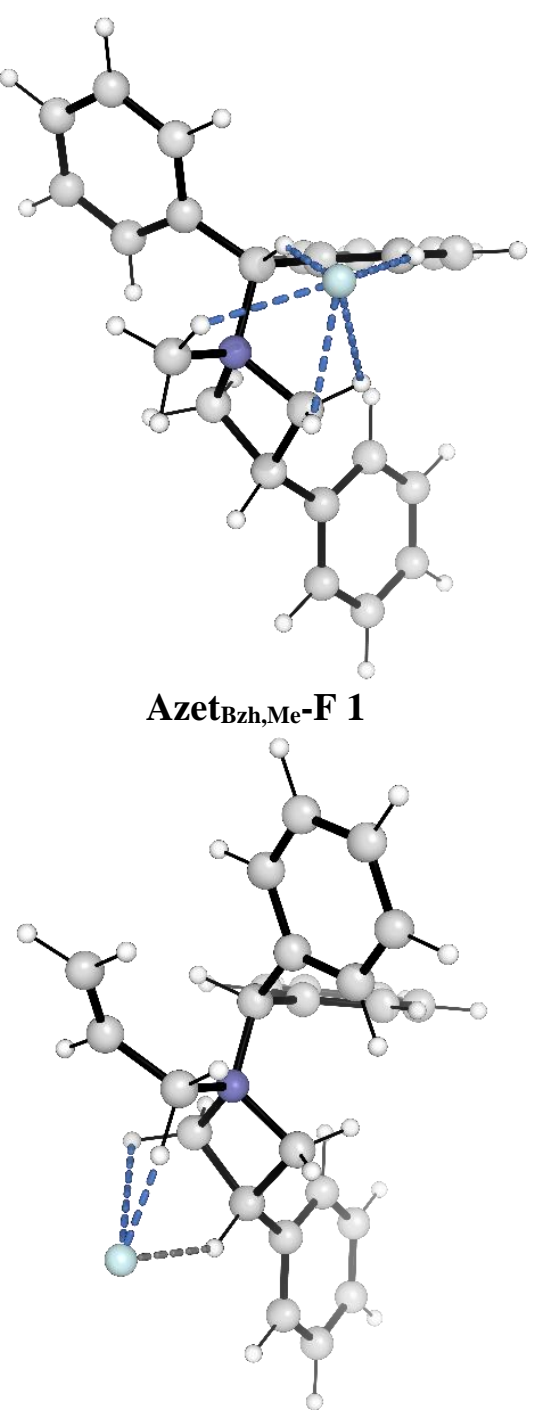

Azet $_{\text {Bzh,Allyl-F } 1}$ 


\section{Azetidinium-Triflate Ion Pairs}

Ion pairs with triflate counterion have larger conformational space due to the rotation of the triflate ion. However, the ion pairs favor the negatively charged oxygen atoms in similar positions to the fluoride ion in

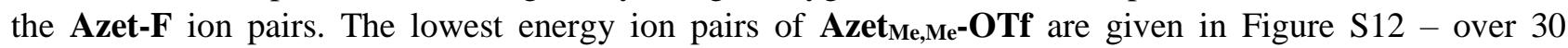
geometries were optimized within $5 \mathrm{~kJ} / \mathrm{mol}$.

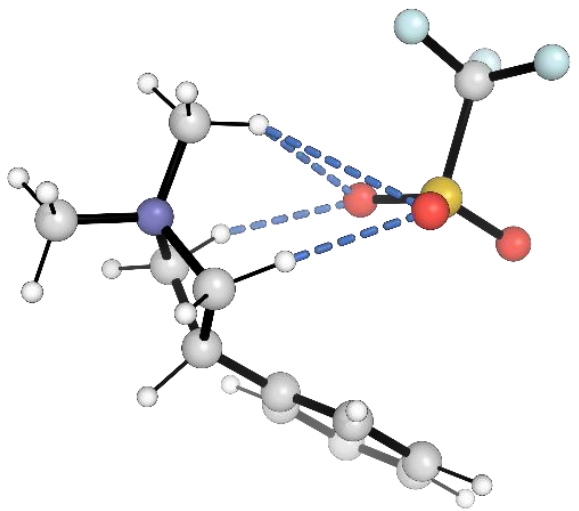

Azet $_{\mathrm{Me}, \mathrm{Me}-\mathrm{OTf} 1}$

$\mathrm{G}_{\text {rel }}=0.0 \mathrm{~kJ} / \mathrm{mol}$

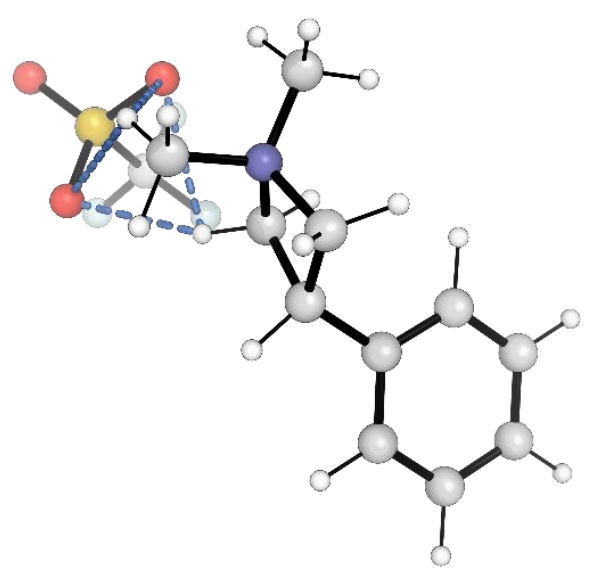

Azet $_{\mathrm{Me}, \mathrm{Me}-\mathrm{OTf} 3}$

$\mathrm{G}_{\text {rel }}=+0.8 \mathrm{~kJ} / \mathrm{mol}$

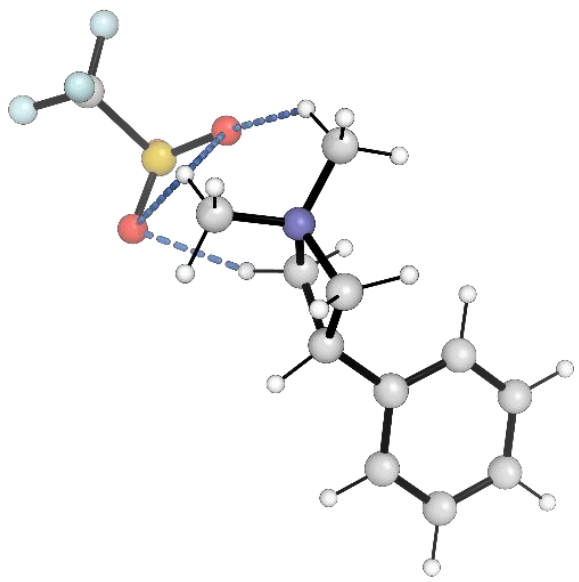

Azet $_{\mathrm{Me}, \mathrm{Me}-O T f} 5$

$\mathrm{G}_{\text {rel }}=+1.2 \mathrm{~kJ} / \mathrm{mol}$

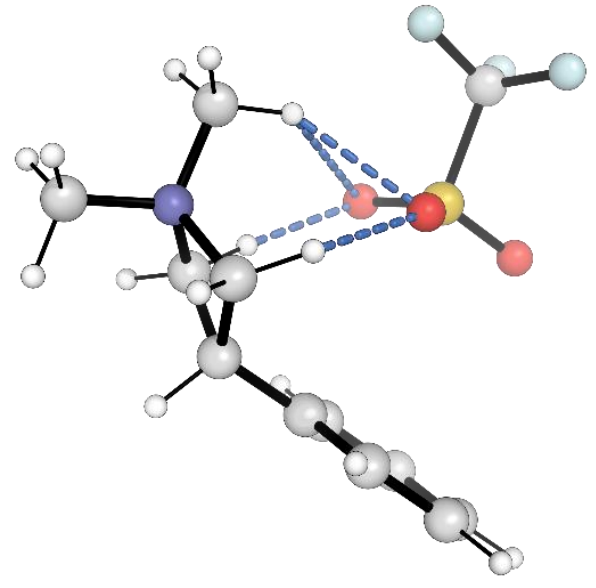

Azet $_{\mathrm{Me}, \mathrm{Me}}$-OTf 2

$\mathrm{G}_{\text {rel }}=+0.1 \mathrm{~kJ} / \mathrm{mol}$

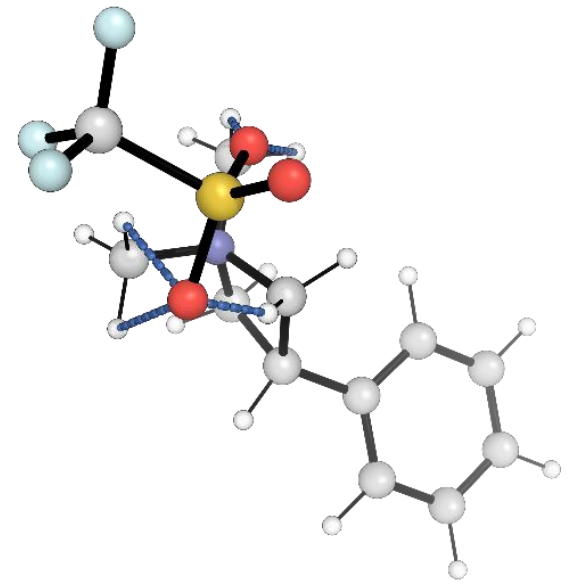

Azet $_{\mathrm{Me}, \mathrm{Me}-\mathrm{OTf}} 4$

$\mathrm{G}_{\mathrm{rel}}=+1.1 \mathrm{~kJ} / \mathrm{mol}$

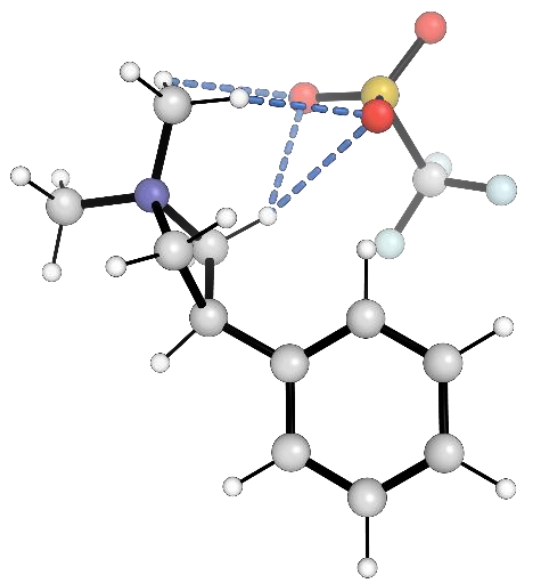

Azet $_{\mathrm{Me}, \mathrm{Me}-\mathrm{OTf}} 6$

$\mathrm{G}_{\mathrm{rel}}=+1.6 \mathrm{~kJ} / \mathrm{mol}$ 


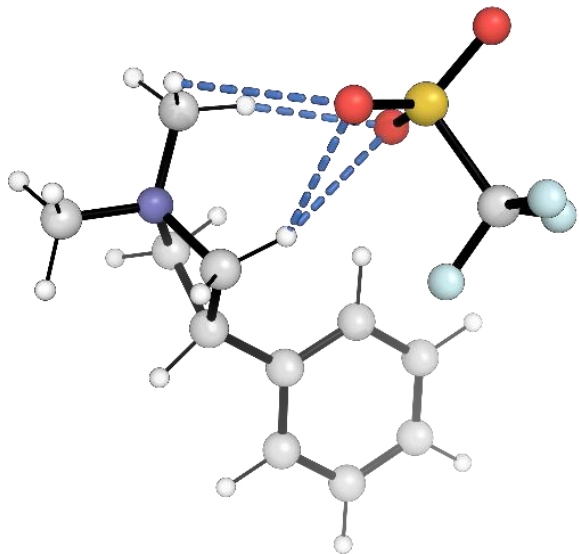

Azet $_{\mathrm{Me}, \mathrm{Me}-\mathrm{OTf}} 7$

$\mathrm{G}_{\mathrm{rel}}=+1.6 \mathrm{~kJ} / \mathrm{mol}$

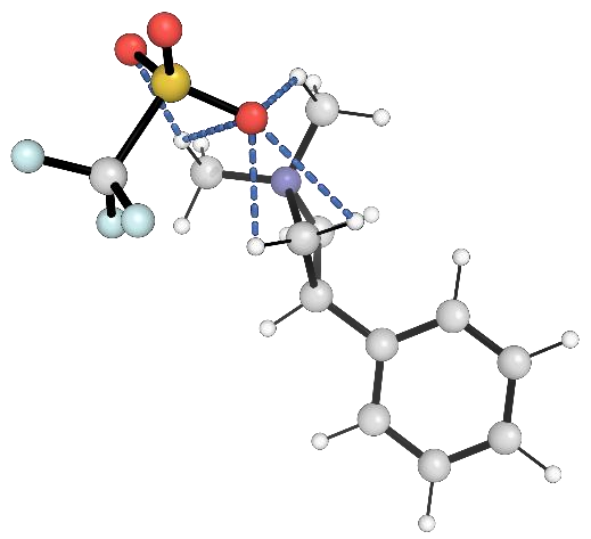

Azet $_{\mathrm{Me}, \mathrm{Me}-\mathrm{OTf} 8} 8$

$\mathrm{G}_{\mathrm{rel}}=+1.6 \mathrm{~kJ} / \mathrm{mol}$

Figure S12: Geometries of lowest energy Azet $_{\mathrm{Me}, \mathrm{Me}}$ ion pairs with fluoride

Ion pairs of the azetidinium ions with triflate counterion are given in Figure S13. In all cases, the triflate ion associates closely with the formally positive nitrogen, and surrounding $\mathrm{C}-\mathrm{H}$ bonds, with many geometries within computational error. Sampling of larger, more flexible, azetidinium ions was guided by the lower energy structures of the less complex ions, as more exhaustive sampling was prohibitive.

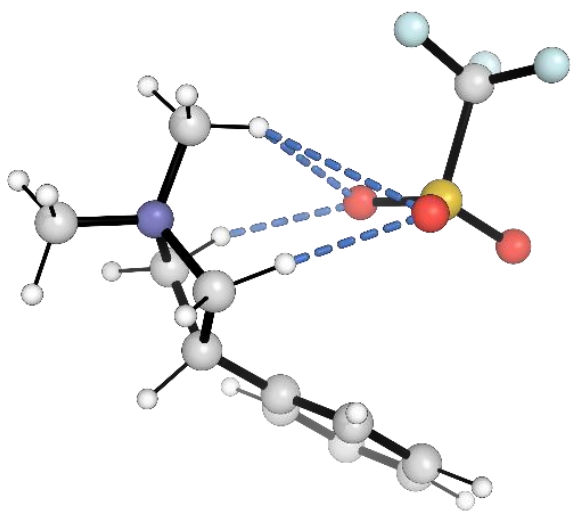

Azet $_{\mathrm{Me}, \mathrm{Me}-O T f} 1$

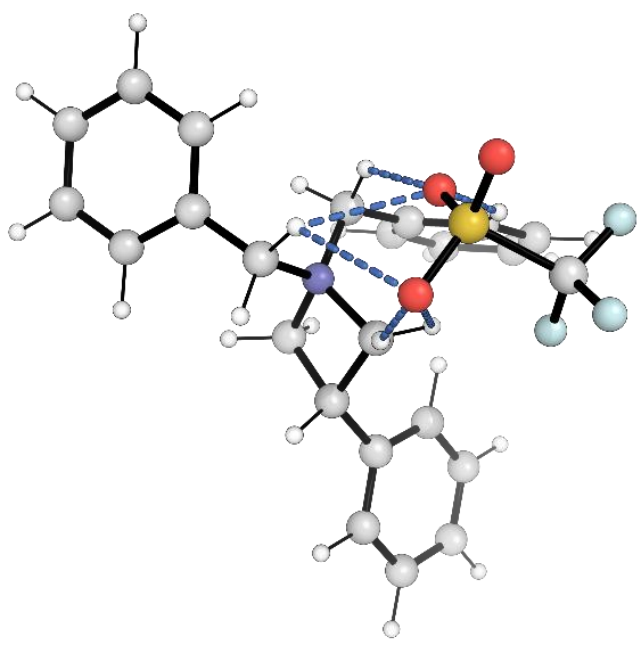

Azet $_{B n, B n-O T f} 1$

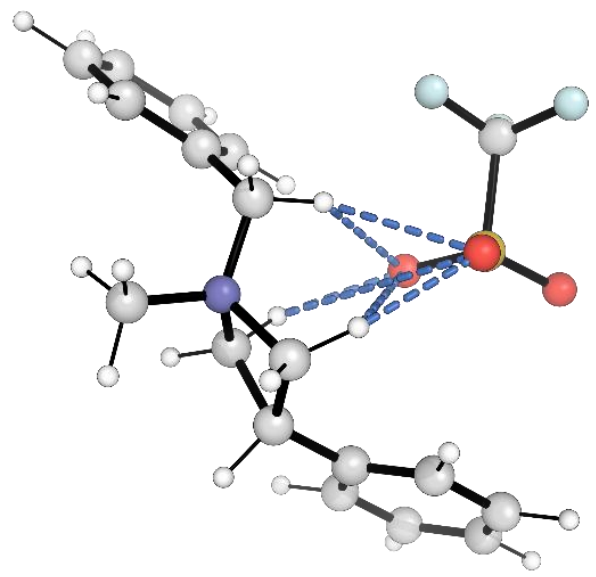

Azet $_{\text {Bn,Me}}$-OTf 1

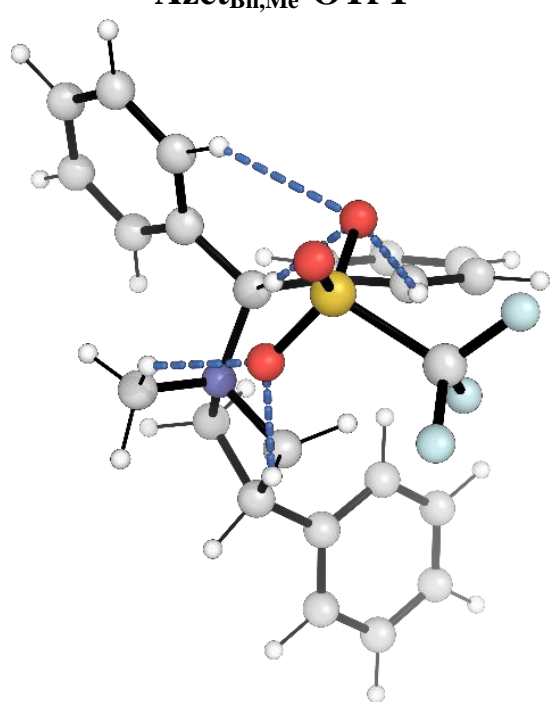

Azet $_{\text {Bzh,Me-OTf } 1}$ 


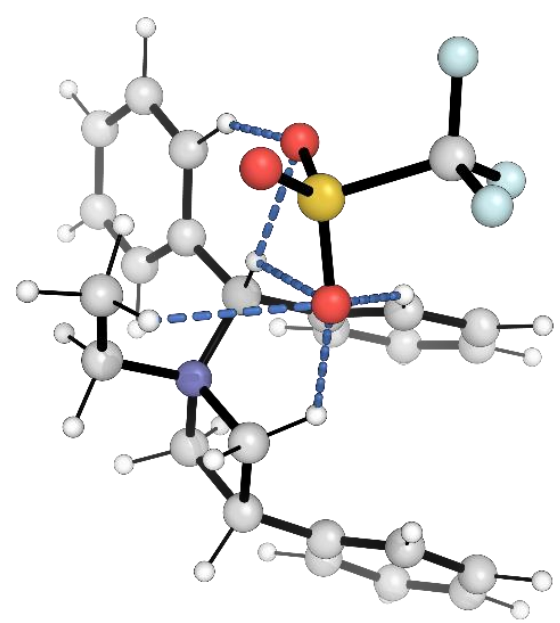

Azet $_{\text {Bzh,Et-OTf } 1}$

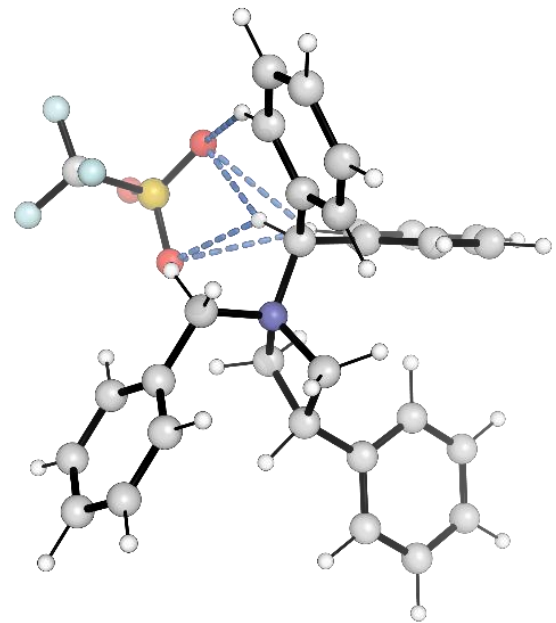

Azet $_{\text {Bzh,Bn-OTf } 1}$

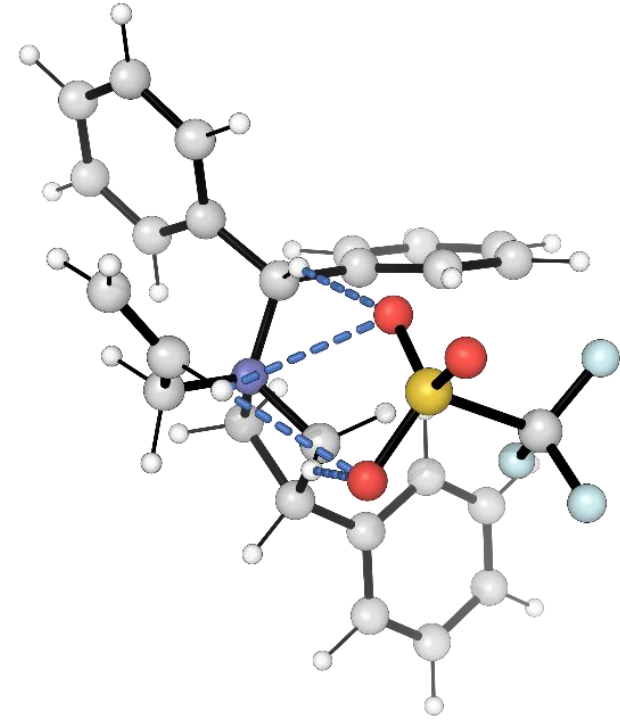

Azet $_{\text {Bzh,Allyl-OTf } 1}$

Figure S13: Azet OTf ion pairs

\section{Azetidinium Fluorination Transition State Structures}

In the fluoride delivery transition state structures conformational space is greatly reduced over the ion pair and the relative energies of the TS conformations mirror those in the ion. The conformations of the TS with Azet $_{\mathbf{M e}, \mathbf{M e}}$ and the lowest energy conformations of the TS with Azet Bzh,Me are given in Figure S14 and Figure S15 respectively. 


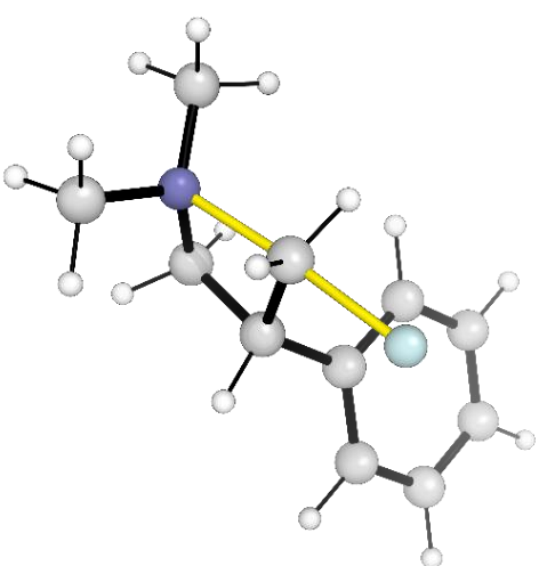

TS-Azet $_{\mathrm{Me}, \mathrm{Me}}-\mathbf{F} 1$

$\mathrm{G}_{\mathrm{rel}}=0.0 \mathrm{~kJ} / \mathrm{mol}$

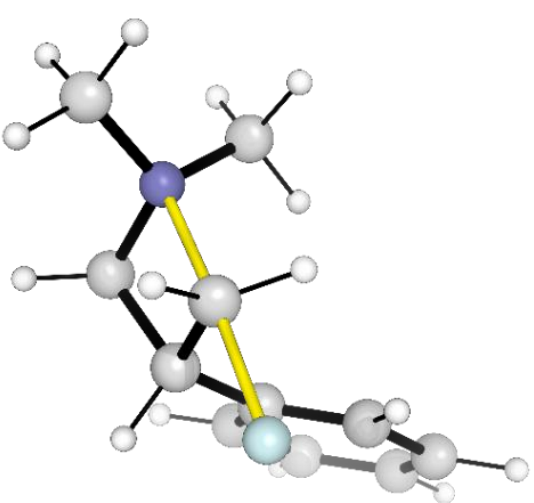

TS-Azet ${ }_{\mathrm{Me}, \mathrm{Me}}-\mathbf{F} 2$

$\mathrm{G}_{\mathrm{rel}}=+3.6 \mathrm{~kJ} / \mathrm{mol}$

Figure S14: TS conformations for delivery of fluoride to Azet $_{\mathbf{M e}, \mathbf{M e}}$

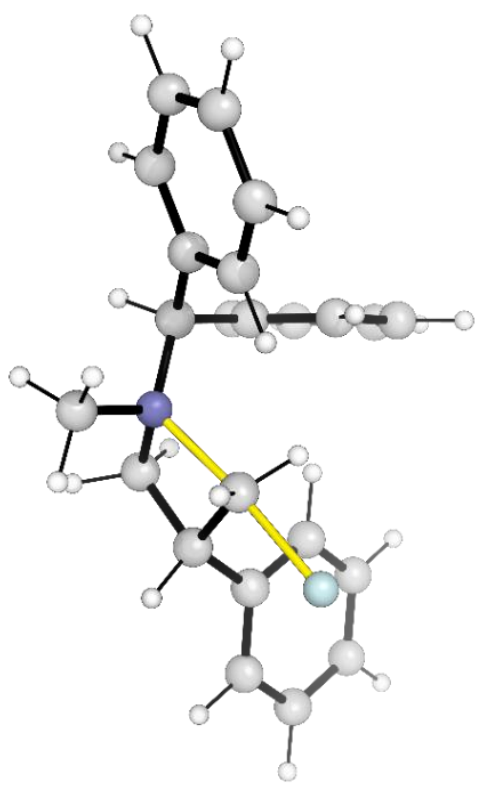

TS-Azet ${ }_{\mathrm{Bzh}, \mathrm{Me}}-\mathbf{F} 1$

$\mathrm{G}_{\text {rel }}=0.0 \mathrm{~kJ} / \mathrm{mol}$

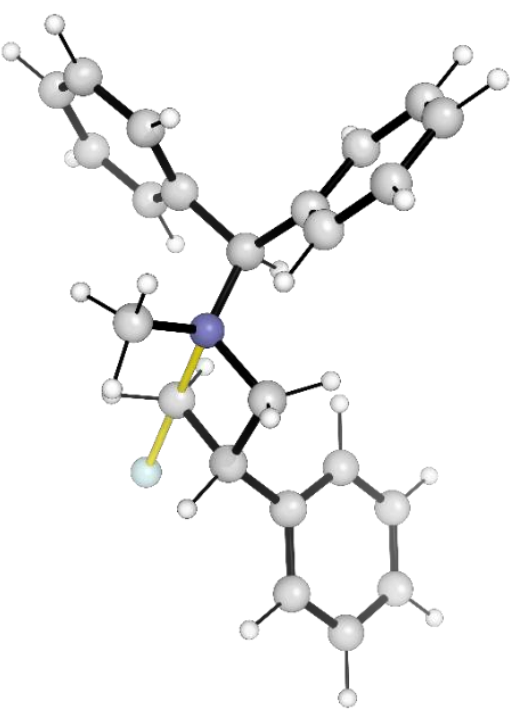

TS-Azet $_{\mathrm{Bzh}, \mathrm{Me}-\mathrm{F} 3} 3$

$\mathrm{G}_{\text {rel }}=+2.3 \mathrm{~kJ} / \mathrm{mol}$

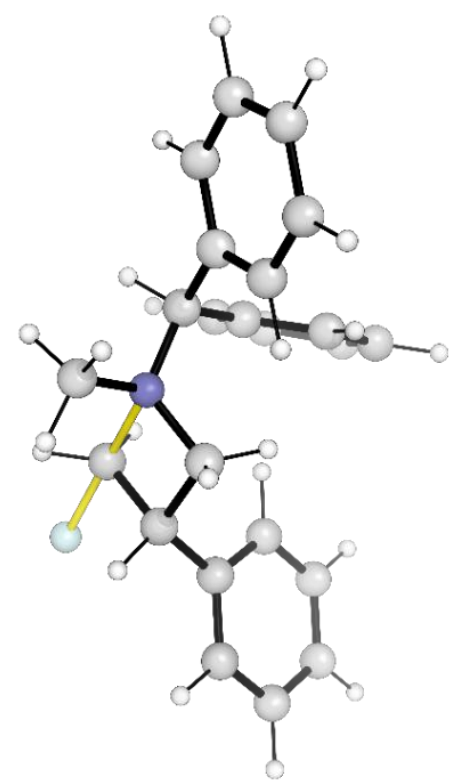

TS-Azet ${ }_{\mathrm{Bzh}, \mathrm{Me}}-\mathbf{F} 2$

$\mathrm{G}_{\text {rel }}=+0.2 \mathrm{~kJ} / \mathrm{mol}$

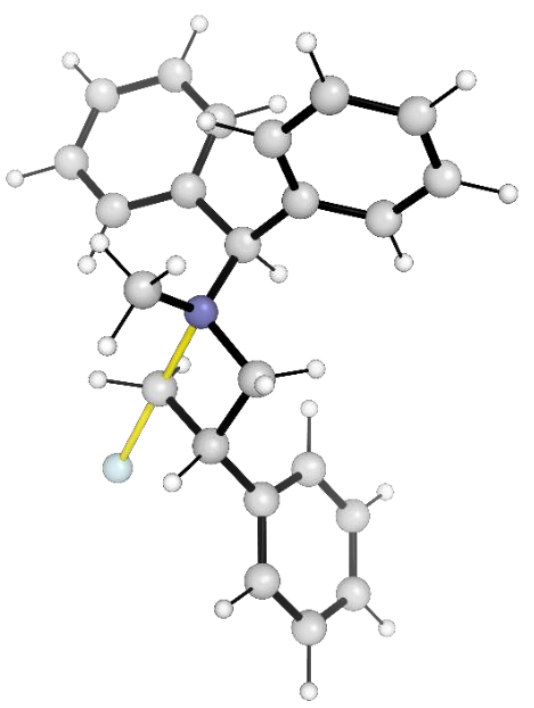

TS-Azet ${ }_{\text {Bzh,Me }}-\mathbf{F} 4$ $\mathrm{G}_{\mathrm{rel}}=+3.5 \mathrm{~kJ} / \mathrm{mol}$ 


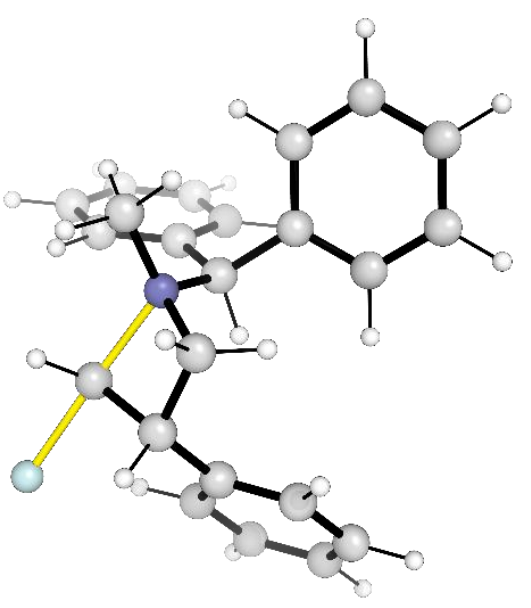

TS-Azet ${ }_{\mathrm{Bzh}, \mathrm{Me}}-\mathbf{F} 5$

$\mathrm{G}_{\mathrm{rel}}=+6.0 \mathrm{~kJ} / \mathrm{mol}$

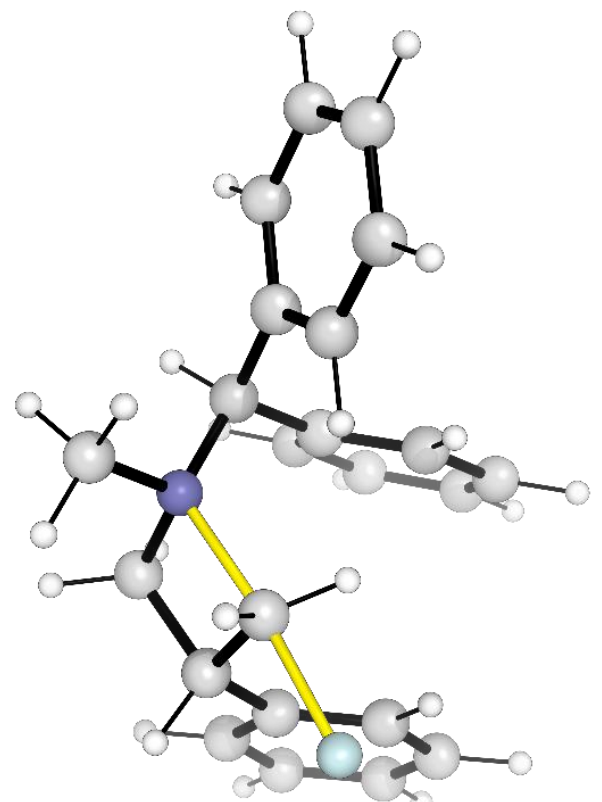

TS-Azet ${ }_{\text {Bzh,Me-F } 7}$

$\mathrm{G}_{\mathrm{rel}}=+8.3 \mathrm{~kJ} / \mathrm{mol}$

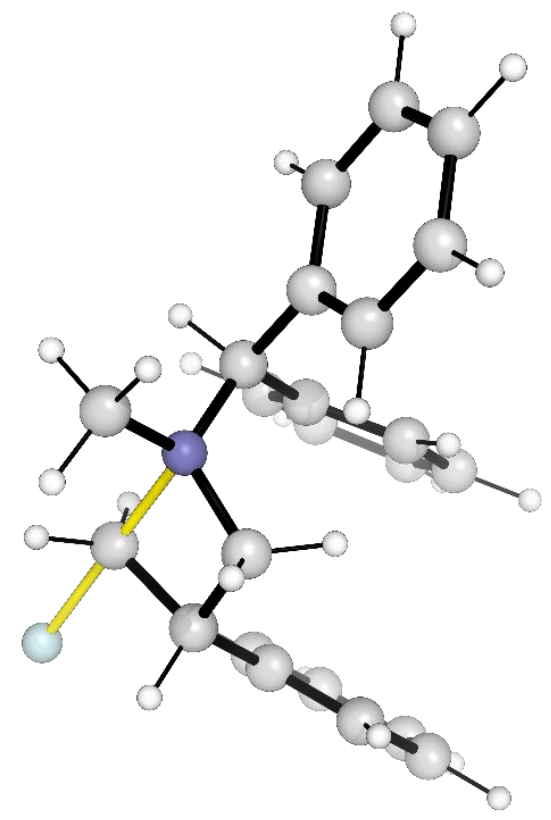

TS-Azet ${ }_{\mathrm{Bzh}, \mathrm{Me}}-\mathbf{F} 6$

$\mathrm{G}_{\mathrm{rel}}=+8.2 \mathrm{~kJ} / \mathrm{mol}$

Figure S15: Geometries of TSs with Azet Bzh,Me.

The lowest energy TSs with all azetidinium ions are given in Figure S16. All azetidinium ions, with the exception of Azet $_{\mathbf{B n}, \mathbf{B n}}$, adopt the same lowest energy conformation as in the free ion (the geometry adopted in

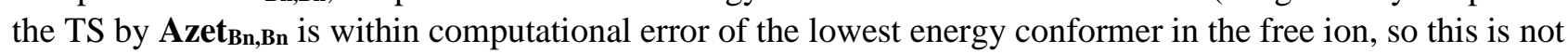
significant). 


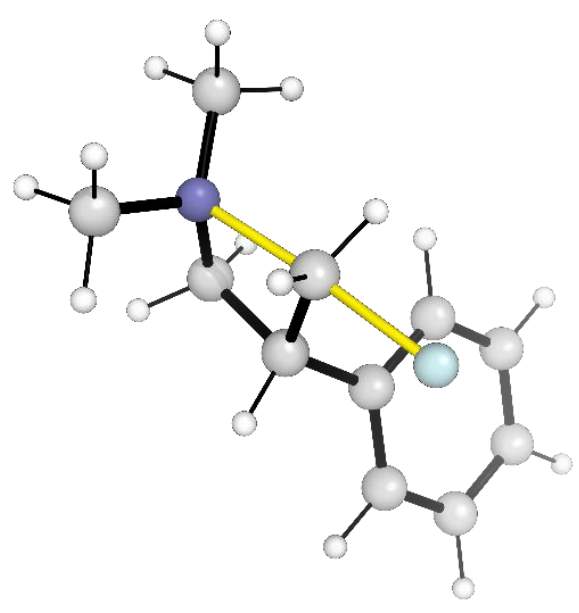

TS-Azet $_{\mathrm{Me}, \mathrm{Me}}-\mathrm{F} 1$

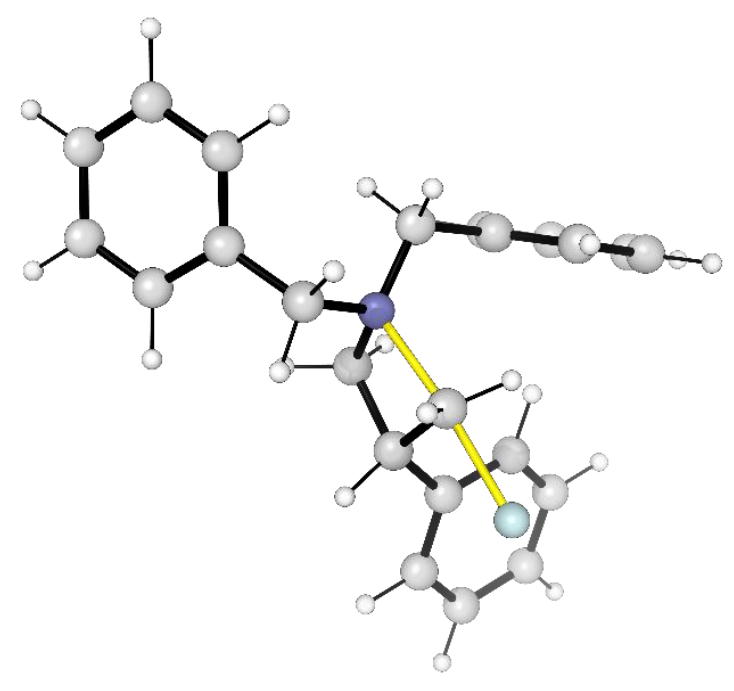

TS-Azet $_{\text {Bn,Bn }}-\mathbf{F} 1$

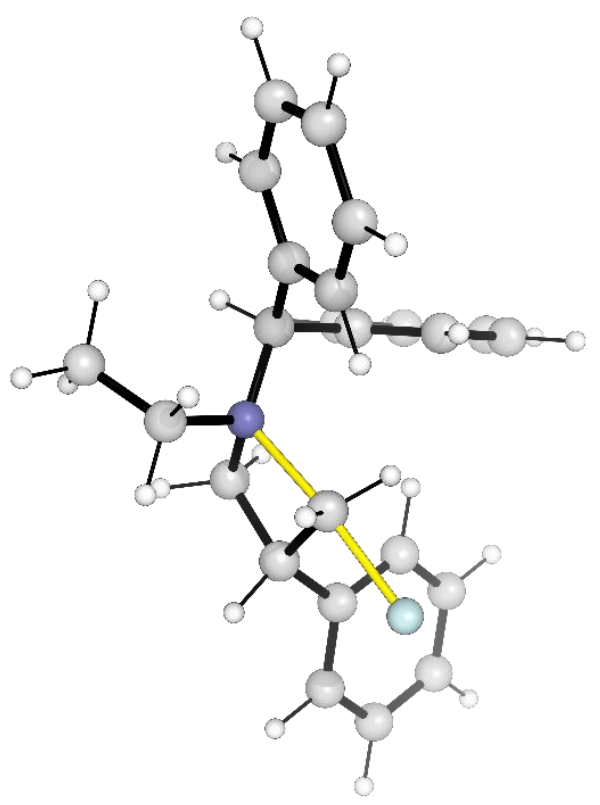

TS-Azet $_{\text {Bzh,Et-F } 1}$

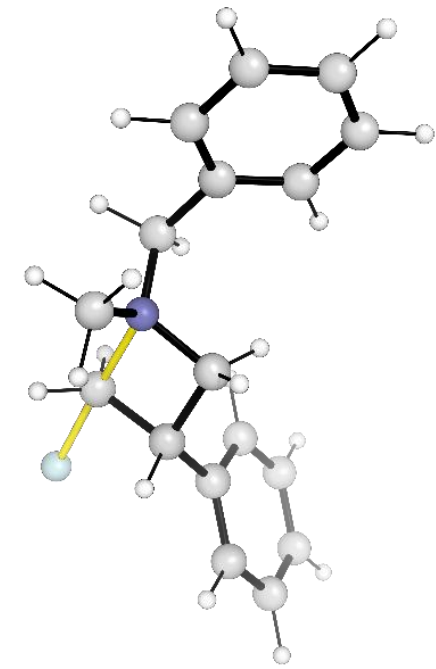

TS-Azet $_{\mathrm{Bn}, \mathrm{Me}}-\mathbf{F} 1$

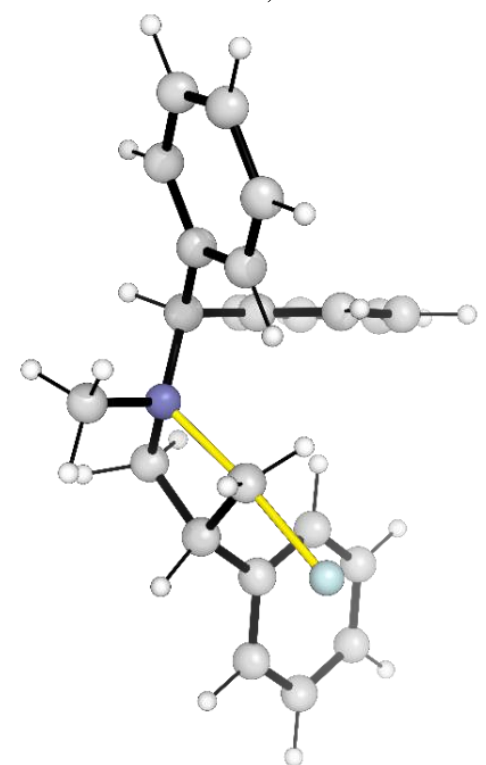

TS-Azet ${ }_{\text {Bzh,Me-F } 1}$

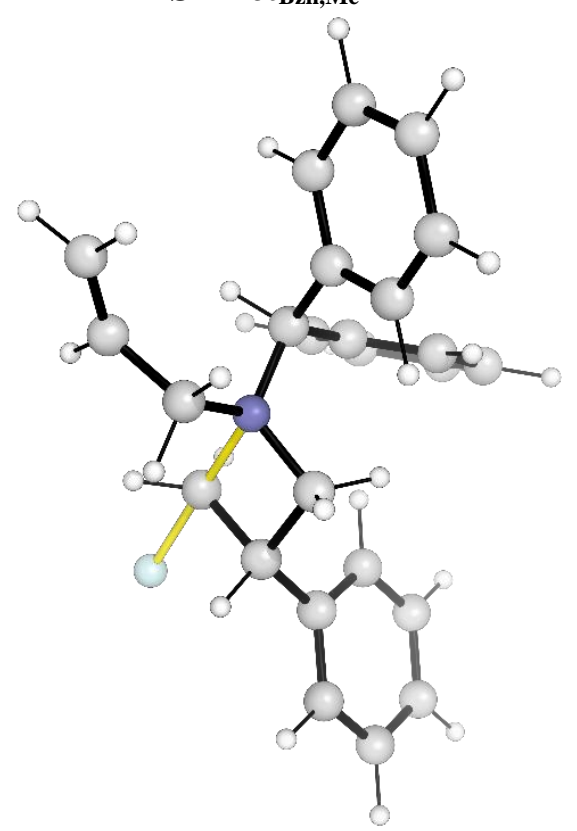

TS-Azet $_{\text {Bzh,Allyl-F } 1}$ 


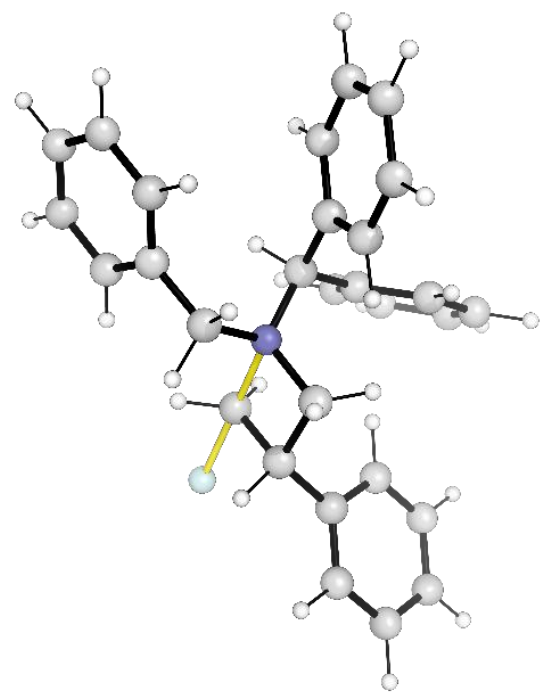

TS-Azet ${ }_{\text {Bzh,Bn-F } 1}$

Figure S16: Geometries of lowest energy TSs with fluoride

\section{Azetidinium Fluorination Energetics}

The computed energies of key states for fluorination of the azetidinium ions are given in Table S17. Species in curly braces, \{\} , indicate a tight ion pair. Lowest energy conformers are used.

Table S17: Gibbs free energies (298.15 K, 1 M) of key species for reactivity of cis-azetidinium ions with fluoride without urea catalyst.

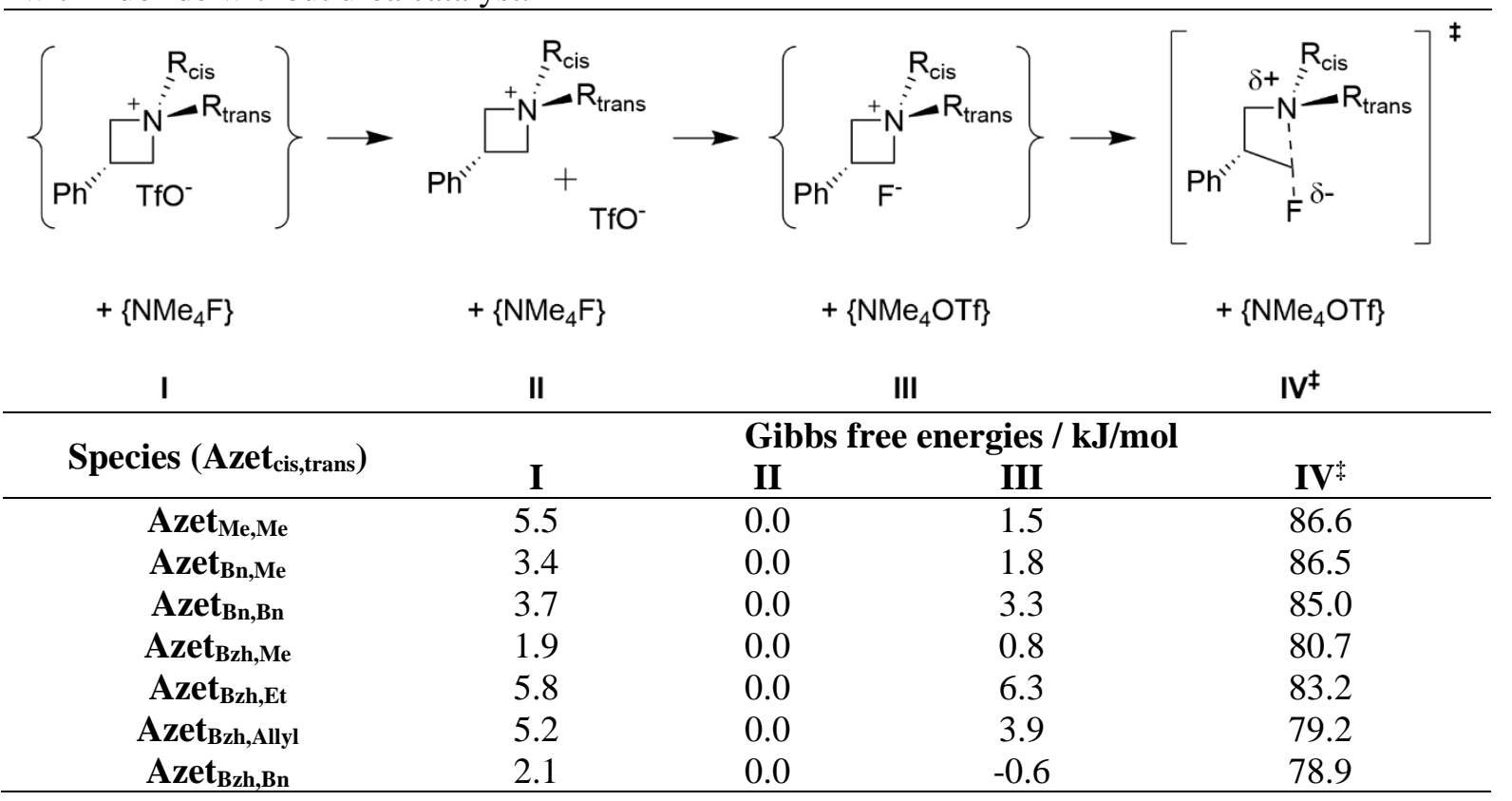

Binding of the azetidinium triflate ion pair is relatively weak in all cases (I-II). Formation of the reactive ion pair from TBAF and the free azetidinium ion (II-III) is approximately neutral in Gibbs free energy, indicating similar affinity for fluoride of $\mathrm{TBA}^{+}$and azetidinium ions. Fluoride delivery then proceeds with a barrier of approximately $75-85 \mathrm{~kJ} / \mathrm{mol}$.

The Gibbs free energy changes and activation barriers for various processes related to fluorination are tabulated in Table S18, with the appropriate kinetic barrier dependent on the reaction mechanism. Activation barriers are plotted against the yields of product obtained under HB PTC conditions (with catalyst) and with TBAF in Figure S17. 
Table S18: Gibbs free energy changes and activation barriers for reactivity of azetidinium ions with fluoride without urea catalyst.

\begin{tabular}{ccccccccc}
\hline & Barrier & \multicolumn{7}{c}{ Azetidinium Gibbs free energies / kJ/mol } \\
& Me,Me & Bn,Me & Bn,Bn & Bzh,Me & Bzh,Et & Bzh,All & Bzh,Bn \\
\hline 1 & $\left\{\right.$ Azet $\left.^{+} \mathrm{OTf}^{-}\right\} \rightarrow$ Azet $^{+}+{ }^{-} \mathrm{OTf}$ & -5.5 & -3.4 & -3.7 & -1.9 & -5.8 & -5.2 & -2.1 \\
2 & $\left\{\mathrm{Azet}^{+} \mathrm{F}^{-}\right\} \rightarrow \mathrm{Azet}^{+}+\mathrm{F}$ & 26.3 & 26.0 & 24.5 & 27.1 & 21.6 & 24.0 & 28.5 \\
3 & $\left\{\mathrm{TBA}^{+} \mathrm{F}^{-}\right\}+\left\{\mathrm{Azet}^{+} \mathrm{OTf}^{-}\right\}$ & -4.0 & -1.6 & -0.3 & -1.1 & 0.4 & -1.3 & -2.7 \\
& $\left\{\mathrm{TBA}^{+} \mathrm{OTf}^{-}\right\}+\left\{\right.$Azet $\left.^{+} \mathrm{F}^{-}\right\}$ & & & & & & & \\
4 & $\left\{\mathrm{TBA}^{+} \mathrm{F}^{-}\right\}+\mathrm{Azet}^{+} \rightarrow \mathrm{TBA}^{+}+$ & -1.6 & -1.3 & 0.2 & -2.4 & 3.1 & 0.7 & -3.8 \\
5 & $\left\{\mathrm{TBA}^{+} \mathrm{F}^{-}\right\}+\mathrm{Azet}^{+} \rightarrow \mathrm{TS}+\mathrm{TBA}^{+}$ & 83.5 & 83.3 & 81.9 & 77.5 & 80.0 & 76.1 & 75.7 \\
6 & $\left\{\mathrm{TBA}^{+} \mathrm{F}^{-}\right\}+\left\{\mathrm{Azet}^{+} \mathrm{OTf}\right\} \rightarrow$ & 81.1 & 83.1 & 81.3 & 78.8 & 77.4 & 74.0 & 76.8 \\
7 & $\left\{\mathrm{TBA}^{+} \mathrm{OTf}^{-}\right\}+\mathrm{TS}$ & 85.1 & 84.6 & 81.7 & 79.9 & 76.9 & 75.3 & 79.5 \\
\hline
\end{tabular}
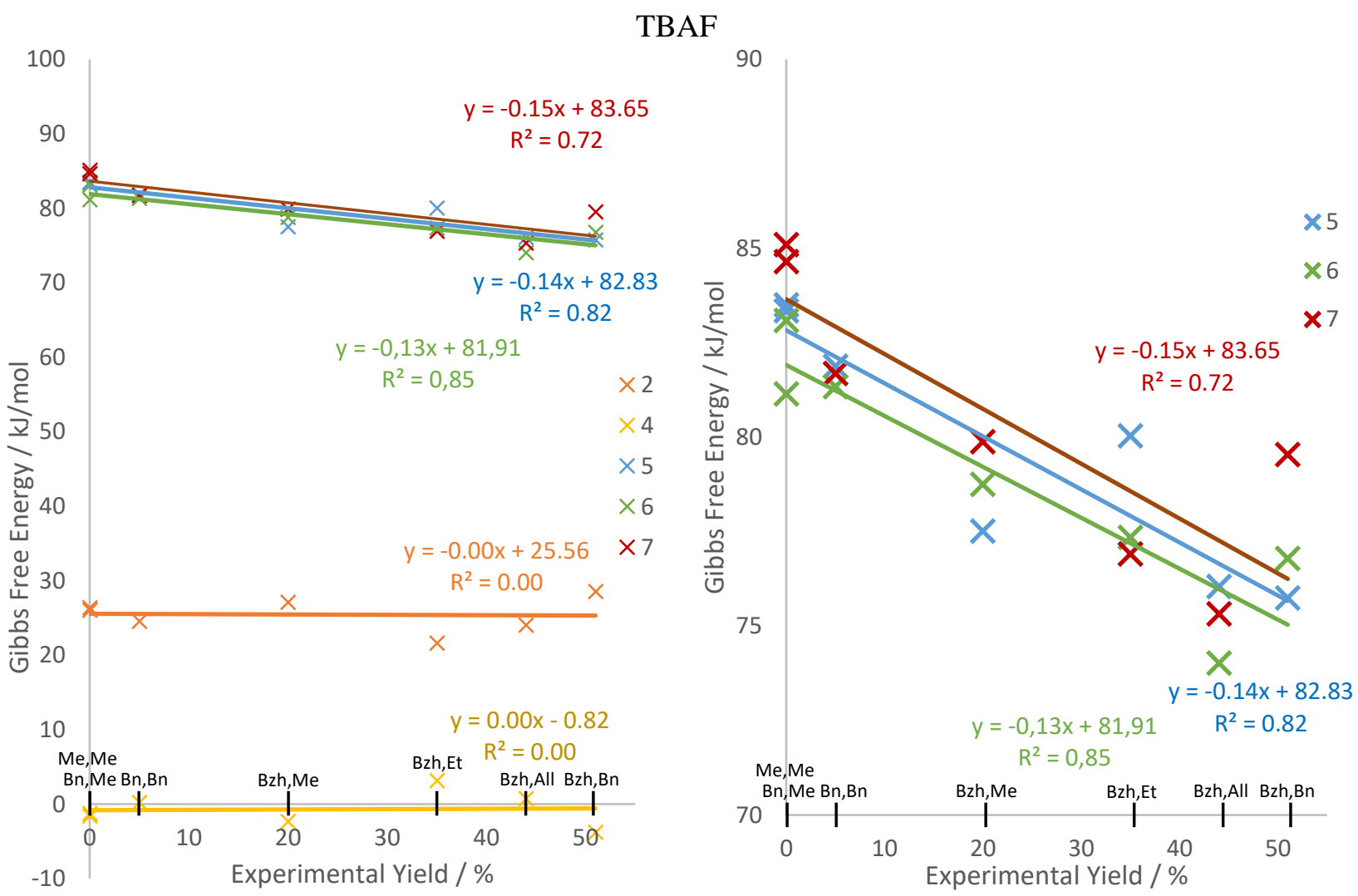


\section{HB PTC}
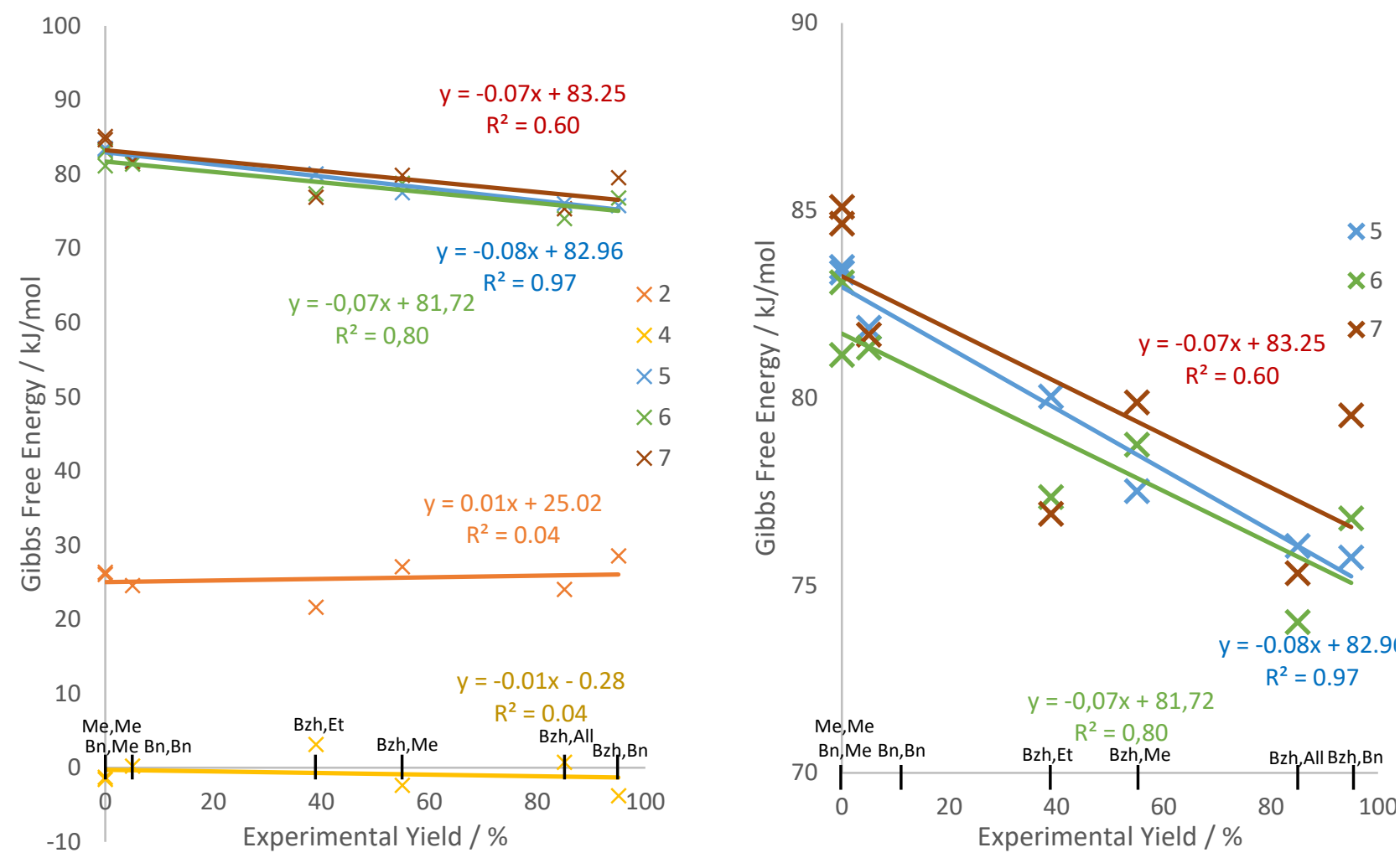

Figure S17: Plot of activation barriers/dissociation energies against experimental fluorination yields. Reactions yielding 'traces' are plotted as 5\% yield. Linear trendlines are plotted to highlight general trends.

Both plots give qualitatively similar results. The uncertainty in computational results should be born in mind when interpreting the results, however, the correlation of several values reduces this. Activation barriers from free azetidinium ion (5), azetidinium triflate ion pair (6) and the reactive ion pair with fluoride (7) correlate negatively against reaction yield under both TBAF and HB PTC conditions, demonstrating consistency of computation and experiment. In contrast, there is no correlation (gradient $\leq 0.01, \mathrm{R}^{2} \leq 0.04$ ) between fluoride binding energy (2) and experimental yield with either fluoride source, suggesting that fluoride binding strength does not strongly influence reaction rate with either fluoride source.

\section{HB PTC Mechanism}

The lack of correlation of experimental yield (for a given reaction time) with the binding strength of azetidinium ion for fluoride suggests that the azetidinium-fluoride ion pair is not present in either of the turnover determining states in the catalyzed reaction (neither TDI nor TDTS). This supports that the azetidinium ion does not participate in a turnover determining phase-transfer process by binding fluoride. For

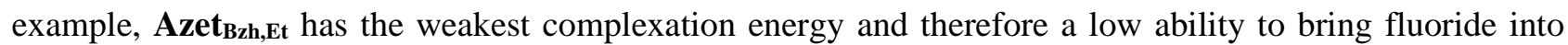
solution, but has a moderate yield. Note that the thermodynamics of phase-transfer may still contribute to the energetic span of the reaction, however the contribution is consistent for all of the azetidinium substrates.

In contrast, the good correlation of yield with the Gibbs free energy barrier to fluoride delivery relative to separated fluoride (5) supports that fluoride delivery by the catalyst in the catalyzed reaction is the TDTS, backed experimentally by the strong dependence of yield on substrate identity and similar reactivity profiles for TBAF and HB PTC conditions.

Additionally, as the intrinsic barrier to fluorination of the azetidinium ion is high, and approaching the maximum energetic span for a viable catalytic reaction at room temperature, the likelihood that one or the other of these states is turnover limiting is high (when coordinated by catalyst). 


\section{The Effect of the Benzhydryl $N$-Substituent}

Experimental yields demonstrate that having a benzhydryl substituent on nitrogen is important for reactivity. This is reflected in computed activation barriers where these substrates have transition state structures lower in Gibbs free energy (relative to separated ion, with TBAF) than those without a benzhydryl group. The series

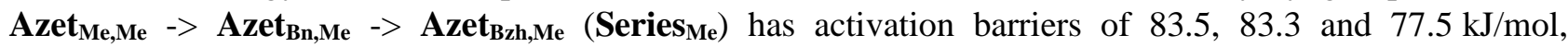
respectively, showing little change $(<1 \mathrm{~kJ} / \mathrm{mol})$ in barrier on conversion of methyl to benzyl, and a much larger change $(6 \mathrm{~kJ} / \mathrm{mol})$ from benzyl to benzhydryl. A similar difference of $6 \mathrm{~kJ} / \mathrm{mol}$ is seen between Azet Bn,Bn $_{\text {and }}$ Azet $_{\mathbf{B z h}, \mathbf{B n}}\left(\right.$ Series $\left._{\mathbf{B n}}\right)$ with barriers of 81.9 and $75.7 \mathrm{~kJ} / \mathrm{mol}$ respectively, indicating a specific effect of the benzhydryl group worth a rate acceleration on the order of 10x over benzyl at room temperature. Critical bond distances are given in Table S19. Both minimum energy conformer and Boltzmann ensemble averaged distances are given.

As reaction yield increases (activation barrier decreases), the $\mathrm{C}-\mathrm{N}$ distance in the ground state ion is increased. In Series $_{M e}$, the average C-N distance in the ground state ions are 1.517, 1.518 and $1.521 \AA$ respectively. Likewise for Series ${ }_{B n}$, the distances are 1.518 and $1.523 \AA$. In both cases, the C-N distance in the benzhydryl substituted ions are significantly longer than those without. C-N distance correlates positively with experimental fluorination yield.

In the TSs, C-F separation increases as experimental yield increases, though without an orderly correlation. C$\mathrm{N}$ separation of the breaking bond demonstrates no clear trend with experimental yield. This corresponds to a more ion pair like TS with increasing experimental yield. Increasing ground state $\mathrm{C}-\mathrm{N}$ bond distances and more ion pair like TS positions are consistent with the Hammond postulate, where the TS more strongly resembles a more strained (closer in energy) ion. This is further supported by the increase in thermodynamic favorability of the ring opening reaction with fluoride in Series Me $_{\text {of }}-85.5,-94.9$ and $-109.2 \mathrm{~kJ} / \mathrm{mol}$, where opening of the benzhydryl substituted ion is most exergonic.

Computation therefore supports that benzhydryl substituted azetidinium ions are intrinsically significantly more reactive than similarly substituted ions (such as benzyl) due to the ion being more strained.

Table S19: Key bond distances with azetidinium ions

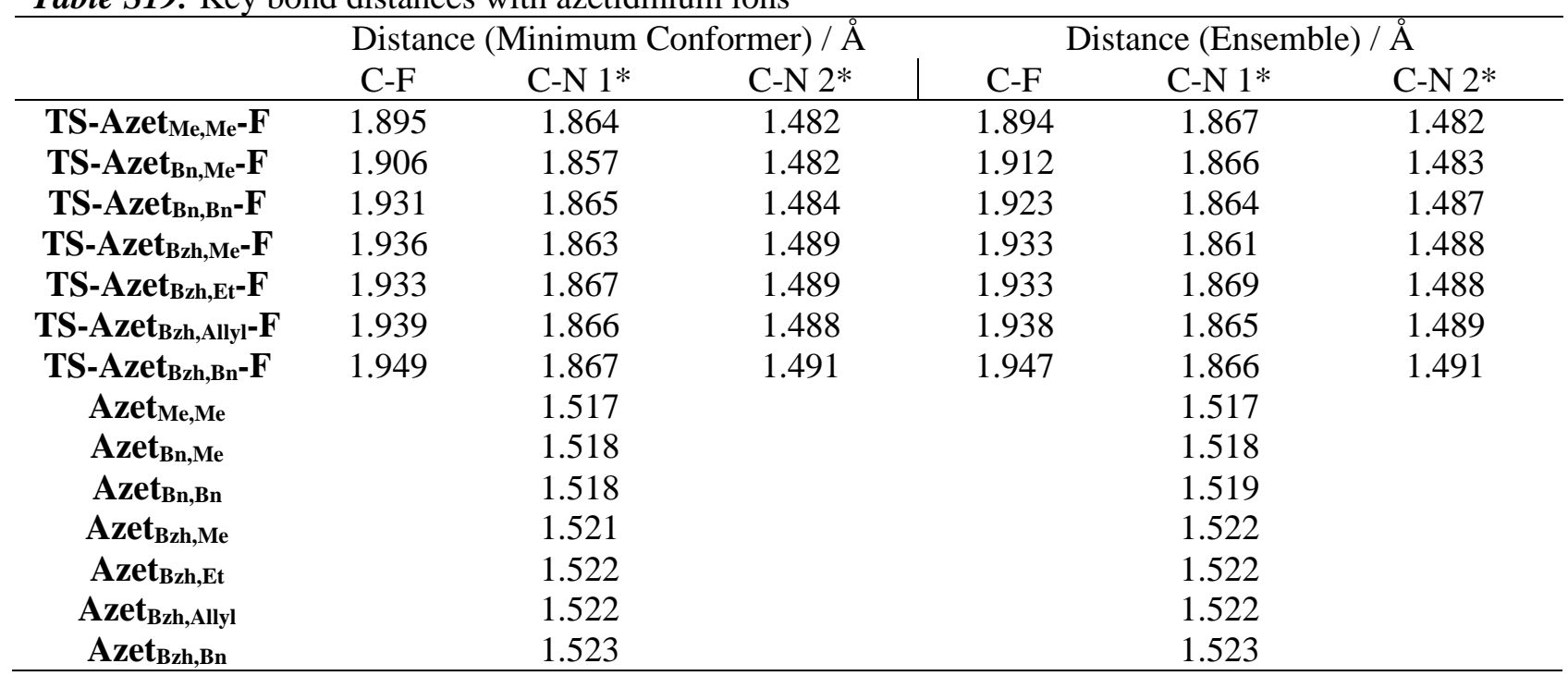



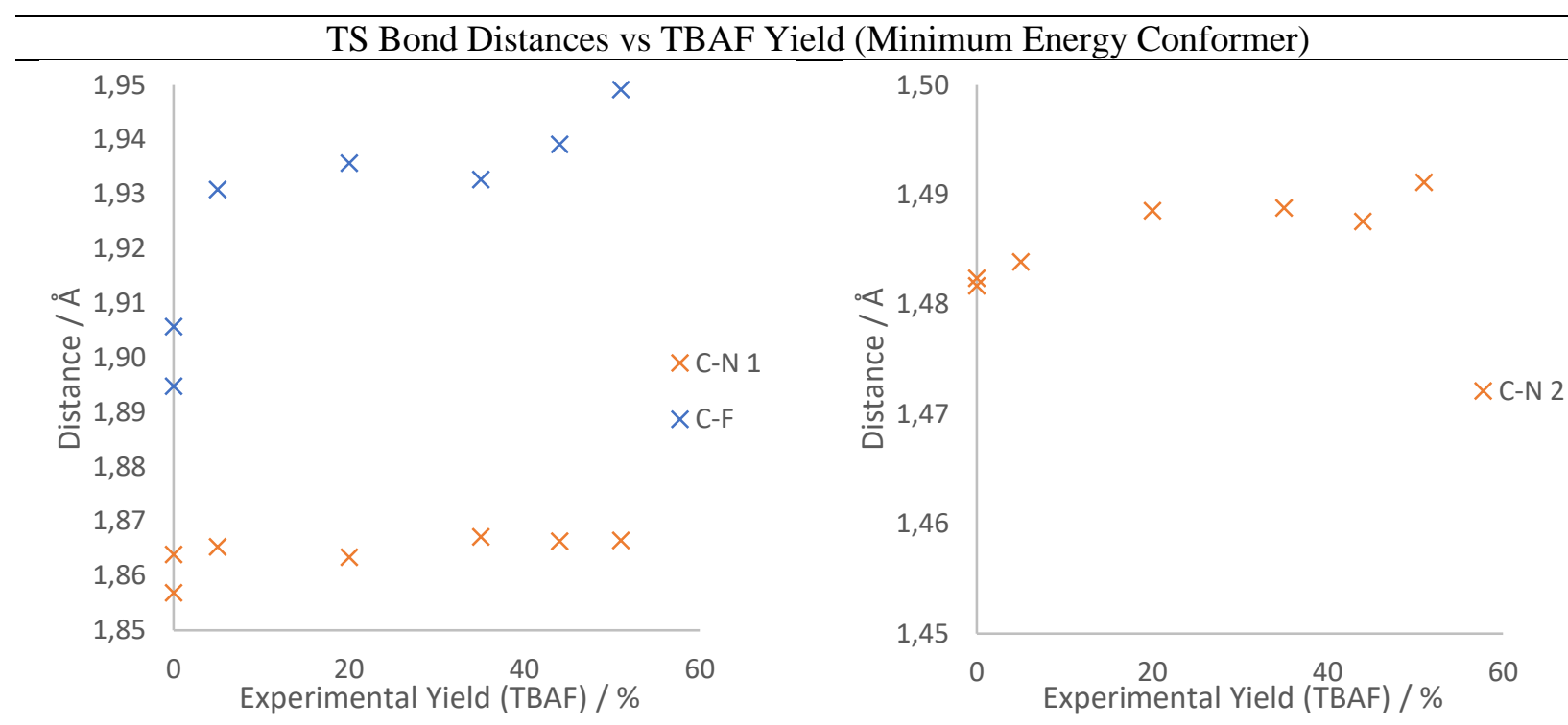

TS Bond Distances vs TBAF Yield (Ensemble)
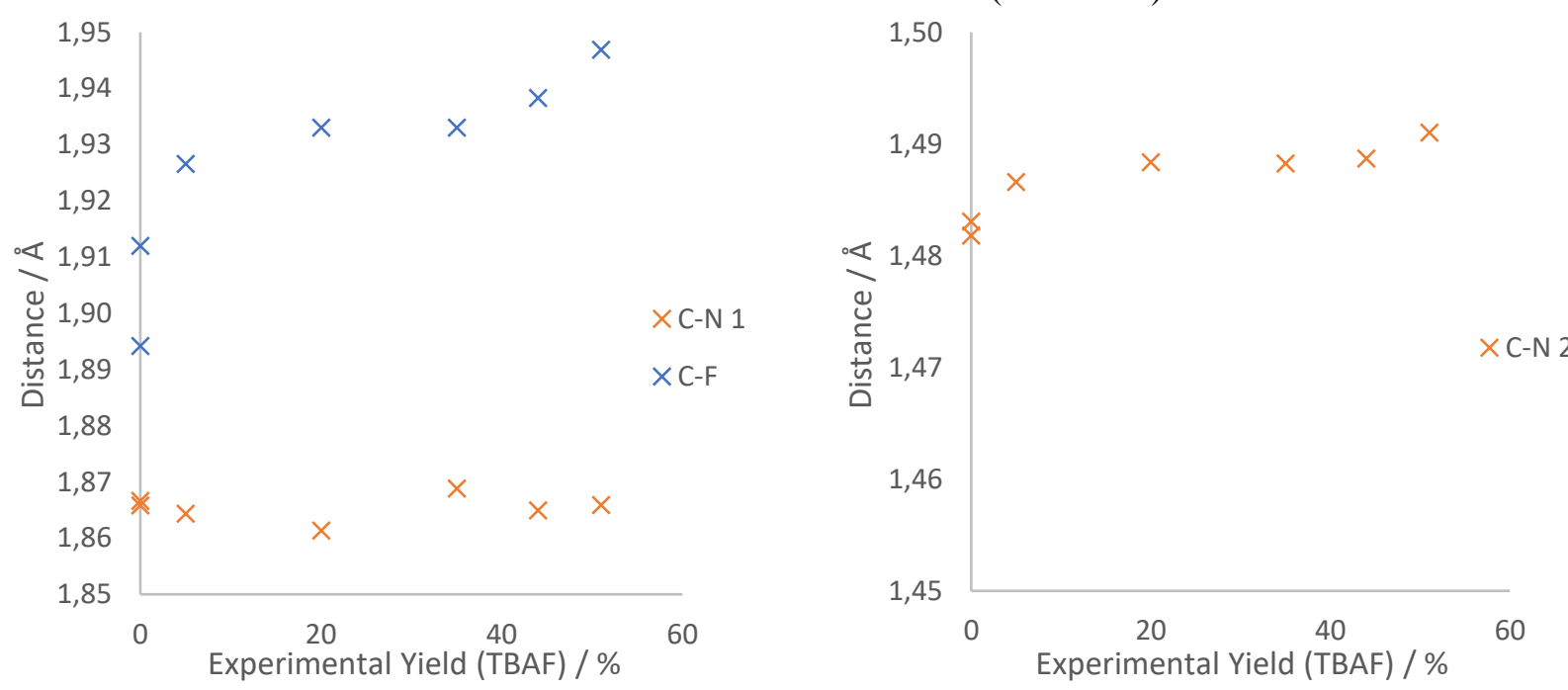

TS Bond Distances vs HBPTC Yield (Minimum Energy Conformer)
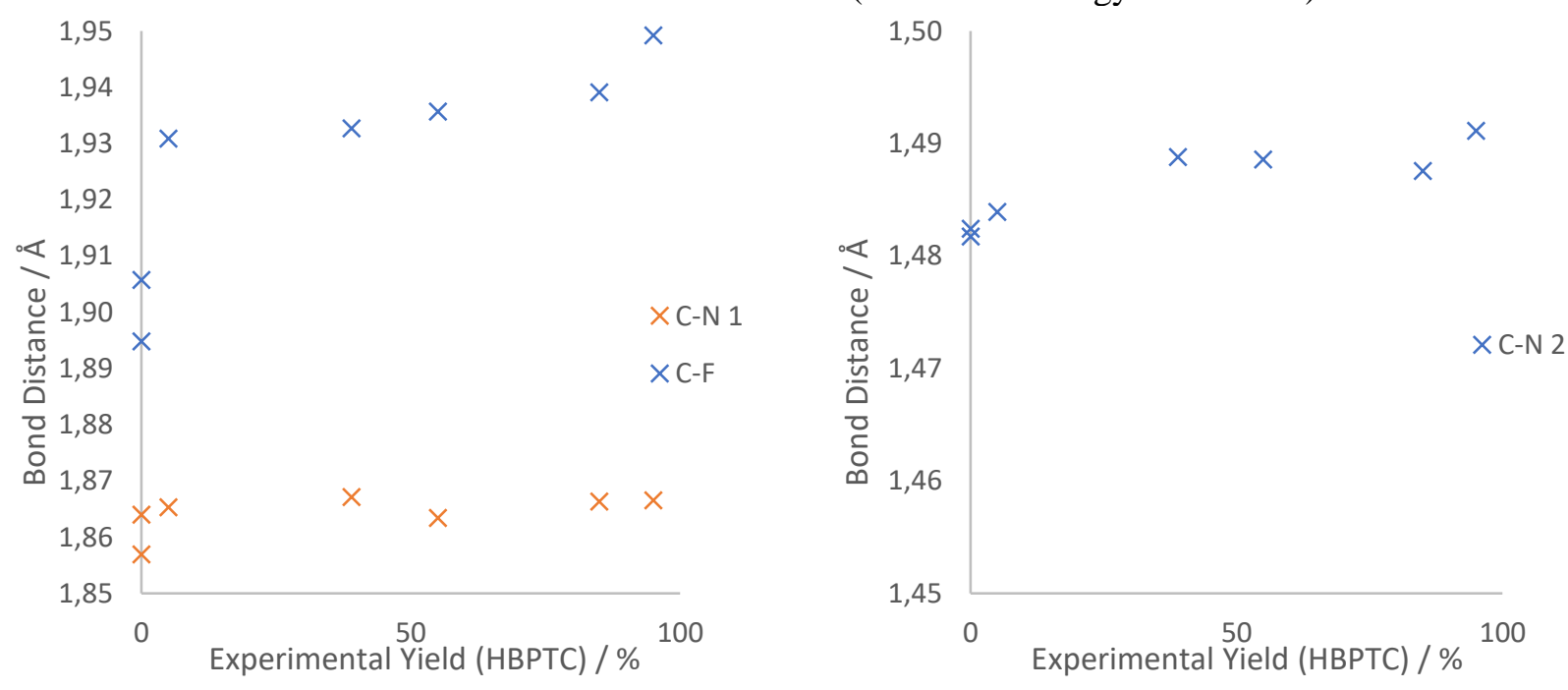


\section{TS Bond Distances vs HBPTC Yield (Ensemble)}
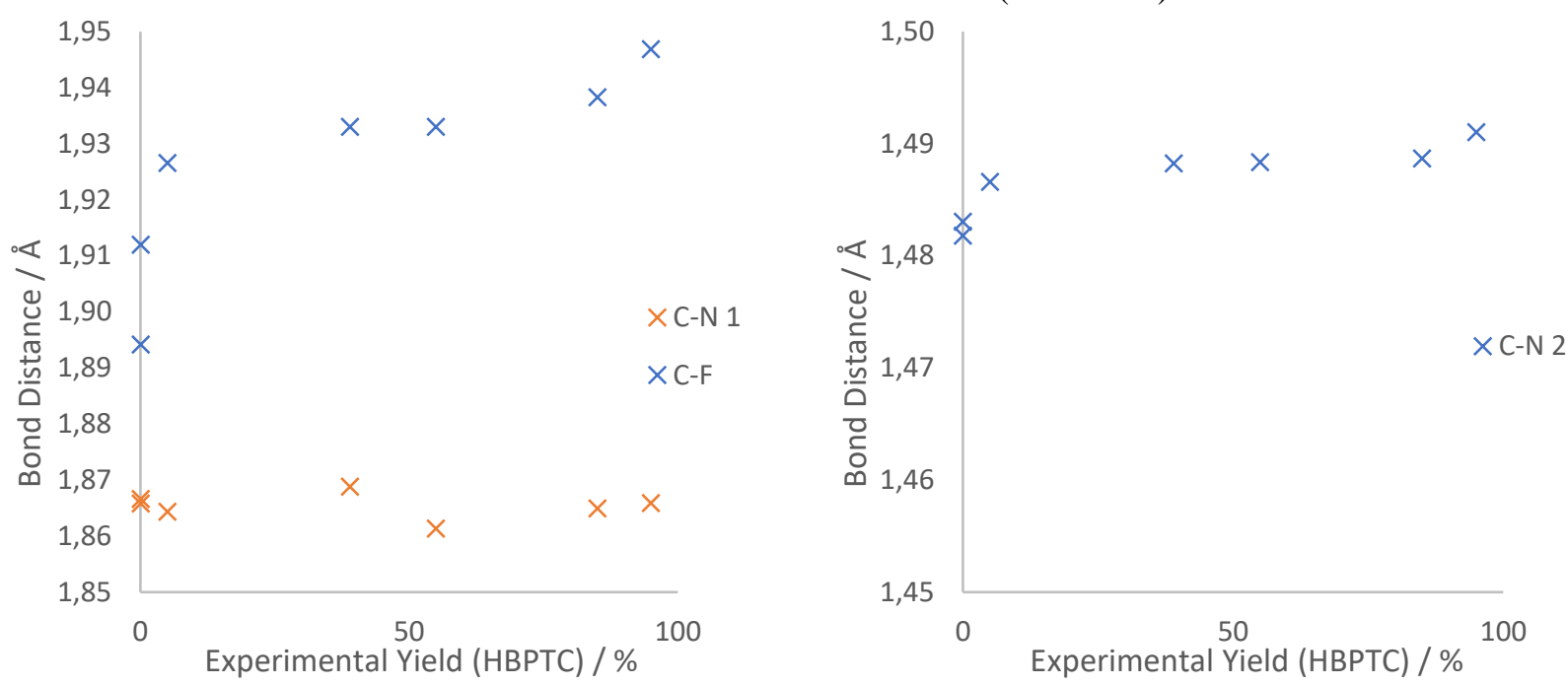

Azetidinium Ion Distances vs TBAF Yield

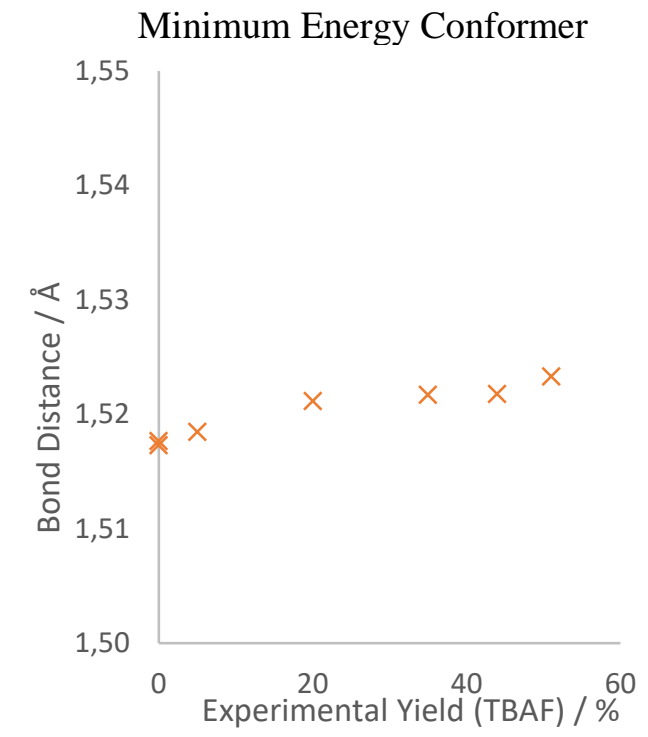

Ensemble

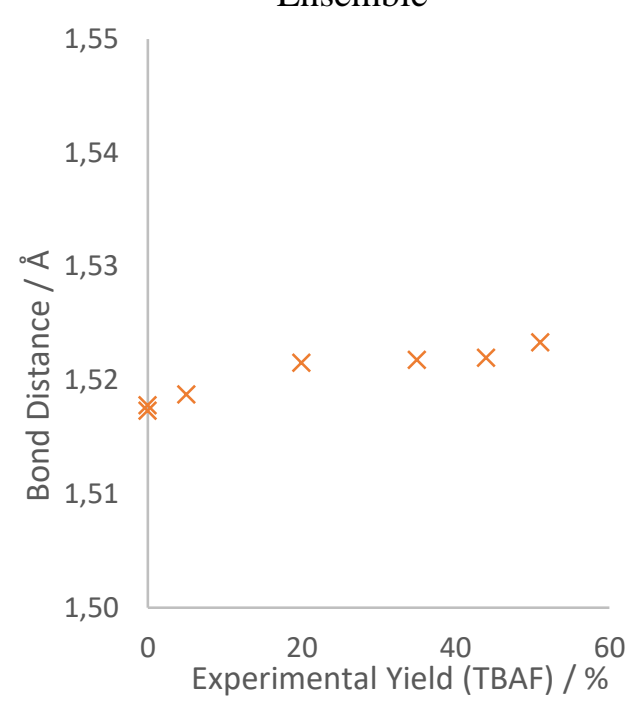

Azetidinium Ion Distances vs HBPTC Yield

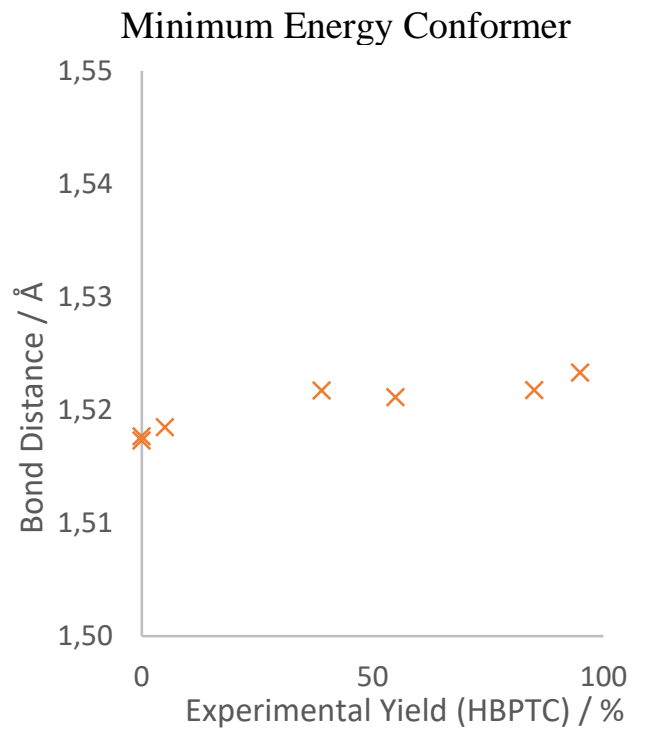

Ensemble

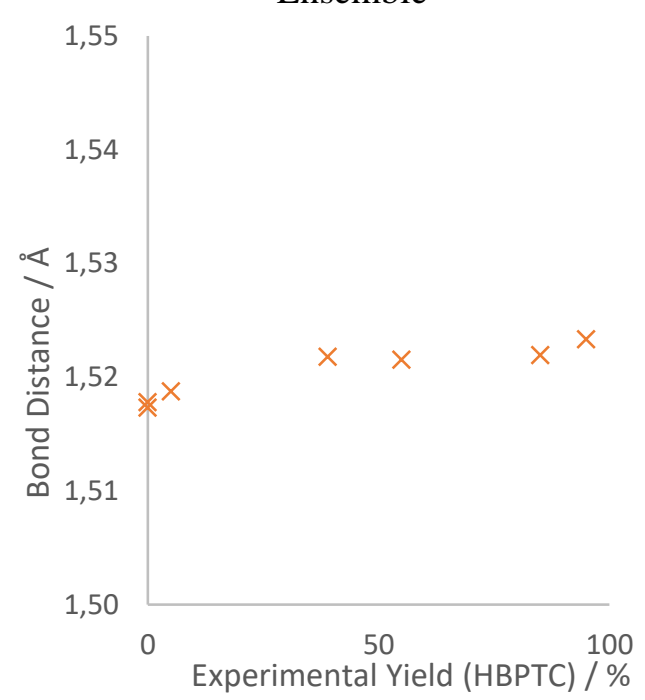




\section{CsF Phase-Transfer}

To further investigate the relative abilities of azetidinium ions and urea hydrogen bond donors to act as phase-transfer catalysts for CsF, the binding energies of Azet $_{\mathbf{M e}, \mathbf{M e}}$ and catalyst $\mathbf{B}$ with a solvated CsF formula unit were evaluated (Figure S18).

Azet $_{\mathrm{Me}, \mathrm{Me}}$ binds a CsF ion pair weakly, with $\Delta \mathrm{G}=-14.0 \mathrm{~kJ} / \mathrm{mol}$, slightly overcoming the entropic penalty of coordination. Binding is weaker over the naked fluoride ion due to competition with the cesium ion and loss of rotational entropy of the CsF unit on coordination. Catalyst $\mathbf{B}$ on the other hand binds CsF much more strongly with $\Delta \mathrm{G}=-69 \mathrm{~kJ} / \mathrm{mol}$, originating from the three strong hydrogen bonds to fluoride. These results demonstrate that the neutral hydrogen bonding urea catalyst has a significantly higher potential to act as a CsF phase-transfer catalyst than the azetidinium ion.

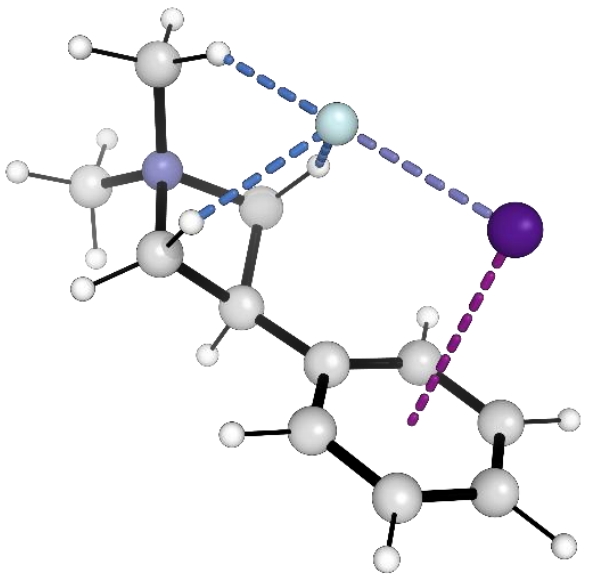

Azet $_{\mathrm{Me}, \mathrm{Me}-\mathrm{FCs} 1}$

$\mathrm{G}_{\mathrm{rel}}=0.0 \mathrm{~kJ} / \mathrm{mol}$

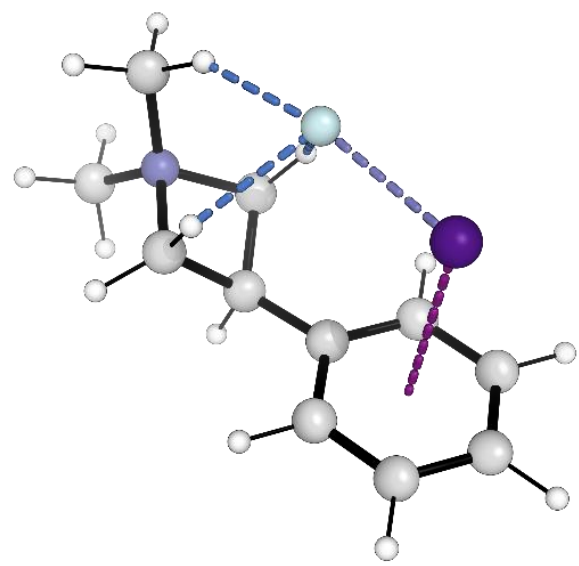

Azet $_{\mathrm{Me}, \mathrm{Me}}-\mathrm{FCs} 3$

$\mathrm{G}_{\text {rel }}=+3.0 \mathrm{~kJ} / \mathrm{mol}$

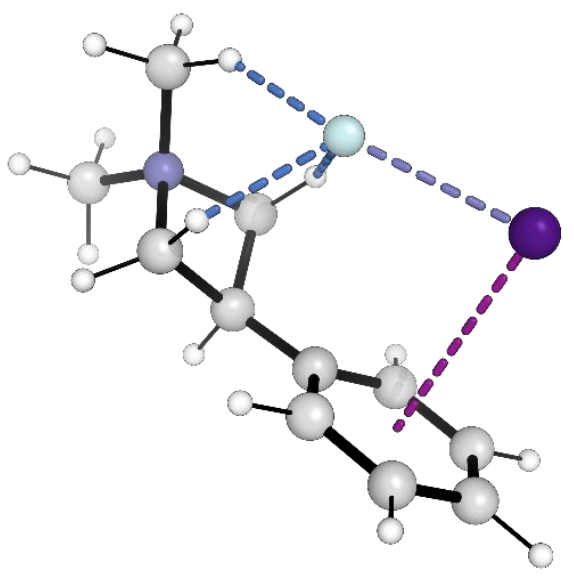

Azet $_{\mathrm{Me}, \mathrm{Me}-\mathrm{FCs} 2}$

$\mathrm{G}_{\mathrm{rel}}=+2.8 \mathrm{~kJ} / \mathrm{mol}$

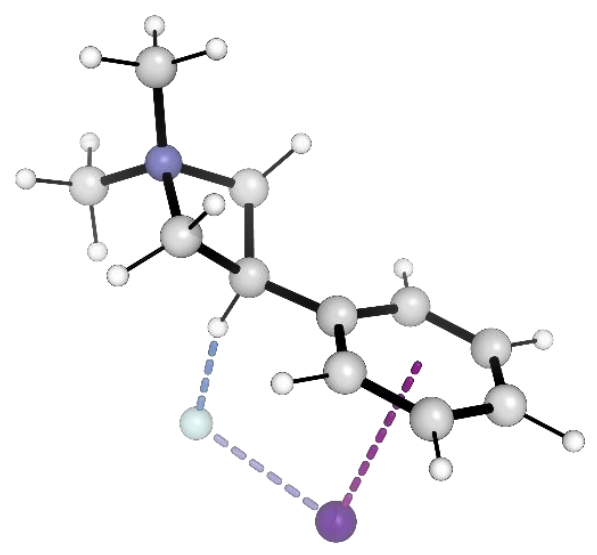

Azet $_{\mathrm{Me}, \mathrm{Me}}-\mathrm{FCs} 4$

$\mathrm{G}_{\text {rel }}=+11.3 \mathrm{~kJ} / \mathrm{mol}$ 


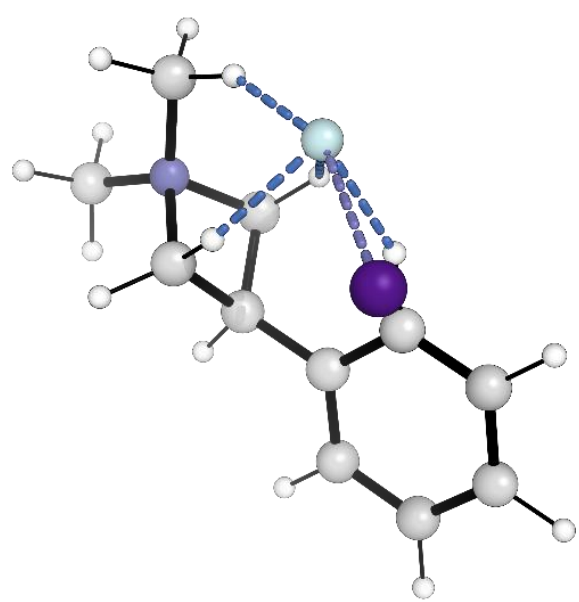

Azet $_{\mathrm{Me}, \mathrm{Me}-\mathrm{FCs} 5}$

$\mathrm{G}_{\text {rel }}=+12.3 \mathrm{~kJ} / \mathrm{mol}$

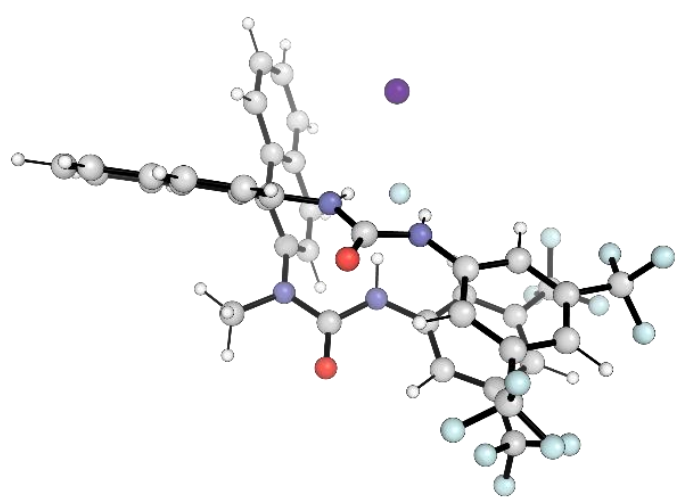

Cat B-FCs 1

$\mathrm{G}_{\text {rel }}=0.0 \mathrm{~kJ} / \mathrm{mol}$

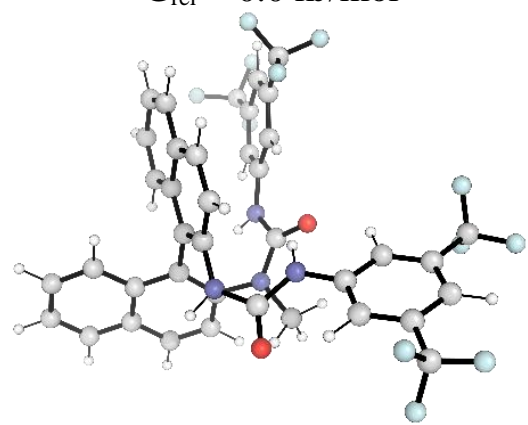

Cat B

Figure S18: Geometries of CsF complexes.

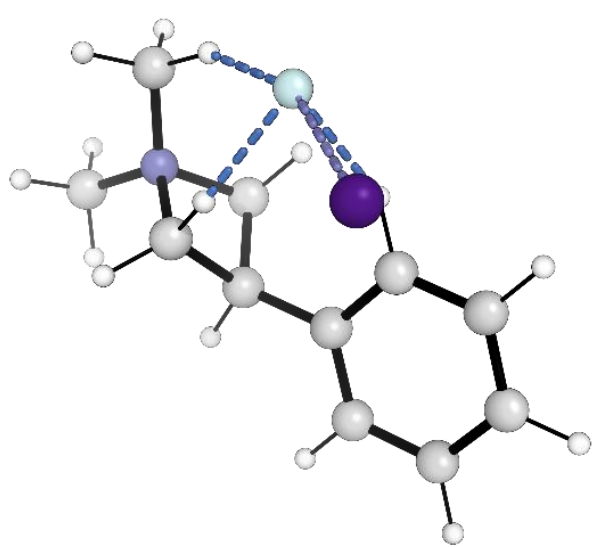

Azet $_{\mathrm{Me}, \mathrm{Me}-\mathrm{FCs} 6}$

$\mathrm{G}_{\mathrm{rel}}=+12.6 \mathrm{~kJ} / \mathrm{mol}$

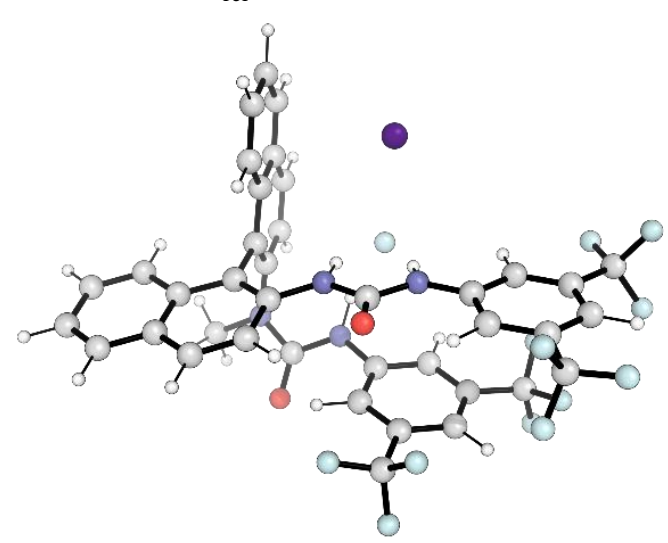

Cat B-FCs 2

$\mathrm{G}_{\mathrm{rel}}=+2.6 \mathrm{~kJ} / \mathrm{mol}$ 
Enantioselectivity was first evaluated on substrate Azet $_{\text {Bzh,Me }}$ with $(\boldsymbol{S})$-D catalyst. Gibbs free energies are evaluated at $298.15 \mathrm{~K}$ and $1 \mathrm{M}$ concentration unless otherwise stated.<smiles>CN1C[C@@H](c2ccccc2)C1c1ccccc1</smiles>

Azet $_{\text {Bzh,Me }}$<smiles>CN(Cc1c(NC(=O)Nc2cc(C(F)(F)F)cc(C(F)(F)F)c2)ccc2ccccc12)C(=O)Nc1ccc2ccccc2c1C(F)(F)F</smiles>

$(S)-\mathrm{D}$

\section{Transition State Structures}

The transition state structures to both enantiomers of product with cis substrate were simulated using classical molecular dynamics for $300 \mathrm{~ns}$, leading to a total simulation time of $600 \mathrm{~ns}$. All frames with F---C---N > 150 in each trajectory were used for clustering with RMSD cutoff of $1.0 \AA$. For attack at the carbon leading to $(R)$ product, herein referred to as $R$-attack, 21796 frames were clustered, resulting in 10 clusters of greater than $1 \%$ weight. Lower weight clusters were manually inspected and verified that they contained no additional conformations of interest. The central frame from each cluster was then optimized using DFT, resulting in 10 unique TSs to $(R)$ product. The same procedure was followed for S-attack, with 20495 frames clustered, resulting in 8 clusters of over $1 \%$ weighting, and 8 unique DFT optimized TSs to (S) product. The intramolecular T-shape $\mathrm{CH}-\pi$ interaction of the azetidinium was sampled manually. A total of 24 TSs were optimized - 14 to $(S)$ product and 10 to $(R)$ product - spanning an energy range of $41 \mathrm{~kJ} / \mathrm{mol}$. The ensemble indicates that $(S)$ catalyst affords $(S)$ product in $54: 46$ e.r. in 1,2-dichloroethane at $298.15 \mathrm{~K}$. The experimental enantioinduction is 80.5:19.5 e.r., consistent within computational error (approximately $3.5 \mathrm{~kJ} / \mathrm{mol}$ deviation).

The Gibbs free energy distribution of the low energy TSs is illustrated in Figure S19. Key geometric parameters are tabulated in Table S20. The highest contributing TSs to major and minor product are shown in more detail in Figure S20.

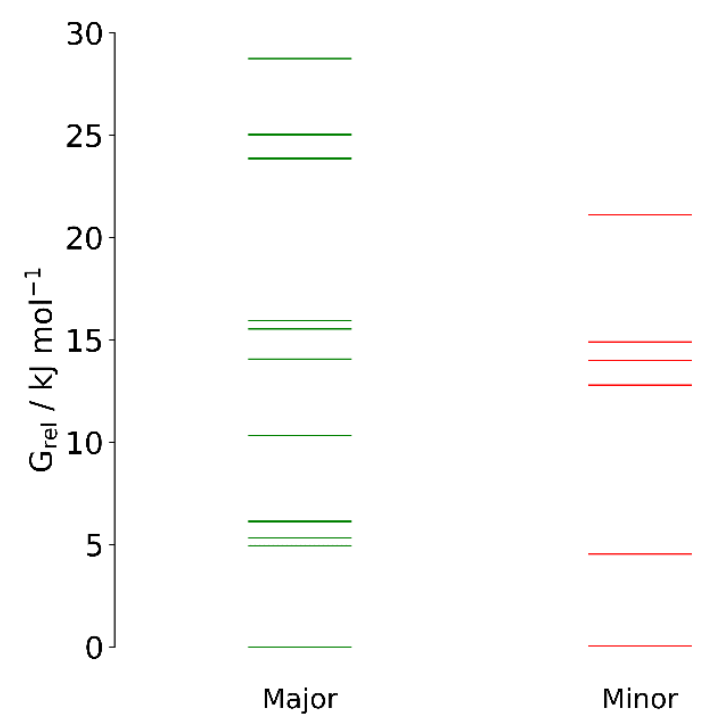

Figure S19: Gibbs free energy distribution of TSs in the ensemble. 


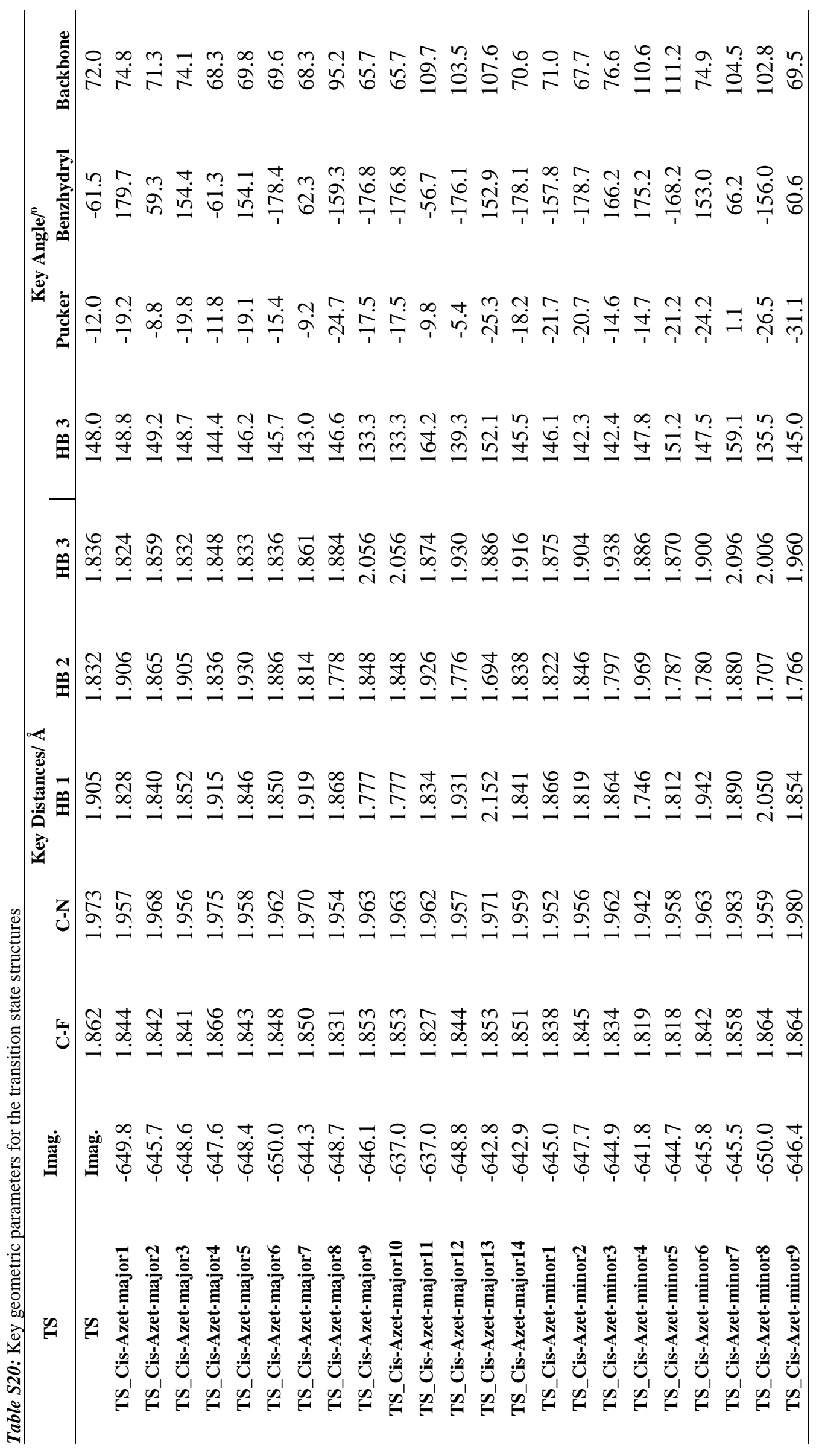


TS $_{\text {Major-Cis }}$

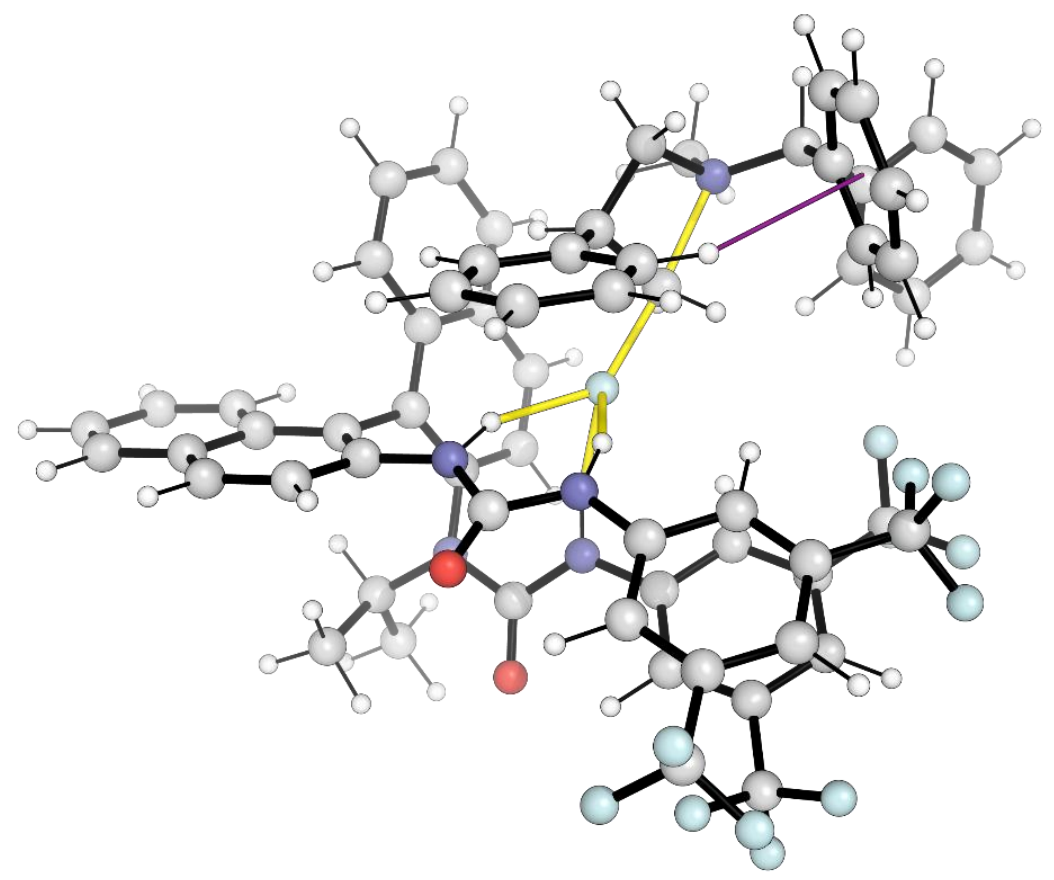

TS $_{\text {Minor-Cis }}$

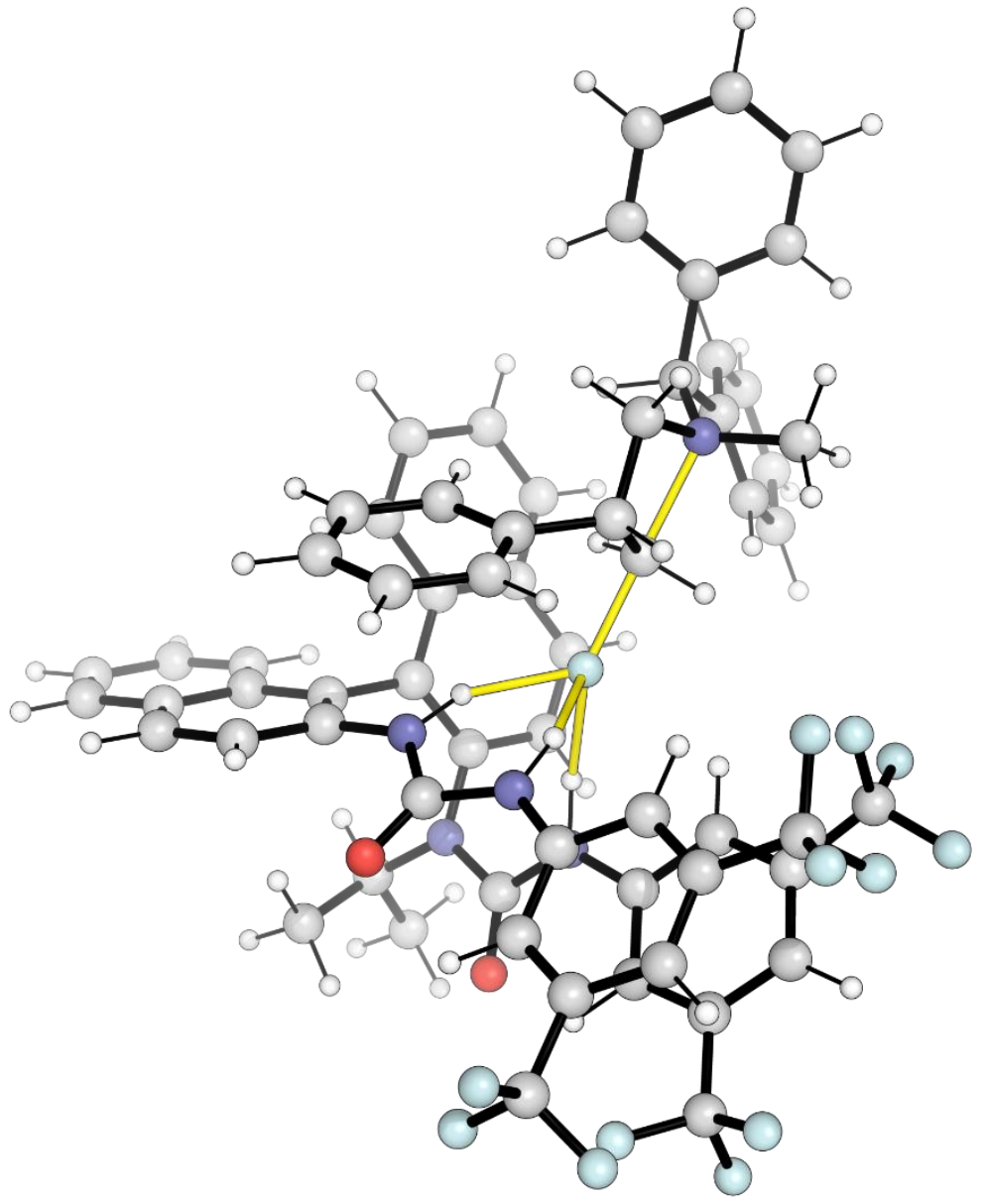

Figure S20: Lowest energy TSs to major and minor product. Azetidinium intramolecular T-shape $\mathrm{CH}-\pi$ interaction illustrated in purple in $\mathbf{T} \mathbf{S}_{\text {Major-Cis. }}$. 


\section{Origins of Enantioselectivity}

The two lowest energy TSs, with $\Delta \Delta \mathrm{G}^{\ddagger}=0.0 \mathrm{~kJ} / \mathrm{mol}$, are superimposed in Figure S21. Catalyst geometry superimposes well. The phenyl group bonded to the azetidinium ring sits in the same place in both TSs, and results in a slight twist of the azetidinium ring. The benzhydryl group points in different directions in the two TSs.

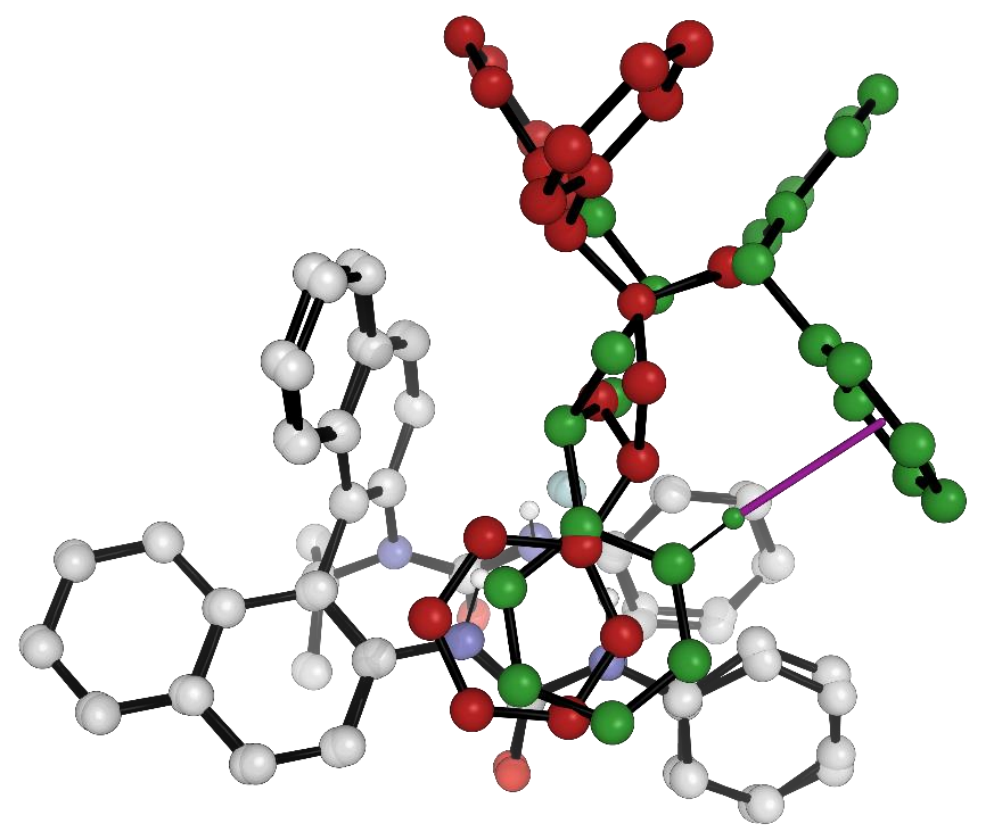

Figure S21: Superposition of $\mathbf{T S}_{\text {Major-Cis (green) and } \mathbf{T S}}$ Minor-Cis (red). Intramolecular T-shape CH$\pi$ interaction is shown in purple.

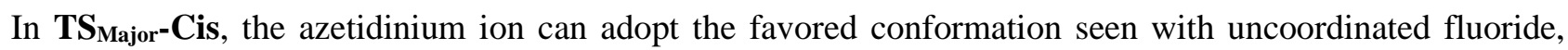
forming an intramolecular $\mathrm{T}$-shape $\mathrm{CH}-\pi$ interaction. The shape of the azetidinium ion, determined primarily by the phenyl group bonded to the azetidinium ring, fits neatly into the catalytic pocket, with phenyl group lying parallel to the bidentate urea. In contrast, the favored $\mathrm{CH}-\pi$ conformation cannot be adopted when delivering fluoride to the other carbon. In the lowest energy minor TS, the proximity of the binam backbone prevents formation of the $\mathrm{CH}-\pi$ interaction due to steric clash, however this must be compensated by stronger substrate-catalyst interactions. The difference in energy between the two azetidinium conformers in the absence of fluoride is $4.3 \mathrm{~kJ} / \mathrm{mol}$, reduced to $2.3 \mathrm{~kJ} / \mathrm{mol}$ on uncoordinated fluoride delivery.

\section{Distortion/Interaction-Activation Strain Analysis}

Intrinsic reaction coordinate (IRC) calculations were run for $\mathbf{T S}_{\text {Major-Cis }}$ and $\mathbf{T S}_{\text {Minor-Cis }}$ and the distortion/interaction-activation strain analysis of Bickelhaupt and Houk applied. ${ }^{51}$ The system was partitioned into $\{$ catalyst-fluoride complex $\}+\{$ azetidinium substrate $\}$. The results of the analysis are given in Figure S22.

In both cases the total energy begins negative, as bringing the negatively charged fluoride complex and positively charged substrate together involves favorable coulombic interactions. In general, the profiles are very similar for both IRCs. IRC $\mathbf{I}_{\text {Minor }}$ consistently has stronger interaction than $\mathbf{I R C}_{\text {Major }}$, however this is accompanied by higher distortion. Interestingly, towards the TS positions, there is little difference in distortion despite the substrate adopting a less favored conformer along $\mathbf{I R C}_{\text {Minor. }}$. The TS positions are very similar, so this is not a significant factor when comparing the distortions at the respective TS positions. 


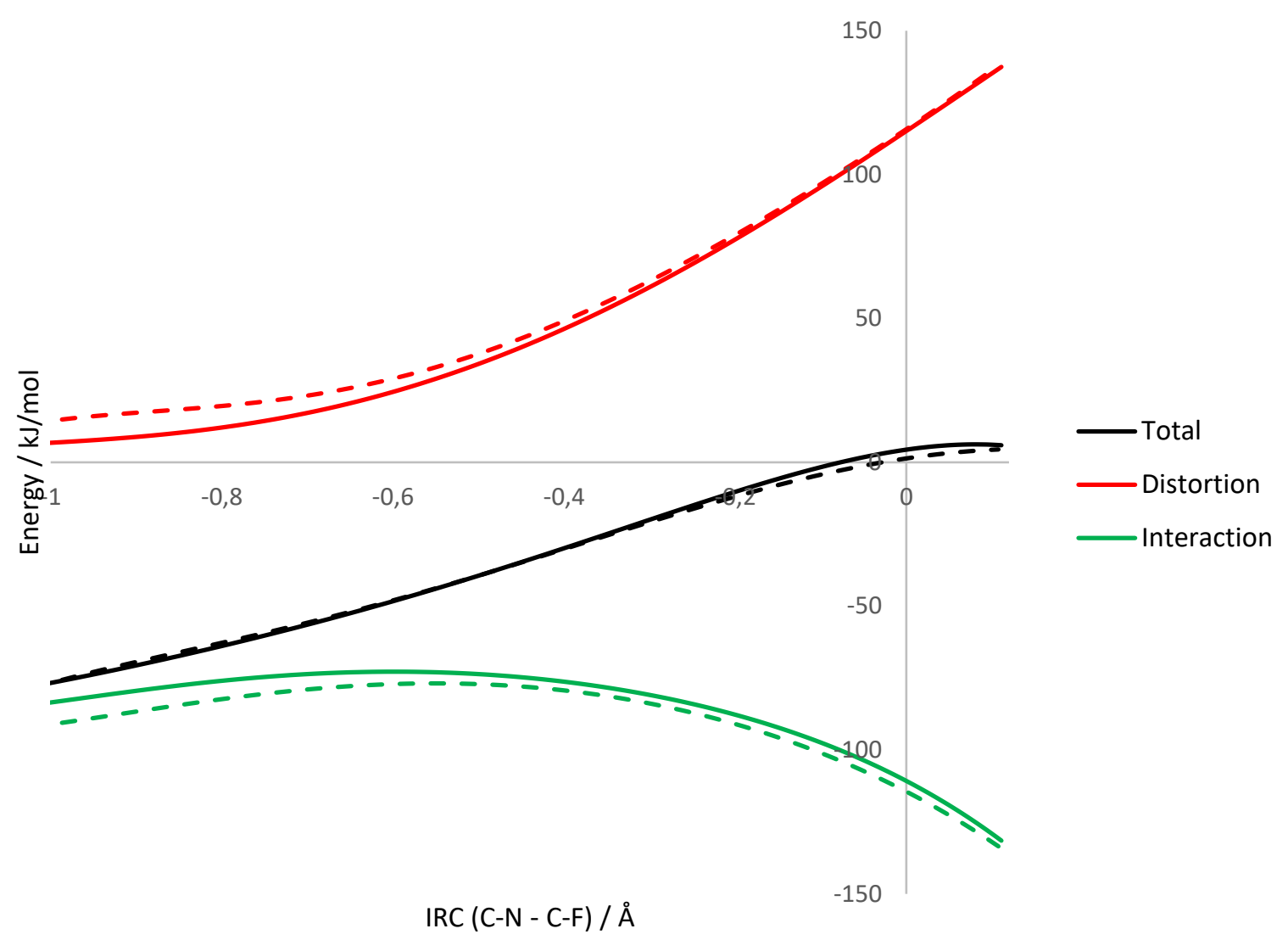

Figure S22: Distortion/interaction-activation strain analysis of fluoride delivery in $\mathbf{T} \mathbf{S}_{\text {Major-Cis }}$ and

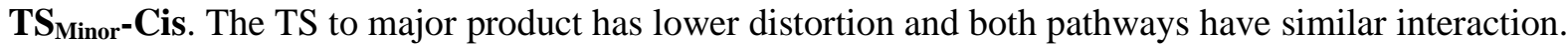
The difference in TS position is minimal. 


\section{Truncated Models}

To probe the contributions of different structural elements to enantioselectivity, the TSs were truncated in various ways, and the energy re-evaluated without optimization (Figure S23). Energies are tabulated in Table S21. Whilst the error in $\Delta \Delta \mathrm{G}^{\ddagger}$ is significant compared to its magnitude, how $\Delta \Delta \mathrm{G}^{\ddagger}$ changes on truncation of different groups can still yield qualitative insight.
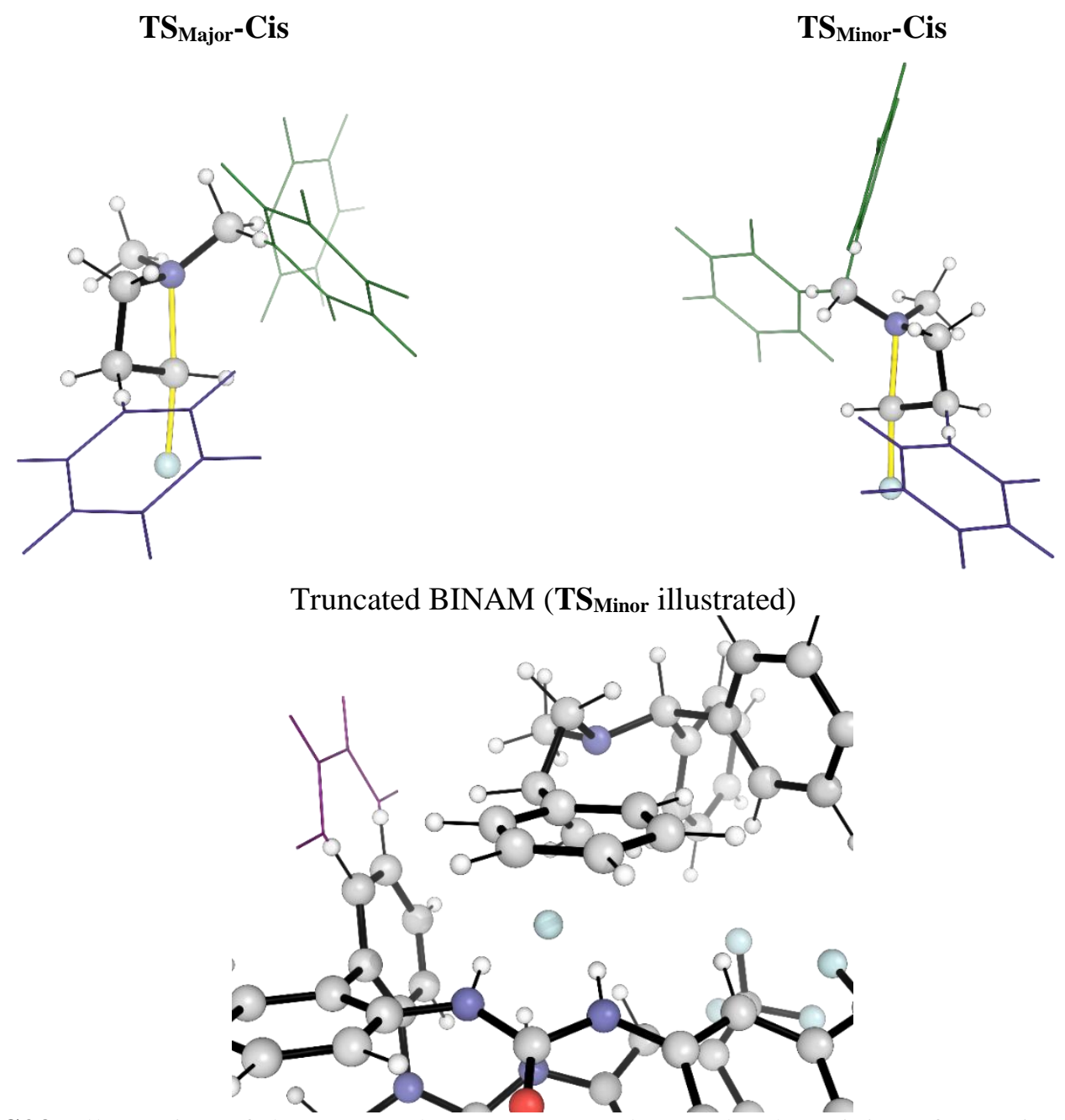

Figure S23: Illustration of the groups that are truncated to probe the origins of enantioselectivity. Green - benzhydryl, Blue - phenyl.

Table S21: $\Delta \Delta \mathrm{E}^{\ddagger}$ selectivities with truncation of the system

\begin{tabular}{cccc}
\hline Entry & & Truncated Groups & $\Delta \Delta \mathrm{E}^{\ddagger} / \mathrm{kJ} / \mathrm{mol}$ \\
\hline 1 & None & & $1.5^{*}$ \\
2 & $\mathrm{Ph}$ & & 4.5 \\
3 & $\mathrm{Bzh}$ & Bzh & 0.2 \\
4 & $\mathrm{Ph}$ & & -2.7 \\
5 & BINAM & & -2.8 \\
6 & Azet & Fluoride & 1.1 \\
7 & Cat & & -1.6 \\
8 & Cat & Ph & -4.7 \\
9 & Cat & Bzh & -0.2 \\
10 & Cat & Ph & 0.0 \\
11 & Cat & Bzh & -1.4 \\
\hline$* \Delta \Delta \mathrm{E}^{\ddagger}$ favors the minor enantiomer due to exclusion of additional terms in $\Delta \Delta \mathrm{G}^{\ddagger}$ \\
\hline \multicolumn{7}{r}{} \\
\hline \multicolumn{7}{r}{}
\end{tabular}


Truncation of the azetidinium phenyl group $(1 \rightarrow 2)$ results in a $\Delta \Delta \mathrm{E}^{\ddagger}$ of $4.5 \mathrm{~kJ} / \mathrm{mol}$ indicating a shift towards minor product of $3 \mathrm{~kJ} / \mathrm{mol}$. This suggests that interactions of the $\mathrm{Ph}$ group are more favorable in $\mathbf{T} \mathbf{S}_{\text {Major }}$ than

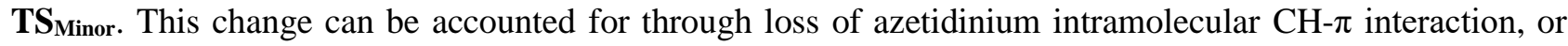
catalyst-substrate interactions. If the same truncation is performed with the catalyst also removed $(8 \rightarrow 9)$, there is an energy change of $4.5 \mathrm{~kJ} / \mathrm{mol}$ towards the minor, demonstrating that the major contributor is the loss of the intramolecular $\mathrm{CH}-\pi$ interaction in $\mathbf{T} \mathbf{S}_{\text {Major }}$.

Truncation of the benzhydryl group in the presence of catalyst $(1 \rightarrow 3)$ leads to a small change in $\Delta \Delta \mathrm{E}^{\ddagger}$ of $1.3 \mathrm{~kJ} / \mathrm{mol}$, to $0.2 \mathrm{~kJ} / \mathrm{mol}$, suggesting the Bzh group has interactions of similar strength in both TSs. In the absence of catalyst, truncation of the Bzh group results in $\Delta \Delta \mathrm{E}^{\ddagger}$ shifting towards minor product by $4.7 \mathrm{~kJ} / \mathrm{mol}$,

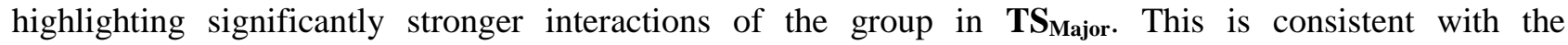
intramolecular $\mathbf{C H}-\pi$ interaction only present in $\mathbf{T S} \mathbf{S}_{\text {Major. }}$. The large difference in behavior of truncating this group in the presence and absence of catalyst is consistent with stronger interactions of the Bzh group with the catalyst in $\mathbf{T S}$ Minor - supported by NCI plots. In the absence of catalyst, truncation of the Ph group or the Bzh group have a similar effect on $\Delta \Delta \mathrm{E}^{\ddagger}$, consistent with the dominant effect being loss of the intramolecular $\mathrm{CH}$ $\pi$ interaction.

If one of the aromatic rings of the binam backbone is truncated $(1 \rightarrow 5)$, selectivity increases by $4.3 \mathrm{~kJ} / \mathrm{mol}$, demonstrating a stronger interaction of the substrate with this group in the $\mathbf{T} \mathbf{S}_{\text {Minor }}$. This arises from interaction with the benzhydryl group of the substrate in $\mathbf{T} \mathbf{S}_{\text {Minor, }}$, despite a shortened cation- $\pi$ in $\mathbf{T} \mathbf{S}_{\text {Major }}$.

\section{Non-covalent Interactions}

Non-covalent interactions plays a key role in docking the substrate with the catalyst-fluoride complex. These non-covalent interactions are visualized in Figure S24 using the non-covalent interaction index. Both TSs have face-to-face $\pi$-interactions between azetidinium phenyl group and catalyst urea group, and a cation- $\pi$ interaction between azetidinium and BINAM backbone. TS $_{\text {Major }}$ benefits from an intramolecular T-shape CH$\pi$ interaction, which is not achieved in $\mathbf{T} \mathbf{S}_{\text {Minor. }}$. However, $\mathbf{T S}_{\text {Minor }}$ can achieve an edge-to-face $\pi$-interaction between the catalyst BINAM backbone and one of the benzhydryl phenyl rings. 

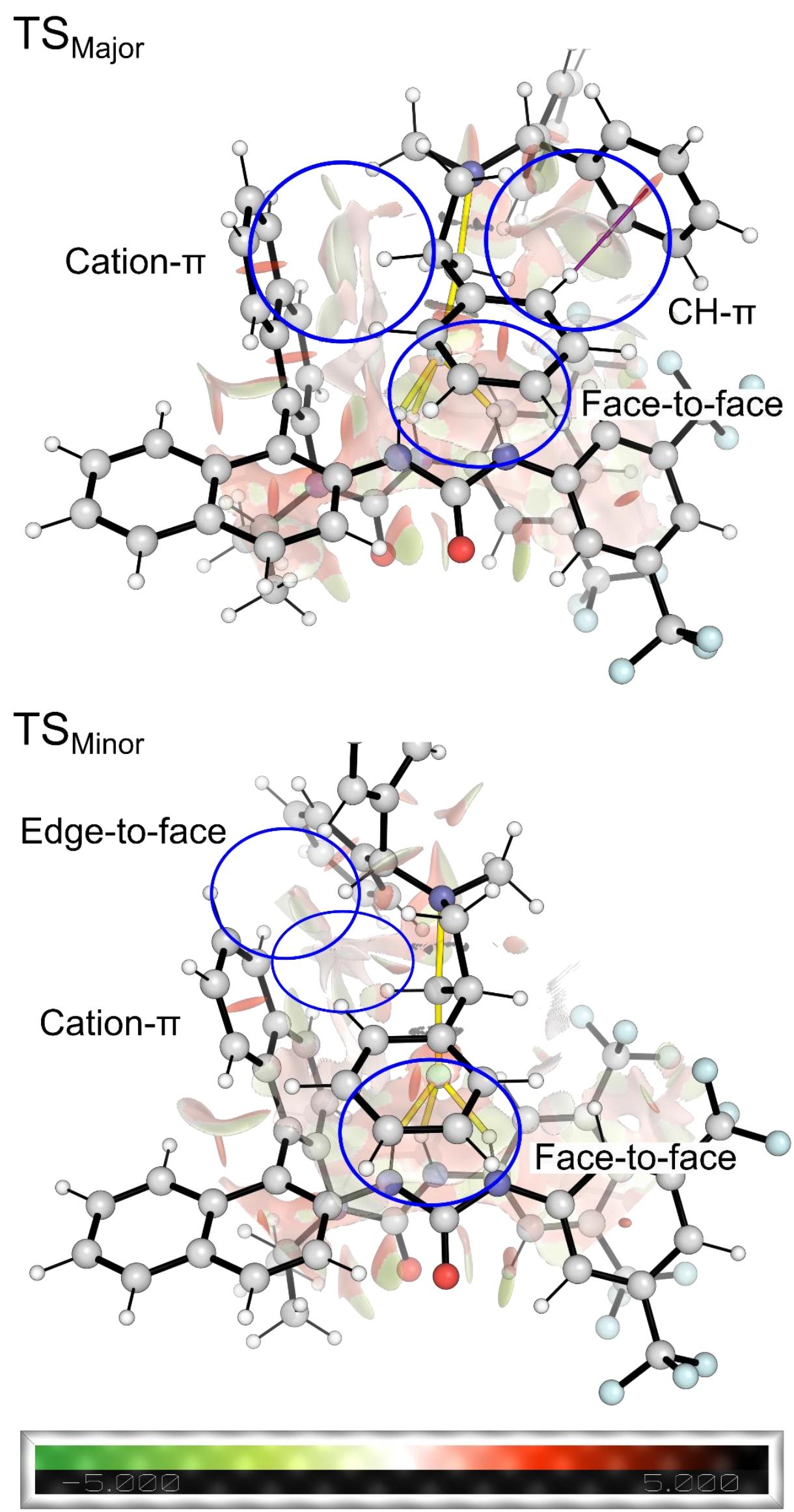

Figure S24: Non-covalent Interaction plots for $\mathbf{T} \mathbf{S}_{\text {Major }}-\mathbf{C i s}$ and $\mathbf{T S} \mathbf{S}_{\text {Minor- }}-\mathbf{C i s}$ 


\section{Geometry Evolution over the Intrinsic Reaction Coordinate}

Distances were monitored over the IRC pathway to major and minor products up to the TSs (IRC Major-Cis and

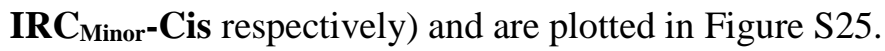

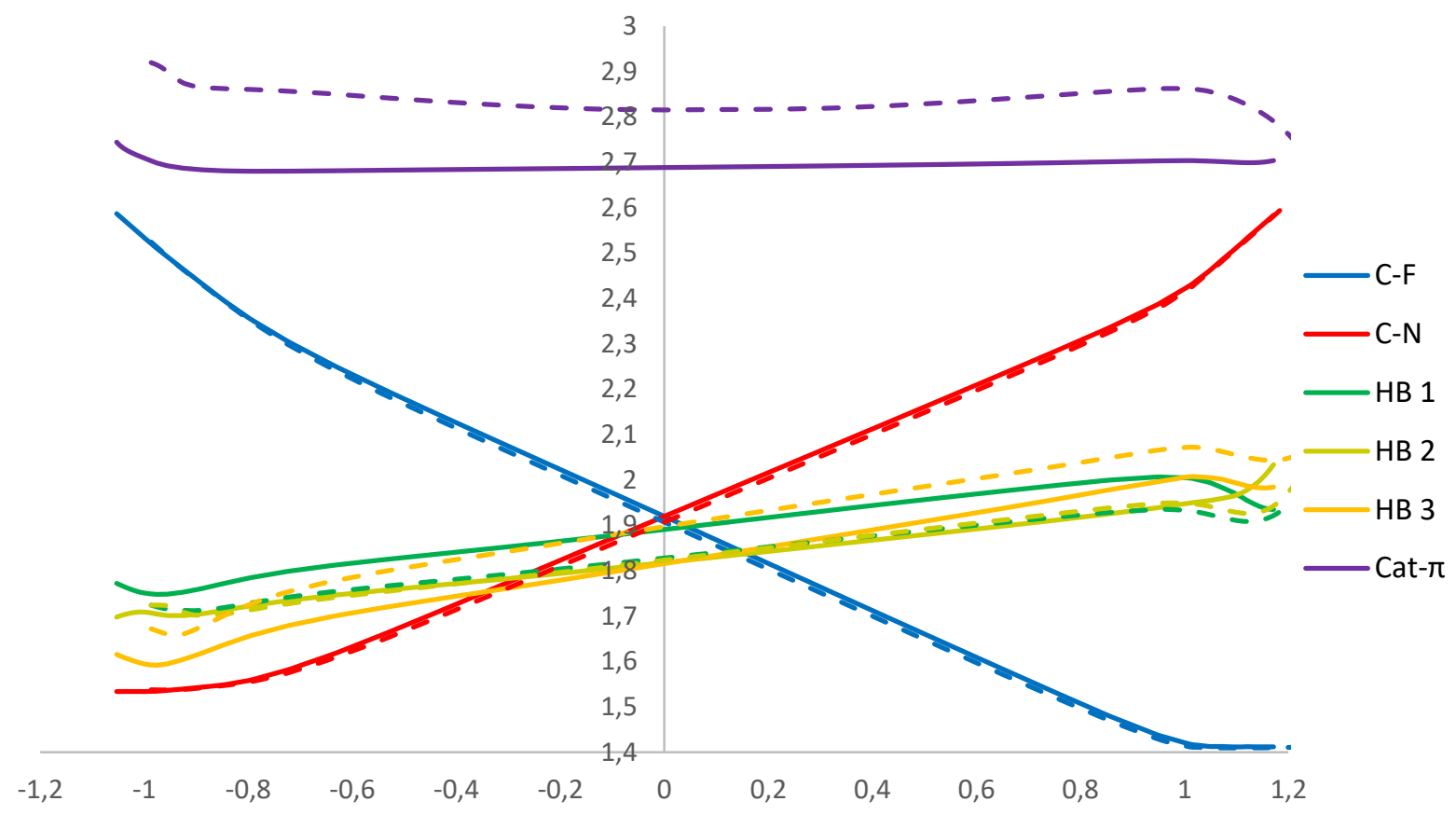

Figure S25: Plot of key geometric parameters over the IRC pathways to major and minor products. Distances on the pathway to major product

i) Forming $\mathrm{C}-\mathrm{F}$ and breaking $\mathrm{C}-\mathrm{N}$ bonds are similar lengths along both IRC pathways, with IRC $_{\text {Major }}$ slightly looser.

ii) The azetidinium-binam cation- $\pi$ interaction, measured from closest hydrogen atom, to the centroid

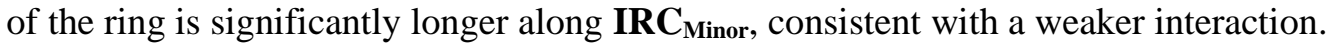

iii) $\quad \mathrm{HB} 2$ is the same length and evolves at the same rate along both IRC pathways.

iv) $\mathrm{HB} 1$ is longer in $\mathbf{I R C}_{\text {Major }}$ and HB 3 is shorter, with the bonds increasing in length at a similar rate along both pathways. HB 3 elongates at the fastest rate along both pathways.

Points iii-iv indicate that fluoride is positioned further from the BINAM backbone along IRC $_{\text {Major }}$ and is positioned further towards the monodentate alkylated urea hydrogen bond donor. 


\section{Effect of the Bzh group}

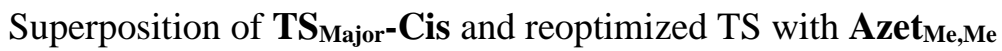

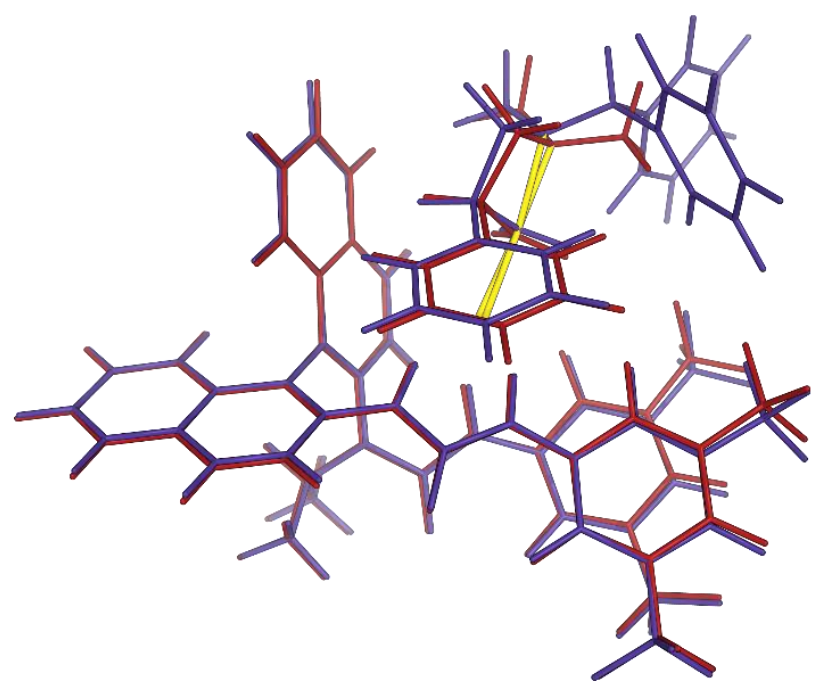

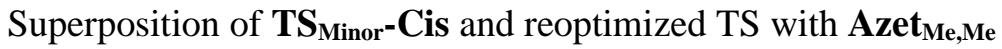

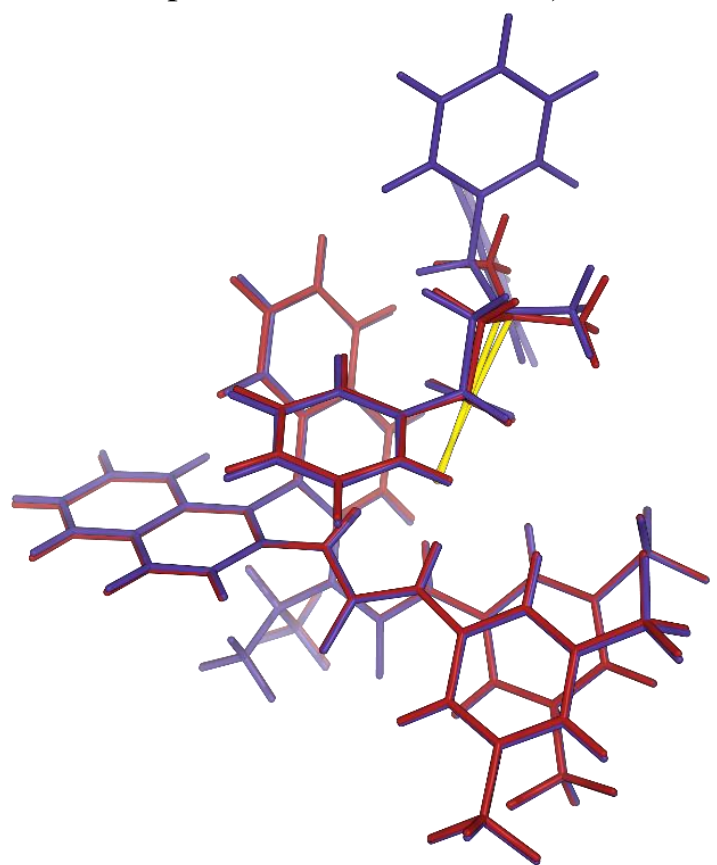

Figure S26: Reoptimization of $\mathbf{T S}_{\text {Major-Cis and }} \mathbf{T} \mathbf{S}_{\text {Minor }}-\mathbf{C i s}$ without benzhydryl group demonstrates minimal change in TS geometries.

Replacement of the Bzh group in the two lowest energy TSs with a Me group (converting the substrate to Azet $_{\text {Me,Me }}$ ) followed by reoptimization yields TSs that superimpose well with the Bzh starting points, demonstrating that the Bzh group has minimal role in determining the substrate docking pose.

\section{Enantioselectivity with Azet Bzh,Et}

To investigate the increased level of enantioselectivity with Azet $_{\text {Bzh,Et }}$ over Azet $_{\text {Bzh,Me, }}$ TS Major-Cis and

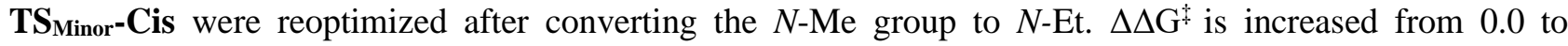
$6.4 \mathrm{~kJ} / \mathrm{mol}$. This is consistent in direction and qualitative magnitude with experimental results where e.r. is 
increased from $81: 19$ to $96: 4$ corresponding to $\Delta \Delta \mathrm{G}^{\ddagger}$ of approximately 3.6 and $7.9 \mathrm{~kJ} / \mathrm{mol}$. The TSs are superimposed in Figure S27.

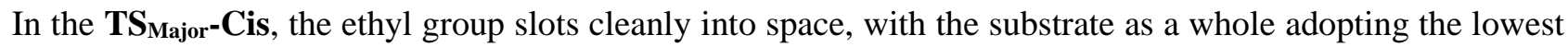
energy conformation seen in the TS with free fluoride. No change in catalyst or substrate geometry is seen, with perfect superposition of both (Figure S27, left). In contrast, in $\mathbf{T} \mathbf{S}_{\text {Minor-Cis, the ethyl group cannot adopt }}$ a conformation without requiring distortion and movement of the substrate. The ethyl group adopts a less favored conformation, eclipsing the azetidinium ring. Figure S27, right, shows translation of the substrate and distortion of the azetidinium ring. As the ethyl groups fits so perfectly into the lowest energy major TS, all other TSs are expected to be destabilized relative to this, consistent with the increase in enantioselectivity seen experimentally.
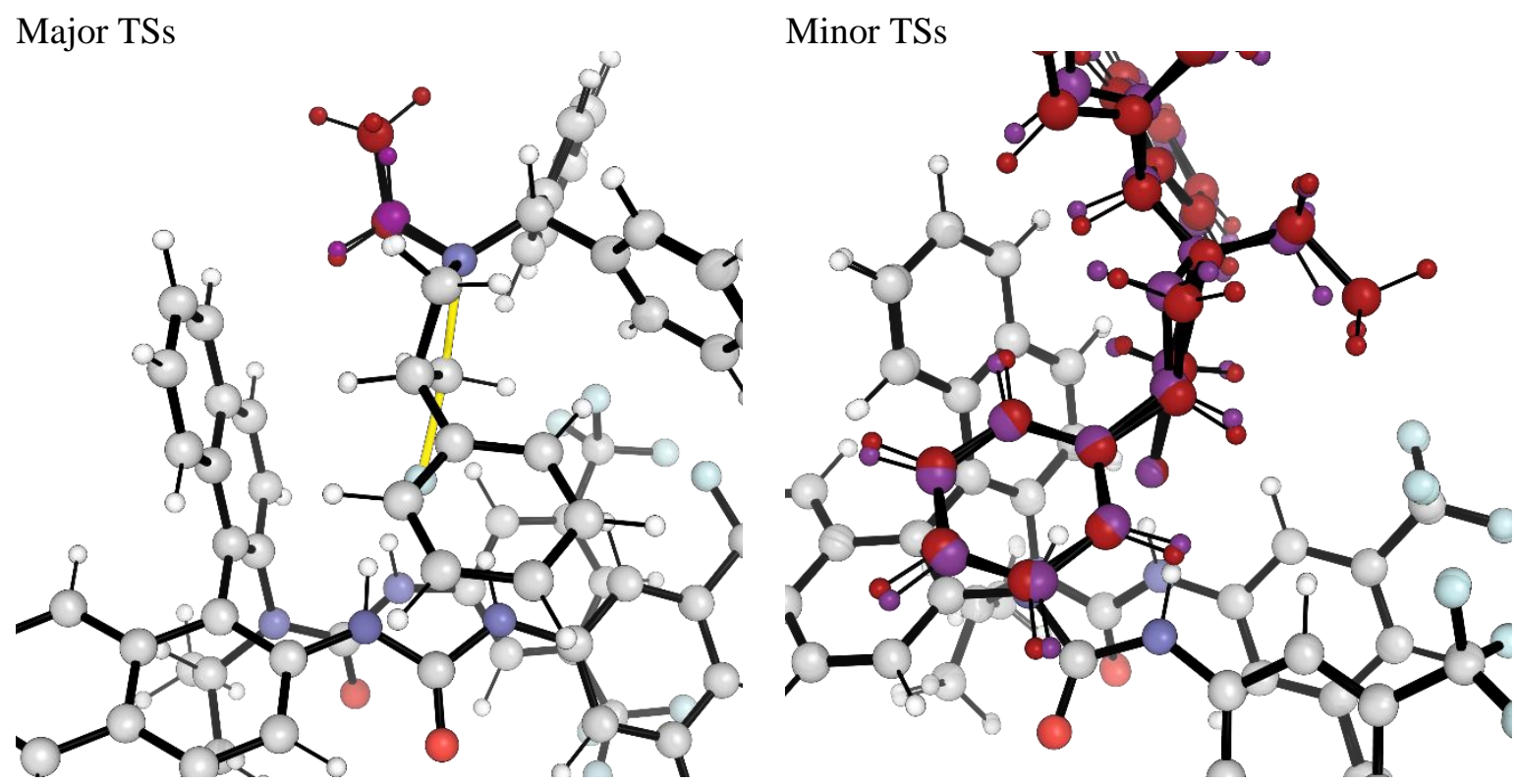

Figure S27: Superposition of lowest energy TSs with Azet $_{\text {Bzh,Me }}$ (purple) and reoptimization with

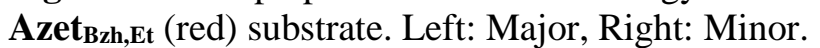

\section{Summary}

The catalyst adopts the same conformation in both the lowest energy TS to major and minor product, eliminating this as a major factor in enantioselectivity.

The docking pose of the substrate into the catalyst-fluoride complex in both cases is determined by face-to-face $\pi$-interactions between azetidinium phenyl ring and bidentate urea, in addition to the stereoelectronic requirements of the $\mathrm{S}_{\mathrm{N}} 2$ mechanism.

The position of the large benzhydryl group has surprisingly little effect on TS geometries exemplified by completely different positioning in the lowest energy TSs to major and minor product.

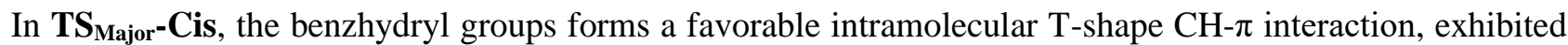
in the TS with naked fluoride, and worth approximately $2-5 \mathrm{~kJ} / \mathrm{mol}$. In $\mathbf{T S}_{\text {Minor- }}$-Cis, however, the benzhydryl group can form an edge-to-face $\pi$-interaction with the BINAM backbone, compensating for this loss.

The cation- $\pi$ distance in $\mathbf{T} \mathbf{S}_{\text {Major-Cis }}$ is shorter, consistent with a stronger interaction. 


\section{Enantioselective Fluorination of trans-Azet $\underline{B z h}$, Me $_{\text {en }}$}

\section{Transition State Structures}

The transition state structures to both enantiomers of product with trans substrate were simulated for $300 \mathrm{~ns}$, leading to a total simulation time of $600 \mathrm{~ns}$. All frames with F---C---N $>150^{\circ}$ in each trajectory were used for clustering with RMSD cutoff of $1.0 \AA$. For R-attack, all frames were clustered, resulting in 10 clusters of greater than $1 \%$ weight. Lower weight clusters were manually inspected and verified that they contained no additional conformations of interest. The central frame from each cluster was then optimized using DFT, resulting in 9 unique TSs to $(R)$ product. The same procedure was followed for S-attack, with all frames clustered, resulting in 9 clusters of over $1 \%$ weighting, and 9 unique DFT optimized TSs to (S) product. Manual sampling was used to augment the low energy conformers of the ensemble, resulting in a total of 37 TSs.

The TSs span an energy range of $46 \mathrm{~kJ} / \mathrm{mol}$. The ensemble indicates that $(S)$ catalyst affords $(S)$ product, however with greatly overestimated enantioselectivity of 98.6:1.4 e.r. in 1,2-dichloroethane at $298.15 \mathrm{~K}\left(\Delta \Delta \mathrm{G}^{\star}\right.$ $=12.6 \mathrm{~kJ} / \mathrm{mol}$ compared to experiment of approximately $3.5 \mathrm{~kJ} / \mathrm{mol}$ ). The major enantiomer is in agreement with crystallography, suggesting that a low energy TS to minor product is missing despite extensive effort to locate it. The structural similarity of the lowest energy TS to major product to the lowest energy TSs with cis substrate, and its low energy compared to all the located minor TSs with trans substrate gives confidence that the lowest energy TS to major product has indeed been located. The Gibbs free energy distribution of the low energy TSs is illustrated in Figure S28. Key geometric parameters for the lowest energy TSs are tabulated in

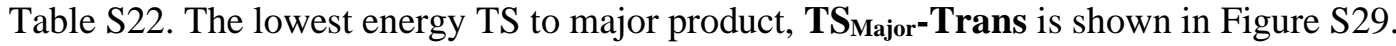

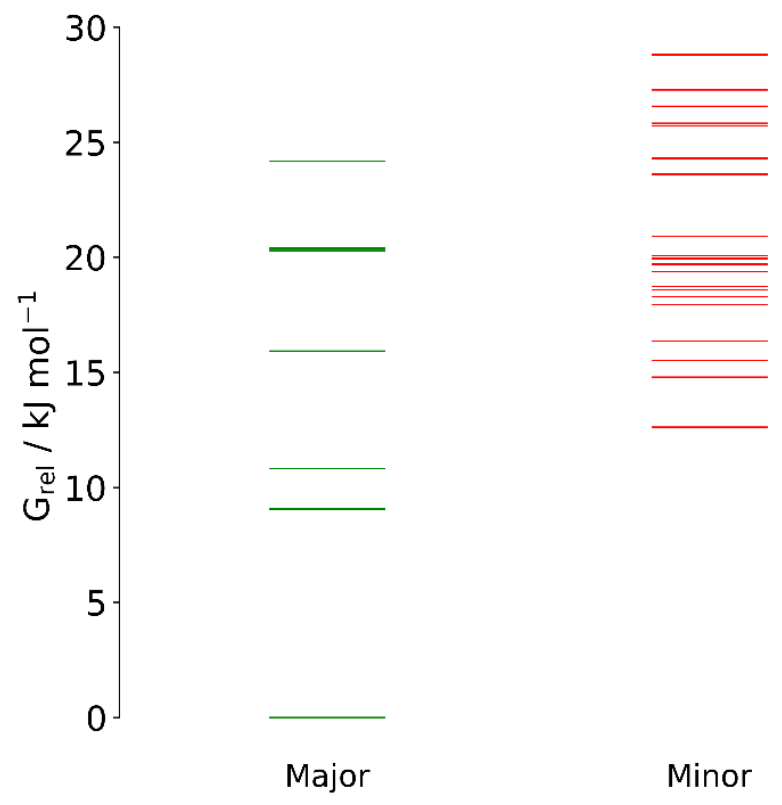

Figure S28: Gibbs free energy distribution of TSs in the ensemble. $\mathbf{T S}_{\text {Major-Trans is significantly }}$ lower in Gibbs free energy than all other TSs located. 


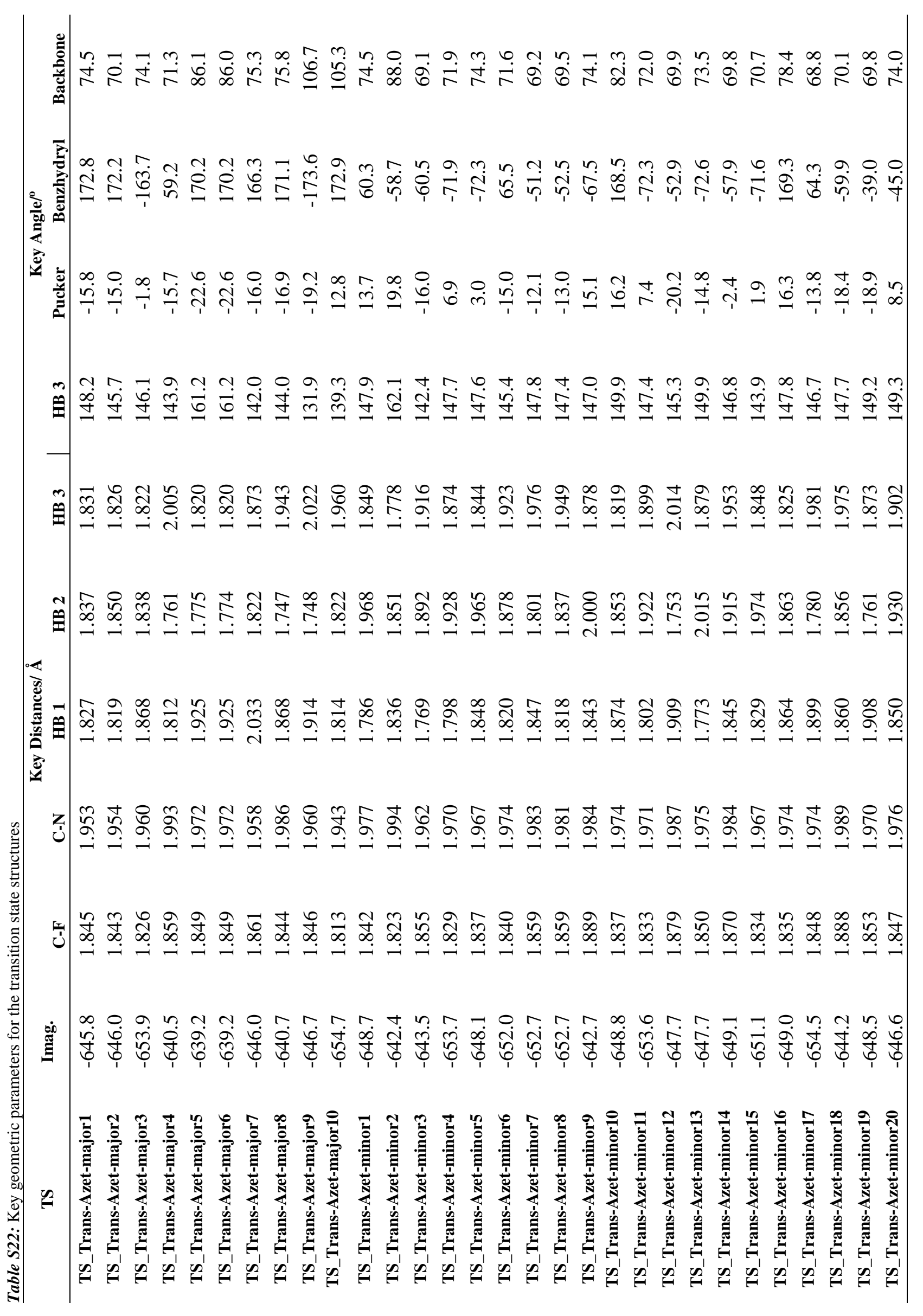


TS Major-Trans

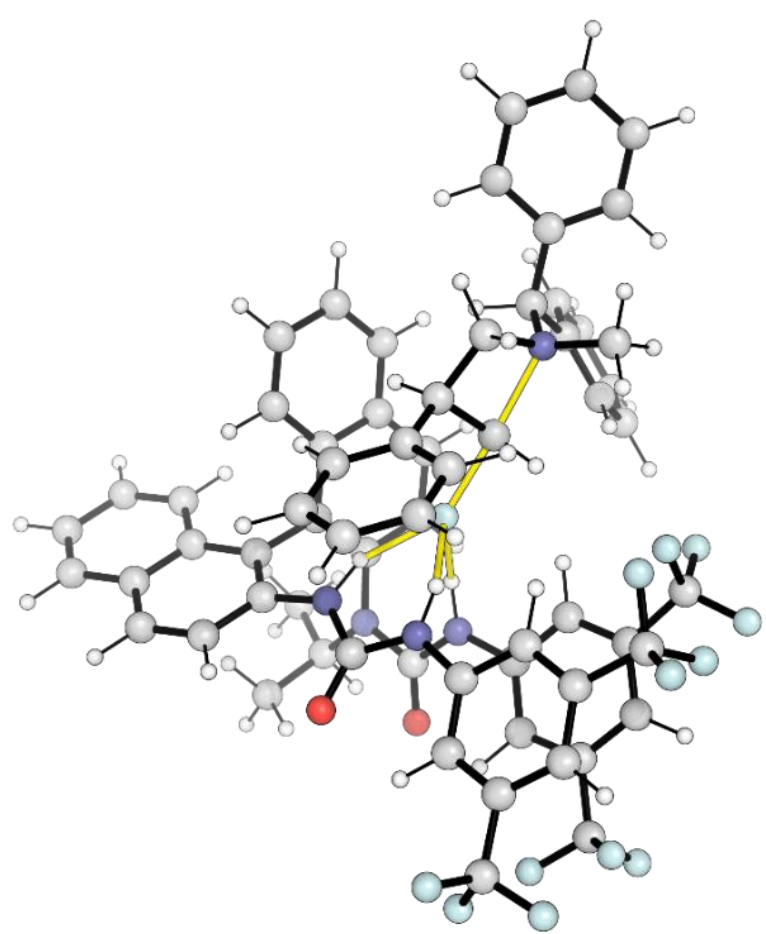

Figure S29: Lowest energy TS to major product

\section{Non-covalent Interactions}

Non-covalent interactions plays a key role in docking the substrate with the catalyst-fluoride complex. These non-covalent interactions are visualized in Figure S30 using the non-covalent interaction index. $\mathbf{T S}_{\text {Major-Trans }}$ features similar NCIs to the TSs with cis substrate, with face-to-face $\pi$-interaction between azetidinium phenyl group and catalyst urea group, and a cation- $\pi$ interaction between azetidinium and BINAM backbone.

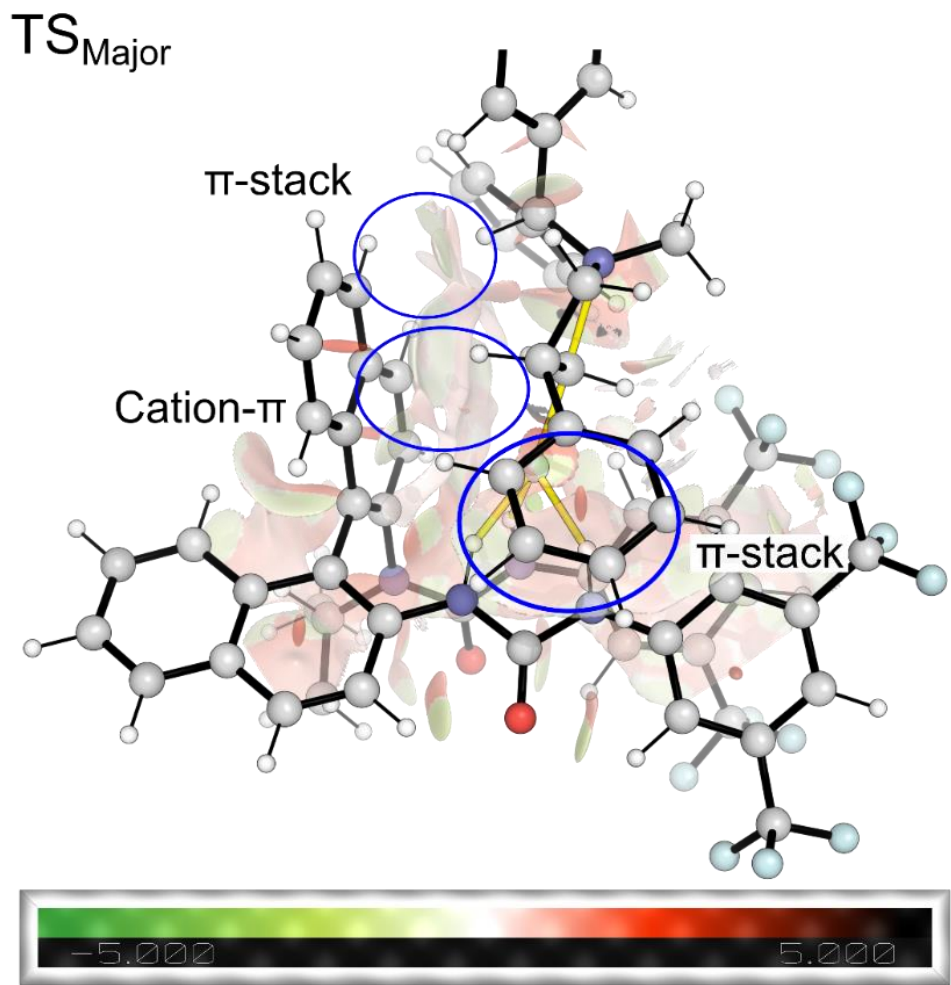

Figure S30: Non-covalent Interaction plots for $\mathbf{T S}_{\text {Major-Trans }}$ 


\section{Comparison of Cis and Trans TSs}

Transition state structures with cis and trans substrate are superimposed in Figure S31.

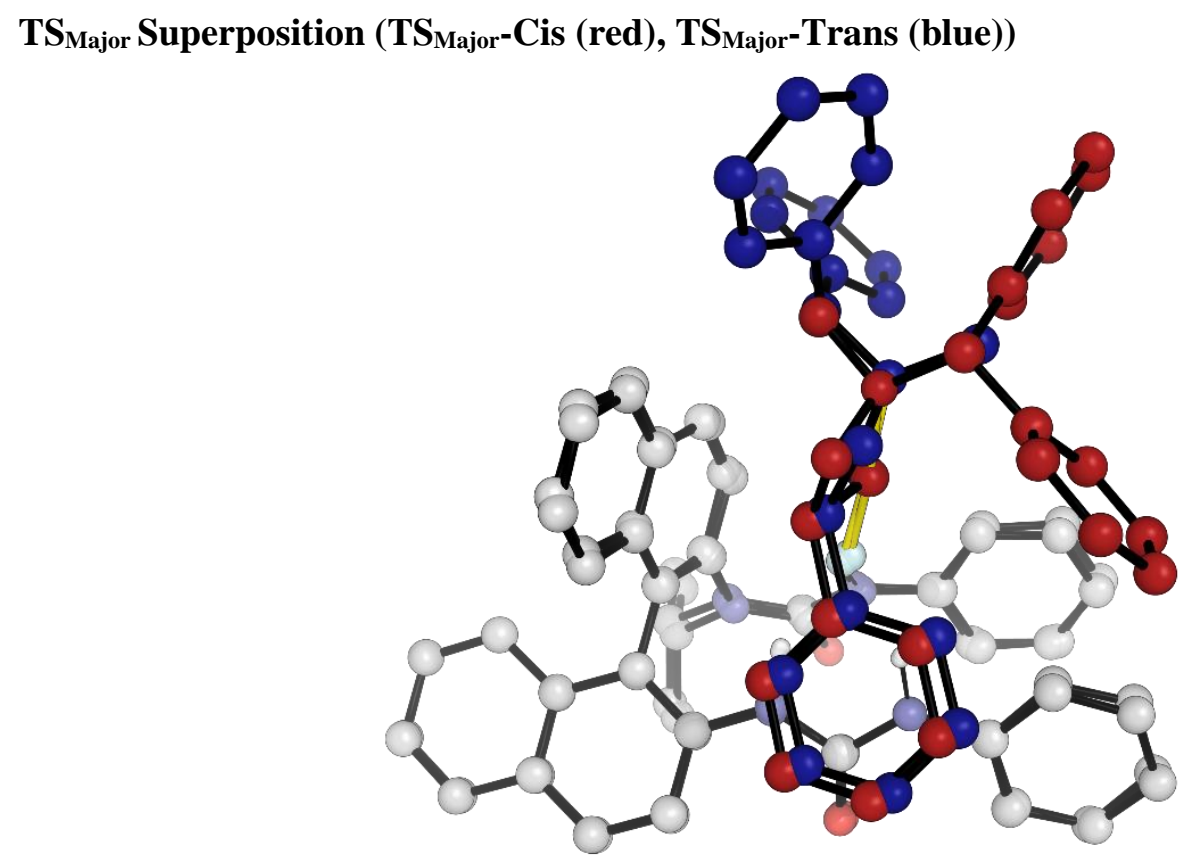

Figure S31: Superpositions of major and minor TSs with cis and trans substrates. Cis in green, trans in blue.

The major TSs with cis and trans substrate superimpose very well. Catalyst geometry is identical, and the substrates also superimpose very well, with the exception of the benzhydryl group pointing in opposite directions. The phenyl group directly bonded to the azetidinium aligns very well, forming a face-to-face interaction with the bidentate urea in both cases and dominating the docking pose of the substrate. The benzhydryl group, despite its steric bulk, projects away from the catalyst and into solvent, having a relatively minor effect on the TSs and enantioselectivity. In the TS with cis substrate, the benzhydryl group can form an intramolecular $\mathrm{CH}-\pi$ interaction whereas with the trans substrate, it can form an edge-to-face $\pi$-interaction with the BINAM backbone of the catalyst.

\section{Origins of enantioconvergence of cis and trans azetidinium ions}

The key reason for enantioconvergence is the importance of the azetidinium phenyl group in orienting the azetidinium ion in the catalytic pocket, and the lower importance of the orientation of the benzhydryl. The importance of the former is due to the shape complementarity of the ion and the catalyst, with phenyl group lying across the face of the bidentate urea. The lower importance of the latter is due to nitrogen substituents pointing out of the catalyst pocket and into solvent, despite the steric bulk of the group. The stereochemistry of the product is determined by the position of fluoride attack relative to the orientation of the azetidinium phenyl ring (the stereochemistry about nitrogen is lost in the product), and so fluoride favors attack at the same carbon regardless of whether the ion is cis or trans.

\section{Generalization to Other Azetidinium Ions}

A diverse range of azetidinium ions feature in the manuscript, not all of which have a phenyl group directly bonded to the azetidinium ring, nor Me/Et and Bzh nitrogen substituents. The TS model described above may be safely generalized to some substrates but can only give qualitative insight into others.

Substrates with different nitrogen substituents: The allyl group behaves similarly to the ethyl group in calculations with uncoordinated fluoride and so the TS model is expected to generalize well to $N$-allyl substrates. An $N$-Bn group is substantially larger and can form $\mathrm{CH}-\pi$ interactions, potentially having a large 
effect. However, the overlay of the major TSs for cis and trans substrates shows an almost perfect overlay (Figure S31), demonstrating that the catalytic pocket is able to accommodate a Bzh group in both cis and trans positions simultaneously, ignoring intramolecular clash. Envisaging the substrate with $N$-Bn and Bzh (minus one phenyl group), suggests directly TSs for both the cis and trans substrates, avoiding significant reorganization of the TS, and avoiding further steric clash (Figure S32). It is therefore plausible that these remain the lowest energy TSs.

Plausible Major TS for Azet $_{\text {Bzh.Bn, with Bzh group }}$ cis - Red and purple.

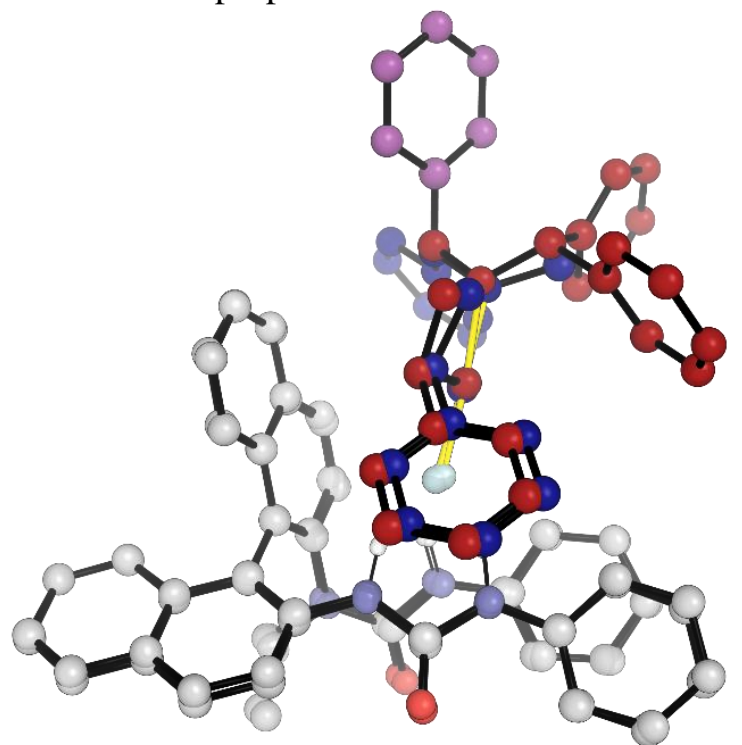

Plausible Major TS for Azet $_{\text {Bzh.Bn, with Bzh group }}$ trans - Blue and purple.

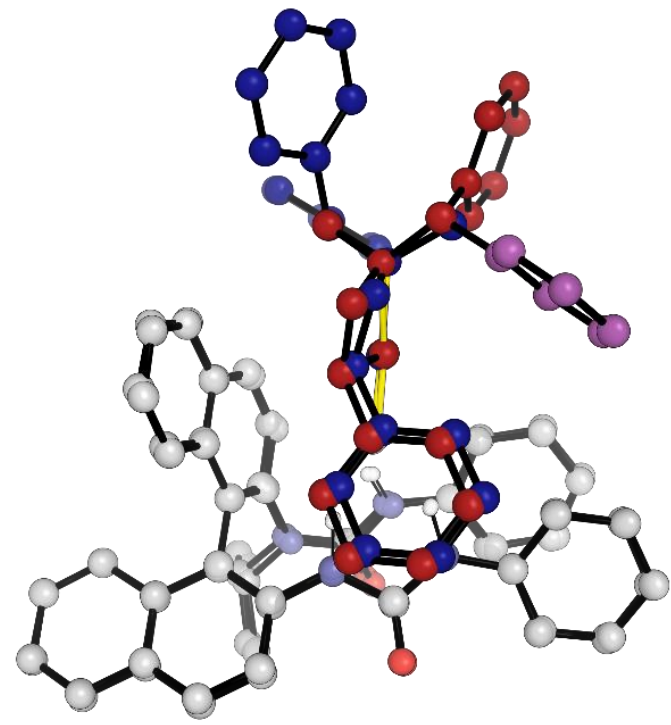

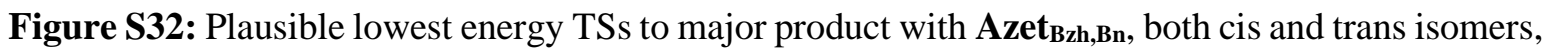
as implied by the lowest energy TSs to major product with Azet $_{\text {Bzh,Me. }}$ Cis substrate is given in red, and trans in blue. The perfect overlay of the substrates suggests plausible positions for the additional $\mathrm{N}$-Bn group in place of methyl (purple).

Substrates with different substituents bonded to the azetidinium ring (in place of phenyl): When this substituent is a substituted aryl group it is unlikely that the TSs will change significantly as the substituents can point away from the catalyst. All are also capable of forming the same $\pi$ interactions. With other substituents without a directly bonded aromatic, such as OBn and OMe, it is likely that the TSs will change significantly, due to different favored conformations and an inability to form an intramolecular $\mathrm{CH}-\pi$ interaction. The general factors that govern the TS geometries are, however, likely to be conserved i) the broad shape of the substrate is unchanged (the substituent projects from the azetidinium ion at the same angle), so similar docking poses will be favored ii) the lack of $\pi$-interactions with the catalyst for substituents such as OMe affects TSs to major and minor products in the same way iii) the $\pi$-interactions and the general positioning of the Bzh groups are unchanged iv) most TSs do not feature an intramolecular $\mathrm{CH}-\pi$ interaction, which this substrate cannot form.

\section{Summary of Computational Work}

i) The difference in reactivity of different $N$-substituted azetidinium ions is explained by the relative energies of the transition state structures to fluoride delivery. Good correlation is seen between increasing experimental yield and decreasing reaction barrier.

ii) Azetidinium ions with $N$-benzhydryl group have lower energy, and earlier fluoride delivery TSs. The ground state $\mathrm{C}-\mathrm{N}$ bond length are also elongated, consistent with a less thermodynamically stable ion.

iii) Much smaller differences are seen changing azetidinium $\mathrm{N}$-substituents from $\mathrm{Me}$ to $\mathrm{Bn}$, than converting Bn to Bzh, consistent with experimental observation. 
iv) Poor correlation is seen between azetidinium-fluoride ion pair binding energy and experimental yield under HB PTC. The $N$-substituents determine how closely, and the geometry in which, the fluoride can approach the quaternary nitrogen.

v) Good correlation between fluoride delivery TS energies and yield supports that fluoride delivery in the catalyzed reaction is turnover determining (TDTS). Poor correlation of azetidinium fluoride binding energies with yield is inconsistent with a turnover determining azetidinium assisted phase-transfer process.

vi) Relatively weak binding of the azetidinium ion to fluoride and a $\mathrm{CsF}$ ion pair - far inferior to the urea hydrogen bond donor - is not consistent with the azetidinium ion playing a significant role in $\mathrm{CsF}$ phase-transfer.

vii) Enantioconvergence of cis and trans substrates occurs as the dominant factor in orienting the azetidinium ion in the catalyst pocket is the phenyl ring directly bonded to the azetidinium ring, whereas the benzhydryl substituent largely projects into solvent and has lower geometric preference. As the position of fluoride delivery relative to the former determines the configuration of the product, the major product is invariant to the configuration at nitrogen.

\section{Tabulated Thermochemical Data}

Conformers containing a single low imaginary frequency are identified by * next to the name. Only significant conformers were reoptimized to remove these frequencies.

\section{Azetidinium ions}

Table S23: Azet Me,Me

\begin{tabular}{lccccc}
\hline & Energy (opt) & G (opt) & G-qh (opt) & E (sp) & G-qh (sp) \\
\hline Azet_Me_Me_1 & -482.790099 & -482.570035 & -482.569047 & -483.425336 & -483.204285 \\
Azet_Me_Me_2 & -482.789334 & -482.570039 & -482.568864 & -483.424241 & -483.203771 \\
Azet_Me_Me_3 & -482.789113 & -482.569459 & -482.568327 & -483.423418 & -483.202631 \\
\hline
\end{tabular}

Table S24: Azet ${ }_{\mathrm{Bn}, \mathrm{Me}}$

\begin{tabular}{lccccc}
\hline & Energy (opt) & G (opt) & G-qh (opt) & E (sp) & G-qh (sp) \\
\hline Azet_Bn_Me_1 & -713.579911 & -713.285084 & -713.282285 & -714.492942 & -714.195317 \\
Azet_Bn_Me_2 & -713.579911 & -713.285070 & -713.282279 & -714.492943 & -714.195312 \\
Azet_Bn_Me_3 & -713.582384 & -713.286139 & -713.284038 & -714.493448 & -714.195102 \\
Azet_Bn_Me_4 & -713.579912 & -713.284325 & -713.281781 & -714.492821 & -714.194690 \\
Azet_Bn_Me_5 & -713.579167 & -713.285321 & -713.282123 & -714.491623 & -714.194578 \\
Azet_Bn_Me_6 & -713.579276 & -713.283691 & -713.281285 & -714.490948 & -714.192956 \\
Azet_Bn_Me_7 & -713.579276 & -713.283688 & -713.281284 & -714.490948 & -714.192955 \\
Azet_Bn_Me_8 & -713.581435 & -713.284250 & -713.282685 & -714.491593 & -714.192843 \\
\hline
\end{tabular}

Table S25: Azet ${ }_{\mathrm{Bn}, \mathrm{Bn}}$

\begin{tabular}{cccccc}
\hline & Energy (opt) & G (opt) & G-qh (opt) & E (sp) & G-qh (sp) \\
\hline Azet_Bn_Bn_1 & -944.368987 & -943.998373 & -943.993976 & -945.559212 & -945.184201 \\
Azet_Bn_Bn_2 & -944.371126 & -943.999223 & -943.995352 & -945.559951 & -945.184177 \\
Azet_Bn_Bn_3 & -944.371126 & -943.999216 & -943.995347 & -945.559949 & -945.184170 \\
Azet_Bn_Bn_4 & -944.368192 & -943.998609 & -943.993911 & -945.557888 & -945.183606 \\
Azet_Bn_Bn_5 & -944.371771 & -943.998686 & -943.995264 & -945.559695 & -945.183188 \\
Azet_Bn_Bn_6 & -944.369345 & -943.998408 & -943.994220 & -945.558219 & -945.183093 \\
Azet_Bn_Bn_7 & -944.370320 & -943.998548 & -943.994997 & -945.558203 & -945.182880 \\
Azet_Bn_Bn_8* & -944.369739 & -943.997336 & -943.993743 & -945.558843 & -945.182847 \\
Azet_Bn_Bn_9 & -944.370131 & -943.997804 & -943.994383 & -945.558109 & -945.182361 \\
Azet_Bn_Bn_10 & -944.366682 & -943.996835 & -943.992094 & -945.556741 & -945.182152 \\
Azet_Bn_Bn_11 & -944.365984 & -943.995179 & -943.990918 & -945.556731 & -945.181665 \\
Azet_Bn_Bn_12 & -944.365892 & -943.996849 & -943.991874 & -945.555458 & -945.181441 \\
Azet_Bn_Bn_13 & -944.367747 & -943.996478 & -943.992364 & -945.556799 & -945.181416
\end{tabular}




\begin{tabular}{llllll} 
Azet_Bn_Bn_14 & -944.365167 & -943.994946 & -943.990554 & -945.555535 & -945.180921 \\
Azet_Bn_Bn_15 & -944.366130 & -943.995337 & -943.991017 & -945.555642 & -945.180530 \\
Azet_Bn_Bn_16 & -944.366095 & -943.994688 & -943.990647 & -945.555396 & -945.179948 \\
Azet_Bn_Bn_17 & -944.366713 & -943.995279 & -943.991179 & -945.555415 & -945.179881 \\
Azet_Bn_Bn_18 & -944.366713 & -943.995277 & -943.991177 & -945.555413 & -945.179877 \\
Azet_Bn_Bn_19 & -944.365366 & -943.995070 & -943.990597 & -945.554360 & -945.179591 \\
Azet_Bn_Bn_20 & -944.364853 & -943.993512 & -943.989366 & -945.554607 & -945.179119 \\
Azet_Bn_Bn_21 & -944.364898 & -943.993277 & -943.989487 & -945.554226 & -945.178815 \\
Azet_Bn_Bn_22 & -944.364160 & -943.993126 & -943.989146 & -945.553013 & -945.178000 \\
Azet_Bn_Bn_23 & -944.363099 & -943.992226 & -943.988165 & -945.552587 & -945.177653 \\
Azet_Bn_Bn_24 & -944.364800 & -943.992489 & -943.988868 & -945.552737 & -945.176806 \\
Azet_Bn_Bn_25 & -944.362337 & -943.991912 & -943.987738 & -945.551286 & -945.176686 \\
Azet_Bn_Bn_26 & -944.362136 & -943.990564 & -943.986702 & -945.550720 & -945.175285 \\
\hline
\end{tabular}

Table S26: Azet Bzh,Me

\begin{tabular}{lccccc}
\hline & Energy (opt) & G (opt) & G-qh (opt) & E (sp) & G-qh (sp) \\
\hline Azet_Bzh_Me_1 & -944.367813 & -943.995205 & -943.991987 & -945.555383 & -945.179557 \\
Azet_Bzh_Me_2 & -944.367813 & -943.995205 & -943.991986 & -945.555383 & -945.179556 \\
Azet_Bzh_Me_3 & -944.366038 & -943.993893 & -943.990579 & -945.553972 & -945.178513 \\
Azet_Bzh_Me_4 & -944.366038 & -943.993890 & -943.990578 & -945.553971 & -945.178512 \\
Azet_Bzh_Me_5 & -944.362396 & -943.991924 & -943.987628 & -945.552681 & -945.177913 \\
Azet_Bzh_Me_6 & -944.362396 & -943.991920 & -943.987626 & -945.552680 & -945.177909 \\
Azet_Bzh_Me_7 & -944.366247 & -943.993747 & -943.990578 & -945.553124 & -945.177455 \\
Azet_Bzh_Me_8 & -944.361644 & -943.992594 & -943.987735 & -945.551288 & -945.177379 \\
Azet_Bzh_Me_9 & -944.363196 & -943.992637 & -943.988472 & -945.551935 & -945.177210 \\
Azet_Bzh_Me_10 & -944.361654 & -943.991789 & -943.987313 & -945.551219 & -945.176878 \\
Azet_Bzh_Me_11 & -944.361674 & -943.991951 & -943.987432 & -945.550688 & -945.176446 \\
Azet_Bzh_Me_12 & -944.362361 & -943.991696 & -943.987464 & -945.550999 & -945.176102 \\
Azet_Bzh_Me_13 & -944.362419 & -943.992271 & -943.988023 & -945.550454 & -945.176058 \\
Azet_Bzh_Me_14 & -944.362419 & -943.992270 & -943.988023 & -945.550452 & -945.176056 \\
Azet_Bzh_Me_15 & -944.362405 & -943.990428 & -943.986820 & -945.549572 & -945.173988 \\
Azet_Bzh_Me_16 & -944.362405 & -943.990426 & -943.986819 & -945.549573 & -945.173987 \\
\hline
\end{tabular}

Table S27: Azet $_{\text {Bzh,Et }}$

\begin{tabular}{cccccc}
\hline & Energy (opt) & G (opt) & G-qh (opt) & E (sp) & G-qh (sp) \\
\hline Azet_Bzh_Et_1 & -983.634301 & -983.234541 & -983.231149 & -984.876229 & -984.473076 \\
Azet_Bzh_Et_2 & -983.633131 & -983.233601 & -983.230142 & -984.874432 & -984.471443 \\
Azet_Bzh_Et_3 & -983.629538 & -983.232088 & -983.227647 & -984.872557 & -984.470666 \\
Azet_Bzh_Et_4 & -983.629537 & -983.232073 & -983.227640 & -984.872551 & -984.470654 \\
Azet_Bzh_Et_5 & -983.631423 & -983.231881 & -983.228419 & -984.873657 & -984.470654 \\
Azet_Bzh_Et_6 & -983.625744 & -983.228246 & -983.223676 & -984.870710 & -984.468641 \\
Azet_Bzh_Et_7 & -983.627180 & -983.229348 & -983.224994 & -984.870643 & -984.468457 \\
Azet_Bzh_Et_8 & -983.624960 & -983.228869 & -983.223695 & -984.869247 & -984.467982 \\
Azet_Bzh_Et_9 & -983.628084 & -983.228649 & -983.225140 & -984.870477 & -984.467533 \\
Azet_Bzh_Et_10 & -983.626448 & -983.229029 & -983.224576 & -984.869283 & -984.467410 \\
Azet_Bzh_Et_11 & -983.626939 & -983.227929 & -983.224037 & -984.868776 & -984.465874 \\
Azet_Bzh_Et_12 & -983.626939 & -983.227928 & -983.224036 & -984.868776 & -984.465872 \\
Azet_Bzh_Et_13 & -983.627110 & -983.226587 & -983.223583 & -984.868821 & -984.465294 \\
Azet_Bzh_Et_14 & -983.624401 & -983.226104 & -983.221969 & -984.867620 & -984.465188 \\
Azet_Bzh_Et_15 & -983.624881 & -983.225629 & -983.221792 & -984.868242 & -984.465153 \\
Azet_Bzh_Et_16 & -983.624445 & -983.226586 & -983.222421 & -984.866967 & -984.464943 \\
Azet_Bzh_Et_17* & -983.625266 & -983.225268 & -983.222119 & -984.868057 & -984.464910 \\
Azet_Bzh_Et_18 & -983.624682 & -983.226170 & -983.222267 & -984.866452 & -984.464036 \\
Azet_Bzh_Et_19 & -983.623107 & -983.224651 & -983.220555 & -984.866506 & -984.463954 \\
Azet_Bzh_Et_20 & -983.623107 & -983.224647 & -983.220553 & -984.866506 & -984.463952 \\
Azet_Bzh_Et_21 & -983.623655 & -983.225371 & -983.221231 & -984.866216 & -984.463792 \\
Azet_Bzh_Et_22 & -983.622222 & -983.225061 & -983.220516 & -984.865165 & -984.463459 \\
Azet_Bzh_Et_23 & -983.621959 & -983.222202 & -983.218684 & -984.863978 & -984.460703 \\
\hline
\end{tabular}


Table S28: Azet $_{\text {Bzh,Ally }}$

\begin{tabular}{|c|c|c|c|c|c|}
\hline & Energy (opt) & G (opt) & G-qh (opt) & $\mathrm{E}(\mathrm{sp})$ & G-qh (sp) \\
\hline Azet_Bzh_All_1 & -1021.669513 & -1021.266432 & -1021.262689 & -1022.954453 & -1022.547630 \\
\hline Azet_Bzh_All_2 & -1021.668952 & -1021.265715 & -1021.261847 & -1022.954069 & -1022.546964 \\
\hline Azet_Bzh_All_3 & -1021.667432 & -1021.266083 & -1021.261336 & -1022.952648 & -1022.546552 \\
\hline Azet_Bzh_All_4 & -1021.668367 & -1021.265589 & -1021.261678 & -1022.952824 & -1022.546136 \\
\hline Azet_Bzh_All_5 & -1021.668367 & -1021.265485 & -1021.261628 & -1022.952811 & -1022.546072 \\
\hline Azet_Bzh_All_6 & -1021.667438 & -1021.265278 & -1021.260962 & -1022.952287 & -1022.545810 \\
\hline Azet_Bzh_All_7 & -1021.666874 & -1021.264513 & -1021.260509 & -1022.952173 & -1022.545808 \\
\hline Azet_Bzh_All_8 & -1021.667048 & -1021.264337 & -1021.260393 & -1022.952333 & -1022.545678 \\
\hline Azet_Bzh_All_9 & -1021.665329 & -1021.263801 & -1021.259262 & -1022.951132 & -1022.545065 \\
\hline Azet_Bzh_All_10 & -1021.665329 & -1021.263800 & -1021.259261 & -1022.951131 & -1022.545063 \\
\hline Azet_Bzh_All_11 & -1021.665293 & -1021.263354 & -1021.258896 & -1022.950289 & -1022.543892 \\
\hline Azet_Bzh_All_12 & -1021.665293 & -1021.263347 & -1021.258893 & -1022.950287 & -1022.543887 \\
\hline Azet_Bzh_All_13 & -1021.662626 & -1021.261632 & -1021.256792 & -1022.949346 & -1022.543511 \\
\hline Azet_Bzh_All_14 & -1021.662626 & -1021.261621 & -1021.256786 & -1022.949346 & -1022.543506 \\
\hline Azet_Bzh_All_15 & -1021.661259 & -1021.259743 & -1021.255026 & -1022.949473 & -1022.543241 \\
\hline Azet_Bzh_All_16 & -1021.662724 & -1021.261068 & -1021.256334 & -1022.949402 & -1022.543012 \\
\hline Azet_Bzh_All_17 & -1021.664677 & -1021.261520 & -1021.257602 & -1022.949758 & -1022.542682 \\
\hline Azet_Bzh_All_18 & -1021.664591 & -1021.261601 & -1021.257736 & -1022.949511 & -1022.542655 \\
\hline Azet_Bzh_All_19 & -1021.661995 & -1021.261353 & -1021.256275 & -1022.947997 & -1022.542276 \\
\hline Azet_Bzh_All_20 & -1021.663834 & -1021.260471 & -1021.256965 & -1022.949144 & -1022.542275 \\
\hline Azet_Bzh_All_21 & -1021.661977 & -1021.261033 & -1021.256163 & -1022.947914 & -1022.542100 \\
\hline Azet_Bzh_All_22 & -1021.661342 & -1021.260574 & -1021.255873 & -1022.947441 & -1022.541972 \\
\hline Azet_Bzh_All_23 & -1021.661191 & -1021.260183 & -1021.255264 & -1022.947729 & -1022.541802 \\
\hline Azet_Bzh_All_24 & -1021.663626 & -1021.260723 & -1021.256901 & -1022.948072 & -1022.541348 \\
\hline Azet_Bzh_All_25 & -1021.663626 & -1021.260722 & -1021.256901 & -1022.948071 & -1022.541346 \\
\hline Azet_Bzh_All_26 & -1021.660556 & -1021.260120 & -1021.255306 & -1022.946188 & -1022.540939 \\
\hline Azet_Bzh_All_27 & -1021.660344 & -1021.258506 & -1021.253921 & -1022.947109 & -1022.540685 \\
\hline Azet_Bzh_All_28 & -1021.662009 & -1021.260021 & -1021.255543 & -1022.947110 & -1022.540645 \\
\hline Azet_Bzh_All_29 & -1021.662008 & -1021.260015 & -1021.255541 & -1022.947109 & -1022.540642 \\
\hline Azet_Bzh_All_30 & -1021.662662 & -1021.259206 & -1021.255897 & -1022.947363 & -1022.540598 \\
\hline Azet_Bzh_All_31 & -1021.661729 & -1021.259922 & -1021.255309 & -1022.946867 & -1022.540446 \\
\hline Azet_Bzh_All_32 & -1021.659624 & -1021.258276 & -1021.253515 & -1022.946247 & -1022.540138 \\
\hline Azet_Bzh_All_33 & -1021.663135 & -1021.259035 & -1021.255543 & -1022.947714 & -1022.540122 \\
\hline Azet_Bzh_All_34 & -1021.663135 & -1021.259032 & -1021.255542 & -1022.947712 & -1022.540118 \\
\hline Azet_Bzh_All_35 & -1021.659987 & -1021.257785 & -1021.253336 & -1022.946653 & -1022.540002 \\
\hline Azet_Bzh_All_36 & -1021.658996 & -1021.257885 & -1021.253598 & -1022.945268 & -1022.539870 \\
\hline Azet_Bzh_All_37 & -1021.659455 & -1021.257899 & -1021.253346 & -1022.945898 & -1022.539790 \\
\hline Azet_Bzh_All_38 & -1021.659455 & -1021.257898 & -1021.253346 & -1022.945897 & -1022.539788 \\
\hline Azet_Bzh_All_39 & -1021.660452 & -1021.259319 & -1021.254590 & -1022.945464 & -1022.539602 \\
\hline Azet_Bzh_All_40 & -1021.659222 & -1021.258287 & -1021.253224 & -1022.945310 & -1022.539312 \\
\hline Azet_Bzh_All_41 & -1021.660221 & -1021.258182 & -1021.253604 & -1022.945711 & -1022.539094 \\
\hline Azet_Bzh_All_42 & -1021.657300 & -1021.257446 & -1021.252287 & -1022.944094 & -1022.539080 \\
\hline Azet_Bzh_All_43 & -1021.658108 & -1021.257811 & -1021.253173 & -1022.944001 & -1022.539065 \\
\hline Azet_Bzh_All_44 & -1021.658530 & -1021.256227 & -1021.251924 & -1022.945512 & -1022.538906 \\
\hline Azet_Bzh_All_45 & -1021.659586 & -1021.257500 & -1021.253253 & -1022.945027 & -1022.538693 \\
\hline Azet_Bzh_All_46 & -1021.658580 & -1021.257067 & -1021.252572 & -1022.944543 & -1022.538535 \\
\hline Azet_Bzh_All_47 & -1021.659290 & -1021.256835 & -1021.253046 & -1022.944322 & -1022.538078 \\
\hline Azet_Bzh_All_48 & -1021.658779 & -1021.257368 & -1021.252841 & -1022.943929 & -1022.537991 \\
\hline Azet_Bzh_All_49 & -1021.658874 & -1021.256044 & -1021.251998 & -1022.944802 & -1022.537926 \\
\hline Azet_Bzh_All_50 & -1021.658413 & -1021.254738 & -1021.251171 & -1022.943559 & -1022.536317 \\
\hline Azet_Bzh_All_51 & -1021.658413 & -1021.254736 & -1021.251172 & -1022.943552 & -1022.536312 \\
\hline Azet_Bzh_All_52 & -1021.655555 & -1021.252235 & -1021.248250 & -1022.943295 & -1022.535989 \\
\hline Azet_Bzh_All_53 & -1021.657153 & -1021.254366 & -1021.250285 & -1022.942619 & -1022.535751 \\
\hline Azet_Bzh_All_54 & -1021.654302 & -1021.253332 & -1021.248303 & -1022.940491 & -1022.534492 \\
\hline Azet_Bzh_All_55 & -1021.655652 & -1021.253294 & -1021.248989 & -1022.940096 & -1022.533433 \\
\hline Azet_Bzh_All_56 & -1021.656226 & -1021.253050 & -1021.249393 & -1022.939827 & -1022.532993 \\
\hline
\end{tabular}


Table S29: Azet ${ }_{\mathrm{Bzh}, \mathrm{Bn}}$

\begin{tabular}{|c|c|c|c|c|c|}
\hline & Energy (opt) & G (opt) & G-qh (opt) & $\mathrm{E}(\mathrm{sp})$ & G-qh (sp) \\
\hline Azet_Bzh_Bn_1 & -1175.156809 & -1174.709106 & -1174.703834 & -1176.621556 & -1176.168581 \\
\hline Azet_Bzh_Bn_2 & -1175.156448 & -1174.708435 & -1174.703410 & -1176.621277 & -1176.168239 \\
\hline Azet_Bzh_Bn_3 & -1175.156515 & -1174.707931 & -1174.703259 & -1176.620081 & -1176.166825 \\
\hline Azet_Bzh_Bn_4 & -1175.156515 & -1174.707925 & -1174.703255 & -1176.620080 & -1176.166820 \\
\hline Azet_Bzh_Bn_5 & -1175.152032 & -1174.706731 & -1174.700431 & -1176.618411 & -1176.166809 \\
\hline Azet_Bzh_Bn_6 & -1175.152032 & -1174.706687 & -1174.700409 & -1176.618411 & -1176.166788 \\
\hline Azet_Bzh_Bn_7 & -1175.154226 & -1174.707105 & -1174.701663 & -1176.618903 & -1176.166339 \\
\hline Azet_Bzh_Bn_8 & -1175.151323 & -1174.705157 & -1174.699222 & -1176.616910 & -1176.164809 \\
\hline Azet_Bzh_Bn_9 & -1175.151324 & -1174.705015 & -1174.699152 & -1176.616915 & -1176.164744 \\
\hline 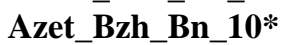 & -1175.150684 & -1174.701586 & -1174.696897 & -1176.618414 & -1176.164627 \\
\hline Azet_Bzh_Bn__11 & -1175.149918 & -1174.703972 & -1174.697845 & -1176.616605 & -1176.164532 \\
\hline Azet_Bzh_Bn_12 & -1175.153734 & -1174.704987 & -1174.700500 & -1176.617666 & -1176.164433 \\
\hline Azet_Bzh_Bn_13* & -1175.149846 & -1174.701752 & -1174.696687 & -1176.616912 & -1176.163753 \\
\hline Azet_Bzh_Bn_14 & -1175.149981 & -1174.703267 & -1174.697500 & -1176.616198 & -1176.163718 \\
\hline Azet_Bzh_Bn_15 & -1175.149869 & -1174.703039 & -1174.697240 & -1176.616018 & -1176.163388 \\
\hline Azet_Bzh_Bn_16 & -1175.149623 & -1174.702937 & -1174.697470 & -1176.614989 & -1176.162835 \\
\hline Azet_Bzh_Bn_17 & -1175.150583 & -1174.703600 & -1174.697904 & -1176.615162 & -1176.162483 \\
\hline Azet_Bzh_Bn_18 & -1175.152544 & -1174.702858 & -1174.698926 & -1176.615723 & -1176.162105 \\
\hline Azet_Bzh_Bn_19 & -1175.147141 & -1174.699977 & -1174.694432 & -1176.613926 & -1176.161217 \\
\hline Azet_Bzh_Bn_20 & -1175.148805 & -1174.701643 & -1174.696022 & -1176.613888 & -1176.161105 \\
\hline Azet_Bzh_Bn_21 & -1175.148663 & -1174.699907 & -1174.695296 & -1176.613882 & -1176.160514 \\
\hline Azet_Bzh_Bn_22 & -1175.148333 & -1174.699865 & -1174.695127 & -1176.613014 & -1176.159808 \\
\hline Azet_Bzh_Bn_23 & -1175.146934 & -1174.698457 & -1174.693794 & -1176.612543 & -1176.159402 \\
\hline Azet_Bzh_Bn_24 & -1175.146934 & -1174.698440 & -1174.693784 & -1176.612540 & -1176.159389 \\
\hline Azet_Bzh_Bn_25 & -1175.147325 & -1174.699867 & -1174.694669 & -1176.611572 & -1176.158915 \\
\hline Azet_Bzh_Bn_26 & -1175.148472 & -1174.698747 & -1174.694417 & -1176.612604 & -1176.158549 \\
\hline Azet_Bzh_Bn_27 & -1175.148472 & -1174.698749 & -1174.694418 & -1176.612602 & -1176.158548 \\
\hline Azet Bzh Bn 28 & -1175.146116 & -1174.698070 & -1174.693306 & -1176.611063 & -1176.158253 \\
\hline Azet_Bzh_Bn_29 & -1175.148535 & -1174.697963 & -1174.693964 & -1176.610848 & -1176.156276 \\
\hline
\end{tabular}

Azetidinium-Fluoride Ion Pairs

Table S30: Azet ${ }_{\mathrm{Me}, \mathrm{Me}}$

\begin{tabular}{lccccc}
\hline & Energy (opt) & G (opt) & G-qh (opt) & E (sp) & G-qh (sp) \\
\hline Azet_Me_Me_F_1 & -582.677639 & -582.460844 & -582.458859 & -583.439845 & -583.221065 \\
Azet_Me_Me_F_2 & -582.673553 & -582.456974 & -582.455031 & -583.439000 & -583.220478 \\
Aze__Me_Me_F_3 & -582.672790 & -582.456562 & -582.454584 & -583.437988 & -583.219782 \\
Azet_Me_Me_F_4 & -582.674890 & -582.457172 & -582.455733 & -583.438576 & -583.219418 \\
Azet_Me_Me_F_5 & -582.674890 & -582.457167 & -582.455730 & -583.438574 & -583.219415 \\
Azet_Me_Me_F_6 & -582.674159 & -582.457594 & -582.455722 & -583.437323 & -583.218885 \\
Azet_Me_Me_F_7 & -582.674710 & -582.457174 & -582.455690 & -583.437505 & -583.218485 \\
Azet_Me_Me_F_8 & -582.675630 & -582.458535 & -582.456846 & -583.437266 & -583.218482 \\
Azet_Me_Me_F_9 & -582.673867 & -582.456746 & -582.455008 & -583.436531 & -583.217672 \\
Azet_Me_Me_F_10 & -582.673868 & -582.456723 & -582.454995 & -583.436522 & -583.217649 \\
Azet_Me_Me_F_11 & -582.672584 & -582.455852 & -582.454026 & -583.435937 & -583.217379 \\
Azet_Me_Me_F_12 & -582.672584 & -582.455783 & -582.453989 & -583.435942 & -583.217347 \\
Azet_Me_Me_F_13 & -582.659366 & -582.443906 & -582.441487 & -583.428883 & -583.211004 \\
Azet_Me_Me_F_14 & -582.658450 & -582.444154 & -582.441198 & -583.427697 & -583.210445 \\
Azet_Me_Me_F_15 & -582.659493 & -582.443072 & -582.441224 & -583.428357 & -583.210087 \\
Azet_Me_Me_F_16 & -582.659682 & -582.443009 & -582.441092 & -583.427670 & -583.209080 \\
Azet_Me_Me_F_17 & -582.658670 & -582.443162 & -582.440636 & -583.426981 & -583.208948 \\
\hline
\end{tabular}

Table S31: Azet ${ }_{\mathrm{Bn}, \mathrm{Me}}$

\begin{tabular}{cccccc}
\hline & Energy (opt) & G (opt) & G-qh (opt) & E (sp) & G-qh (sp) \\
\hline Azet_Bn_Me_F_1 & -813.470129 & -813.176288 & -813.173393 & -814.508720 & -814.211984 \\
Azet_Bn_Me_F_2 & -813.470130 & -813.176295 & -813.173396 & -814.508717 & -814.211983 \\
Azet_Bn_Me_F_3 & -813.465220 & -813.173055 & -813.169316 & -814.506990 & -814.211087
\end{tabular}




\begin{tabular}{llllll} 
Azet_Bn_Me_F_4 & -813.463354 & -813.172073 & -813.168022 & -814.506330 & -814.210998 \\
Azet_Bn_Me_F_5 & -813.465038 & -813.173390 & -813.169671 & -814.505656 & -814.210289 \\
Azet_Bn_Me_F_6 & -813.466822 & -813.174664 & -813.171093 & -814.505844 & -814.210115 \\
Azet_Bn_Me_F_7 & -813.466822 & -813.174647 & -813.171087 & -814.505838 & -814.210103 \\
Azet_Bn_Me_F_8 & -813.462575 & -813.171197 & -813.167316 & -814.505312 & -814.210053 \\
Azet_Bn_Me_F_9 & -813.465445 & -813.171673 & -813.168773 & -814.506683 & -814.210011 \\
Azet_Bn_Me_F_10 & -813.469365 & -813.174314 & -813.172014 & -814.506927 & -814.209576 \\
Azet_Bn_Me_F_11 & -813.464877 & -813.172602 & -813.169052 & -814.504892 & -814.209066 \\
Azet_Bn_Me_F_12 & -813.464877 & -813.172599 & -813.169051 & -814.504890 & -814.209064 \\
Azet_Bn_Me_F_13 & -813.461444 & -813.169364 & -813.165877 & -814.504608 & -814.209041 \\
Azet_Bn_Me_F_14 & -813.461444 & -813.169350 & -813.165871 & -814.504602 & -814.209029 \\
Azet_Bn_Me_F_15 & -813.465758 & -813.172394 & -813.169392 & -814.504837 & -814.208471 \\
Azet_Bn_Me_F_16 & -813.465759 & -813.172380 & -813.169385 & -814.504832 & -814.208458 \\
Azet_Bn_Me_F_17 & -813.460921 & -813.168671 & -813.165207 & -814.503616 & -814.207903 \\
Azet_Bn_Me_F_18 & -813.468388 & -813.172872 & -813.171021 & -814.505046 & -814.207680 \\
Azet_Bn_Me_F_19 & -813.466638 & -813.171397 & -813.169401 & -814.504230 & -814.206993 \\
Azet_Bn_Me_F_20 & -813.466638 & -813.171393 & -813.169397 & -814.504227 & -814.206985 \\
Azet_Bn_Me_F_21 & -813.455903 & -813.164287 & -813.160580 & -814.499197 & -814.203874 \\
Azet_Bn_Me_F_22 & -813.453644 & -813.162043 & -813.158201 & -814.498328 & -814.202885 \\
Azet_Bn_Me_F_23 & -813.456697 & -813.162916 & -813.160019 & -814.498425 & -814.201746 \\
Azet_Bn_Me_F_24 & -813.456697 & -813.162907 & -813.160014 & -814.498424 & -814.201741 \\
\hline
\end{tabular}

Table S32: Azet $_{\mathrm{Bn}, \mathrm{Bn}}$

\begin{tabular}{|c|c|c|c|c|c|}
\hline & Energy (opt) & $\mathrm{G}(\mathrm{opt})$ & G-qh (opt) & $E(s p)$ & G-qh (sp) \\
\hline Azet_Bn_Bn_F_1 & -1044.258298 & -1043.888433 & -1043.884060 & -1045.574538 & -1045.200300 \\
\hline Azet_Bn_Bn_F_2 & -1044.258298 & -1043.888431 & -1043.884059 & -1045.574537 & -1045.200298 \\
\hline Azet_Bn_Bn_F_3 & -1044.256859 & -1043.888147 & -1043.883205 & -1045.573531 & -1045.199876 \\
\hline Azet_Bn_Bn_F_4 & -1044.259784 & -1043.889429 & -1043.885193 & -1045.574247 & -1045.199655 \\
\hline Azet_Bn_Bn_F_5 & -1044.259034 & -1043.888722 & -1043.884569 & -1045.573802 & -1045.199338 \\
\hline Azet_Bn_Bn_F_6 & -1044.256096 & -1043.888205 & -1043.883022 & -1045.572236 & -1045.199162 \\
\hline Azet_Bn_Bn_F_7 & -1044.256048 & -1043.887360 & -1043.882385 & -1045.572729 & -1045.199065 \\
\hline Azet_Bn_Bn_F_8 & -1044.256826 & -1043.886532 & -1043.882393 & -1045.573377 & -1045.198943 \\
\hline Azet_Bn_Bn_F_9 & -1044.256728 & -1043.887379 & -1043.882696 & -1045.572439 & -1045.198407 \\
\hline Azet_Bn_Bn_F_10 & -1044.258313 & -1043.888057 & -1043.884041 & -1045.572610 & -1045.198337 \\
\hline Azet_Bn_Bn_F_11 & -1044.255213 & -1043.885606 & -1043.881249 & -1045.572216 & -1045.198252 \\
\hline Azet_Bn_Bn_F_12 & -1044.257794 & -1043.887009 & -1043.883305 & -1045.572255 & -1045 \\
\hline Azet_Bn_Bn_F_13 & -1044.257794 & -1043.887005 & -1043.883303 & -1045.572250 & -1045.197759 \\
\hline Azet_Bn_Bn_F_14 & -1044.254700 & -1043.886484 & -1043.881338 & -1045.570764 & -1045.197401 \\
\hline Azet_Bn_Bn_F_15 & -1044.255838 & -1043.885943 & -1043.881552 & -1045.571682 & -1045.197395 \\
\hline Azet_Bn_Bn_F_16 & -1044.257696 & -1043.886991 & -1043.883266 & -1045.571634 & -1045.197204 \\
\hline Azet_Bn_Bn_F_17 & -1044.252393 & -1043.883308 & -1043.878833 & -1045.570626 & -1045.197067 \\
\hline Azet_Bn_Bn_F_18 & -1044.257964 & -1043.887083 & -1043.883361 & -1045.571598 & -1045.196994 \\
\hline Azet_Bn_Bn_F_19 & -1044.255587 & -1043.886092 & -1043.881601 & -1045.570936 & -1045.196950 \\
\hline Azet_Bn_Bn_F_20 & -1044.254154 & -1043.884733 & -1043.880575 & -1045.570521 & -1045.196942 \\
\hline Azet_Bn_Bn_F_21 & -1044.252944 & -1043.884948 & -1043.879792 & -1045.570037 & -1045.196886 \\
\hline Azet_Bn_Bn_F_22 & -1044.255346 & -1043.886223 & -1043.881515 & -1045.570702 & -1045.196871 \\
\hline Azet_Bn_Bn_F_23 & -1044.252781 & -1043.884822 & -1043.879641 & -1045.569967 & -1045.196827 \\
\hline Azet_Bn_Bn_F_24 & -1044.253746 & -1043.884104 & -1043.879788 & -1045.570229 & -1045.196271 \\
\hline Azet_Bn_Bn_F_25 & -1044.251027 & -1043.883801 & -1043.878512 & -1045.568671 & -1045.196156 \\
\hline Azet_Bn_Bn_F_26 & -1044.251027 & -1043.883801 & -1043.878512 & -1045.568671 & -1045.196155 \\
\hline Azet_Bn_Bn_F_27 & -1044.251563 & -1043.882768 & -1043.878093 & -1045.569273 & -1045.195803 \\
\hline Azet_Bn_Bn_F_28 & -1044.253432 & -1043.885131 & -1043.879969 & -1045.569009 & -1045.195546 \\
\hline Azet_Bn_Bn_F_29 & -1044.253383 & -1043.885134 & -1043.879977 & -1045.568873 & -1045.195466 \\
\hline Azet_Bn_Bn_F_30 & -1044.253383 & -1043.885131 & -1043.879975 & -1045.568873 & -1045.195464 \\
\hline
\end{tabular}

Table S33: Azet $_{\mathrm{Bzh}, \mathrm{Me}}$

\begin{tabular}{lccccc}
\hline & Energy (opt) & G (opt) & G-qh (opt) & E (sp) & G-qh (sp) \\
\hline Azet_Bzh_Me_F_1 & -1044.255741 & -1043.885965 & -1043.881860 & -1045.570510 & -1045.196628 \\
Azet_Bzh_Me_F_2 & -1044.254795 & -1043.885884 & -1043.881433 & -1045.569762 & -1045.196401
\end{tabular}




\begin{tabular}{|c|c|c|c|c|c|}
\hline 3 & 3 & 8 & 2 & 50 & -1045.196050 \\
\hline zet_Bzh_Me_F_44 & -1044.253813 & -1043.885075 & -1043.880472 & -1045.568841 & -1045195500 \\
\hline zet_Bzh_Me_F_5 & 4.250705 & 3.881568 & +3.877207 & & \\
\hline Azet_Bzh_Me_F_6 & & & & & \\
\hline Azet_Bzh_Me_F_7 & 44.253892 & & 3.880708 & 5.568064 & -104 \\
\hline Azet_Bzh_M & & & & & \\
\hline Azet_Bzh_Me_F_9 & 4.251168 & 2011 & & & 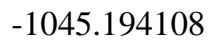 \\
\hline Azet_Bzh_Me_F_10 & 044.249725 & 3.881576 & & 66664 & 93752 \\
\hline zet_Bzh_Me_F_11 & 4.251369 & 3.883230 & & 45.566406 & \\
\hline e_F_12 & & & & & \\
\hline zet_Bzh_Me_F_13 & & & & & \\
\hline Azet_Bzh_I & & & & 6253 & \\
\hline Azet_Bzh_Me_F_15 & & & & & \\
\hline _16 & & & & & \\
\hline _17 & & & & & 80 \\
\hline _F_18 & & & & & \\
\hline F_19 & & & & & \\
\hline & & & & & \\
\hline F_21 & & & & & \\
\hline _22 & & & & & \\
\hline 23 & & & & & \\
\hline & & & & & \\
\hline F_25 & & & & & \\
\hline 26 & & & & & \\
\hline$\_27$ & & & & & \\
\hline & & & & & \\
\hline 29 & & & & & \\
\hline 30 & & & & & \\
\hline & & & & & \\
\hline & & & & & \\
\hline & & & & & \\
\hline & & & & & \\
\hline & & & & & \\
\hline & & & & & \\
\hline & & & & & \\
\hline & & & & & \\
\hline _39 & & & & & 607 \\
\hline & & & & & \\
\hline & & & & & \\
\hline $\mathbf{A}$ & & & & & \\
\hline F_43 & & & & & 894 \\
\hline & & & & & \\
\hline & & & & & \\
\hline _46 & & & & 704 & SOS \\
\hline _Bzh_I & & & 8 & 64 & 84644 \\
\hline & & & & & \\
\hline & & & & & \\
\hline Azet_Bzh_Me_F_50 & -1044.235447 & -1043.868057 & -1043.863033 & -1045.556379 & -1045.1839 \\
\hline
\end{tabular}

Table S34: Azet ${ }_{\mathrm{Bzh}, \mathrm{Et}}$

\begin{tabular}{lccccc}
\hline & Energy (opt) & G (opt) & G-qh (opt) & E (sp) & G-qh (sp) \\
\hline Azet_Bzh_Et_F_1 & -1083.516936 & -1083.120077 & -1083.115816 & -1084.889173 & -1084.488053 \\
Azet_Bzh_Et_F_2 & -1083.517995 & -1083.120794 & -1083.116805 & -1084.889082 & -1084.487892 \\
Azet_Bzh_Et_F_3 & -1083.519086 & -1083.122576 & -1083.118179 & -1084.888760 & -1084.487854 \\
Azet_Bzh_Et_F_4 & -1083.519050 & -1083.124015 & -1083.119091 & -1084.887686 & -1084.487728 \\
Azet_Bzh_Et_F_5 & -1083.520493 & -1083.122445 & -1083.118801 & -1084.889189 & -1084.487497 \\
Azet_Bzh_Et_F_6 & -1083.519057 & -1083.122789 & -1083.118462 & -1084.888017 & -1084.487422 \\
Azet_Bzh_Et_F_7 & -1083.517708 & -1083.120376 & -1083.116452 & -1084.888613 & -1084.487357 \\
Azet_Bzh_Et_F_8 & -1083.519085 & -1083.121395 & -1083.117591 & -1084.888773 & -1084.487280 \\
Azet_Bzh_Et_F_9 & -1083.517111 & -1083.121436 & -1083.116645 & -1084.887504 & -1084.487038
\end{tabular}


Azet_Bzh_Et_F_10 Azet_Bzh_Et_F_11 Azet_Bzh_Et_F_12 Azet_Bzh_Et_F_13 Azet_Bzh_Et_F_14 Azet_Bzh_Et_F_15 Azet_Bzh_Et_F_16 Azet_Bzh_Et_F_17 Azet_Bzh_Et_F_18 Azet_Bzh_Et_F_19 Azet_Bzh_Et_F_20 Azet_Bzh_Et_F_21 Azet_Bzh_Et_F_22 Azet_Bzh_Et_F_23 Azet_Bzh_Et_F_24 Azet_Bzh_Et_F_25 Azet_Bzh_Et_F_26 Azet_Bzh_Et_F_27 Azet_Bzh_Et_F_28
$-1083.517111$ $-1083.519320$ $-1083.518160$ $-1083.515910$ $-1083.515909$ $-1083.517724$ $-1083.516638$ $-1083.517570$ $-1083.517570$ $-1083.510786$ $-1083.516933$ $-1083.515256$ $-1083.514536$ $-1083.513777$ $-1083.516079$ $-1083.513130$ $-1083.514332$ $-1083.514534$ $-1083.511334$
$-1083.121421$ $-1083.121717$ $-1083.119604$ $-1083.118796$ $-1083.118787$ $-1083.120321$ $-1083.118953$ $-1083.119697$ $-1083.119696$ $-1083.115543$ $-1083.119489$ $-1083.118054$ $-1083.118511$ $-1083.117058$ $-1083.118381$ $-1083.116474$ $-1083.116211$ $-1083.116840$ $-1083.114822$
$-1083.116638$ $-1083.117720$ $-1083.116376$ $-1083.114771$ $-1083.114767$ $-1083.116059$ $-1083.115374$ $-1083.116107$ $-1083.116105$ $-1083.110441$ $-1083.115409$ $-1083.114005$ $-1083.113775$ $-1083.112620$ $-1083.114499$ $-1083.112060$ $-1083.112643$ $-1083.113088$ $-1083.110289$
$-1084.887509$ $-1084.888604$ $-1084.888648$ $-1084.887226$ $-1084.887227$ $-1084.887617$ $-1084.886914$ $-1084.886521$ $-1084.886516$ $-1084.885109$ $-1084.886156$ $-1084.885726$ $-1084.885197$ $-1084.885183$ $-1084.885382$ $-1084.884017$ $-1084.884362$ $-1084.883678$ $-1084.879812$
$-1084.487035$ $-1084.487004$ $-1084.486865$ $-1084.486088$ $-1084.486085$ $-1084.485952$ $-1084.485651$ $-1084.485058$ $-1084.485051$ $-1084.484764$ $-1084.484633$ $-1084.484474$ $-1084.484436$ $-1084.484026$ $-1084.483802$ $-1084.482947$ $-1084.482674$ $-1084.482233$ $-1084.478767$

Table S35: Azet ${ }_{\text {Bzh,Allyl }}$

\begin{tabular}{|c|c|c|c|c|c|}
\hline & Energy (opt) & G (opt) & G-qh (opt) & $\mathrm{E}(\mathrm{sp})$ & G-qh (sp) \\
\hline Azet_Bzh_All_F_1 & -1121.553328 & -1121.153166 & -1121.148583 & -1122.968267 & -1122.563522 \\
\hline Azet_Bzh_ & 53527 & -1121.153645 & -1121.148725 & -1122.967762 & -1122.562960 \\
\hline Azet_Bzh_All_F_3 & -1121.551635 & -1121.152095 & -1121.147051 & -1122.967482 & -1122.562899 \\
\hline Azet_Bzh_ & & 75 & & -1122.9 & \\
\hline Azet_Bzh_All_F_5 & 55501 & -1121.155053 & -1121.150477 & -1122.967751 & -1122.562726 \\
\hline Azet Bzh All F 6 & -1121.554111 & -1121.154183 & -1121.149530 & -1122.967156 & -1122.562575 \\
\hline Azet_Bzh_All_F_7 & -112 & -1121.1 & 03 & -1122.96 & 2571 \\
\hline Azet_Bzh_- & -11 & -1121 & 73 & -1122.9 & 555 \\
\hline Azet_Bzh_All_F_9 & & & -11 & 242 & 242 \\
\hline Azet_Bzh_All_F_10 & & & & & \\
\hline Azet_Bzh_ & & -1 & & 64 & 891 \\
\hline Azet_Bzh_ & & -112 & 69 & -1122 & \\
\hline Azet_Bzh_All_F_13 & 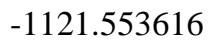 & -1 & 38 & 731 & 61203 \\
\hline Azet_B & & & & & \\
\hline Azet_Bzh & -11 & -11 & -11 & -1122. & 055 \\
\hline Azet_Bzh_ & -112 & -1121 & -112 & -1122.965611 & 560683 \\
\hline Azet_Bzh_All_F_17 & & 61 & 6147 & -1122.965219 & -1122.560636 \\
\hline Azet & & & & & \\
\hline F_19 & & & 3 & -11 & 878 \\
\hline Azet_Bzh_All_F_20 & -1121.553202 & -1121.152252 & -1121.147683 & -1122.965290 & -1122.559771 \\
\hline Azet_Bzh_All_F_21 & & & & & -1122.559702 \\
\hline Azet_Bzh_t & & & & & \\
\hline Azet_Bzh_- & -11 & -1 & -112 & -1122.9 & 558262 \\
\hline Azet_Bzh_All_F_24 & -1121.550752 & -1121.150122 & -1121.145800 & -1122.963210 & -1122.558259 \\
\hline Azet_Bzh_All_F_25 & -1121.551236 & -1121.150697 & -1121.145945 & -1122.963387 & -1122.558097 \\
\hline
\end{tabular}

Table S36: Azet ${ }_{\mathrm{Bzh}, \mathrm{Bn}}$

\begin{tabular}{cccccc}
\hline & Energy (opt) & G (opt) & G-qh (opt) & E (sp) & G-qh (sp) \\
\hline Azet_Bzh_Bn_F_1 & -1275.045778 & -1274.600736 & -1274.594651 & -1276.637323 & -1276.186195 \\
Azet_Bzh_Bn_F_2 & -1275.042985 & -1274.597830 & -1274.591827 & -1276.635679 & -1276.184521 \\
Azet_Bzh_Bn_F_3 & -1275.042985 & -1274.597820 & -1274.591821 & -1276.635682 & -1276.184518 \\
Azet_Bzh_Bn_F_4 & -1275.043430 & -1274.597931 & -1274.592187 & -1276.635001 & -1276.183758 \\
Azet_Bzh_Bn_F_5 & -1275.043429 & -1274.597925 & -1274.592182 & -1276.635002 & -1276.183754 \\
Azet_Bzh_Bn_F_6 & -1275.041916 & -1274.596424 & -1274.590678 & -1276.634742 & -1276.183505 \\
Azet_Bzh_Bn_F_7 & -1275.041998 & -1274.596518 & -1274.590397 & -1276.634946 & -1276.183346 \\
Azet_Bzh_Bn_F_8* & -1275.043421 & -1274.596709 & -1274.591444 & -1276.635146 & -1276.183170 \\
Azet_Bzh_Bn_F_9 & -1275.043041 & -1274.597600 & -1274.592030 & -1276.634142 & -1276.183132
\end{tabular}




\begin{tabular}{|c|c|c|c|c|c|}
\hline Azet_Bzh_Bn_F_10 & -1275.042301 & -1274.595783 & -1274.590588 & -1276.634749 & -1276.183036 \\
\hline Azet_Bzh_Bn_F_11 & -1275.043394 & -1274.597995 & -1274.592098 & -1276.634219 & -1276.182922 \\
\hline Azet_Bzh_Bn_F_12 & -1275.039765 & -1274.595734 & -1274.589350 & -1276.633252 & -1276.182836 \\
\hline Azet_Bzh_Bn_F_13 & -1275.042375 & -1274.596017 & -1274.590571 & -1276.634434 & -1276.182631 \\
\hline Azet_Bzh_Bn_F_14 & -1275.042375 & -1274.596016 & -1274.590571 & -1276.634434 & -1276.182631 \\
\hline Azet_Bzh_Bn_F_15 & -1275.040178 & -1274.595420 & -1274.589270 & -1276.633063 & -1276.182154 \\
\hline Azet_Bzh_Bn_F_16 & -1275.043486 & -1274.596464 & -1274.591477 & -1276.634116 & -1276.182107 \\
\hline Azet_Bzh_Bn_F_17 & -1275.043486 & -1274.596453 & -1274.591470 & -1276.634116 & -1276.182100 \\
\hline Azet_Bzh_Bn_F_18 & -1275.042732 & -1274.596270 & -1274.591127 & -1276.633590 & -1276.181985 \\
\hline Azet_Bzh_Bn_F_19 & -1275.040928 & -1274.594468 & -1274.589317 & -1276. & 181505 \\
\hline Azet_Bzh_Bn_F_20 & -1275.040584 & -1274.593483 & 88573 & -1276. & 81475 \\
\hline Azet_Bzh_Bn_F_21 & -1275.038186 & -1274.594317 & -1274.587763 & -1276.631456 & -1276.181032 \\
\hline Azet_Bzh_Bn_F_22 & -1275.039839 & -1274.593167 & -1274.588044 & -1276.6 & -1276.180937 \\
\hline Azet_Bzh_Bn_F_23 & -1275.040956 & -1274.594560 & -1274.589390 & -1276.632405 & -1276.180840 \\
\hline Azet_Bzh_Bn_F_24 & -1275.039839 & -1274.593286 & -1274.588091 & -1276.632392 & -1276.180644 \\
\hline Azet_Bzh_Bn_F_25 & -1275.041920 & -1274.594692 & -1274.589906 & -1276.632571 & -1276.180557 \\
\hline Azet_Bzh_Bn_F_26 & -1275.041920 & -1274.594692 & -1274.589907 & -1276.632568 & -1276.180555 \\
\hline Azet_Bzh_Bn_F_27 & -1275.041301 & -1274.594478 & -1274.589701 & -1276.631593 & -1276.179993 \\
\hline Azet_Bzh_Bn_F_28 & -1275.036808 & -1274.591981 & -1274.586000 & -1276.630468 & -1276.179659 \\
\hline Azet_Bzh_Bn_F_29 & -1275.038785 & -1274.593028 & -1274.587554 & -1276.630729 & -1276.179498 \\
\hline Azet_Bzh_Bn_F_30 & -1275.037959 & -1274.592208 & -1274.586597 & -1276.630683 & -1276.179321 \\
\hline Azet_Bzh_Bn_F_31 & -1275.038536 & -1274.592660 & -1274.587295 & -1276.630152 & -1276.178911 \\
\hline Azet_Bzh_Bn_F_32 & -1275.035376 & -1274.590608 & -1274.584297 & -1276.628703 & -1276.177624 \\
\hline Azet_Bzh_Bn_F_33 & -1275.039469 & -1274.592627 & -1274.587712 & -1276.629130 & -1276.177372 \\
\hline Azet_Bzh_Bn_F_34 & -1275.039469 & -1274.592606 & -1274.587701 & -1276.629130 & -1276.177362 \\
\hline
\end{tabular}

Azetidinium-Triflate Ion Pairs

Table S37: Azet ${ }_{M e, M e}$

\begin{tabular}{|c|c|c|c|c|c|}
\hline & Energy (opt) & $\mathrm{G}$ (opt) & G-qh (opt) & $E(s p)$ & G-qh (sp) \\
\hline Azet_Me_Me_OTf_1 & -1443.698994 & -1443.465156 & -1443.460315 & -1445.227439 & -1444.988760 \\
\hline Azet_Me_Me_OTf_2 & -1443.698995 & -1443.465082 & -1443.460277 & -1445.227434 & -1444.988717 \\
\hline Azet_Me_Me_OTf_3 & -1443.697351 & -1443.464071 & -1443.459021 & -1445.226776 & -1444.988446 \\
\hline Azet_Me_Me_OTf_4 & -1443.697124 & -1443.464116 & -1443.458962 & -1445.226488 & -1444.988326 \\
\hline Azet_Me_Me_OTf_5 & -1443.697125 & -1443.464096 & -1443.458952 & -1445.226485 & -1444.988312 \\
\hline Azet_Me_Me_OTf_6 & -1443.697384 & -1443.463708 & -1443.458847 & -1445.226707 & -1444.988169 \\
\hline Azet_Me_Me_OTf_7 & -1443.697384 & -1443.463702 & -1443.458844 & -1445.226701 & -1444.988161 \\
\hline Azet_Me_Me_OTf_8 & -1443.696697 & -1443.463804 & -1443.458512 & -1445.226338 & -1444.988153 \\
\hline Azet_Me_Me_OTf_9 & -1443.695979 & -1443.464797 & -1443.458813 & -1445.225260 & -1444.988093 \\
\hline Azet_Me_Me_OTf_10 & -1443.697071 & -1443.463874 & -1443.458751 & -1445.226379 & -1444.988059 \\
\hline Azet_Me_Me_OTf_11 & -1443.697596 & -1443.464068 & -1443.459133 & -1445.226512 & -1444.988049 \\
\hline Azet_Me_Me_OTf_12 & -1443.697879 & -1443.463161 & -1443.458838 & -1445.227075 & -1444.988034 \\
\hline Azet_Me_Me_OTf_13 & -1443.697317 & -1443.464265 & -1443.459006 & -1445.226314 & -1444.988003 \\
\hline Azet_Me_Me_OTf_14 & -1443.697317 & -1443.464271 & -1443.459009 & -1445.226306 & -1444.987997 \\
\hline Azet_Me_Me_OTf_15 & -1443.696869 & -1443.464040 & -1443.458920 & -1445.225848 & -1444.987899 \\
\hline Azet_Me_Me_OTf_16 & -1443.697403 & -1443.464460 & -1443.459275 & -1445.226024 & -1444.987897 \\
\hline Azet_Me_Me_OTf_17 & -1443.696869 & -1443.464037 & -1443.458918 & -1445.225847 & -1444.987896 \\
\hline Azet_Me_Me_OTf_18* & -1443.697543 & -1443.462833 & -1443.458445 & -1445.226982 & -1444.987884 \\
\hline Azet_Me_Me_OTf_19 & -1443.696432 & -1443.464302 & -1443.458853 & -1445.225442 & -1444.987863 \\
\hline Azet_Me_Me_OTf_20 & -1443.696432 & -1443.464299 & -1443.458852 & -1445.225441 & -1444.987861 \\
\hline Azet_Me_Me_OTf_21 & -1443.695568 & -1443.463337 & -1443.457817 & -1445.225597 & -1444.987846 \\
\hline Azet_Me_Me_OTf_22 & -1443.696218 & -1443.462864 & -1443.457812 & -1445.226180 & -1444.987774 \\
\hline Azet_Me_Me_OTf_23 & -1443.697266 & -1443.463176 & -1443.458599 & -1445.226435 & -1444.987768 \\
\hline Azet_Me_Me_OTf_24 & -1443.695909 & -1443.463790 & -1443.458351 & -1445.225314 & -1444.987756 \\
\hline Azet_Me_Me_OTf_25 & -1443.695568 & -1443.462866 & -1443.457577 & -1445.225595 & -1444.987604 \\
\hline Azet_Me_Me_OTf_26 & -1443.696479 & -1443.463493 & -1443.458325 & -1445.225717 & -1444.987562 \\
\hline Azet_Me_Me_OTf_27 & -1443.697273 & -1443.462797 & -1443.458468 & -1445.226326 & -1444.987521 \\
\hline Azet_Me_Me_OTf_28 & -1443.696655 & -1443.463940 & -1443.458562 & -1445.225592 & -1444.987500 \\
\hline Azet_Me_Me_OTf_29 & -1443.695544 & -1443.462630 & -1443.457402 & -1445.225588 & -1444.987447 \\
\hline Azet_Me_Me_OTf_30 & -1443.696017 & -1443.462439 & -1443.457486 & -1445.225967 & -1444.987437 \\
\hline
\end{tabular}


Azet_Me_Me_OTf_31

Azet_Me_Me_OTf_32

Azet_Me_Me_OTf_33*

Azet_Me_Me_OTf_34

Azet_Me_Me_OTf_35

Azet_Me_Me_OTf_36

Azet_Me_Me_OTf_37

Azet_Me_Me_OTf_38

Azet_Me_Me_OTf_39

Azet_Me_Me_OTf_40

Azet_Me_Me_OTf_41

Azet_Me_Me_OTf_42

Azet_Me_Me_OTf_43

Azet_Me_Me_OTf_44

Azet_Me_Me_OTf_45

Azet_Me_Me_OTf_46*

Azet_Me_Me_OTf_47

Azet_Me_Me_OTf_48

Azet_Me_Me_OTf_49*

Azet_Me_Me_OTf_50

Azet_Me_Me_OTf_51

Azet_Me_Me_OTf_52*
$-1443.695533$

$-1443.695568$

$-1443.695903$

$-1443.696822$

$-1443.696822$

$-1443.696884$

$-1443.697232$

$-1443.697925$

$-1443.696788$

$-1443.696904$

$-1443.697490$

$-1443.695813$

$-1443.697490$

$-1443.697498$

$-1443.696203$

$-1443.696863$

$-1443.697114$

$-1443.697114$

$-1443.697109$

$-1443.696584$

$-1443.697800$

$-1443.697112$
$-1443.462652$

$-1443.462461$

$-1443.462987$

$-1443.464509$

$-1443.464506$

$-1443.462725$

$-1443.464458$

$-1443.464147$

$-1443.462470$

$-1443.464291$

$-1443.463969$

$-1443.463857$

$-1443.463890$

$-1443.463594$

$-1443.462791$

$-1443.461996$

$-1443.463844$

$-1443.463831$

$-1443.461772$

$-1443.463346$

$-1443.462859$

$-1443.461424$
$-1443.457389$

$-1443.457362$

$-1443.457596$

$-1443.459083$

$-1443.459082$

$-1443.458003$

$-1443.459173$

$-1443.459321$

$-1443.457847$

$-1443.458843$

$-1443.458964$

$-1443.458185$

$-1443.458920$

$-1443.458706$

$-1443.457825$

$-1443.457656$

$-1443.458651$

$-1443.458645$

$-1443.457718$

$-1443.458105$

$-1443.458655$

$-1443.457362$
$-1445.225572$

$-1445.225586$

$-1445.225574$

$-1445.224975$

$-1445.224972$

$-1445.226007$

$-1445.225185$

$-1445.225577$

$-1445.225828$

$-1445.224923$

$-1445.225303$

$-1445.224400$

$-1445.225295$

$-1445.225440$

$-1445.224962$

$-1445.225740$

$-1445.224961$

$-1445.224959$

$-1445.225200$

$-1445.224221$

$-1445.224859$

$-1445.224907$
$-1444.987428$

$-1444.987380$

$-1444.987268$

$-1444.987237$

$-1444.987231$

$-1444.987126$

$-1444.987125$

$-1444.986974$

$-1444.986887$

$-1444.986862$

$-1444.986778$

$-1444.986772$

$-1444.986726$

$-1444.986648$

$-1444.986584$

$-1444.986532$

$-1444.986498$

$-1444.986489$

$-1444.985810$

$-1444.985742$

$-1444.985714$

$-1444.985156$

Table S38: Azet ${ }_{\mathrm{Bn}, \mathrm{Me}}$

\begin{tabular}{|c|c|c|c|c|c|}
\hline & Energy (opt) & $\mathrm{G}(\mathrm{opt})$ & G-qh (opt) & $\mathrm{E}(\mathrm{sp})$ & G-qh (sp) \\
\hline Azet_Bn_Me_OTf_1 & -1674.491463 & -1674.182673 & -1674.175550 & -1676.296505 & -1675.980591 \\
\hline Azet_Bn_Me_OTf_2 & -1674.492066 & -1674.181088 & -1674.175123 & -1676.296734 & -1675 \\
\hline Azet_Bn_Me_OTf_3 & -1674.492040 & -1674.181250 & -1674.175017 & -1676.296439 & -1675.979416 \\
\hline Azet_Bn_Me_OTf_4 & -1674.488535 & -1674.179304 & -1674.172359 & -1676.295558 & -1675.979382 \\
\hline Azet_Bn_Me_OTf_5 & -1674.492040 & -1674.181182 & -1674.174984 & -1676.296433 & -1675.979376 \\
\hline Azet_Bn_Me_OTf_6 & -1674.488535 & -1674.179273 & -1674.172342 & -1676.295556 & -1675.979363 \\
\hline Azet_Bn_Me_OTf_7 & -1674.490859 & -1674.179959 & -1674.173822 & -1676.296085 & -1675 \\
\hline Azet_Bn_Me_OTf_8 & -1674.490859 & -1674.179952 & -1674.173818 & -1676.296081 & -1675.979041 \\
\hline Azet_Bn_Me_OTf_9 & -1674.489442 & -1674.179735 & -1674.173054 & -1676.295379 & -1675.978991 \\
\hline Azet_Bn_Me_OTf_10 & -167 & -1674.1 & -167 & -1676.2 & -1675 \\
\hline Azet_Bn_Me_OTf_11 & -1674.491208 & -1674.180901 & -1674.174499 & -1676.295473 & -1675.978764 \\
\hline Azet_Bn_Me_OTf_12 & -167 & -1674 & -167 & -1676 & -167 \\
\hline Azet_Bn_Me_OTf_13 & -167 & -1674 & -167 & -1676 . & -167 \\
\hline Azet_Bn_Me_OTf_14 & -1674 & -1674.179078 & 71977 & -1676.2 & -1675 \\
\hline Azet_Bn_Me_OTf_15 & -167 & -1674. & -167 & -1676 & -167 \\
\hline Azet_Bn_Me_OTf_16 & -167 & -1674 & 1748 & -1676 & -167 \\
\hline Azet_Bn_Me_OTf_17 & -167 & -1674 & -167 & -1676 & -167 \\
\hline Azet_Bn_Me_OTf_18 & -167 & -1674 & -16 & -1676 . & -167 \\
\hline Azet_Bn_Me_OTf_19 & -167 & -1674 & -167 & -1676. & -1675 \\
\hline Azet_Bn_Me_OTf_20 & -1674.491000 & -1674.180199 & -1674.174020 & -1676 & -167 \\
\hline Azet_Bn_Me_OTf_21 & -167 & -1674 & -167 & -1676 . & -167 \\
\hline Azet_Bn_Me_OTf_22 & -167 & -1674.179303 & -1674.172932 & -1676.2 & -1675 \\
\hline Azet_Bn_Me_OTf_23 & -167 & -1674.179295 & -167 & -1676.2 & -167 \\
\hline Azet_Bn_Me_OTf_24 & -1674.491366 & -1674.180150 & -1674.174144 & -1676.295715 & -167 \\
\hline Azet_Bn_Me_OTf_25 & -1674.488458 & -1674.179121 & -1674.172205 & -1676.294677 & -167 \\
\hline Azet_Bn_Me_OTf_26 & -1674.487254 & -1674.178641 & -1674.171575 & -1676.293819 & -1675.978140 \\
\hline Azet_Bn_Me_OTf_27 & -1674.487165 & -1674.179872 & -1674.172239 & -1676.292964 & 78038 \\
\hline Azet_Bn_Me_OTf_28 & -167 & -1674 & -1674.174302 & -1676.294832 & 8004 \\
\hline Azet_Bn_Me_OTf_29 & -1674.490582 & -1674.180843 & -1674.174364 & -1676.294082 & -1675.977864 \\
\hline Azet_Bn_Me_OTf_30 & -1674.487117 & -1674.178618 & -1674.171576 & -1676.293335 & 77793 \\
\hline Azet_Bn_Me_OTf_31 & -1674.491042 & -1674.180031 & -1674.174247 & -1676.294483 & -1675.977688 \\
\hline Azet_Bn_Me_OTf_32 & -1674.491046 & -1674.179960 & -1674.174206 & -1676.294477 & -1675.977638 \\
\hline Azet_Bn_Me_OTf_33 & -1674.491495 & -1674.179406 & -1674.174047 & -1676.295064 & -1675.977615 \\
\hline Azet_Bn_Me_OTf_34 & -1674.491495 & -1674.179396 & -1674.174041 & -1676.295063 & -1675.977610 \\
\hline Azet_Bn_Me_OTf_35 & -1674.487823 & -1674.179023 & -1674.171930 & -1676.293464 & -1675.977570 \\
\hline
\end{tabular}




\begin{tabular}{llllll} 
Azet_Bn_Me_OTf_36 & -1674.491654 & -1674.179510 & -1674.174133 & -1676.295023 & -1675.977502 \\
Azet_Bn_Me_OTf_37 & -1674.491654 & -1674.179488 & -1674.174121 & -1676.295021 & -1675.977487 \\
Azet_Bn_Me_OTf_38 & -1674.490534 & -1674.179896 & -1674.173677 & -1676.294292 & -1675.977435 \\
Azet_Bn_Me_OTf_39 & -1674.490533 & -1674.179884 & -1674.173666 & -1676.294282 & -1675.977414 \\
Azet_Bn_Me_OTf_40 & -1674.491207 & -1674.179773 & -1674.174026 & -1676.294260 & -1675.977079 \\
Azet_Bn_Me_OTf_41 & -1674.487177 & -1674.177733 & -1674.170953 & -1676.293047 & -1675.976822 \\
Azet_Bn_Me_OTf_42 & -1674.490515 & -1674.179042 & -1674.173396 & -1676.293777 & -1675.976659 \\
Azet_Bn_Me_OTf_43 & -1674.490515 & -1674.179034 & -1674.173392 & -1676.293767 & -1675.976645 \\
Azet_Bn_Me_OTf_44 & -1674.487768 & -1674.178178 & -1674.171644 & -1676.292304 & -1675.976181 \\
\hline
\end{tabular}

Table S39: Azet $_{\mathrm{Bn}, \mathrm{Bn}}$

\begin{tabular}{|c|c|c|c|c|c|}
\hline & Energy (opt) & $\mathrm{G}(\mathrm{opt})$ & G-qh (opt) & $\mathrm{E}(\mathrm{sp})$ & G-qh (sp) \\
\hline Azet_Bn_Bn_OTf_1 & -1905.281263 & -1904.895744 & -1904.887165 & 07.363466 & -1906.969369 \\
\hline Azet_Bn_Bn_OTf_2 & -1905 & 96229 & 176 & -1907.363383 & -190 \\
\hline Azet_Bn_Bn_OTf_3 & -1905.280321 & 1904.894997 & -1904.886465 & -1907.362427 & -1906 \\
\hline Azet_Bn_Bn_OTf_4 & -1905 & 3874 & 39 & & -10 \\
\hline Azet_Bn_Bn_OTf_5 & -1905.2 & 94991 & -1904.888004 & -1907.3 & -190 \\
\hline Azet_Bn_Bn_OTf_6 & -190 & 904. & & -1907 & -19 \\
\hline Azet_Bn_Bn_C & -190 & -1904 & 00 & & -19 \\
\hline Azet_Bn_Bn_OTf_8 & -190 & -1904. & -190 & -1907. & -19 \\
\hline Azet_Bn_Bn_OTf_9 & -190 & 26 & 69 & & -19 \\
\hline Azet_Bn_Bn_OTf_10 & -1905. & -1904.8 & -1904.88 & & -190 \\
\hline Azet_Bn_I & -190 & -190 & -19 & -19 & -19 \\
\hline Azet_Bn_Bn_OTf_12 & -190 & & & & \\
\hline Azet_Bn_Bn_OTf_13 & -190 & 09 & & -1907 & -19 \\
\hline Bn_Bn_OTf_14 & -190 & -15 & & & \\
\hline Azet & -190 & & & & \\
\hline OTf_16 & -190 & -19 & -19 & -19 & -19 \\
\hline Azet_Bn_Bn_OTf_17 & -190 & & & & \\
\hline Azet_Bn_Bn_OTf_18 & -19 & & & & \\
\hline Azet_Bn_Bn_OTf_19 & -190 & -190 & & -19 & \\
\hline Azet_Bn_Bn_OTf_20 & -19 & & & & \\
\hline Azet_Bn_Bn_OTf_21 & -190 & & & & \\
\hline & & & & & \\
\hline 1OTf_23 & & & & & \\
\hline n_OTf_24 & -190 & -1 & & -19 & \\
\hline Azet_Bn_Bn_OTf_25 & -19 & -1 & & -19 & -19 \\
\hline Azet_Bn_Bn_OTf_26 & & & & & \\
\hline Azet_Bn_Bn_OTf_27 & & & & & \\
\hline Tf_28 & -19 & -1 & 39 & -1 & -19 \\
\hline Azet_Bn_Bn_OTf_29 & -190 & & & & \\
\hline & & & & & \\
\hline Azet_Bn_Bn_OTf_31 & -190 & -1904 & & -1907 & -19 \\
\hline Azet_Bn_Bn_OTf_32 & & & & & \\
\hline Azet_Bn_Bn_OTf_33 & -190 & -190 & -19 & -190 & -19 \\
\hline Azet_Bn_Bn_OTf_34 & -190 & -1904. & -1904. & -1907.360676 & -190 \\
\hline Azet_Bn_Bn_OTf_35 & & & & & \\
\hline Azet_Bn_Bn_OTf_36 & -190 & -1904 & & -1907. & -190 \\
\hline Azet_Bn_Bn_OTf_37 & -1905.279370 & -1904.891647 & -1904.884281 & -1907.360189 & -1906.965101 \\
\hline
\end{tabular}

Table S40: Azet ${ }_{\text {Bzh,Me }}$

\begin{tabular}{lccccc}
\hline & Energy (opt) & G (opt) & G-qh (opt) & E (sp) & G-qh (sp) \\
\hline Azet_Bzh_Me_OTf_1 & -1905.277419 & -1904.891864 & -1904.883723 & -1907.359096 & -1906.965400 \\
Azet_Bzh_Me_OTf_2 & -1905.278552 & -1904.890803 & -1904.883712 & -1907.359352 & -1906.964512 \\
Azet_Bzh_Me_OTf_3 & -1905.277939 & -1904.890957 & -1904.883405 & -1907.358768 & -1906.964235 \\
Azet_Bzh_Me_OTf_4 & -1905.277939 & -1904.890947 & -1904.883401 & -1907.358762 & -1906.964225 \\
Azet_Bzh_Me_OTf_5 & -1905.278861 & -1904.891035 & -1904.883986 & -1907.359052 & -1906.964177 \\
Azet_Bzh_Me_OTf_6 & -1905.278861 & -1904.891026 & -1904.883983 & -1907.359054 & -1906.964175 \\
Azet_Bzh_Me_OTf_7 & -1905.278095 & -1904.890608 & -1904.883358 & -1907.358729 & -1906.963992
\end{tabular}


Azet_Bzh_Me_OTf 8 Azet_Bzh_Me_OTf_9 Azet_Bzh_Me_OTf_10 Azet_Bzh_Me_OTf_11 Azet_Bzh_Me_OTf_12 Azet_Bzh_Me_OTf_13 Azet_Bzh_Me_OTf_14 Azet_Bzh_Me_OTf_15 Azet_Bzh_Me_OTf_16 Azet_Bzh_Me_OTf_17 Azet_Bzh_Me_OTf_18 Azet_Bzh_Me_OTf_19 Azet_Bzh_Me_OTf_20 Azet_Bzh_Me_OTf_21 Azet_Bzh_Me_OTf_22 Azet_Bzh_Me_OTf_23 Azet_Bzh_Me_OTf_24 Azet_Bzh_Me_OTf_25 Azet_Bzh_Me_OTf_26 Azet_Bzh_Me_OTf_27 Azet_Bzh_Me_OTf_28 Azet_Bzh_Me_OTf_29 Azet_Bzh_Me_OTf_30 Azet_Bzh_Me_OTf_31 Azet_Bzh_Me_OTf_32 Azet_Bzh_Me_OTf_33 Azet_Bzh_Me_OTf_34* Azet_Bzh_Me_OTf_35* Azet_Bzh_Me_OTf_36 Azet_Bzh_Me_OTf_37 Azet_Bzh_Me_OTf_38
$-1905.277302$ $-1905.276235$ $-1905.276236$ $-1905.276110$ $-1905.276502$ $-1905.276966$ $-1905.277602$ $-1905.277601$ $-1905.273710$ $-1905.277155$ $-1905.275893$ $-1905.274455$ $-1905.276548$ $-1905.274455$ $-1905.274399$ $-1905.274242$ $-1905.276089$ $-1905.276089$ $-1905.275226$ $-1905.275674$ $-1905.275674$ $-1905.276077$ $-1905.274465$ $-1905.276768$ $-1905.276769$ $-1905.274226$ $-1905.274535$ $-1905.275808$ $-1905.276767$ $-1905.274964$ $-1905.275830$
$-1904.891346$ $-1904.889919$ $-1904.889904$ $-1904.890898$ $-1904.889808$ $-1904.890469$ $-1904.890649$ $-1904.890654$ $-1904.890075$ $-1904.890284$ $-1904.890177$ $-1904.889194$ $-1904.890096$ $-1904.888955$ $-1904.888502$ $-1904.887899$ $-1904.889678$ $-1904.889683$ $-1904.889882$ $-1904.888644$ $-1904.888622$ $-1904.889701$ $-1904.889827$ $-1904.888647$ $-1904.888590$ $-1904.887634$ $-1904.886298$ $-1904.887882$ $-1904.889055$ $-1904.887330$ $-1904.888937$
$-1904.883271$ $-1904.882197$ $-1904.882191$ $-1904.882523$ $-1904.882260$ $-1904.882821$ $-1904.883096$ $-1904.883100$ $-1904.880906$ $-1904.882661$ $-1904.882014$ $-1904.880902$ $-1904.882327$ $-1904.880782$ $-1904.880451$ $-1904.880247$ $-1904.882135$ $-1904.882136$ $-1904.881718$ $-1904.881299$ $-1904.881290$ $-1904.881927$ $-1904.881054$ $-1904.881943$ $-1904.881909$ $-1904.879975$ $-1904.879163$ $-1904.880848$ $-1904.881804$ $-1904.880207$ $-1904.881481$
$-1907.357996$ $-1907.357834$ $-1907.357837$ $-1907.357232$ $-1907.357760$ $-1907.357659$ $-1907.358018$ $-1907.358013$ $-1907.356177$ $-1907.357663$ $-1907.356817$ $-1907.356410$ $-1907.356949$ $-1907.356401$ $-1907.356573$ $-1907.356495$ $-1907.356420$ $-1907.356417$ $-1907.355901$ $-1907.356560$ $-1907.356556$ $-1907.356289$ $-1907.355460$ $-1907.356705$ $-1907.356693$ $-1907.356056$ $-1907.357161$ $-1907.356645$ $-1907.356560$ $-1907.356256$ $-1907.355631$
$-1906.963965$ $-1906.963796$ $-1906.963792$ $-1906.963645$ $-1906.963519$ $-1906.963514$ $-1906.963511$ $-1906.963511$ $-1906.963373$ $-1906.963169$ $-1906.962937$ $-1906.962857$ $-1906.962729$ $-1906.962727$ $-1906.962624$ $-1906.962500$ $-1906.962466$ $-1906.962464$ $-1906.962394$ $-1906.962185$ $-1906.962172$ $-1906.962138$ $-1906.962048$ $-1906.961879$ $-1906.961832$ $-1906.961805$ $-1906.961789$ $-1906.961685$ $-1906.961597$ $-1906.961499$ $-1906.961282$

Table S41: Azet $_{\mathrm{Bzh}, \mathrm{Et}}$

\begin{tabular}{|c|c|c|c|c|c|}
\hline & Energy (opt) & $\mathrm{G}(\mathrm{opt})$ & G-qh (opt) & $\mathrm{E}(\mathrm{sp})$ & G-qh (sp) \\
\hline Azet_Bzh_Et_OTf_1 & -1944.543731 & -1944.130145 & -1944.122221 & -1946.678935 & -1946.257424 \\
\hline Azet_Bzh_Et_OTf_2 & -1944.543731 & -1944.129954 & -1944.122126 & -1946.678936 & -1946 \\
\hline Azet_Bzh_Et_OTf_3 & -1944.545202 & -1944.129845 & -1944.122693 & -1946.679704 & -1946.257195 \\
\hline Azet_Bzh_Et_OTf_4 & -1944.540849 & -1944.129061 & -1944.120357 & -1946.677667 & -1946.257175 \\
\hline Azet_Bzh_Et_OTf_5 & -1944.544017 & -1944.128834 & -1944.121691 & -1946.679370 & -1946.257043 \\
\hline Azet_Bzh_Et_OTf_6 & -1944.544019 & -1944.128830 & -1944.121684 & -1946.679376 & -1946.257041 \\
\hline Azet_Bzh_Et_OTf_7 & -1944.543606 & -1944.130213 & -1944.122253 & -1946.678285 & -1946. \\
\hline Azet_Bzh_Et_OTf_8 & -1944.542924 & -1944.130025 & -1944.121875 & -1946.677862 & -1946.256814 \\
\hline Azet_Bzh_Et_OTf_9 & -1944.544305 & -1944.129230 & -1944.121977 & -1946.679119 & -1946 \\
\hline Azet_Bzh_Et_OTf_10 & -1944.542006 & -1944.128631 & -1944 & -1946.6 & -1946 \\
\hline Azet_Bzh_Et_OTf_11 & -1944.543650 & -1944.129354 & -1944.121647 & -1946.678674 & -1946.256671 \\
\hline Azet_Bzh_Et_OTf_12 & -194 & -1944 & -194 & -1946. & -1946 \\
\hline Azet_Bzh_Et_OTf_13 & -1944 & -1944.129345 & -1944 & -1946.6 & -1946 \\
\hline Azet_Bzh_Et_OTf_14 & -1944.542831 & -1944.128302 & -1944.120831 & -1946.678297 & -1946. \\
\hline Azet_Bzh_Et_OTf_15 & -1944.543289 & -1944.129314 & -1944.121489 & -1946.678086 & -1946.256286 \\
\hline Azet_Bzh_Et_OTf_16 & -1944.542646 & -1944.127705 & -1944.120430 & -1946.678350 & -1946. \\
\hline Azet_Bzh_Et_OTf_17 & -1944.542586 & -1944.127850 & -1944.120569 & -1946.678110 & -1946. \\
\hline Azet_Bzh_Et_OTf_18 & -1944.542142 & -1944.127669 & -1944.120194 & -1946.677890 & -1946.255941 \\
\hline Azet_Bzh_Et_OTf_19 & -1944.540726 & -1944.127209 & -1944.119342 & -1946.677013 & -1946.255630 \\
\hline Azet_Bzh_Et_OTf_20 & -1944.542188 & -1944.128002 & -1944.120387 & -1946.677368 & -1946.255566 \\
\hline Azet_Bzh_Et_OTf_21 & -1944.540458 & -1944.127768 & -1944.119484 & -1946.676422 & -1946.255448 \\
\hline Azet_Bzh_Et_OTf_22 & -1944.542691 & -1944.126914 & -1944.120263 & -1946.677850 & -1946.255422 \\
\hline Azet_Bzh_Et_OTf_23 & -1944.540185 & -1944.126876 & -1944.118821 & -1946.676722 & -1946.255358 \\
\hline Azet_Bzh_Et_OTf_24 & -1944.540915 & -1944.127814 & -1944.119559 & -1946.676444 & -1946.255088 \\
\hline Azet_Bzh_Et_OTf_25 & -1944.541946 & -1944.127757 & -1944.120055 & -1946.676630 & -1946.254739 \\
\hline Azet_Bzh_Et_OTf_26 & -1944.540491 & -1944.126397 & -1944.118730 & -1946.675810 & -1946.254050 \\
\hline
\end{tabular}


Azet_Bzh_Et_OTf_27 Azet_Bzh_Et_OTf_28 Azet_Bzh_Et_OTf_29 Azet_Bzh_Et_OTf_30 Azet_Bzh_Et_OTf_31 Azet_Bzh_Et_OTf_32 Azet_Bzh_Et_OTf_33 Azet_Bzh_Et_OTf_34 Azet_Bzh_Et_OTf_35 Azet_Bzh_Et_OTf_36
$-1944.540049$ $-1944.540416$ $-1944.540416$ $-1944.539514$ $-1944.539966$ $-1944.539966$ $-1944.540462$ $-1944.540462$ $-1944.540333$ $-1944.537381$
$-1944.125384$ $-1944.127053$ $-1944.127061$ $-1944.127184$ $-1944.125798$ $-1944.125784$ $-1944.125382$ $-1944.125374$ $-1944.125930$ $-1944.124575$
$-1944.117856$ $-1944.119061$ $-1944.119056$ $-1944.118595$ $-1944.118156$ $-1944.118148$ $-1944.118191$ $-1944.118186$ $-1944.118216$ $-1944.116078$
$-1946.675961$ $-1946.674835$ $-1946.674812$ $-1946.674362$ $-1946.674860$ $-1946.674857$ $-1946.675290$ $-1946.675291$ $-1946.674987$ $-1946.673429$
$-1946.253768$ $-1946.253480$ $-1946.253452$ $-1946.253444$ $-1946.253051$ $-1946.253040$ $-1946.253019$ $-1946.253015$ $-1946.252870$ $-1946.252125$

Table S42: Azet $_{\mathrm{Bzh}, \mathrm{Allyl}}$

\begin{tabular}{|c|c|c|c|c|c|}
\hline & Energy (opt) & $\mathrm{G}(\mathrm{opt})$ & G-qh (opt) & $\mathrm{E}(\mathrm{sp})$ & G-qh (sp) \\
\hline Azet_Bzh_ & -1982.579455 & -1982.161508 & -1982.153719 & 984.757965 & -1984.33222 \\
\hline Azet_Bzh_All_OTf_2 & 1982.577067 & 982.162086 & -1982.152819 & -1984.756146 & -198 \\
\hline Azet_Bzh_All_OTf_3 & -1982.577067 & -1982.162037 & -1982.152794 & -1984.756147 & -198 \\
\hline Azet_Bzh_All_OTf_4 & -198 & -1982.161333 & -198 & & \\
\hline Azet_Bzh_All_OTf_5 & -1982.578507 & -1982.160933 & -198 & -1984.757306 & -198 \\
\hline Azet_Bzh_All_OTf_6 & -198 & -1982 & -19 & -1984 & \\
\hline Azet_Bzh_All_C & -198 & -1982 & -19 & -1984. & \\
\hline Azet_Bzh_All_OTf_8 & -198 & -1982.161320 & -198 & -1984.756765 & -198 \\
\hline Azet_Bzh_All_OTf_9 & -198 & -1982 & -19 & 83 & \\
\hline Azet_Bzh_All_OTf_10 & -198 & -1982. & -198 & -1984 & -198 \\
\hline Azet_Bzh_All_OTf_11 & -198 & -1982 & -19 & -1984 & -19 \\
\hline Azet_Bzh_All_OTf_12 & -19 & & & & \\
\hline Azet_Bzh_A & -19 & -1982 & -19 & -1984. & -19 \\
\hline Azet_Bzh_t & -19 & -198 & -19 & -19 & -19 \\
\hline Azet_Bzh_ & -19 & & & & \\
\hline Azet_Bzh_A & -19 & -198 & -19 & -19 & -19 \\
\hline Azet_Bzh_All_C & & & & & \\
\hline Azet_Bzh_t & & & & & \\
\hline Azet_Bzh_A & -19 & -198 & -19 & -19 & -15 \\
\hline Azet_Bzh_- & & & & & \\
\hline Azet_Bzh_All_OTf_21 & & -198 & & & \\
\hline f_22 & & & & & \\
\hline Azet_Bzh_A & & & & & \\
\hline Azet_Bzh_A & -19 & -198 & -19 & -19 & \\
\hline Azet_Bzh_A & & & & & \\
\hline Azet_Bzh_All_OTf_26 & & -1982 & & -1984. & \\
\hline Azet_Bzh_All_OTf_27 & & & & & \\
\hline Azet_Bzh_- & -15 & 03 & & 78 & -15 \\
\hline Azet_Bzh_All_OTf_29 & & & & & \\
\hline & & & & & \\
\hline Azet_Bzh_All_OTf_31 & & -1982.1 & & -1984. & -19 \\
\hline Azet_Bzh_All_OTf_32 & & & & & \\
\hline Azet_Bzh_A & -19 & -198 & -19 & -198 & -19 \\
\hline Azet_Bzh_All_OTf_34 & -198 & -1982.1 & -1982. & -1984. & -198 \\
\hline Azet_Bzh_All_OTf_35* & & & & & -19 \\
\hline Azet_Bzh_All_OTf_36 & -198 & -1982. & & -1984. & -19 \\
\hline Azet_Bzh_A & .576214 & -1982. & -1982.150916 & -1984. & -19 \\
\hline Azet_Bzh_All_OTf_38 & & & & & -198 \\
\hline Azet_Bzh_All_OTf_39 & -1982.576583 & -1982.159206 & -1982.150882 & -1984.754737 & -1984.329037 \\
\hline
\end{tabular}

Table S43: Azet $_{\text {Bzh,Bn }}$

\begin{tabular}{lccccc}
\hline & Energy (opt) & G (opt) & G-qh (opt) & E (sp) & G-qh (sp) \\
\hline Azet_Bzh_Bn_OTf_1 & -2136.068799 & -2135.605692 & -2135.596489 & -2138.426659 & -2137.954349 \\
Azet_Bzh_Bn_OTf_2 & -2136.068799 & -2135.605685 & -2135.596486 & -2138.426661 & -2137.954348 \\
Azet_Bzh_Bn_OTf_3 & -2136.068857 & -2135.605911 & -2135.596687 & -2138.426414 & -2137.954244 \\
Azet_Bzh_Bn_OTf_4 & -2136.067984 & -2135.605590 & -2135.595977 & -2138.425917 & -2137.953910
\end{tabular}




\begin{tabular}{|c|c|c|c|c|c|}
\hline Azet_Bzh_Bn_OTf_5 & 36.067766 & 606168 & 6211 & 180 & \\
\hline Azet_Bzh_Bn_OTf_6 & & 604495 & 595714 & -2138.426310 & \\
\hline Azet_Bzh_Bn_O & & & & & \\
\hline Tf_8 & & & & & \\
\hline Azet_Bzh_Bn_OTf_9 & 5.066663 & 033 & 5073 & 709 & 53119 \\
\hline Rah Bn OTf 10 & 6066663 & & & & \\
\hline & & & & & \\
\hline & & & & & \\
\hline & & & & & \\
\hline & & & & & \\
\hline & & & & & \\
\hline & & & & & \\
\hline & & & & & \\
\hline & & & & & \\
\hline & & & & & \\
\hline & & & & & \\
\hline & & & & & \\
\hline & & & & & \\
\hline & & & & & 33 \\
\hline & & & & & \\
\hline & & & & & \\
\hline & & & & & \\
\hline & & & & & \\
\hline & & & & & \\
\hline & & & & & \\
\hline 30 & & & & & 970 \\
\hline & & & & & \\
\hline & & & & & \\
\hline & & & & & \\
\hline & & & & & 882 \\
\hline & & & & & \\
\hline & & & & & \\
\hline & & & & & \\
\hline Azet_B & -2136.063836 & -2135.599653 & -2135.591300 & -2138.419610 & -2137.9470 \\
\hline
\end{tabular}

\section{Azetidinium Fluorination Transition State Structures}

Table S44: Azet ${ }_{\mathrm{Me}, \mathrm{Me}}$

\begin{tabular}{lccccc}
\hline & Energy (opt) & G (opt) & G-qh (opt) & E (sp) & G-qh (sp) \\
\hline TS_Me_Me_F_1 & -582.644436 & -582.428903 & -582.427323 & -583.405767 & -583.188653 \\
TS_Me_Me_F_2 & -582.645614 & -582.428995 & -582.427974 & -583.404913 & -583.187272 \\
TS_Me_Me_F_3 & -582.645614 & -582.428991 & -582.427971 & -583.404908 & -583.187264 \\
\hline
\end{tabular}

Table S45: Azet $_{\mathrm{Bn}, \mathrm{Me}}$

\begin{tabular}{cccccc}
\hline & Energy (opt) & G (opt) & G-qh (opt) & E (sp) & G-qh (sp) \\
\hline TS_Bn_Me_F_1 & -813.435421 & -813.144173 & -813.140916 & -814.474250 & -814.179744 \\
TS_Bn_Me_F_2 & -813.434691 & -813.144325 & -813.140638 & -814.473635 & -814.179582 \\
TS_Bn_Me_F_3 & -813.436899 & -813.143884 & -813.141469 & -814.474775 & -814.179345 \\
TS_Bn_Me_F_4 & -813.436899 & -813.143882 & -813.141468 & -814.474769 & -814.179338 \\
TS_Bn_Me_F_5 & -813.436899 & -813.143879 & -813.141466 & -814.474770 & -814.179337 \\
TS_Bn_Me_F_6 & -813.437395 & -813.144843 & -813.142228 & -814.474045 & -814.178878 \\
TS_Bn_Me_F_7 & -813.437395 & -813.144842 & -813.142228 & -814.474043 & -814.178876 \\
TS_Bn_Me_F_8 & -813.435712 & -813.143502 & -813.140737 & -814.472622 & -814.177647 \\
TS_Bn_Me_F_9 & -813.435712 & -813.143499 & -813.140735 & -814.472619 & -814.177641 \\
TS_Bn_Me_F_10 & -813.436557 & -813.142480 & -813.140660 & -814.472194 & -814.176297 \\
TS_Bn_Me_F_11 & -813.436557 & -813.142480 & -813.140660 & -814.472194 & -814.176297 \\
TS_Bn_Me_F_12 & -813.432898 & -813.142201 & -813.138815 & -814.469521 & -814.175438 \\
TS_Bn_Me_F_13 & -813.433285 & -813.141334 & -813.138401 & -814.470115 & -814.175231 \\
\hline
\end{tabular}


Table S46: Azet ${ }_{\mathrm{Bn}, \mathrm{Bn}}$

\begin{tabular}{|c|c|c|c|c|c|}
\hline & Energy (opt) & G (opt) & G-qh (opt) & E (sp) & G-qh (sp) \\
\hline I_F_1 & -1044.226744 & -1043.857865 & -1043.853752 & 45.542179 & 045.169187 \\
\hline TS_Bn_Bn_F_2 & -1044.224829 & -1043.857864 & -1043.852778 & -1045.540916 & -1045.168865 \\
\hline TS_Bn_Bn_F_3 & 44.227483 & & & 629 & 68725 \\
\hline TS_Bn_Bn_F_4 & 44.223862 & & -1043 & -1045.540148 & 68329 \\
\hline TS_Bn_Bn_F_5 & -1044.2 & & & & 8224 \\
\hline TS_Bn_Bn_F_6 & -1044.225211 & -1043.856521 & -1043.852388 & -1045.541041 & -1045.168218 \\
\hline TS_Bn & & & & & \\
\hline TS_Br & & & -10 & & \\
\hline TS_Bn_Bn_F_9 & & & & & \\
\hline 1_F_10 & & & & & \\
\hline _11 & & & 52 & 09 & \\
\hline 12 & & & & & \\
\hline TS_Bn_Bn_F_13 & & & & & 009 \\
\hline 14 & & & & & \\
\hline 15 & & & & & \\
\hline E_F_16 & -104 & & -10 & -104 & -10 \\
\hline TS & & & & & \\
\hline TS_l & & & & & 09 \\
\hline TS_E & & & -10 & -104 & 6509 \\
\hline TS_Bn_Bn_F_20 & & & -1043 & -1045.5 & -104 \\
\hline & & & & & \\
\hline TS & & & & & \\
\hline F_23 & -1044.2 & -1043 & -1043 & -1045.53 & 65671 \\
\hline TS_Bn_Bn_F_24 & & & & & -1045.165358 \\
\hline & & & & & \\
\hline 26 & & & & & 65 \\
\hline F_27 & -1044.223383 & -1043.8 & -1043.850819 & -1045.536663 & -1045.164099 \\
\hline & & & & & \\
\hline TS_Bn_Bn_F_29 & -1044.223628 & -1043.8 & -1043.850690 & -1045.536919 & -1045.163980 \\
\hline
\end{tabular}

Table S47: Azet ${ }_{\text {Bzh,Me }}$

\begin{tabular}{lccccc}
\hline & Energy (opt) & G (opt) & G-qh (opt) & E (sp) & G-qh (sp) \\
\hline TS_Bzh_Me_F_1 & -1044.224371 & -1043.855352 & -1043.851688 & -1045.538887 & -1045.166204 \\
TS_Bzh_Me_F_2 & -1044.224412 & -1043.855370 & -1043.851700 & -1045.538842 & -1045.166130 \\
TS_Bzh_Me_F_3 & -1044.221393 & -1043.854836 & -1043.849986 & -1045.536720 & -1045.165313 \\
TS_Bzh_Me_F_4 & -1044.221932 & -1043.854513 & -1043.849970 & -1045.536836 & -1045.164873 \\
TS_Bzh_Me_F_5 & -1044.224260 & -1043.855111 & -1043.851244 & -1045.536947 & -1045.163931 \\
TS_Bzh_Me_F_6 & -1044.223795 & -1043.854197 & -1043.850953 & -1045.535921 & -1045.163079 \\
TS_Bzh_Me_F_7 & -1044.222760 & -1043.852724 & -1043.849596 & -1045.536192 & -1045.163027 \\
TS_Bzh_Me_F_8 & -1044.222769 & -1043.852695 & -1043.849633 & -1045.535182 & -1045.162046 \\
TS_Bzh_Me_F_9 & -1044.222020 & -1043.853298 & -1043.849350 & -1045.534636 & -1045.161966 \\
\hline
\end{tabular}

Table S48: Azet ${ }_{\mathrm{Bzh}, \mathrm{Et}}$

\begin{tabular}{cccccc}
\hline & Energy (opt) & G (opt) & G-qh (opt) & E (sp) & G-qh (sp) \\
\hline TS_Bzh_Et_F_1 & -1083.490333 & -1083.093341 & -1083.089800 & -1084.859294 & -1084.458761 \\
TS_Bzh_Et_F_2 & -1083.490148 & -1083.093588 & -1083.089832 & -1084.858983 & -1084.458666 \\
TS_Bzh_Et_F_3 & -1083.489687 & -1083.092297 & -1083.089010 & -1084.856603 & -1084.455925 \\
TS_Bzh_Et_F_4 & -1083.488428 & -1083.091873 & -1083.087960 & -1084.856110 & -1084.455642 \\
TS_Bzh_Et_F_5 & -1083.486271 & -1083.089829 & -1083.086104 & -1084.855553 & -1084.455385 \\
TS_Bzh_Et_F_6 & -1083.484842 & -1083.089655 & -1083.085140 & -1084.855001 & -1084.455299 \\
TS_Bzh_Et_F_7 & -1083.486301 & -1083.089857 & -1083.086054 & -1084.855459 & -1084.455211 \\
TS_Bzh_Et_F_8 & -1083.489195 & -1083.091394 & -1083.088387 & -1084.855926 & -1084.455117 \\
TS_Bzh_Et_F_9 & -1083.484283 & -1083.090003 & -1083.085053 & -1084.854069 & -1084.454839 \\
TS_Bzh_Et_F_10 & -1083.485359 & -1083.089059 & -1083.085187 & -1084.854884 & -1084.454712 \\
TS_Bzh_Et_F_11 & -1083.486526 & -1083.090298 & -1083.086389 & -1084.854129 & -1084.453992 \\
TS_Bzh_Et_F_12 & -1083.482936 & -1083.088047 & -1083.083433 & -1084.852914 & -1084.453410 \\
TS_Bzh_Et_F_13 & -1083.483152 & -1083.087401 & -1083.083158 & -1084.852592 & -1084.452598
\end{tabular}




\begin{tabular}{llllll} 
TS_Bzh_Et_F_14 & -1083.482972 & -1083.086768 & -1083.082808 & -1084.852693 & -1084.452529 \\
TS_Bzh_Et_F_15 & -1083.483922 & -1083.088626 & -1083.084183 & -1084.852136 & -1084.452397 \\
TS_Bzh_Et_F_16 & -1083.483147 & -1083.087012 & -1083.082776 & -1084.852622 & -1084.452250 \\
TS_Bzh_Et_F_17 & -1083.483751 & -1083.088049 & -1083.084019 & -1084.851901 & -1084.452169 \\
TS_Bzh_Et_F_18 & -1083.481776 & -1083.086692 & -1083.082301 & -1084.851531 & -1084.452056 \\
TS_Bzh_Et_F_19 & -1083.485114 & -1083.088927 & -1083.084802 & -1084.852269 & -1084.451957 \\
TS_Bzh_Et_F_20 & -1083.482132 & -1083.086438 & -1083.082076 & -1084.851984 & -1084.451928 \\
TS_Bzh_Et_F_21 & -1083.483617 & -1083.087307 & -1083.083312 & -1084.852033 & -1084.451728 \\
TS_Bzh_Et_F_22 & -1083.484602 & -1083.087729 & -1083.084242 & -1084.852034 & -1084.451673 \\
TS_Bzh_Et_F_23 & -1083.486026 & -1083.088842 & -1083.085436 & -1084.852214 & -1084.451624 \\
TS_Bzh_Et_F_24 & -1083.486026 & -1083.088840 & -1083.085435 & -1084.852213 & -1084.451623 \\
TS_Bzh_Et_F_25 & -1083.481898 & -1083.086289 & -1083.081922 & -1084.851135 & -1084.451160 \\
TS_Bzh_Et_F_26 & -1083.481222 & -1083.086395 & -1083.081605 & -1084.850054 & -1084.450437 \\
TS_Bzh_Et_F_27 & -1083.481088 & -1083.084740 & -1083.080937 & -1084.850209 & -1084.450058 \\
TS_Bzh_Et_F_28 & -1083.481545 & -1083.085209 & -1083.081073 & -1084.850257 & -1084.449785 \\
TS_Bzh_Et_F_29 & -1083.478935 & -1083.083483 & -1083.079046 & -1084.848424 & -1084.448535 \\
TS_Bzh_Et_F_30 & -1083.480468 & -1083.084219 & -1083.080061 & -1084.848851 & -1084.448443 \\
\hline
\end{tabular}

Table S49: Azet $_{\text {Bzh,Allyl }}$

\begin{tabular}{|c|c|c|c|c|c|}
\hline & Energy (opt) & $\mathrm{G}(\mathrm{opt})$ & G-qh (opt) & $\mathrm{E}(\mathrm{sp})$ & G-qh (sp) \\
\hline TS_Bzh_All_F_1 & -1121.526327 & -1121.126787 & -1121.122438 & -1122.938723 & -1122.534834 \\
\hline TS_Bzh_All_F_2 & -1121.526411 & -1121.126266 & -1121.122155 & -1122.938657 & -1122.534401 \\
\hline TS_Bzh_All_F_3 & -1121.525680 & -1121.125495 & -1121.121303 & -1122.938076 & -1122.533699 \\
\hline TS_Bzh_All_F_4 & -1121.524757 & -1121.124885 & -1121.120553 & -1122.937064 & -1122.532860 \\
\hline TS_Bzh_All_F_5 & -1121.526005 & -1121.125300 & -1121.121487 & -1122.936425 & -1122.531907 \\
\hline TS_Bzh_All_F_6 & -1121.522829 & -1121.123475 & -1121.119013 & -1122.935275 & -1122.531459 \\
\hline TS_Bzh_All_F_7 & -1121.522772 & -1121.123112 & -1121.118861 & -1122.935332 & -1122.531421 \\
\hline TS_Bzh_All_F_8 & -1121.522511 & -1121.123362 & -1121.118826 & -1122.934836 & -1122.531151 \\
\hline TS_Bzh_All_F_9 & -1121.522505 & -1121.123011 & -1121.118619 & -1122.935029 & -1122.531143 \\
\hline TS_Bzh_All_F_10 & -1121.524148 & -1121.124245 & -1121.119739 & -1122.935325 & -1122.530917 \\
\hline TS_Bzh_All_F_11 & -1121.520993 & -1121.122711 & -1121.117547 & -1122.934354 & -1122.530909 \\
\hline TS_Bzh_All_F_12 & -1121.520109 & -1121.123301 & -1121.117507 & -1122.933437 & -1122.530835 \\
\hline TS_Bzh_All_F_13 & -1121.521602 & -1121.122238 & -1121.117886 & -1122.934540 & -1122.530824 \\
\hline TS_Bzh_All_F_14 & -1121.522177 & -1121.122584 & -1121.118096 & -1122.934854 & -1122.530772 \\
\hline TS_Bzh_All_F_15 & -1121.525266 & -1121.124004 & -1121.120570 & -1122.935412 & -1122.530717 \\
\hline TS_Bzh_All_F_16 & -1121.521105 & -1121.122356 & -1121.117456 & -1122.934330 & -1122.530680 \\
\hline TS_Bzh_All-F_F_17 & -1121.523974 & -1121.124223 & -1121.119709 & -1122.934896 & -1122.530632 \\
\hline TS_Bzh_All_F_18 & -1121.520679 & -1121.122873 & -1121.117451 & -1122.933858 & -1122.530630 \\
\hline TS_Bzh_All-F_F_19 & -1121.523467 & -1121.123851 & -1121.119414 & -1122.934327 & -1122.530273 \\
\hline TS_Bzh_All_F_20 & -1121.524408 & -1121.123400 & -1121.119730 & -1122.934824 & -1122.530146 \\
\hline TS_Bzh_All_F_21 & -1121.519950 & -1121.121628 & -1121.116559 & -1122.933149 & -1122.529758 \\
\hline TS_Bzh_All_F_22 & -1121.524105 & -1121.123208 & -1121.119293 & -1122.934549 & -1122.529737 \\
\hline TS_Bzh_All_F_23 & -1121.518836 & -1121.121561 & -1121.116292 & -1122.932198 & -1122.529654 \\
\hline TS_Bzh_All_F_24 & -1121.520204 & -1121.121299 & -1121.116631 & -1122.933122 & -1122.529549 \\
\hline TS_Bzh_All-F_F_25 & -1121.519911 & -1121.120460 & -1121.116116 & -1122.932964 & -1122.529170 \\
\hline TS_Bzh_All_F_26 & -1121.520694 & -1121.121500 & -1121.116947 & -1122.932733 & -1122.528985 \\
\hline TS_Bzh_All_F_27 & -1121.518344 & -1121.120676 & -1121.115343 & -1122.931931 & -1122.528930 \\
\hline TS_Bzh_All_F_28 & -1121.520652 & -1121.121929 & -1121.117128 & -1122.932435 & -1122.528911 \\
\hline TS_Bzh_All_F_F-29 & -1121.520652 & -1121.121868 & -1121.117098 & -1122.932430 & -1122.528877 \\
\hline TS_Bzh_All_F_30 & -1121.521158 & -1121.122147 & -1121.117745 & -1122.932208 & -1122.528795 \\
\hline TS_Bzh_All_F_31 & -1121.521127 & -1121.121832 & -1121.117421 & -1122.932489 & -1122.528782 \\
\hline TS_Bzh_All_F_32 & -1121.517775 & -1121.120180 & -1121.115323 & -1122.931134 & -1122.528682 \\
\hline TS_Bzh_All_F_F3 & -1121.518463 & -1121.120107 & -1121.115090 & -1122.931884 & -1122.528511 \\
\hline TS_Bzh_All_F_34 & -1121.520553 & -1121.121784 & -1121.116979 & -1122.931847 & -1122.528272 \\
\hline TS_Bzh_All_F_35 & -1121.519540 & -1121.119789 & -1121.115370 & -1122.932193 & -1122.528023 \\
\hline TS_Bzh_All_F_36 & -1121.520149 & -1121.120896 & -1121.116626 & -1122.931496 & -1122.527973 \\
\hline TS_Bzh_All-F_F_37 & -1121.520612 & -1121.120679 & -1121.116440 & -1122.932108 & -1122.527936 \\
\hline TS_Bzh_All_F_38 & -1121.520852 & -1121.120864 & -1121.116875 & -1122.931903 & -1122.527926 \\
\hline TS_Bzh_All_F_39 & -1121.521044 & -1121.121928 & -1121.117152 & -1122.931785 & -1122.527894 \\
\hline TS_Bzh_All_F_40 & -1121.522294 & -1121.121890 & -1121.117999 & -1122.932077 & -1122.527781 \\
\hline
\end{tabular}




\begin{tabular}{llllll} 
TS_Bzh_All_F_41 & -1121.519323 & -1121.119576 & -1121.114889 & -1122.932082 & -1122.527649 \\
TS_Bzh_All_F_42 & -1121.517465 & -1121.119201 & -1121.114481 & -1122.930532 & -1122.527548 \\
TS_Bzh_All_F_43 & -1121.521188 & -1121.120690 & -1121.116672 & -1122.931819 & -1122.527303 \\
TS_Bzh_All_F_44 & -1121.520429 & -1121.120778 & -1121.116146 & -1122.931128 & -1122.526844 \\
TS_Bzh_Al_F_45 & -1121.520041 & -1121.120100 & -1121.116004 & -1122.930513 & -1122.526477 \\
TS_Bzh_AlI_F_46 & -1121.518071 & -1121.117381 & -1121.113701 & -1122.929764 & -1122.525394 \\
\hline
\end{tabular}

Table S50: Azet ${ }_{\mathrm{Bzh}, \mathrm{Bn}}$

\begin{tabular}{|c|c|c|c|c|c|}
\hline & Energy (opt) & G (opt) & G-qh (opt) & $\mathrm{E}(\mathrm{sp})$ & G-qh (sp) \\
\hline TS_Bzh_Bn_F_1 & -1275.014246 & -1274.569739 & -1274.564087 & -1276.606060 & -1276.155901 \\
\hline TS_Bzh_Bn_F_2 & -1275.014410 & -1274.569500 & -1274.563963 & -1276.605929 & -1276.155482 \\
\hline TS_Bzh_Bn_F_3 & -1275.015001 & -1274.569547 & -1274.564416 & -1276.604562 & -1276.153977 \\
\hline TS_Bzh_Bn_F_4 & -1275.012089 & -1274.567064 & -1274.561705 & -1276.604157 & -1276.153772 \\
\hline TS_Bzh_Bn_F_5 & -1275.012080 & -1274.567353 & 51816 & -1276.603952 & 153688 \\
\hline TS_Bzh_Bn_F_6 & -1275 & -1274 & -1274.560440 & -1276.603355 & -1276.153416 \\
\hline TS_Bzh_Bn_F_7 & -1275.009693 & -1274.567250 & -1274.560575 & -1276.602436 & -1276.153317 \\
\hline TS_Bzh_Bn_F_8 & & & & -1276 & 268 \\
\hline F_9 & & & & & \\
\hline TS_Bzh_Bn_F_10 & -12 & -12 & -127 & $-127 c$ & -12 \\
\hline TS_Bzh_Bn_F_11 & & & -127 & -1276 & -127 \\
\hline TS_I & & & -127 & -1276 & $-127 t$ \\
\hline TS_B & -121 & 4488 & -127 & -1276.6 & 139 \\
\hline TS_Bzh_Bn_F_14 & -1275.0 & -127 & -127 & -1276.600735 & -1276.150800 \\
\hline TS_I & & & & 97 & \\
\hline TS_B & & & $-12+2 x+3$ & -1276 & -127 \\
\hline TS_Bzh_Bn_F_17 & -1275.010522 & -1274.566154 & -1274.560504 & -1276.600264 & -1276.150246 \\
\hline TS_Bzh_Bn_F_18 & -1275.011748 & -1274.565545 & -1274.561032 & -1276.600847 & -1276.150131 \\
\hline TS_I & & & & & \\
\hline TS_B & -12 & -127 & -127 & -1276. & -127 \\
\hline TS_Bzh_Bn_F_21 & -1275.006434 & -1274.561510 & -1274.556112 & -1276.598598 & -1276.148276 \\
\hline TS_Bzh_Bn_F_22 & 1134 & -1274.564005 & -1274.559 & -1276.599943 & -1276.148263 \\
\hline 1_Bn_F_23 & & & & & -1276.147943 \\
\hline TS_B & -12 & -1274 & -1274 & -1276.5 & -1276.147845 \\
\hline TS_Bzh_Bn_F_25 & -1275.008435 & -1274.562912 & -1274.557743 & -1276.598341 & -1276.147648 \\
\hline
\end{tabular}

\section{Azetidinium Fluorination Products}

Table S51: Azet ${ }_{\mathrm{Me}, \mathrm{Me}}$

\begin{tabular}{lccccc}
\hline & Energy $(\mathrm{opt})$ & $\mathrm{G}(\mathrm{opt})$ & $\mathrm{G}-\mathrm{qh}(\mathrm{opt})$ & $\mathrm{E}(\mathrm{sp})$ & $\mathrm{G}-\mathrm{qh}(\mathrm{sp})$ \\
\hline Prod_Me_Me_1 & -582.708649 & -582.492942 & -582.490973 & -583.461258 & -583.243582 \\
Prod_Me_Me_2 & -582.708489 & -582.492633 & -582.490690 & -583.461308 & -583.243509 \\
Prod_Me_Me_3 & -582.708375 & -582.492466 & -582.490536 & -583.461300 & -583.243461 \\
Prod_Me_Me_4 & -582.708933 & -582.492694 & -582.490879 & -583.460845 & -583.242791 \\
Prod_Me_Me_5 & -582.708704 & -582.492630 & -582.490682 & -583.460549 & -583.242528 \\
Prod_Me_Me_6 & -582.707018 & -582.491058 & -582.489134 & -583.459804 & -583.241920 \\
Prod_Me_Me_7 & -582.707167 & -582.490762 & -582.489146 & -583.459361 & -583.241339 \\
Prod_Me_Me_8 & -582.706151 & -582.490525 & -582.488433 & -583.459015 & -583.241297 \\
Prod_Me_Me_9 & -582.706093 & -582.490345 & -582.488560 & -583.458489 & -583.240956 \\
Prod_Me_Me_10 & -582.707046 & -582.490220 & -582.488733 & -583.459111 & -583.240799 \\
Prod_Me_Me_11 & -582.706825 & -582.490873 & -582.488924 & -583.458389 & -583.240489 \\
Prod_Me_Me_12 & -582.706351 & -582.490133 & -582.488218 & -583.458491 & -583.240358 \\
Prod_Me_Me_13 & -582.706054 & -582.490507 & -582.488352 & -583.457865 & -583.240164 \\
Prod_Me_Me_14 & -582.707221 & -582.490332 & -582.488840 & -583.458393 & -583.240012 \\
Prod_Me_Me_15 & -582.704668 & -582.488806 & -582.486760 & -583.457782 & -583.239874 \\
Prod_Me_Me_16 & -582.704530 & -582.488352 & -582.486502 & -583.457569 & -583.239542 \\
Prod_Me_Me_17 & -582.703981 & -582.488009 & -582.486071 & -583.456137 & -583.238226 \\
Prod_Me_Me_18 & -582.704105 & -582.487730 & -582.485982 & -583.456167 & -583.238044 \\
Prod_Me_Me_19 & -582.703397 & -582.487382 & -582.485367 & -583.455251 & -583.237221 \\
Prod_Me_Me_20 & -582.703356 & -582.487107 & -582.485586 & -583.454930 & -583.237160
\end{tabular}




\begin{tabular}{llllll} 
Prod_Me_Me_21 & -582.703034 & -582.485967 & -582.484438 & -583.455006 & -583.236410 \\
Prod_Me_Me_22 & -582.702512 & -582.486705 & -582.484754 & -583.454000 & -583.236242 \\
Prod_Me_Me_23 & -582.703387 & -582.486031 & -582.484660 & -583.454566 & -583.235839 \\
Prod_Me_Me_24 & -582.702799 & -582.485538 & -582.484164 & -583.453825 & -583.235190 \\
Prod_Me_Me_25 & -582.703258 & -582.485801 & -582.484421 & -583.453662 & -583.234825 \\
Prod_Me_Me_26 & -582.701842 & -582.484796 & -582.483284 & -583.452360 & -583.233802 \\
Prod_Me_Me_27 & -582.701838 & -582.484066 & -582.482725 & -583.452817 & -583.233705 \\
\hline
\end{tabular}

Table S52: Azet ${ }_{\mathrm{Bn}, \mathrm{Me}}$

\begin{tabular}{|c|c|c|c|c|c|}
\hline & Energy (opt) & $\mathrm{G}$ (opt) & G-qh (opt) & $\mathrm{E}(\mathrm{sp})$ & G-qh (sp) \\
\hline Prod_Bn_Me_1 & -813.502216 & -813.211663 & -813.207690 & -814.532734 & -814.238208 \\
\hline Prod_Bn_Me_2 & -813.502523 & -813.210508 & -813.207381 & -814.532668 & -814.237526 \\
\hline Prod_Bn_Me_3 & -813.502442 & -813.211223 & -813.207604 & -814.532283 & -814.237444 \\
\hline Prod_Bn_Me_4 & -813.501457 & -813.211418 & -813.207166 & -814.531628 & -814.237337 \\
\hline Prod_Bn_Me_5 & -813.502589 & -813.211801 & -813.207831 & -814.532049 & -814.237291 \\
\hline Prod_Bn_Me_6 & -813.500917 & -813.210564 & -813.206513 & -814.531158 & -814.236754 \\
\hline Prod_Bn_Me_7 & -813.501971 & -813.210465 & -813.207072 & -814.531463 & -814.236564 \\
\hline Prod_Bn_Me_8 & -813.500755 & -813.210738 & -813.206492 & -814.530707 & -814.236444 \\
\hline Prod_Bn_Me_9 & -813.500462 & -813.210408 & -813.206201 & -814.530508 & -814.236247 \\
\hline Prod_Bn_Me_10 & -813.501815 & -813.210793 & -813.206884 & -814.530918 & -814.235988 \\
\hline Prod_Bn_Me_11 & -813.500322 & -813.209634 & -813.205564 & -814.530704 & -814.235945 \\
\hline Prod_Bn_Me_12 & -813.500346 & -813.208859 & -813.205223 & -814.530655 & -814.235533 \\
\hline Prod_Bn_Me_13 & -813.500314 & -813.209519 & -813.205542 & -814.530189 & -814.235417 \\
\hline Prod_Bn_Me_14 & -813.499197 & -813.208850 & -813.204807 & -814.529803 & -814.235413 \\
\hline Prod_Bn_Me_15 & -813.501524 & -813.209494 & -813.206314 & -814.530540 & -814.235330 \\
\hline Prod_Bn_Me_16 & -813.502244 & -813.209826 & -813.206865 & -814.530595 & -814.235216 \\
\hline Prod_Bn_Me_17 & -813.500156 & -813.209614 & -813.205673 & -814.529678 & -814.235195 \\
\hline Prod_Bn_Me_18 & -813.500378 & -813.208672 & -813.205019 & -814.530523 & -814.235164 \\
\hline Prod_Bn_Me_19 & -813.500919 & -813.209609 & -813.205803 & -814.530161 & -814.235045 \\
\hline Prod_Bn_Me_20 & -813.500232 & -813.209116 & -813.205256 & -814.529923 & -814.234948 \\
\hline Prod_Bn_Me_21 & -813.499464 & -813.208947 & -813.204952 & -814.529439 & -814.234928 \\
\hline Prod_Bn_Me_22 & -813.500032 & -813.209149 & -813.205365 & -814.529531 & -814.234864 \\
\hline Prod_Bn_Me_23 & -813.499644 & -813.208837 & -813.205040 & -814.529146 & -814.234542 \\
\hline Prod_Bn_Me_24 & -813.500618 & -813.208699 & -813.205211 & -814.529544 & -814.234137 \\
\hline Prod_Bn_Me_25 & -813.499446 & -813.208245 & -813.204590 & -814.528892 & -814.234037 \\
\hline Prod_Bn_Me_26 & -813.500364 & -813.208287 & -813.204845 & -814.529552 & -814.234034 \\
\hline Prod_Bn_Me_27 & -813.498446 & -813.208839 & -813.204279 & -814.528094 & -814.233926 \\
\hline Prod_Bn_Me_28 & -813.500219 & -813.209046 & -813.205295 & -814.528833 & -814.233909 \\
\hline Prod_Bn_Me_29 & -813.498692 & -813.208329 & -813.204200 & -814.528124 & -814.233632 \\
\hline Prod_Bn_Me_30 & -813.498704 & -813.207245 & -813.203493 & -814.528656 & -814.233444 \\
\hline Prod_Bn_Me_31 & -813.499657 & -813.208195 & -813.204627 & -814.528242 & -814.233212 \\
\hline Prod_Bn_Me_32 & -813.500900 & -813.208266 & -813.205069 & -814.529024 & -814.233193 \\
\hline Prod_Bn_Me_33 & -813.500579 & -813.208422 & -813.204956 & -814.528793 & -814.233170 \\
\hline Prod_Bn_Me_34 & -813.498116 & -813.206145 & -813.202713 & -814.528174 & -814.232771 \\
\hline Prod_Bn_Me_35 & -813.500135 & -813.205824 & -813.203599 & -814.528391 & -814.231855 \\
\hline Prod_Bn_Me_36 & -813.499746 & -813.205566 & -813.203201 & -814.528075 & -814.231529 \\
\hline Prod_Bn_Me_37 & -813.499776 & -813.205683 & -813.203322 & -814.527201 & -814.230747 \\
\hline
\end{tabular}

Table S53: Azet $_{\text {Bzh,Me }}$

\begin{tabular}{lccccc}
\hline & Energy (opt) & G (opt) & G-qh (opt) & E (sp) & G-qh (sp) \\
\hline Prod_Bzh_Me_1 & -1044.293768 & -1043.926966 & -1043.921938 & -1045.599729 & -1045.227898 \\
Prod_Bzh_Me_2 & -1044.291928 & -1043.926607 & -1043.920787 & -1045.598807 & -1045.227665 \\
Prod_Bzh_Me_3 & -1044.292568 & -1043.925762 & -1043.920615 & -1045.599618 & -1045.227665 \\
Prod_Bzh_Me_4 & -1044.293776 & -1043.926585 & -1043.921766 & -1045.599546 & -1045.227536 \\
Prod_Bzh_Me_5 & -1044.293643 & -1043.926221 & -1043.921402 & -1045.599457 & -1045.227217 \\
Prod_Bzh_Me_6 & -1044.292969 & -1043.926189 & -1043.920936 & -1045.598859 & -1045.226825 \\
Prod_Bzh_Me_7 & -1044.291627 & -1043.925416 & -1043.920050 & -1045.597598 & -1045.226022 \\
Prod_Bzh_Me_8 & -1044.293808 & -1043.925704 & -1043.921314 & -1045.598504 & -1045.226011 \\
Prod_Bzh_Me_9 & -1044.293539 & -1043.925043 & -1043.920839 & -1045.598547 & -1045.225847
\end{tabular}




\begin{tabular}{|c|c|c|c|c|c|}
\hline Prod_Bzh_Me_10 & -1044.291513 & -1043.925306 & -1043.919821 & -1045.597462 & -1045.225770 \\
\hline Prod_Bzh_Me_11 & -1044.292696 & -1043.924765 & -1043.920134 & -1045.598072 & -1045.225511 \\
\hline Prod_Bzh_Me_12 & -1044.290150 & -1043.923953 & -1043.918459 & -1045.597099 & -1045.225408 \\
\hline Prod_Bzh_Me_13 & -1044.290416 & -1043.924075 & -1043.918763 & -1045.596973 & -1045.225320 \\
\hline Prod_Bzh_Me_14 & -1044.292313 & -1043.924266 & -1043.919739 & -1045.597791 & -1045.225217 \\
\hline Prod_Bzh_Me_15 & -1044.292590 & -1043.924150 & -1043.919754 & -1045.598030 & -1045.225194 \\
\hline Prod_Bzh_Me_16 & -1044.291997 & -1043.923881 & -1043.919285 & -1045.597778 & -1045.225065 \\
\hline Prod_Bzh_Me_17 & -1044.291737 & -1043.924434 & -1043.919447 & -1045.597328 & -1045.225038 \\
\hline Prod_Bzh_Me_18 & -1044.291201 & -1043.924176 & -1043.918995 & -1045.597086 & -1045.224880 \\
\hline Prod_Bzh_Me_19 & -1044.289820 & -1043.923228 & -1043.917955 & -1045.596700 & -1045.224835 \\
\hline Prod_Bzh_Me_20 & -1044.289619 & -1043.923571 & -1043.918221 & -1045.596171 & -1045.224773 \\
\hline Prod_Bzh_Me_21 & -1044.292907 & -1043.924711 & -1043.920160 & -1045.597427 & -1045.224680 \\
\hline Prod_Bzh_Me_22 & -1044.292612 & -1043.924125 & -1043.919845 & -1045.597117 & -1045.224350 \\
\hline Prod_Bzh_Me_23 & -1044.291197 & -1043.923423 & -1043.918522 & -1045.596791 & -1045.224116 \\
\hline Prod_Bzh_Me_24 & -1044.290997 & -1043.924061 & -1043.918908 & -1045.596116 & -1045.224028 \\
\hline Prod_Bzh_Me_25 & -1044.290511 & -1043.922670 & -1043.917872 & -1045.596565 & -1045.223926 \\
\hline Prod_Bzh_Me_26 & -1044.292119 & -1043.924078 & -1043.919280 & -1045.596691 & -1045.223852 \\
\hline Prod_Bzh_Me_27 & -1044.291503 & -1043.923654 & -1043.918814 & -1045.596428 & -1045.223739 \\
\hline Prod_Bzh_Me_28 & -1044.289632 & -1043.922824 & -1043.917621 & -1045.595576 & -1045.223564 \\
\hline Prod_Bzh_Me_29 & -1044.290036 & -1043.922529 & -1043.917612 & -1045.595739 & -1045.223315 \\
\hline Prod_Bzh_Me_30 & -1044.291393 & -1043.922001 & -1043.918094 & -1045.595765 & -1045.222467 \\
\hline Prod_Bzh_Me_31 & -1044.290065 & -1043.921388 & -1043.916944 & -1045.595511 & -1045.222390 \\
\hline Prod_Bzh_Me_32 & -1044.288578 & -1043.921249 & -1043.916241 & -1045.593453 & -1045.221116 \\
\hline Prod_Bzh_Me_33 & -1044.287460 & -1043.920568 & -1043.915537 & -1045.592898 & -1045.220976 \\
\hline Prod_Bzh_Me_34 & -1044.289761 & -1043.920646 & -1043.916346 & -1045.594300 & -1045.220885 \\
\hline Prod_Bzh_Me_35 & -1044.288864 & -1043.920578 & -1043.916167 & -1045.593293 & -1045.220595 \\
\hline
\end{tabular}

Asymmetric Fluorination Transition State Structures

Table S54: Azet ${ }_{\text {Bzh,Me }}$

\begin{tabular}{|c|c|c|c|c|c|}
\hline & Energy (opt) & G (opt) & G-qh (opt) & $\mathrm{E}(\mathrm{sp})$ & G-qh (sp) \\
\hline TS Major-Cis-1 & -4187.904254 & -4186.958416 & -4186.940769 & -4193.014743 & -4192.051258 \\
\hline TS $_{\text {Major-Cis-2 }}$ & -4187.902588 & -4186.957131 & -4186.939106 & -4193.012853 & -4192.049371 \\
\hline TS $_{\text {Major-Cis-3 }}$ & -4187.903028 & -4186.957249 & -4186.939442 & -4193.012822 & -4192.049235 \\
\hline TS & -4187.900377 & -4186.956354 & -4186.937643 & -4193.011656 & -4192.048923 \\
\hline TS Major-Cis-5 & -4187.903048 & -4186.955345 & -4186.938193 & -4193.012178 & -4192.047323 \\
\hline TS $_{\text {Major-Cis-6 }}$ & -4187.899365 & -4186.954568 & -4186.936071 & -4193.009194 & -4192.045900 \\
\hline TS Major-Cis-7 & -4187.901646 & -4186.954038 & -4186.936445 & -4193.010540 & -4192.045339 \\
\hline TS & -4187.901408 & -4186.953317 & -4186.936213 & -4193.010378 & -4192.045183 \\
\hline TS $_{\text {Major-Cis-9 }}$ & -4187.893810 & -4186.948599 & -4186.929989 & -4193.005990 & -4192.042169 \\
\hline TS Major-Cis-10 & -4187.897339 & -4186.950562 & -4186.932517 & -4193.006553 & -4192.041731 \\
\hline TS Major-Cis-11 $_{11}$ & -4187.897339 & -4186.950560 & -4186.932515 & -4193.006549 & -4192.041726 \\
\hline TS Major-Cis-12 & -4187.888635 & -4186.946068 & -4186.925600 & -4193.003343 & -4192.040309 \\
\hline TS Major-Cis-13 & -4187.895675 & -4186.949604 & -4186.931273 & -4193.003238 & -4192.038836 \\
\hline TS & -4187.886415 & -4186.944989 & -4186.924476 & -4193.000511 & -4192.038572 \\
\hline TS $_{\text {Minor-Cis-1 }}$ & -4187.905521 & -4186.958870 & -4186.941462 & -4193.015301 & -4192.051242 \\
\hline TS Minor-Cis-2 & -4187.901322 & -4186.956487 & -4186.938179 & -4193.012674 & -4192.049531 \\
\hline TS $_{\text {Minor-Cis-3 }}$ & -4187.900990 & -4186.955505 & -4186.936718 & -4193.010657 & -4192.046384 \\
\hline TS $_{\text {Minor-Cis-4 }}$ & -4187.899292 & -4186.952503 & -4186.934907 & -4193.010312 & -4192.045926 \\
\hline TS $_{\text {Minor-Cis-5 }}$ & -4187.896368 & -4186.952598 & -4186.932663 & -4193.009285 & -4192.045580 \\
\hline TS $_{\text {Minor-Cis-6 }}$ & -4187.889391 & -4186.948794 & -4186.927601 & -4193.005009 & -4192.043219 \\
\hline TS $_{\text {Minor-Cis-7 }}$ & -4187.891753 & -4186.945306 & -4186.927335 & -4193.003092 & -4192.038675 \\
\hline TS $_{\text {Minor-Cis-8 }}$ & -4187.887141 & -4186.944431 & -4186.924367 & -4193.001446 & -4192.038672 \\
\hline TS $_{\text {Minor-Cis-9 }}$ & -4187.890274 & -4186.945841 & -4186.927181 & -4193.001015 & -4192.037921 \\
\hline TS Minor-Cis-10 & -4187.894899 & -4186.942936 & -4186.927355 & -4193.003041 & -4192.035498 \\
\hline
\end{tabular}

Table S55: Azet ${ }_{\mathrm{Bzh}, \mathrm{Et}}$

\begin{tabular}{cccccc}
\hline & Energy (opt) & G (opt) & G-qh (opt) & E (sp) & G-qh (sp) \\
\hline TS $_{\text {Major-Cis-1-Ethyl-1 }}$ & -4227.170278 & -4226.197188 & -4226.179290 & -4232.335611 & -4231.344623
\end{tabular}




\begin{tabular}{|c|c|c|c|c|c|}
\hline TS Major-Cis-1-Ethyl-2 & -4227.164451 & -4226.190737 & -4226.172949 & -4232.330775 & -4231.339274 \\
\hline TS Major-Cis-1-Ethyl-3 & -4227.164319 & -4226.188732 & -4226.171693 & -4232.330099 & -4231.337474 \\
\hline TS Minor-Cis-1-Ethyl-1 & -4227.168998 & -4226.194329 & -4226.177033 & -4232.334152 & -4231.342187 \\
\hline TS Minor-Cis-1-Ethyl-2 & -4227.166980 & -4226.193250 & -4226.175415 & -4232.332037 & -4231.340473 \\
\hline TS Minor-Cis-1-Ethyl-3 & -4227.164560 & -4226.191401 & -4226.173213 & -4232.330216 & -4231.338868 \\
\hline TSMinor-Cis-2-Ethyl-1 & -4227.163884 & -4226.190944 & -4226.172576 & -4232.330271 & -4231.338962 \\
\hline TS Minor-Cis-2-Ethyl-2 & -4227.160871 & -4226.188861 & -4226.170188 & -4232.327466 & -4231.336782 \\
\hline TSMinor-Cis-2-Ethyl-3 & -4227.163653 & -4226.190470 & -4226.172172 & -4232.330386 & -4231.338905 \\
\hline
\end{tabular}

Table S56: Azet Bzh,Me $_{\text {B. }}$

\begin{tabular}{|c|c|c|c|c|c|}
\hline & Energy (opt) & $\mathrm{G}(\mathrm{opt})$ & G-qh (opt) & $\mathrm{E}(\mathrm{sp})$ & G-qh (sp) \\
\hline TS Major-Trans-1 & -4187.906300 & -4186.961432 & -4186.942932 & -4193.017984 & -4192.054616 \\
\hline TS Major-Trans-2 & -4187.904980 & -4186.958483 & -4186.940490 & -4193.015650 & -4192.051160 \\
\hline TSMajor-Trans-3 & -4187.901550 & -4186.957388 & -4186.938780 & -4193.013264 & -4192.050494 \\
\hline TS Major-Trans-4 & -4187.901172 & -4186.956080 & -4186.937598 & -4193.012123 & -4192.048549 \\
\hline TS Major-Trans-5 & -4187.893903 & -4186.952134 & -4186.931890 & -4193.008899 & -4192.046886 \\
\hline TS Major-Trans-6 & -4187.893903 & -4186.952057 & -4186.931851 & -4193.008897 & -4192.046846 \\
\hline TS Major-Trans-7 & -4187.896600 & -4186.949868 & -4186.932015 & -4193.009991 & -4192.045406 \\
\hline TS Major-Trans-8 & -4187.892564 & -4186.947150 & -4186.928749 & -4193.006197 & -4192.042382 \\
\hline TS Major-Trans-9 & -4187.887409 & -4186.944195 & -4186.924335 & -4193.001313 & -4192.038239 \\
\hline TS Major-Trans-10 & -4187.894851 & -4186.948029 & -4186.929774 & -4193.002894 & -4192.037817 \\
\hline TS Minor-Trans-1 & -4187.899930 & -4186.957014 & -4186.937528 & -4193.012209 & -4192.049807 \\
\hline TS Minor-Trans-2 & -4187.894128 & -4186.953579 & -4186.933096 & -4193.010013 & -4192.048981 \\
\hline TS Minor-Trans-3 & -4187.901958 & -4186.956438 & -4186.937731 & -4193.012930 & -4192.048704 \\
\hline TS Minor-Trans-4 & -4187.900386 & -4186.956443 & -4186.937532 & -4193.011239 & -4192.048385 \\
\hline TS Minor-Trans-5 & -4187.900206 & -4186.956120 & -4186.937371 & -4193.010613 & -4192.047779 \\
\hline TS Minor-Trans-6 & -4187.900019 & -4186.955845 & -4186.937368 & -4193.010297 & -4192.047646 \\
\hline TSMinor-Trans-7 & -4187.899421 & -4186.954517 & -4186.936254 & -4193.010704 & -4192.047536 \\
\hline TS Minor-Trans-8 & -4187.899686 & -4186.953001 & -4186.935626 & -4193.011540 & -4192.047479 \\
\hline TS Minor-Trans-9 & -4187.896802 & -4186.953341 & -4186.934371 & -4193.009664 & -4192.047233 \\
\hline TSMinor-Trans-10 & -4187.896587 & -4186.953591 & -4186.934528 & -4193.009168 & -4192.047109 \\
\hline TSMinor-Trans-11 & -4187.900309 & -4186.954689 & -4186.936676 & -4193.010651 & -4192.047017 \\
\hline TSMinor-Trans-12 & -4187.897677 & -4186.953011 & -4186.934666 & -4193.009981 & -4192.046969 \\
\hline TSMinor-Trans-13 & -4187.898909 & -4186.953881 & -4186.935226 & -4193.010333 & -4192.046651 \\
\hline TSMinor-Trans-14 & -4187.897524 & -4186.951264 & -4186.933777 & -4193.009363 & -4192.045616 \\
\hline TSMinor-Trans-15 & -4187.899780 & -4186.954993 & -4186.935997 & -4193.009137 & -4192.045354 \\
\hline TS Minor-Trans-16 & -4187.894892 & -4186.951313 & -4186.932382 & -4193.007333 & -4192.044823 \\
\hline TS Minor-Trans-17 & -4187.896789 & -4186.952900 & -4186.934439 & -4193.007129 & -4192.044778 \\
\hline TS Minor-Trans-18 & -4187.898485 & -4186.950531 & -4186.933677 & -4193.009301 & -4192.044494 \\
\hline TS Minor-Trans-19 & -4187.898350 & -4186.951462 & -4186.934183 & -4193.008391 & -4192.044224 \\
\hline TS Minor-Trans-20 & -4187.896470 & -4186.951406 & -4186.932998 & -4193.007115 & -4192.043643 \\
\hline TSMinor-Trans-21 & -4187.890583 & -4186.945910 & -4186.927264 & -4193.006153 & -4192.042834 \\
\hline TSMinor-Trans-22 & -4187.885516 & -4186.945170 & -4186.924292 & -4193.003980 & -4192.042756 \\
\hline TS Minor-Trans-23 & -4187.898829 & -4186.951560 & -4186.934242 & -4193.006945 & -4192.042359 \\
\hline TS Minor-Trans-24 & -4187.893252 & -4186.947821 & -4186.928800 & -4193.004564 & -4192.040112 \\
\hline TS Minor-Trans-25 & -4187.892450 & -4186.948890 & -4186.929381 & -4193.003038 & -4192.039970 \\
\hline TS Minor-Trans-26 & -4187.891363 & -4186.947716 & -4186.928204 & -4193.002697 & -4192.039538 \\
\hline TSMinor-Trans-27 & -4187.888556 & -4186.944575 & -4186.925201 & -4193.000575 & -4192.037221 \\
\hline
\end{tabular}




\section{Copies of NMR Spectra}
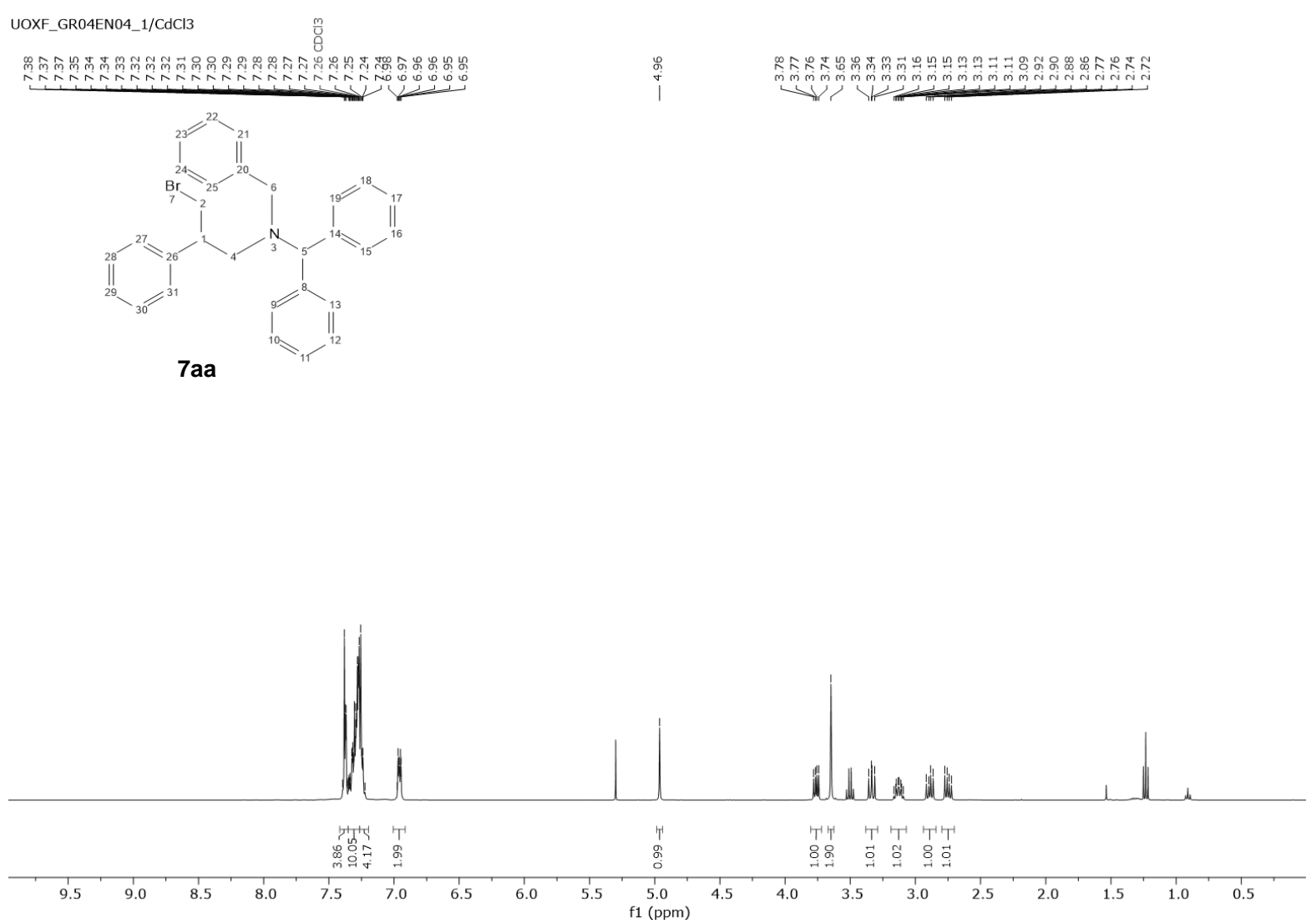

UOXF_GR04EN04_1/CdCl3
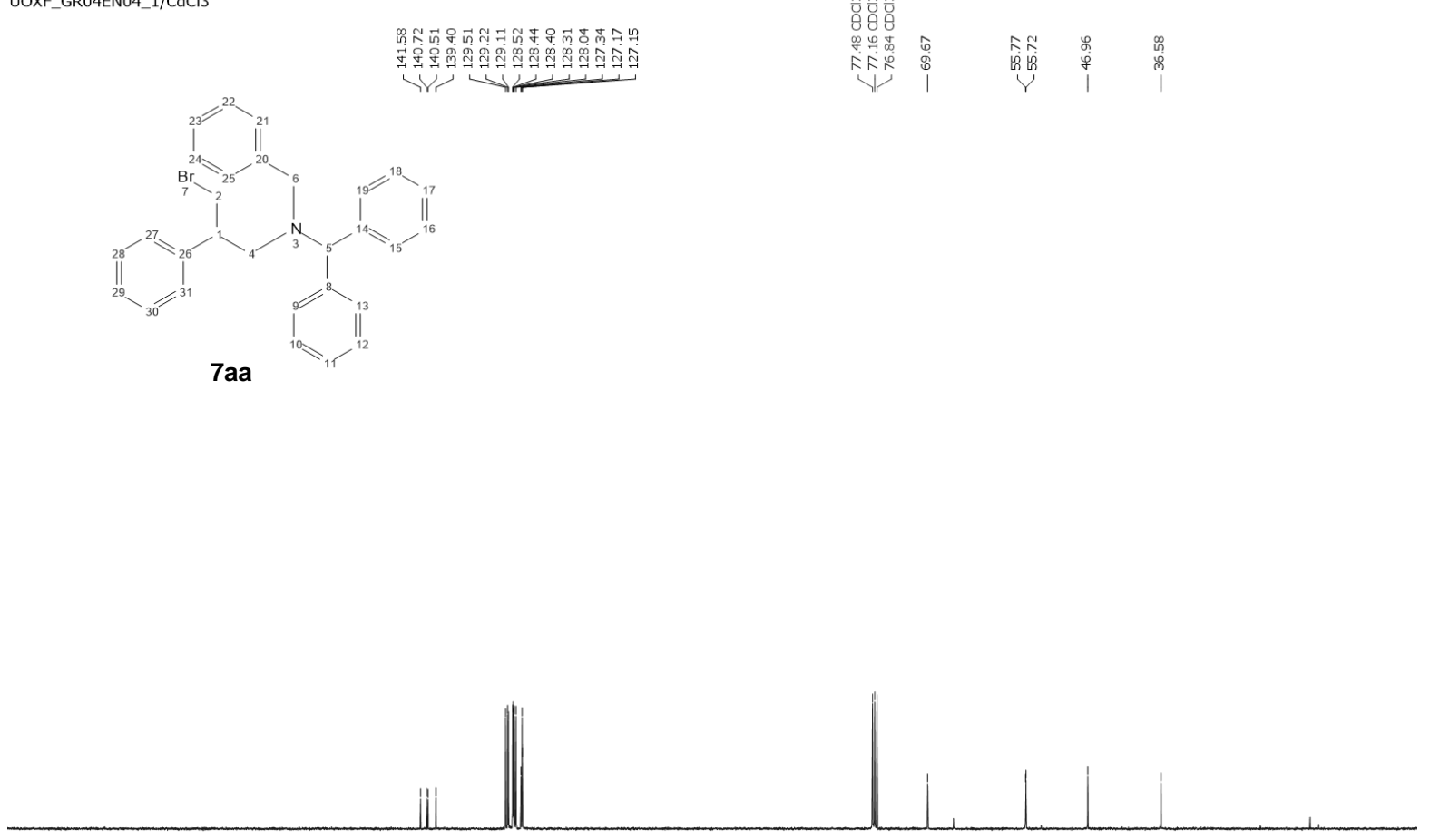

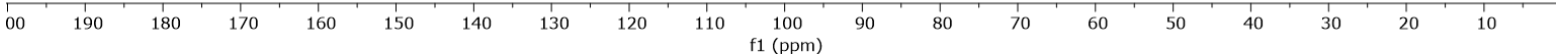




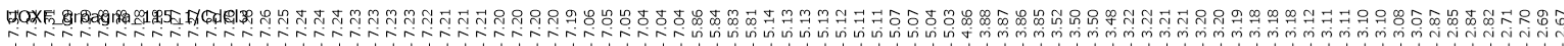<smiles></smiles>

\section{$\int \quad \int 1 / 1 / 11$}

$7 a b$

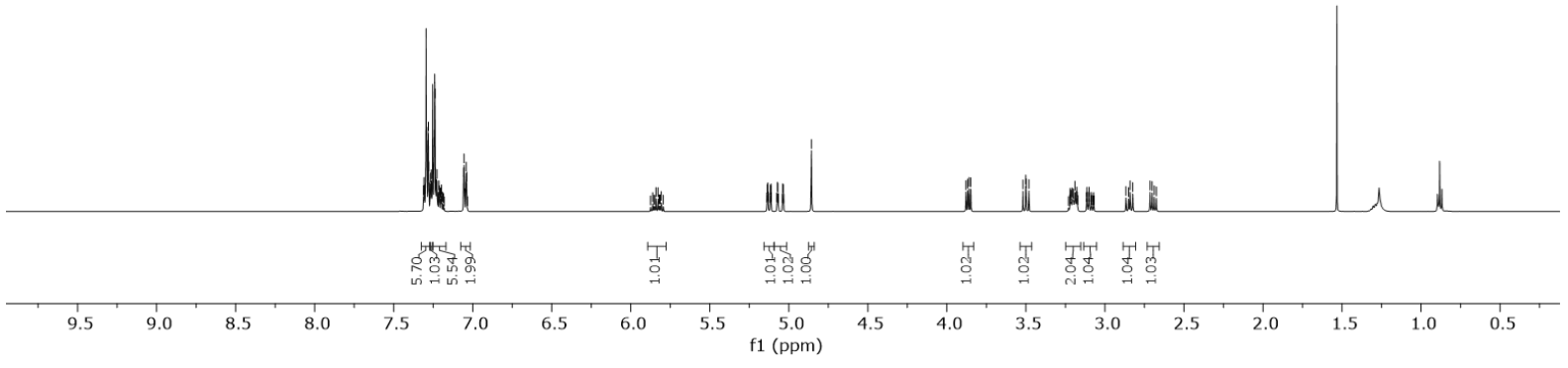

UOXF_groagna_115_1/CdCl3

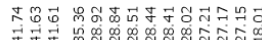

理主

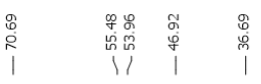<smiles>C=CNN(Cc1ccncn1)N(c1cnnnn1)n1nnnn1</smiles>

$7 a b$

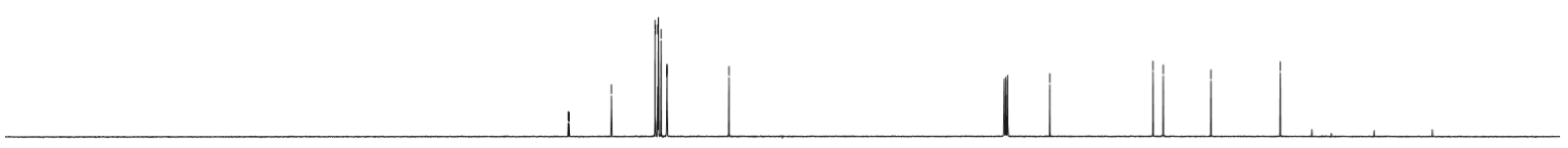

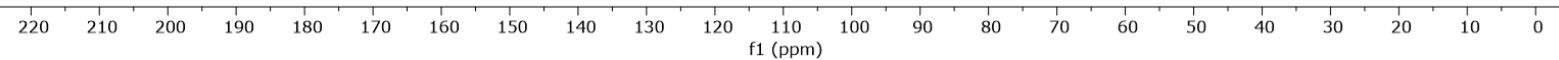


UOXF_groagna_331_1/CdC్

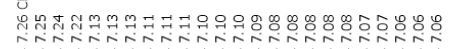

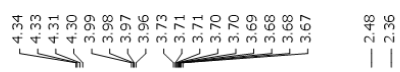<smiles>C=S(C)C1CN(C(=O)OC(C)(C)C)C1</smiles>

$5 b$

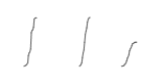

$\mathrm{CH}_{3}$

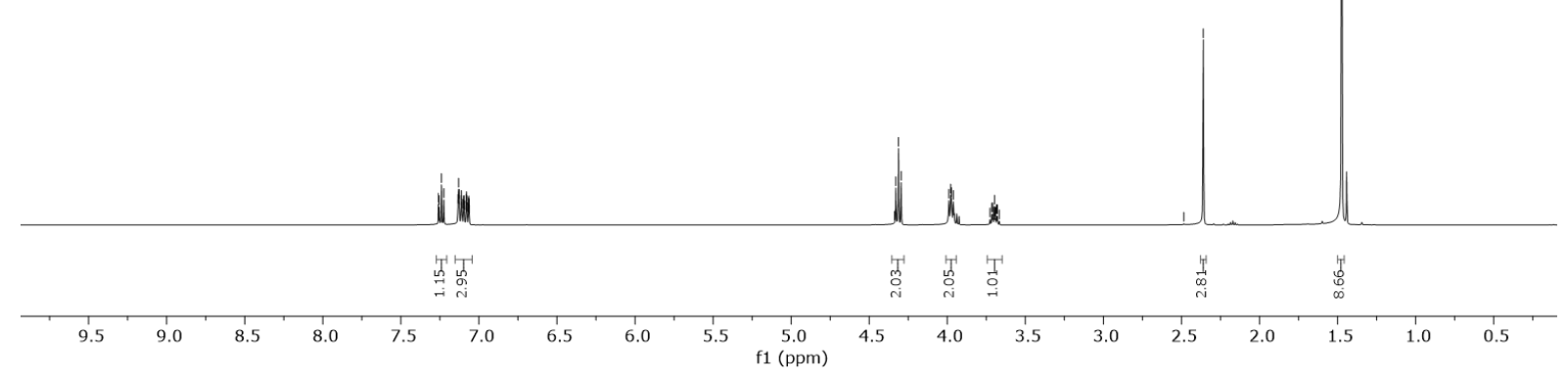

UOXF_groagna_331_1/CdCl3

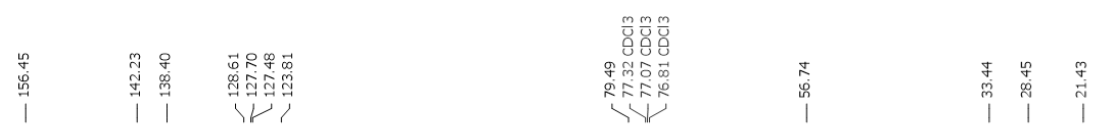
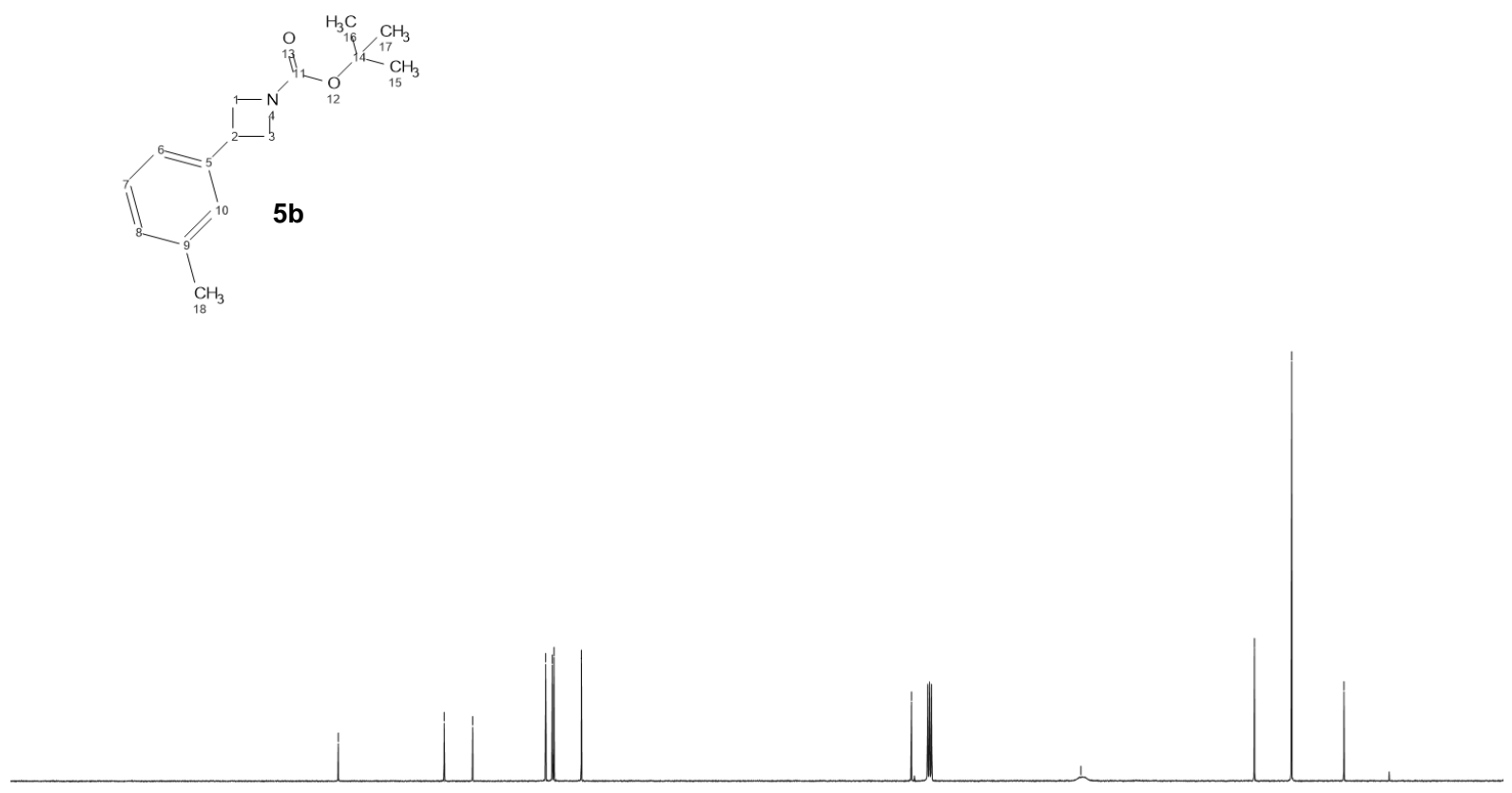

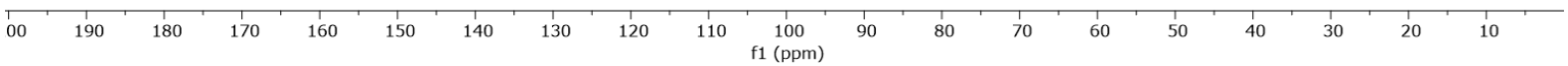


UOXF_groagna_351_1/CdCl3

$$
\frac{\int_{0}^{\circ}}{m}
$$

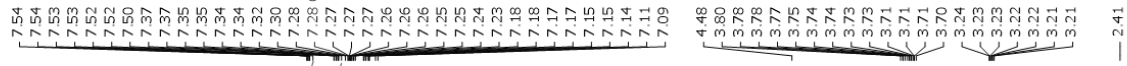
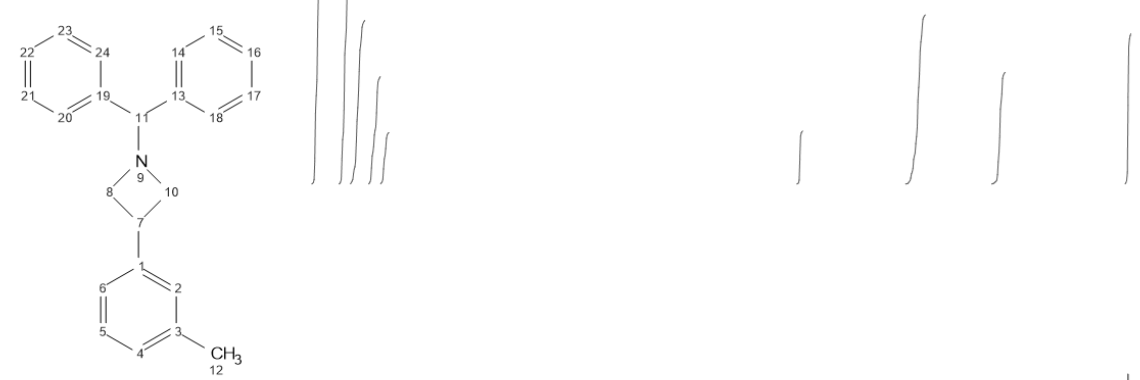

$6 b$

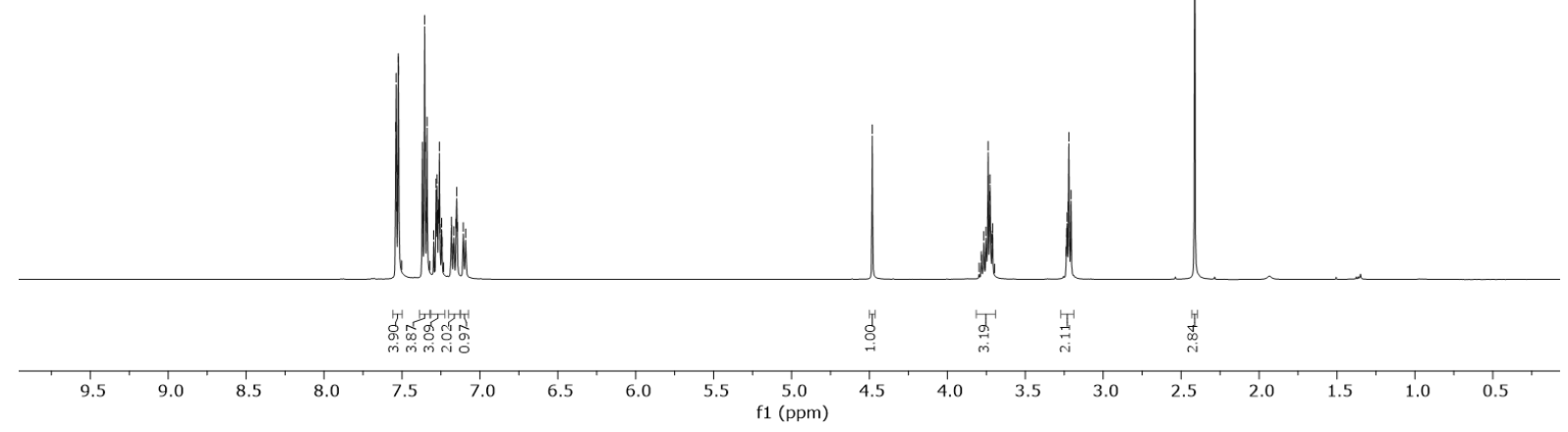

UOXF_groagna_351_1/CdCl3

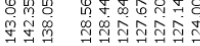

$\frac{m}{0} \frac{m}{4}$
8
8

1,1

年

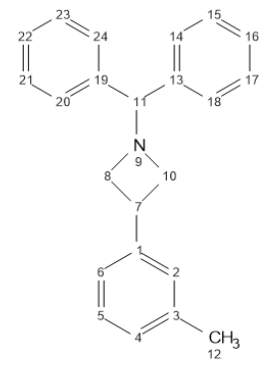

$6 b$
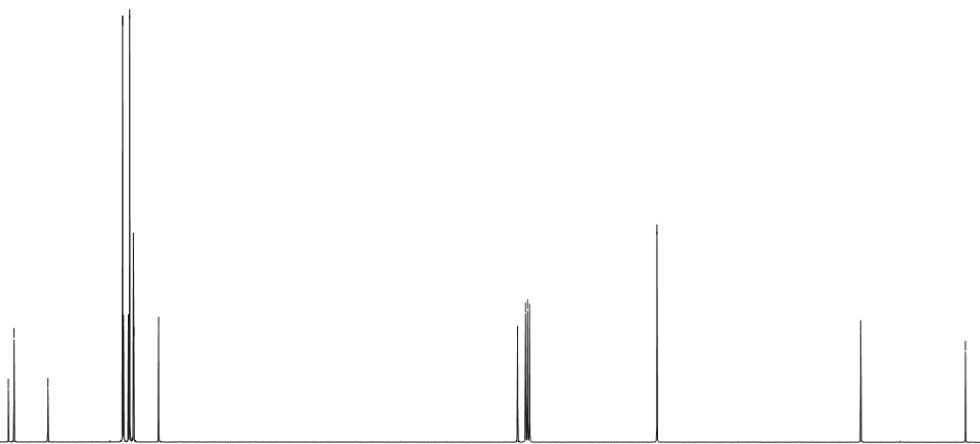

$190 \quad 180$

170
160

150

130
120
110

100
$\mathrm{f} 1(\mathrm{ppm})$ 
UOXF_groagna_362_1/Cdeg/3

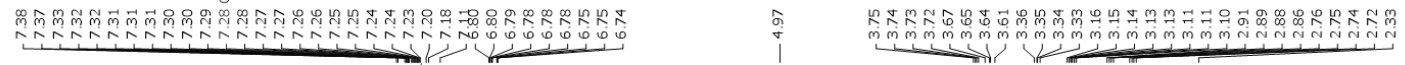

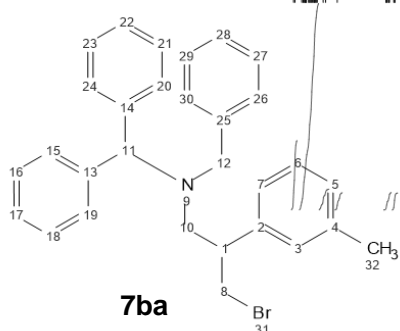

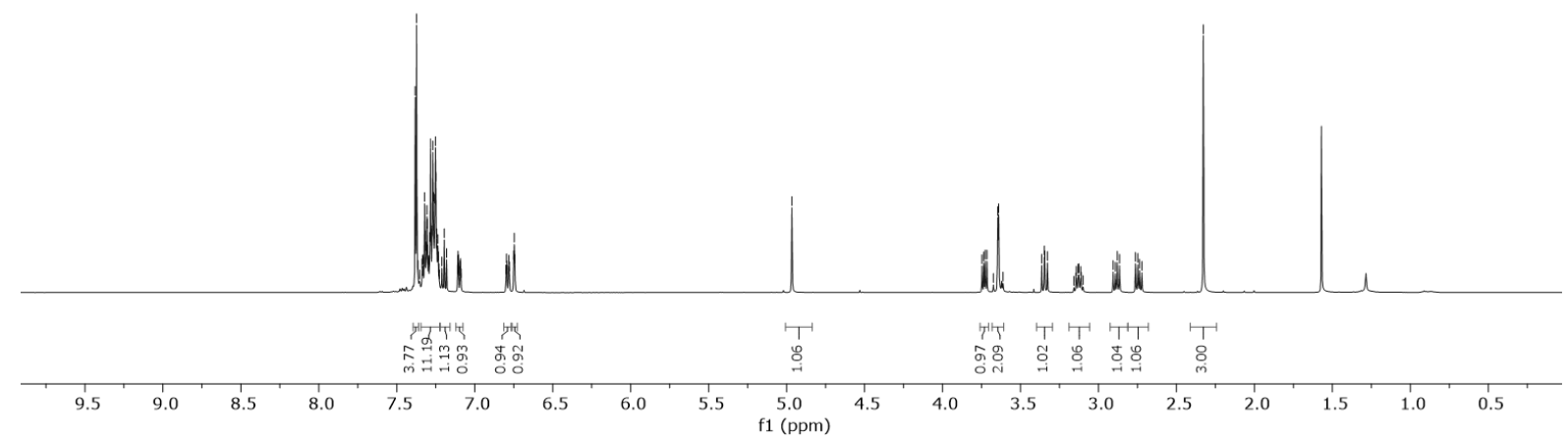

UOXF_groagna_362_1/CdCl3

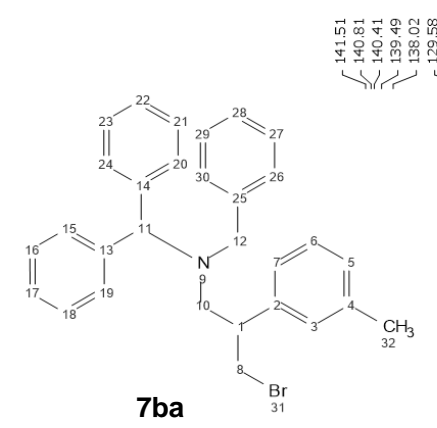

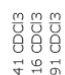

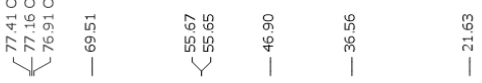

$7 b a$

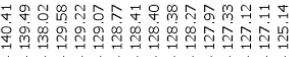
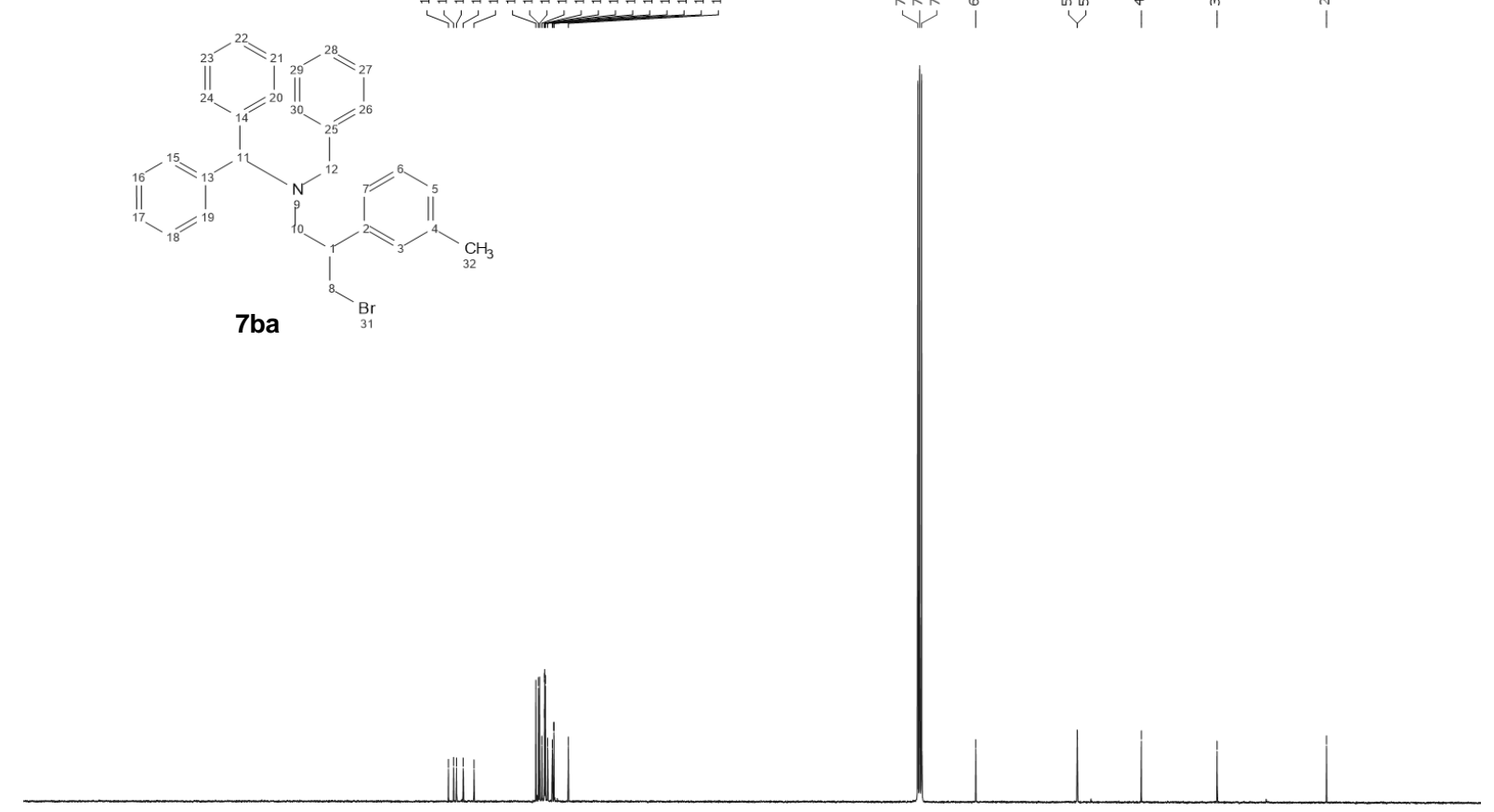

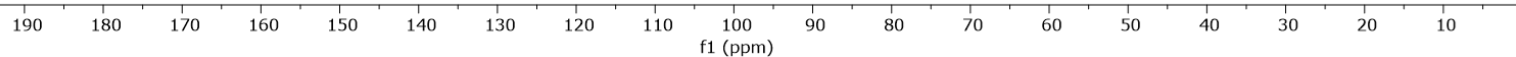


UOXF_groagna_330_1/CdCl3
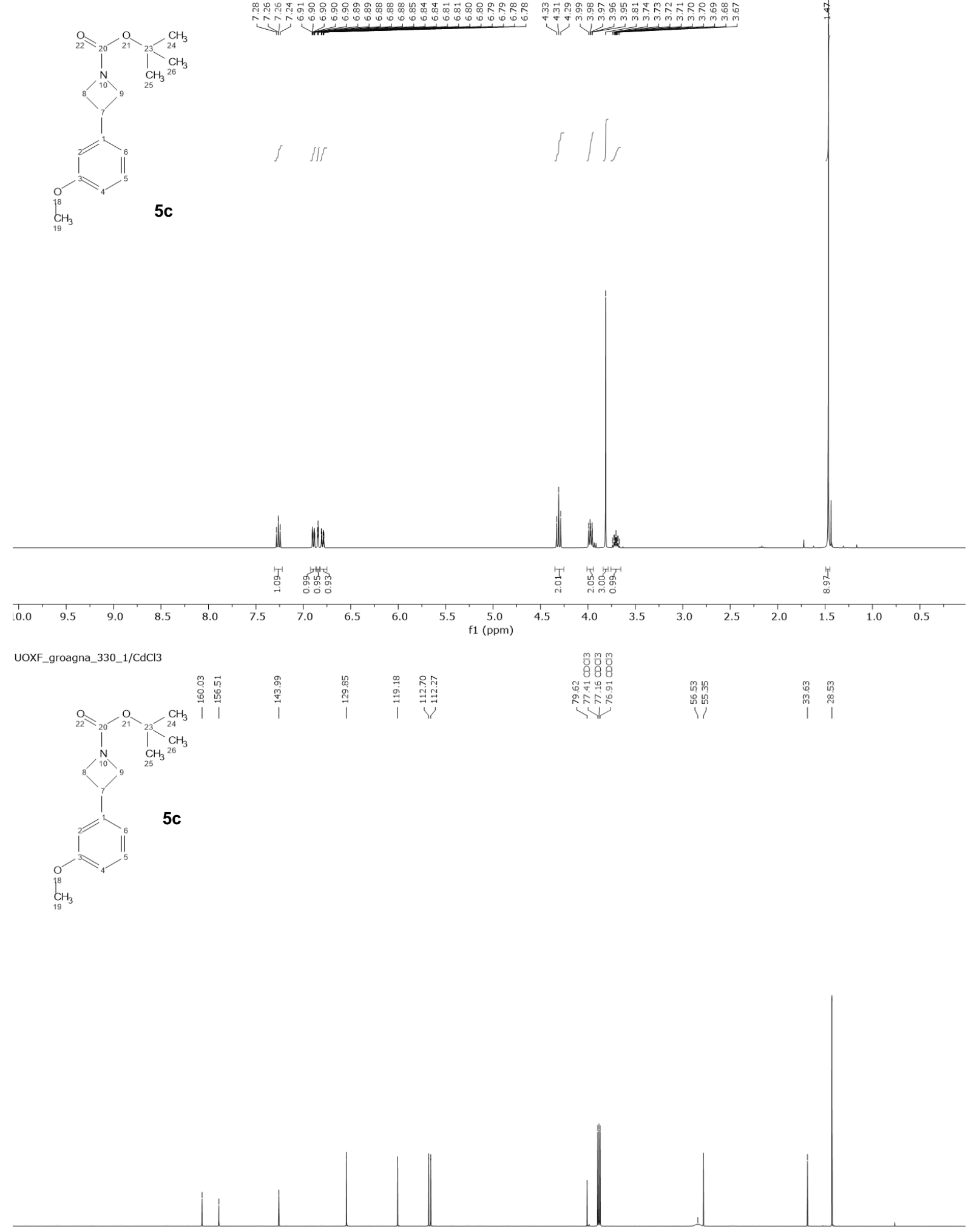

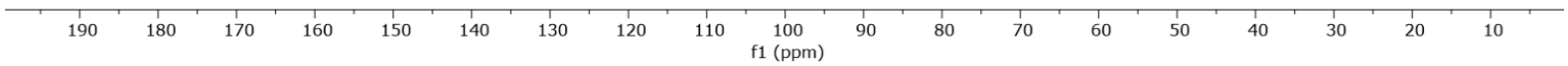



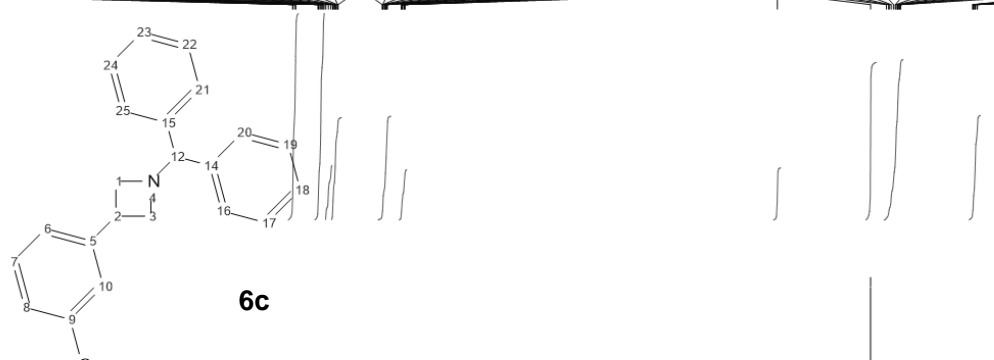

$\mathrm{H}^{3} \mathrm{C}^{\prime}{ }^{1}$

$6 c$

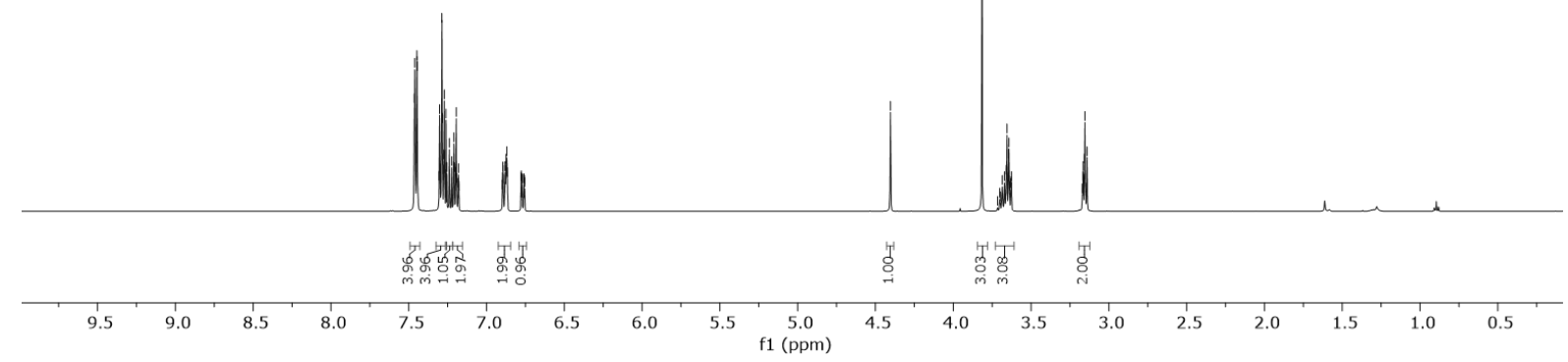

UOXF_groagna_352_A_1/CdCl3

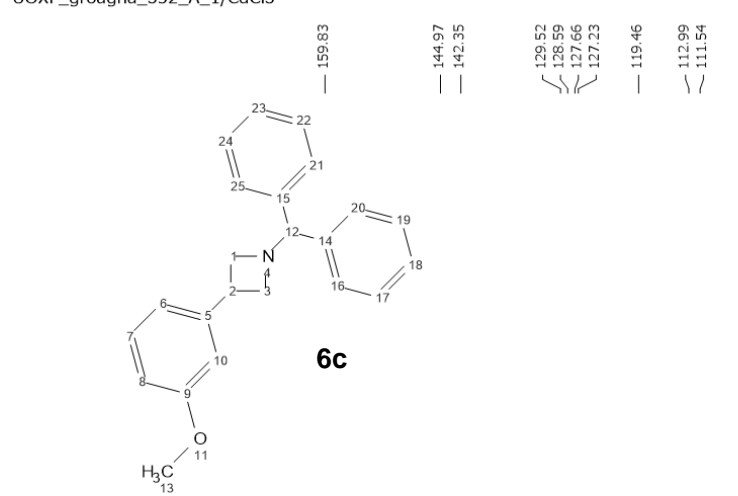

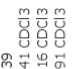

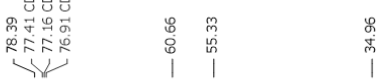

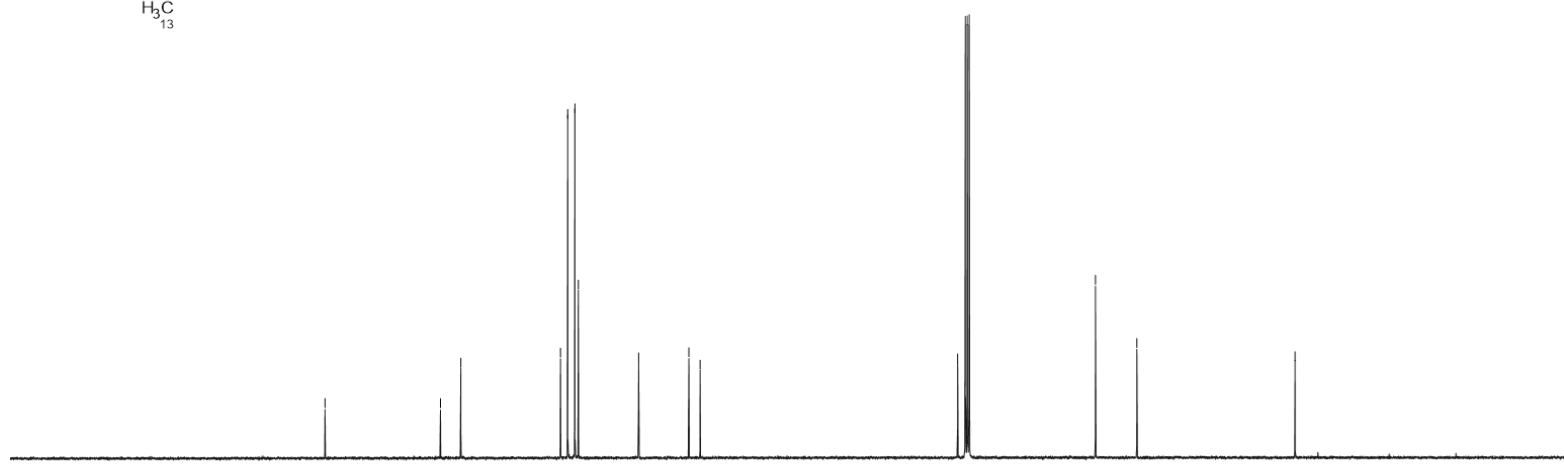

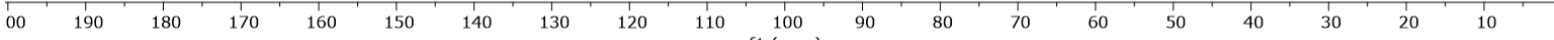


UOXF_groagna_364_1/CdCl3 $\frac{m}{\mathrm{~g}}$

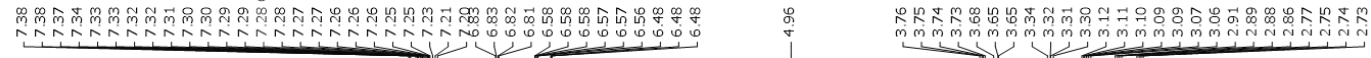

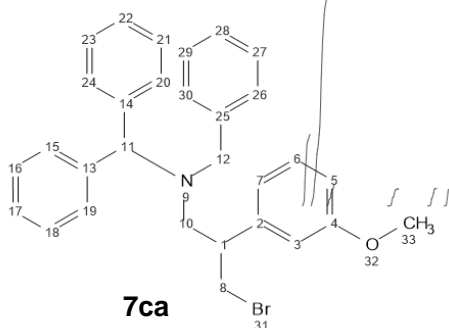

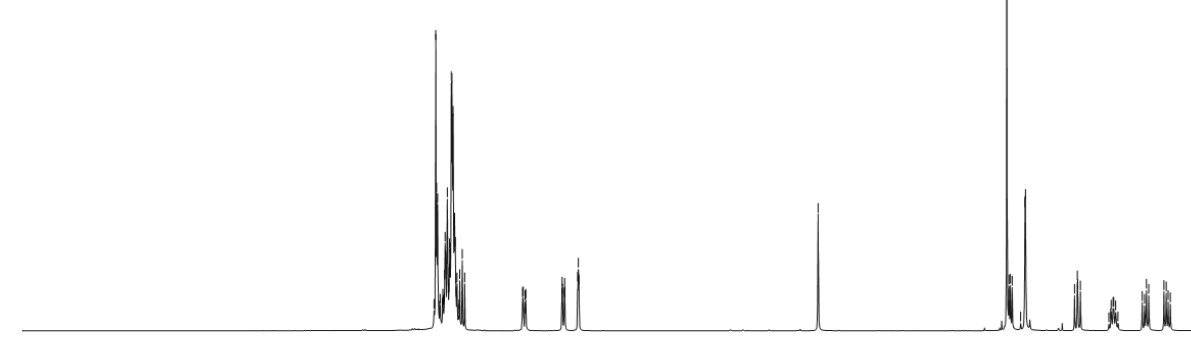

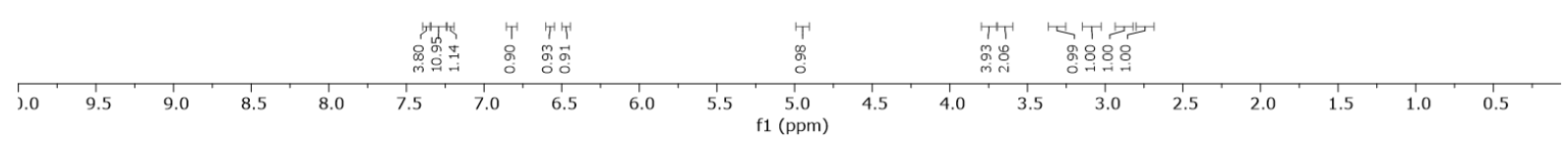

UOXF_groagna_364_1/CdCl3

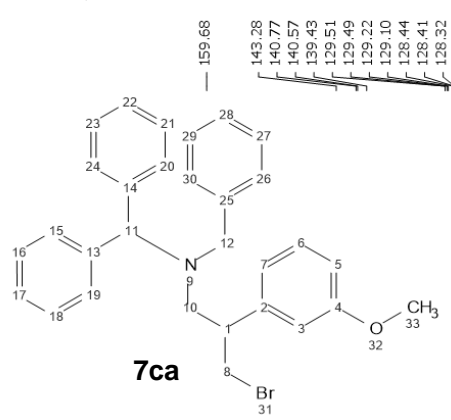

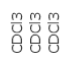

棺

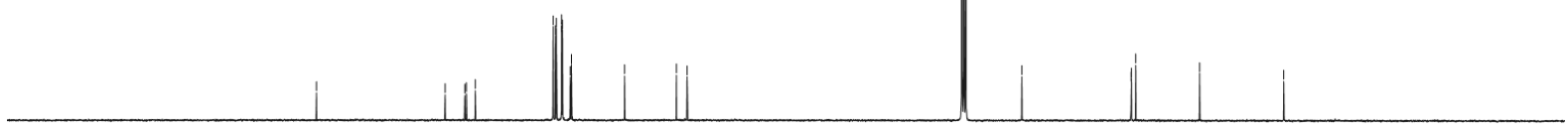


UOXF_groagna_326_1_B/CdCl3

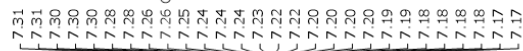

(1)

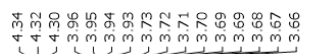
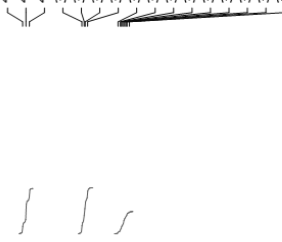
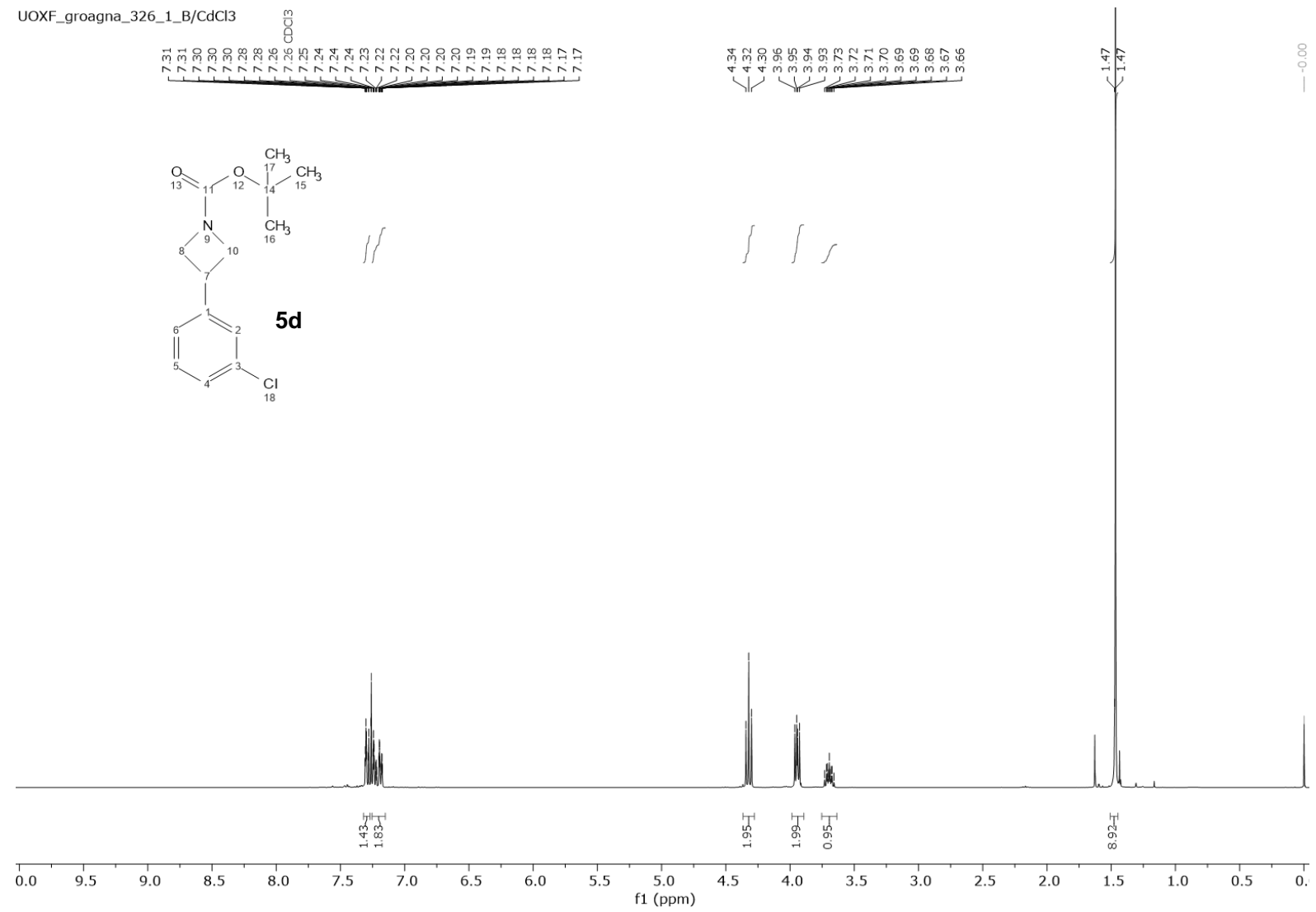

UOXF_groagna_326_1_B/CdCl3

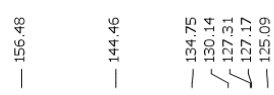

$\frac{m}{u} \frac{m}{u}$
080
080
$\infty$
$\infty$
$\infty$

5
5

$\underset{\substack{n \\ 0}}{\substack{0 \\ \text { in }}}$

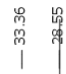

Sd

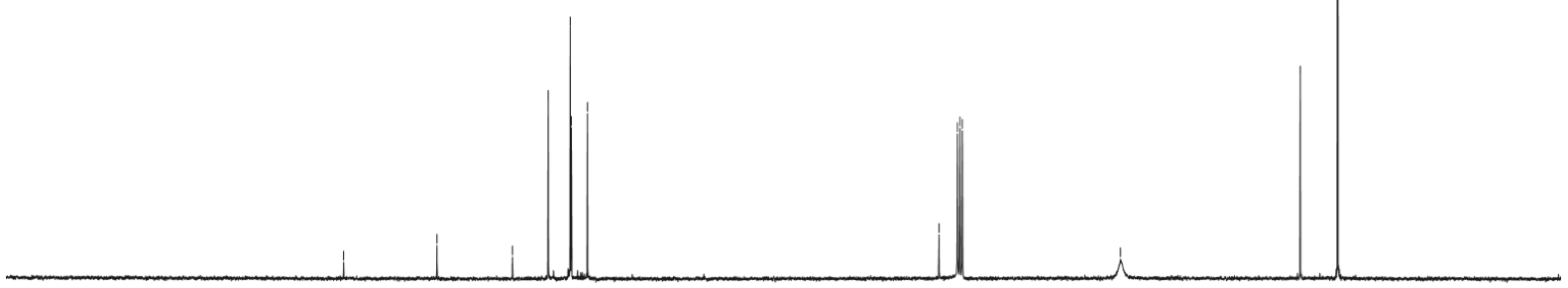

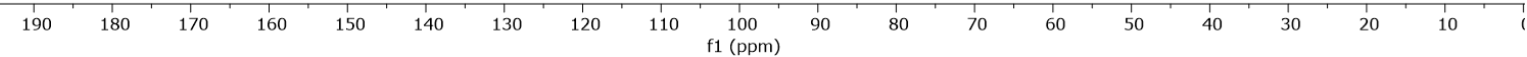




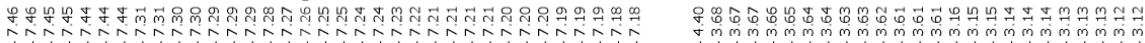

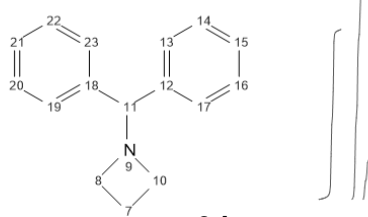

$6 d$<smiles>Clc1ccccc1</smiles>

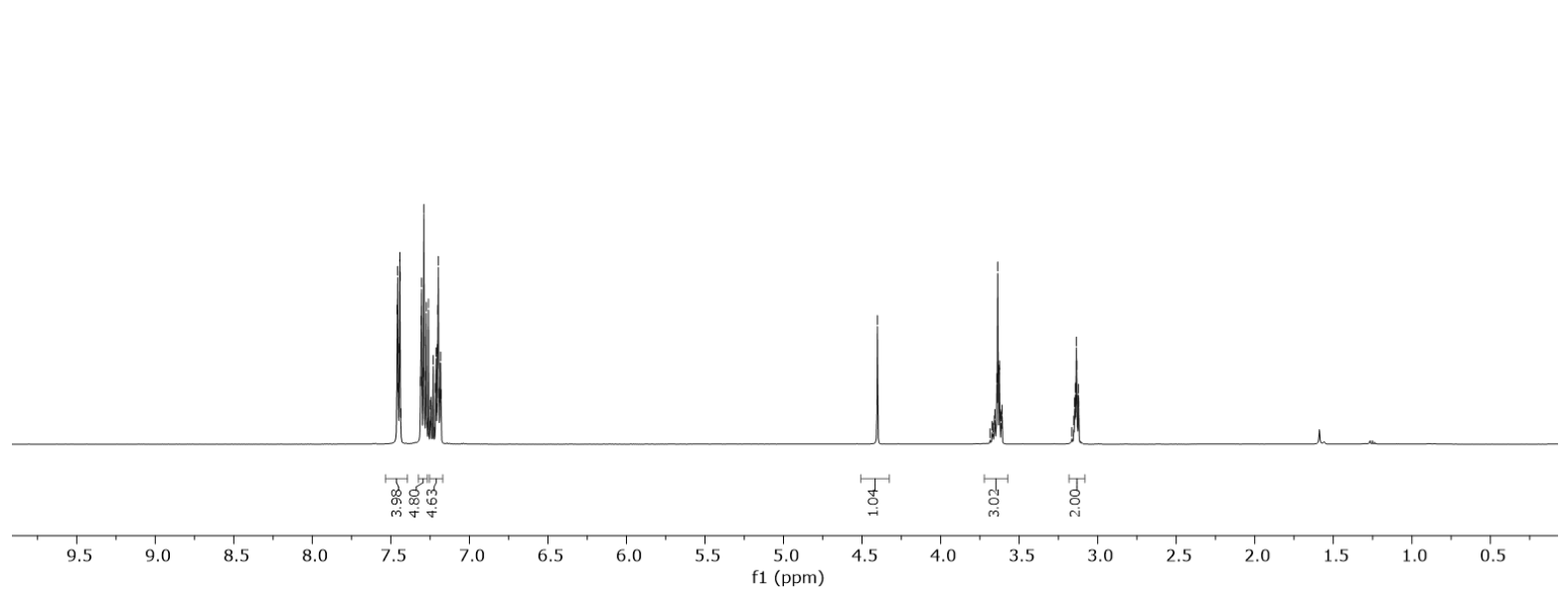

UOXF_groagna_336_1/CdCl3

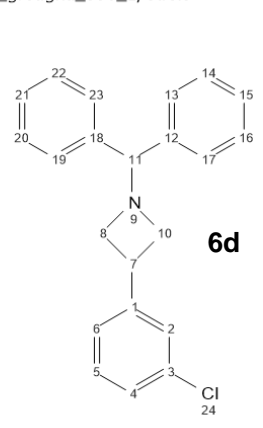

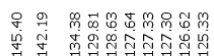

88

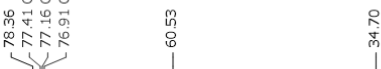

।

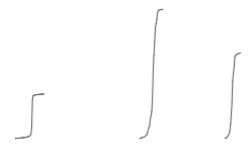




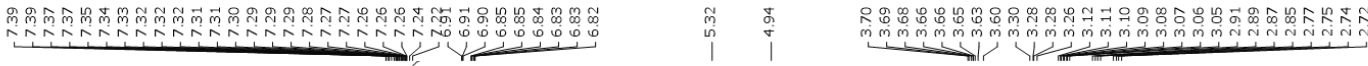

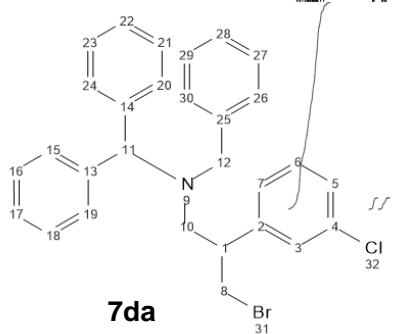

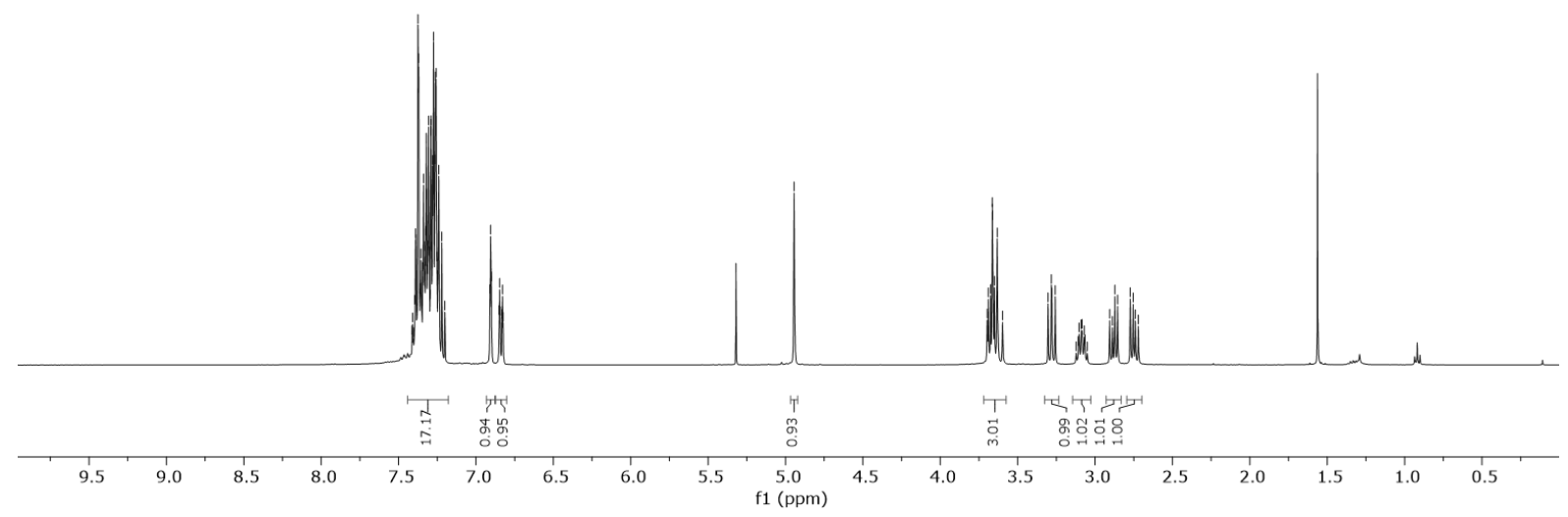

GR03EN55_1/CdCl3

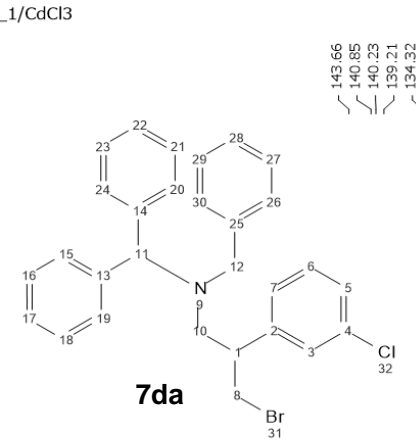

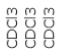

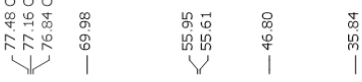

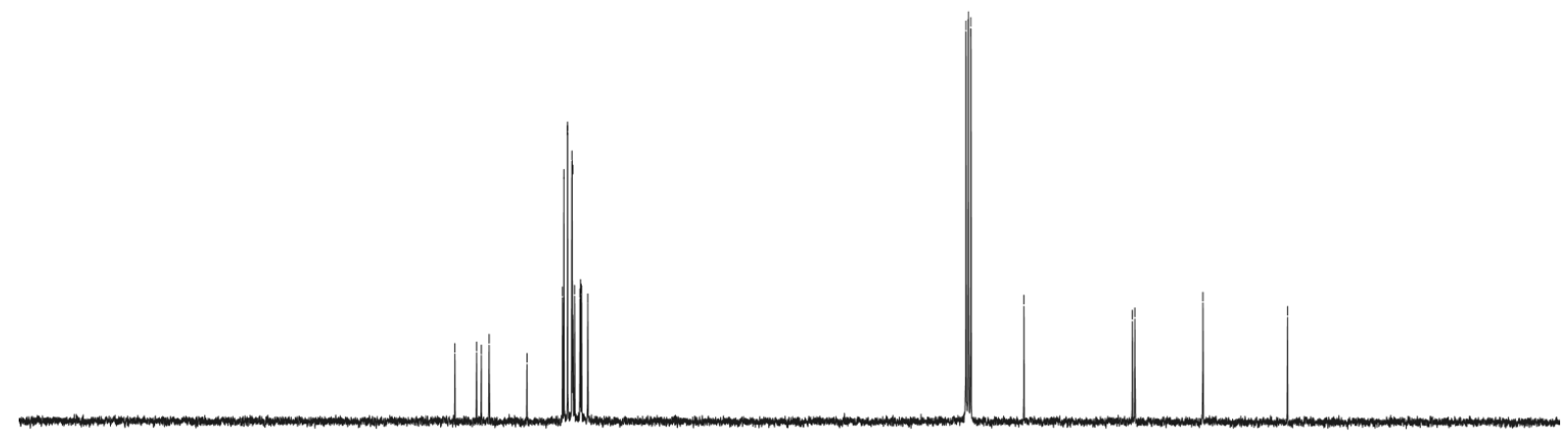

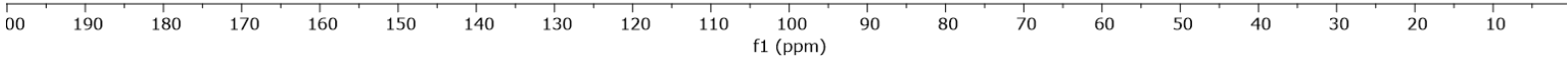


UOXF_groagna_430_1/CdCl3?

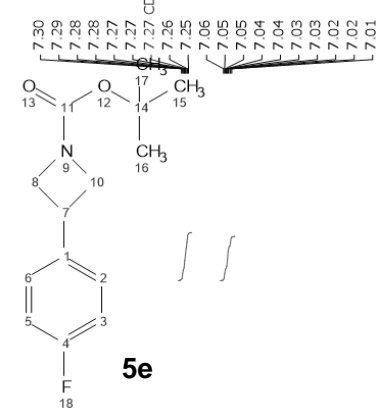

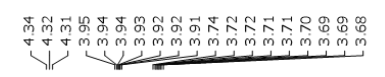

$\stackrel{\circ}{\circ}$
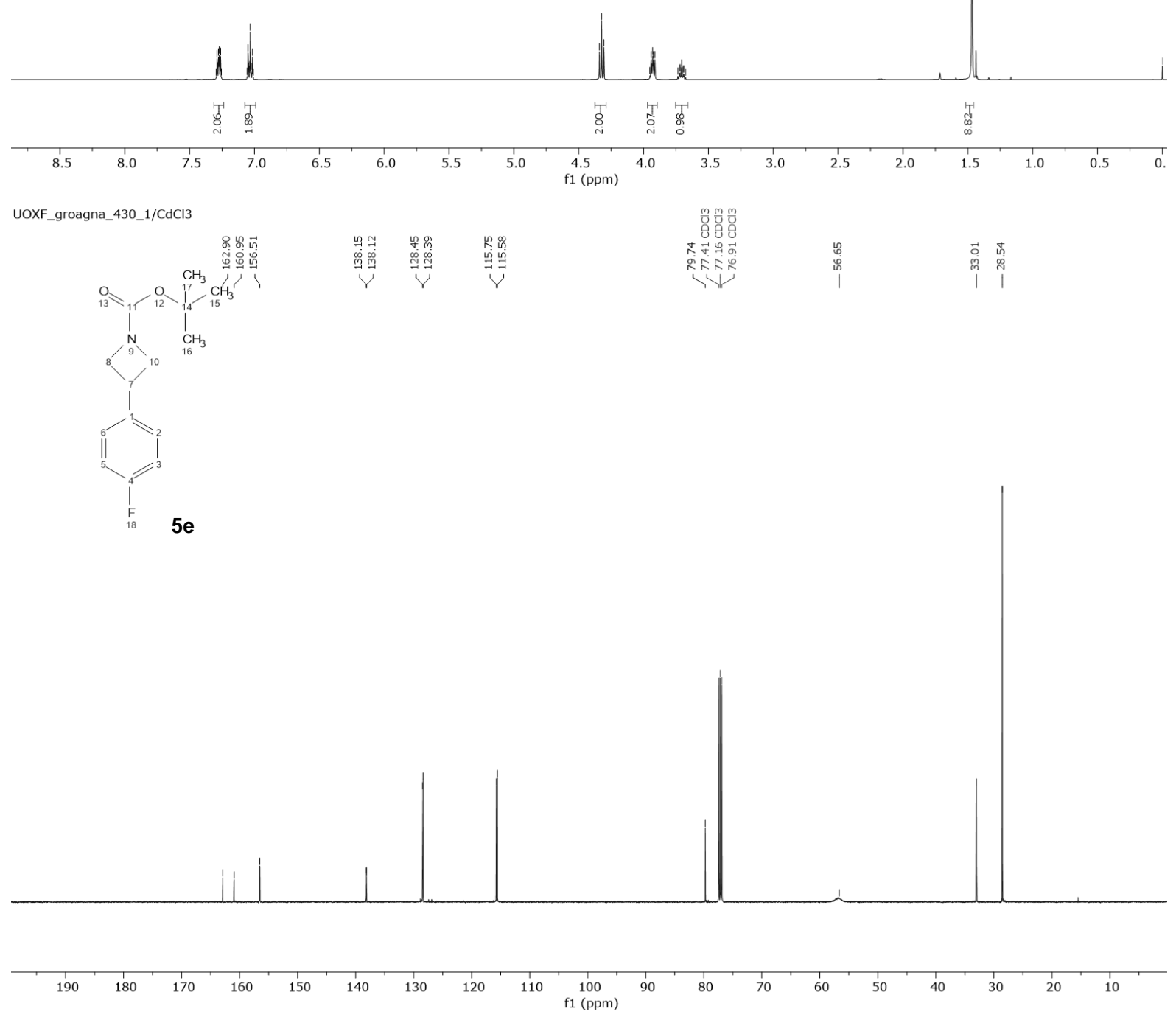

S146 

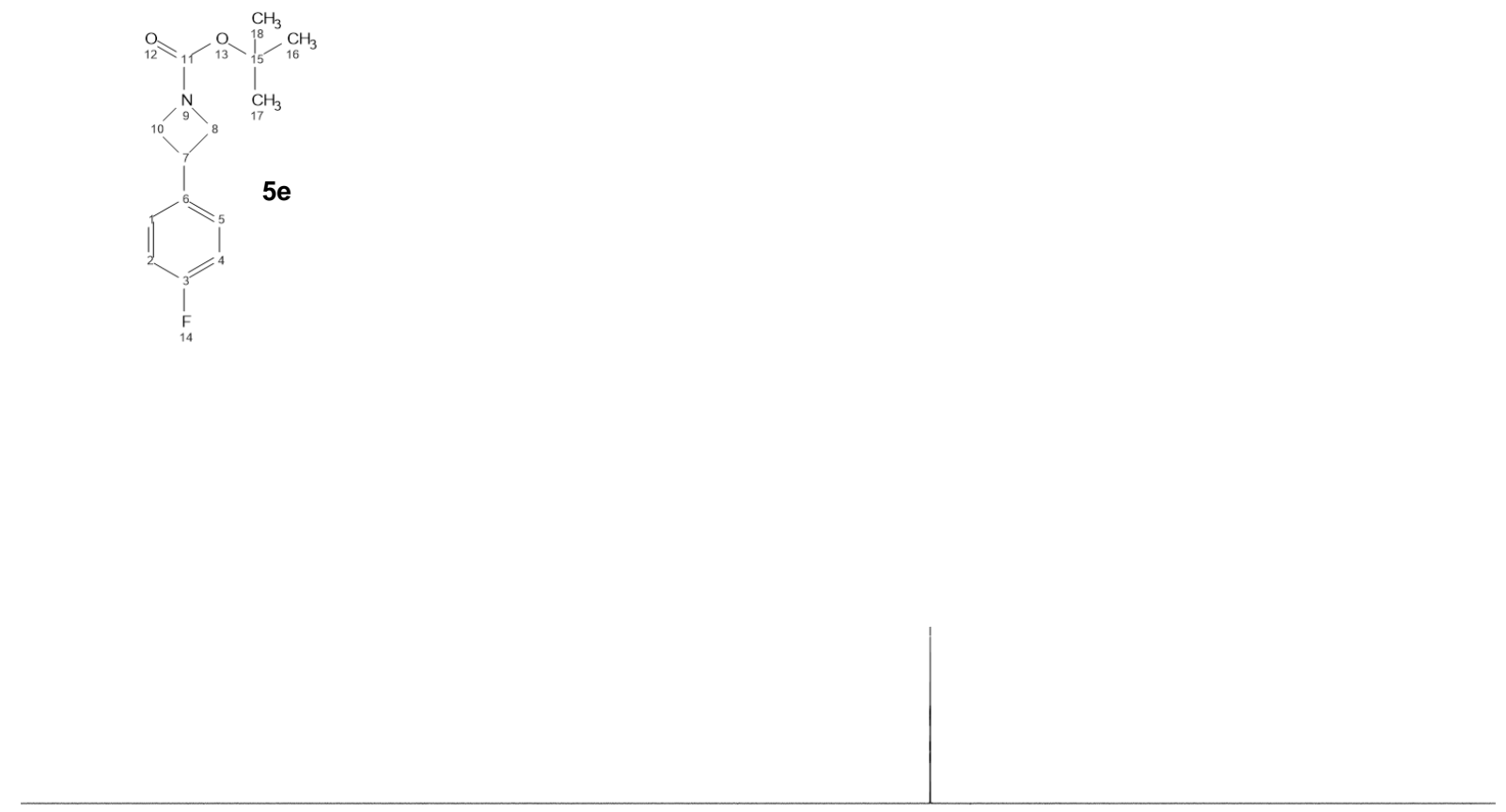

$\begin{array}{lllllllllllllllllllllllllllllllllllllllll}1 & 80 & 80 & 70 & 60 & 50 & 40 & 30 & 20 & 10 & 0 & -10 & -20 & -30 & -40 & -50 & -60 & -70 & -80 & -90 & -100 & -110 & -120 & -130 & -140 & -150 & -160 & -170 & -180 & -190 & -200 & -210 & -220 & -230 & -240\end{array}$ 
GR03EN59_1/CdCl3

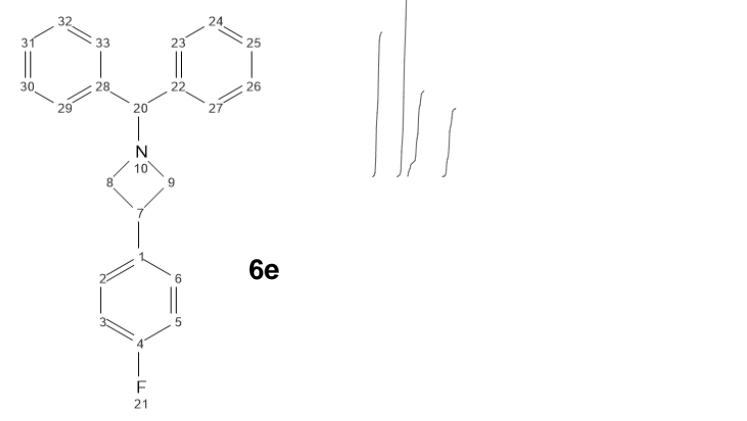

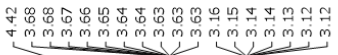

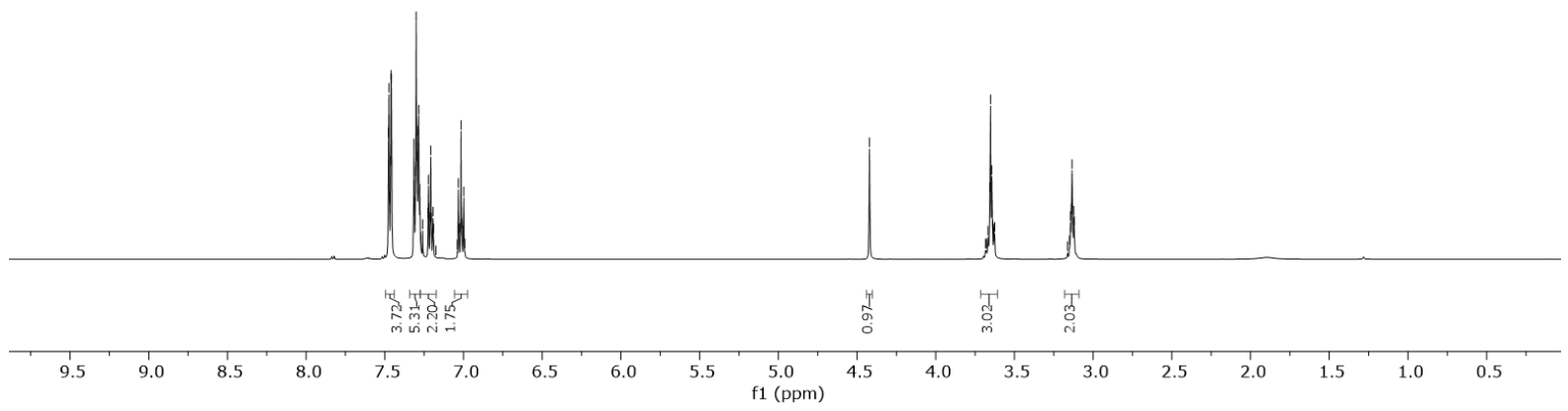

GR03EN59_1/CdCl3

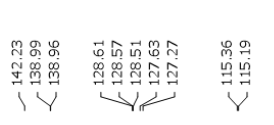

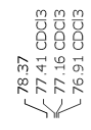

$\sigma$
0
0
1

偙

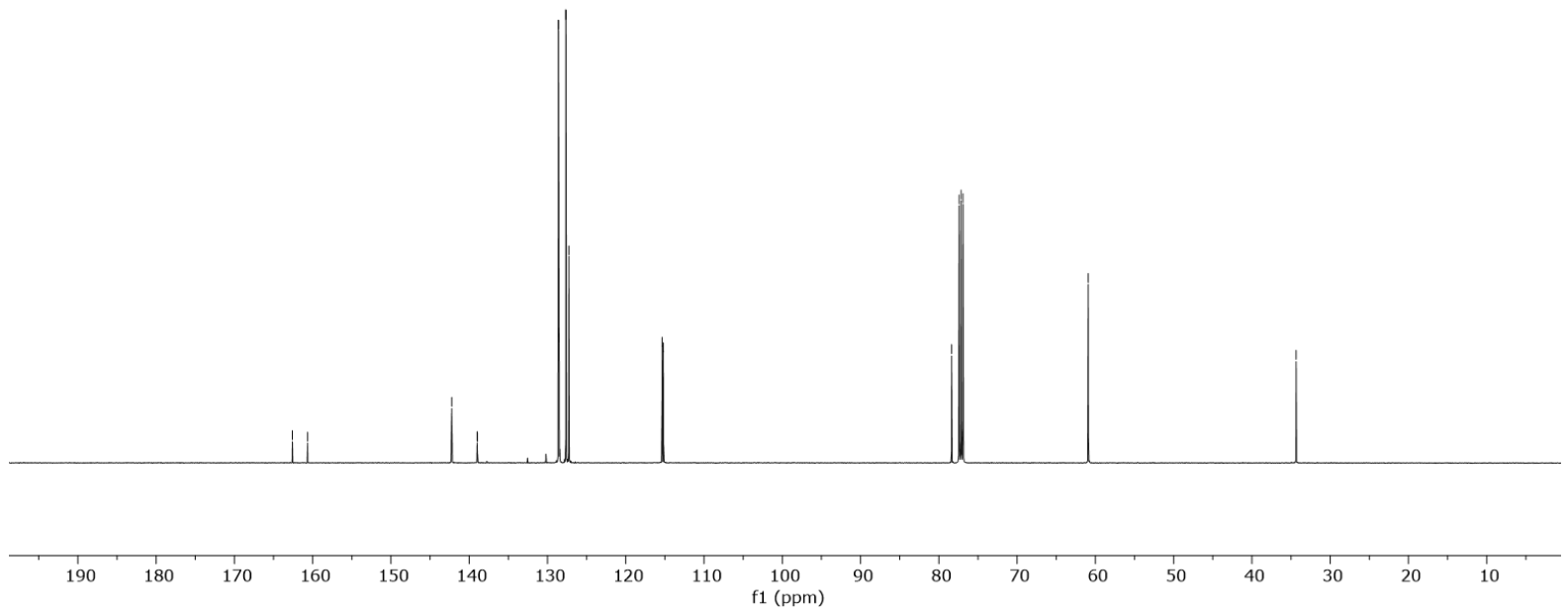

S148 


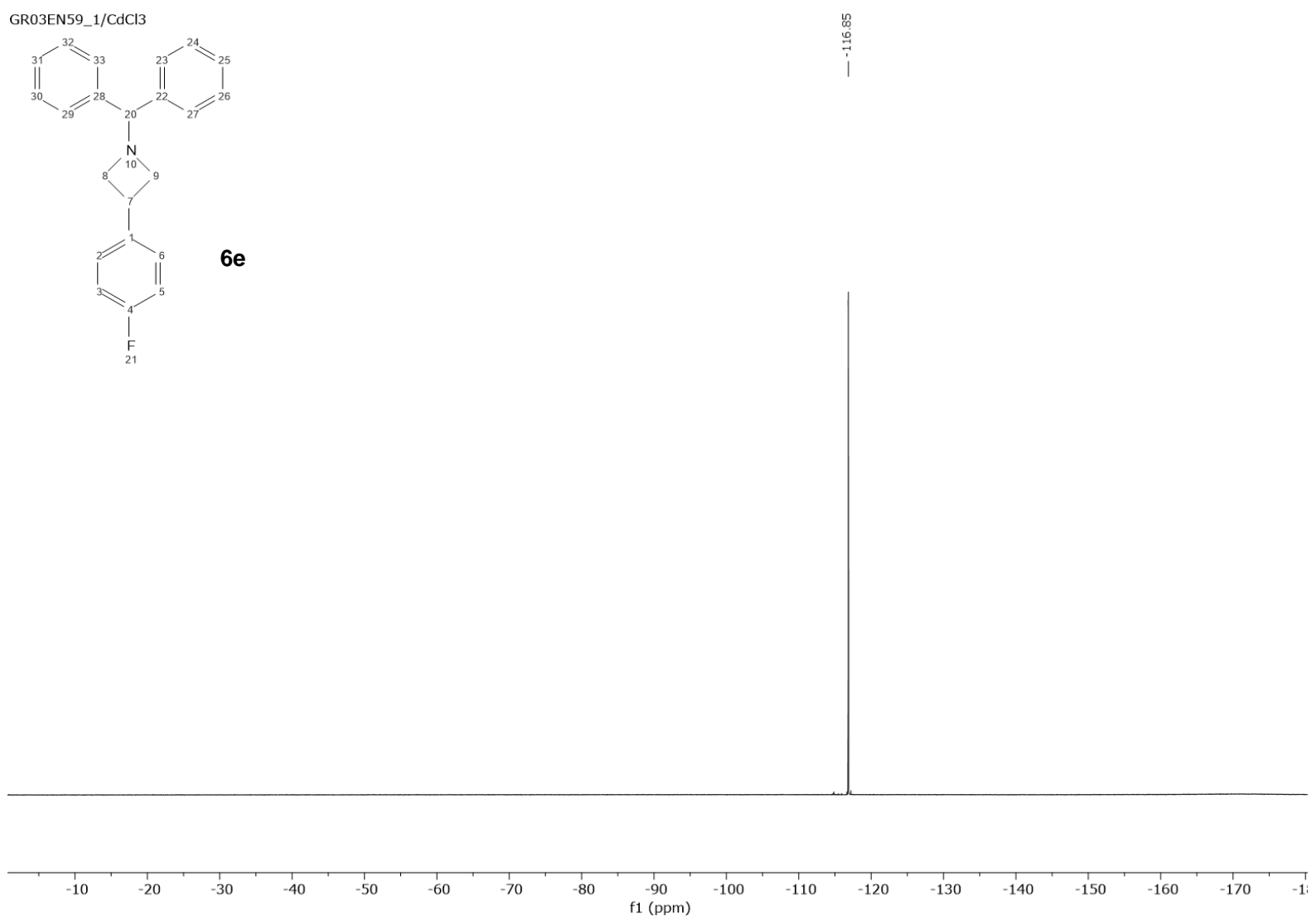



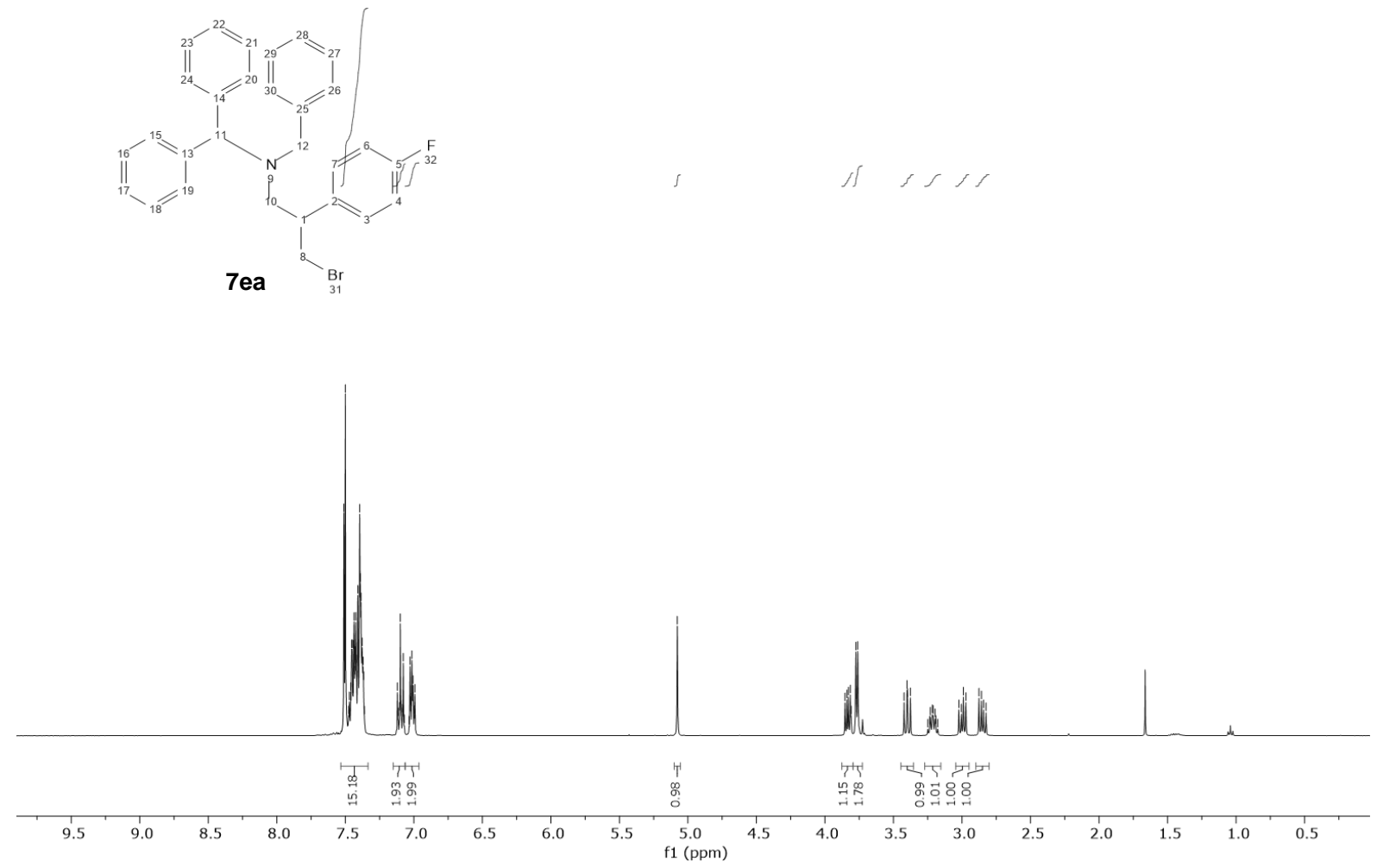

GR03EN79_1/CdCl3

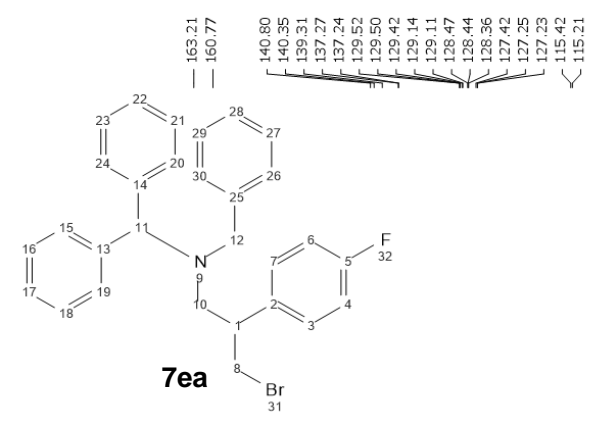

$m$
0
8

V|l

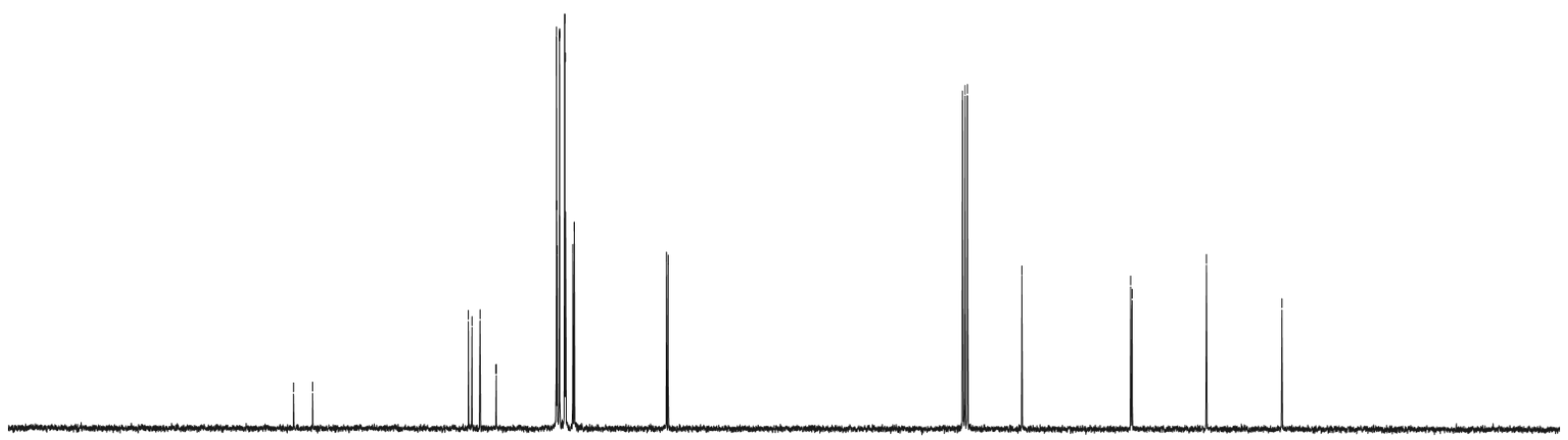

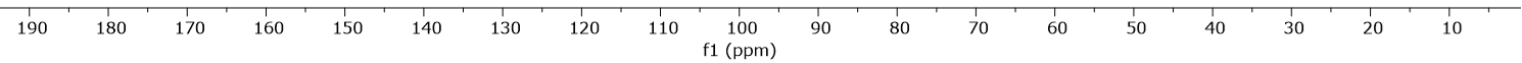




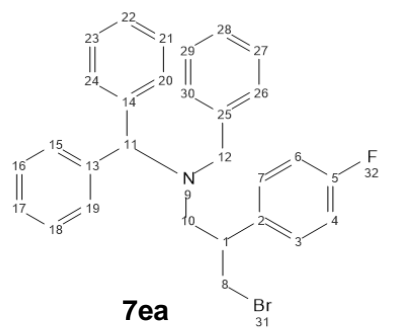

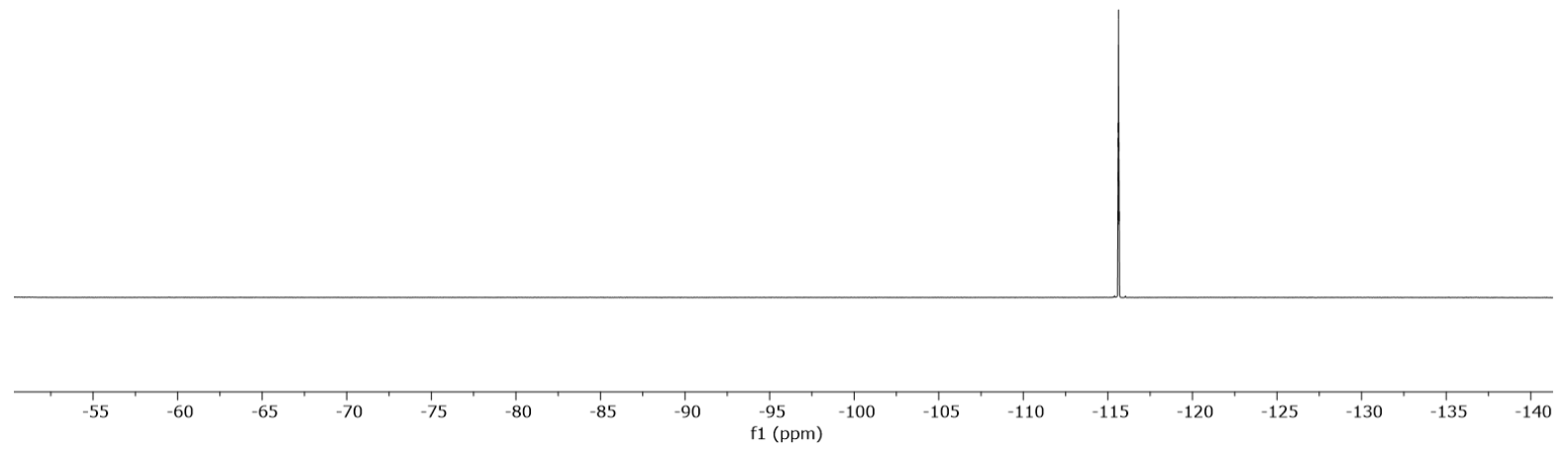


UOXF_groagna_426_1/CdCl3

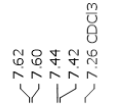
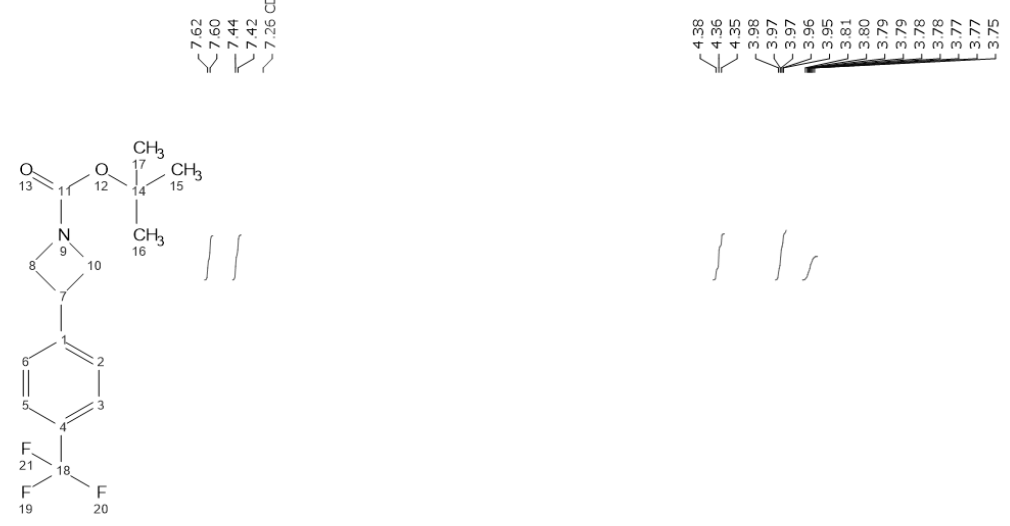

$5 f$
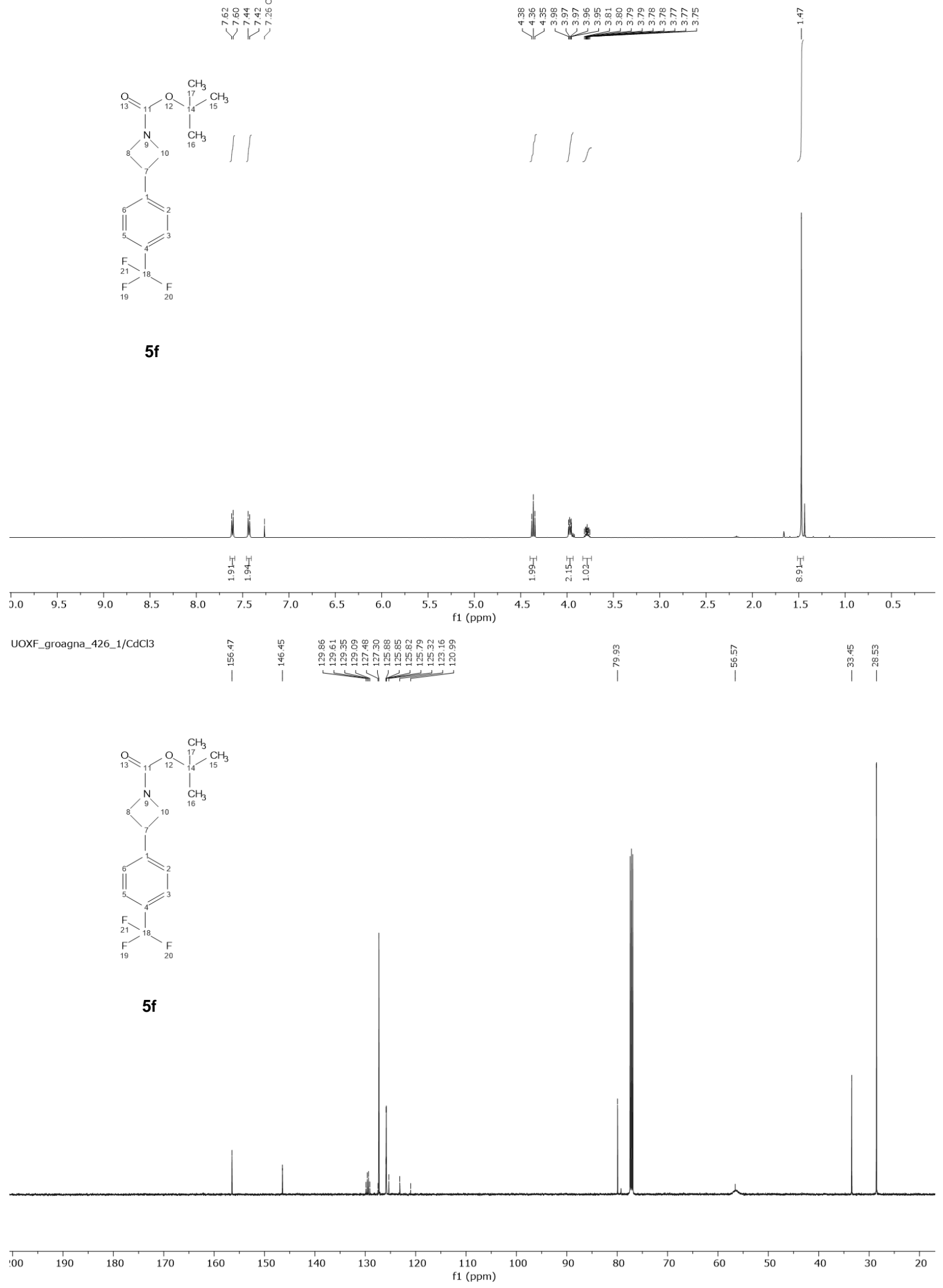

S152 


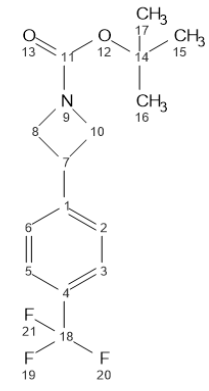

$5 f$

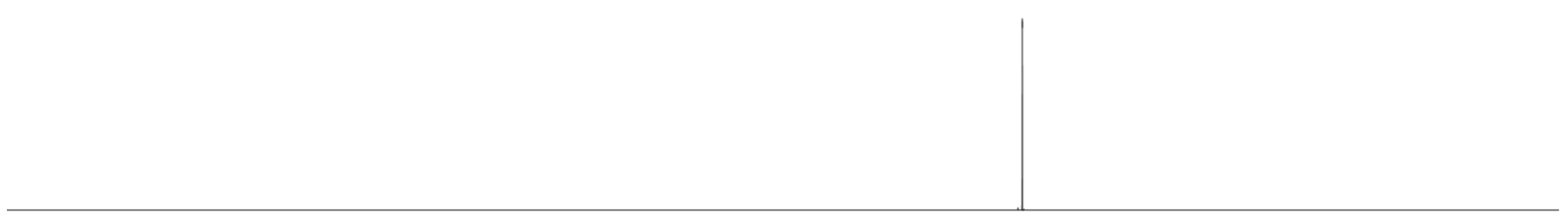

$200 \quad 180 \quad 160 \quad 140 \quad 120 \quad 100 \quad 80 \quad 60 \quad 40 \quad 20 \begin{gathered}0 \\ \mathrm{f} 1(\mathrm{ppm})\end{gathered}$ 
UOXF_groagna_389_1/CdCl3

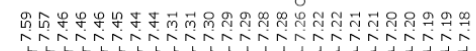

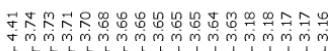

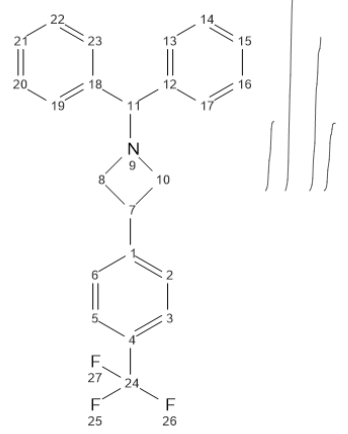

$6 f$

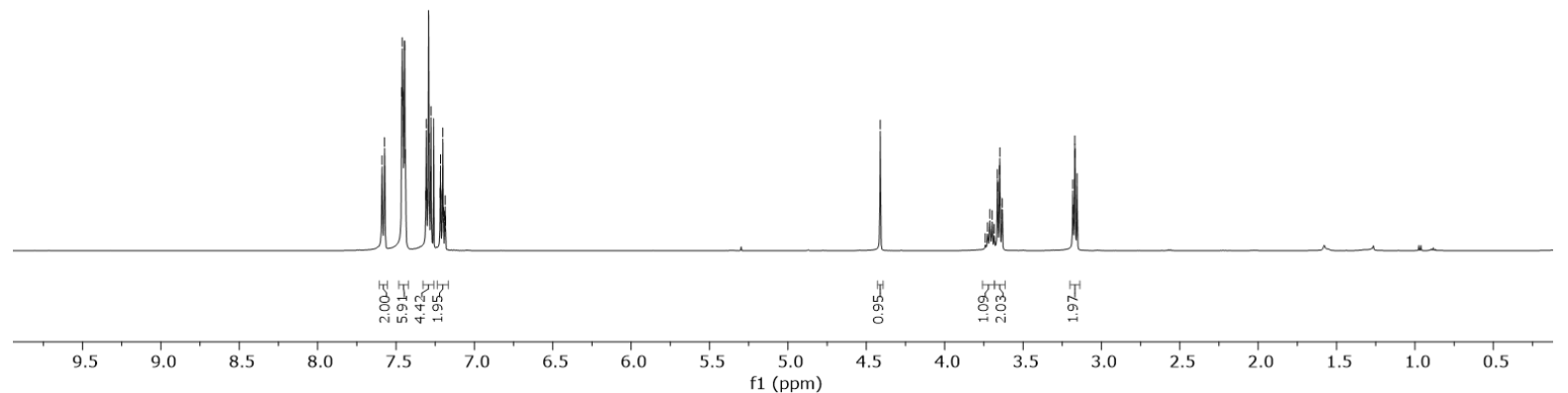

UOXF_groagna_389_1/CdCl3

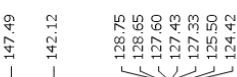

$m$
0
80
80

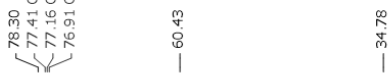<smiles></smiles>

$6 f$

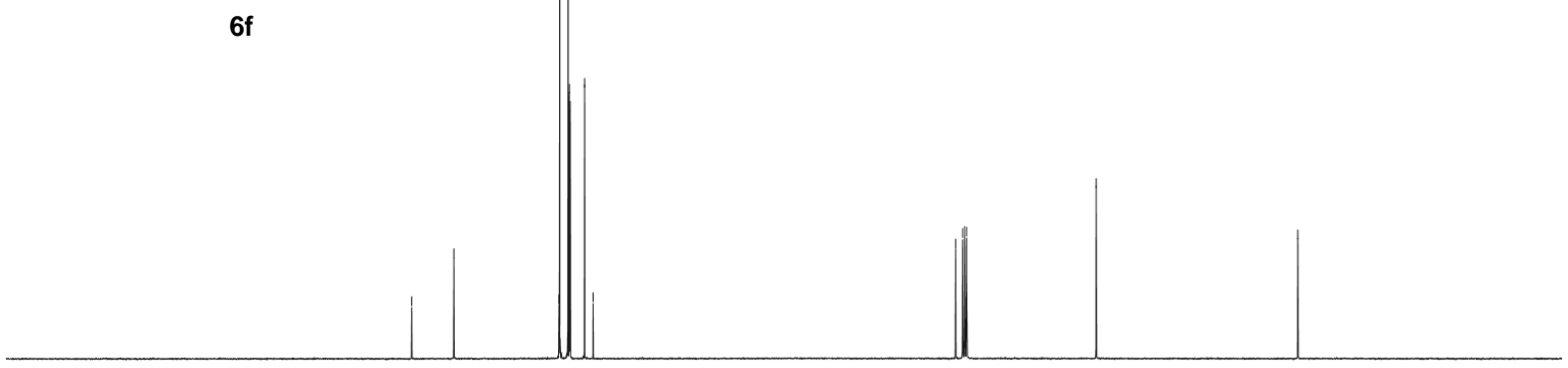

190

$180 \quad 170 \quad 160$

$150 \quad 140$

$110 \quad 100$

1
90
80

70

50

$30 \quad 20,10$ 
UOXF_groagna_389_1/CdCl3

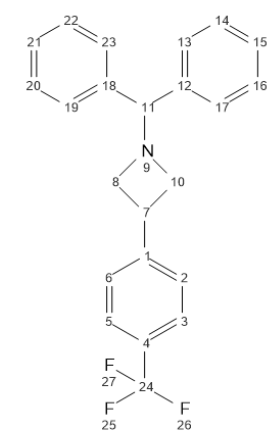

$6 f$

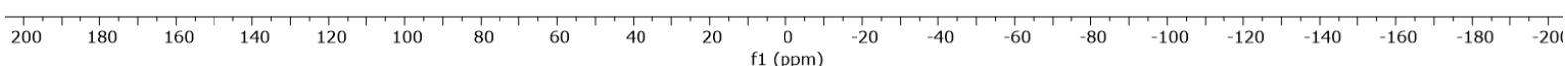


GR03EN78_1/CdCl3 $\frac{\pi}{\square}$

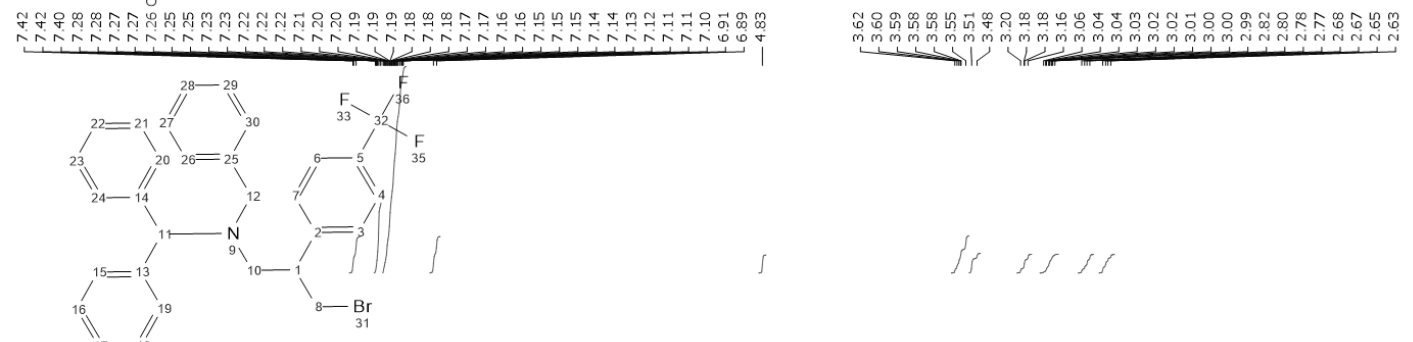

$7 f a$

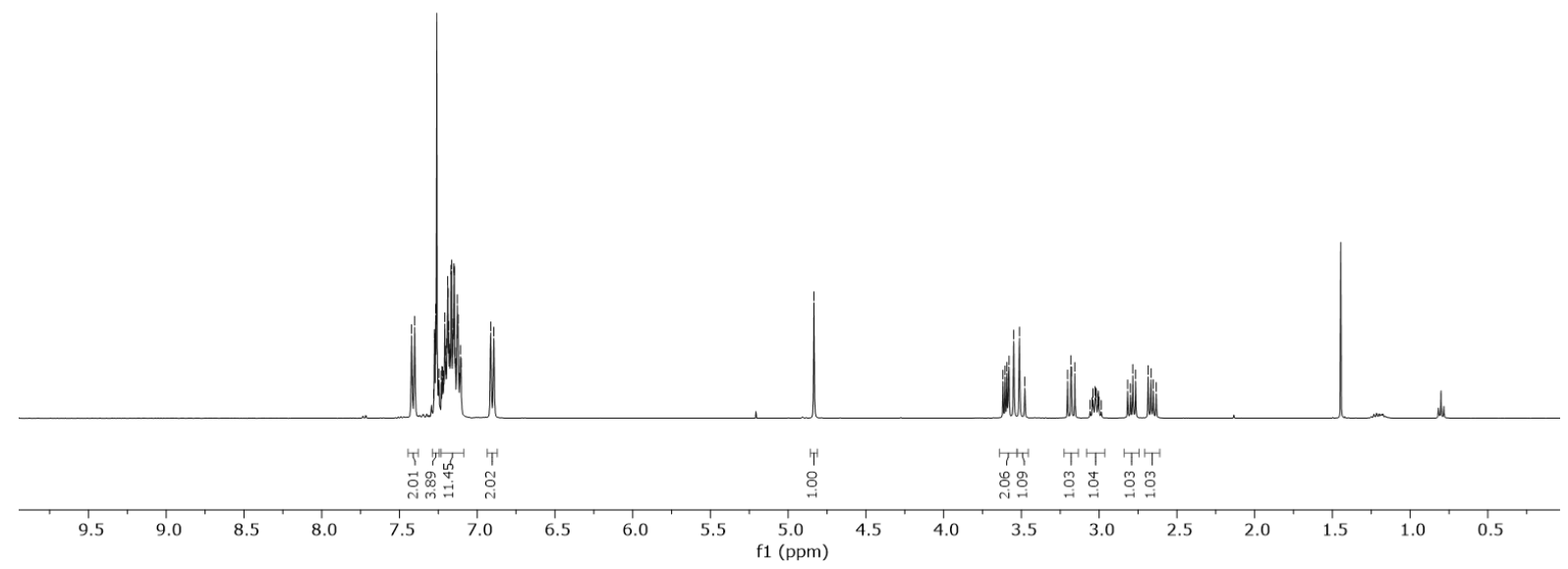

GR03EN78_1/CdCl3

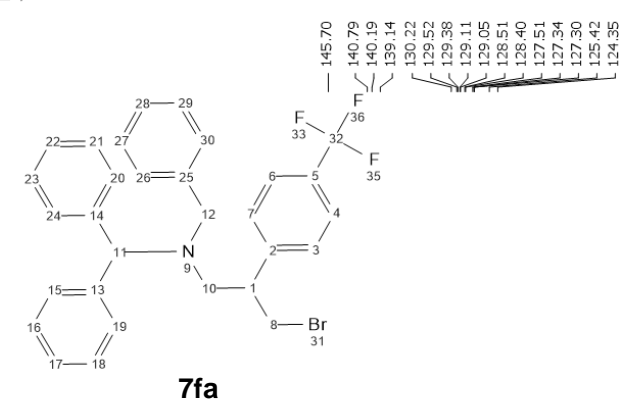

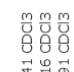

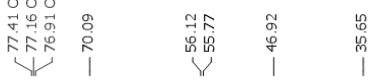

$7 f a$

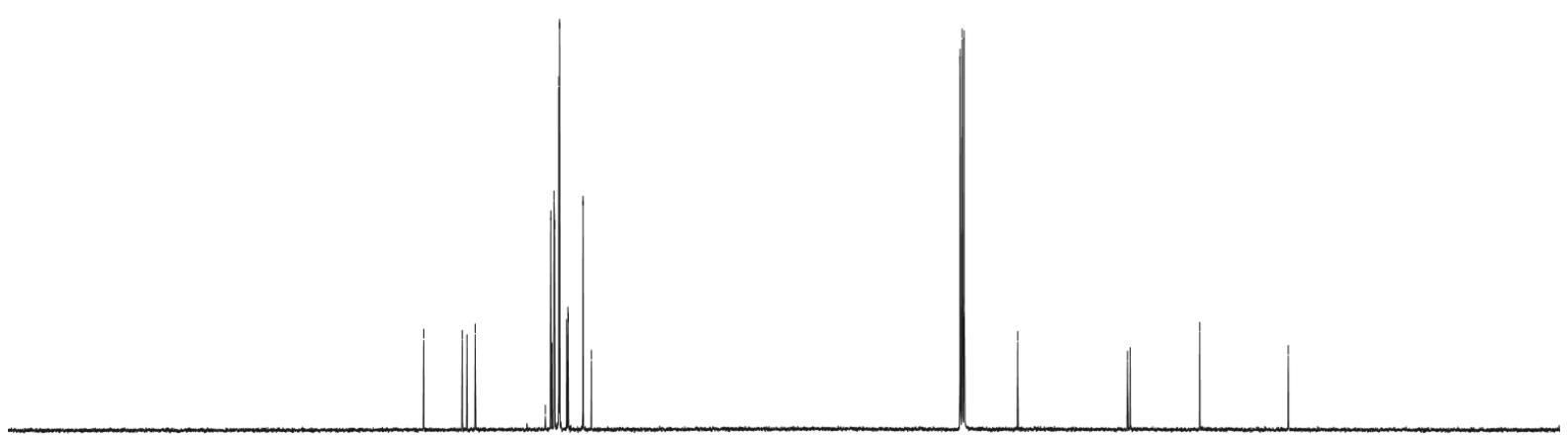

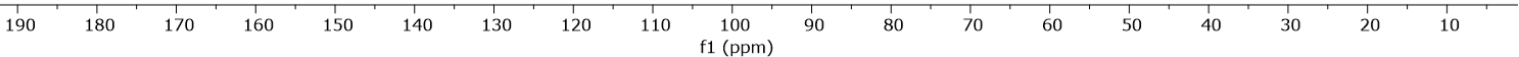


GR03EN78_1/CdCl3

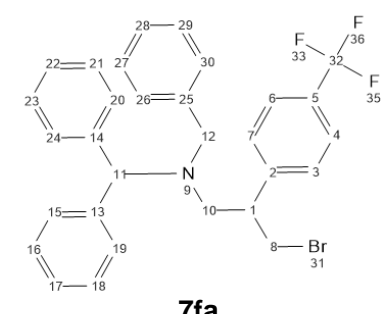

\section{$7 \mathrm{fa}$}

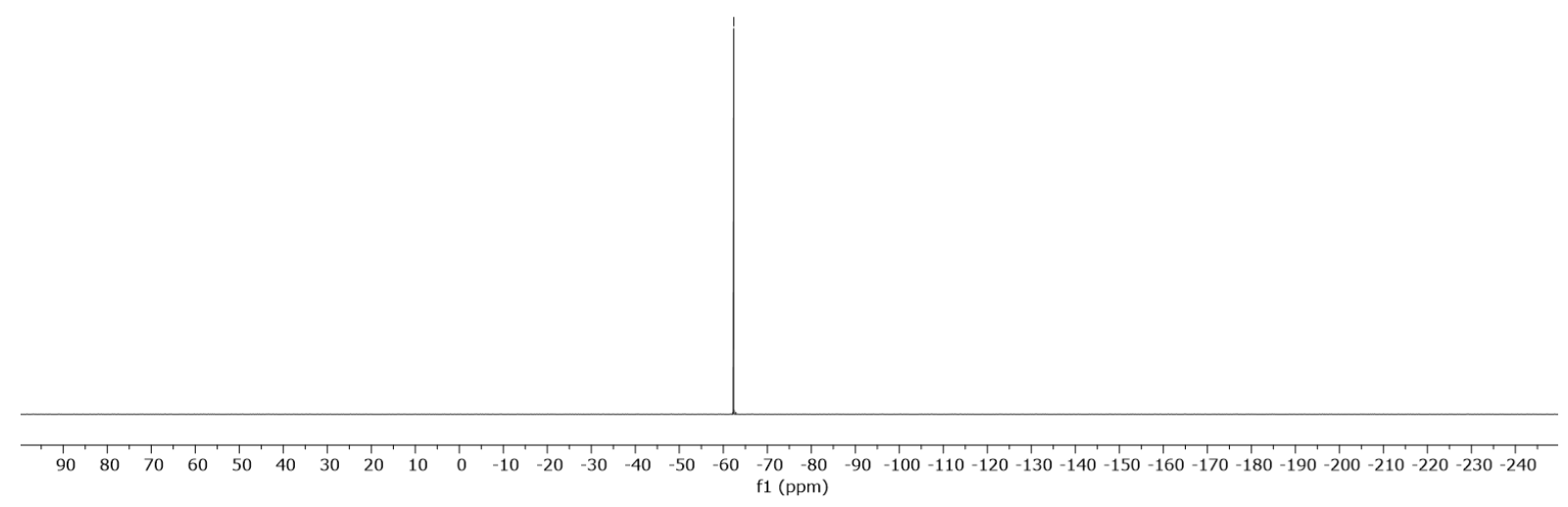




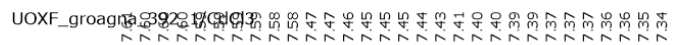

$\underbrace{m}$

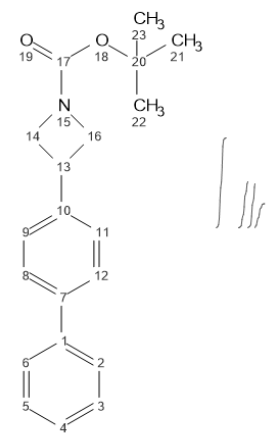

$5 g$
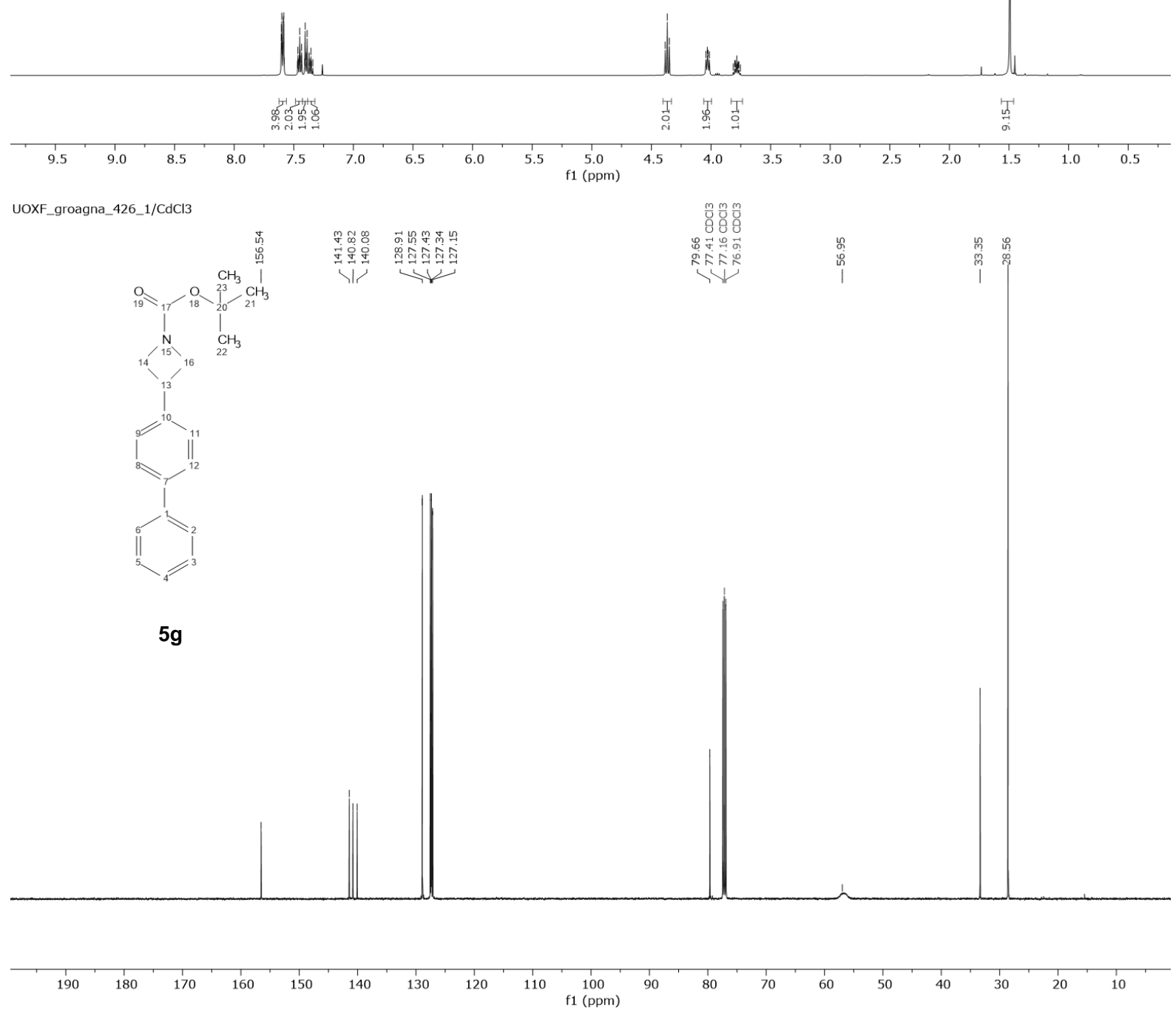

$5 g$

S158 
UOXF_groagna_411_1/CdCl3

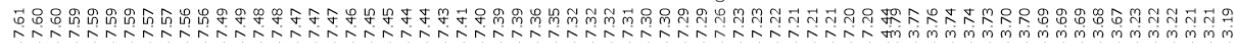

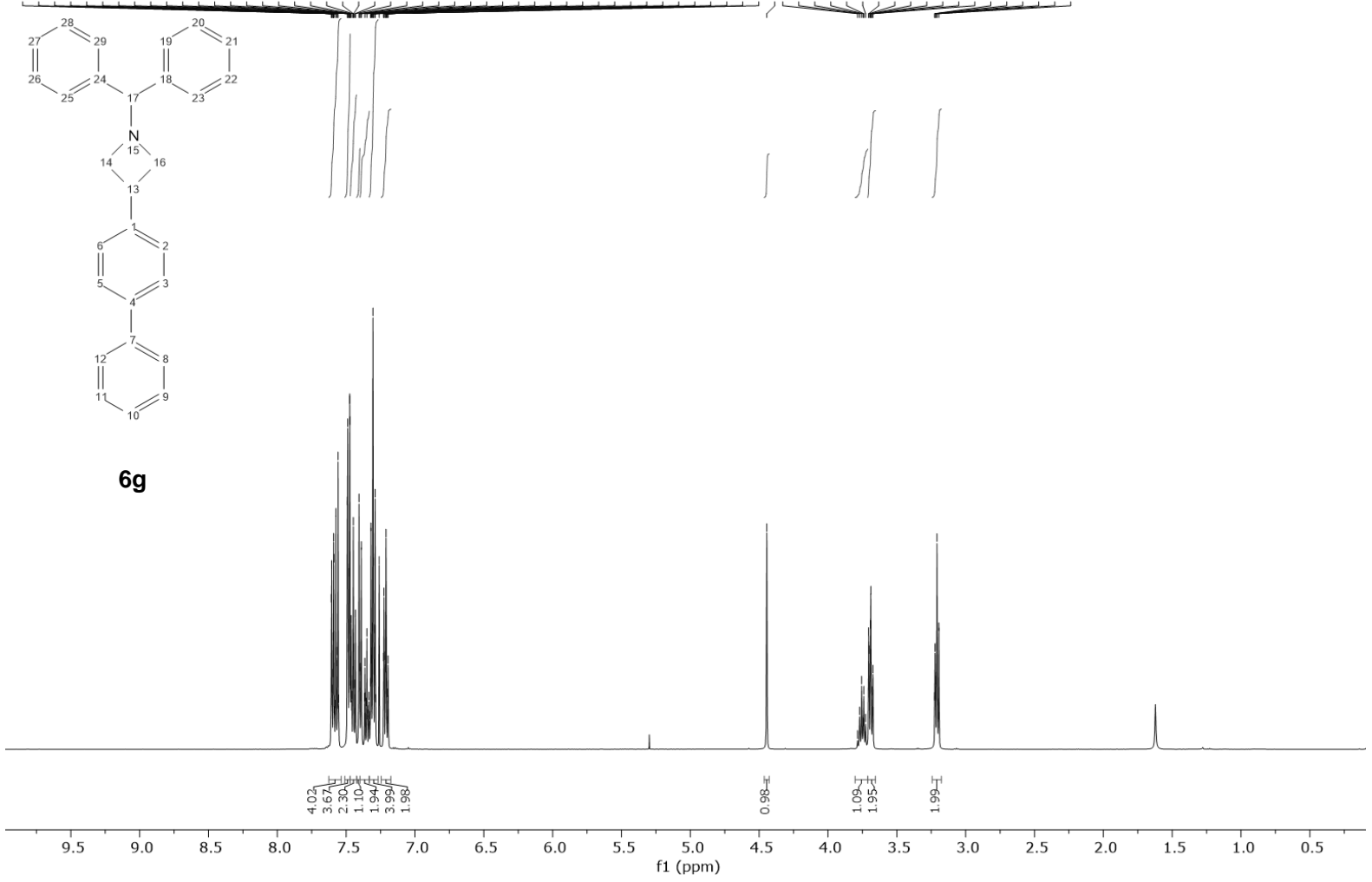

UOXF_groagna_411_1/CdCl3

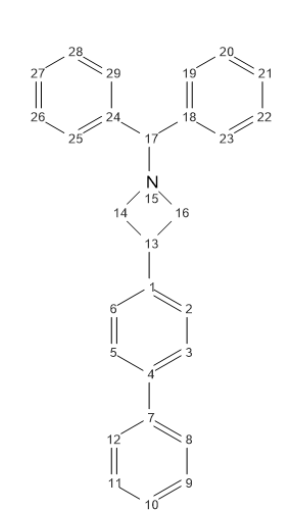

$\frac{m}{0}{ }^{m} \frac{m}{0}$
80

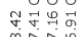

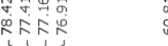

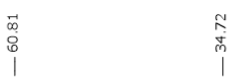

$6 g$

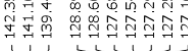

(1)

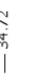
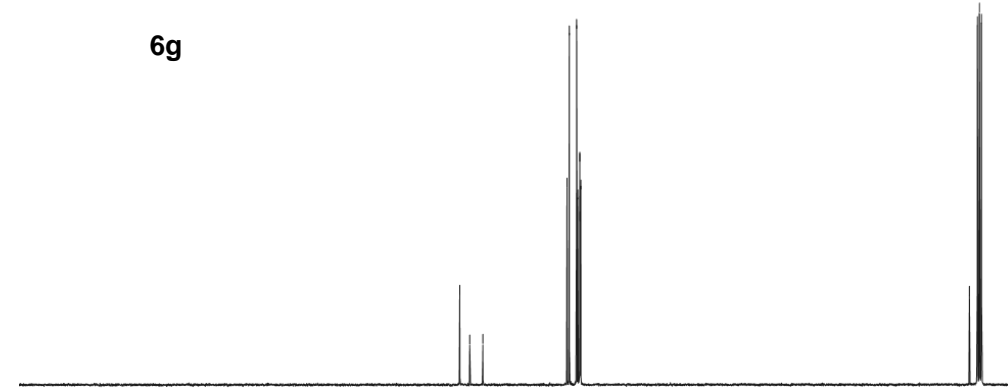


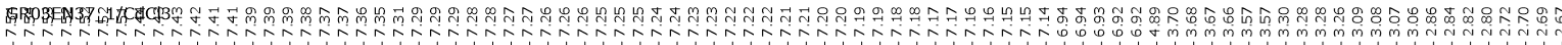

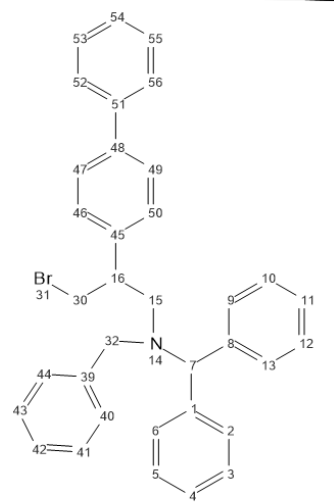

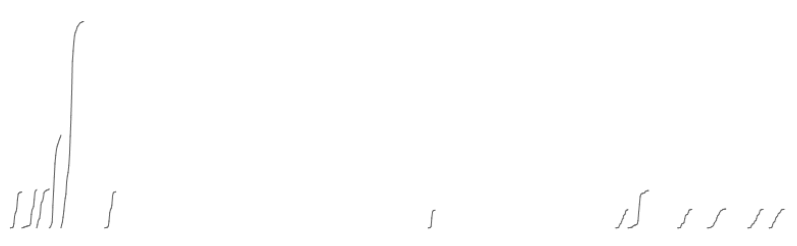

$7 g a$
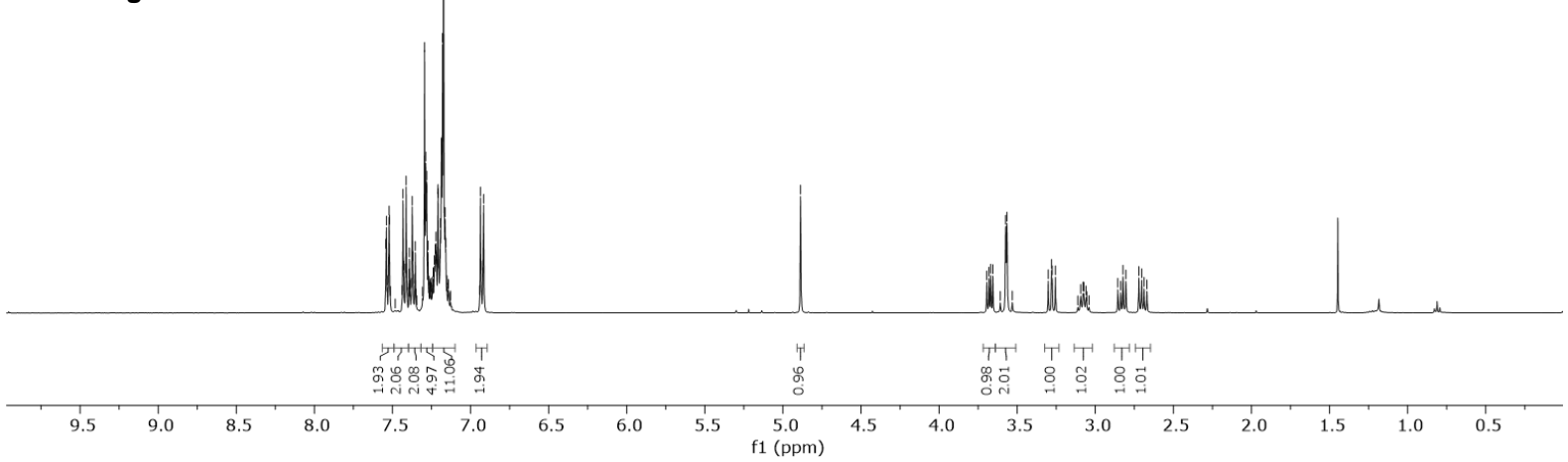

GR03EN37_1/CdCl3

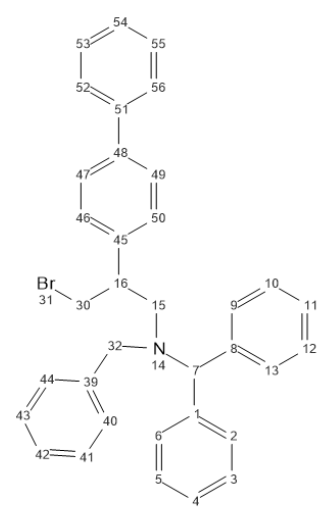

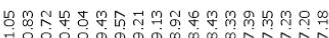

盟盟照

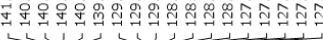

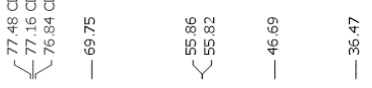

7ga

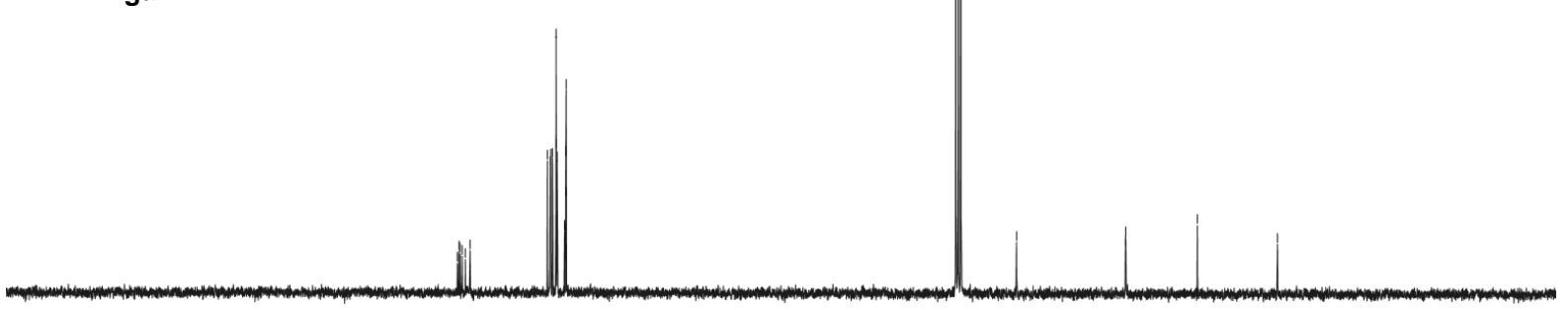

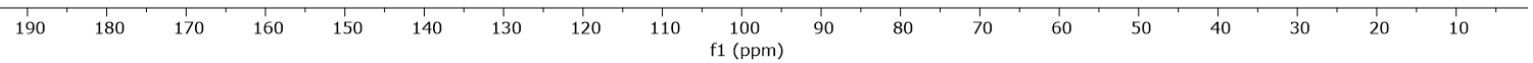



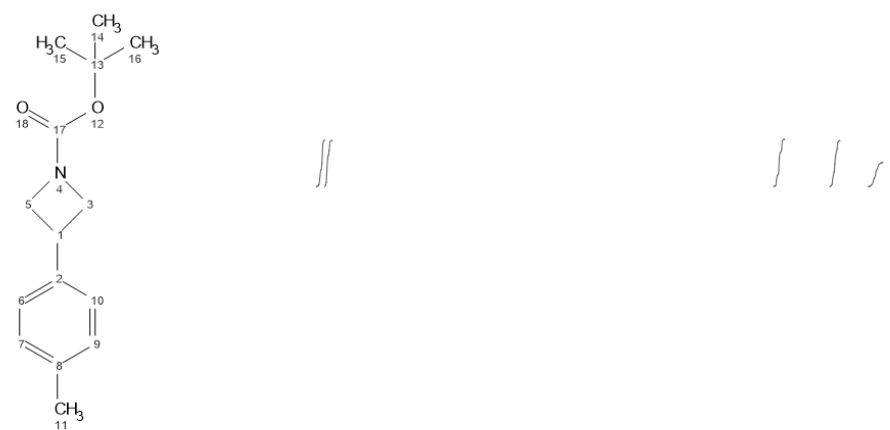

$5 \mathrm{~h}$

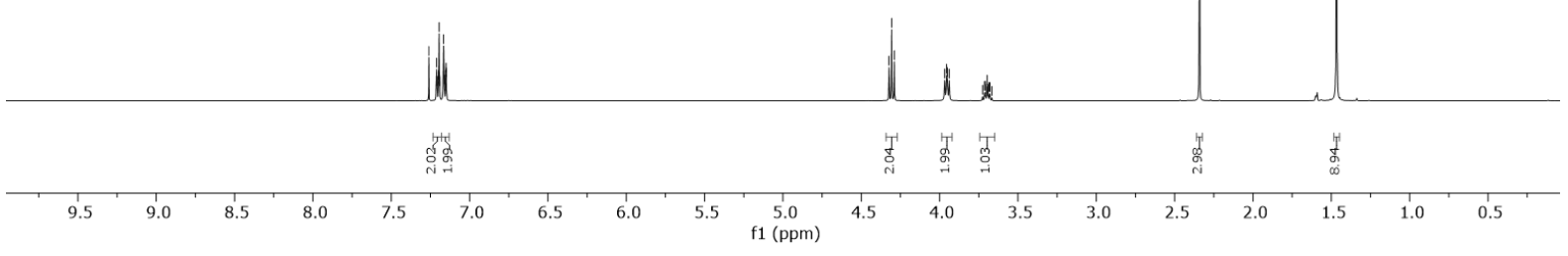

UOXF_groagna_333_1/CdCl3

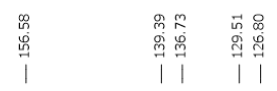

$m$
$\overline{0}$
0

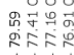

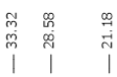

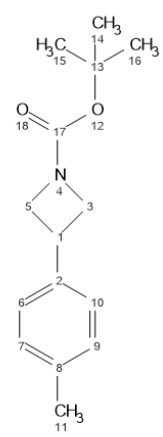

$5 h$

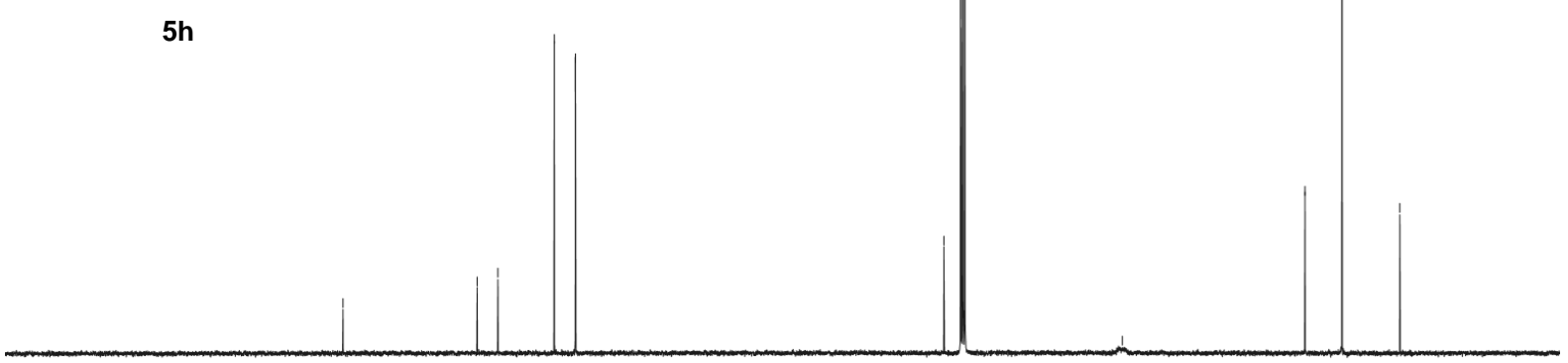

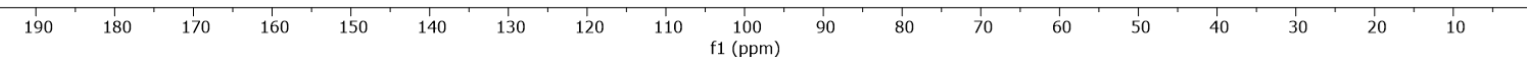


UOXF_groagna_398_1/CdCl3

若

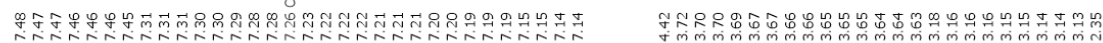

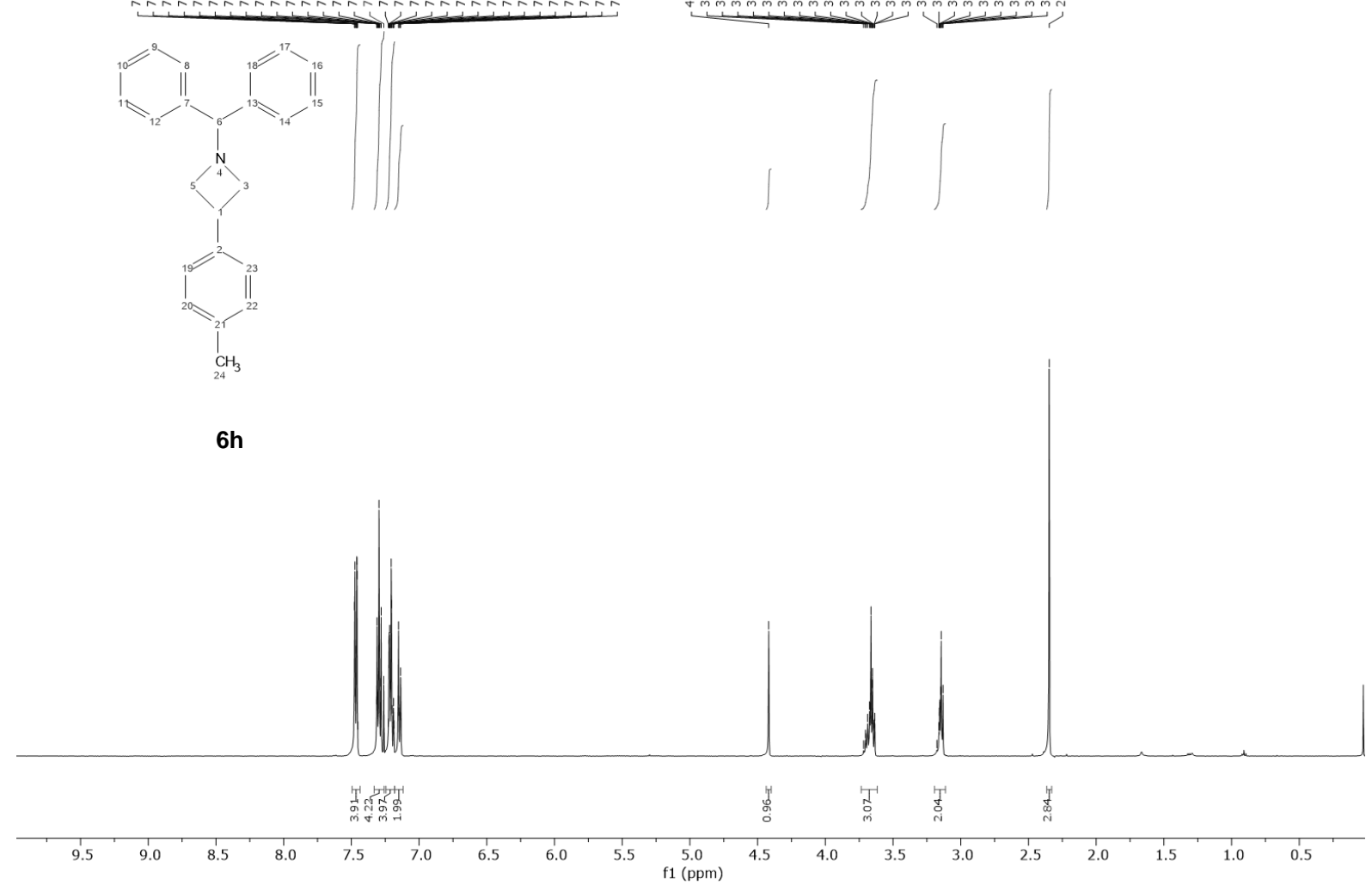

$6 \mathrm{~h}$

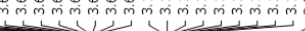

UOXF_groagna_398_1/CdCl3

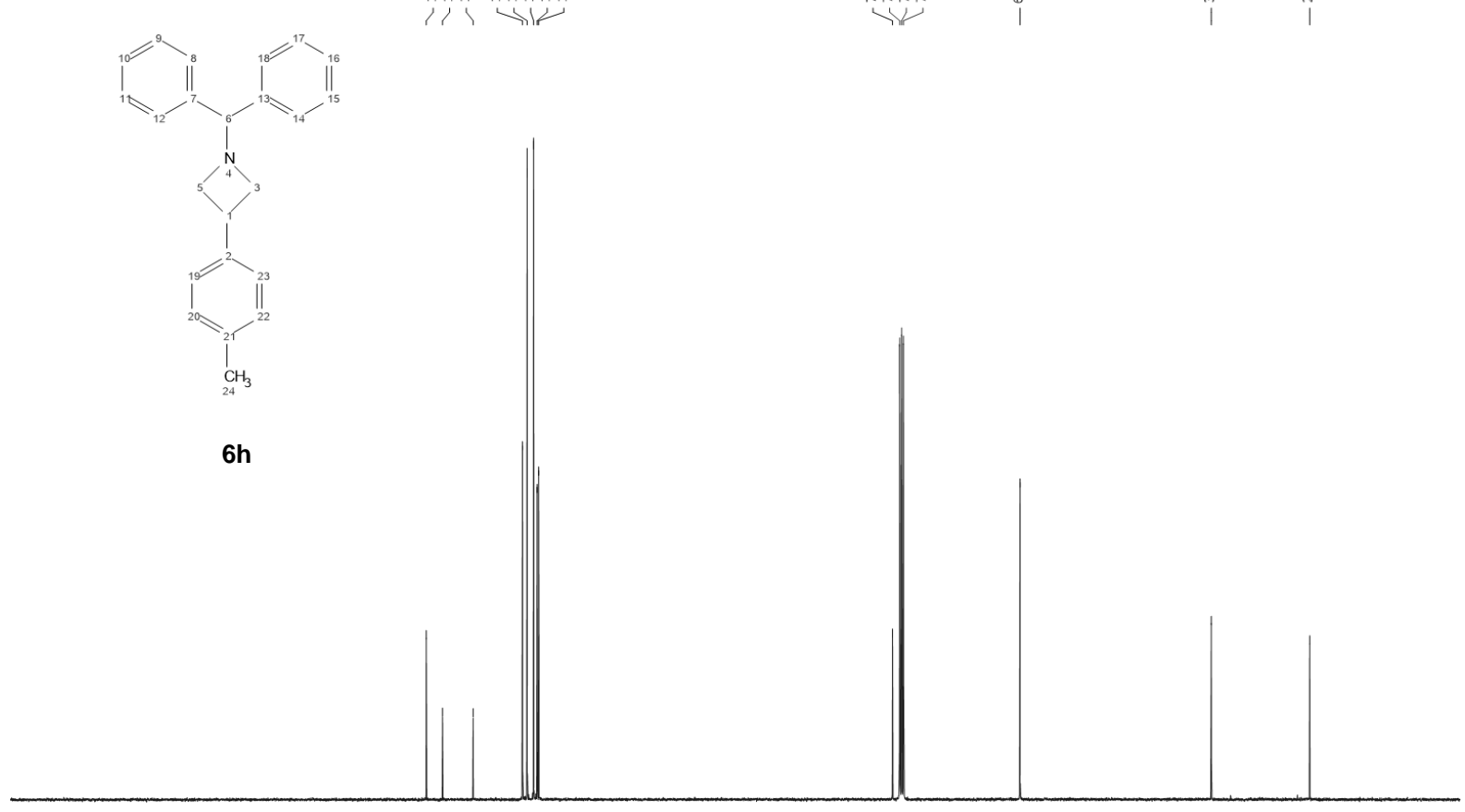

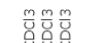

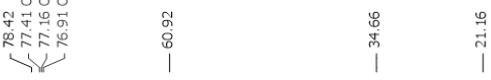

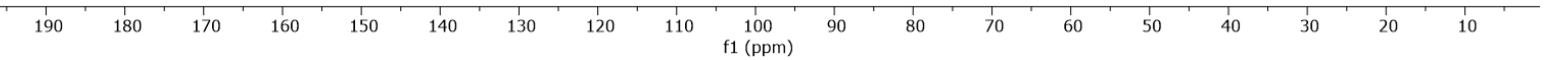


UOXF_groagna_407_1/CdCl3

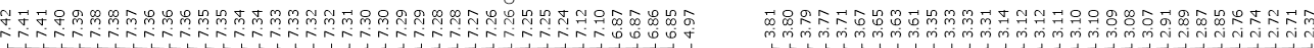

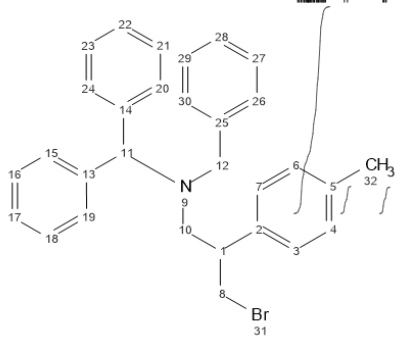

7ha

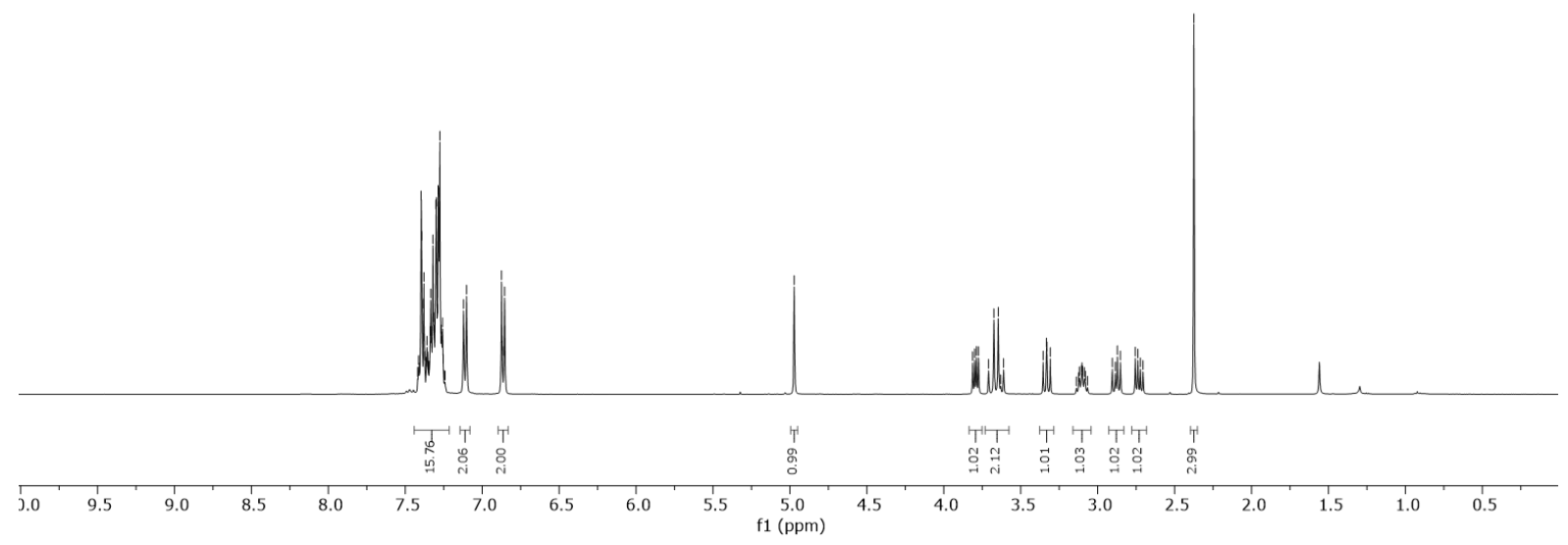

UOXF_groagna_407_1/CdCl3

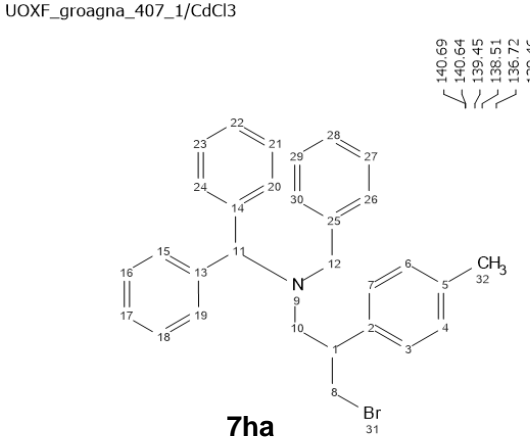

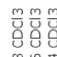

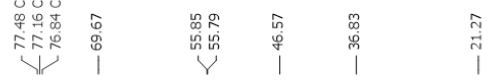

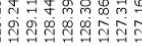

1.1.7.
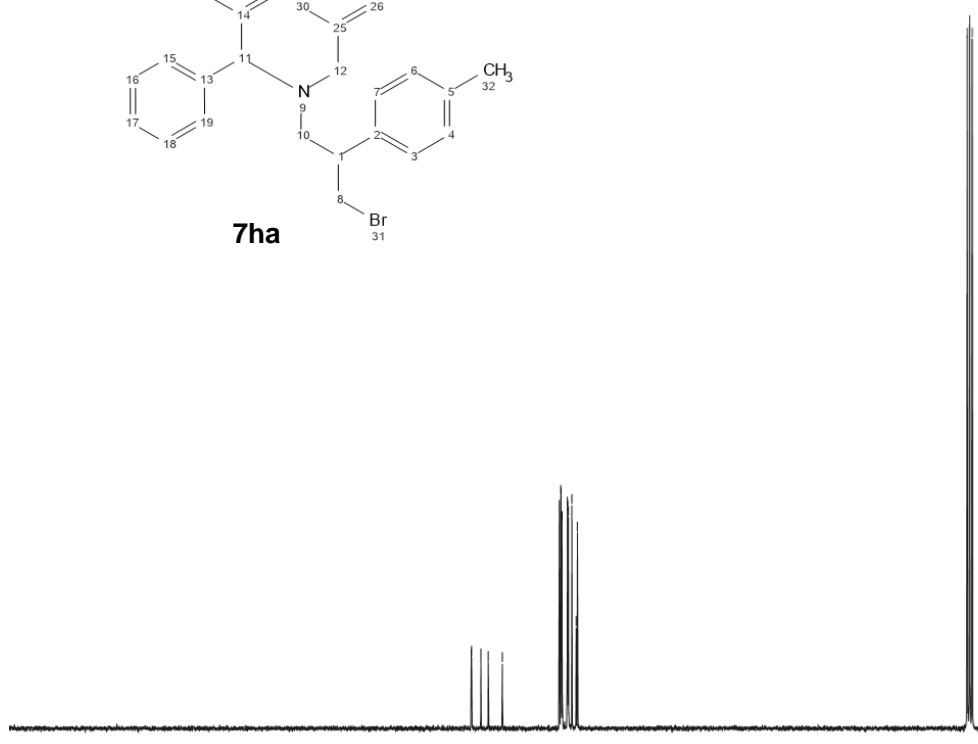

190

$\begin{array}{rrrr}180 & 170 & 160 & 150\end{array}$

$\quad 140 \quad 130$

110

100
$\mathrm{f} 1(\mathrm{ppm})$ 


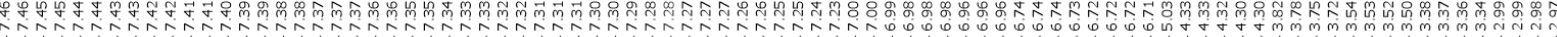<smiles></smiles>

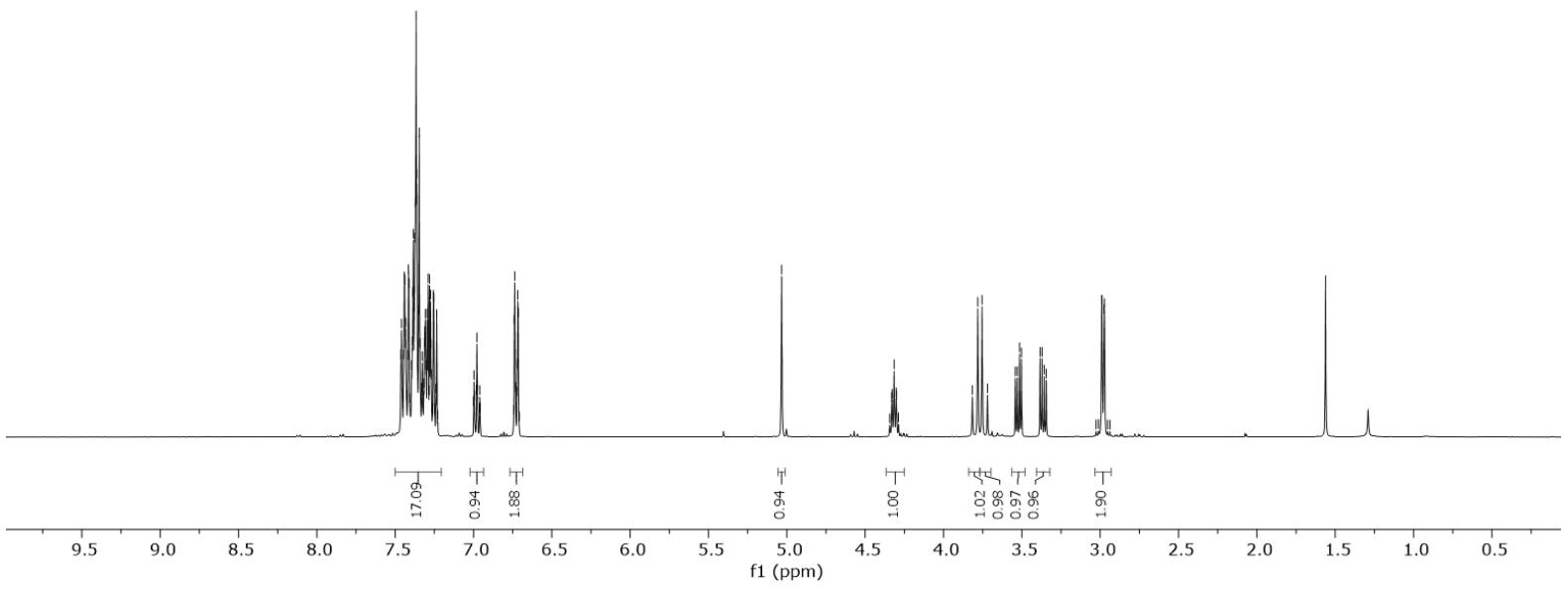

GR03EN56_1/CdCl3

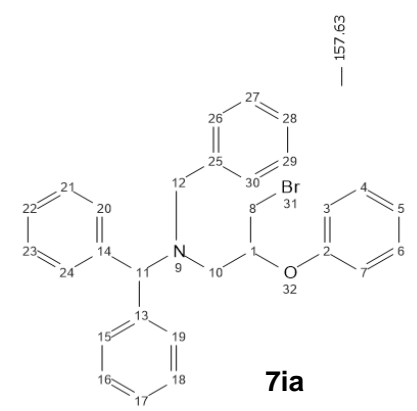

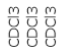

|

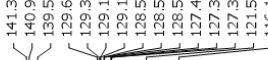

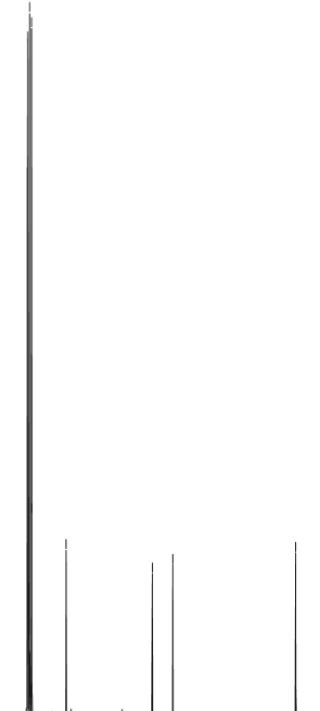

$\begin{array}{lllllllllllll}220 & 210 & 200 & 190 & 180 & 170 & 160 & 150 & 140 & 130 & 120 & 110 & 100 \\ \mathrm{f} 1(\mathrm{pp})\end{array}$ 
<smiles>C=CNN(NC(=O)O)N(NCBr)c1cnnnn1</smiles>
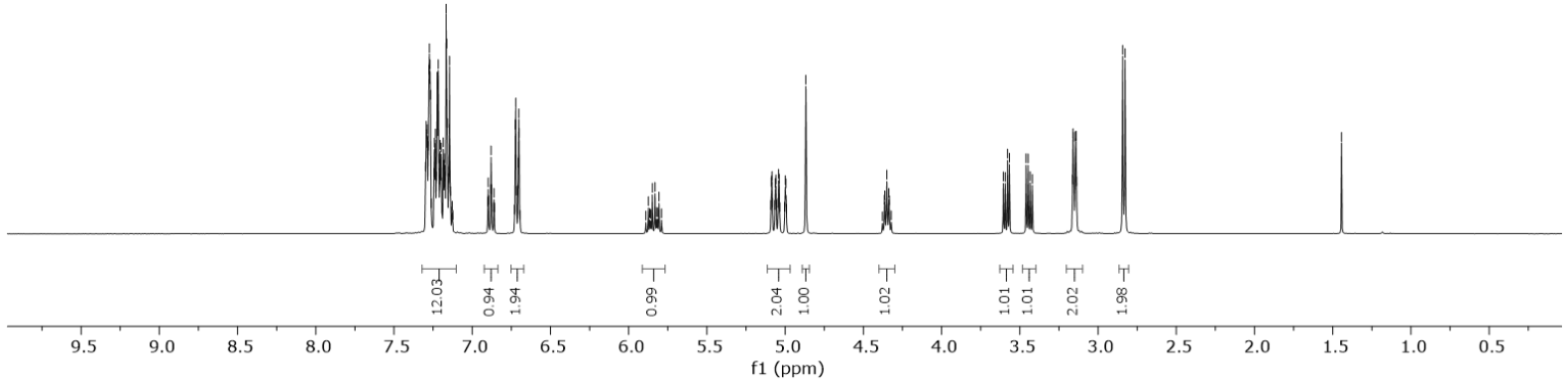

GR03EN77_1/CdCl3

\begin{tabular}{|c|c|c|c|c|}
\hline "ָ. & 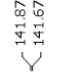 & 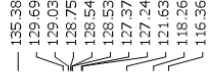 & | & 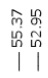 \\
\hline
\end{tabular}

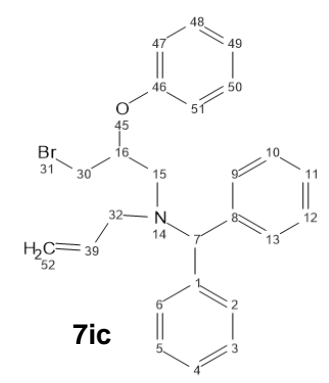

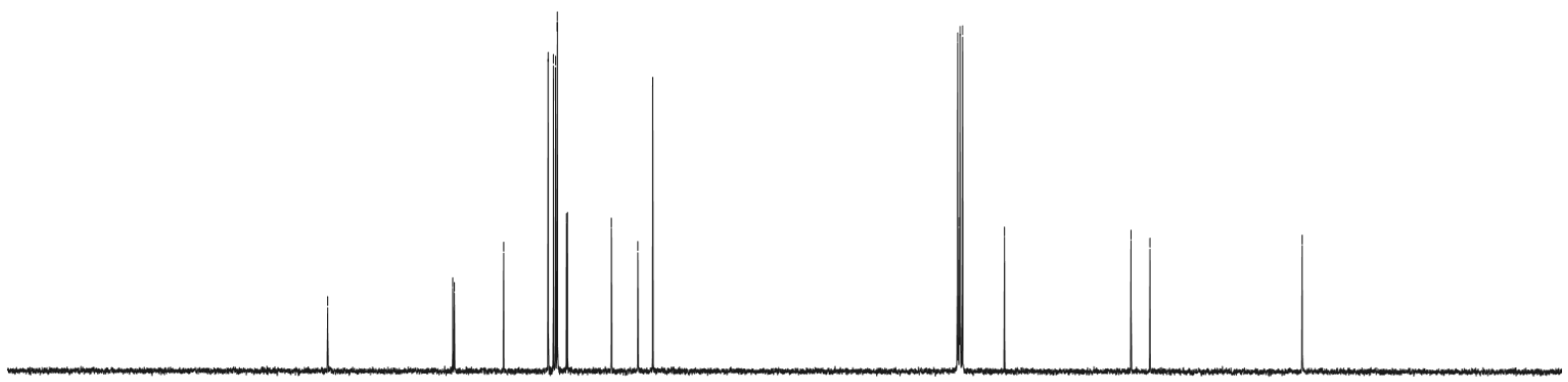

$\begin{array}{lllll}190 & 180 & 170 & 160 & 150\end{array}$

$100 \quad 90$

$\begin{array}{llllllll}1 & 1 & 1 & 1 & 1 & 1 & 1 & 1\end{array}$ 
UOXF_groagna_318_1/CdCl3

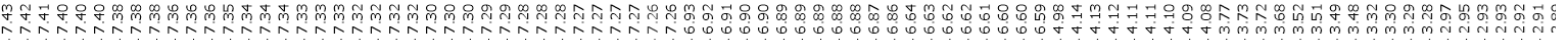

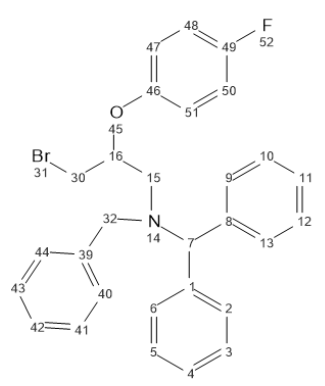

7ja

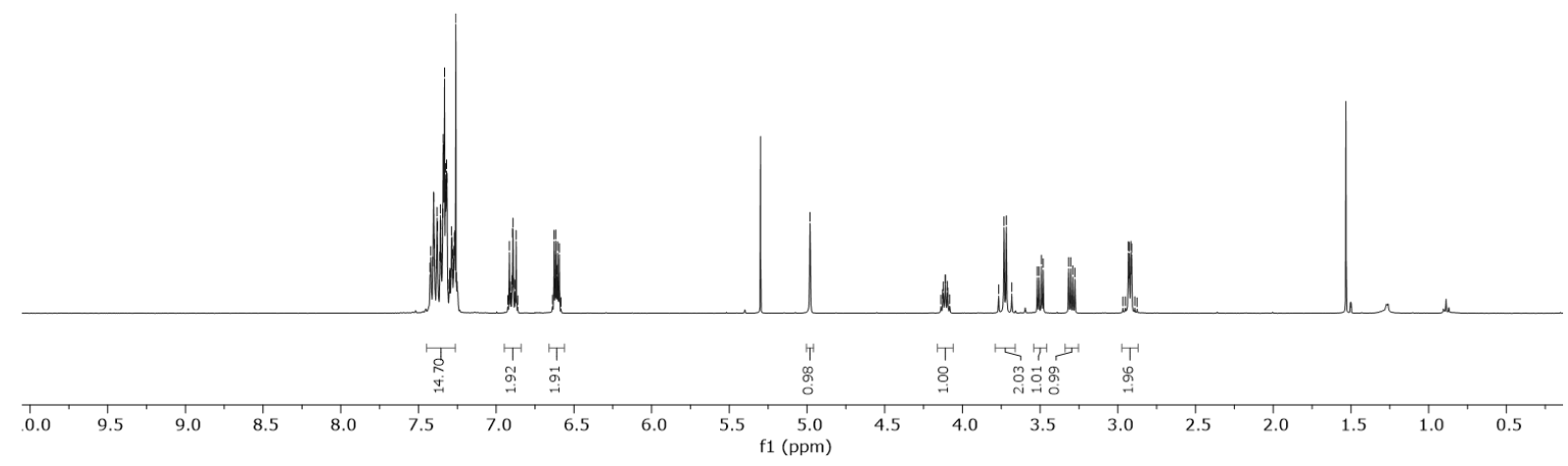

UOXF_groagna_318_1/CdCl3

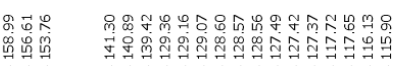

111 年

\section{$\frac{m}{\mathrm{C}} \frac{m}{\mathrm{C}} \frac{m}{\mathrm{u}}$
$8 \mathrm{0}$}

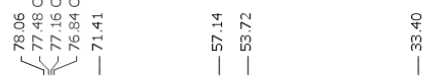<smiles>Fc1ccc(OP(CBr)CN(Cc2ccncn2)N(Cc2cccnc2)P(c2cccnc2)c2ccncn2)cc1</smiles>

7ja

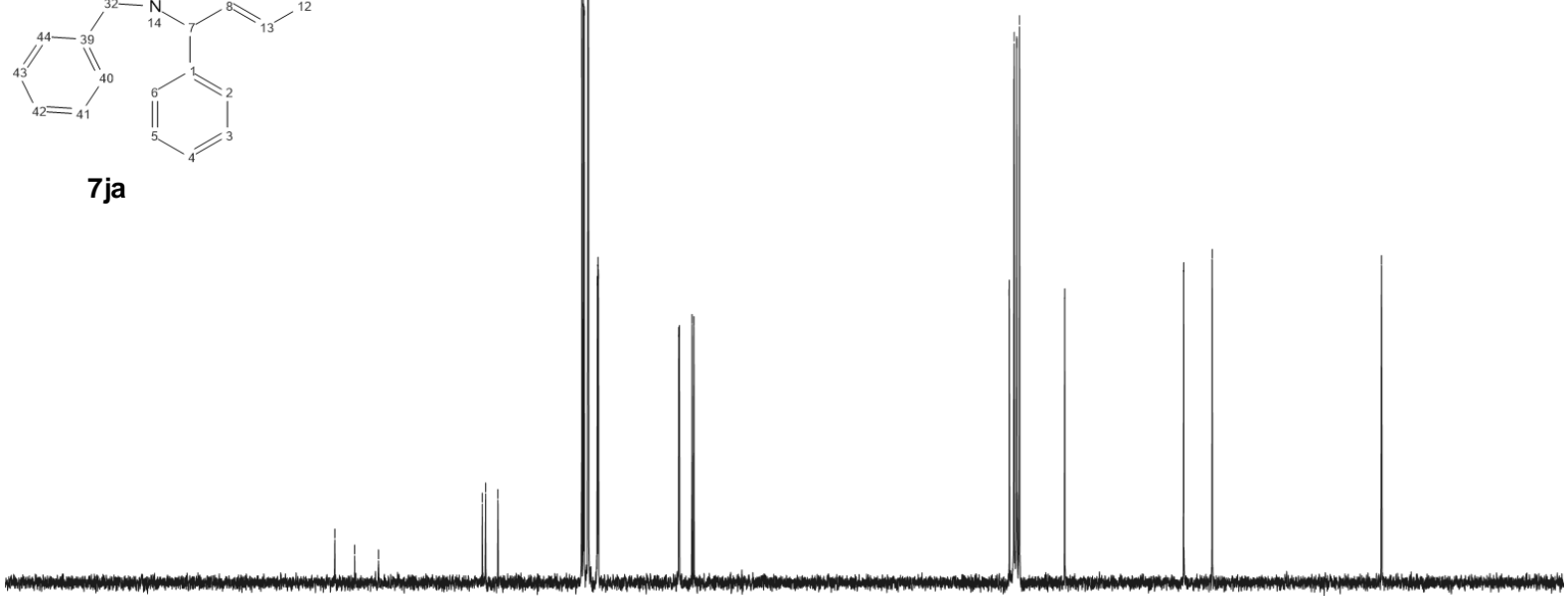

$190 \quad 180 \quad 170 \quad 160$ $150 \quad 140$ 130 $120 \quad 110 \quad 100$
$\mathrm{f} 1(\mathrm{ppm})$ 
UOXF_groagna_318_1/CdCl3<smiles>Fc1cnc(OC(CBr)CN(Cn2cccn2)C(c2nncnn2)c2nnnnn2)cn1</smiles>

7ja

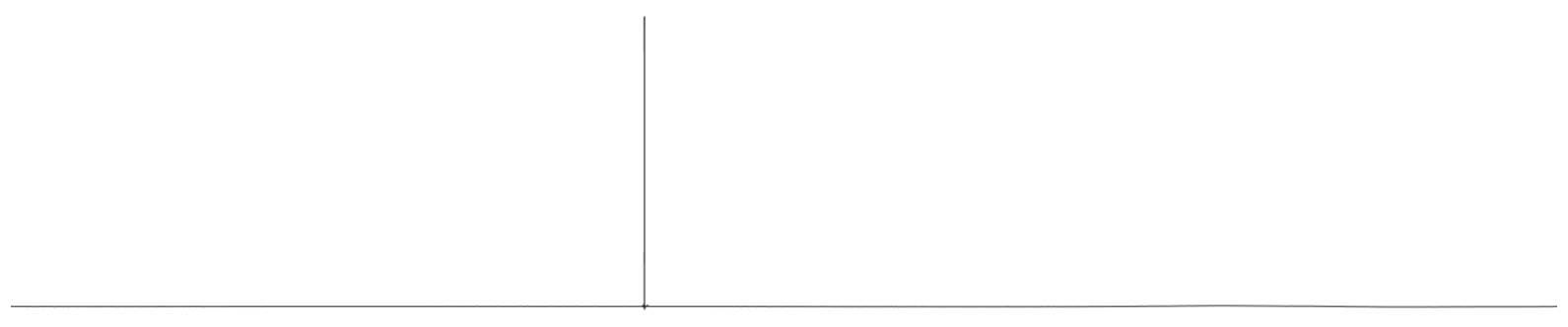

\begin{tabular}{lllllllllllllllllllllllllllll}
1 & 10 & 10 & 0 & -10 & -20 & -30 & -40 & -50 & -60 & -70 & -80 & -90 & -100 & -110 & -120 & -130 & -140 & -150 & -160 & -170 & -180 & -190 & -200 & -210 & -2 \\
\hline
\end{tabular} 
UOXF_groagna_258_1/CdCl3

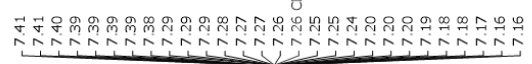

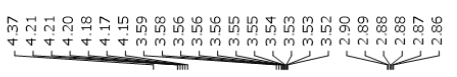
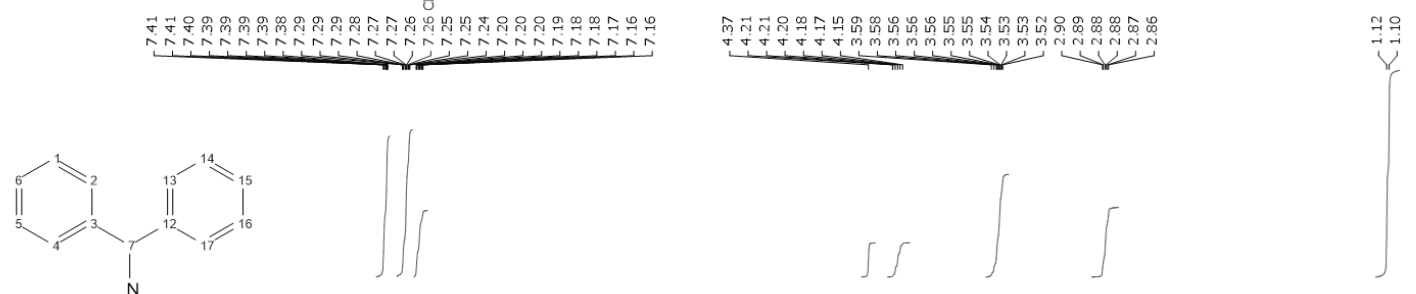

$$
\iiint \int
$$
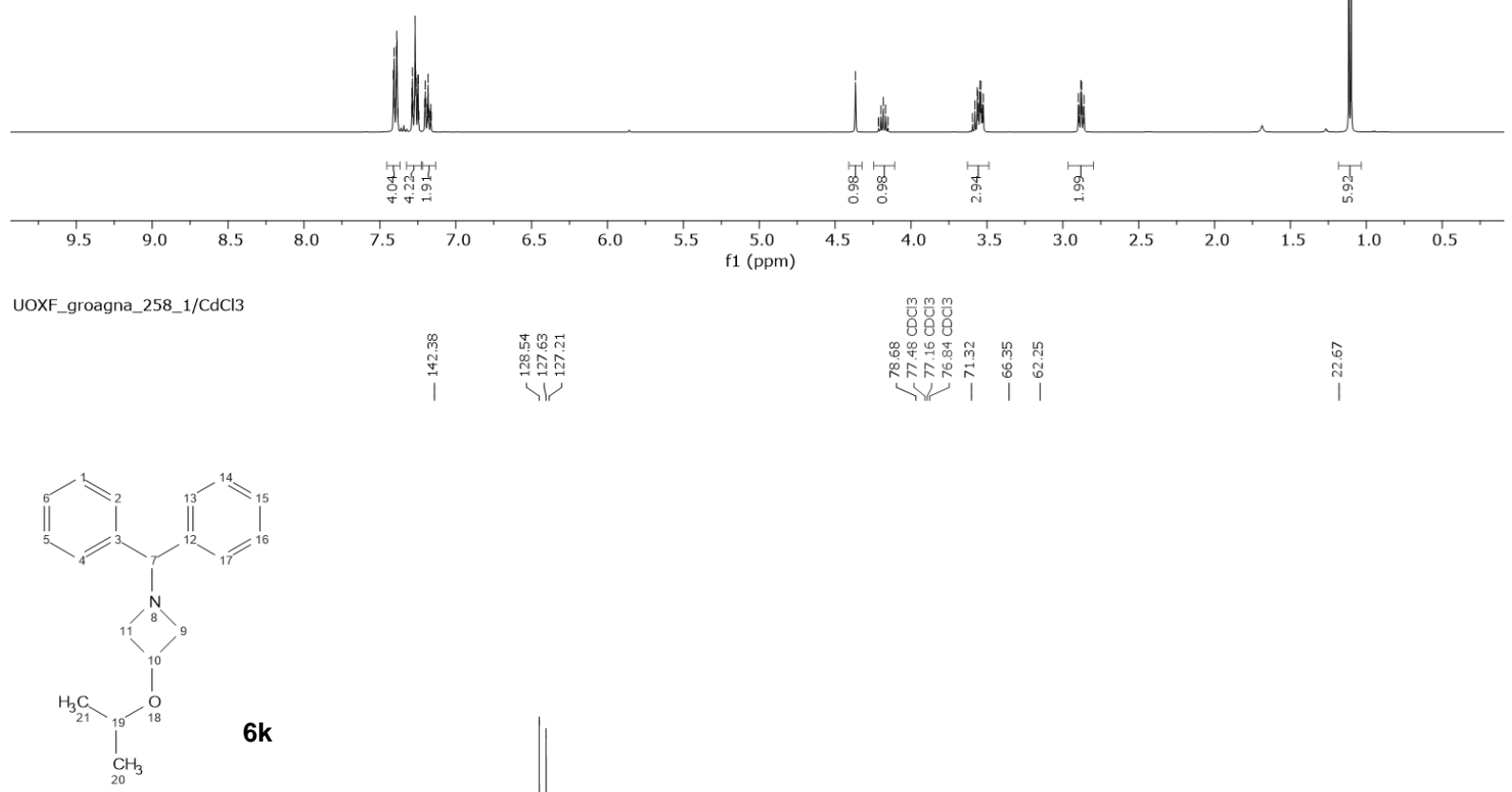

S168 

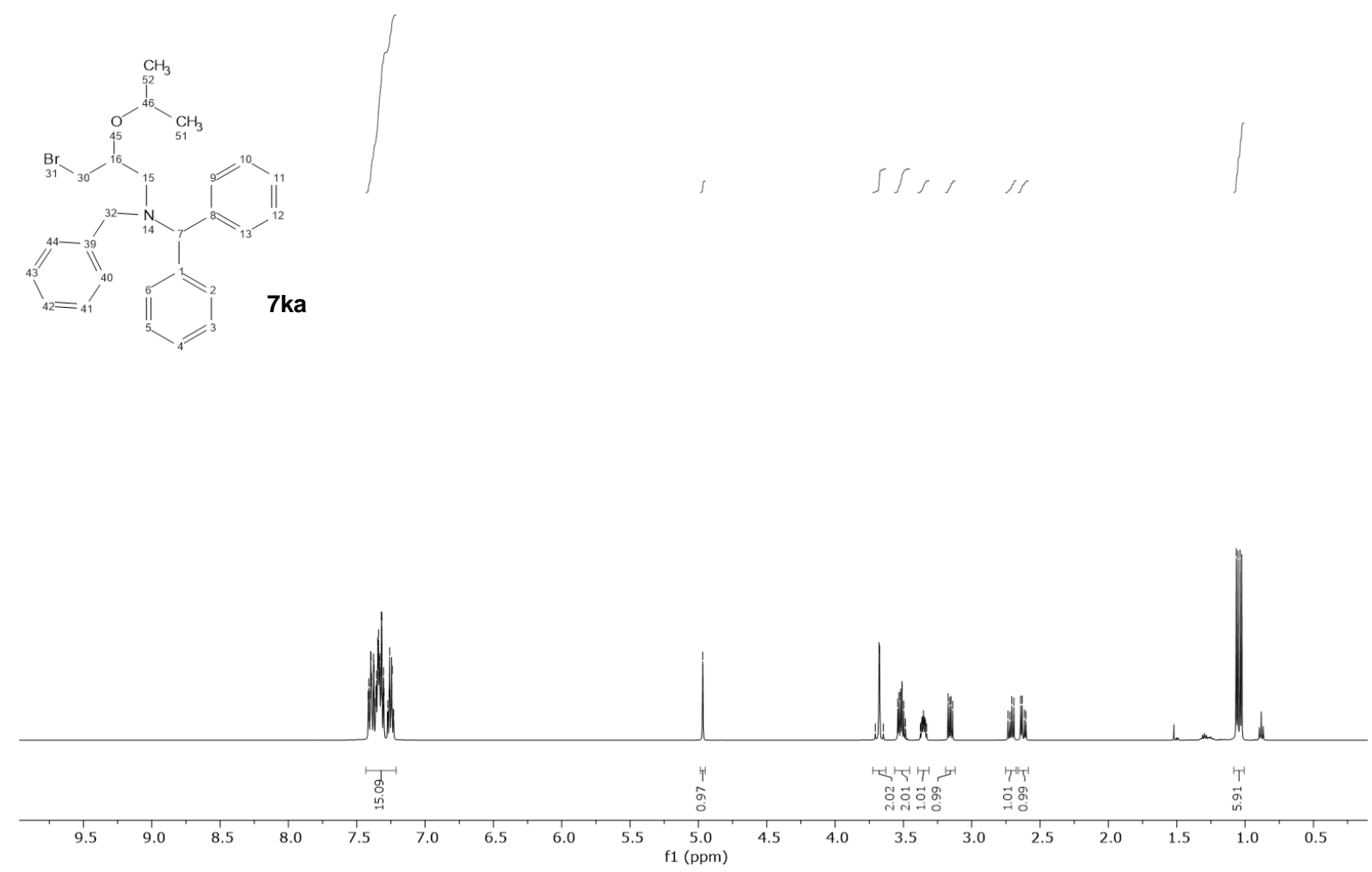

UOXF_groagna_394_1/CdCl3
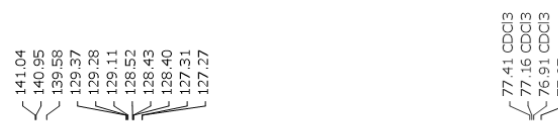

|

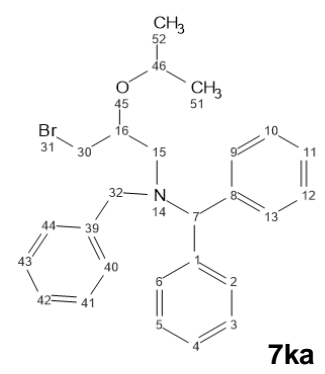

$7 \mathrm{ka}$

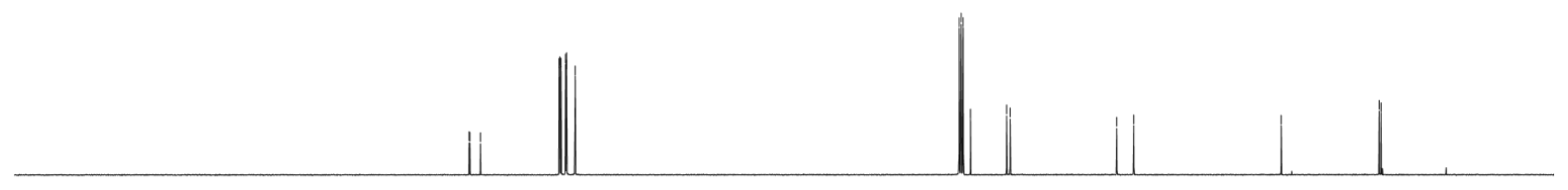

\begin{tabular}{|llllllllllllllllllll}
\hline 00 & 190 & 180 & 170 & 160 & 150 & 140 & 130 & 120 & 110 & 100 & 90 & 80 & 70 & 60 & 50 & 40 & 30 & 20 & 10
\end{tabular} 


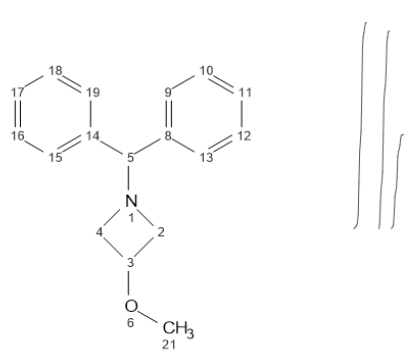

61

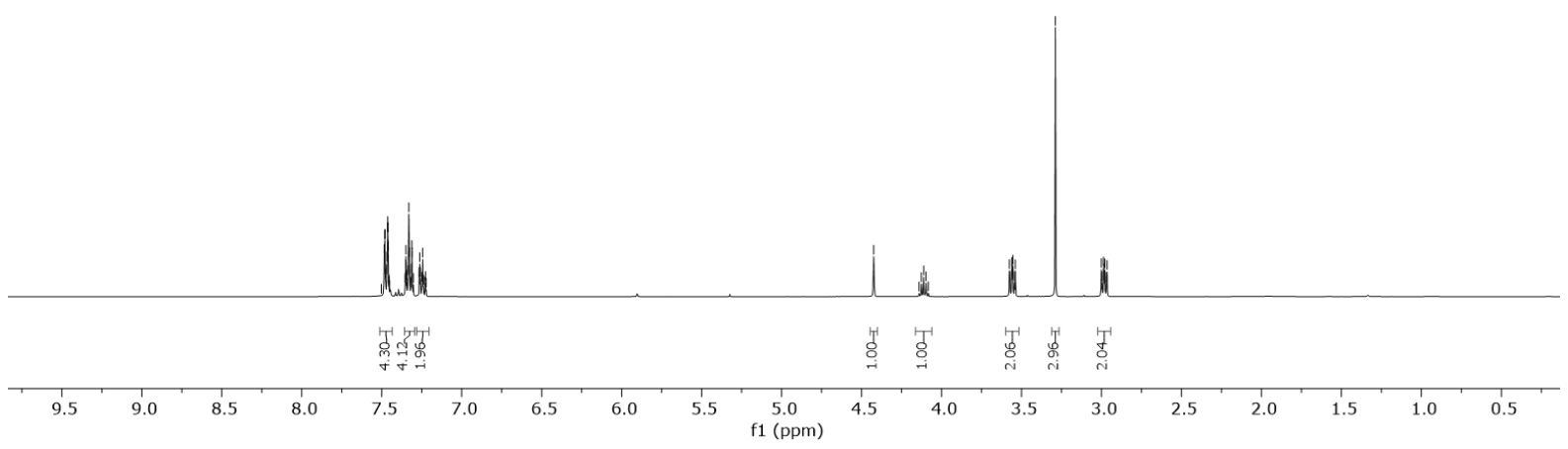

UOXF_groagna_257_1/CdCl3

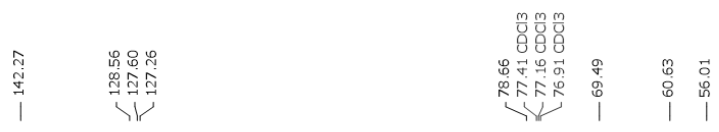

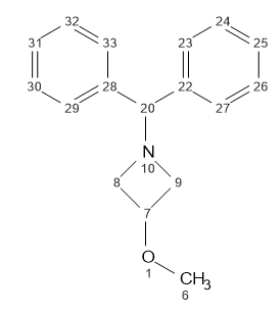

6l

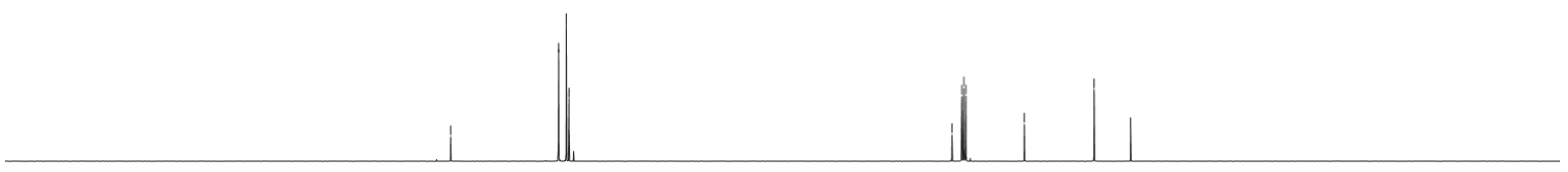

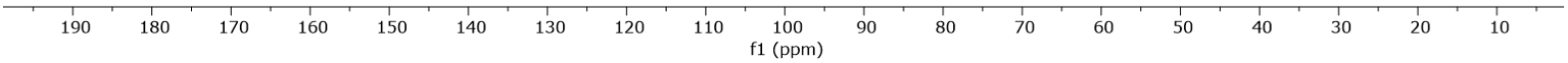



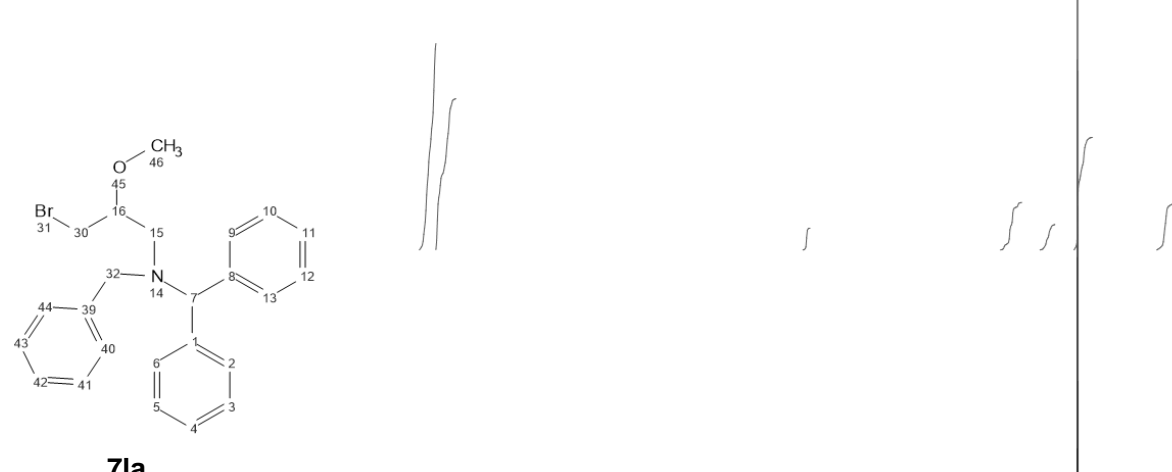

7la

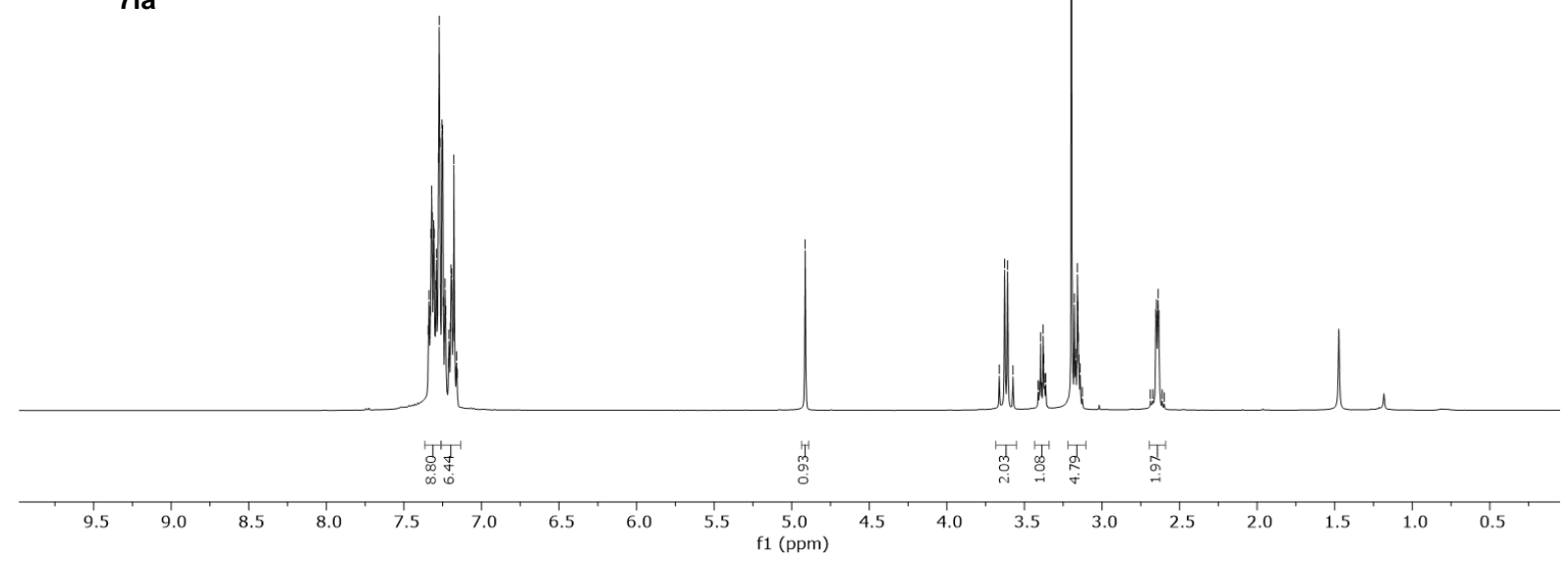

UOXF_groagna_335_1/CdCl3

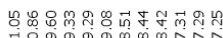

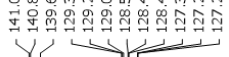

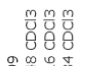

|
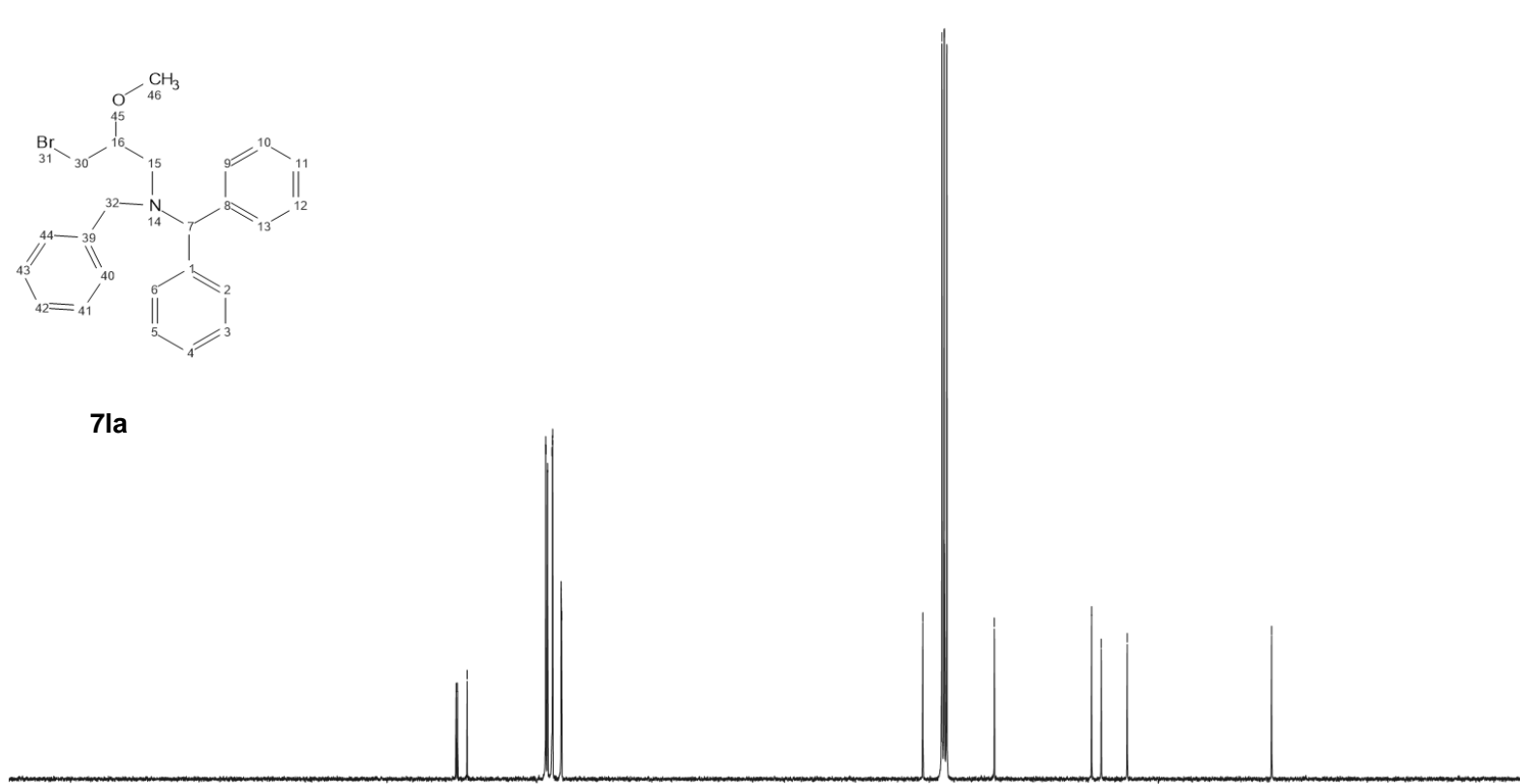

7la

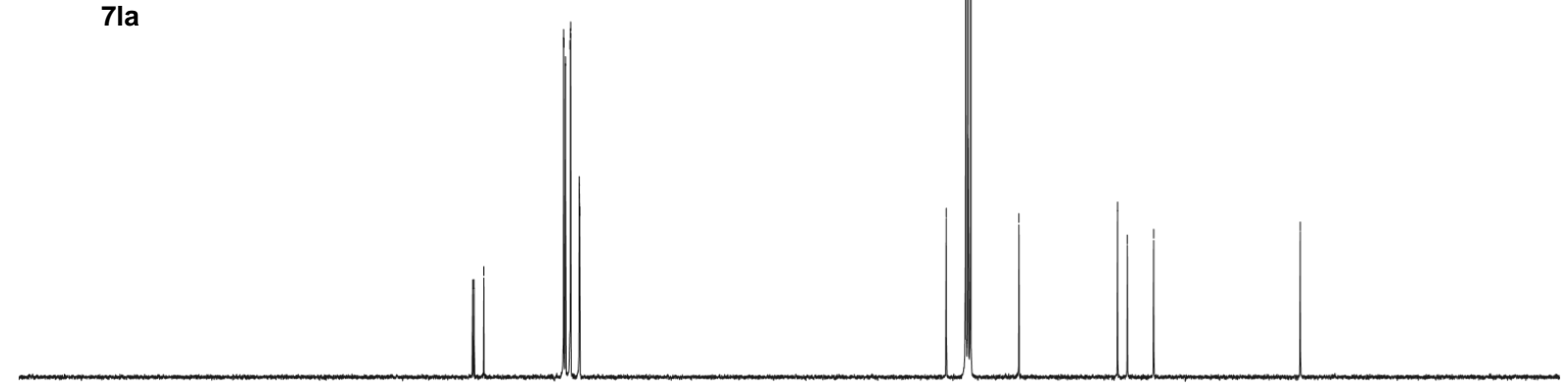

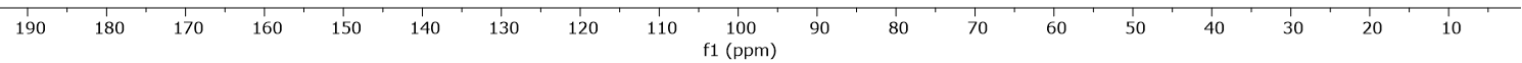




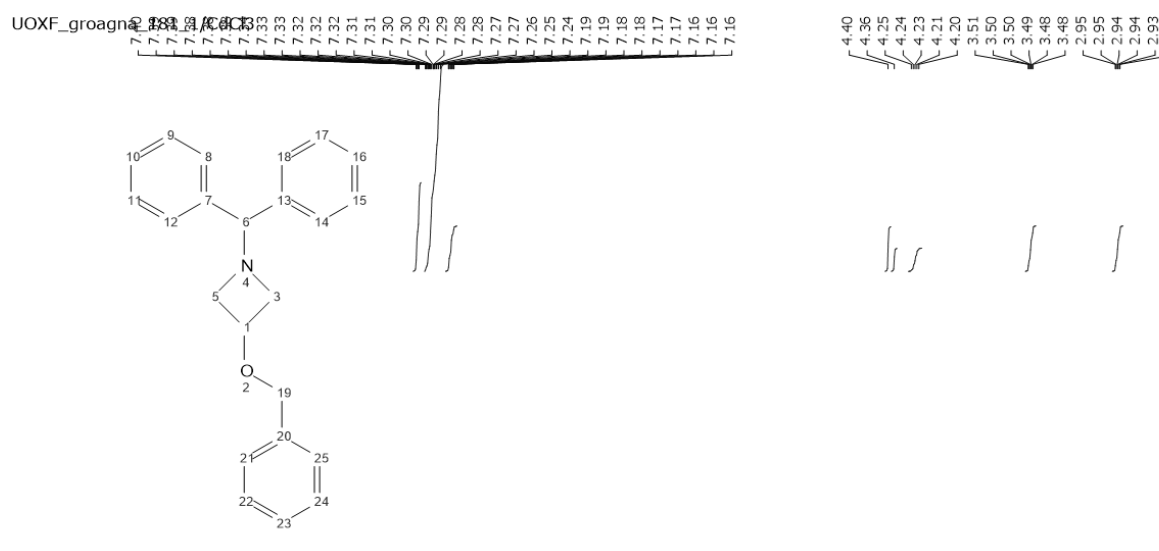

$6 m$

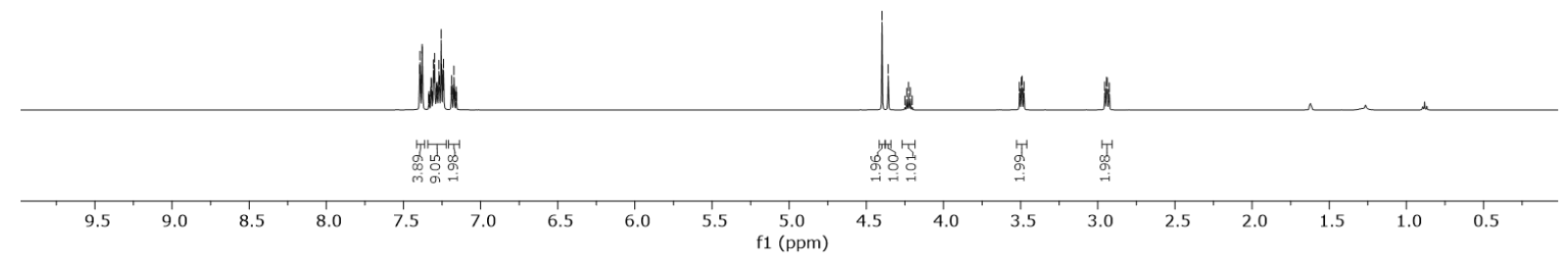

UOXF_groagna_181_1/CdCl3

\begin{tabular}{|c|c|c|}
\hline 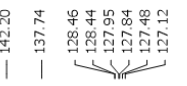 & $\stackrel{\substack{n+\\
\infty}}{\stackrel{\infty}{\mid}}$ & $\begin{array}{lll}8 & 0 \\
0 & 0 \\
2 & 0 \\
1 & 1\end{array}$ \\
\hline
\end{tabular}

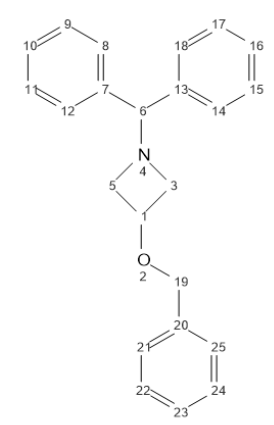

$6 m$

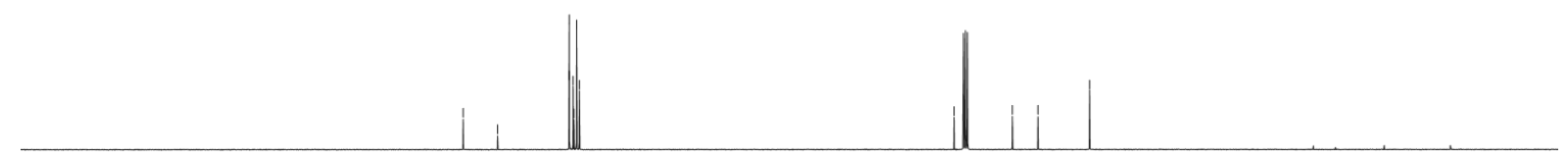

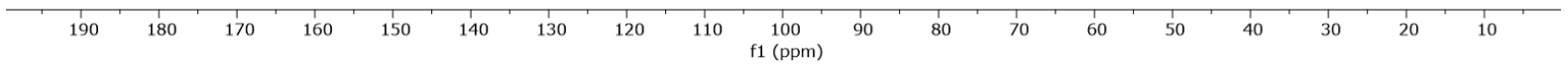




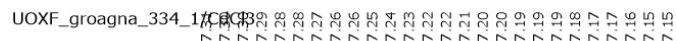

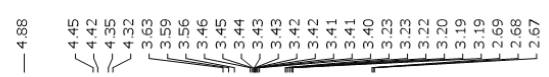

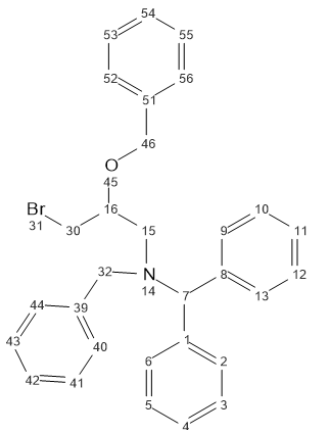

$\int$

$7 m a$

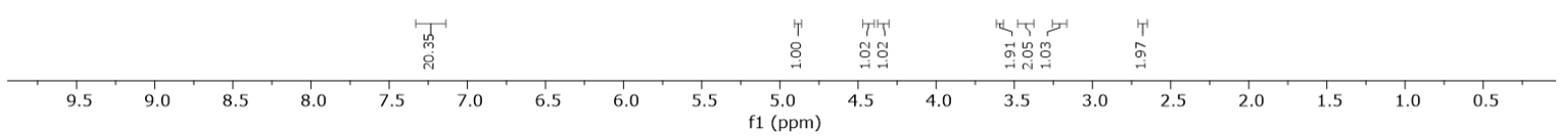

UOXF_groagna_334_1/CdCl3

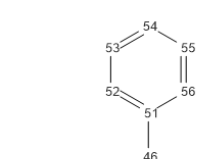

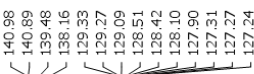

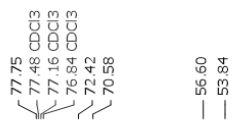

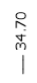

(1)

7ma
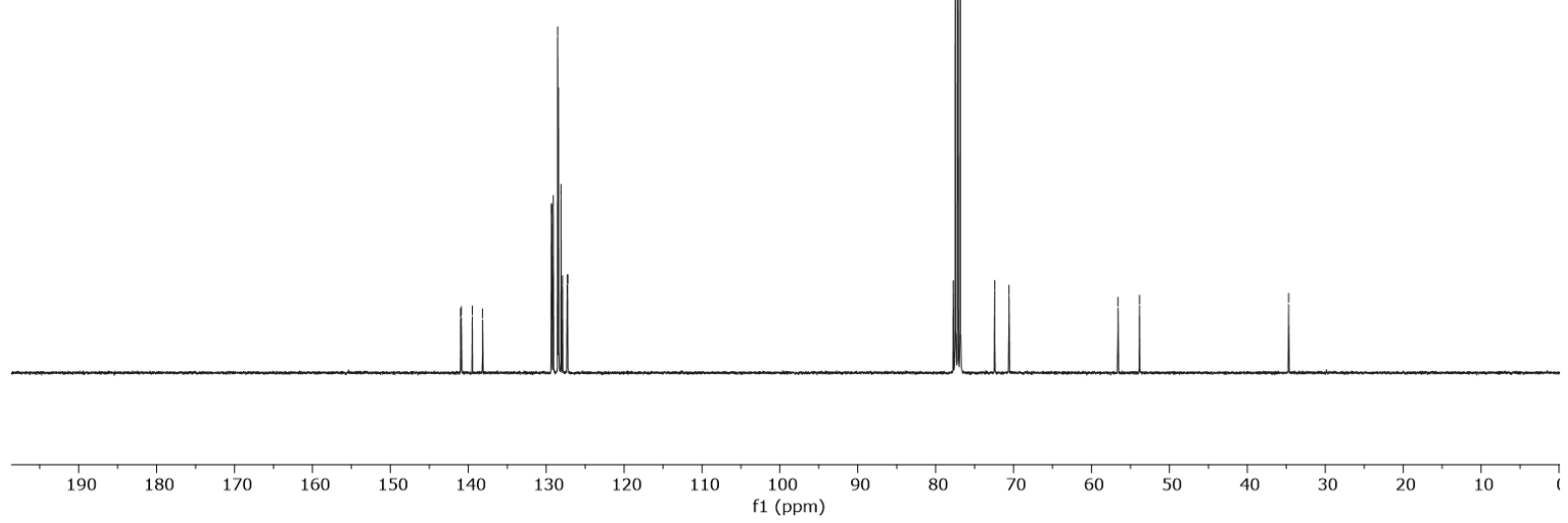

S173 


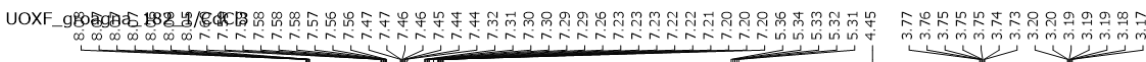

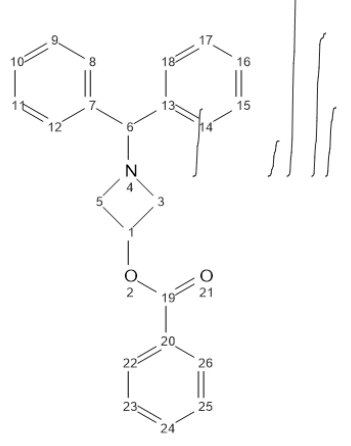

$6 n$

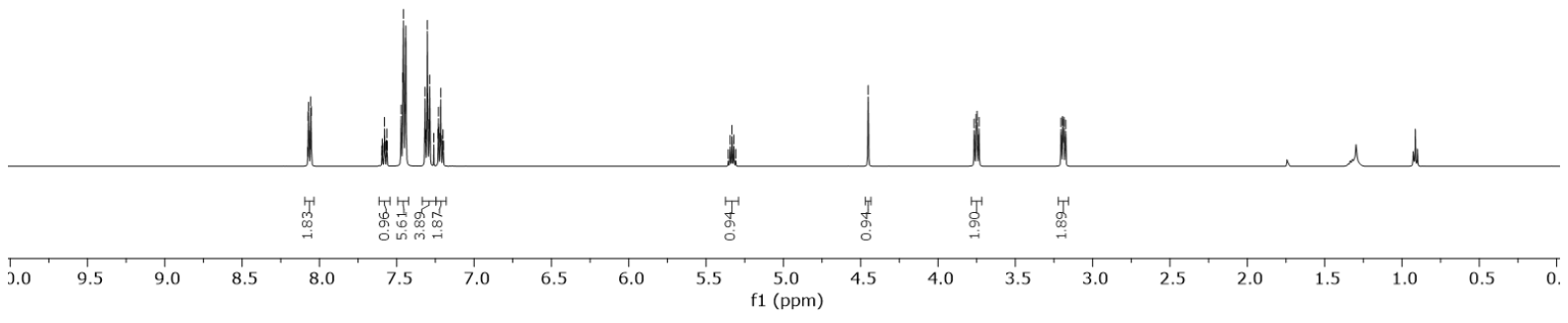

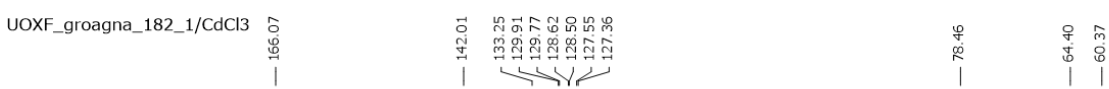

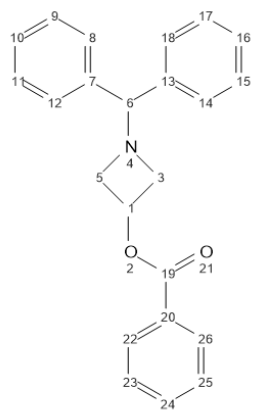

$6 n$

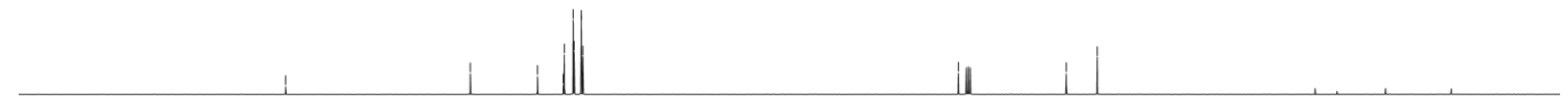

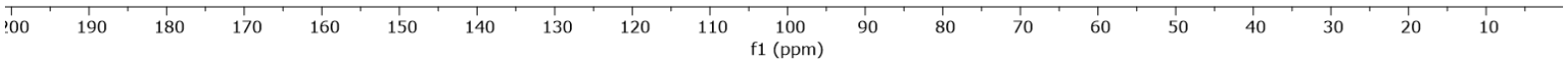




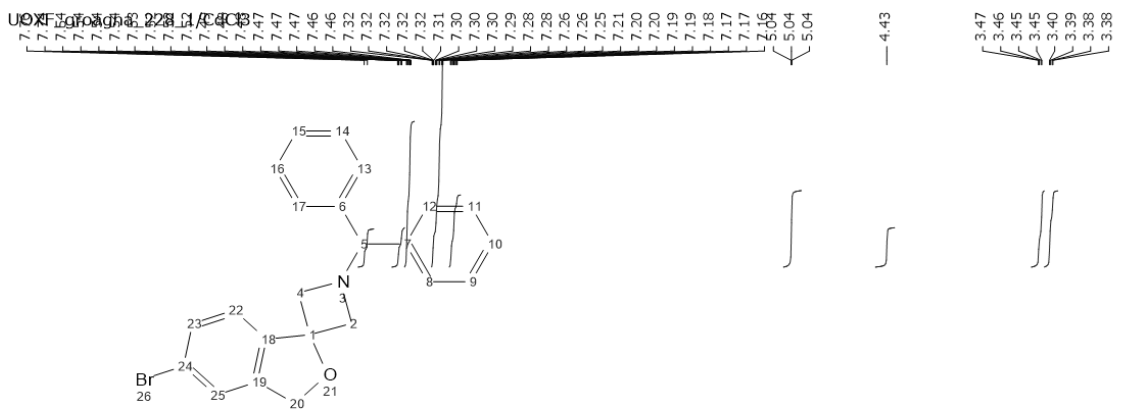

60
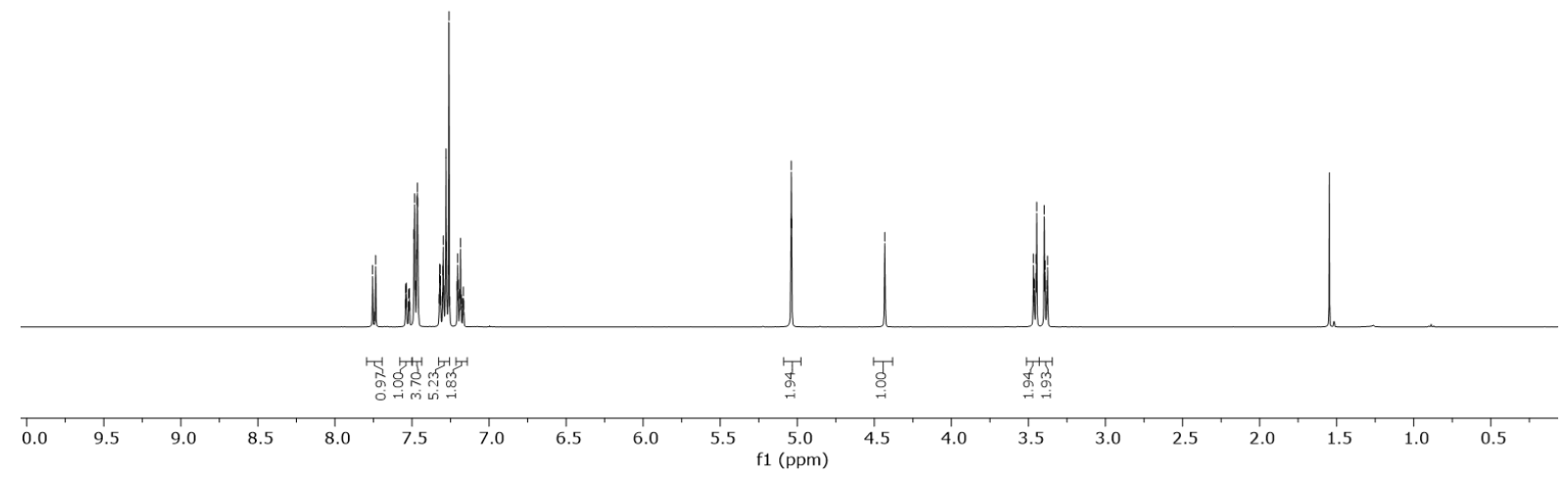

UOXF_groagna_228_1/CdCl3

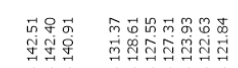

V) रो人।

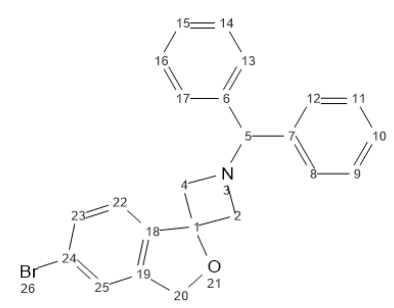

60

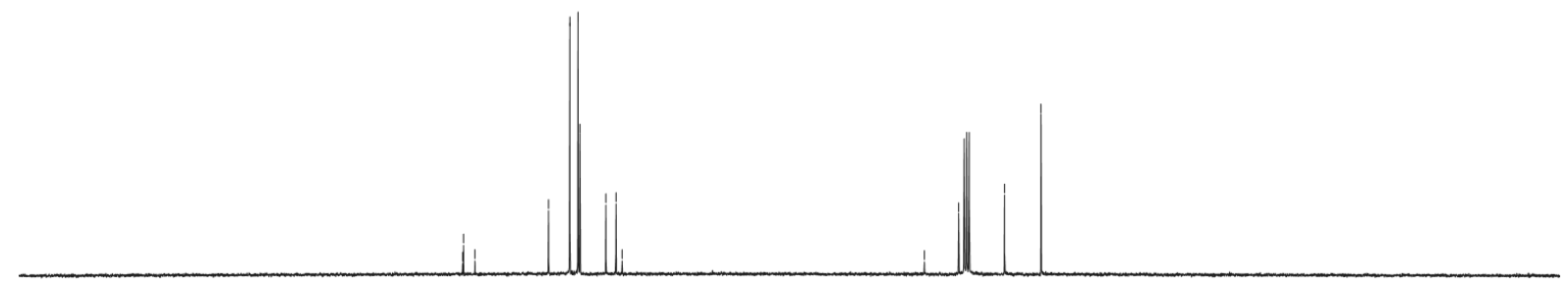

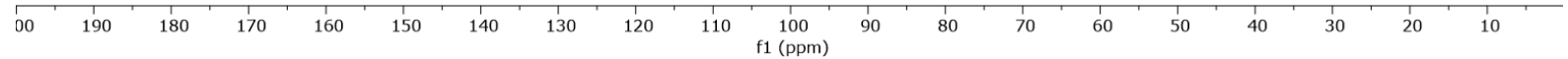


UOXF_groagna_222_1/CdCl3

$\frac{m_{0}^{2}}{8}$

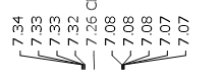

$\underbrace{\vec{m}}$

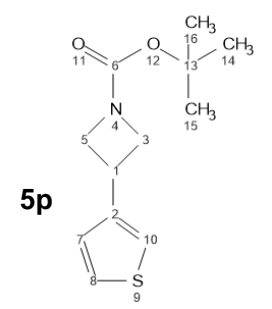

j

$\iint s$
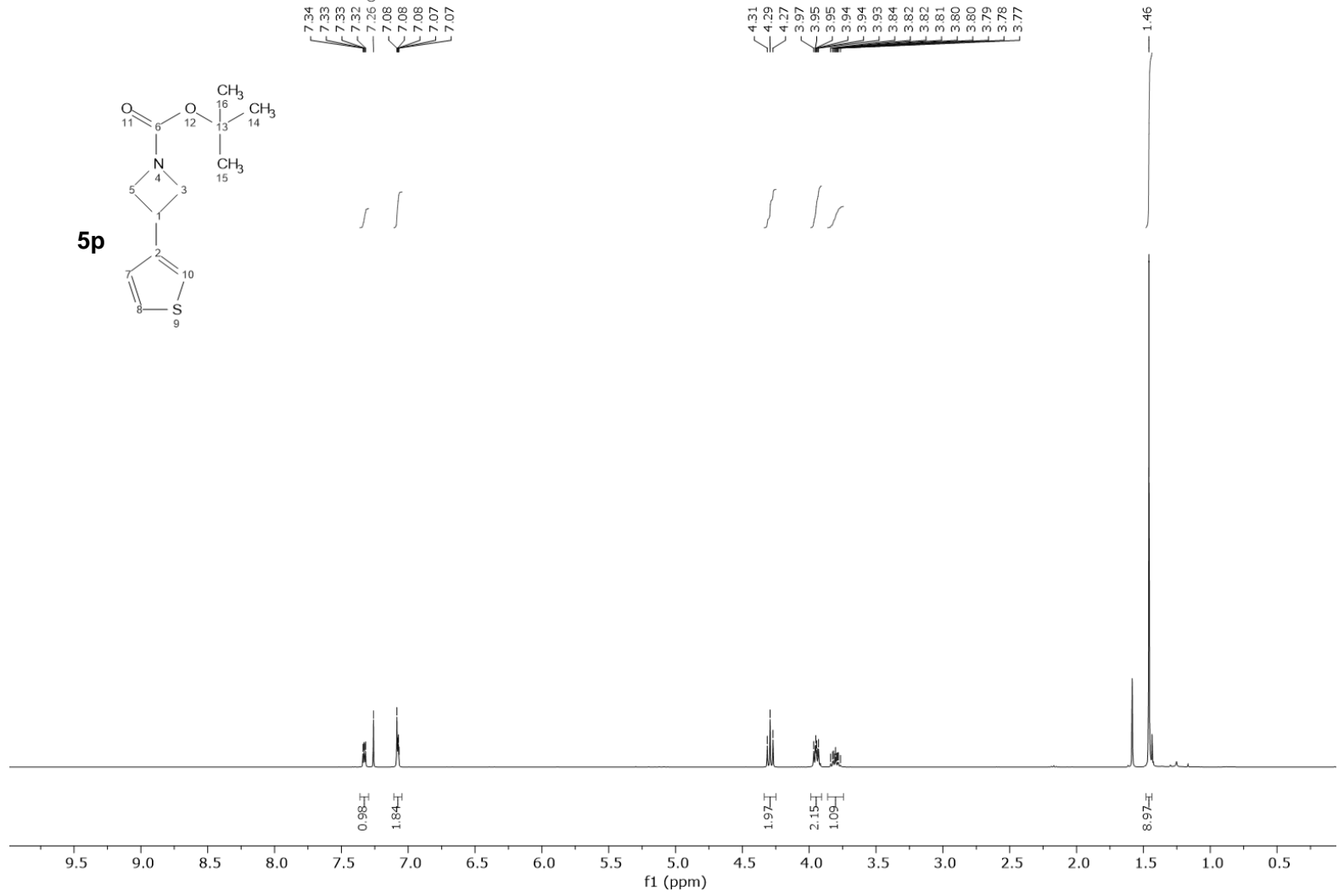

UOXF_groagna_222_1/CdCl3

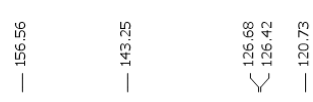
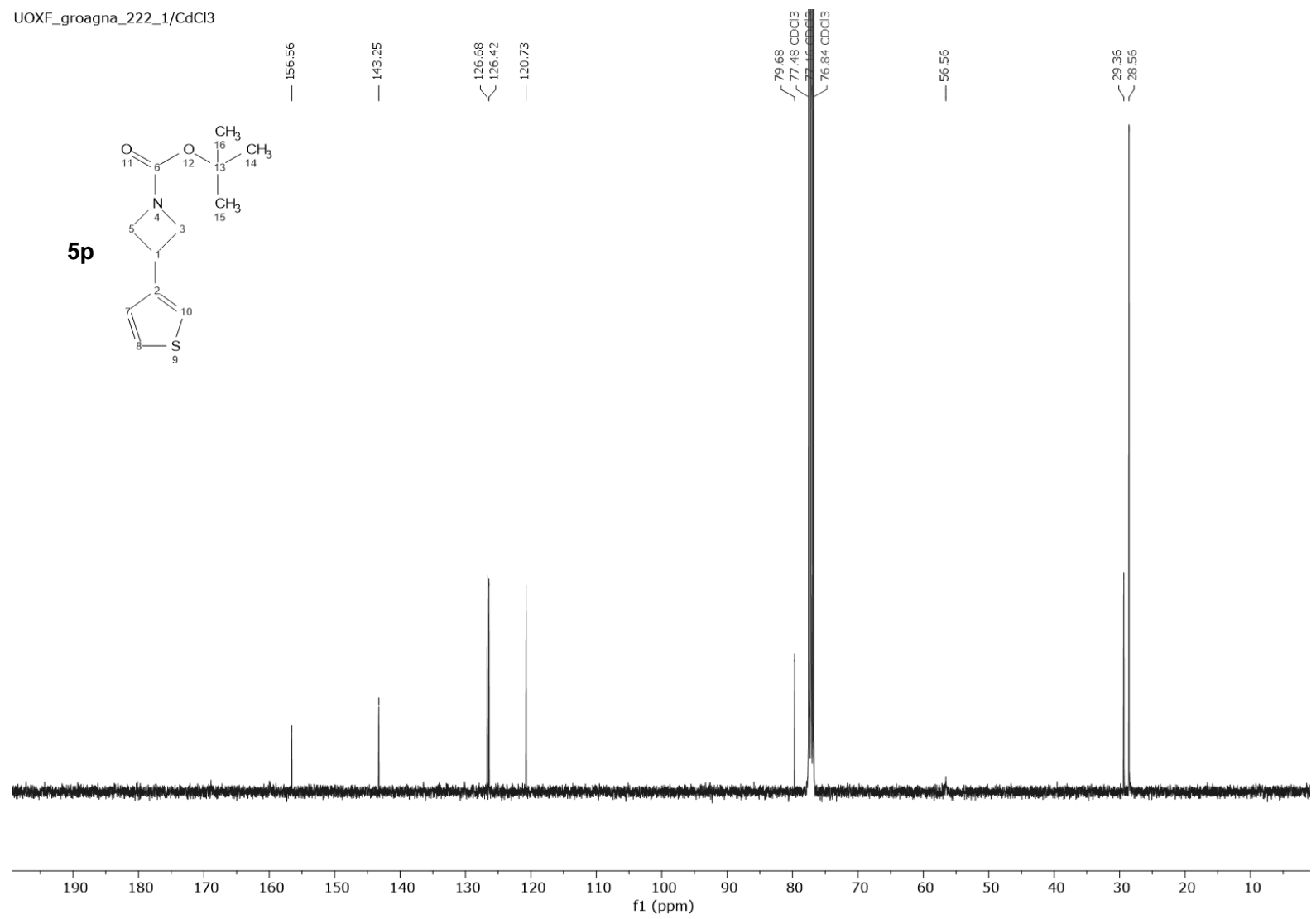

S176 
UOXF_groagna_245_1/CdCl3

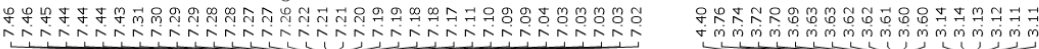
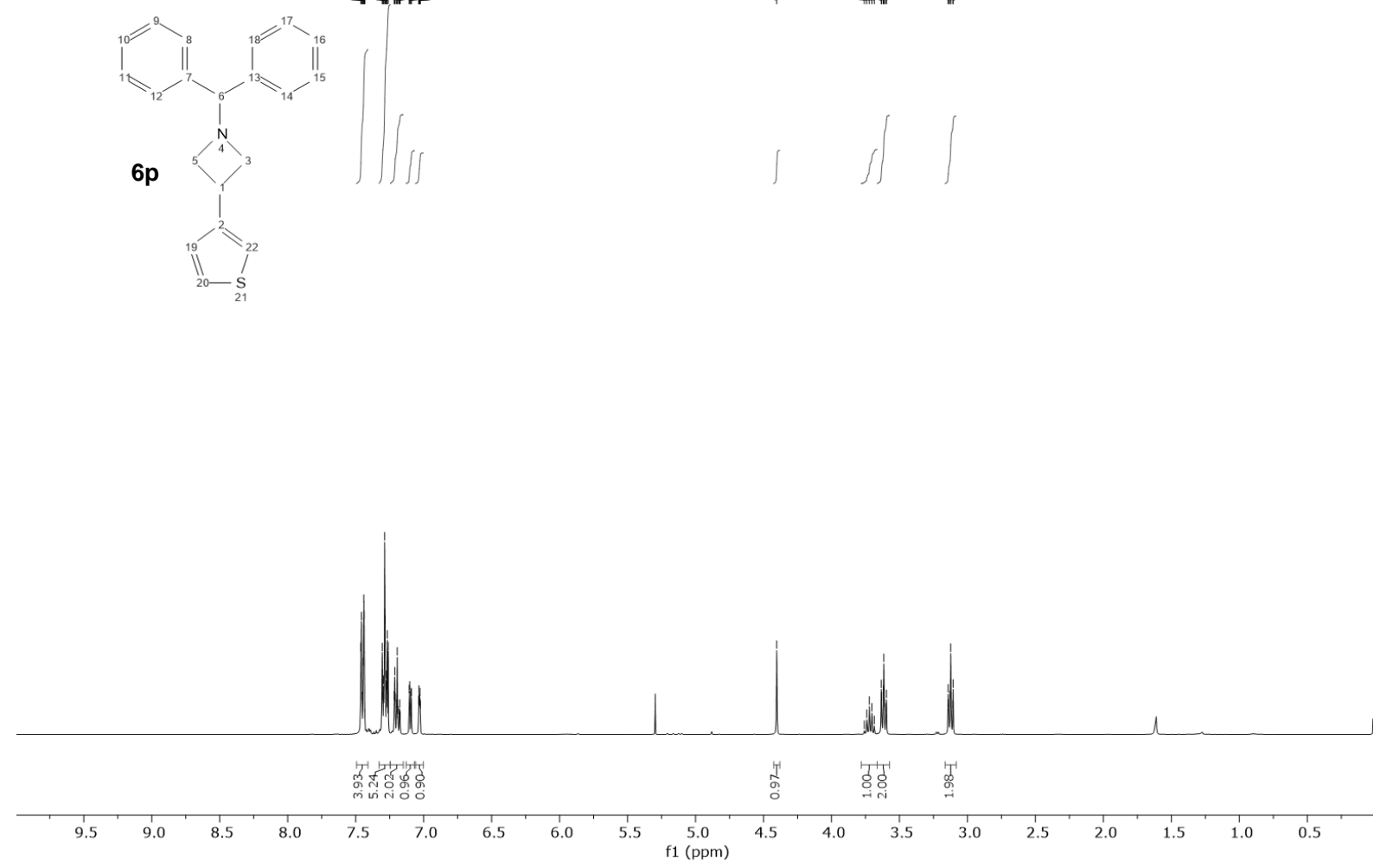

UOXF_groagna_245_1/CdCl3
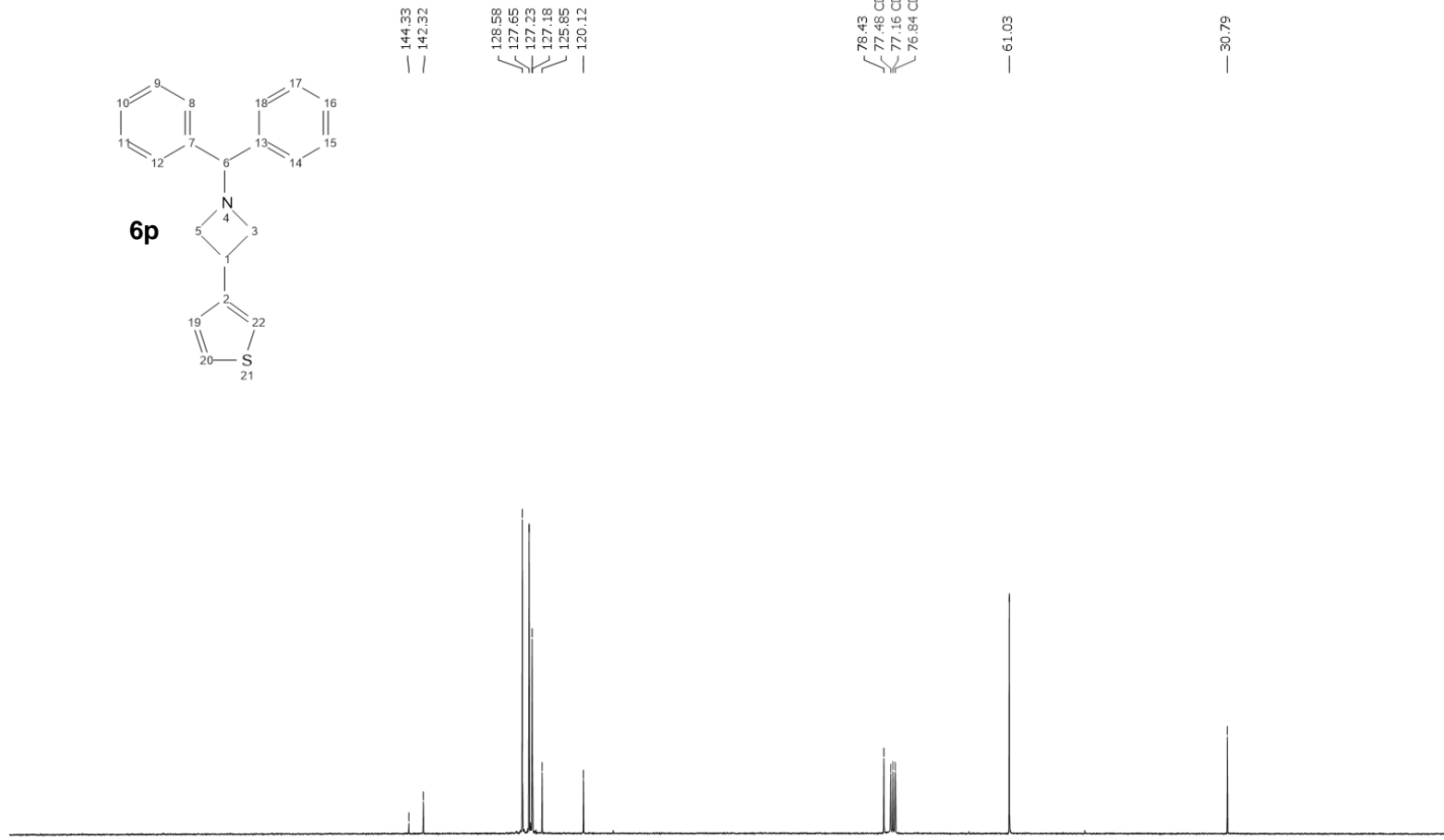

100

1
$80 \quad 70$

60,50

$\begin{array}{lll}1 & 1 & 1 \\ 30 & 20 & 10\end{array}$ 
UOXF_groagna_338_1/CdCl3

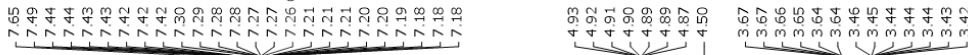

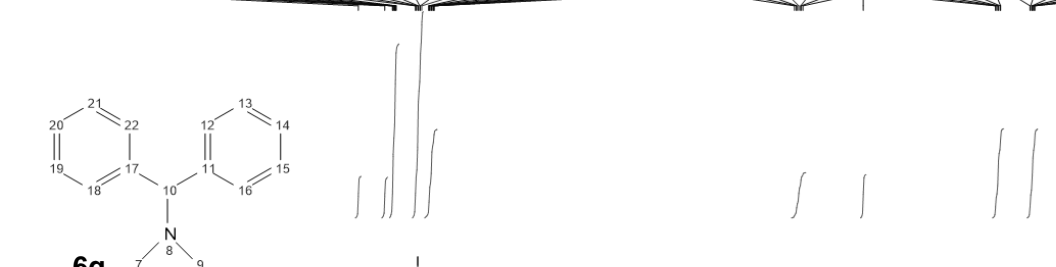

$6 q$

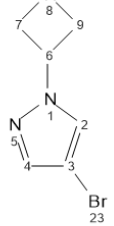

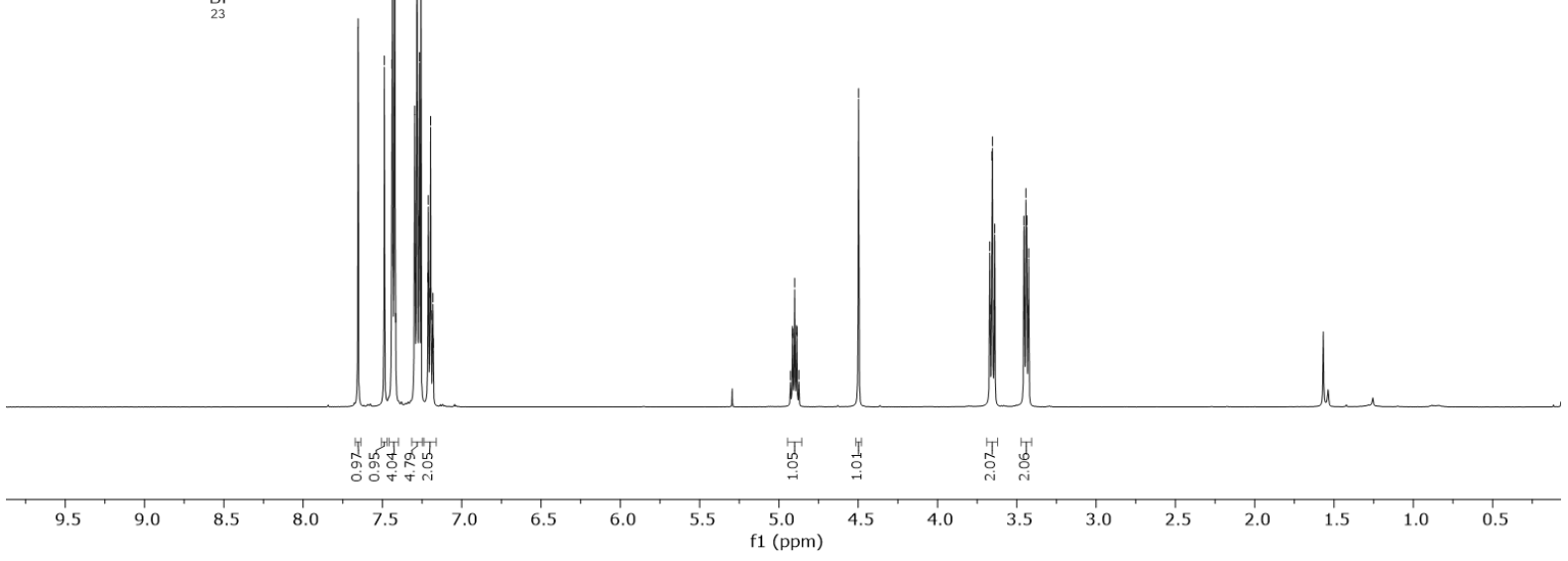

UOXF_groagna_338_1/CdCl3

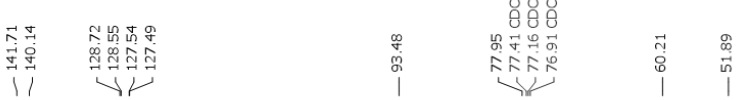
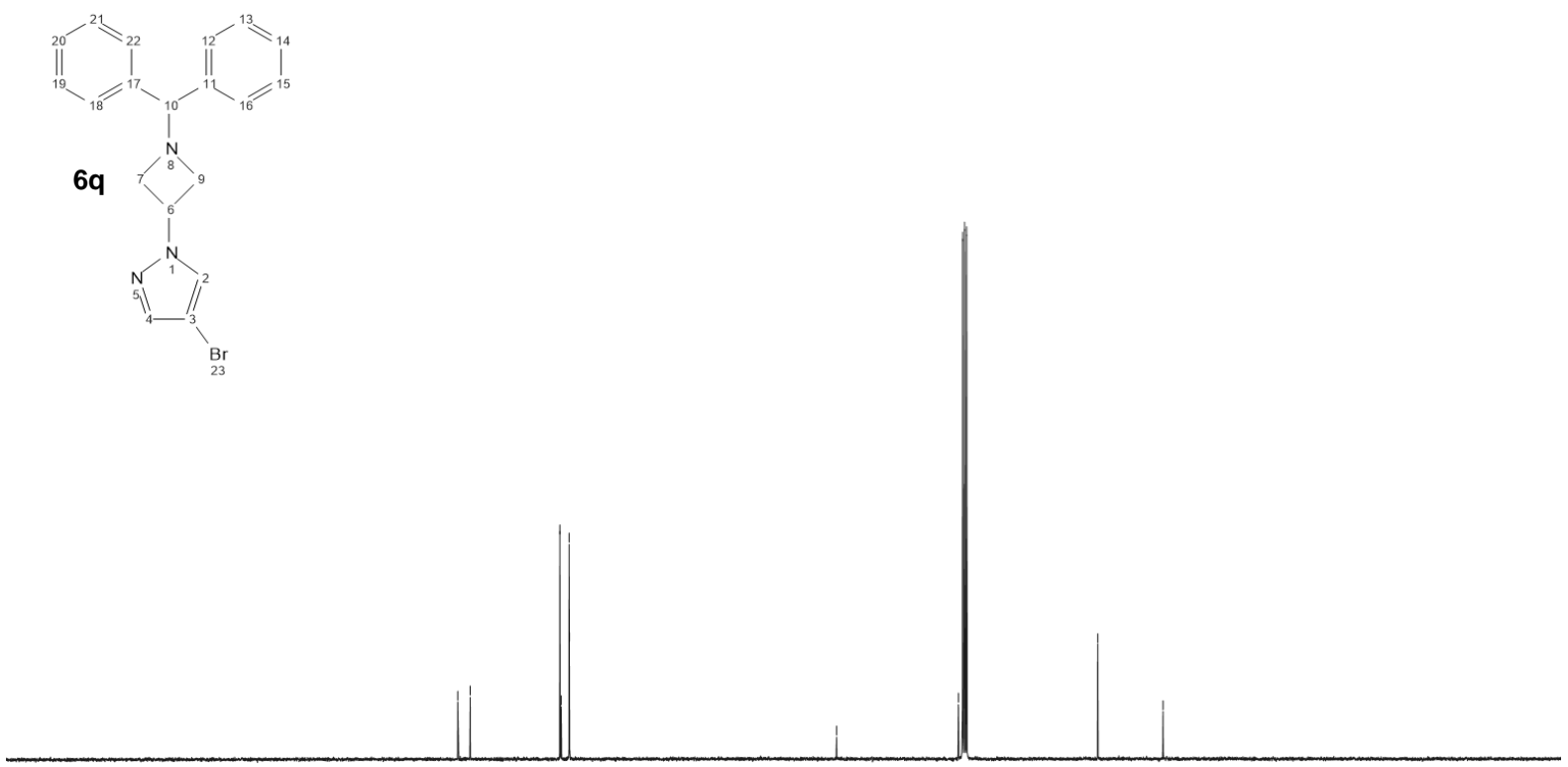

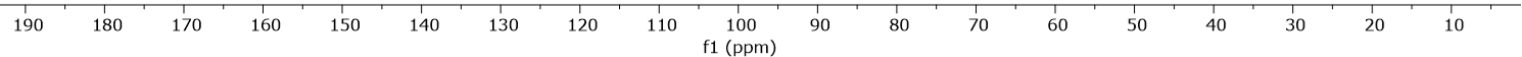


UOXF_groagna_361_1/CdCl3

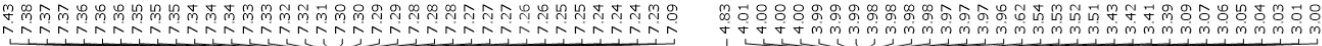

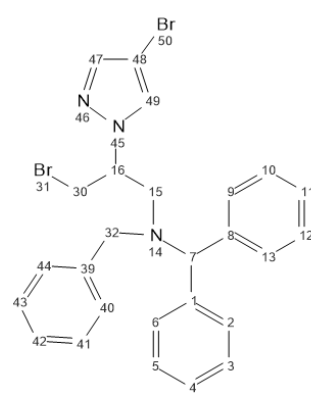

7qa

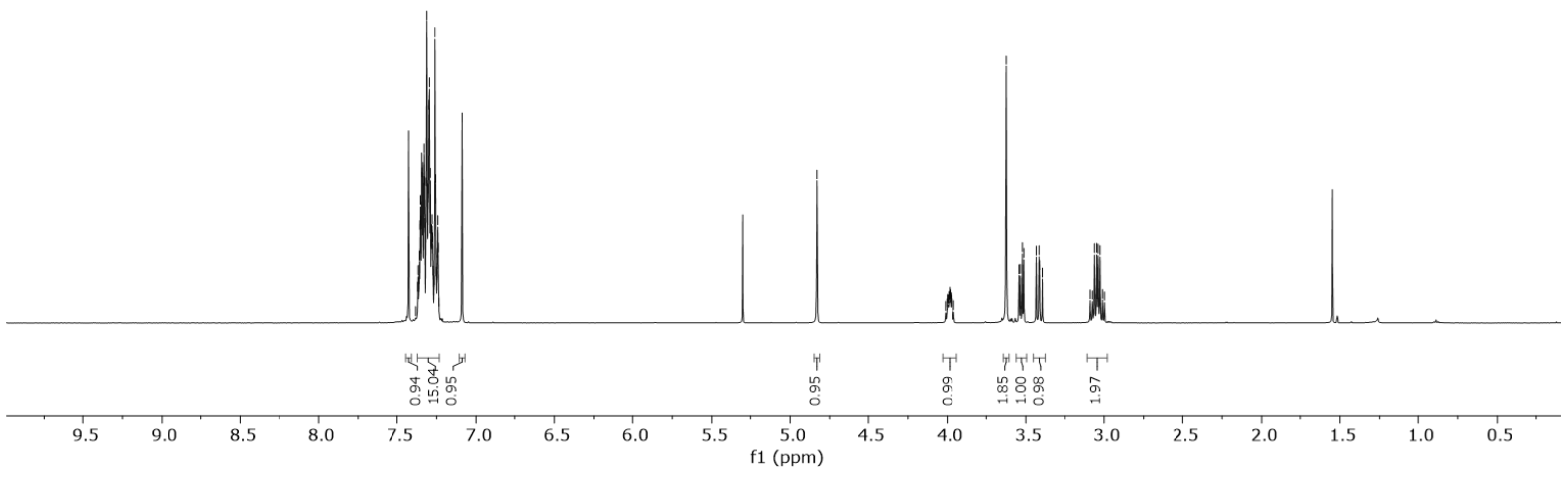

UOXF_groagna_361_1/CdCl3

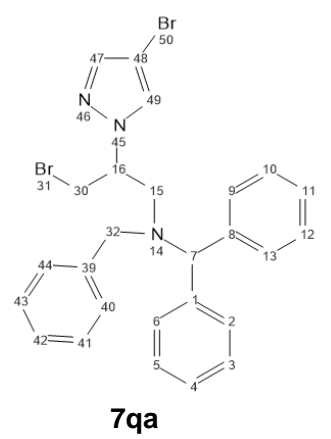

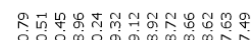

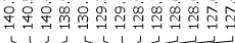

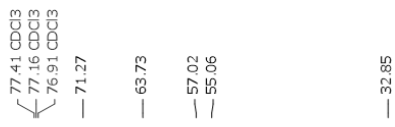

$7 q a$

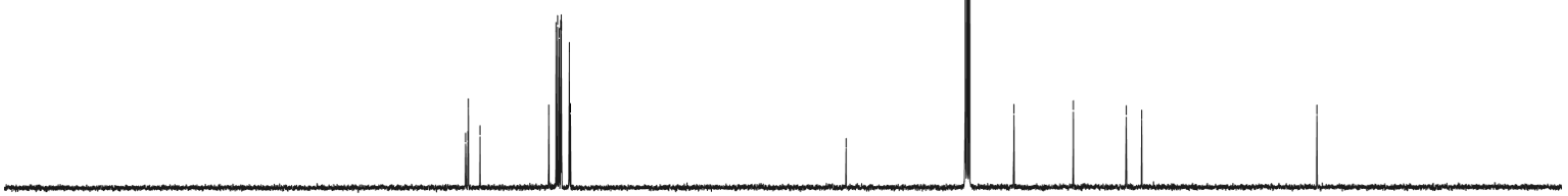



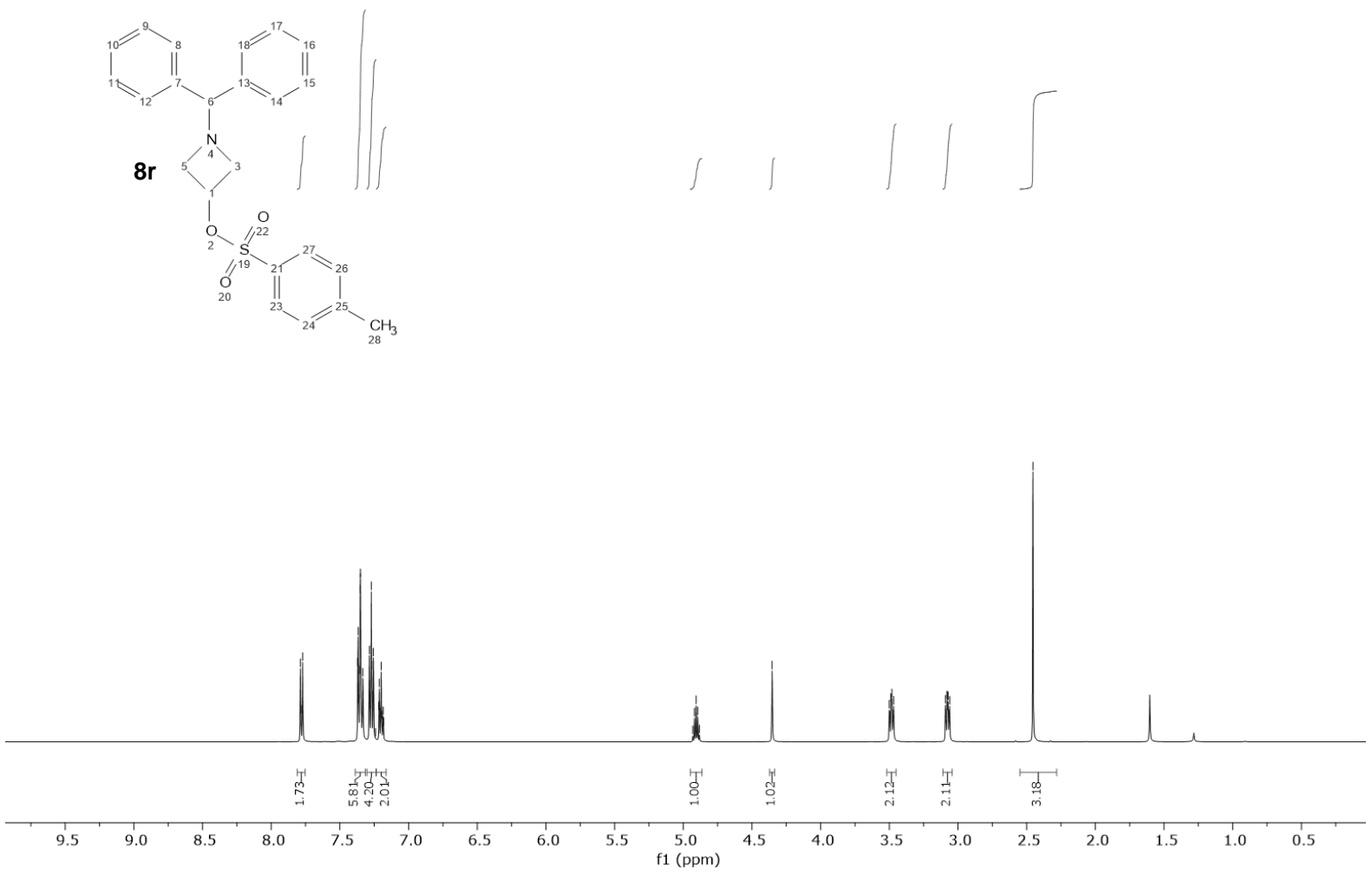

UOXF_groagna_280_1/CdCl3

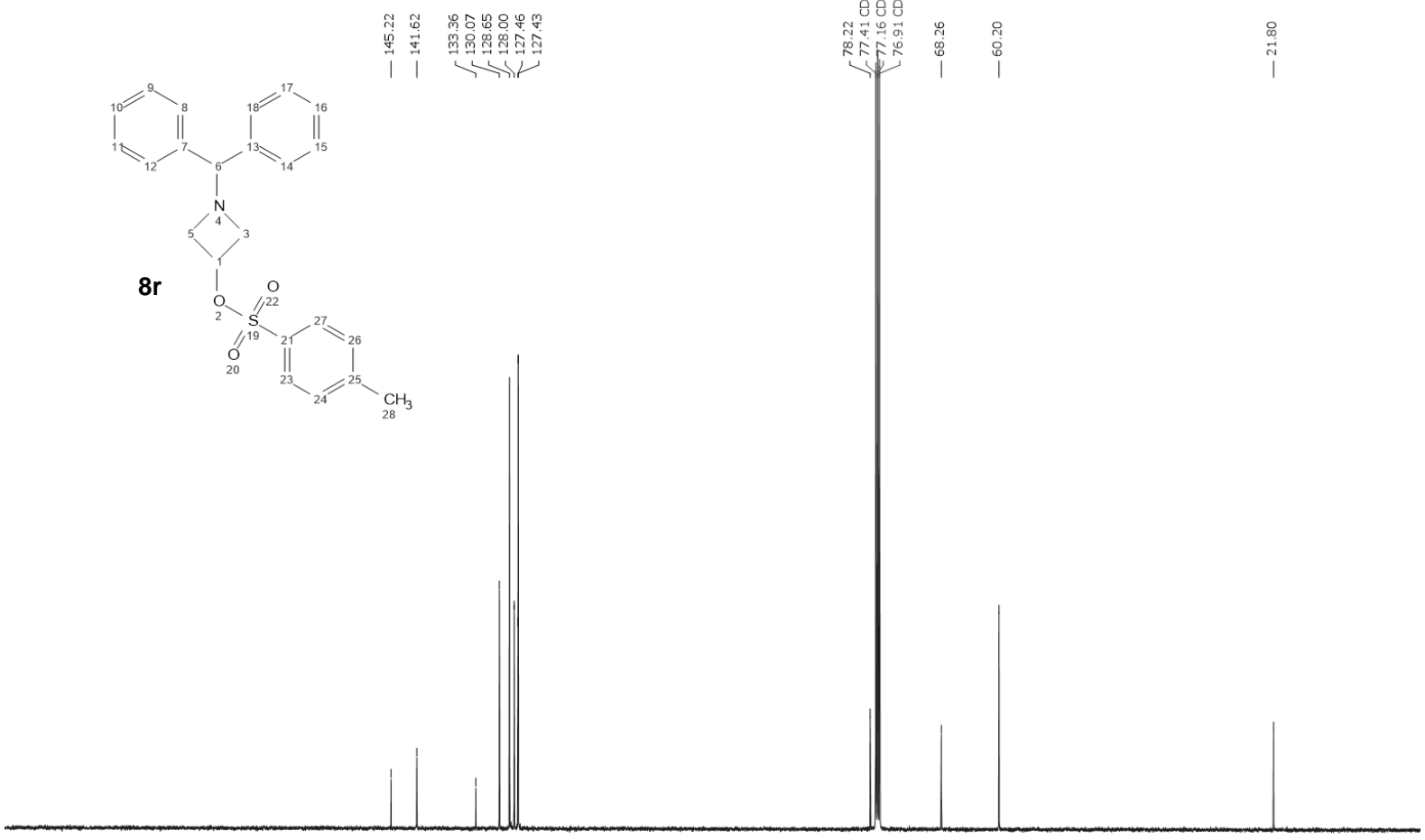

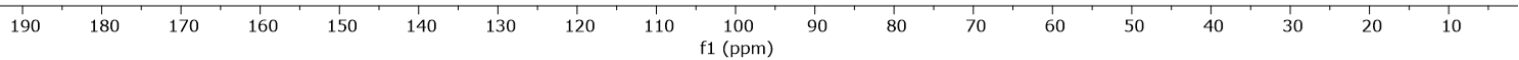


UOXF_groagna_298_1/CdCl3

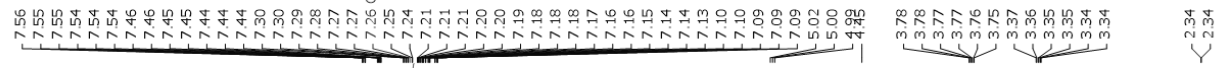
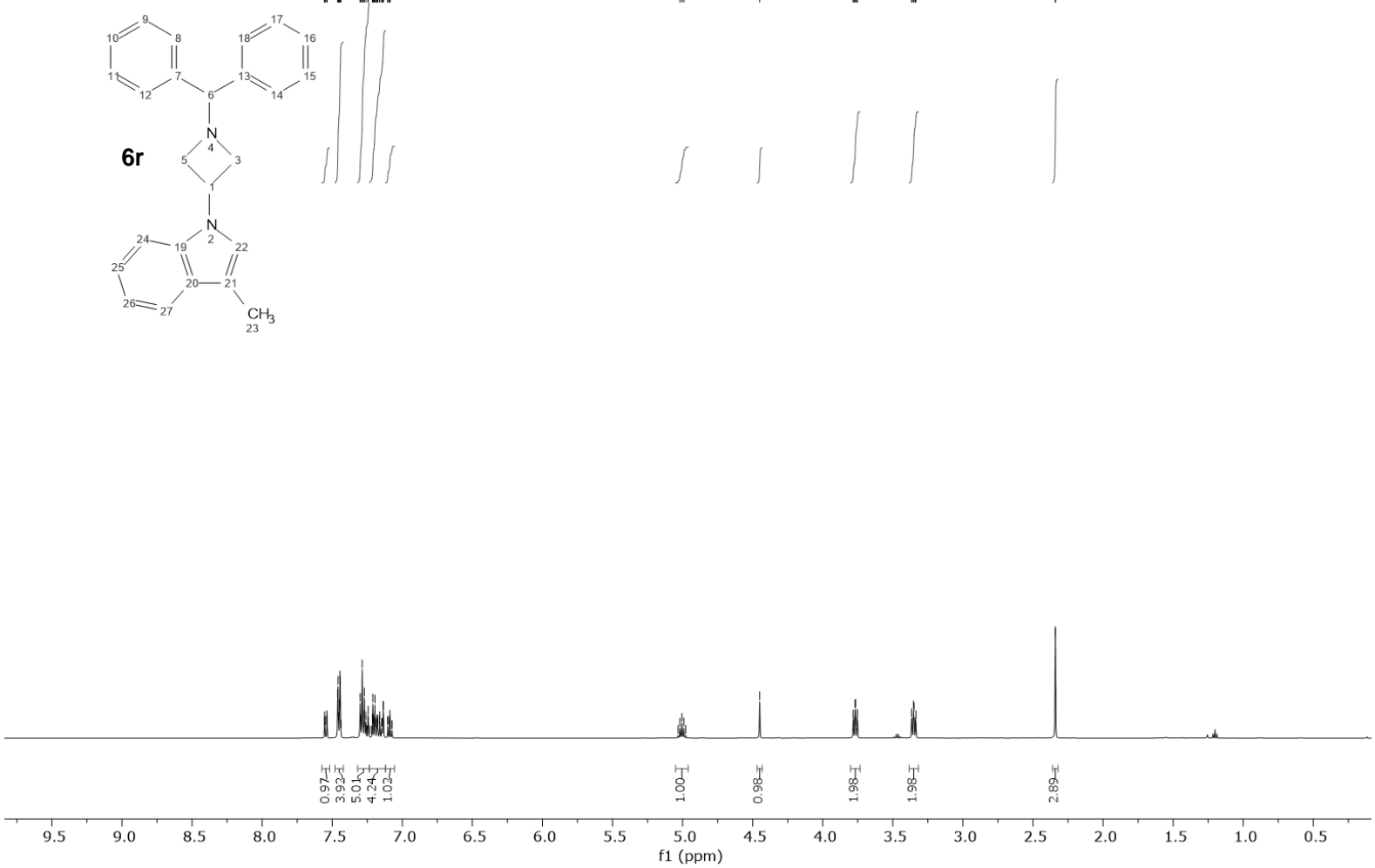

UOXF_groagna_298_1/CdCl3

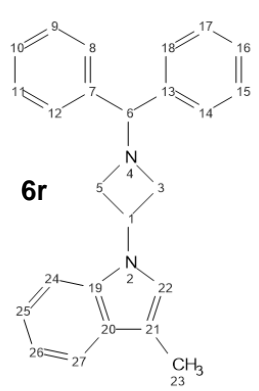

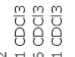

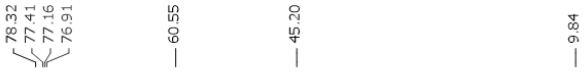

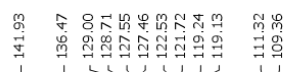

।

3

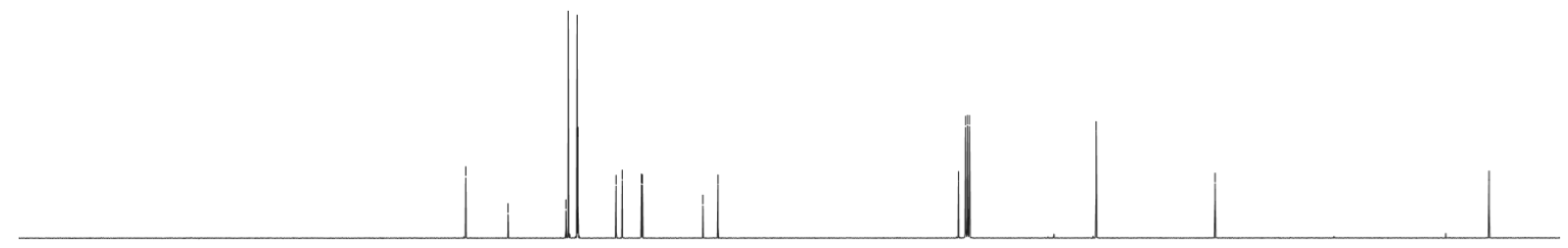

$190 \quad 180 \quad 170 \quad 160 \quad 150$

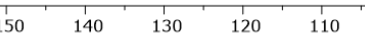

f1 100 
UOXF_groagna_344_1/CdCl3

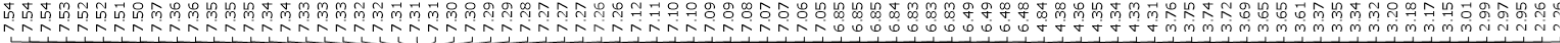<smiles></smiles>

$7 \mathrm{ra}$

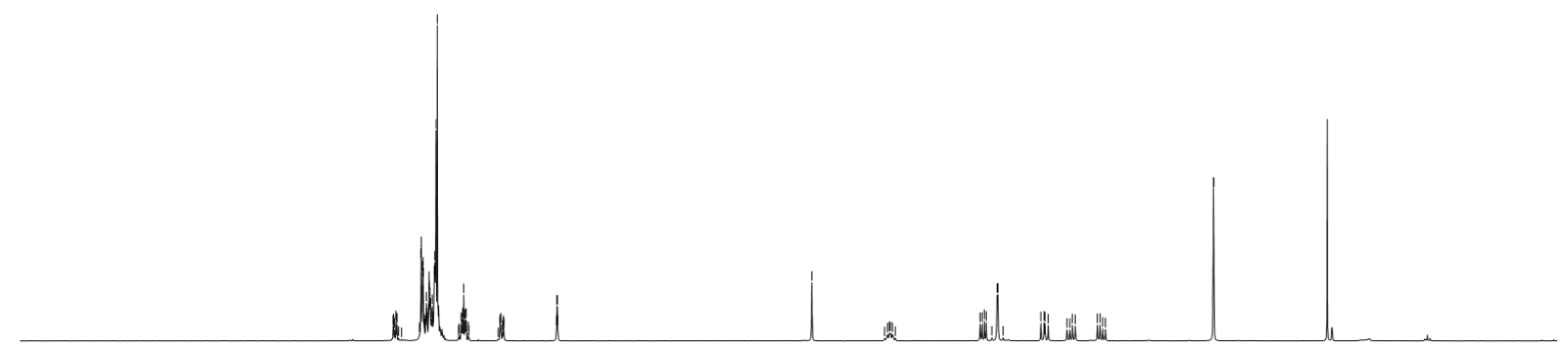

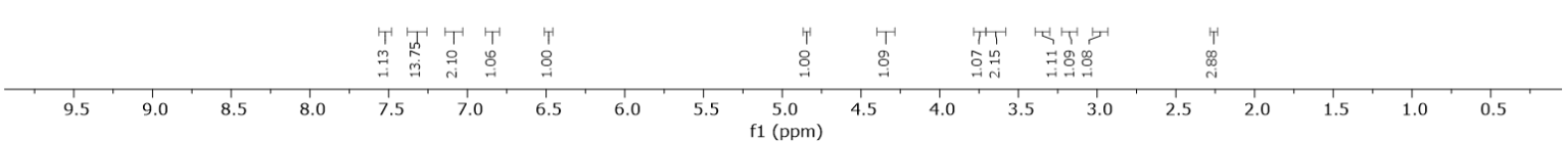

UOXF_groagna_344_1/CdCl3<smiles>Cc1nn(NCBr)c2nnnnc12</smiles>

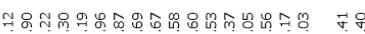

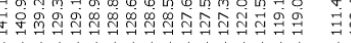

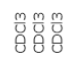

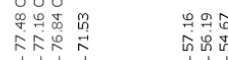

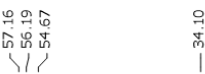

\section{㔛}

$\substack{0 \\ 0 \\ 0 \\ \mid}$
$\mid$

7ra

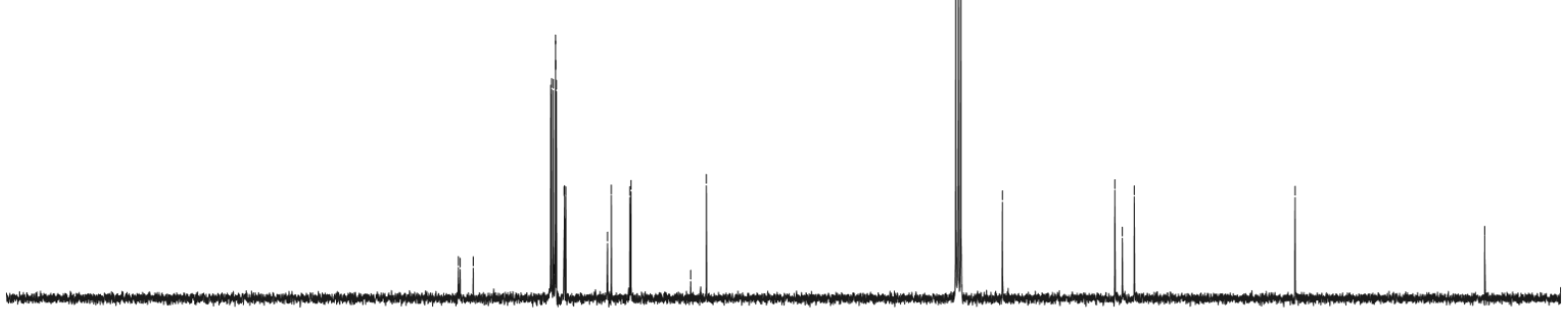

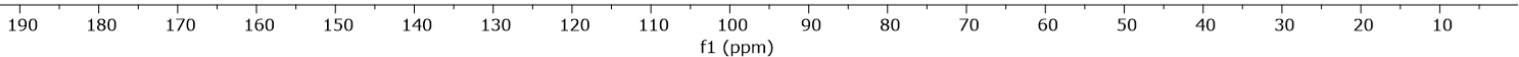


GR03EN34_1/CdCl3

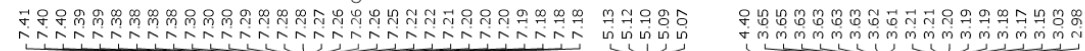

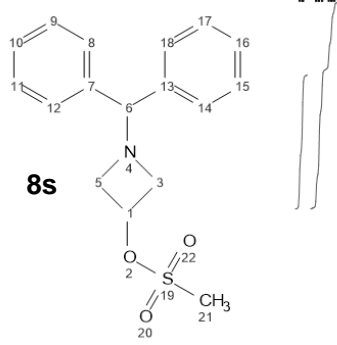

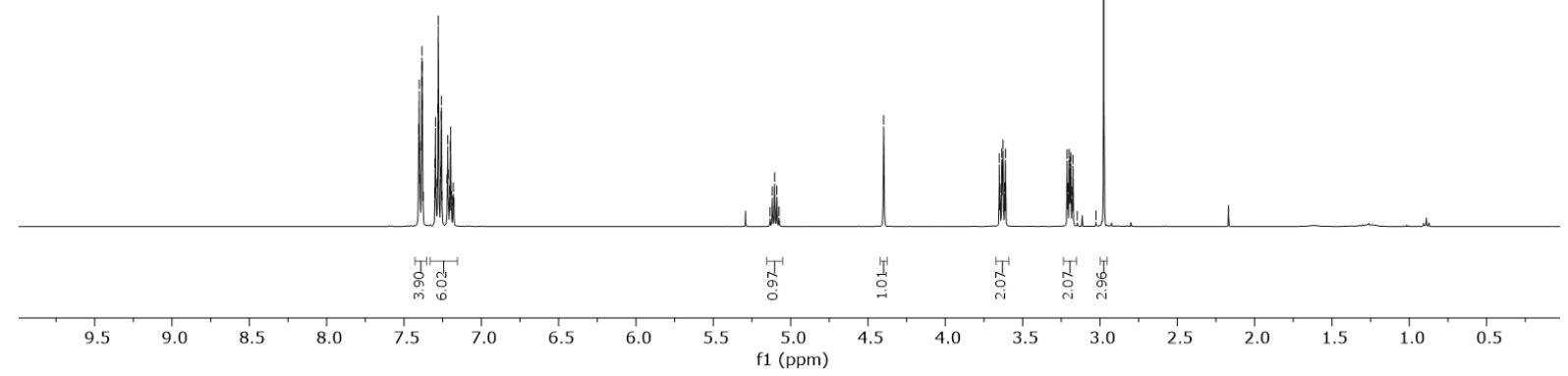

GR03EN34_1/CdCl3

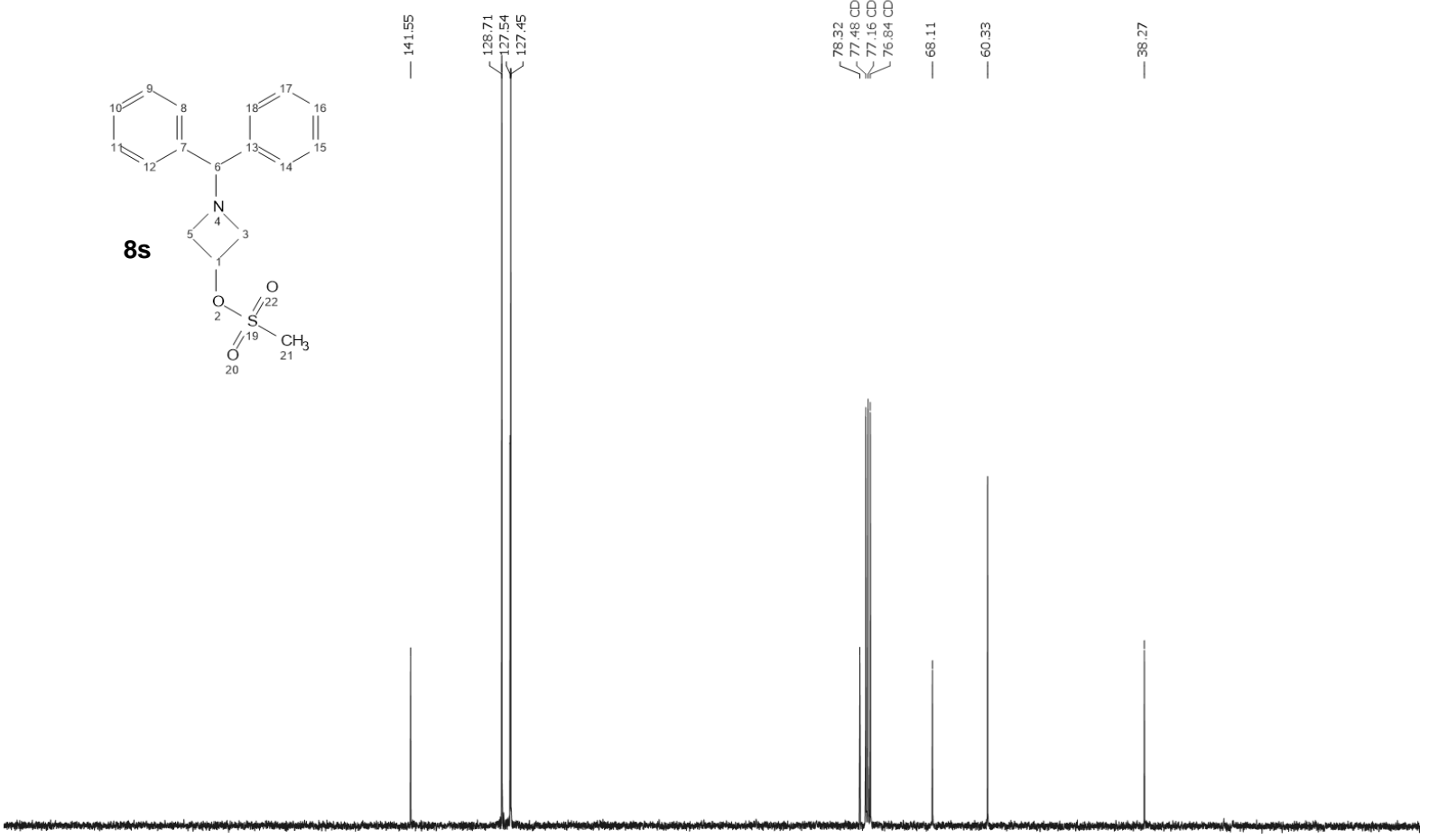

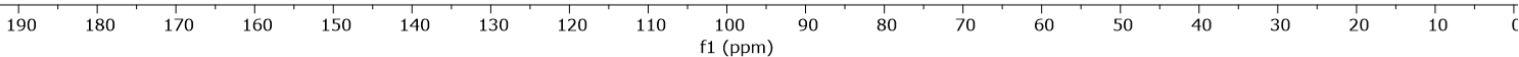


GR03EN35_1/CdCl3

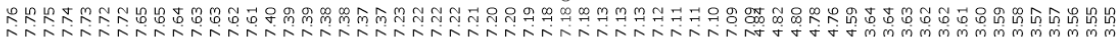
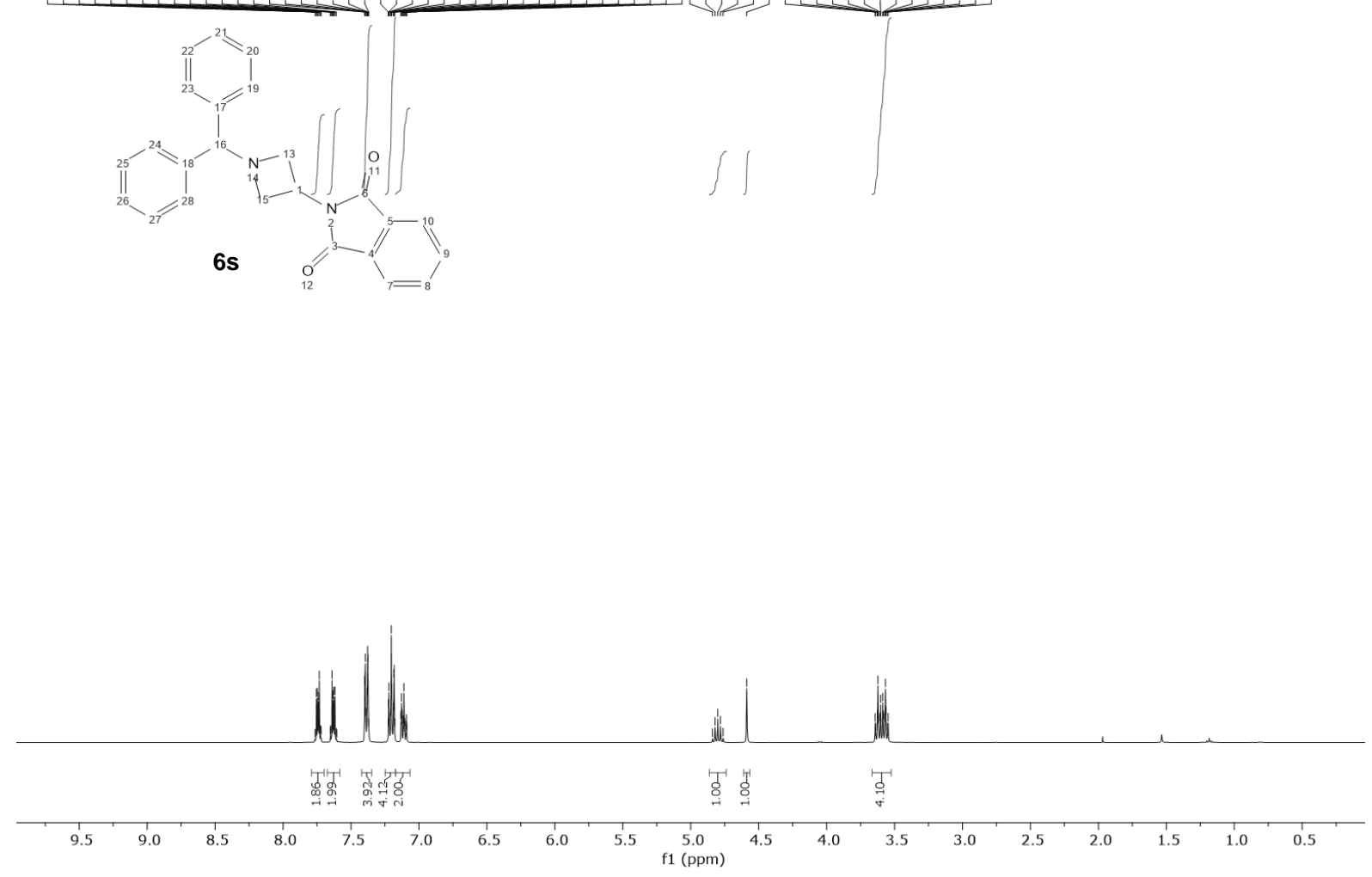

GR03EN35_1/CdCl3

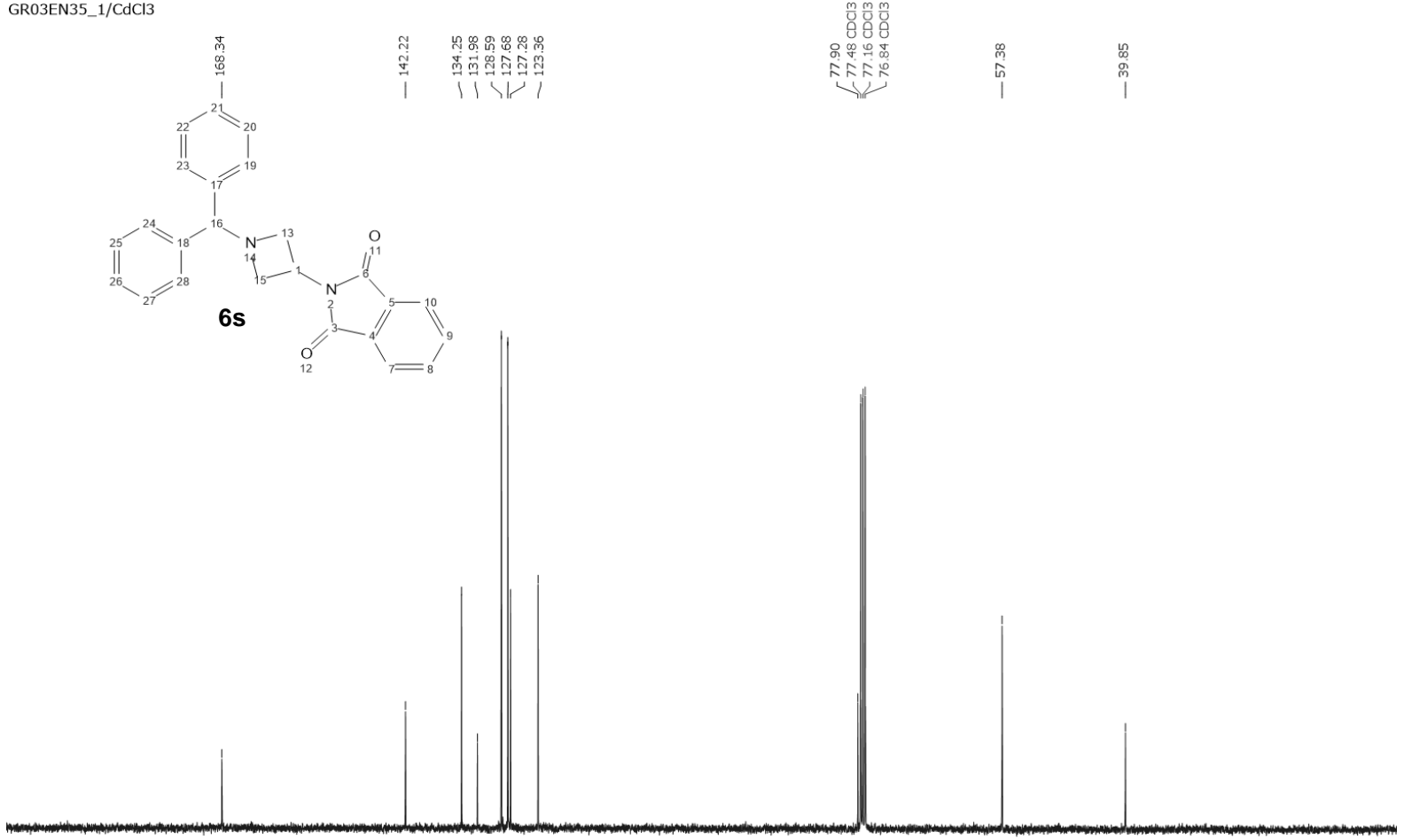

190

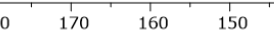

$\begin{array}{lll}130 & 120 & 110\end{array}$

100
$\mathrm{f} 1(\mathrm{ppm})$

90

$70+60$

50

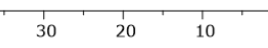



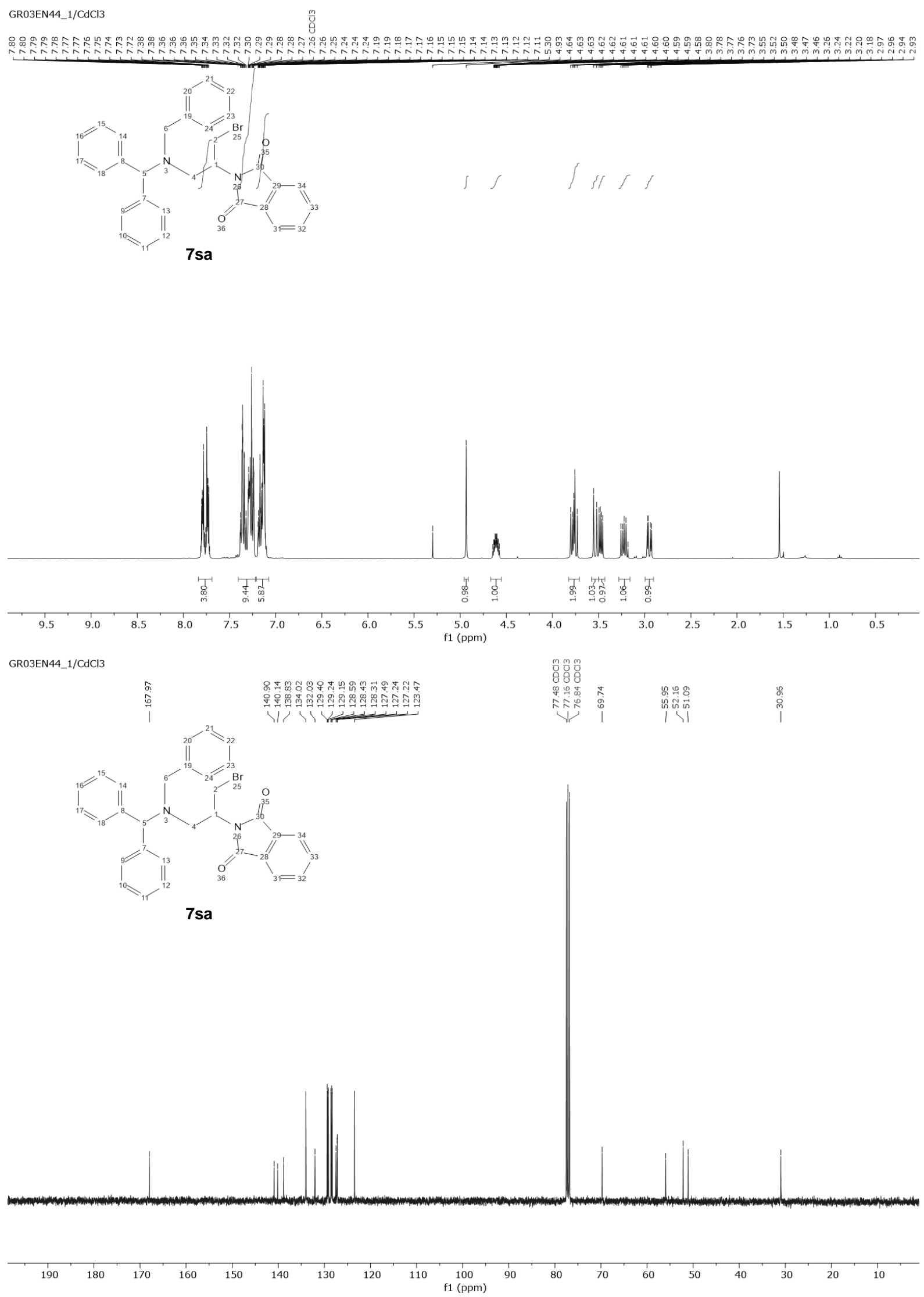

S185 

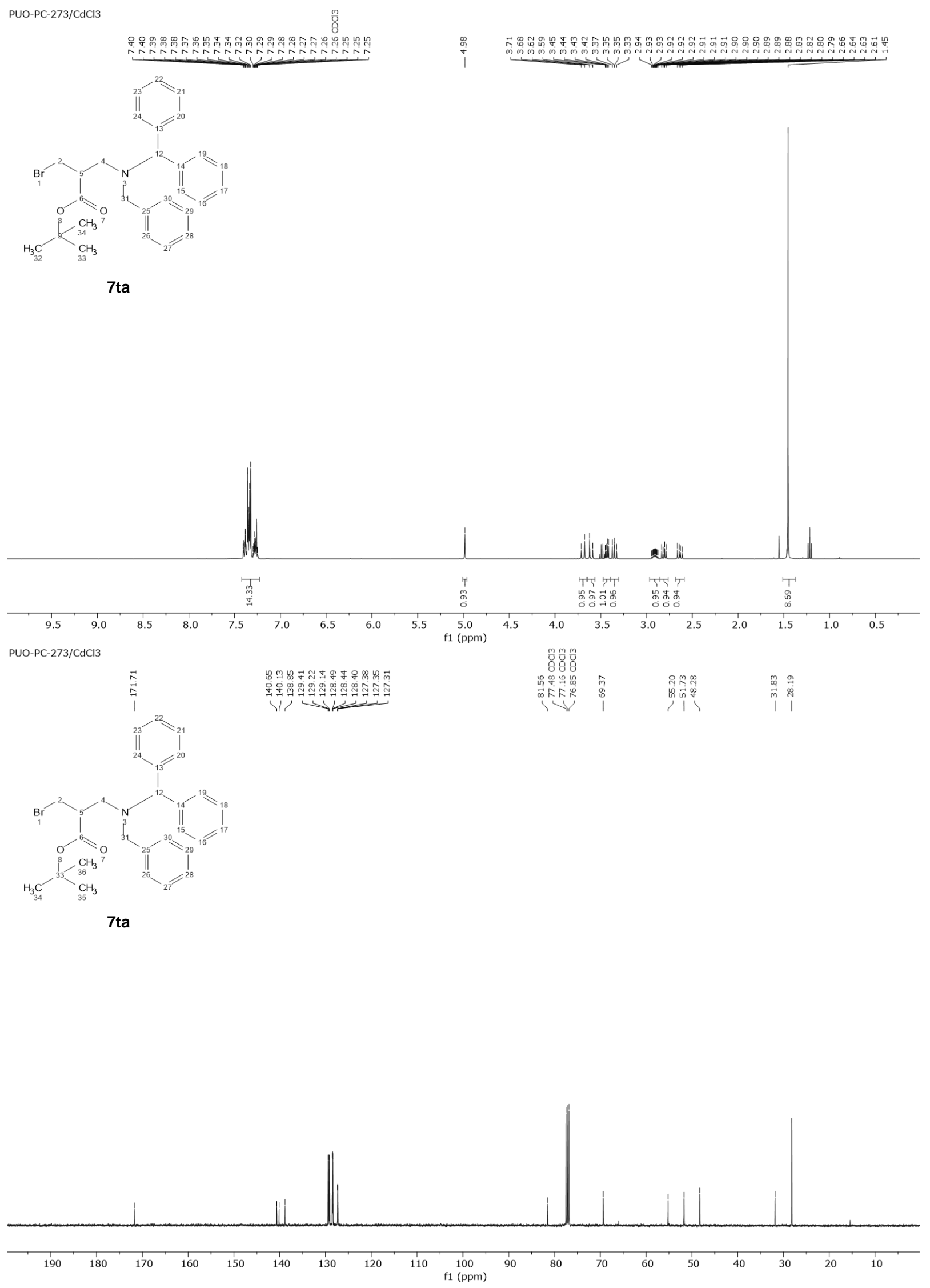


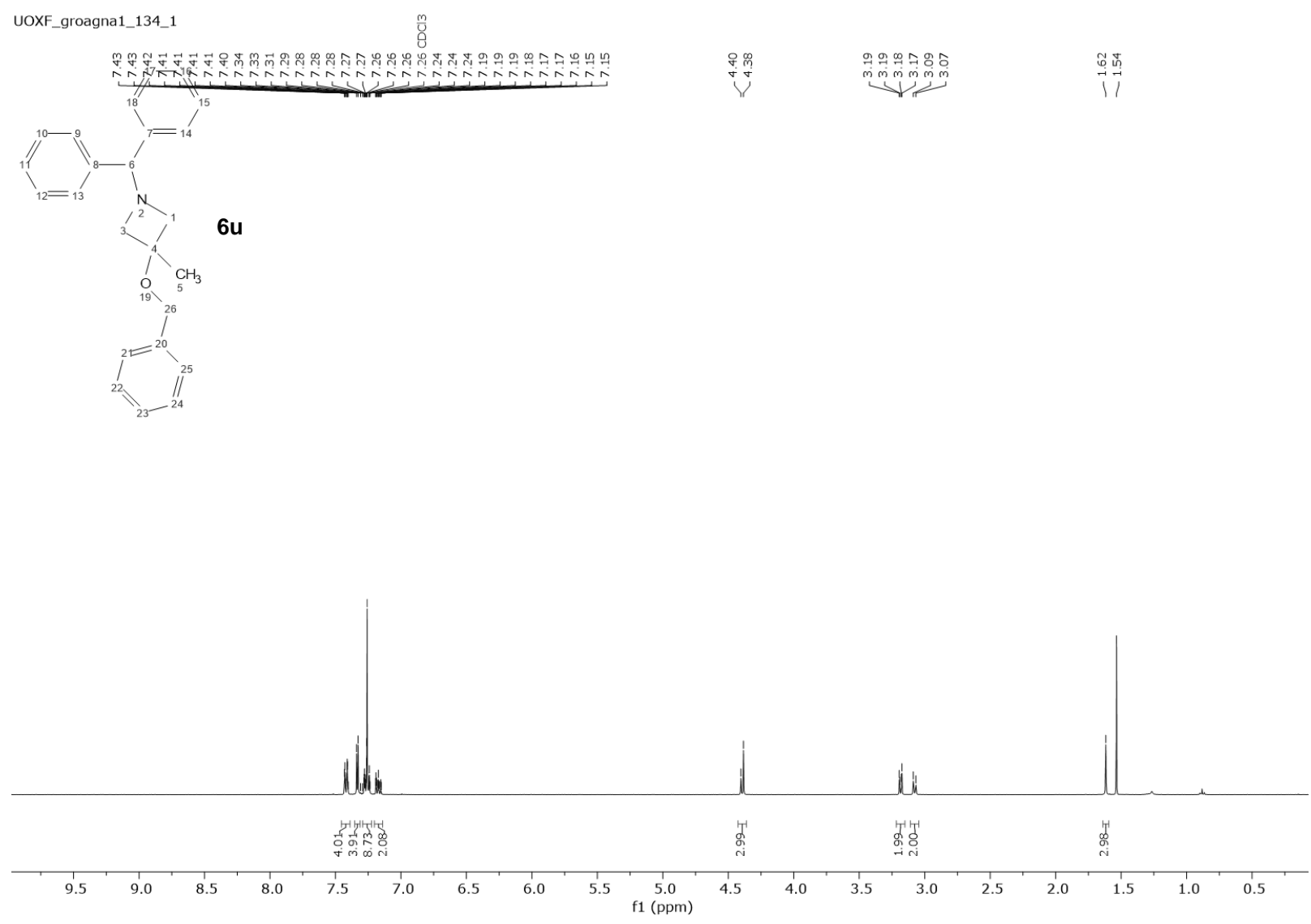

UOXF_groagna1_134_1
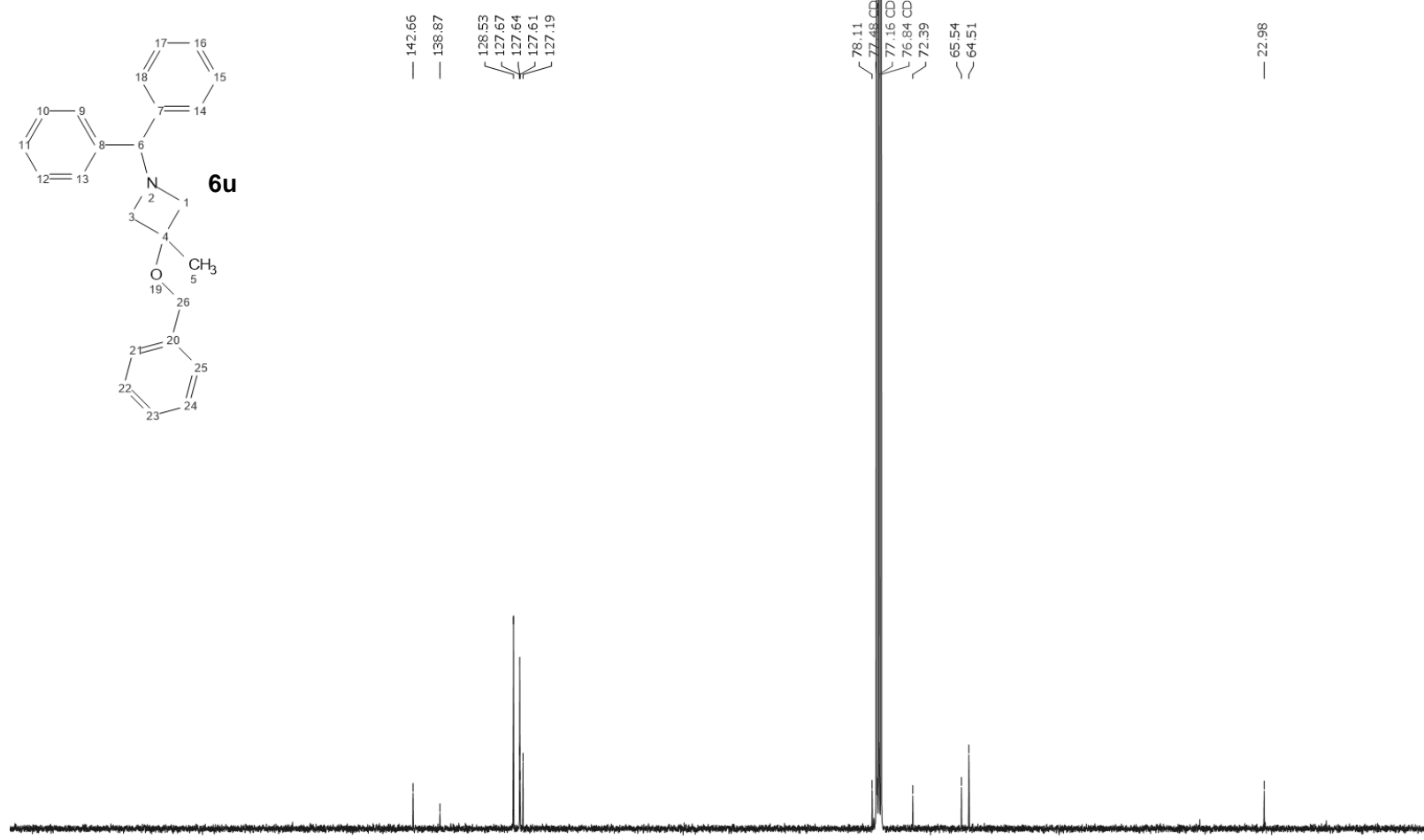

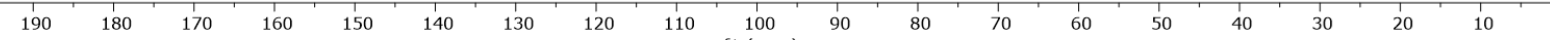


UOXF_groagna1_144_1

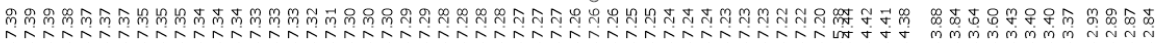

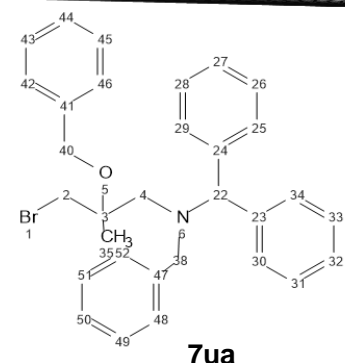

7ua

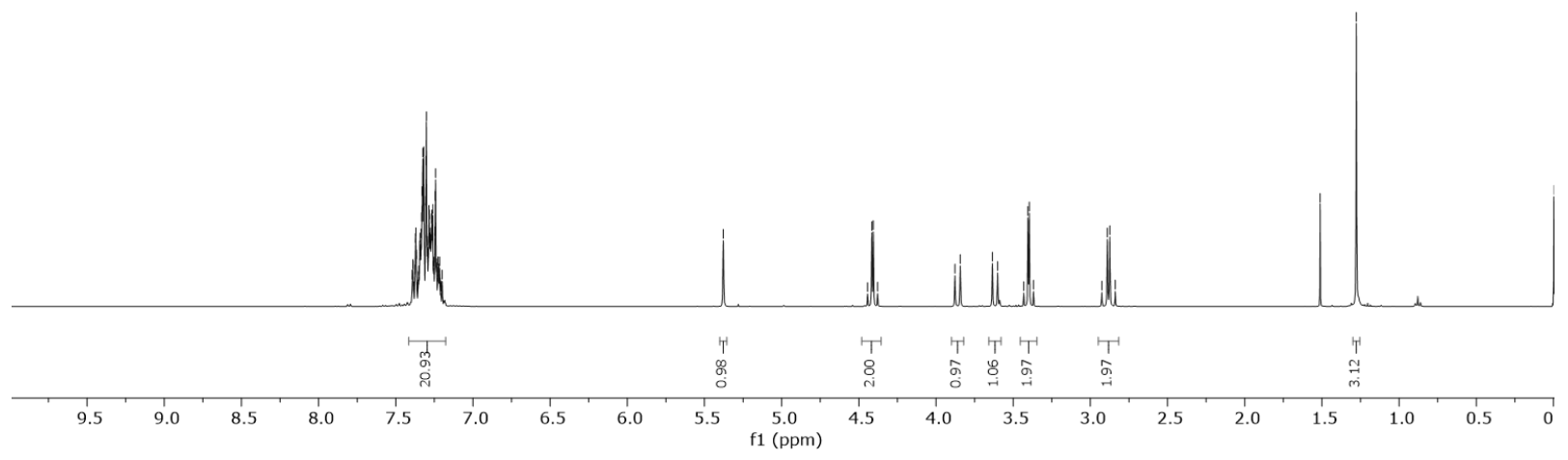

UOXF_groagna1_144_1

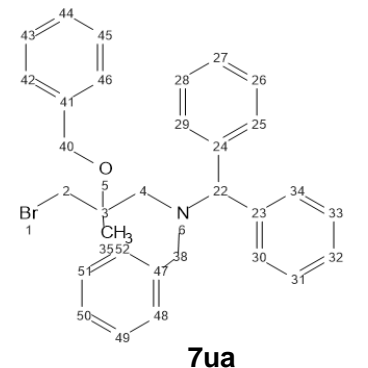

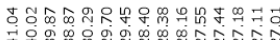

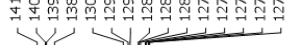

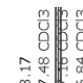

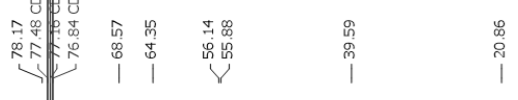

7ua

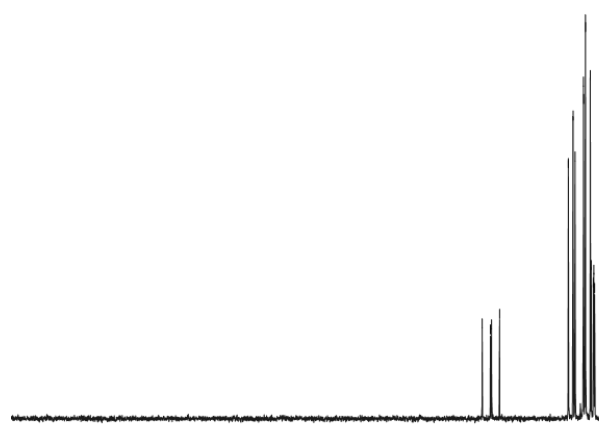

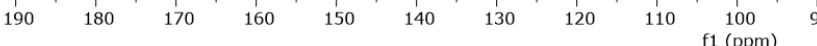

$80 \quad 70,60,50$

$40 \quad 30 \quad 20 \quad 10$ 


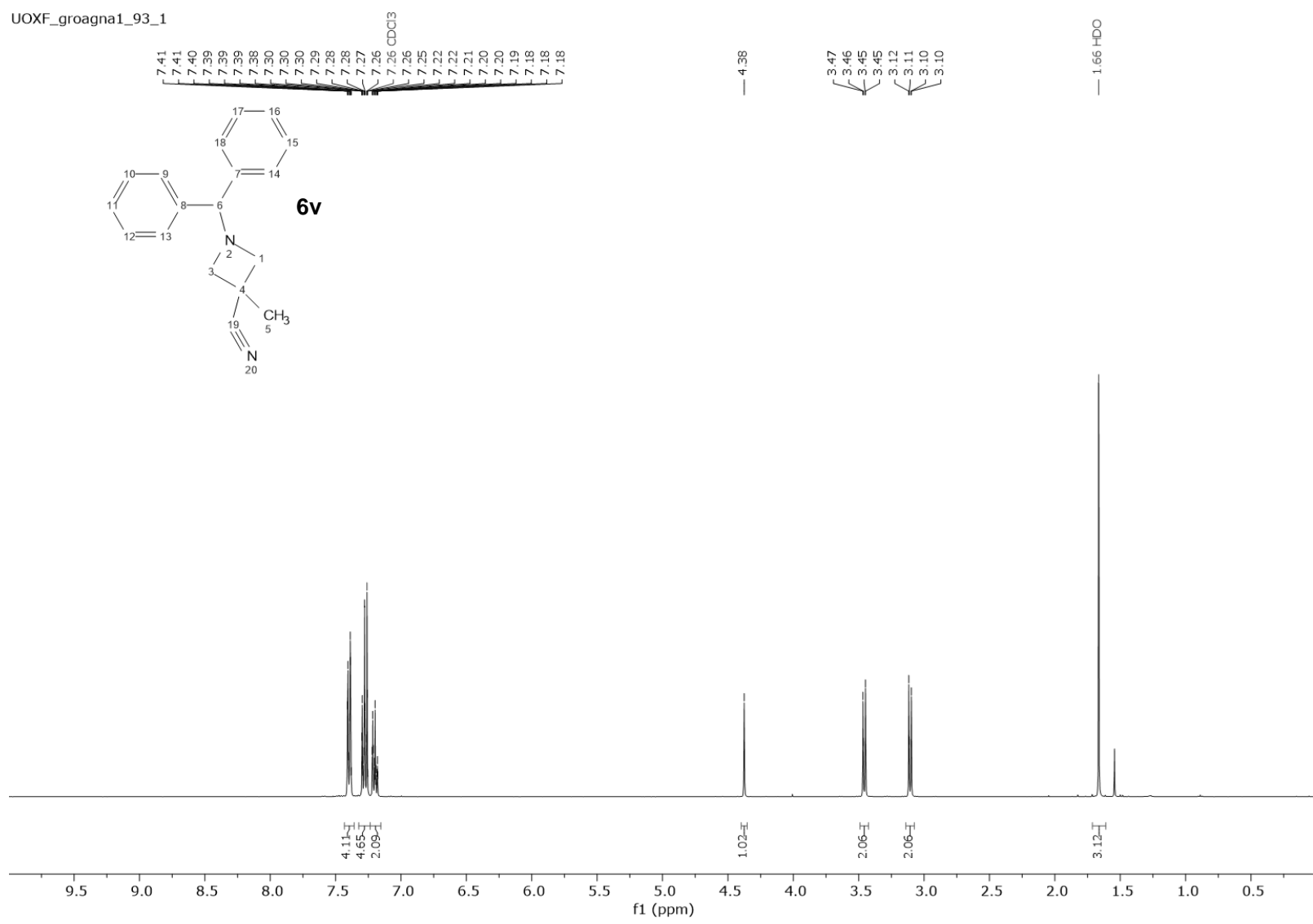

UOXF_groagna1_93_1

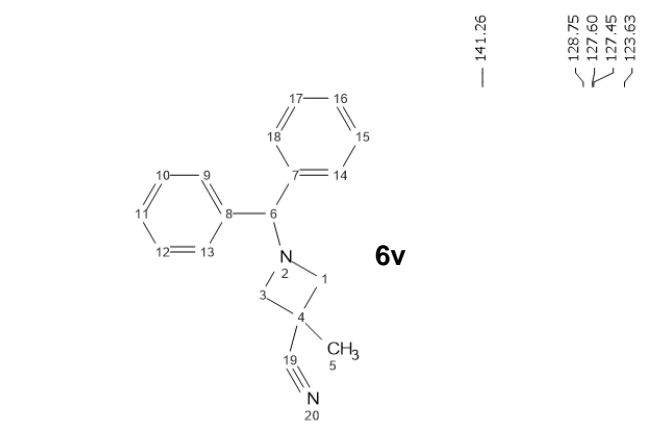

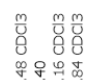

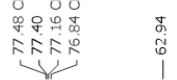

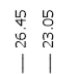

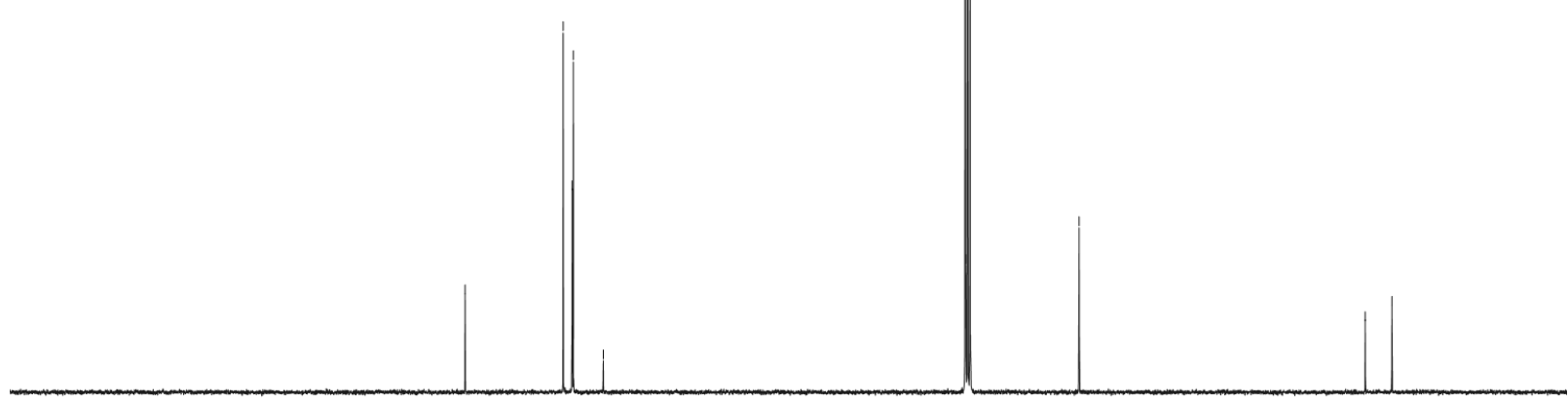

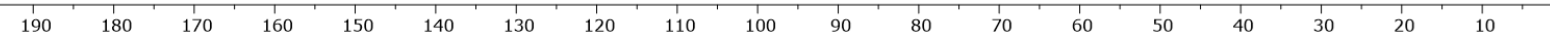
f1 (ppm) 

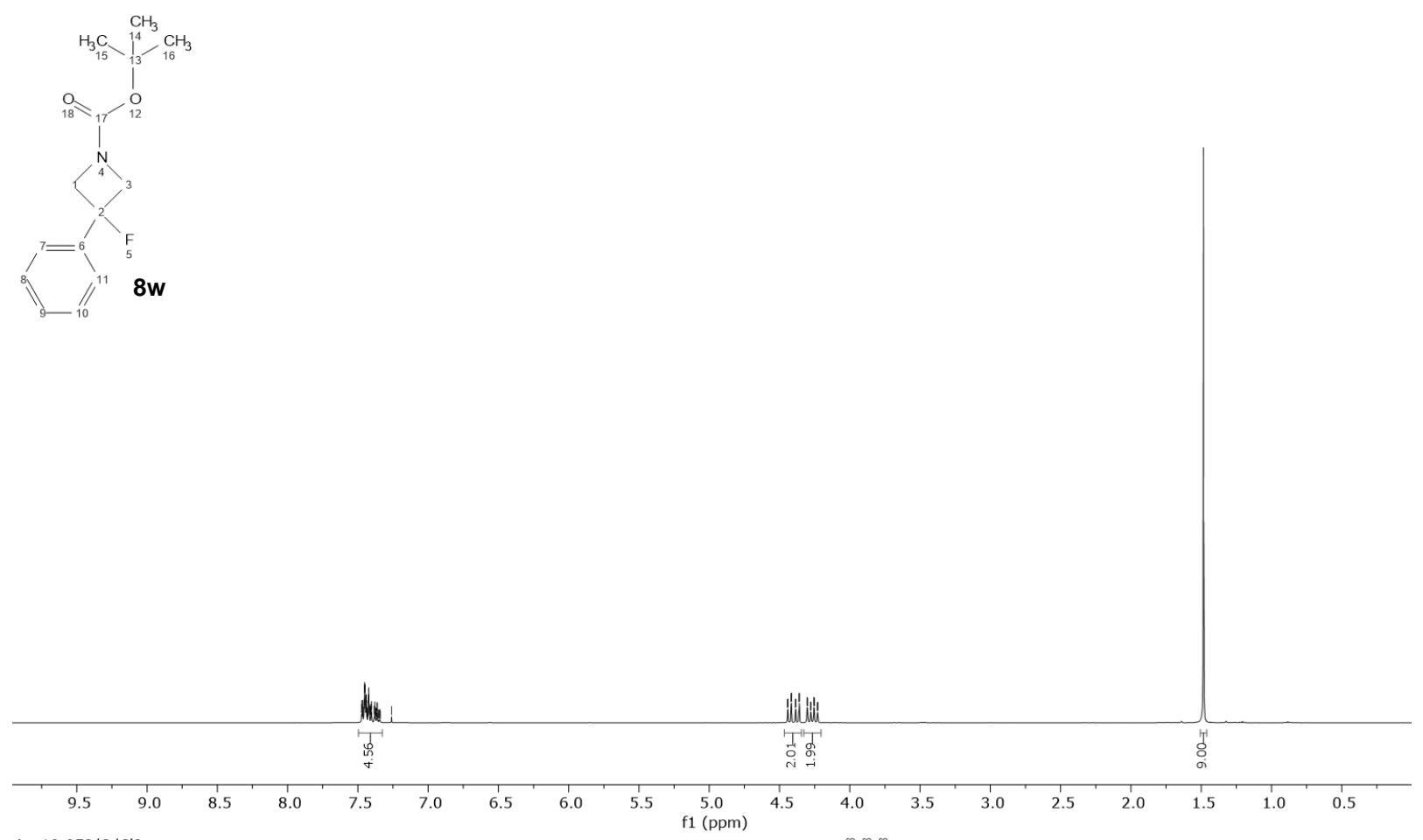

Acv10-052/CdCl3

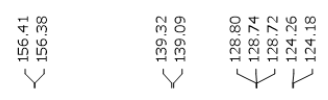
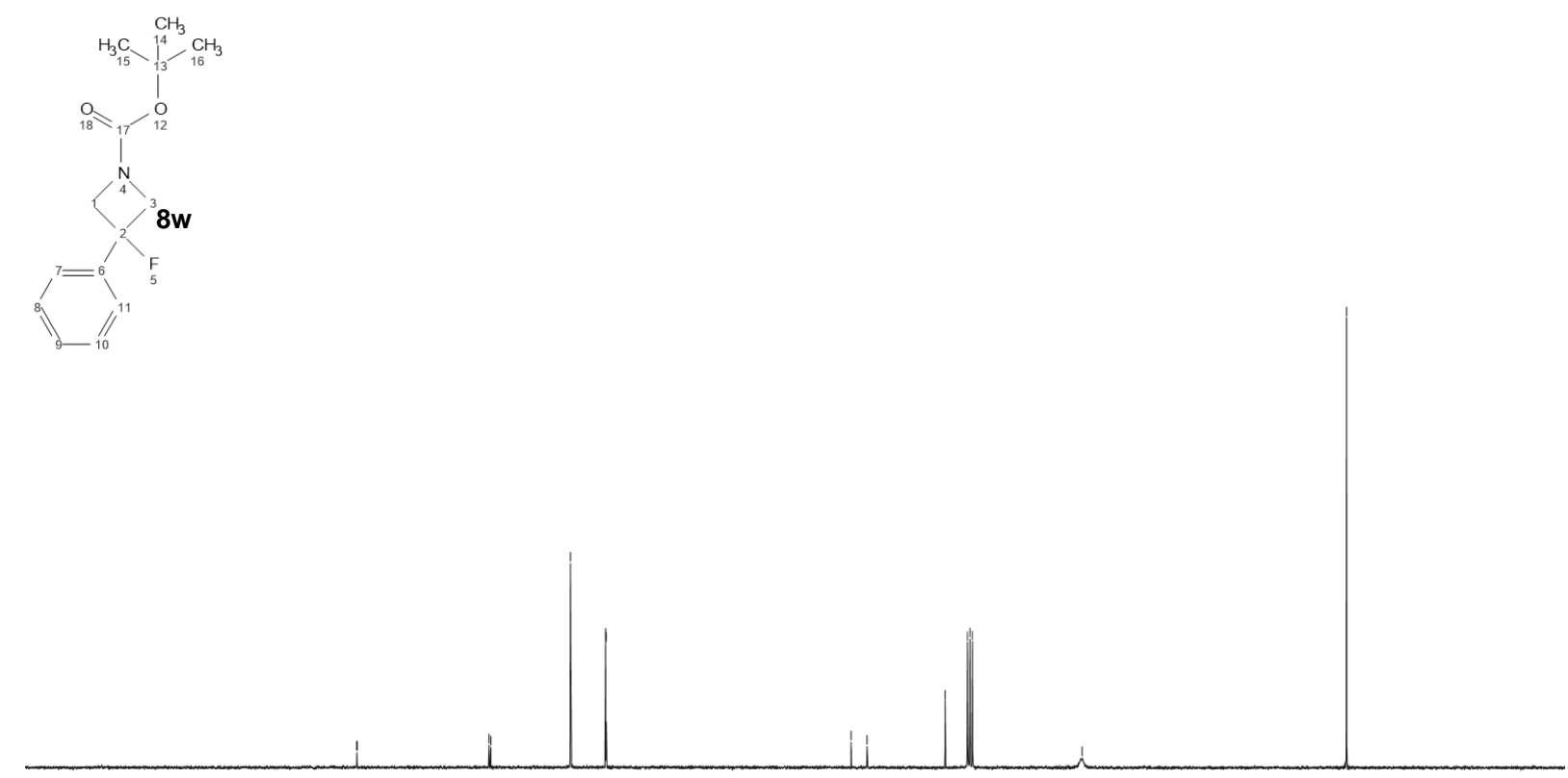

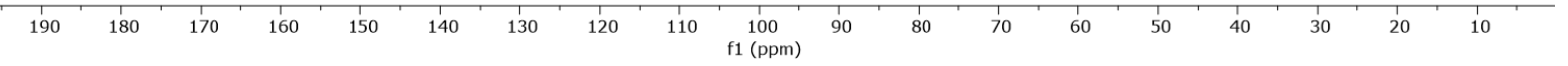




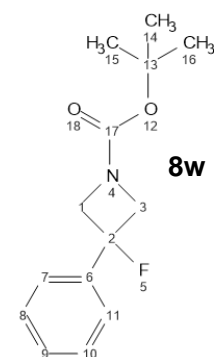

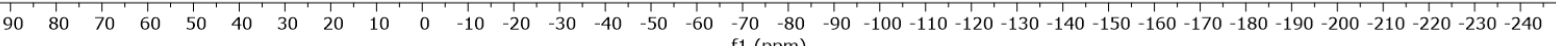
f1 (ppm) 


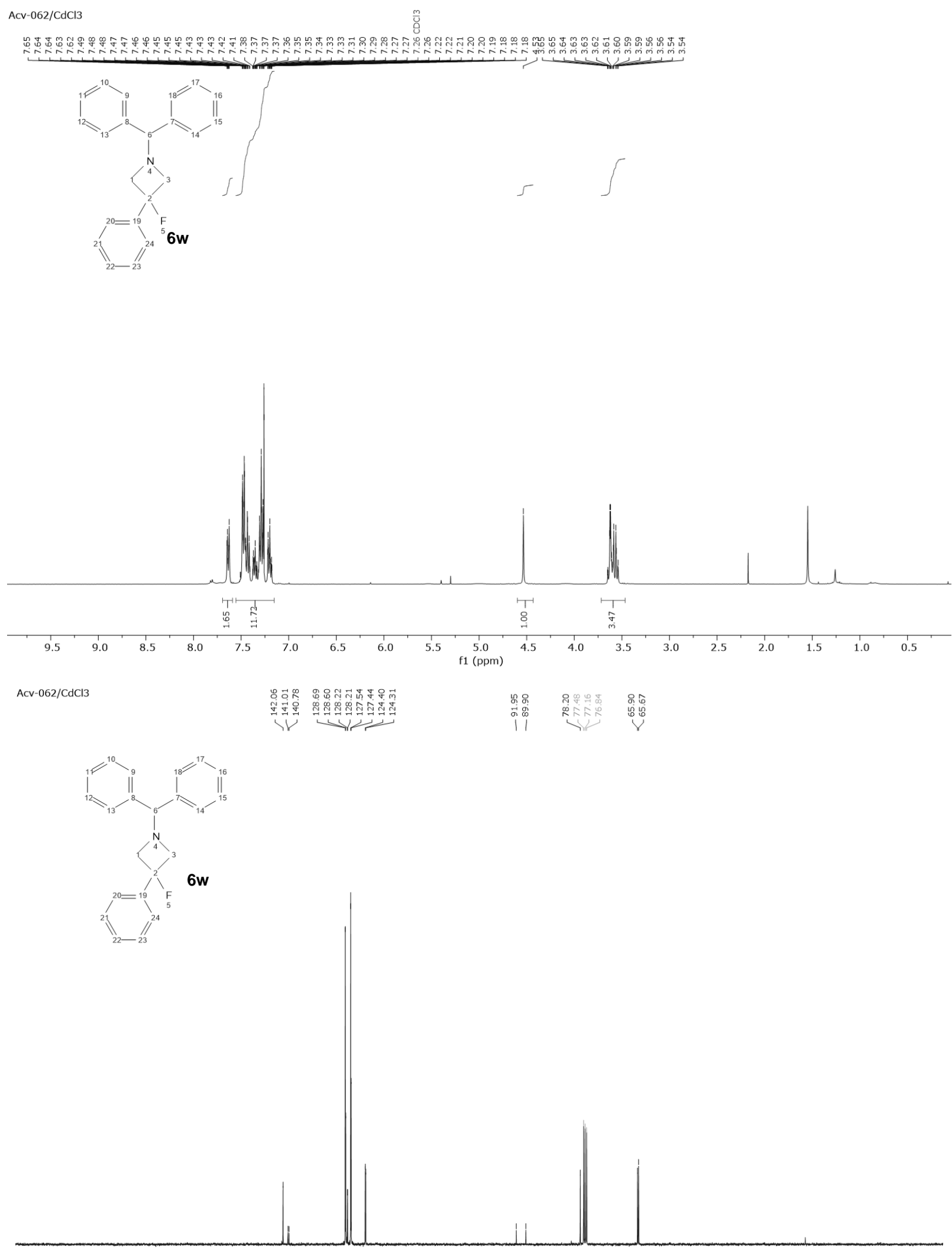

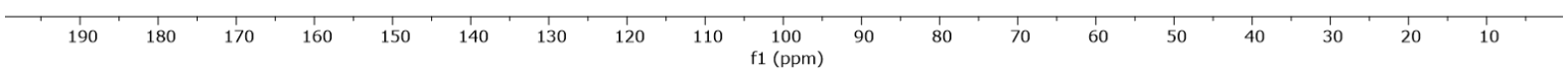




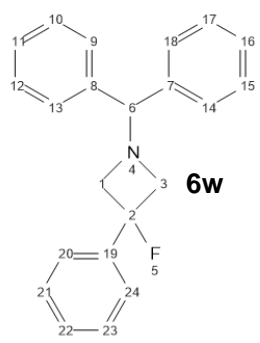

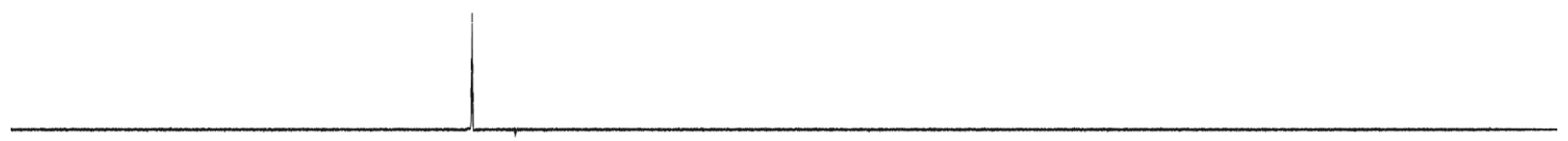

\begin{tabular}{lllllllllllllllllllllllllllllllllll}
\hline 00 & -105 & -110 & -115 & -120 & -125 & -130 & -135 & -140 & -145 & -150 & -155 & -160 & -165 & -170 & -175 & -180 & -185 & -190 & -195 & -200 & -205 & -210 & -215 & -220 & -225 & -230 & -235 & -240 & -245
\end{tabular} 

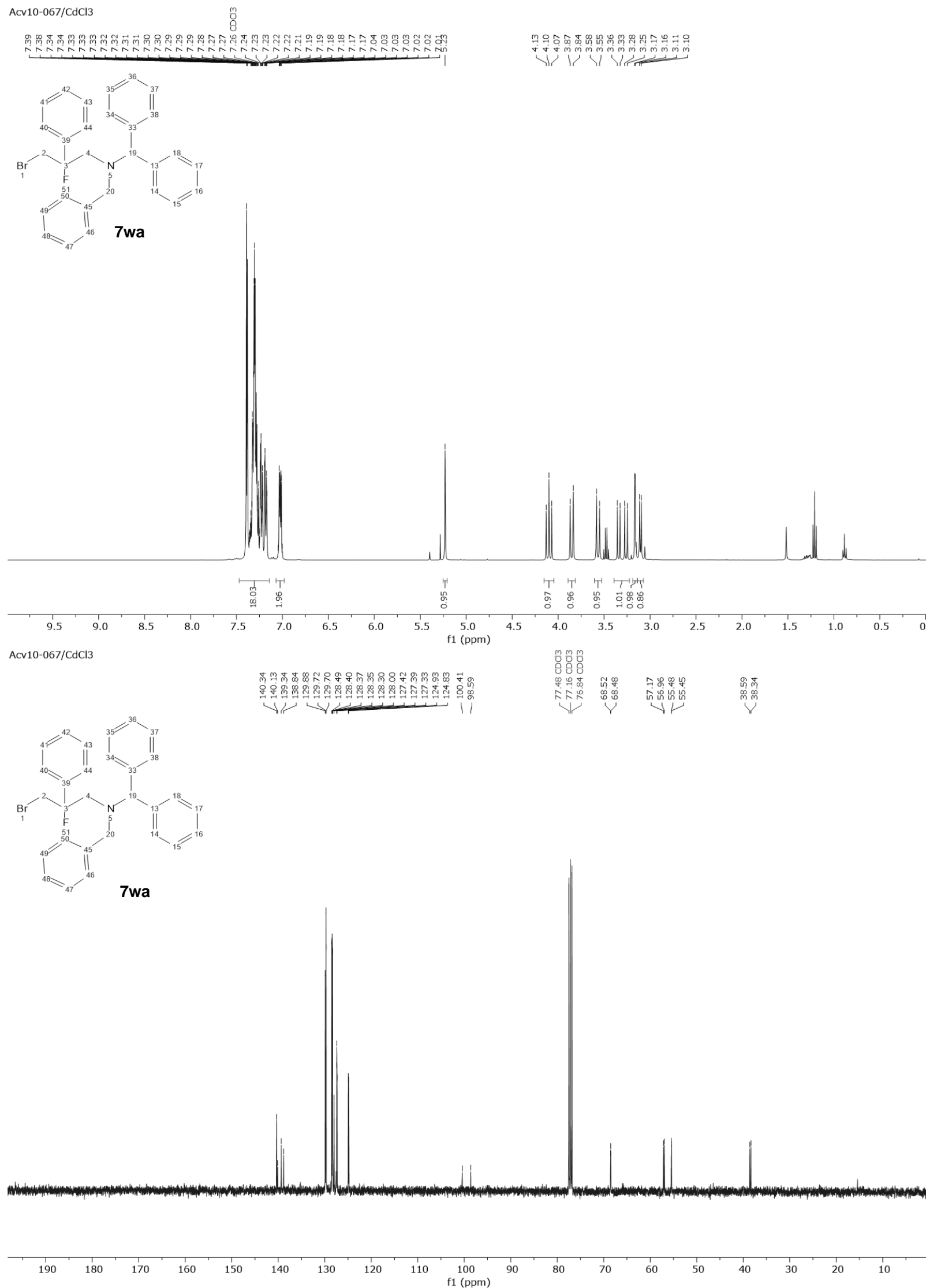

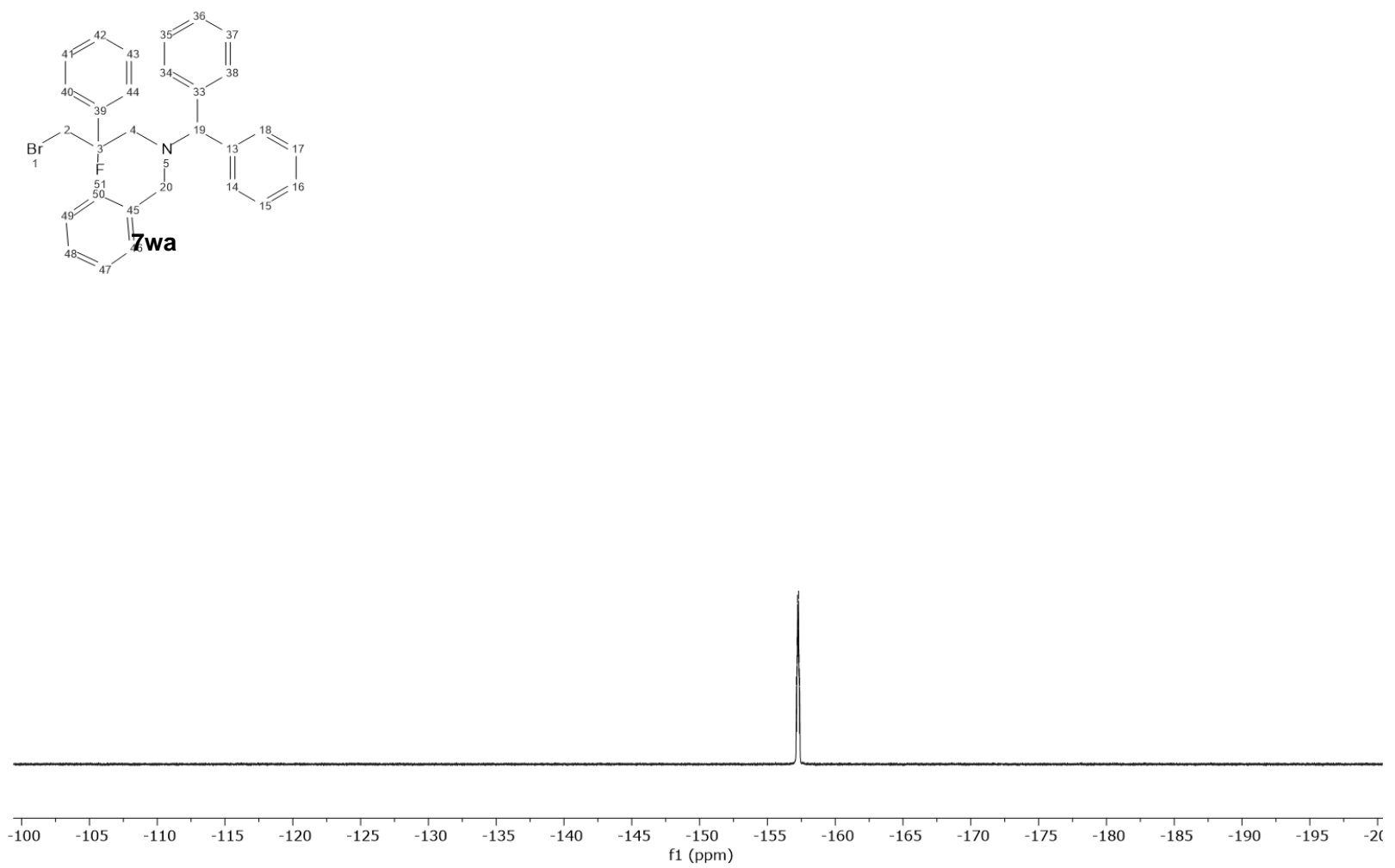

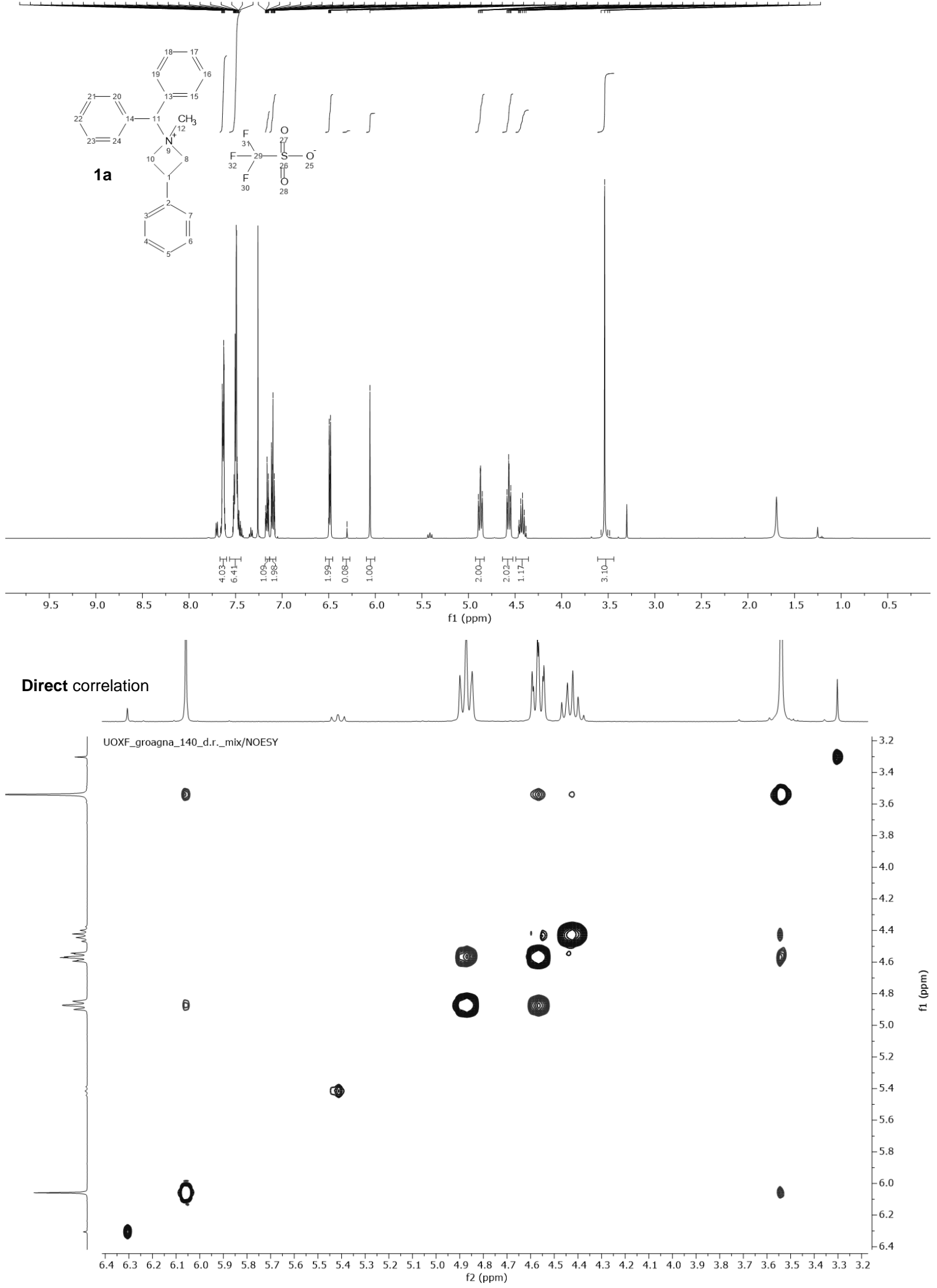
UOXF_groagna_140_d.r._mix/CdCl3
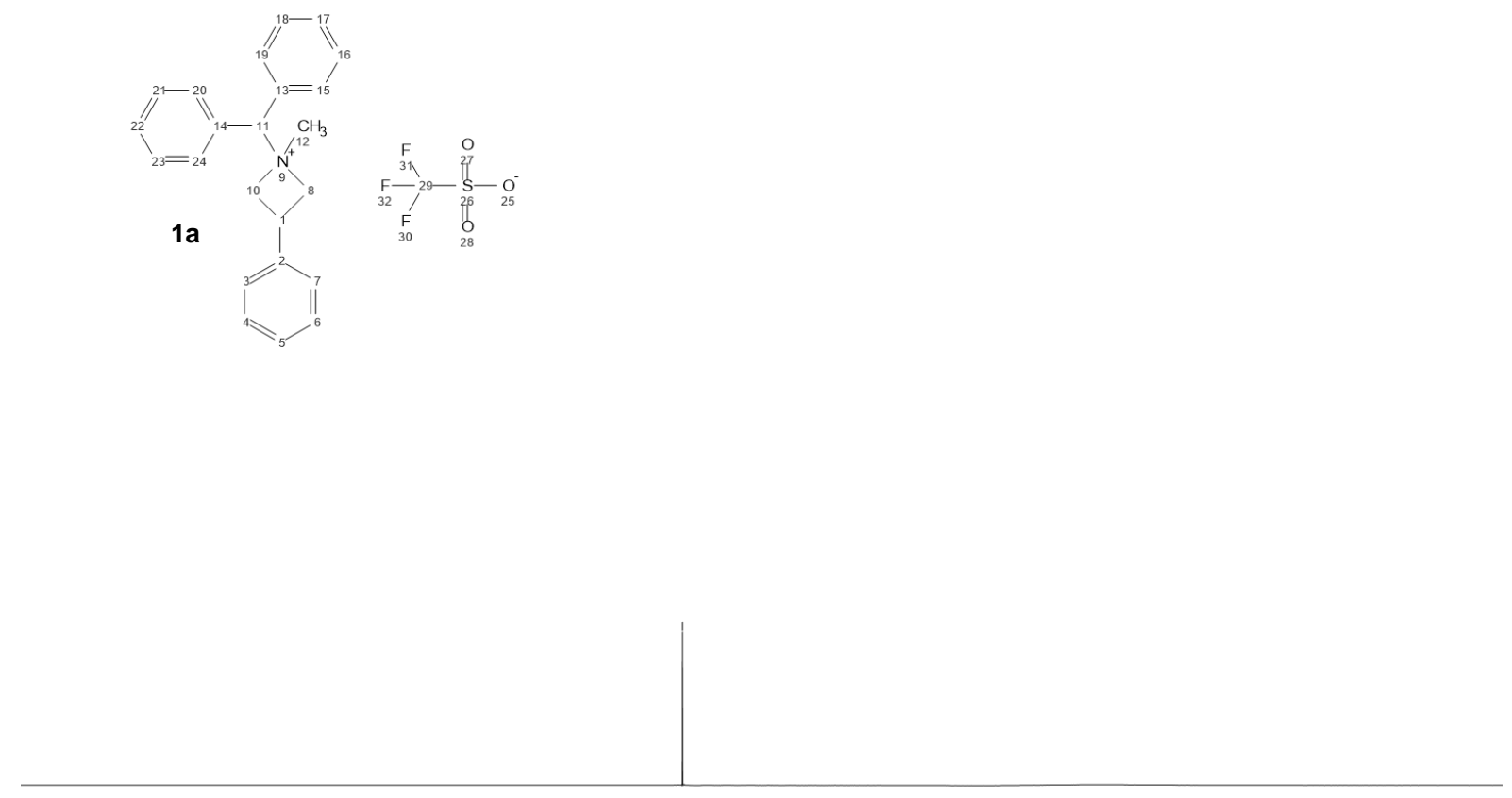

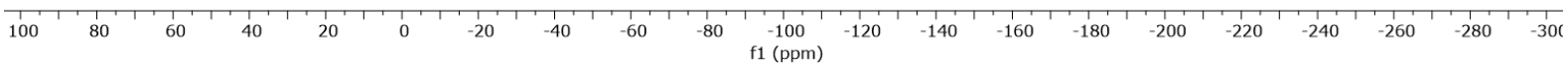



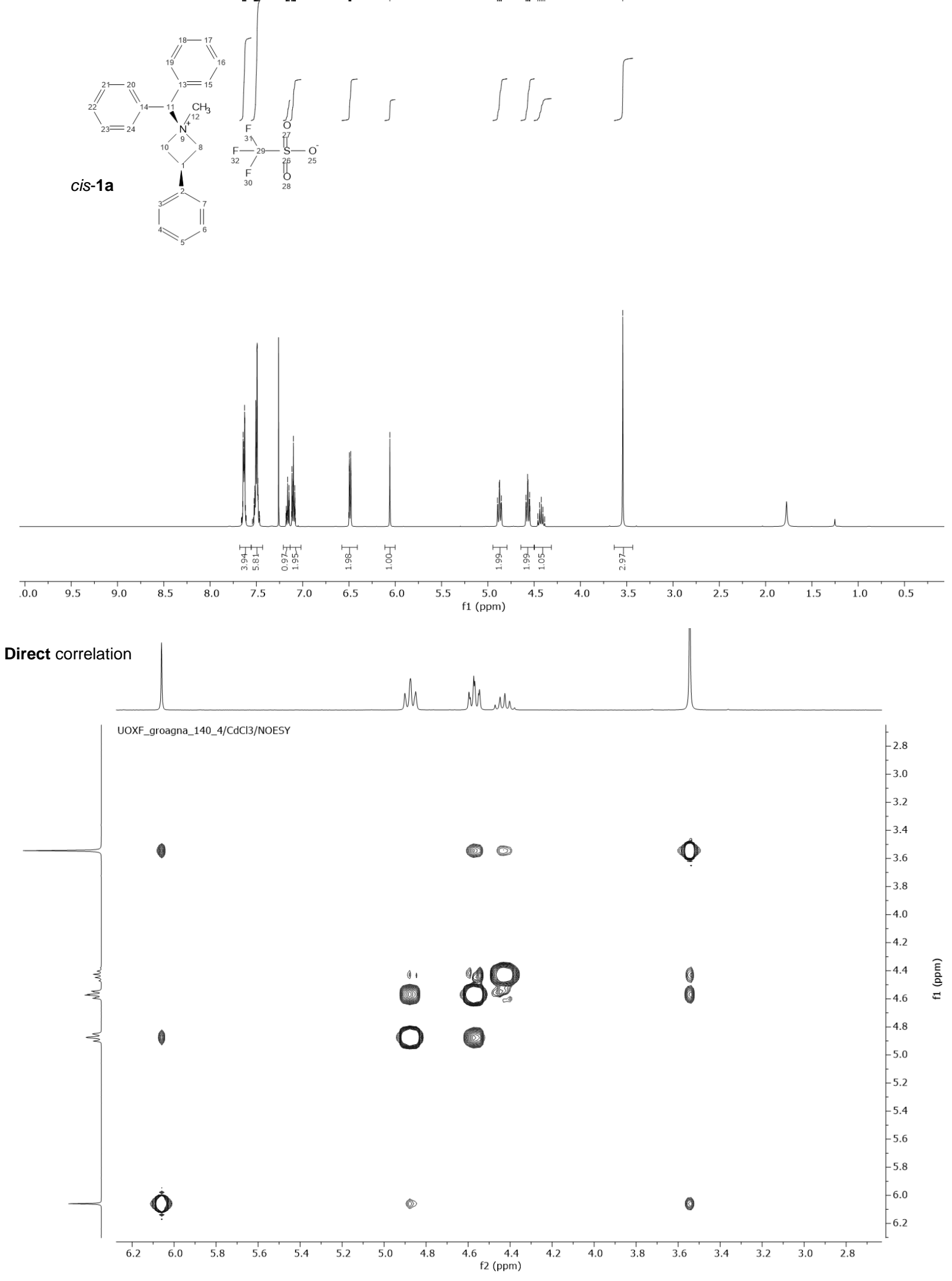


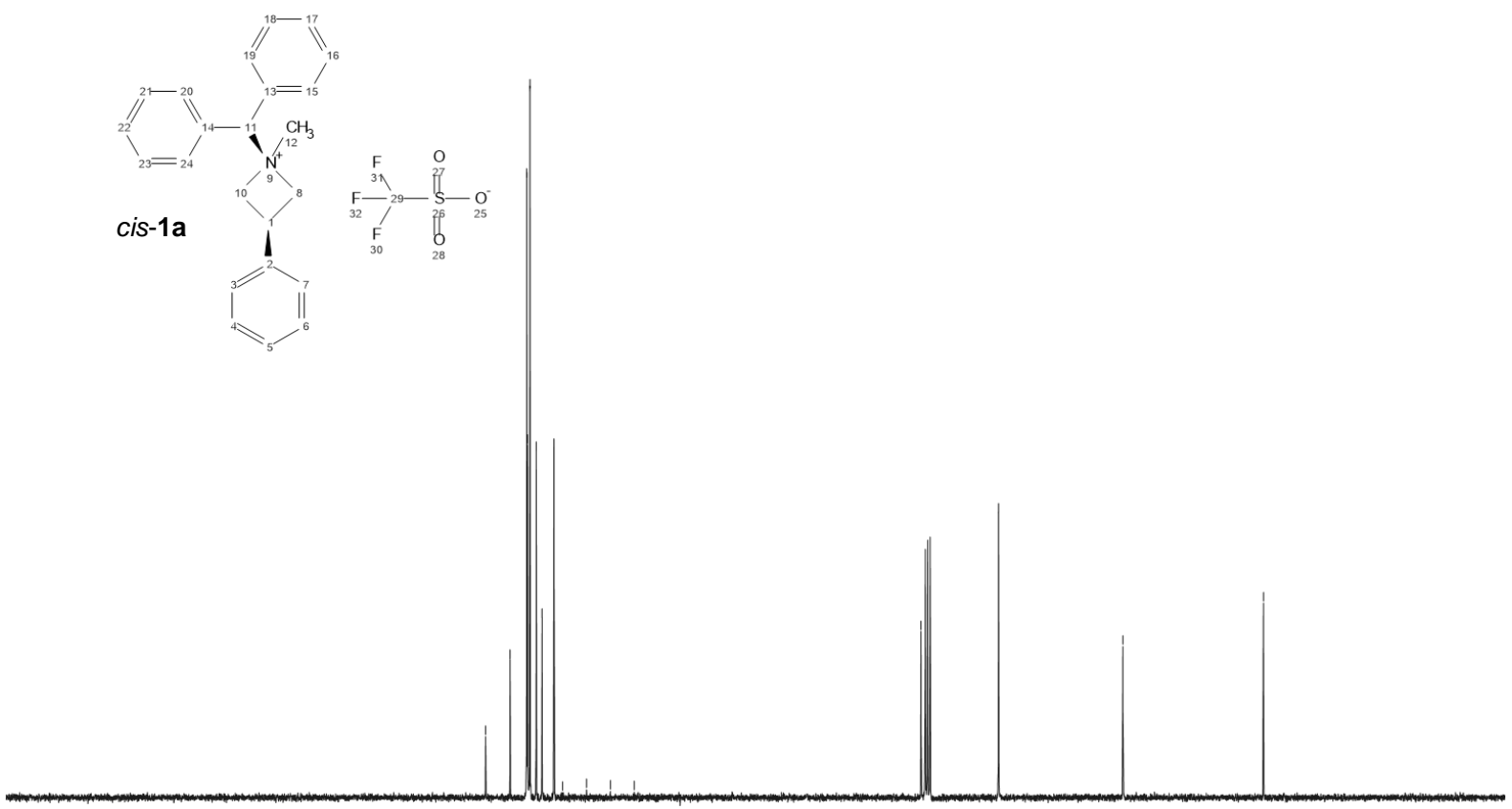

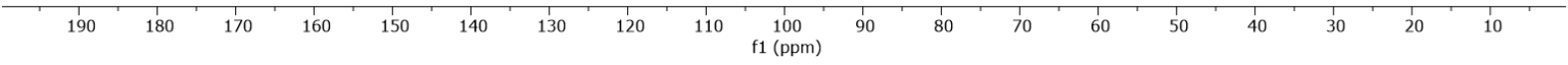

UOXF_groagna_140_4/CdCl3
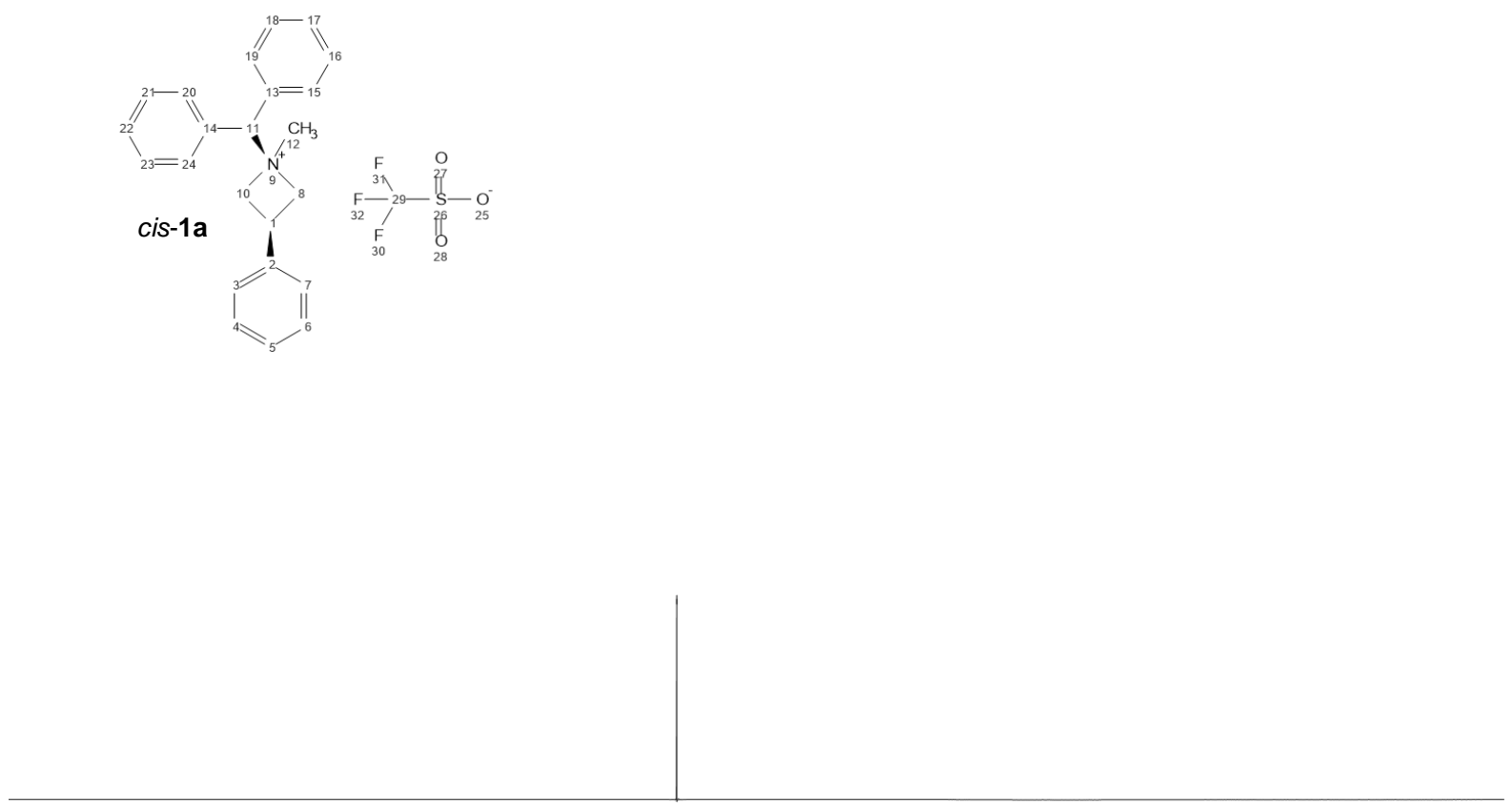

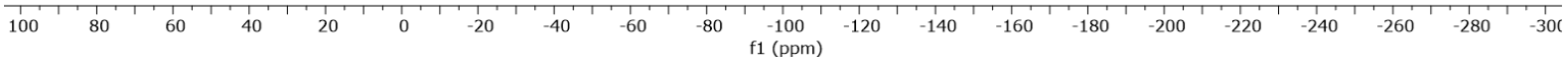



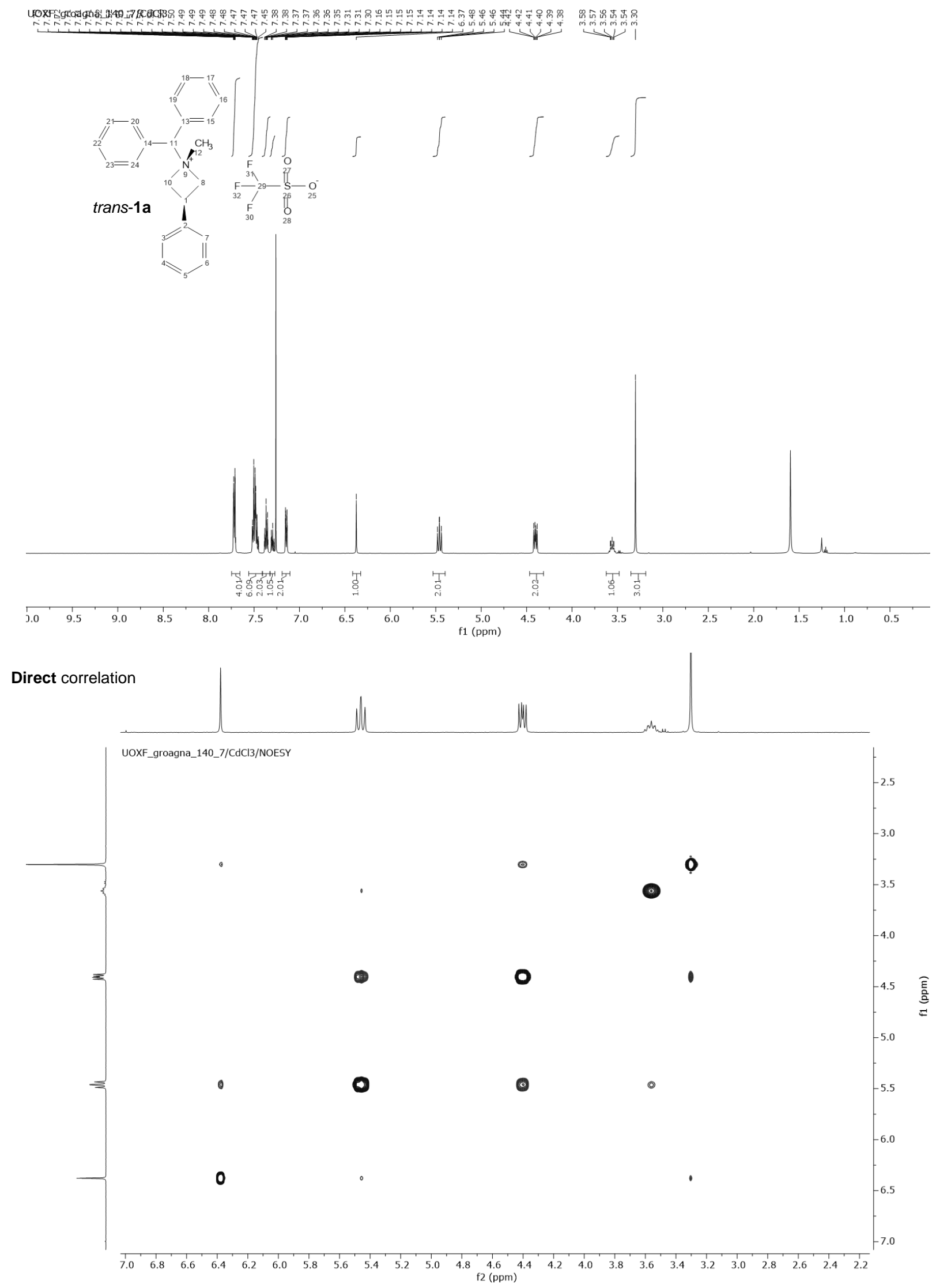
은

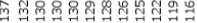

$1 \times$
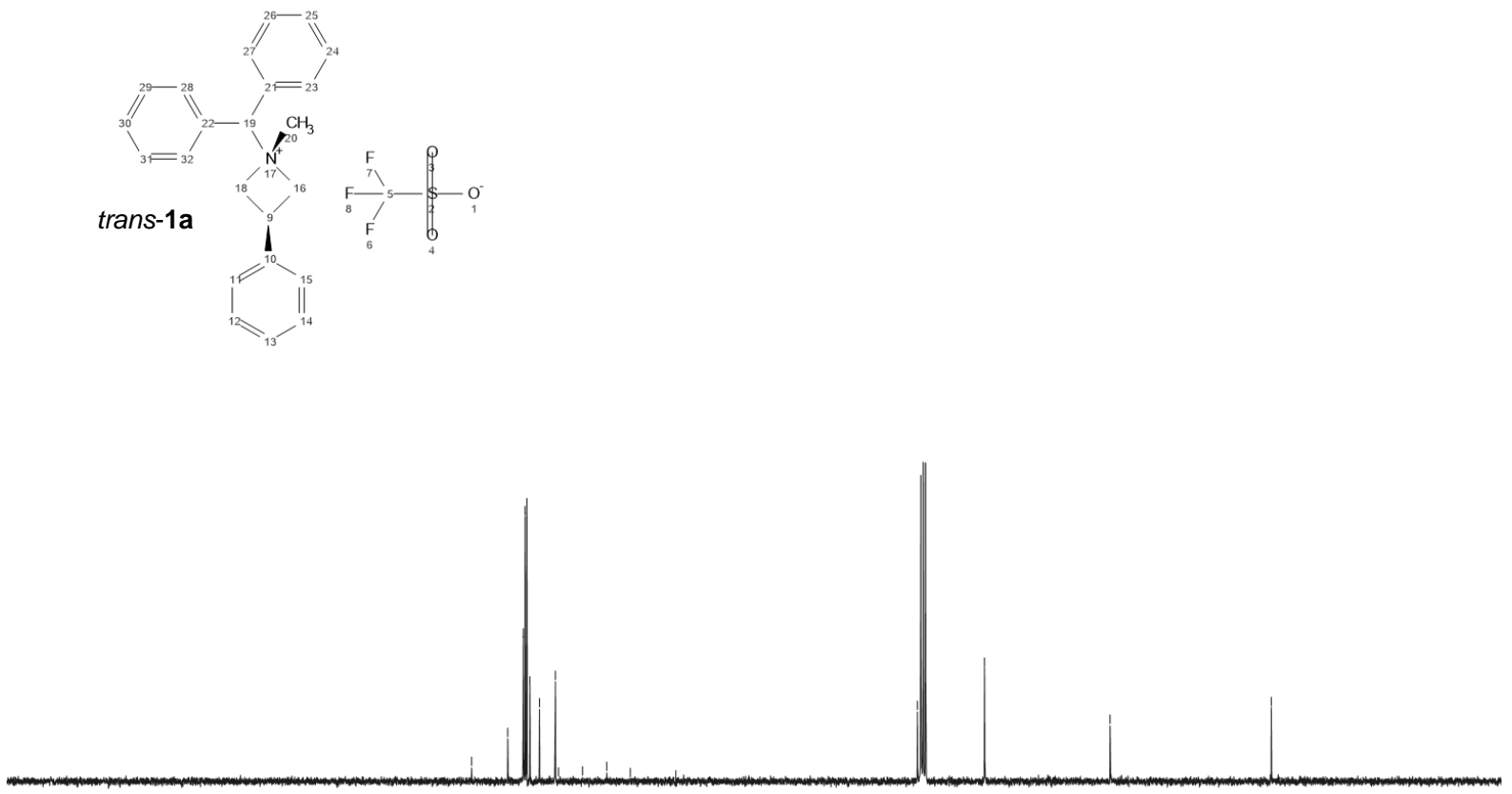

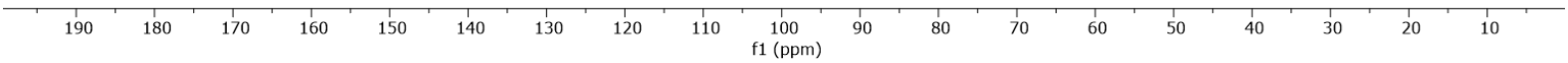

UOXF_groagna_140_7/CdCl3
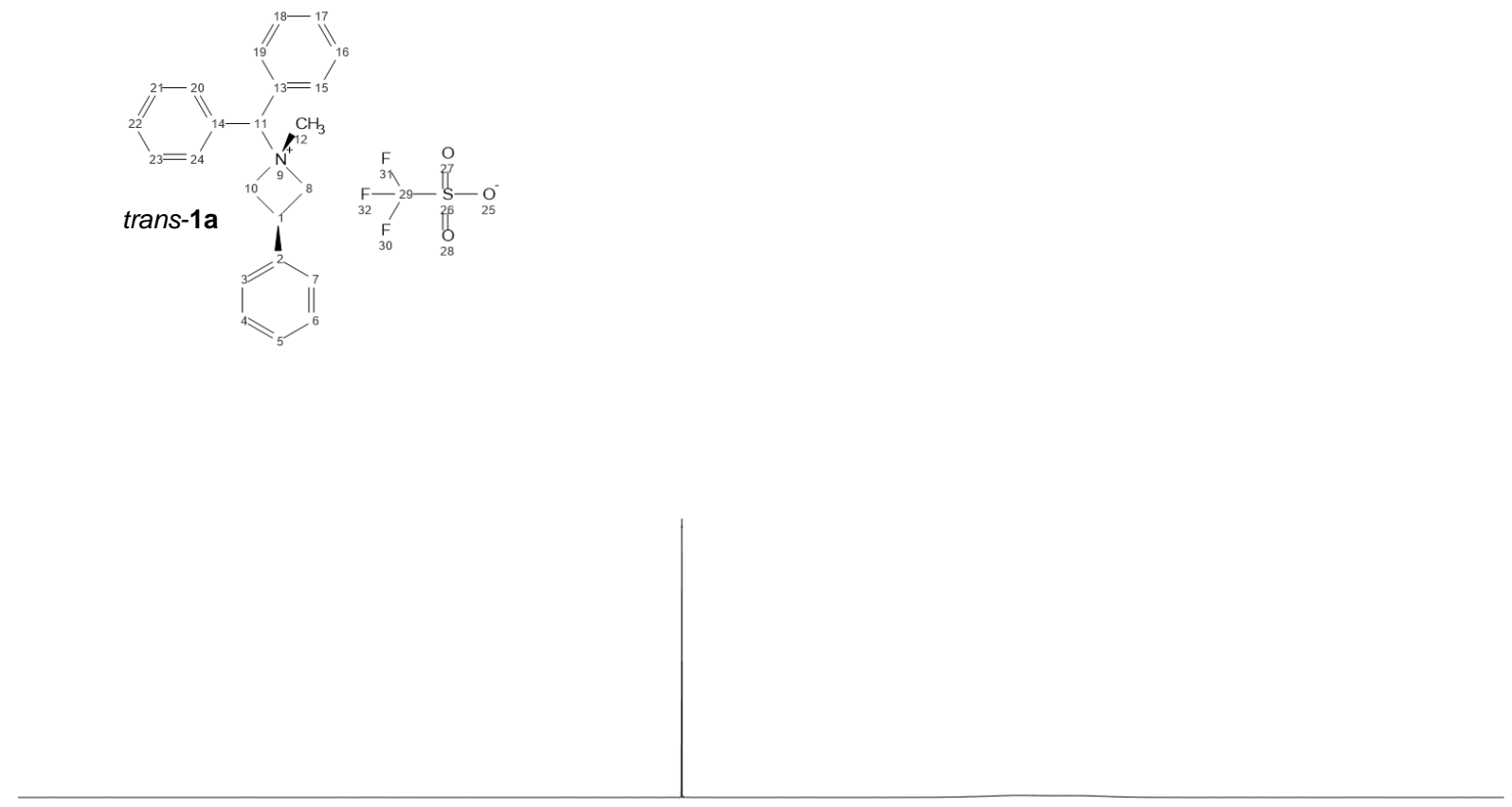

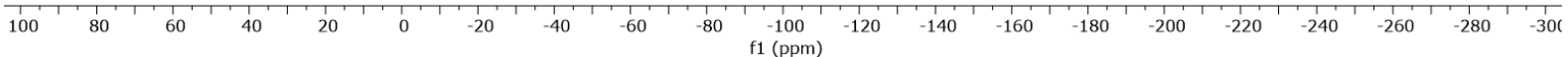




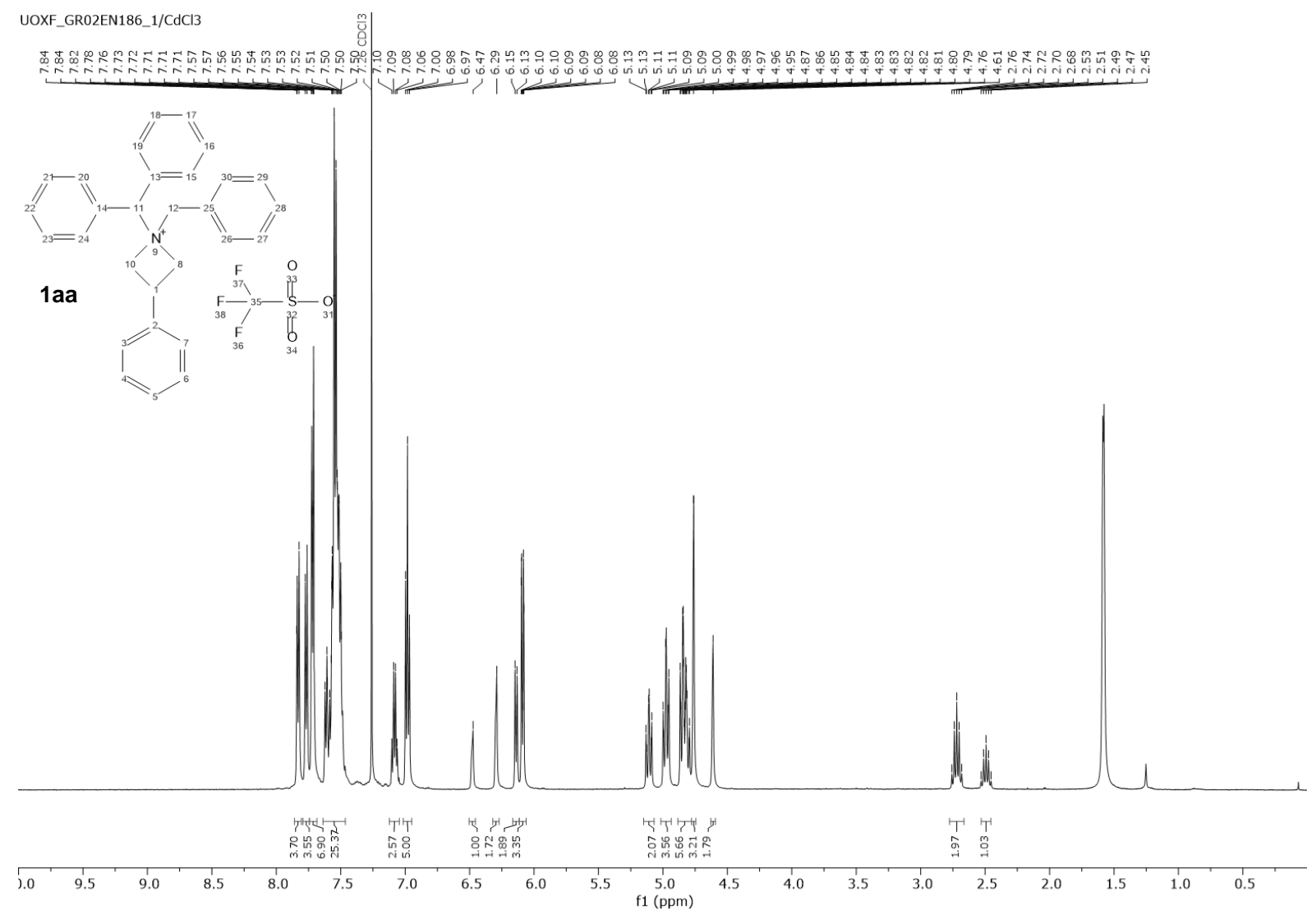

Direct and Indirect correlation

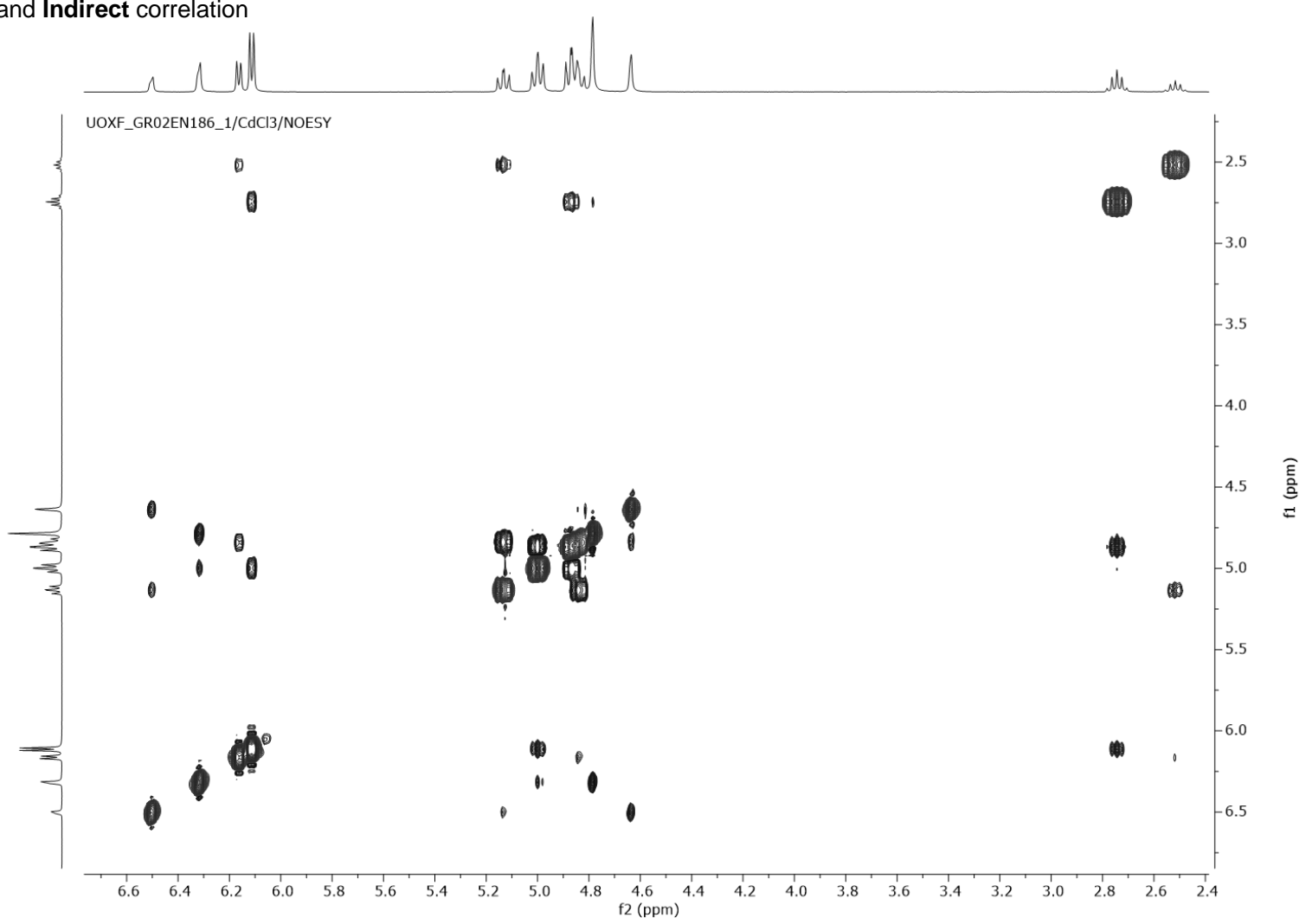



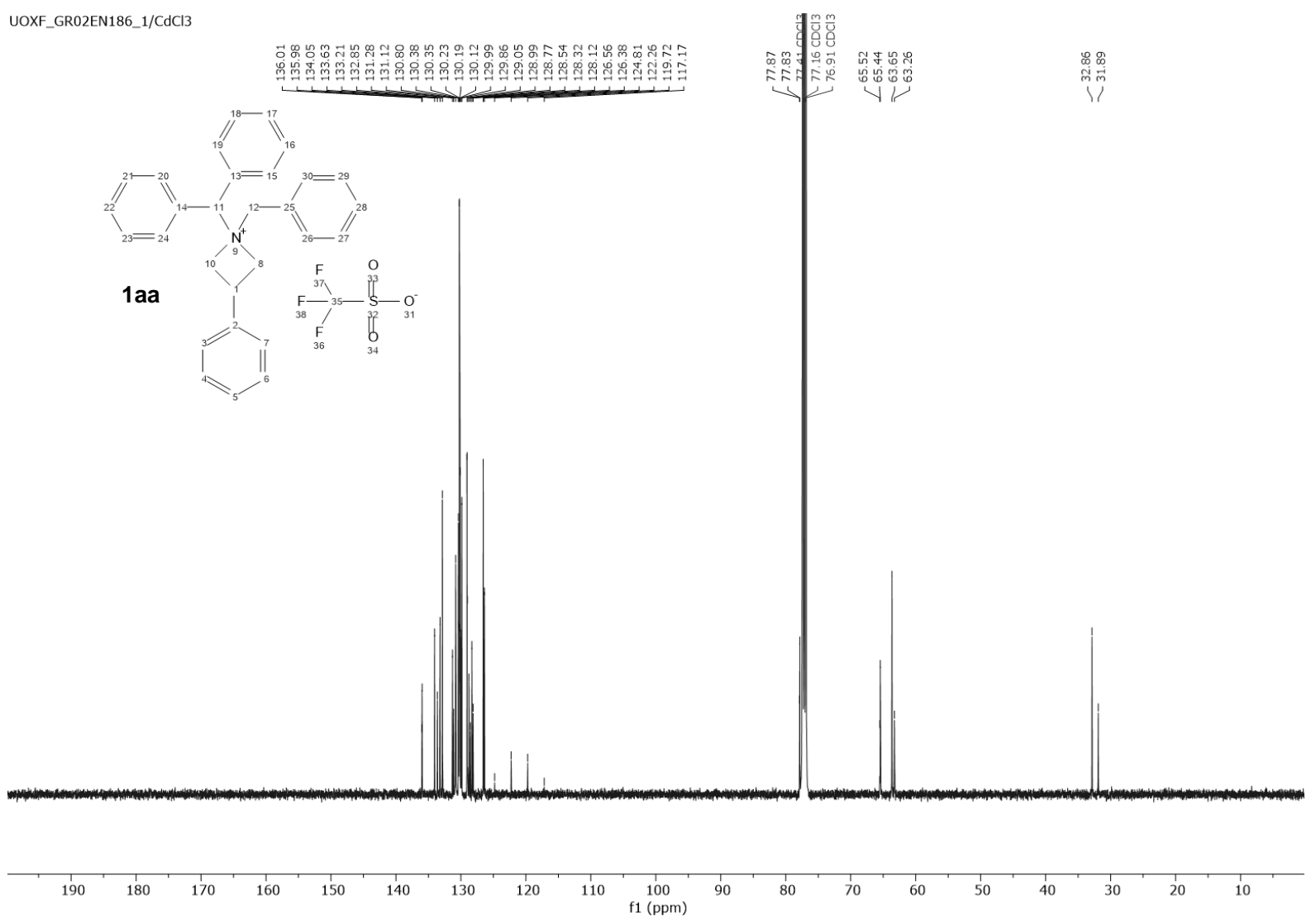

UOXF_groagna_186_1/CdCl3
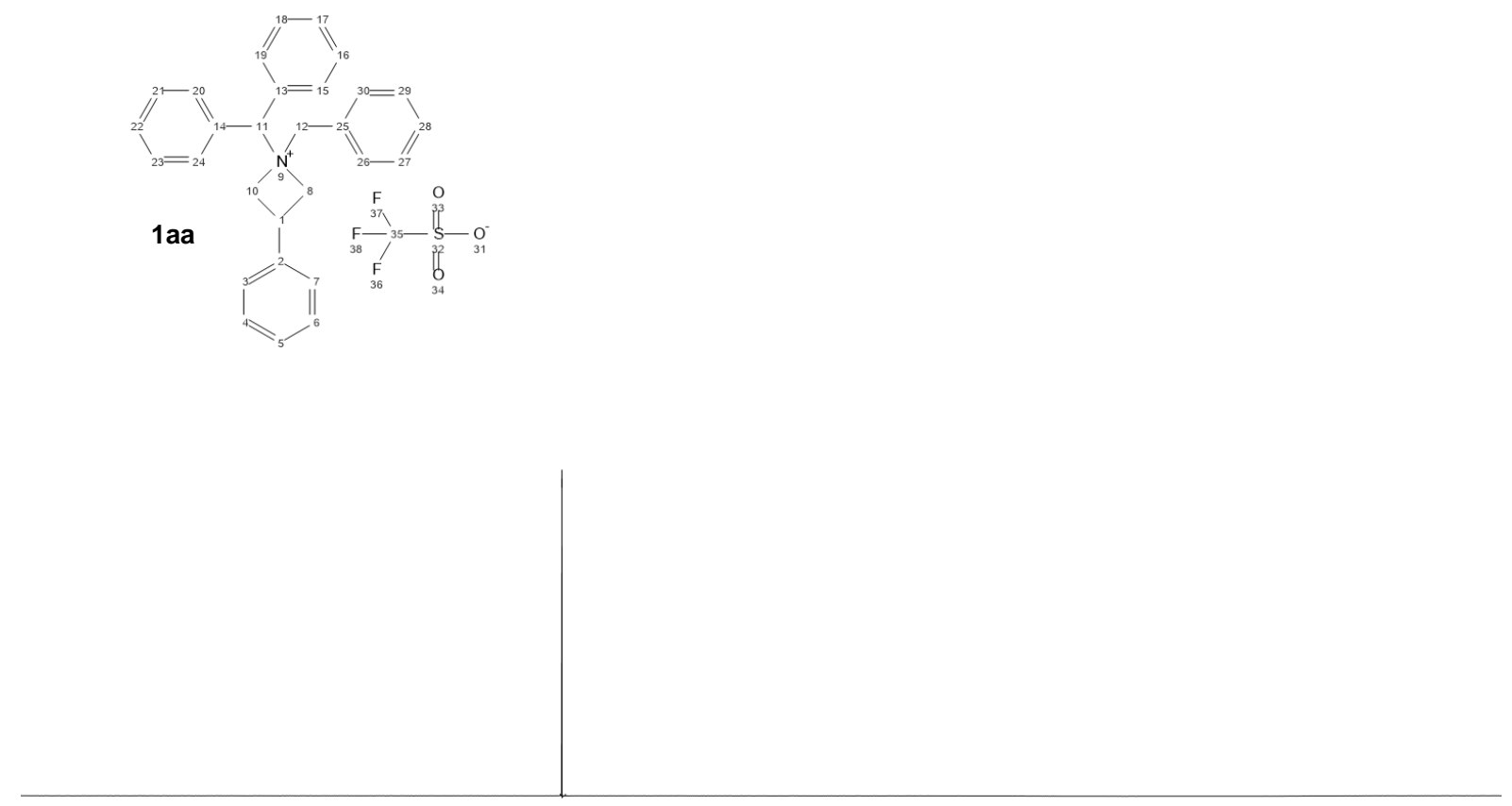

\begin{tabular}{lllllllllllllllllllllllll}
\hline & 1 & -10 & -20 & -30 & -40 & -50 & -60 & -70 & -80 & -90 & -100 & -110 & -120 & -130 & -140 & -150 & -160 & -170 & -180 & -190 & -200 & -210 & -220
\end{tabular} 

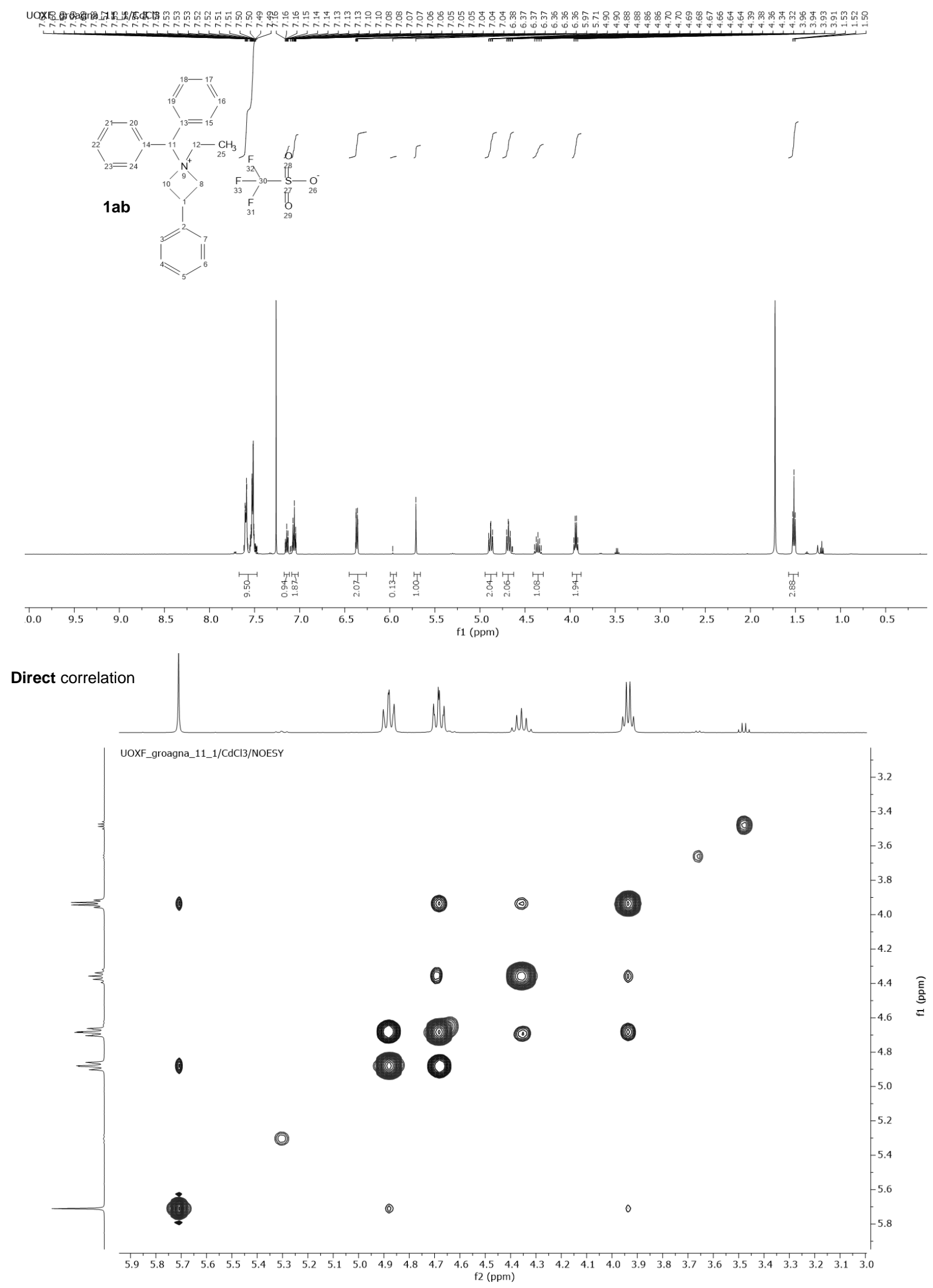


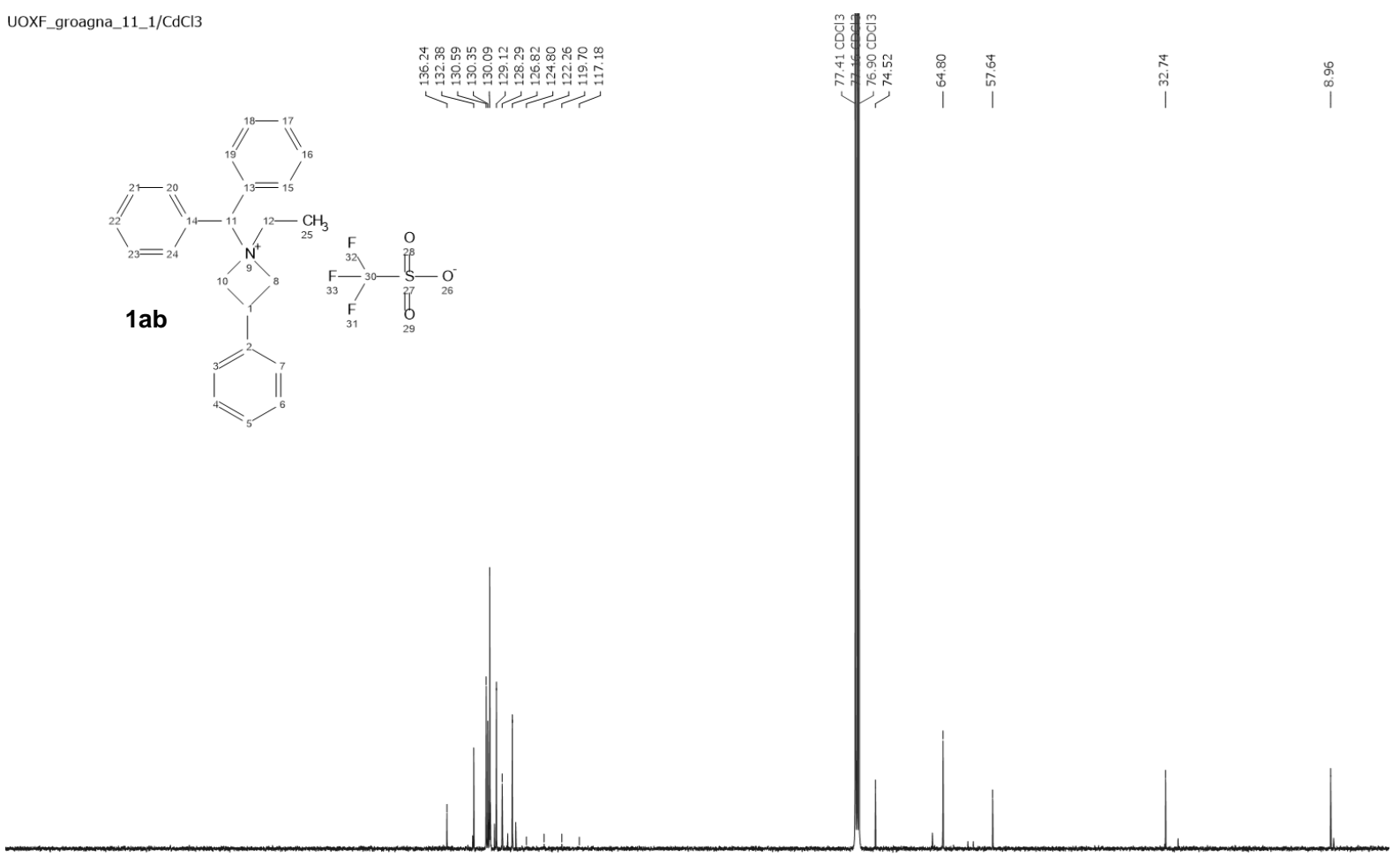

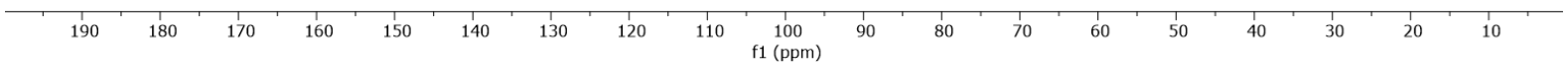

UOXF_groagna_11_1/CdCl3

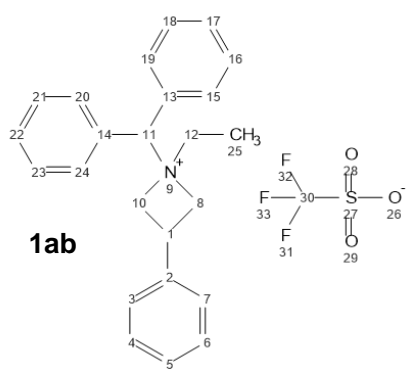

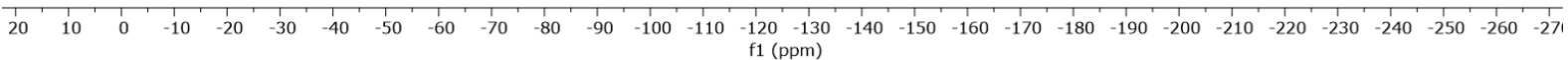


UOXF_groagna_116_1/CdCl3

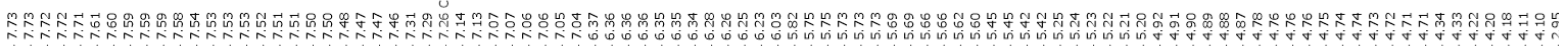
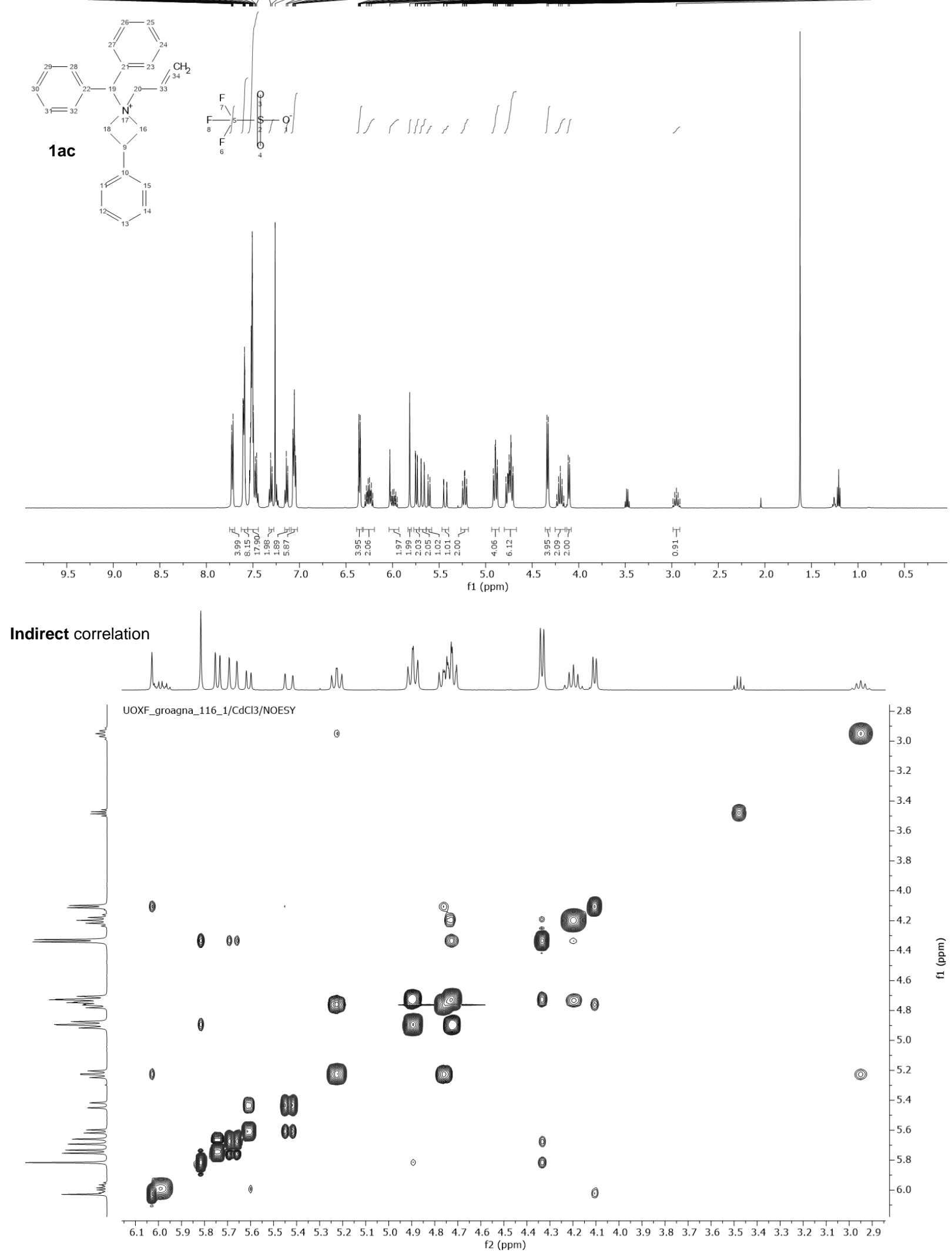

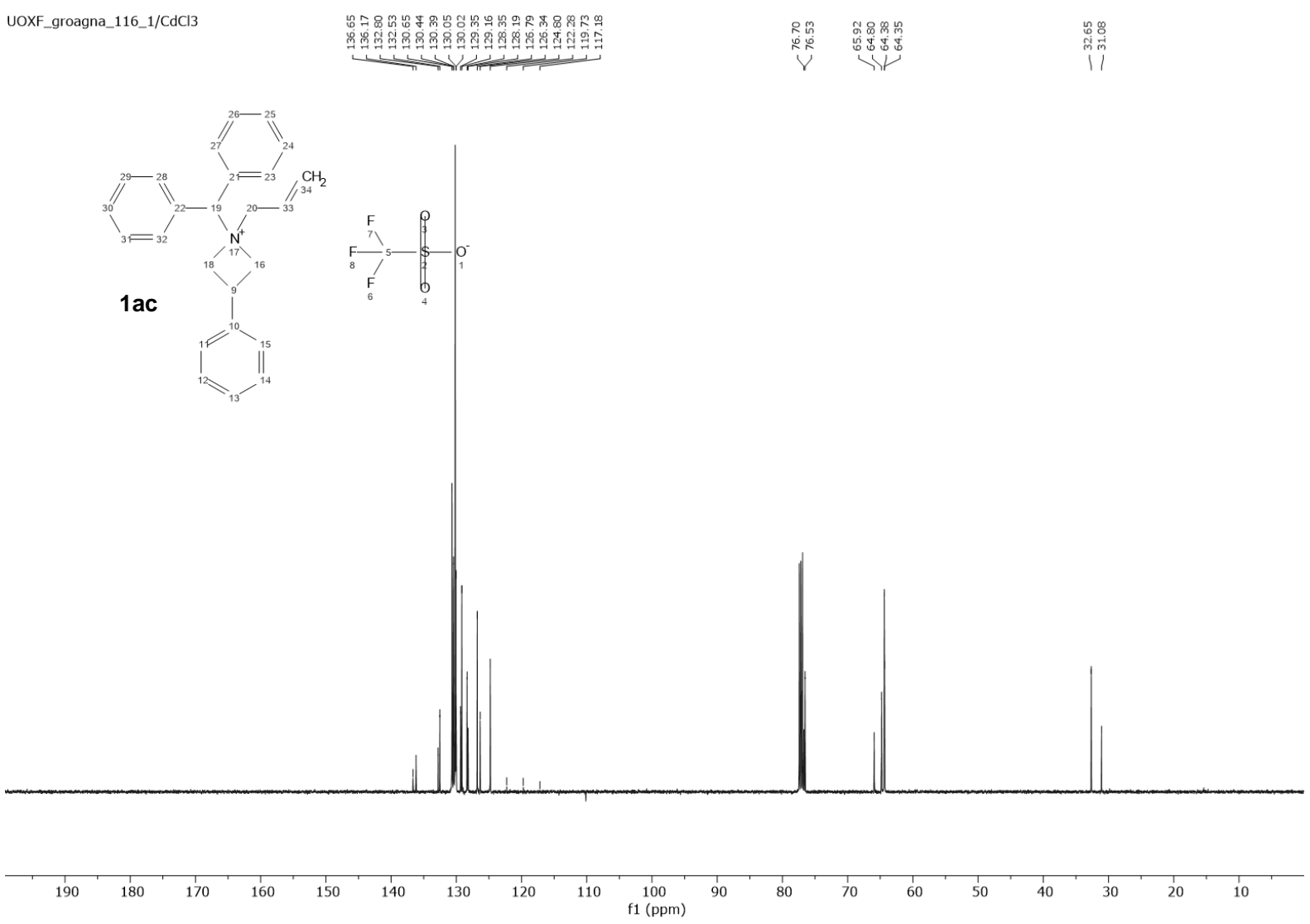

UOXF_groagna_116_1/CdCl3
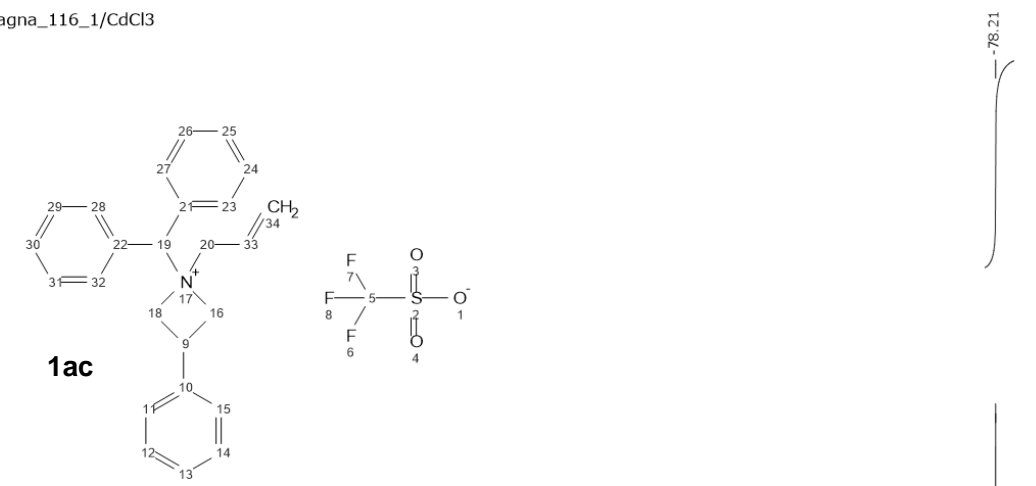

's

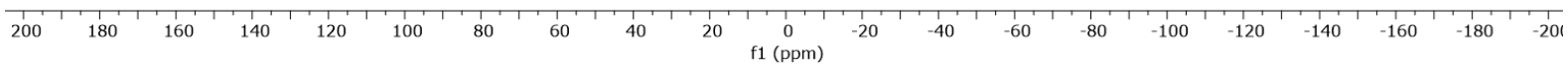



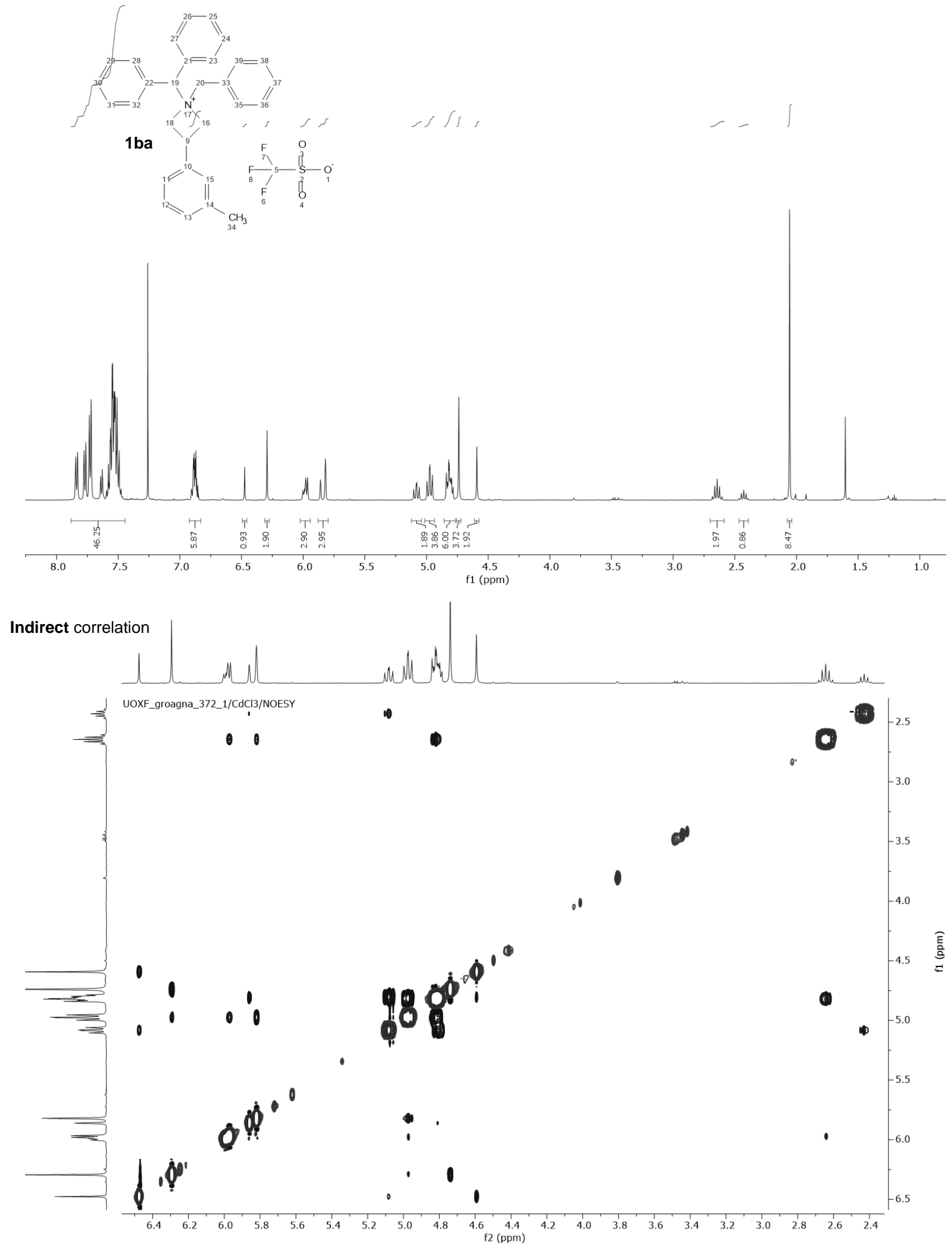

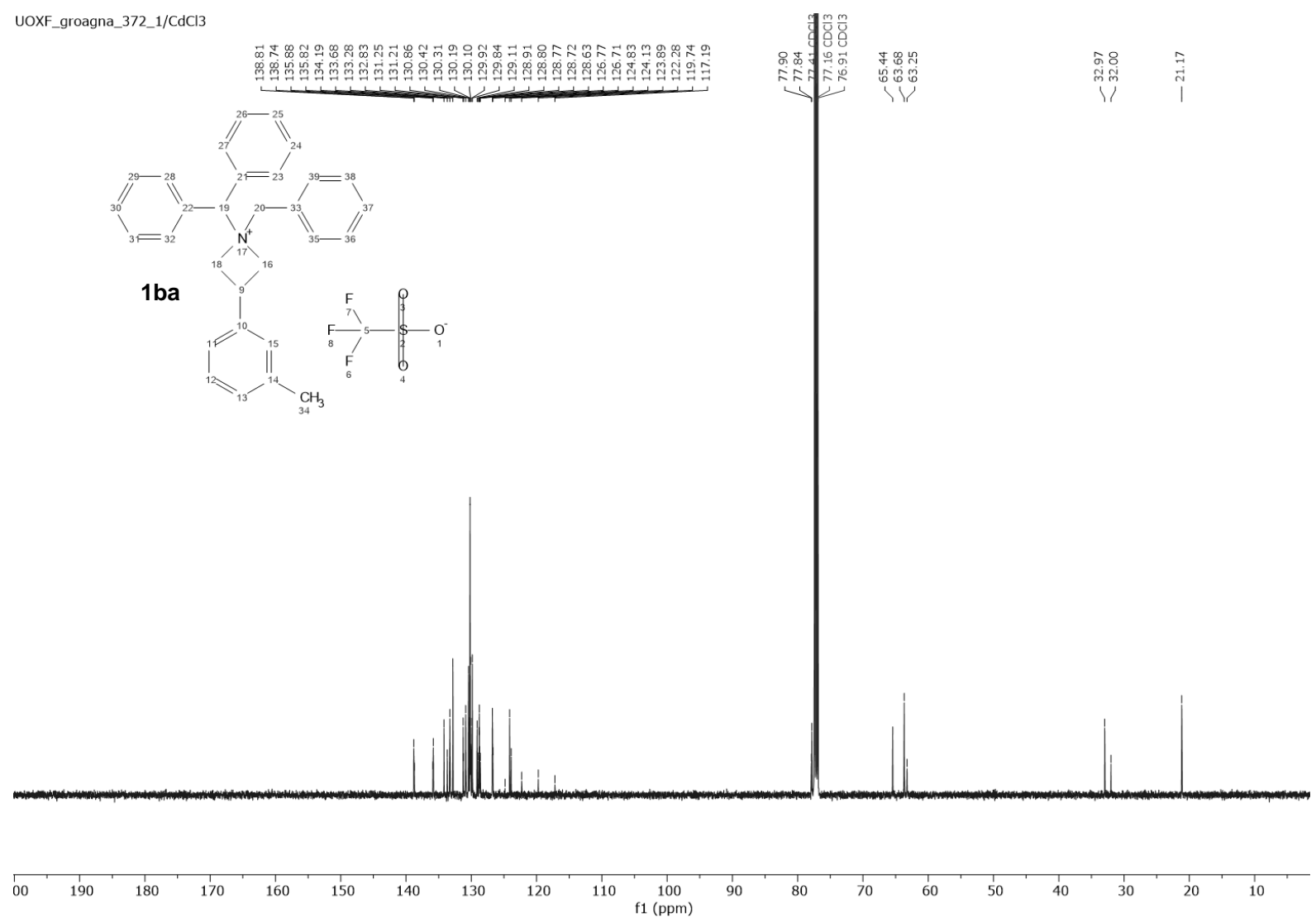

UOXF_groagna_372_1/CdCl3
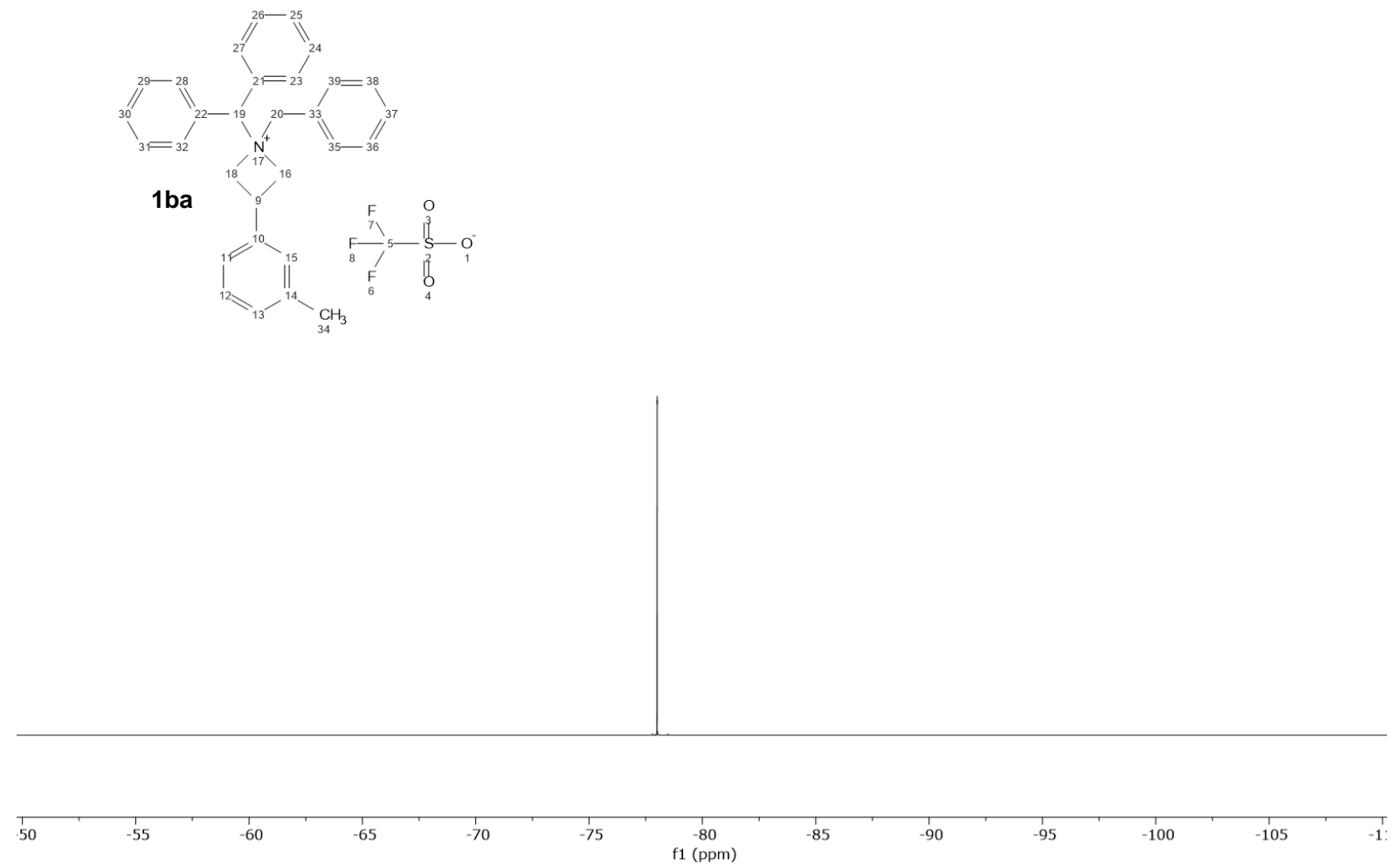
UOXF_groagna_373_1/CdCl3

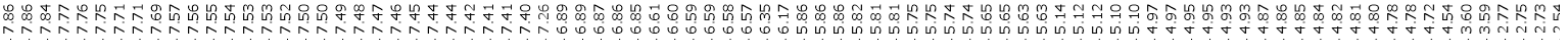

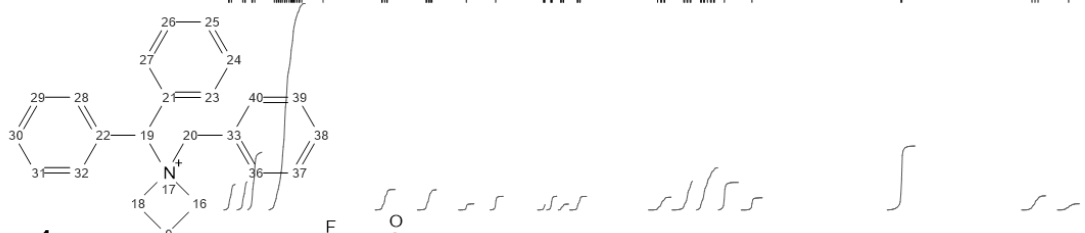

$1 \mathrm{ca}$
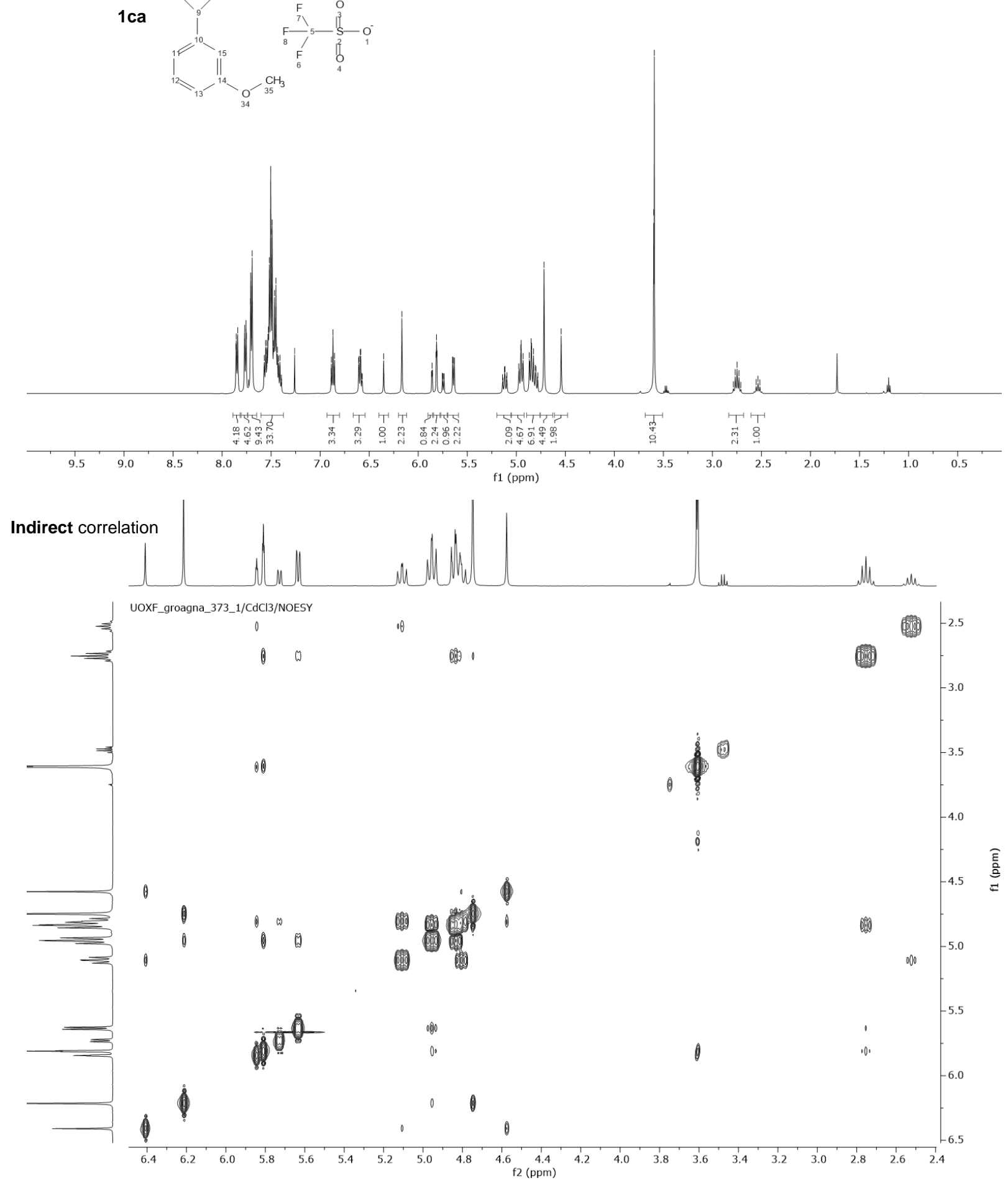

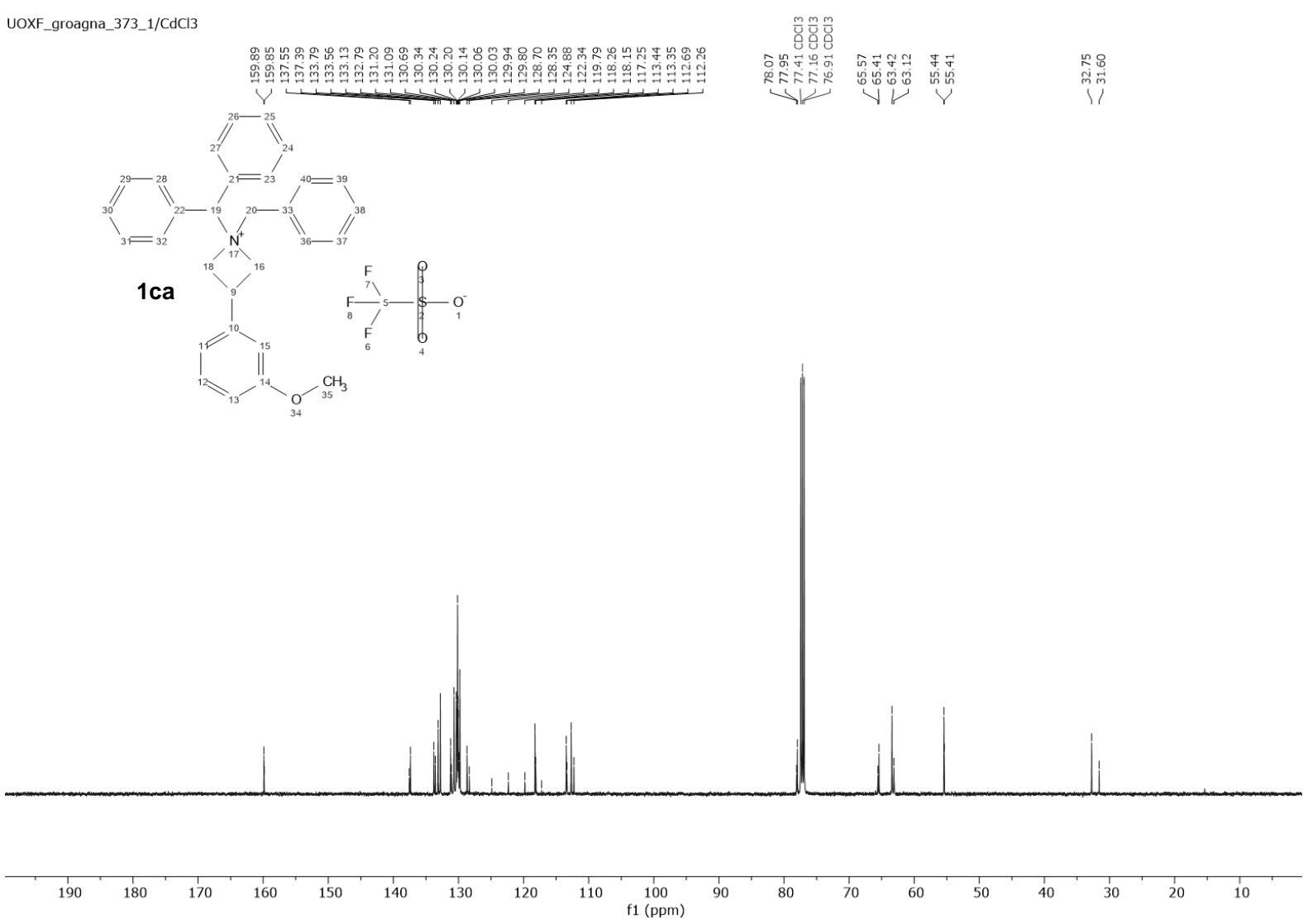

UOXF_groagna_373_1/CdCl3

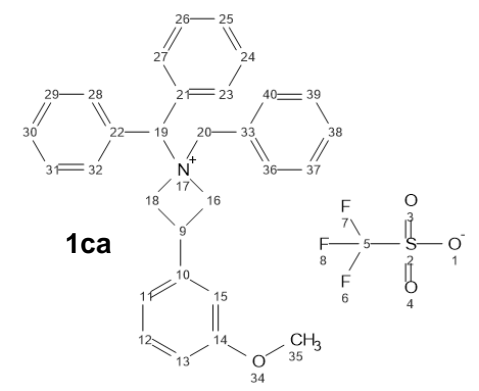

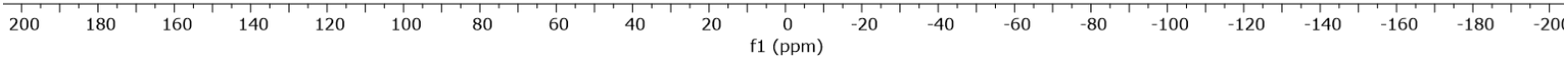




\section{UOXF_groagna_370_1/CdCl3}

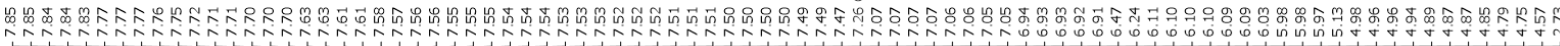
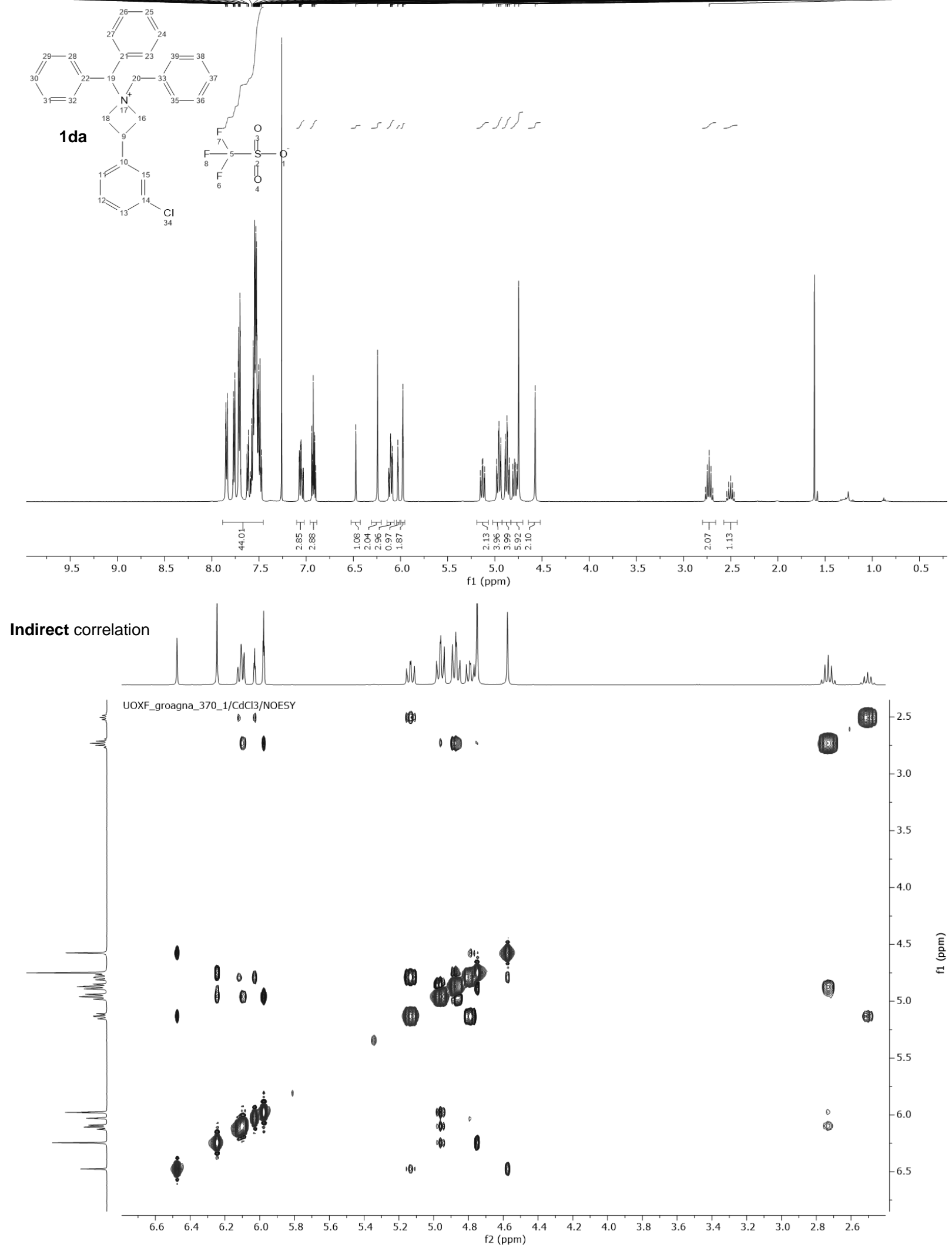

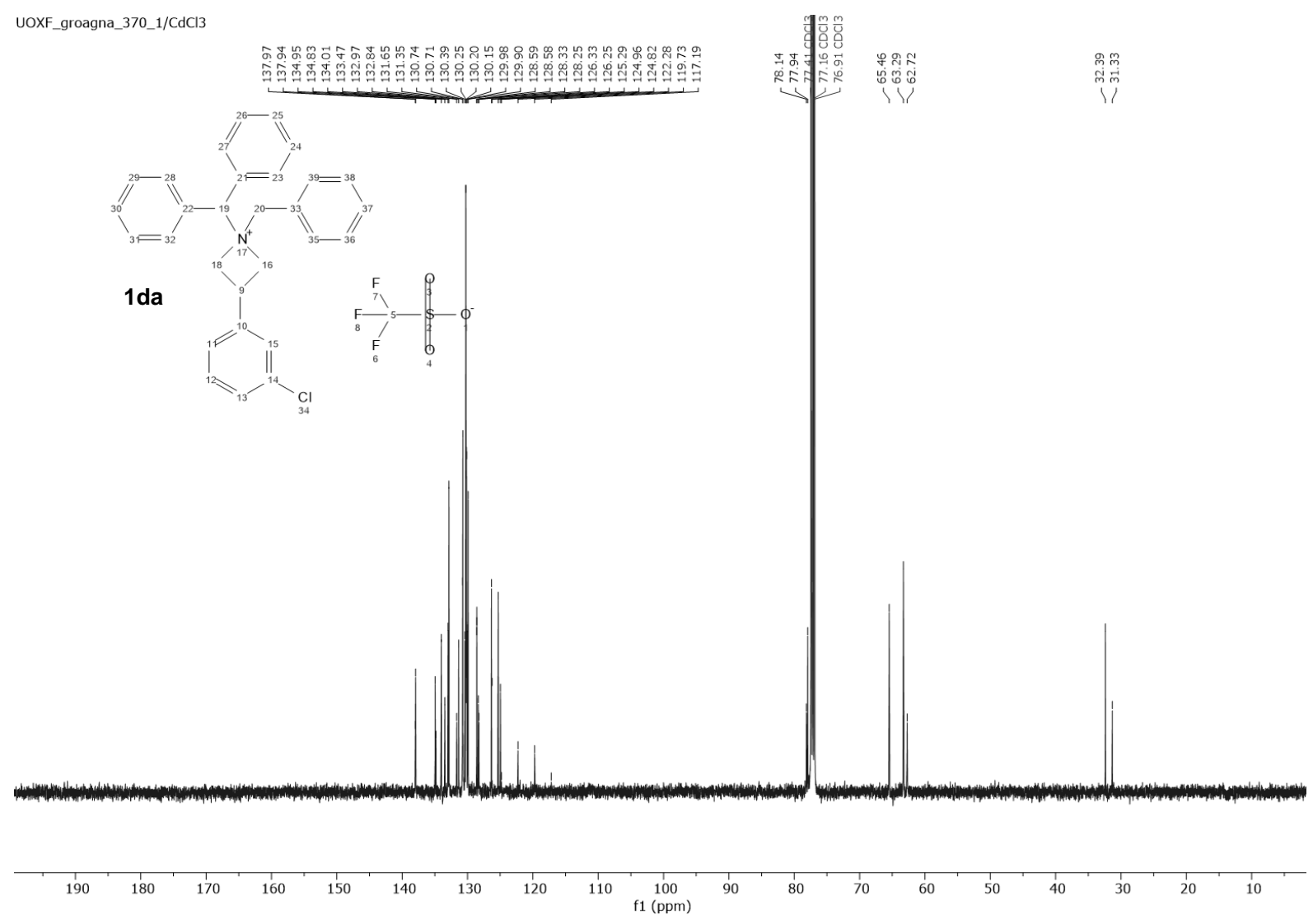

UOXF_groagna_370_1/CdCl3
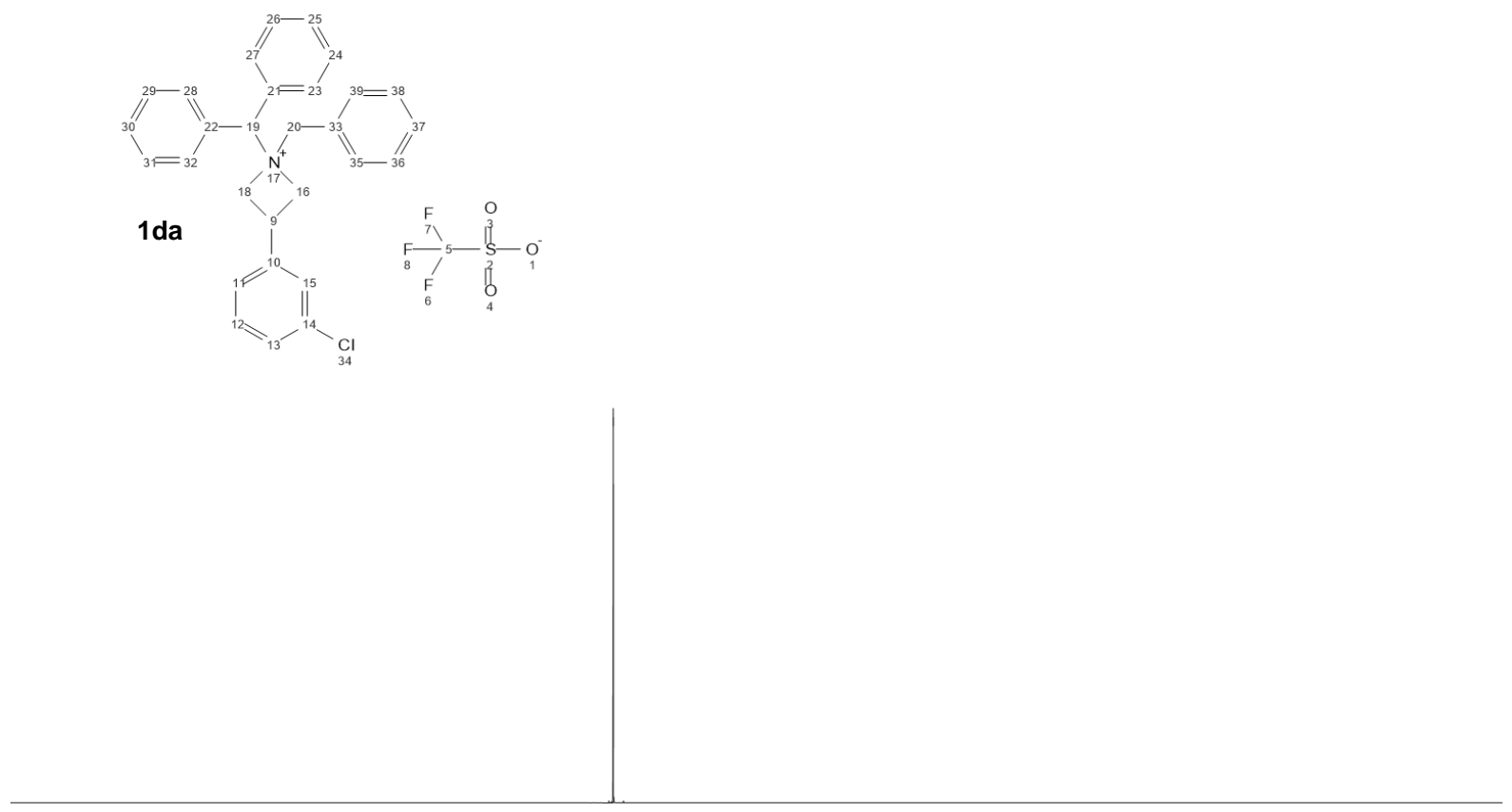

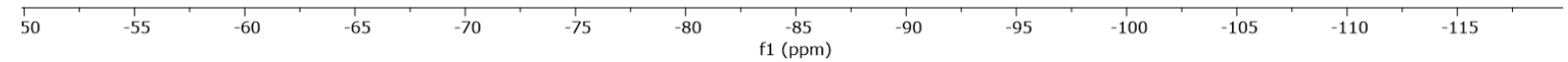




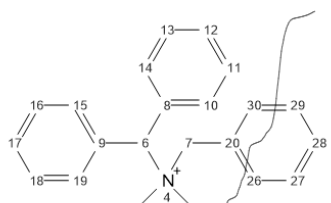

1ea<smiles>O=S(=O)(O)N(F)C(F)(F)F</smiles>
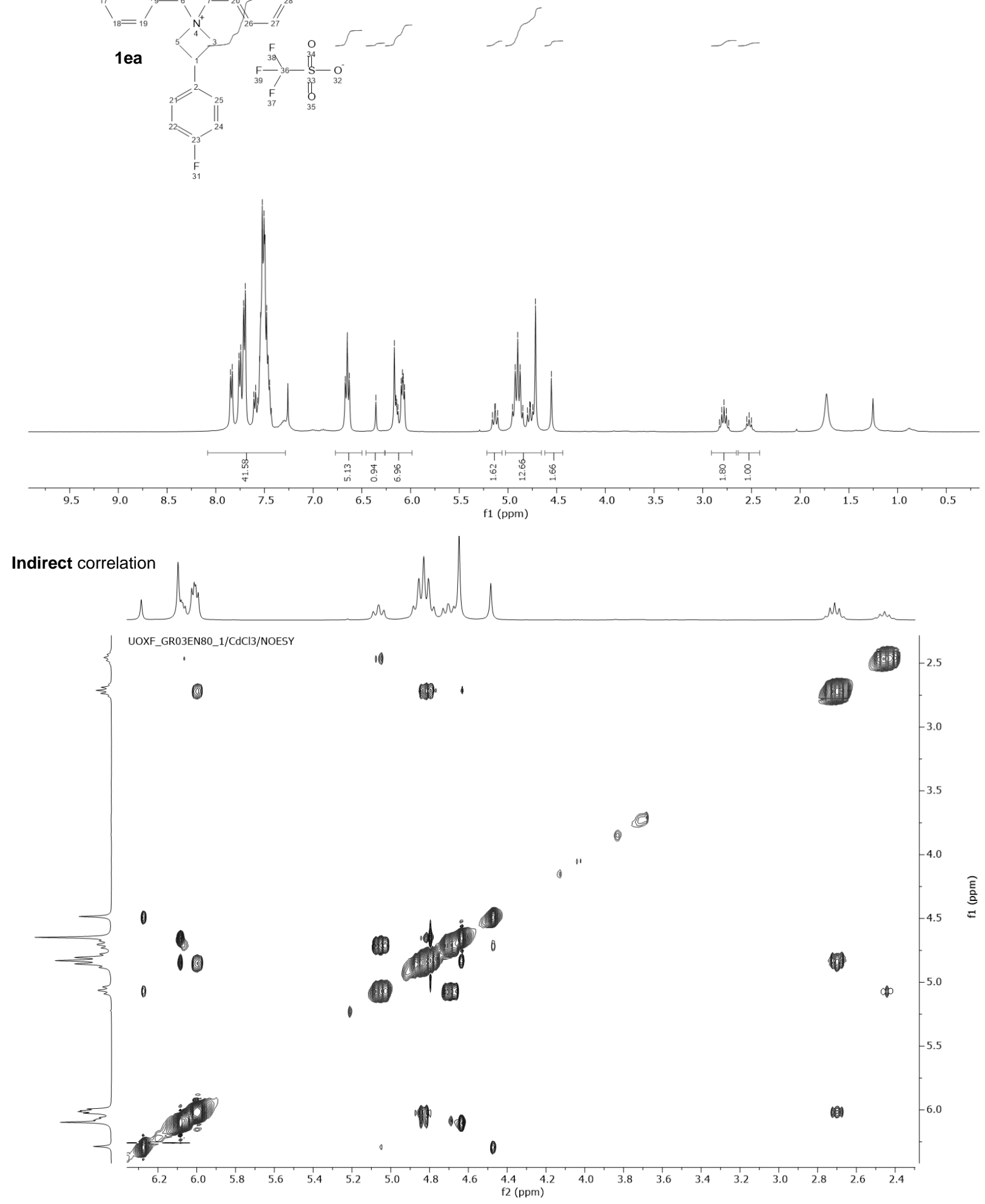

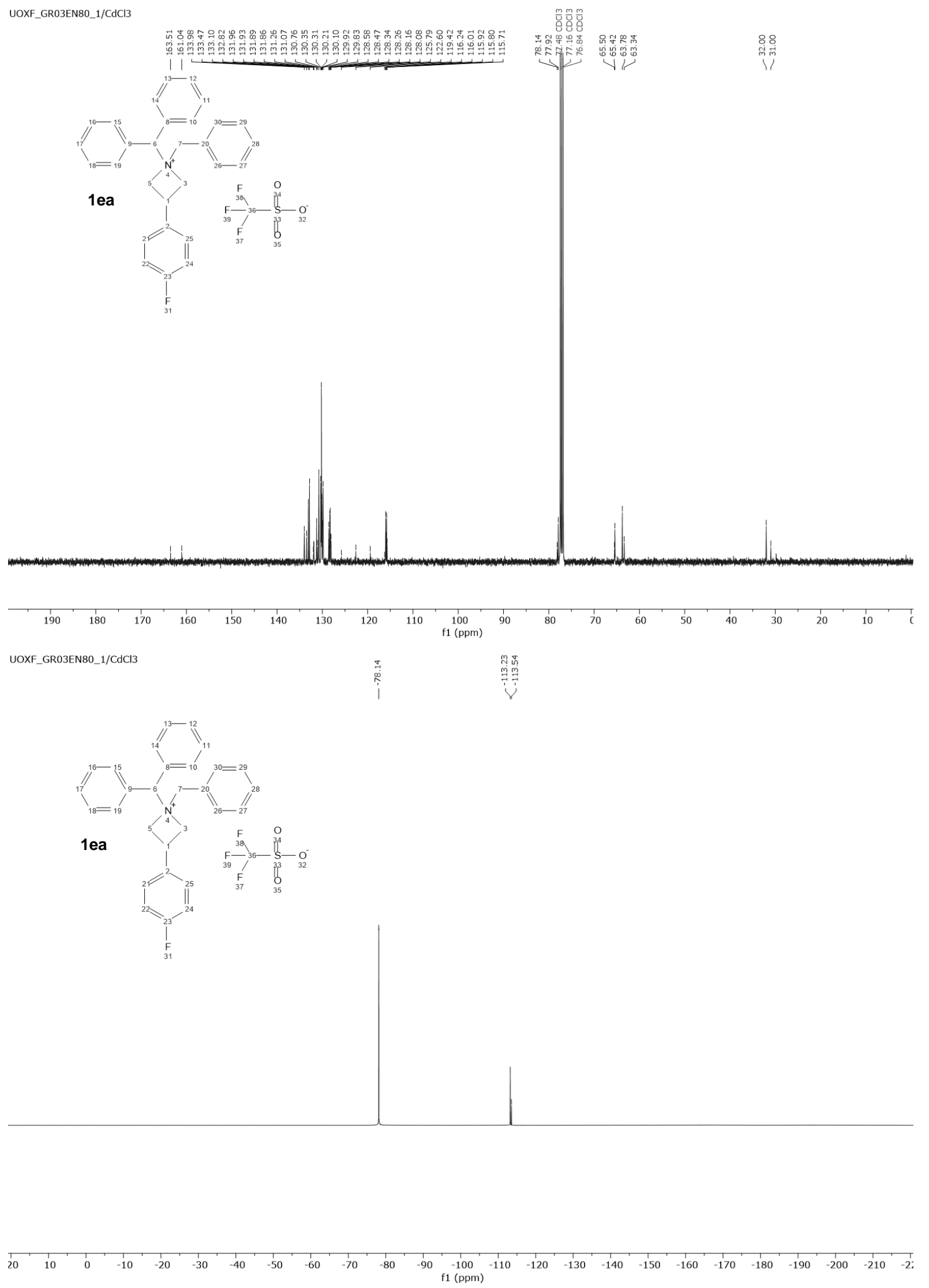

S215 
UOXF_GR03EN85_1/CdCl3

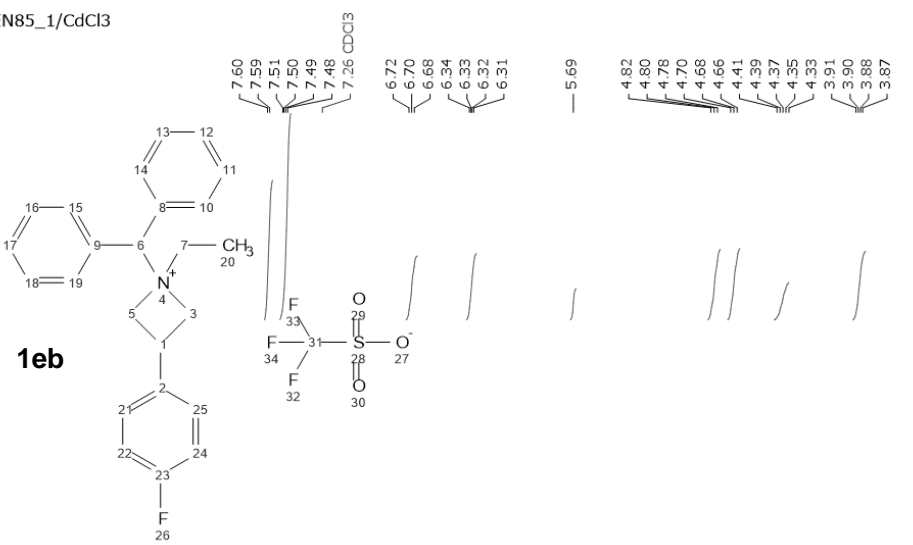

紫早

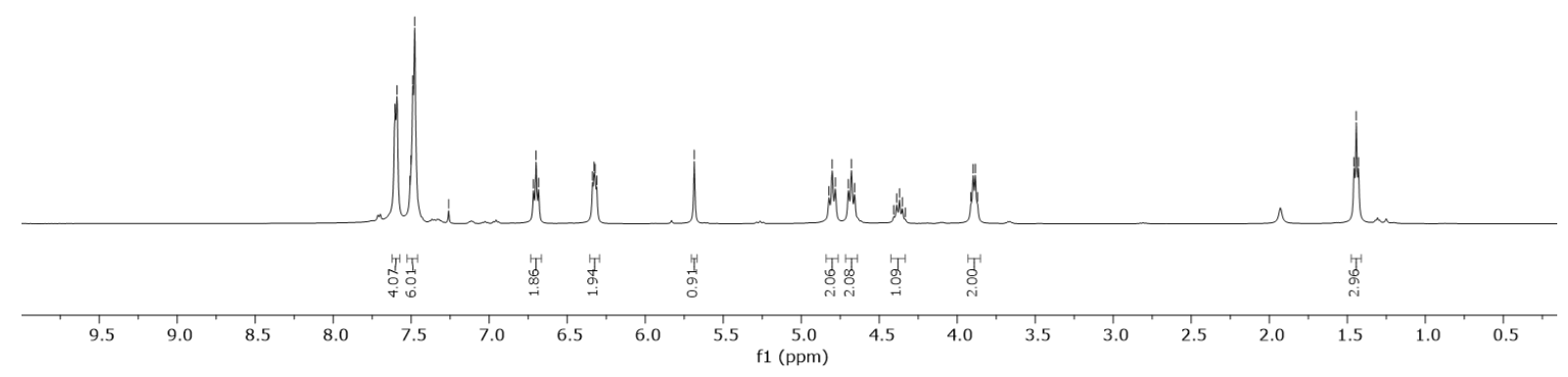

\section{Direct correlation}
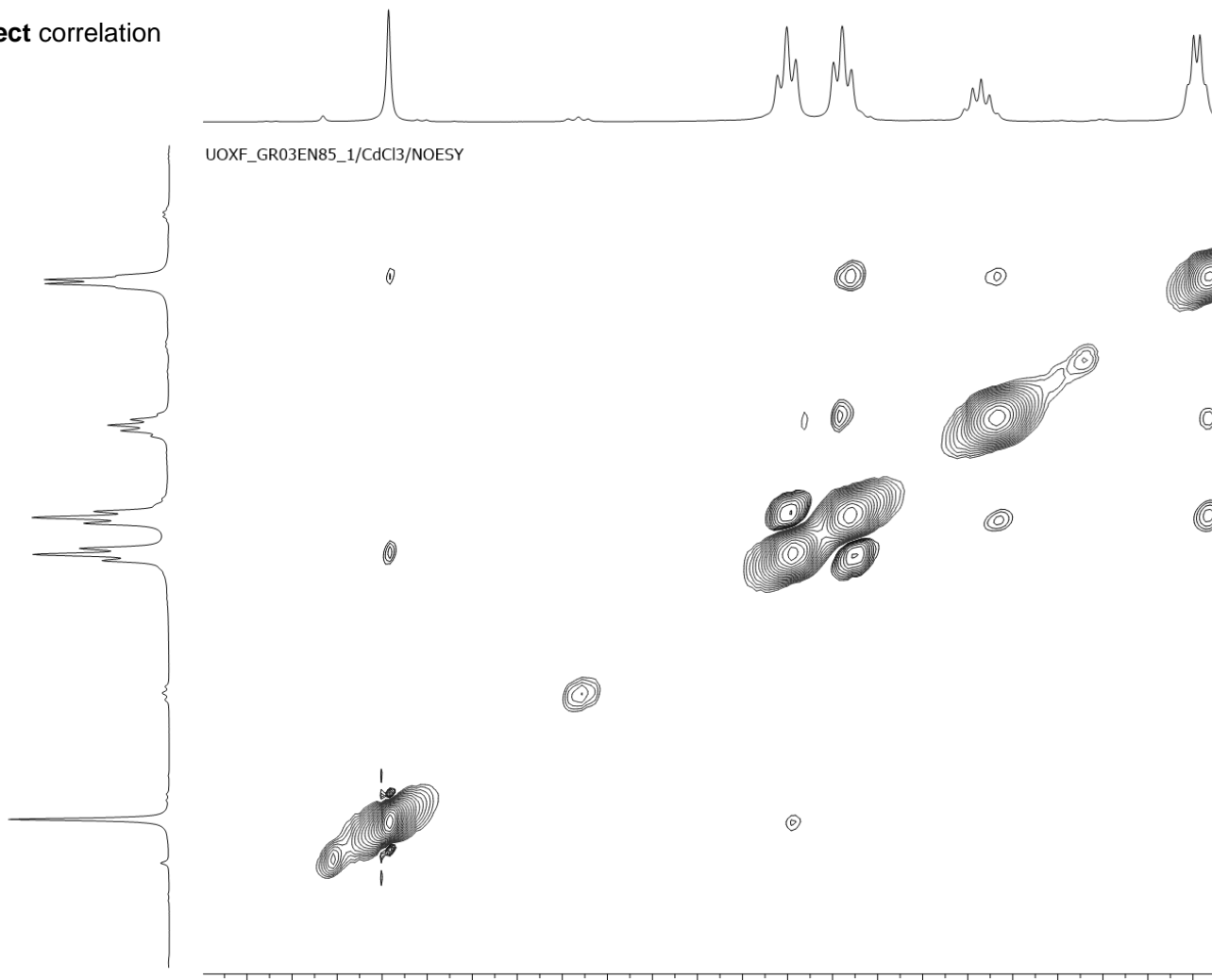

UOXF_GR03EN85_1/CdCI3/NOESY

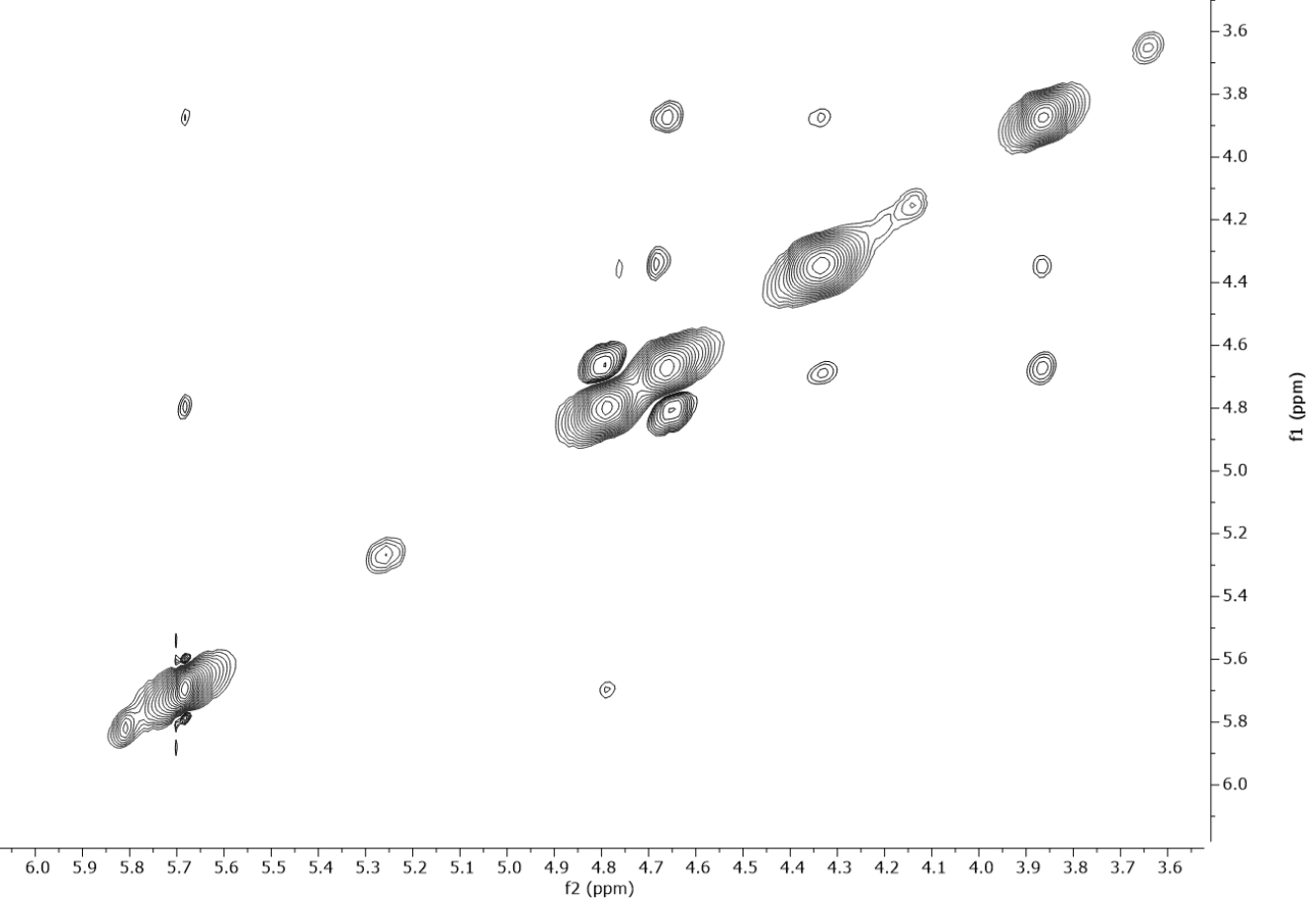




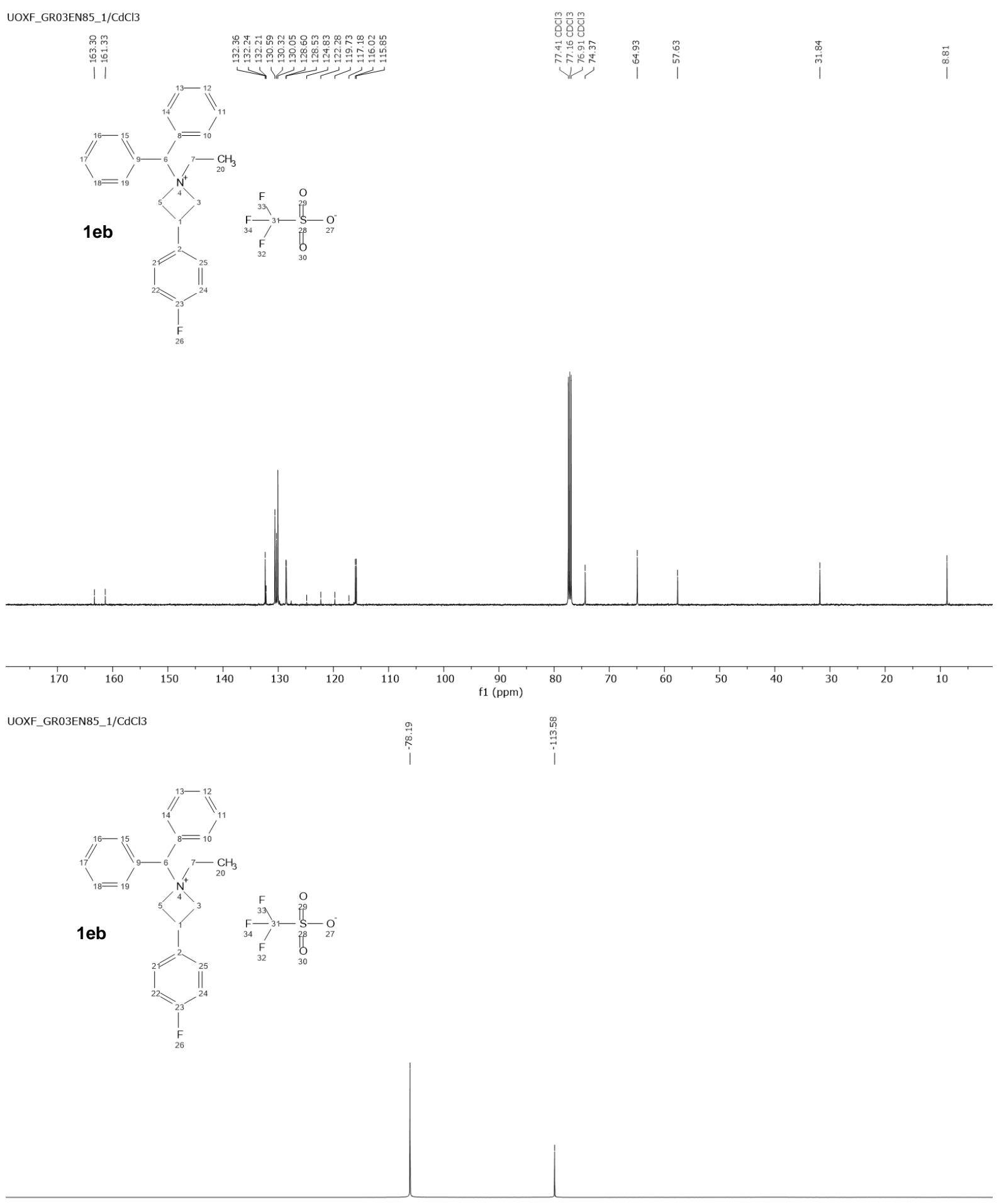

\begin{tabular}{lllllllllllllllllllllllllllllll}
\hline & 10 & 10 & 0 & -10 & -20 & -30 & -40 & -50 & -60 & -70 & -80 & -90 & -100 & -1 & 1 & -120 & -130 & -140 & -150 & -160 & -170 & -180 & -190 & -200 & -210 & -2 \\
\hline
\end{tabular} 
UOXF_GR03EN81_1/CdCl3

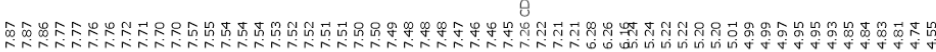

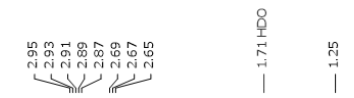
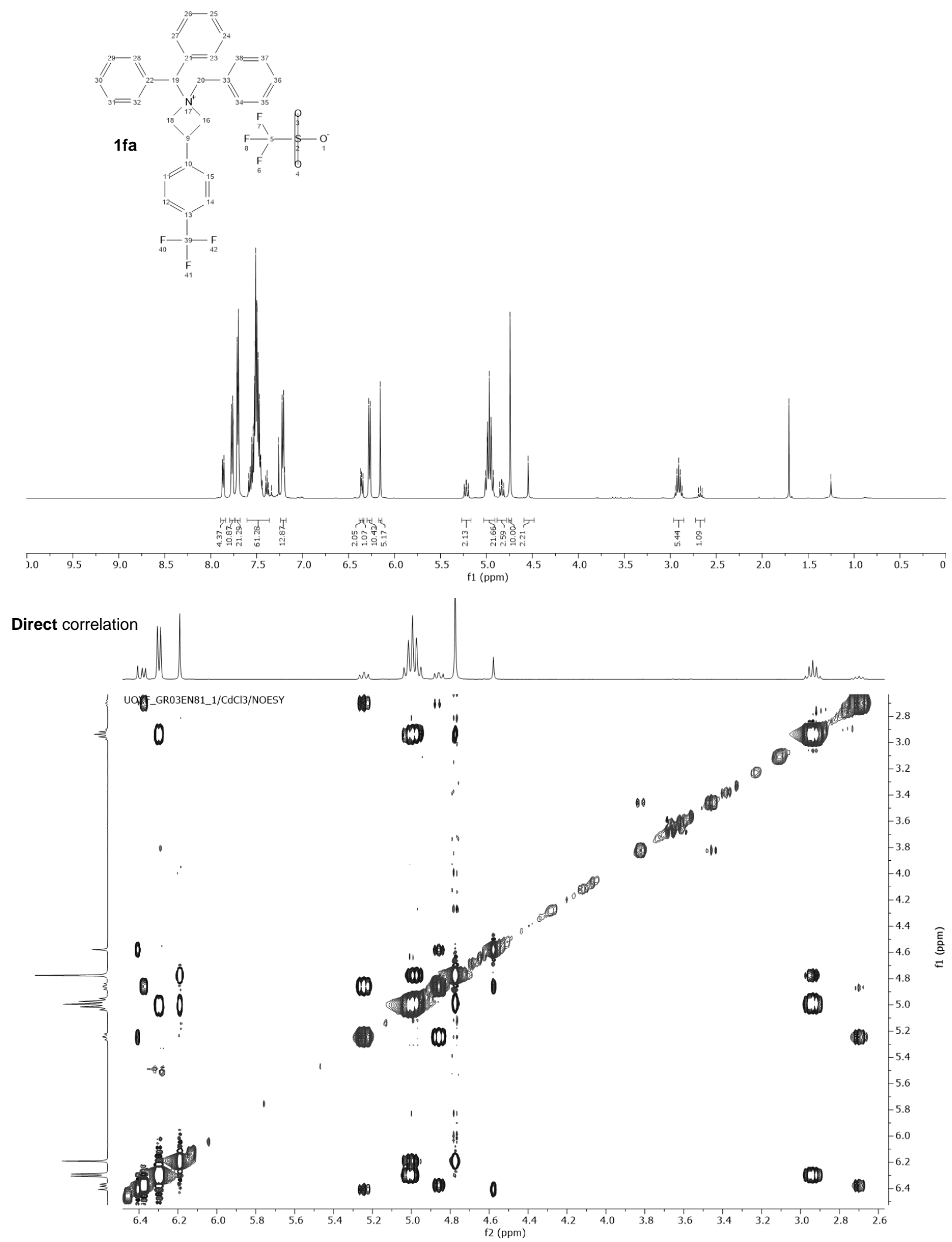

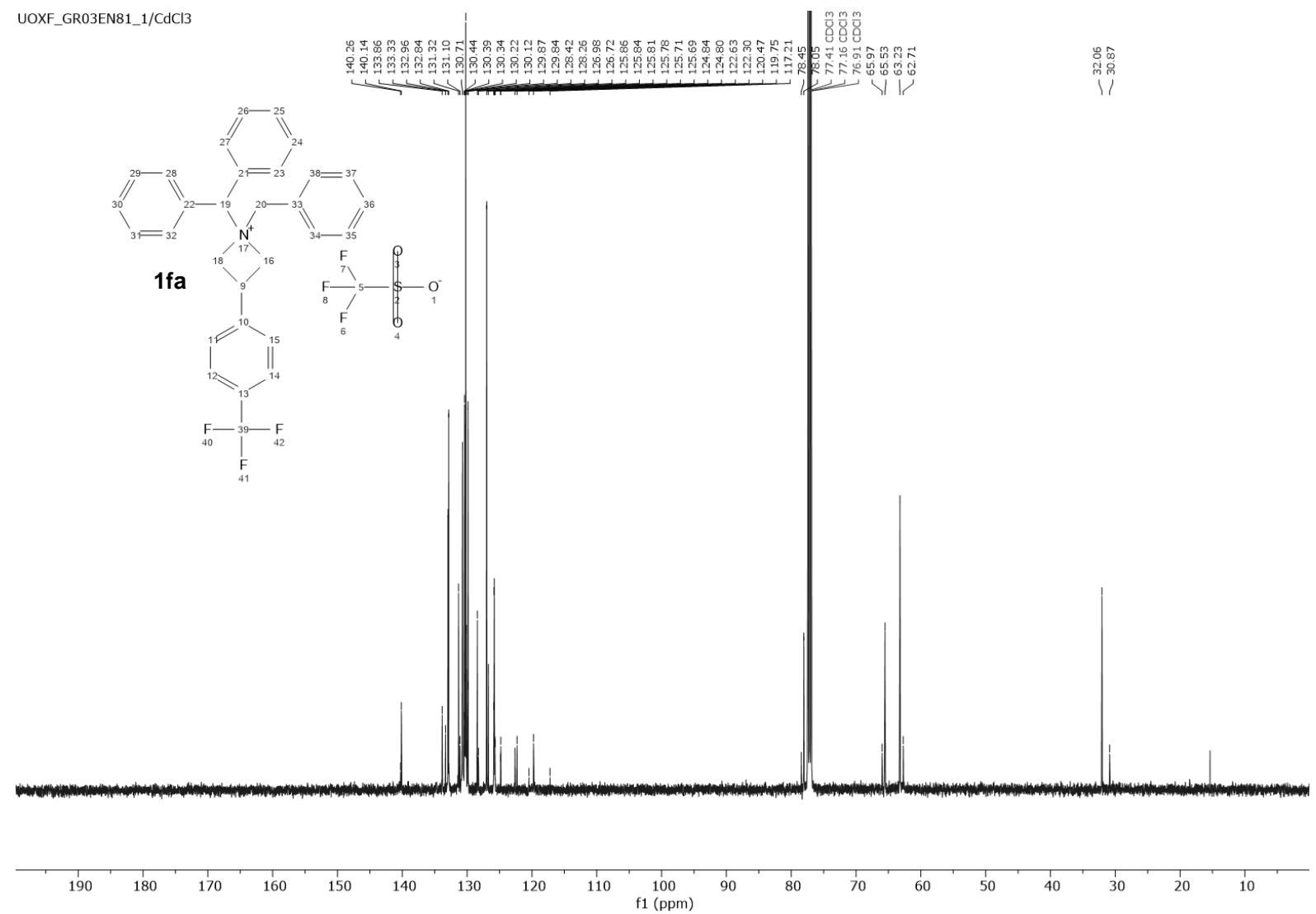

UOXF_GR03EN81_1/CdCI3

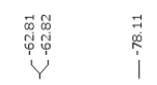
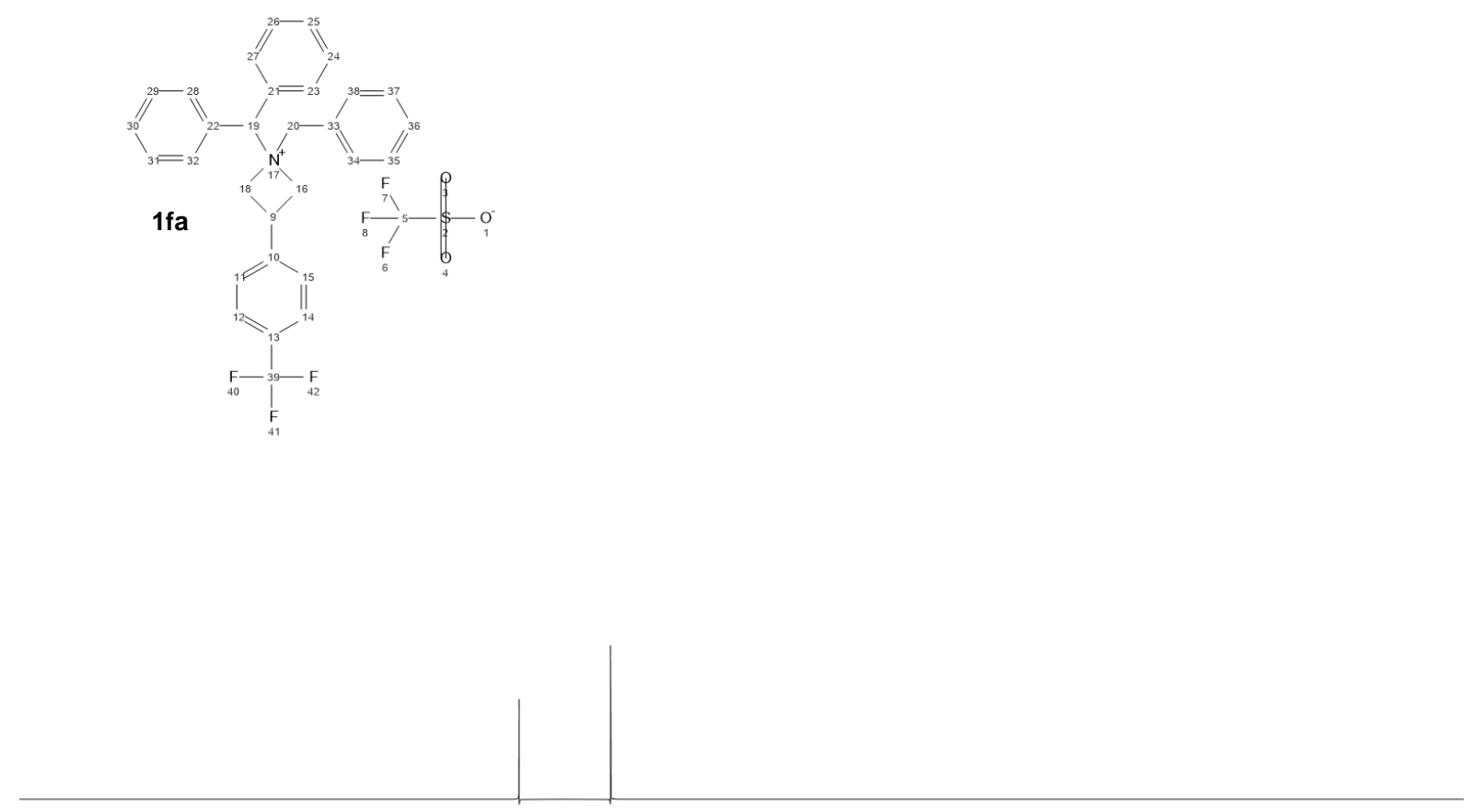

\begin{tabular}{rlllllllllllllllllllllllllll}
\hline & 10 & 10 & 0 & -10 & -20 & -30 & -40 & -50 & -60 & -70 & -80 & -90 & -100 & -110 & -120 & -130 & -140 & -150 & -160 & -170 & -180 & -190 & -200 & -210 & -2
\end{tabular} 

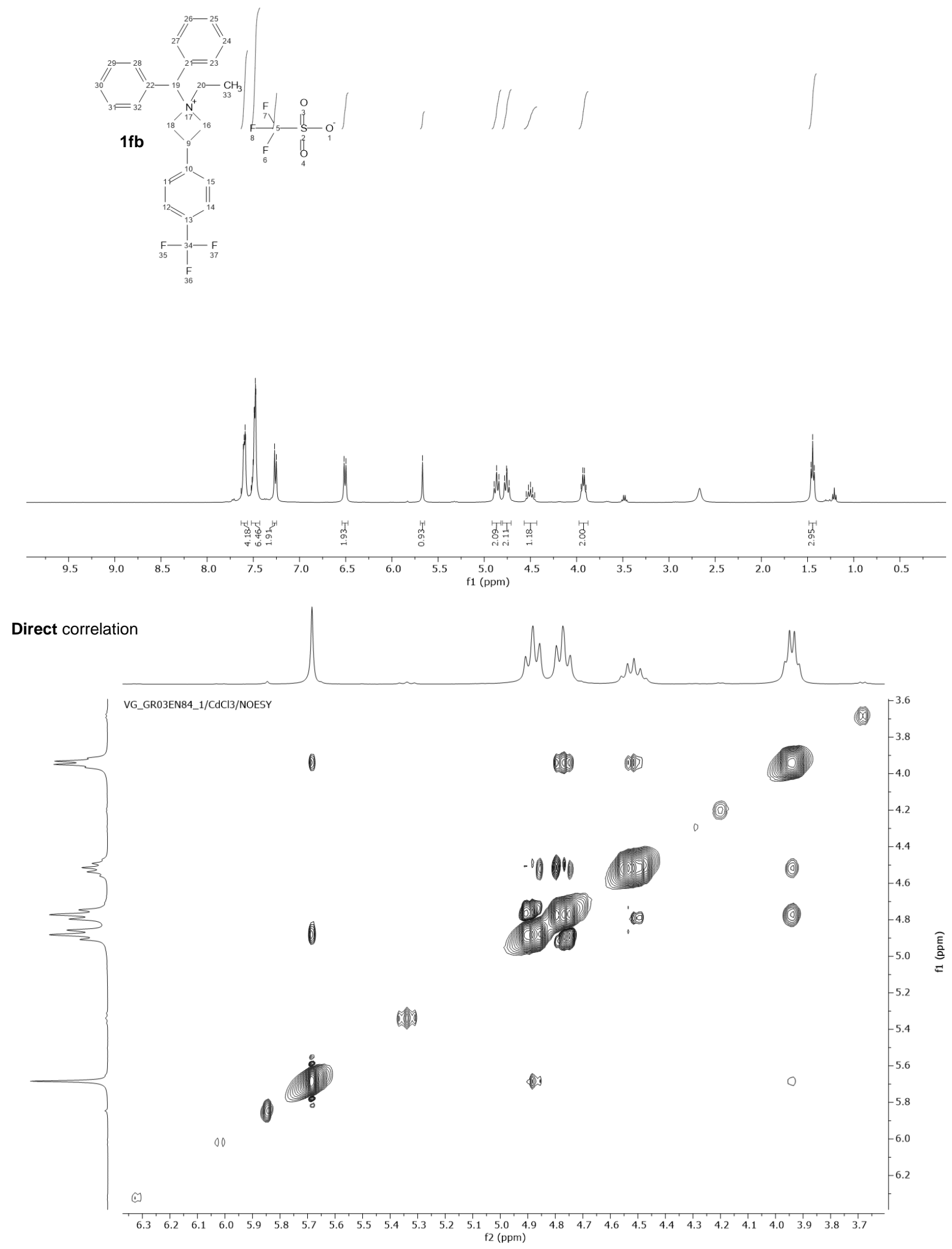
UOXF_GR03EN84_1/CdCl3

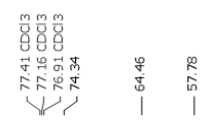

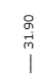

$\underset{\substack{* \\ \infty}}{\substack{0 \\ \mid}}$
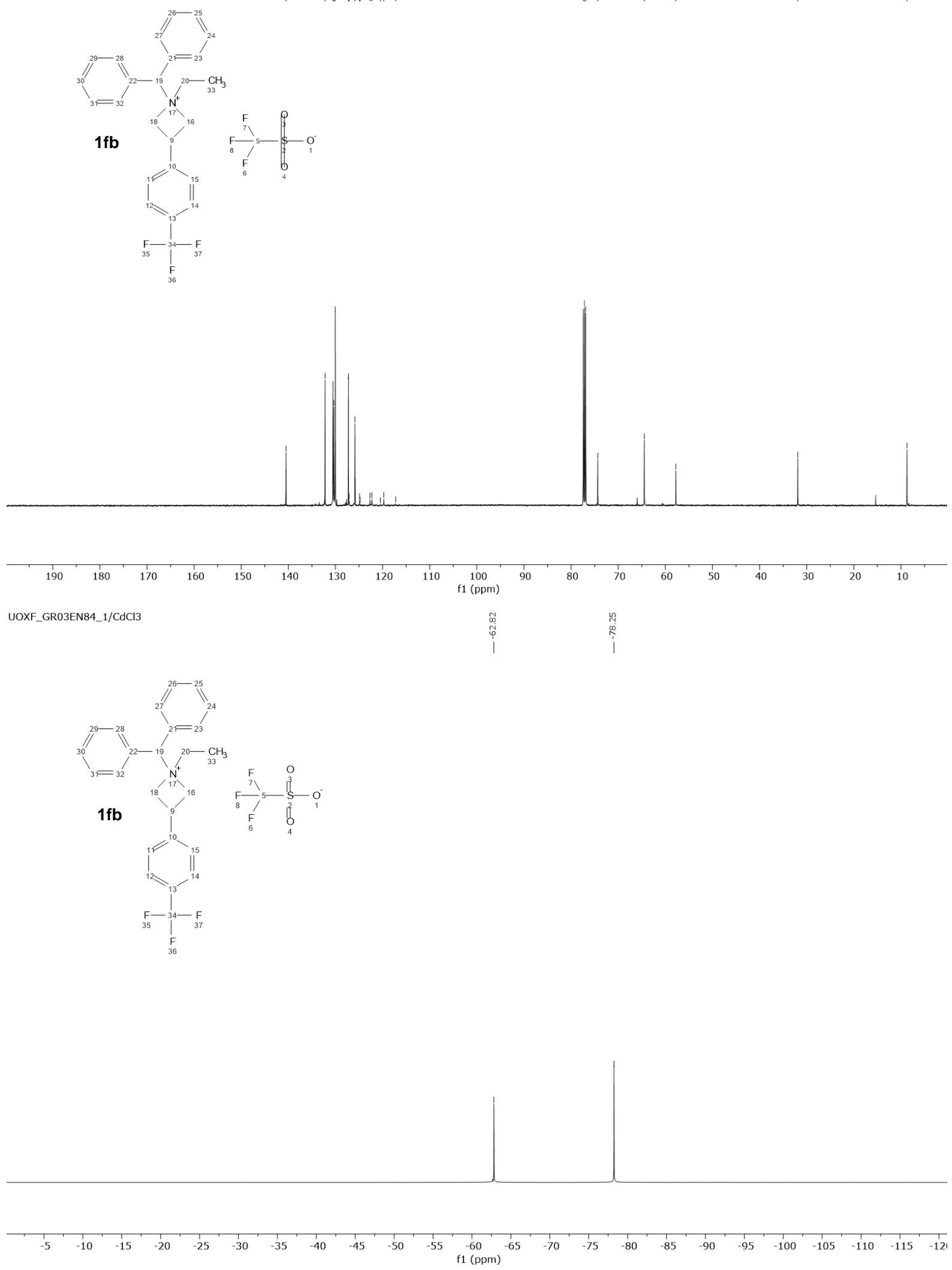

S221 

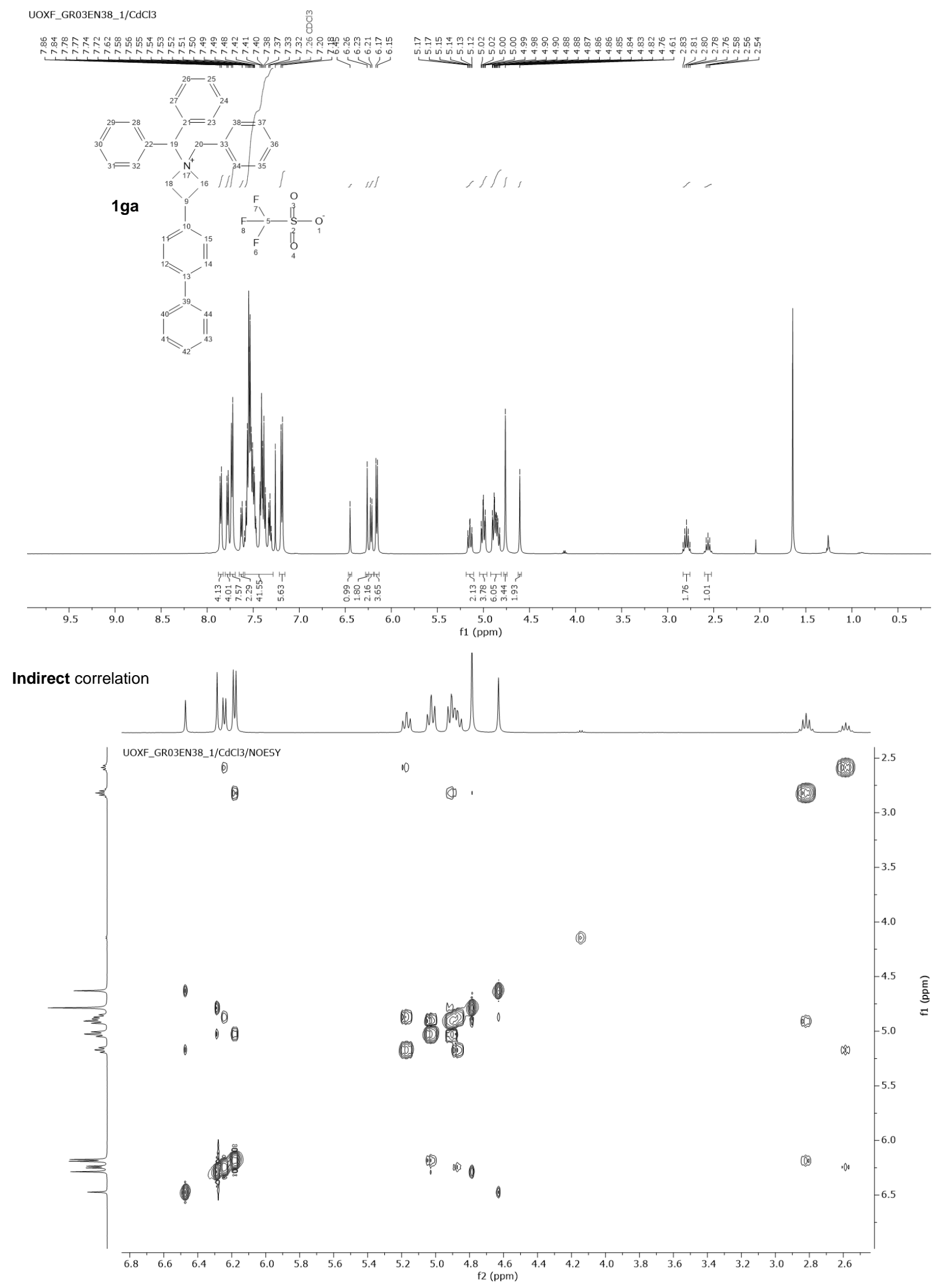
UOXF_GR03EN38_1/CdCl3

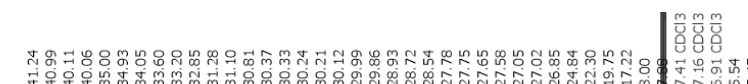

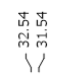

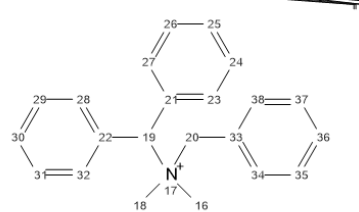

1ga
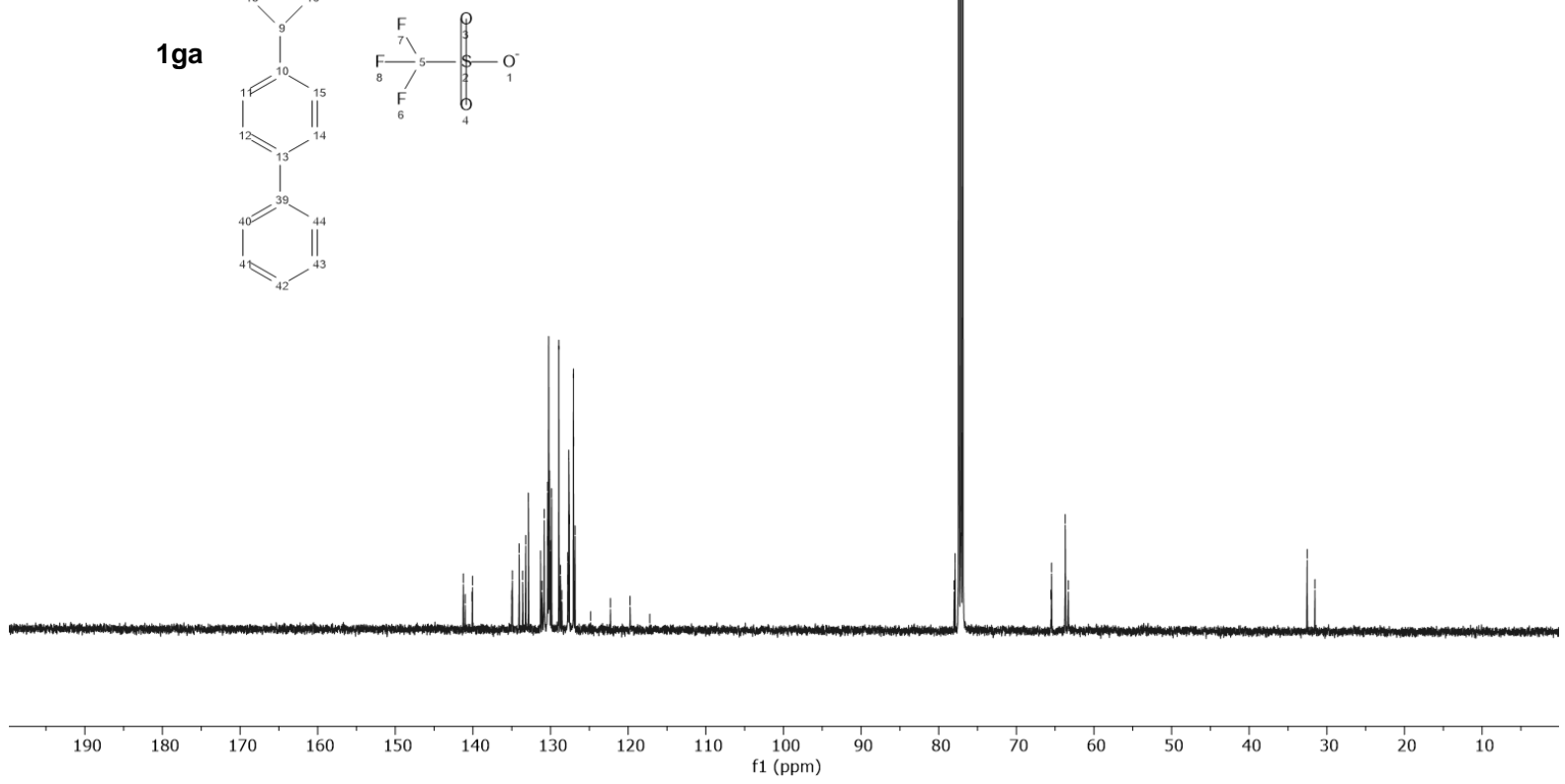

UOXF_GR03EN38_1/CdCl3

$\underset{i}{\stackrel{\vec{\infty}}{\sim}}$
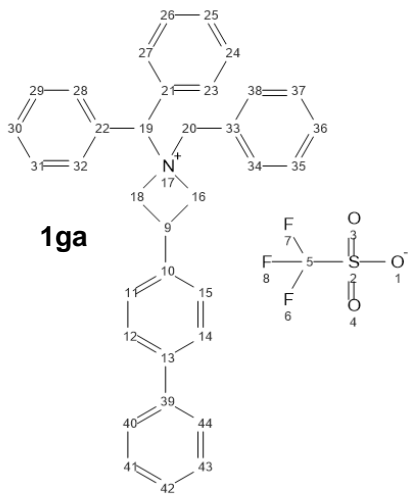

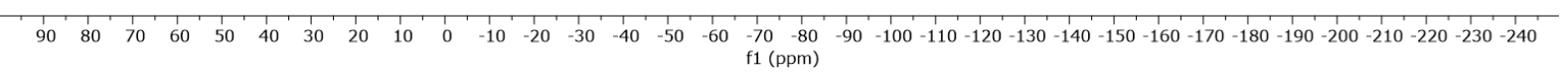

S223 

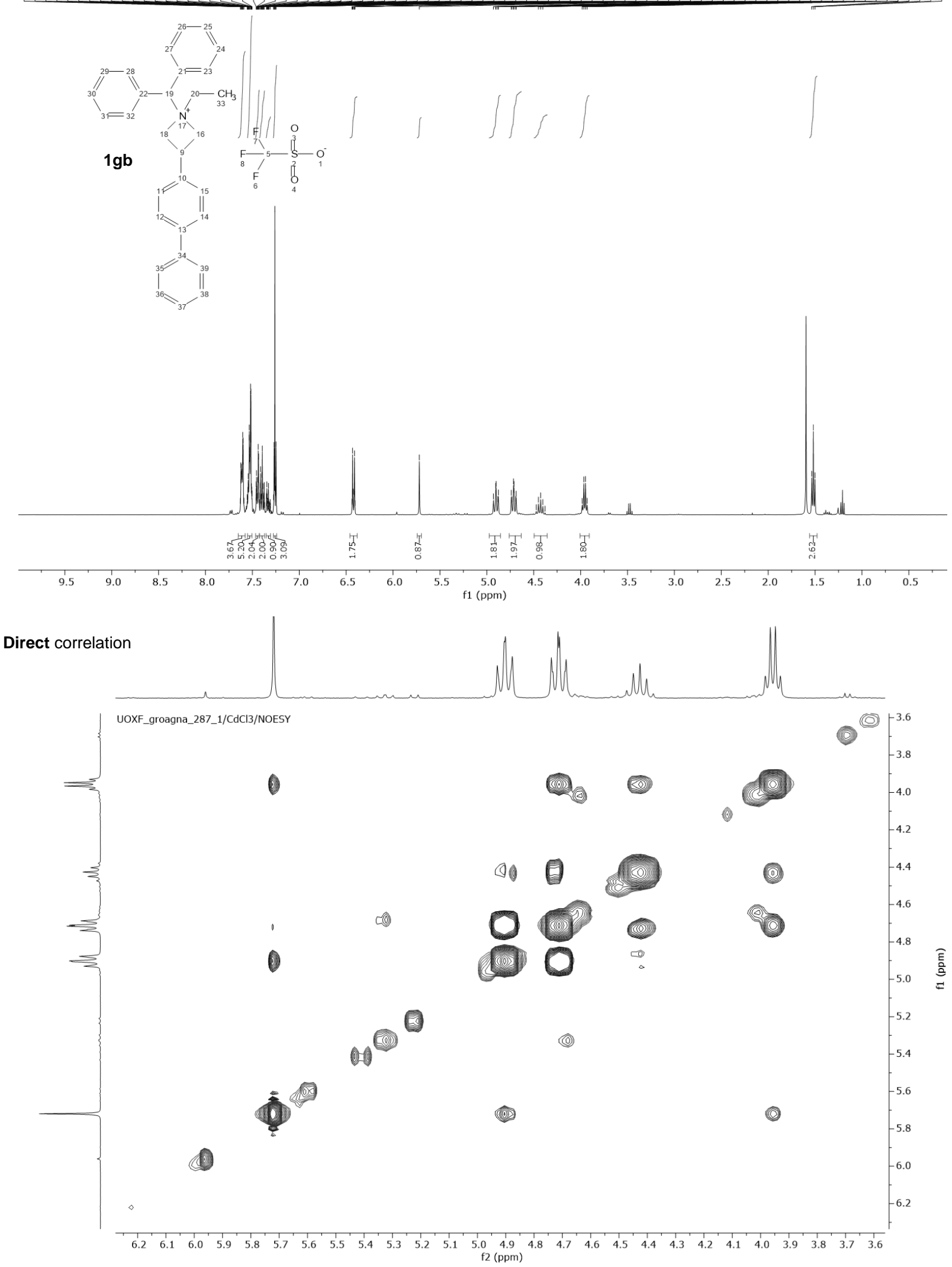

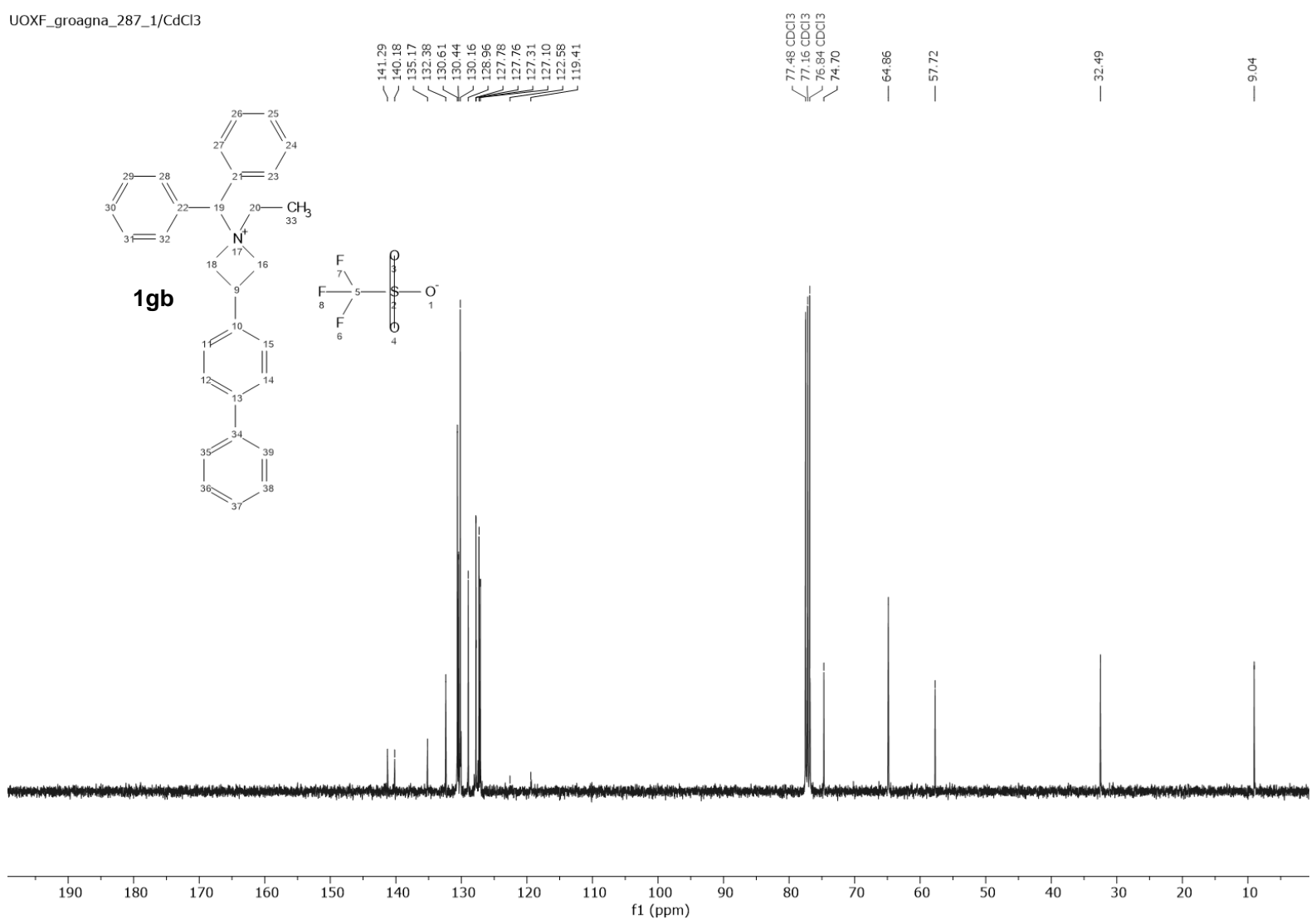

UOXF_groagna_287_1/CdCl3

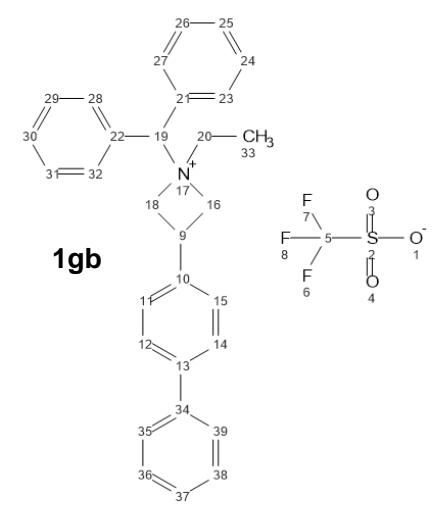

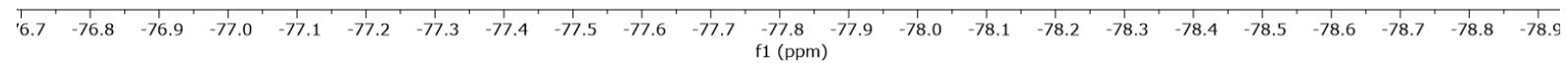



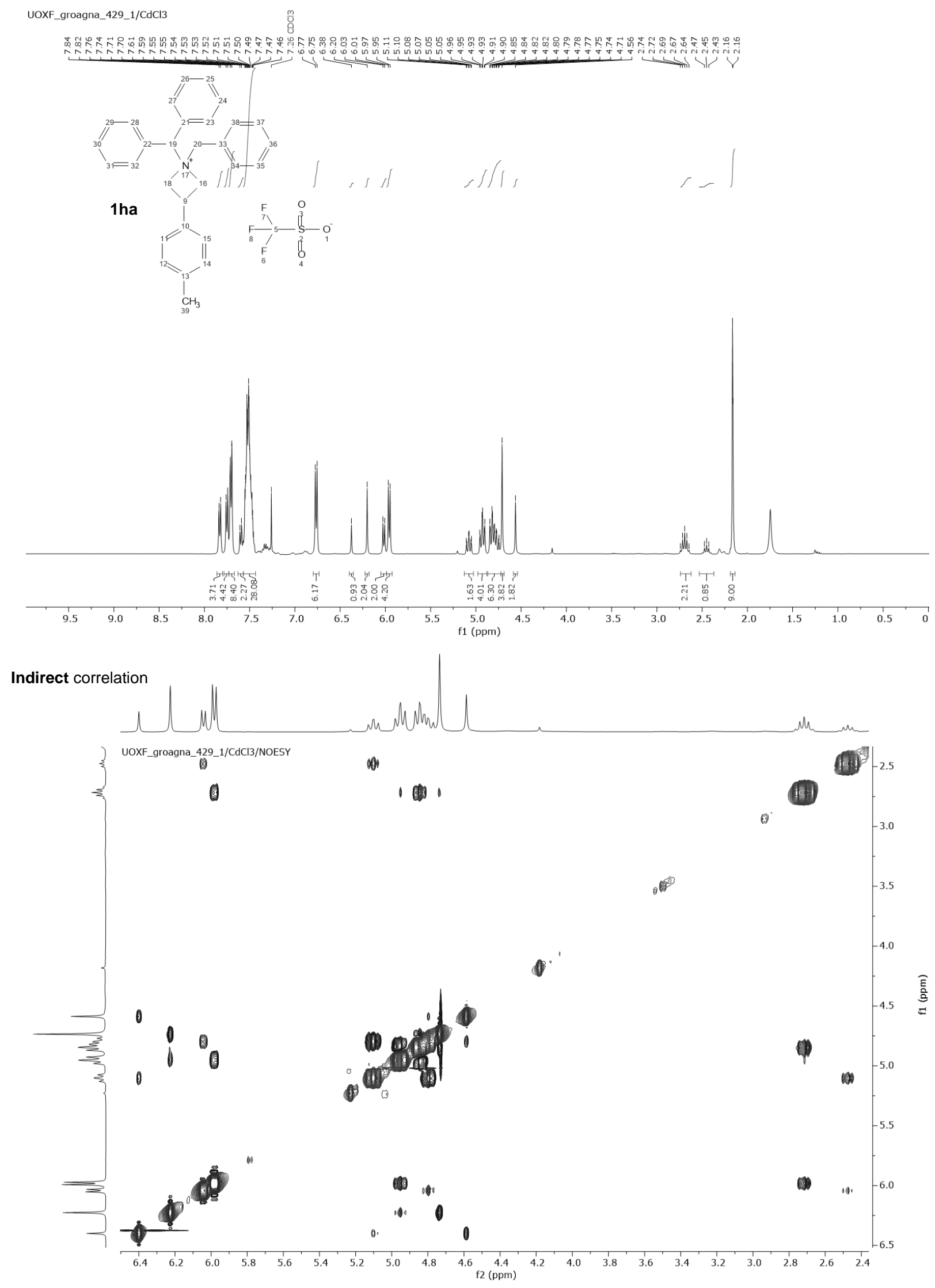

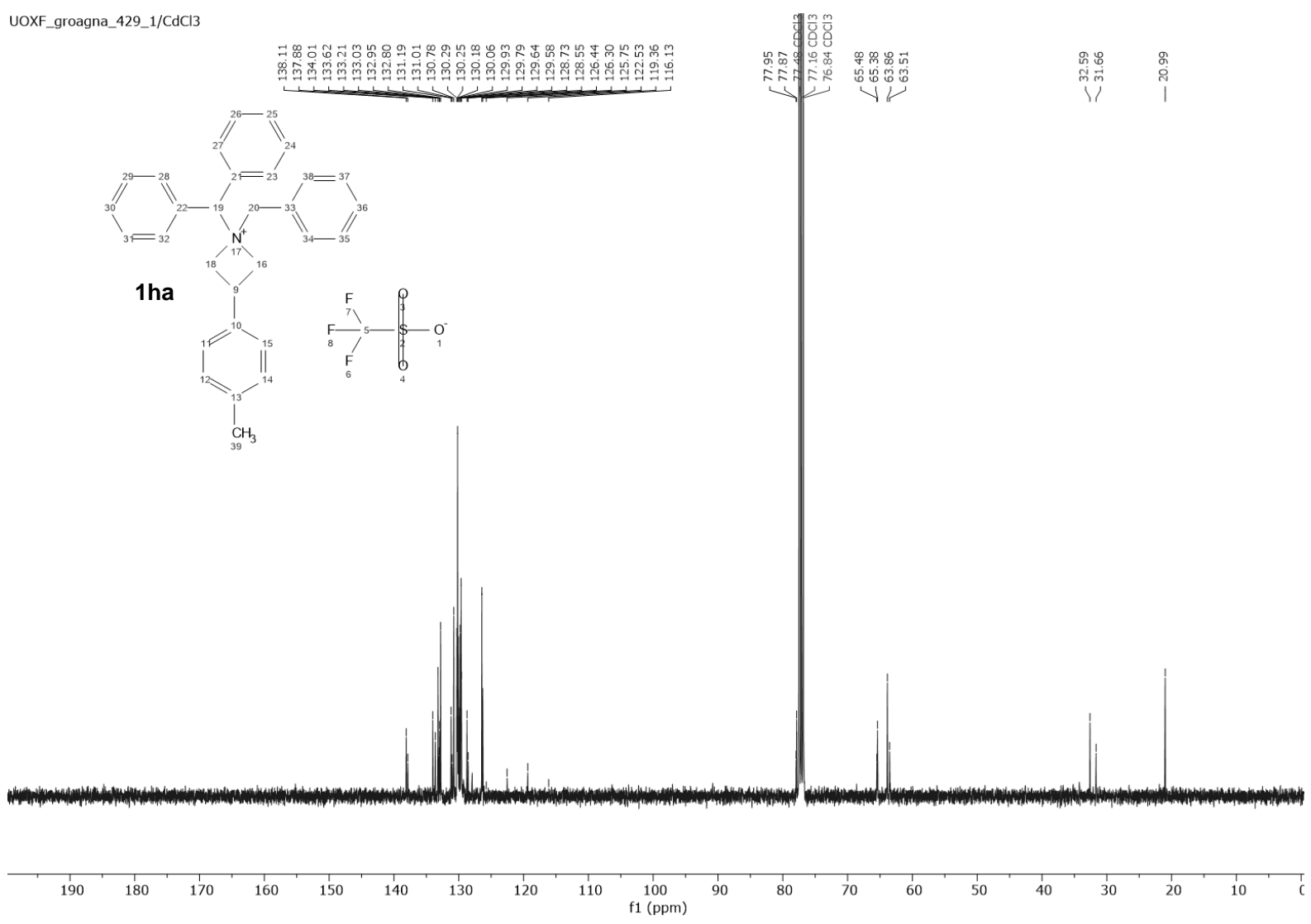

UOXF_groagna_429_1/CdCl3
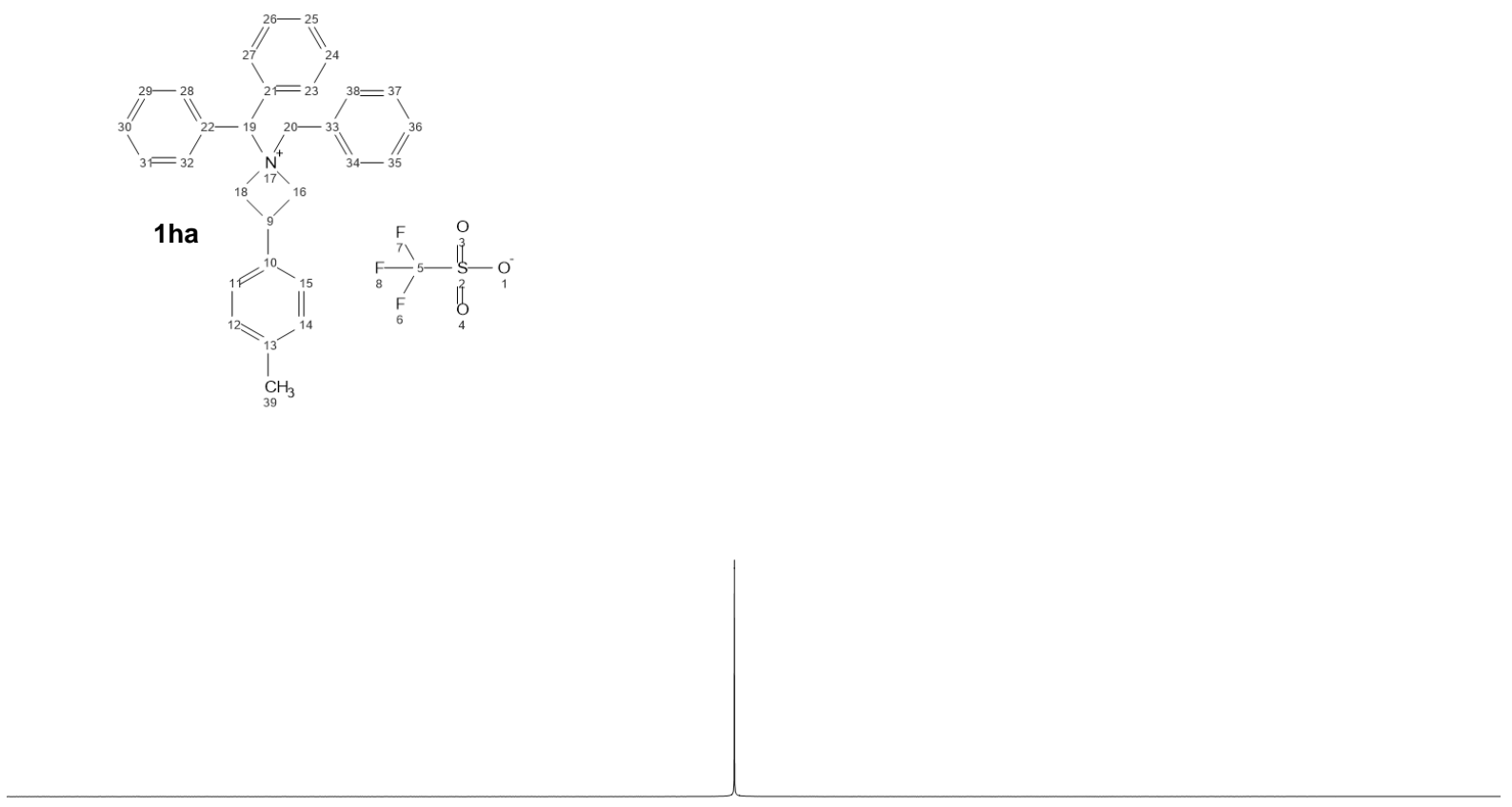

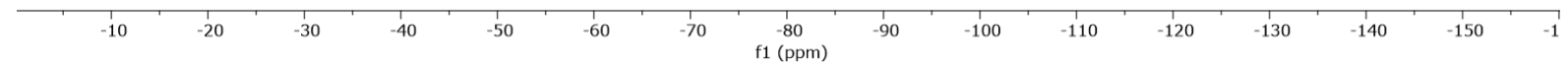



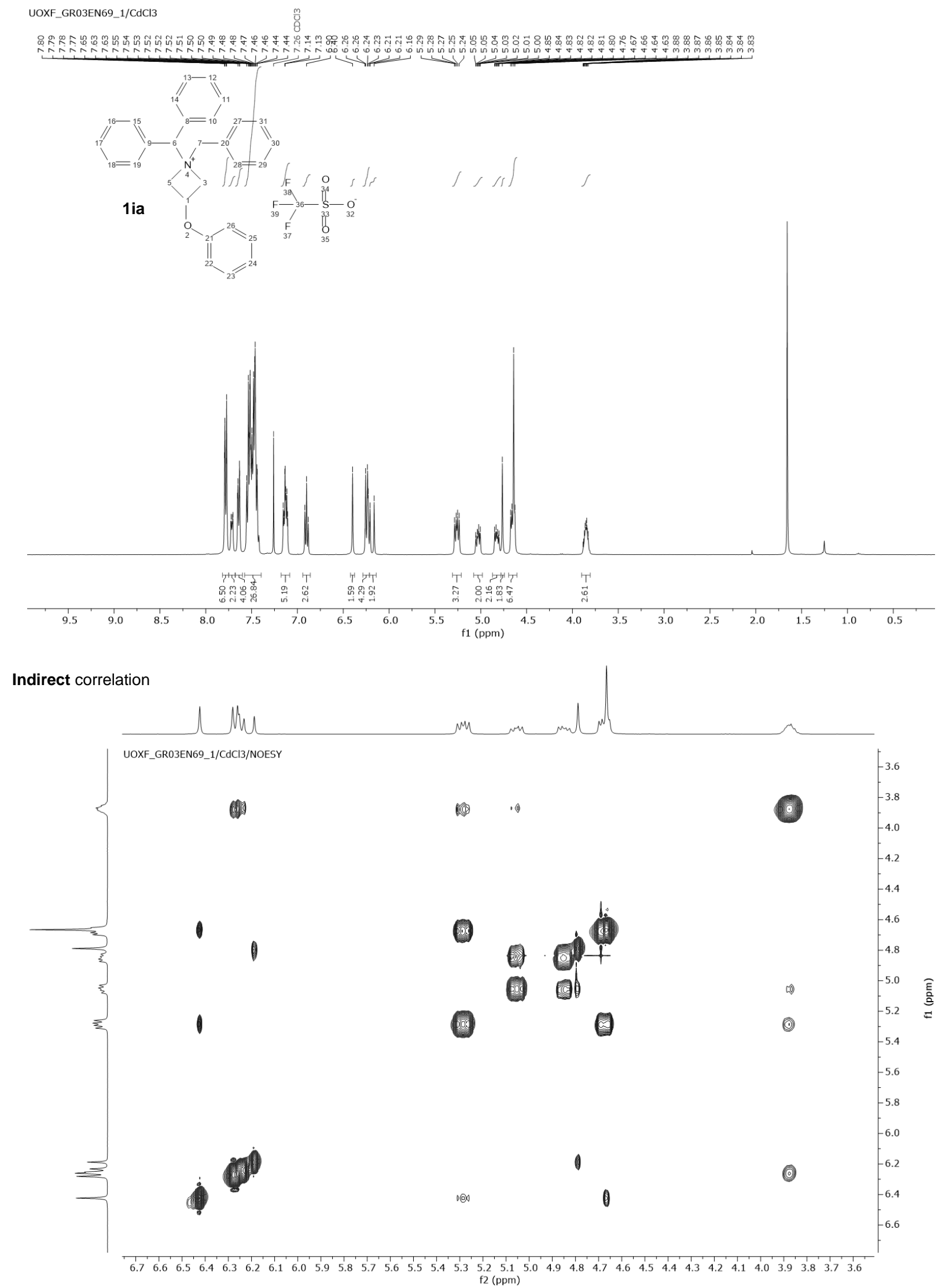

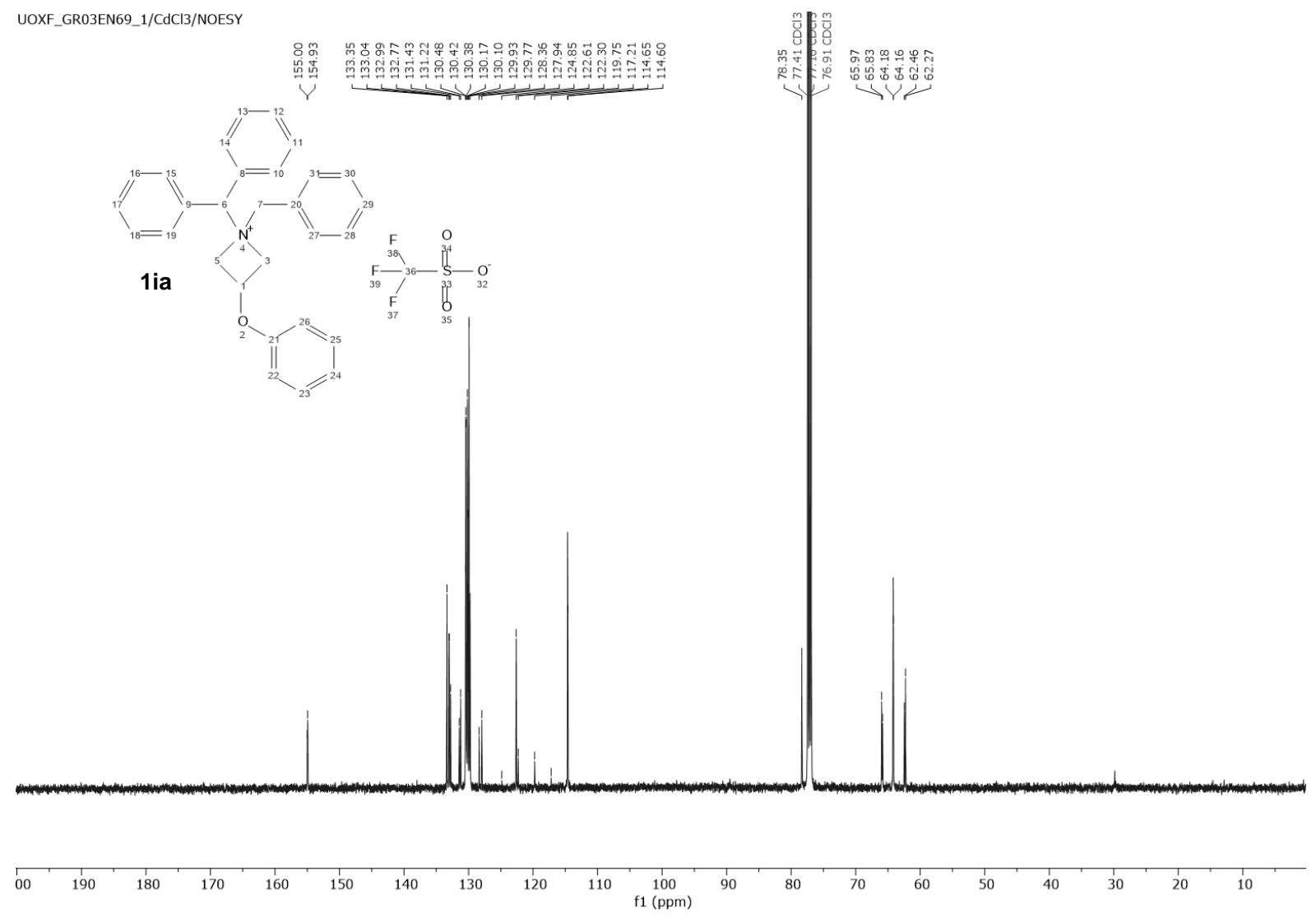

UOXF_GR03EN69_1/CdCl3

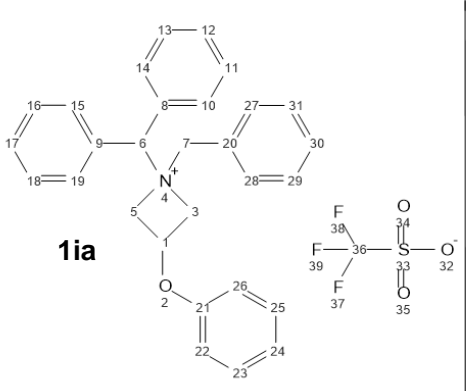

\begin{tabular}{llllllllllllllllllllllllllllll}
\hline 10 & 0 & -10 & -20 & -30 & -40 & -50 & -60 & -70 & -80 & -90 & -100 & -110 & -120 & -130 & -140 & -150 & -160 & -170 & -180 & -190 & -200 & -210 & -220 & -2
\end{tabular} 

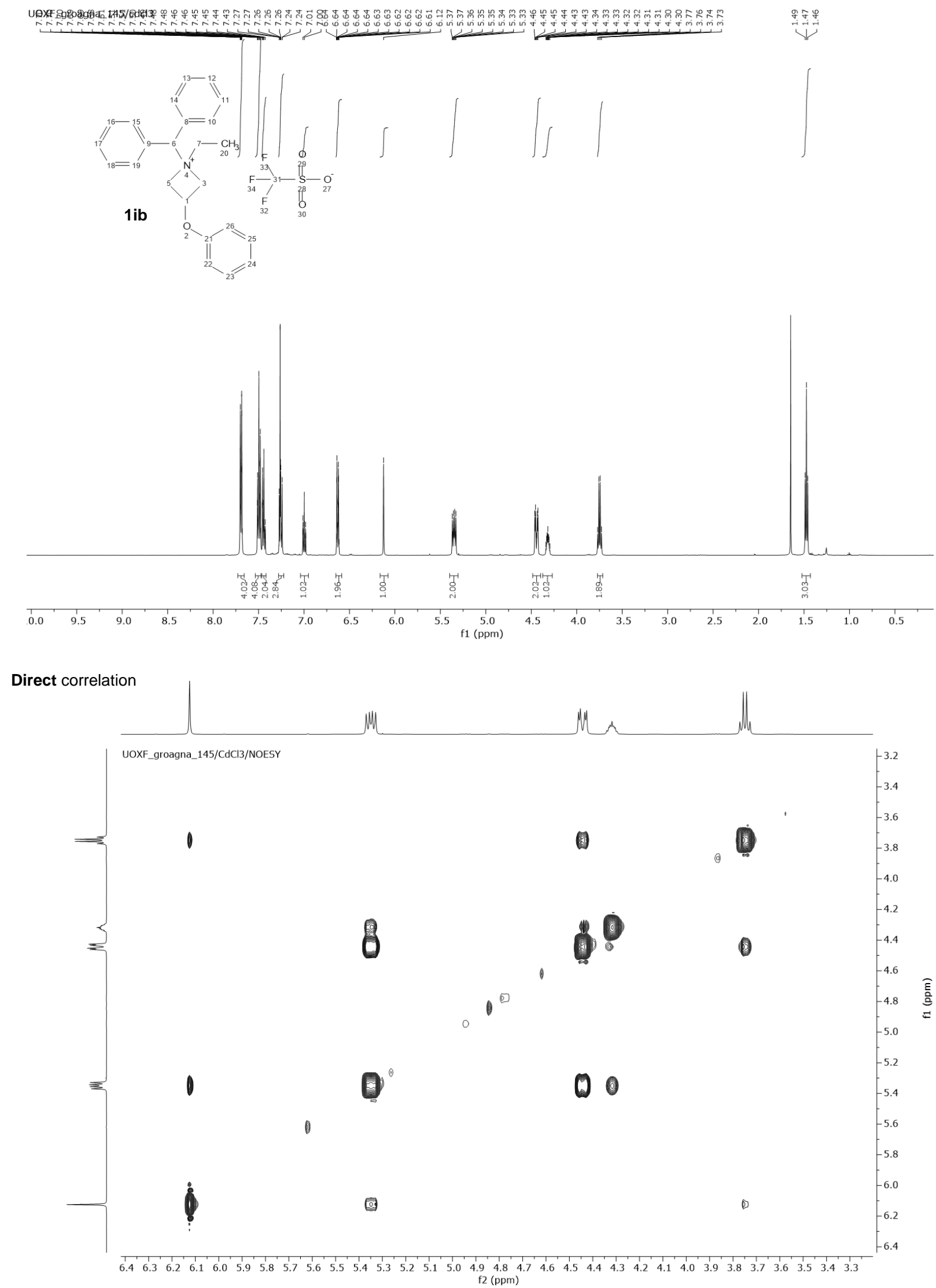
UOXF_groagna_145_1/CdCl3

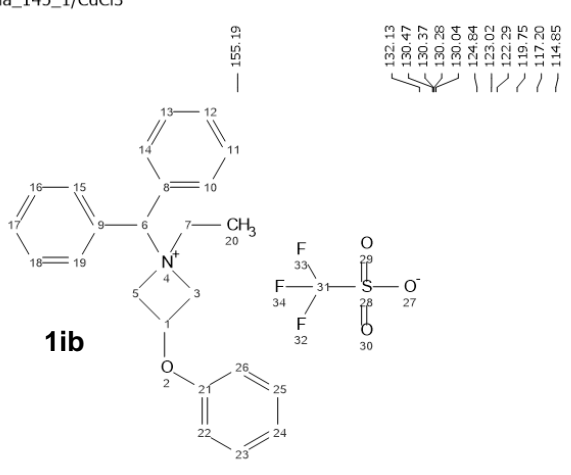

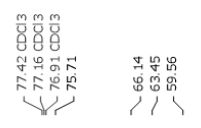

$\stackrel{\substack{\infty \\ \infty}}{1}$
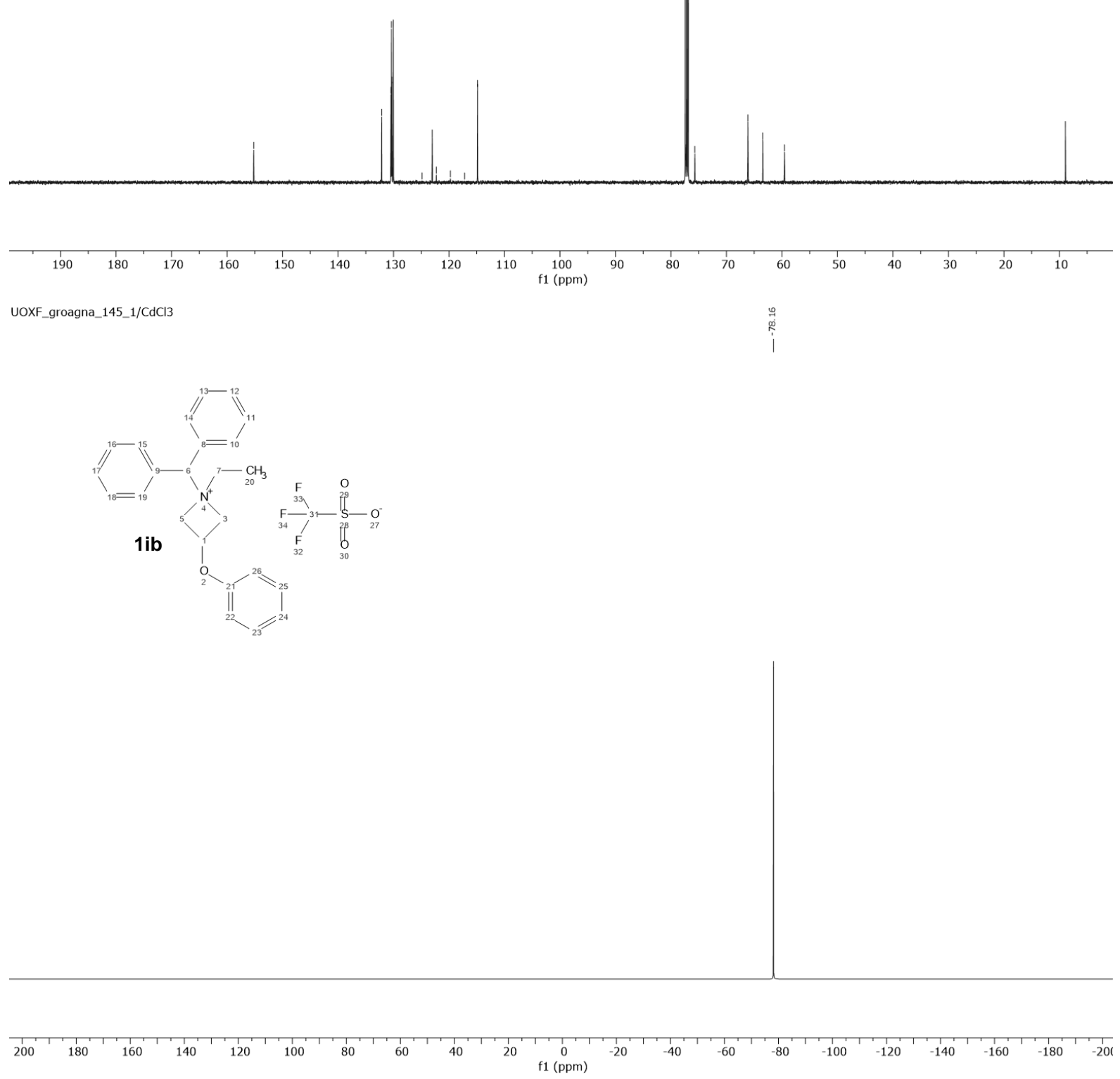

S231 

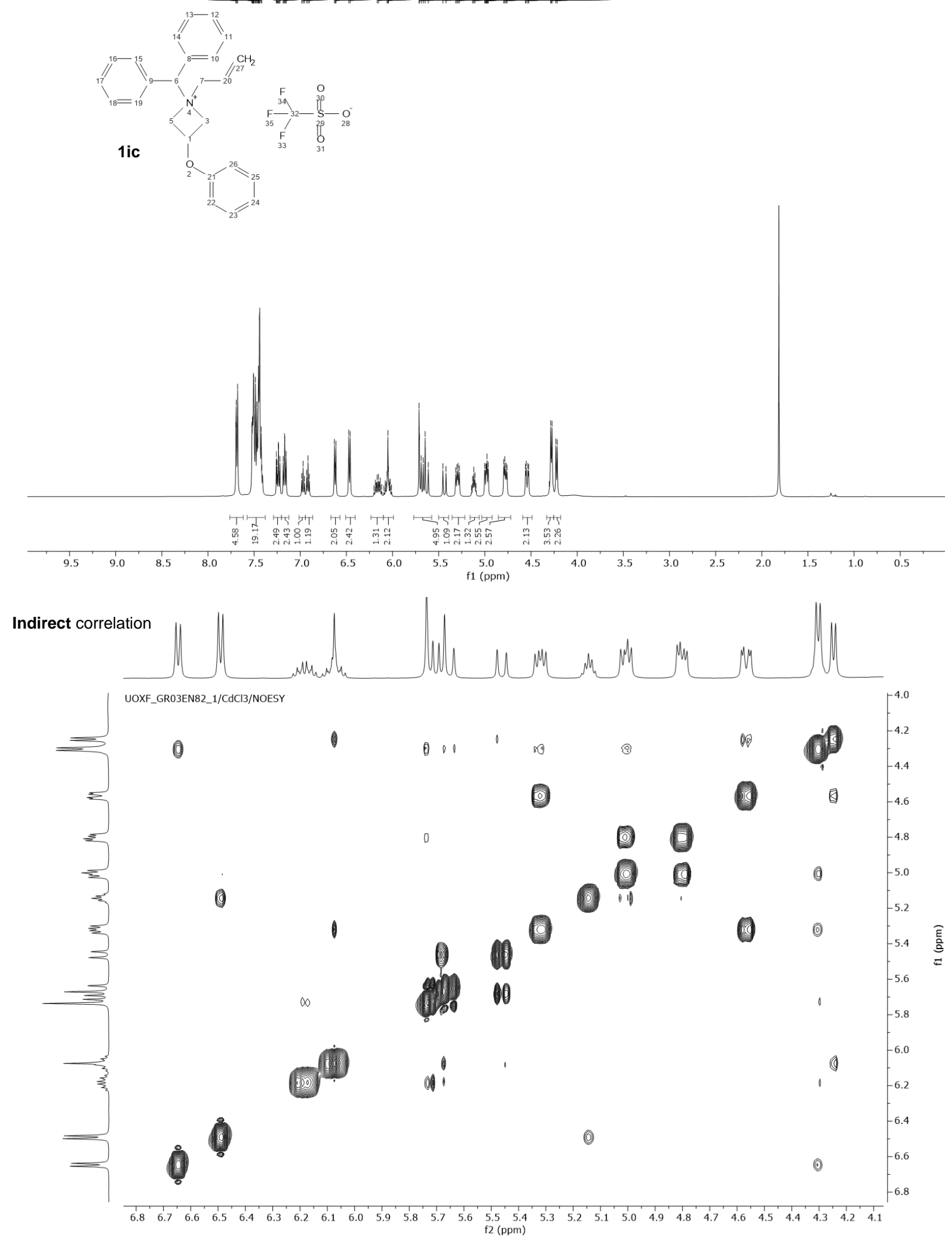
UOXF_GR03EN82_1/CdCl3
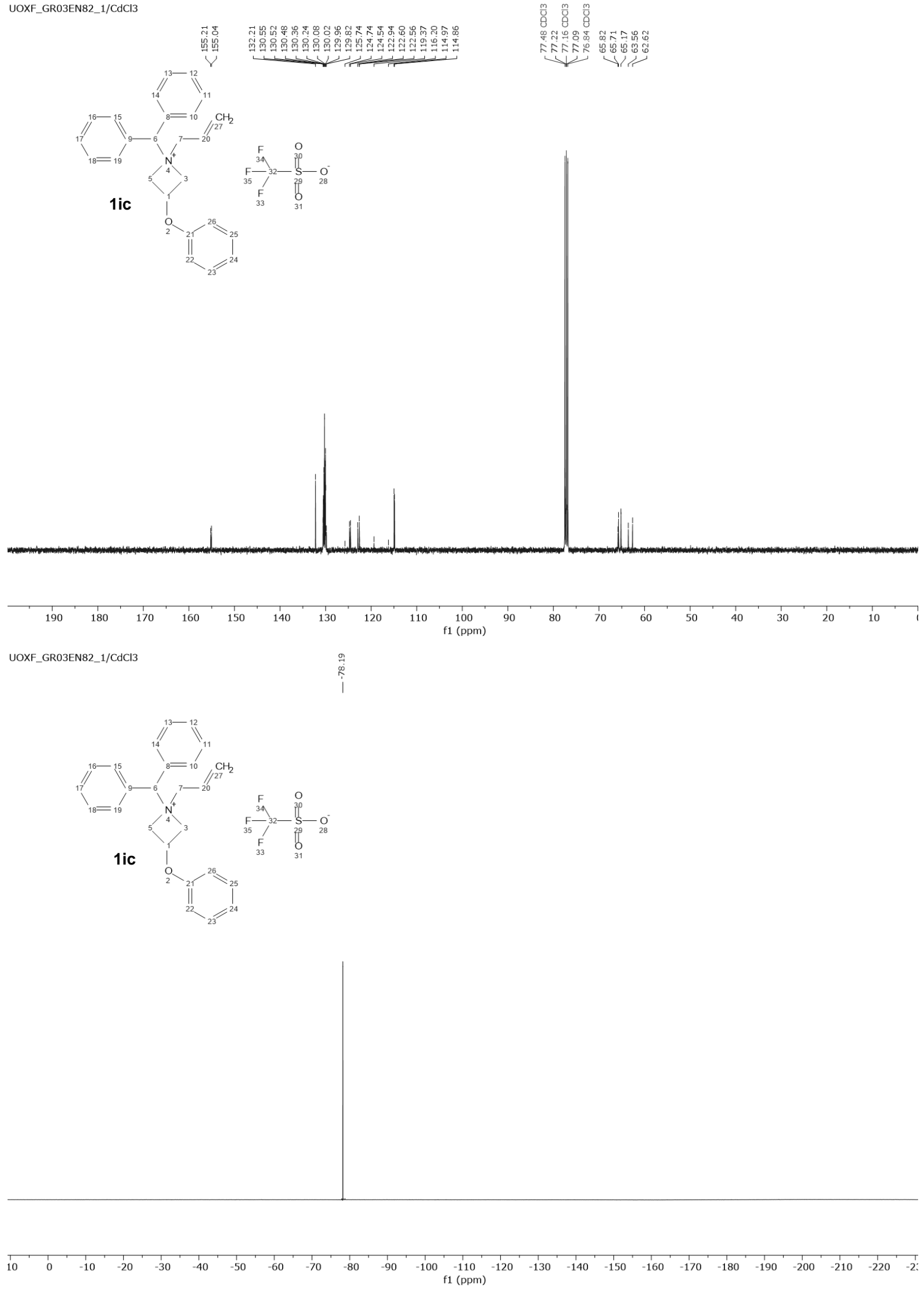

S233 
UOXF_groagna_368_1/CdCl3

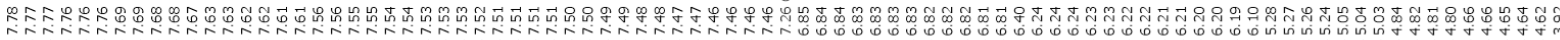
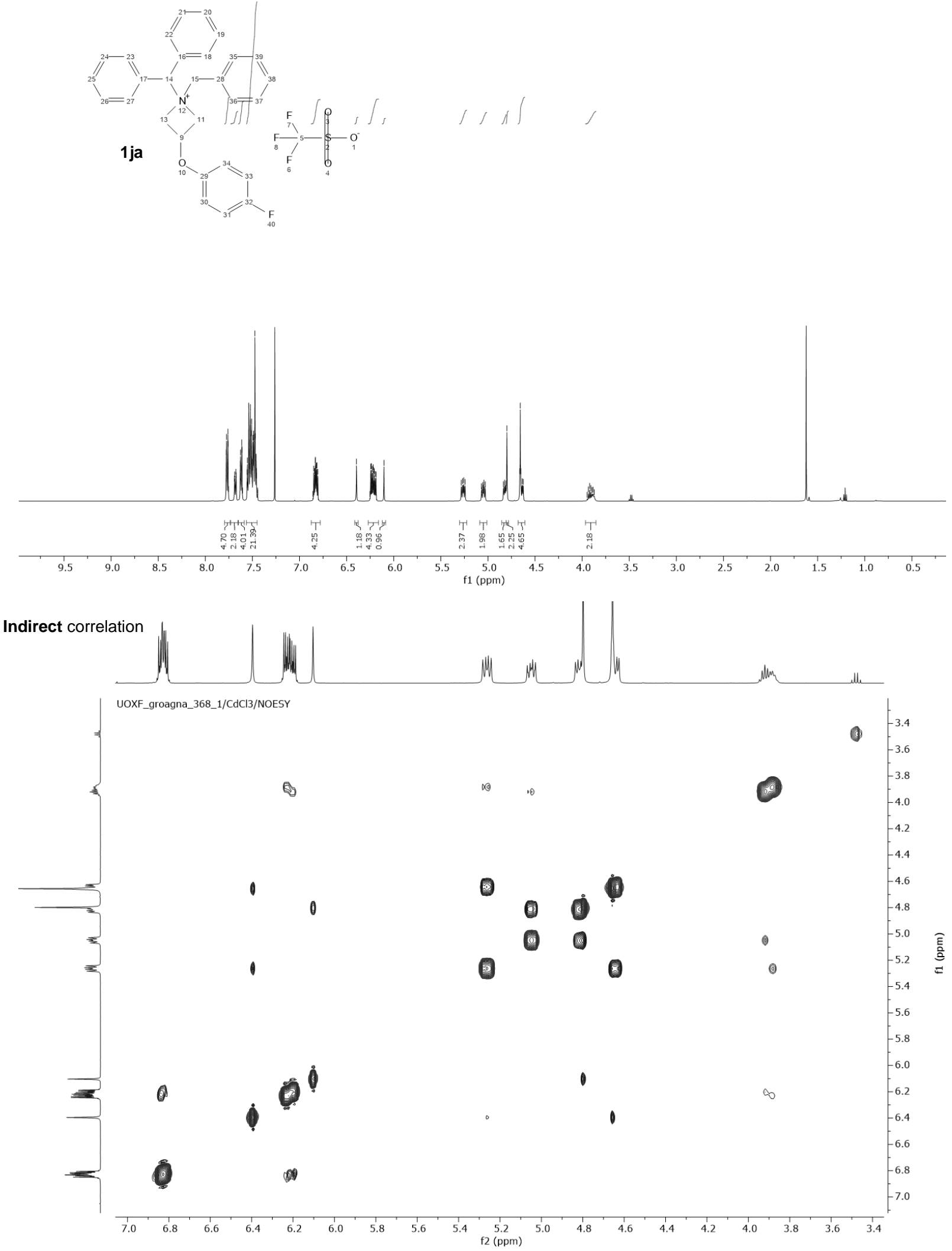

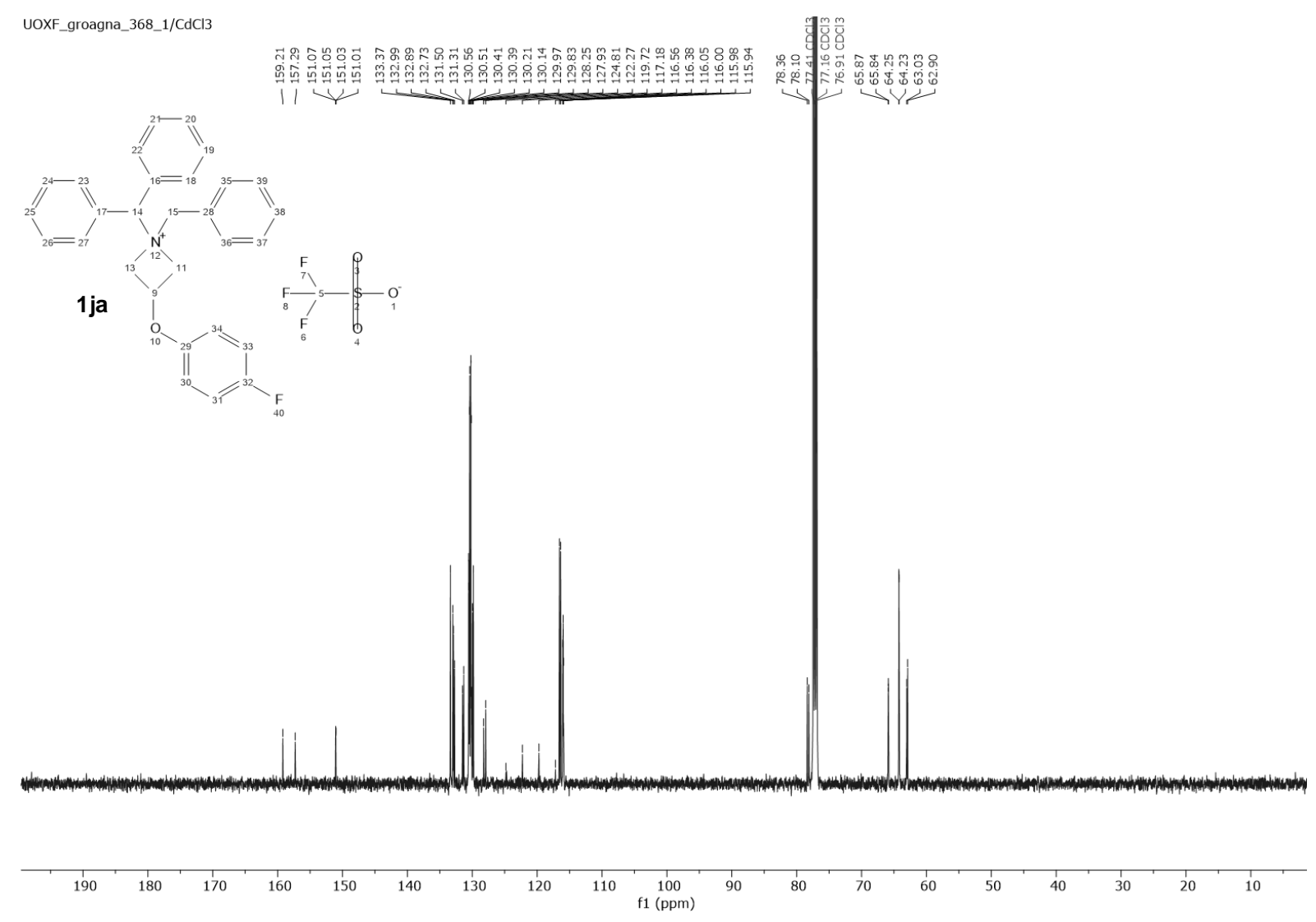

UOXF_groagna_368_t/ $1 / \mathrm{CdCl} 3$

$\stackrel{\infty}{i}$
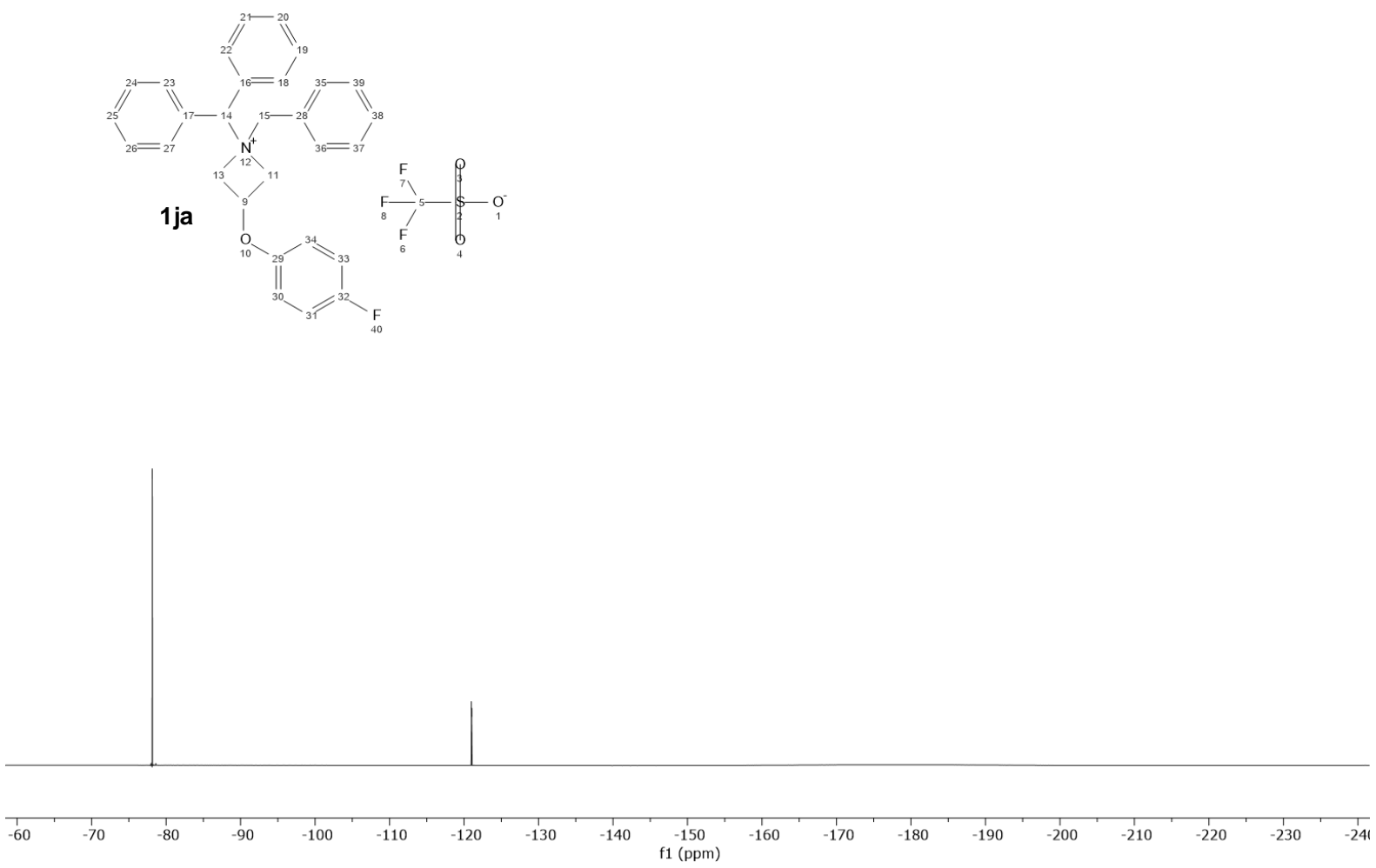
UOXF_groagna_410_1/CdCl3

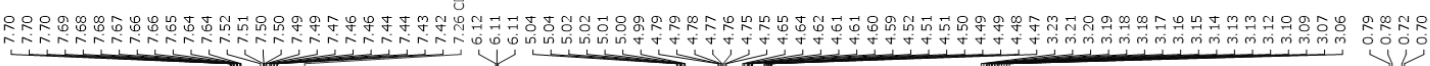

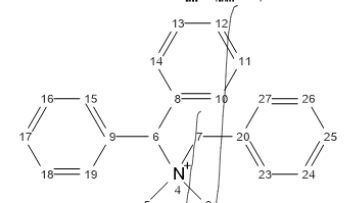

1 ka
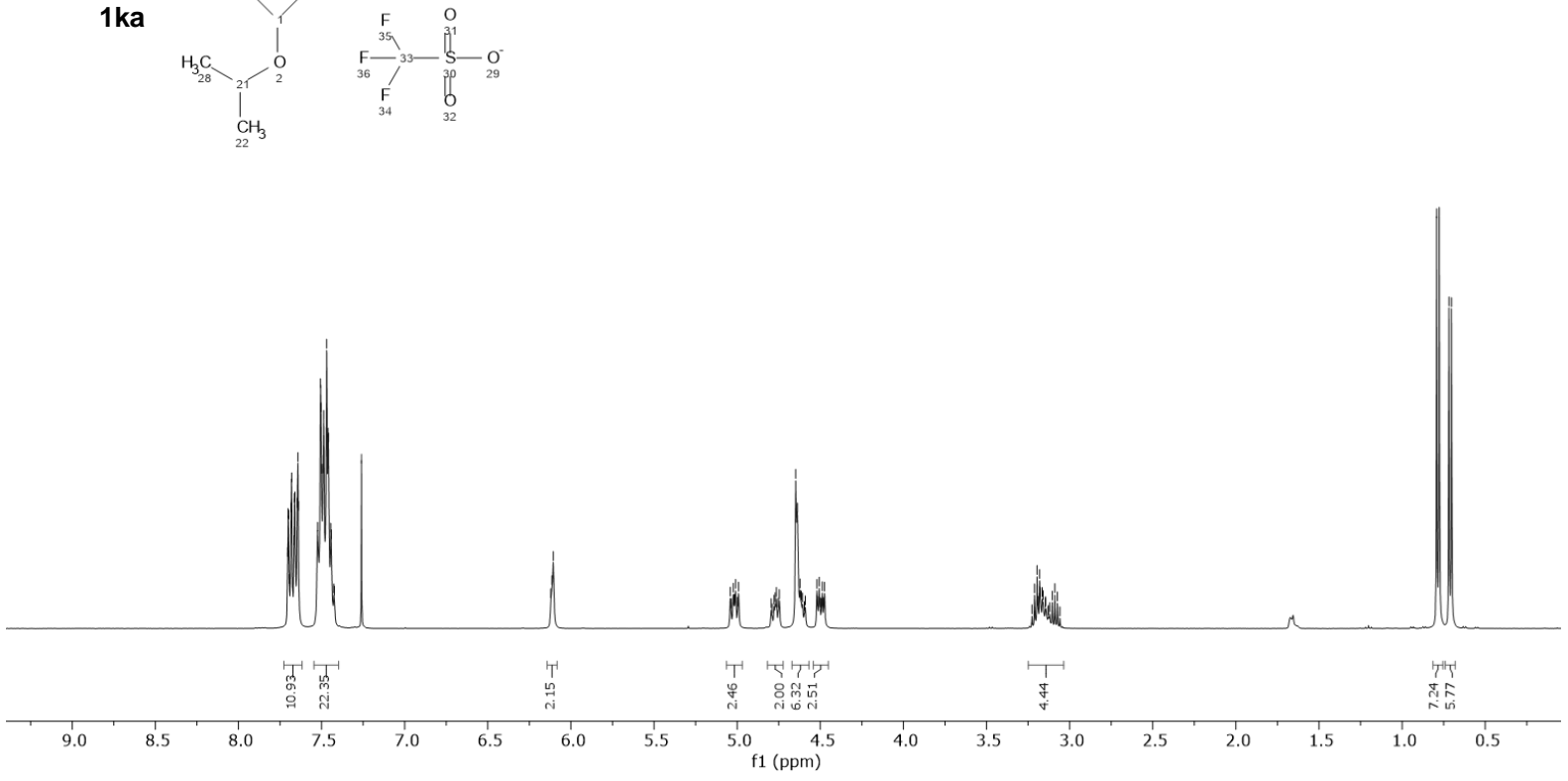

Indirect correlation

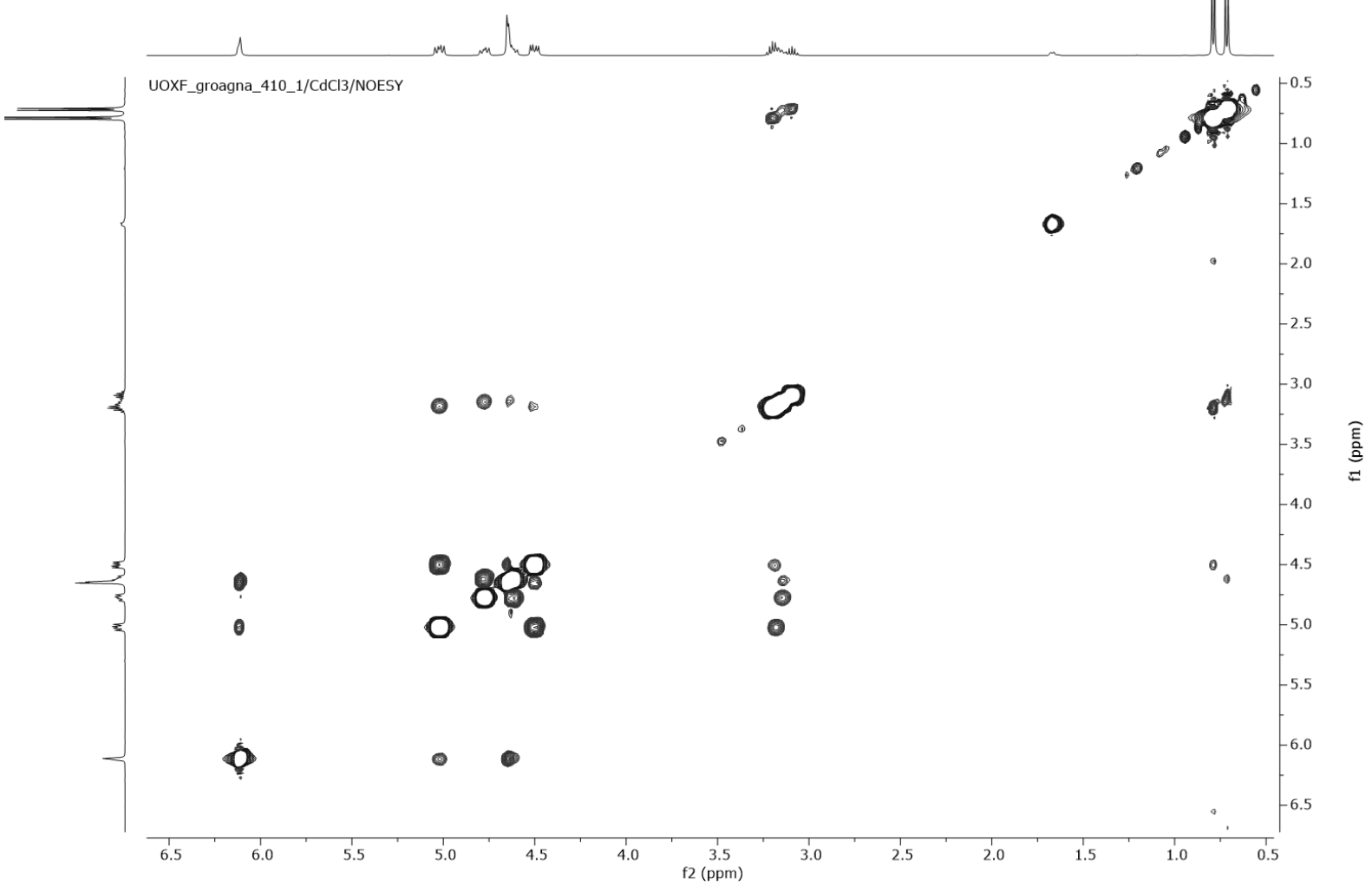




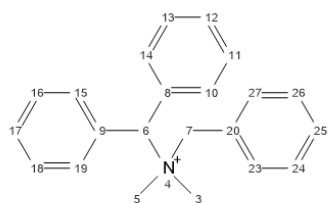

$1 \mathrm{ka}$<smiles>CC(C)OOC(C)C</smiles>

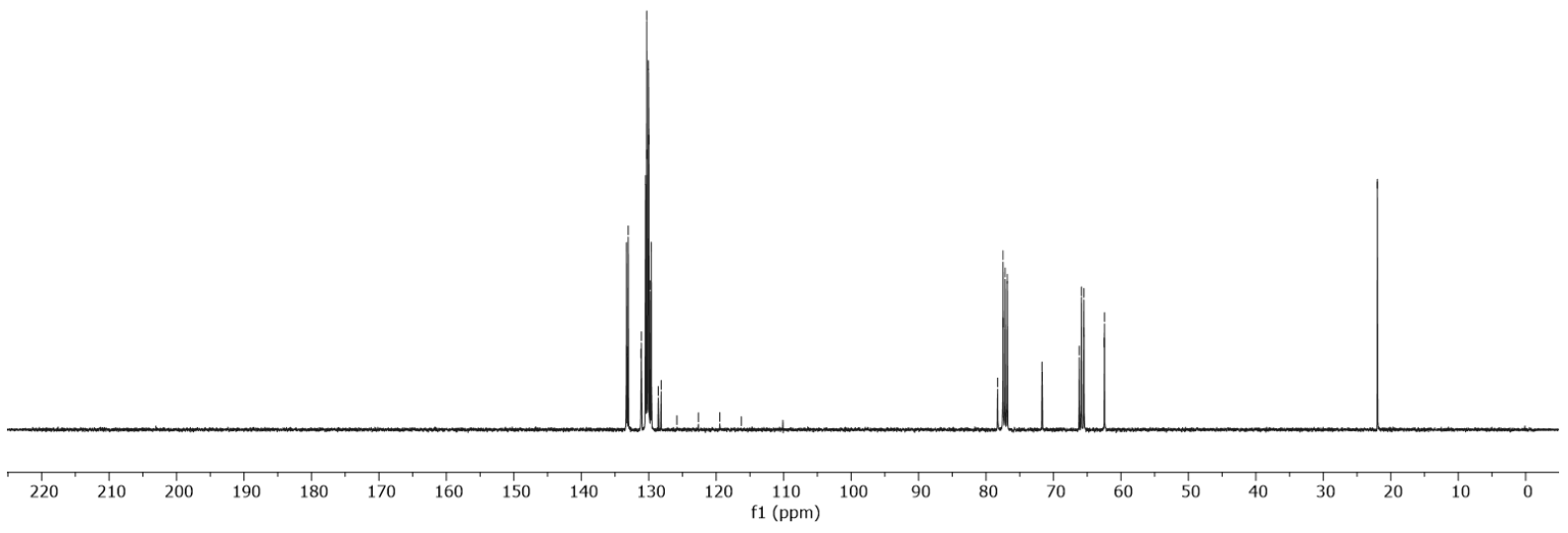

UOXF_groagna_410_1/CdCl3
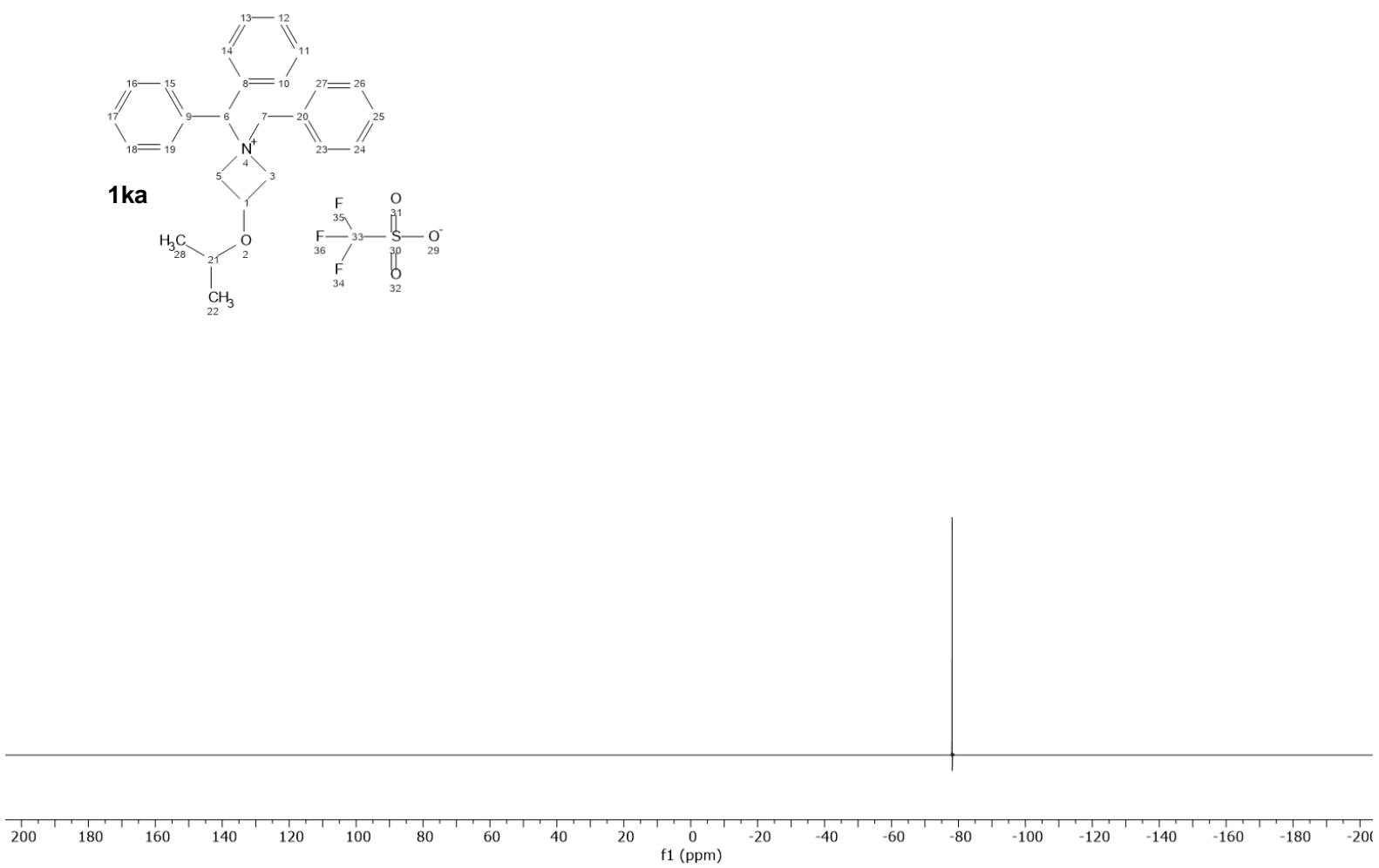
UOXF_groagna_265_1/CdCl3

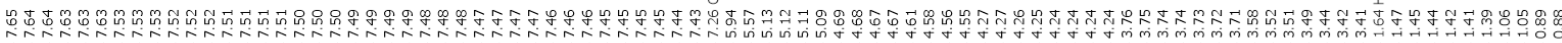

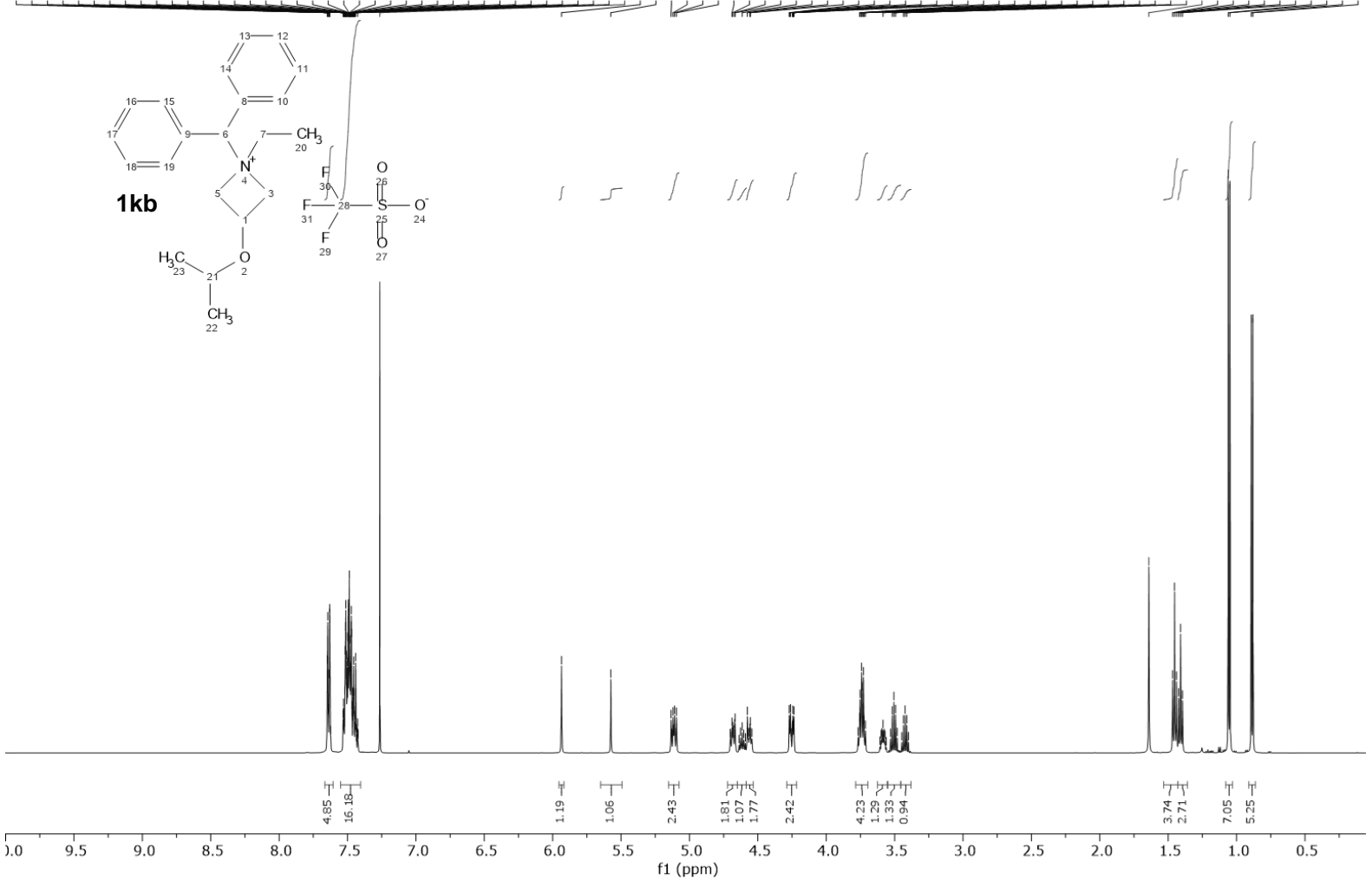

Indirect correlation

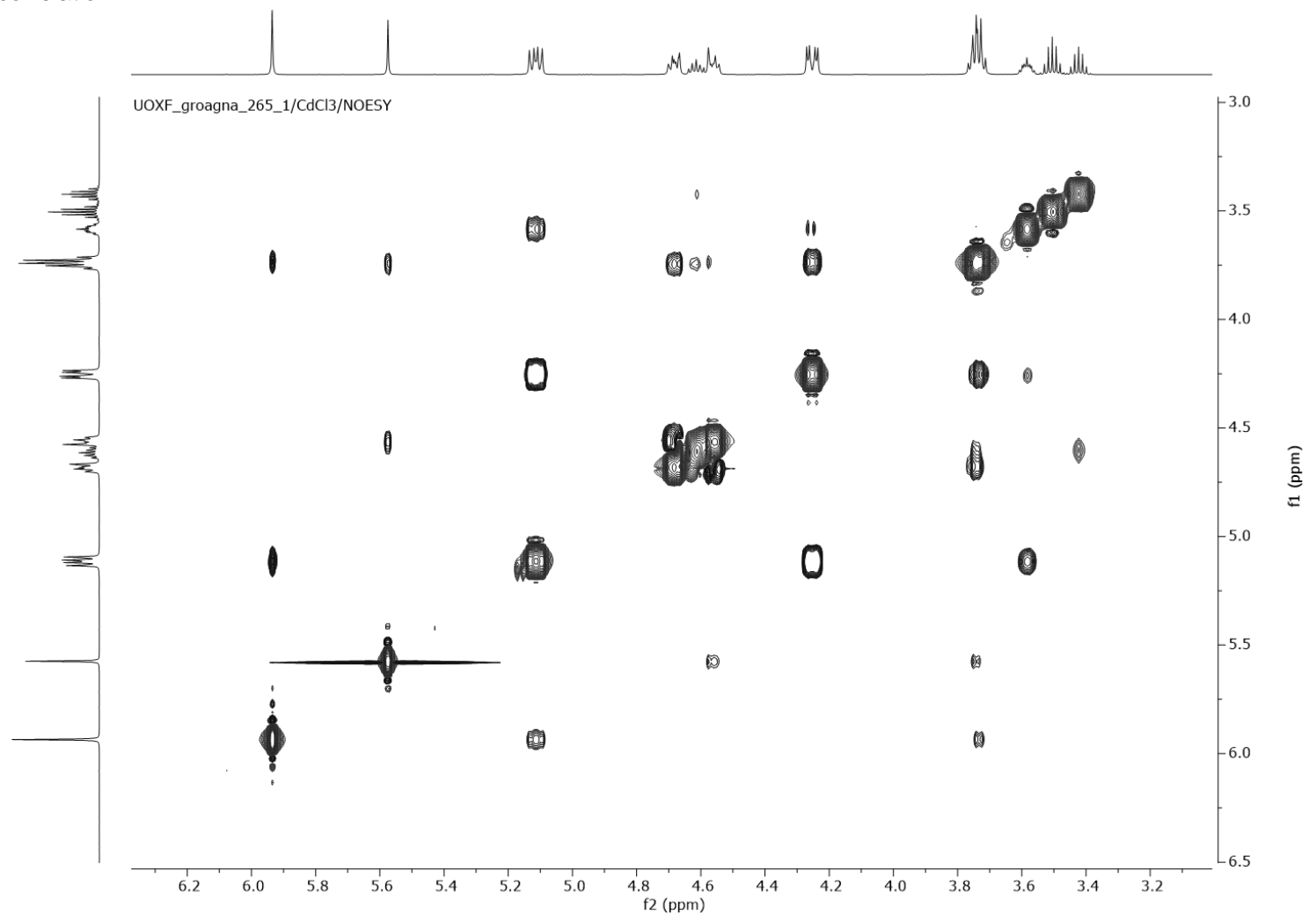


UOXF_groagna_265_1/CdCl3

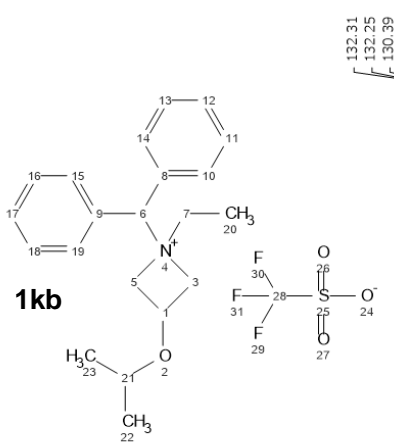

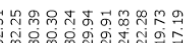

N 1111

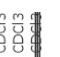

年

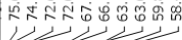

กิ่

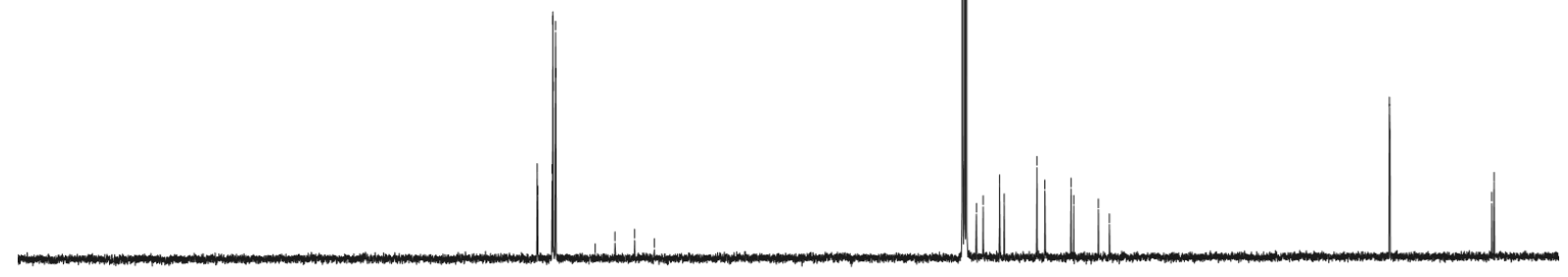

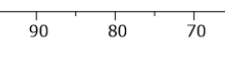

UOXF_groagna_265_1/CdCl3
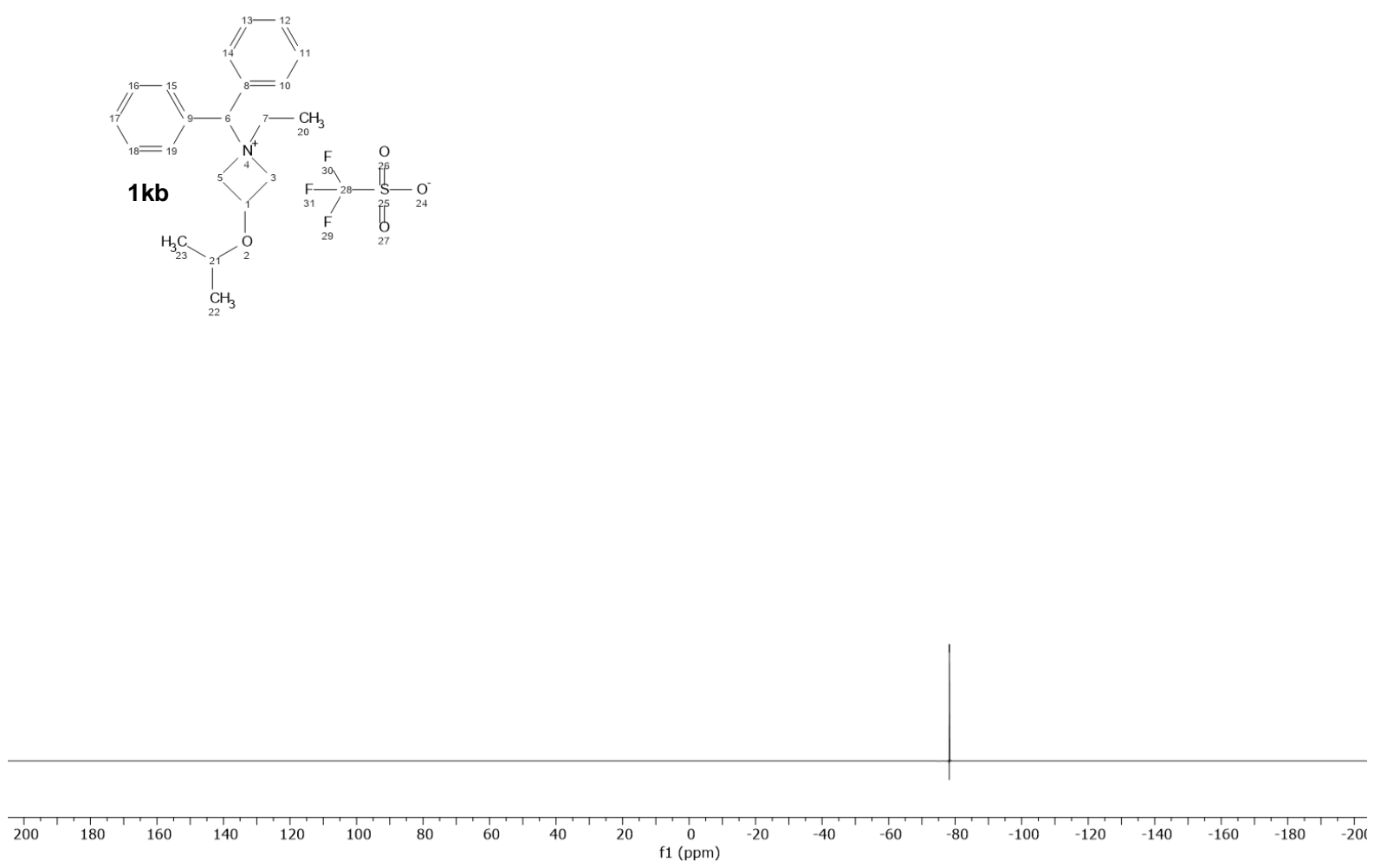
UOXF_groagna_341_1/CdCl3

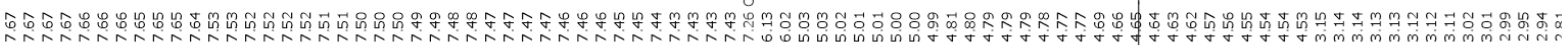

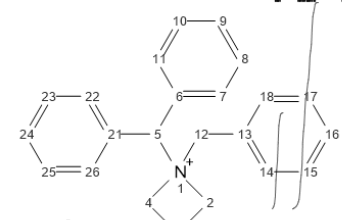

1 la
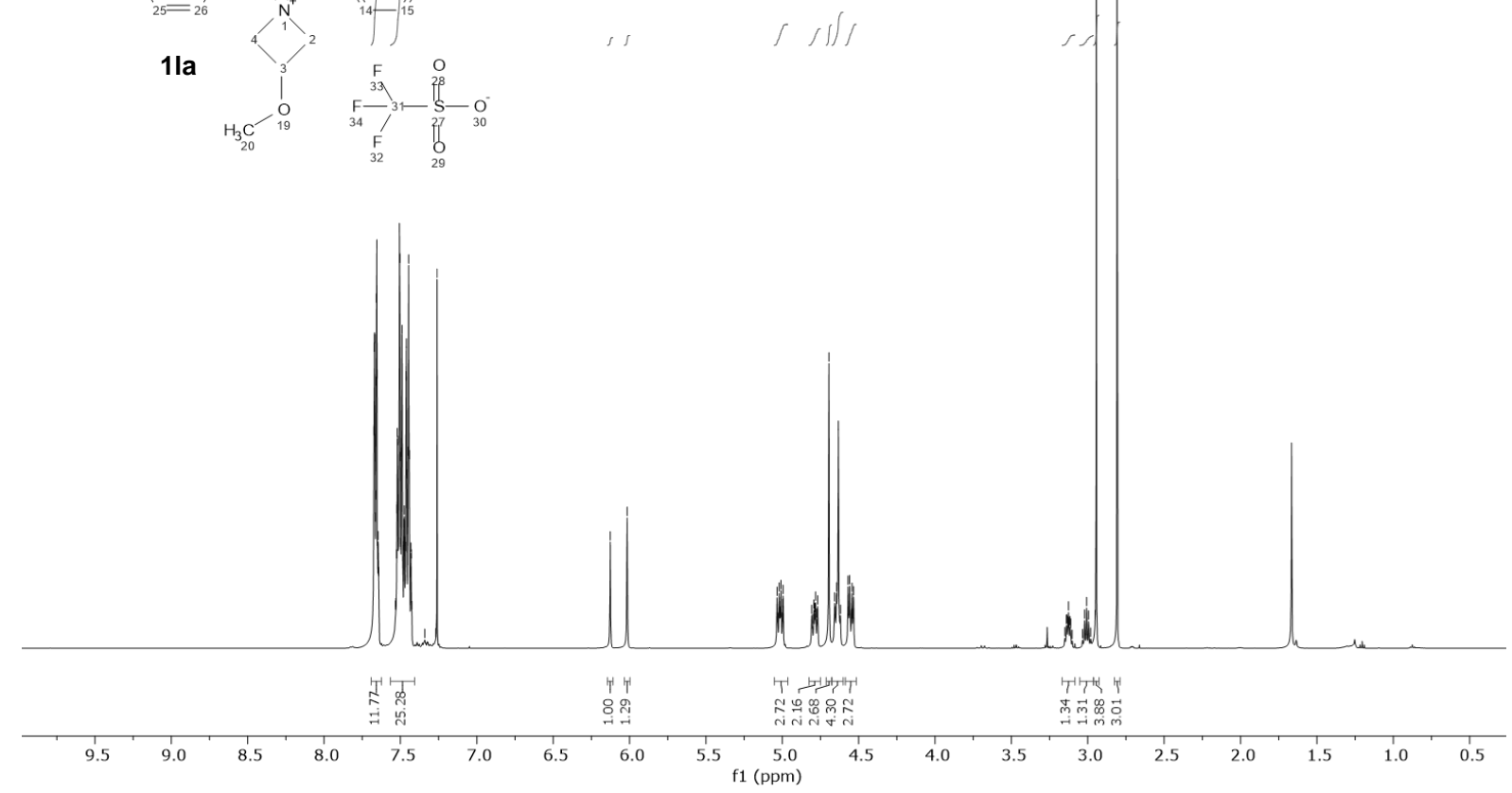

Indirect correlation

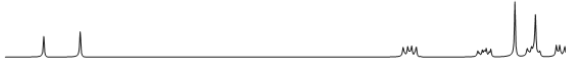

UOXF_groagna_341_1/CdC13/NOESY

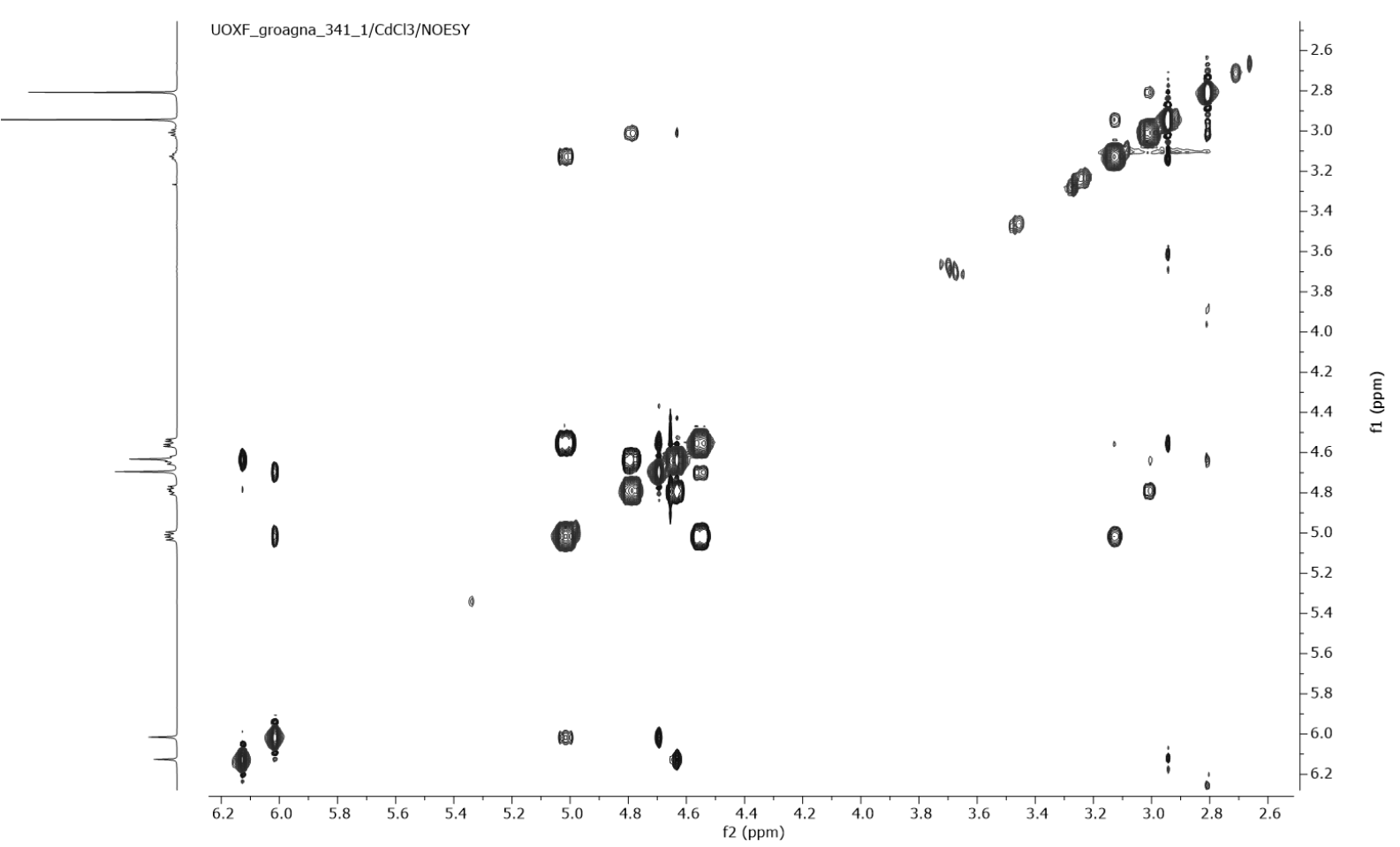


UOXF_groagna_341_1/CdCl3

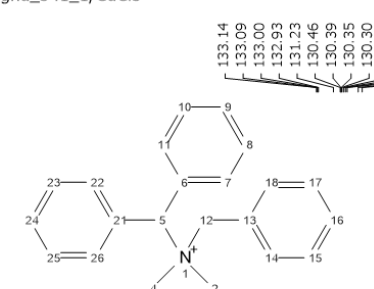

1 la

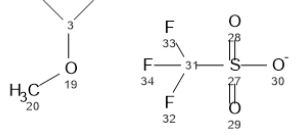

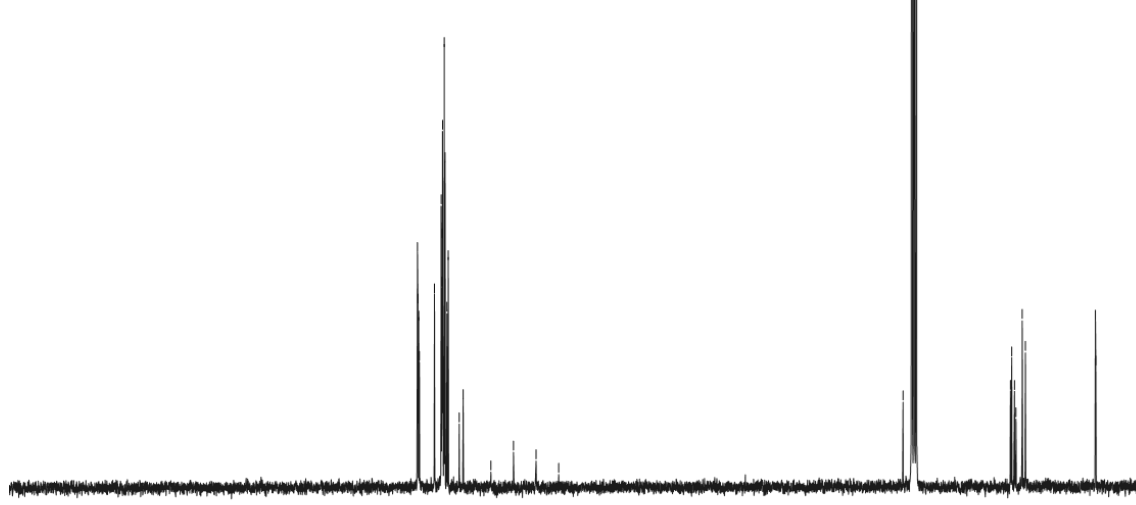

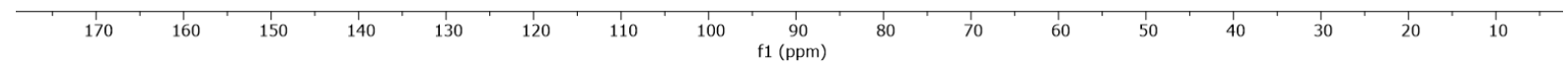

UOXF_groagna_341_1/CdCl3

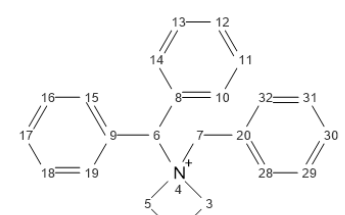

$11 a$

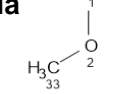

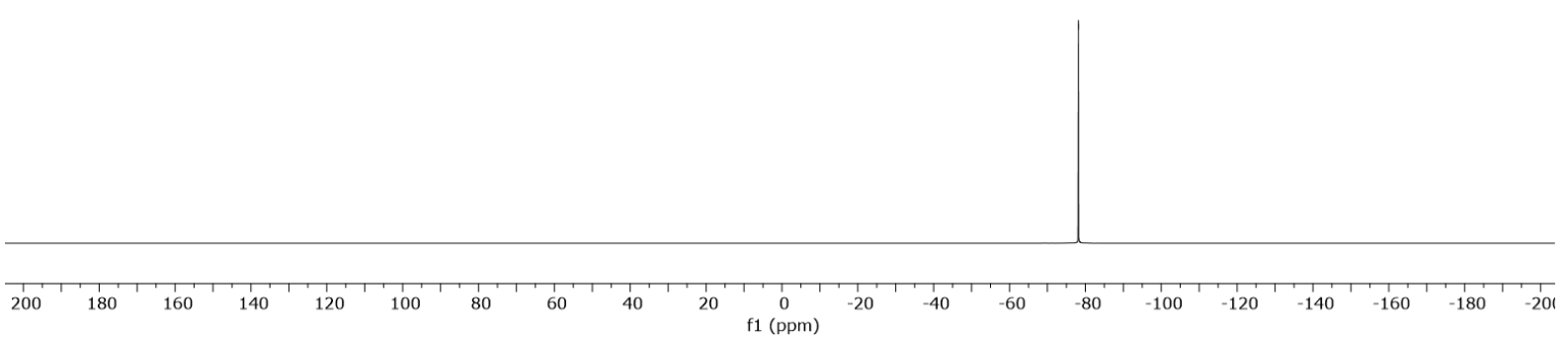


UOXF_groagna_264_1/CdCl3

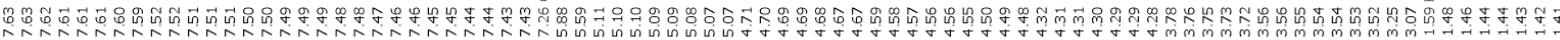
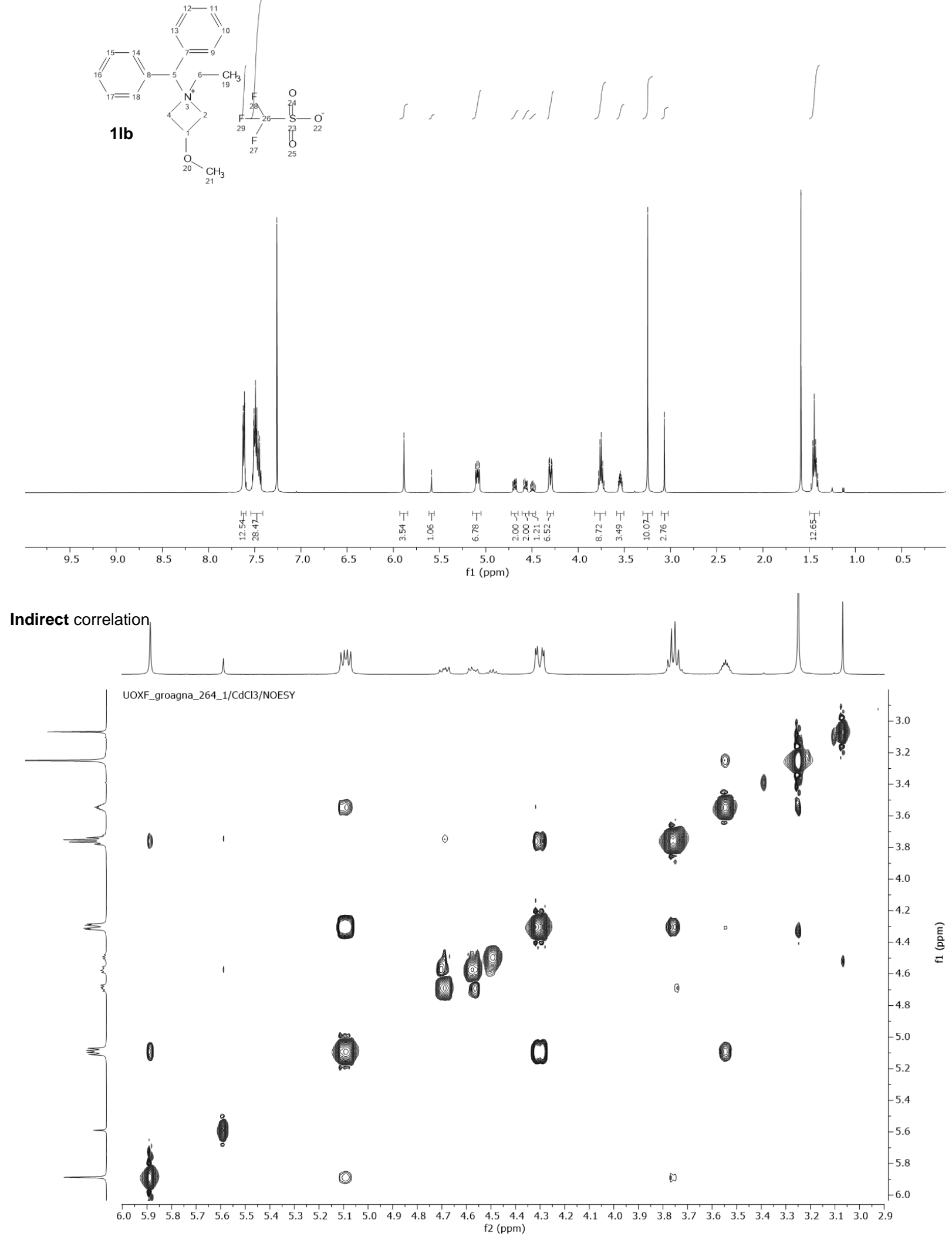
UOXF_groagna264_1/CdCl3/

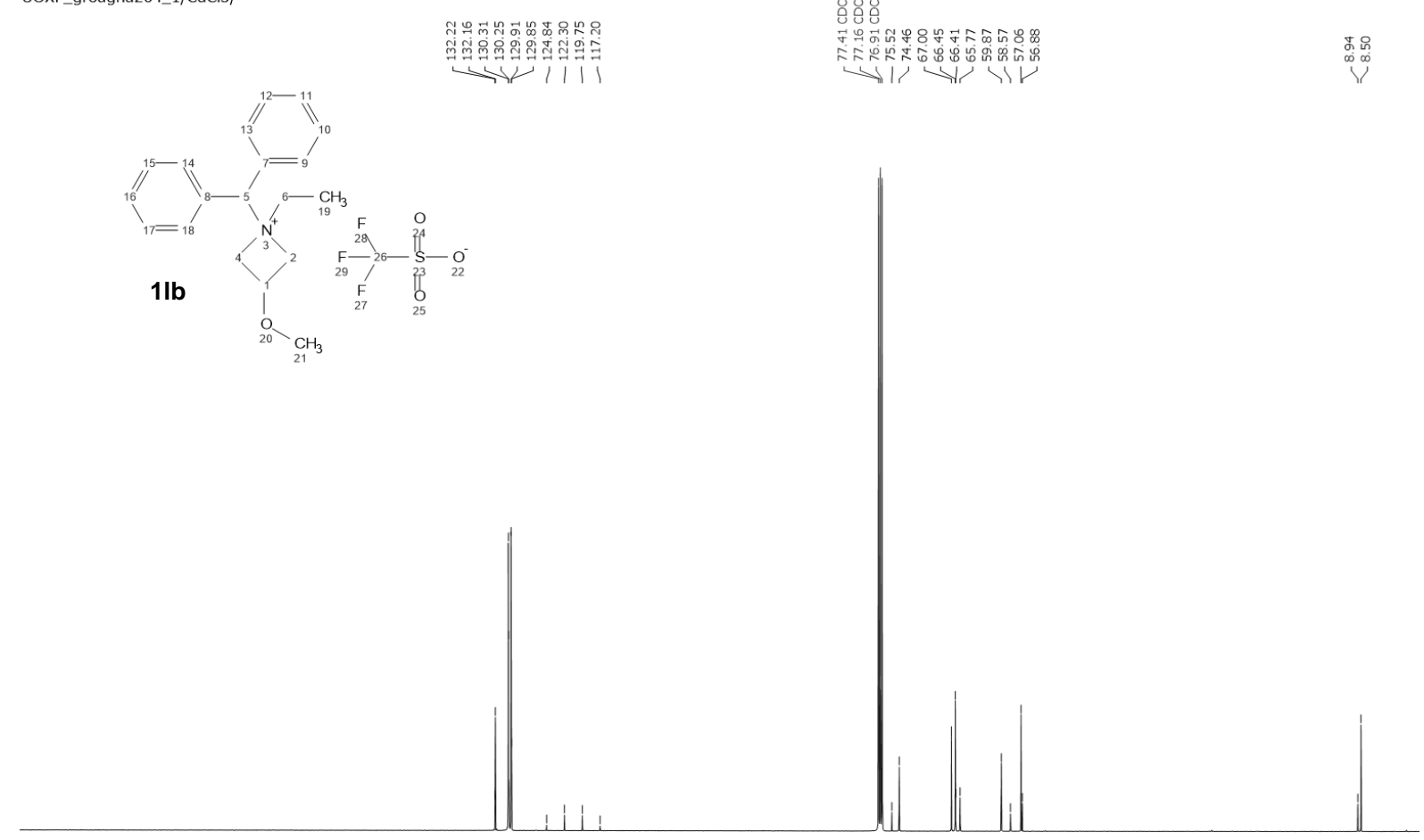

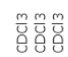

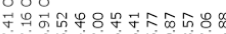

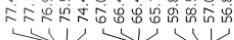

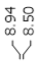

管।|1।

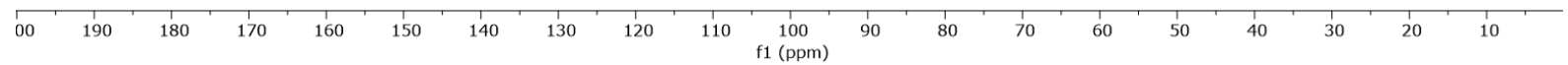

UOXF_groagna_264_1/CdCl3

$n$
$n$
$\infty$
0
0
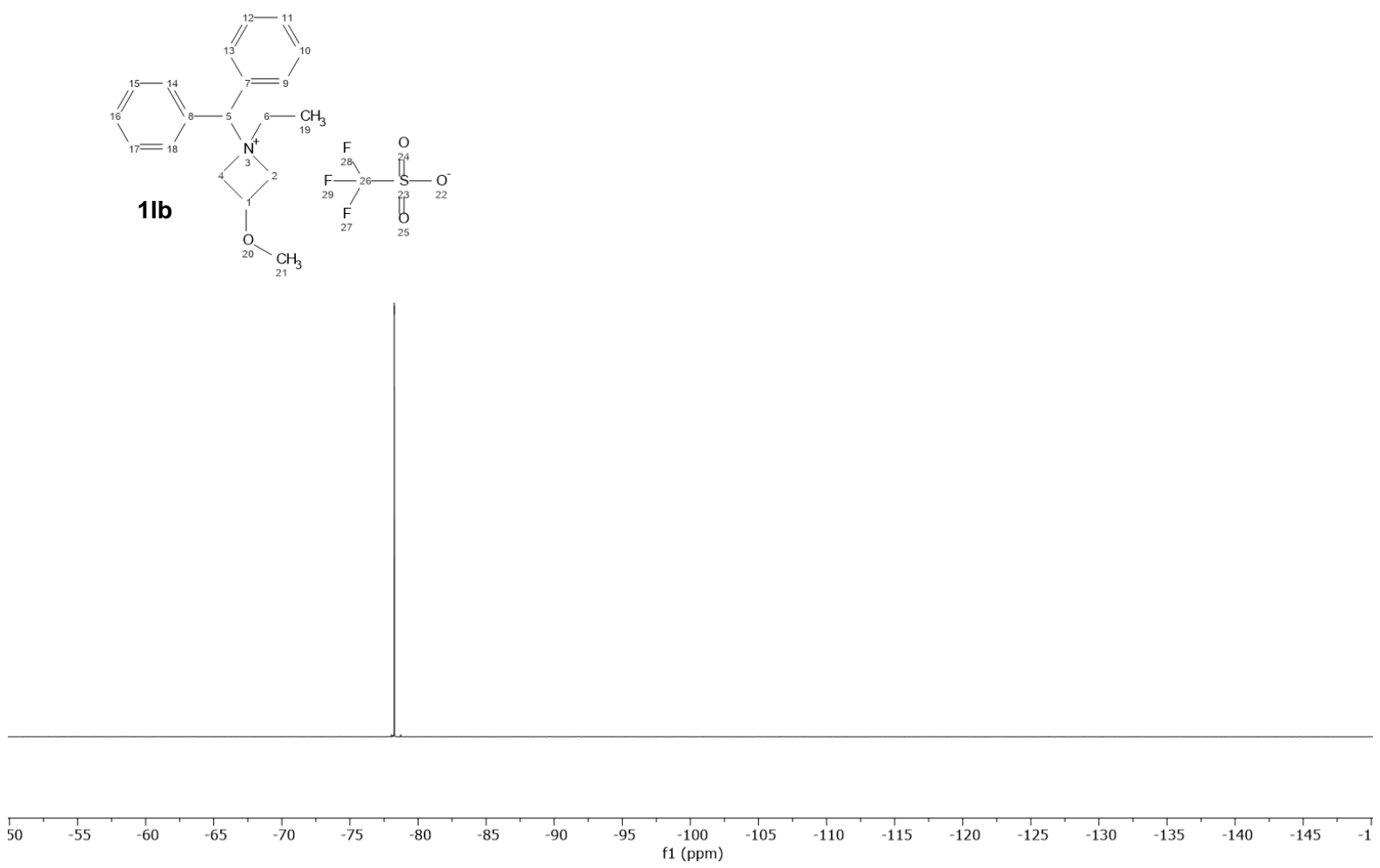
UOXF_groagna_340_1/CdCl3

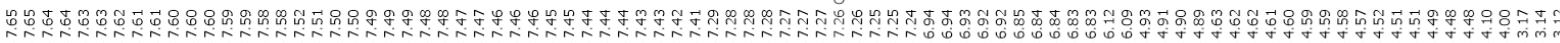
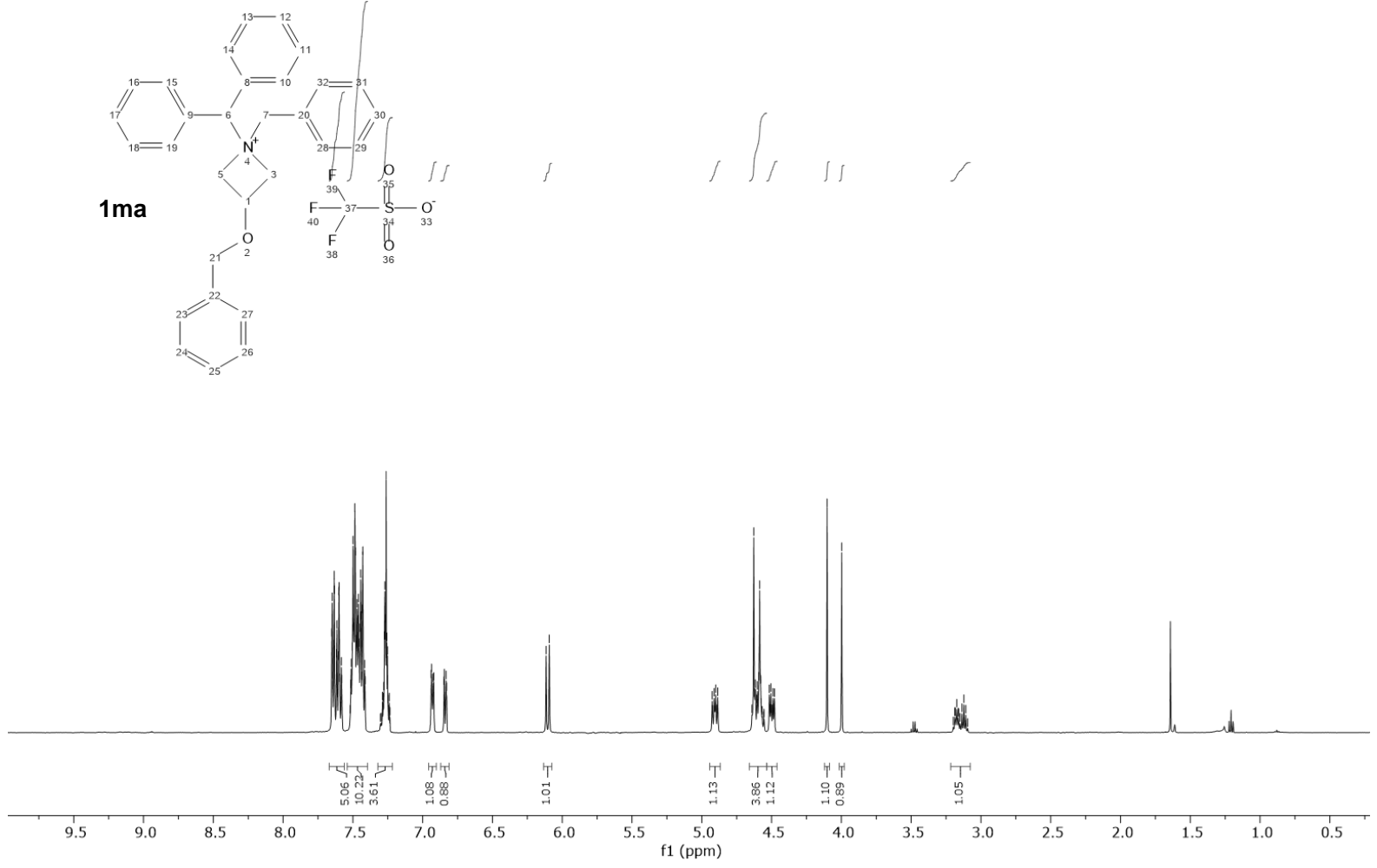

Indirect correlation
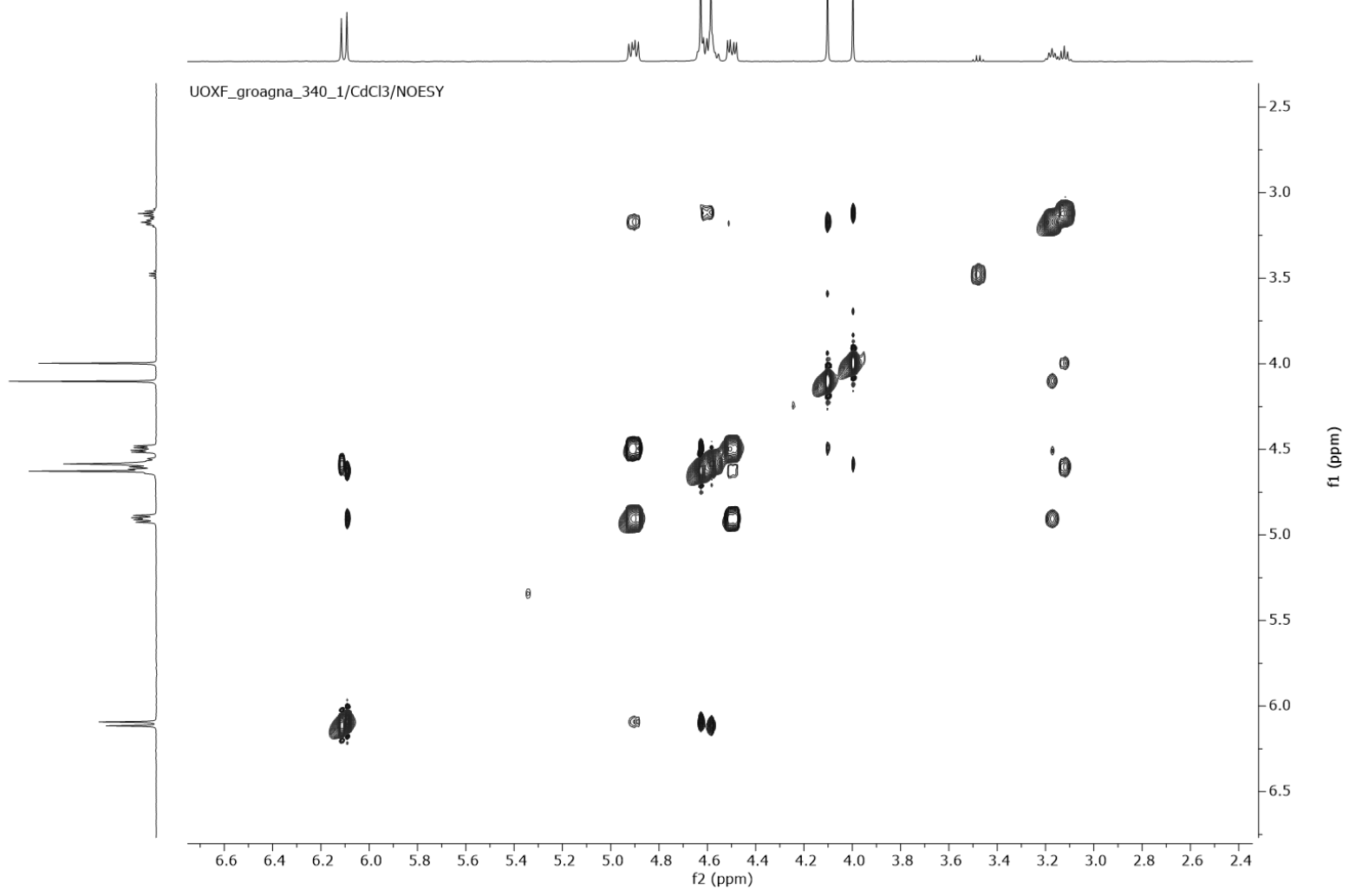


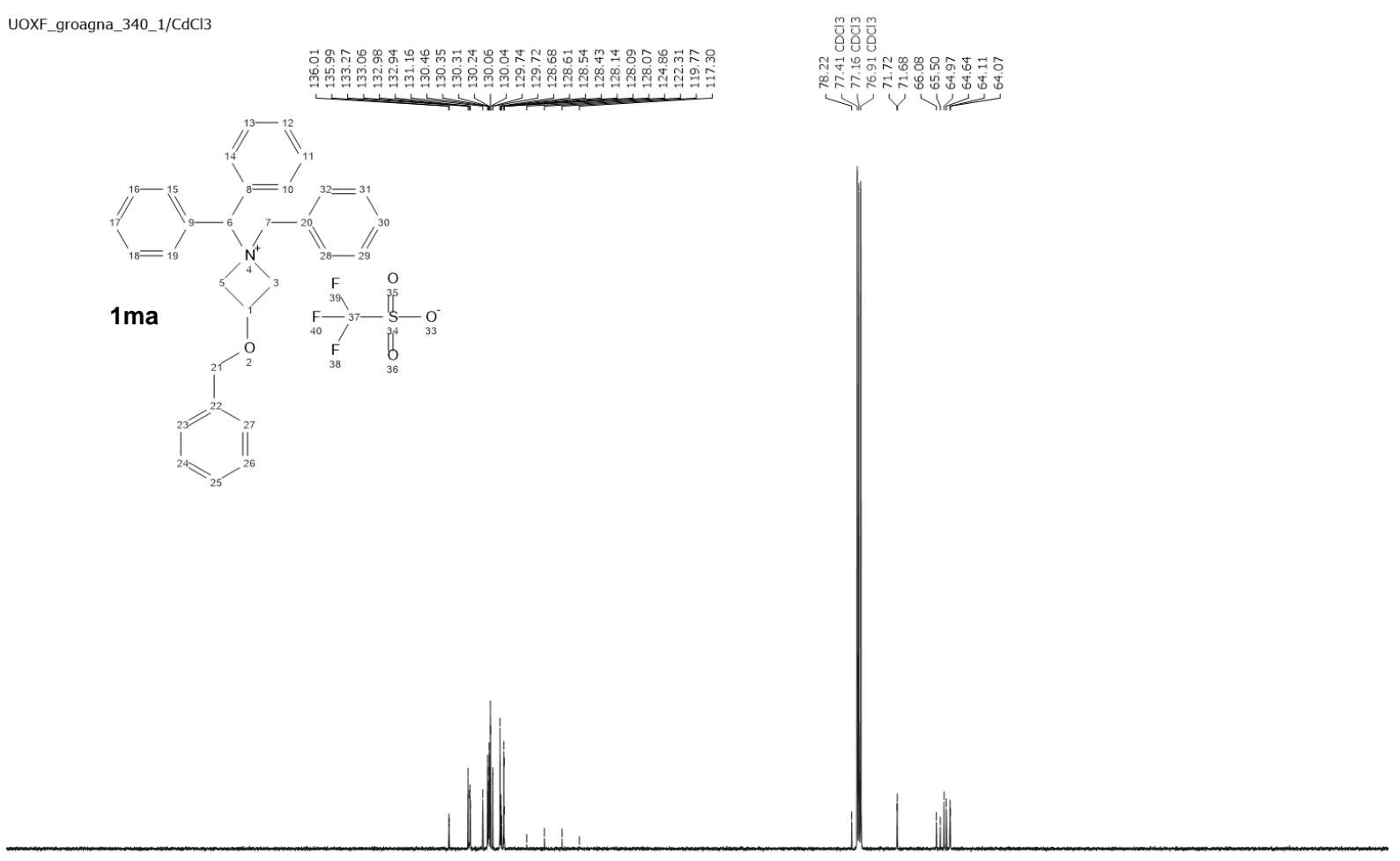

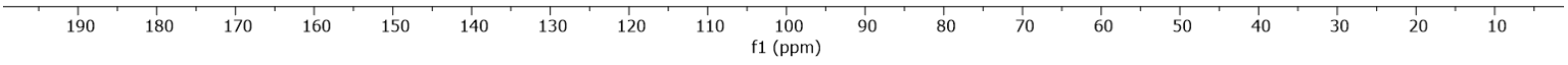

UOXF_groagna_340_1/CdCl3

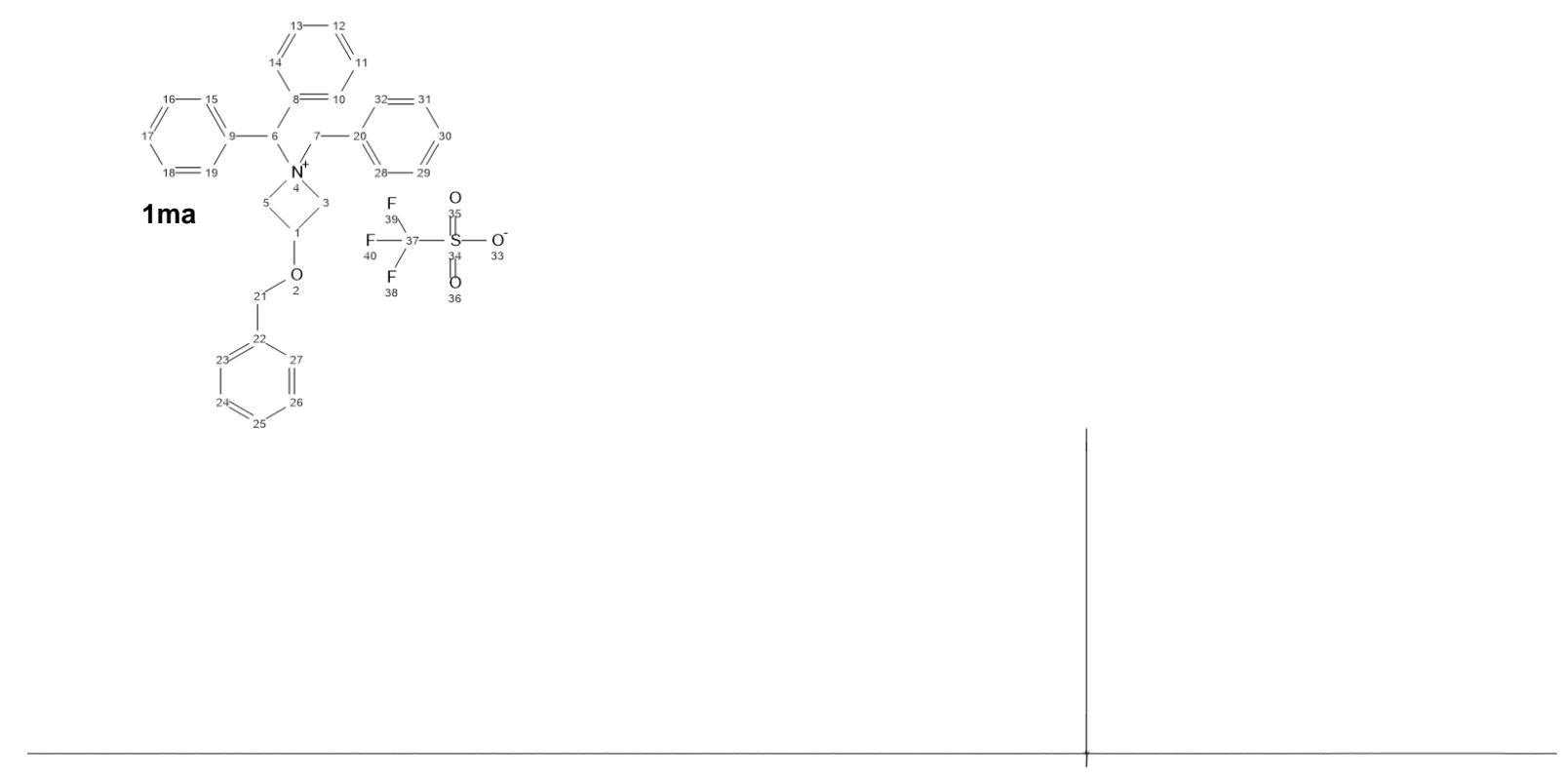

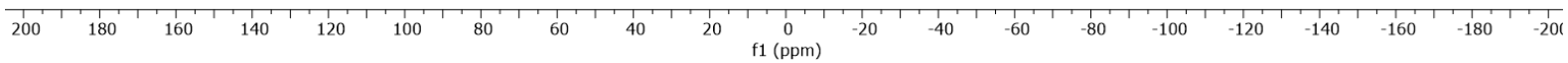



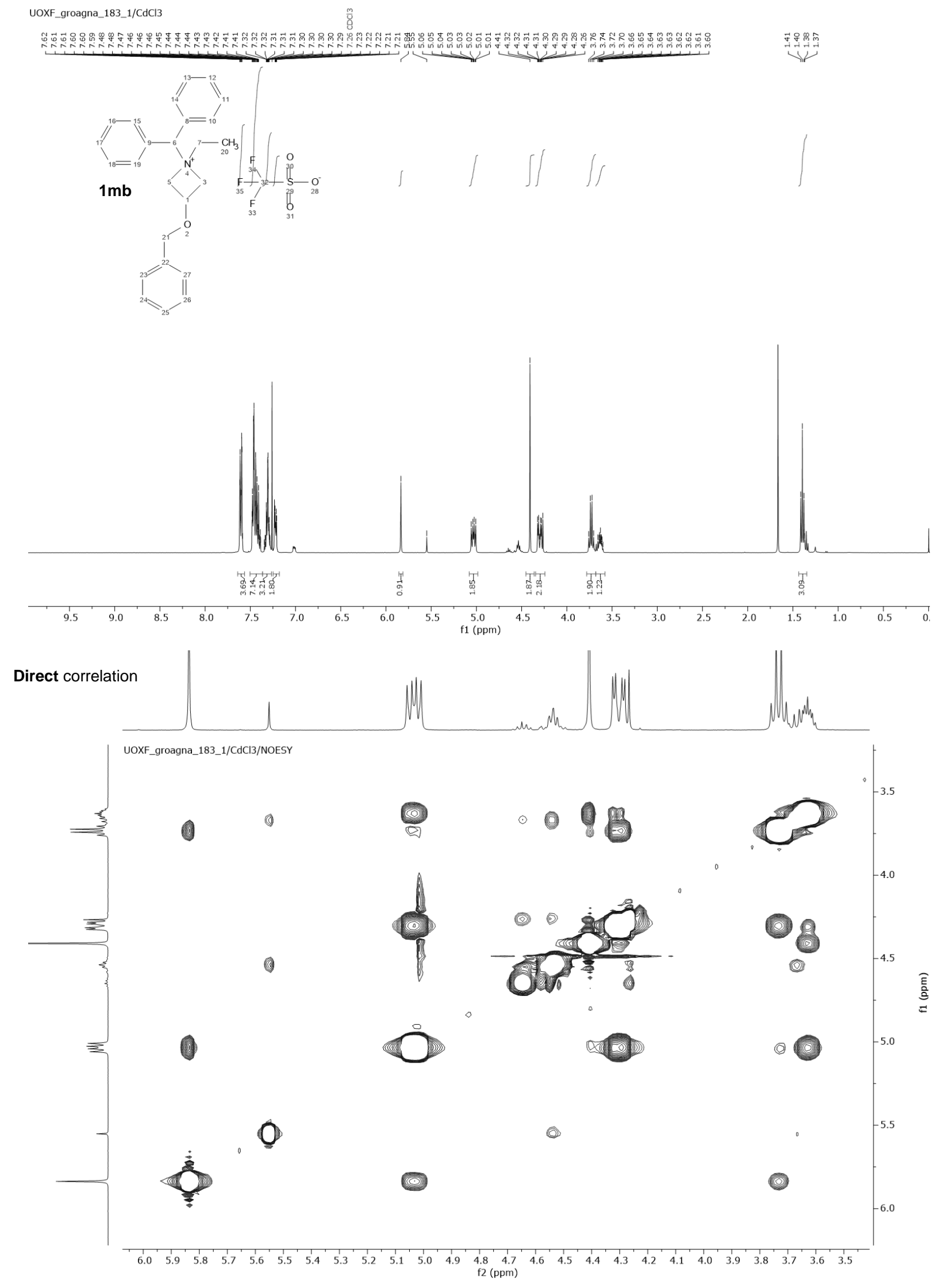


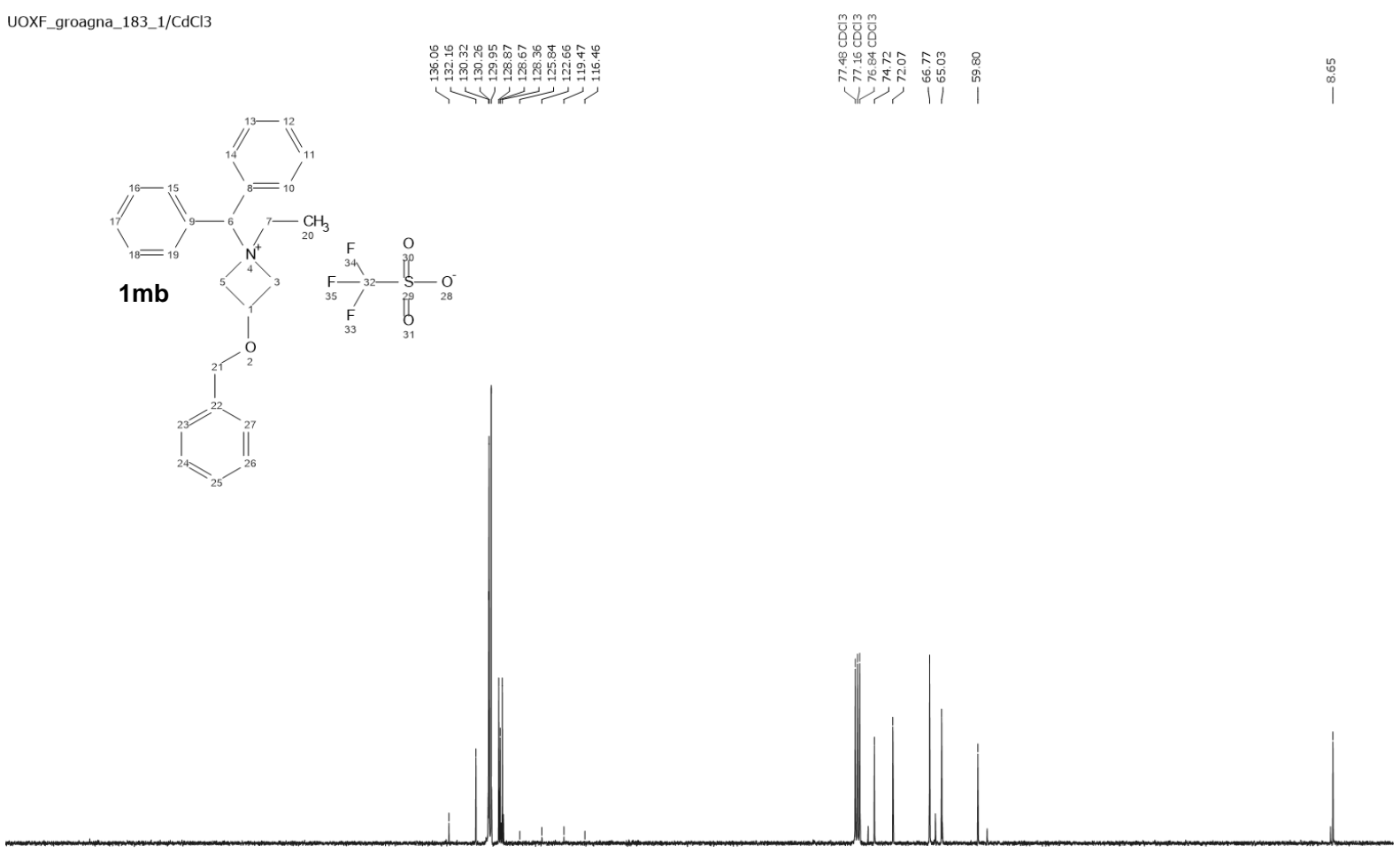

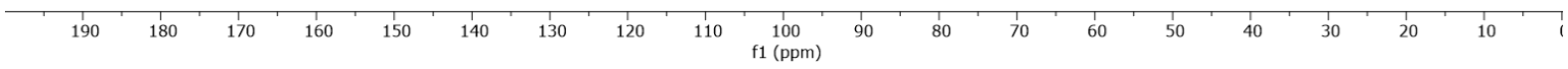

UOXF_groagna_183_1/CdCl3
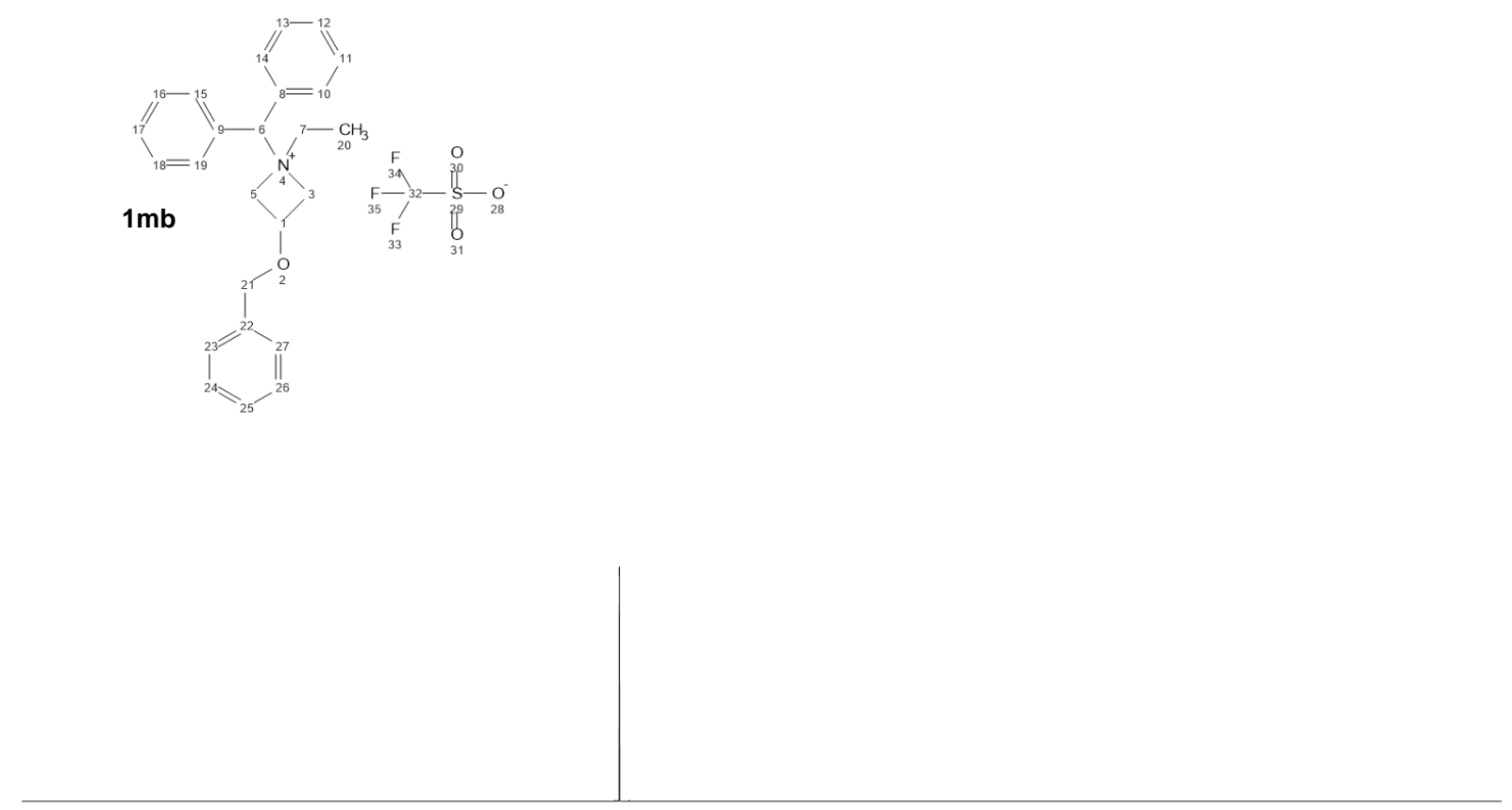

\begin{tabular}{|c|c|c|c|c|c|c|c|c|c|c|c|c|c|}
\hline 50 & -55 & -60 & -65 & -70 & -75 & -80 & $\begin{array}{c}-85 \\
\mathrm{f} 1(\mathrm{ppm})\end{array}$ & -90 & -95 & -100 & -105 & -110 & -115 \\
\hline
\end{tabular}



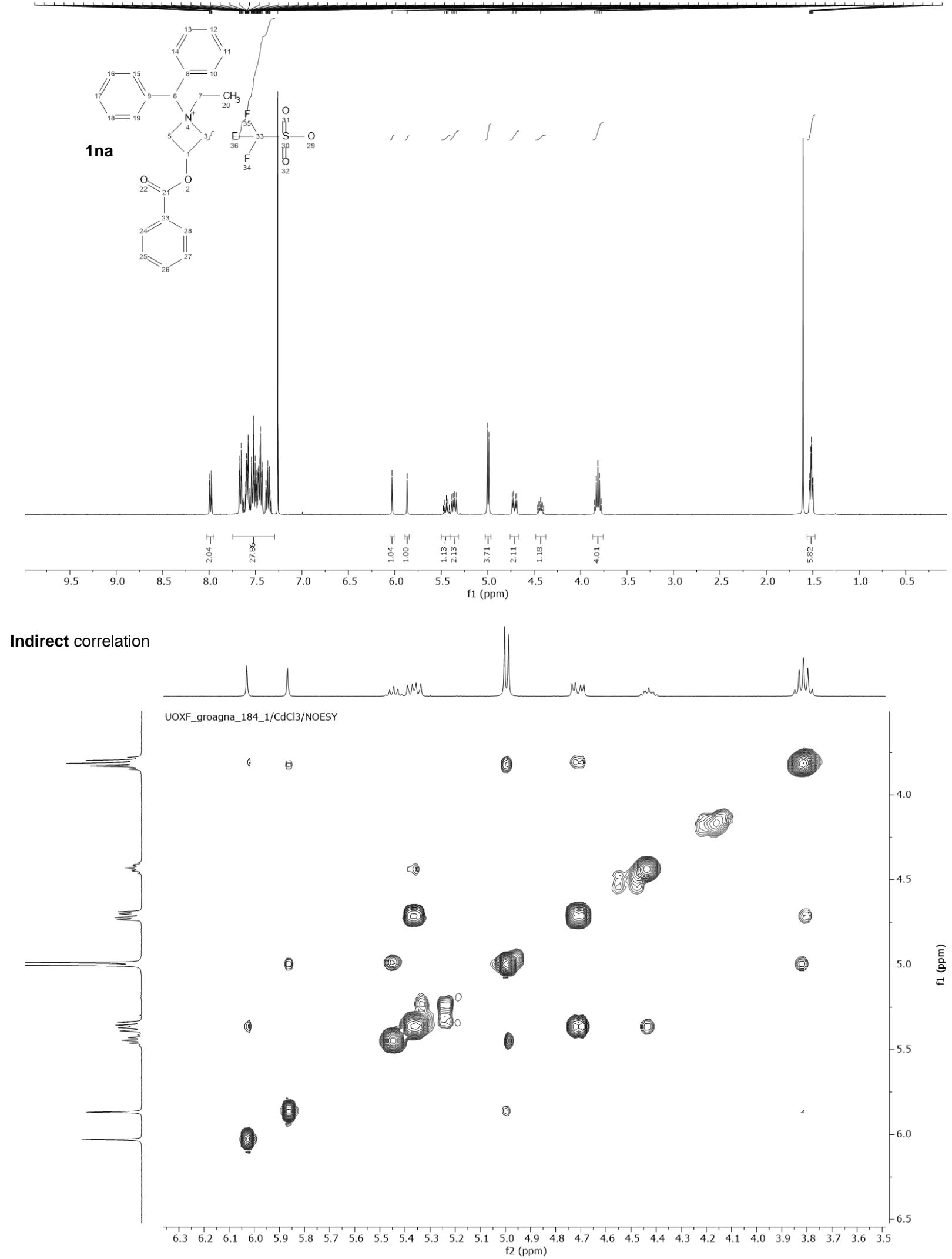


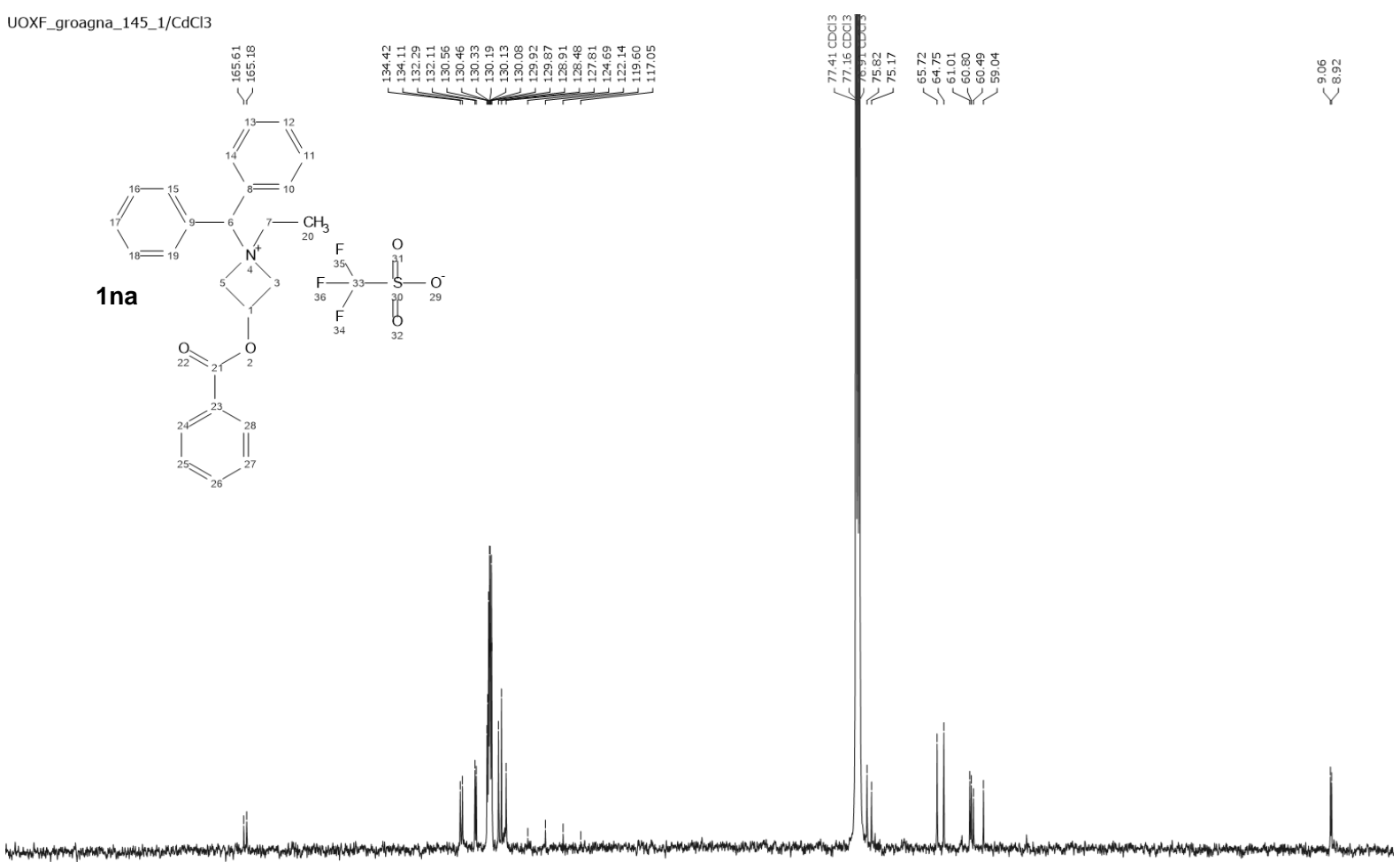

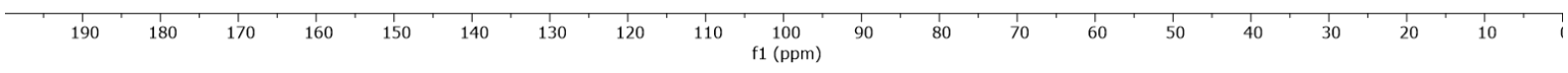

UOXF_groagna_184_1/CdCl3

$\underset{\substack{n \\ \infty}}{\substack{a \\ j}}$
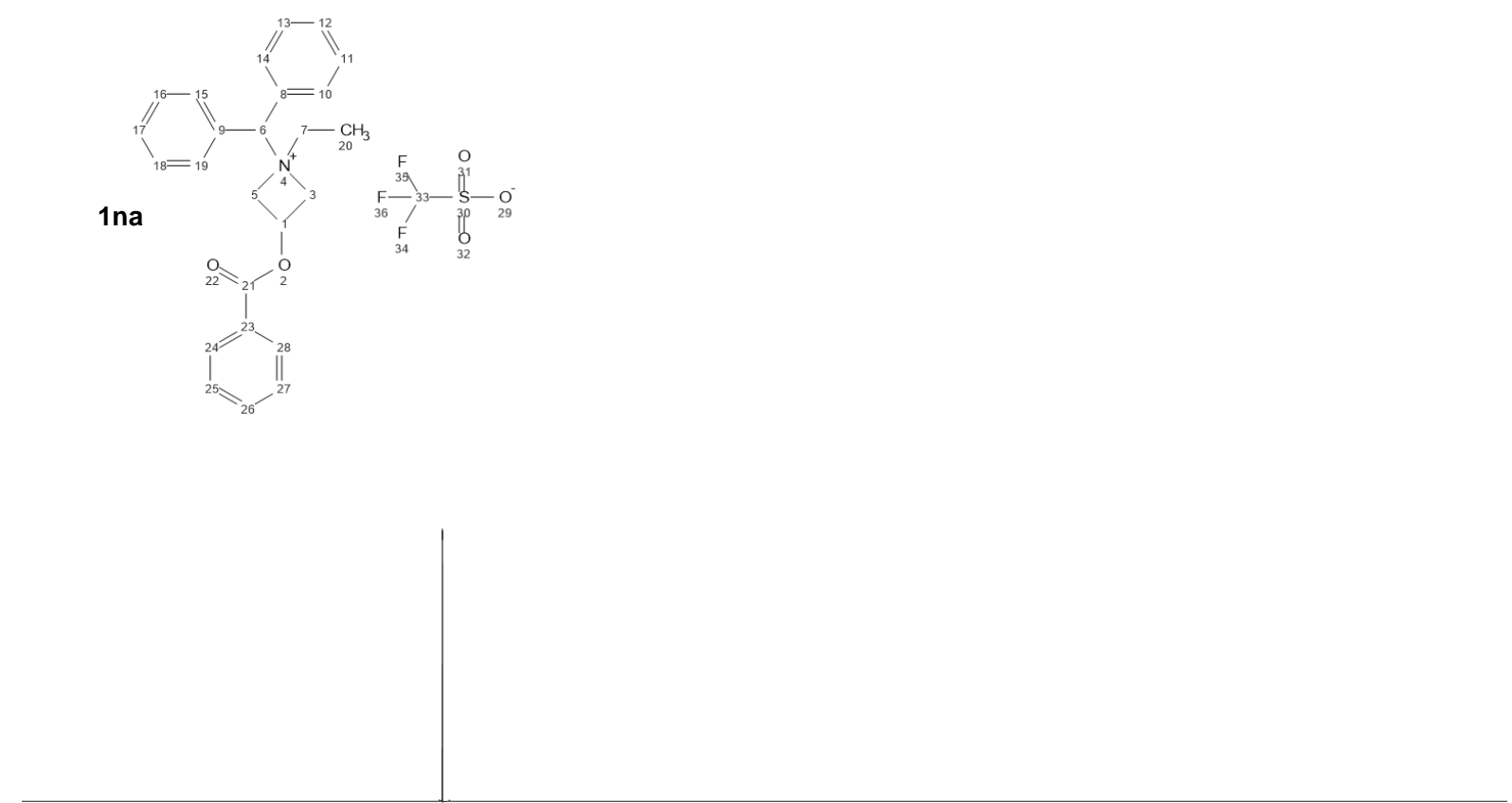

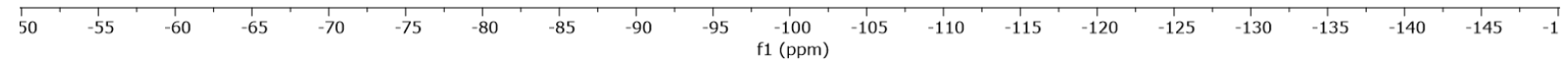


UOXF_groagna_231_1/DMSO-d

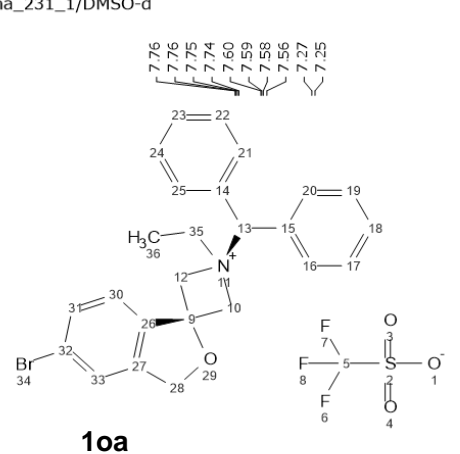

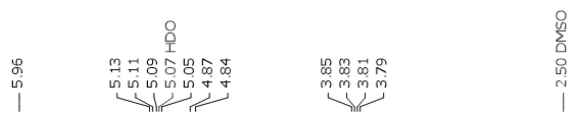

$\sqrt[5]{\frac{5}{2}}$

1oa
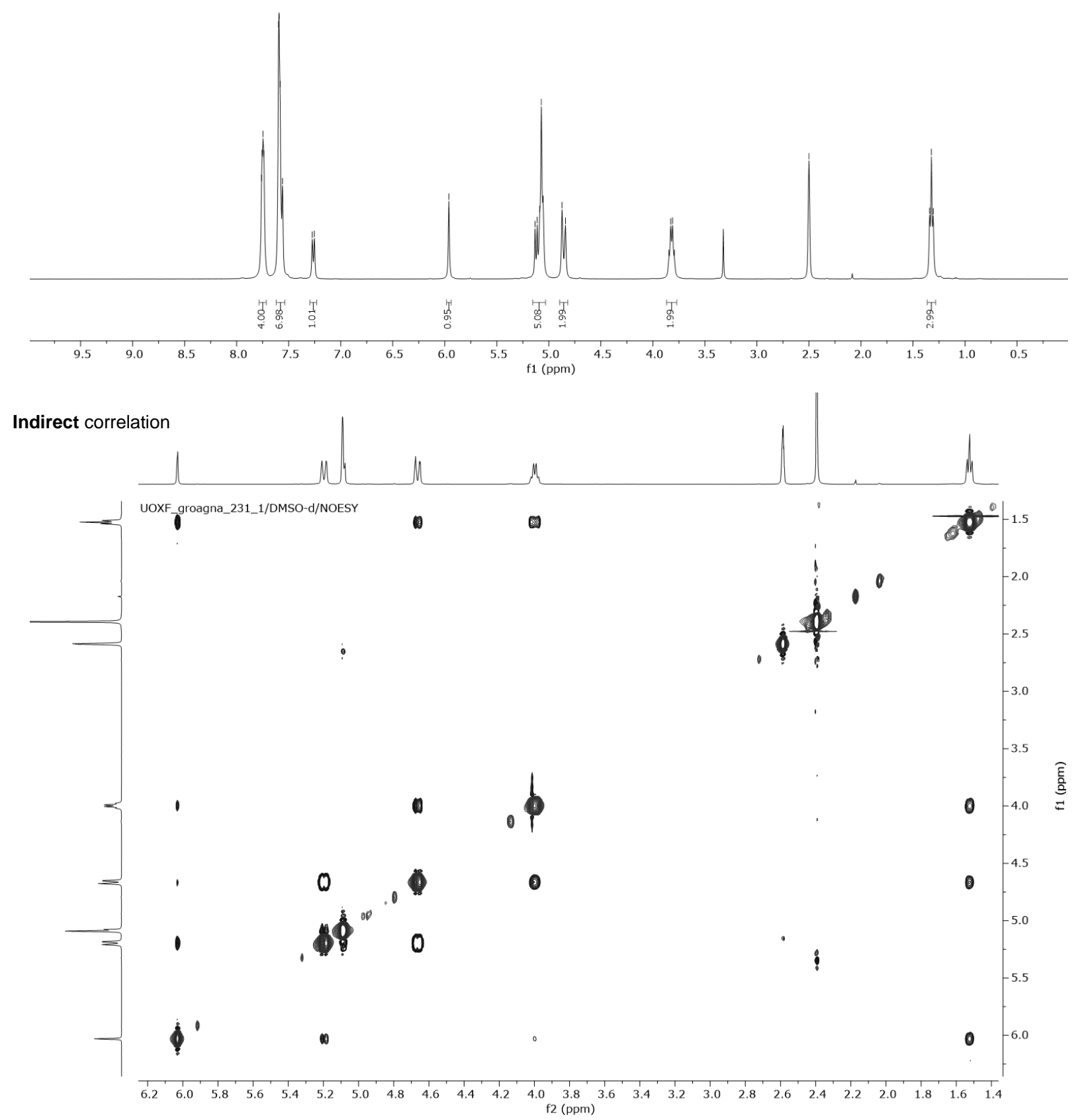


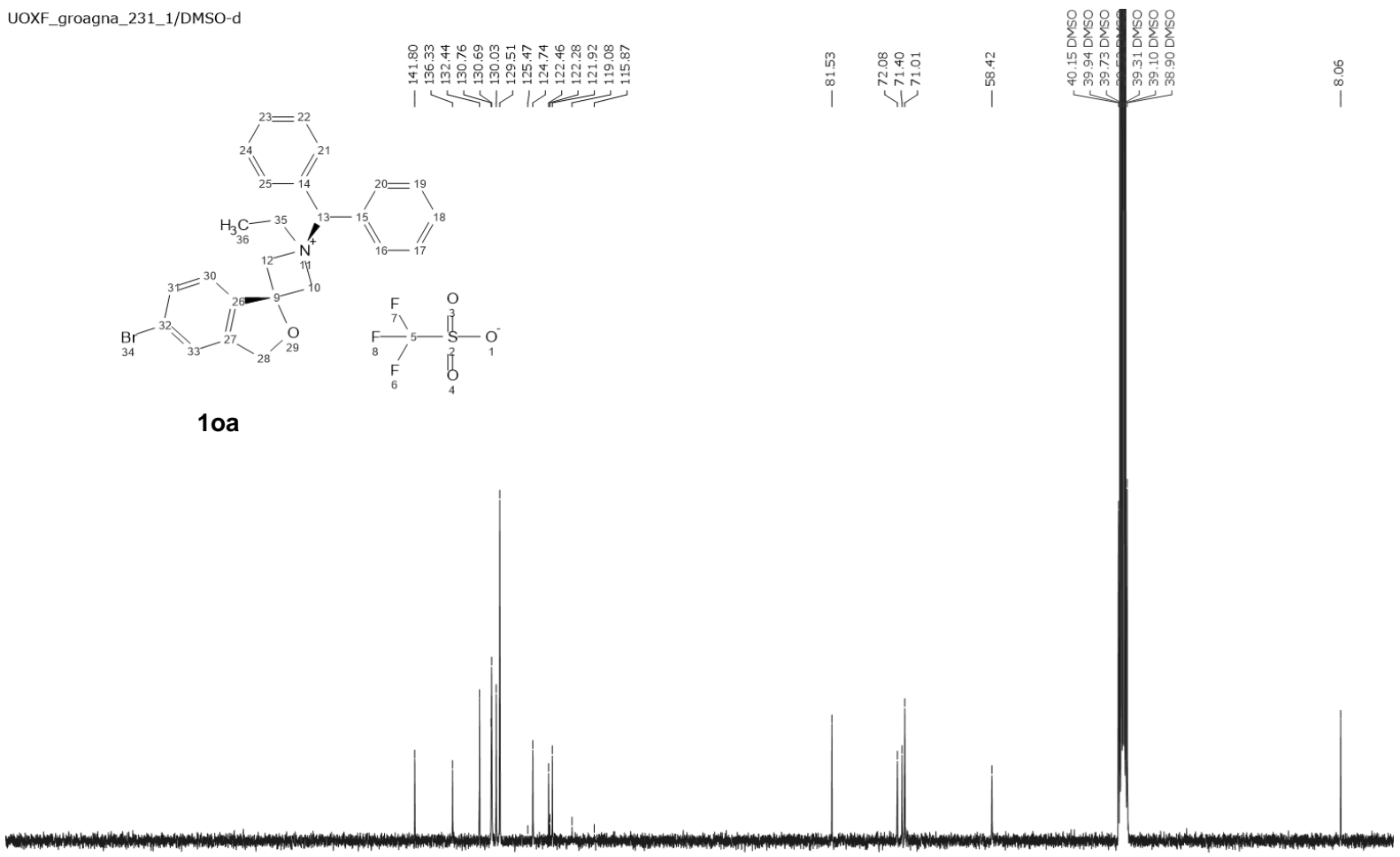

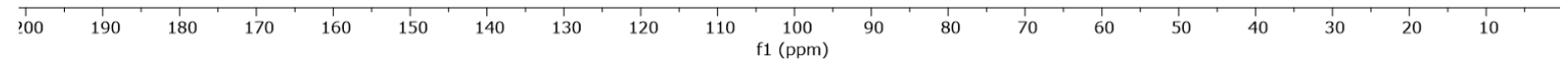

UOXF_groagna_231_1/CdCl3

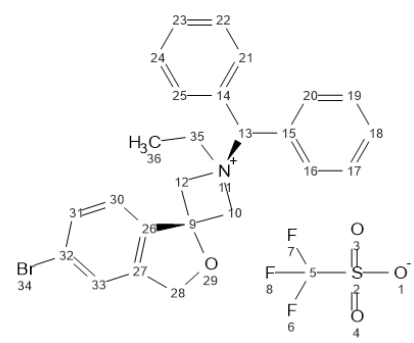

$10 a$

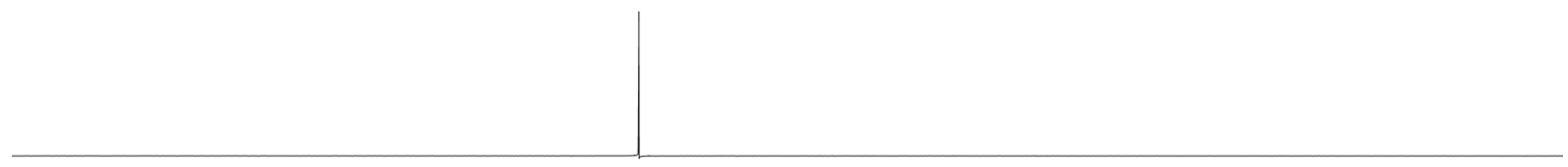

\begin{tabular}{|c|c|c|c|c|c|c|c|c|c|c|c|c|}
\hline 50 & -55 & -60 & -65 & -70 & -75 & -80 & $\begin{array}{c}-85 \\
\mathrm{f} 1(\mathrm{ppm})\end{array}$ & -90 & -95 & -100 & -105 & -110 \\
\hline
\end{tabular}



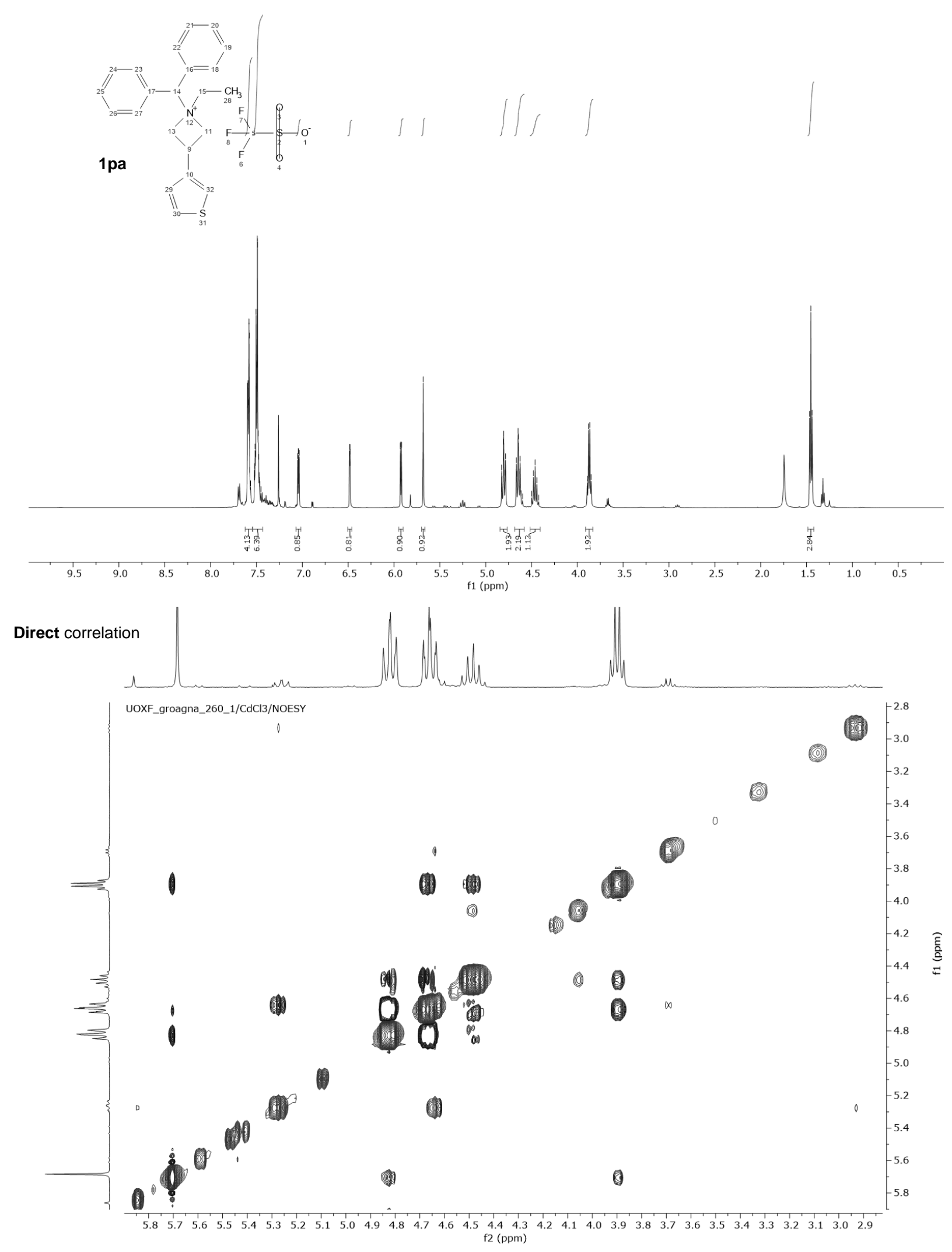


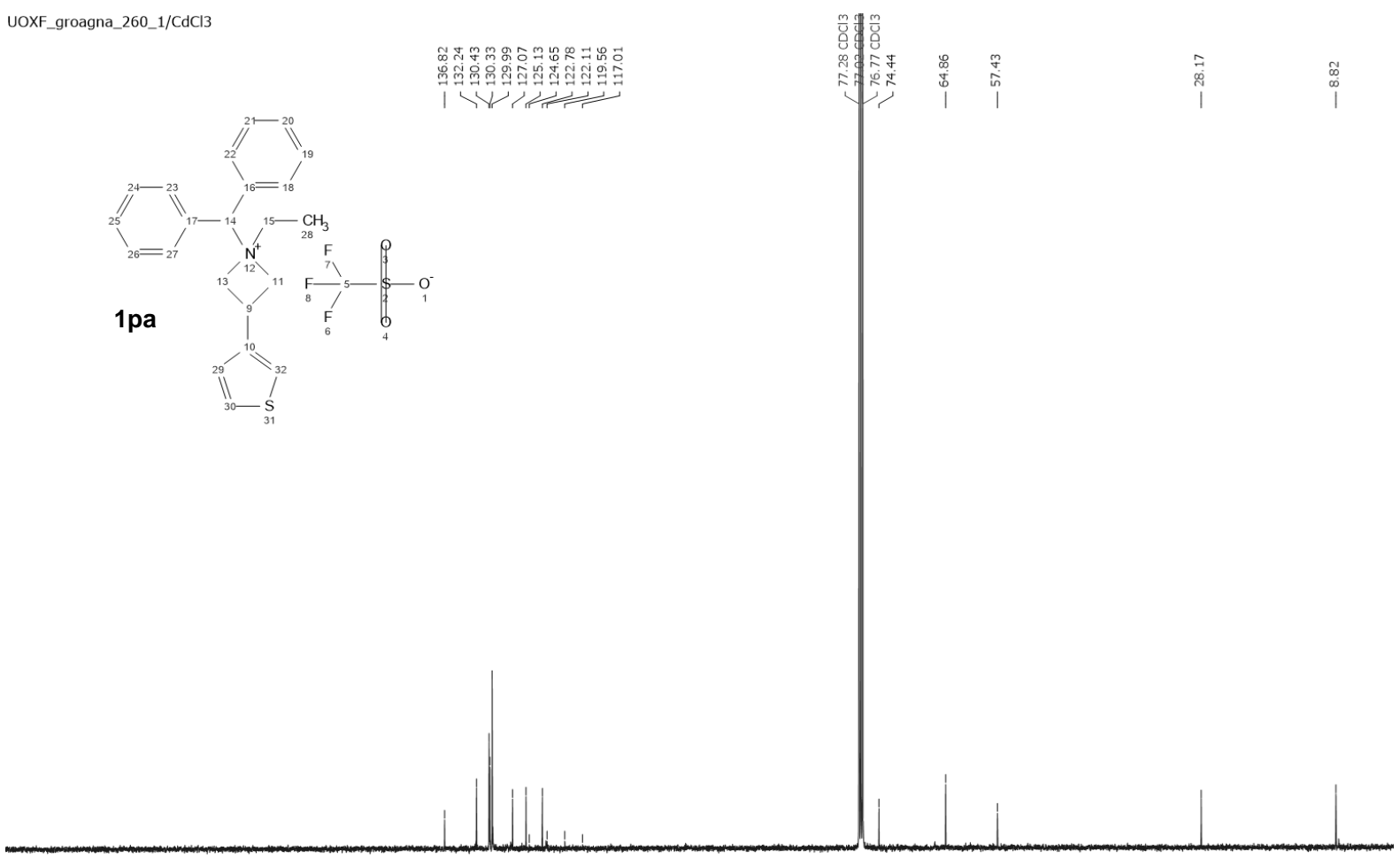

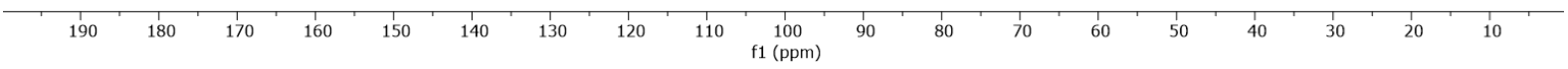
UOXF_groagna_260_1/CdCl3

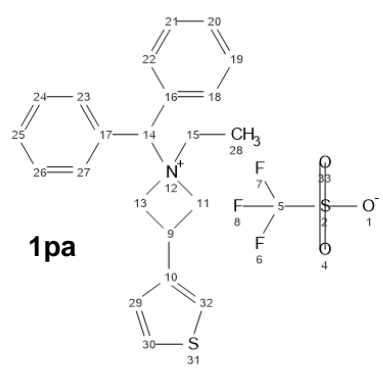

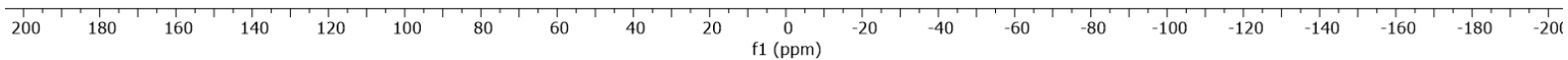




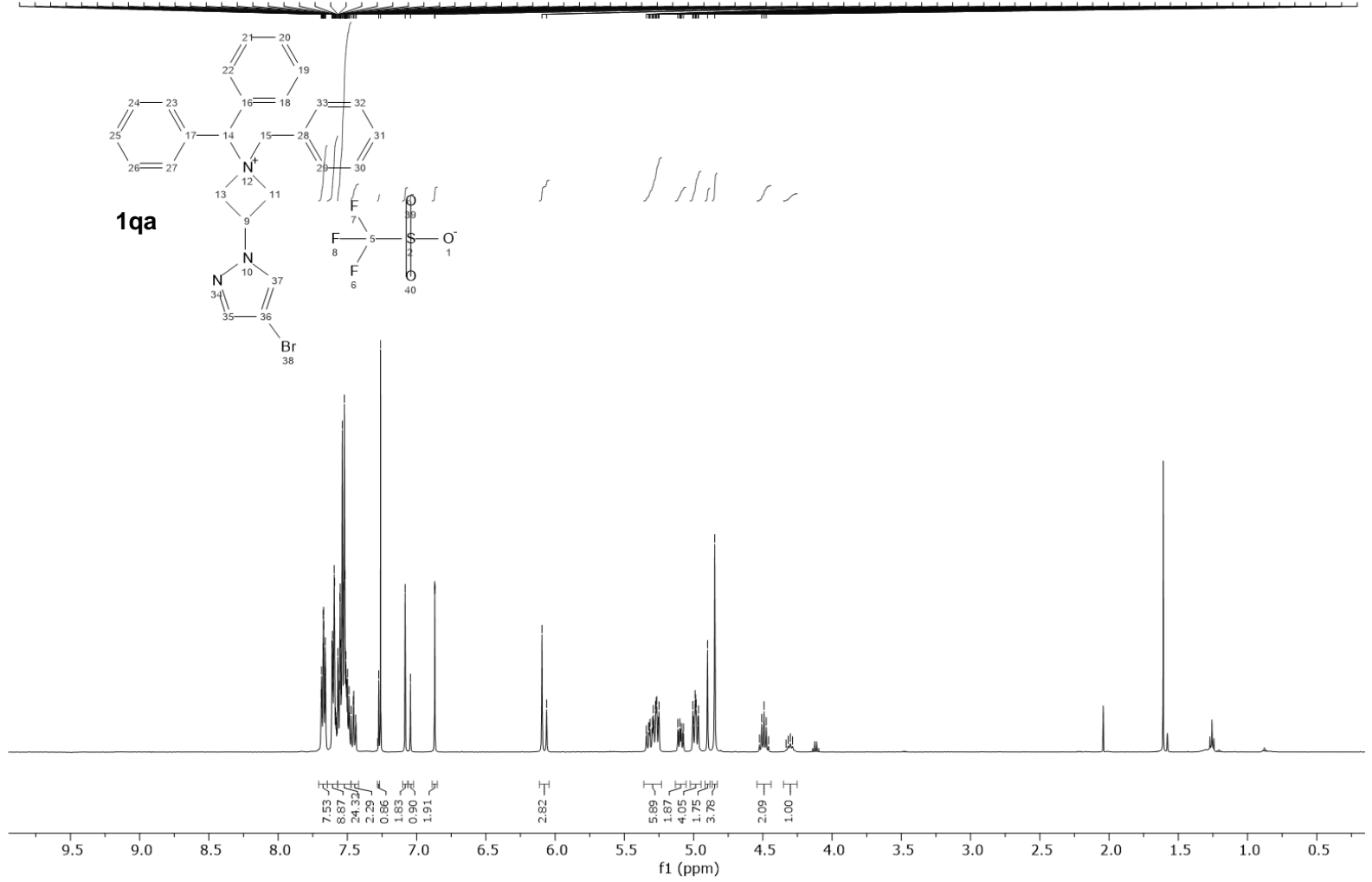

Indirect correlation
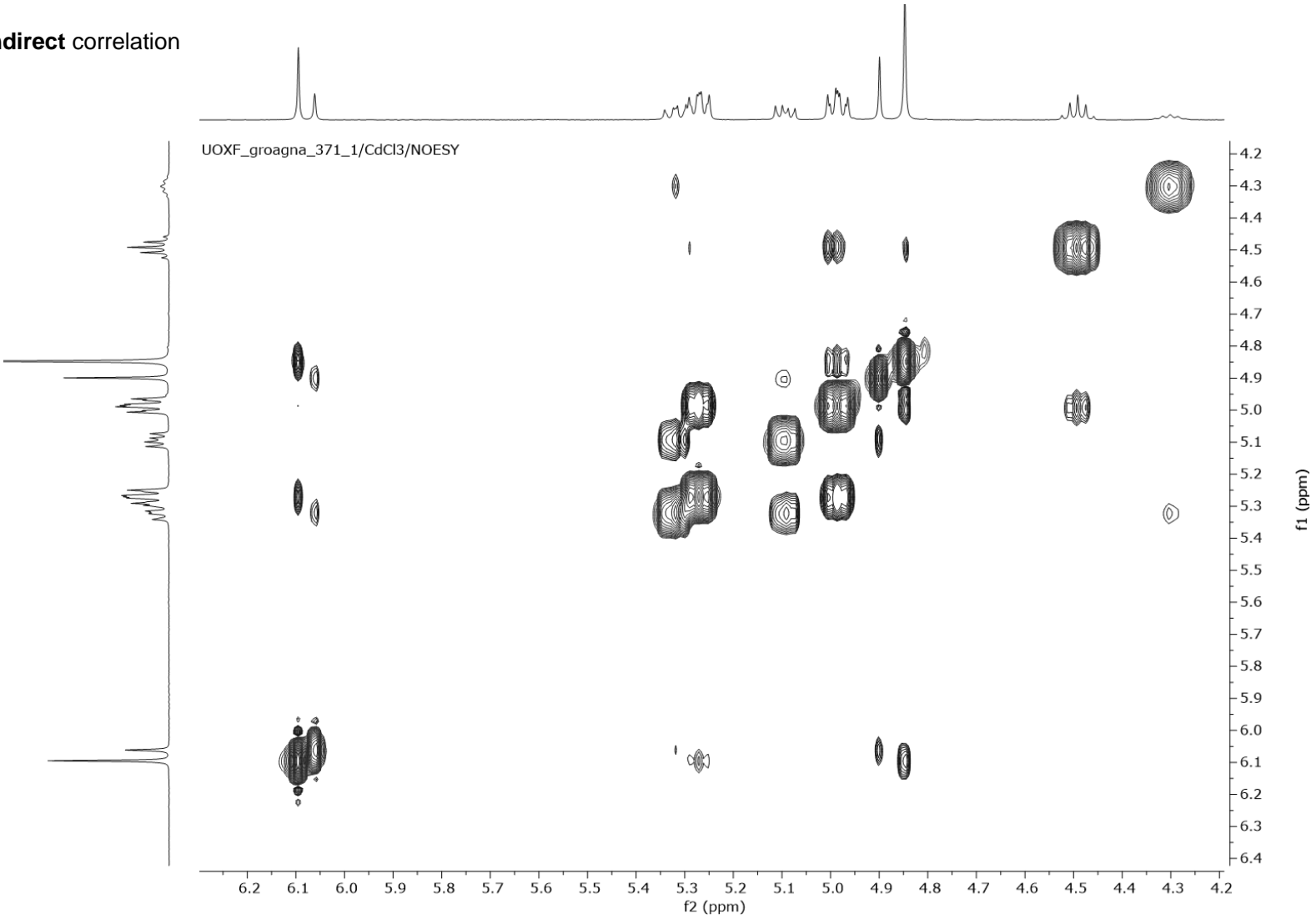

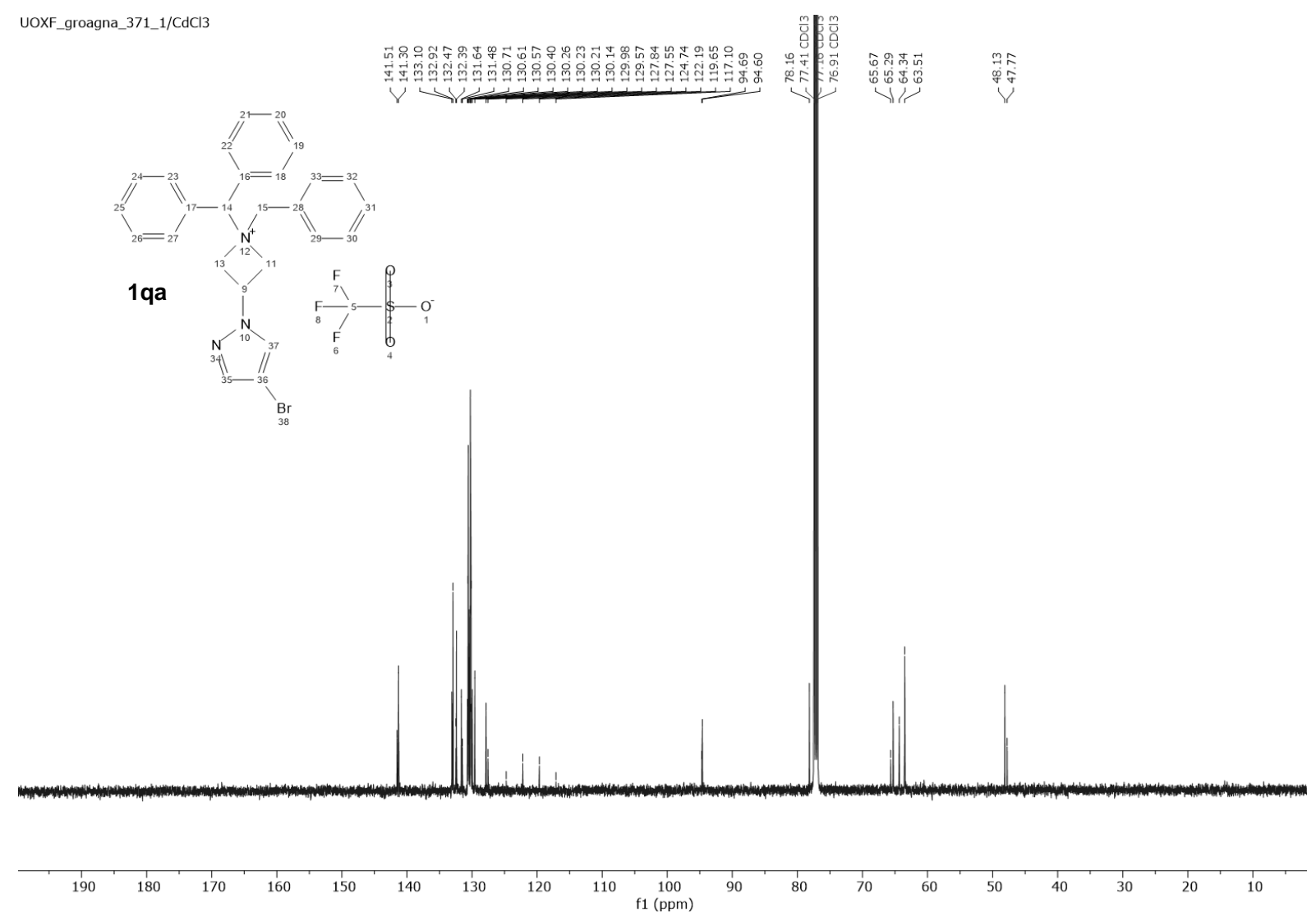

UOXF_groagna_371_1/CdCl3

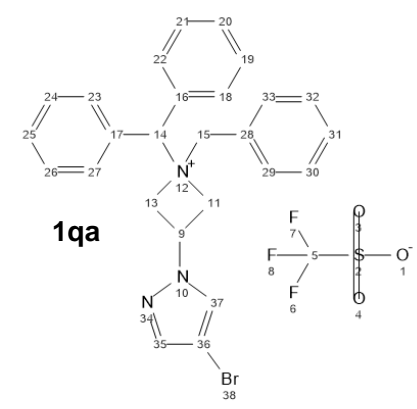

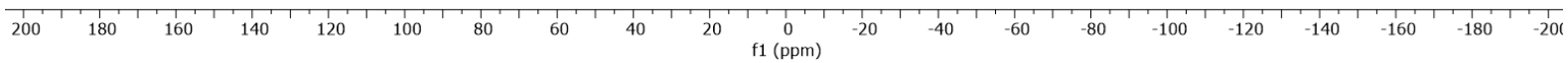


UOXF_groagna_358_1/CdCl3

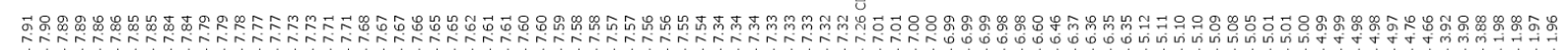

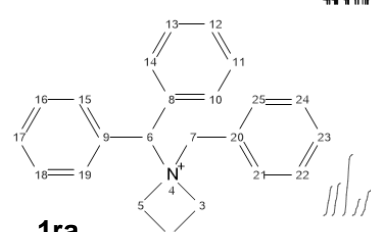

$1 \mathrm{ra}$

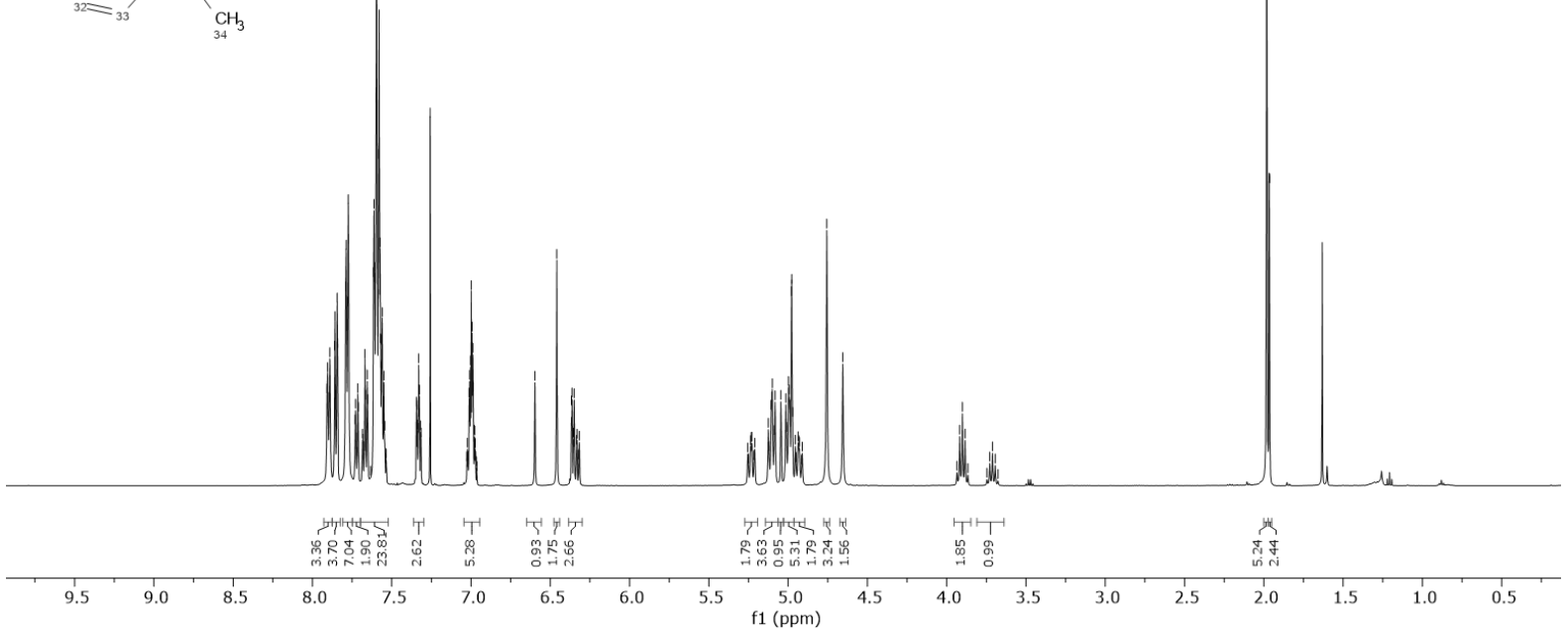

Indirect correlation
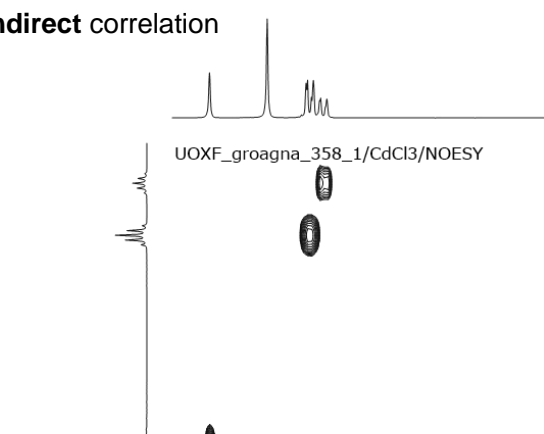

(क)
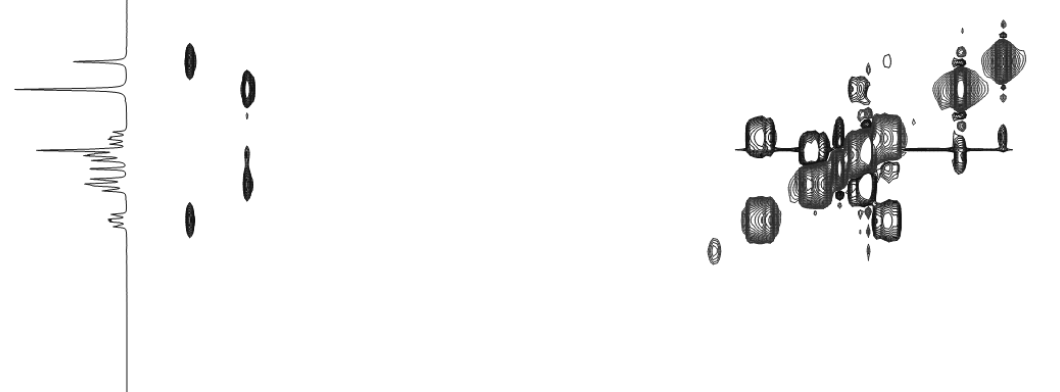

MWNin

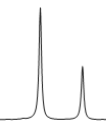

10

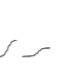


UOXF_groagna_358_1/CdCl3

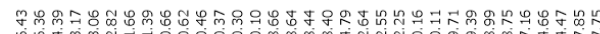

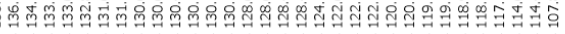

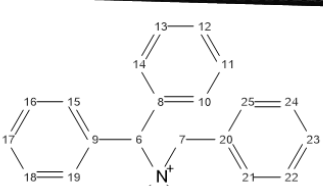

1ra
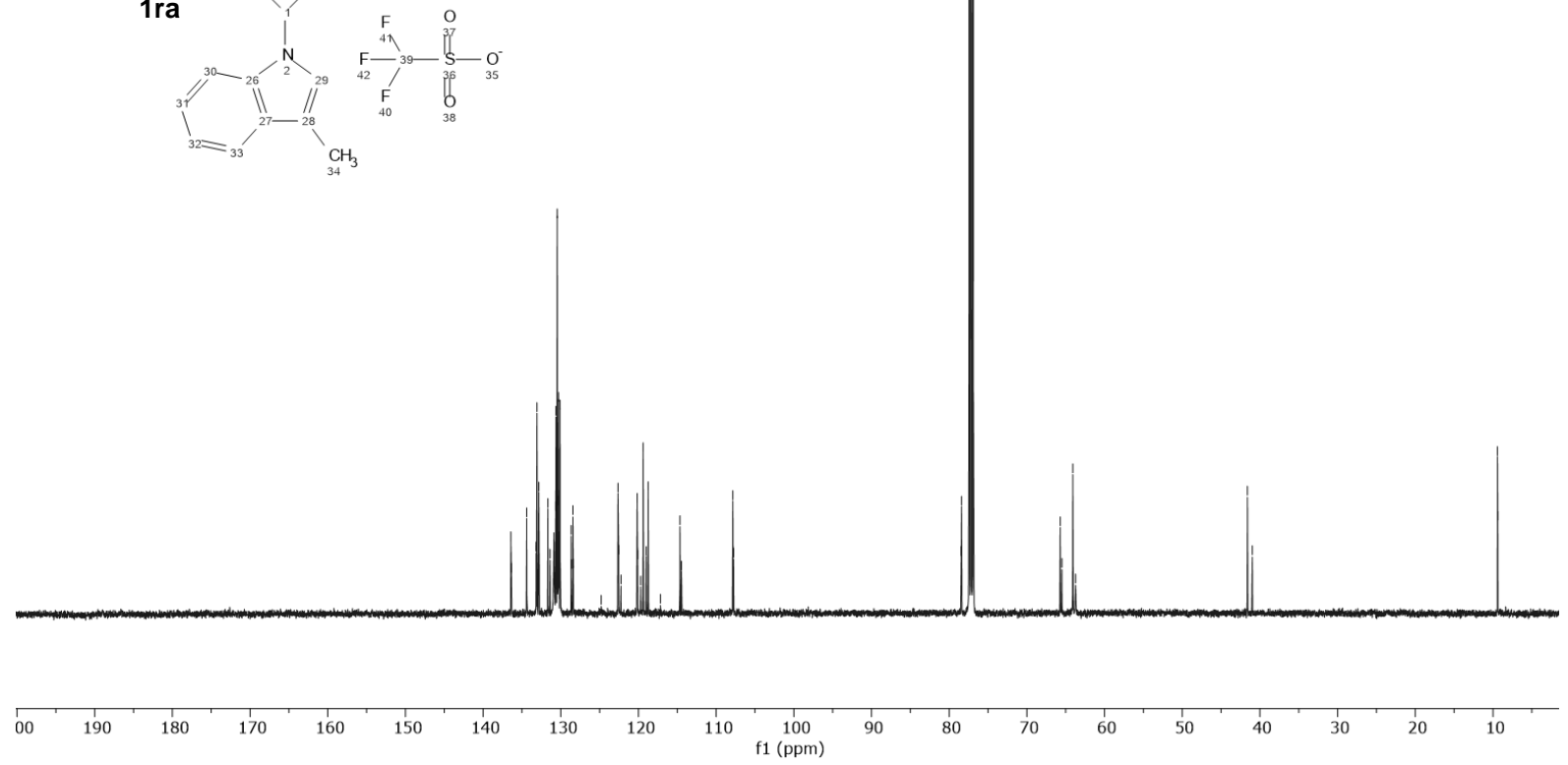

UOXF_groagna_358_1/CdCl3

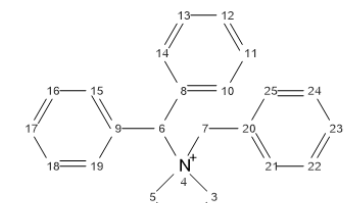

1ra

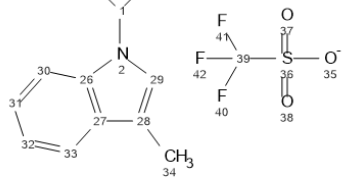

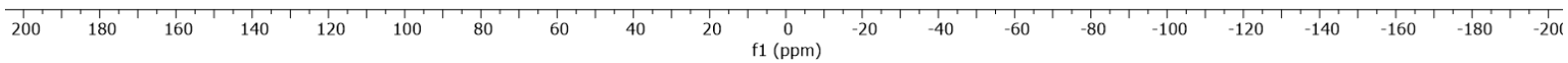



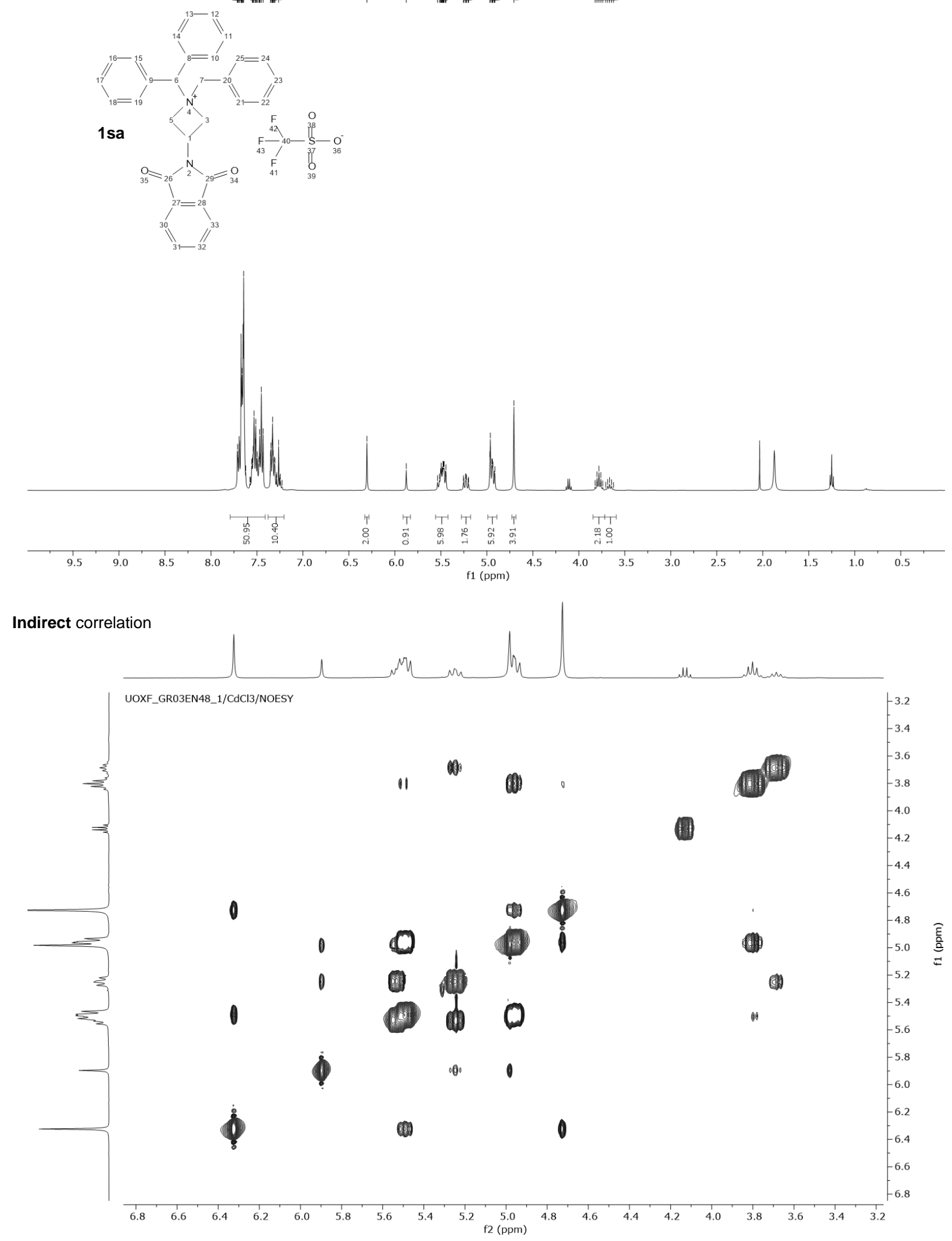


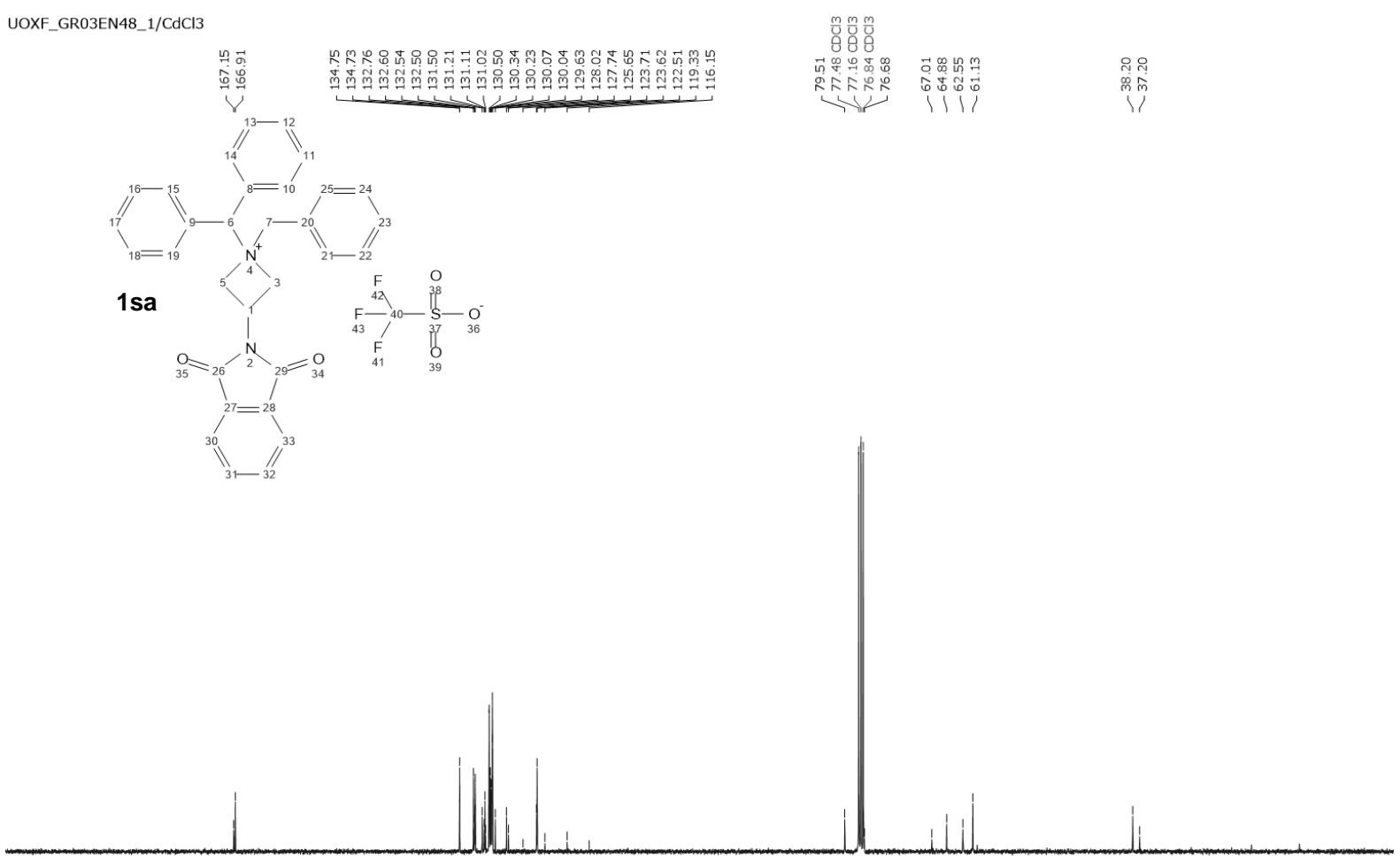

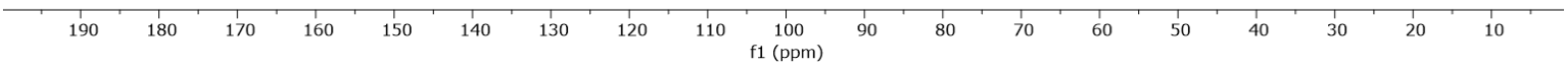

UOXF_GR03EN48_1/CdCI3

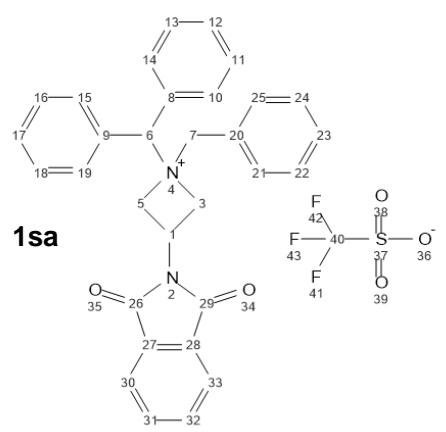

$\begin{array}{lllllllllllllllllllllllllllllllllllllll}1 & 80 & 80 & 70 & 60 & 50 & 40 & 30 & 20 & 10 & 0 & -10 & -20 & -30 & -40 & -50 & -60 & -70 & -80 & -90 & -100 & -110 & -120 & -130 & -140 & -150 & -160 & -170 & -180 & -190 & -200 & -210 & -220 & -230 & -240\end{array}$ 


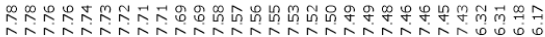

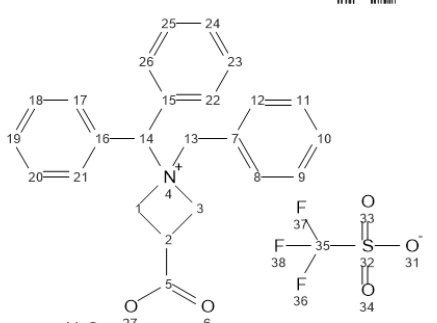

$\mathrm{H}_{33} \mathrm{C}_{43}{ }_{40}^{27}$

$\mathrm{H}_{41} \mathrm{C}_{42}{ }_{42}$

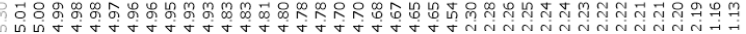

1 ta
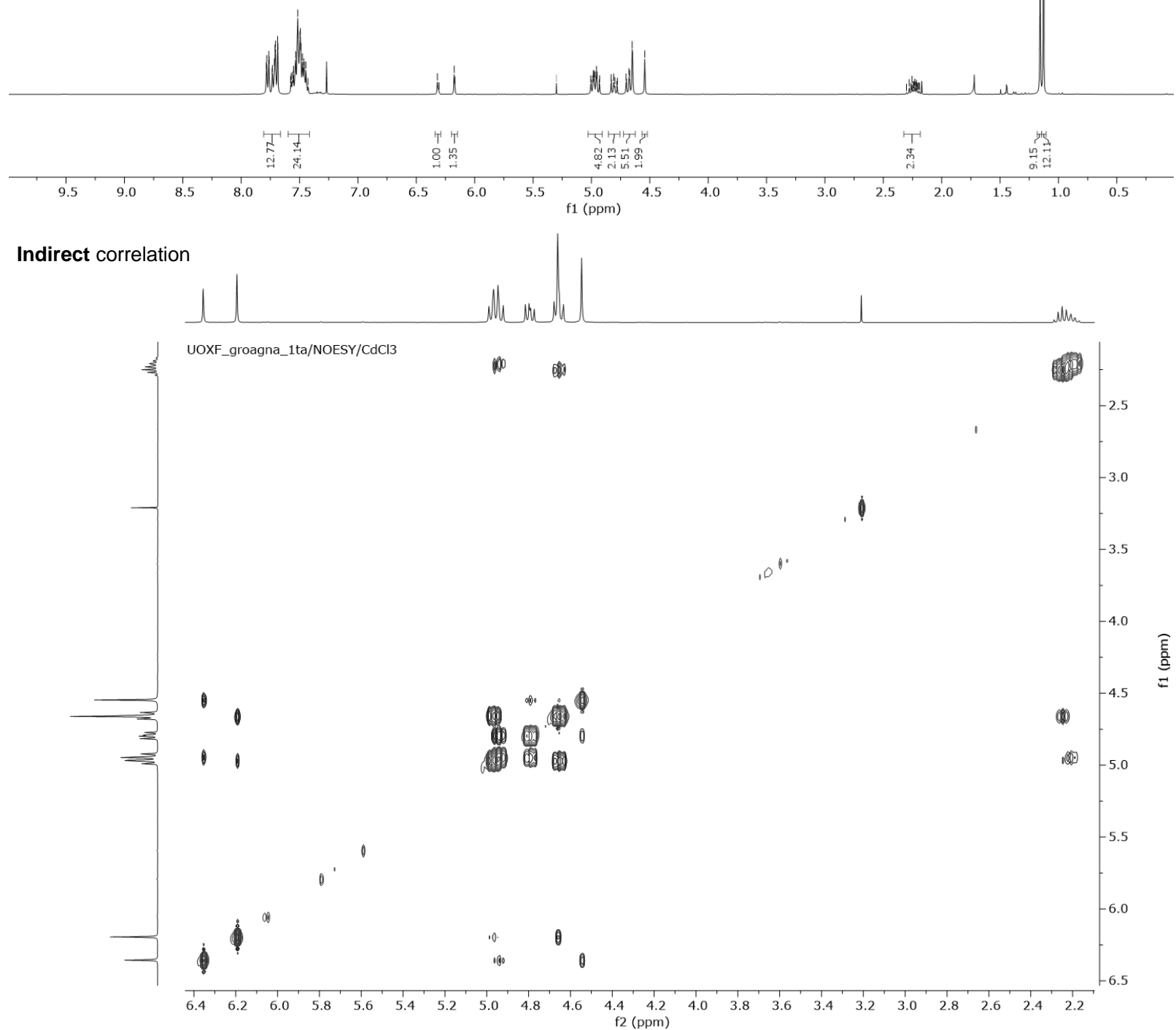

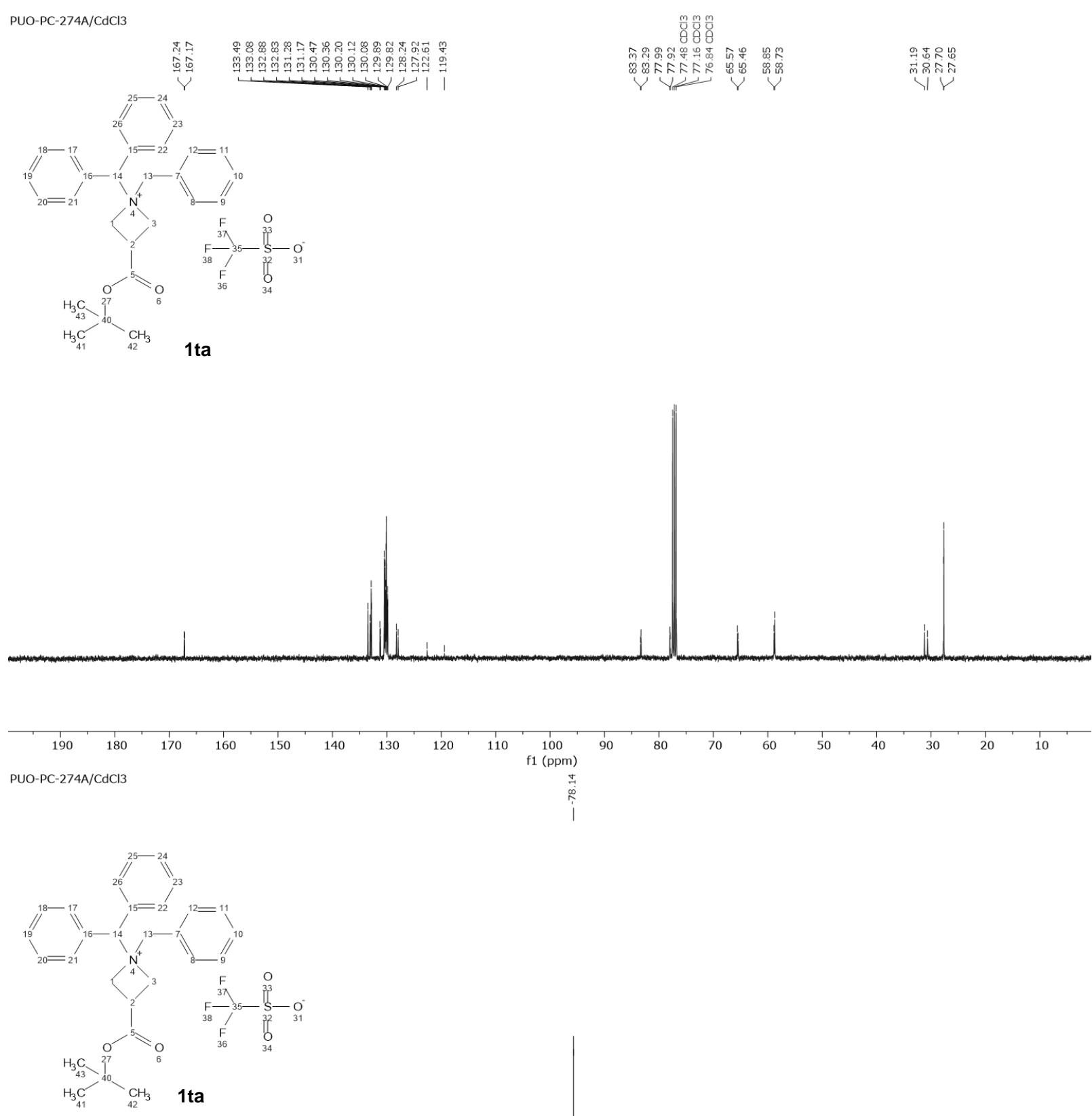

\begin{tabular}{llllllllllllllllllllllllllllllllllllllllllllll}
\hline & -5 & -10 & -15 & -20 & -25 & -30 & -35 & -40 & -45 & -50 & -55 & -60 & -65 & -70 & -75 & -80 & -85 & -90 & -95 & -100 & -105 & -110 & -115 & -120 & -125 & -130 & -135 & -140 & -145
\end{tabular} 

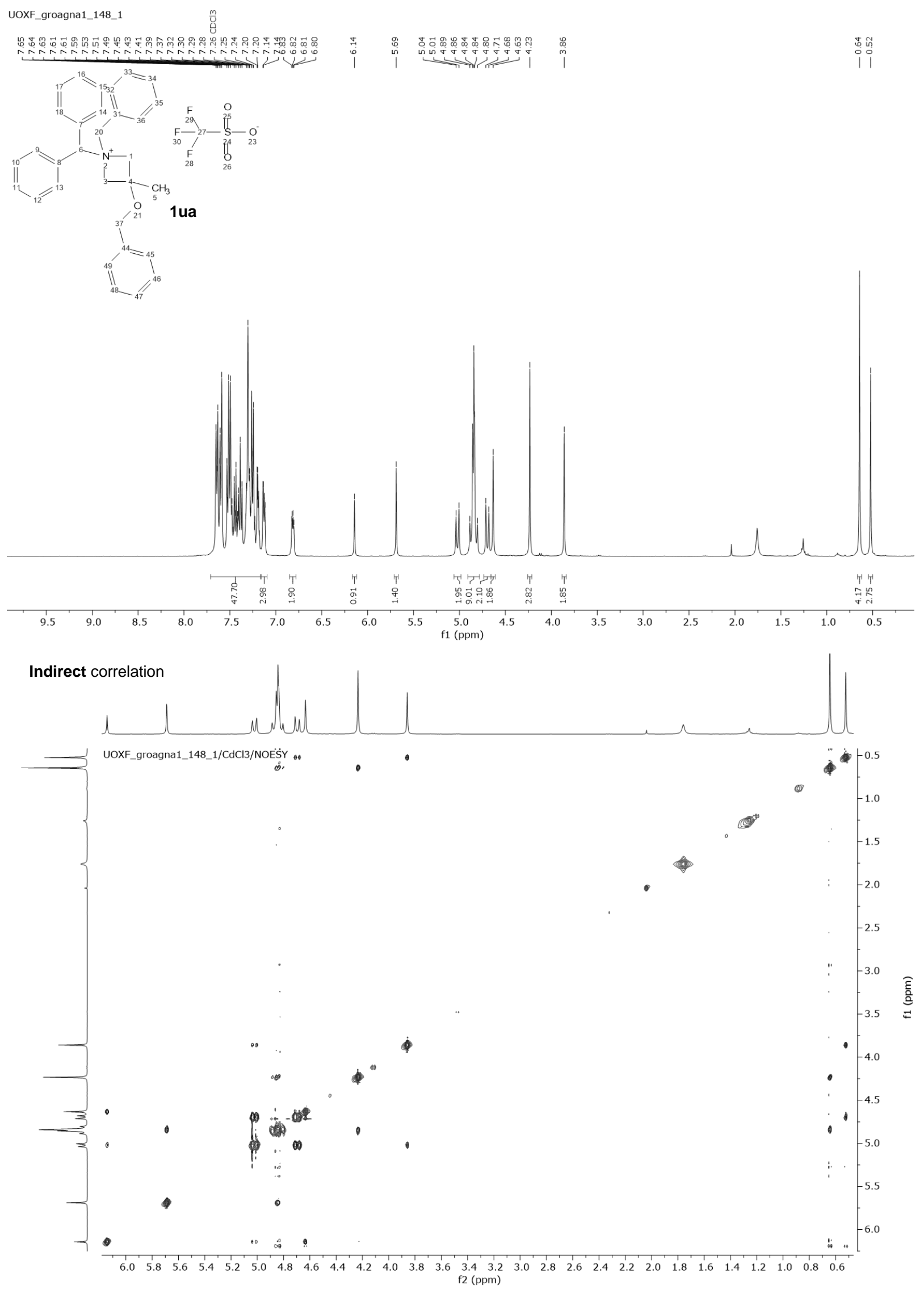


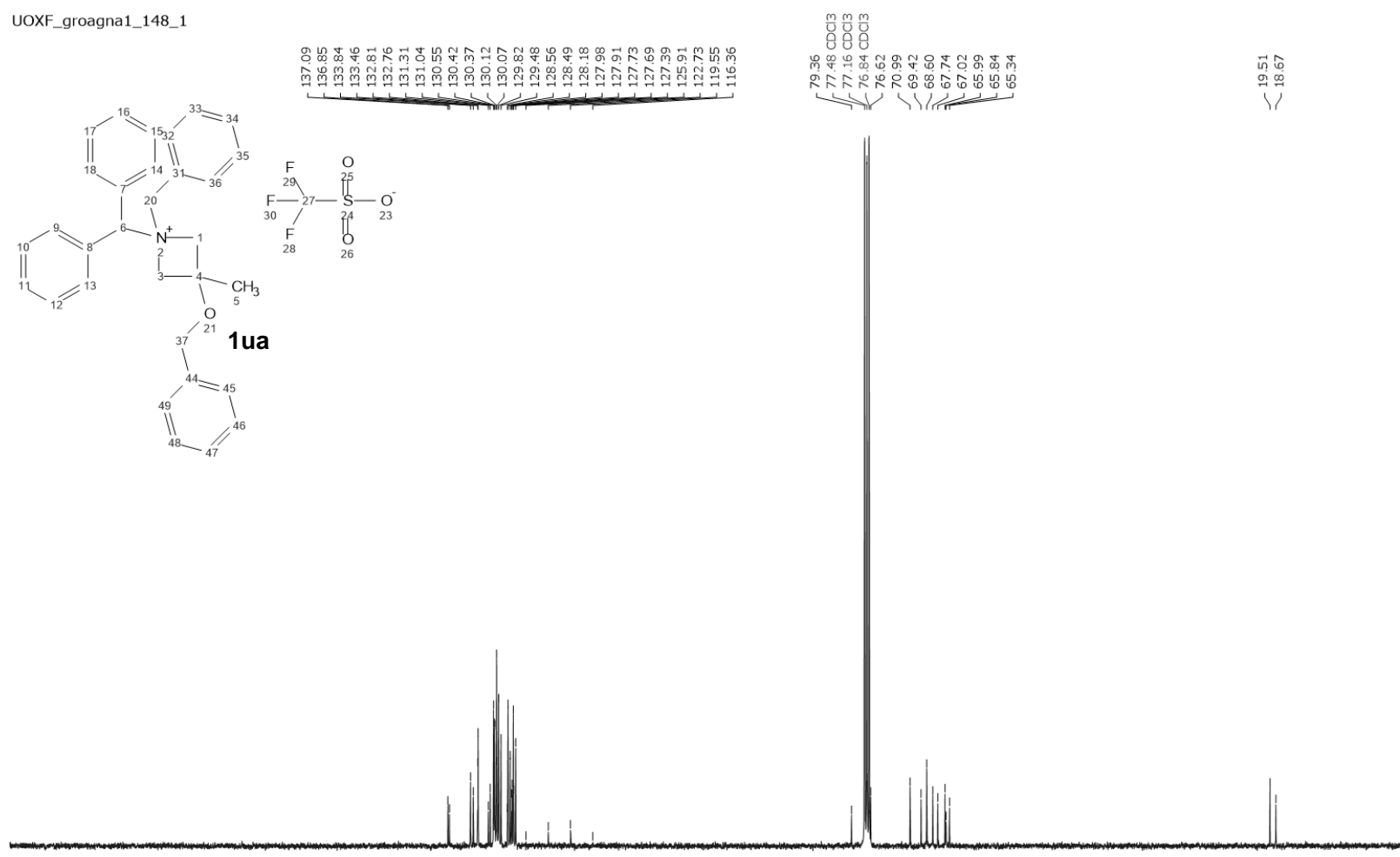

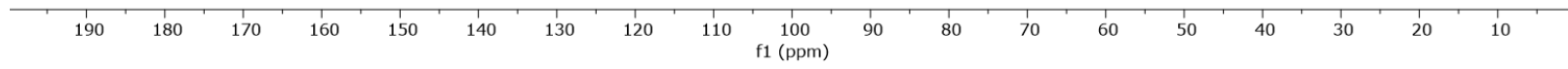
UOXF_groagna1_148_1

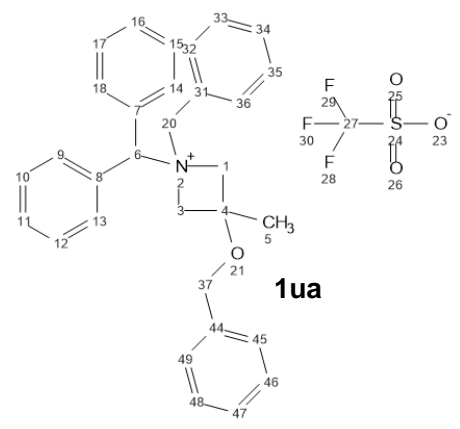

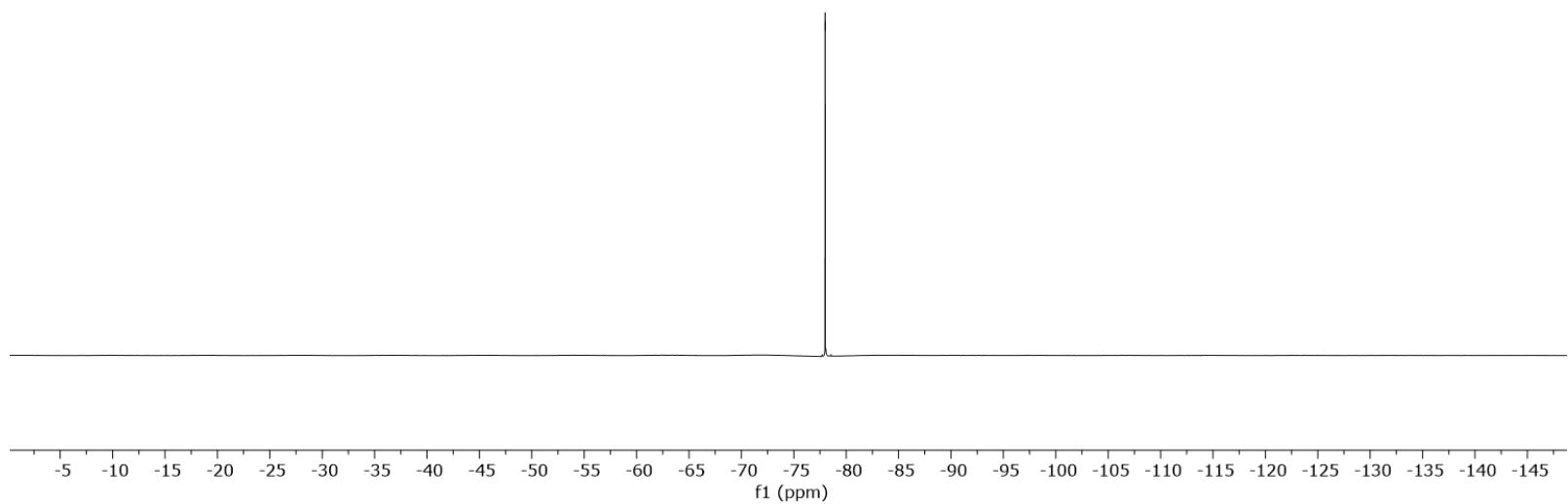



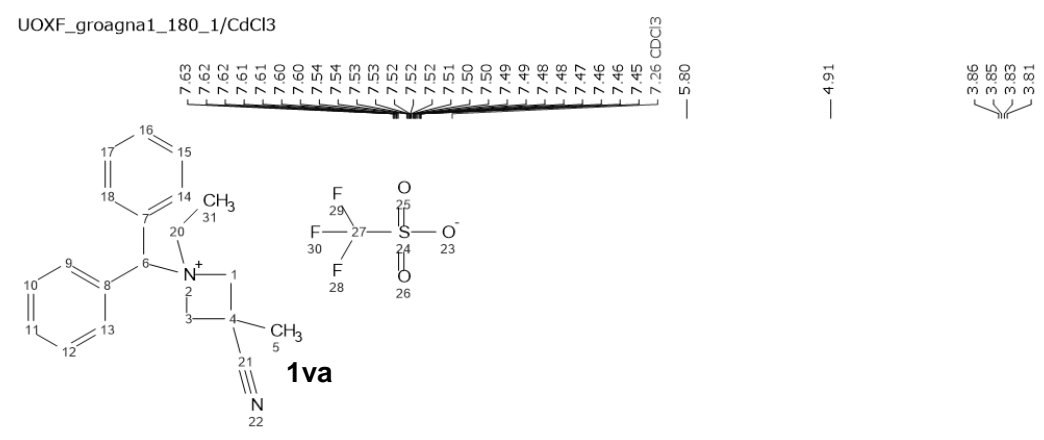

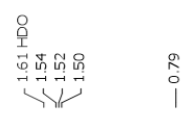
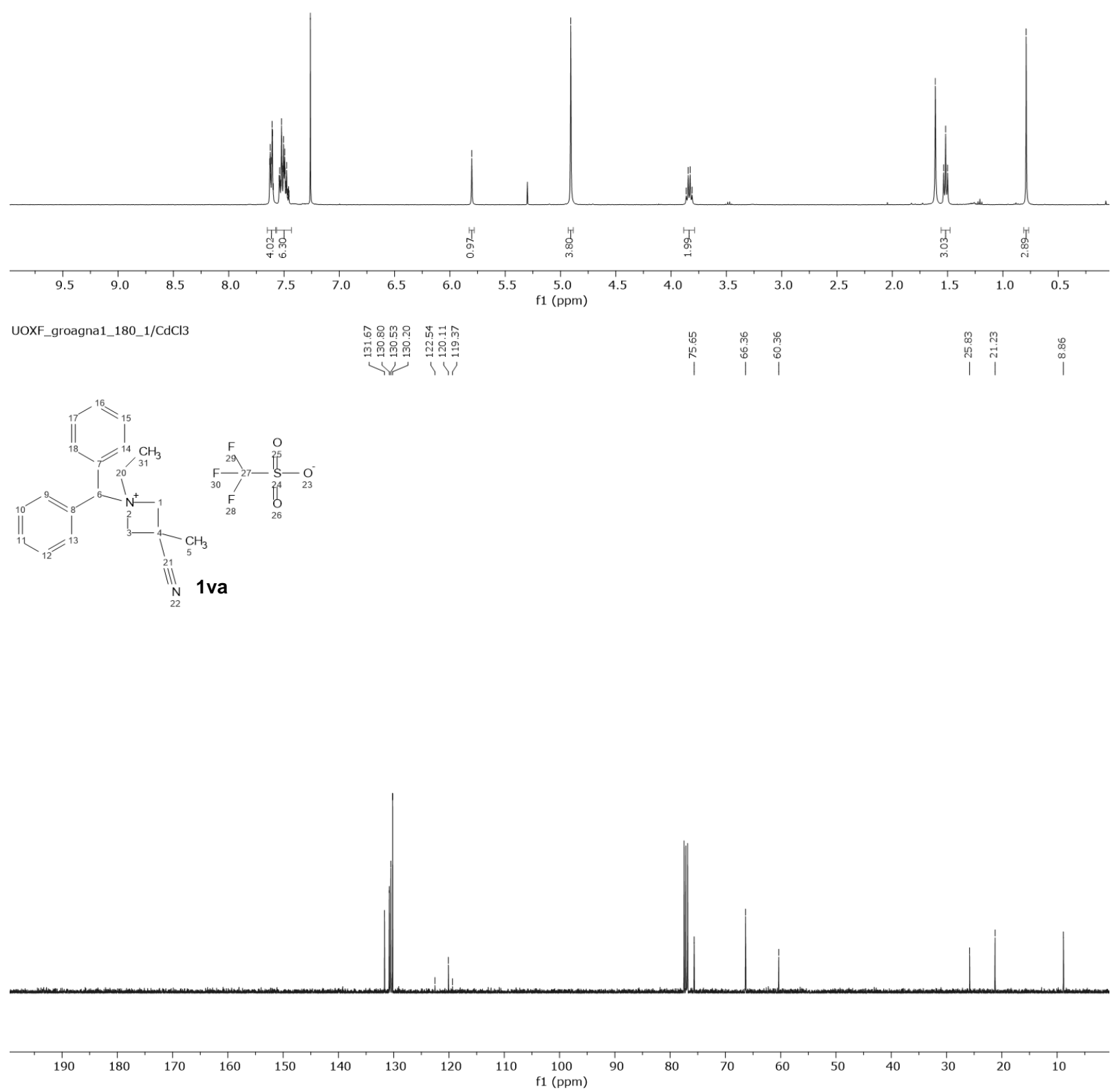

S264 
UOXF_groagna1_180_1/CdCl3
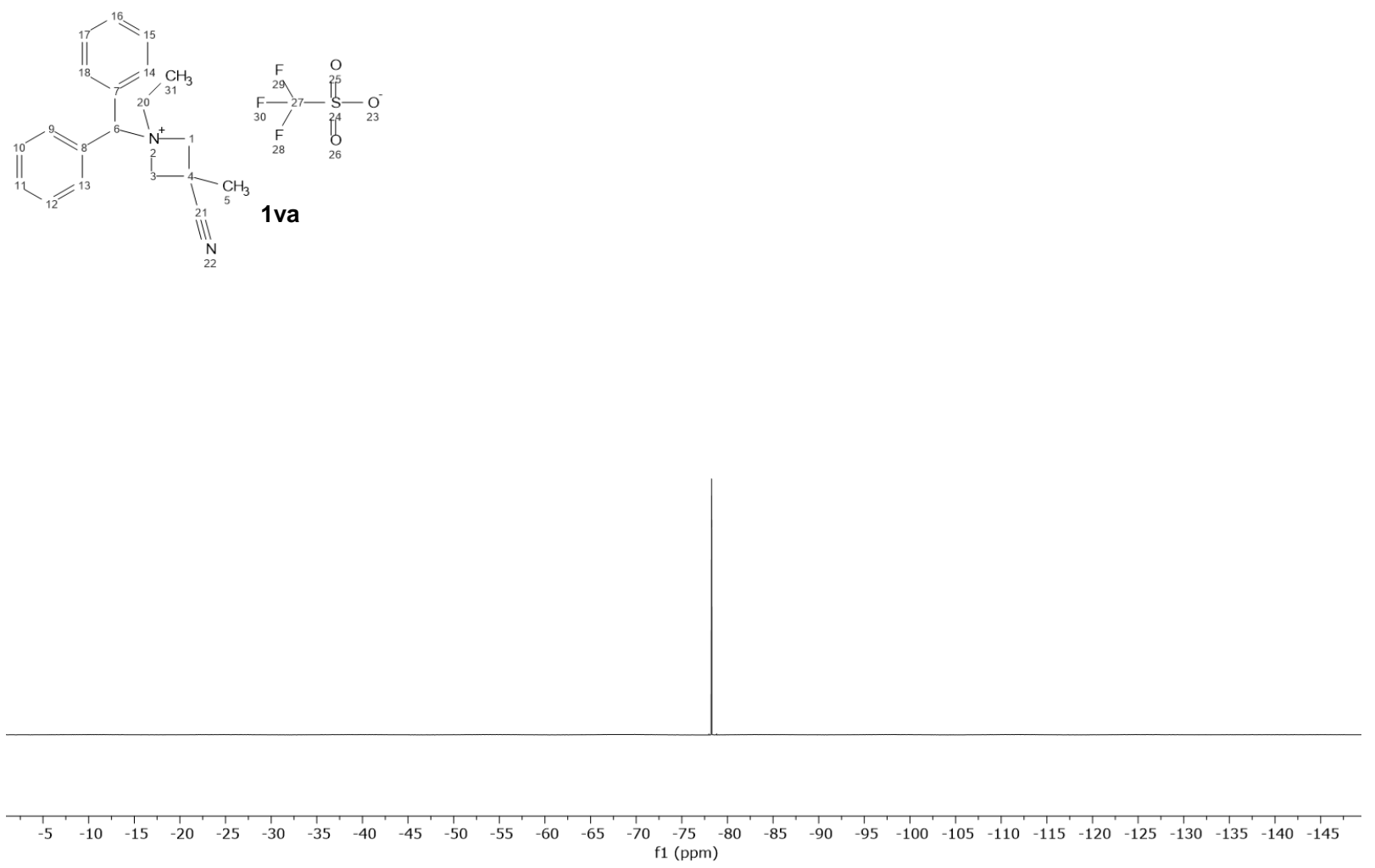


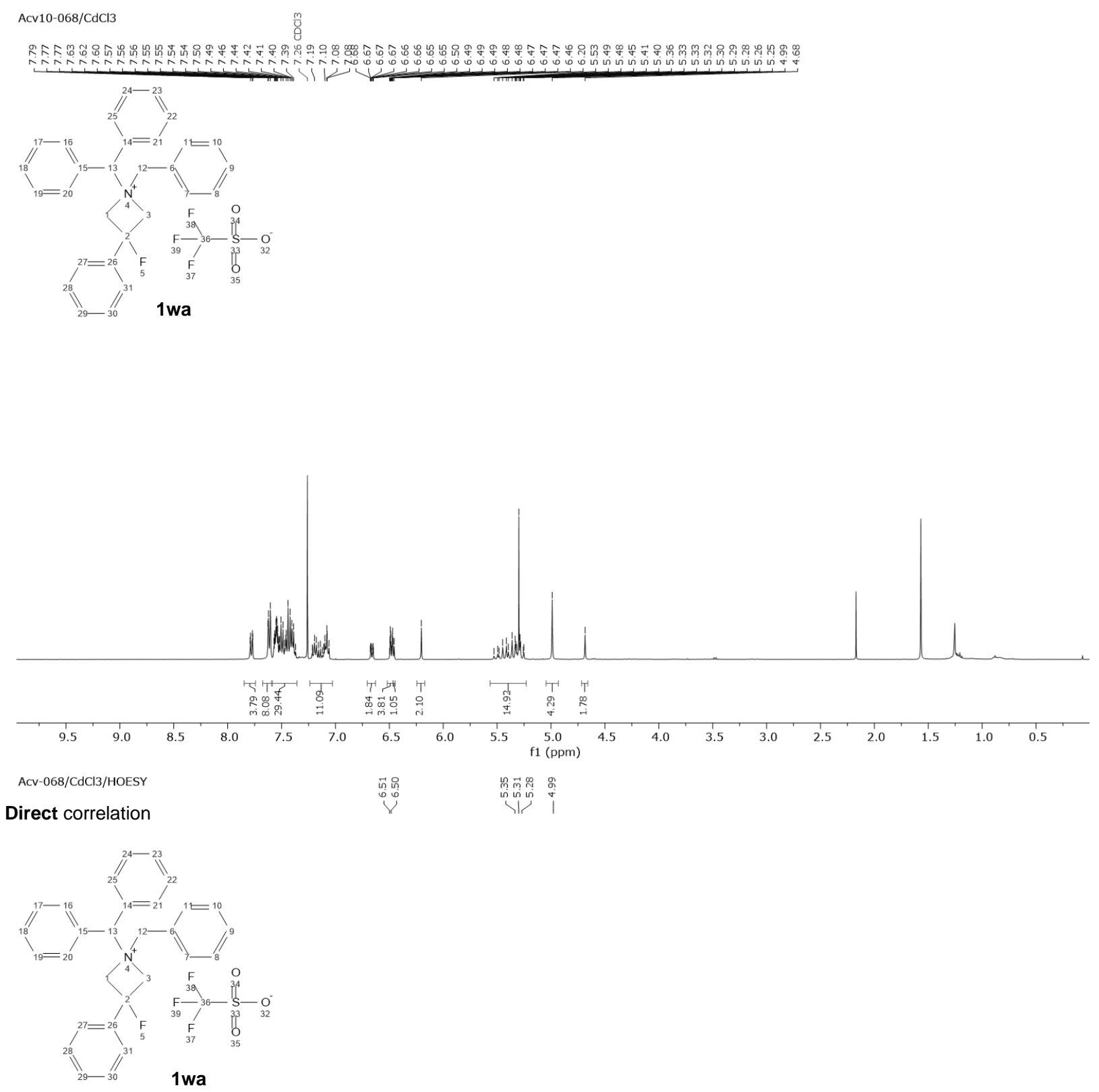

wom \begin{tabular}{ccccc}
\hline 1 & & & & \\
9.5 & 9.0 & 8.5 & 8.0 & 7.5
\end{tabular} $5 \stackrel{5.0}{5.0}$ 

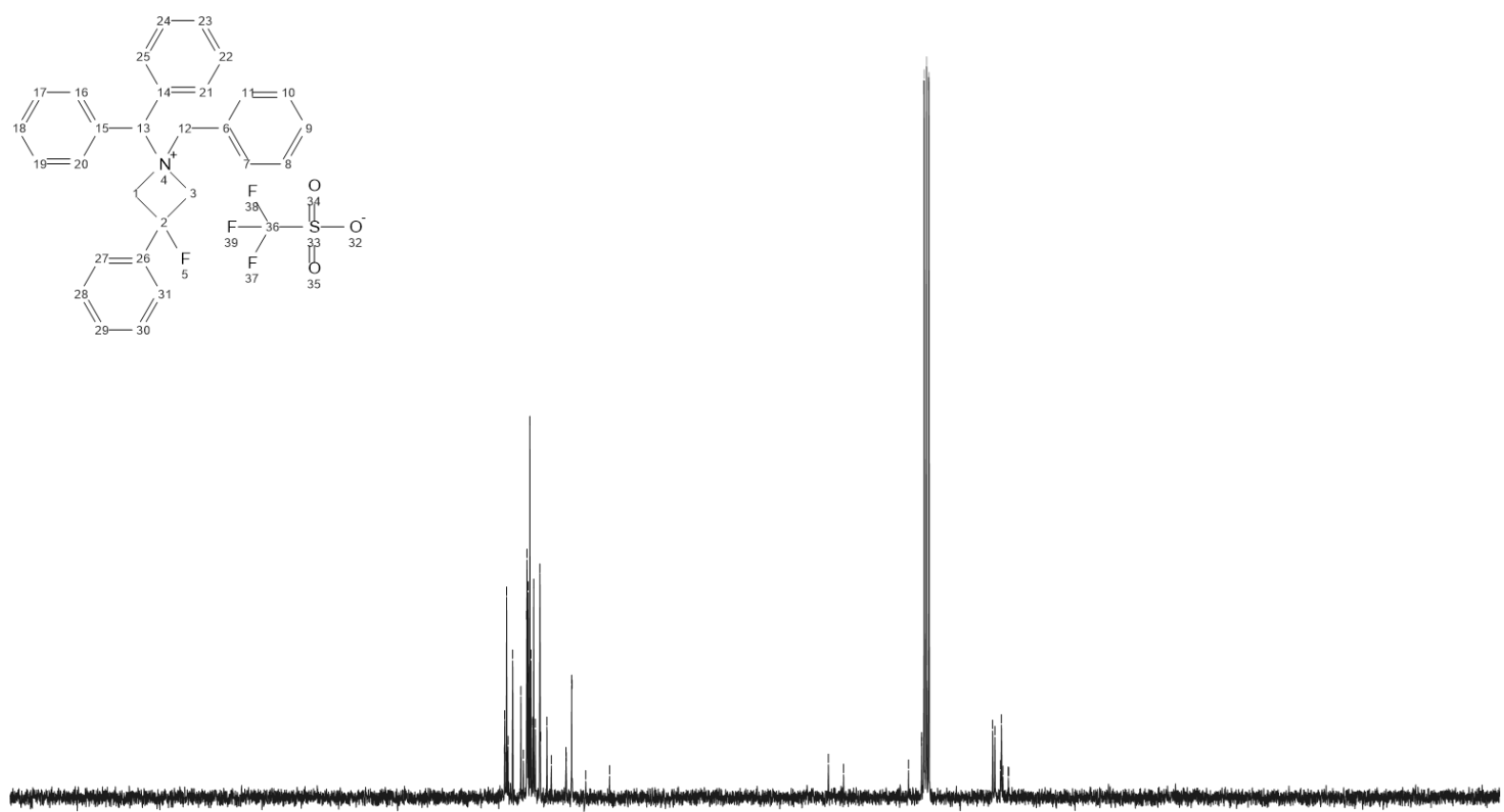

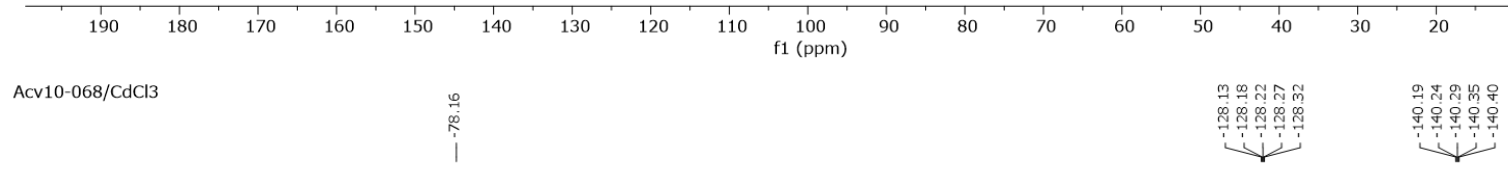

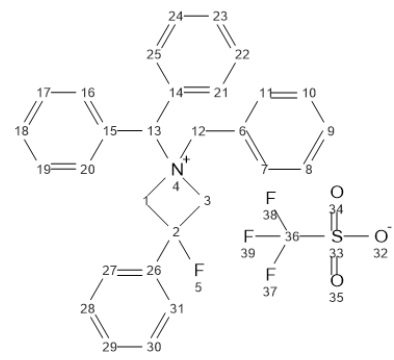

1wa

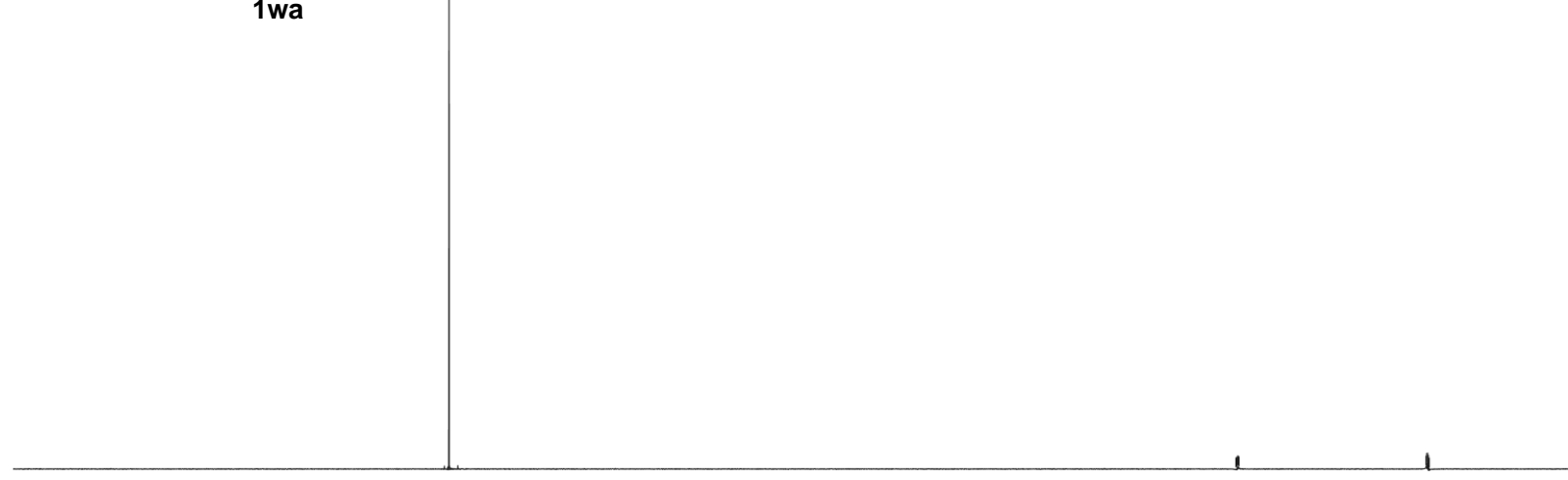

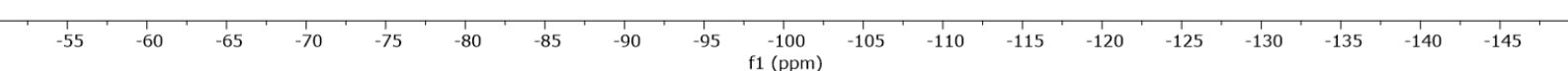




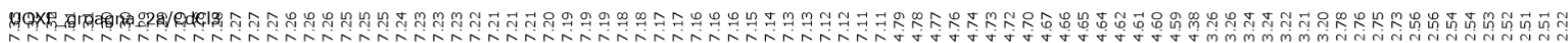
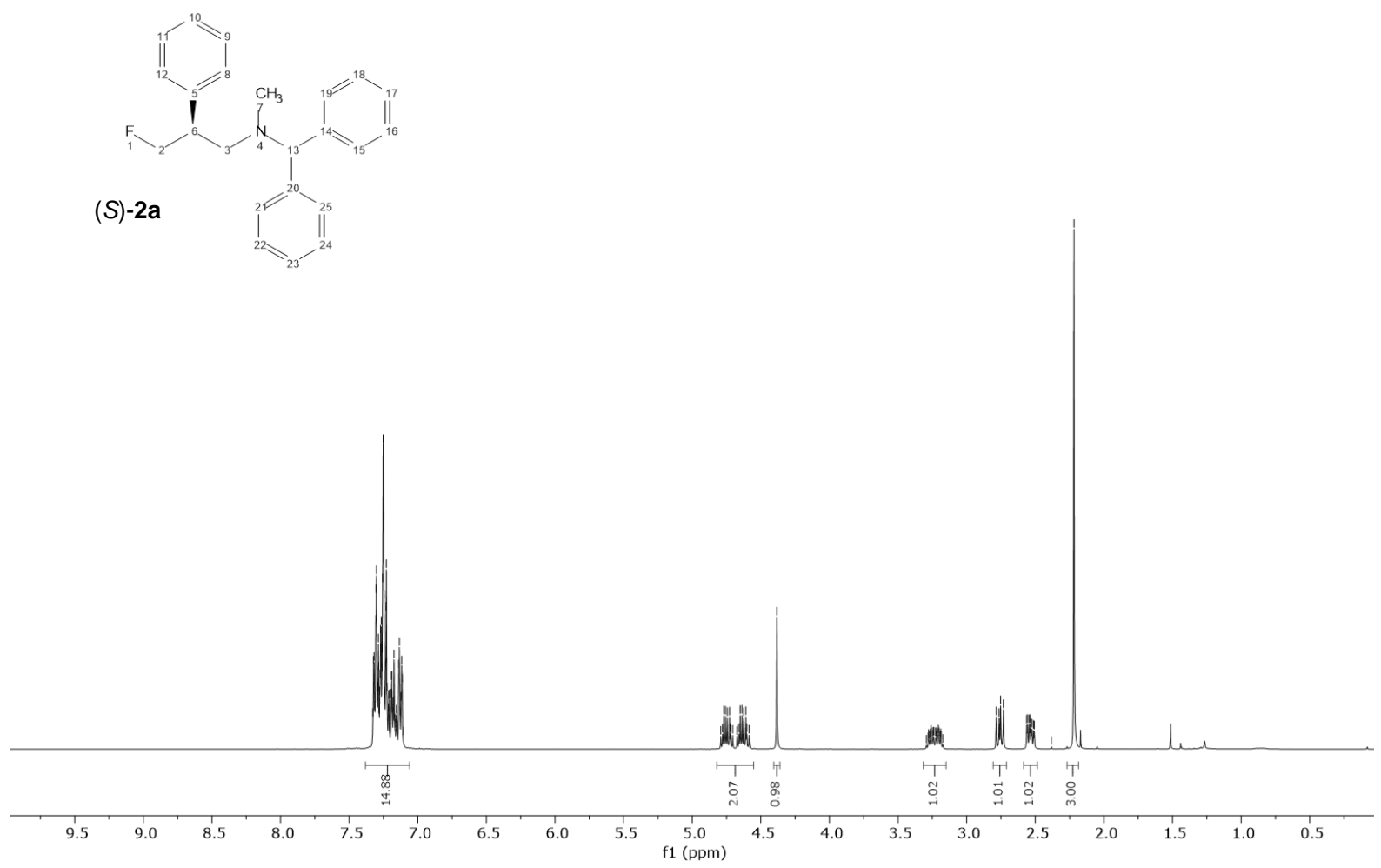

UOXF_groagna_2a/CdCl3
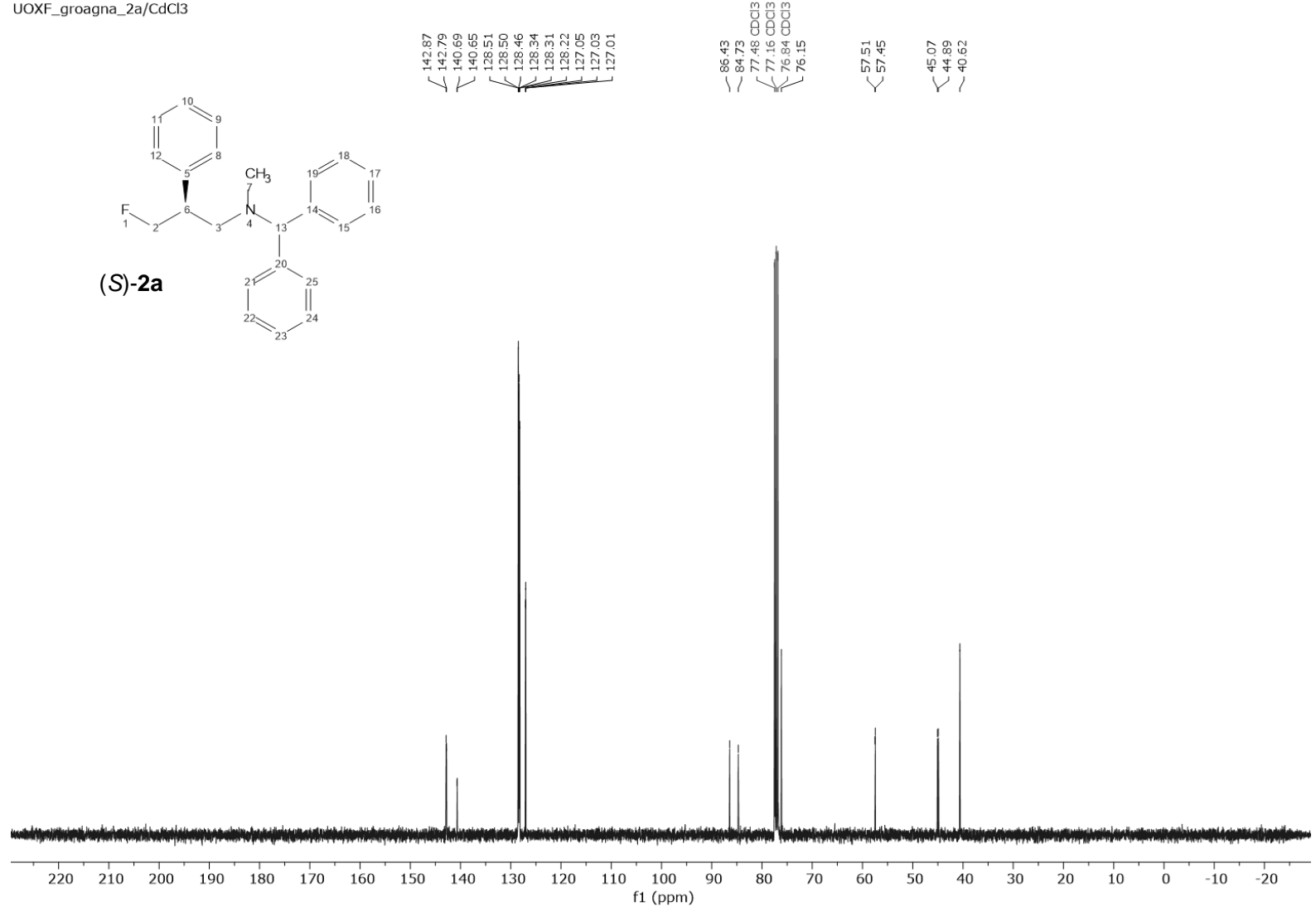

S268 
<smiles>CN(CC(CF)c1ccnnc1)C(c1ccnnc1)c1ccncn1</smiles>

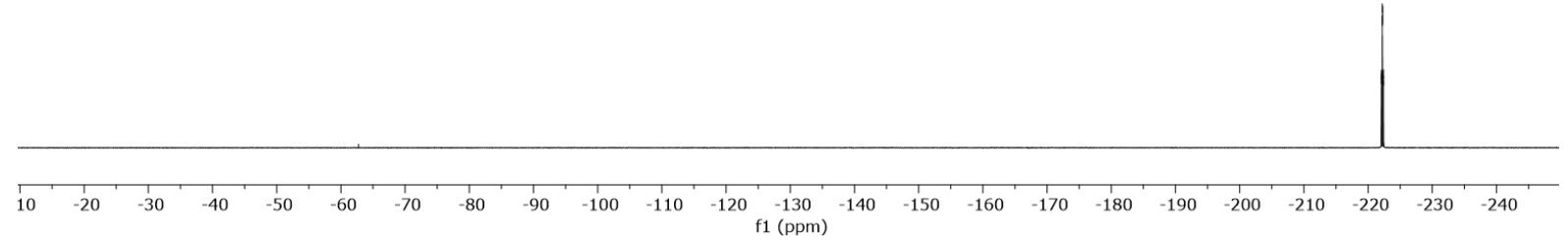


UOXF_groagna_2aa/Cdब

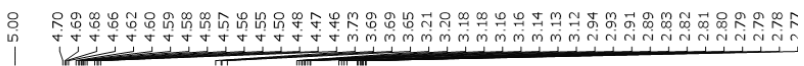
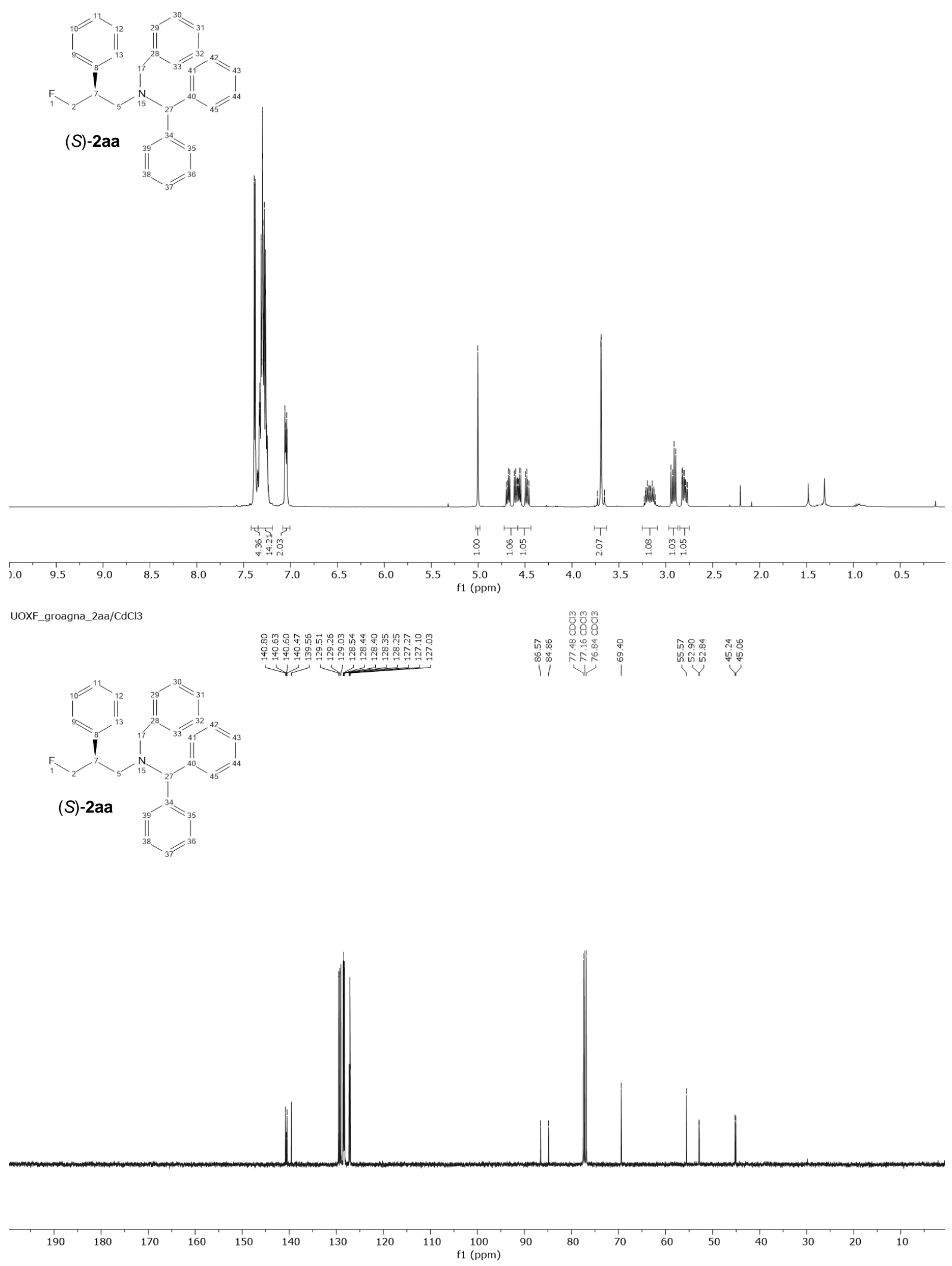

S270 

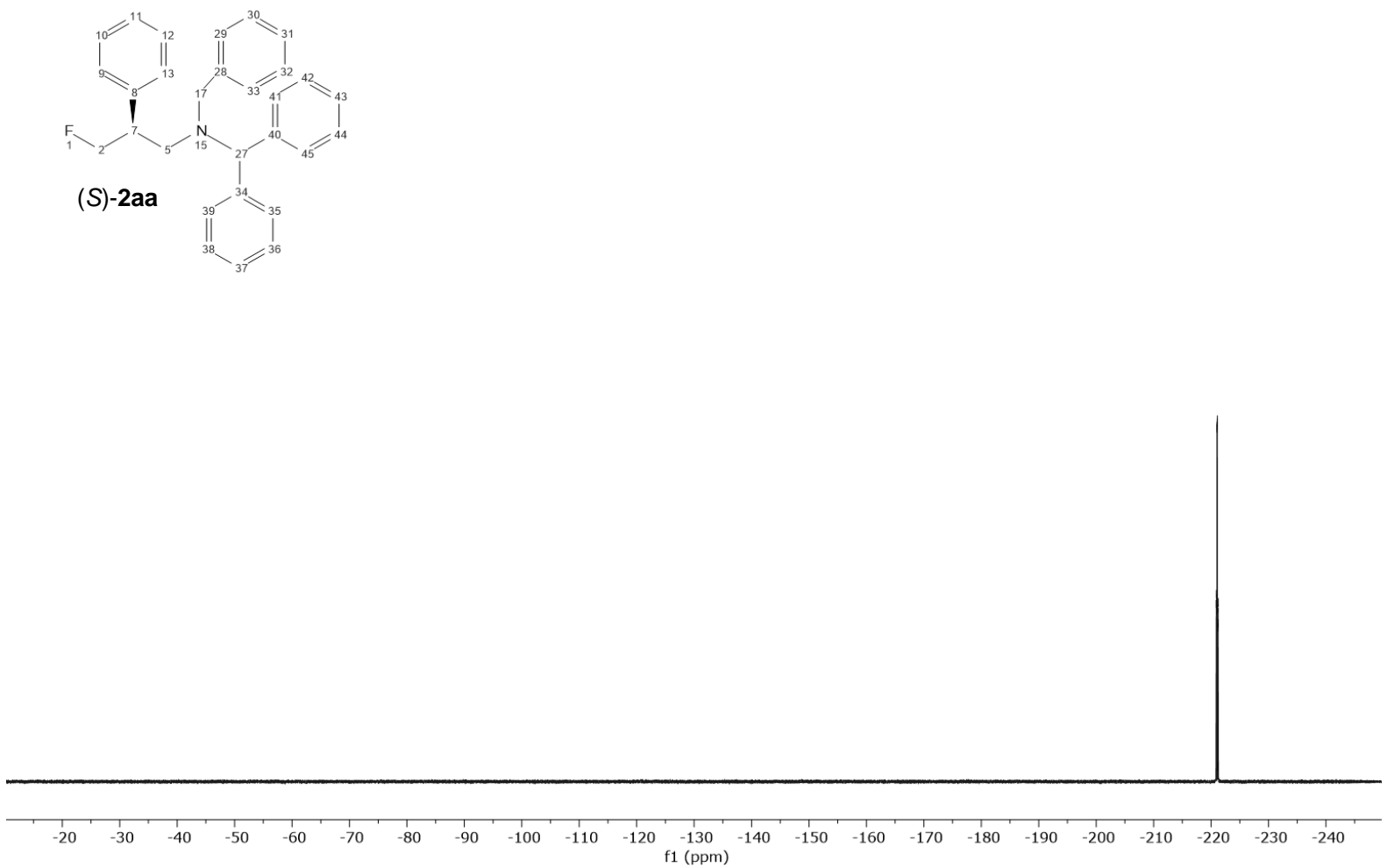

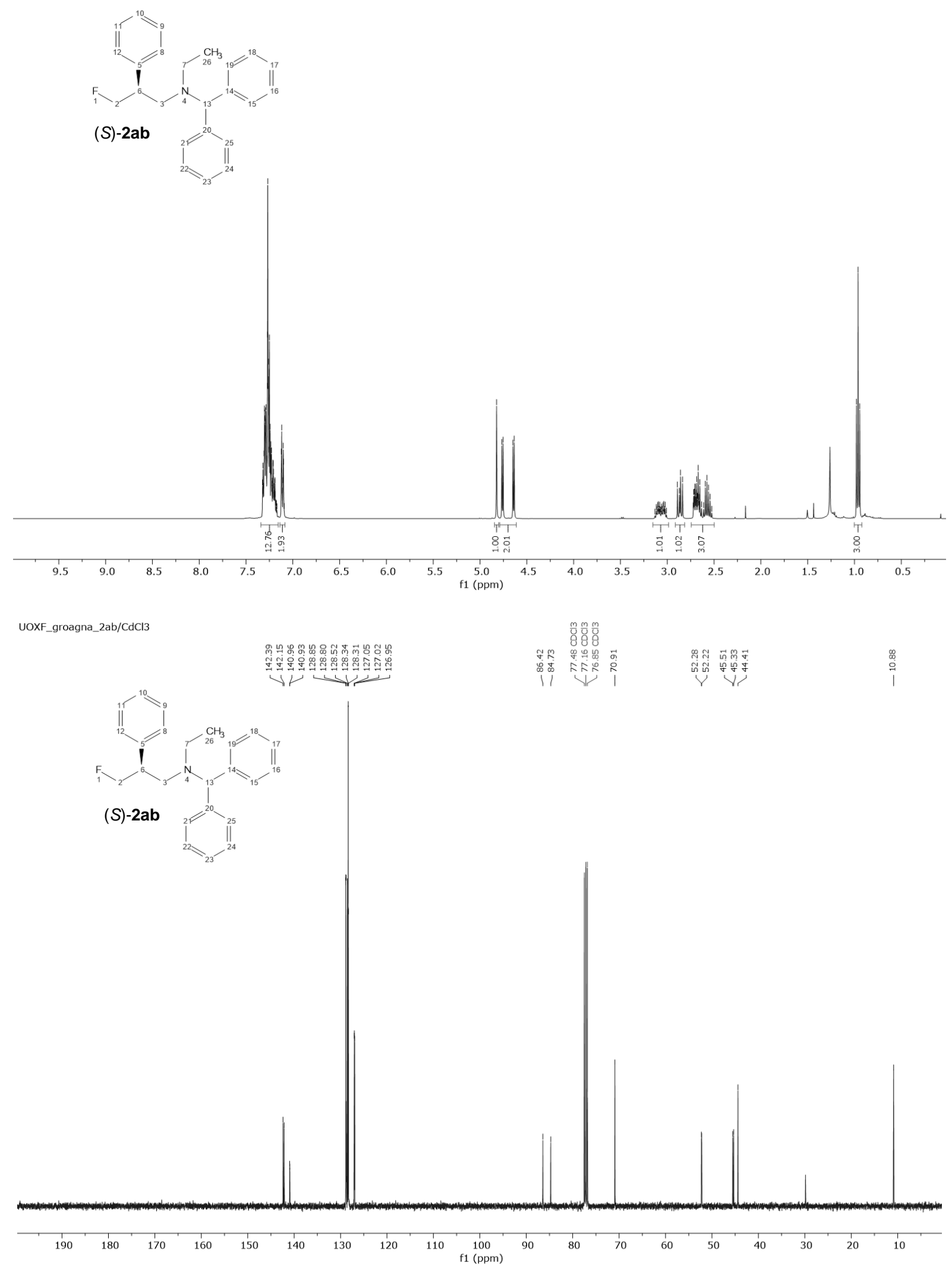

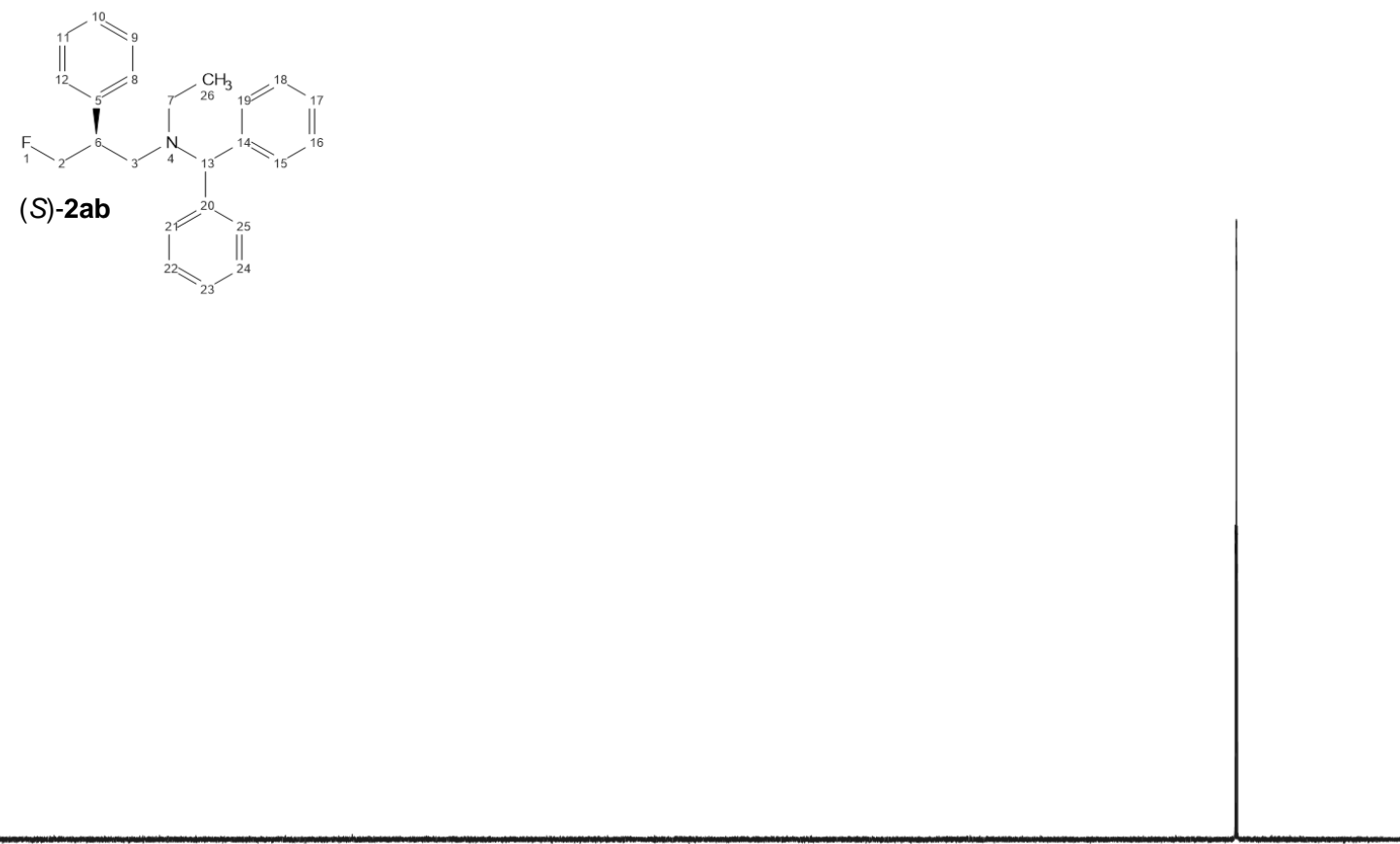

$\begin{array}{lllllllllllllllllllllllllll}-20 & -30 & -40 & -50 & -60 & -70 & -80 & -90 & -100 & -110 & -120 & -130 & -140 & -150 & -160 & -170 & -180 & -190 & -200 & -210 & -220 & -230 & -240 & 1\end{array}$ 


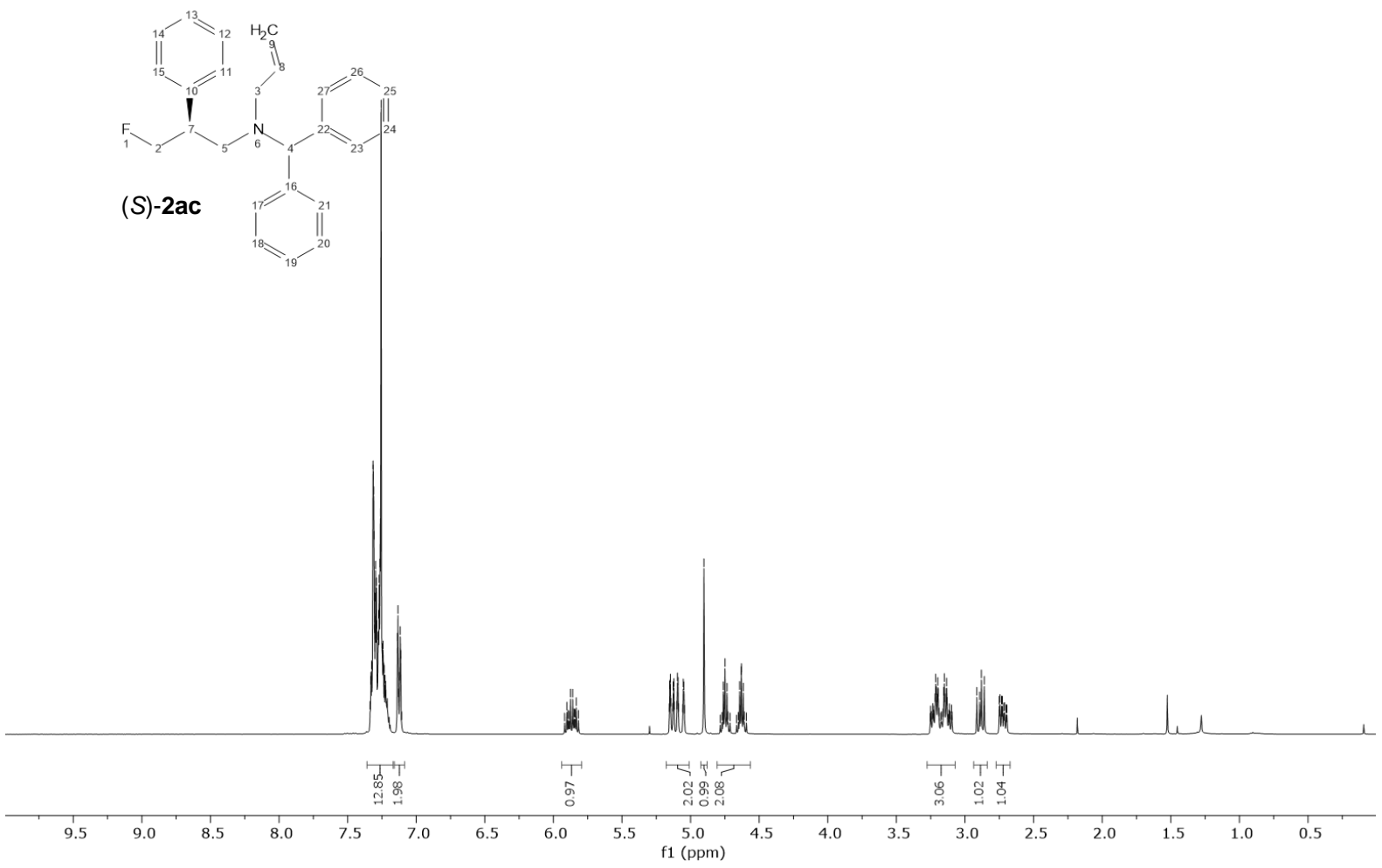

UOXF_groagna_2ac/CdCl3

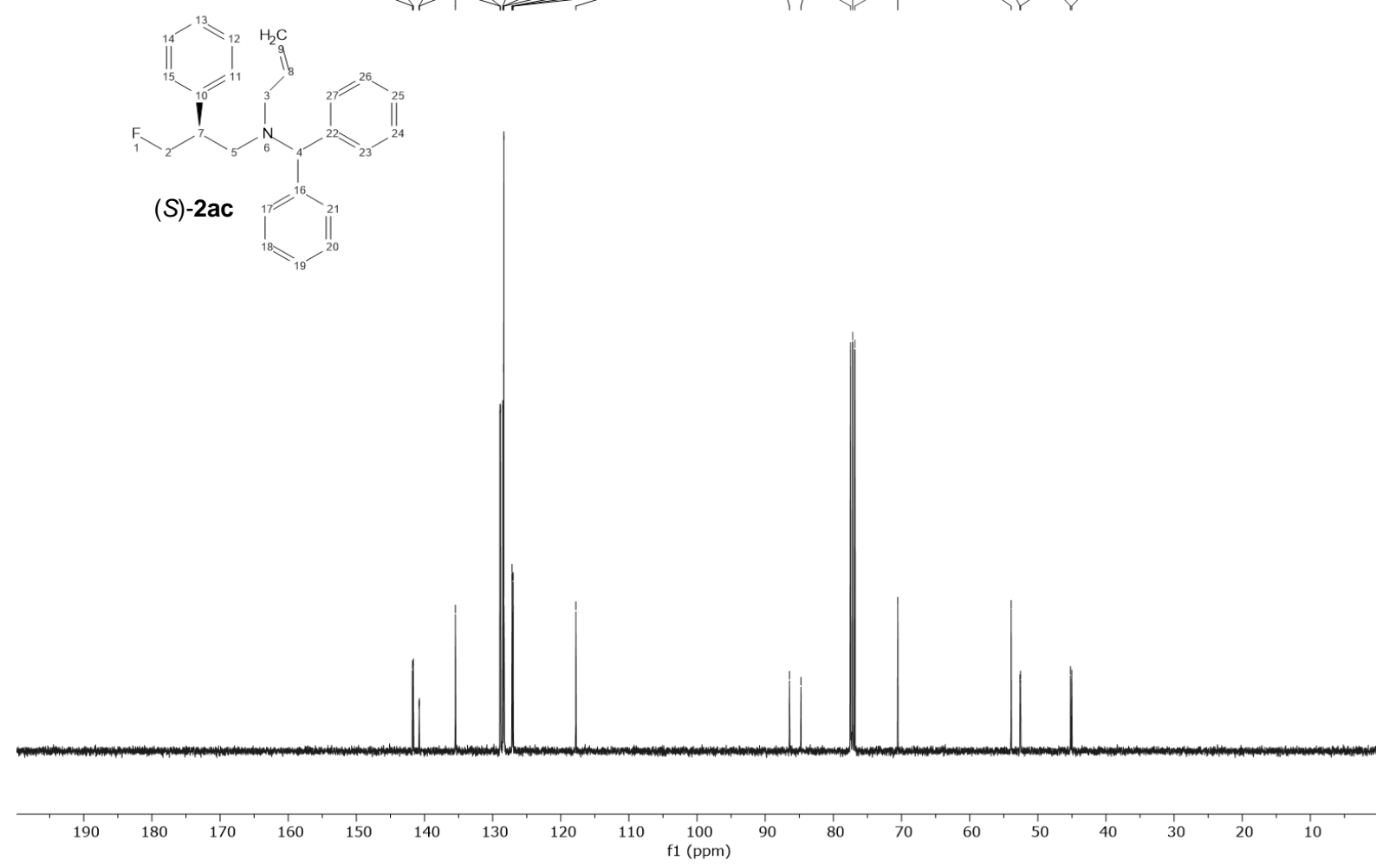

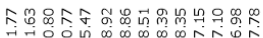

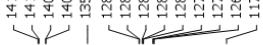

$m$
0
0
8
8
8

プ 


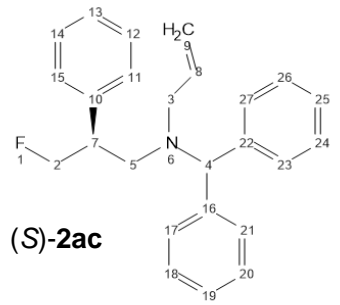

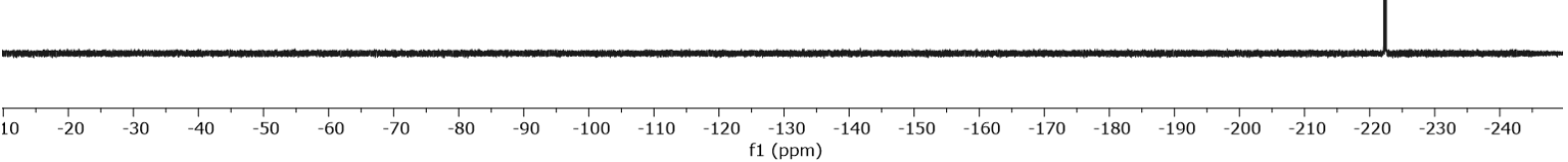




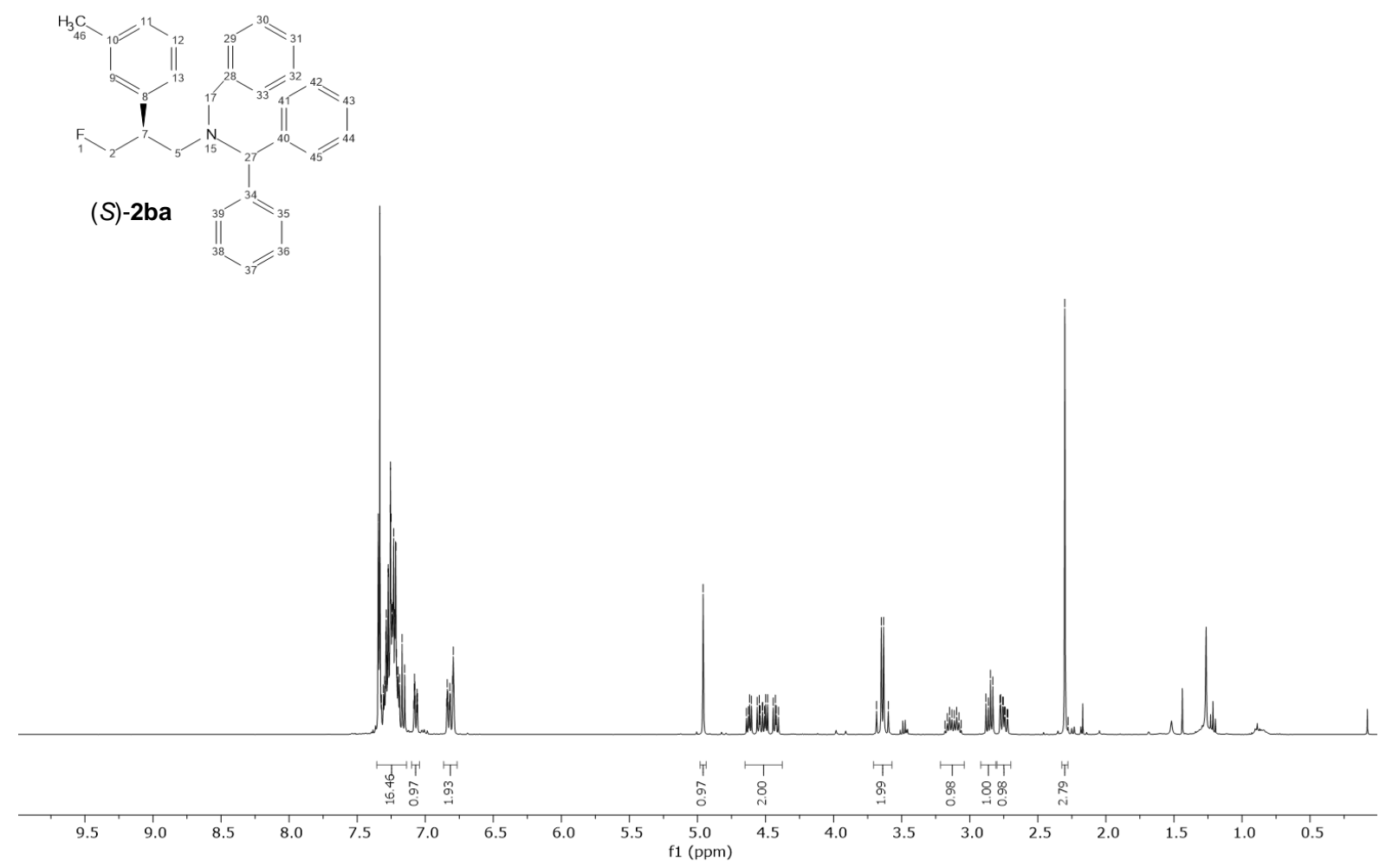

UOXF_groagna_2ba/CdCl3

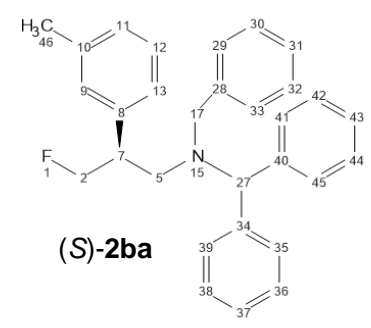

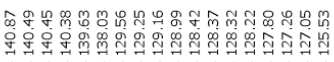

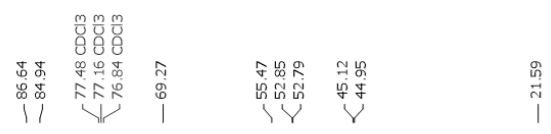

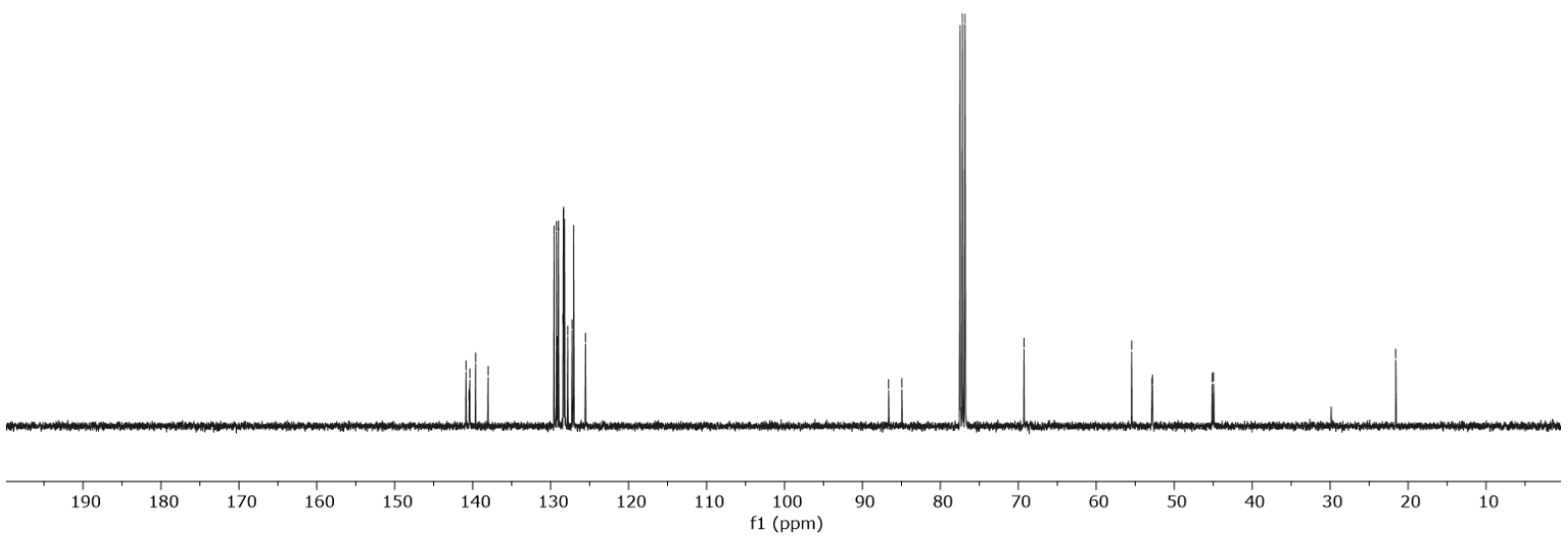



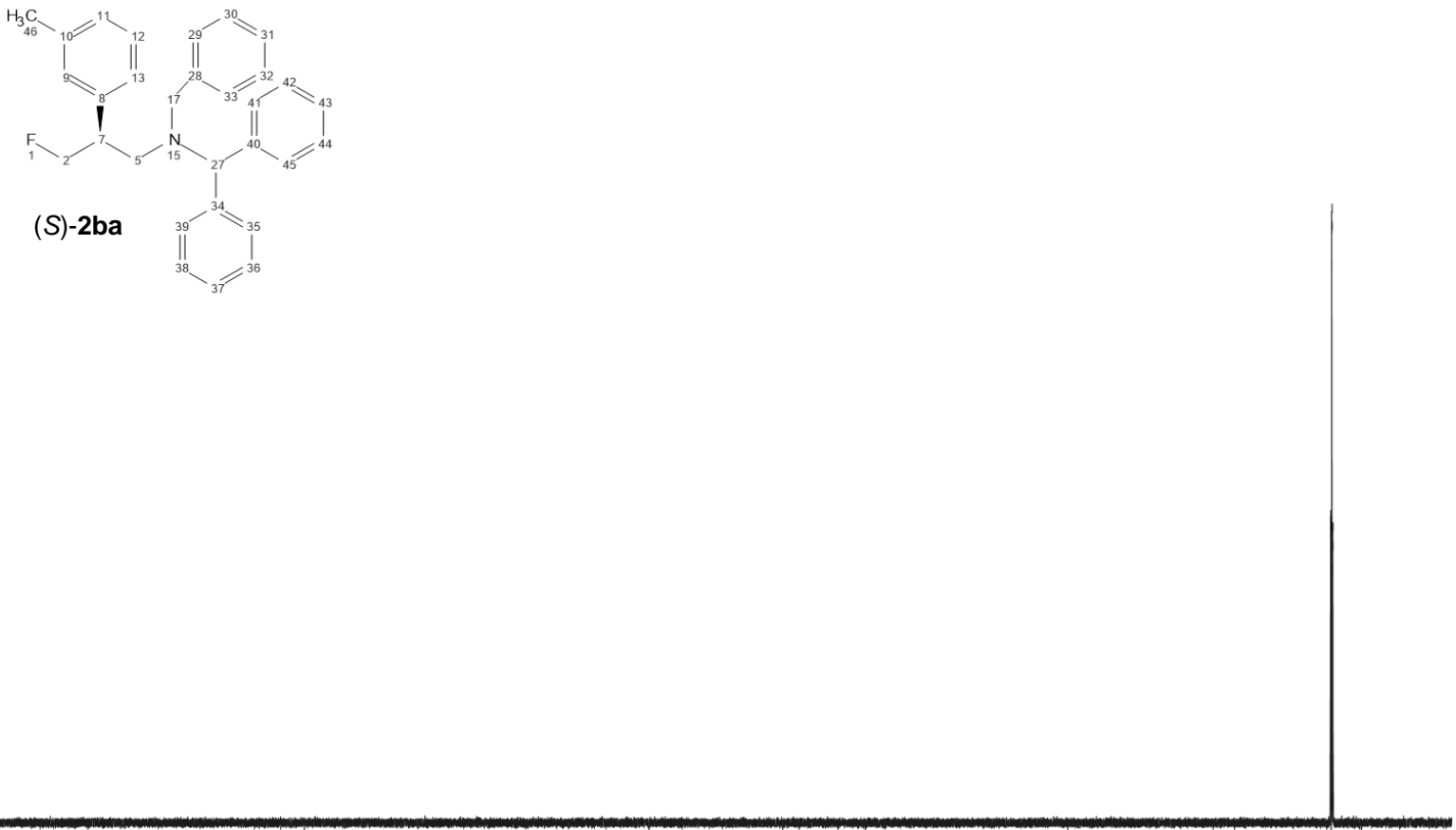

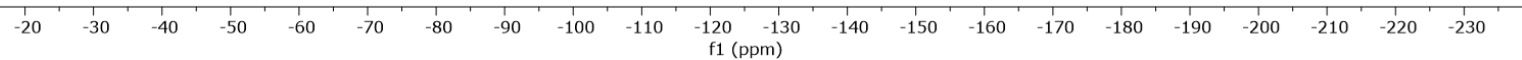


UOXF_groagna_2ca/CdCl3

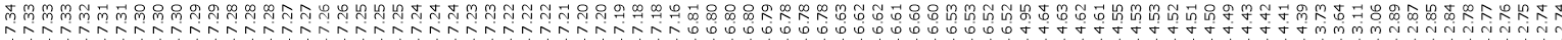
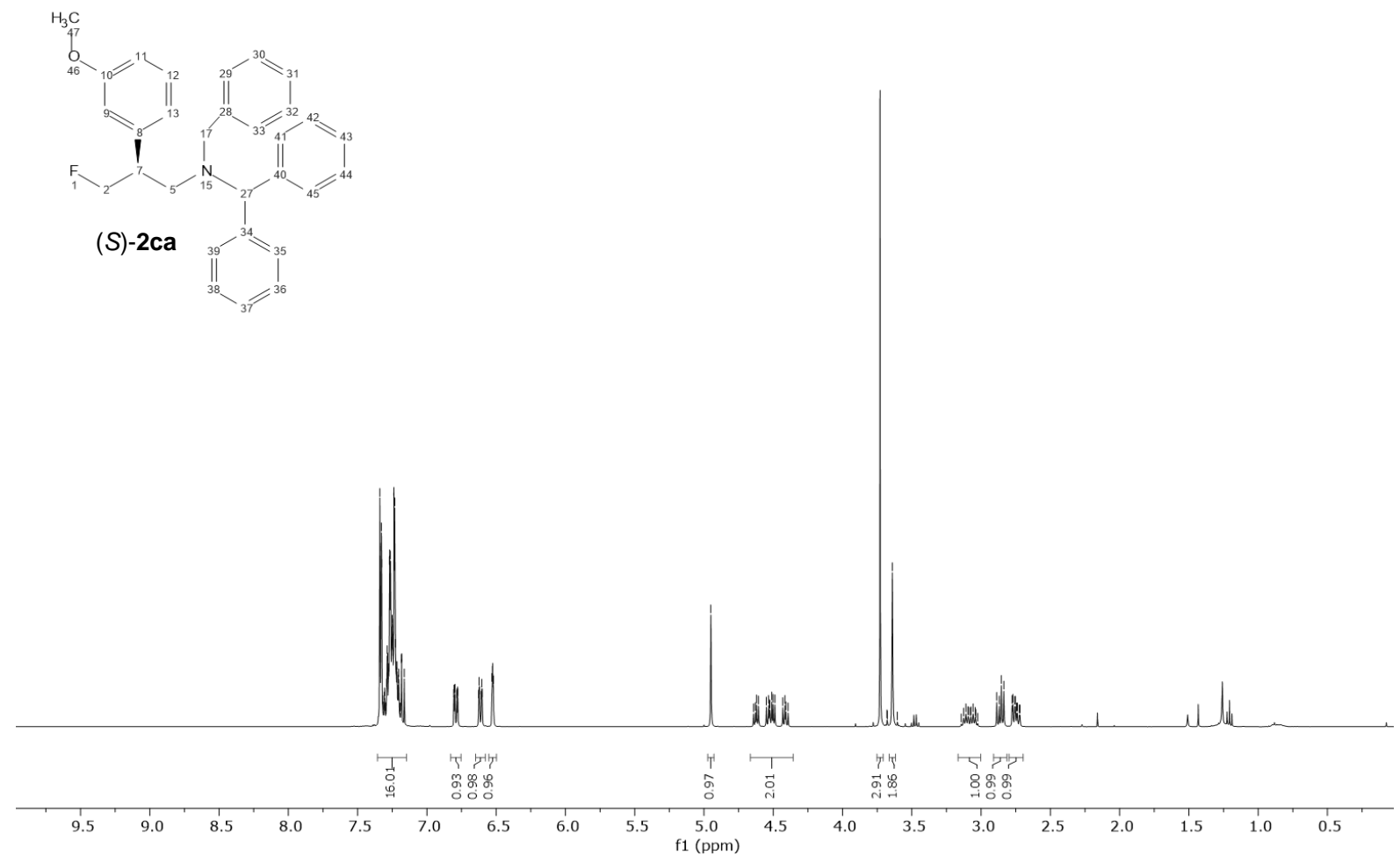

UOXF_groagna_2ca/CdCl3

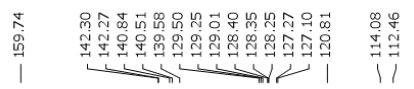

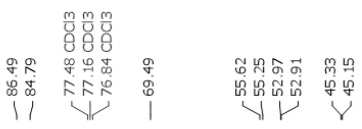<smiles>COc1cncnc1P(CF)SN(Cc1cccnc1)C(c1cccnc1)c1ccccn1</smiles>

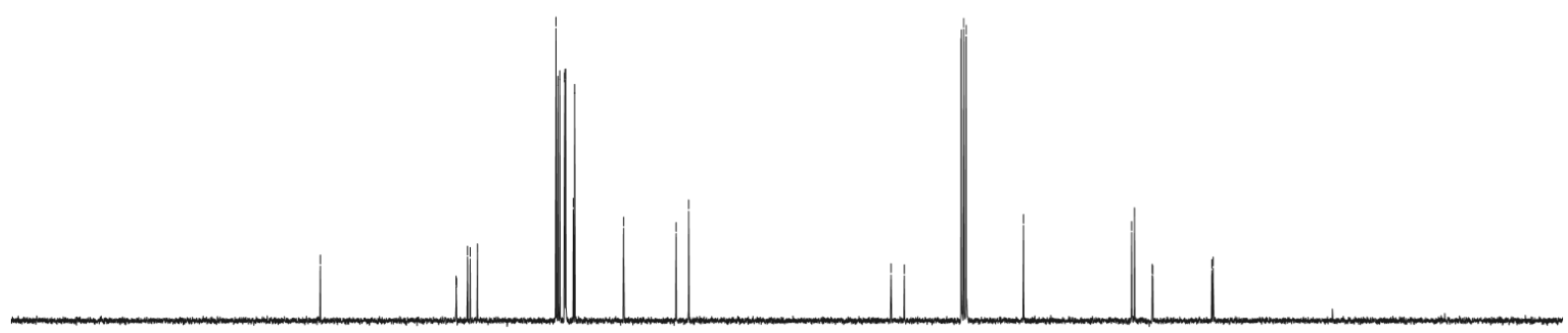

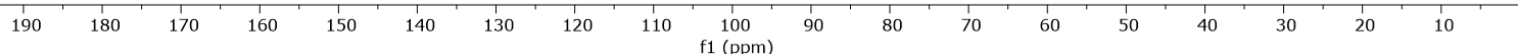




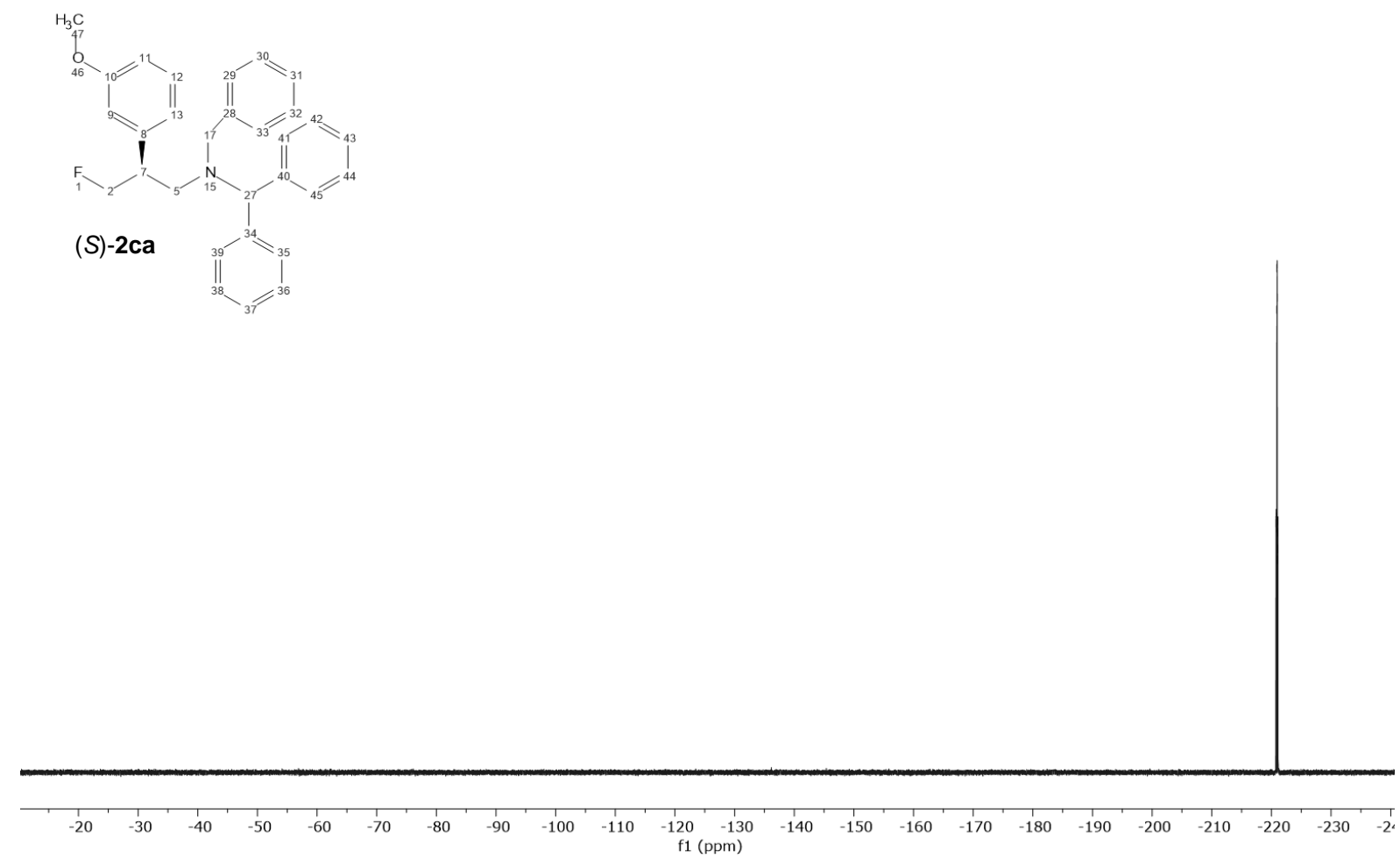


UOXF_groagna_2da/CdCl3

$\stackrel{m}{8}$

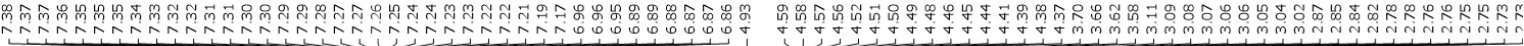
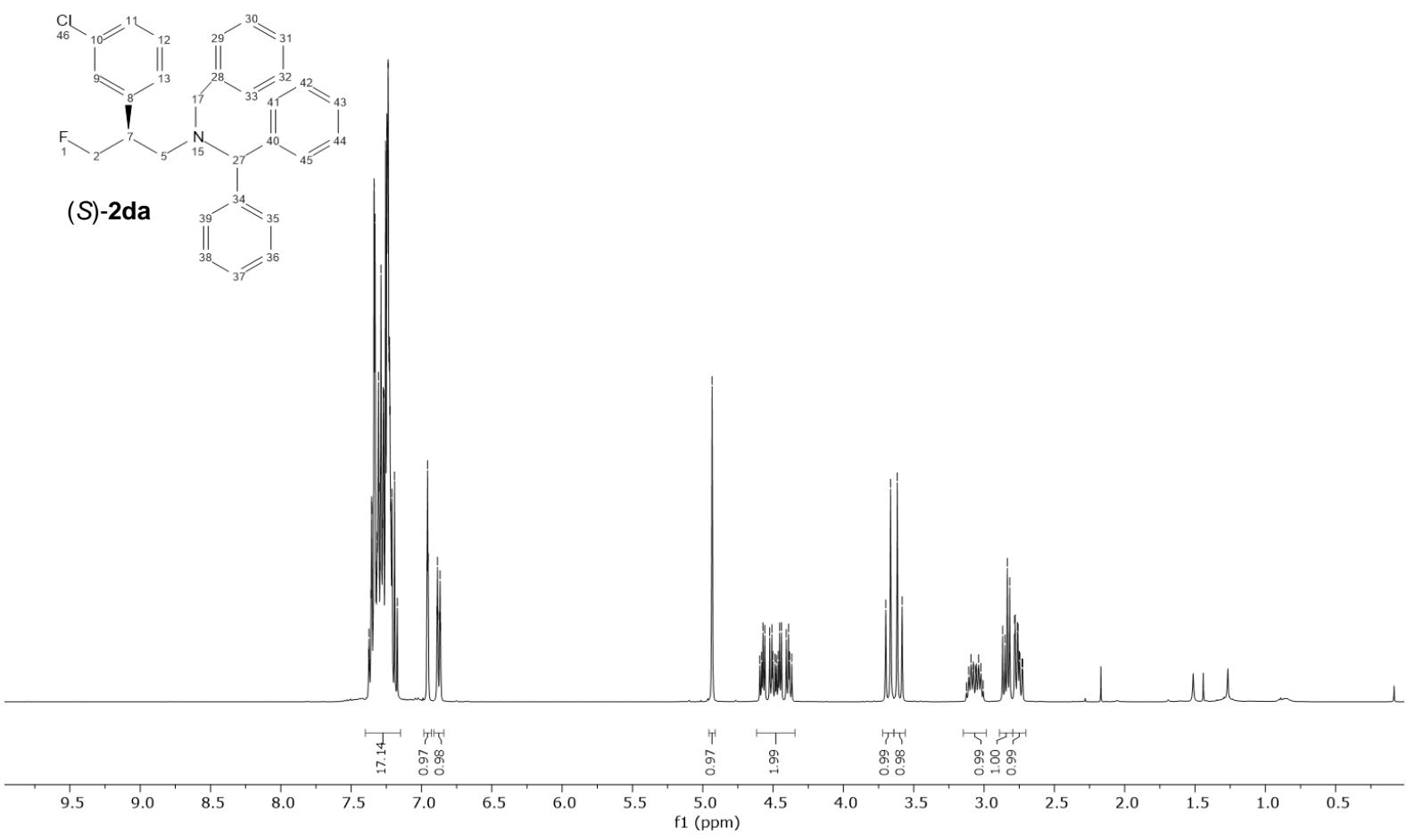

UOXF_groagna_2da/CdCl3

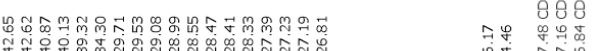

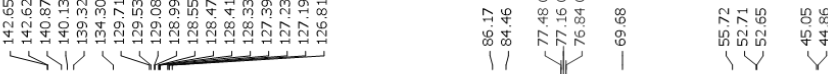
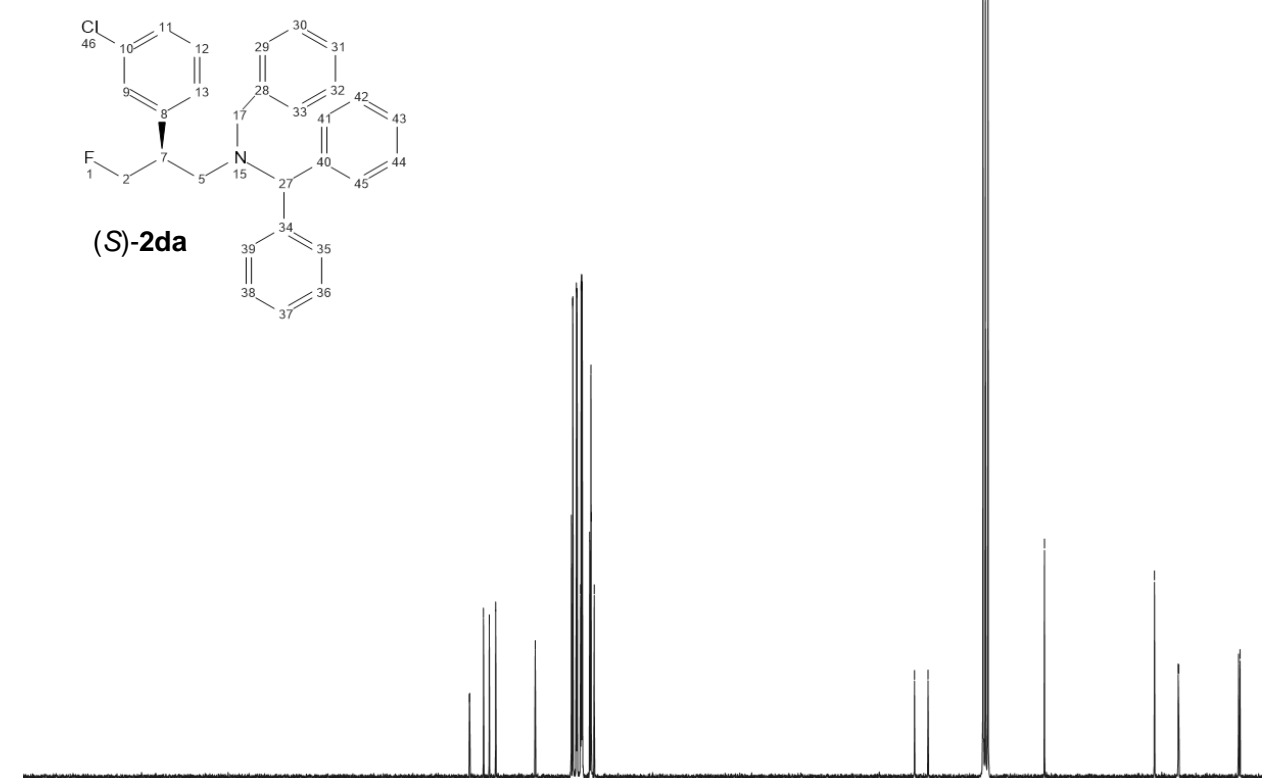

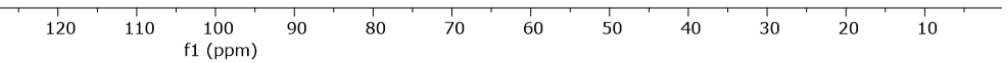




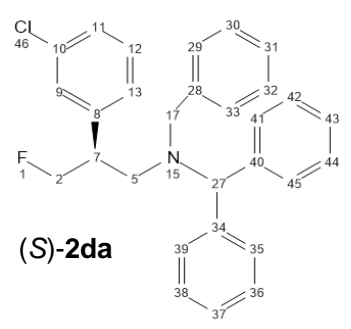

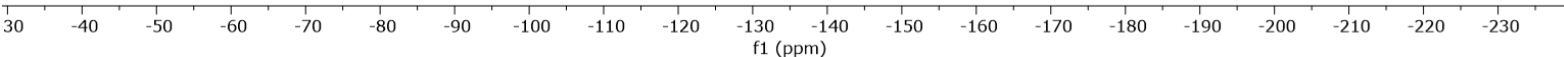




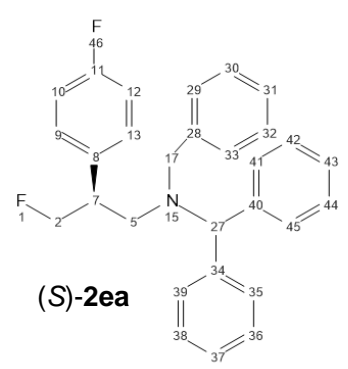

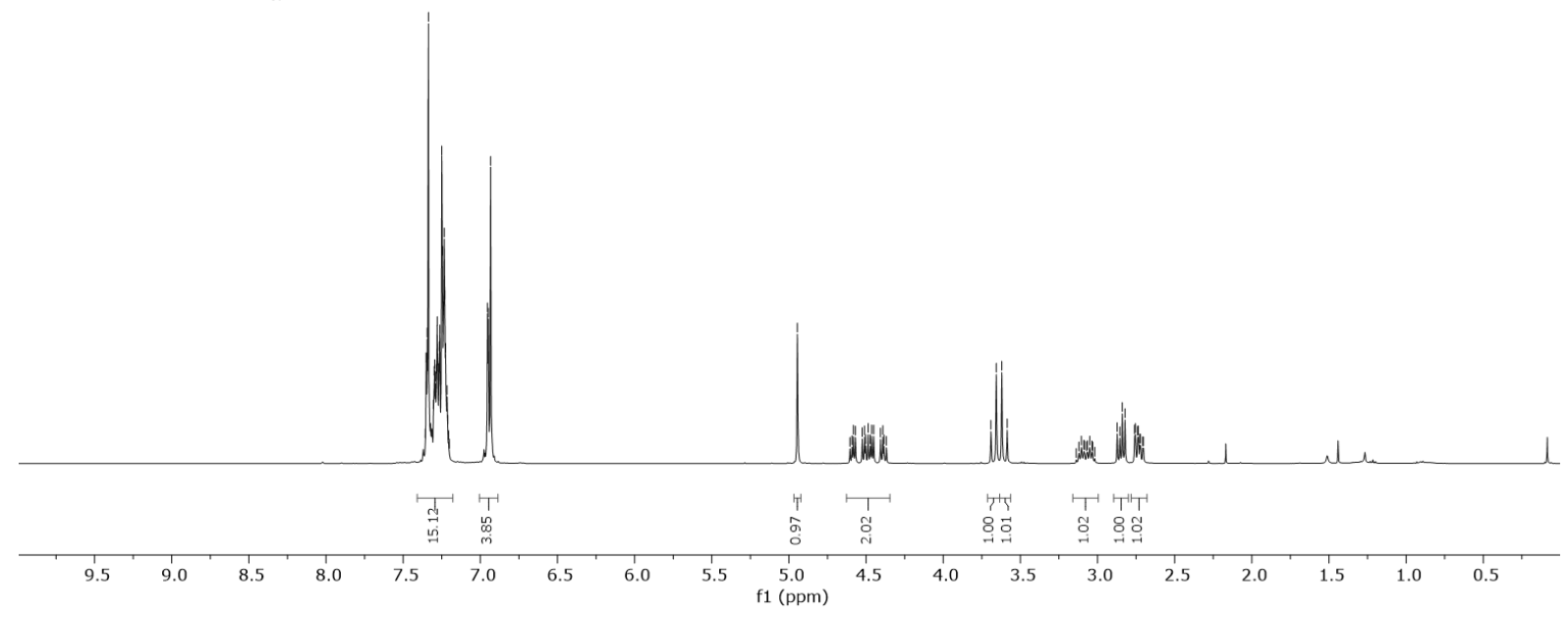

UOXF_groagna_2ea/CdCl3

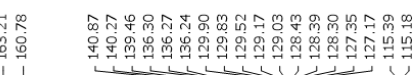
1 |

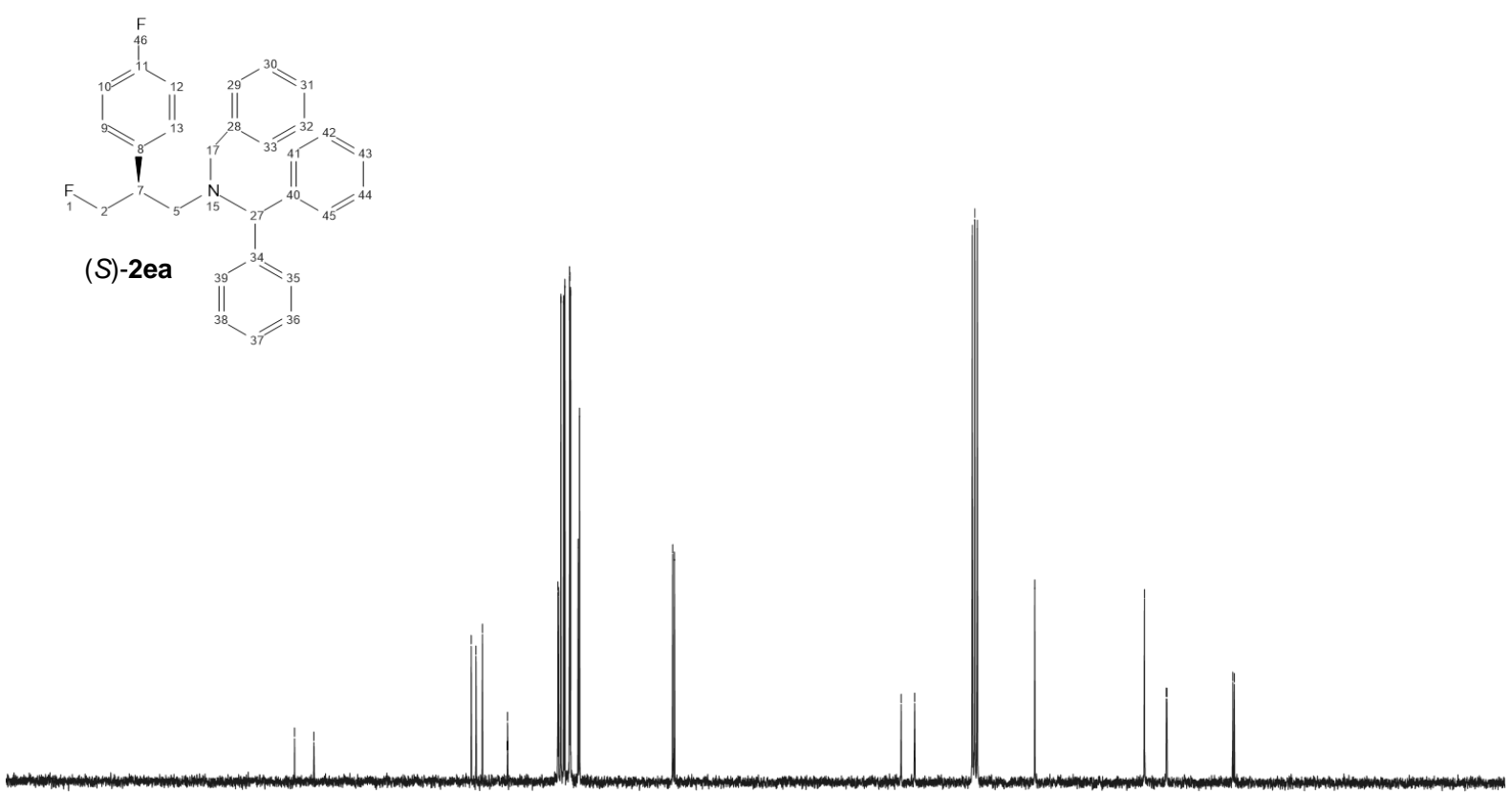

$\begin{array}{lllllllll}190 & 180 & 170 & 160 & 150 & 140 & 130 & 120 & 110 \\ \mathrm{f} 1(\mathrm{ppm})\end{array}$ 
<smiles>Fc1ccc(N(CN(c2cncnc2)c2ccccn2)c2cccnc2)nc1</smiles>

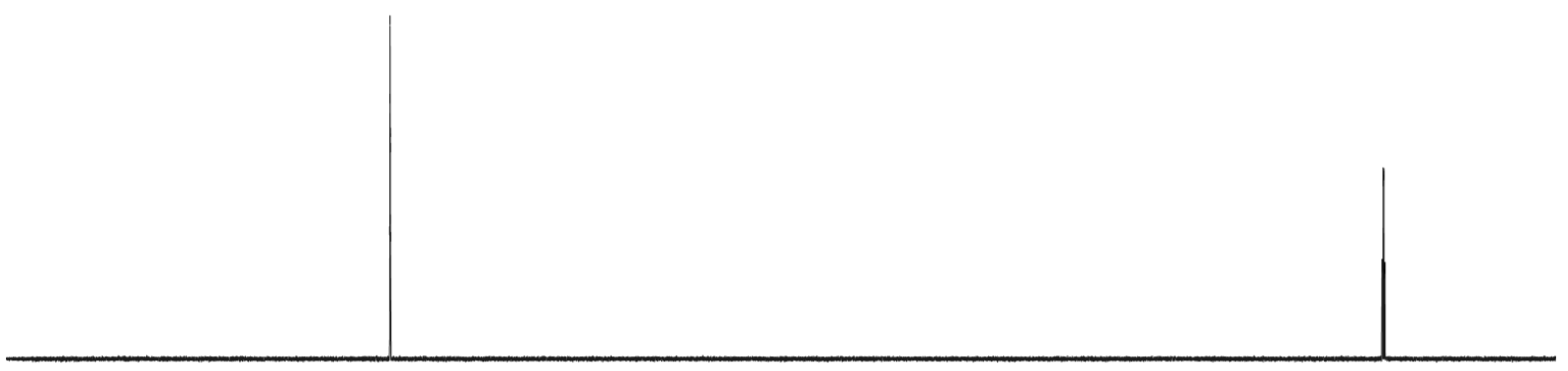

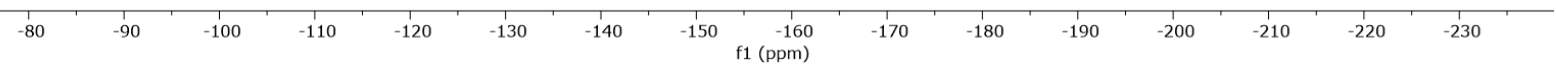




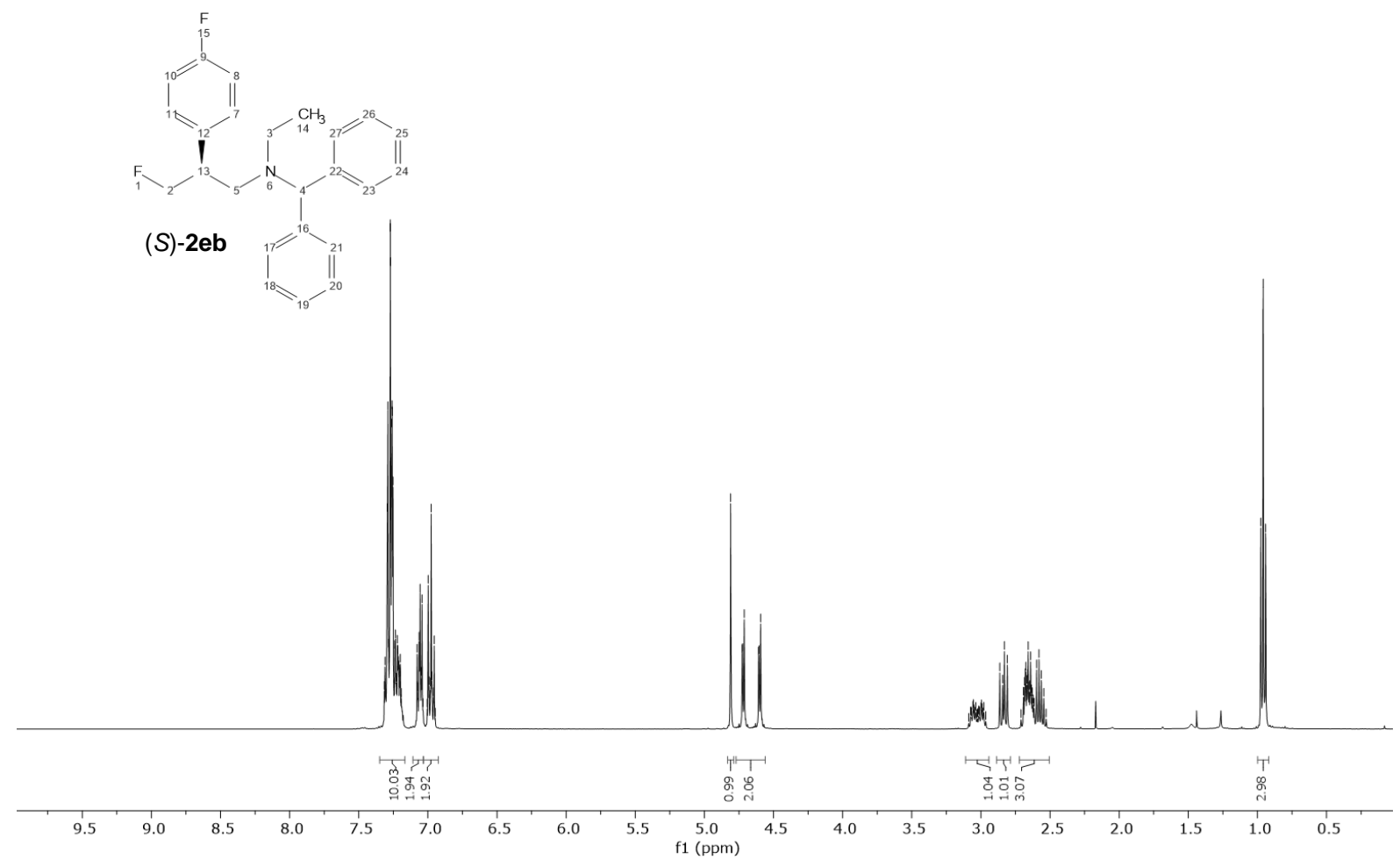

UOXF_groagna_2eb/CdCl3

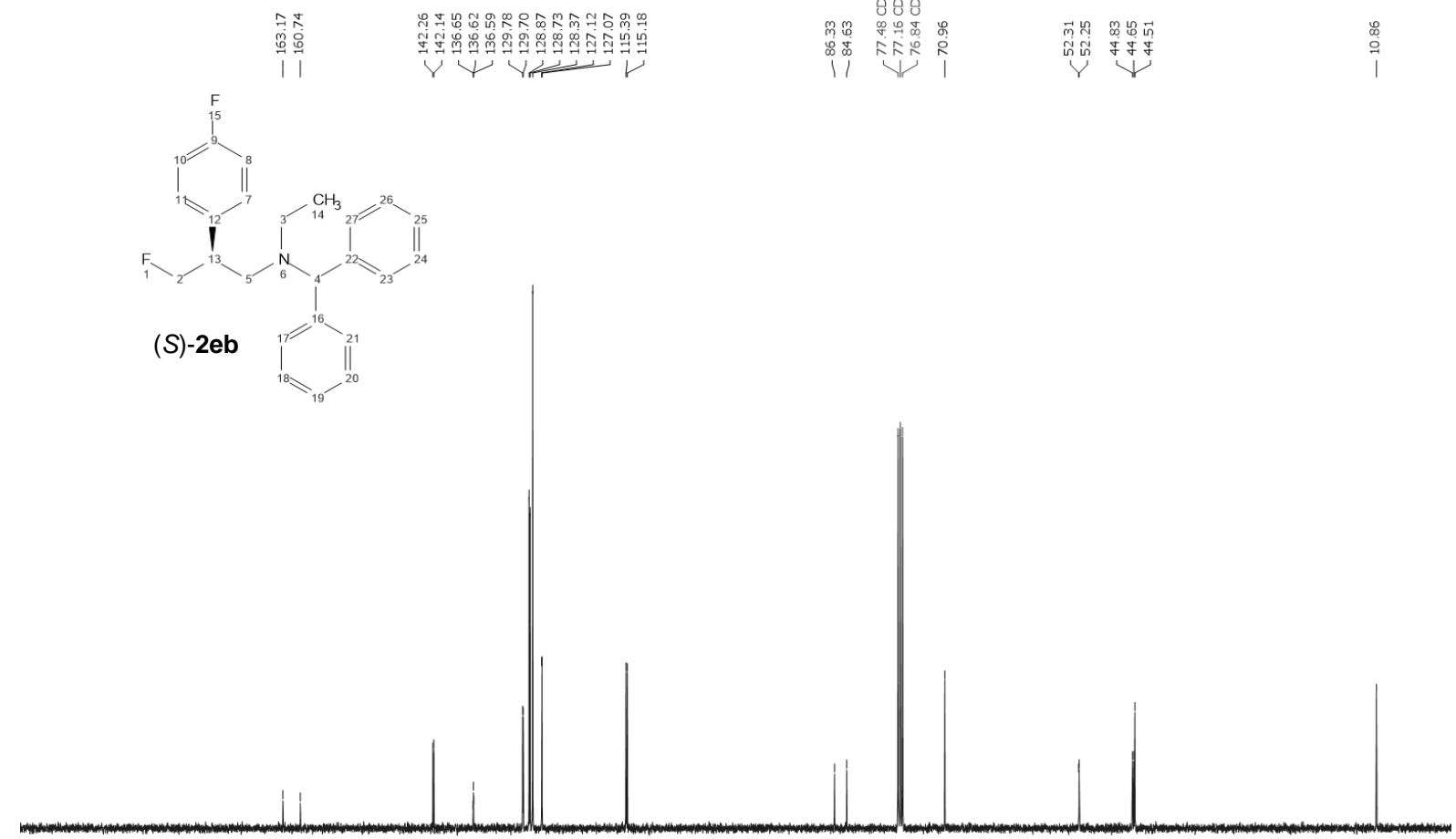

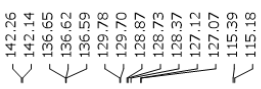

$\frac{m}{0}{ }^{m} \frac{m}{0}$

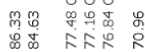

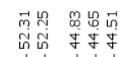

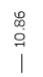

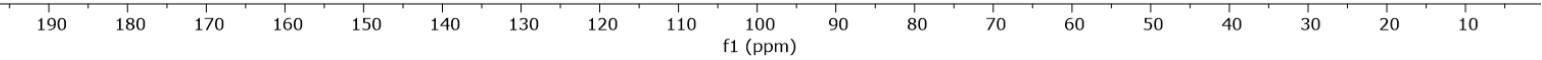


<smiles>CCN(CN(CF)c1ccncn1)c1cccnc1</smiles>

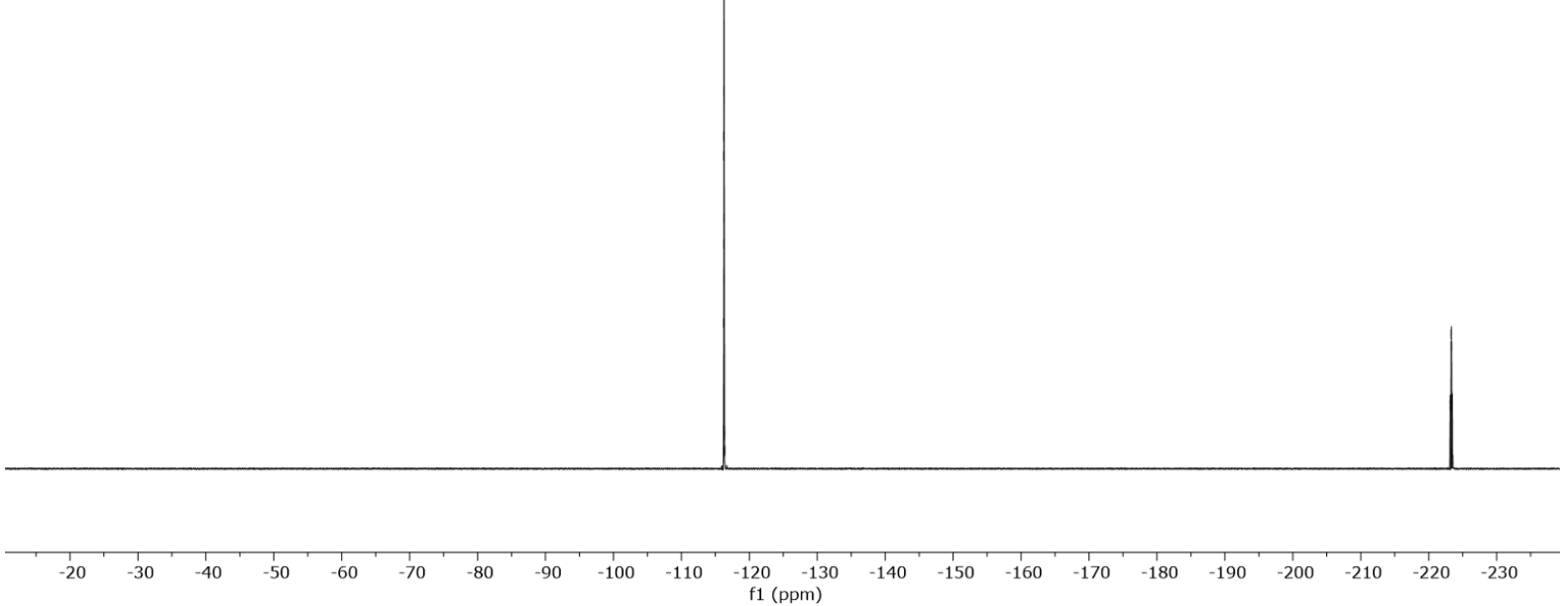




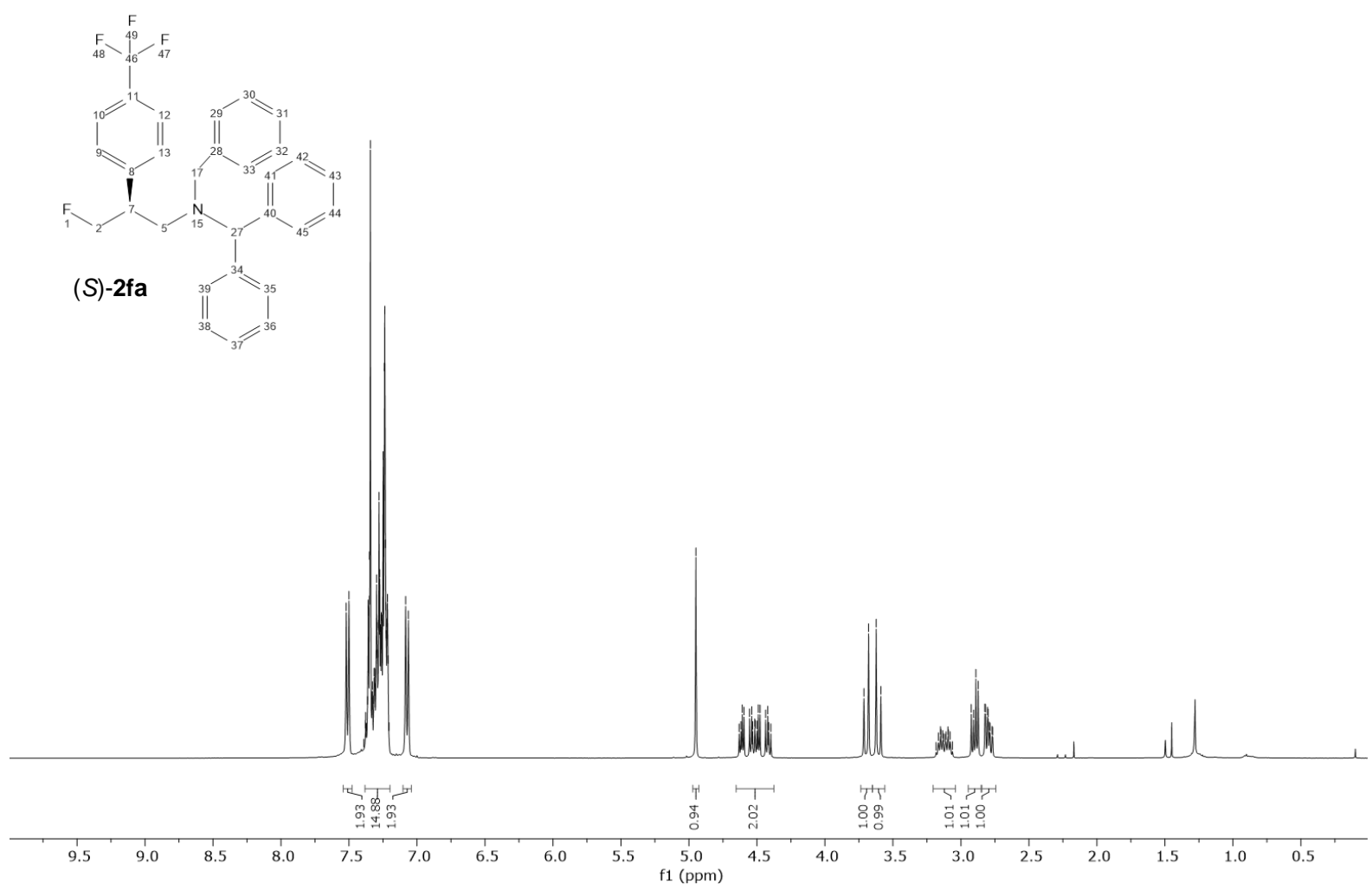

UOXF_groagna_2fa/CdCl3

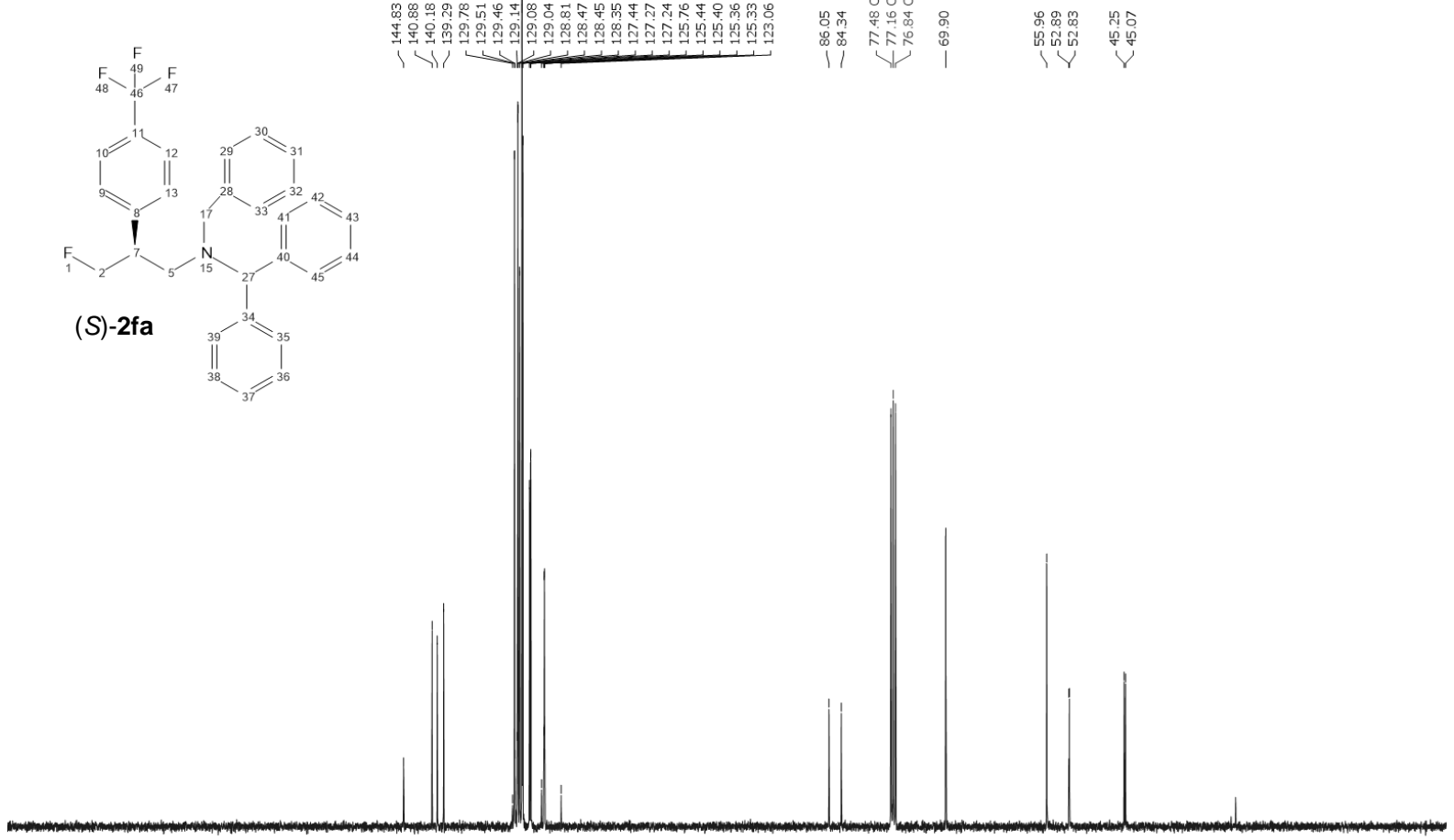

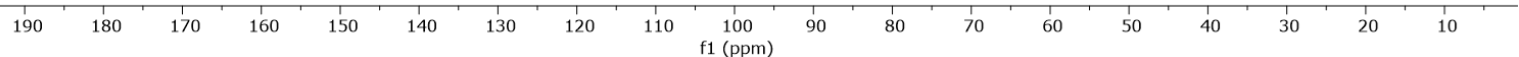




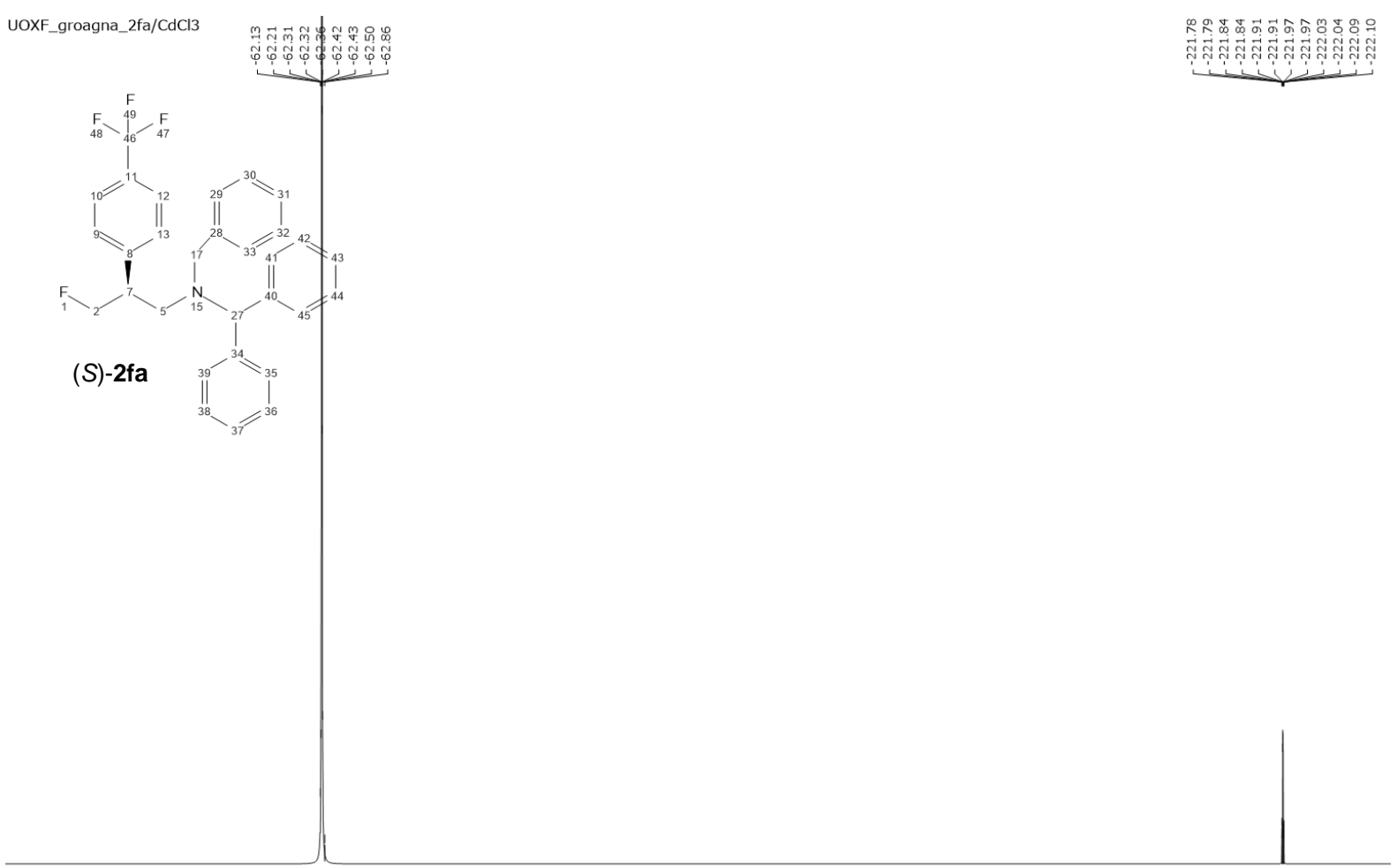

\begin{tabular}{llllllllllllllllllllllllllllllllll}
\hline 10 & -20 & -30 & -40 & -50 & -60 & -70 & -80 & -90 & -100 & -110 & -120 & -130 & -140 & 1 & 150 & -160 & -170 & -180 & -190 & -200 & -210 & -220 & -230
\end{tabular} 


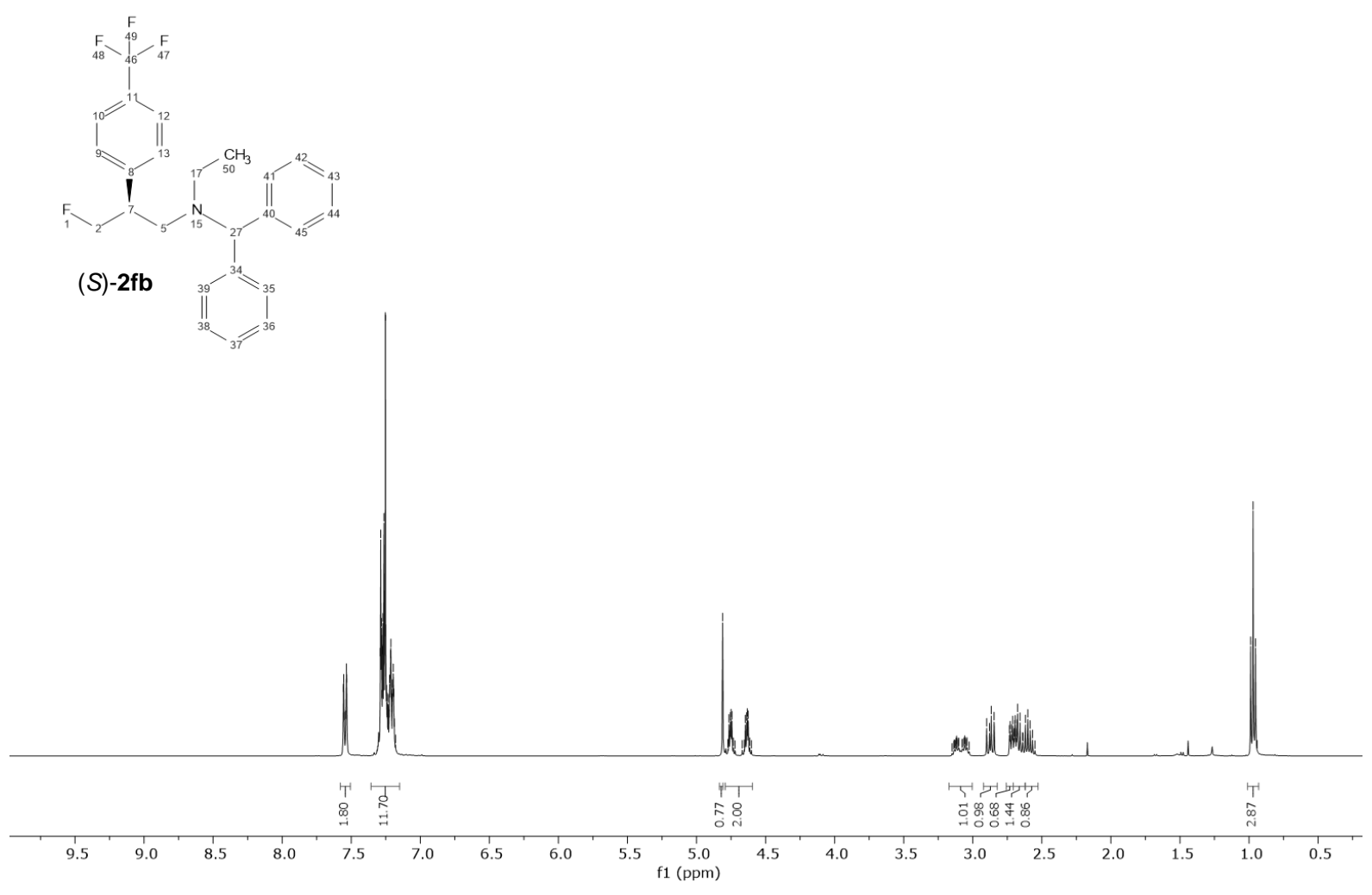

UOXF_groagna_2fb/CdCl3

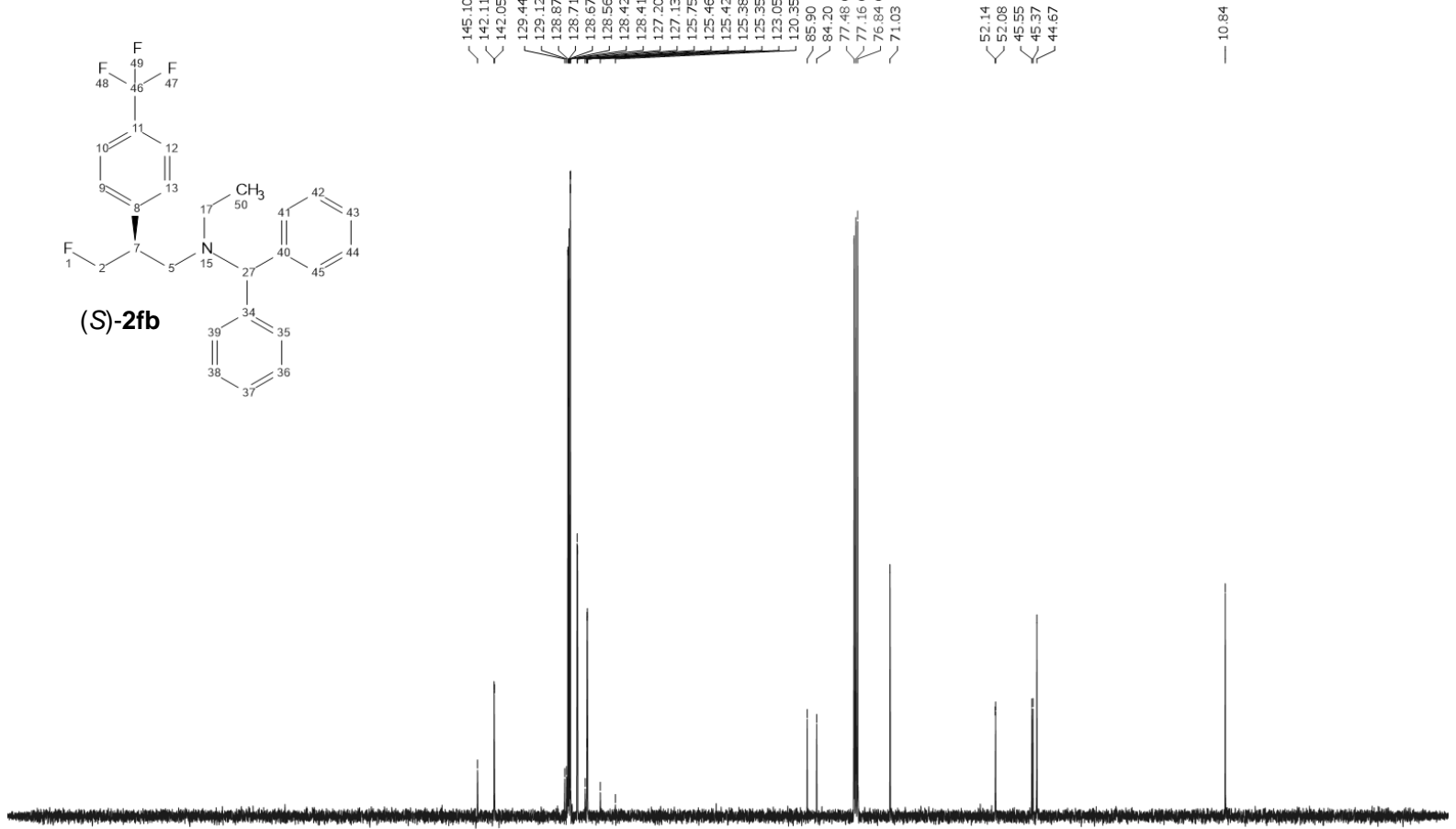

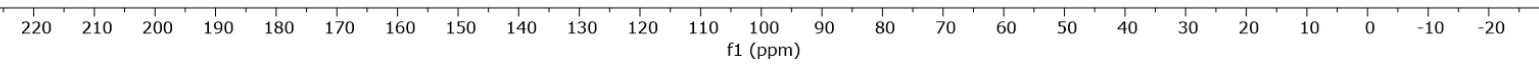




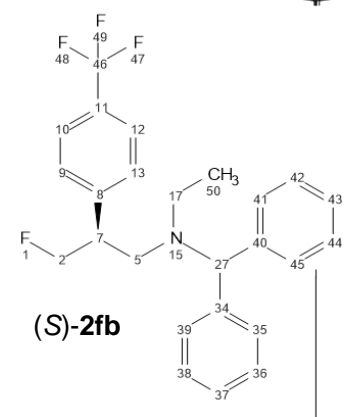

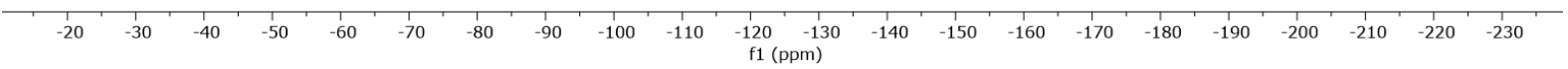




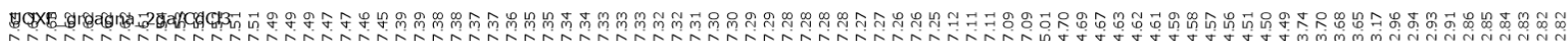
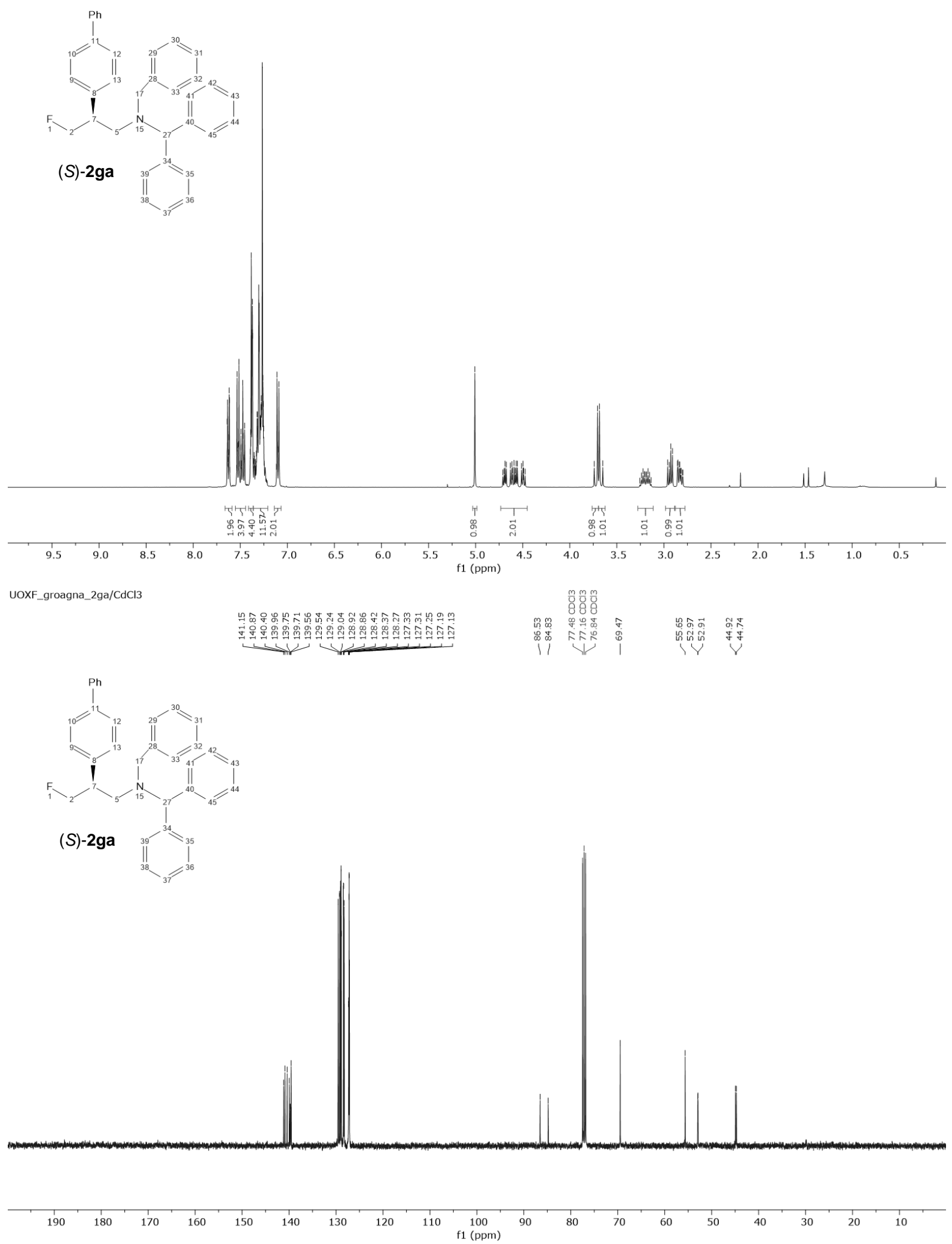

S290 

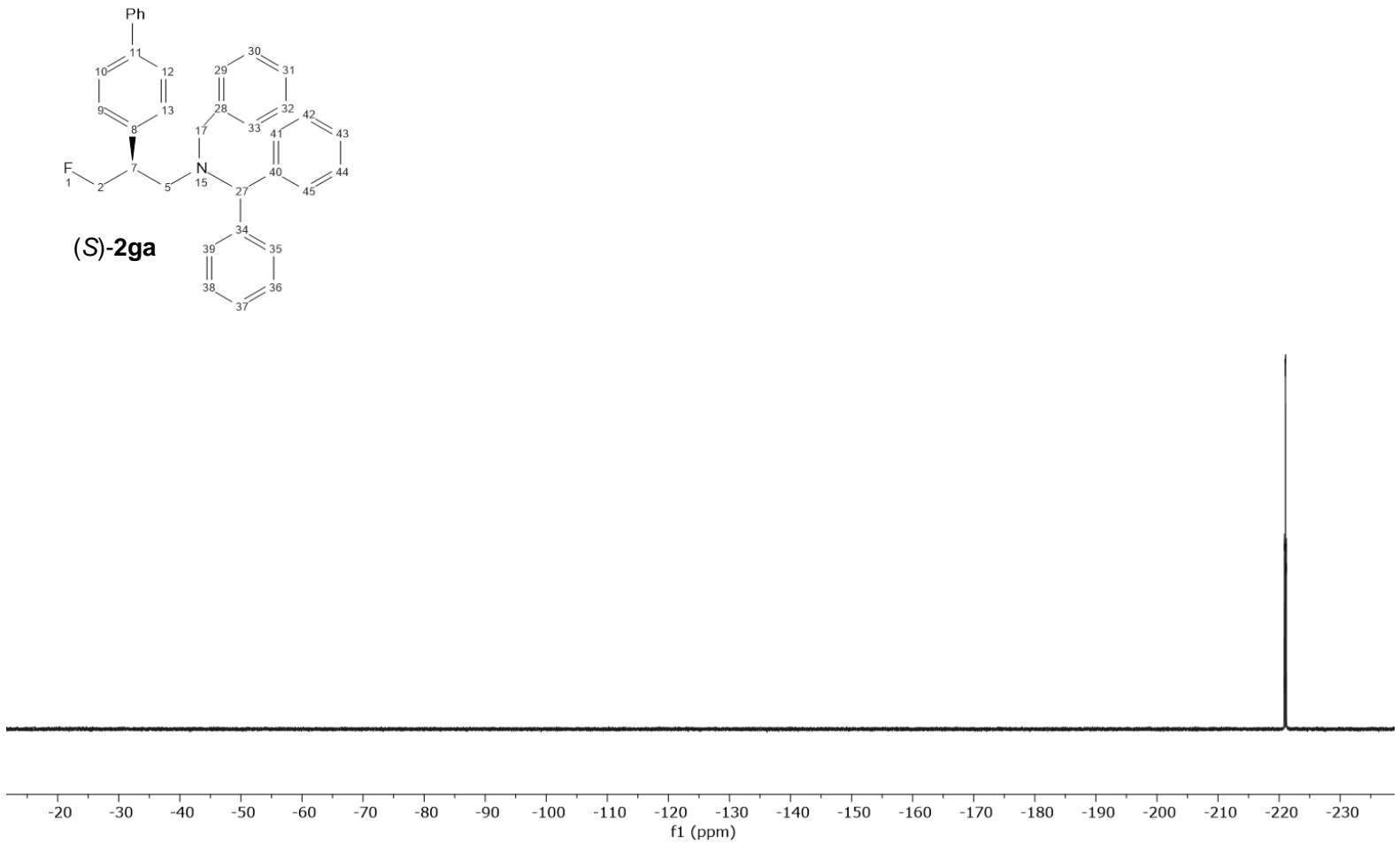


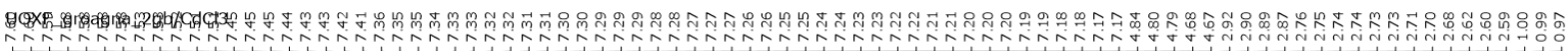
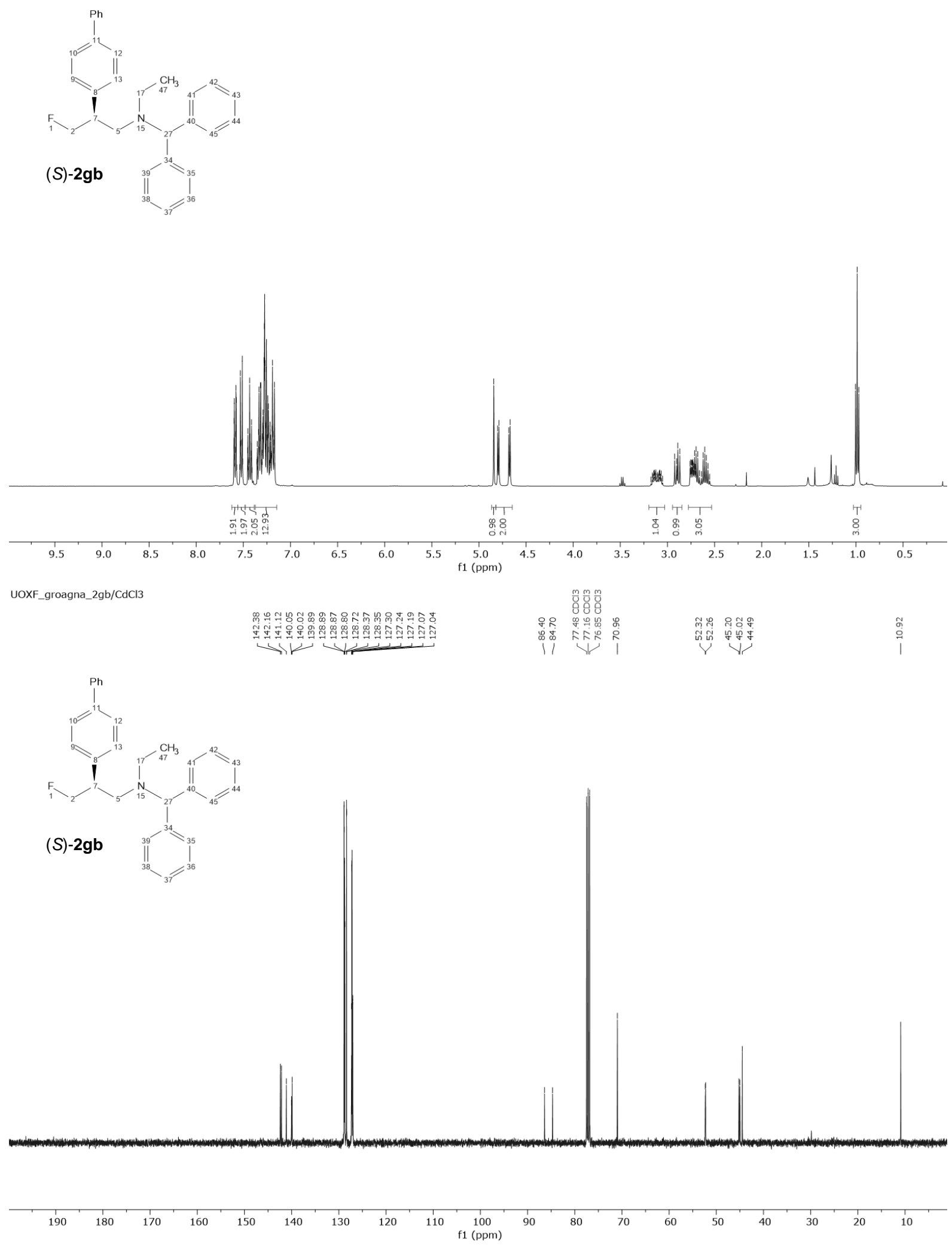

S292 


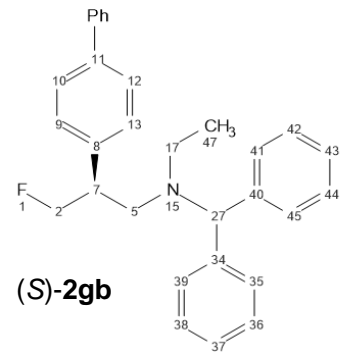

\begin{tabular}{lllllllllllllllllllllllllllllll}
-20 & -30 & -40 & -50 & -60 & -70 & -80 & -90 & -100 & -110 & -120 & -130 & -140 & -150 & -160 & -170 & -180 & -190 & -200 & -210 & -220 & -230 & -2 \\
\hline
\end{tabular} 


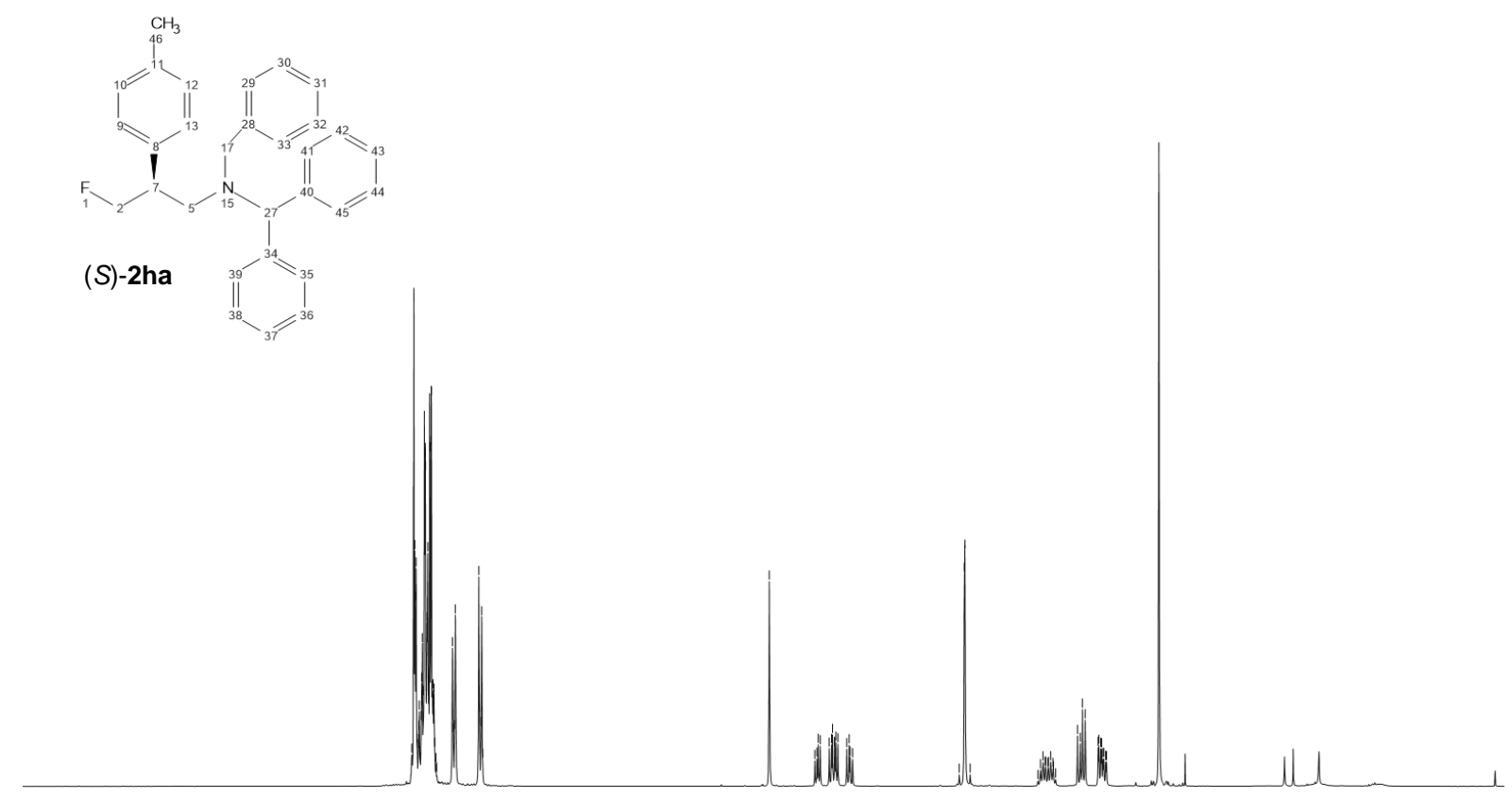

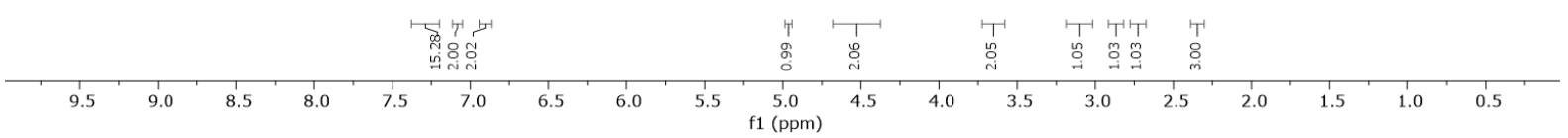

UOXF_groagna_2ha/CdCl3

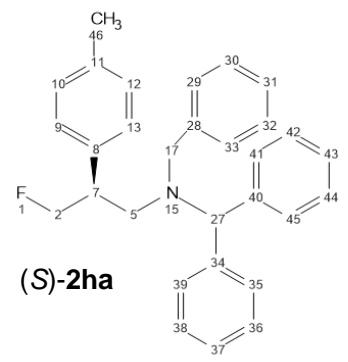

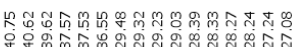

तन

iv $m$
0
0
0
0
0
0
0
0

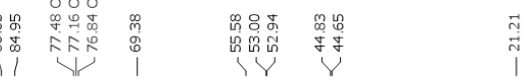

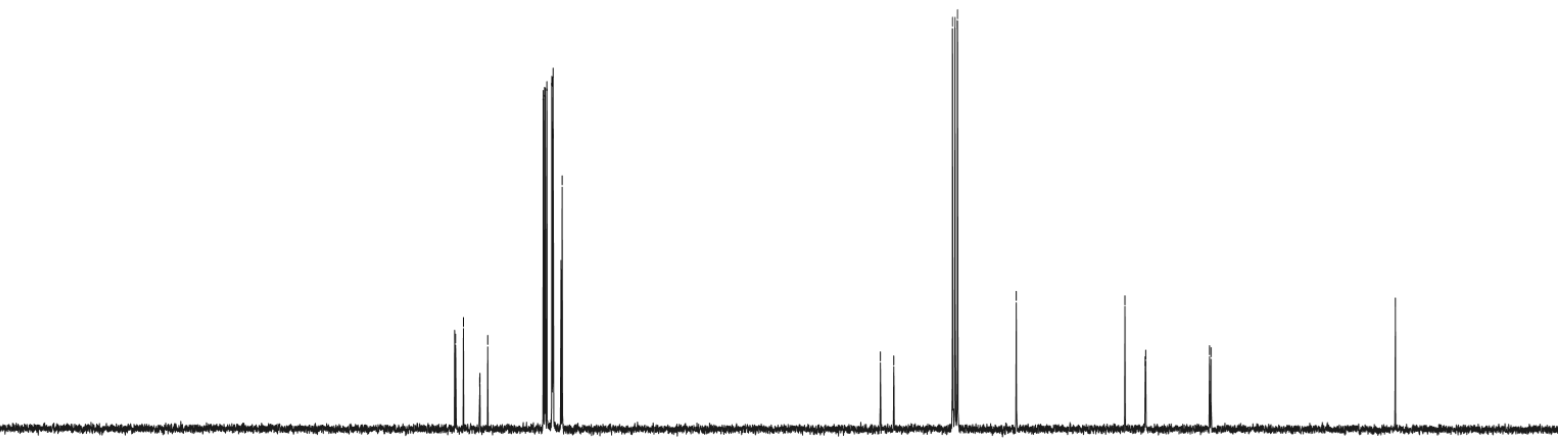
$10 \begin{gathered}100 \\ \mathrm{f} 1(\mathrm{ppm})\end{gathered}$ 


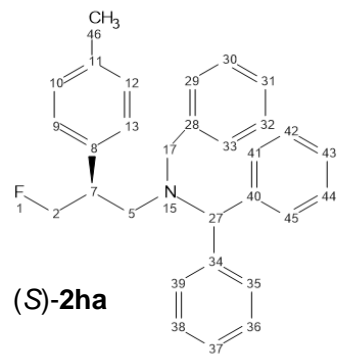

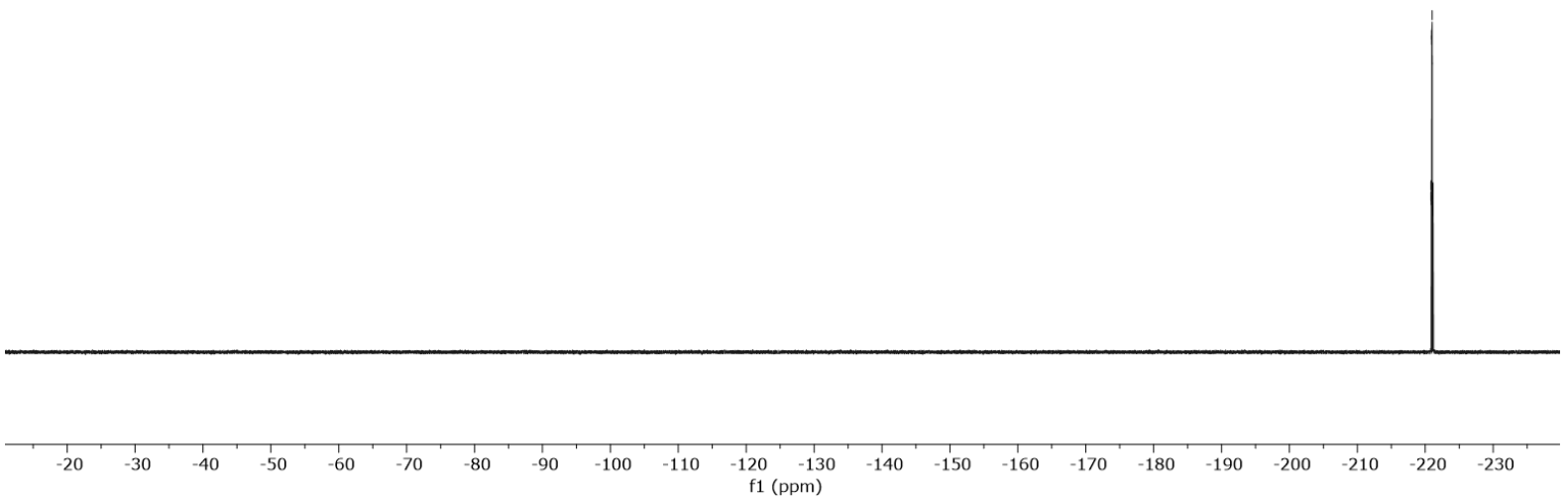


<smiles>FCP(Oc1cccnc1)SN(Cc1cccnc1)C(c1cccnc1)c1ccccn1</smiles>
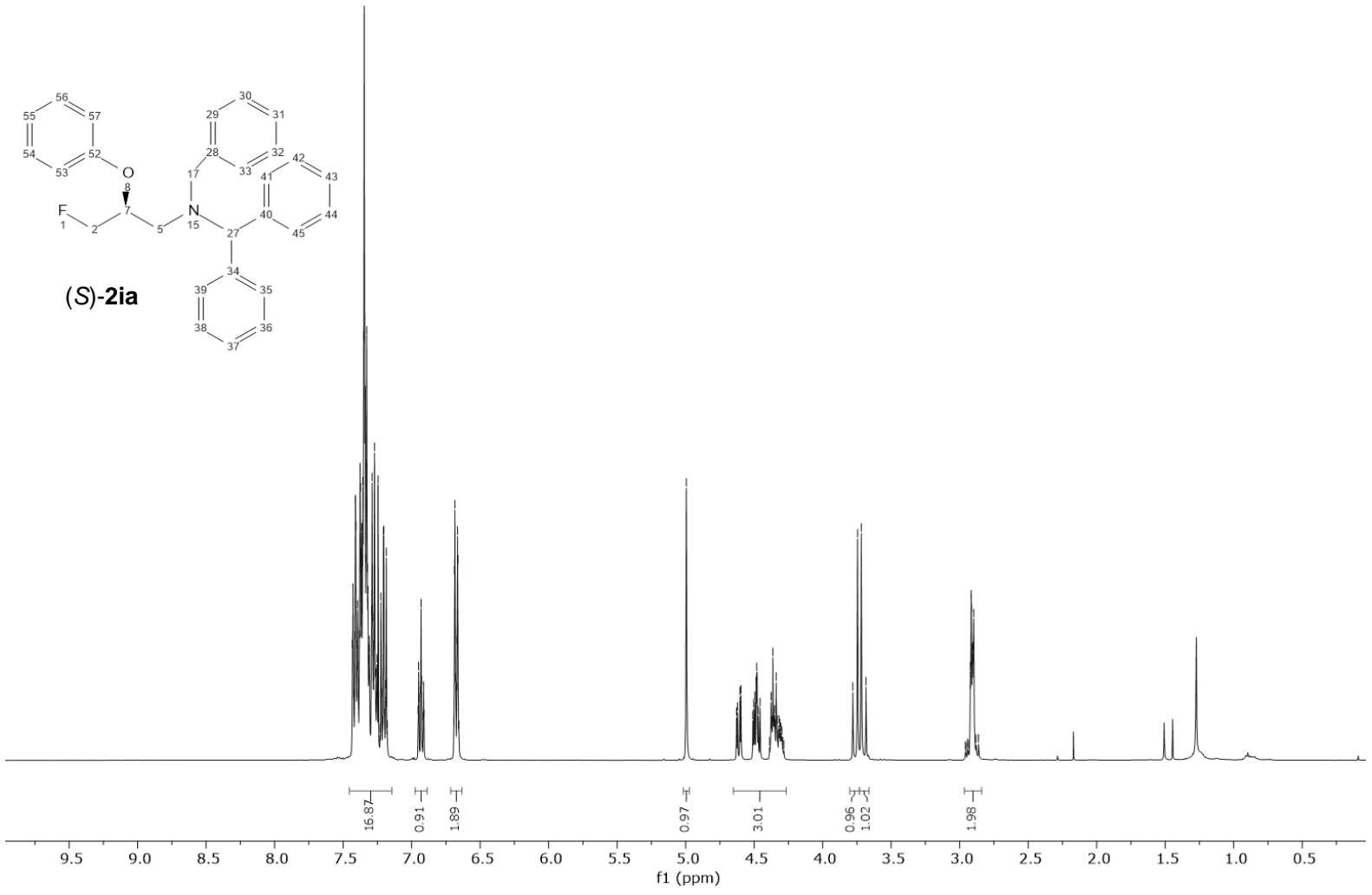

UOXF_groagna_2ia/CdCl3

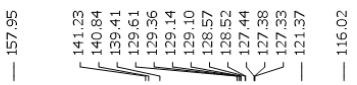
$\begin{array}{ll}\frac{m}{\mathrm{C}} & \frac{m}{\mathrm{C}} \\ \mathrm{C} & 8\end{array}$

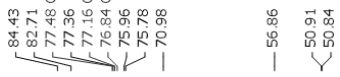<smiles>FCP(Oc1ccncn1)SN(Cc1cnccn1)C(c1cccnc1)c1ccccn1</smiles>

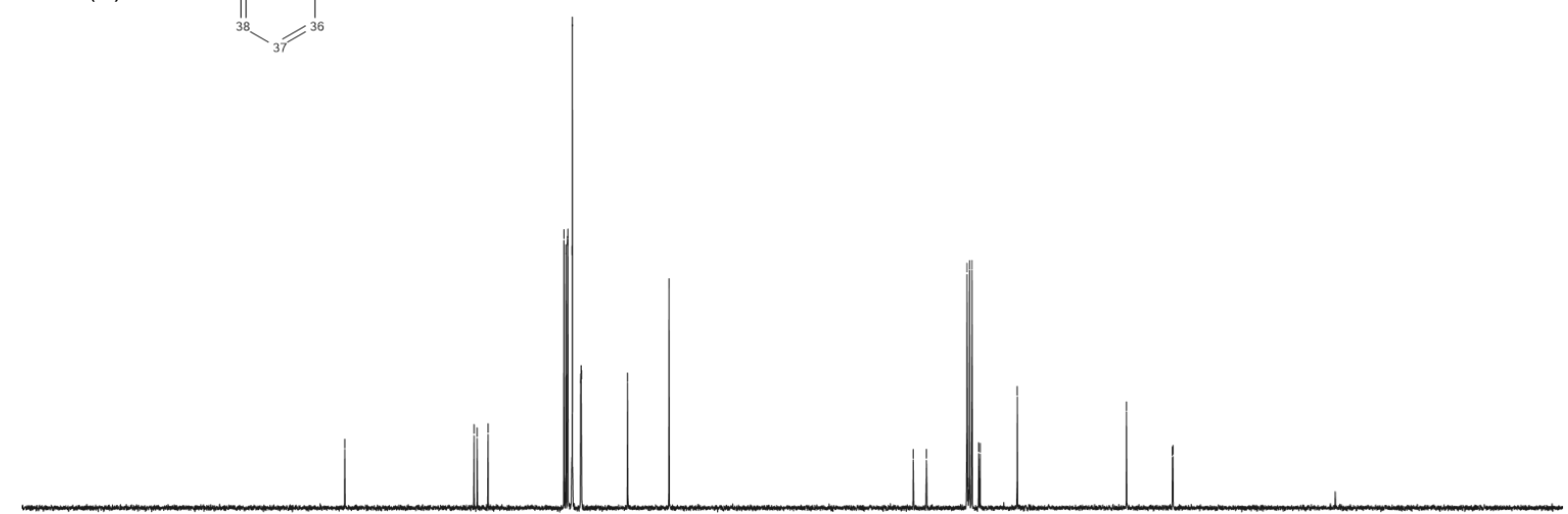

$\begin{array}{lllllll}190 & 180 & 170 & 160 & 150 & 140 & 130\end{array}$ 

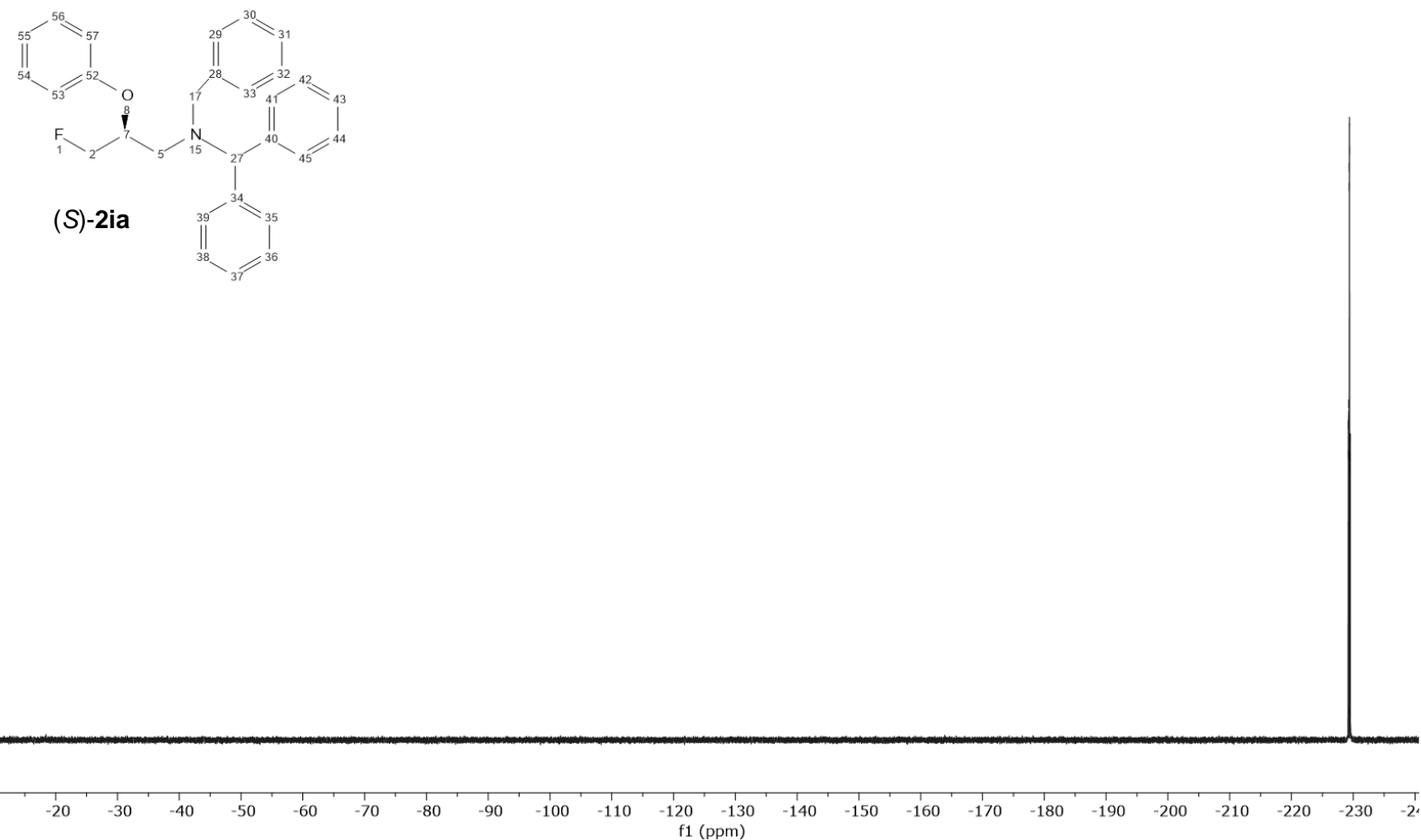


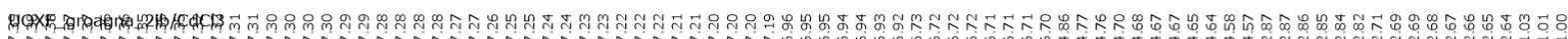
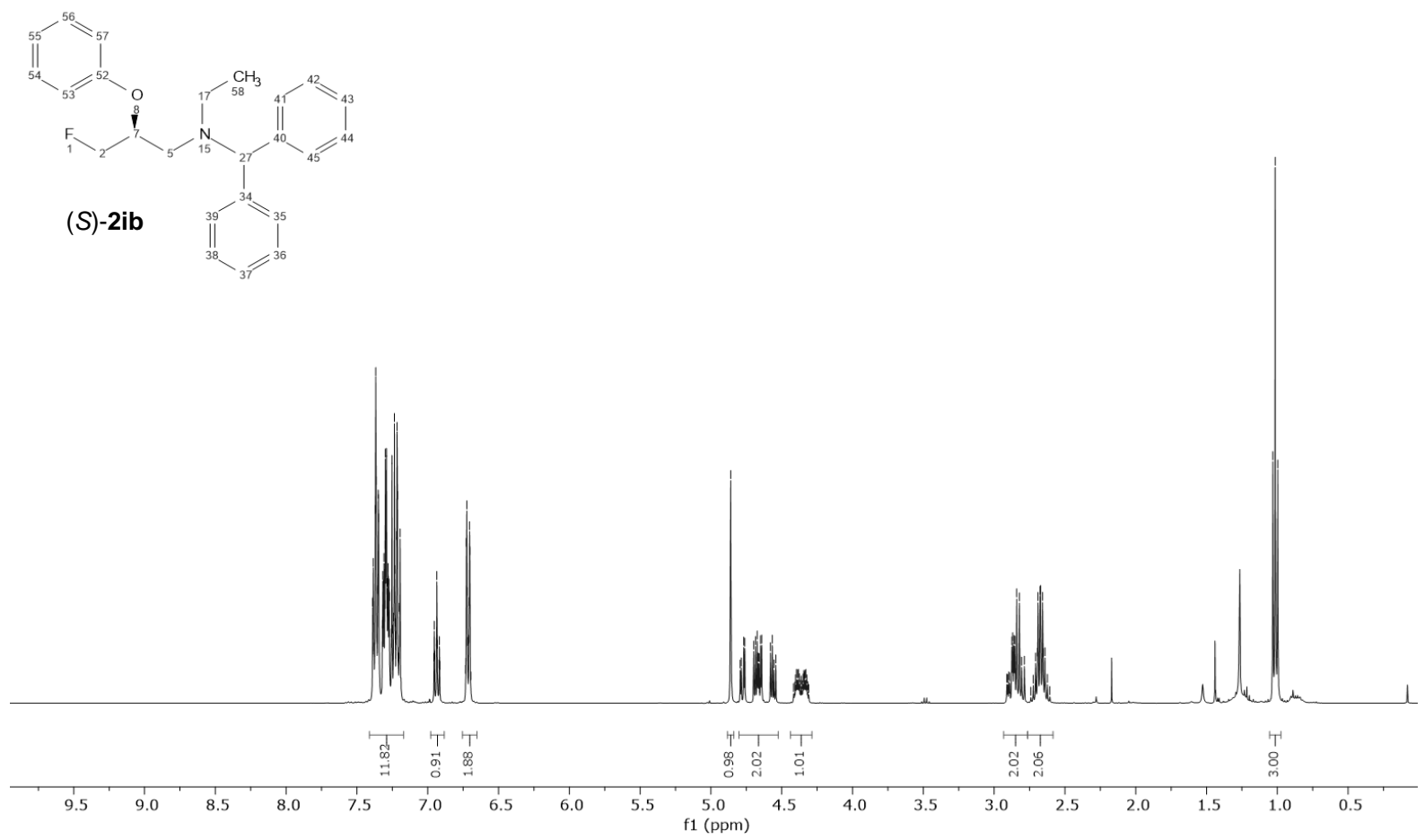

UOXF_groagna_2ib/CdCl3

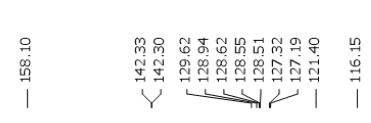

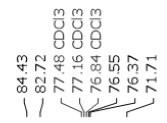

レ゙

굴
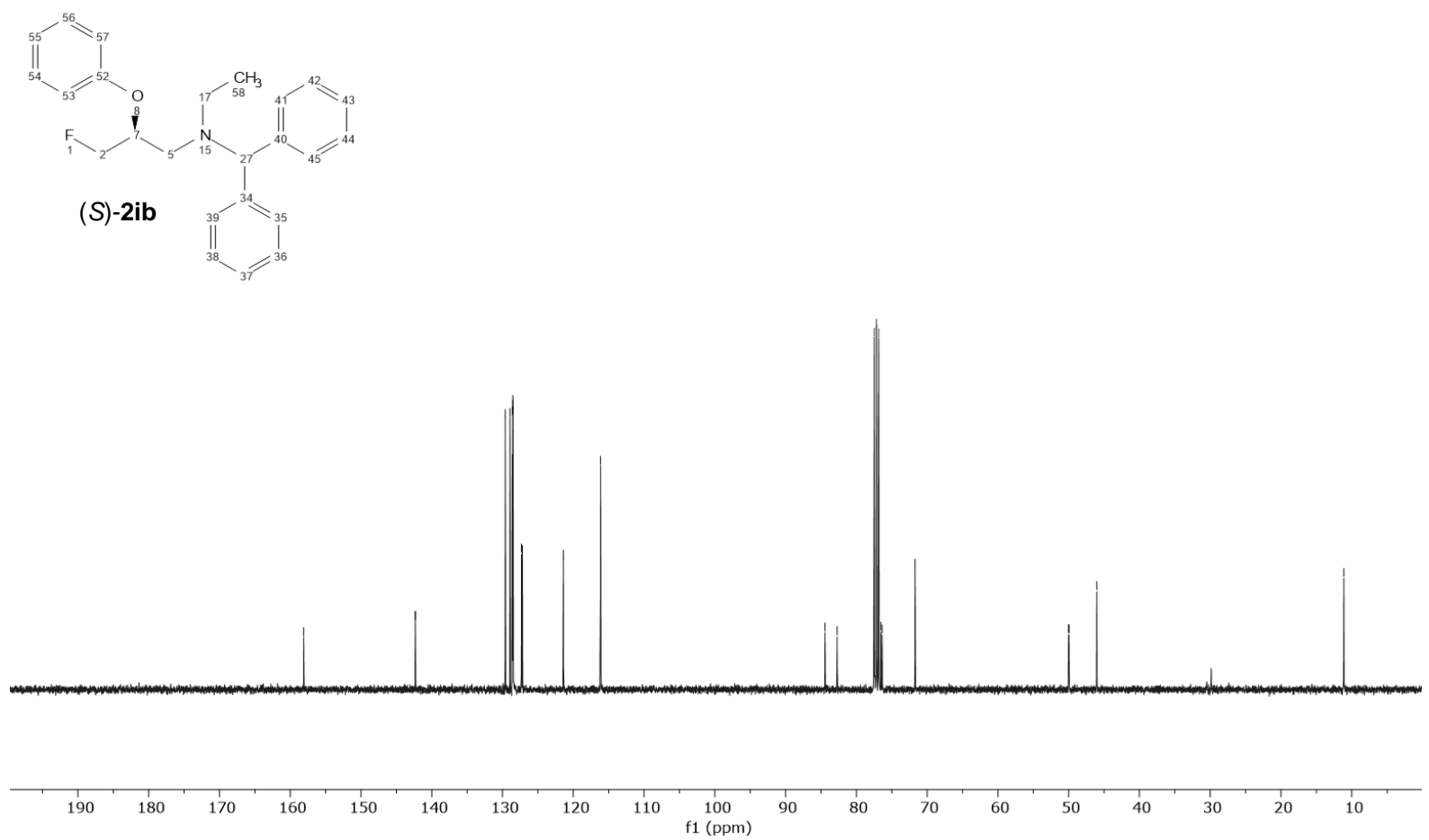

S298 


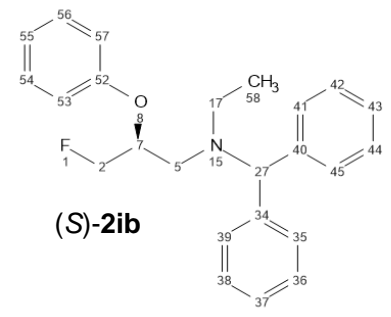

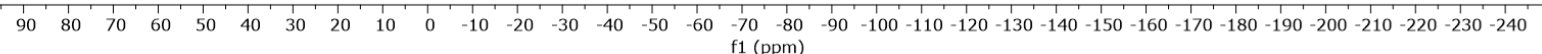


UOXF_groagna_2ic/CdCl3

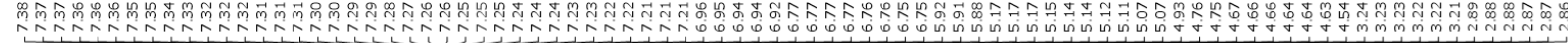
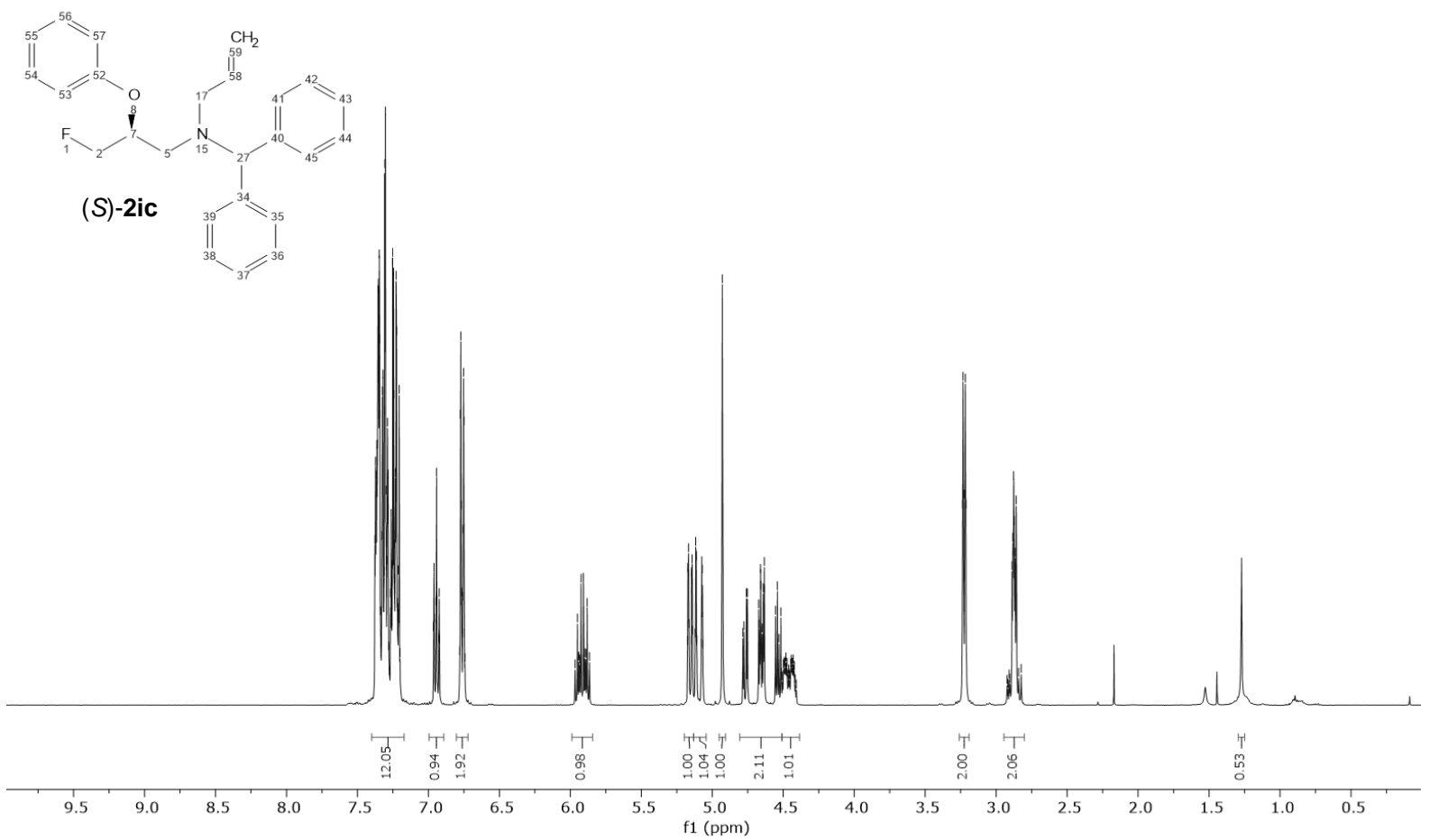

UOXF_groagna_2ic/CdCl3

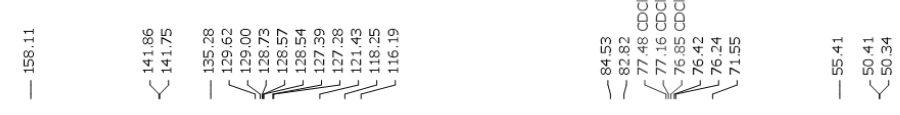
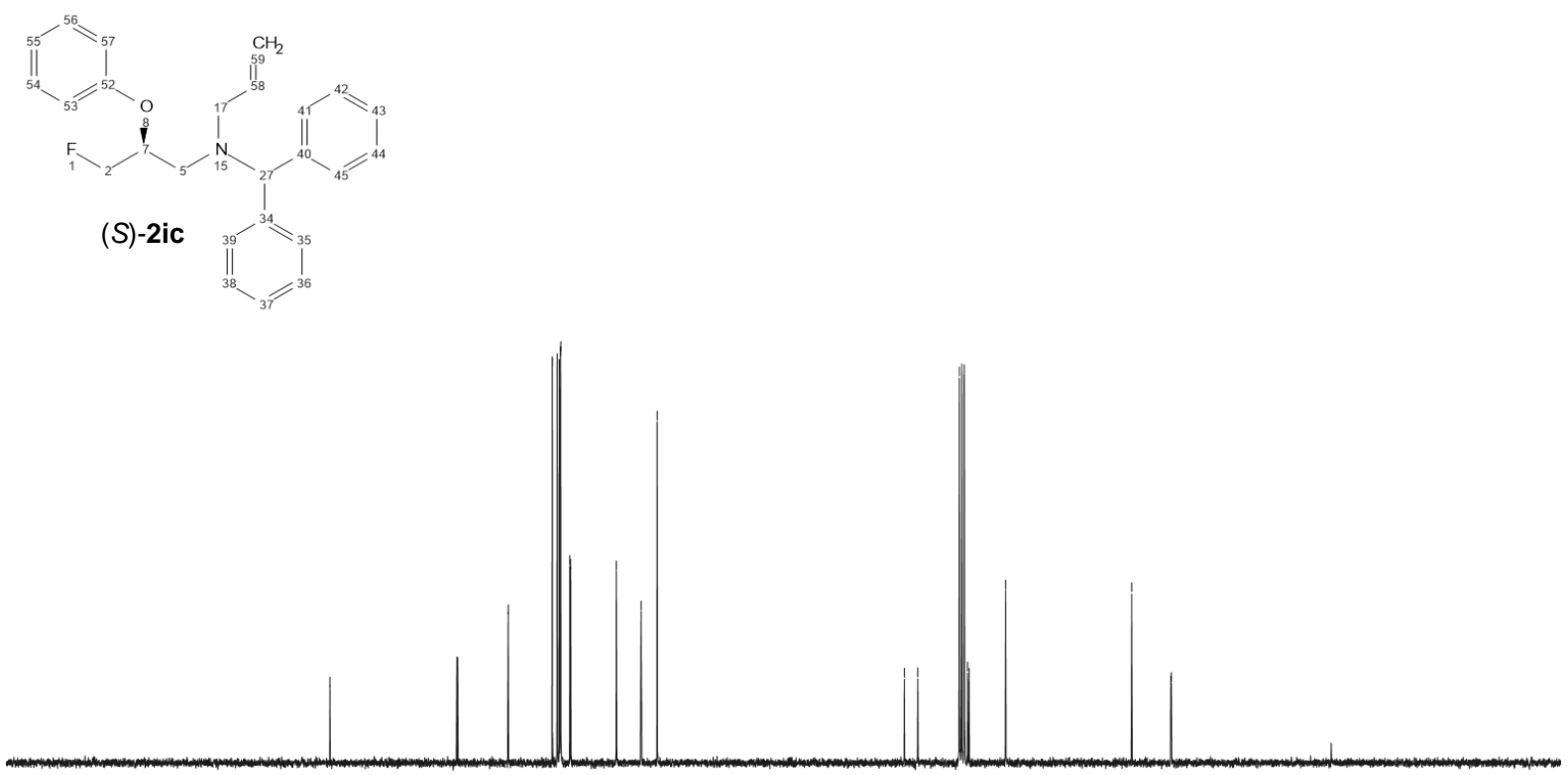

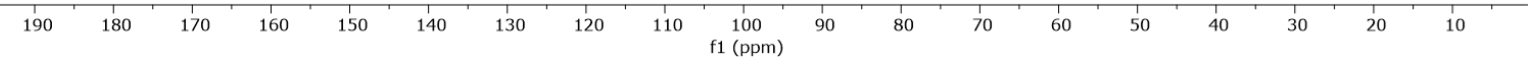


<smiles>C=CN(NP(CF)Oc1nnnnn1)N(c1cncnn1)c1nncnn1</smiles>

\begin{tabular}{rllllllllllllllllllllllllllllllll}
\hline-20 & -30 & -40 & -50 & -60 & -70 & -80 & -90 & -100 & -110 & -120 & -130 & -140 & -150 & -160 & -170 & -180 & -190 & -200 & -210 & -220 & -230 & -240
\end{tabular} 
UOXF_groagna_2ja/CdCl3

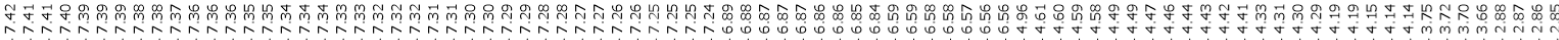

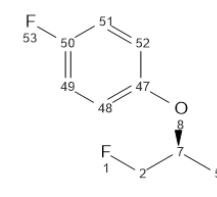

(S)-2ja

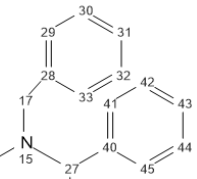<smiles>Cc1nnnn1C1C2CC3CC(C2)C1C3</smiles>

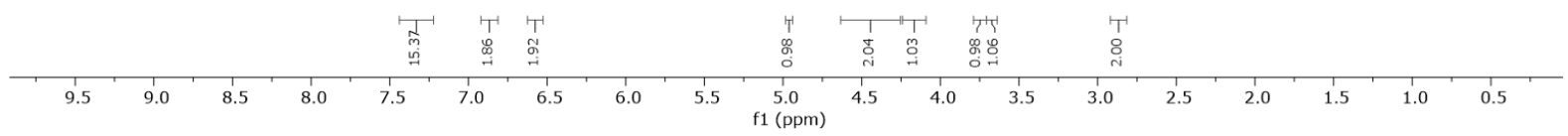

UOXF_groagna_2ja/CdCl3

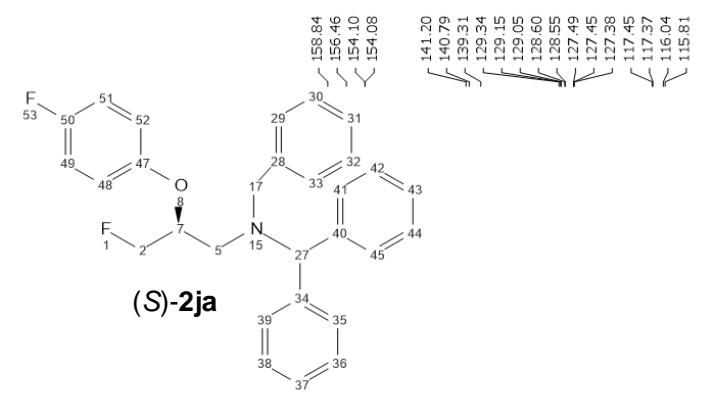

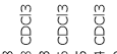

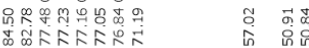

可ARERT की

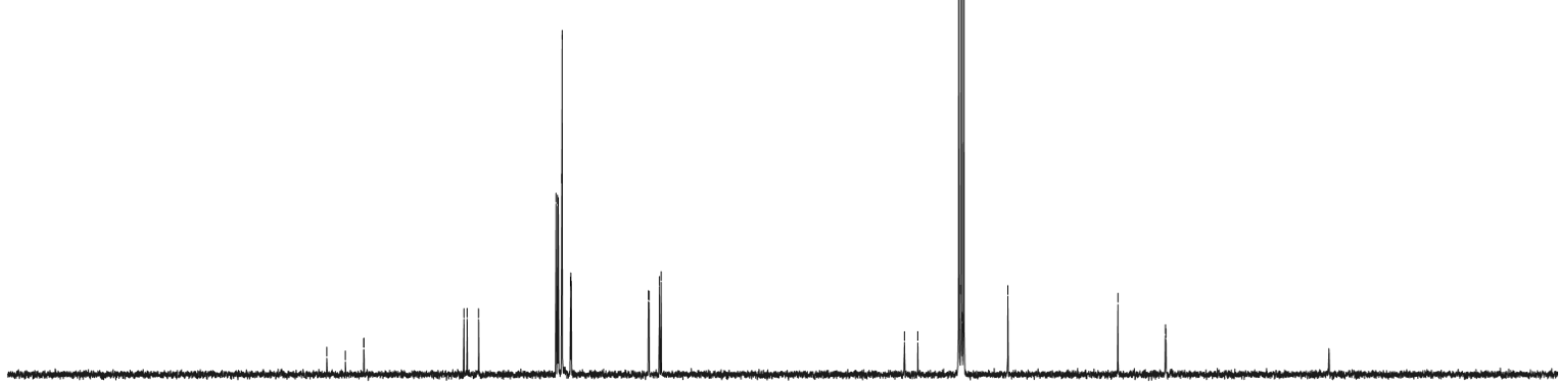

$\begin{array}{lllll}190 & 180 & 170 & 160 & 150\end{array}$ 

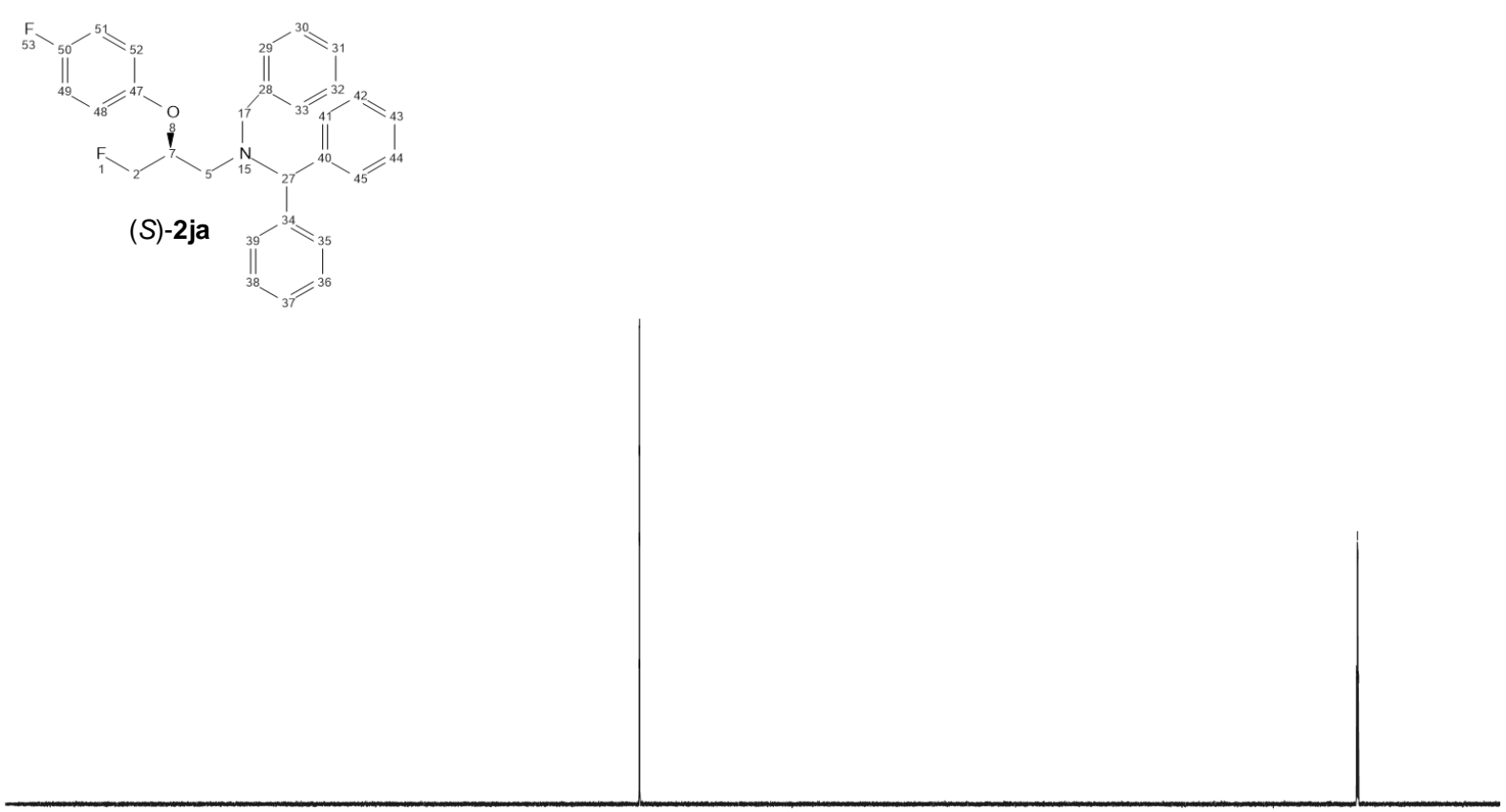

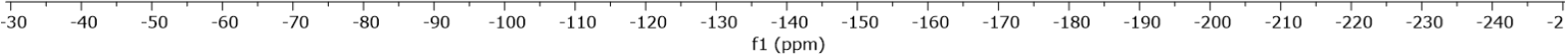




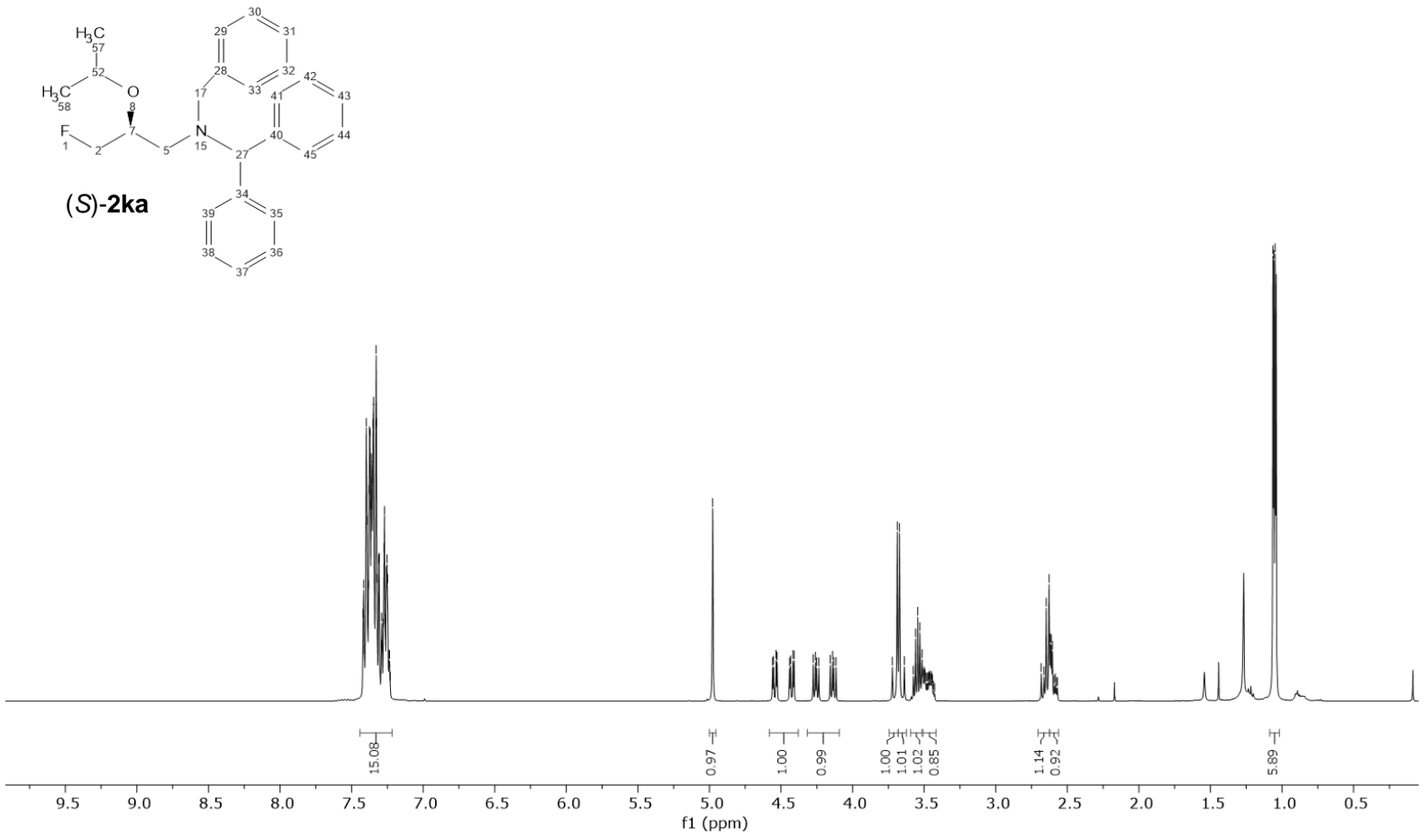

UOXF_groagna_2ka/CdCl3

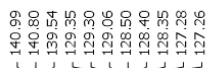

$\frac{m}{0} \frac{m}{\square}$
8
8

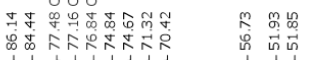

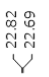

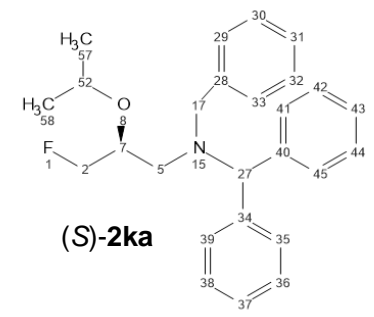

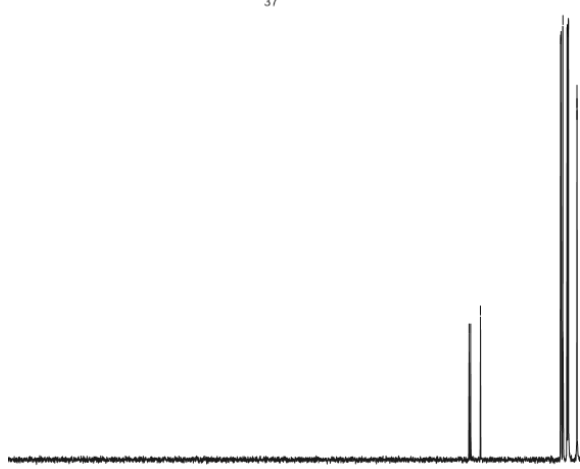

11 W 


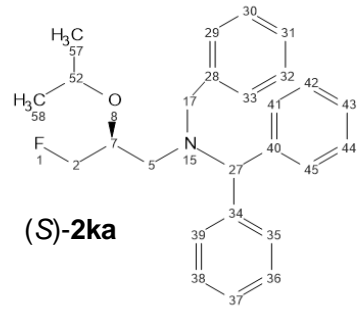

\begin{tabular}{llllllllllllllllllllllllllllllllll}
\hline 30 & -40 & -50 & -60 & -70 & -80 & -90 & -100 & -110 & -120 & -130 & -140 & -150 & -160 & -170 & -180 & -190 & -200 & -210 & -220 & -230 & -240 & -250 & -260 & -2 \\
\hline
\end{tabular} 


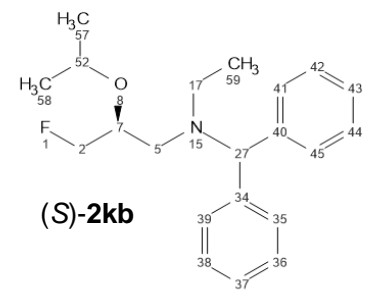
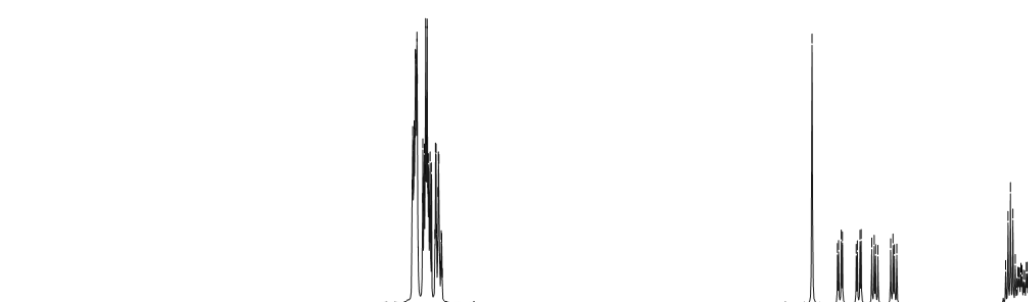

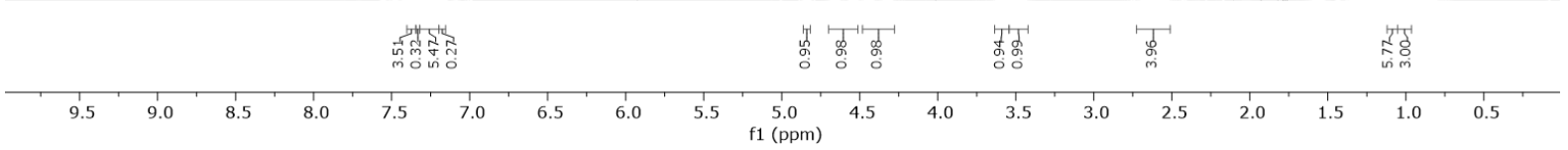

UOXF_groagna_2kb/CdCl3

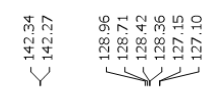

11<smiles>C=[PH](C)OP(CF)SN(CC)C(c1cccnc1)c1ccccn1</smiles>

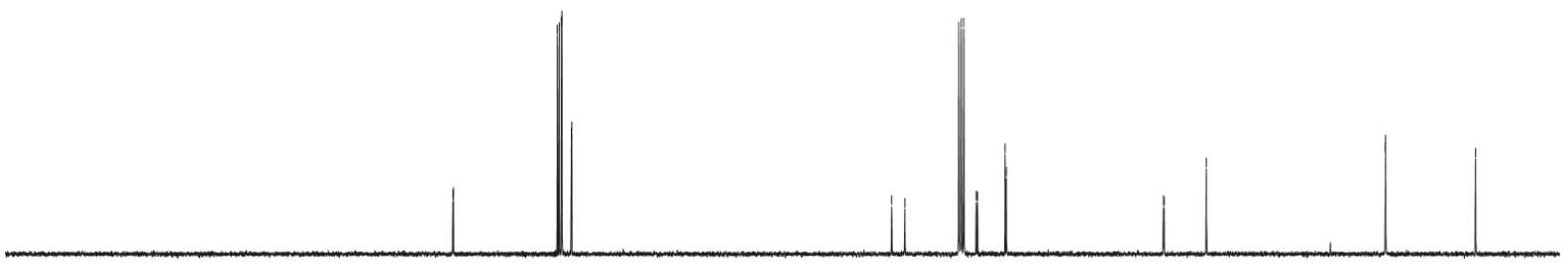

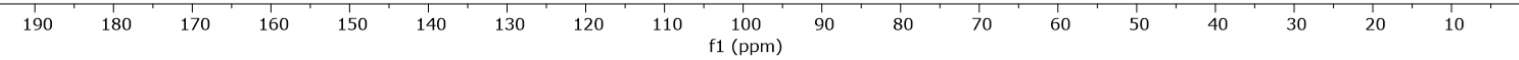




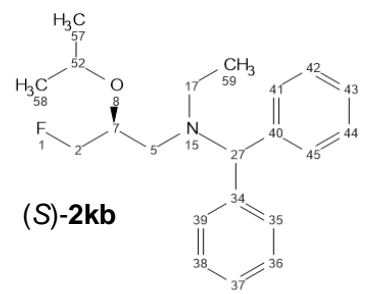

$\begin{array}{llllllllllllllllllllllllllllllllllllllllllll} & 90 & 80 & 70 & 60 & 50 & 40 & 30 & 20 & 10 & 0 & -10 & -20 & -30 & -40 & -50 & -60 & -70 & -80 & -90 & -100 & -110 & -120 & -130 & -140 & -150 & -160 & -170 & -180 & -190 & -200 & -210 & -220 & -230 & -240\end{array}$ 
<smiles>COP(CF)SN(Cc1cncnc1)Nc1cnncn1</smiles>

(S)-2la<smiles>Cc1nnnn1C1CC2CCCCC21</smiles>
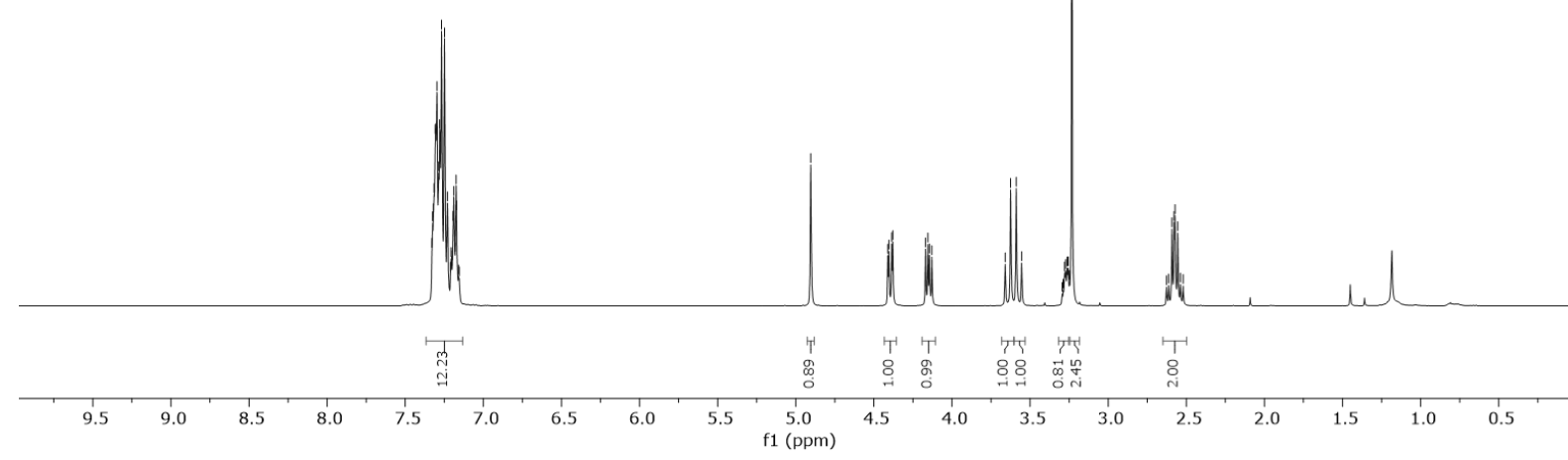
UOXF_groagna_2la/CdCl3<smiles>COP(CF)SN(Cc1ccccn1)C(c1ccccn1)c1ccccn1</smiles>

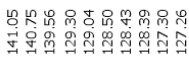
i)

$\mathrm{H}_{3} \mathrm{C}$

S)-2la

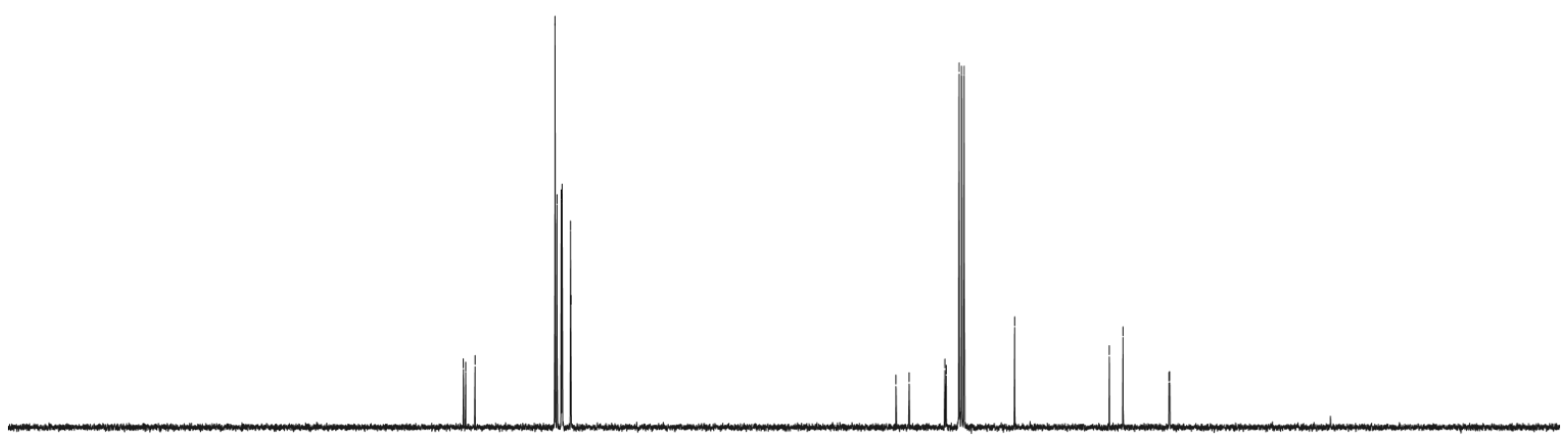

\section{$120 \quad 110$}




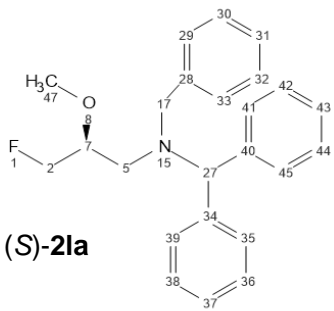

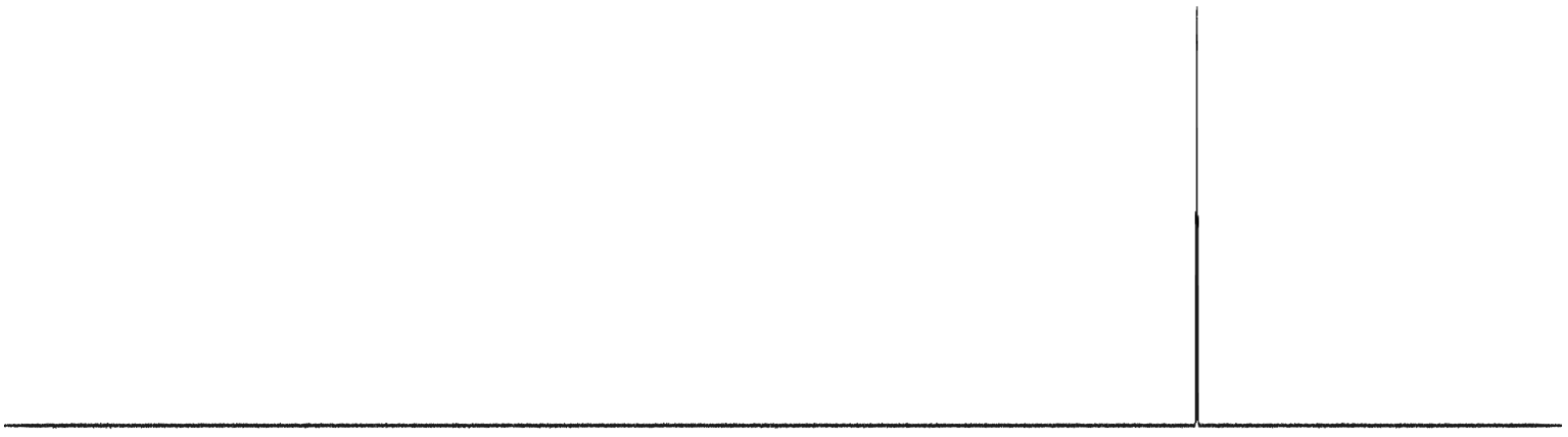

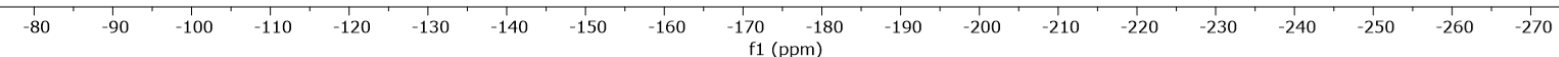


UOXF_groagna_2lb/CdCl3
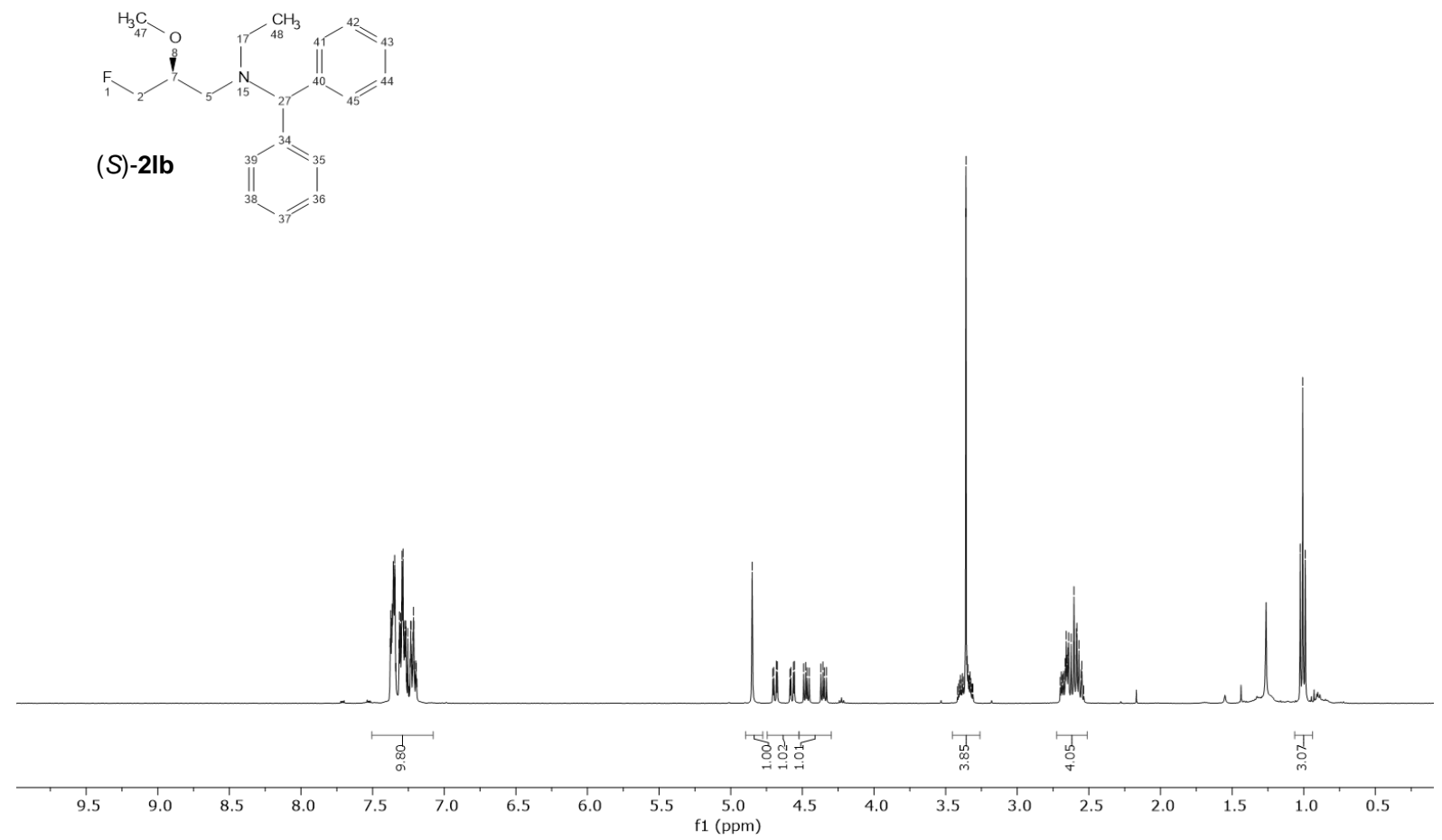

UOXF_groagna_2lb/CdCl3

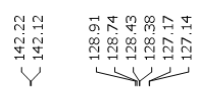

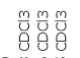

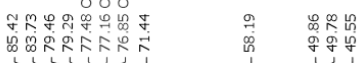

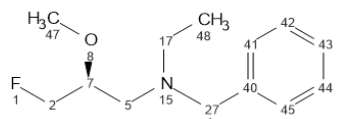

(S)-2lb<smiles></smiles>

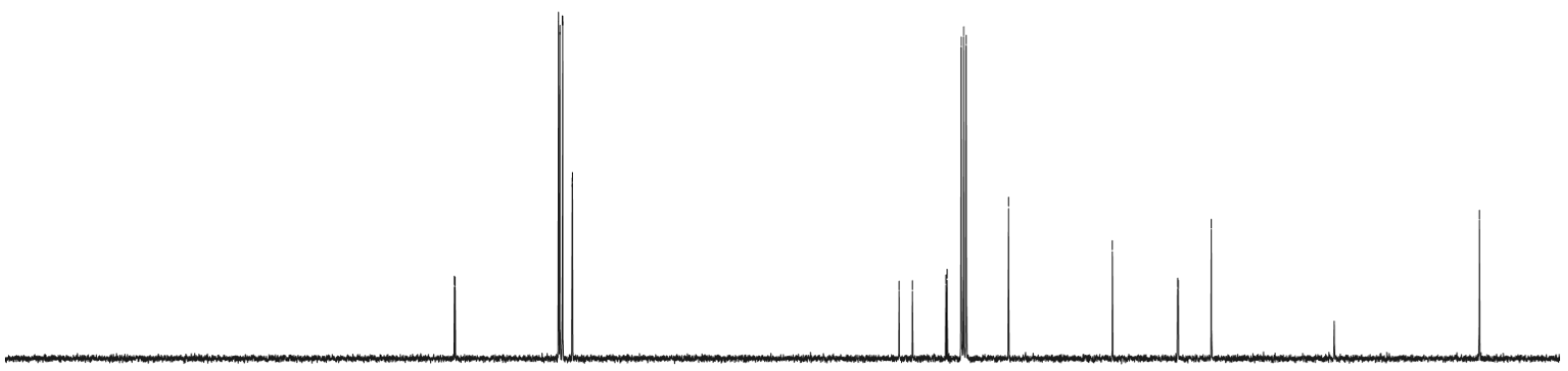

$\begin{array}{rrrrr}190 & 180 & 170 & 160 & 150\end{array}$ 
<smiles>CCN(POC)P(CF)N(CC)c1cccnn1</smiles> 


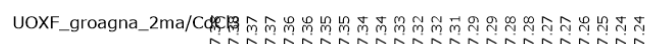

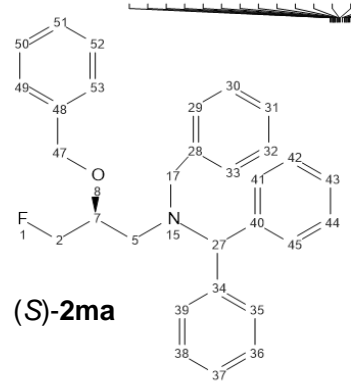

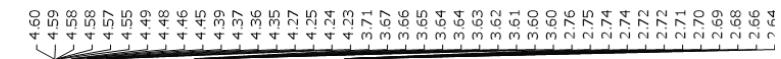

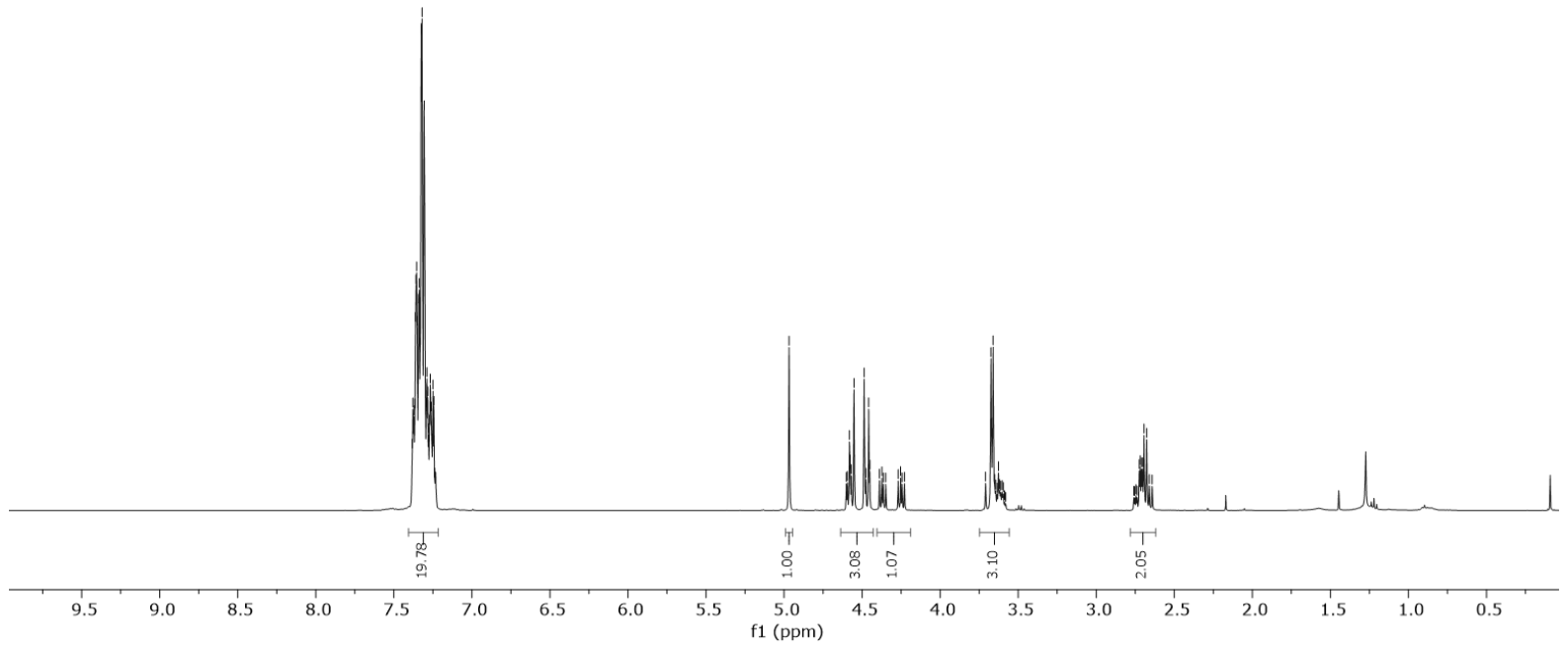

UOXF_groagna_2ma/CdCl3

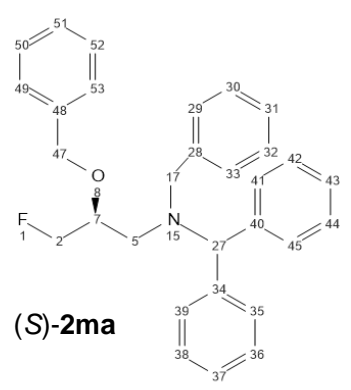

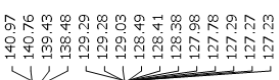

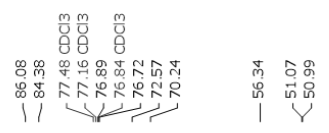

S312 

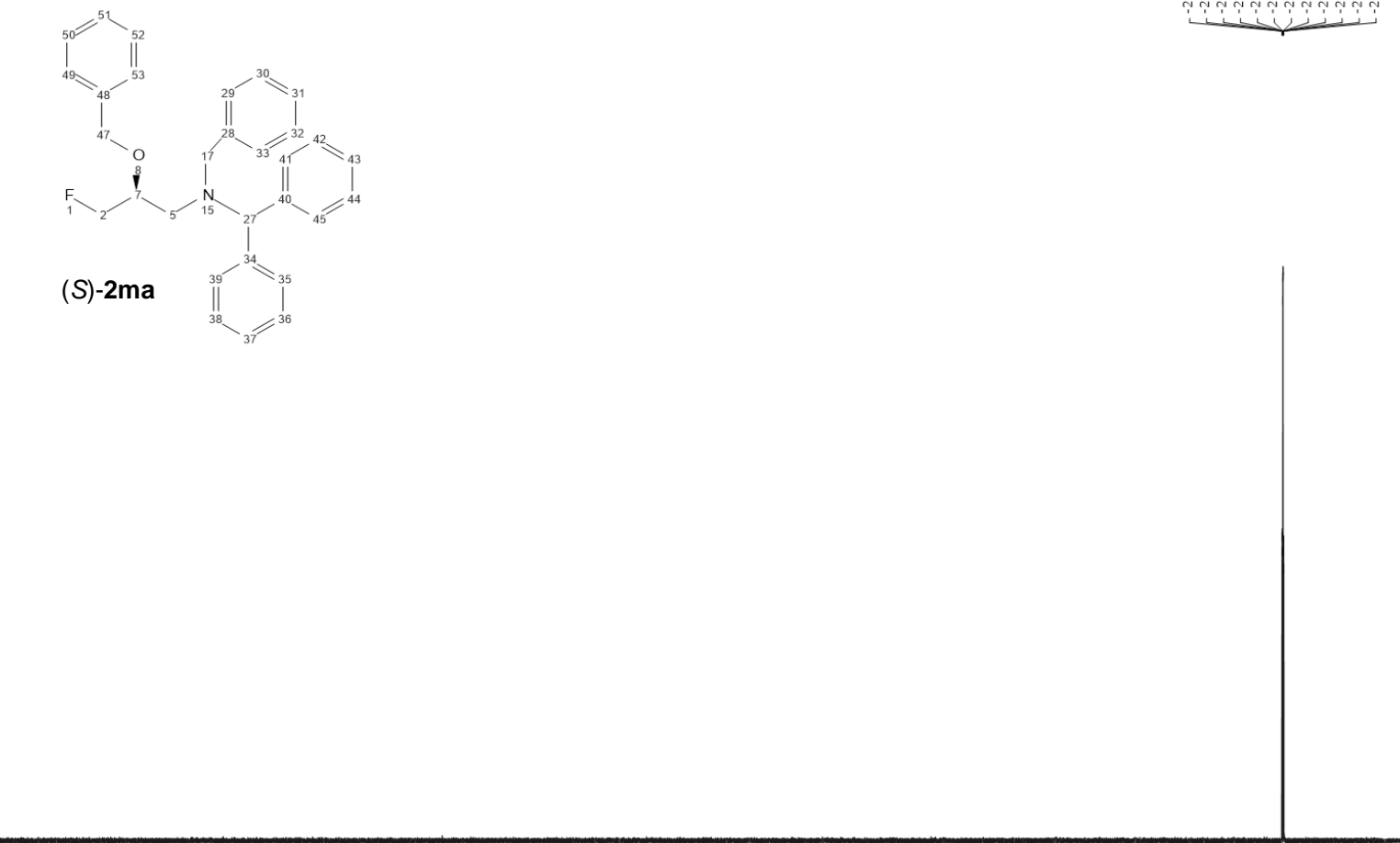

\begin{tabular}{|l|l|l|l|lllllllllllllllllllllll}
\hline & -20 & -30 & -40 & -50 & -60 & -70 & -80 & -90 & -100 & -110 & -120 & -130 & -140 & -150 & -160 & -170 & -180 & -190 & -200 & -210 & -220 & -230 & -240
\end{tabular} 
UOXF_groagna_2lb/CdCl3

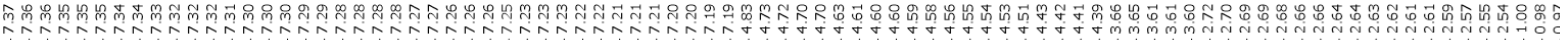

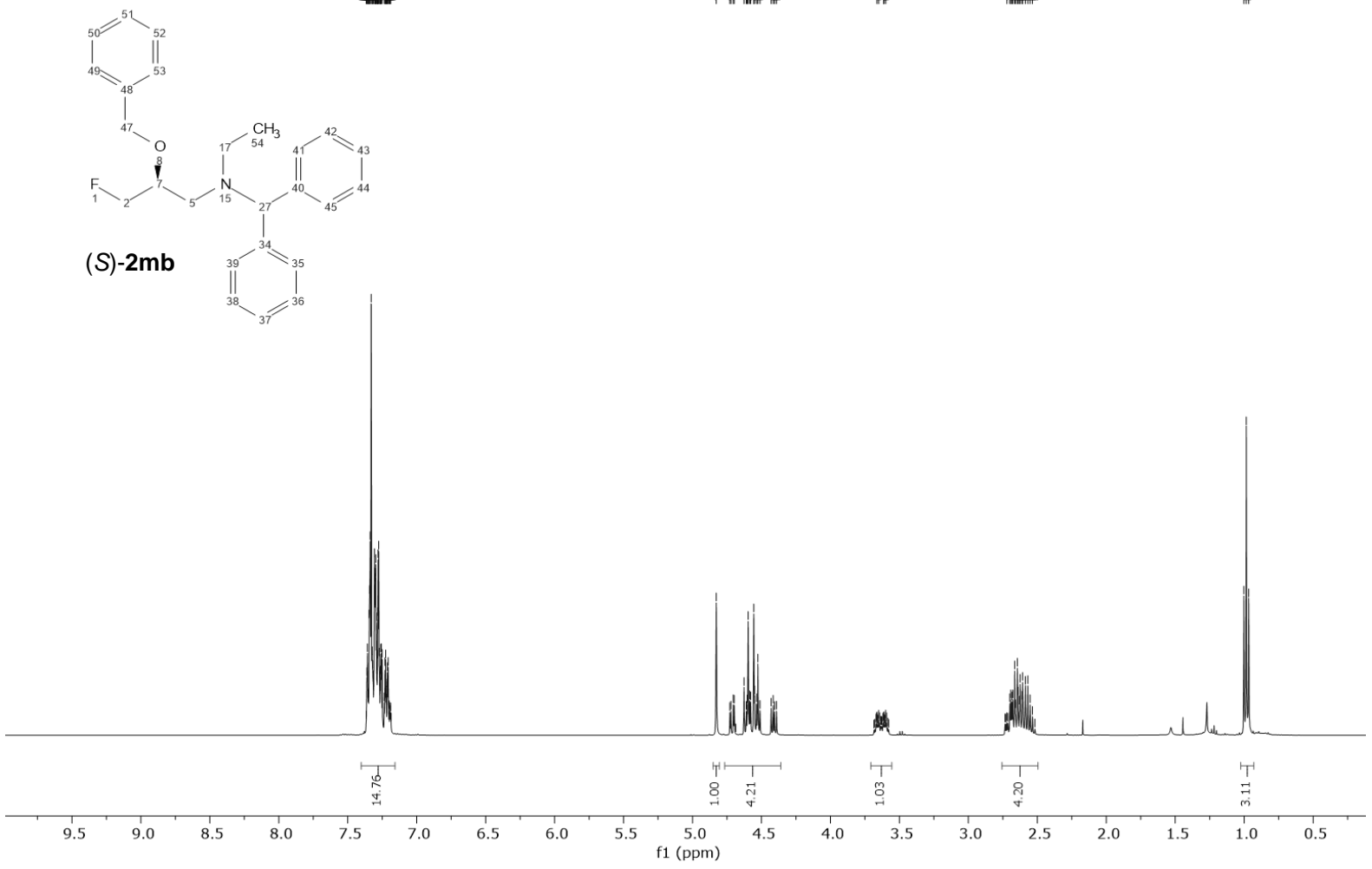

UOXF_groagna_2lb/CdCl3

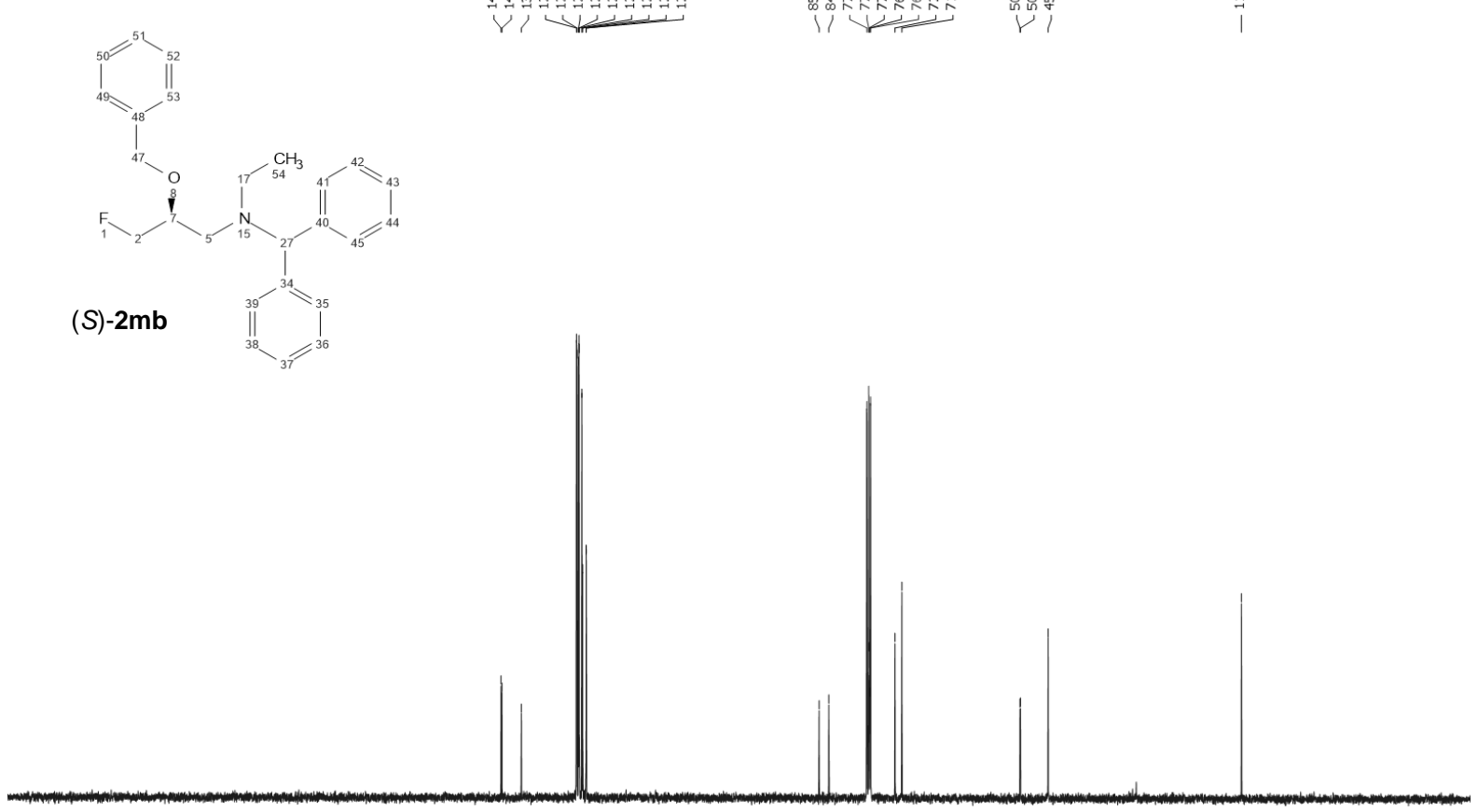

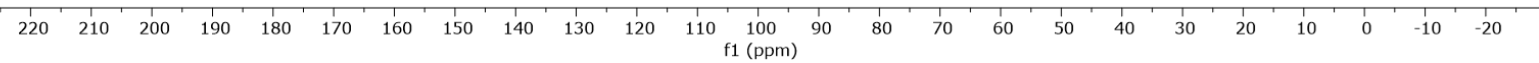



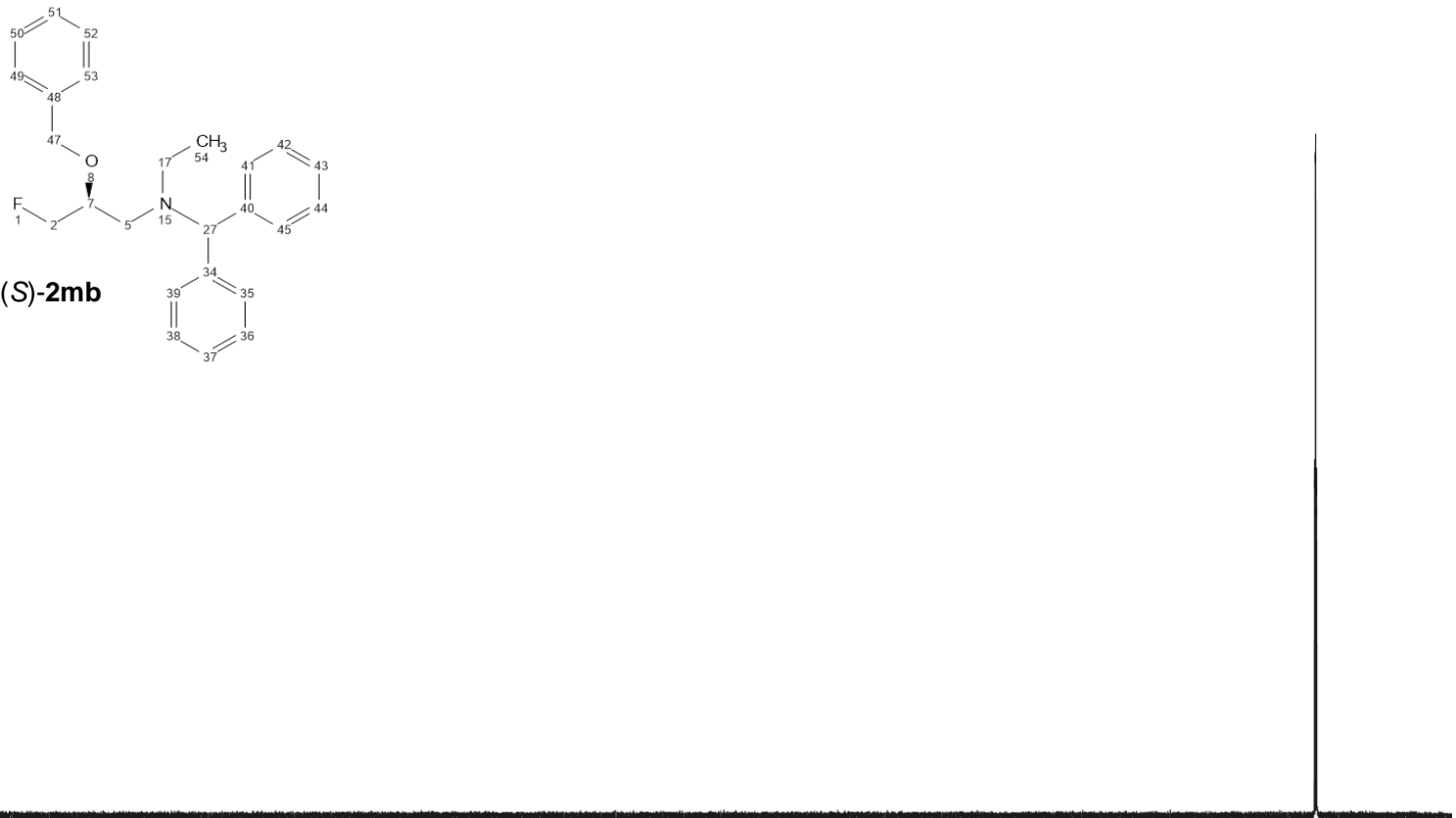

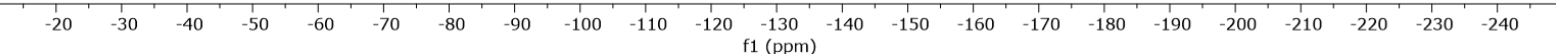



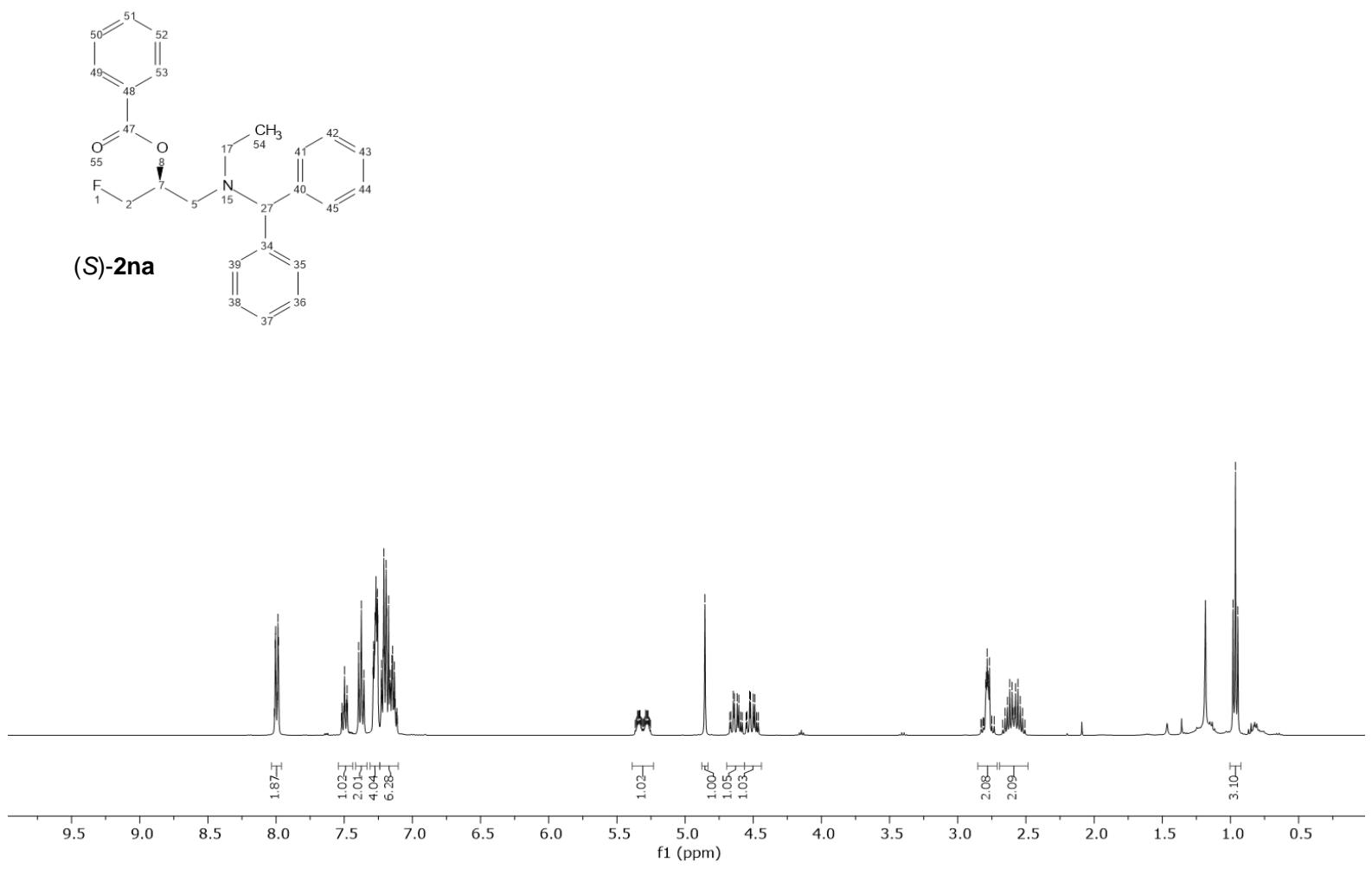

UOXF_groagna_2na/CdCl3

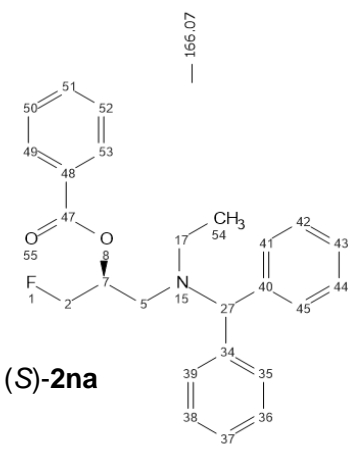

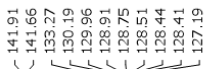

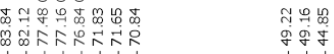

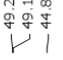

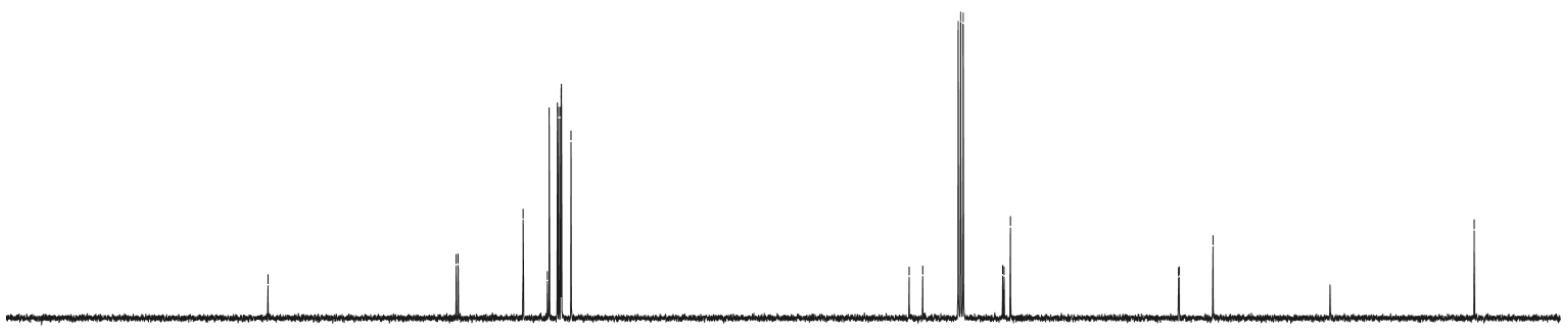

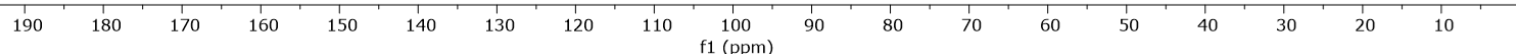




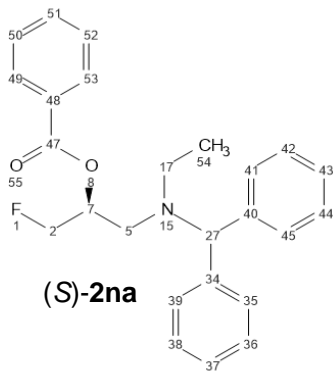

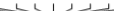

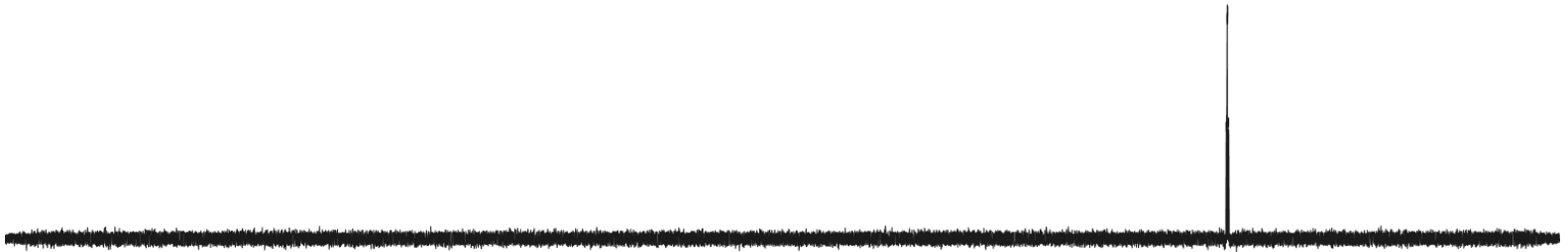

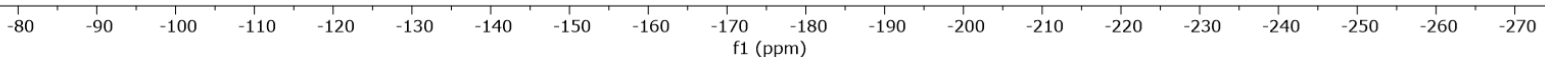




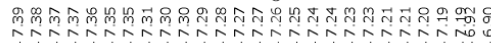

$\underset{17}{B r}$

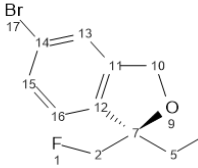

(S)-2oa<smiles>c1cnc2c(c1)NCNC2</smiles><smiles></smiles>

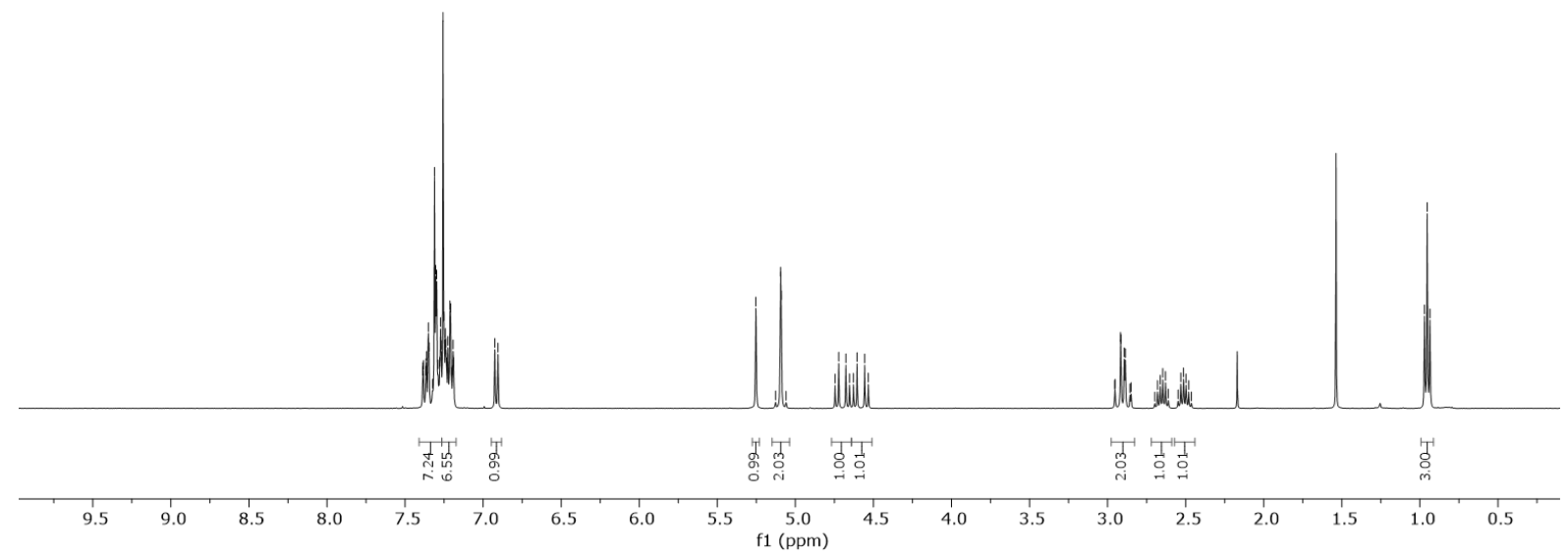

UOXF_groagna_2oa/CdCl3

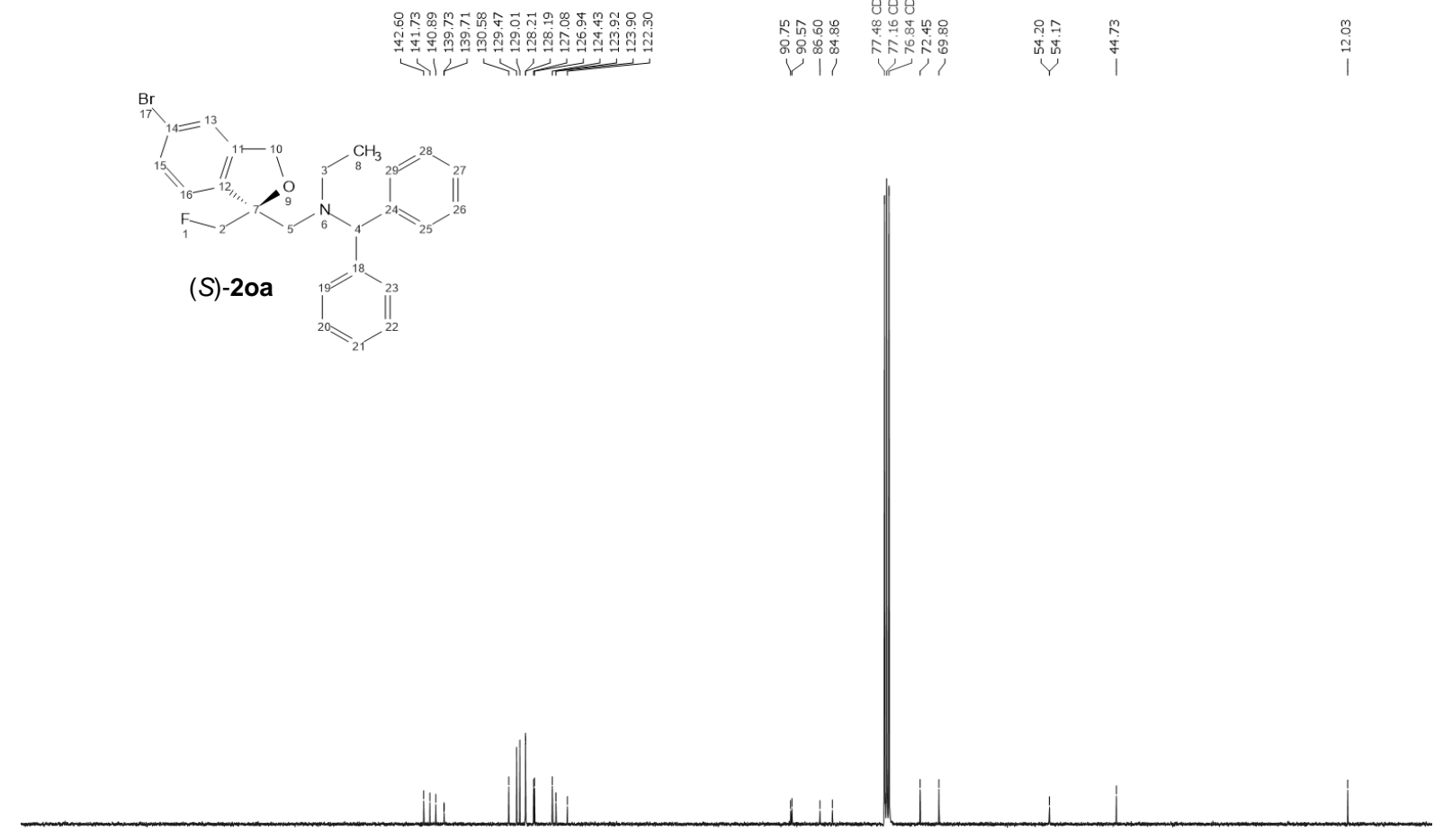

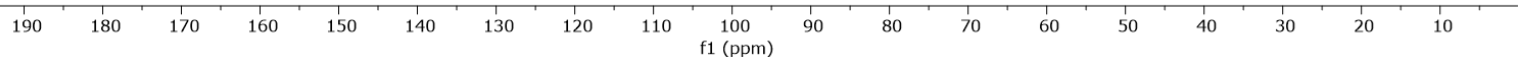




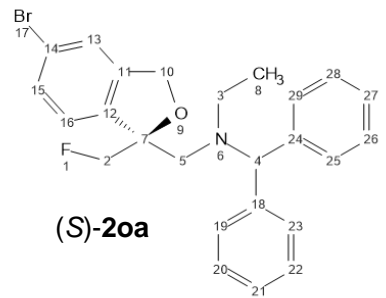

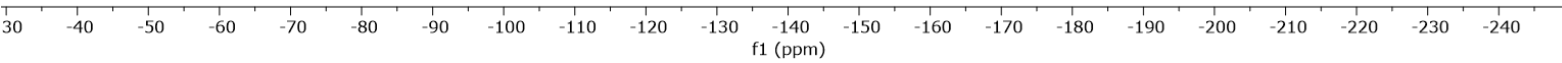



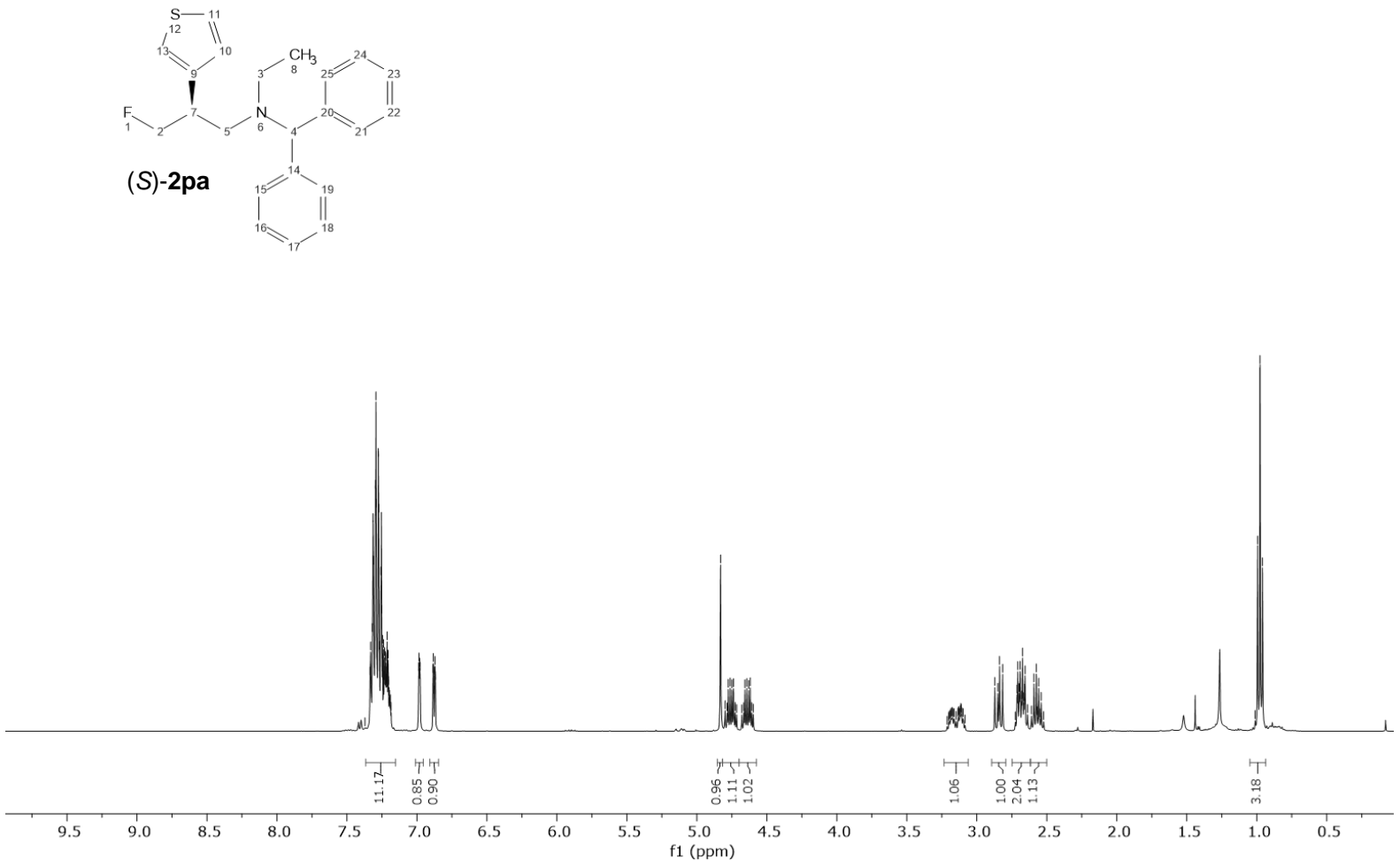

UOXF_groagna_2pa/CdCl3

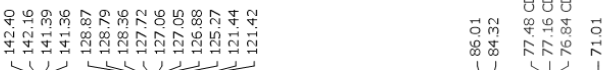
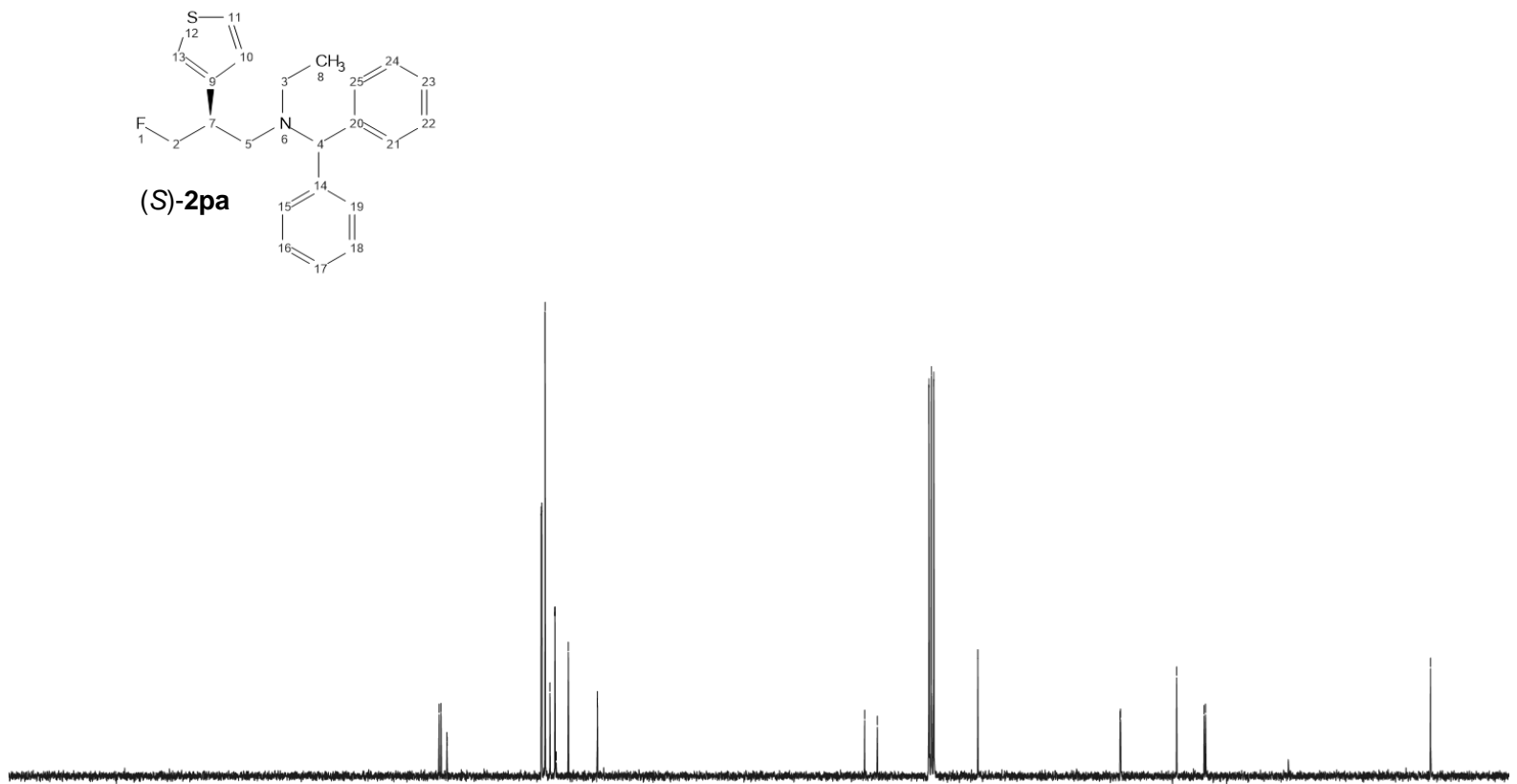


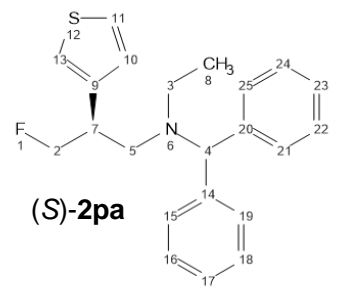

\begin{tabular}{lllllllllllllllllllllllllllll}
\hline-20 & -30 & -40 & -50 & -60 & -70 & -80 & -90 & -100 & -110 & -120 & -130 & -140 & -150 & -160 & -170 & -180 & -190 & -200 & -210 & -220 & -230 & -240
\end{tabular} 


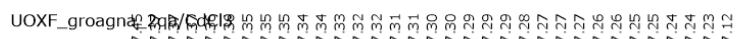

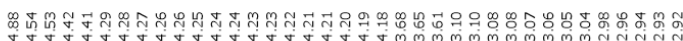
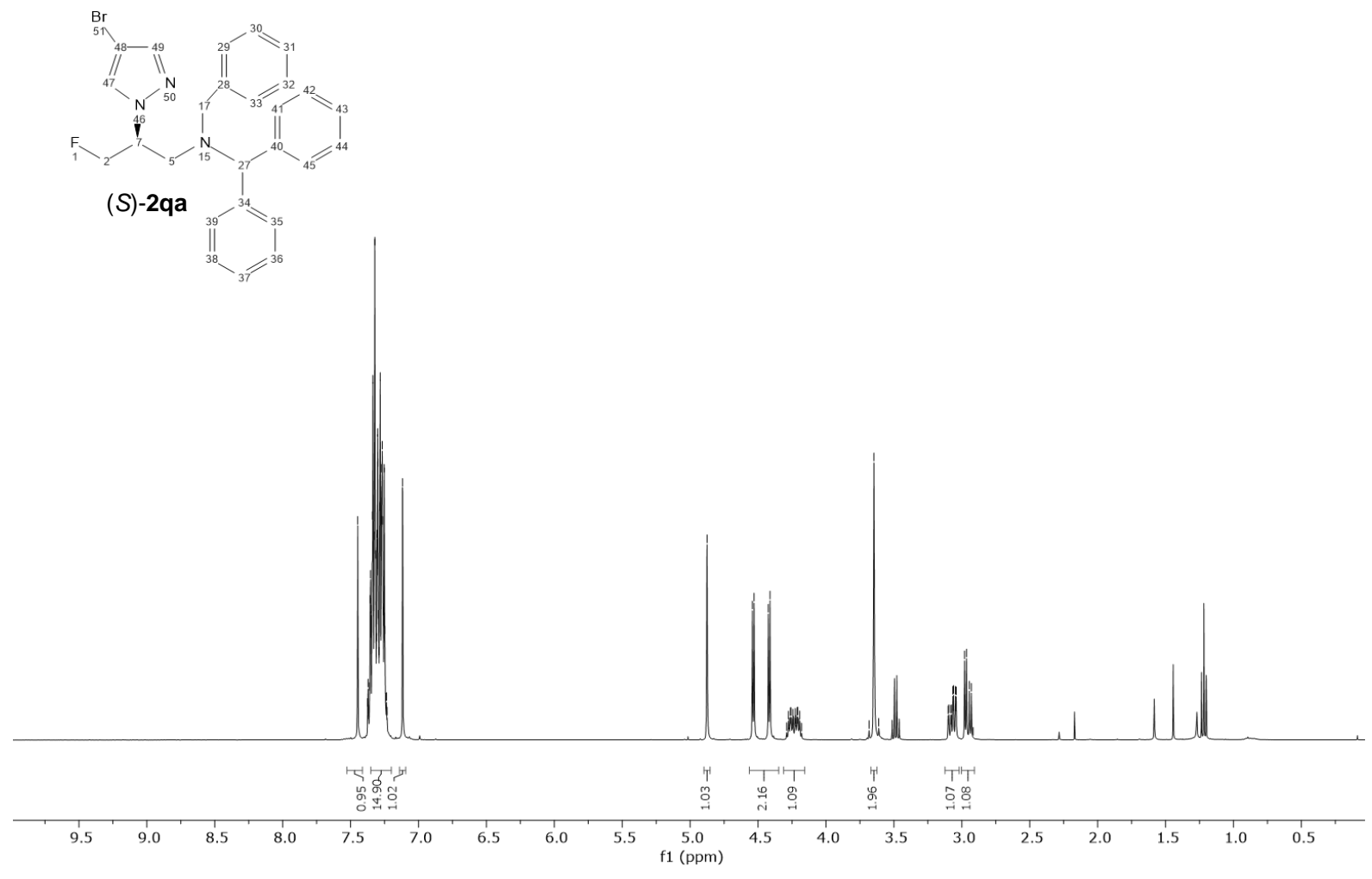

UOXF_groagna_2qa/CdCl3

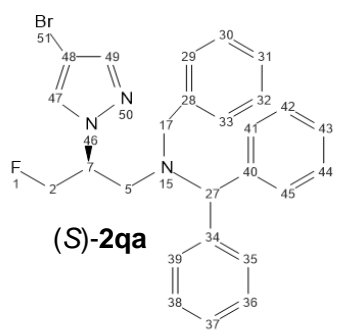

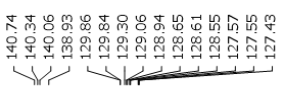
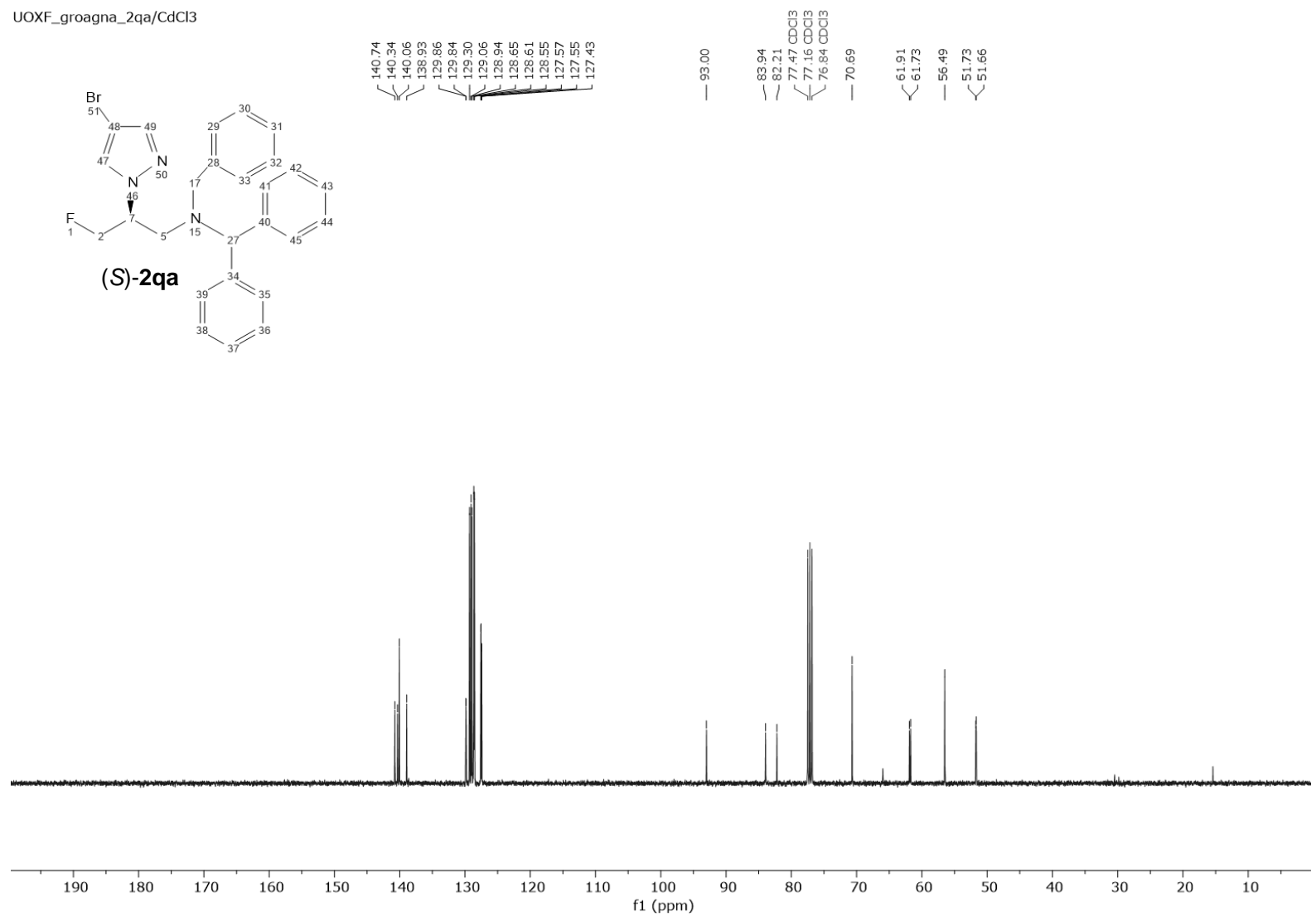

S322 

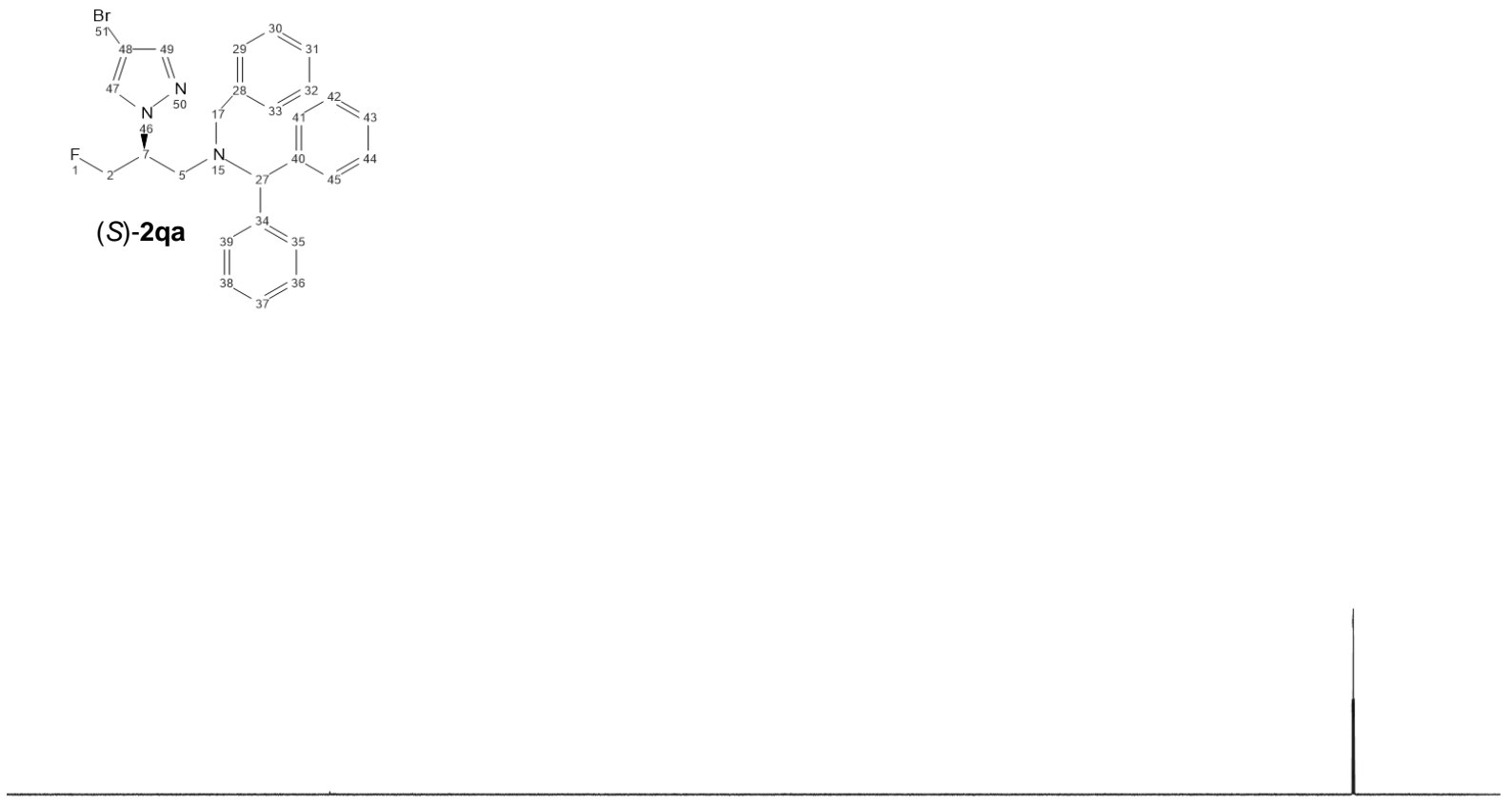

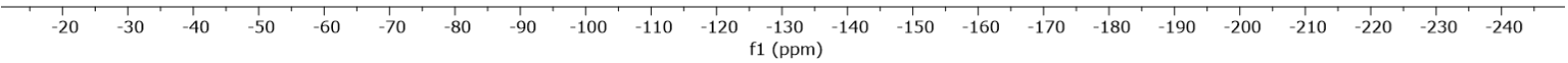




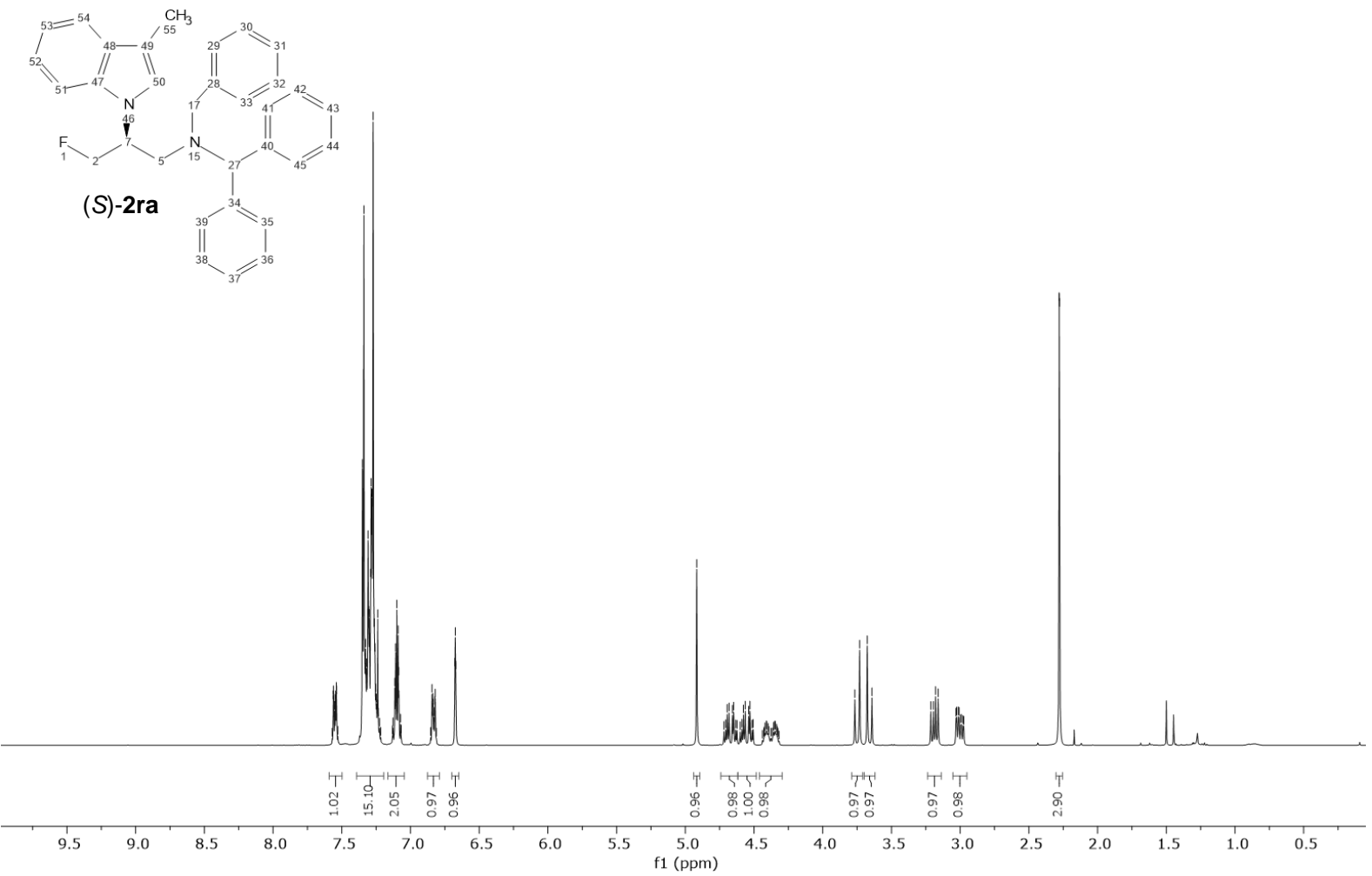

UOXF_groagna_2ra/CdCl3
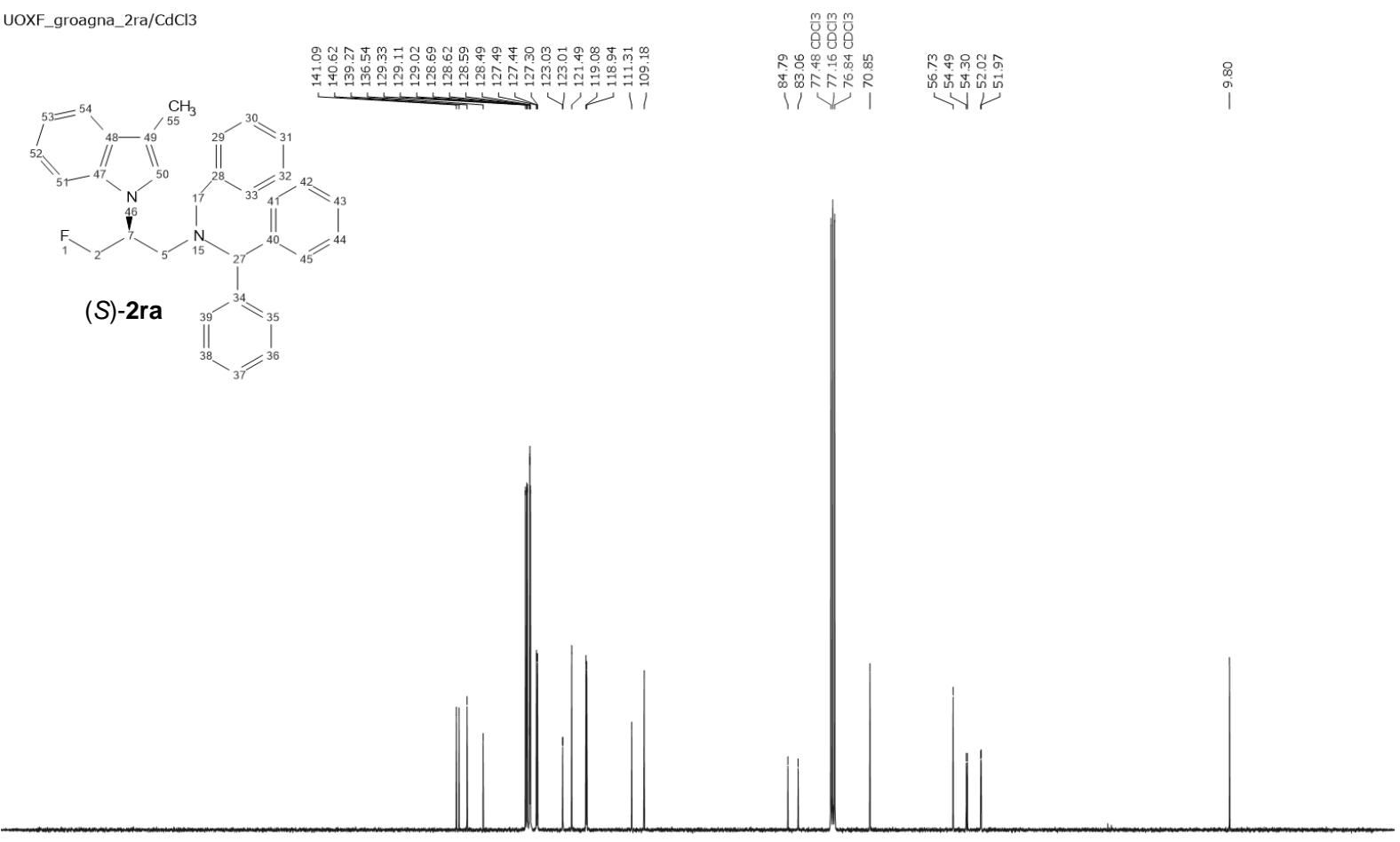

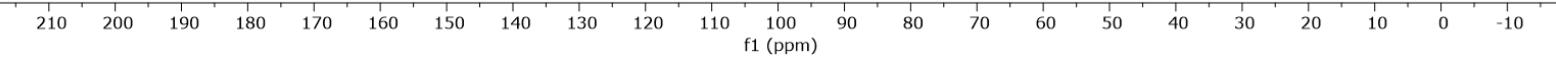



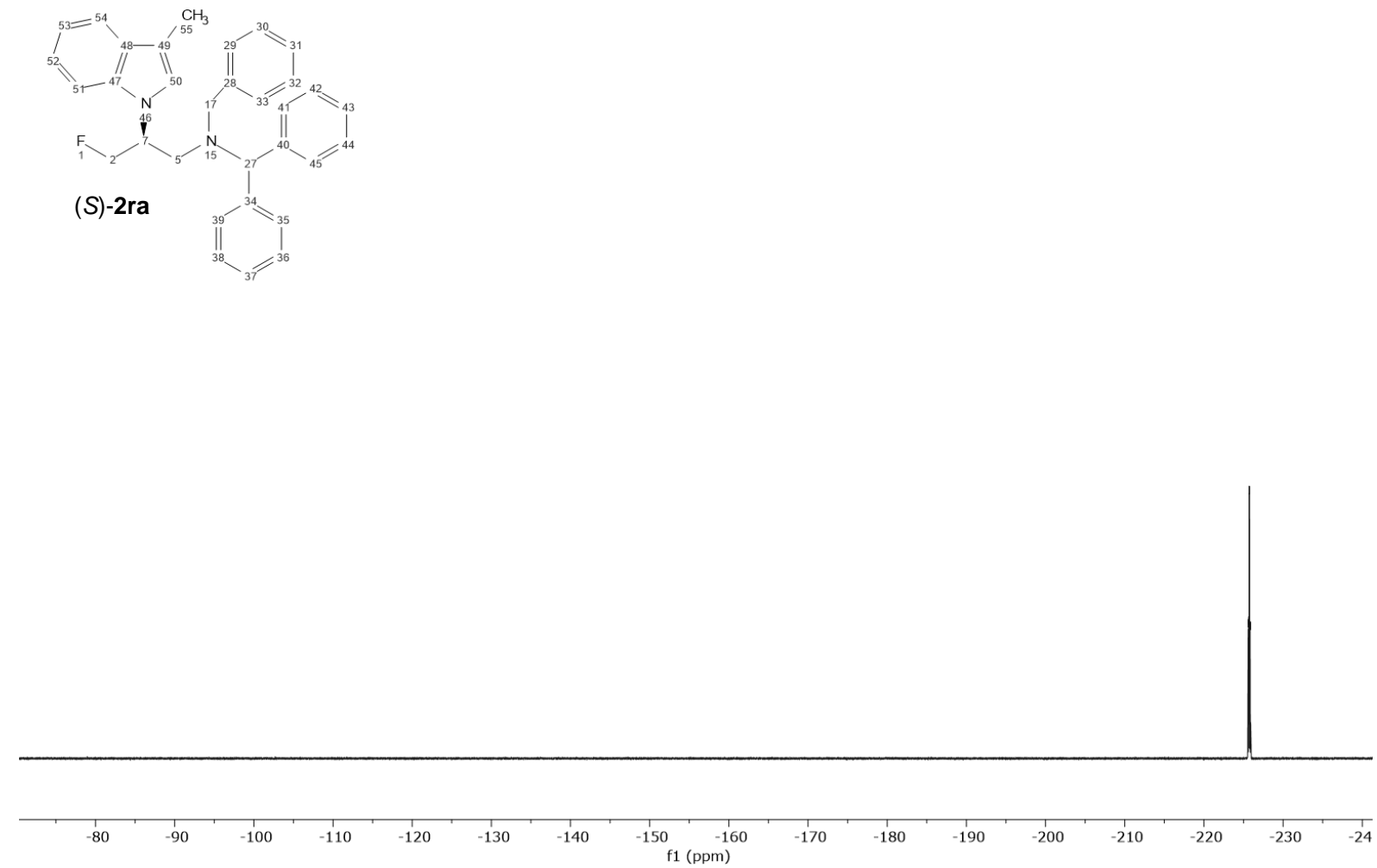
UOXF_groagna_2sa/CdCl3

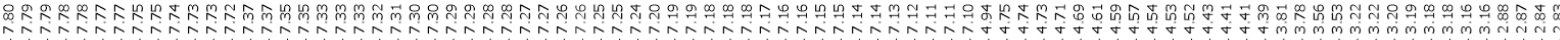

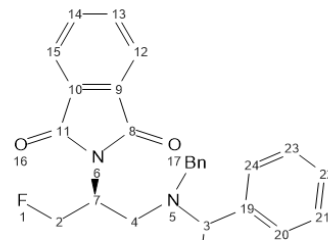

(S)-2sa<smiles>c1ncnpn1</smiles>

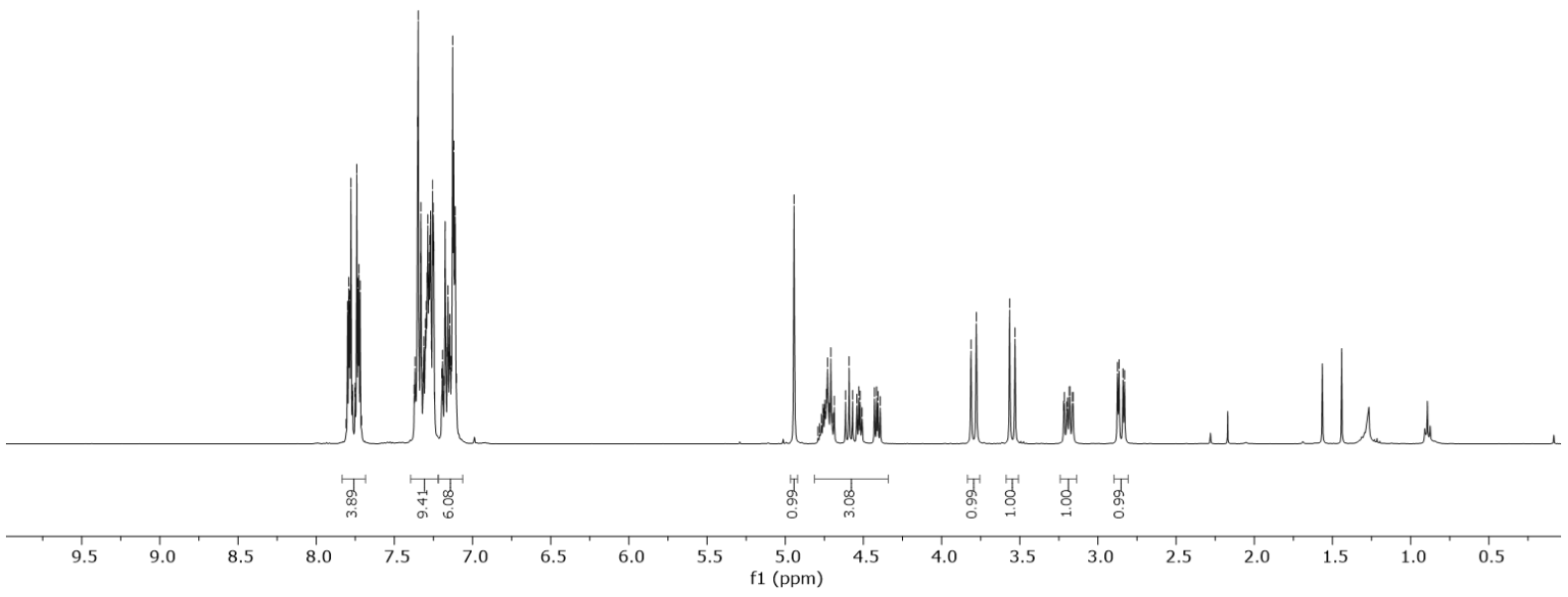

UOXF_groagna_2sa/CdCl3

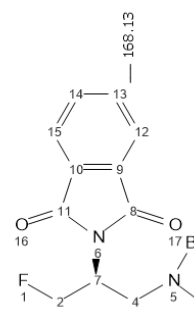

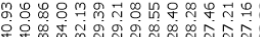

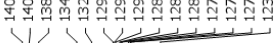

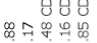

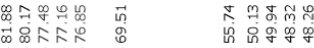

\1, 11110

(S)-2sa

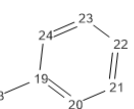

$\prod_{27}^{26} \|^{25} \backslash 10$

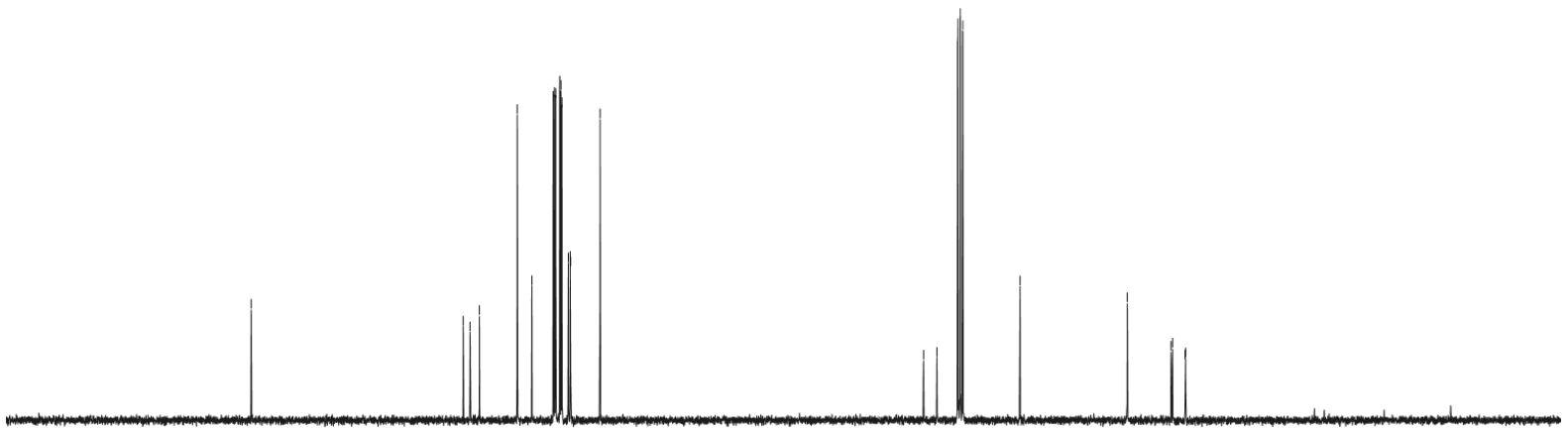

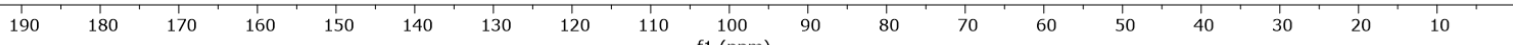



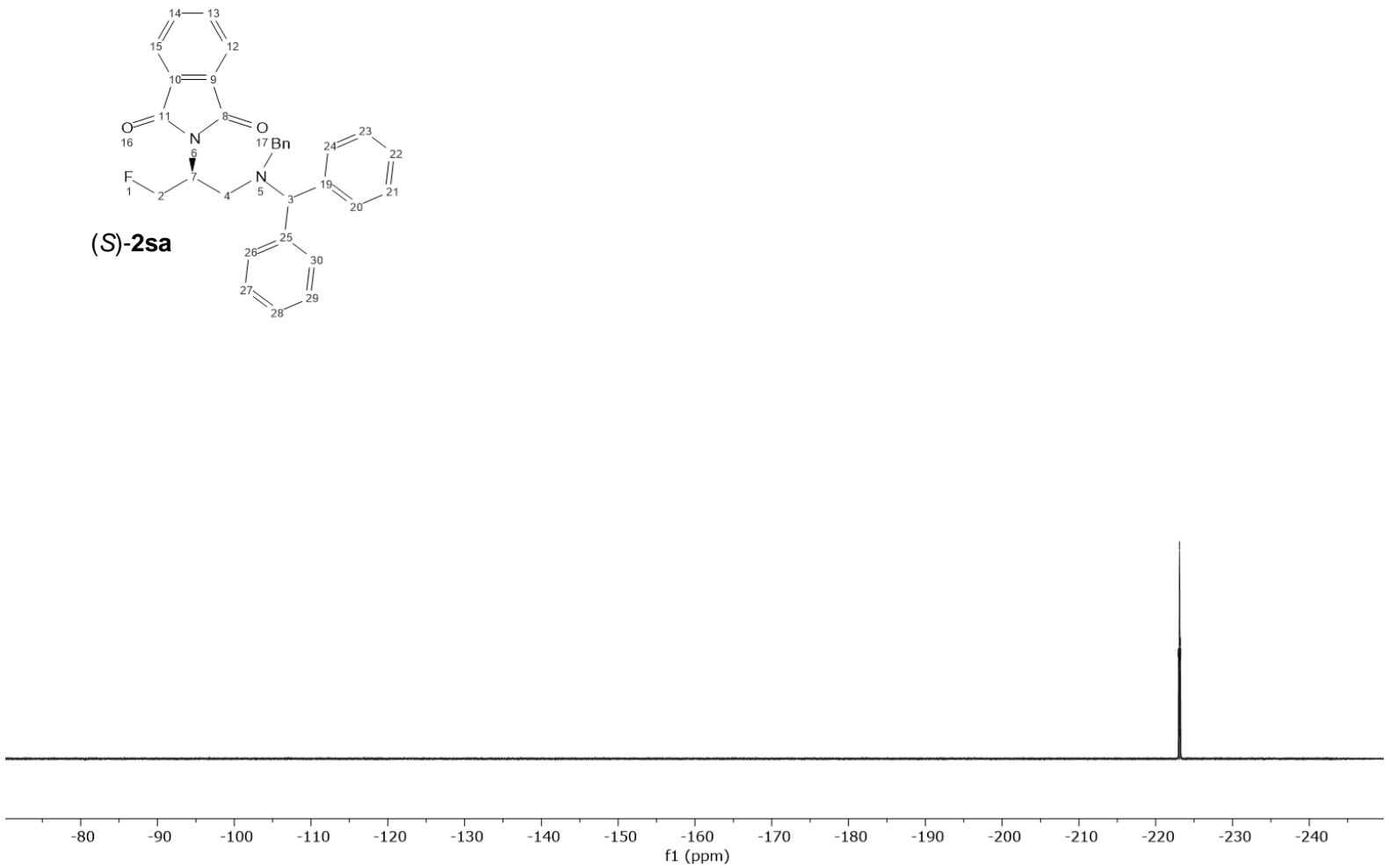


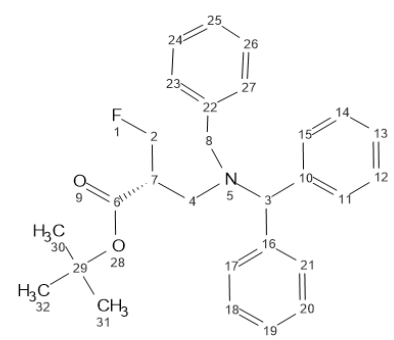

$(R)-2$ ta

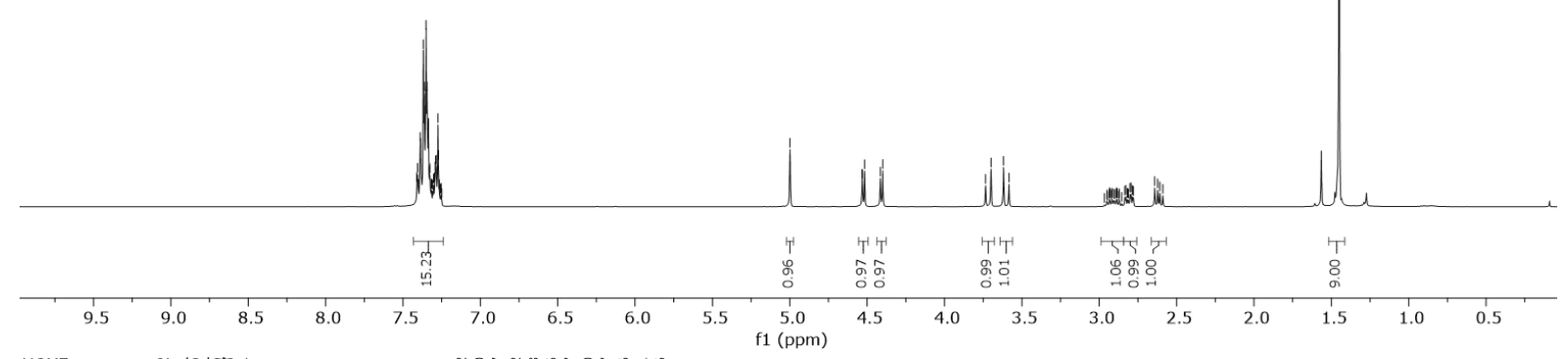
UOXF_groagna_ta/CdGB-

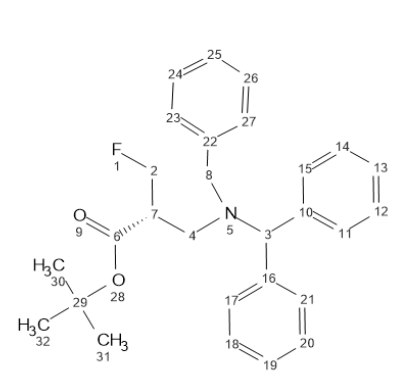

(R)-2ta

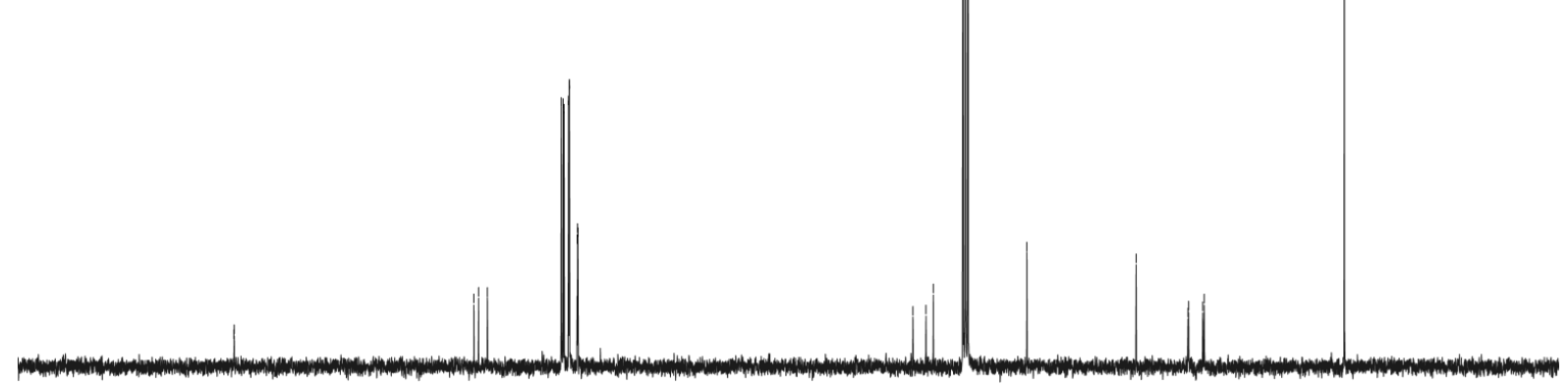

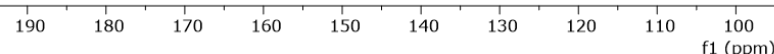
$90 \quad 80$ $70 \quad 60$ $50 \quad 40$ $30 \quad 20 \quad 10$ 


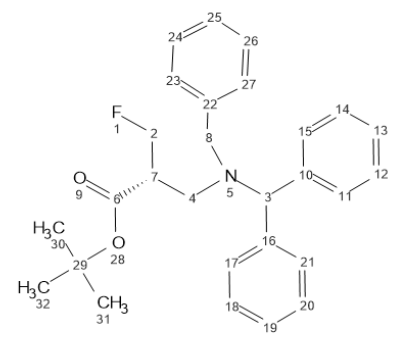

(R)-2ta

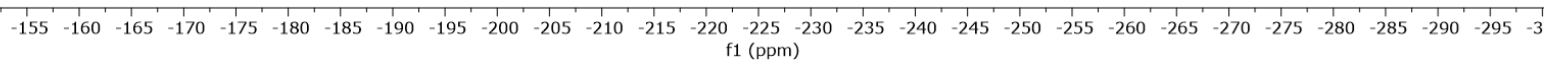


UOXF_groagna_2ua/CdCl3

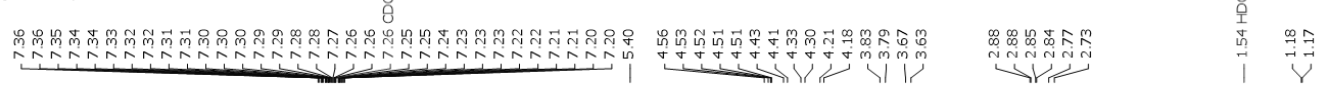
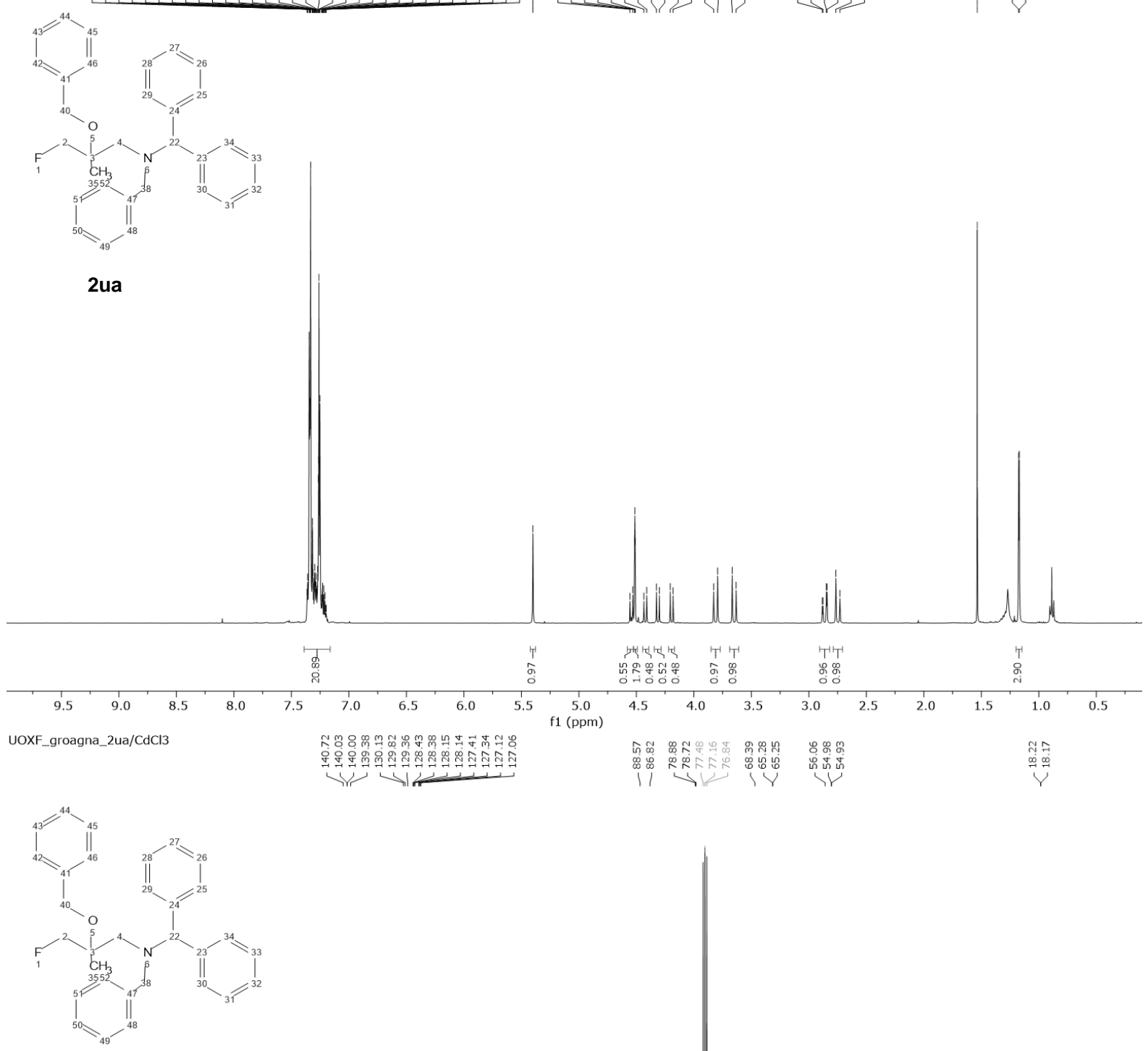

2ua

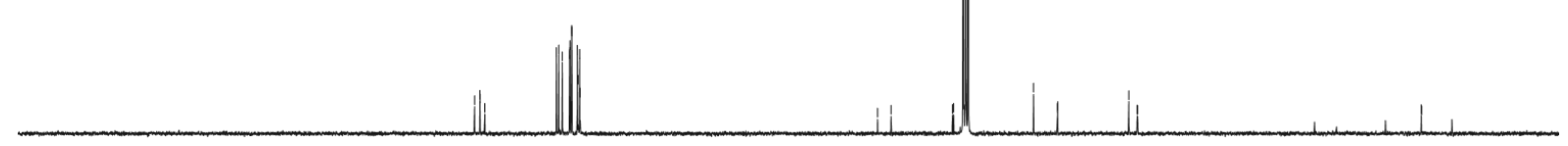

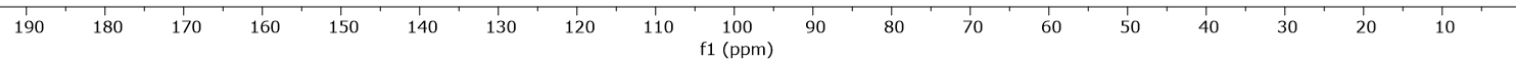




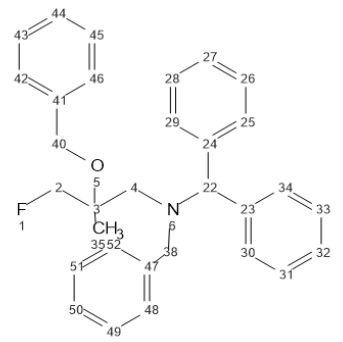

2ua

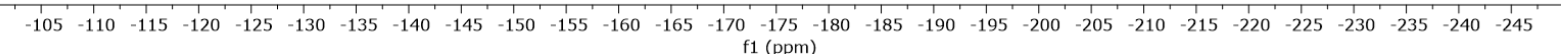




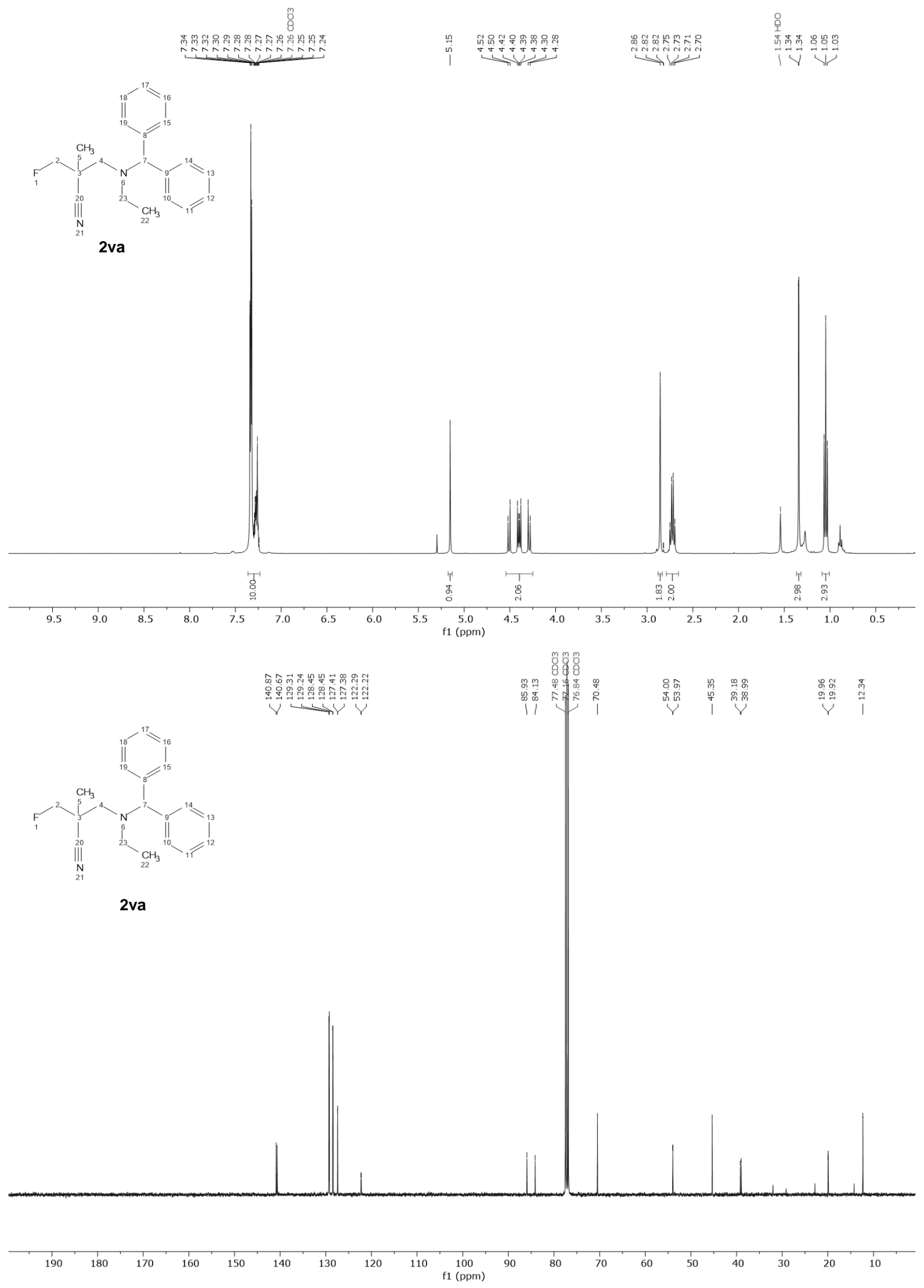

S332 


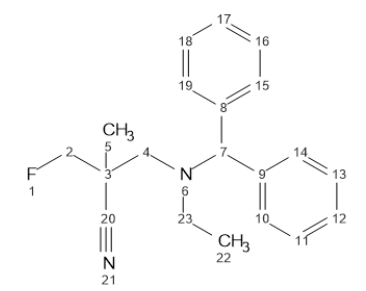

2va

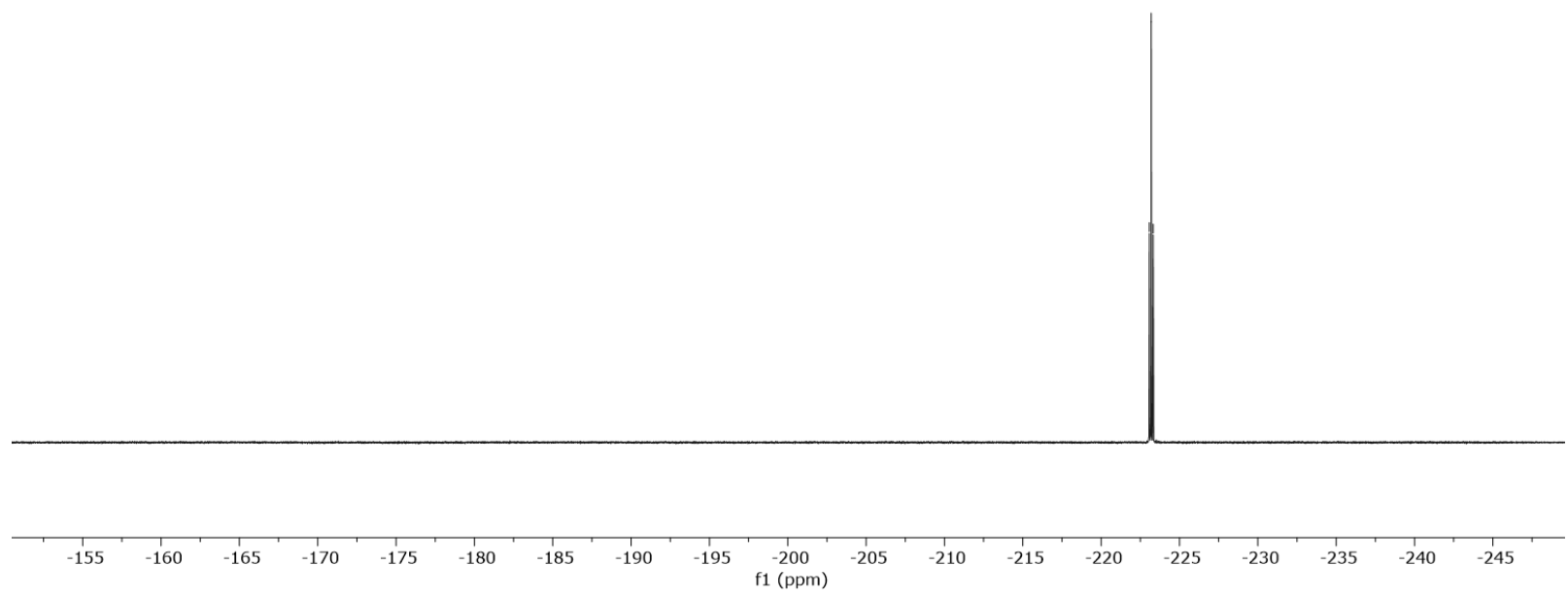




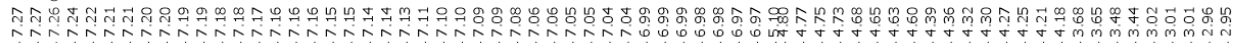

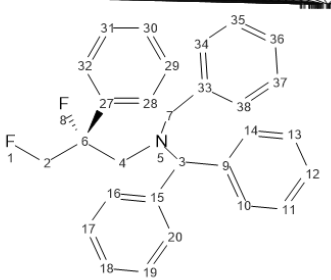

2wa

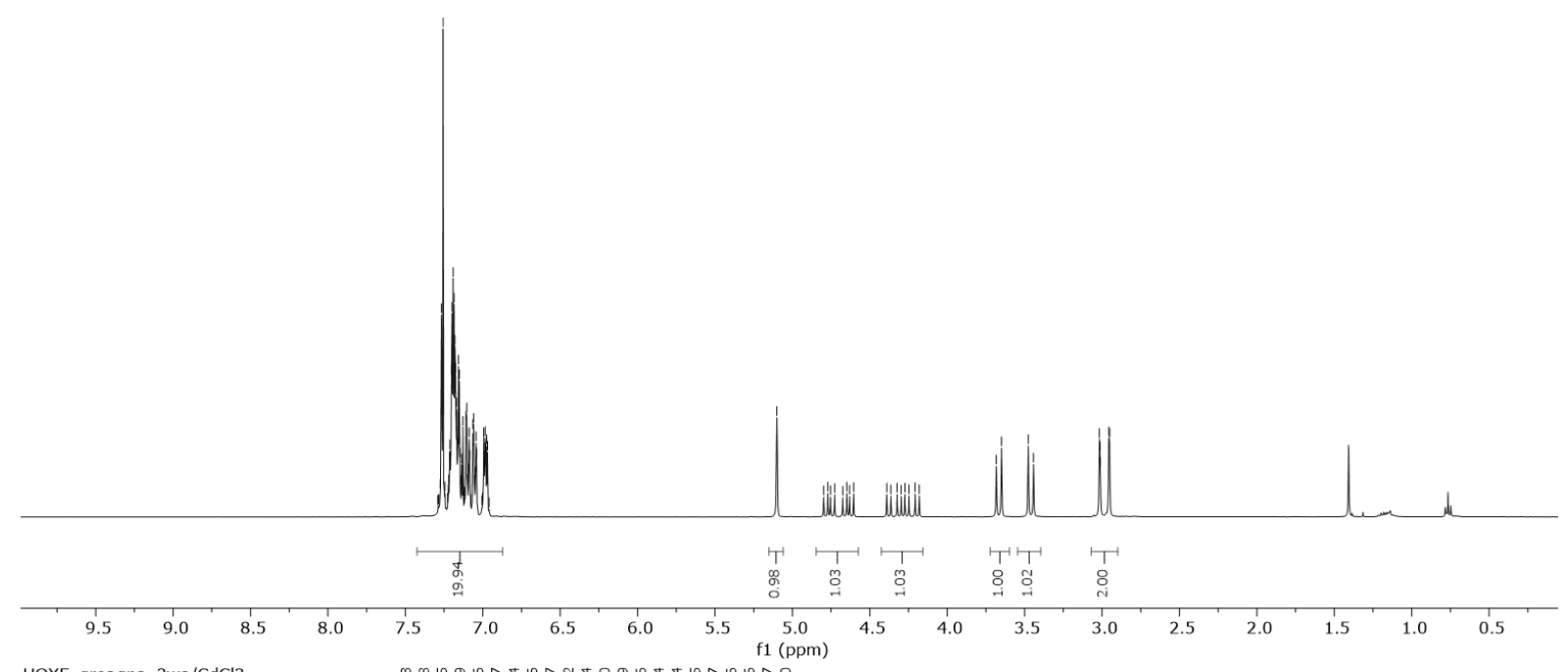

UOXF_groagna_2wa/CdCl3

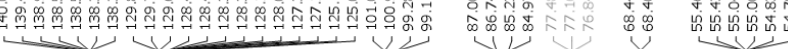

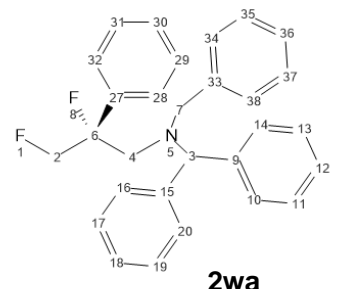

2wa

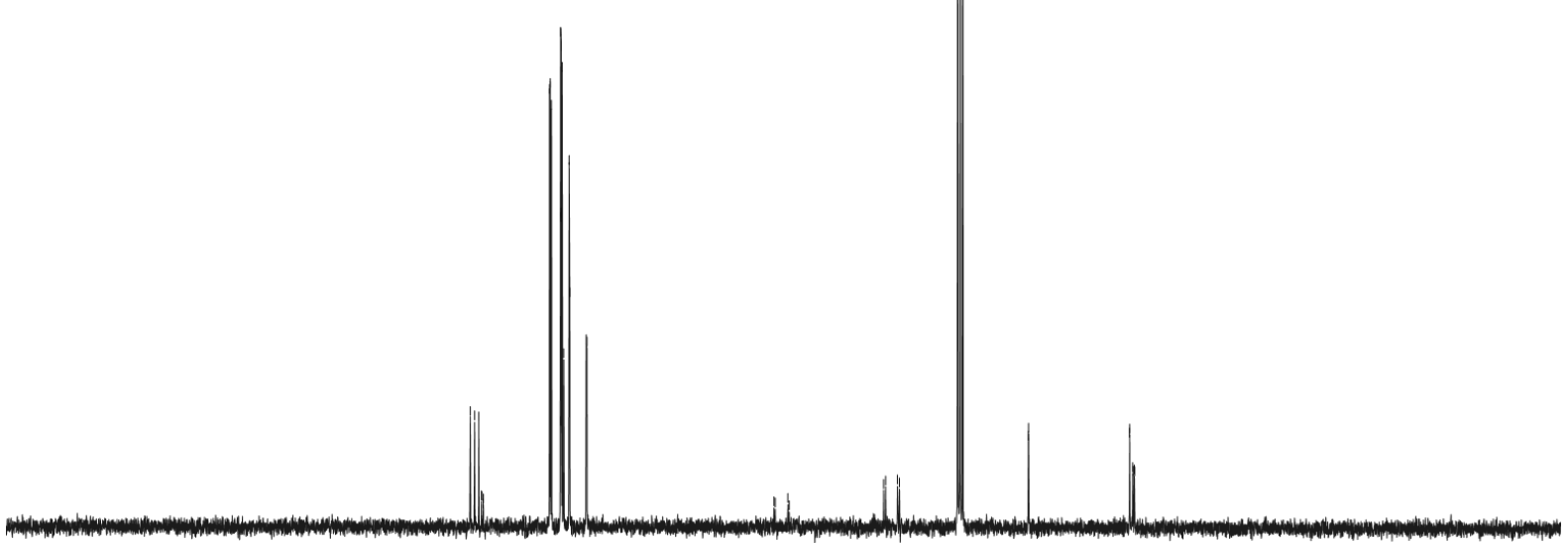

100
$f 1(\mathrm{ppm})$

$90 \lcm{10}$

70

50

$\begin{array}{llll}1 & 1 & 1 \\ 40 & 20 & 10\end{array}$ 


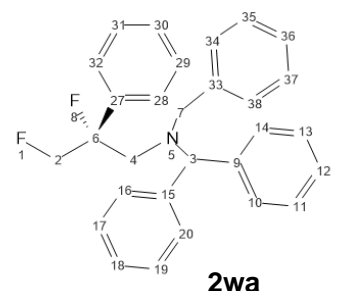

2wa

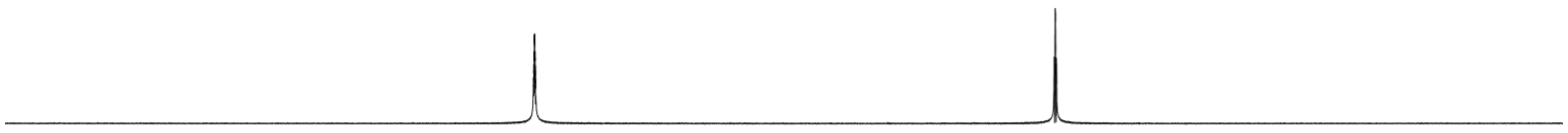

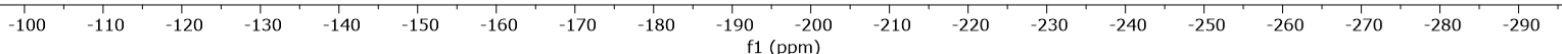


<smiles></smiles>

(S)-3aa-HCl

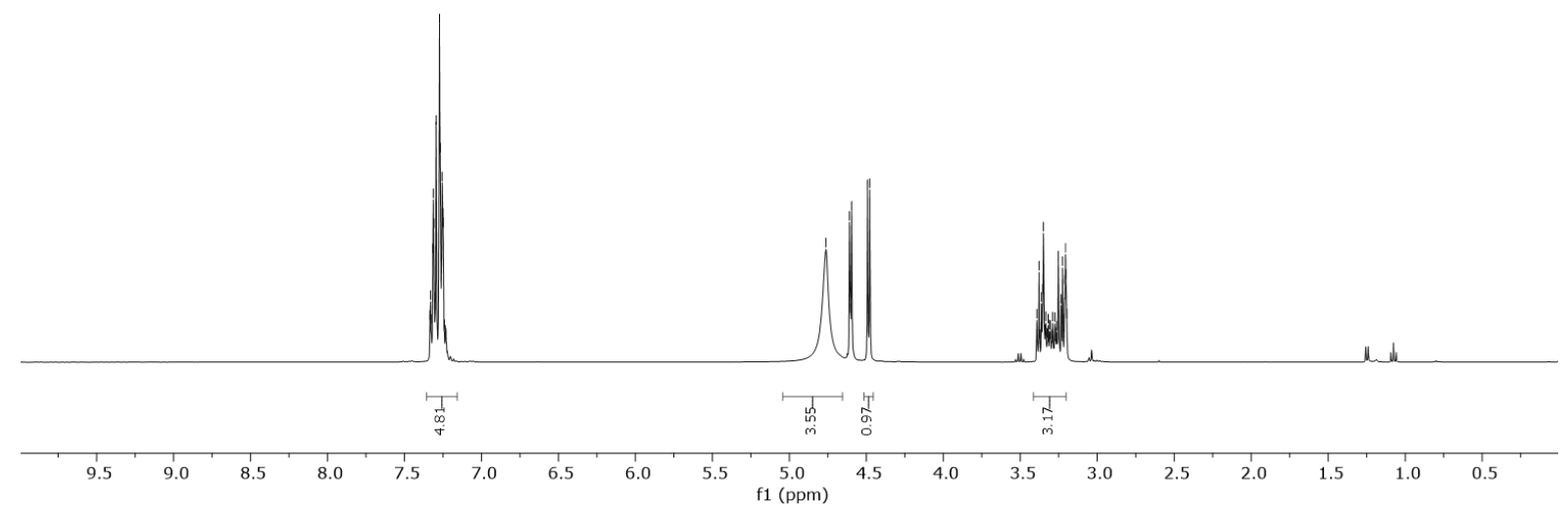
UoxF_groagna_3aa/CdCl3<smiles></smiles>

(S)-3aa $\cdot \mathbf{H C l}$

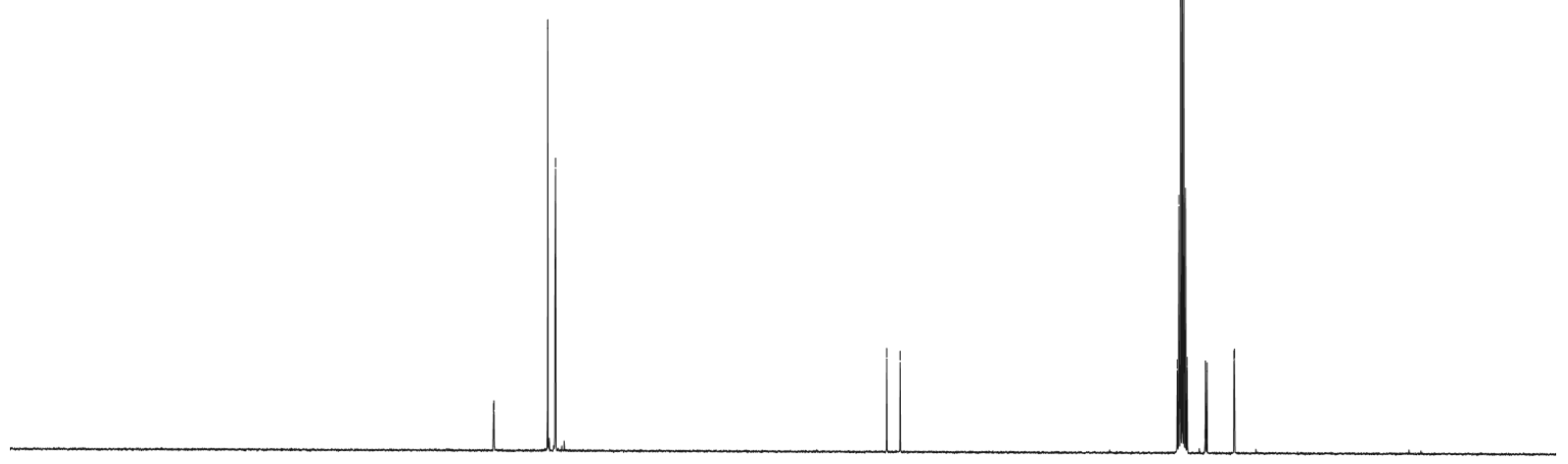

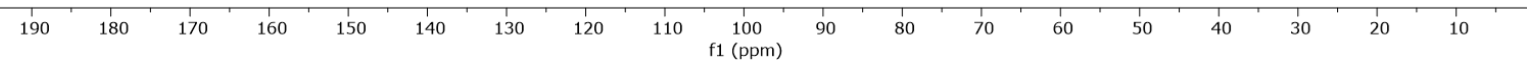




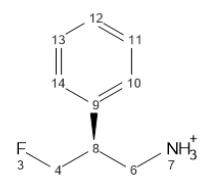

(S)-3aa $\cdot \mathbf{H C l}$

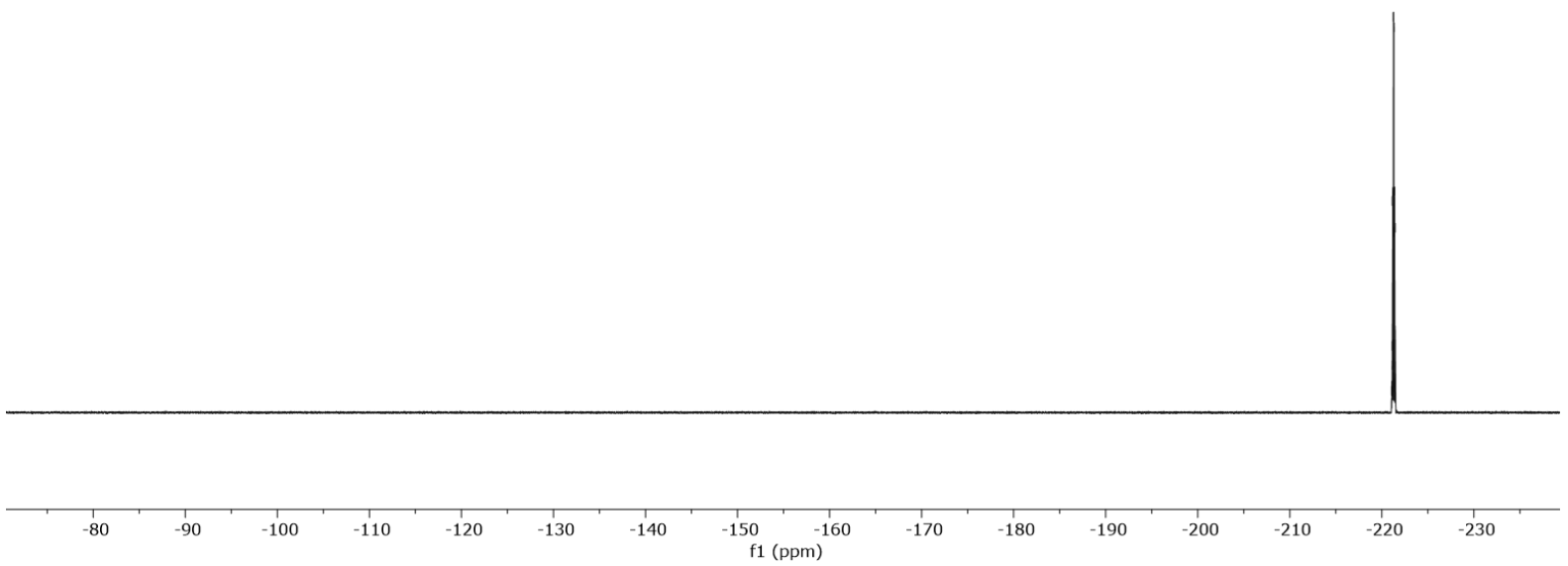


UOXF_groagna_3ab/CdCl3

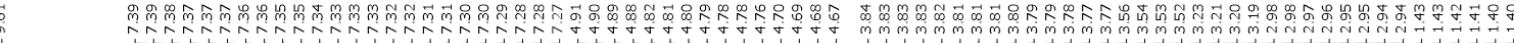<smiles>CCNCC(CF)Nc1nnnnn1</smiles>

$(R)-\mathbf{3} \mathbf{a b} \cdot \mathbf{H C l}$

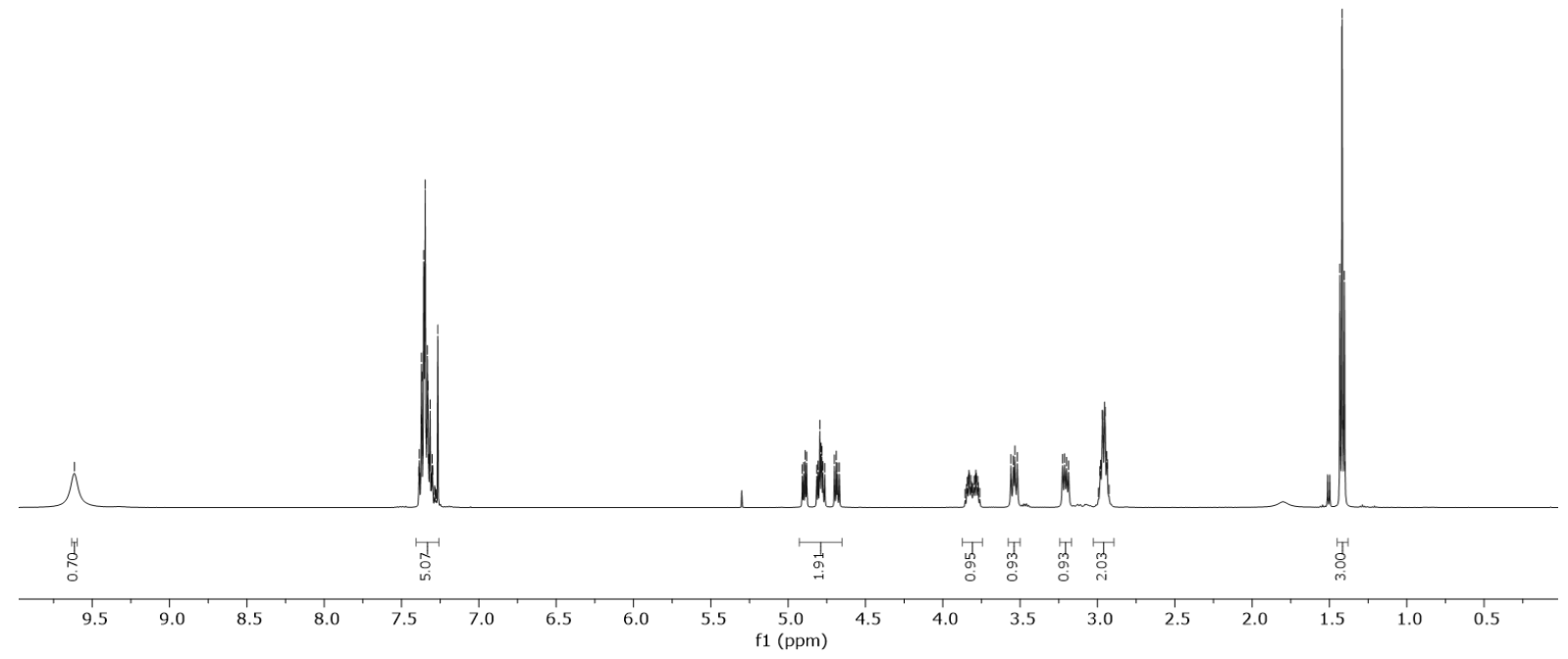

UOXF_groagna_3ab/CdCl3
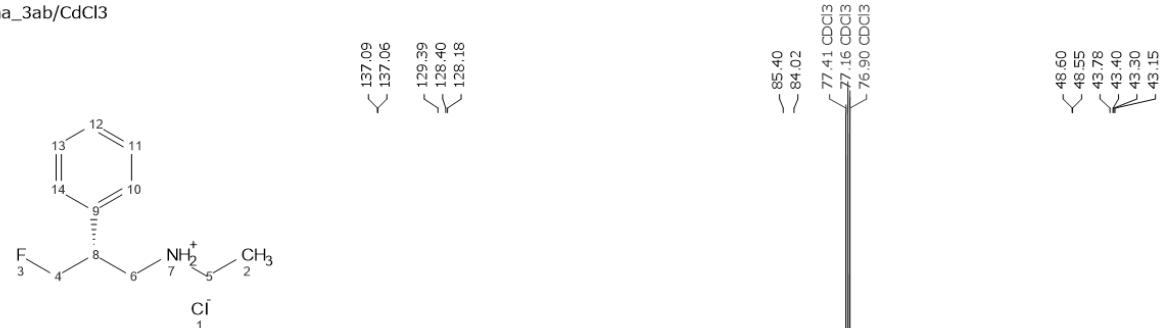

$(R)-3 \mathrm{ab} \cdot \mathrm{HCl}$
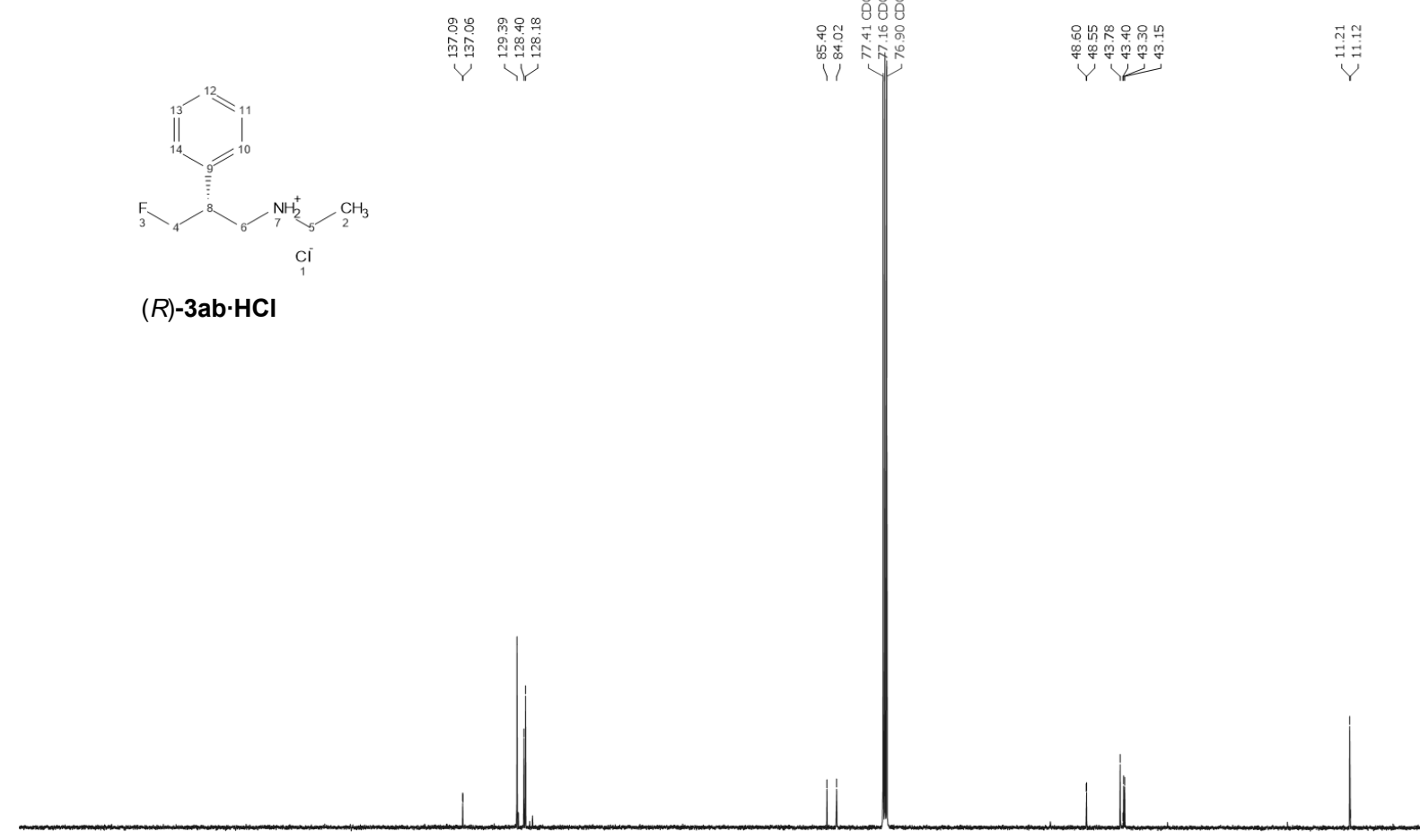

190

180

$170 \quad 160$

150

140 


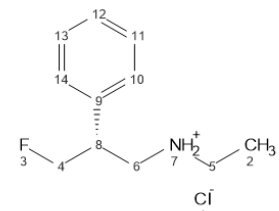

$(R)-3 \mathbf{a b} \cdot \mathbf{H C l}$

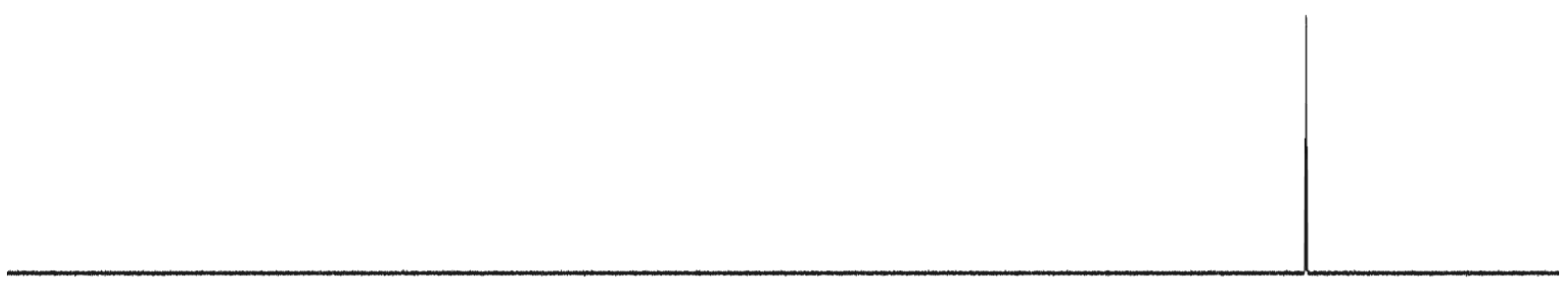

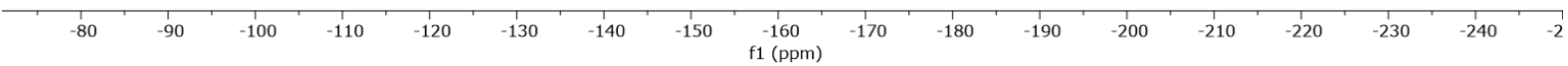


<smiles>CCC(CN)c1cc(Cl)cnn1</smiles>

$4 a$

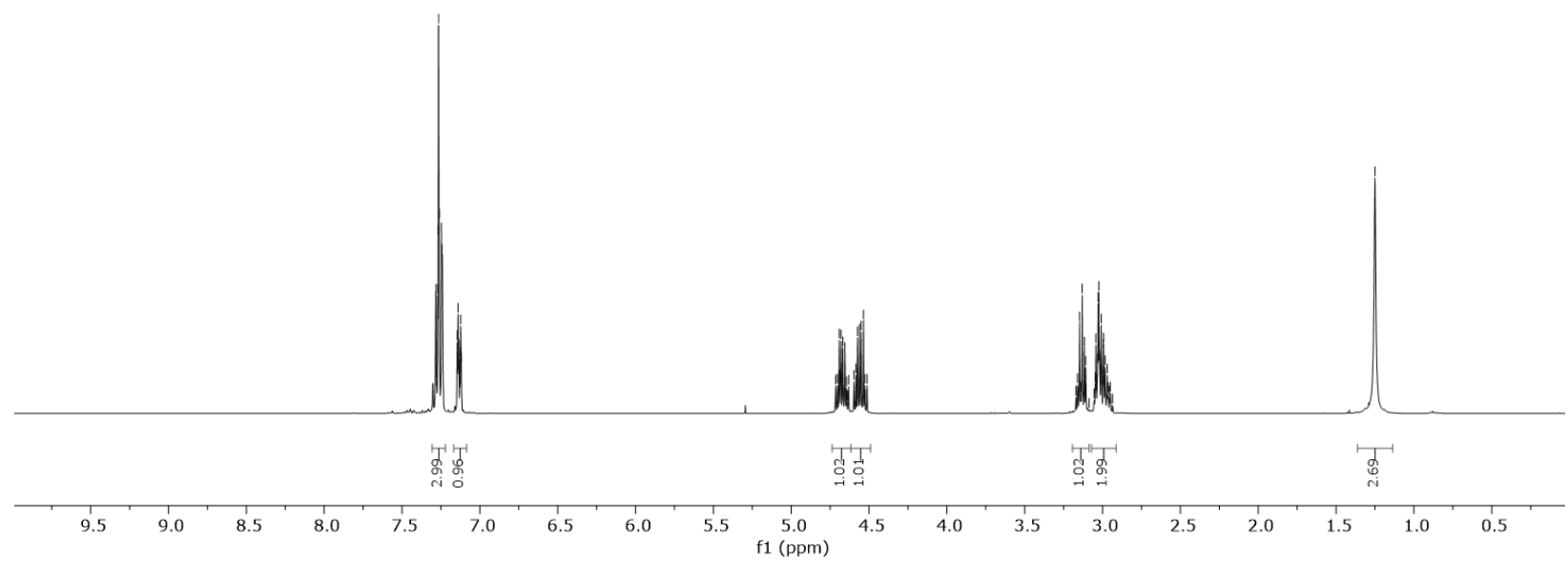

UOXF_groagna1_4a/CdCl3

$4 a$

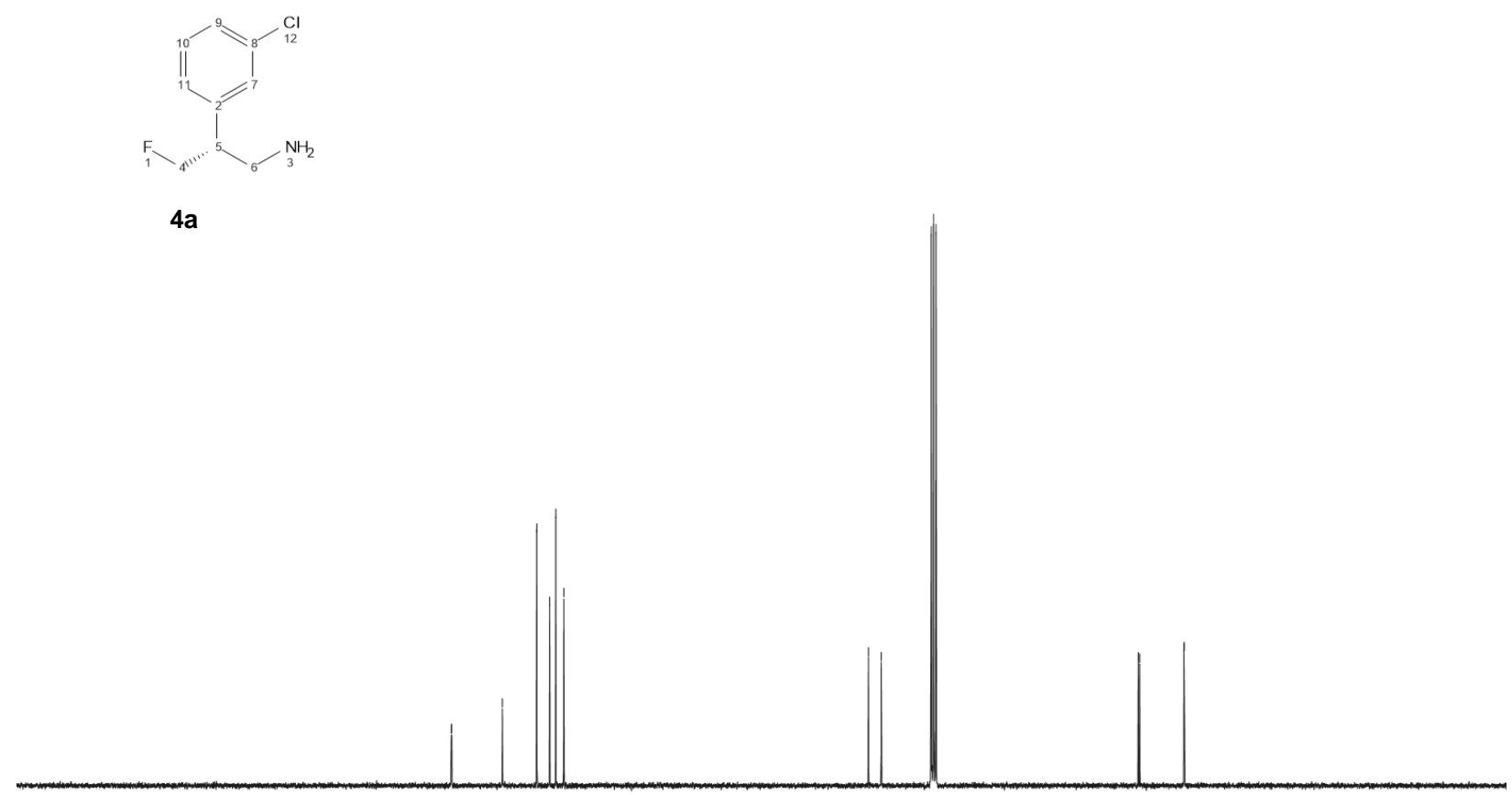

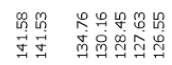

I

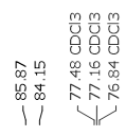

11

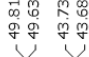

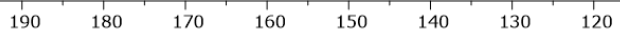
$\begin{array}{ccc}110 & 100 & 9 \\ & f 1(p p m)\end{array}$ 


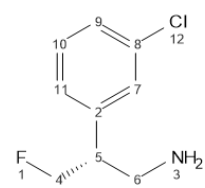

$4 a$

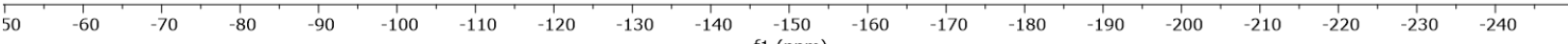




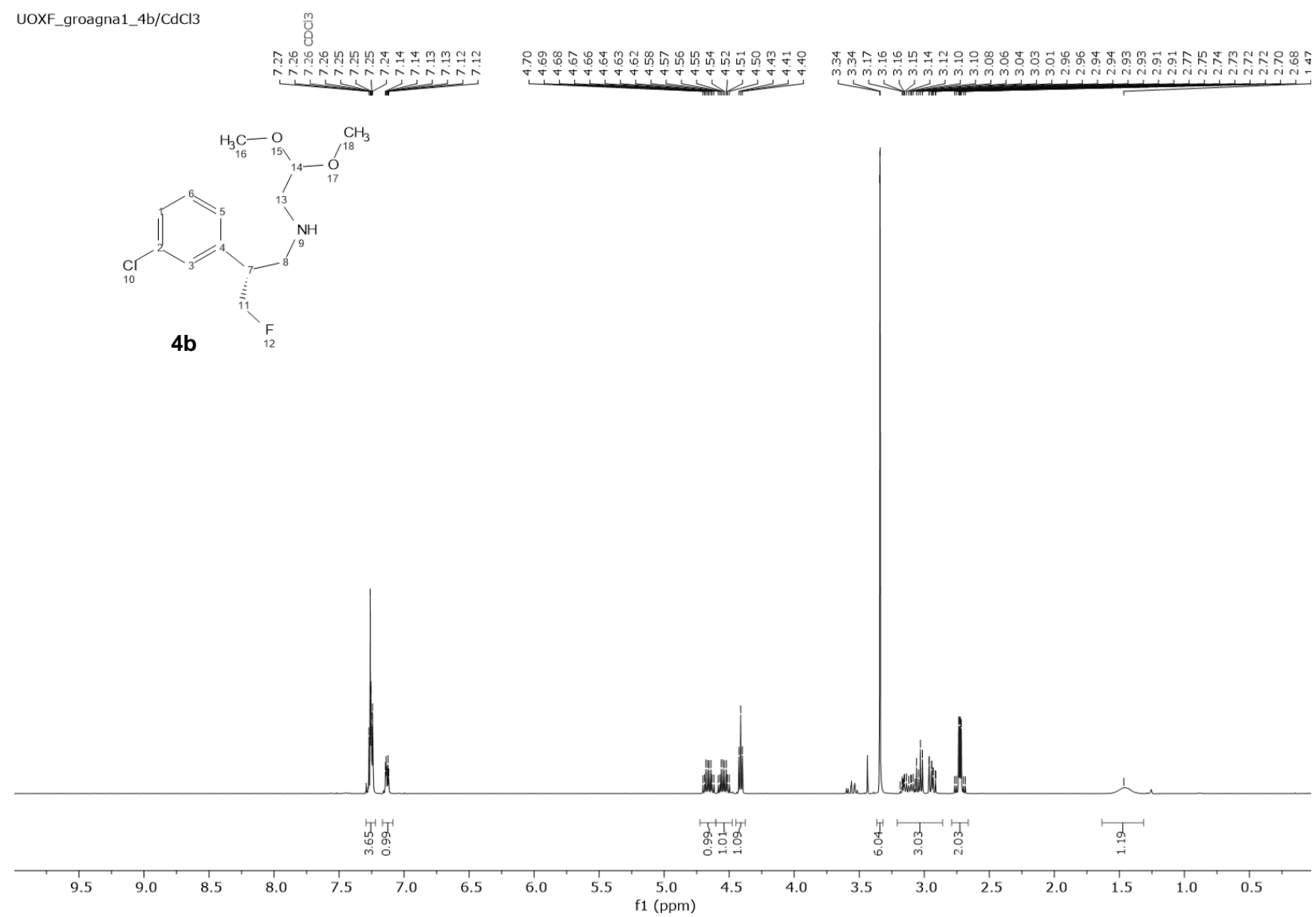

UOXF_groagna1_4b/CdCl3

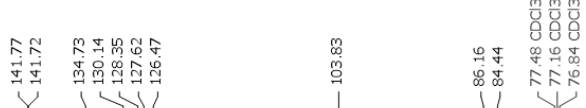

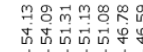

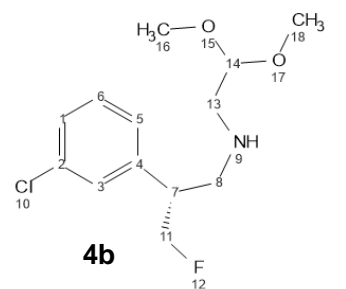

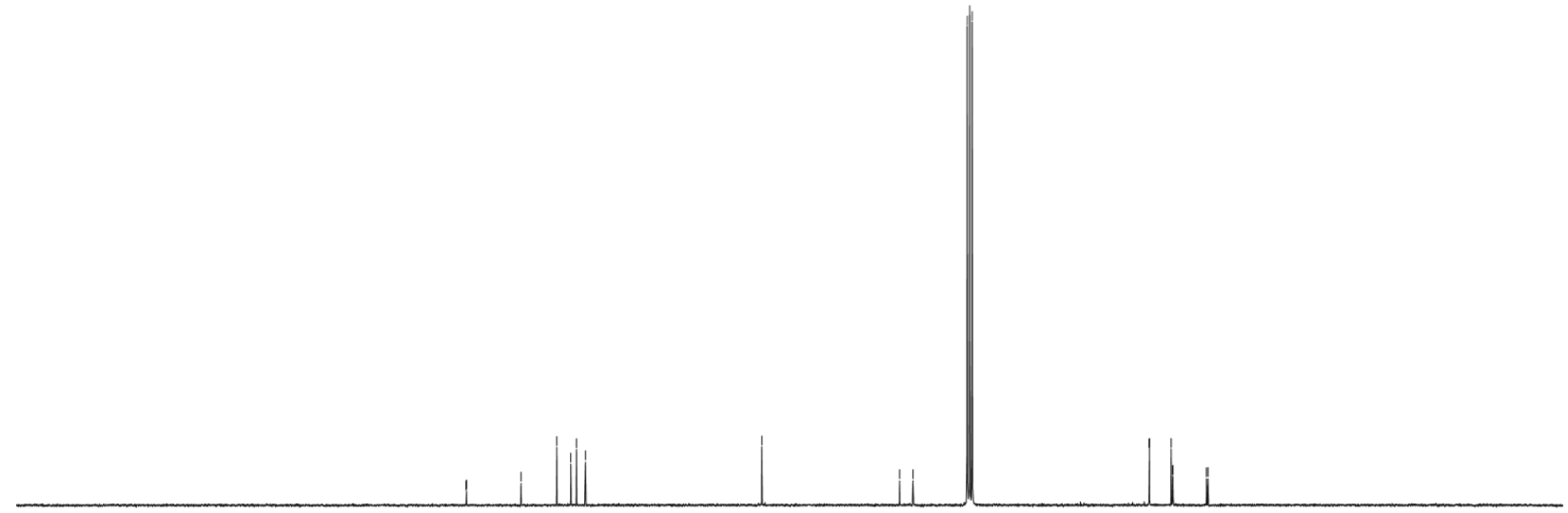

190

180

160

140

130

$120 \quad 11$

100
$\mathrm{f} 1(\mathrm{ppm})$ 

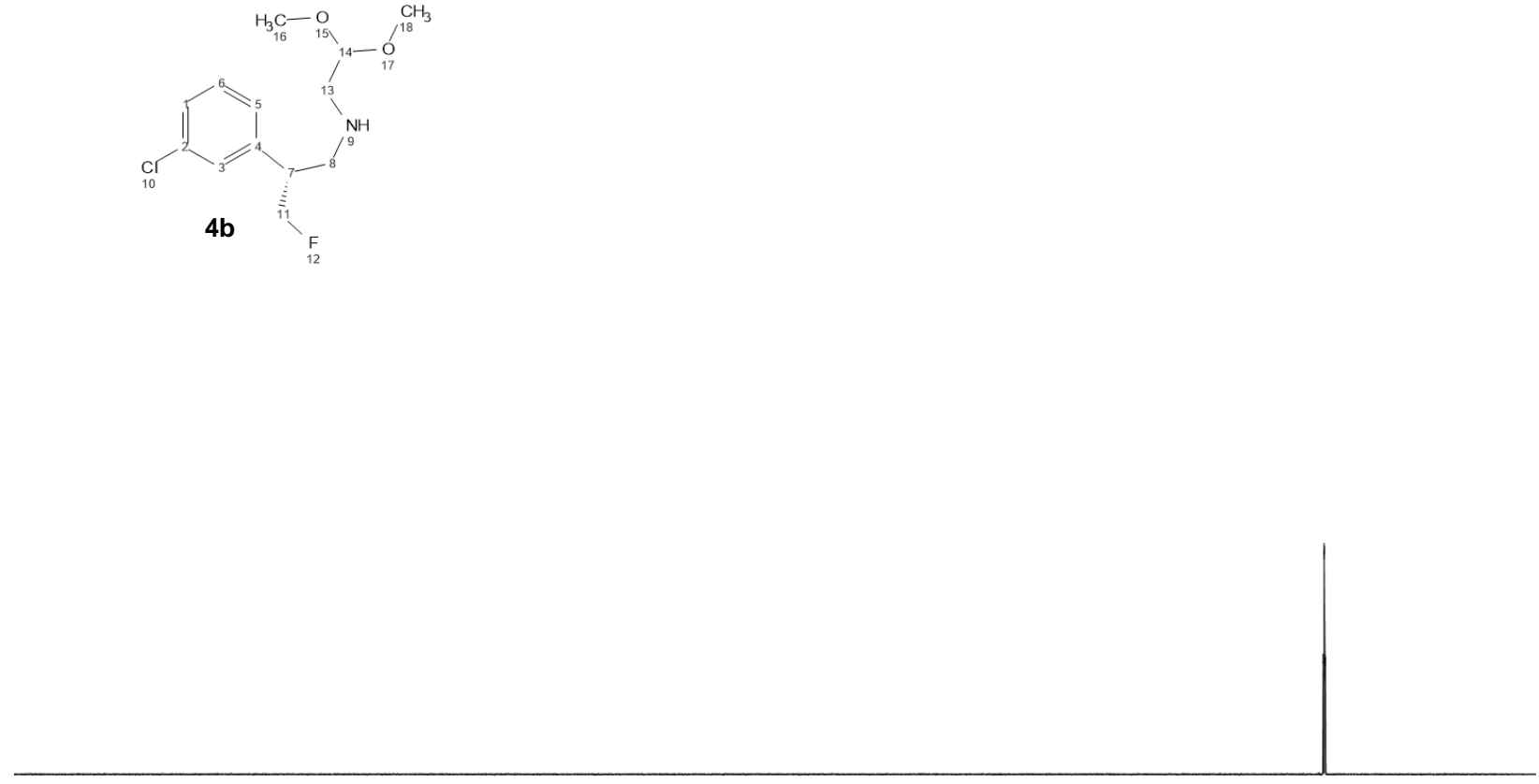

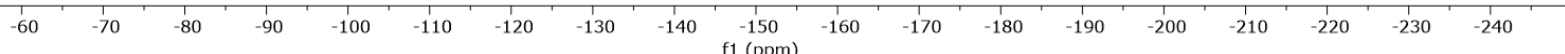


UOXF_groagna1_4cgCdCl3

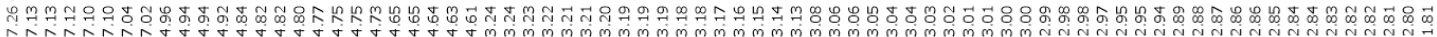<smiles>FCP1ONNNS1</smiles>

Fluorinated Lorcaserin

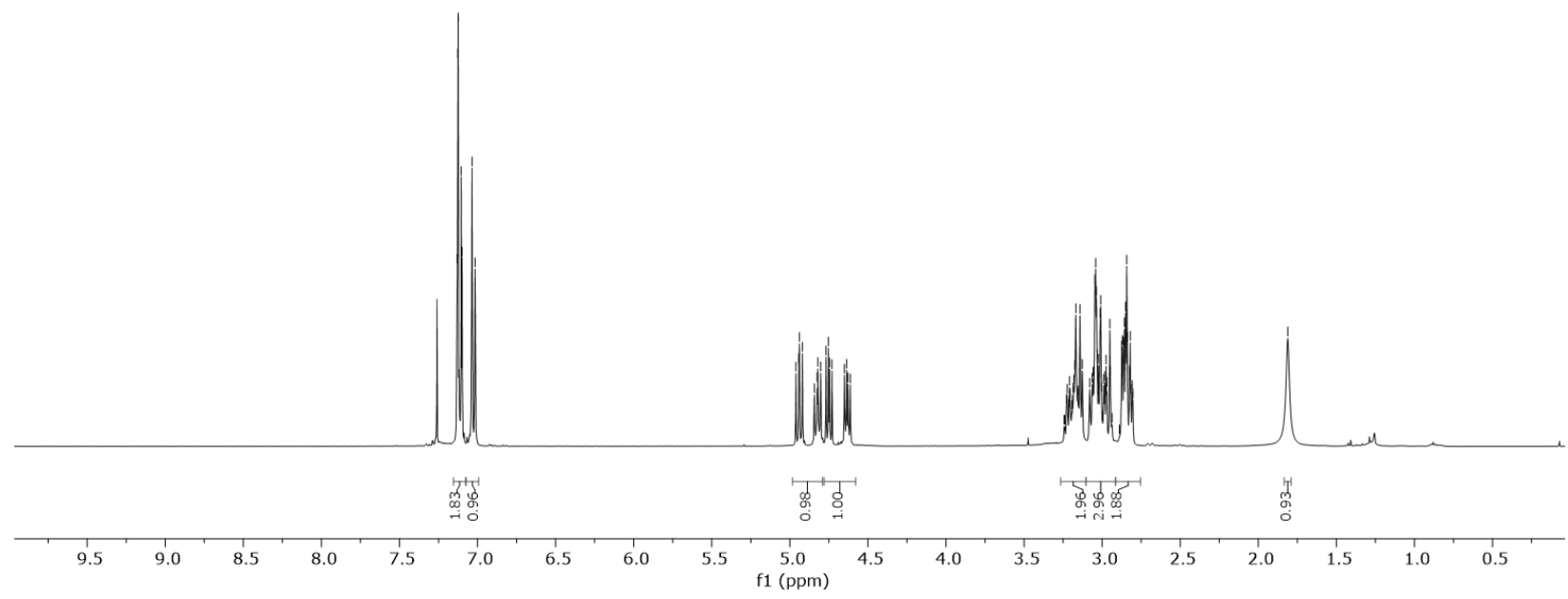

UOXF_groagna1_4c/CdCl3

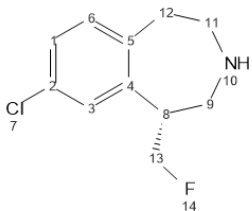

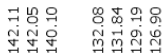

जन

Fluorinated Lorcaserin

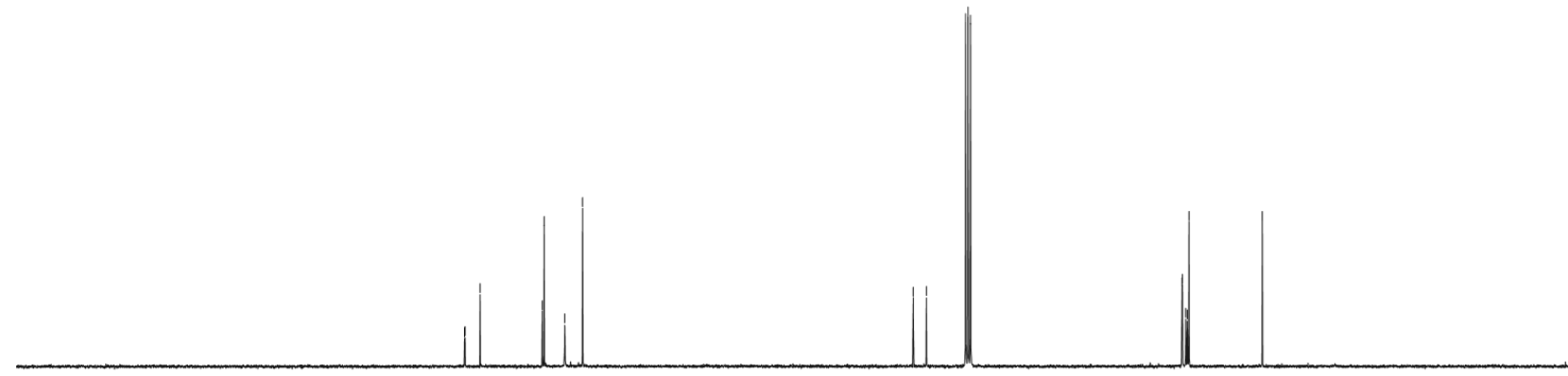

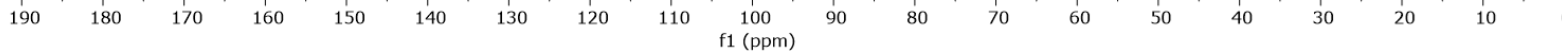

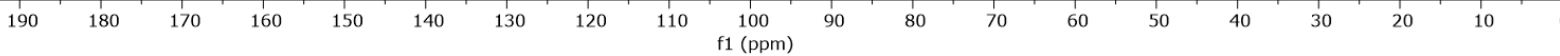

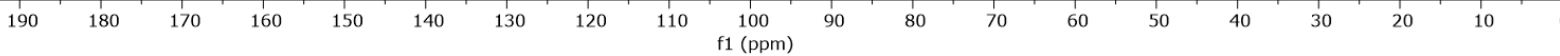

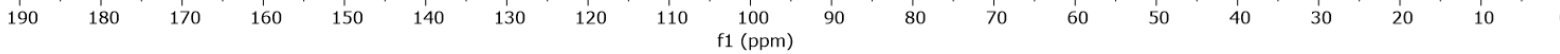

$\cos 20$

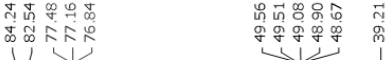

$11<$ 


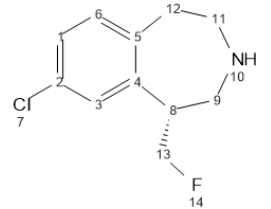

Fluorinated Lorcaserin

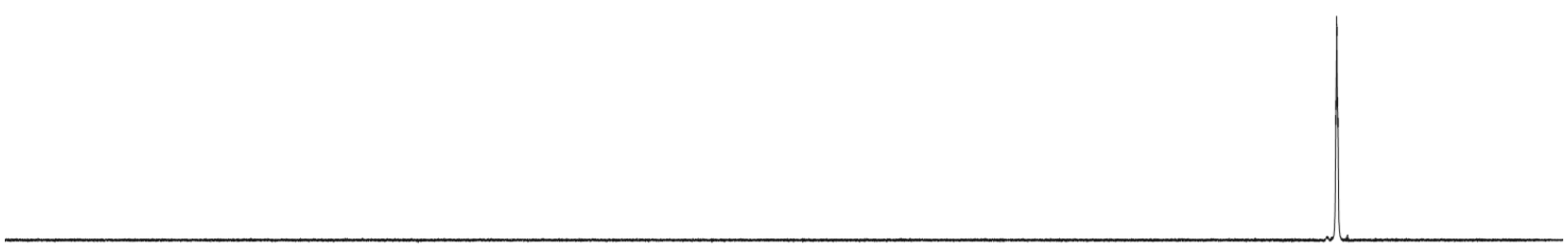

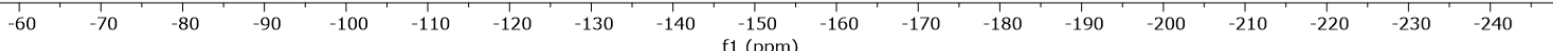




\section{Copies of HPLC traces}

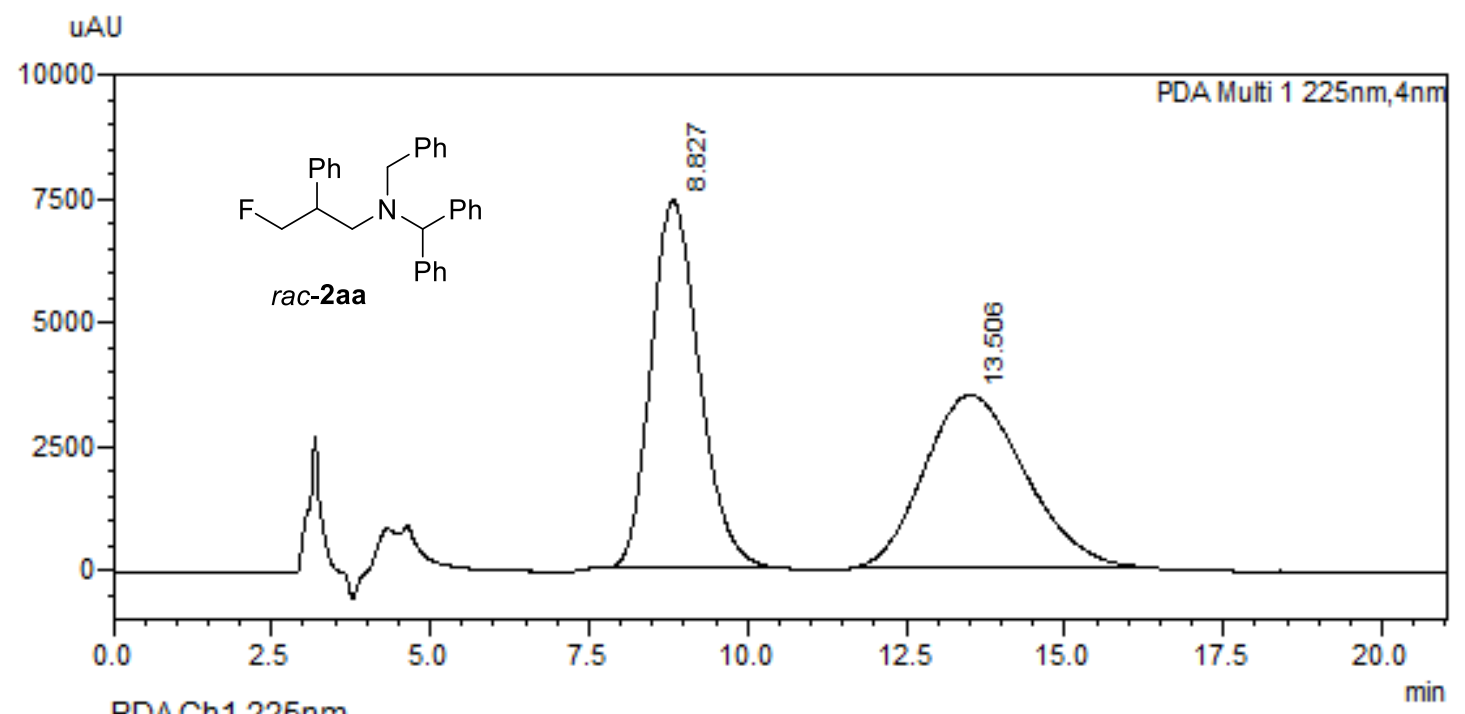

PDAch $225 \mathrm{~nm}$

Peakf Ret. Time $\quad$ Area\%

\begin{tabular}{r|r|r|}
1 & 8.827 & 50.420 \\
\hline 2 & 13.506 & 49.580 \\
\hline
\end{tabular}

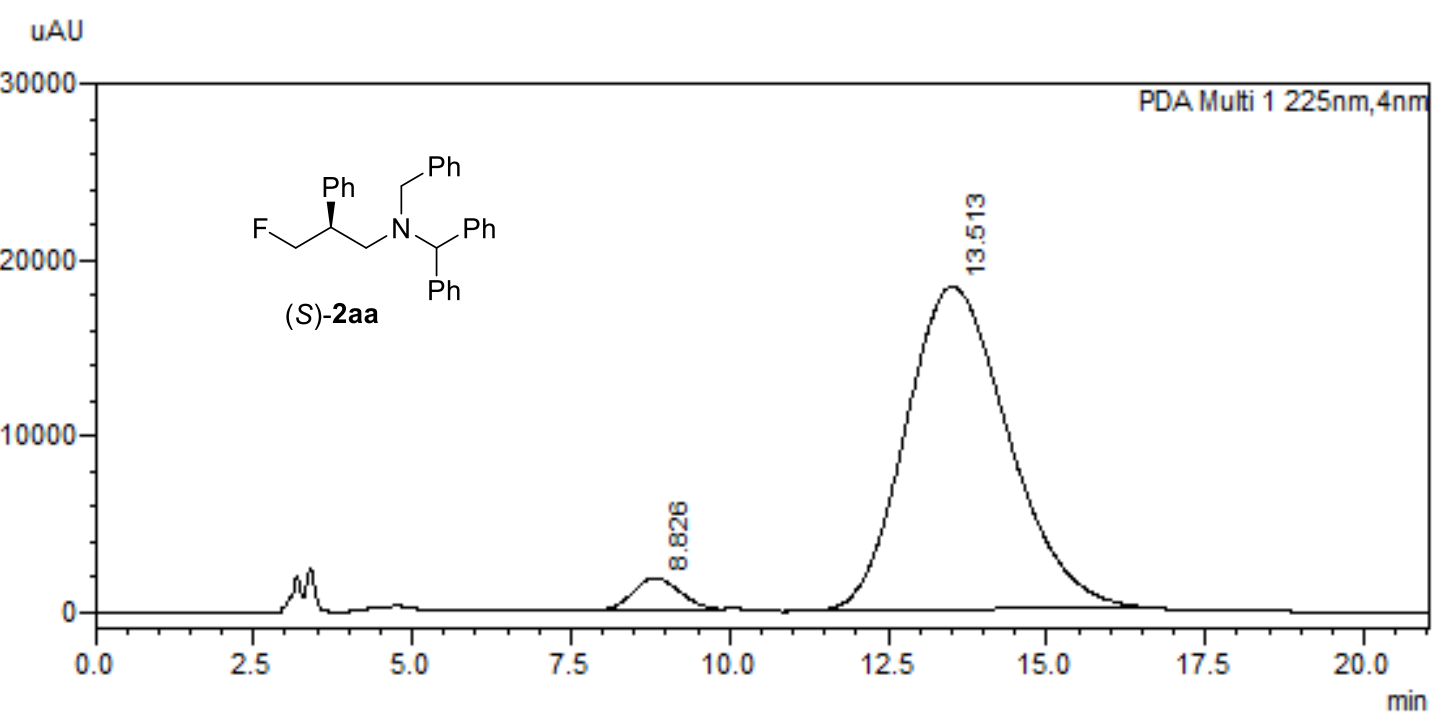

\begin{tabular}{|c|c|c|}
\hline \multicolumn{3}{|c|}{ PDACh1225nm } \\
\hline Peakł & \# Ret. Time & Area $\%$ \\
\hline 1 & 8.826 & 4.081 \\
\hline 2 & 13.513 & 95.919 \\
\hline
\end{tabular}




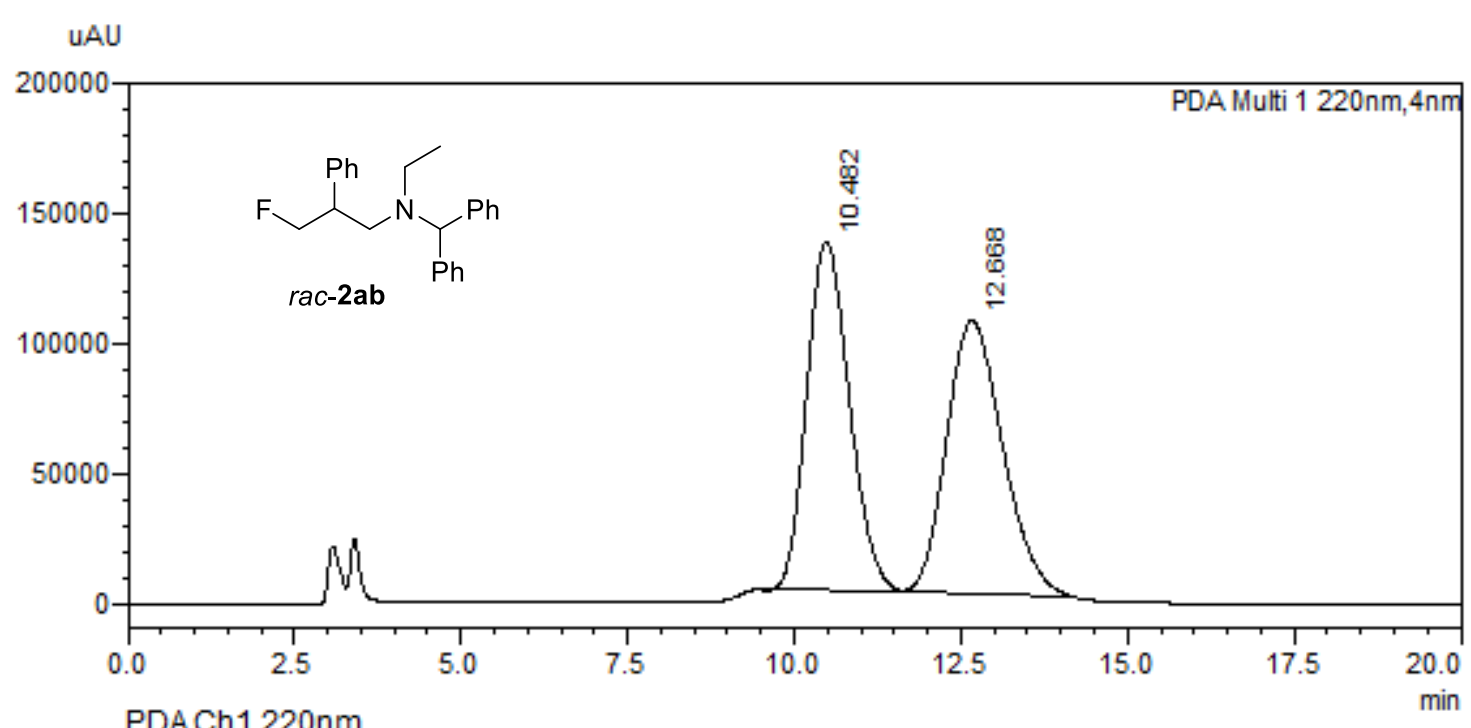

PRACh1 $220 \mathrm{~nm}$

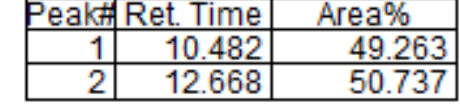

mAL

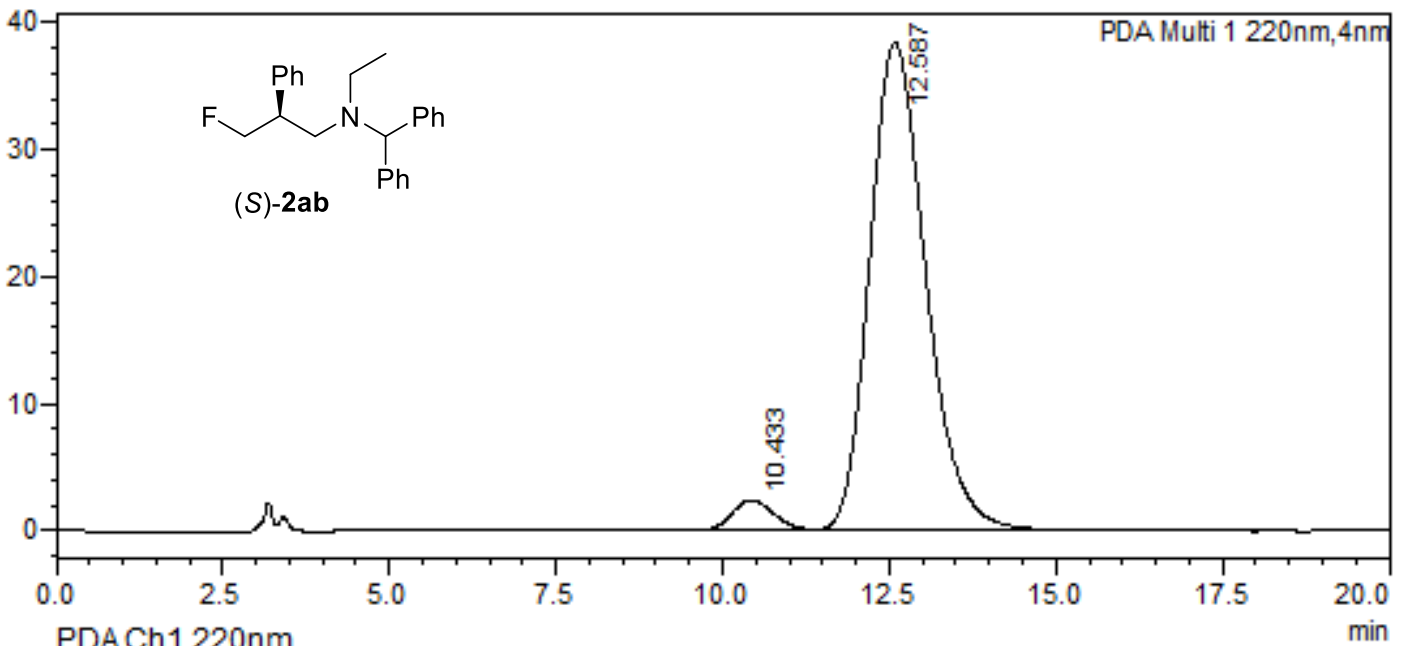

PDACh1220nm

49.263

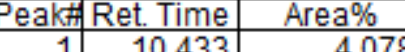

\begin{tabular}{l|r|r|}
\hline 2 & 12.587 & 95.922 \\
\hline
\end{tabular} 


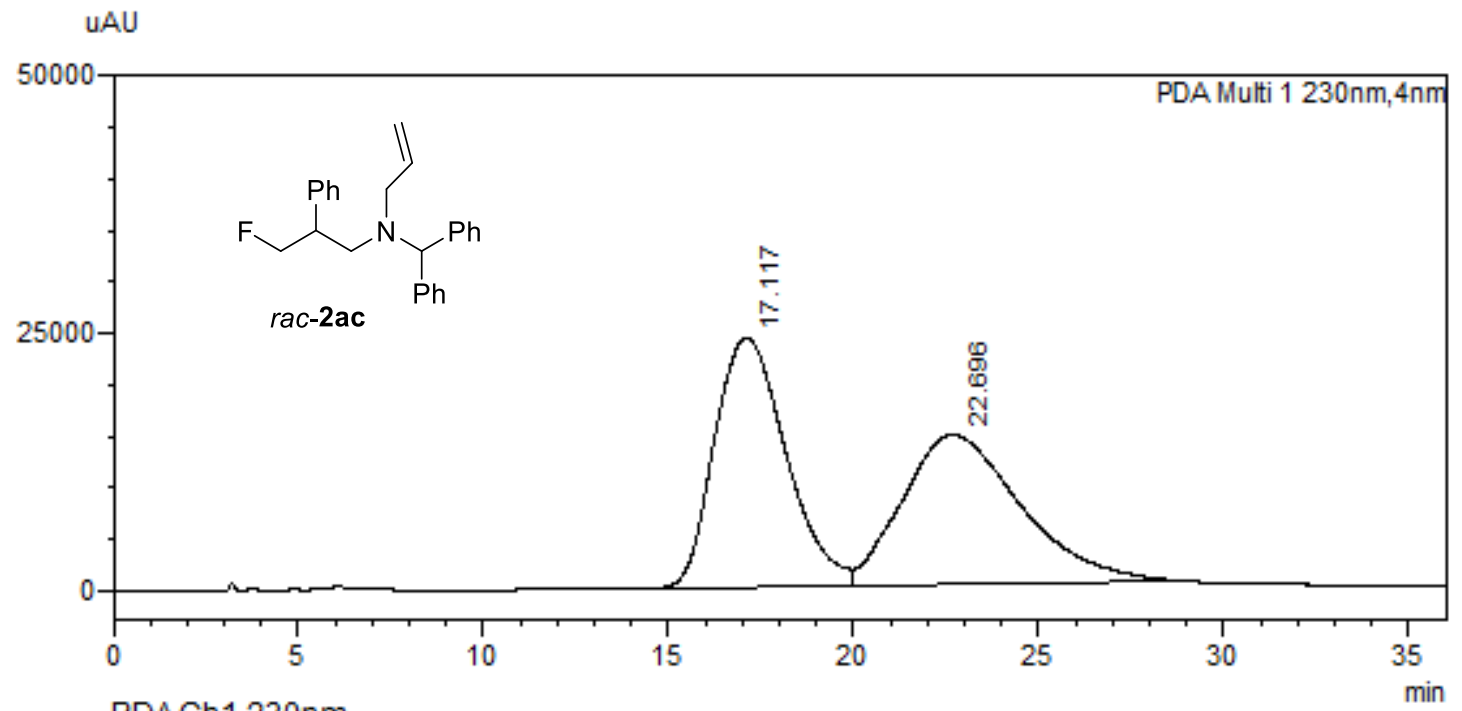

PDACh1230nm

Peak\# Ret. Time $\quad$ Area\%

\begin{tabular}{l|l|l|}
1 & 17.117 & 49.884 \\
\hline 2 & 22.696 & 50.116 \\
\hline
\end{tabular}

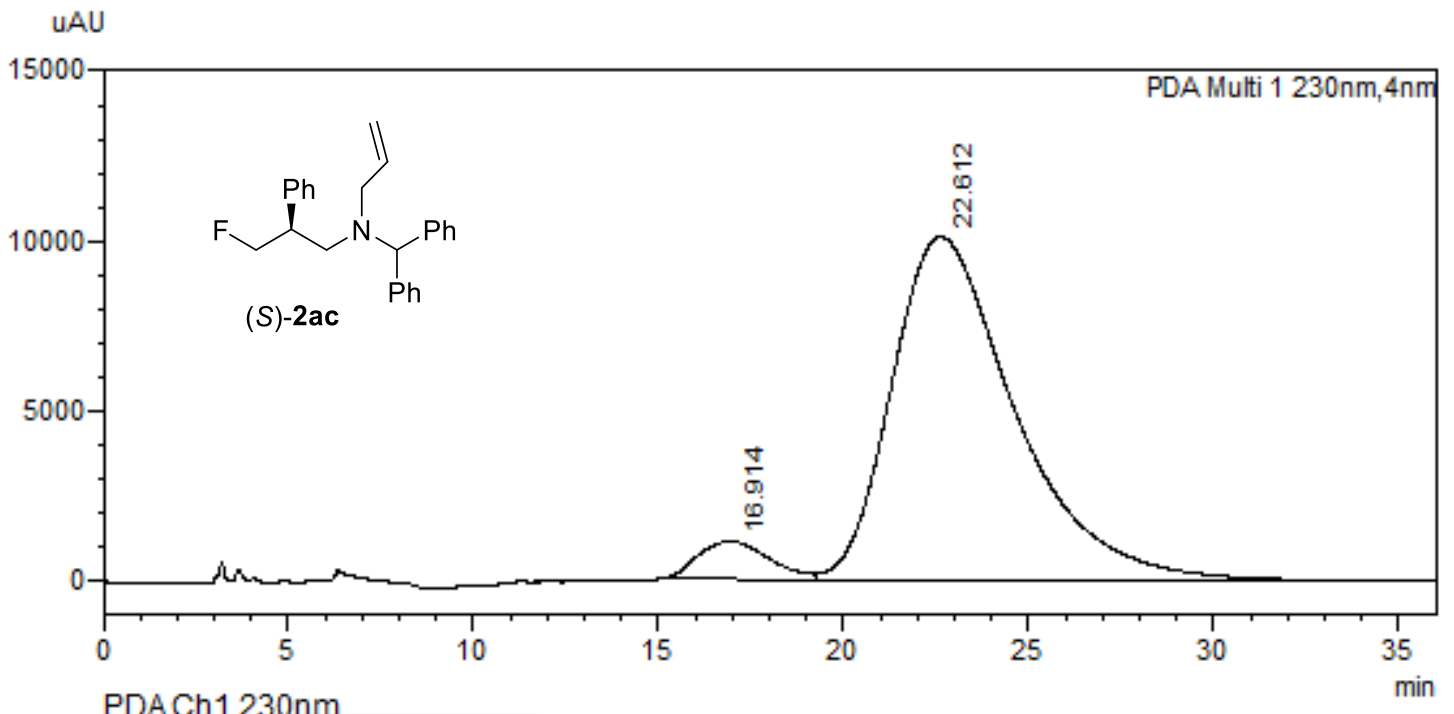

PDACh1230nm

Peakf Ret. Time 1 Area\%

\begin{tabular}{ll|l|}
1 & 16.914 & 5.998 \\
\hline
\end{tabular}

94.002 


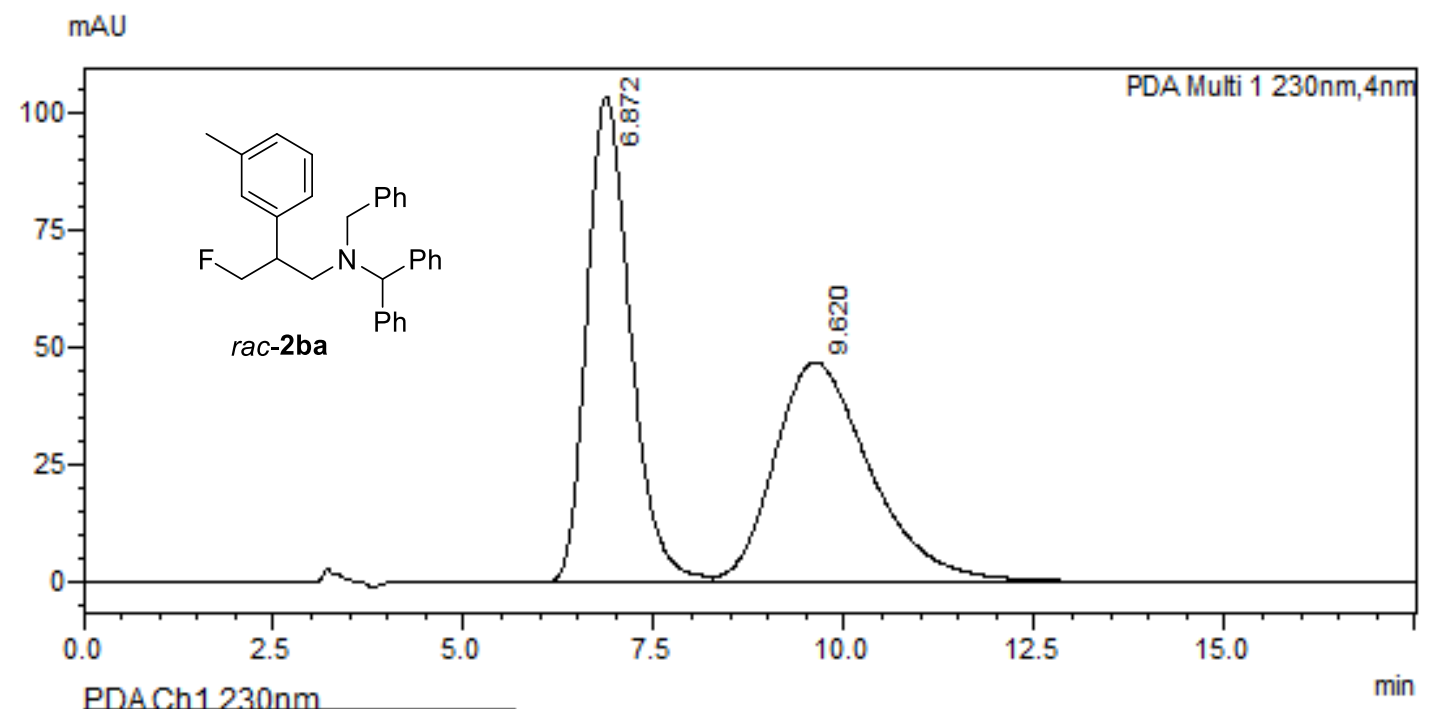

Peak\#Ret. Time Area\%

\begin{tabular}{l|l|l|}
1 & 6.872 & 50.081 \\
2 & 9.620 & 49.919 \\
\hline
\end{tabular}

mAU

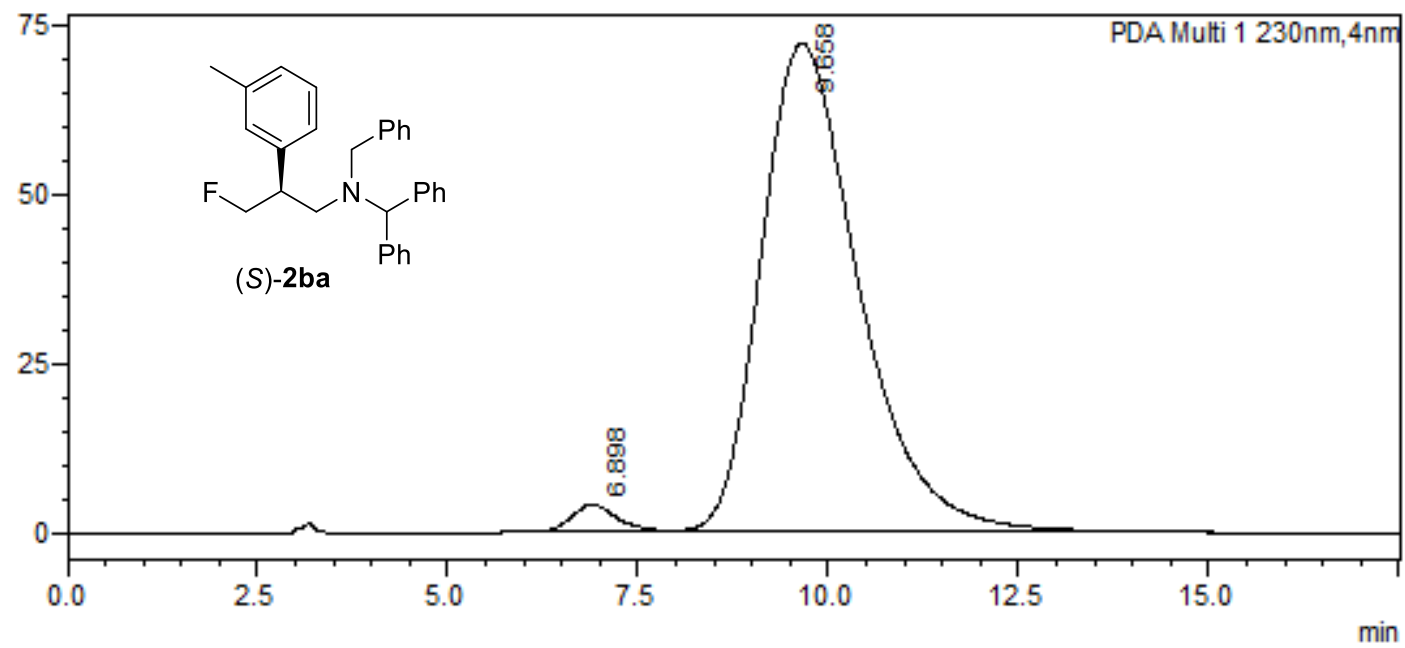

PDACh1230nm

Peakf Ret. Time $\quad$ Area\%

\begin{tabular}{r|r|r|}
\hline 1 & 6.898 & 2.276 \\
\hline 2 & 9.658 & 97.724 \\
\hline
\end{tabular}




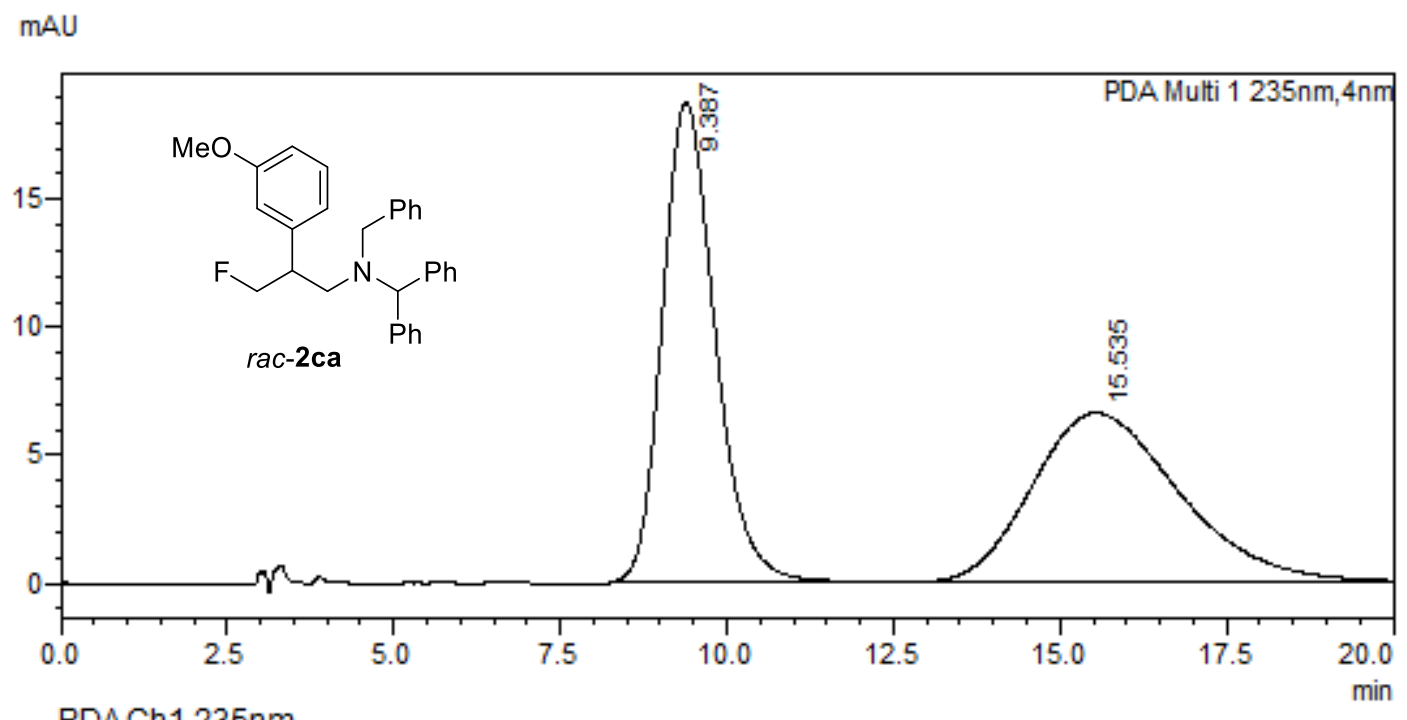

PDACh1 23.5nm

Peak\# Ret. Time Area\%

\begin{tabular}{r|r|r|}
1 & 9.387 & 50.641 \\
\hline 2 & 15.535 & 49.359 \\
\hline
\end{tabular}

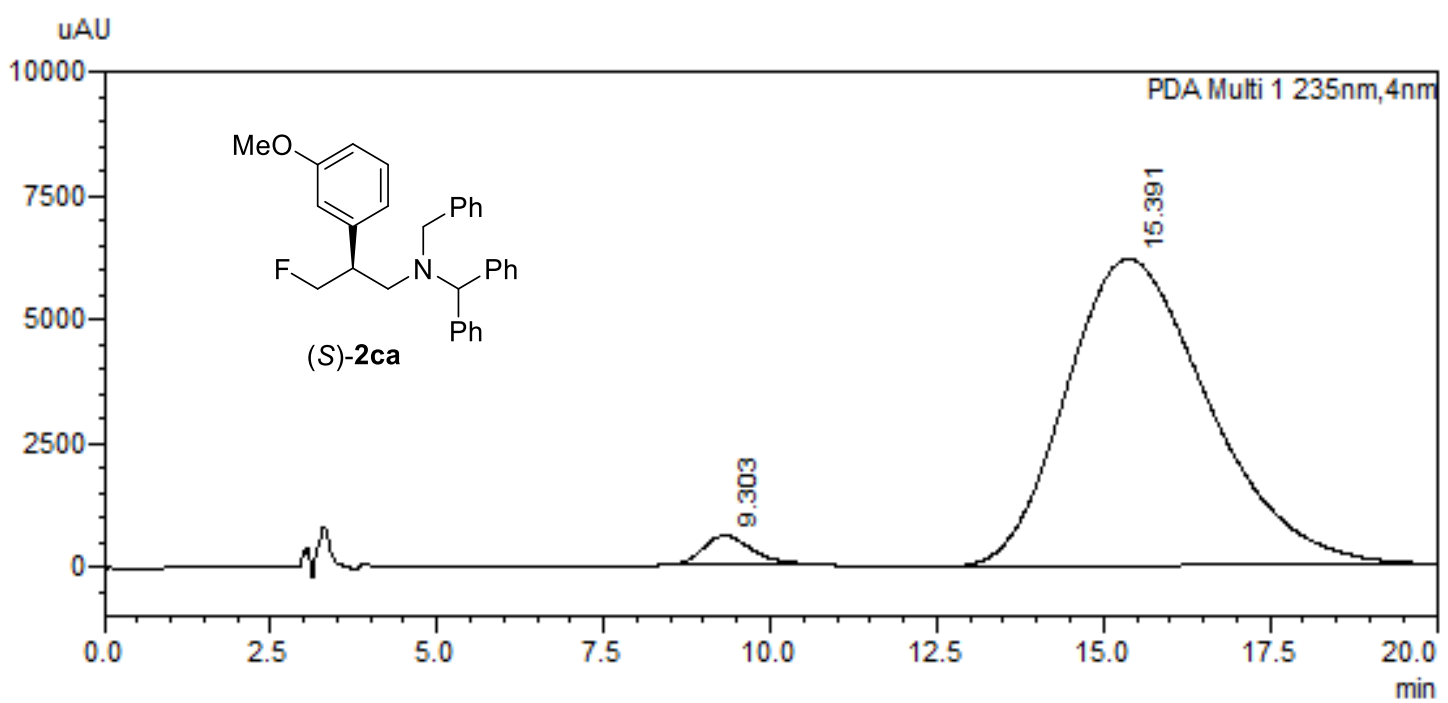

PDACh1235nm

Peakfl Ret. Time Area\%

\begin{tabular}{|r|r|r|}
\hline 1 & 9.303 & 2.842 \\
\hline 2 & 15.391 & 97.158 \\
\hline
\end{tabular} 


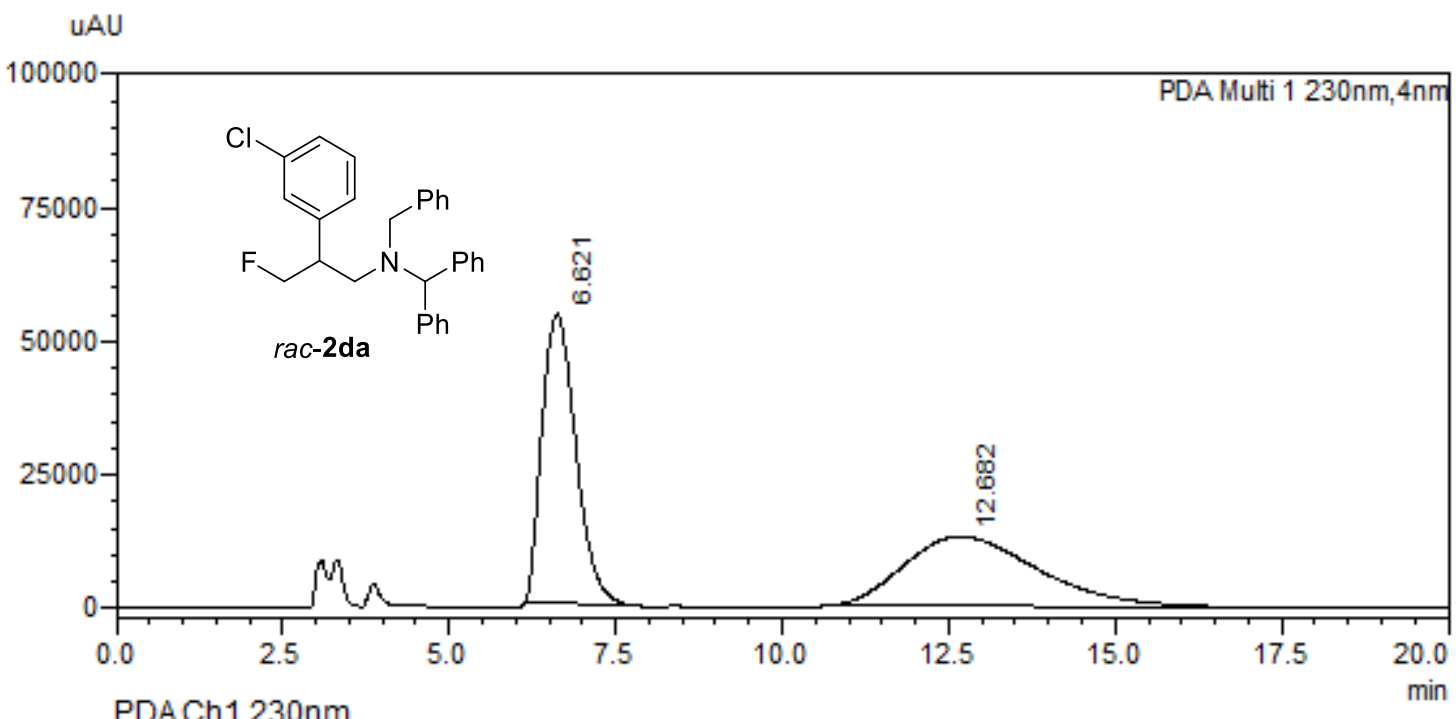

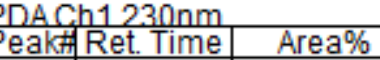
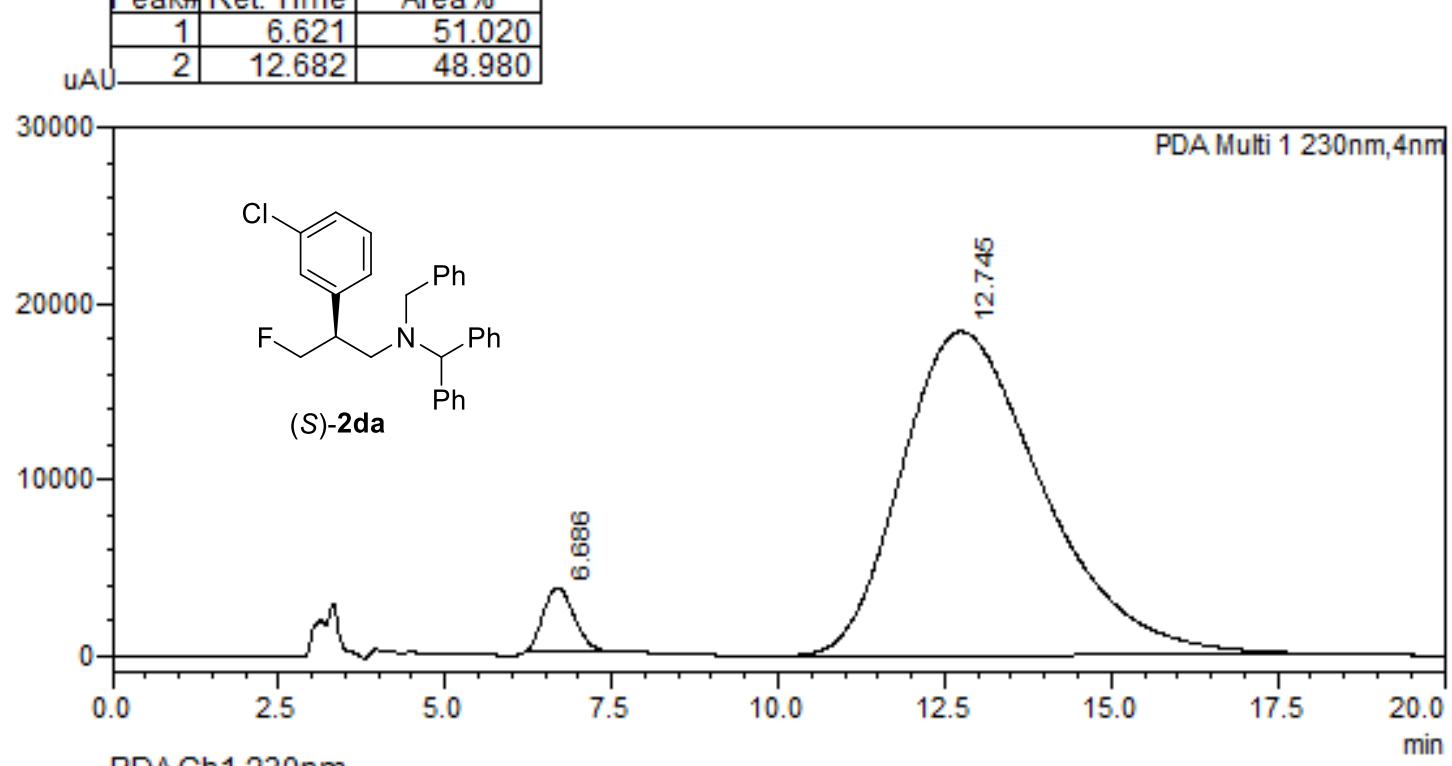

PDACh1230nm

Peakf Ret. Time $\quad$ Area\%

\begin{tabular}{r|r|r|}
1 & 6.686 & 4.135 \\
\hline 2 & 12.745 & 95.865 \\
\hline
\end{tabular} 


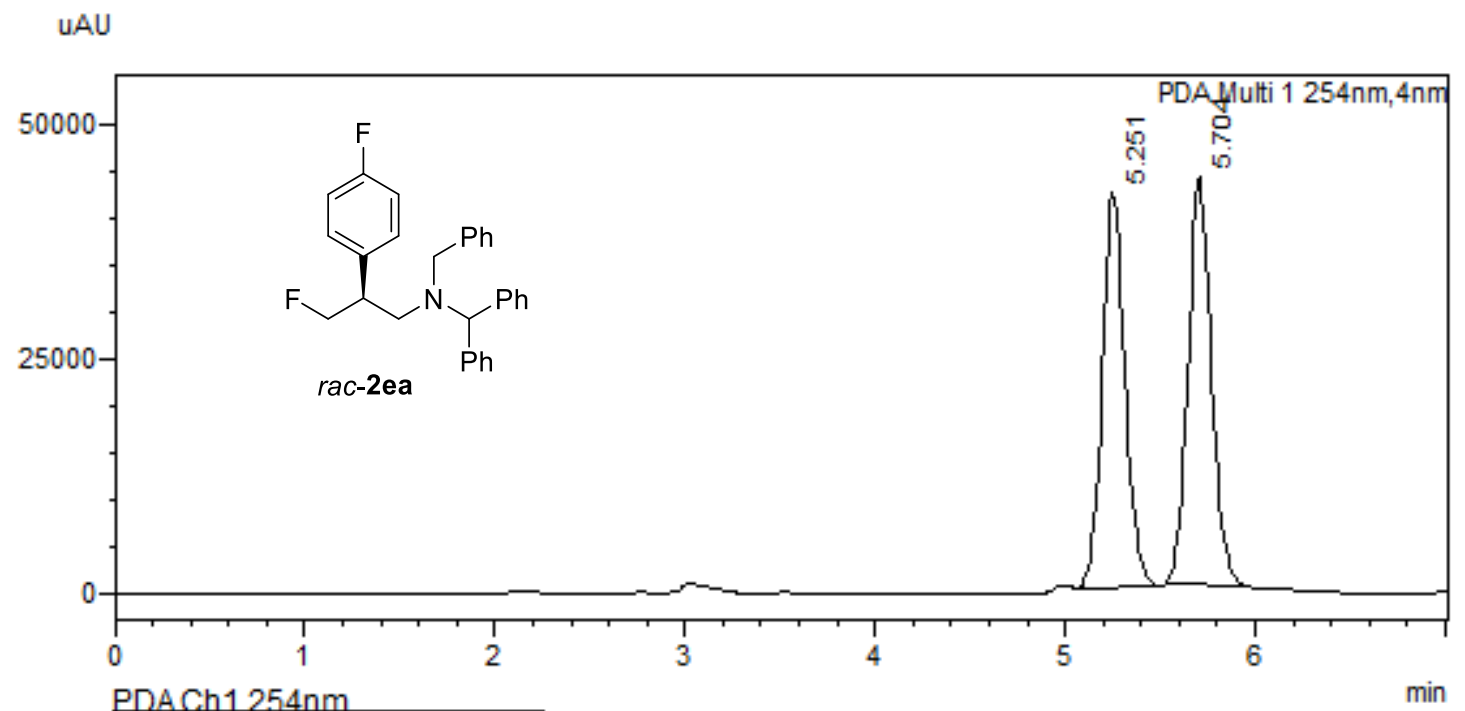

Peakf Ret. Time 1 Area\%

\begin{tabular}{|r|r|r|}
\hline 1 & 5.251 & 48.719 \\
\hline 2 & 5.704 & 51.281 \\
\hline
\end{tabular}

UAU

150000

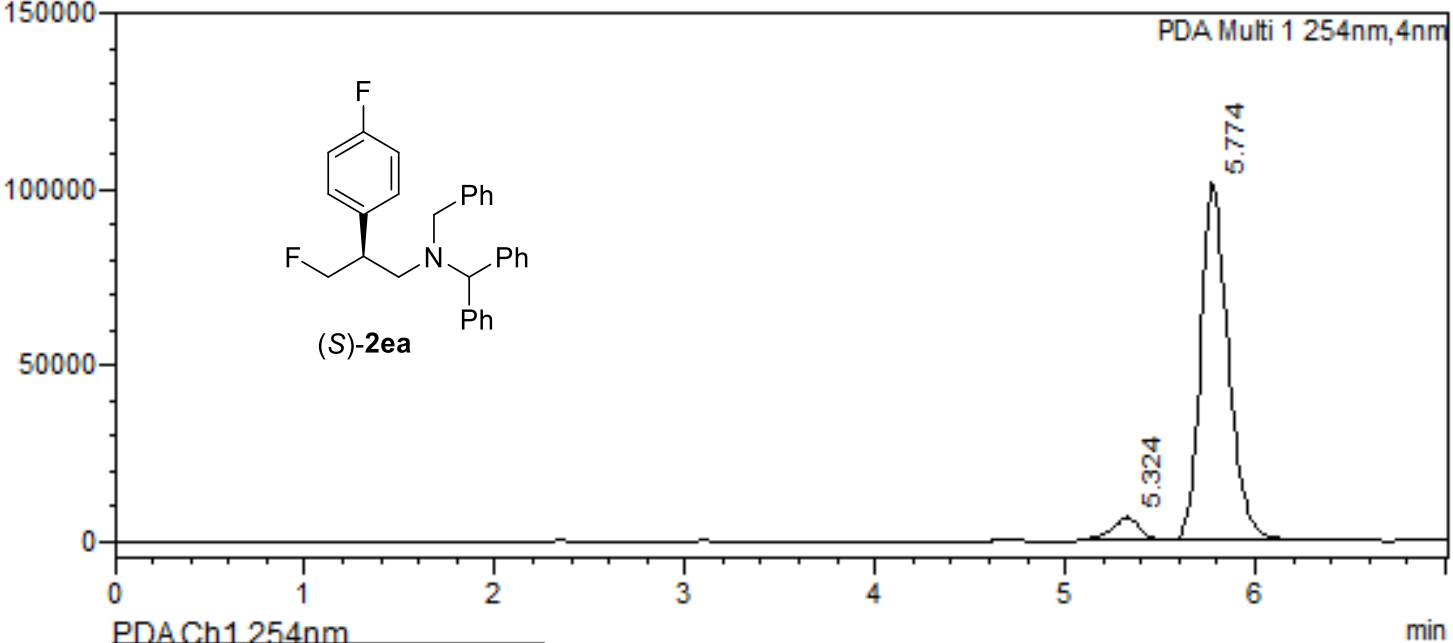

Peak\#Ret. Time

\begin{tabular}{r|r|r|}
1 & 5.324 & 5.645 \\
\hline 2 & 5.774 & 94.355 \\
\hline
\end{tabular} 


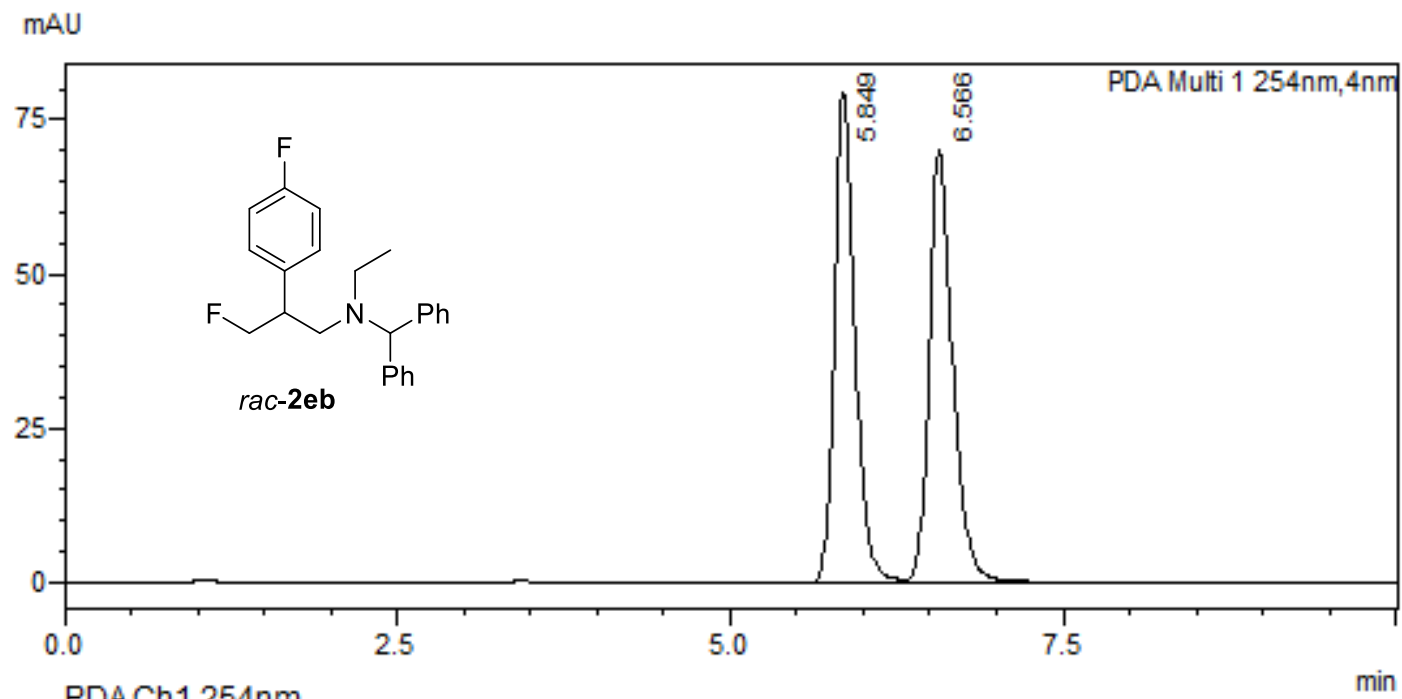

\begin{tabular}{l|l}
\hline Peakf Ret. Time & Area $\%$ \\
\hline
\end{tabular}
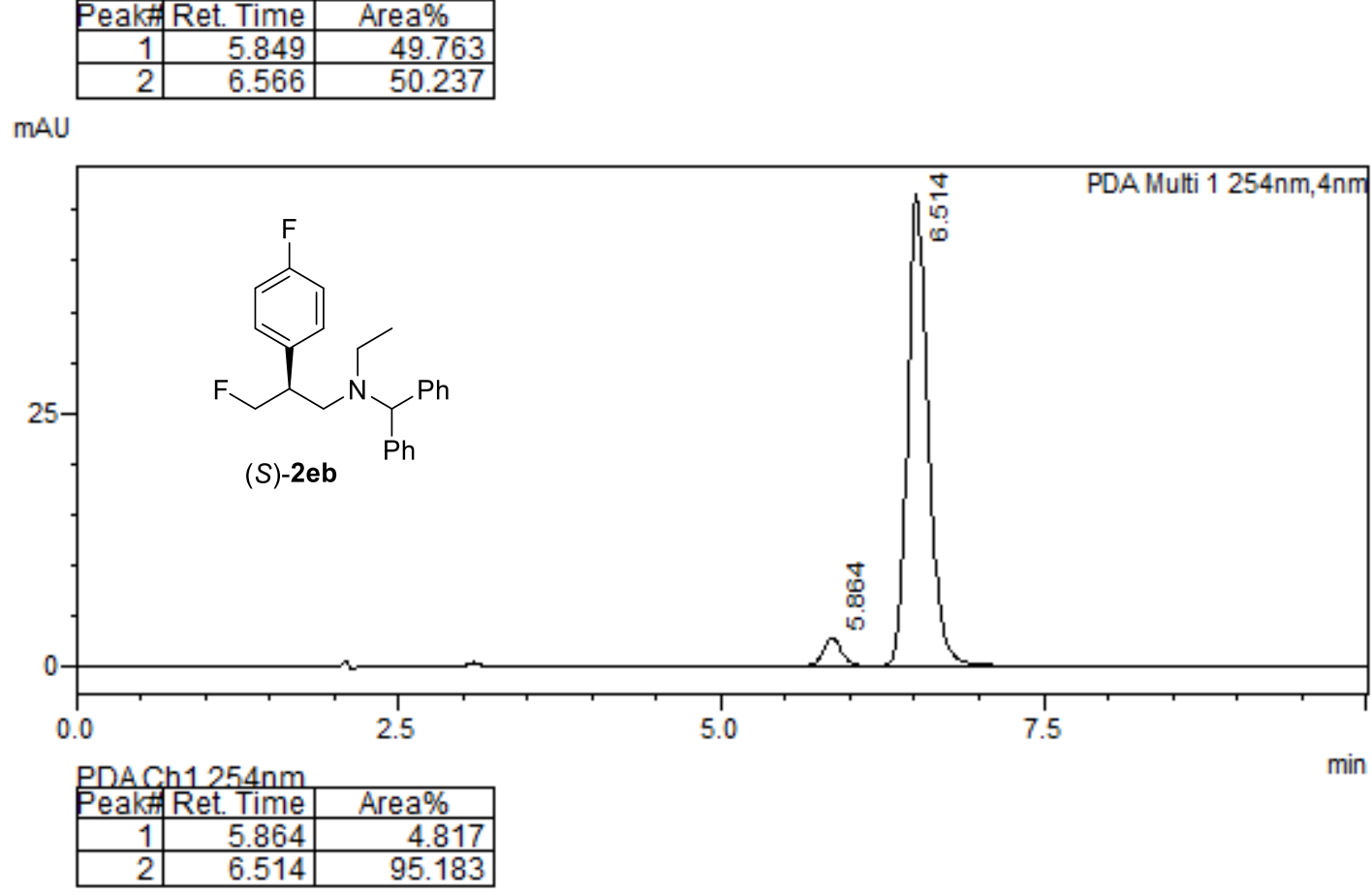


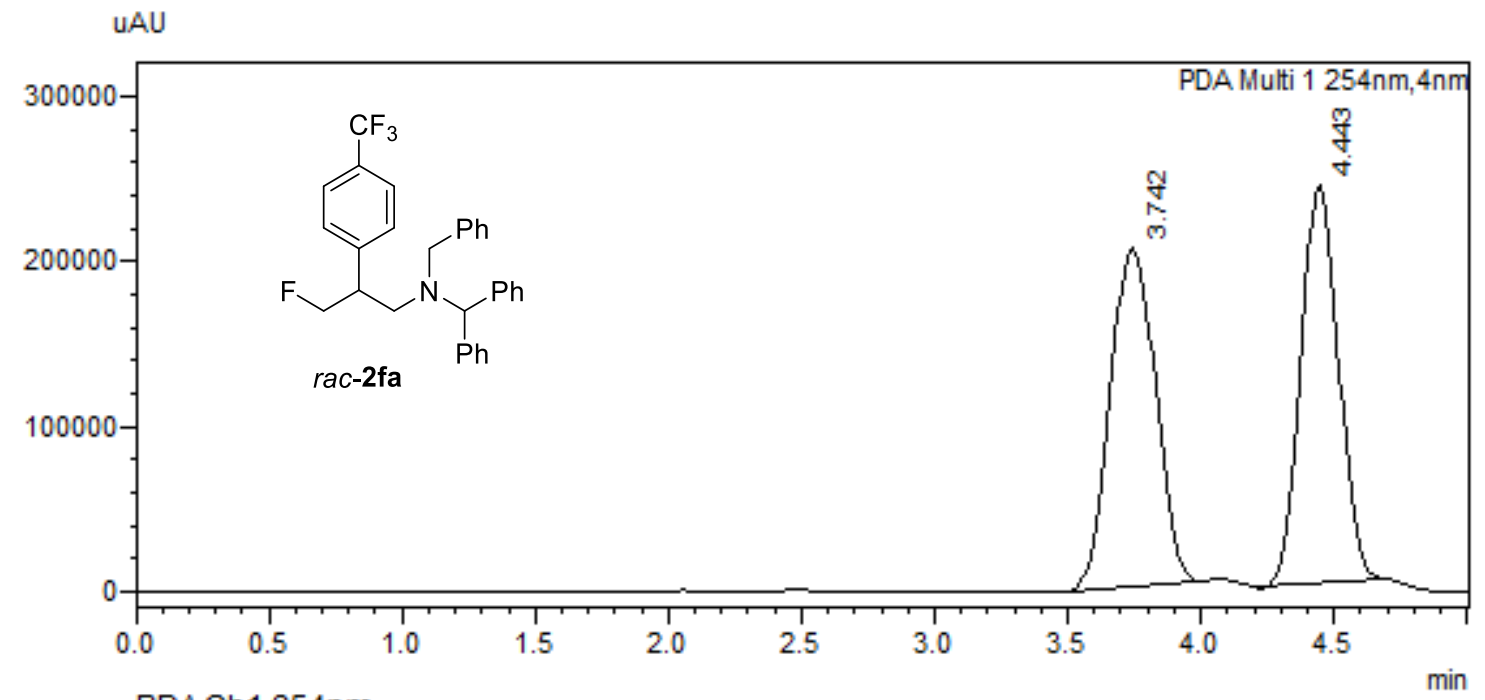

PDACh1254nm

\begin{tabular}{|r|r|r|}
\hline Peakff Ret. Time & Area\% \\
\hline 1 & 3.742 & 50.579 \\
\hline 2 & 4.443 & 49.421 \\
\hline
\end{tabular}

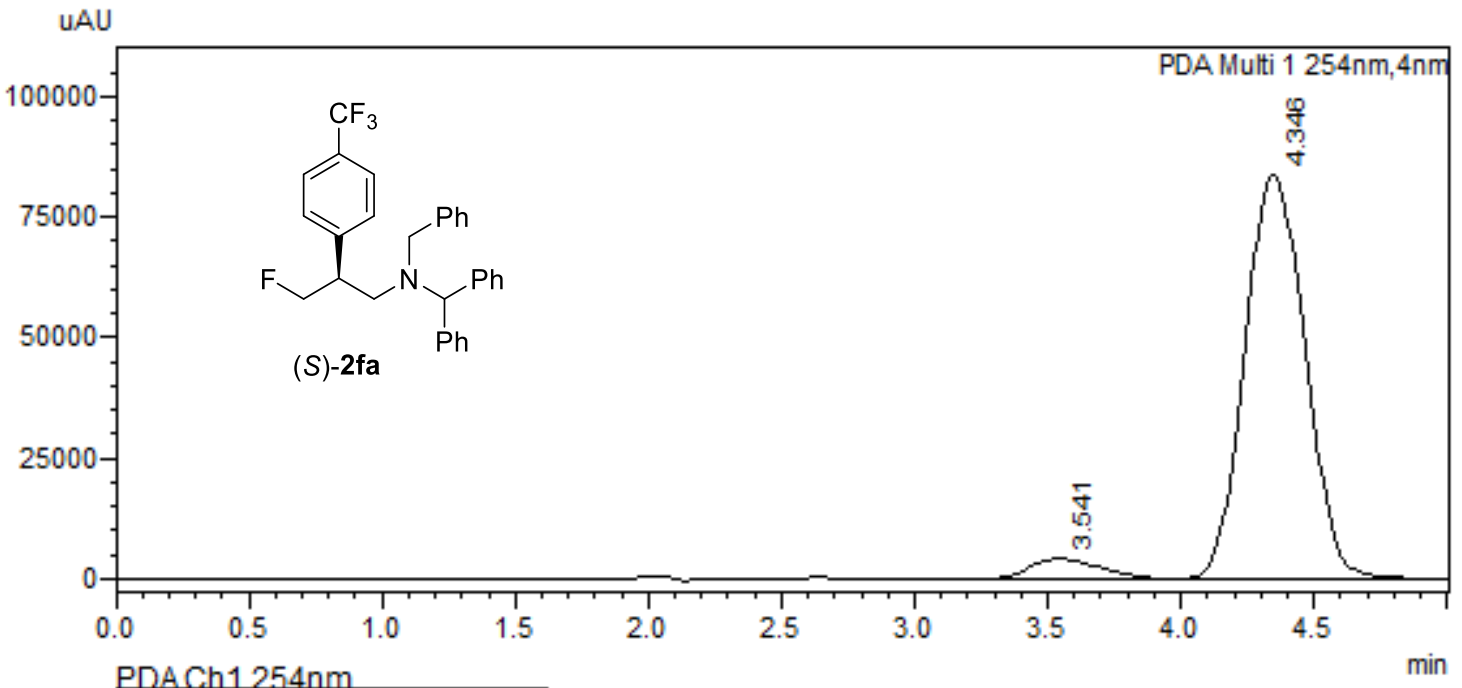

PDACh1254nm

\begin{tabular}{r|r|r|}
\hline 1 & 3.541 & 5.085 \\
\hline 2 & 4.346 & 94.915 \\
\hline
\end{tabular}



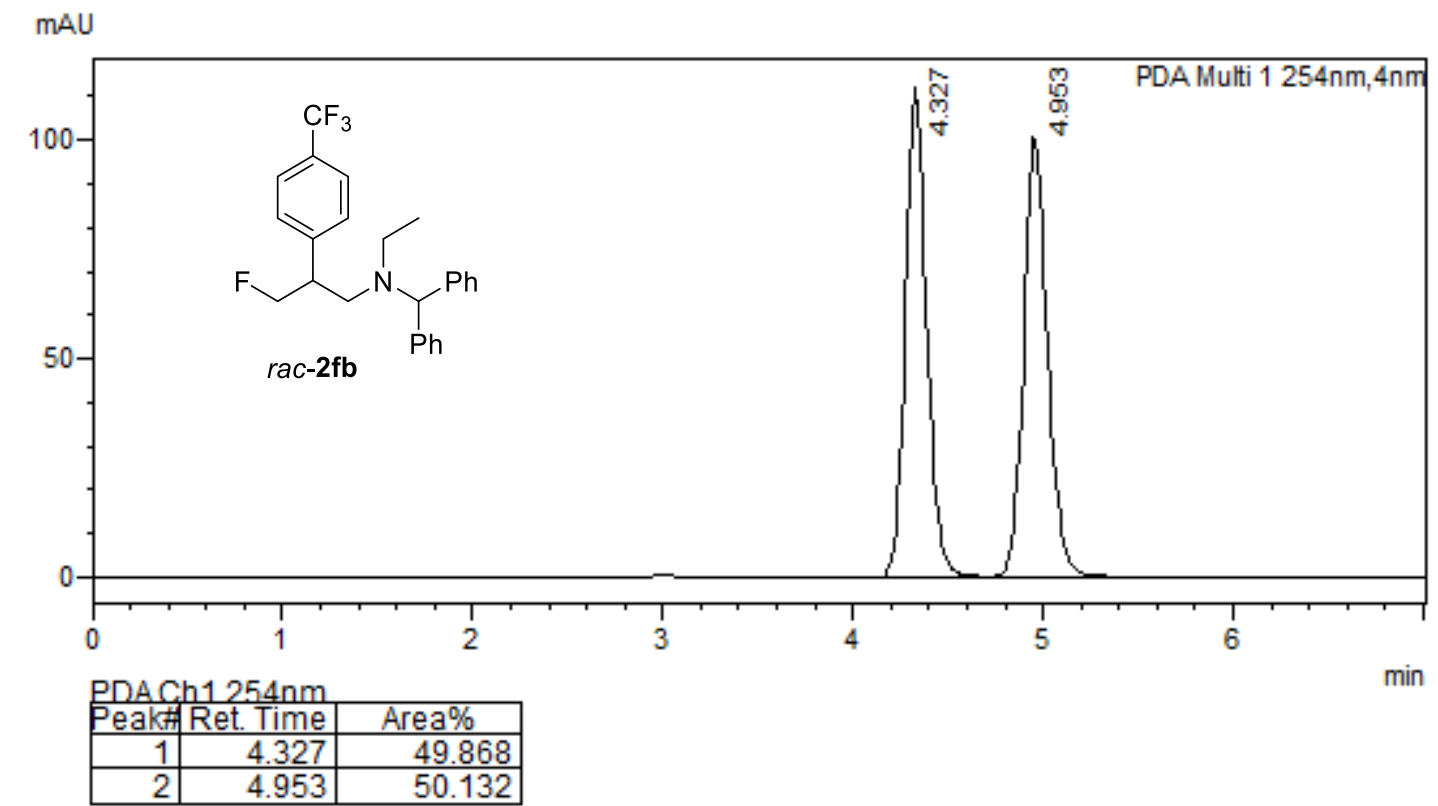

mAU

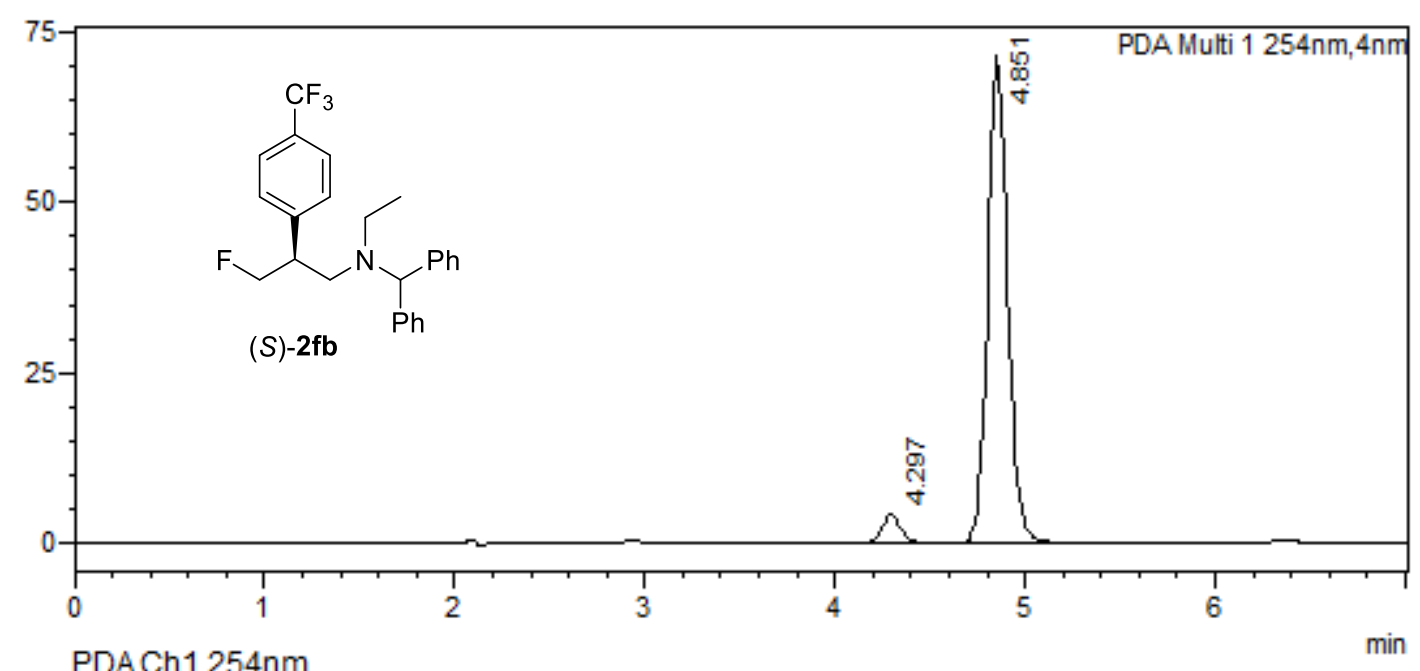

DACh1254nm

\begin{tabular}{|r|r|r|}
\hline Peakftret Time & \multicolumn{1}{c|}{ Area\% } \\
\hline 1 & 4.297 & 4.953 \\
\hline 2 & 4.851 & 95.047 \\
\hline
\end{tabular}



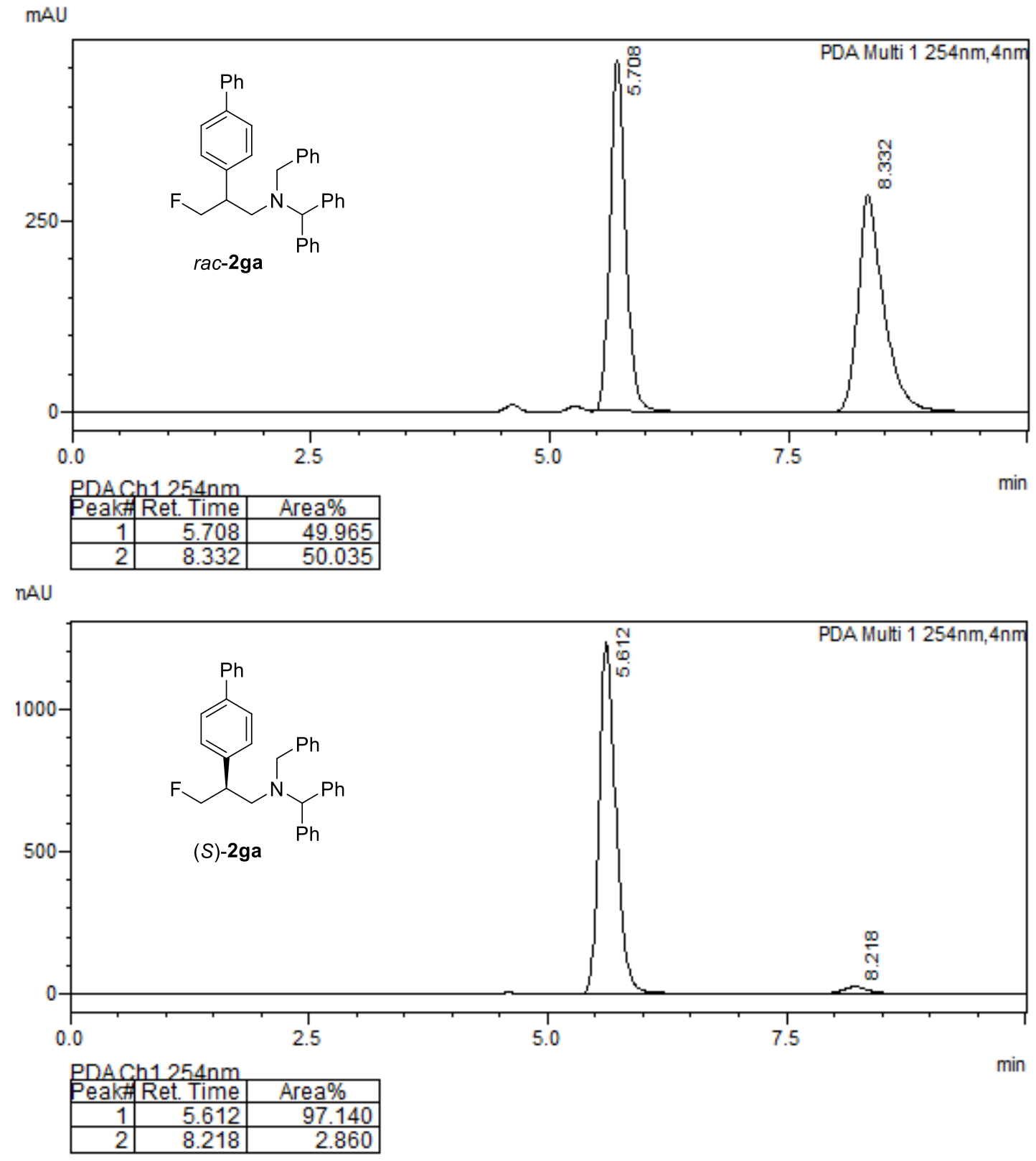


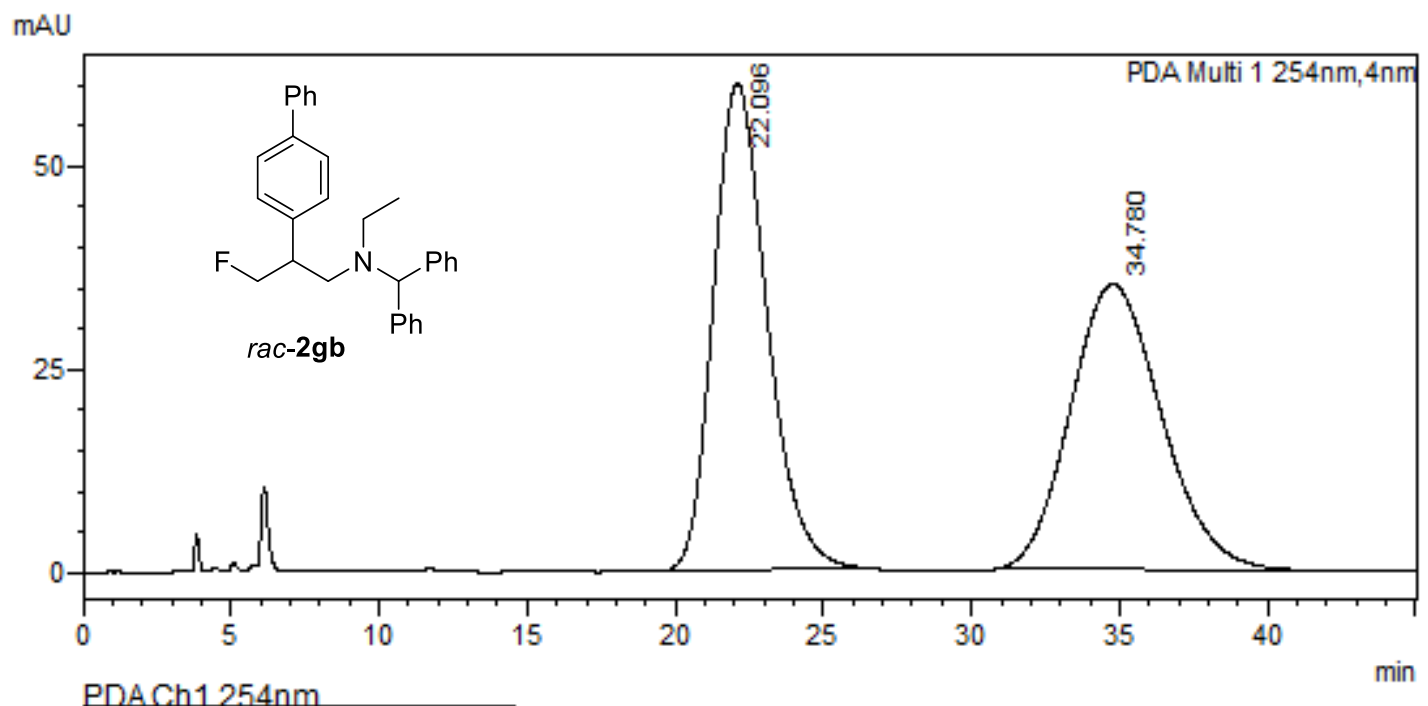

PDACh1254nm Area\%

\begin{tabular}{r|r|r|}
\hline 1 & 22.096 & 50.197 \\
\hline 2 & 34.780 & 49.803 \\
\hline
\end{tabular}

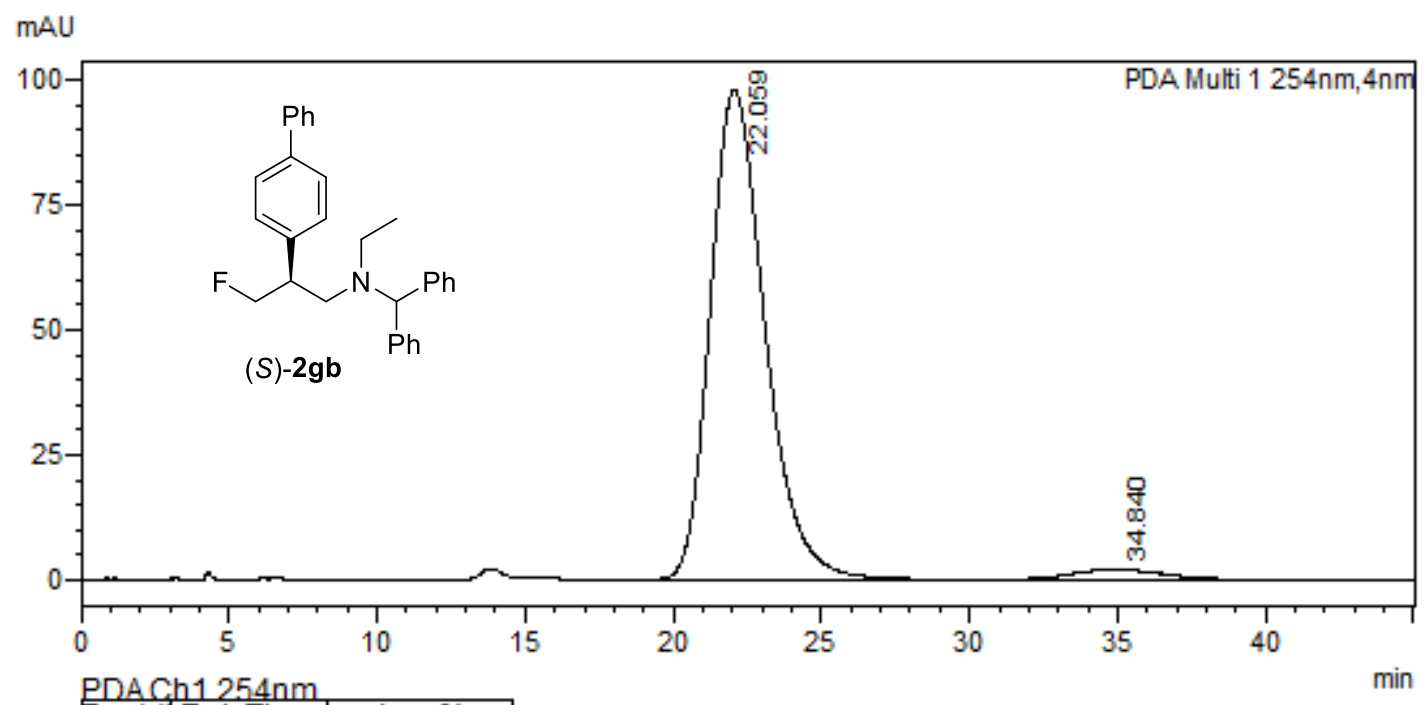

\begin{tabular}{|l|l}
\hline PeakA Ret. Time & Area\% \\
\hline
\end{tabular}

\begin{tabular}{r|r|r|}
1 & 22.059 & 96.772 \\
\hline 2 & 34.840 & 3.228 \\
\hline
\end{tabular} 


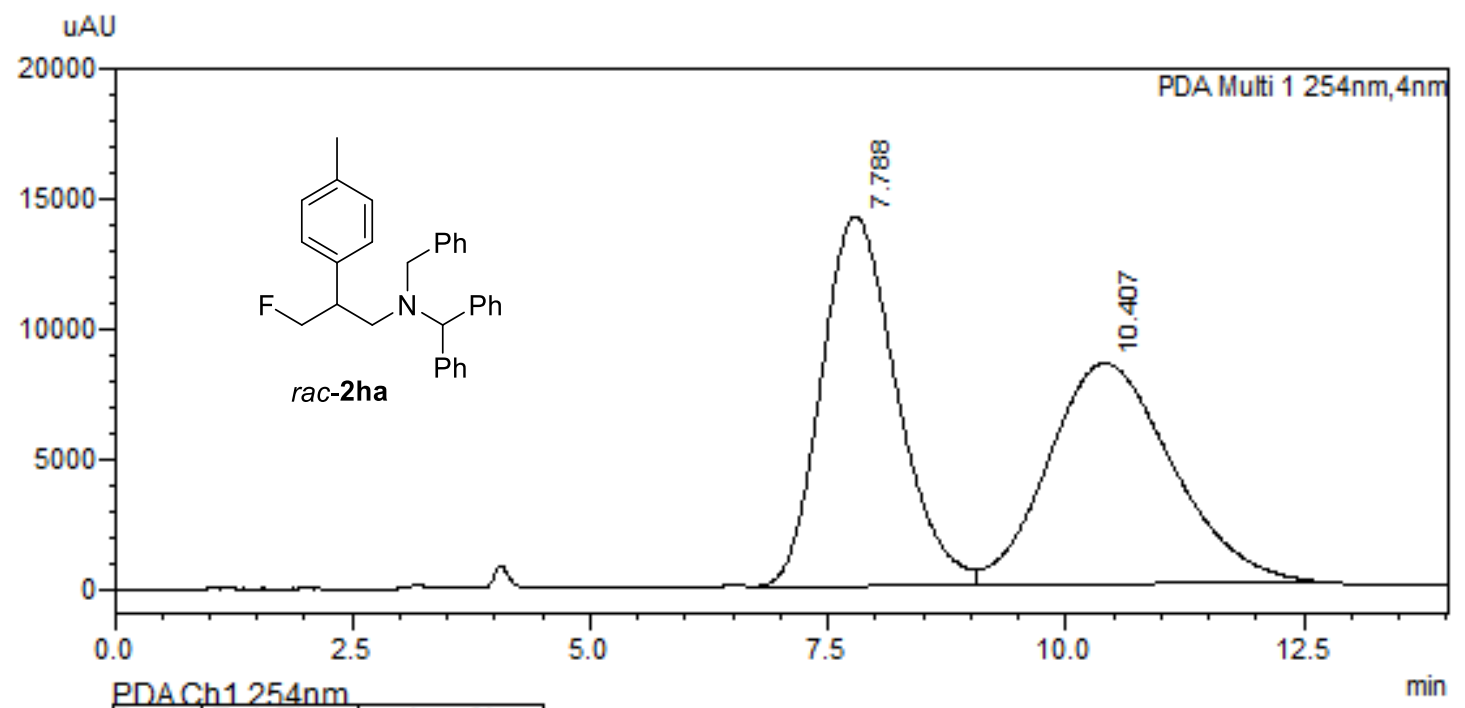

PDACh1254nm
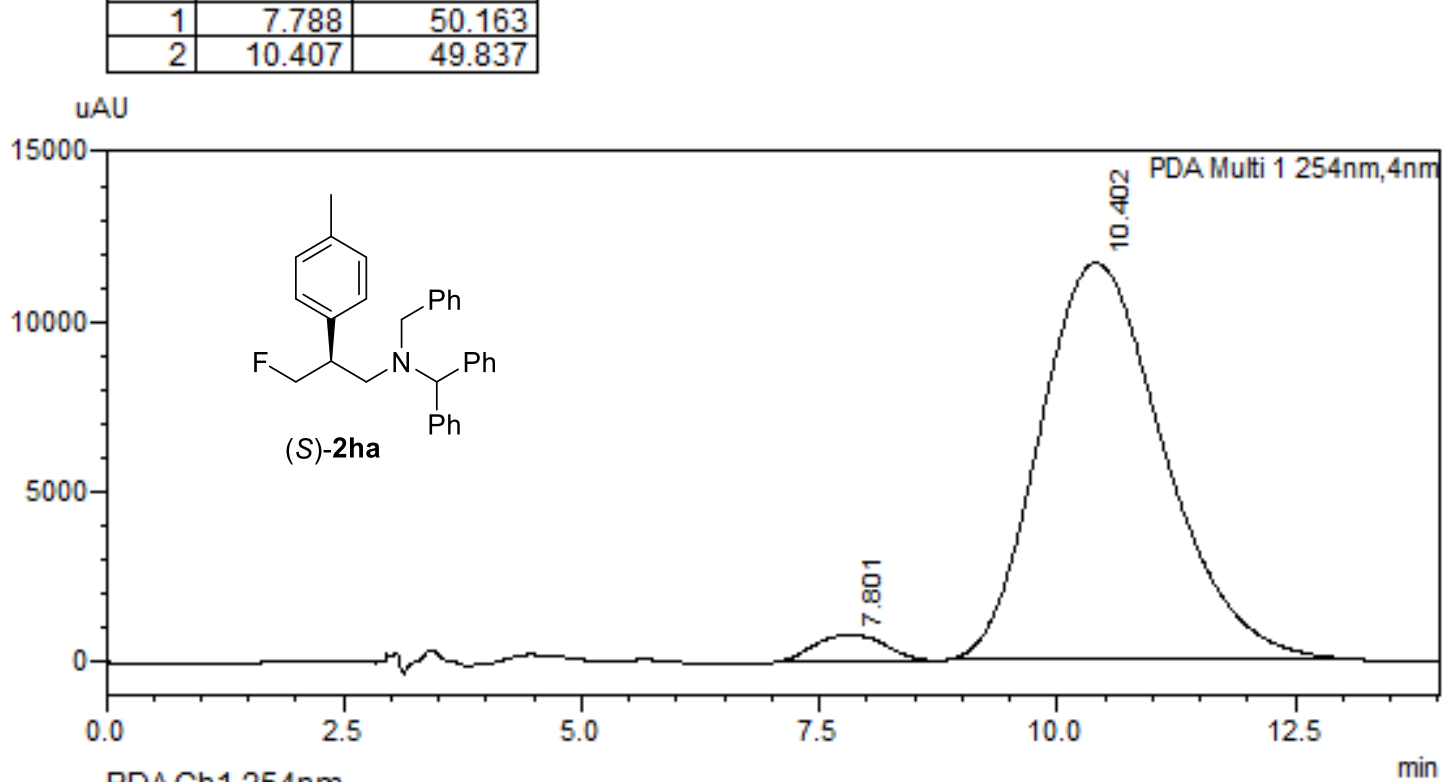

PDACh1 254nm

Peak\# Ret. Time $\quad$ Area\%

\begin{tabular}{|r|r|r|}
1 & 7.801 & 3.499 \\
\hline 2 & 10.402 & 96.501 \\
\hline
\end{tabular} 

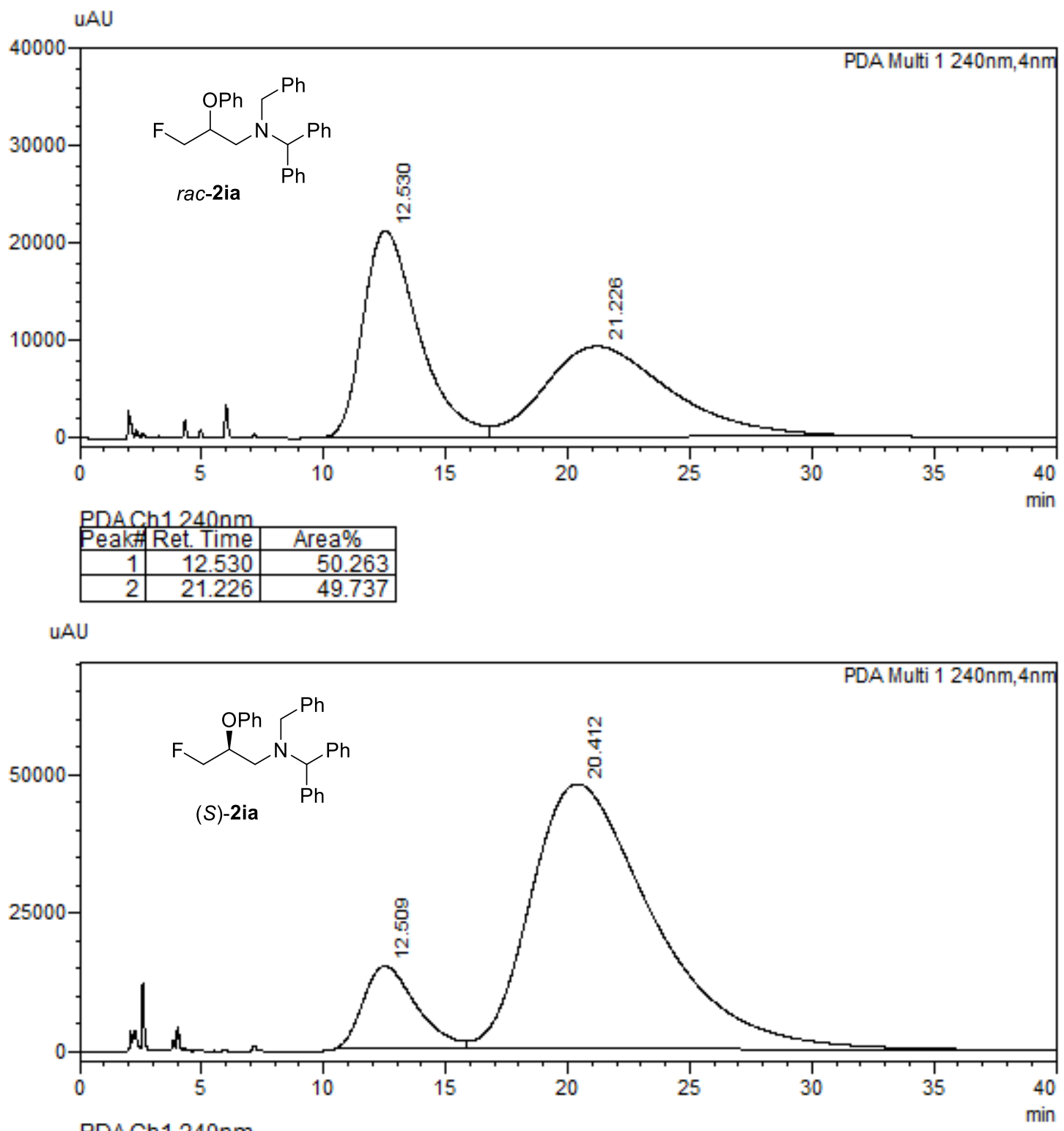

PRACh1 240nm

\begin{tabular}{|r|r|r|}
\hline Peakf Ret. Time & Area\% \\
\hline 1 & 12.509 & 11.923 \\
\hline 2 & 20.412 & 88.077 \\
\hline
\end{tabular} 

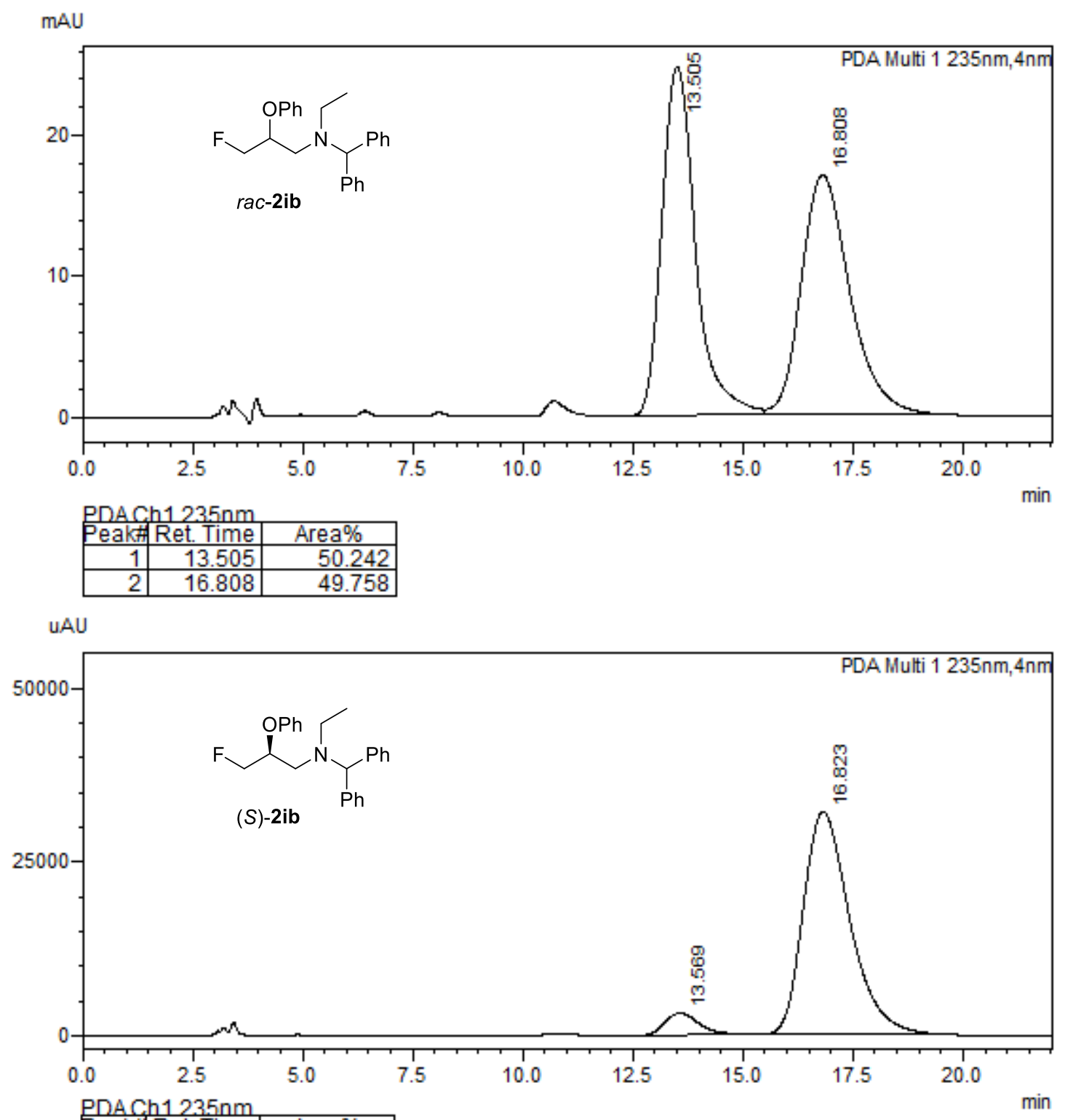

PDACh123.5nm

\begin{tabular}{r|r|r|}
\hline 1 & 13.569 & 6.534 \\
\hline 2 & 16.823 & 93.466 \\
\hline
\end{tabular} 


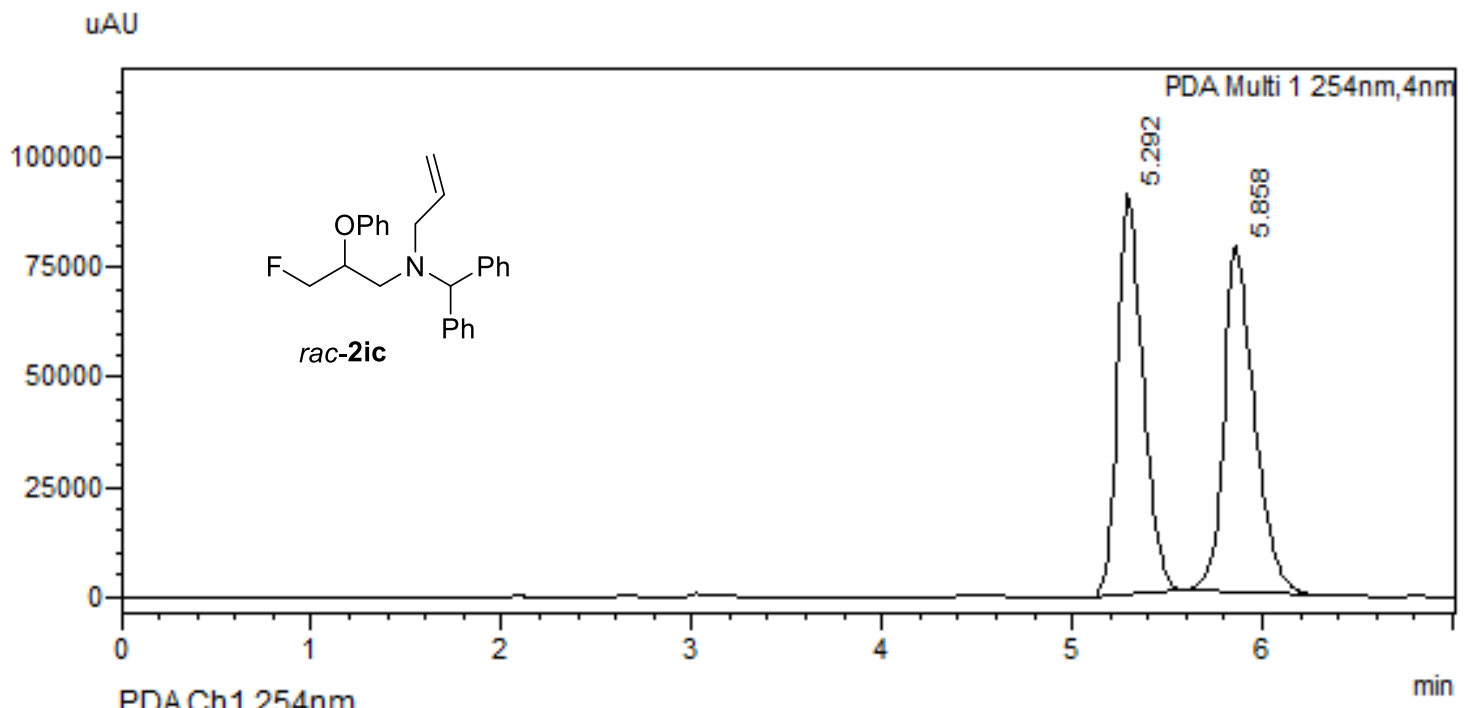

PDACh1 254nm

Peak\# Ret. Time Area\%

\begin{tabular}{l|l|l|}
1 & 5.292 & 49.052 \\
\hline 2 & 5.858 & 50.948 \\
\hline
\end{tabular}

UAU

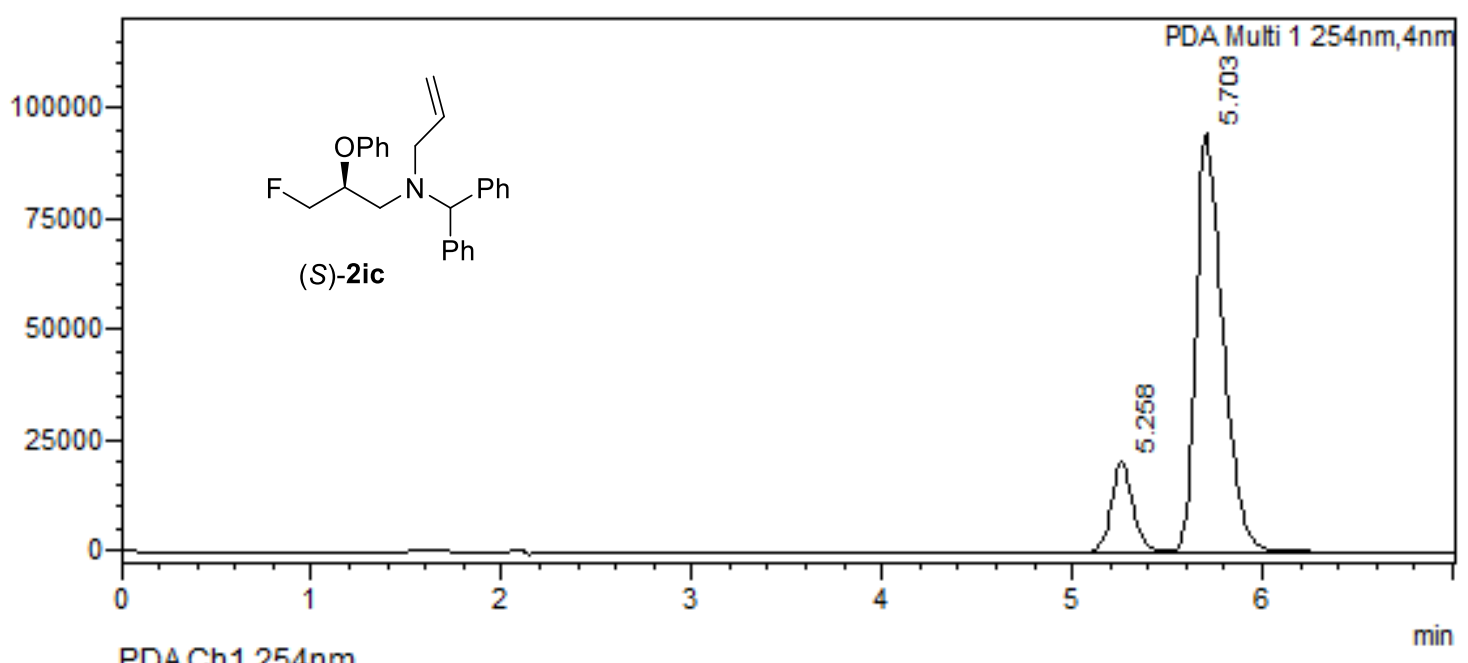

PDA Ch1 254nm

Peak\# Ret. Time Area\%

\begin{tabular}{l|l|l|}
1 & 5.258 & 14.81 \\
\hline 2 & 5.703 & 85.184 \\
\hline
\end{tabular}

\begin{tabular}{ll}
5.703 & 85.184 \\
\hline
\end{tabular} 

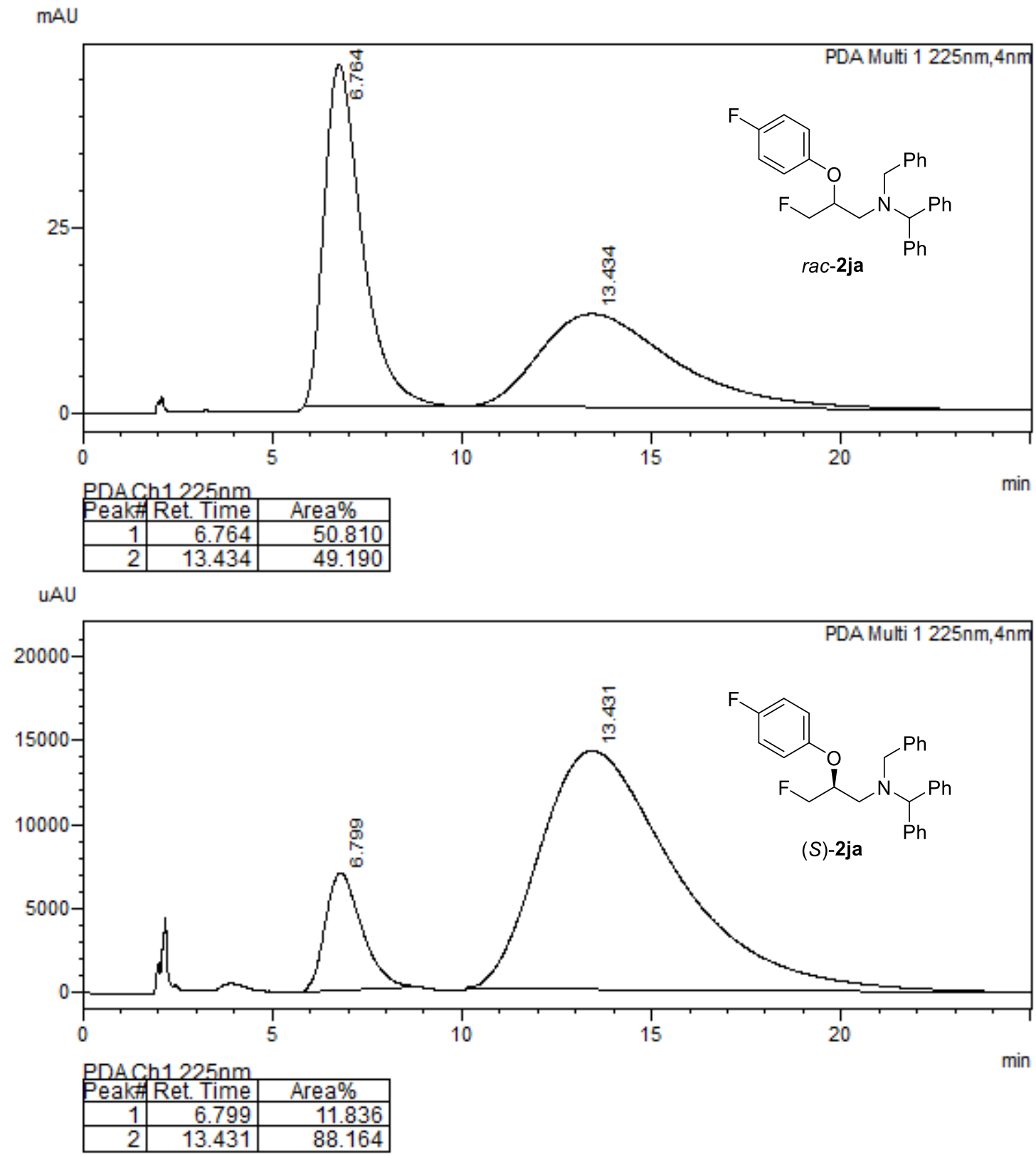


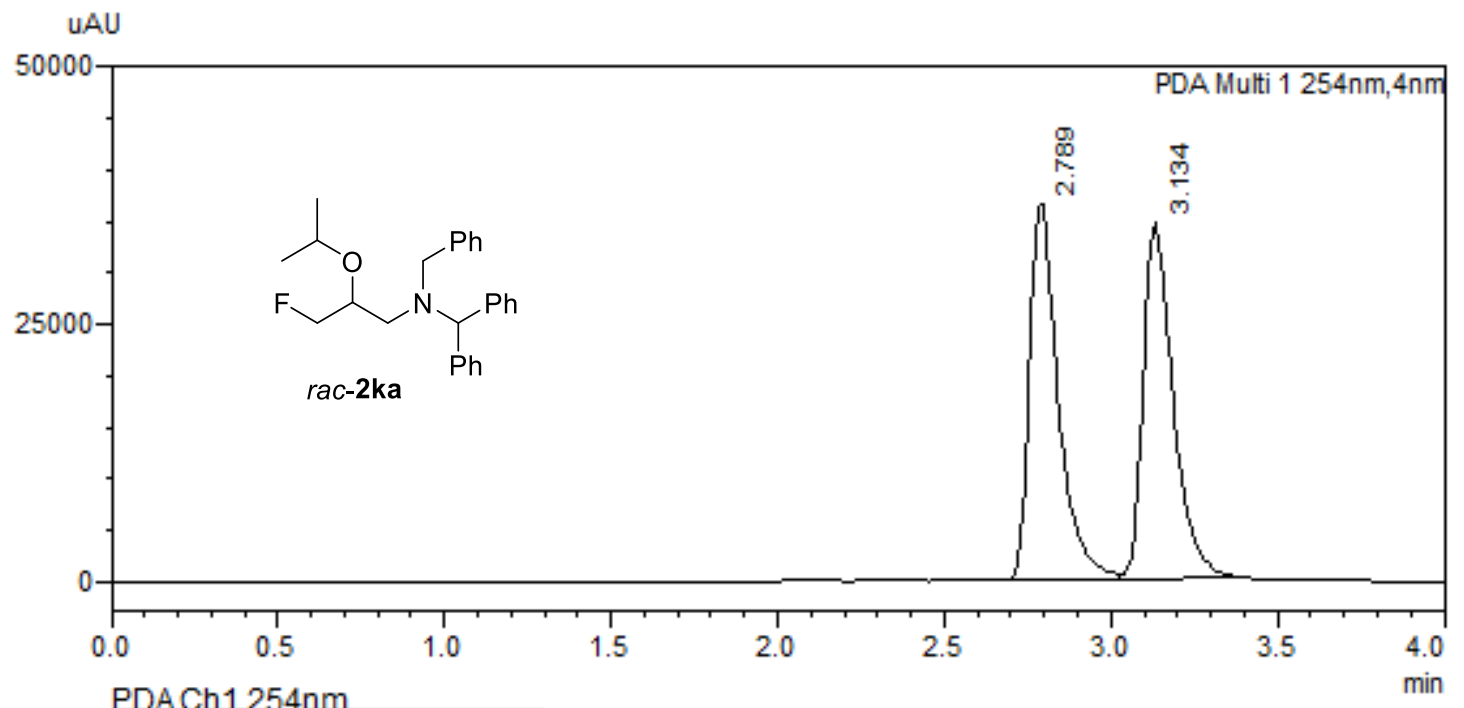

PDACh1254nm

\begin{tabular}{l|l} 
Peakf Ret. Time & Area\%
\end{tabular}

\begin{tabular}{l|l|l|}
1 & 2.789 & 50.217 \\
\hline 2 & 3.134 & 49.783
\end{tabular}

UAU

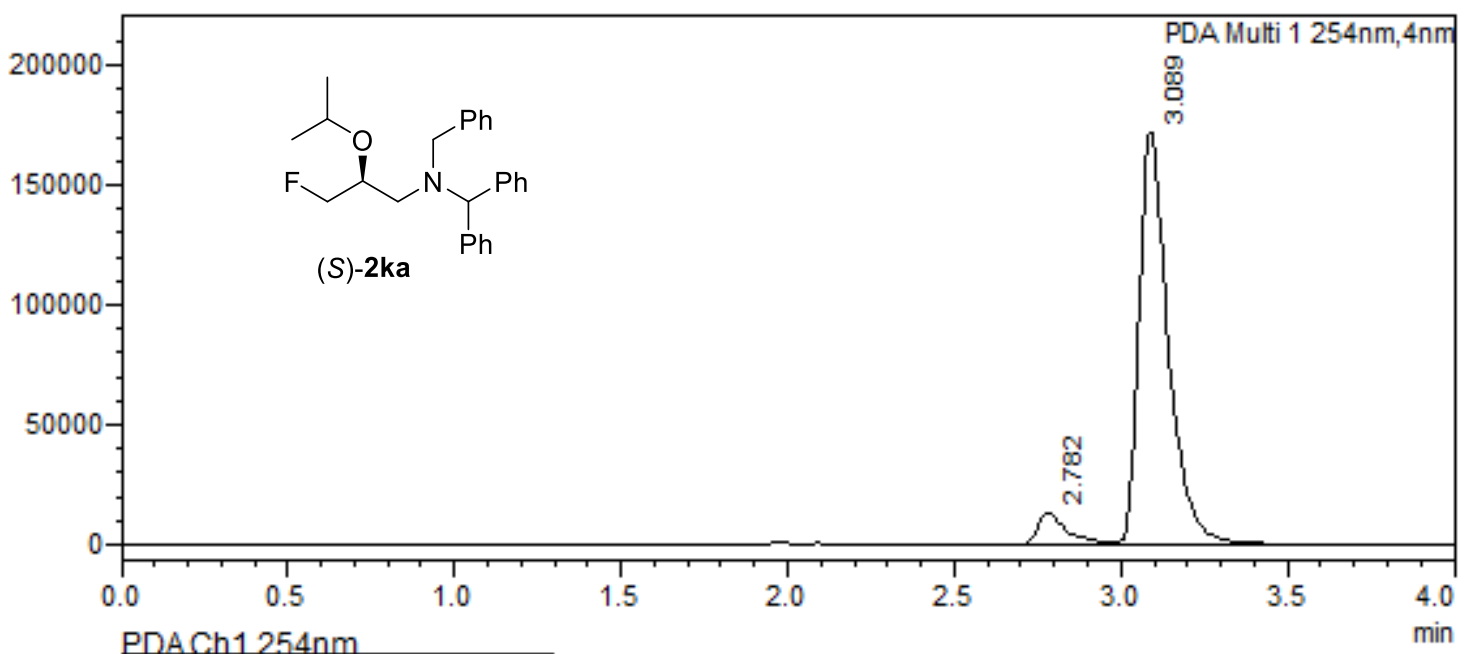

PDACh1254nm

Area\%

\begin{tabular}{r|r|r|}
\hline 1 & 2.782 & 7.111 \\
\hline & 3.089
\end{tabular}

\begin{tabular}{l|l|l|}
\hline 2 & 3.089 & 92.889 \\
\hline
\end{tabular} 


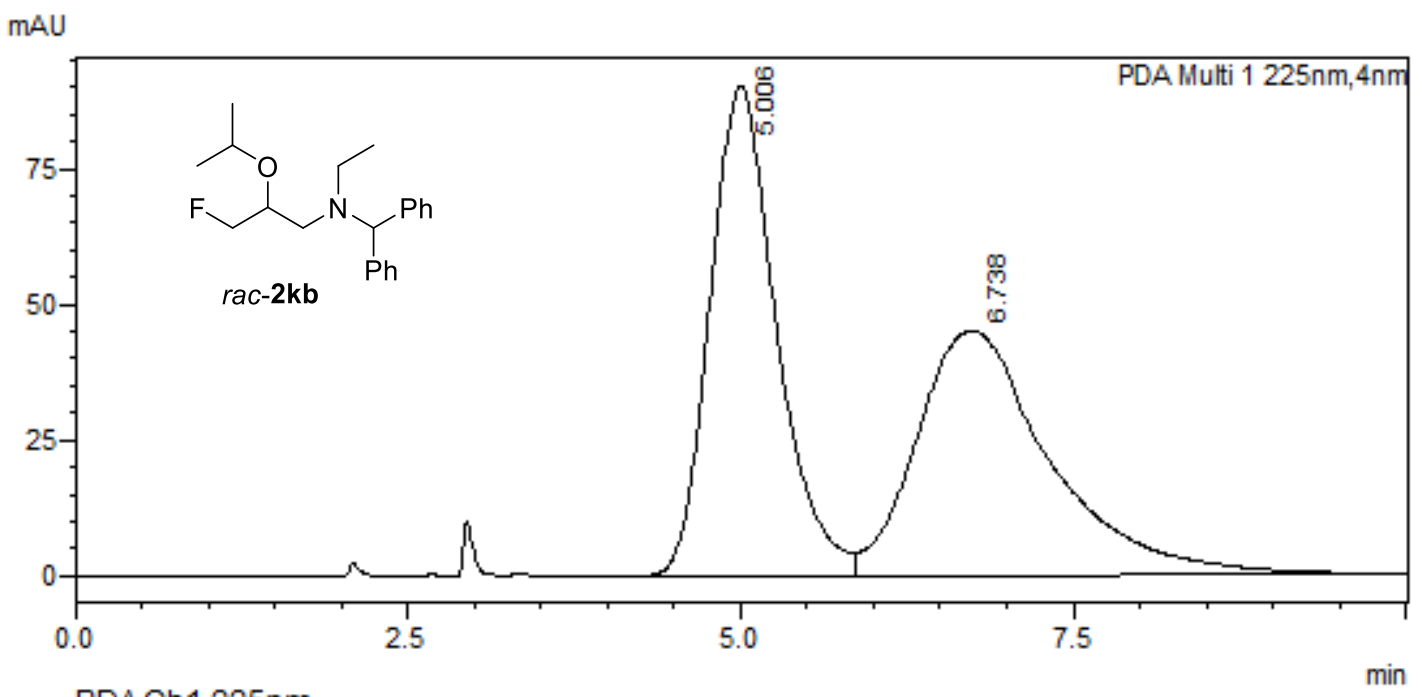

PRACh $1225 \mathrm{~nm}$

\begin{tabular}{|r|r|r|}
\hline Peakftrime & Ret. Tirea\% \\
\hline 1 & 5.006 & 49.962 \\
\hline 2 & 6.738 & 50.038 \\
\hline
\end{tabular}

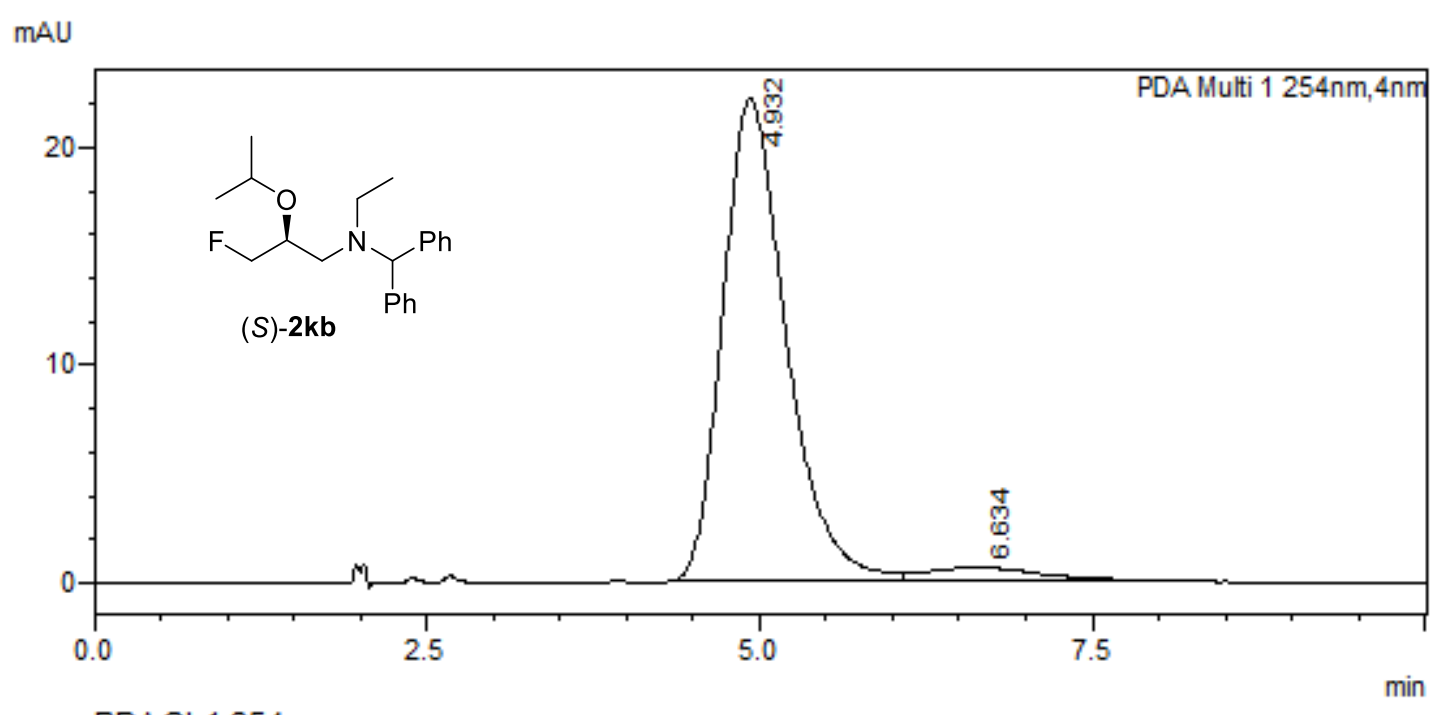

PDACh1254nm

\begin{tabular}{|l|l|}
\hline Peakf Ret. Time & Area\% \\
\hline 1 &
\end{tabular}

\begin{tabular}{l|l|r|}
1 & 4.932 & 94.796 \\
\hline
\end{tabular}

5.204 


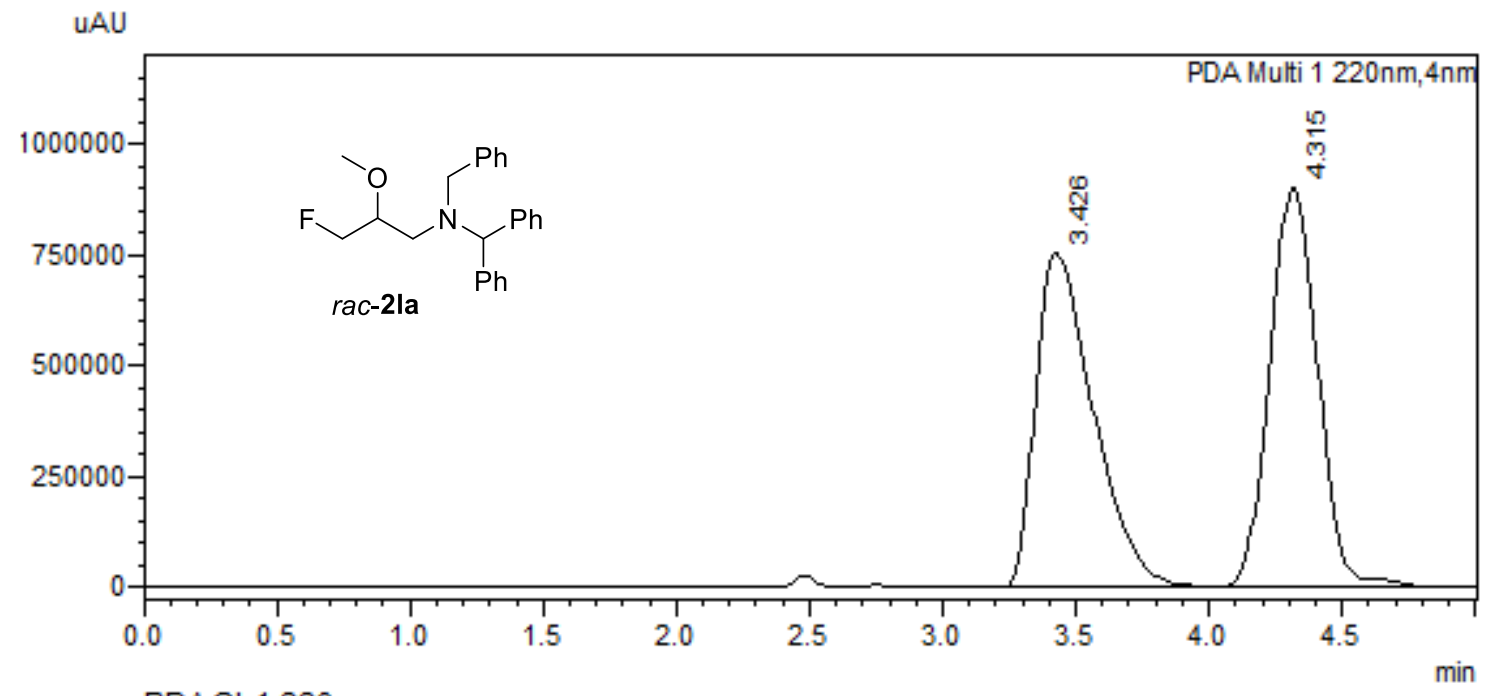

PRACh1 $220 \mathrm{~nm}$

PeakA Ret. Time Area\%

\begin{tabular}{l|l|l|}
1 & 3.426 & 50.265 \\
\hline 2 & 4.315 & 49.735 \\
\hline
\end{tabular}

UAU

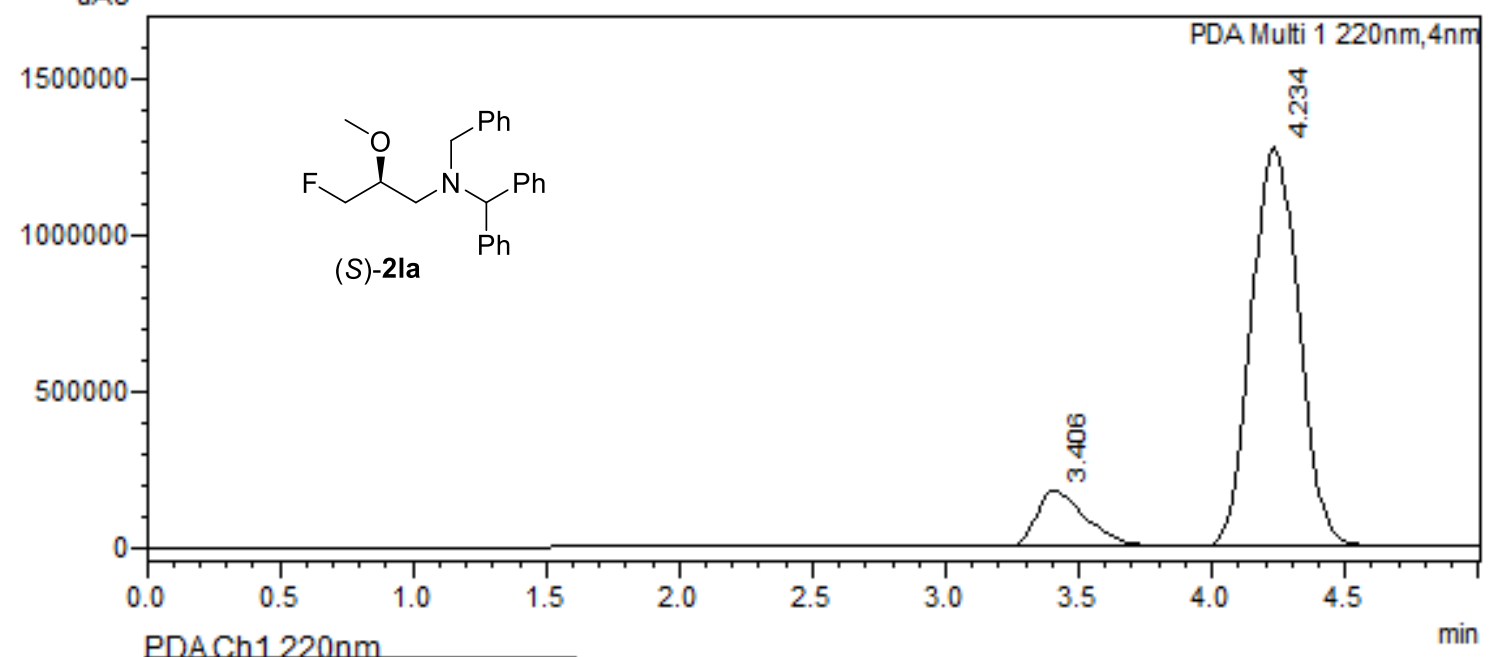

PDACh1220nm

Peak\# Ret. Time 1 Area\%

\begin{tabular}{l|r|r}
1 & 3.406 & 12.011 \\
\hline 2 & 4.234 & 87.989 \\
\hline
\end{tabular}



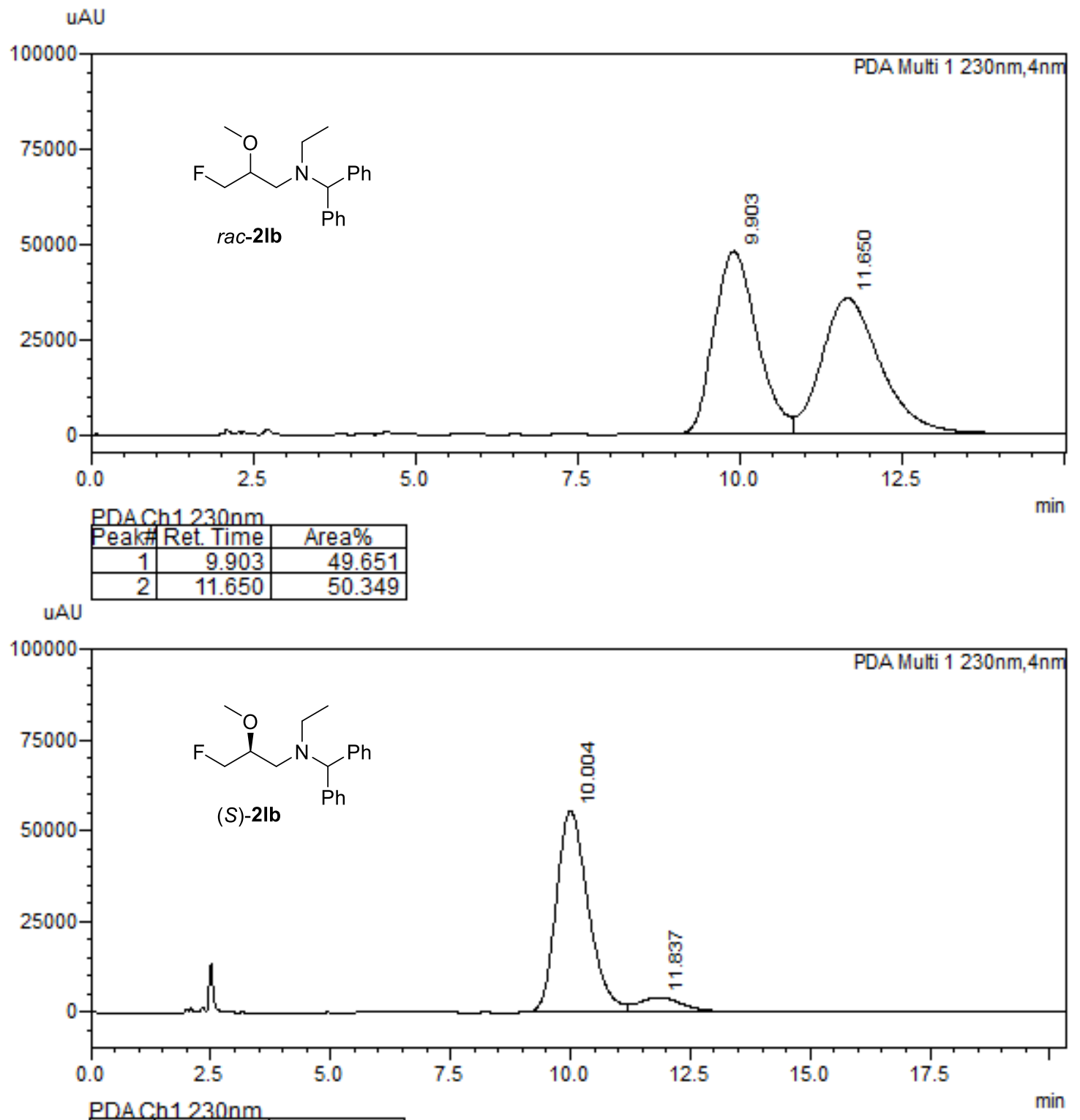

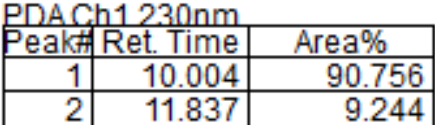




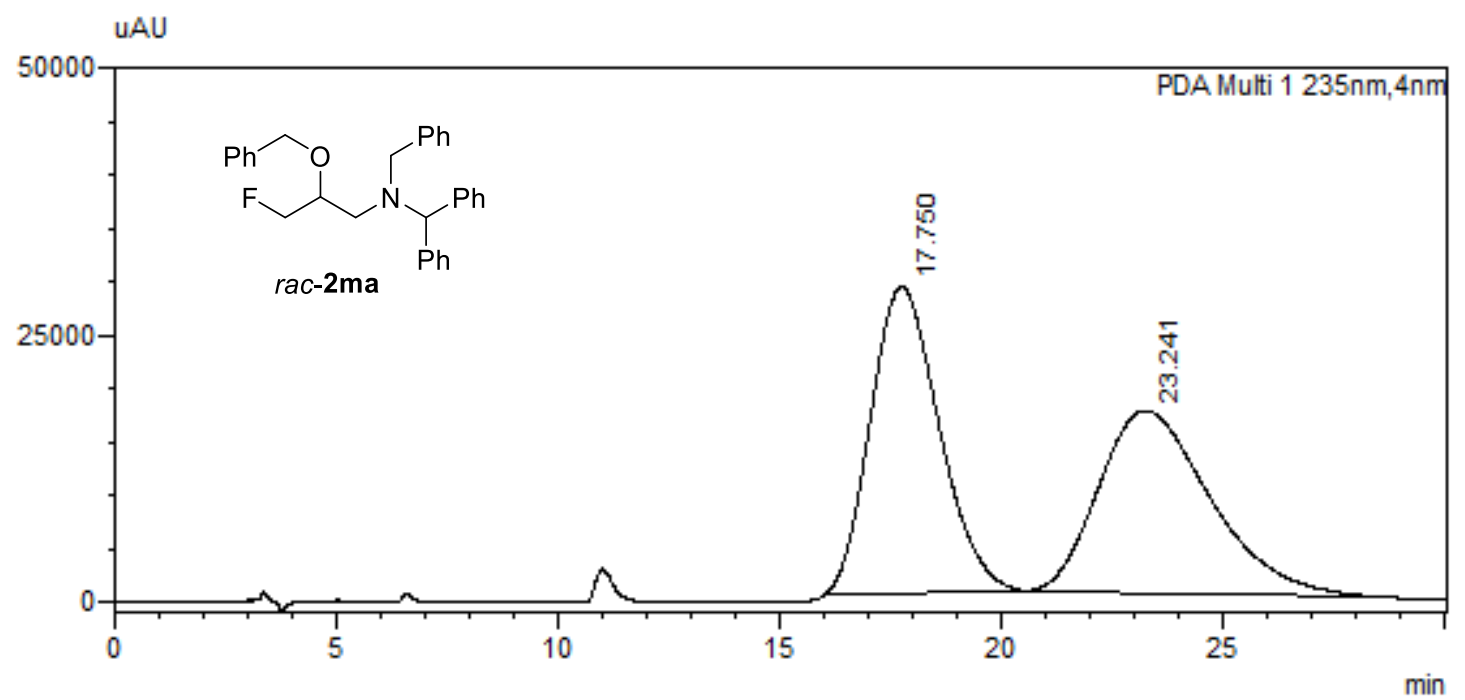

PDACh123.5nm

Peak\#Ret. Time $\quad$ Area\%

\begin{tabular}{l|l|l|}
1 & 17.750 & 50.853 \\
\hline 2 & 23.241 & 49.147 \\
\hline
\end{tabular}

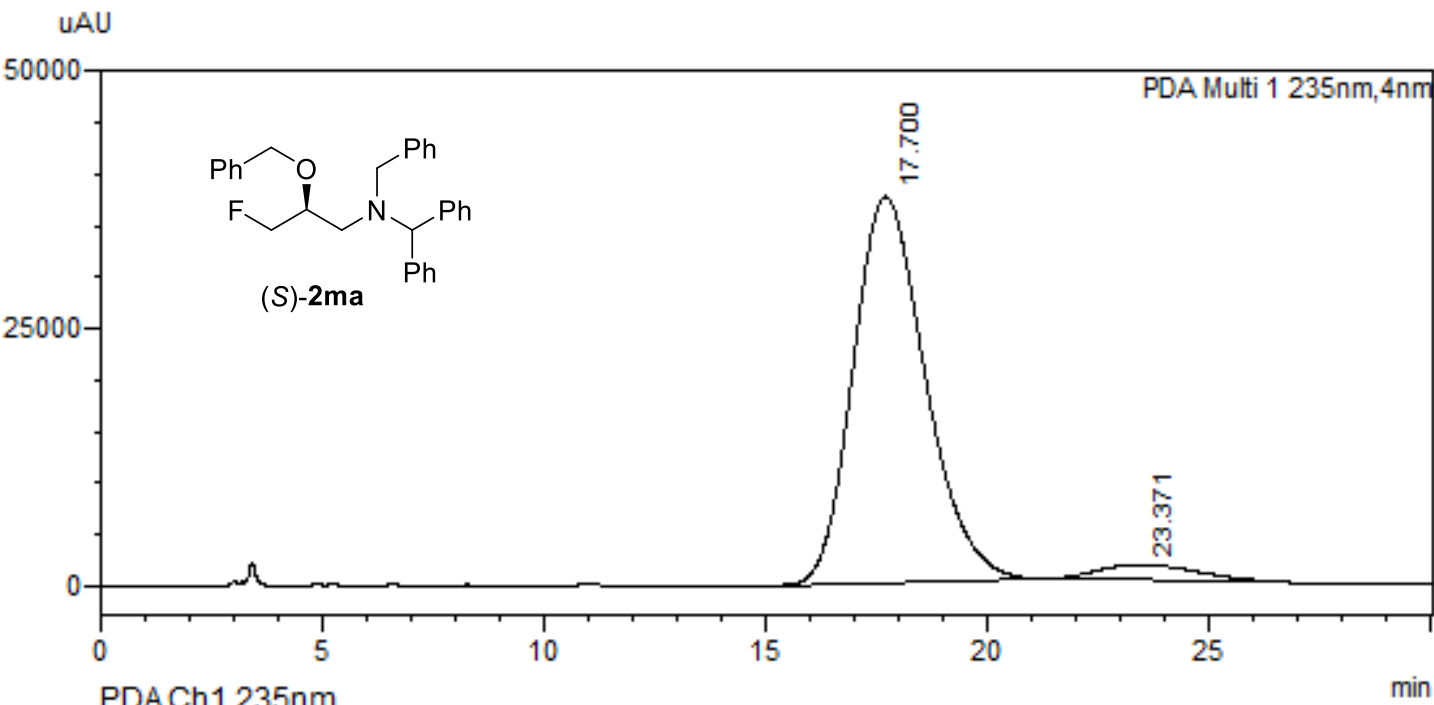

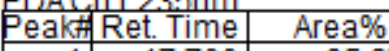




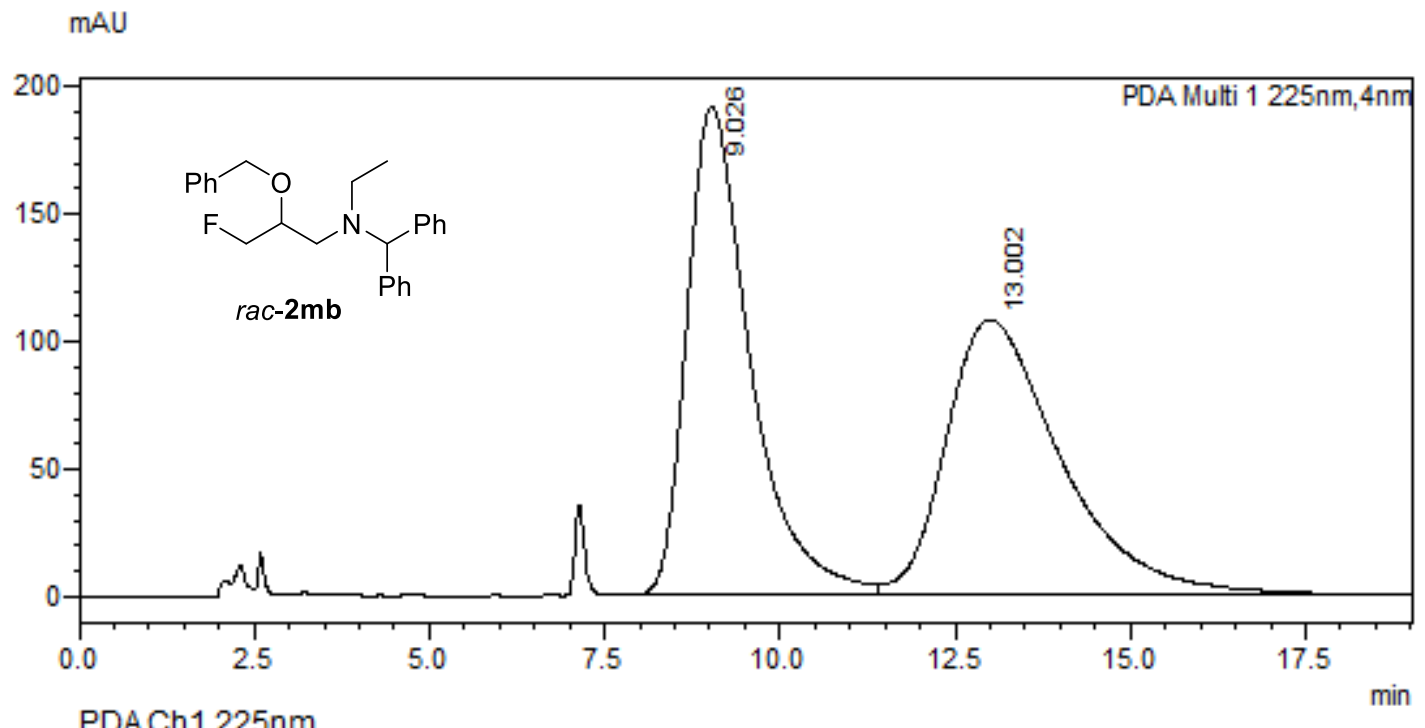

PDACh1225nm

\begin{tabular}{|r|r|r|}
\hline 1 & 9.026 & 49.371 \\
\hline 2 & 13.002 & 50.629 \\
\hline
\end{tabular}

mAU

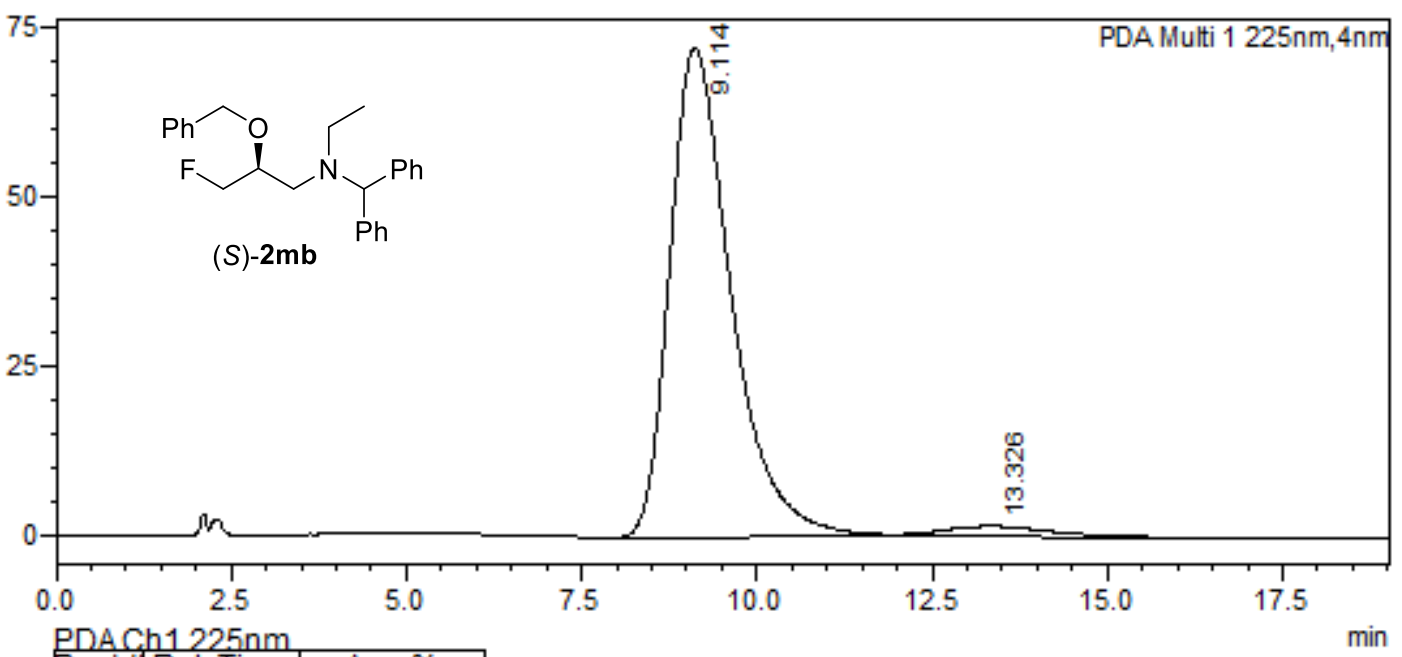

PDACh1 $225 \mathrm{~nm}$ Preakf Ret. Time

\begin{tabular}{|r|r|r|}
1 & 9.114 & 96.998 \\
\hline
\end{tabular}

\begin{tabular}{|r|r|r|}
\hline 2 & 13.326 & 3.002 \\
\hline
\end{tabular} 


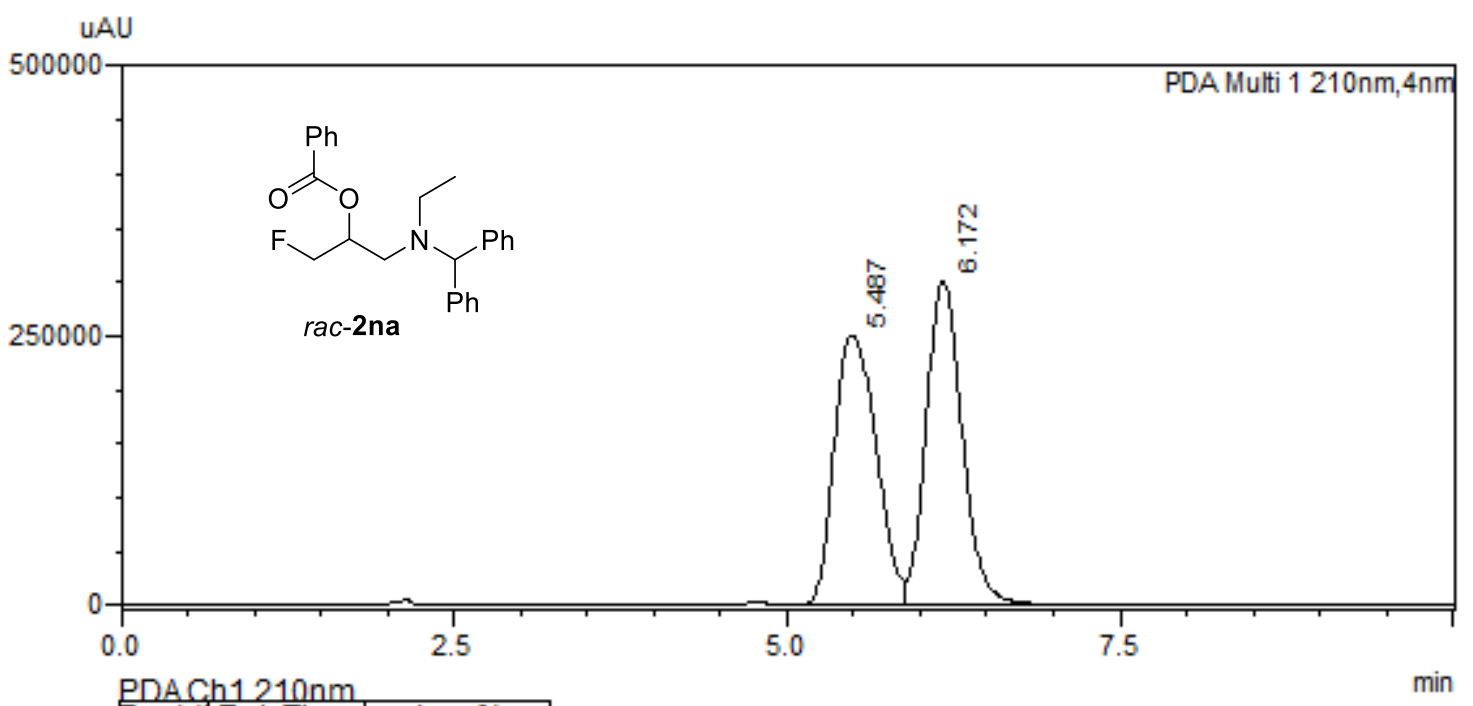

PDA.ah $210 \mathrm{~nm}$

Peak\#Ret. Time $\quad$ Area \%

\begin{tabular}{r|r|r|}
1 & 5.487 & 49.268 \\
\hline 2 & 6.172 & 50.732 \\
\hline
\end{tabular}

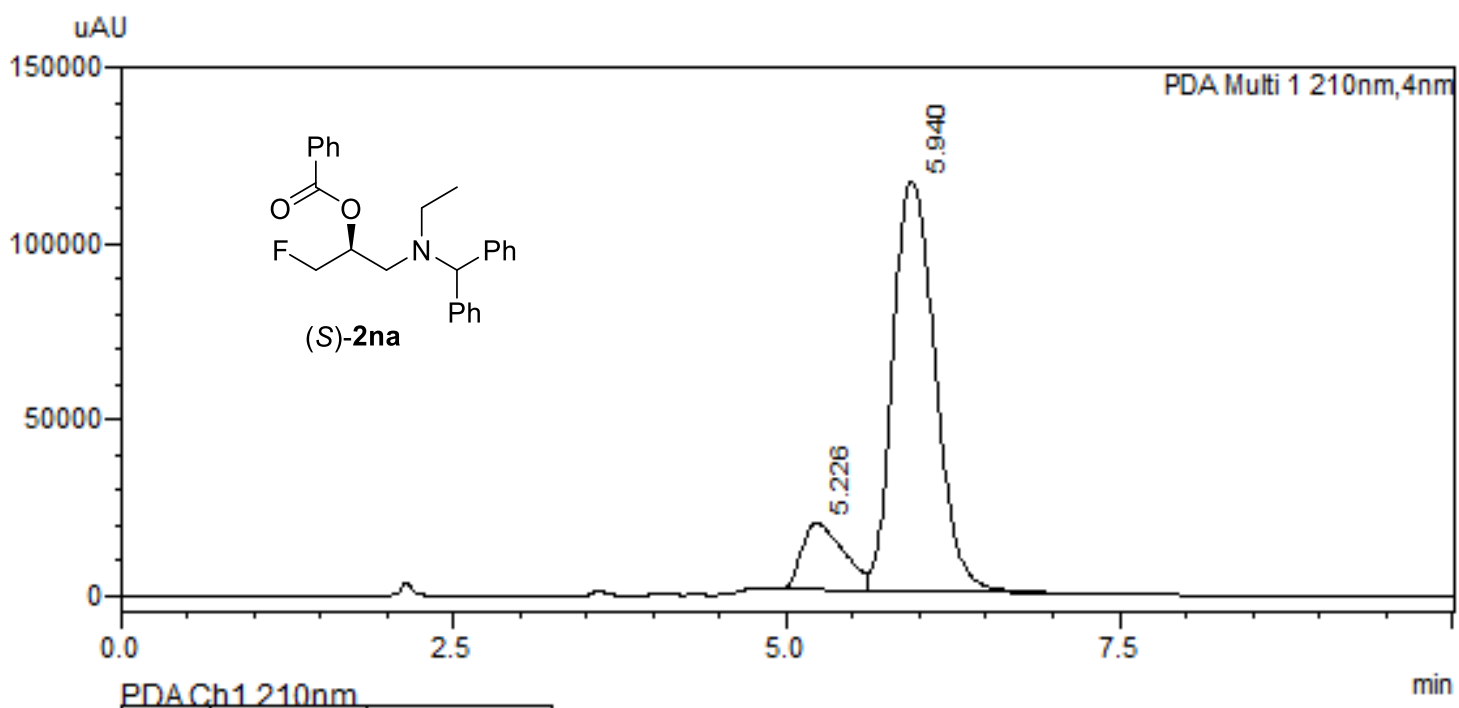

Peakf Ret. Time $\quad$ Area\%

$1 \quad 5.226$

13.72

86.277 

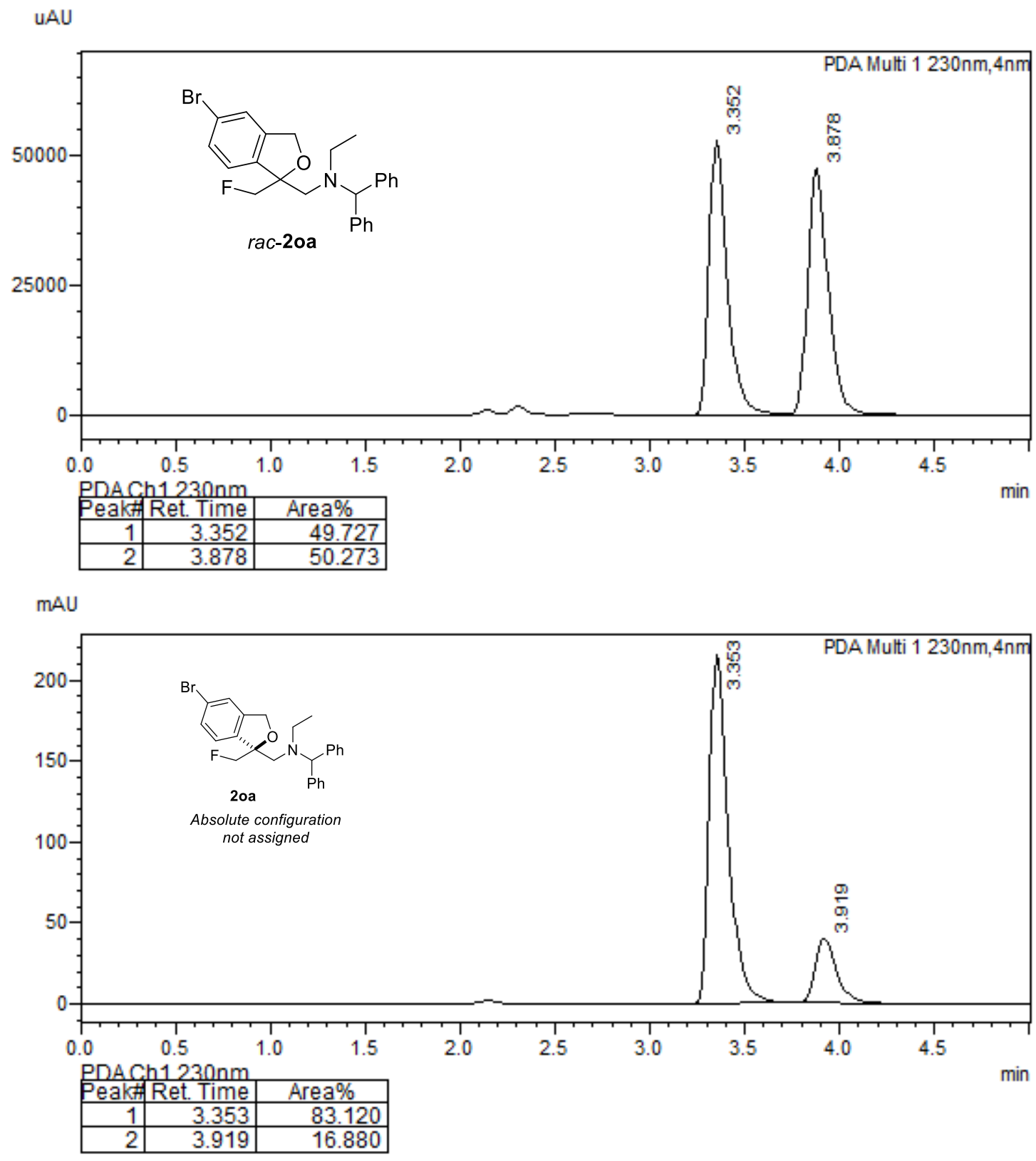

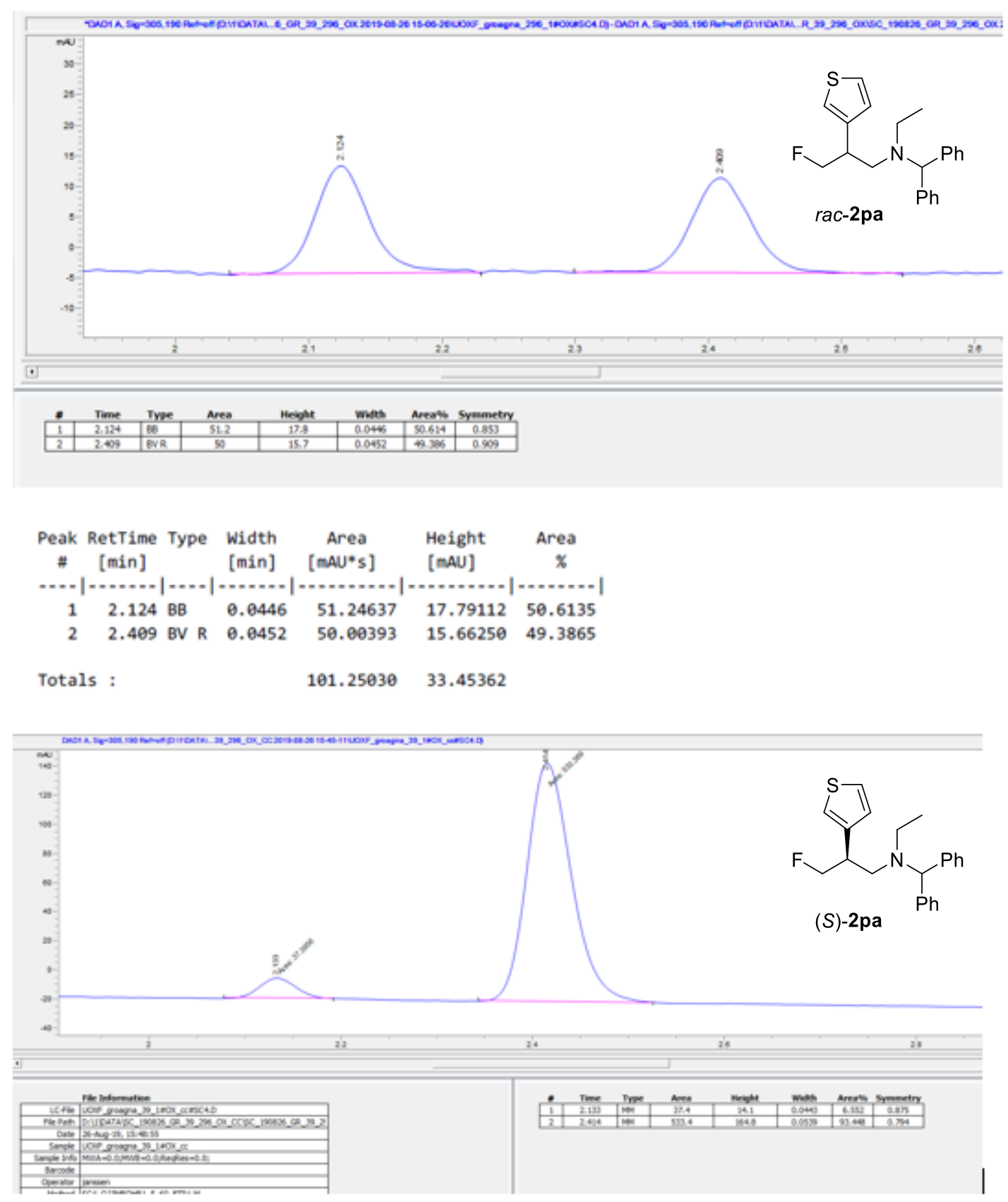

Signal 1: DAD1 A, Sig=305, 190 Ref=off

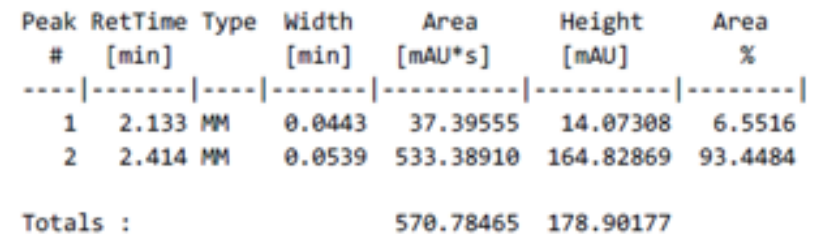




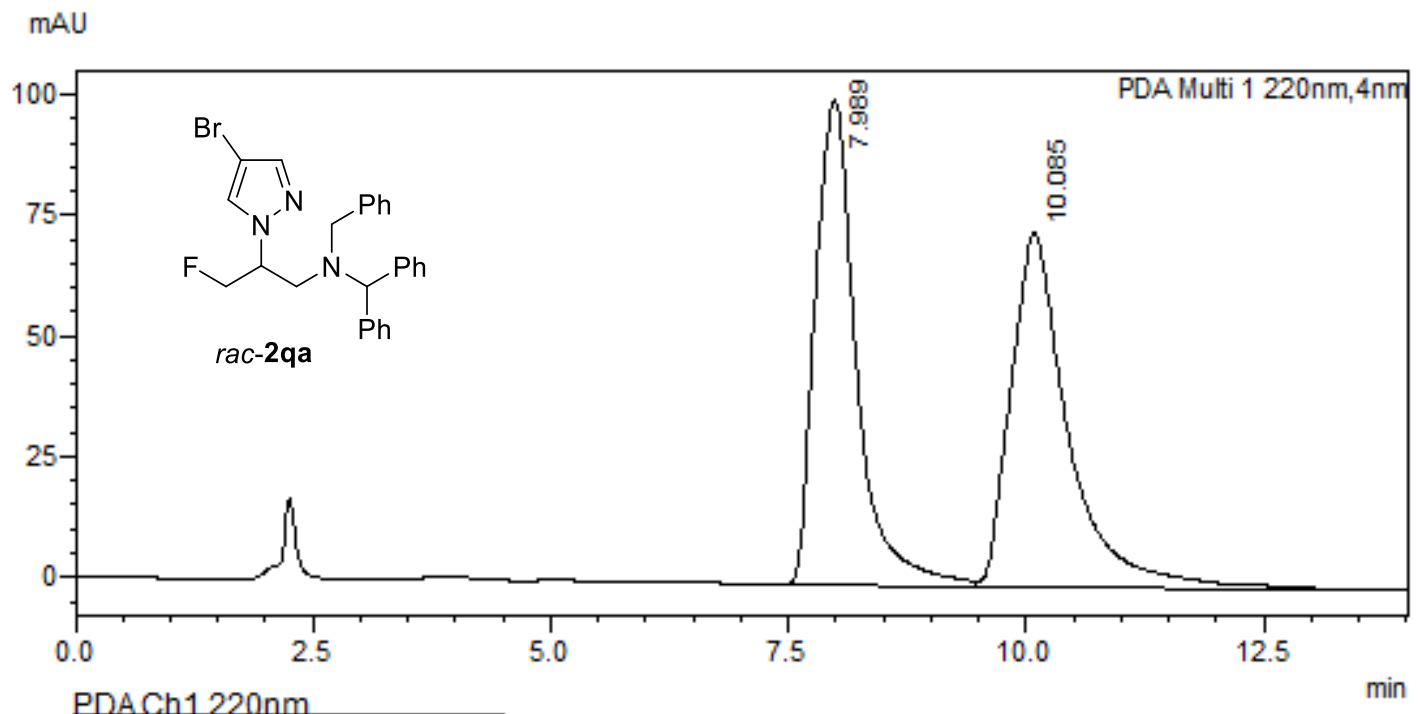

\begin{tabular}{l|l} 
Peakf Ret. Time & Area\%
\end{tabular}
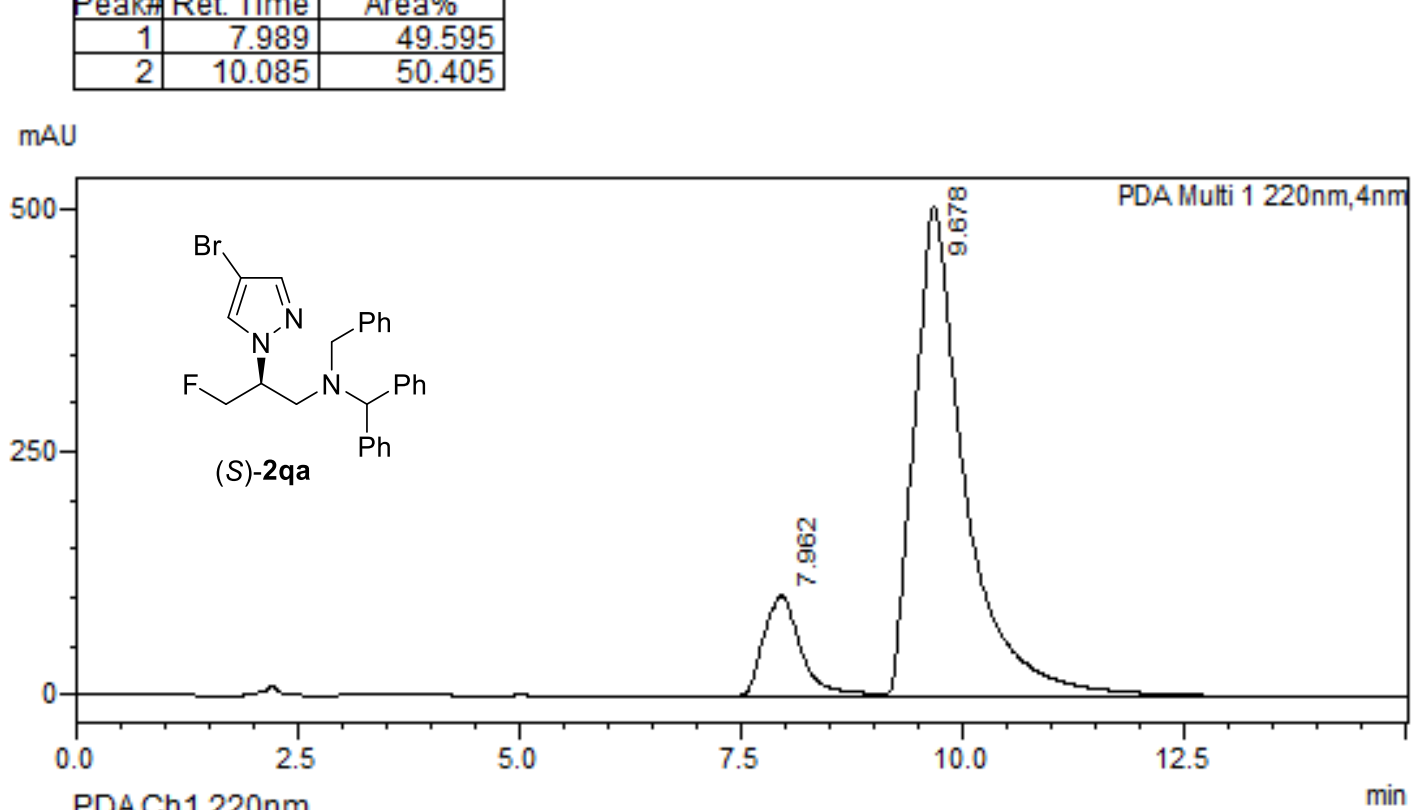

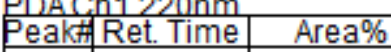

\begin{tabular}{r|r|r|}
\hline 1 & 7.962 & 13.106 \\
\hline
\end{tabular}

86.894 


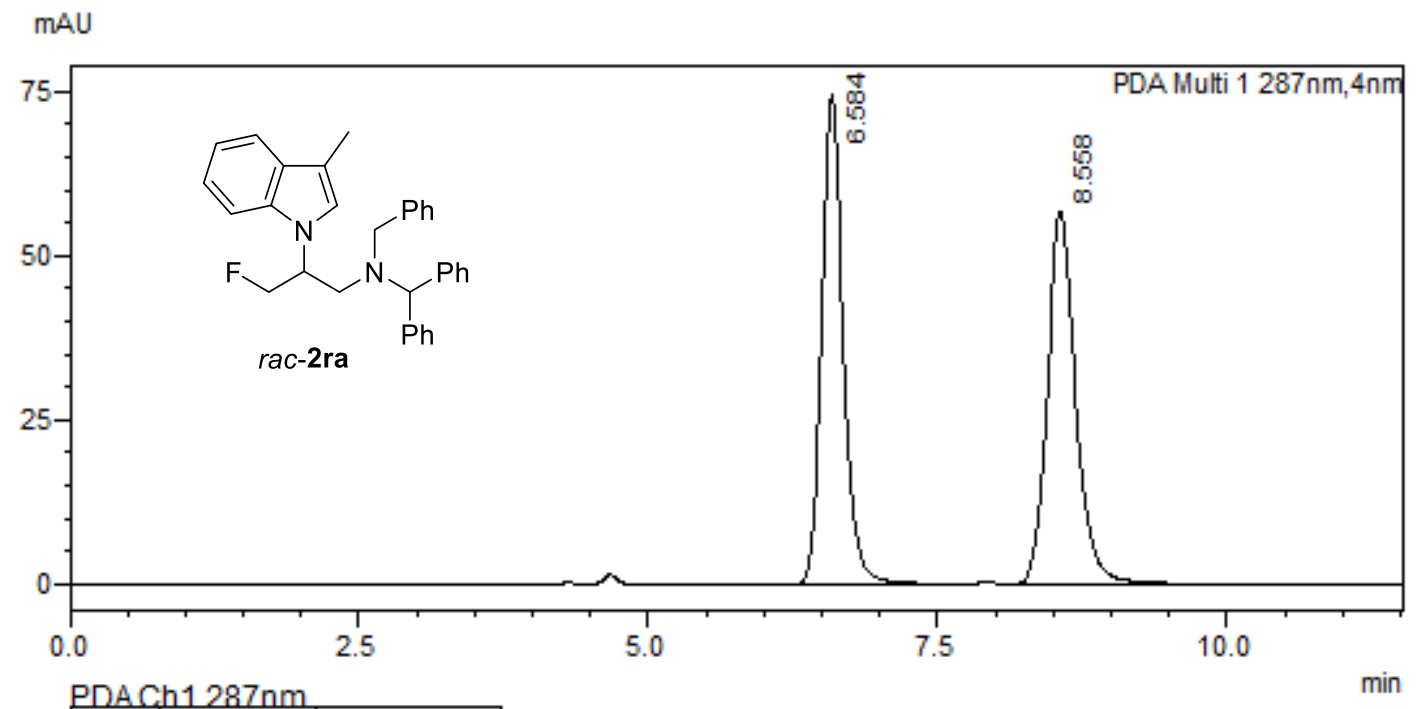

Peakf Ret. Time Area\%

\begin{tabular}{r|r|r|}
\hline 1 & 6.584 & 49.960 \\
\hline 2 & 8.558 & 50.040 \\
\hline
\end{tabular}

mAU

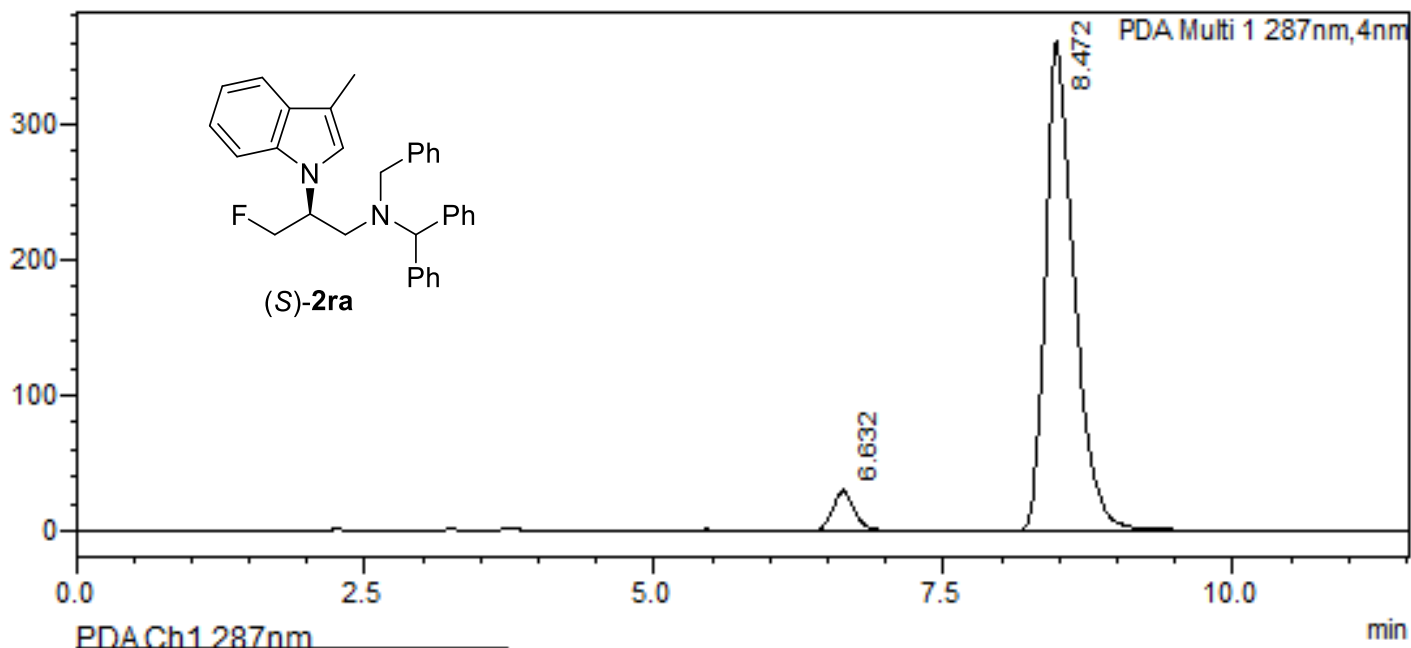

DACh1 $287 \mathrm{~nm}$

\begin{tabular}{|r|r|r|}
\hline 1 & 6.632 & 5.741 \\
\hline 2 & 8.472 & 94.259 \\
\hline
\end{tabular}



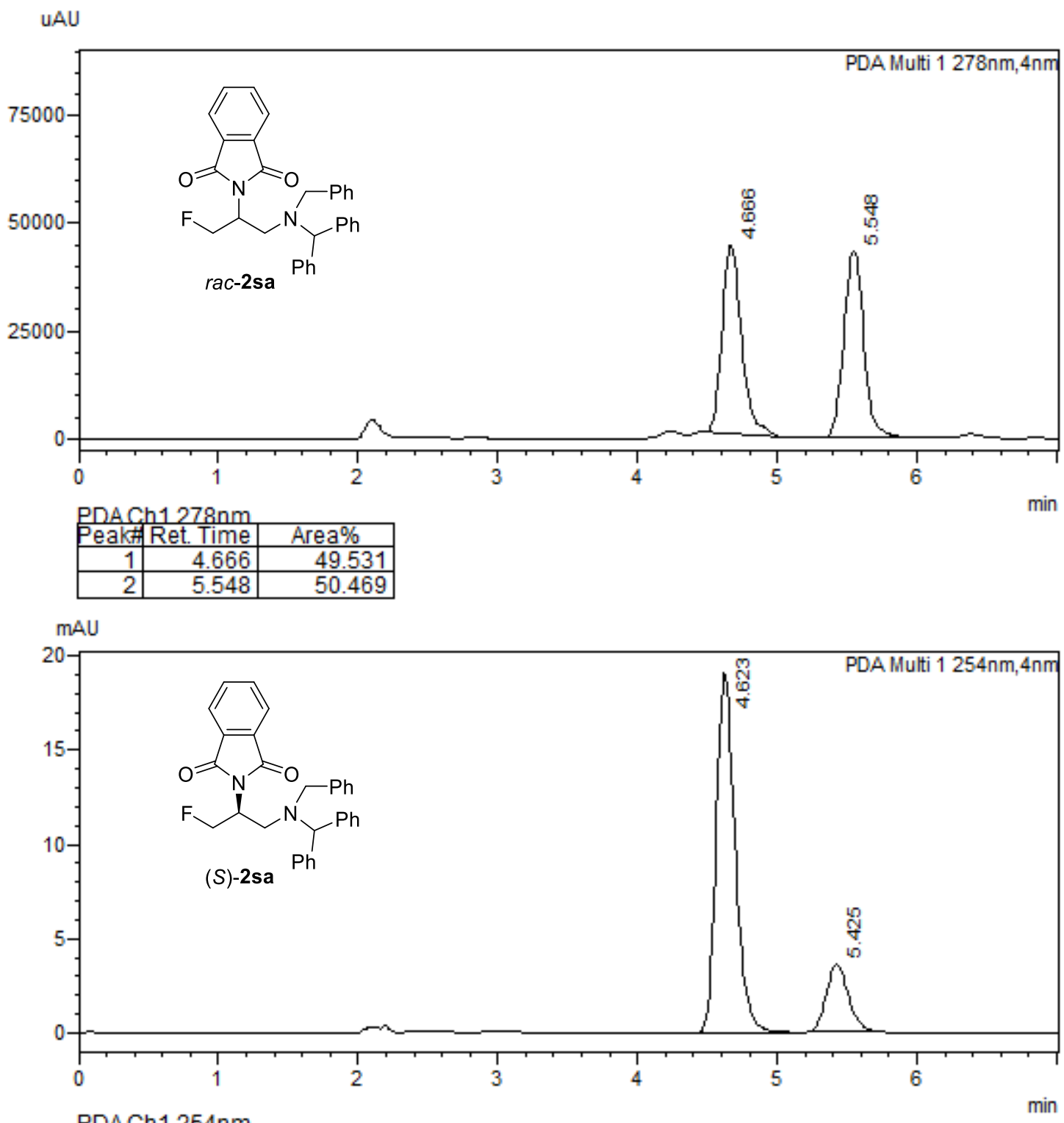

Peakf Ret. Time

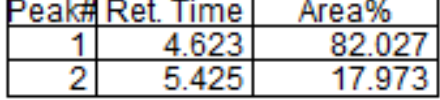

mAU

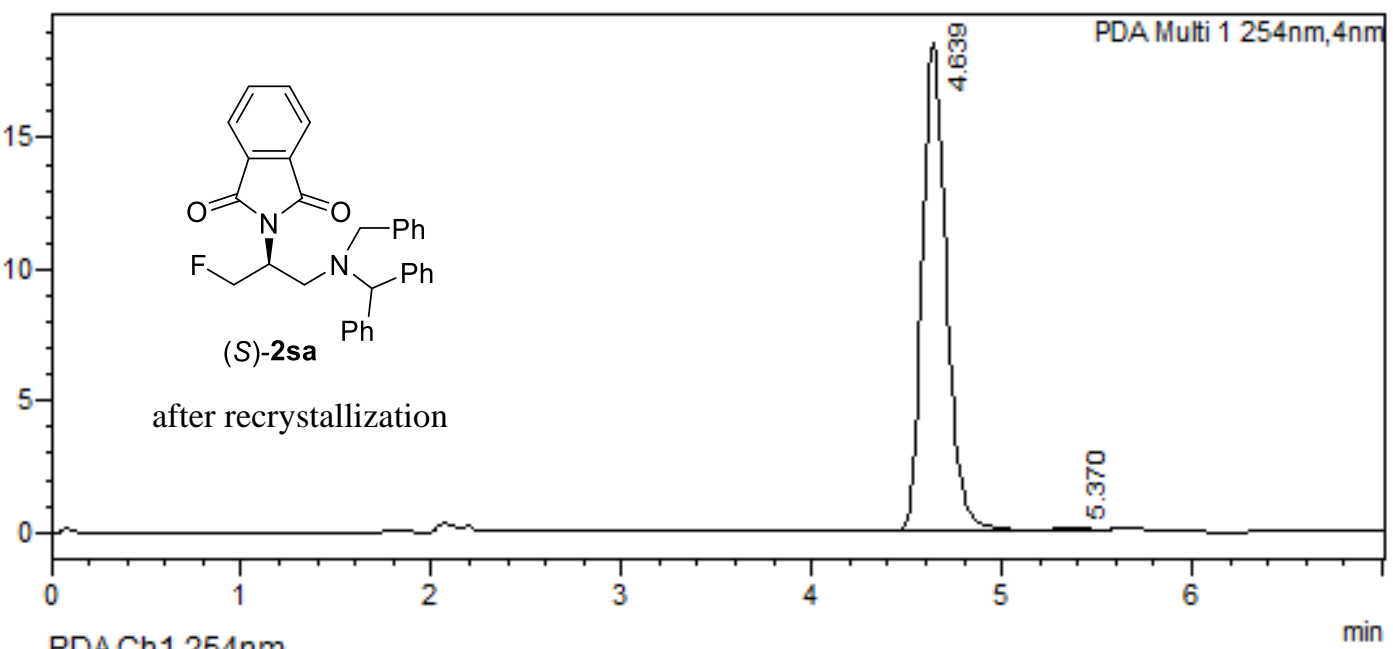

PDACh1254nm

Area $\%$

\begin{tabular}{|c|c|c|}
\hline eakm & $\mathrm{Re}$ & Area $\%$ \\
\hline 1 & 4.639 & 99.645 \\
\hline 2 & 5.370 & \\
\hline
\end{tabular}



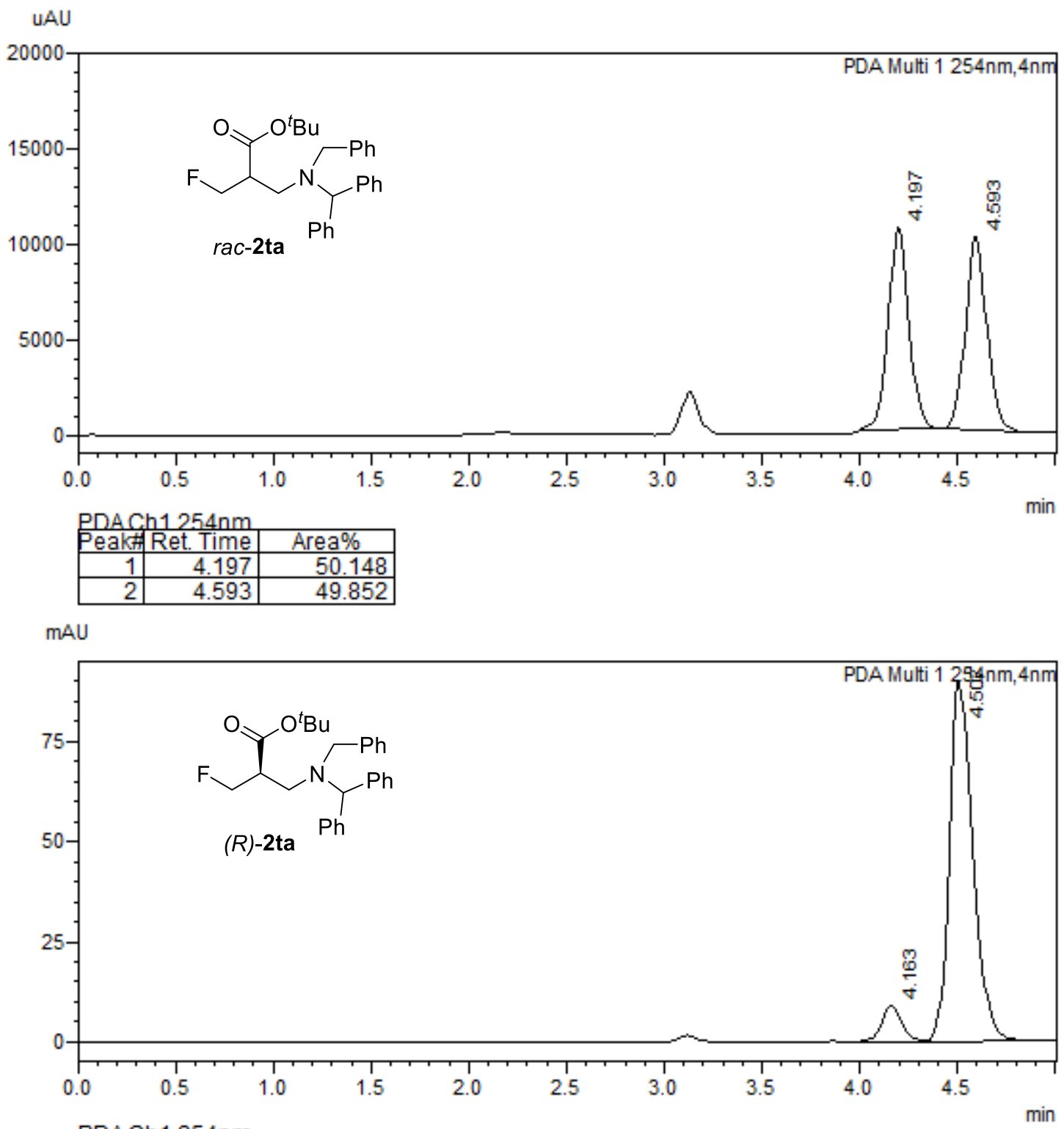

PDACh1254nm

Peak\#Ret. Time Area $\%$

\begin{tabular}{r|r|r|}
\hline 1 & 4.163 & 7.738 \\
\hline 2 & 4.507 & 92.262 \\
\hline
\end{tabular}

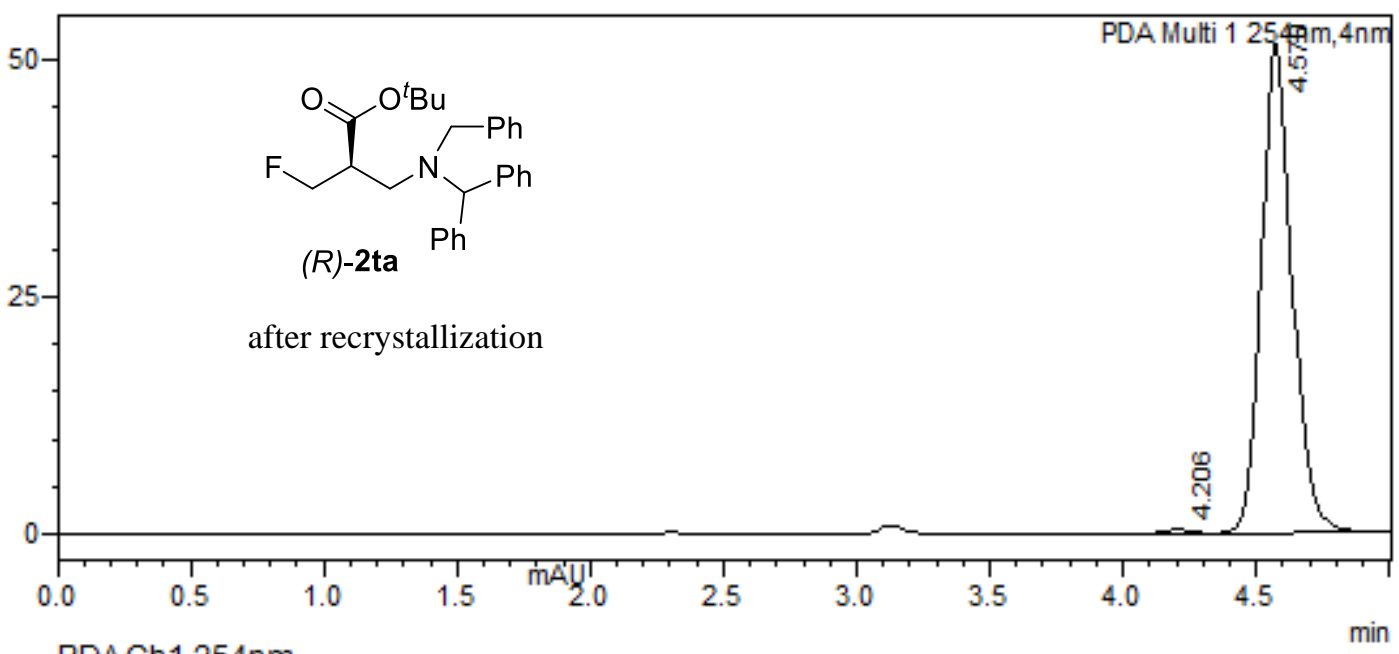

PRACh1 $254 \mathrm{~nm}$

Peak+f Ret. Time Area \%

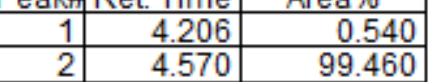




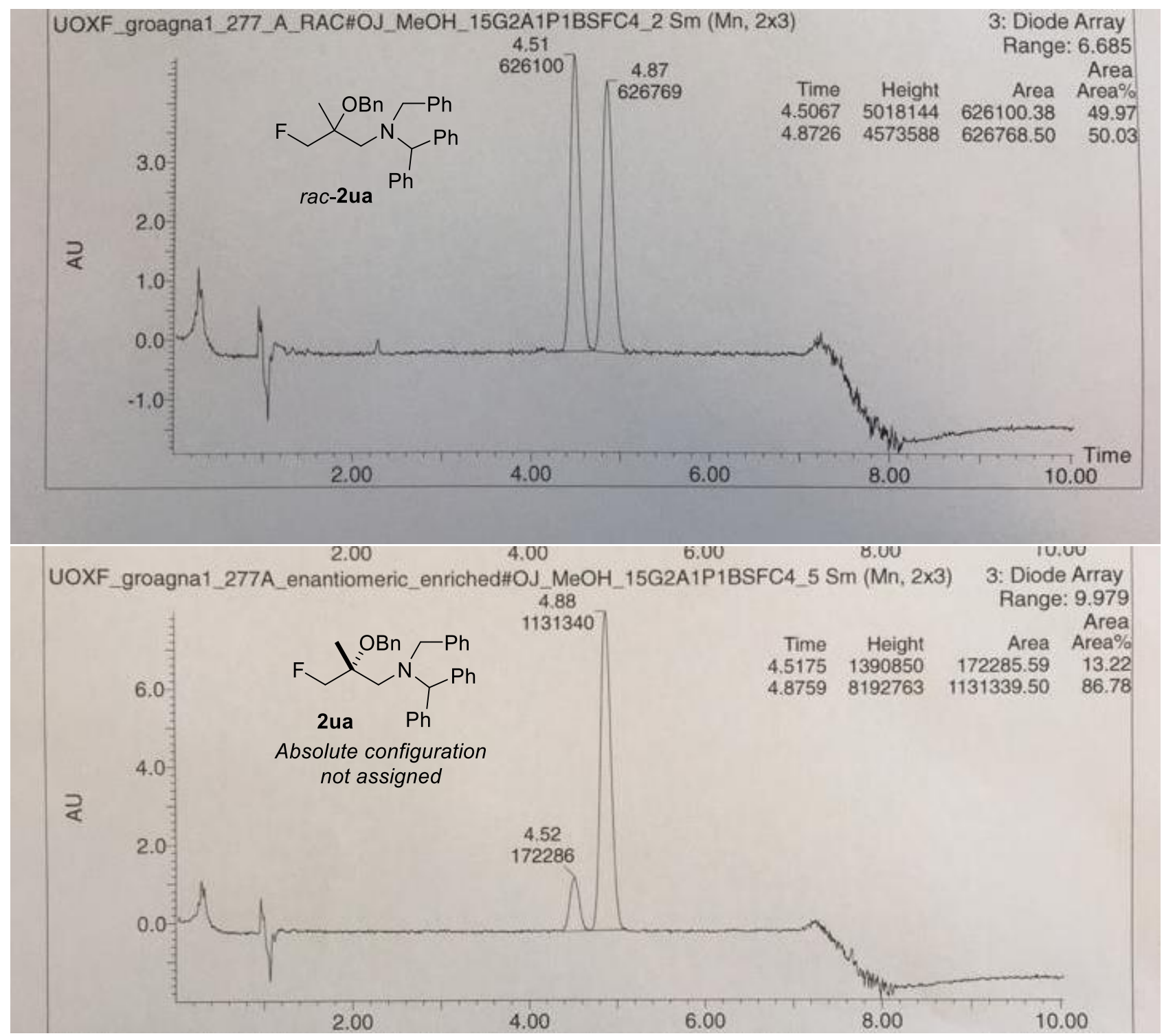



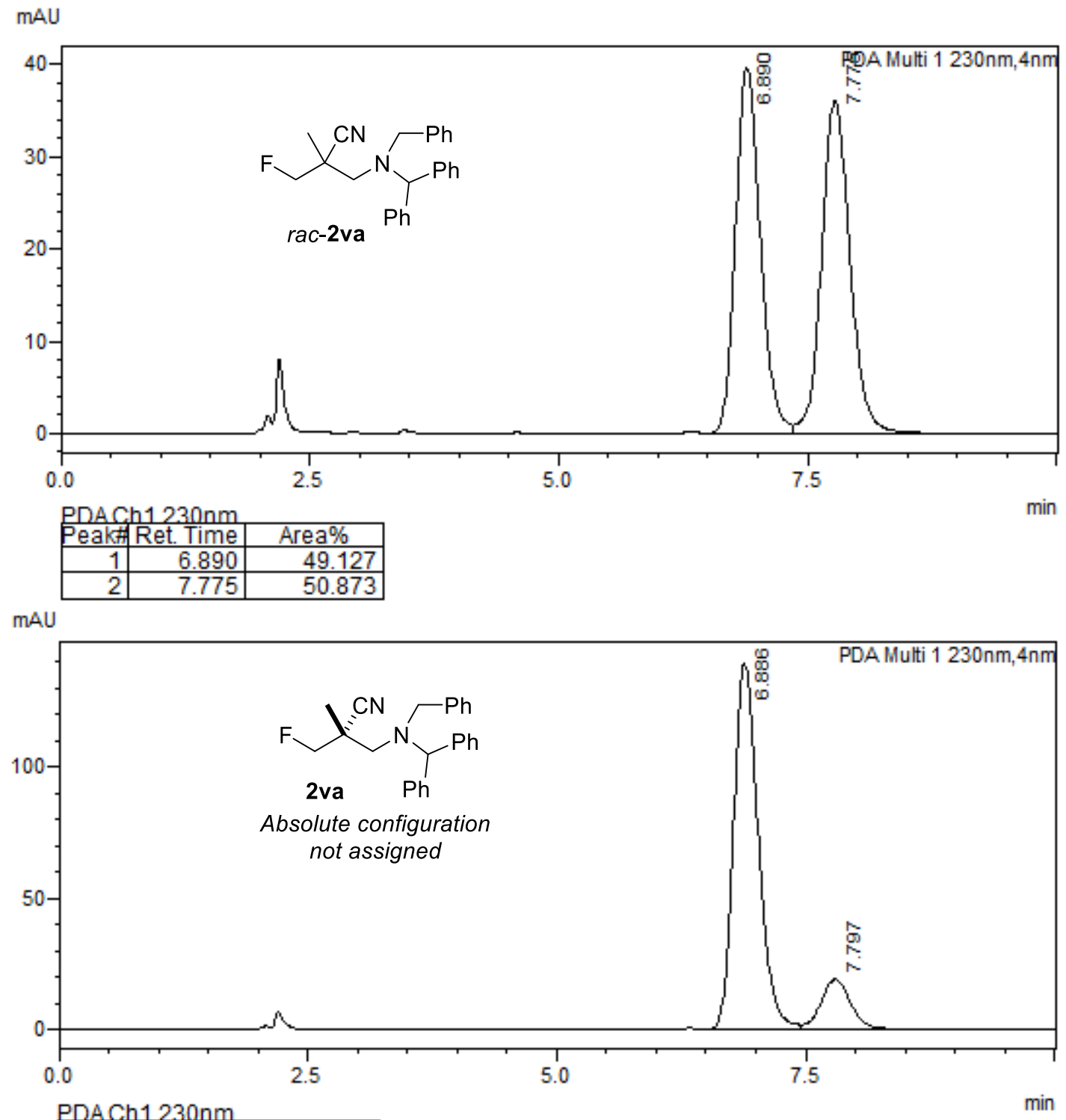

Area \%

86.02

13.974 


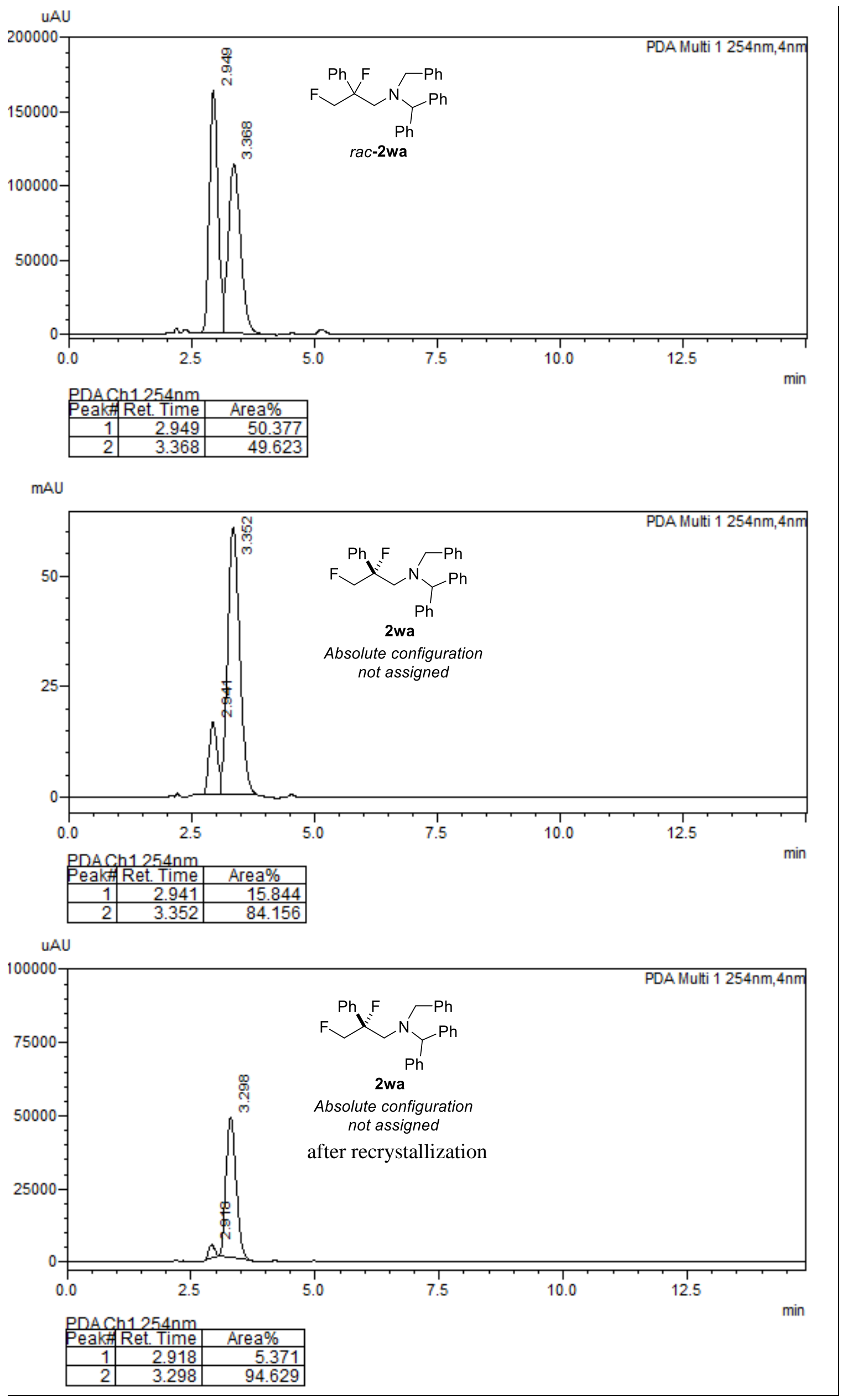




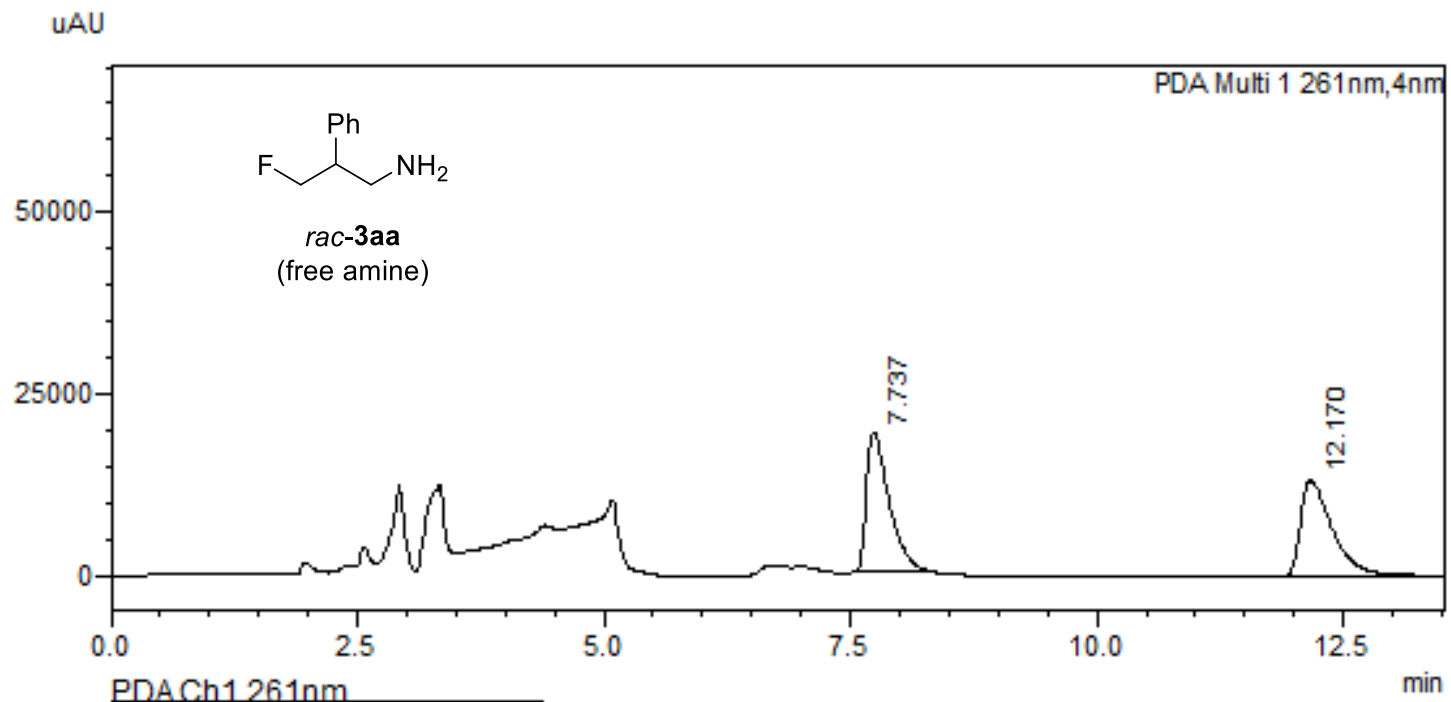

PDACh1261nm

Peakf Ret. Time $\quad$ Area \%

\begin{tabular}{|r|r|r|}
\hline 1 & 7.737 & 51.203 \\
\hline 2 & 12.170 & 48.797 \\
\hline
\end{tabular}

UAL

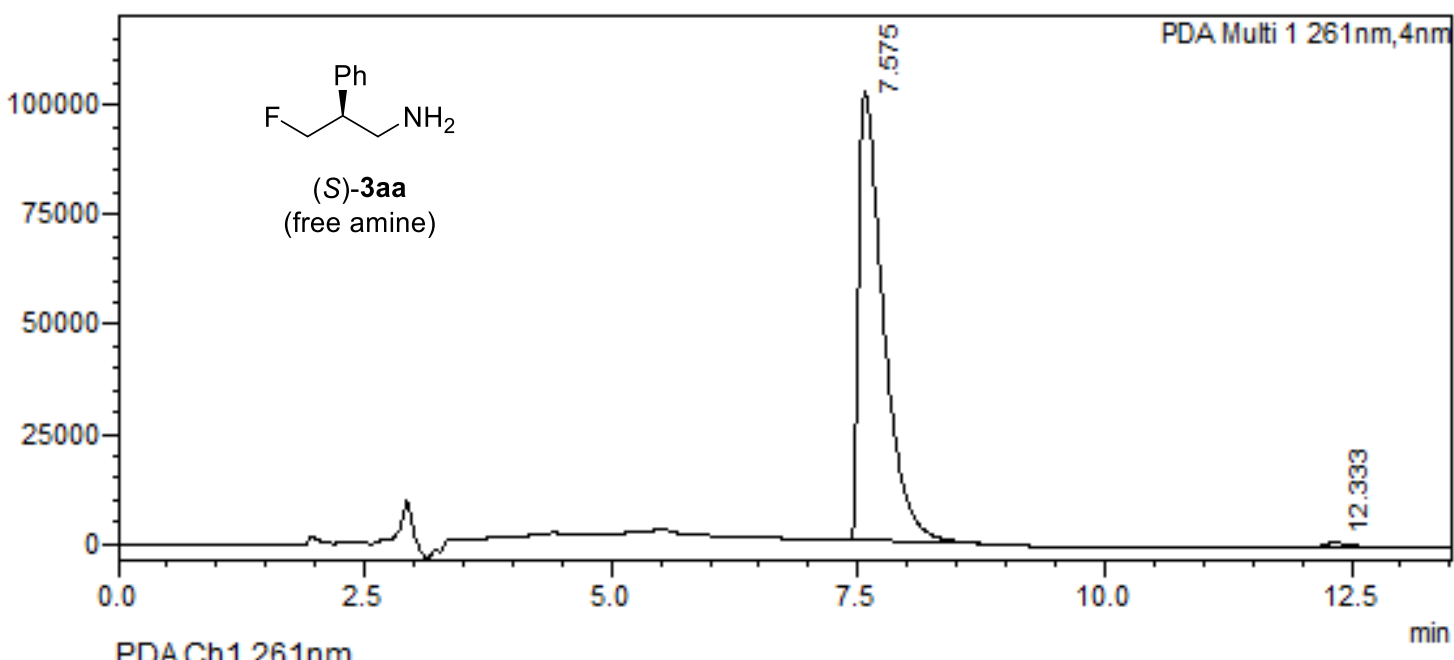

DAG $1261 \mathrm{~nm}$

Peak\# Ret. Time $\quad$ Area \%

\begin{tabular}{r|r|r|}
1 & 7.575 & 99.090 \\
\hline 2 & 12.333
\end{tabular}

0.910 


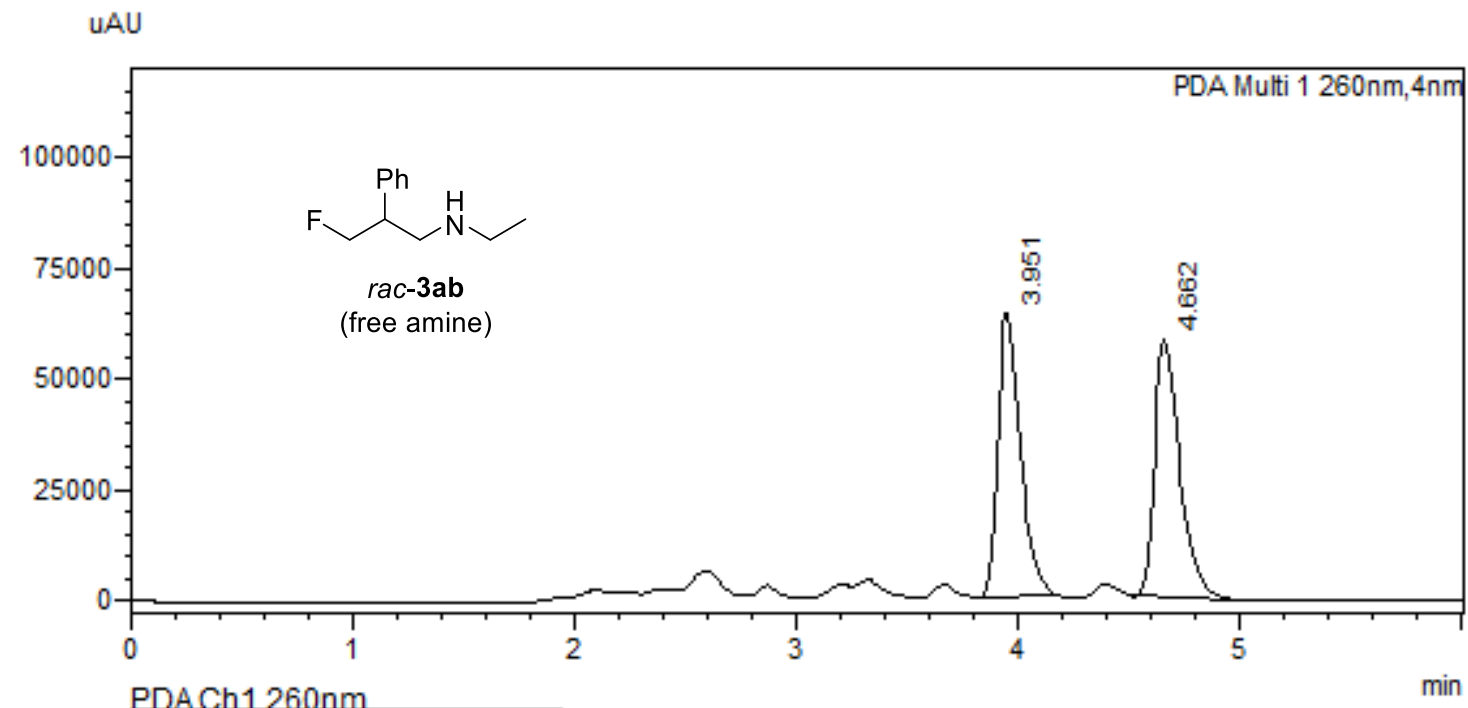

Peakf Ret. Time Area\%

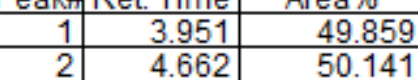

UAU

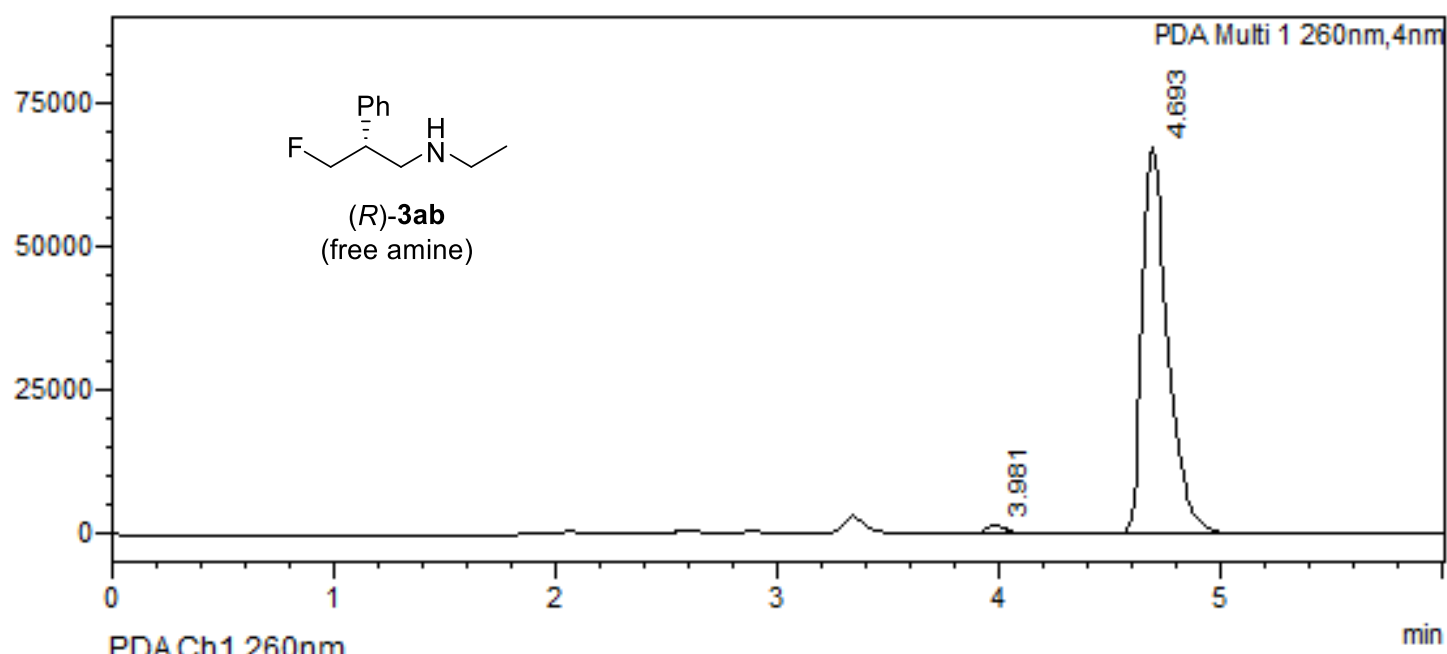

Peak\#Ret. Time $\quad$ Area\%

\begin{tabular}{r|r|r|}
\hline 1 & 3.981 & 1.639 \\
\hline 2 & 4.693 & 98.361 \\
\hline
\end{tabular} 
UOXF_groagna1_72_1\#ID_EtOH_15G1A1P1BSFC2_1

$$
\text { \& } 2
$$

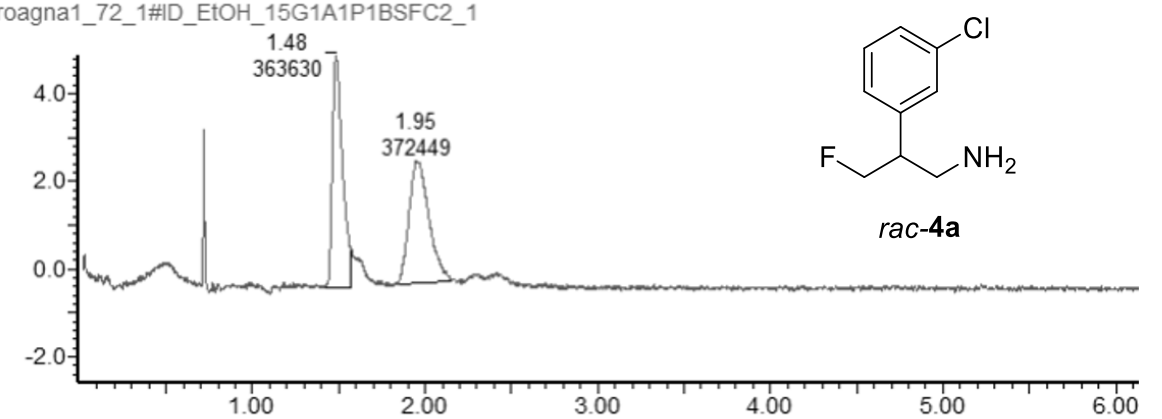

UOXF_groagna1|88_1\#ID_EtOH_15G1A1P1BSFC2_1912_2

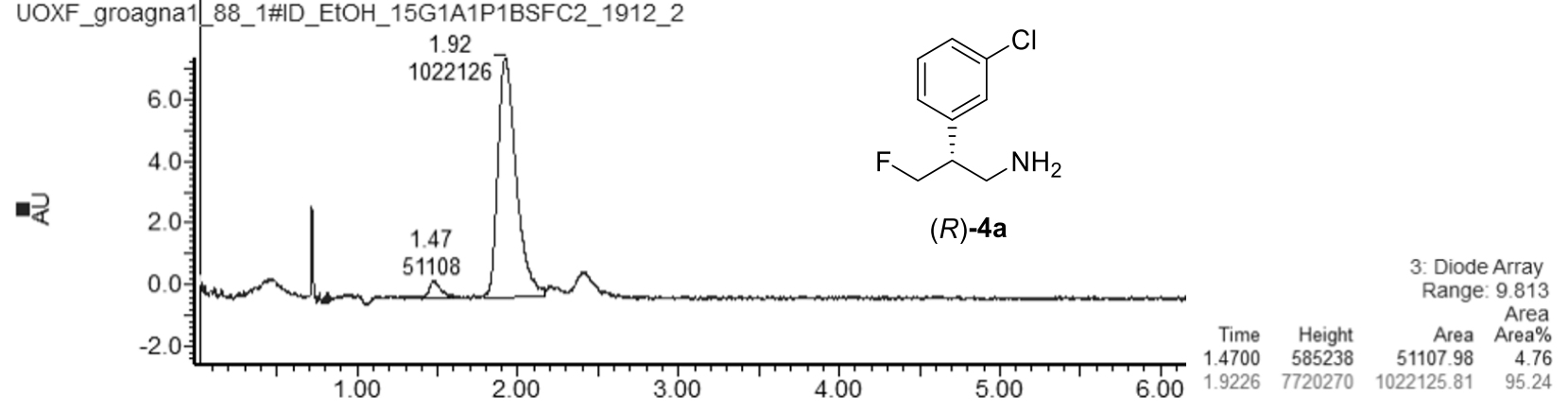

UOXF_groagna1_82_1\#IG_MeOH_15G1A1P1BSFC2
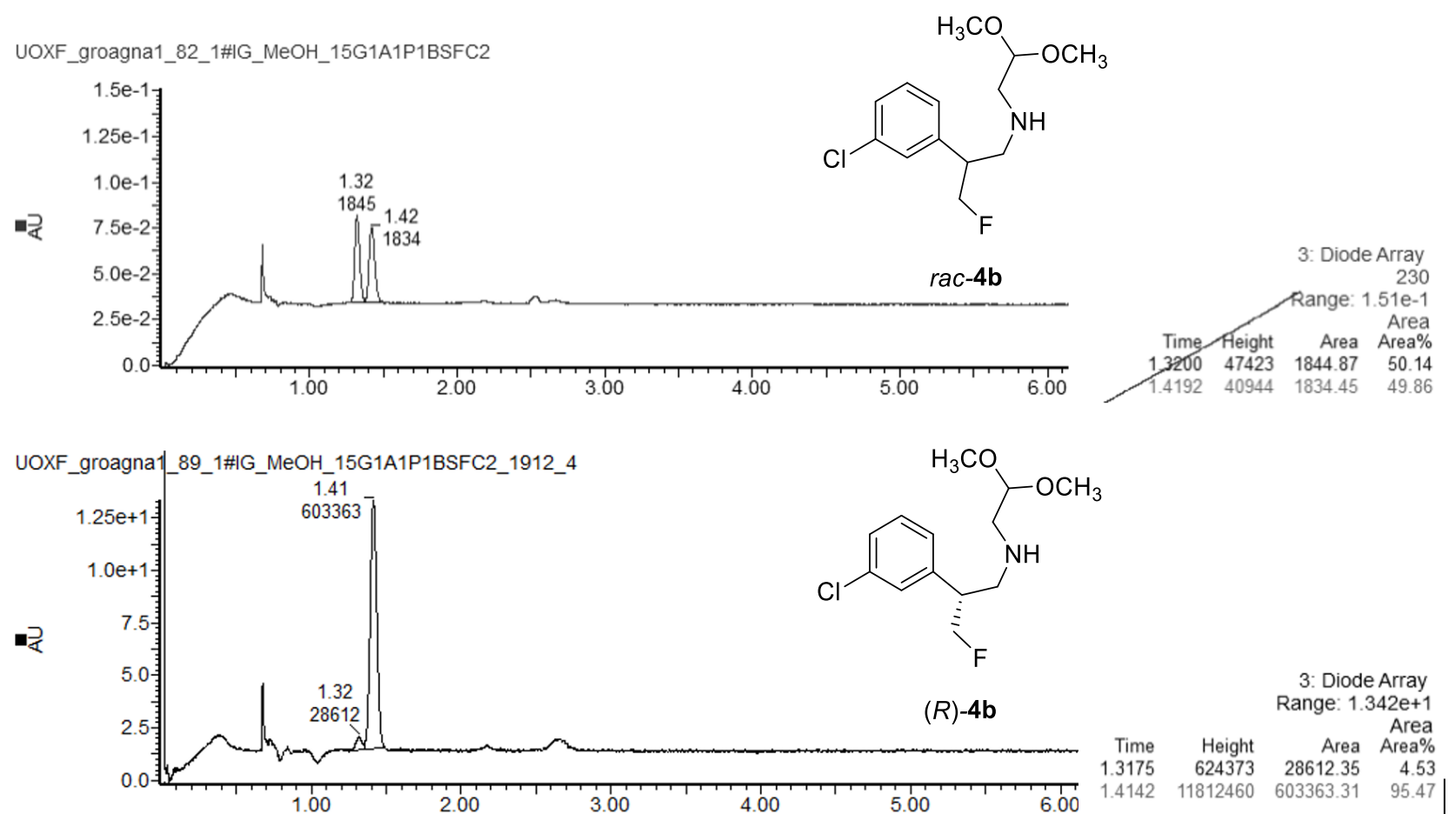
UOXF_groagna1_83_1\#IG_EtOH_20G1A1P1BSFC2_1

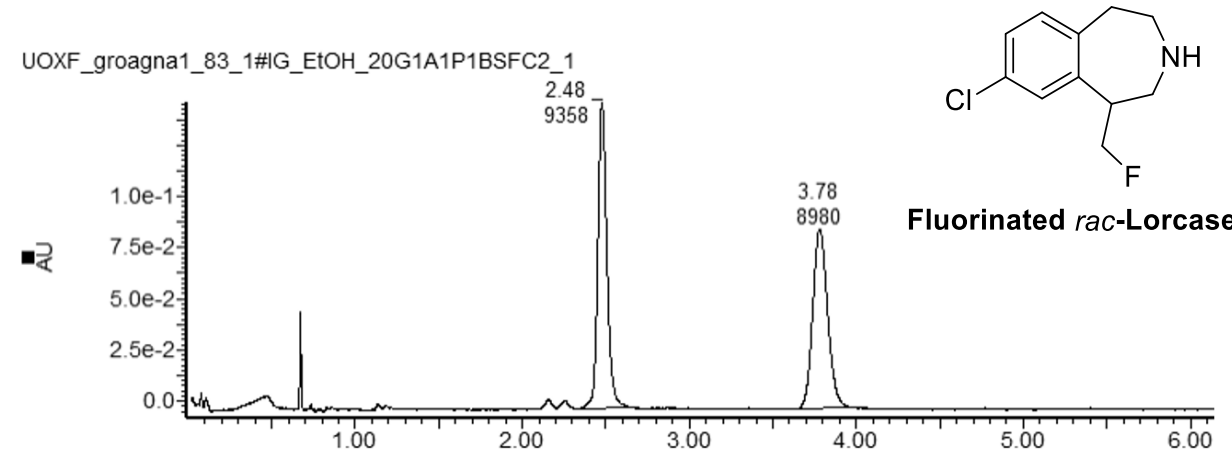

UOXF_groagna T90_1\#IG_EtOH_20G1A1P1BSFC2_1912_2

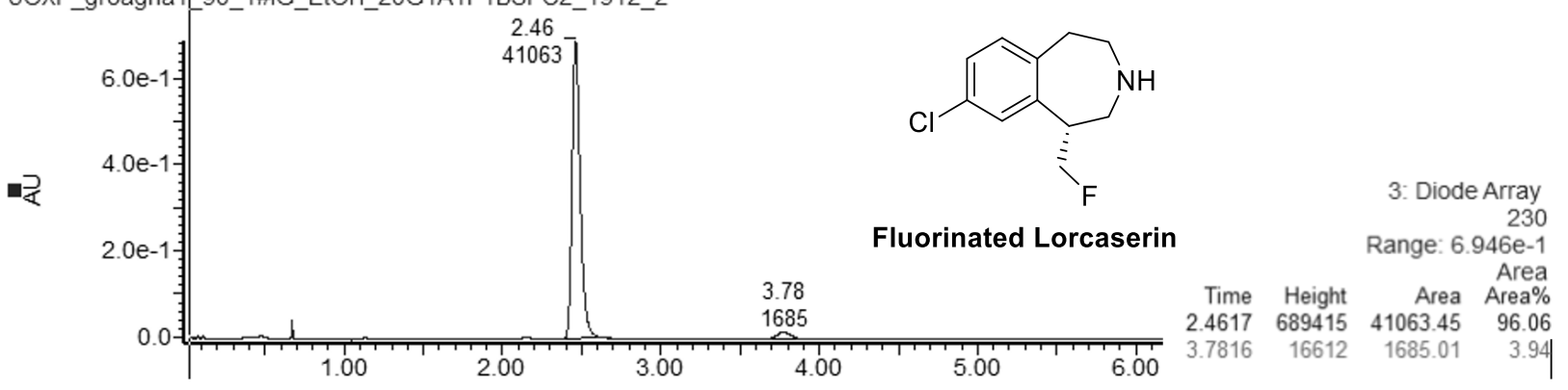




\section{$\underline{\text { References }}$}

1) Pupo, G.; Ibba, F.; Ascough, D. M. H.; Vicini, A. C.; Ricci, P.; Christensen, K.; Pfifer, L.; Morphy, J. R.; Brown, J. M.; Paton, R.; Gouverneur, V. Asymmetric Nucleophilic Fluorination under Hydrogen Bonding Phase-transfer Catalysis. Science 2018, 360, 638.

2) Adams, R. W.; Holroyd, C. M.; Aguilar, J. A.; Nilsson, M.; Morris, G. A. "Perfecting” WATERGATE: Clean Proton NMR Spectra from Aqueous Solution. Chem. Commun. 2013, 49, 358

3) a) Brynn Hibbert, D.; Thordarson, P. The Death of the Job Plot, Transparency, Open Science and Online Tools, Uncertainty Estimation Methods and Other Developments in Supramolecular Chemistry Data Analysis. Chem. Commun. 2016, 52, 12792; b) Thordarson, P. Determining Association Constants from Titration Experiments in Supramolecular Chemistry. Chem. Soc. Rev. 2011, 40, 1305.

4) Brak, K.; Jacobsen, E. N. Asymmetric Ion-Pairing Catalysis. Angew. Chem. Int. Ed. 2013, 52, 534.

5) Qian, D.; Chen. M.; Bissember, A. C.; Sun, J. Counterion-Induced Asymmetric Control in RingOpening of Azetidiniums: Facile Access to Chiral Amines. Angew. Chem. Int. Ed. 2018, 57, 3763.

6) Parmar, D.; Henkel, L. Dib, J.; Rueping, M. Iron Catalysed Cross-couplings of Azetidines Application to the Formal Synthesis of a Pharmacologically Active Molecule. Chem. Comm. 2015, 51,2111

7) Barré, B.; Gonnard, L.; Campagne, R.; Reymond, S.; Marin, J.; Ciapetti, P.; Brellier, M.; Guérinot A.; Cossy, J. Iron- and Cobalt-Catalyzed Arylation of Azetidines, Pyrrolidines, and Piperidines with Grignard Reagents. Org. Lett. 2014, 16, 6160.

8) Dequirez, G.; Bourotte, M.; Porras de Francisco, E.; Remuiñan, M. J.; Déprez, B.; Willand Microwave-Assisted Suzuki-Miyaura Cross Coupling using Nickel as Catalyst to Rapidly Access to 3-Arylazetidine. ChemistrySelect 2017, 2, 8841.

9) Hillier, M. C.; Chen, C. -Y. A One-Pot Preparation of 1,3-Disubstituted Azetidines. J. Org. Chem. 2006, 71, 7885 .

10) Berton, M.; Huck, L.; Alcazar, J. On-demand synthesis of organozinc halides under continuous flow conditions. Nature Protoc. 2018, 13, 324

11) a) Nisato, D.; Frigerio, M. Synthèse de 1-amino-3 azétidine. J. Heterocycl. Chem. 1985, 22, 961; b) Davies, S. G.; Fletcher, A. M.; Frost, A. B.; Roberts, P. M.; Thomson, J. E. Asymmetric Synthesis of Substituted anti- $\beta$-Fluorophenylalanines. Org. Lett. 2015, 17, 2254.

12) Cosier, J.; Glazer. A. M. A nitrogen-gas-stream cryostat for general X-ray diffraction studies. J. Appl. Cryst. 1986, 19, 105.

13) Palatinus, L.; Chapuis, G. "SUPERFLIP - a computer program for the solution of crystal structures by charge flipping in arbitrary dimensions. J. Appl. Cryst. 2007, 40, 786.

14) a) Parois, P.; Cooper, R. I.; Thompson, A. L. Crystal structures of increasingly large molecules: meeting the challenges with CRYSTALS software. Chem. Cent. J. 2015, 9, 30; b) Cooper, R. I.; Thompson, A. L.; Watkin, D. J. CRYSTALS Enhancements: Dealing with Hydrogen Atoms in Refinement. J. Appl. Cryst. 2010, 43, 1100. 
15) Frisch, M. J.; Trucks, G. W.; Schlegel, H. B.; Scuseria, G. E.; Robb, M. A.; Cheeseman, J. R.; Scalmani, G.; Barone, V.; Petersson, G. A.; Nakatsuji, H.; Li, X.; Caricato, M.; Marenich, A.; Bloino, J.; Janesko, B. G.; Gomperts, R.; Mennucci, B.; Hratchian, H. P.; Ortiz, J. V.; Izmaylov, A. F.; Sonnenberg, J. L.; Williams-Young, D.; Ding, F.; Lipparini, F.; Egidi, F.; Goings, J.; Peng, B.; Petrone, A.; Henderson, T.; Ranasinghe, D.; Zakrzewski, V. G.; Gao, J.; Rega, N.; Zheng, G.; Liang, W.; Hada, M.; Ehara, M.; Toyota, K.; Fukuda, R.; Hasegawa, J.; Ishida, M.; Nakajima, T.; Honda, Y.; Kitao, O.; Nakai, H.; Vreven, T.; Throssell, K.; Montgomery Jr., J. A.; Peralta, J. E.; Ogliaro, F.; Bearpark, M.; Heyd, J. J.; Brothers, E.; Kudin, K. N.; Staroverov, V. N.; Keith, T.; Kobayashi, R.; Normand, J.; Raghavachari, K.; Rendell, A.; Burant, J. C.; Iyengar, S. S.; Tomasi, J.; Cossi, M.; Millam, J. M.; Klene, M.; Adamo, C.; Cammi, R.; Ochterski, J. W.; Martin, R. L.; Morokuma, K.; Farkas, O.; Foresman, J. B.; Fox, D. J. Gaussian 16. Gaussian Inc: Wallingford, CT 2016.

16) Zhao, Y.; Truhlar, D. G. The M06 Suite of Density Functionals for Main Group Thermochemistry, Thermochemical Kinetics, Noncovalent Interactions, Excited States, and Transition Elements: Two New Functionals and Systematic Testing of Four M06-Class Functionals and 12 Other Functionals. Theor. Chem. Acc. 2008, 120, 215.

17) Weigend, F.; Ahlrichs, R. Balanced Basis Sets of Split Valence, Triple Zeta Valence and Quadruple Zeta Valence Quality for H to Rn: Design and Assessment of Accuracy. Phys. Chem. Chem. Phys. 2005, 7, 3297.

18) Rappoport, D.; Furche, F. Property-Optimized Gaussian Basis Sets for Molecular Response Calculations. J. Chem. Phys. 2010, 133, 134105.

19) Leininger, T.; Nicklass, A.; Küchle, W.; Stoll, H.; Dolg, M.; Bergner, A. The Accuracy of the Pseudopotential Approximation: Non-Frozen-Core Effects for Spectroscopic Constants of Alkali Fluorides XF (X=K, Rb, Cs). Chem. Phys. Lett. 1996, 255, 274.

20) Wheeler, S. E.; Houk, K. N. Integration Grid Errors for Meta-GGA-Predicted Reaction Energies: Origin of Grid Errors for the M06 Suite of Functionals. J. Chem. Theory Comput. 2010, 6, 395.

21) Barone, V.; Cossi, M. Quantum Calculation of Molecular Energies and Energy Gradients in Solution by a Conductor Solvent Model. J. Phys. Chem. A 1998, 102, 1995.

22) Cossi, M.; Rega, N.; Scalmani, G.; Barone, V. Energies, Structures, and Electronic Properties of Molecules in Solution with the C-PCM Solvation Model. J. Comput. Chem. 2003, 24, 669.

23) Takano, Y.; Houk, K. N. Benchmarking the Conductor-like Polarizable Continuum Model (CPCM) for Aqueous Solvation Free Energies of Neutral and Ionic Organic Molecules. J. Chem. Theory Comput. 2005, 1,70 .

24) Neese, F. The ORCA Program System. Wiley Interdiscip. Rev. Comput. Mol. Sci. 2012, 2, 73.

25) Neese, F. Software Update: The ORCA Program System, Version 4.0. Wiley Interdiscip. Rev. Comput. Mol. Sci. 2018, 8, e1237.

26) Chai, J.-D.; Head-Gordon, M. Long-Range Corrected Hybrid Density Functionals with Damped Atom-Atom Dispersion Corrections. Phys. Chem. Chem. Phys. 2008, 10, 6615. 
27) Lin, Y.-S.; Li, G.-D.; Mao, S.-P.; Chai, J.-D. Long-Range Corrected Hybrid Density Functionals with Improved Dispersion Corrections. J. Chem. Theory Comput. 2013, 9, 263.

28) Grimme, S.; Antony, J.; Ehrlich, S.; Krieg, H. A Consistent and Accurate Ab Initio Parametrization of Density Functional Dispersion Correction (DFT-D) for the 94 Elements H-Pu. J. Chem. Phys. 2010, 132, 154104.

29) Zheng, J.; Xu, X.; Truhlar, D. G. Minimally Augmented Karlsruhe Basis Sets. Theor. Chem. Acc. 2011, 128, 295.

30) Paton Lab; Rodriguez-Guerra, J.; Chen, J. T.; Paton, R. S. Goodvibes 2019 DOI: 10.5281/zenodo.595246.

31) Grimme, S. Supramolecular Binding Thermodynamics by Dispersion-Corrected Density Functional Theory. Chem. Eur. J. 2012, 18, 9955.

32) Grimme, S.; Bannwarth, C.; Dohm, S.; Hansen, A.; Pisarek, J.; Pracht, P.; Seibert, J.; Neese, F. Fully Automated Quantum-Chemistry-Based Computation of Spin-Spin-Coupled Nuclear Magnetic Resonance Spectra. Angew. Chem. Int. Ed. 2017, 56, 14763.

33) Jorgensen, W. L.; Tirado-Rives, J. The OPLS Potential Functions for Proteins. Energy Minimizations for Crystals of Cyclic Peptides and Crambin. J. Am. Chem. Soc. 1988, 110, 1657.

34) Jorgensen, W. L.; Maxwell, D. S.; Tirado-Rives, J. Development and Testing of the OPLS All-Atom Force Field on Conformational Energetics and Properties of Organic Liquids. J. Am. Chem. Soc. 1996, $118,11225$.

35) Bayly, C. I.; Cieplak, P.; Cornell, W. D.; Kollman, P. A. A Well-Behaved Electrostatic Potential Based Method Using Charge Restraints for Deriving Atomic Charges: The RESP Model. J. Phys. Chem. 1993, 97, 10269 .

36) Comell, W. D.; Cieplak, P.; Bayly, C. I.; Kollman, P. A. Application of RESP Charges To Calculate Conformational Energies, Hydrogen Bond Energies, and Free Energies of Solvation. J. Am. Chem. Soc. 1993, 115, 9620.

37) Schrodinger LLC. Schrodinger Release 2017-2. MS Jaguar: New York, NY 2017.

38) Case, D. A.; Cerutti, D. S.; Cheatham III, , T. E.; Darden, T.A.; Duke, R. E.; Giese, T. J.; Gohlke, H.; Goetz, A. W.; Greene, D.; Homeyer, N.; Izadi, S.; Kovalenko, A.; Lee, T.S.; LeGrand, S.; Li, P.; Lin, C.; Liu, J.; Luchko, T.; Luo, R.; Mermelstein, D.; Merz, K.M.; Monard, G. Nguyen, H.; Omelyan, I.; Onufriev, A.; Pan, F.; Qi, R.; Roe, D. R.; Roitberg, A.; Sagui, C.; Simmerling, C. L.; Botello-Smith W. M.; Swails, J. Walker, R. C.; Wang, J.; Wolf, R. M.; Wu, X.; Xiao, L.; York, D. M.; Kollman, P. A.; AMBER 2017. University of California: San Francisco 2017.

39) van der Spoel, D.; van Maaren, P. J.; Caleman, C. GROMACS Molecule \& Liquid Database. Bioinformatics 2012, 28, 752.

40) Chen, S.; Yi, S.; Gao, W.; Zuo, C.; Hu, Z. Force Field Development for Organic Molecules: Modifying Dihedral and 1-n Pair Interaction Parameters. J. Comput. Chem. 2015, 36, 376. 
41) Bussi, G.; Donadio, D.; Parrinello, M. Canonical Sampling through Velocity Rescaling. J. Chem. Phys. 2007, 126, 014101.

42) Parrinello, M.; Rahman, A. Crystal Structure and Pair Potentials: A Molecular Dynamics Study. Phys. Rev. Lett. 1980, 45, 1196.

43) Parrinello, M.; Rahman, A. Polymorphic Transitions in Single Crystals: A New Molecular Dynamics Method. J. Appl. Phys. 1981, 52, 7182.

44) Hess, B.; Bekker, H.; Berendsen, H. J. C.; Fraaije, J. G. E. M. LINCS: A Linear Constraint Solver for Molecular Simulations. J. Comput. Chem. 1997, 18, 1463.

45) Daura, X.; Gademann, K.; Jaun, B.; Seebach, D.; van Gunsteren, W. F.; Mark, A. E. Peptide Folding: When Simulation Meets Experiment. Angew. Chem. Int. Ed. 1999, 38, 236.

46) Kozuch, S.; Shaik, S. A Combined Kinetic-Quantum Mechanical Model for Assessment of Catalytic Cycles: Application to Cross-Coupling and Heck Reactions. J. Am. Chem. Soc. 2006, 128, 3355.

47) Kozuch, S.; Shaik, S. Kinetic-Quantum Chemical Model for Catalytic Cycles: The Haber-Bosch Process and the Effect of Reagent Concentration. J. Phys. Chem. A 2008, 112, 6032.

48) Kozuch, S.; Lee, S. E.; Shaik, S. Theoretical Analysis of the Catalytic Cycle of a Nickel CrossCoupling Process: Application of the Energetic Span Model. Organometallics 2009, 28, 1303.

49) Kozuch, S.; Shaik, S. How to Conceptualize Catalytic Cycles? The Energetic Span Model. Acc. Chem. Res. 2011, 44, 101

50) Kozuch, S. A Refinement of Everyday Thinking: The Energetic Span Model for Kinetic Assessment of Catalytic Cycles. WIREs Comput. Mol .Sci. 2012, 2, 795.

51) Bickelhaupt, F. M.; Houk, K. N. Analyzing Reaction Rates with the Distortion/Interaction-Activation Strain Model. Angew. Chem. Int. Ed. 2017, 56, 10070. 